\title{
The Hydrostatic Mooring System
}

\section{RECEIVED \\ DEC 042000 \\ OSTI}

Final Technical Report

Main Report + Appendices A, B, \& C

Volume 1 of 2

Jens Korsgaard

Jens Korsgaard

Date Published August 8, 2000

Prepared for the United States

Department of Energy

Under Cooperative Agreement

No. DE-FG36-98G010321 


\section{DISCLAIMER}

This report was prepared as an account of work sponsored by an agency of the United States Government. Neither the United States Government nor any agency thereof, nor any of their employees, make any warranty, express or implied, or assumes any legal liability or responsibility for the accuracy, completeness, or usefulness of any information, apparatus, product, or process disclosed, or represents that its use would not infringe privately owned rights. Reference herein to any specific commercial product, process, or service by trade name, trademark, manufacturer, or otherwise does not necessarily constitute or imply its endorsement, recommendation, or favoring by the United States Government or any agency thereof. The views and opinions of authors expressed herein do not necessarily state or reflect those of the United States Government or any agency thereof. 


\section{DISCLAIMER}

Portions of this document may be illegible in electronic image products. Images are produced from the best available original document. 
Jens Korsgaard

318 North Post Road

Princeton Jct. NJ 08550

Phone: 609-936-9568

Fax: 609-936-9373

August 8, 2000

Ms. Roxanne Danz

Project Officer

US Dept. of Energy

Golden Field Office

1617 Cole Blvd.

Golden CO 80401-3393

Reference: Contract DE-FG36-98G010321

The Hydrostatic Mooring

Dear Ms. Danz:

Please find enclosed the final technical report in the captioned matter. I wish to express gratitude for having been granted the grant, which enabled this work. The work carried out under this contract will go a long way toward making the Hydrostatic Mooring a commercial reality.

I also wish to complement everyone in the Department of Energy with whom I have had the fortune of coming into contact. It has been a true pleasure to work with your staff. I have found everyone truly competent and helpful, which is particularly important for an individual like myself.

Sincerely yours

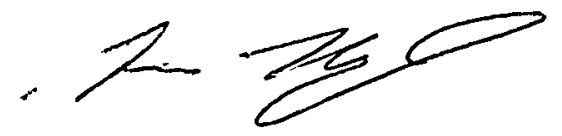

Jens Korsgaard 


\section{EXECUTIVE SUMMARY}

This document reports the work carried out by Jens Korsgaard under the U.S. Department of Energy (DOE) Innovation and Energy Program Contract No. DE-FG36-98G010321. The work was carried out in the period October 10, 1998, to August 10, 2000. The object of the contract was proving the extraordinary performance of the Hydrostatic Mooring.

The Hydrostatic Mooring is a new type of Single Point Mooring, which moors oil tankers and similar large ships in the open ocean. There is a number of existing Single Point Moorings. All other Single Point Moorings have a mechanical coupling between the vessel and the mooring. This may be a gripping mechanism or an ordinary mooring hawser. Common for all Single Point Moorings including the Hydrostatic Mooring, is that they permit the ship to weathervane $360^{\circ}$ about the mooring, thereby enabling it to assume a position that minimizes the forces from the winds, waves and currents.

All other Single Point Moorings have limitations on mooring operations due to weather, which limits the maximum sea state to between 2 and $4.5 \mathrm{~m}$ significant wave height. In cases with particularly favorable current conditions the operations limits may be somewhat higher for the other moorings. These limitations cause typical weather related downtimes between $5 \%$ and $30 \%$ in the open ocean. The Hydrostatic Mooring holds out the promise of being able to moor in $8 \mathrm{~m}$ significant wave height, thereby virtually eliminating weather downtime.

The mechanism that moors the Hydrostatic Mooring to the vessel is the suction cup principle, in which the mooring buoy itself is a giant suction cup and the vessel provides the power to reduce the pressure between the buoy and the vessel. The resulting compressive force between the vessel and the buoy is very large and the friction that results from this compressive force is the one that actually restrains the vessel. 
All other Single Point Moorings have retrieval lines, which are retrieved by the vessel and hauled in, in order to effect the mooring. The Hydrostatic Mooring is brought into contact with the ship by variable buoyancy. This is remotely actuated from the ship when it maneuvers above the buoy at its resting position, $8 \mathrm{~m}$ below the keel. Consequently, there is no guiding force that will guide the mooring into its proper position. The ship must therefore be carefully navigated to a position above the mooring buoy and the proper time selected for actuating the variable buoyancy system aboard the buoy. The principal objective of this contract was to prove or disprove that this operation is feasible and reliable. A number of real time tests using the true real time performance of a tanker were carried out at Nichols Advanced Marine Sciences in Arlington, Virginia. A 150,000 DWT oil tanker was used for the tests. The oil tanker used had the minimum maneuvering characteristics allowed by the classification societies. Consequently, any real tanker would have the same or better maneuvering characteristics than the tanker used in the tests. The tanker was equipped with the standard equipment that is required for the use with the Hydrostatic Mooring, which includes an omni-directional bow thruster, in this case with a $200 \mathrm{kN}$ thrust force.

Twenty-five tests were carried out in weather conditions ranging from flat calm to very stormy conditions with a significant wave height of $8 \mathrm{~m}$. Unsteady winds and currents were also present in most of the tests. The mooring was successful in $100 \%$ of the twenty-five tests made. In $20 \%$ of the tests, the final location of the mooring buoy on the bottom of the ship would not permit the connection of the fluid transfer equipment. Consequently, in those cases, the mooring attempt would have to be repeated. It was found, however, in the tests, that the navigator continued to learn throughout the test period. In the final sixteen tests, which were made in the most adverse weather conditions, there was only one instance in which the buoy was not placed with the proper accuracy on the bottom of the ship. In the first 9 tests in more benign conditions there were 4 such instances. This buoy has, in its standard configuration, sufficient power aboard the buoy to perform eight mooring attempts. It was therefore concluded that the 
mooring between a large oil tanker and the Hydrostatic Mooring could be carried out with extreme reliability in all wave conditions up to $8 \mathrm{~m}$ significant wave height.

The second objective of the project was to gain approval by the American Bureau of Shipping for the buoy concept and design. This entails carrying out preliminary design work, which was done by the author of this report and by Han-Padron Associates Consulting Engineers in New York through a subcontract. The design package was submitted to the ABS in two stages. The first package was submitted in April 2000, consisting of all the tests that were made at Nichols Advanced Marine Sciences and prior tests made at the Danish Maritime Institute in Denmark. The second submittal was on July 7, 2000 containing all the design work carried out in connection with this project.

As of the date of the publication of this report, the American Bureau of Shipping (ABS) had not completed their review of the submitted material and $A B S$ has requested some supplemental calculations that will shortly be furnished to the ABS. 


\section{THE HYDROSTATIC MOORING SYSTEM}

\section{TABLE OF CONTENTS}

Page No.

TRANSMITTAL LETTER

EXECUTIVE SUMMARY

LIST OF FIGURES

vi

LIST OF TABLES

vii

1. INTRODUCTION

$1-1$

2. OVERVIEW OF THE HYDROSTATIC MOORING SYSTEM

2.1 CONCEPT

$2-1$

2.2 APPLICATION EXAMPLE

2-4

2.2.1 Met-Ocean Conditions Ashkelon

$2-4$

2.2.2 Mooring Design Conditions

$2-5$

2.2.3 Cargo Transfer

2-7

2.3 PETROLEUM CARGO TRANSFER

$2-8$

3. MANEUVERING TESTS

4. PRELIMINARY DESIGN

4.1 SEALING SYSTEM BETWEEN THE HULL AND THE BUOY

4.2 BUOYANCY CONTROL SYSTEM

4.3 MAIN BEARING

4.4 STRUCTURAL DESIGN

4.5 FAILURE TREE ANALYSIS

5. AMERICAN BUREAU OF SHIPPING APPROVAL

6. CONCLUSIONS 


\section{TABLE OF CONTENTS (Cont'd)}

APPENDICES:

A - MANEUVERING TEST REPORT BY NICHOLS

B - REPORT ON APPLICATION TO THE LIVERPOOL BAY PROJECT

C - MAIN REPORT FROM PHYSICAL MODEL TESTS AT THE DANISH MARITIME INSTITUTE

D - SUBMITTAL TO THE AMERICAN BUREAU OF SHIPPING

F - CORRESPONDENCE WITH THE AMERICAN BUREAU OF SHIPPING

G - EVALUATION OF THE MOORING BY THE NAVIGATOR, CAPTAIN JAMES ATKINSON

H - OFFSHORE TECHNOLOGY CONFERENCE PAPER NUMBER 7711

I-HYDROSTATIC MOORING BROCHURE 


\section{LIST OF FIGURES}

Page No.

FIG 2-1 HYDROSTATIC MOORING PRINCIPLE 2-1

FIG 2-2 HYDROSTATIC MORING BUOY CROSS-SECTION 2-3

FIG 2-3 FRESH WATER TRANSFER SYSTEM 2-7

FIG 2-4 PETROLEUM CARGO TRANSFER FLUID PATHS 2-9

FIG 2-5 PETROLEUM TRANSFER SYSTEM 2-10

GENERAL ARRANGEMENT

FIG 4-1 START OF RAPID ASCENT 4- 4

FIG 4- 2 RAPID ASCENT 4-5

FIG 4- 3 MAXIMUM BUOYANCY 4-6 


\section{LIST OF TABLES}

Page No.

TABLE 3-1 TEST CONDITIONS

3-4

TABLE 3-2 TESTS RESULTS

$3-6$ 


\section{INTRODUCTION}

This report is the technical report from a contract between the Department of Energy and Jens Korsgaard contract number DE-FG36-98G010321.

The principal objective of the contract is to develop the Hydrostatic Mooring further. The project had two main components:

- To prove in a maneuvering simulator that a seafarer could in fact navigate the vessel with the required precision to mate to the Hydrostatic Mooring.

- To obtain the approval of the American Bureau of Shipping (ABS) for the mooring.

The ship maneuvering study was carried out at Nichols Advanced Marine, in Arlington, Virginia, in 1999.

In order to gain the ABS approval of the design, the existing design had to be developed further. This was done partly through a contract with Han-Padron Associates (HPA) in New York, and was partly done by the Author.

At the date of publication of this report, the ABS had not yet completed their review of the design.

The system of units used in this document is the SI system. 


\section{OVERVIEW OF THE HYDROSTATIC MOORING SYSTEM}

\subsection{CONCEPT}

The Hydrostatic Mooring is a single point mooring of the type that attaches to the keel of the vessel. Existing similar concepts comprise the Submerged Turret Loading (STL) provided by Advanced Product Loading (Norway) and the Turret-Collet type buoy moorings provided by Single Buoy Moorings (Monaco) and SOFEC (USA). These existing systems rely on structural connectors of the hydraulic clamp type to effect the structural connection between the buoy and the vessel. The STL has the structural swivel built into the buoy whereas the turret-collet type buoy mooring has the structural swivel built into the vessel.

The Hydrostatic Mooring has the structural swivel built into the buoy, but in contrast to the other submerged systems relies on a combination of compressive and friction forces between the hull of the vessel and the buoy to transmit the mooring forces from the buoy to the vessel. The Hydrostatic Mooring is shaped like a giant suction cup and reducing the hydrostatic pressure in the volume between the buoy and the hull creates a large compressive force between the buoy and the vessel hull. The mooring is attached to the ship's hull as shown on Fig 2-1 below.

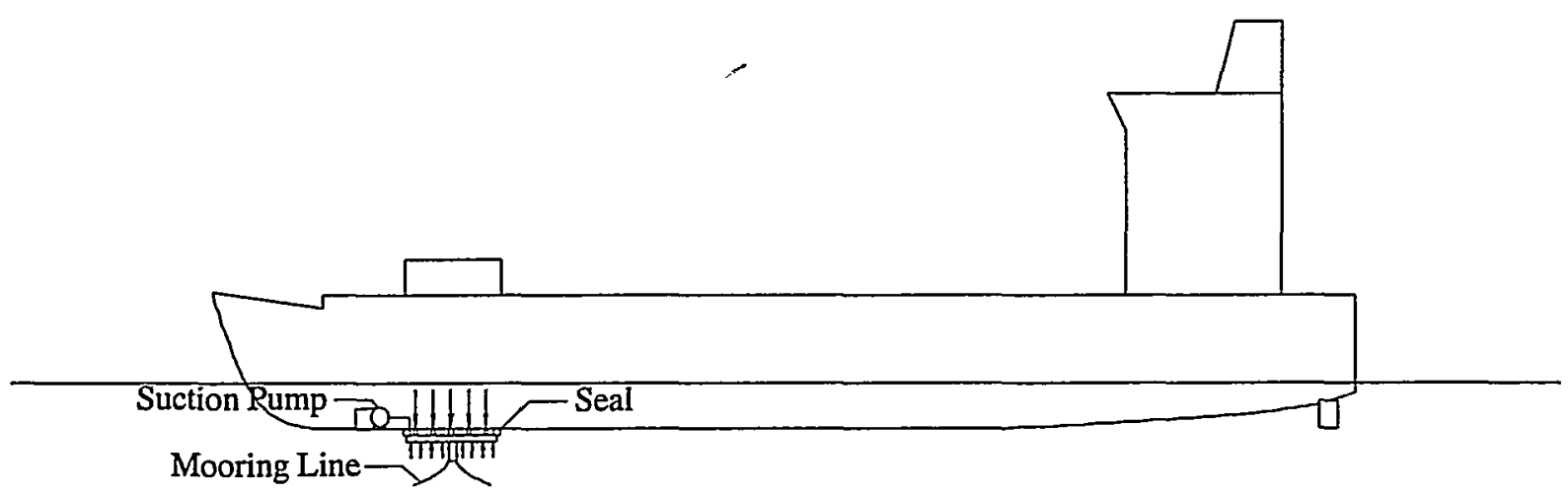

Fig 2-1 Hydrostatic Mooring Principle 
The volume between the ship's hull and the mooring is isolated from the sea by the circular seal shown on Fig 2-1. The suction pump takes suction within this volume and lowers the absolute pressure to for example $50 \mathrm{kPa}$ within the volume. The exterior pressure is at least atmospheric (100 $\mathrm{kPa}$ ) thus the disk is pressed upward as indicated by the upward arrows. This upward force is resisted by the compression elements within the volume. The downward pointing arrows illustrate the compressive forces. These compressive forces resist any vertical mooring forces. There is friction between the hull and the compression elements. This friction provides the means to resist horizontal forces between the mooring buoy and the hull. The mooring lines shown in Fig 2-1 provide the means to transmit the mooring forces to the seabed.

The potential of this mooring system is illustrated by the following numerical example:

Ships draft in the mooring area: 5 meters

Sealing ring diameter

Mooring pressure:

External pressure:

Friction coefficient:
20 meters

$50 \mathrm{kPa}$ (absolute)

$150 \mathrm{kPa}$ (absolute)

$20 \%$

Mooring area calculated:

Compressive force calculated: $\quad 314^{*}(150-50)=31400 \mathrm{kN}$

Max. horizontal force calculated: $20 \% * 31400=6280 \mathrm{kN}$

The maximum horizontal force in this example is comparable to a normal design force for a Floating Production, Storage, and Offloading vessel (FPSO) in an extreme ocean environment. Several avenues are available to increase the force capability of the above sample mooring. The sealing ring diameter can in most cases be doubled, the friction coefficient can be increased by proper materials selection and finally ballasting operations can increase the pressure differential. In this manner this mooring can resist even 
extreme ice forces. Consequently, the Hydrostatic Mooring has the capability of mooring all large ships in the Arctic or ocean environment.

A more detailed view of the Mooring concept is shown in Fig 2-2 below:

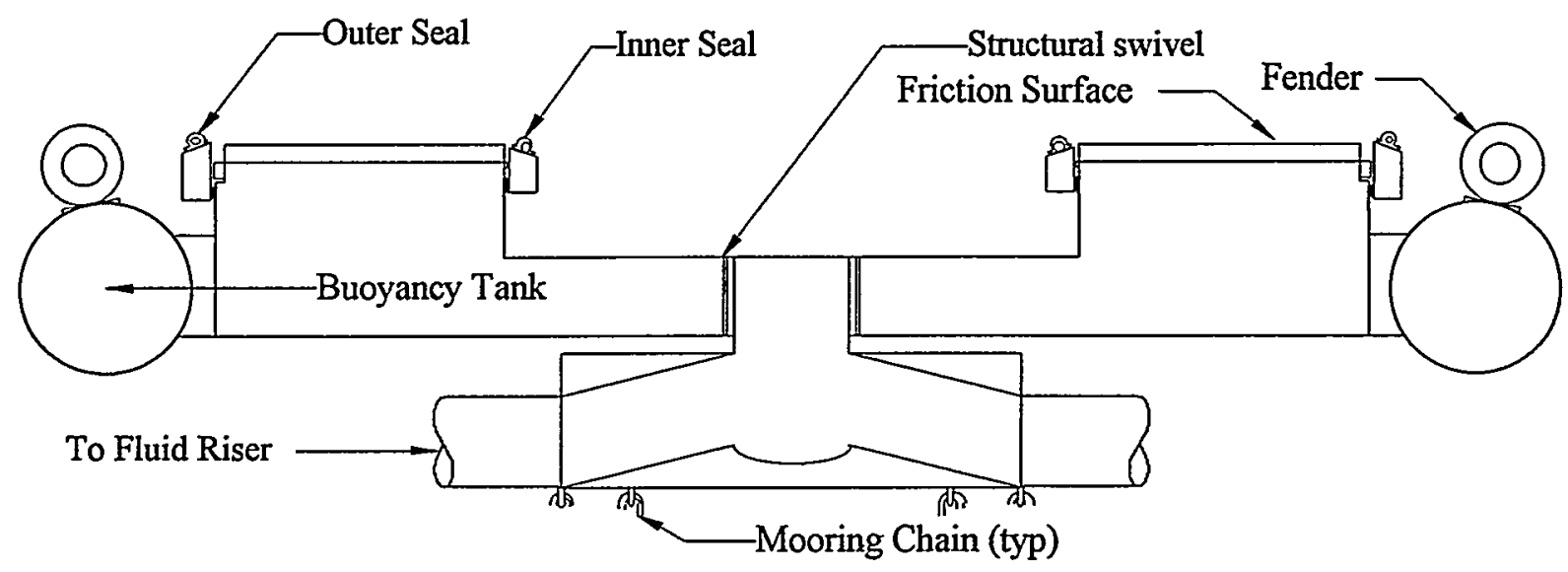

Fig 2-2 Hydrostatic Mooring Buoy Cross-Section

This more detailed view shows two annular seals (outer and inner seals) between which the hydrostatic pressure is lowered. This reduces the compressive force somewhat, but then permits the maintenance of for example atmospheric pressure in the center area of the buoy. The fluid connection is made within the structural swivel area. The fluid swivel is on the vessel. This is similar to the STL arrangement. Also note the fenders that prevent damaging impacts between the buoy and the vessel. 


\subsection{APPLICATION EXAMPLE}

The application example is for a transport system in which water is transported from Manavgat in Turkey to Ashkelon in Israel. The vessels used are either VLCC (approximately 250,000 DWT) or ULCC (approximately 450,000 DWT).

The following describes only the Ashkelon mooring. The mooring in Turkey is similar. A buoy that has an outer sealing ring diameter of $21 \mathrm{~m}$ is chosen for either class of vessel. The vessel must have flat bottom plating in the mooring area with a width on the order of $28 \mathrm{~m}$ or more for this to work effectively and safely. The width of either class of vessel is more than $40 \mathrm{~m}$, therefore, this is not a problem. Tests show that the mooring operation can easily be performed with a precision of 2 meters in conditions with wave heights of $\mathrm{Hs}=5 \mathrm{~m}$ or below regardless of cross currents and wind. Mooring is consistently possible on the first attempt in all wave heights up to $\mathrm{Hs}=8$ meters regardless of wind and cross current conditions. The accuracy deteriorates to a typical error of 3 meters at 8 meter wave heights. Mooring operations are probably feasible in even higher wave heights, but this may be of theoretical interest only, because operations on the vessel will be curtailed in any event at these extreme conditions.

\subsubsection{Met-Ocean Conditions Ashkelon}

The environmental condition in Ashkelon and in Manavgat is described by the following:

- $\mathrm{Hs}=2.0$ meters

Exceeded 50 days per year

- $\mathrm{Hs}=5.0$ meters

Exceeded 3.8 days per year

- $\mathrm{Hs}=6.0$ meters

Exceeded 0.6 days per year

- $\mathrm{Hs}=8.0$ meters

Return Period 50 years 
The current at Ashkelon is reported to be usually north going with a speed of 0.5 knots. For design purposes at this time a design current of 1.5 knots is assumed.

The wind speed is generally quite low at Ashkelon and the maximum recorded wind speed is 35 knots.

\subsubsection{Mooring Design Conditions}

A system designed for a maximum wave height of $\mathrm{Hs}=5.0 \mathrm{~m}$ will have $99 \%$ availability and weather delays is not a concern. The required precision for mooring can be set to be $2.0 \mathrm{~m}$ in accordance with the results from the maneuvering tests performed. In the actual design a 3.0 meter tolerance will be provided.

For purposes of this document the mooring is designed for a maximum horizontal mooring force of $6000 \mathrm{kN}$.

The buoy is secured to the ship by differential hydrostatic pressure. The present buoy is designed for water transport. It has two sealing rings, one at $10.5 \mathrm{~m}$ radius and another at 4 meter radius. The pressure in the area between the two sealing rings is typically maintained at $50 \mathrm{kPa}$ (absolute). This is done through pumps aboard the moored vessel that take suction within the space between the two sealing rings. The vessels to be moored by this buoy must have a minimum width of flat bottom plate of 28 meters $(60,000 \mathrm{DWT})$. There is no upper limit on ship size other than the limit on force caused by the combination of ship size and environmental conditions and potential water depth limitations.

The submergence of the hull may vary between 4 meters and 25 meters depending on the ship size and the loading condition. This corresponds to absolute hydrostatic pressures at the keel between $140 \mathrm{kPa}$ and $290 \mathrm{kPa}$. The pressure within the sealing area is $50 \mathrm{kPa}$. Assuming a 4 meter submergence the differential hydrostatic pressure is $90 \mathrm{kPa}$. The area 
is $\square\left(10.5^{*} 10.5-4^{*} 4\right)=295 \mathrm{~m} 2$. The resulting compressive force between the buoy and the vessel is then $27000 \mathrm{kN}$. The net buoyancy of the combined buoy/mooring system must be added. This is normally negative but close to zero and is ignored. Consequently, a friction coefficient of 0.3 between the keel and the buoy permits a horizontal force of $8100 \mathrm{kN}$ at a draft of 4 meters. At larger drafts the possible horizontal force increases to as much as $18000 \mathrm{kN}$. In consequence the vessel can control the friction capacity of the buoy and may in principle avoid dangerous combinations of force capability versus possible mooring force by increasing its draft.

The friction coefficient between the ship's bottom and the buoy is believed to be in the $50 \%$ to $80 \%$ range. Standard ablating anti-fouling paint coats the ship's bottom. The surfaces on the buoy that touch the ship are primarily composed of rubber fender elements. The rubber in these fender elements is specially formulated to have a large friction coefficient relative to wet anti-fouling paint.

The friction coefficient that is assumed for computational purposes is ordinarily in the $10 \%$ to $30 \%$ range, thereby providing an adequate safety factor against mooring failure. It should be noted that it is possible to determine the actual friction coefficient during unmooring by recording the combination of mooring force and pressure at the time of initiation of the separation process, i.e., when the buoy starts moving on the bottom of the vessel. Therefore, it is possible to mónitor the efficacy of the system by measuring the buoy loading. condition at initiation of motion upon separation at unmooring. This measurement may be done at each unmooring thereby providing for each ship that uses the mooring a continuous record of the friction coefficient between the buoy and the vessel.

The vessels arriving in Ashkelon are loaded and will discharge their cargo. For purposes of this document it is assumed that the maximum draft of the arriving vessel is 25 meters. The maximum wave condition for approach and mooring is set to $\mathrm{Hs}=5$ meters in this case. A safety margin 
between the vessel and the buoy of $7.0 \mathrm{~m}$ is set. The buoy is 8 meters high requiring a minimum water depth in the mooring area of $25+7+8=40 \mathrm{~m}$.

The water depth in the chosen mooring area is only about $37 \mathrm{~m}$. Therefore, in order to provide space for the buoy a circular area with a diameter of $100 \mathrm{~m}$ will be dredged to a depth of $44 \mathrm{~m}$. The additional $4 \mathrm{~m}$ over depth allows for sedimentation for several years until maintenance dredging is required.

\subsubsection{Cargo Transfer}

The cargo to be transferred in the case at hand is fresh water and therefore unique in the sense that it is nonpolluting and normally can be discharged freely to the sea. This makes it possible to use simpler designs in the cargo transfer system with fewer safeguards against leakage and spills compared to petroleum transfer systems. Later in this section a normal petroleum or gas transfer system will be described.

The proposed water transfer system is shown in Fig 2-3.

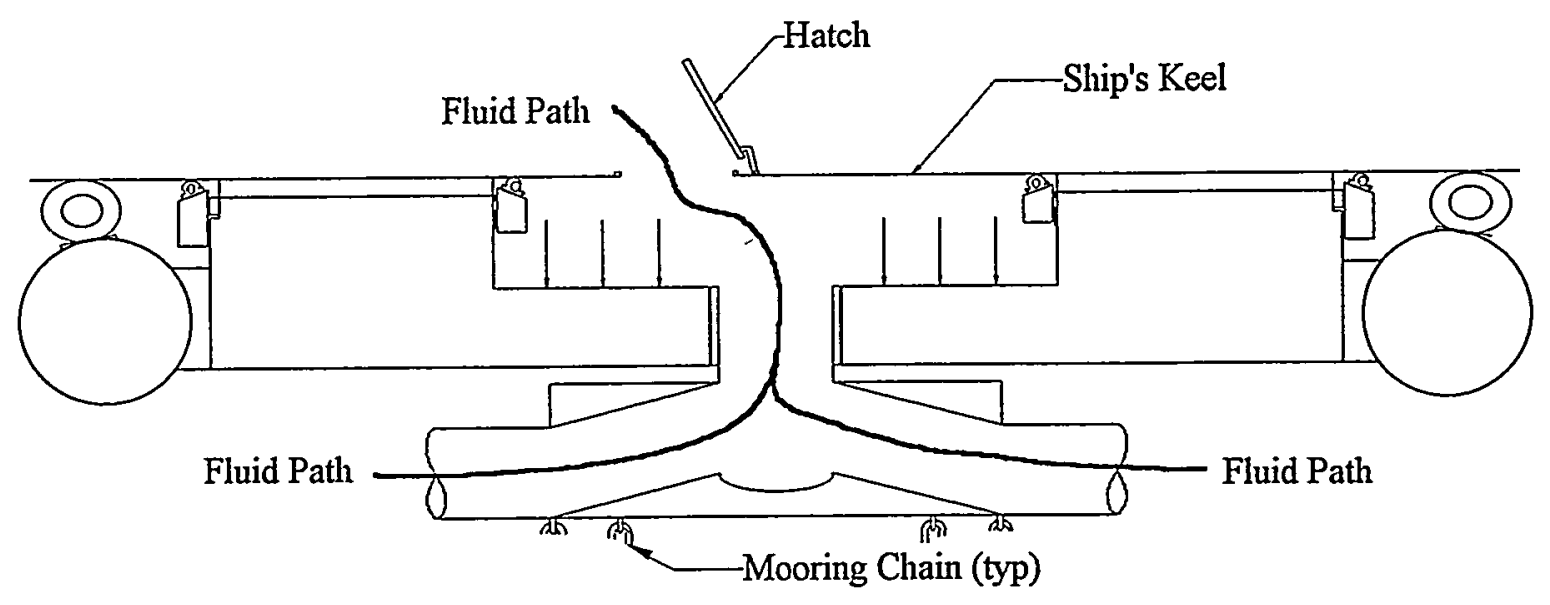

Fig 2-3 Fresh Water Transfer System 
ship. One disadvantage of the arrangement shown in Fig 2-3 is the downward pressure indicated by the arrows in the center of the buoy. This pressure counteracts the suction that presses the buoy onto the ship's keel. However, it acts on a much smaller area than the suction. As an example consider that the suction acts between two sealing circles with diameters 21 $\mathrm{m}$ and $8 \mathrm{~m}$ respectively. Assume that the differential hydrostatic pressure is $90 \mathrm{kPa}$, then the compressive force is $27000 \mathrm{kN}$ as previously calculated. Assume further that the differential hydrostatic pressure in the center of the buoy is $+200 \mathrm{kPa}$, then the repulsive force in the center of the buoy is $\pi^{*} 4^{*} 4^{*} 200=10000 \mathrm{kN}$ resulting in a minimum net attractive force between the buoy and the vessel of $17000 \mathrm{kN}$. The latter only occurs at the end of the discharge process or in the beginning of the loading process when the vessel is ballasted. At any other time the compressive force and thereby the mooring capacity is larger.

The vessel monitors the mooring force at all times. Monitoring the mooring buoy position through the GPS positioning system does this. The known mooring response curve then provides the force. Therefore, the vessel can continuously monitor dangerous combinations of center well pressure and mooring capacity. In the event that such dangerous combination is approached then the vessel may take action by reducing the center well pressure. This would in turn reduce the cargo transfer rate.

\subsection{PETROLEUM CARGO TRANSFER}

A more common use of the buoy is for the transfer of oil and/or gas between the vessel and the riser. In this case the pressure in the center well of the buoy would normally be maintained at close to atmospheric pressure. In this case the center of the buoy would provide additional compressive force and thereby assist in the mooring. 


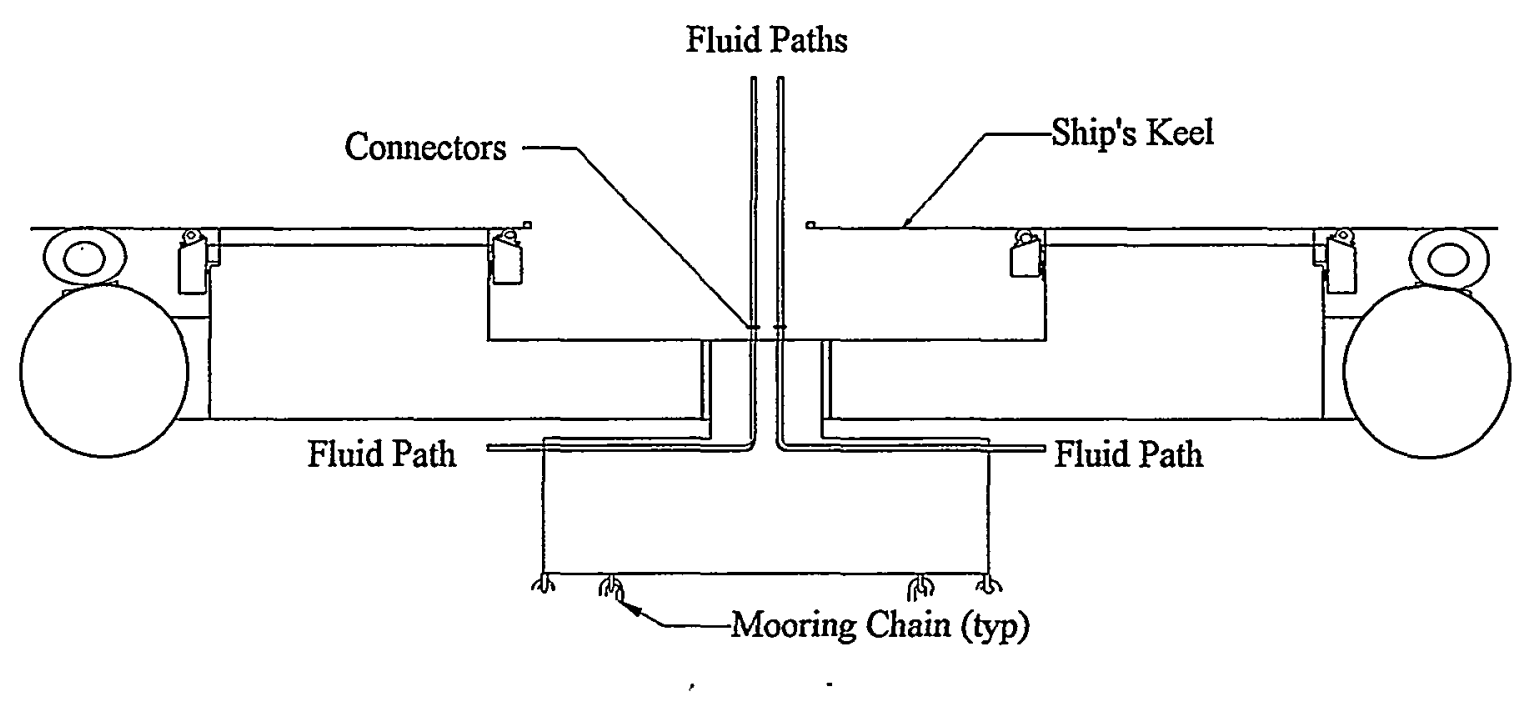

Fig 2-4 Petroleum Cargo Transfer Fluid Paths

The mooring shown in Fig 2-4 is similar to the mooring shown in Fig 23 except that the fluids are transferred in separate pipes (Fluid Paths) as shown on Fig 2-4. In this case a fluid connection is established after mooring by connecting the pipes in the vessel to the pipes in the buoy at the connectors shown on Fig 2-4. Note that the buoy is not centered in the opening in the ship's bottom. Positioning the ship over the buoy for the mooring during the mooring operation cannot be done with complete accuracy. This is particularly so because of the 13 seconds delay between the decision to make the mooring and the actual mating between the buoy and the vessel. Test performed at Nichol's Advanced Marine in Arlington showed that the mating can be consistently performed with an accuracy of about 3 meters in conditions with $\mathrm{Hs}=8 \mathrm{~m}$. In lower wave heights the operation can be performed with better accuracy. It is expected that experienced operators of the system will consistently be able to perform the mooring with a deviation between the center of the buoy and the center of the hull opening of less than 2 meters.

Assume that the fluid pipe assembly has a diameter of $1.5 \mathrm{~m}$ and that the diameter of the inner sealing circle is $10 \mathrm{~m}$. An opening in the bottom of 
the vessel of $5.8 \mathrm{~m}$ then assures that it is possible to make the fluid connection whenever the deviation of the buoy is less than $2.1 \mathrm{~m}$ from the centered position. Check calculations: Opening $2 \star 2.1 m+1.5 m=5.7 m<5.8$ $m$, Sealing circle $2^{\star} 2.1 m+5.8 m=10 m$.

A $13.5 \mathrm{~m}$ diameter inner sealing circle with $7.5 \mathrm{~m}$ opening in the bottom of the ship would in this case ensures a $3 \mathrm{~m}$ tolerance on the positioning of the buoy on the bottom of the ship.

The fluid paths rotate relative to the ship when the ship changes heading. Therefore, a fluid swivel is required. This is located on the vessel. This fluid swivel may be an ordinary swivel stack permitting unlimited rotation. Alternatively, the fluid swivel may be of the limited number of rotations kind. There are several such types. The limit on the number of revolutions is 1.5 in the case of a drag chain and may be 12 or more in the case of another swivel. Power to turn the swivel must be provided. A powered servomechanism may be used or torque may be transferred from the buoy to the swivel. The system proposed here use torque transfer. A long member of triangular cross section will serve the dual purpose of supporting

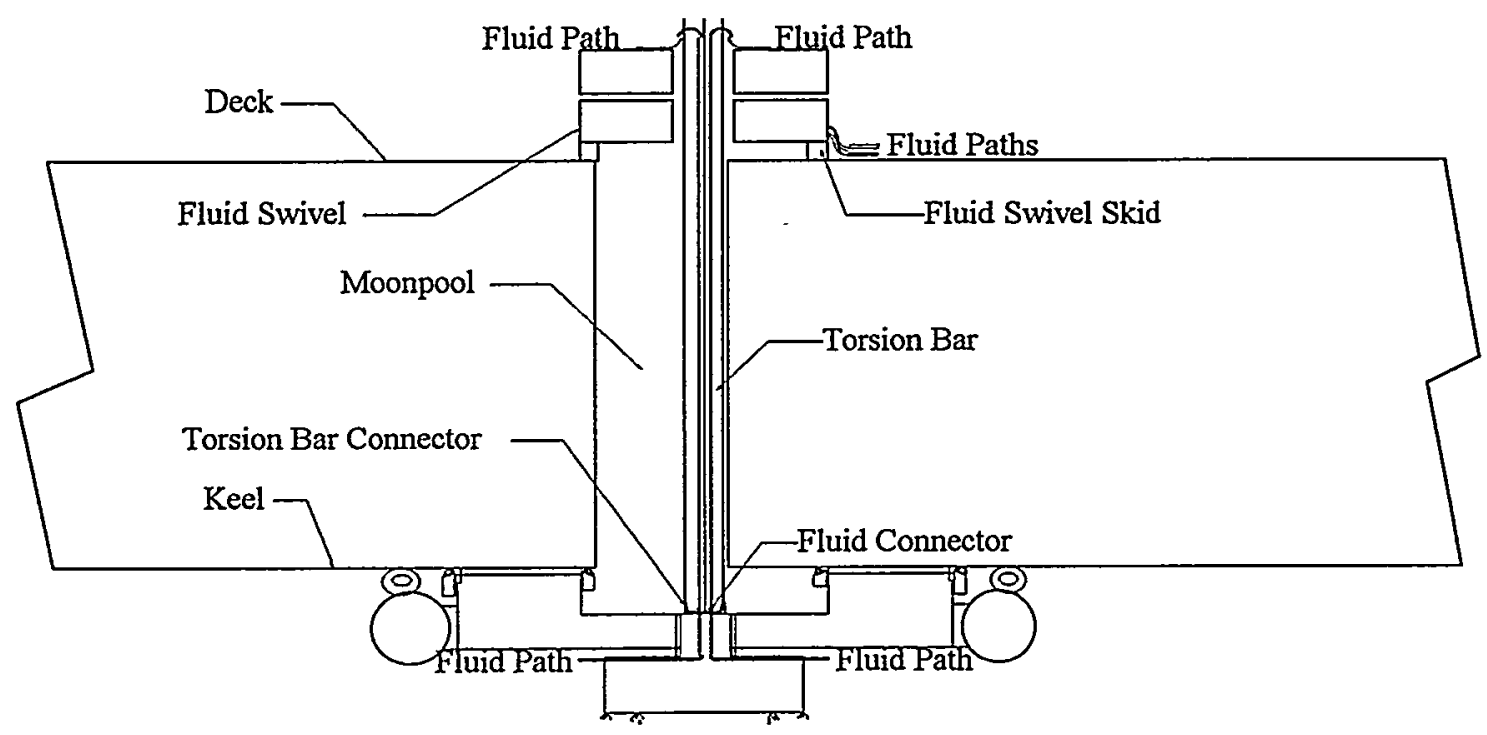

Fig 2-5 Petroleum Transfer System General Arrangement 
the fluid paths and to serve as a torque bar. The buoy is placed on the bottom of the ship at random within the mooring area. Therefore, the swivel can be moved such that it is always placed vertically above the center of the buoy.

Fig 2-5 shows the general arrangement of the fluid transfer system. A fluid swivel on the deck of the ship is connected through a moonpool to the center of the buoy by means of a triangular torsion bar. The torsion bar is mated at the buoy to a triangular female receptacle. The fluid pipes are held to the torsion bar by sleeves. Thus alignment with the fluid connectors on the buoy is automatically achieved. The torsion bar can be raised and lowered relative to the fluid swivel. The fluid pipes can be raised or lowered relative to the torsion bar. During the mooring operation the torsion bar and the pipes are all in their upper position. Following the mooring the fluid swivel skid is centered above the buoy. Then the torsion bar is turned to align with the buoy orientation. The torsion bar is then guided into and seated in the torsion bar receptacle on the buoy. The fluid pipes are now lowered individually to each connector and connected. Each pipe is pressurized with nitrogen and the seal integrity is tested. Following the seal integrity test the nitrogen pressure is increased further. The sealing area is in fluid communication with the valve actuator in the corresponding valve in the buoy. The nitrogen pressure actuates the valve to open. The valve is equipped with spring return for automatic closure upon loss of sealing pressure. At disconnect the operation is reversed. Instead of the testing of seal integrity the pipes are flushed and cleaned such that no product escapes upon disconnect.

The fluid pipes are shown located on the exterior surface of the torsion bar in Fig 2-5. However, they would ordinarily be located inside the torsion bar. This location inside the torsion bar creates several advantages:

- $\quad$ The fluid pipes are shielded from impact damage

- The atmosphere inside the torsion bar may be maintained inert at low cost and with high reliability. 
- Any leaking fluid is contained within the torsion bar. This provides a double barrier against escape.

- The torsion bar may have a larger cross section for the same overall assembly diameter. This enhances the structural capacity of the torsion bar.

The fluid pipes are equipped with several safety devices that ensure against all but minimal spills during emergency and/or accidental disconnect. Controlled emergency disconnect is initiated if:

- The buoy moves on the bottom of the ship. This is detected two ways, optically by an automatic monitoring system and mechanically by a continuous comparison between tilt sensors within the torsion bar and on deck.

- The mooring maintenance pressure rises to above a preset level. This pressure is measured continuously at all 3 pressure maintenance pumps.

- The vessel has an excessive excursion, either due to chain failure or due to excessive weather conditions. This is detected by the differential GPS system, which determines buoy deflection. Backup systems may consist of acoustic systems detecting movement relative to beacons on the seabed or electronic triangulation systems such as for example Motorola Miniranger.

- Emergency conditions aboard the vessel such as fire or collision.

Controlled emergency disconnect comprises the following steps:

- Reducing nitrogen seal pressure. This causes closure of the valves on both sides of the connector, because these valves are maintained open by the seal pressure.

- If time permits purging of the fluid paths by nitrogen

- Blow down of Seal nitrogen and release of couplers 
The vessel may now disconnect the mooring. In the event of accidental violent moves of the mooring buoy the nitrogen seal pressure maintenance system will automatically release all pressure and automatically cause closure of both the automatic valves on both sides of the coupler and the release of the coupler. This is automatic upon loss of sealing pressure. The content of approximately $20 \mathrm{~m}$ of pipe will be released within the torsion bar. Please note that all or nearly all this liquid will be trapped within the mooring moonpool and may be cleaned up by the ship's crew before the next mooring.

Uncontrolled disconnect is forced by the release of the seal pressure, either through rupture of the nitrogen piping or through the activation of emergency blowdown systems. The seal pressure release causes automatic valve closure $(7-10 \mathrm{sec})$ and automatic release of couplers. The pipes within the torsion bar may optionally be equipped with break-away couplers that are actuated by the torsion bar being let down below a preset level. If so equipped, the actuation causes a short time release of the fluids $(7-10 \mathrm{sec})$, closure of valves on both sides of the coupler and release of the couplers. All couplers will likely be lost in this event, the torsion bar will likely be damaged, and there may be some superficial hull damage in the moonpool area. The buoy will in this case drop to its storage position well clear of the vessel. 


\section{MANEUVERING TESTS}

The initial development of the Hydrostatic Mooring was model tested at the Danish Maritime Institute in Denmark, which proved that it was physically possible to perform the mooring operation in adverse weather conditions with up to $7.7 \mathrm{~m}$ of significant wave height. See appendices $B, C$, and $\mathrm{H}$. This was done with a free-floating ship model in the model basin, which was controlled by elastic ropes, in order to emulate the maneuvering required by the vessel prior to the mooring to the buoy. This procedure, while demonstrating that the mooring operation was physically feasible, did not properly account for the limitations of propulsion power and the human intervention factor in making the actual mooring.

The maneuvering that is required by a ship to meet with the Hydrostatic Mooring is to position the mooring area in the ship vertically above the buoy in a circle which has a $3 \mathrm{~m}$ radius. The navigator then judges the position of the vessel 13 seconds later. When this estimated position is ideal, he actuates the buoyancy control system in the mooring buoy by remote control, causing the buoy to rise in the water. Approximately 13 seconds later, the buoy impacts the bottom of the ship, thereby blocking a water intake in the center of the mooring area. This causes the buoy to be sucked on to the bottom of the ship and the mooring is accomplished.

By initial appearance, this procedure is nearly impossible because the mooring area of the vessel has to be bróught into a position which is no more than 3 meters away from the theoretically ideal position in order to moor successfully. When one considers that a 150,000 DWT vessel is $280 \mathrm{~m}$ long and that this operation may take place during storm conditions at sea, this would appear to be an impossible task. In fact, this was the opinion of many experts, prior to the tests made at Nichols Advanced Marine.

The present project included tests of the ability of a practical seafarer to actually perform the maneuvers required to moor successfully to the Hydrostatic Mooring in adverse weather conditions. The Nichols Advanced Marine maneuvering simulator was used to emulate this operation. A 
maneuvering simulator is similar to a flight simulator, and is capable of simulating all motions and to present a true real time response of the ship to the environmental forces and the application of rudder, engine power, and thruster power.

The maneuvering simulator actually existed prior to the tests, and only needed to be programmed with the parameters of the ship used. The actual ship was a 150,000 DWT vessel in partial loaded condition. Only one loading condition was tested. The Hydrostatic Mooring needed to be developed for incorporation in the maneuvering simulator. A mathematical model of the mooring was built to faithfully emulate the behavior of the Hydrostatic Mooring. This included the proper horizontal motions due to direct interaction with the waves and the current. It also included the correct vertical movement of the buoy, including the interaction with the vessel hull when approaching the keel. These features were cross-checked in the maneuvering simulation against the observed motion from the prior physical model test.

The maneuvering that is required to moor to the Hydrostatic Mooring system is a non-standard maneuver, which is not covered in any maneuvering textbook. Therefore, Captain James Atkinson, an experienced tanker navigator, was retained to develop the maneuvering procedures required to meet with the buoy, to train on the simulator for three days prior to the actual tests, and to perform the tests.

The tanker was equipped with an Elliott White Gill bow thruster type T3. This is a thruster that can deliver thrust with a delay of six seconds in any direction. This thruster comes in three versions with a $100 \mathrm{kN}$ thrust, $150 \mathrm{kN}$ thrust, and $200 \mathrm{kN}$ thrust. Only the $150 \mathrm{kN}$ and the $200 \mathrm{kN}$ thrusters were tested. During the training prior to the actual tests, it was determined that the $150 \mathrm{kN}$ thruster had inadequate thrusting power and therefore, the tests were only done with the $200 \mathrm{kN}$ thruster. This is the largest unit that is manufactured by Elliot. 
This is not a limitation on the application of the technology because several thrusters may be built into a large tank ship.

During the training exercises, the results and outcomes were not recorded, other than for training purposes and to improve operations. No report was made concerning the experience during the training exercises.

Following the training exercises, a program for twenty five tests was carried out. The program is listed below, in Table 3-1. All tests were made in deep water. The winds, the waves and the currents that were present in the test area are also listed in Table 3-1. 
TABLE 3-1

Tests Conditions

\begin{tabular}{|c|c|c|c|c|c|c|}
\hline Simulation & \multicolumn{2}{|c|}{ Wind } & \multicolumn{3}{c|}{ Waves } & \multicolumn{2}{c|}{ Current } \\
\hline Run \# & $\begin{array}{c}\text { Speed } \\
\text { (knots) }\end{array}$ & $\begin{array}{c}\text { Direction } \\
\text { (degrees } \\
\text { from) }\end{array}$ & $\begin{array}{c}\text { Significant } \\
\text { Height } \\
\text { (m) }\end{array}$ & $\begin{array}{c}\text { Direction } \\
\text { (degrees } \\
\text { to) }\end{array}$ & $\begin{array}{c}\text { Speed } \\
\text { (knots) }\end{array}$ & $\begin{array}{c}\text { Direction } \\
\text { (degrees } \\
\text { to) }\end{array}$ \\
\hline 10 & 0 & 0 & 0 & 0 & 0 & 0 \\
\hline 11 & 0 & 0 & 0 & 0 & 0 & 0 \\
\hline 12 & 0 & 0 & 0 & 0 & 2 & 180 \\
\hline 13 & 20 & 0 & 3 & 180 & 0 & 0 \\
\hline 14 & 20 & 0 & 3 & 180 & 0 & 0 \\
\hline 15 & 20 & 45 & 3 & 225 & 1 & 180 \\
\hline 16 & 20 & 45 & 3 & 225 & 1 & 180 \\
\hline 17 & 20 & 90 & 3 & 270 & 1 & 180 \\
\hline 18 & 20 & 90 & 3 & 270 & 1 & 180 \\
\hline 19 & 25 & 0 & 5 & 180 & 0 & 0 \\
\hline 20 & 25 & 0 & 5.1 & 180 & 0 & 0 \\
\hline 21 & 25 & 45 & 5 & 225 & 1 & 180 \\
\hline 22 & 25 & 45 & 5 & 225 & 1 & 180 \\
\hline 23 & 25 & 90 & 5 & 270 & 1 & 180 \\
\hline 24 & 25 & 90 & 5 & 270 & 1 & 180 \\
\hline 25 & 25 & 90 & 5 & 270 & 2 & 180 \\
\hline 26 & 25 & 90 & 5 & 180 & 2 & 180 \\
\hline 27 & 40 & 0 & 8 & 180 & 0 & 0 \\
\hline 28 & 40 & 0 & 8 & 180 & 0 & 0 \\
\hline 29 & 40 & 0 & 8 & 180 & 1 & 135 \\
\hline 30 & 40 & 0 & 8 & 180 & 1 & 135 \\
\hline 31 & 40 & 0 & 8 & 180 & 1 & 90 \\
\hline 32 & 40 & 0 & 8 & 180 & 1 & 90 \\
\hline 33 & 40 & 0 & 8 & 180 & 2 & 90 \\
\hline 34 & 40 & 0 & 8 & 180 & 2 & 90 \\
\hline & & & & & & \\
\hline
\end{tabular}

It is notable that no existing mooring system can perform moorings in significant wave heights of $5 \mathrm{~m}$ and $8 \mathrm{~m}$, which were used in the test program. Consequently, sixteen of the twenty-five tests were in conditions under which no existing mooring systems can safely perform the mooring operation.

All tests were successful, in the sense that the buoy safely met with the vessel and was securely held by the vessel following mooring. In order to transfer fluids, the deviation between the centerline of mooring area and the centerline of the buoy, should not exceed $2.8 \mathrm{~m}$. It is seen from Table 32 , that in five instances ( $20 \%$ of the tests) that the final position of the buoy 
on the tanker were outside this margin. In these cases, the buoy would be released and the mooring attempt repeated. Table 3-2 lists the order in which the tests were performed. The tests were made in ever more difficult conditions. It is notable that among the last sixteen tests at $5 \mathrm{~m}$ and $8 \mathrm{~m}$ wave heights, only one failed to place the buoy in a position that petroleum fluid transfer may be made. It is concluded that the navigator was not fully trained at the beginning of the tests. It was further concluded that a fully trained navigator can consistently place the mooring such that the buoy is properly positioned for fluid transfer in more than $90 \%$ of all attempts for conditions where the wave height is between 0 and $8 \mathrm{~m}$.

The Hydrostatic Mooring would ordinarily be equipped with enough compressed air power to make 4 to 8 mooring attempts without being supplied with additional air. The maneuvering tests clearly demonstrate that the mooring between a large vessel of $150,000 \mathrm{DWT}$, and the Hydrostatic Mooring, can be made with extreme reliability, in all wave conditions up to a significant wave height of $\mathrm{Hs}=8 \mathrm{~m}$.

It was not tested, but it is probable in view of the results of these tests, that the mooring operation can be made with reasonable accuracy in even higher waves. This, however, may only have theoretical interest, because the operation of equipment aboard ship degrades at this level of wave height, and it may therefore not have practical interest to be able to moor beyond $\mathrm{Hs}$ $=8 \mathrm{~m}$. Therefore, from a practical point of view, the Hydrostatic Mooring would eliminate weather downtime associated with the operations of a mooring system offshore. The importance of this can be appreciated from the fact that a typical offshore mooring has a downtime of between $5 \%$ and $30 \%$. 
TABLE 3-2

Tests Results

\begin{tabular}{|c|c|c|}
\hline \multicolumn{2}{|c|}{ Simulation } & \multirow{2}{*}{$\begin{array}{c}\text { Mating } \\
\begin{array}{c}\text { Center to Center Distance } \\
(\mathrm{m})\end{array} \\
\end{array}$} \\
\hline Run \# & Duration (sec) & \\
\hline 10 & 1808 & 4.1 \\
\hline 11 & 2156 & 0.9 \\
\hline 12 & 1650 & 0.2 \\
\hline 13 & 1785 & 1.25 \\
\hline 14 & 3310 & 2.9 \\
\hline 15 & 3328 & 3.1 \\
\hline 16 & 2940 & 0.4 \\
\hline 17 & 2872 & 1.1 \\
\hline 18 & 2812 & 3.8 \\
\hline 19 & 2205 & 2.6 \\
\hline 20 & 2280 & 1.8 \\
\hline 21 & 3664 & 2.5 \\
\hline 22 & 1665 & 2.5 \\
\hline 23 & 2160 & 2.0 \\
\hline 24 & 2235 & 2.5 \\
\hline 25 & 3465 & 1.8 \\
\hline 26 & 3912 & 1.5 \\
\hline 27 & 5704 & 5.1 \\
\hline 28 & 2145 & 2.8 \\
\hline 29 & 2595 & 1.8 \\
\hline 30 & 1530 & 2.0 \\
\hline 31 & 4566 & 1.9 \\
\hline 32 & 2760 & 1.5 \\
\hline 33 & 3065 & 1.9 \\
\hline 34 & 3540 & 1.7 \\
\hline
\end{tabular}




\section{PRELIMINARY DESIGN}

Prior to initiating this project, conceptual designs existed for the Hydrostatic Mooring, that are similar to the design that is featured in this report.

The previous designs were not adequate in detail and quality to gain approval by the American Bureau of Shipping (ABS), and therefore, further designs were undertaken to bring the level of design to a level that the approval by $A B S$ can be gained. The specific areas that required further refinement were:

- The sealing system between the ship's hull and the buoy

- The buoyancy control system

- The main bearing between the two buoy parts

- Main structure

- An assessment of risk.

\subsection{SEALING SYSTEM BETWEEN THE HULL AND THE BUOY}

The sealing system between the hull and the buoy are subject to extreme conditions because the bottom of the ship is not designed to be a sealing surface. In addition, significant relative motion takes place during the mooring process, when the buoy initially contacts the bottom of the ship. The buoy is pulled in approximately $300 \mathrm{~mm}$ following contact with the bottom of the ship. Moreover, the bottom of the ship may not be plane. The structural system is designed for an out-of-plane deviation of the ship's bottom of 63 $\mathrm{mm}$. The sealing ring around the perimeter of the buoy must cope with at least this unevenness of the bottom of the ship and must cope with the deflections which take place between the buoy and the vessel, when the buoy is subjected to large horizontal and vertical forces. 
A review of possible structural systems to cope with these extreme conditions, reveal that only three systems were promising:

- Beam elements made from glass reinforced plastic

- Elements made from steel that were all connected to the main structure by hinges

- Elements made from steel that pivot on an edge of the main structure.

These structural elements must be able to resist the maximal external hydrostatic pressure in presence of absolute vacuum on the other side. This is a condition that may be encountered if the structural capacity of the mooring system is exceeded during a tear off of the mooring buoy from the bottom of the ship.

The glass fiber reinforced plastic elements are very attractive because there are no sliding contacts between the structural elements and the main structure. However, it was found that less than normal factors of safety would be required to make the system work. Therefore, this system was discarded.

Steel elements made from steel that are hinged to the main structure were feasible. The main problem with these elements is the very large structural capacity required of the hinges and potential wear of the pins that would constitute the bearings in the hinges.

The third option, structural elements that are loosely held to the structure and that pivots on an edge, was the solution selected. These elements can be made strong enough to cope with all differential pressures at normal factors of safety and can be made to safely deflect a distance which in the actual case exceeds $600 \mathrm{~mm}$. This large deflection capability makes it possible to conform to all unevenness of the hull, and to maintain sealing contact when the buoy is subject to extreme forces. Furthermore, this large deflection prevents the sealing element from being damaged when 
the exterior fenders are fully deflected to protect the buoy and the ship from mutual impact.

The design is featured in Appendix D, on Figs 1-6 through 1-8.

\subsection{BUOYANCY CONTROL SYSTEM}

The buoyancy control system must be capable of increasing the buoyancy of the buoy by approximately 15 metric tons per second for approximately 8 seconds in order to give the buoy the proper upward velocity and in order to constrain the time between actuation and actual mooring. This time should be as short as possible, in order to reduce the uncertainty associated with the navigator having to predict the future position of the ship when he makes the decision to moor.

A working design of the buoyancy control system existed prior to this project. This project refined the buoyancy control system. Refinements comprised:

- Simplification of the control systems previously used

- Provision of an automatic and passive buoyancy removal system

- Determination of the behavior of the system during multiple mooring attempts.

In the prior system, the buoyancy control system consisted of a permanent medium pressure tank, in which the internal water level could be controlled and thereby the buoyancy of the buoy slowly changed. This tank has been eliminated such that the buoyancy of the buoy can only be changed rapidly through the buoyancy control system, and alternatively changed by divers regulating the water level inside trim tanks. This reduces the complexity of the system and the cost. Particular details can be found in Appendix D, Chapter 3. Systems operations are depicted on on Fig 4-1 through 4- 3. 


\section{Hydrostatic Mooring -- Buoyancy Control}

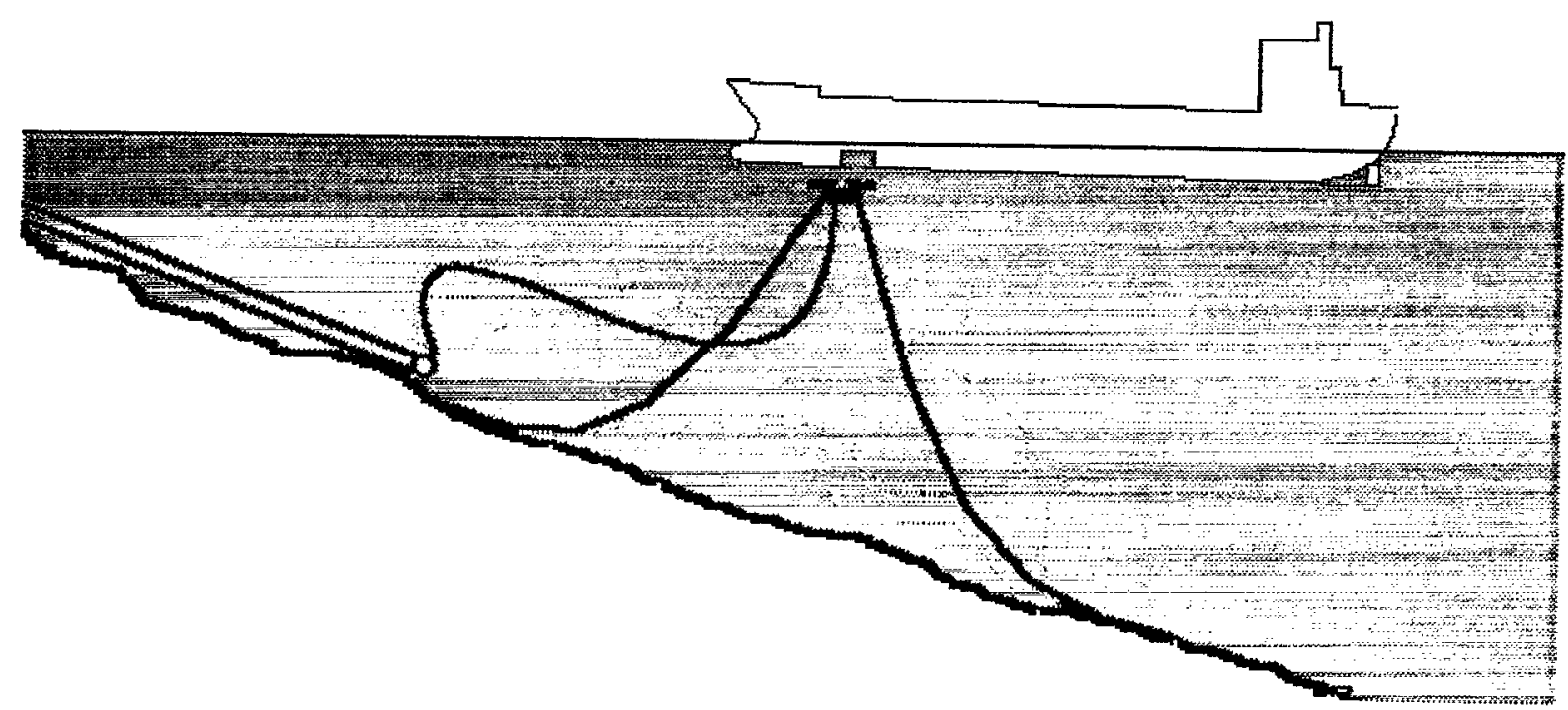

\begin{tabular}{|l|l|}
\hline Mooring Condition: Start of Rapid Ascent & Time $=t+0.5 \mathrm{sec}$ \\
\hline
\end{tabular}

At Time $=t$ the vessel commanded valve 4 to open. Valve 4 opens fully in $0.5 \mathrm{sec}$.

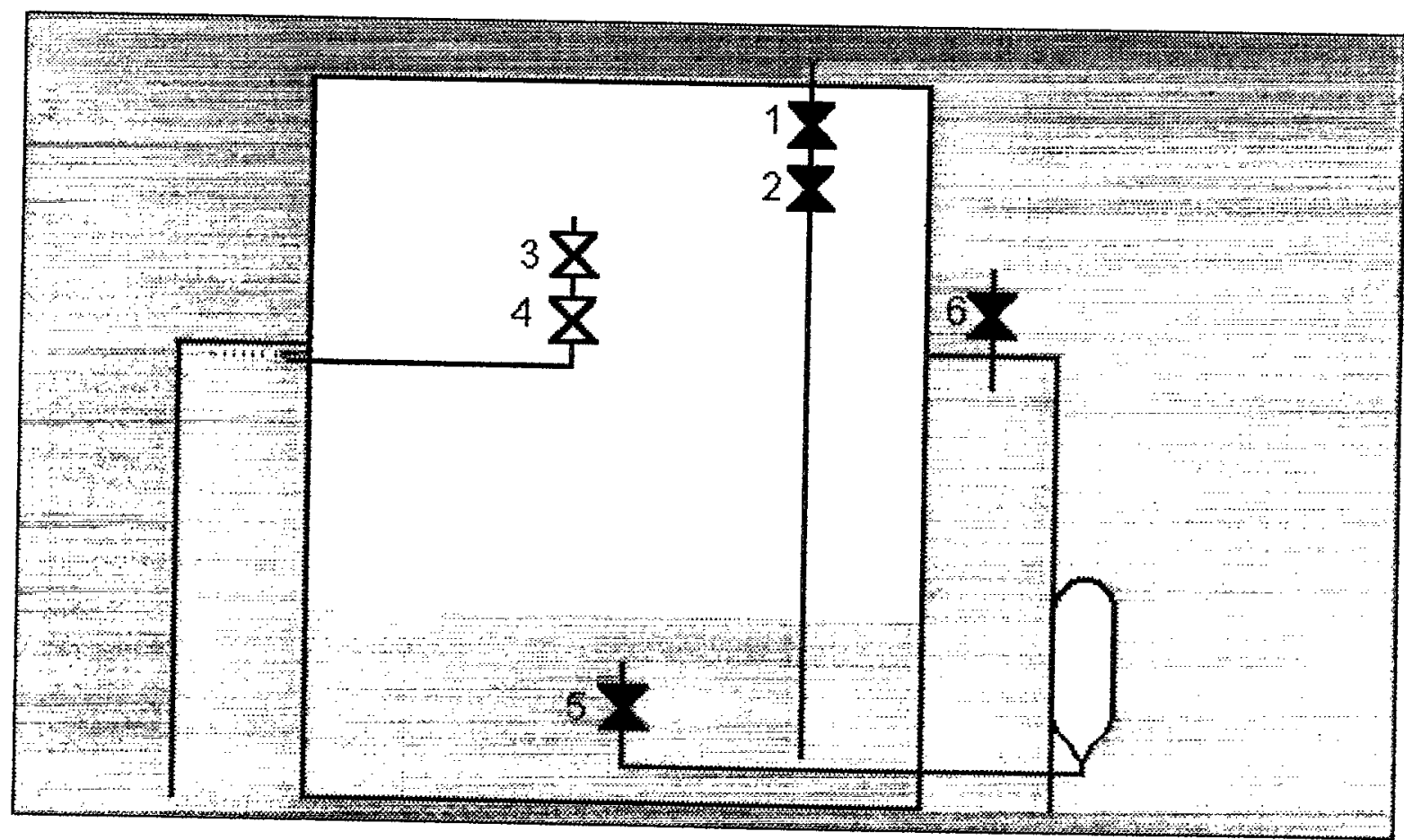

Figure 4-1 Start of Rapid Ascent 


\section{Hydrostatic Mooring - Buoyancy Control}

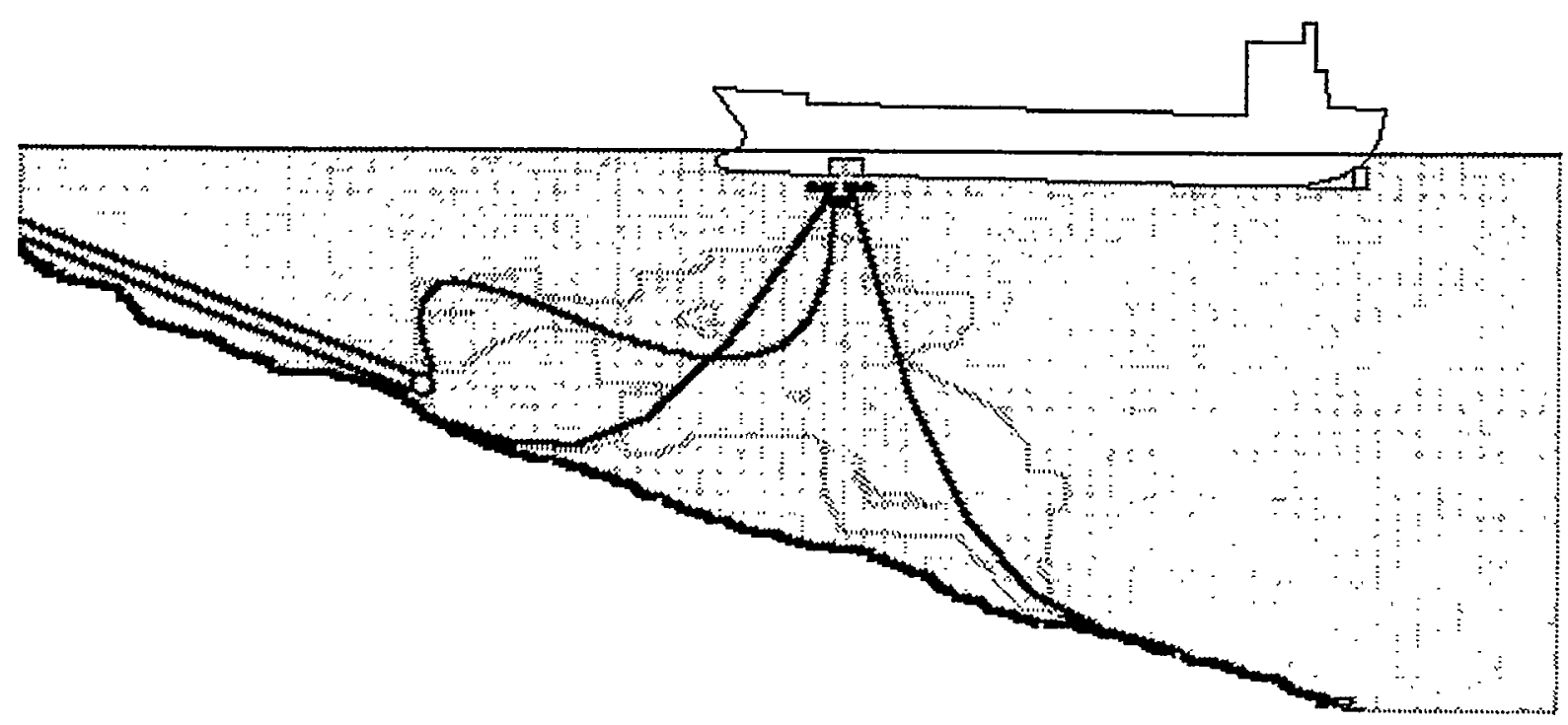

\begin{tabular}{|l|l}
\hline Mooring Condition: Rapid Ascent & Time $=\mathrm{t}+4 \mathrm{sec}$ \\
\hline
\end{tabular}

Rapid Ascent. Air flowing through valye 4 increases the buoyancy at a rate of 15 tonnes/second. Bouy rises in the water at a rate of up to $1.5 \mathrm{~m} / \mathrm{sec}$.

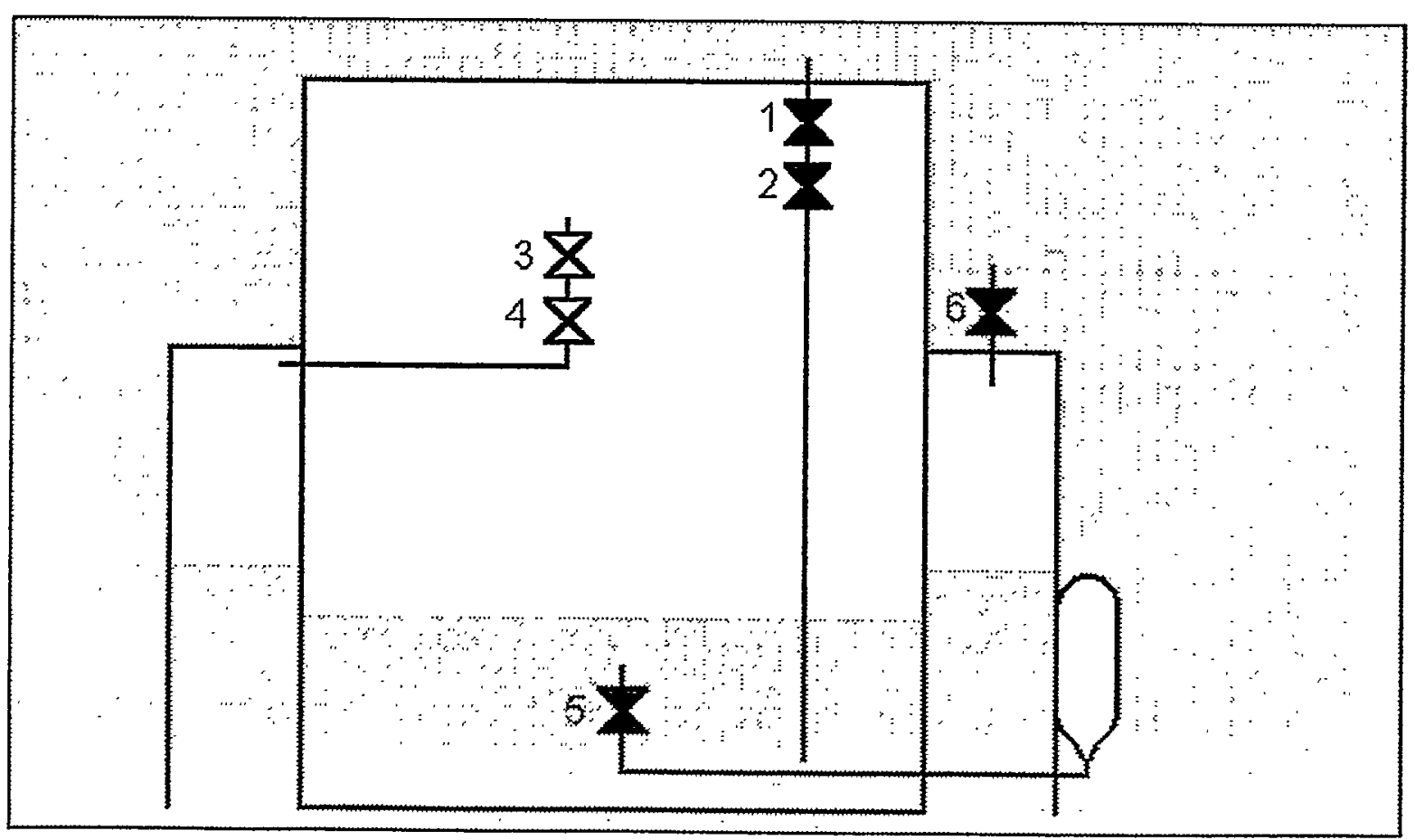

Figure 4- 2 Rapid Ascent 


\section{Hydrostatic Mooring -- Buoyancy Control}

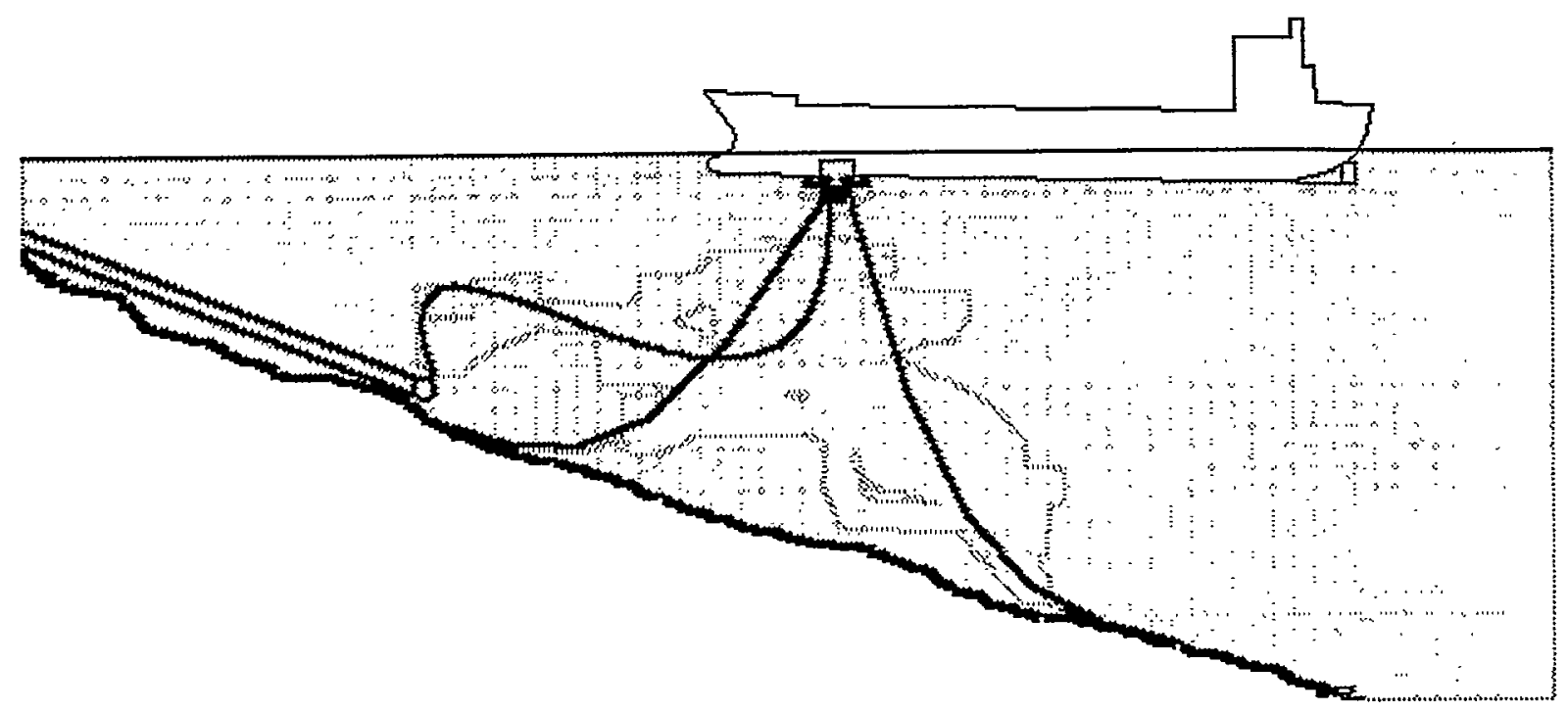

Mooring Condition: Maximum Buoyancy

Time $=\mathrm{t}+9 \mathrm{sec}$

The rapid ascent system automatically closes valve 4 when reaching maximum buoyancy. This is controlled by a timer in the control system.

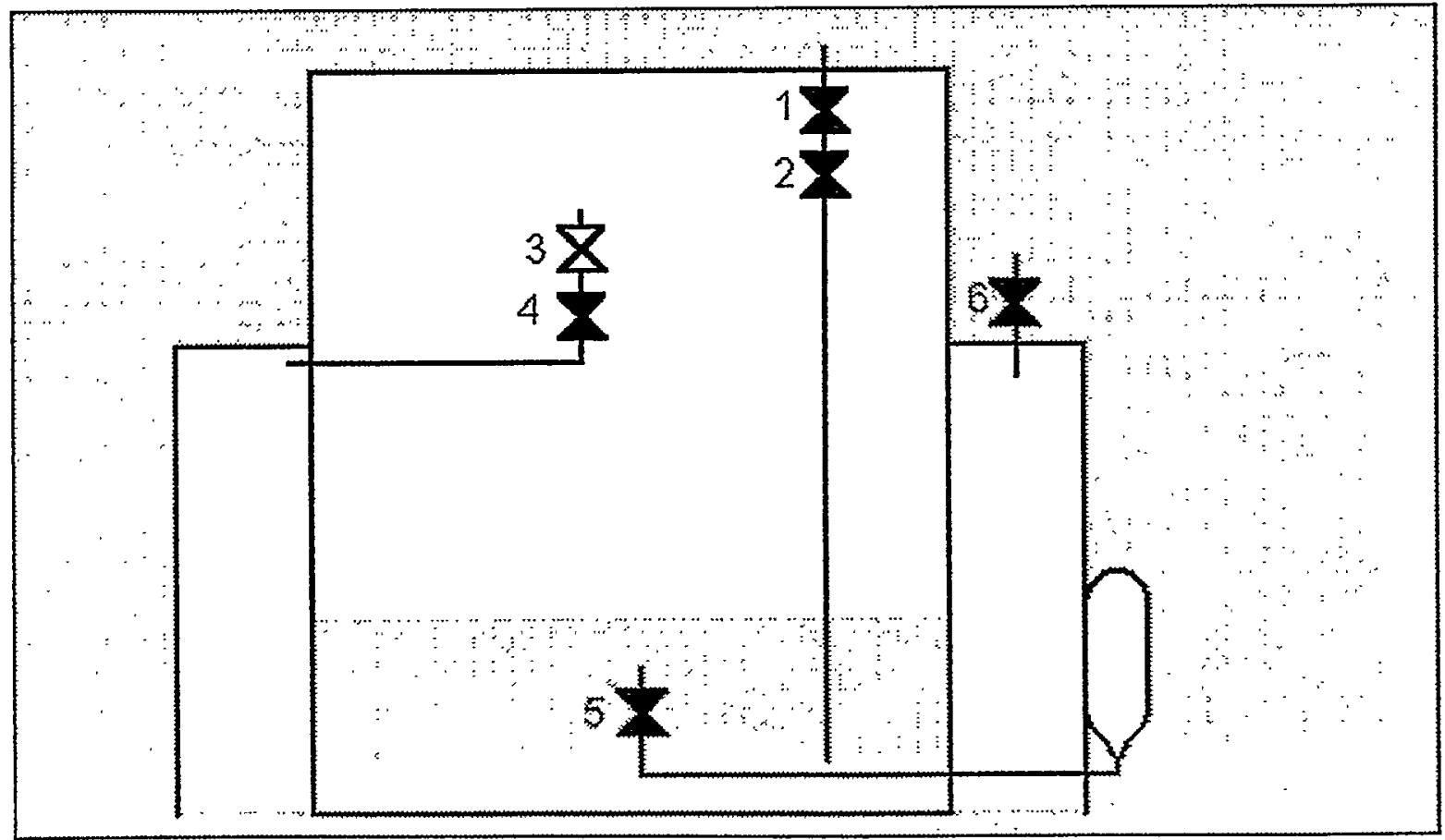

Figure 4- 3 Maximum Buoyancy 
The automatic and passive elimination of the buoyancy created by the buoyancy control system is achieved by having small holes in the rapid ascent buoyancy tank that will let all air out within 15 minutes after the tank is being filled. Since the tank only needs to work for approximately 13 seconds, it does not have to be airtight, and the provision of the controlled leaks greatly enhances the security of the system by making it impossible for biologically generated gases to be captured within the rapid ascent buoyancy volume. Such capture could cause the buoy to surface unexpectedly.

\subsection{MAIN BEARING}

The main bearing design that existed prior to this project was based on LUBRON sliding elements in contact with a ring, which was immersed in lubrication oil. This concept is reliable, however, the tolerance on the bearing ring is very small and represents a manufacturing problem. The tolerance is within what sophisticated machinery can do but it increases the cost of the bearing to have to comply with the tolerance requirements of the LUBRON bearing pads.

The oil bath in the previous design floated on top of the water, and therefore has the surface between the oil and the water pointing downwards. A potential problem is that any gas generated for whatever reason tends to accumulate above the oil bath and will tend to force the oil out of the bearing thereby potentially cause pollution of the water.

Another problem with the LUBRON bearing is that any grit that is present in the water may become mixed with the lubrication oil. This would cause rapid wear of the LUBRON bearing.

For these reasons, alternative options for the design of this bearing were explored in this project. It was found that Hillman rollers manufactured by the Hillman Company in New Jersey would have better characteristics than the LUBRON bearings. 
These rollers could be operated such that they are immersed in seawater continuously. The potential problem that would originate from this is that marine growth would attach itself to the bearing surfaces and hardware. This could possibly interfere with the operation of the chains that guide the rollers. Testing would be required to ascertain whether this is a problem or not, therefore, a solution was found where this potential problem is avoided.

The buoy has a large amount of air available for purposes of providing the variable buoyancy during ascent. A very small fraction, less than $1 \%$ could be used to maintain the Hillman roller bearing in air using a trap of the type used for the lubrication oil in the LUBRON bearings. The Hillman rollers would be subjected to immersion in seawater when the buoy descends and the air in the trap compresses. Following the descent, a small amount of air would then be bled into the roller bearing housing. The marine growth, which might otherwise grow on the bearing elements, is then avoided.

The main disadvantage of this system is that another mechanical system is required to replenish the air that is compressed when the buoy descends.

The proposed mechanical system for this purpose is extraordinarily simple in that the main high-pressure system in the buoy would have an accumulator attached to the high-pressure air replenishing system. This accumulator is furnished with a very small continuous bleed connection into the roller bearing housing. Following disconnect of the buoy, this bleed connection will continue to bleed air into the roller bearing housing for an hour. No valves or any other control systems are required in this system.

\subsection{STRUCTURAL DESIGN}

In a specific structural design the buoy would ordinarily be made for a specific vessel at a specific location. Since the approval by the ABS is an approval in principle, it was agreed with $A B S$ prior to the signing of the 
contract that a sample design using a horizontal mooring force of $6,000 \mathrm{kN}$ would be satisfactory.

The sample design is intended for tankers that transport water between Turkey and Israel. Since it is economical to use the very largest sizes of tankers in this traffic, the buoy was designed for the draft of a 500,000 DWT tanker, which is 25 meters.

The absolute lowest pressure that is possible on the upper surface of the buoy, when being moored to the tanker, is the vapor pressure of seawater, at the ambient temperature. This pressure is very close to absolute vacuum. Consequently, for design purposes it is considered that the hydrostatic pressure above the buoy may reach absolute vacuum.

The external pressure is the absolute hydrostatic pressure at the water depth in question. For the tankers in question, this ranges from $25 \mathrm{~m}$ maximum to a minimum of $4 \mathrm{~m}$ (350 kpa $-140 \mathrm{kpa})$.

In addition, the mooring must be designed for a simultaneous application of a horizontal mooring force ranging from zero to $6,000 \mathrm{kN}$.

The structural system in the buoy must be able to withstand differential forces, which arise from unevenness of the ship's bottom. A general consideration of unevenness is that any point of the ship's bottom may deviate as much as $63 \mathrm{~mm}$ from the plane which most closely represents the position of the ship's bottom in the mooring area.

The contact element between the buoy and the ship's bottom are Trellex Morse Fender Bar fenders. These are solid rubber fenders with very high compressive force capability and relatively limited compression with a maximum compression of $150 \mathrm{~mm}$.

Individual beams to provide the structural strength backing these fenders are provided. In addition, the fender area must be watertight and therefore a steel membrane is placed immediately below the fenders. Refer to the Drawings attached to Section 2 in Appendix D. 
Beams are required to resist the large forces that are involved in this system. A network of steel I-beam resembling a honeycomb is consequently placed immediately below the surface area of the buoy. The voids between the beams could act as traps for gases that are present in the water and may trap some of the air that is let out when the mooring has been accomplished. Such trapped air could add sufficient buoyancy to the buoy that it would not descend after being released from the vessel. Therefore, the voids need to be filled with a material. For the purpose of this report, timber has been selected as the fill material in these areas. The timber provides a small amount of buoyancy, but more importantly, the timber provides structural assist to the I-beams by preventing buckling of the web plating, and also fills in the voids, thereby preventing the accumulation of any gas.

Other elements of the structural design the buoy are standard and are shown in Appendix D, Section 2.

\subsection{FAILURE TREE ANALYSIS}

The $A B S$, as one their requirements, require a failure tree analysis of the buoy to ascertain the sequences of events, which might lead to uncontrolled oil spills or other undesirable consequences of operating the buoy.

This, of course, is not a concern for a buoy design for transferring water. However, since the principal objective of the buoy design is to use it for petroleum transfer operation, the ABS requires such an analysis. This analysis is contained in Section 4 of Appendix D. 


\section{AMERICAN BUREAU OF SHIPPING APPROVAL}

The American Bureau of Shipping (ABS) was retained in December 1999 to provide approval in principle for the mooring system. Two submittals were made to ABS. The initial submittal in April 2000 of the mooring tests and the maneuvering tests that were performed prior to and in connection with this project. This submittal is Appendices $A, B$, and $C$, in this report.

Following on July 7 , Appendix $D$ to this report was submitted to the ABS.

The correspondence by letter and e-mail with ABS is contained in Appendix E.

As of August 5, 2000, ABS requires the following calculations, which we will provide.

- Floating stability calculations of the buoy. This was actually tested in the physical model in Denmark and calculations exist for prior versions of the buoy. However, they need to be updated for the present design.

- Inclusion of corrosion allowance in the design of the members

- Calculation of stake piles used as anchors in the seabed. HanPadron Associates carried out-this work in late July, but has not yet been received.

- An assessment of mechanical wear of the fluid risers and the mooring chains is also required.

It is anticipated that these calculations will be submitted to ABS late August 2000. 


\section{CONCLUSIONS}

The main conclusions from the work carried out under this contract are:

An ordinary seafarer can learn by training on a simulator, to moor large tanker vessels to the Hydrostatic Mooring, safely and quickly, in all weather conditions up to storms generating waves with a significant wave height of $8 \mathrm{~m}$.

Complete conceptual design of the Hydrostatic Mooring buoy was carried out which proved that the buoy could be constructed entirely from commercially available standard components and materials. The design is robust, and damage resistant.

The mooring tests had a $100 \%$ success rate from the point of view of the buoy being securely attached and moored to the vessel following every mooring attempt. The tests had an $80 \%$ success rate from the point of view of the buoy being adequately centered such that petroleum transfer equipment on the vessel could be attached to the corresponding equipment on the buoy.

The results given in Table 3-2 of the mooring tests show a consistently improving performance from test to test by the Captain that performed the mooring operations. This is not surprising, in view of the fact that the Captain had only three days of training on the simulator prior to conducting the tests, that the maneuvering required is non-standard, and the test program itself lasted four days. One conclusion of the test performance is that the Captain was not fully trained at the initiation of the test.

It may therefore be concluded that a thoroughly trained navigator would probably be able to make the mooring such that the fluid transfer equipment can be connected with reliability in excess of $90 \%$. Considering that the typical standard buoy has enough power aboard to make eight mooring attempts, this implies that the probability that the mooring attempt should fail because of the inability to connect the fluid transfer equipment is 
of the order of $10^{-8}$. It may therefore be concluded that the mooring operation between a Hydrostatic Mooring and a large tanker vessel can be carried out with near absolute reliability in all sea states up to a sea state where the significant wave height is $8 \mathrm{~m}$. 


\section{Appendix A}

\section{MANEUVERING TEST REPORT BY NICHOLS}




\title{
SHIP MANEUVERING STUDY $O F$
}

\author{
HYDRO STATIC MOORING
}

Prepared for:

Suction Mooring Technology

318 North Post Road

Princeton Junction, New Jersey 08550

Purchase Order

Letter, dated October 29, 1998

Prepared by:

Nichols Advanced Marine

1725 Jefferson Davis Hwy, Suite 1300

February 1999 


\title{
SHIP MANEUVERING STUDY
}

\author{
OF

\section{MOORING TO HYDROSTATIC BUOY}

\author{
Prepared for: \\ Suction Mooring Technology \\ 318 North Post Road \\ Princeton Junction, New Jersey 08550 \\ Purchase Order \\ Letter, dated October 29, 1998
}

Prepared by:

Nichols Advanced Marine

1725 Jefferson Davis Hwy, Suite 1300

February 1999 


\section{Table of Contents}

Section

Title

Page

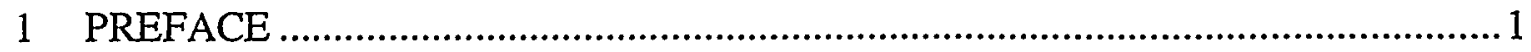

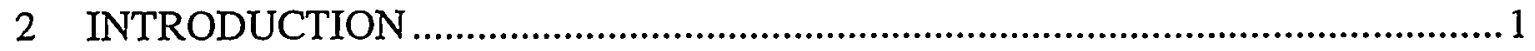

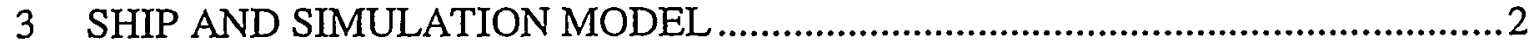

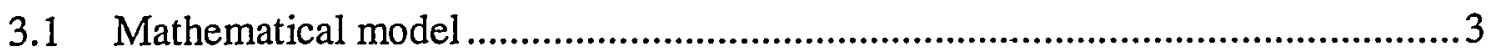

3.2 Ship Characteristics ....................................................................................

3.3 Wave Motions of Tanker ....................................................................... 4

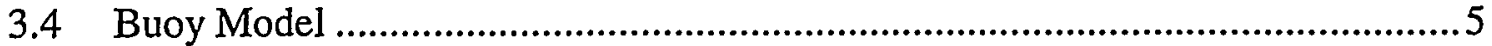

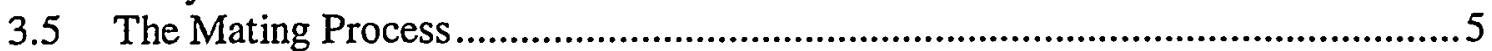

3.6 Buoy Mooring System.........................................................................

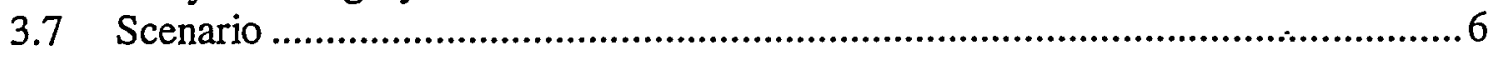

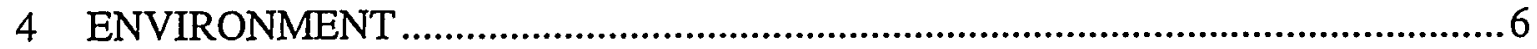

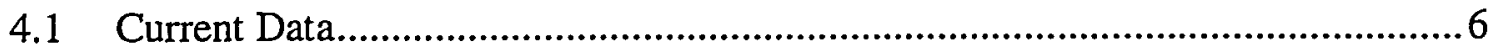

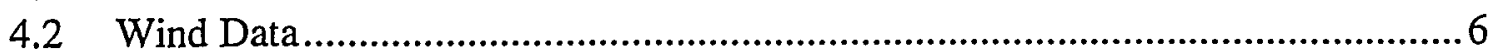

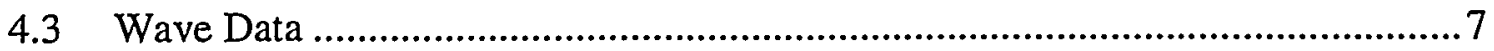

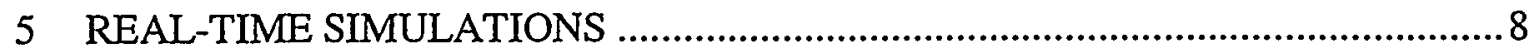

6 AN EXPERIENCED PILOT'S EVALUATION ........................................

7 SUMMARY AND CONCLUSIONS .......................................................... 10

\section{List of Tables}

Section

Title

Page

Table 1 Ship Characteristics .........................................................................

Table 2 Maneuvering Characteristics for tanker................................................... 3

Table 3 Real-Time Simulations ..................................................................... 8

\section{List of Figures}

Section

Title

Page

Figure 1 Wave Drift Forces, Head Seas. ...7 


\section{List of Appendices}

APPENDIX I Maneuvering Characteristics for Tanker

APPENDIX II RAO's for Tanker

APPENDIX III RAO's for Buoy

APPENDIX IV SIMULATION RESULTS 


\section{PREFACE}

This report presents the results from a simulation study performed by Nichols Advanced Marine, Crystal City, Virginia, for Suction'Mooring Technology, Princeton Junction, New Jersey. The study was conducted under funding from the Department of Energy for Energy Related Inventions Program project titled "The Hydrostatic Mooring", DE-FG3698G010321. The objective of the study was to investigate by use of a ship handling simulator how precisely an experienced mariner would be able to position a tanker in the 125.000 to 150.000 DWT range approaching a Hydrostatic Mooring buoy under heavy weather conditions. All simulation runs were conducted at Nichols Advance Marine.

\section{INTRODUCTION}

The hydrostatic mooring concept uses a moored buoy with a buoyancy chamber that enables the control of the vertical location of the buoy. The buoy is located about 6 meter below the draft of tanker during approach. The tanker is equipped with a moon pool, a hole in the bottom of the tanker with a diameter of 5 meter. The moon pool may be connected with a pipe to the free surface, but the moon pool itself is only a few meters high. When the center of the moon pool is above the buoy the mariner will push a button that will activate the buoyancy control system in the buoy. The buoy will move upwards to the bottom of the tanker in about 12 seconds. The water in the moon pool is removed by the bow thruster, which has the water intake from the moon pool. The bow thruster is an Elliot White Gill thruster type. When the buoy is in the mated location under the bottom of the tanker, it is fixed to the tanker by the pressure difference between the surrounding water and the pressure in the moonpool. Water leaking past the buoy / tanker bottom seal is removed continuously by a drainage pump.

The objective of the simulation study was to evaluate the difficulty for an experienced mariner to maneuver the tanker under heavy weather conditions to approach the buoy and connect. The buoy in connected location must cover the moon pool. The distance from the center of the buoy to the center of the moon pool should be less than 5 meters for making a fluid coupling. The buoy will be captured within a distance of 7.5 meters. The speed of the tanker at time of mating should be less than 2 knots.

This report presents results from 25 simulation runs which were carried out during the week of February 1 to 5, 1999 with a 150.000 DWT tanker. During all simulation runs, the simulator was under the command of Capt. James A. Atkinson. He is a very experienced mariner and pilot with more than 30 years of experience. The simulations were supervised by Mr. J. Korsgaard. 


\section{SHIP AND SIMULATION MODEL}

The simulation of the maneuvering of the tanker and the buoy was carried out on Nichols Advanced Marine's engineering shiphandling simulator. The simulator was setup with ship control console and displays as follows:

(1) Normal Ship Control Console with Helm and Engine Throttle, and a button for release of the buoy. The console displays ship heading, rudder angle, engine RPM, Doppler speed, which is the ship speed over ground. Lateral speed is given at the bow and the stern.

(2) A virtual azimuth thruster control. The instruments were shown on a PC monitor, and controlled by a mouse. Thrust direction 0 to 359 degrees and throttle 0 to 100 percent.

(3) Precision Navigation Display on a monitor at the ship control console shows an icon of the ownship and the mooring buoy and response in real time to motions of ship and the mooring buoy. The display simulates the input from DGPS position of the ship and buoy. A red vertical line on the screen was the track line to the buoy. Distances from the buoy to the center of the ship were given in east-north coordinates.

(4) Nichols Advance Marine's Virtual Marine Scene generation program was used to create an Out of Window View from the bridge of the tanker with sea surface (3D waves) displayed with a monitor. The screen included a picture of the camera view from the center of the moon pool 3 meters above the bottom of the tanker. The opening in the bottom of the tanker was set to $10 \mathrm{~m}$ in diameter to give a larger viewing area, and also it is the area, where it is possible to make a fluid coupling. A red circle inside showed the $5 \mathrm{~m}$ diameter of the moon pool. Two thin cross hairs were placed over the opening to help the captain during the mating process. Furthermore, a third picture was provided that showed an underwater camera side view of the buoy and tanker with the moon pool. The last viewing point was detached from the ship and fixed in space, the other two viewing points were moving with the ship. This last picture was only used for the check-out of the simulations and in the familiarization of the mating procedure. In the evaluation simulation phase the picture was changed to an outside view of the tanker. The viewing point was located north of the buoy.

(5) The buoy was white on the top with four concentric circles in black color. The innermost circle was 6 meter in diameter and 1 meter wide. On the edge of the buoy was a 12 sided polygon to show the fender on the top of the buoy. In the center of the buoy was a cross, one by one meter, for the identification of the center of the buoy. 


\subsection{Mathematical model}

The simulator uses a physics-based 4-degree of freedom (4-DOF) hydrodynamic model for the maneuvering of the ship, which has been used for shiphandling bridge training simulators over many years in the U.S. and abroad. The simulator includes wave drift, wind and current forces. Furthermore, the hydrodynamic model contains a model for the Elliot White Gill thruster and machinery dynamics for a slow speed diesel engine.

\subsection{Ship Characteristics}

The selected ship is a tanker with the following characteristics:

Table 1 Ship Characteristics

\begin{tabular}{|l|c|}
\hline Length, overall, $\mathrm{m}$ & 289.59 \\
\hline Length, b.p., $\mathrm{m}$ & 278.90 \\
\hline Beam, mld., $\mathrm{m}$ & 44.20 \\
\hline Draft, $\mathrm{m}$ & 13.72 \\
\hline Propulsion System & Direct Diesel \\
\hline Displacement, tons & 130287 \\
\hline Maximum Speed, knots & 16.00 \\
\hline RPM & 100.00 \\
\hline
\end{tabular}

The main propulsion plant is a slow speed diesel engine, direct reversing, with a minimum engine speed of about 25 RPM, which will give the ship a speed of 4 knots. The number of starts is limited to 12 per hour. Definitive maneuvers were performed for turning, zig-zag, and stopping. A listing of the maneuvering characteristics are shown in Table 2, and plots of the maneuvers are shown in APPENDIX I.

Table 2 Maneuvering Characteristics for tanker

\begin{tabular}{|c|r|}
\hline Turning Maneuver & \\
\hline Approach speed [knots] & 16.00 \\
\hline Rudder angle [degrees] & $35 \mathrm{R}$ \\
\hline Non dimensional advance & 3.28 \\
\hline Non dimensional transfer & 1.31 \\
\hline Non dimensional steady diameter & 2.10 \\
\hline Non dimensional tactical diameter & 3.07 \\
\hline Time at 90 degrees & 155.16 \\
\hline Time at 180 degrees & 324.58 \\
\hline 20/20 Zigzag & 12.00 \\
\hline Approach speed [knots] & 15.15 \\
\hline First overshoot angle [degrees] & 17.38 \\
\hline Second overshoot angle [degrees] & 15.62 \\
\hline Third overshoot angle [degrees] & 14.31 \\
\hline Fourth overshoot angle [degrees] &
\end{tabular}




\begin{tabular}{|c|r|}
\hline Stopping Maneuver & \\
\hline Approach speed [knots] & 16.00 \\
\hline Time to stop ship [sec] & 886.25 \\
\hline Non dimensional advance & 11.55 \\
\hline Non dimensional transfer & 0.08 \\
\hline Final heading change [degrees] & 46.19 \\
\hline
\end{tabular}

The ship was equipped with an Elliot White Gill thruster, which uses a pump to draw water from the moon pool when the buoy is detached from the tanker. When the Gill thruster runs dry from the moon pool a valve will open and supply the thruster from the sea without loosing its thrust. The Gill thruster is able to rotate 360 degrees and deliver a maximum of 15.3 tons thrust. After one day of simulation, it was judged to be too small, and the maximum thrust was increased to 20 tons. This is the largest unit available from the Gill thruster catalogue.

\subsection{Wave Motions of Tanker}

6-DOF ship motions of the tanker were calculated by using a linear strip theory approach to determine the response amplitude operators (RAO's) and phase angles relative to the waves in the frequency domain. The calculations follow normal seakeeping practice using a strip theory method according to Salvesen, et al., published in the Transactions of SNAME 1970. Added mass and damping are approximated by curve fits to model tests by Vugts, "The Hydrodynamic Coefficients for Swaying, Heaving, and Rolling Cylinders in a Free Surface. The on-line calculated frequency domain ship motion RAO's are converted into time domain motions by multiplying each regular wave component (10) of the sea spectrum with the appropriate RAO values and adding the results together. The spectrum is of the Bretschneider type, which matches the visual system.

The 6-DOF RAO's are shown in APPENDIX II. The RAO's are shown for 180, 150, $120,90,60,30,0$ degrees heading, where 180 degrees is head sea, and 0 degrees is following sea. Each RAO is shown for wave length to ship length ratio from 0.4 to 5.0. The simulation program calculates the RAO's online for the actual wave history due to heading, ship speed and wave frequency. 


\subsection{Buoy Model}

The suction mooring buoy main characteristics are two cylinders on top of each other with the following dimensions:

$\begin{array}{lc}\text { Diameter of top cylinder [m] } & 27.0 \\ \text { Height of top cylinder [m] } & 2.0 \\ \text { Diameter of lower cylinder [m] } & 6.0 \\ \text { Height of lower cylinder [m] } & 7.0 \\ \text { The lower cylinder is connected to the top cylinder by a cone. } \\ \text { Total height of the buoy [m] } & 10.0 \\ \text { Displacement [tons] } & 800.0\end{array}$

The buoy was handled as a traffic ship in the simulation, which means that the position will be displayed together with motions due to waves. The buoy will have different motion characteristics in the initial location, which was set to be $20 \mathrm{~m}$ below the water surface (or $6 \mathrm{~m}$ below the keel) than when the buoys moves closer to the bottom of the tanker due to changes in wave motion and mass of the buoy.

A 6-DOF motion program was developed for the buoy motion using a similar linear strip theory approach as described in Section 3.3. However, there is no free surface effect for the buoy since it is submerged all the time. The restoring forces all come from the mooring system. The mass of the buoy was kept constant. The wave excitation forces were made a function of the water depth. Furthermore, the roll and sway RAO's for beam sea are equal to the pitch and surge RAO's for head sea, respectively. The yaw RAO is put to zero since the buoy is free to rotate. When the buoy raises up to $1.5 \mathrm{~m}$ below the tanker bottom the buoy pitch and roll motion are made equal to pitch and roll motion of the tanker, respectively. This is consistant with model test videos provided by Suction Mooring Technology. The RAO's for water depth of 20 meter are shown in APPENDIX III. The RAO's are shown for 180, 150, 120, 90, 60, 30, 0 degrees heading, where 180 degrees is head sea, and 0 degrees is following sea. Each RAO is shown for wave length to ship length ratio from 0.4 to 5.0. The simulation program calculates the RAO online for the immediate wave history due to heading, ship speed, wave frequency. As the buoy moves up towards the bottom of the tanker, the RAO's change as a function of water depth. This change may be insignificant as the transition only takes about 12 seconds, it was not included.

\subsection{The Mating Process}

The mating process is from the time the shiphandler pushes the release button until the buoy is located at the bottom of the tanker. The transition time is about 13 seconds. The simulator uses two input functions, buoy distance and buoy velocity as function of buoy time from release. The first two thirds of the distance from initial location to the tanker the simulator uses interpolation of the first curve. The rest of the vertical transition moves the buoy location by buoy velocity times the simulation time step. The second 
method is added because the tanker and buoy motions change the distance between them due to wave motion during the time of transition, and could be longer than the distance in calm water.

The ship console was equipped with a release button.

The distance between the center of the buoy and the moon pool was calculated, and if the distance was greater than 5 meter the mating process was set to fail. In a similar manner if the speed of the tanker during mating became more than 2 knots the buoy would not hold the tanker and the mooring attempt fails.

\subsection{Buoy Mooring System}

When the buoy is mated the tanker is moored to the buoy. The mooring forces due to excursion of the tanker and buoy were calculated by interpolating a lookup table.

\subsection{Scenario}

The simulations were carried out in an open ocean scenario with 50 meter of water depth. The scenario did not contain any navigational aids.

\section{ENVIRONMENT}

The environment consists of contributions from current, wind, and waves, which are included in the maneuvering model.

\subsection{Current Data}

The maneuvering equations are written in terms of relative velocities and yaw rate to allow the introduction of a current velocity, which may vary along the length of the ship. The relative longitudinal and lateral current velocity in the body axis system are calculated and used in the maneuvering equations.

\subsection{Wind Data}

The wind velocity is assumed to consist of two components having the same direction: a steady or mean velocity and a random, time varying velocity, which is described by the Davenport wind spectrum. The unsteady wind velocity is divided into ten bands of equal energy and by representing each energy band by a sinusoidal velocity having one tenth of the total energy.

The input wind velocity is assumed for 10 meter above the sea level. The wind velocity is adjusted to the height of the wind center of the ship due to the boundary layer. 
The wind related data for the tanker are the following:

Front wind area $\left[\mathrm{m}^{2}\right]$ :

739.2

Lateral wind area $\left[\mathrm{m}^{2}\right]$ :

3572.6

Wind center above water surface $[\mathrm{m}] \quad 6.33$

A 50 knot head wind will result in about 25 tons of nominal longitudinal force.

\subsection{Wave Data}

Ocean waves in a fully or partially developed sea creates two types of forces on the ship:

- First order oscillatory forces centered on the dominant wave encounter frequency.

- Second order drift forces, which consist of a steady (time independent) component and a low frequency component.

The first order forces are much larger in magnitude than the second order forces. The motions that results from the first order forces are included in the 6-DOF ship motion modeling described in Section 3.3 above. The second order wave drift forces are calculated and included as external forces in the simulation.

A calculation of the average drift forces was made based on RAO's from Dr. J.A. Pinkster, Low Frequency Second Order Wave Exciting Forces on Floating Structures, Publication No. 650, NSMB, Wageningen, Netherlands. The head sea RAO's were multiplied with the spectrum used in the simulator, and the results showed a reasonable agreement with simulated values as shown on the Figure 1 below.

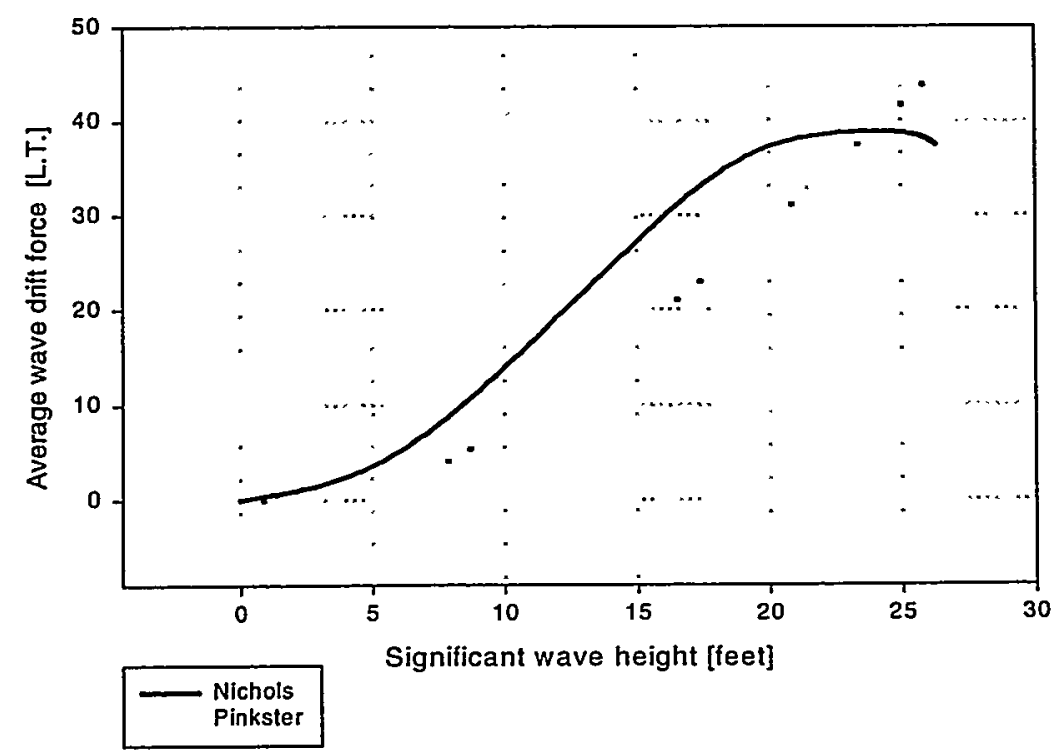

Figure 1 Wave Drift Forces, Head Sea 


\section{REAL-TIME SIMULATIONS}

Twenty five real-time simulation runs, run \#10 to run \#34, were carried out with increasing severity of wind, waves, and current, see Table 3. All simulation runs were carried out twice for the same weather condition, except for one condition, run \# 12, a 2 knots cross current with no wind and waves.

In preparation of real-time simulations, fast-time simulations were run simulating definitive maneuvers, see Section 3.2. Furthermore, deceleration tests, and ship speeds for different weather conditions were simulated. Three days were spent on familiarization of ship behavior with a number of environmental conditions trying. different maneuvering techniques. Different approaches were tried, and the learning continued to the end of all the simulation runs, and more may be learned from continued simulation runs.

The approach was to start about 1500 to $2000 \mathrm{~m}$ south of the buoy depending on the weather condition. The simulation was started with a ship speed of 5 knots, and then the ship was decelerated down to about 0.2 knots when the moon pool of the ship arrived at the buoy, or the buoy came into camera view. Normally the engine was not stopped or reversed except in very few cases. The thruster was used as a braking force only in the initial runs, run \# 10 to run \# 23. Later the thruster was used as the only control device when hovering over the buoy after an equilibrium heading was found that approximately balances the forces. By using this technique the captain was able to stay over the buoy for a long time at almost zero speed (about 0.2 knots). This would allow the captain to make more than one mating attempt if necessary. The hydrostatic buoy allows for mating at ship speed of less than 2 knots. The different technique of using a high speed ( 0.5 to 1.0 knots) and then miss the mating would make it necessary to make a new approach might be more time consuming and less precise. The low speed approach was possible in all weather conditions.

In APPENDIX IV for each simulation run is a the captain's commentary, a log table of position, RPM, Doppler speed, heading, turn rate, rudder angle, Gill thruster, wind and current data, and finally a track plot of the last 15 minutes of the simulation run.

Table 3 Real-Time Simulations

\begin{tabular}{|c|c|c|c|c|c|c|c|c|c|}
\hline \multicolumn{3}{|c|}{ Simulation } & \multicolumn{2}{c|}{ Wind } & \multicolumn{2}{c|}{ Waves } & \multicolumn{2}{c|}{ Current } & Mating \\
\hline $\begin{array}{c}\text { Run } \\
\text { Time }\end{array}$ & $\begin{array}{c}\text { Length } \\
{[\mathrm{secs}]}\end{array}$ & $\begin{array}{c}\text { Speed } \\
{[\mathrm{knots}]}\end{array}$ & $\begin{array}{c}\text { Direction } \\
\text { [degrees] }\end{array}$ & $\begin{array}{c}\text { Significant } \\
\text { Height } \\
{[\mathrm{m}]}\end{array}$ & $\begin{array}{c}\text { Direction } \\
{[\text { degrees] }}\end{array}$ & $\begin{array}{c}\text { Speed } \\
{[\mathrm{knots}]}\end{array}$ & $\begin{array}{c}\text { Direction } \\
\text { [degrees] }\end{array}$ & $\begin{array}{c}\text { Center to } \\
\text { Center dist } \\
{[\mathrm{m}]}\end{array}$ \\
\hline 10 & $8: 25$ & 1808 & 0 & 0 & 0 & 0 & 0 & 0 & 4.1 \\
\hline 11 & $9: 25$ & 2156 & 0 & 0 & 0 & 0 & 0 & 0 & 0.9 \\
\hline 12 & $10: 10$ & 1650 & 0 & 0 & 0 & 0 & 2 & 180 & 0.2 \\
\hline 13 & $10: 45$ & 1785 & 20 & 0 & 3 & 180 & 0 & 0 & 1.25 \\
\hline 14 & $11: 30$ & 3310 & 20 & 0 & 3 & 180 & 0 & 0 & 2.9 \\
\hline 15 & $13: 30$ & 3328 & 20 & 45 & 3 & 225 & 1 & 180 & 3.1 \\
\hline 16 & $14: 30$ & 2940 & 20 & 45 & 3 & 225 & 1 & 180 & 0.4 \\
\hline 17 & $15: 35$ & 2872 & 20 & 90 & 3 & 270 & 1 & 180 & 1.1 \\
\hline 18 & $16: 30$ & 2812 & 20 & 90 & 3 & 270 & 1 & 180 & 3.8 \\
\hline 19 & $8: 00$ & 2205 & 25 & 0 & 5 & 180 & 0 & 0 & 2.6 \\
\hline 20 & $8: 45$ & 2280 & 25 & 0 & 5.1 & 180 & 0 & 0 & 1.8 \\
\hline
\end{tabular}




\begin{tabular}{|c|c|c|c|c|c|c|c|c|c|}
\hline 21 & $9: 30$ & 3664 & 25 & 45 & 5 & 225 & 1 & 180 & 2.5 \\
\hline 22 & $10: 50$ & 1665 & 25 & 45 & 5 & 225 & 1 & 180 & 2.5 \\
\hline 23 & $11: 30$ & 2160 & 25 & 90 & 5 & 270 & 1 & 180 & 2.0 \\
\hline 24 & $14: 00$ & 2235 & 25 & 90 & 5 & 270 & 1 & 180 & 2.5 \\
\hline 25 & $14: 40$ & 3465 & 25 & 90 & 5 & 270 & 2 & 180 & 1.8 \\
\hline 26 & $16: 25$ & 3912 & 25 & 90 & 5 & 270 & 2 & 180 & 1.5 \\
\hline 27 & $9: 25$ & 5704 & 40 & 0 & 8 & 180 & 0 & 0 & 5.1 \\
\hline 28 & $9: 50$ & 2145 & 40 & 0 & 8 & 180 & 0 & 0 & 2.8 \\
\hline 29 & $10: 40$ & 2595 & 40 & 0 & 8 & 180 & 1 & 135 & 1.8 \\
\hline 30 & $11: 30$ & 1530 & 40 & 0 & 8 & 180 & 1 & 135 & 2.0 \\
\hline 31 & $13: 15$ & 4566 & 40 & 0 & 8 & 180 & 1 & 90 & 1.9 \\
\hline 32 & $14: 30$ & 2760 & 40 & 0 & 8 & 180 & 1 & 90 & 1.5 \\
\hline 33 & $15: 25$ & 3065 & 40 & 0 & 8 & 180 & 2 & 90 & 1.9 \\
\hline 34 & $16: 25$ & 3540 & 40 & 0 & 8 & 180 & 2 & 90 & 1.7 \\
\hline
\end{tabular}

The captain was able to make a mating of the buoy within the set criteria of $5 \mathrm{~m}$ from the center of the moon pool to the center of the buoy in all simulation runs except one, run \# 27 (5.1 m off). This simulation run \# 27 was very long, 5704 seconds, and it was stopped before a successful mating was achieved. If the captain had used a little longer time, he would have been able to make a closer mating by repeating the mating.

There is no correlation that shows that the second run with the same weather condition was shorter than the first run. However, in eight out of the twelve repeat runs the second mating was closer or equal to the target than the first one.

The results of all the run used for recording gave the following results:
Minimum distance $\quad 0.2 \mathrm{~m}$
Maximum distance $5.1 \mathrm{~m}$
Average distance $\quad 2.1 \mathrm{~m}$
Standard deviation $1.1 \mathrm{~m}$

The longer simulation time for the higher weather conditions was affected by an effort by the captain to make the mating within the moon pool diameter of $5 \mathrm{~m}$, which was achieved with patience and a large portion of ship handling skills.

In all the recorded simulation runs (run \# 10 to run \# 34), a 20 tonnes static thrust bow thruster was used. The captain felt that the rudder rate, which is the minimum approved by the classification societies (2.33 degrees/min), was too slow. A higher rudder rate (e.g. 3.33 degrees $/ \mathrm{min}$ ) may improve the performance, but it was not tested. A high lift rudder might also improve the performance. The ship, as expected, was very slow to respond when the rudder was hard over.

\section{AN EXPERIENCED PILOT'S EVALUATION}

The following is the opinion of Captain Atkinson:

During the simulation runs of the week of February 1 to 5, 1999, I found the Nichols simulator to be very realistic. I was particularly pleased with how the movement typified how I would have expected a 150.000 TWD tanker to move in the sea states that were 
simulated. Maneuvering was as anticipated for the type, size and draft of ship simulated, and when "hovering" with the moon pool over the buoy, was very much as I would expect with a real ship of the size simulated under the simulated conditions. I found it impossible to hold any balance of forces in equilibrium, as the ship not only moved continuously, but tended to accelerate and decelerate variously with the wave history based on the simulated wave spectra.

For the very stringent requirement of positioning the moon pool over the buoy and to achieve mating within a 5 meter radius of the buoy center, with the buoy also in varying cyclical motion, I found the minimal rudder rate of the simulated ship, while typical of some such ships, not high enough to satisfy me. That is not a criticism of the simulator, but it does relate to desirable design feature of a ship that might be either built or converted to serve as a shuttle vessel for suction mooring.

This week of simulation runs has been a very challenging and satisfying learning experience.

\section{SUMMARY AND CONCLUSIONS}

A simulation study has been carried out with a man-in-the-loop to study how successfully an experienced pilot could position a 150,000 TWD tanker above a hydrostatic buoy and perform the suction mooring mating. A representative 150.000 TWD tanker in a partly loaded condition was selected for use in the study. Definitive maneuvers were performed (turning, zig-zag, and stopping) to check that the mathematical model, used in the simulation study, had realistic maneuvering characteristics.

The most significant conclusions and recommendations include:

- The captain found the simulations with the 150.000 TWD tanker very realistic.

- The captain was able to move the moon pool of the ship over the buoy and keep it there by using small adjustments, waiting for small waves to occur.

- The captain was able to mate the buoy to the tanker within a prescribed limit of $5 \mathrm{~m}$ between the center of the moon pool and the center of the buoy in the majority of test runs, and only in one case, he exceeded $5.0 \mathrm{~m}$ with $5.1 \mathrm{~m}$. This was possible in all the tested weather conditions up to $8 \mathrm{~m}$ significant wave height together with 40 knots wind and 2 knots of current. The wind and waves were collinear. The current direction was chosen for the following three configurations:

1. Collinear with wind and waves.

2. 45 degrees off the wind and wave direction.

3. 90 degrees off the wind and wave direction.

In 75 percent of the repeat runs, the captain was able to mate the buoy to the tanker with a larger or equal accuracy than the previous run. 
- An additional camera mounted in front of the moon pool is recommended. It might give the pilot more time to make appropriate maneuvers.

- A faster rudder rate is recommended, increase the test rate of 2.33 degrees/sec to 3.33 degrees/sec. The higher rate was not tested, but is believed to result in substantial improvement.

- A high lift rudder would be recommended. The ship was very slow to respond to hard over rudder. This change might reduce the mating time.

- The Elliot White Gill thruster used had a maximum static thrust of 20 tons, which is a minimum for proper performance for this type and size of ship. 


\section{APPENDIXI}

\section{Maneuvering Characteristics for Tanker}

Turning Circle Trajectory

Left 20-20 Zig-Zag Maneuver

Stopping Maneuver Trajectory

Stopping Characteristics 


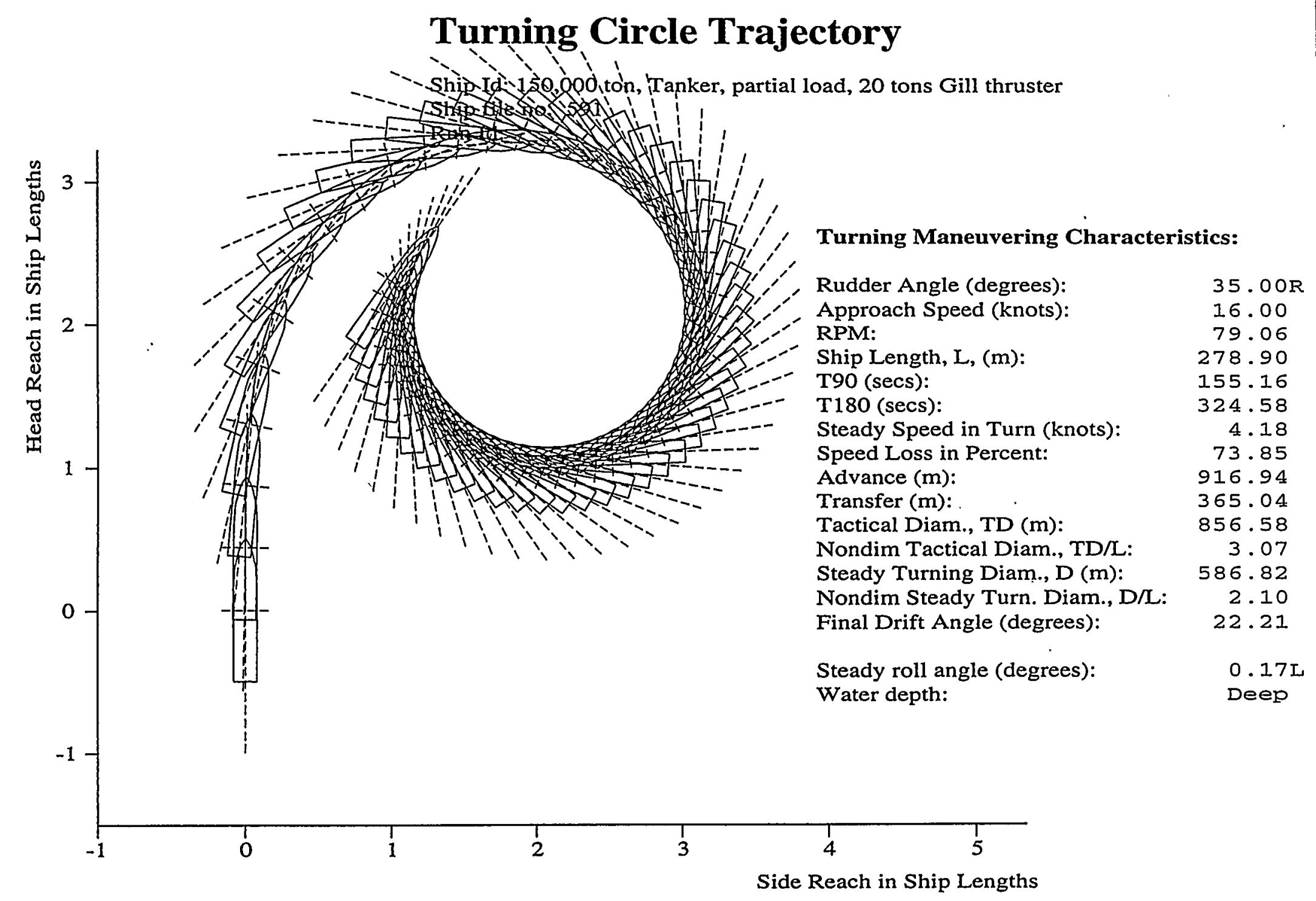

Date: 16-Feb-99 Time: 11:26:41 


\section{Left 20-20 Zig-Zag Maneuver}

Ship Id: 150,000 ton, Tanker, partial load, 20 tons Gill thruster Ship file no: 591

Run Id:

Zig-Zag Maneuvering Characteristics:

Approach Speed (knots)

RPM:

70.09

Ship Length, L, (m):

278.90

Time to reach head. change (sec):

89.47

Overshoot Angle (degrees):

15.15

Oversh):

35.15

Width of Path at Execute (m):

Overshoot Width of Path $(\mathrm{m})$ :

31.05

Total Width of Path (m)

575.34

Reach (seconds):

606.39

2nd Overshoot Heading Angle (deg): $\quad 17.38$

3rd Overshoot Heading Angle (deg.): 15.62

Period (seconds):

603.54

4th Overshoot Heading Angle (deg.):

14.31

Water depth:

Deep

Heading Angle

0

要

$20-1$

$-40$

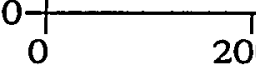

200

400

600

800

1000

1200

Time in Seconds

Date: 16-Feb-99 Time: 11:30:39 


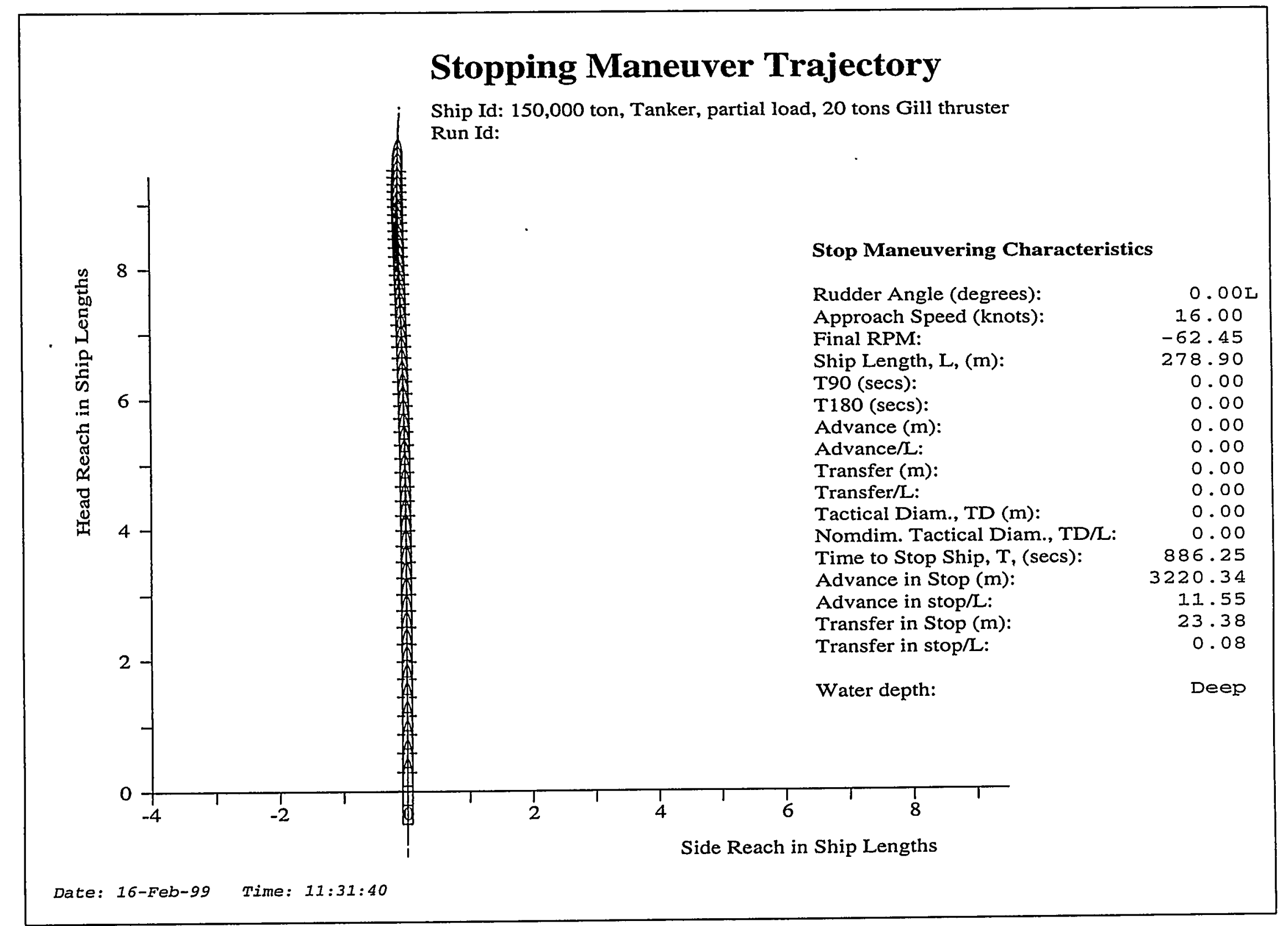




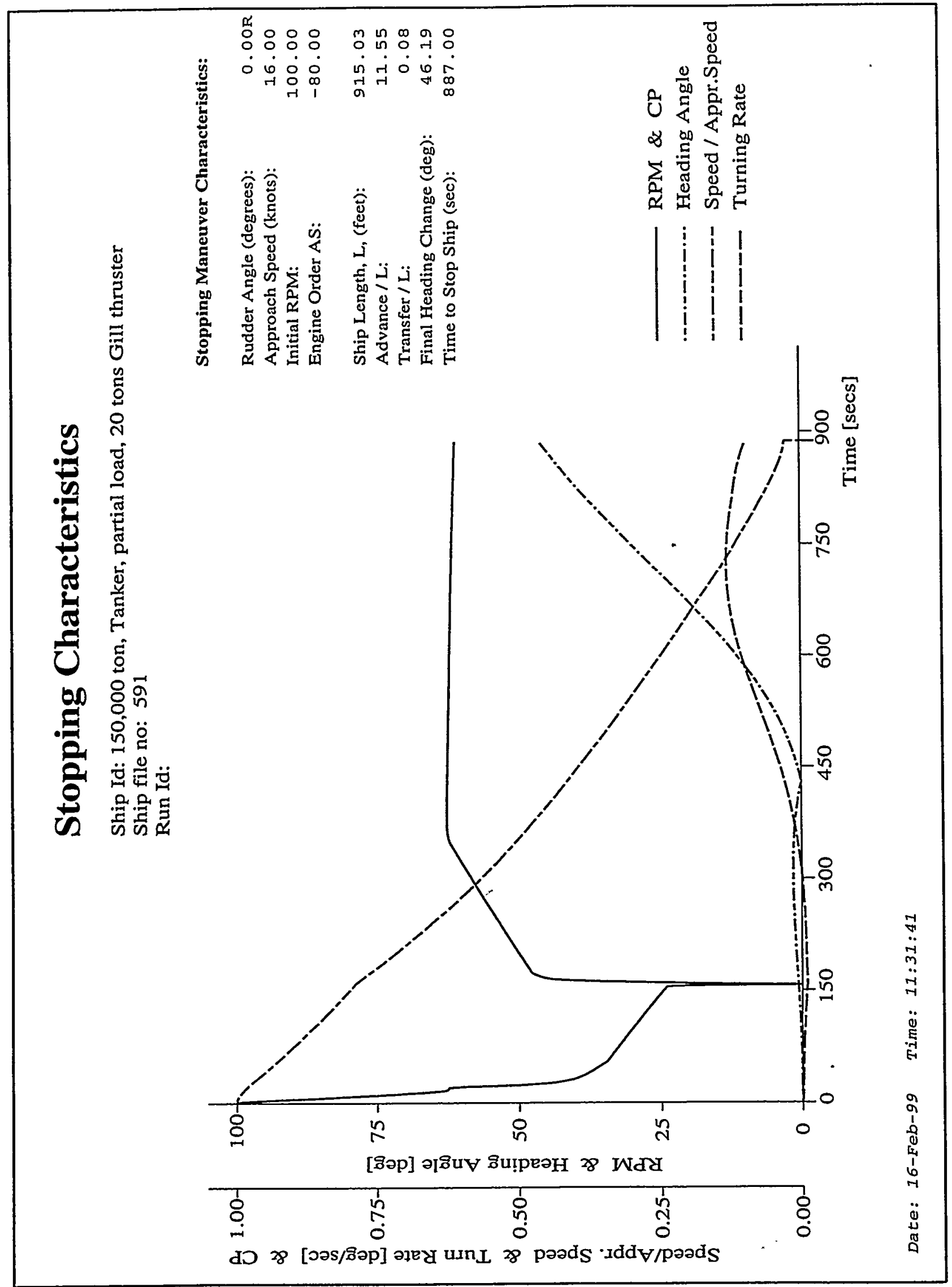




\section{APPENDLX II}

\section{RAO's for Tanker}

Heading $180,150,120,90,60,30,0$ degrees at zero ship speed 
Draft to depth ratio, $h / t=3.64$

Non Dimensional Ship Motions

Ship file: 0591

Shipid: 150,000 ton, Tanker, partial load, 20 tons Gill thruster

Heading (degrees) $\quad=180.0$

Ship speed (knots). $=0.0$

\begin{tabular}{|c|c|c|c|c|c|c|c|c|}
\hline Wave L./ & Omegae & Omega & $\mathrm{He}$ & ave & & tch & Su & ge \\
\hline Ship L. & $2 \mathrm{pi} / \mathrm{s}$ & $2 \mathrm{pi} / \mathrm{s}$ & $z / h$ & Phase & Theta/k & th Phase & $x / h$ & Pha \\
\hline 0.40 & 0.741 & 0.741 & 0.04 & -179.87 & 0.02 & 9.27 & 0.01 & \\
\hline 0.50 & 0.6 & 0.657 & 0.09 & 113.22 & 0.05 & -28.85 & 0.07 & \\
\hline 0.60 & 0.593 & 0.593 & 0.16 & 102.14 & 0.05 & -85.58 & 0.08 & 97. \\
\hline 0.80 & 0.495 & 0.495 & 0.12 & 59.71 & 0.29 & -112.33 & 0.05 & -141.8 \\
\hline 1.00 & 0.423 & 0.423 & 0.25 & 10.96 & 0.47 & -104.20 & 0.22 & -104 \\
\hline 1.20 & 0.368 & 0.368 & 0.40 & 3.31 & 0.58 & -99.93 & 0.40 & -96 \\
\hline 1.40 & 0.324 & 0.324 & 0.51 & 1.43 & 0.66 & -97.55 & 0.59 & -92. \\
\hline 1.60 & 0.289 & 0.289 & 0.59 & 0.78 & 0.71 & -96.09 & 0.77 & -88.9 \\
\hline 1.80 & 0.261 & 0.261 & 0.65 & 0.49 & 0.75 & -95.11 & 0.94 & -86.2 \\
\hline 2.00 & 0.237 & 0.237 & 0.70 & 0.34 & 0.78 & -94.41 & 1.00 & -83. \\
\hline 2.50 & 0.193 & 0.193 & 0.78 & 0.17 & 0.84 & -93.33 & 1.00 & -79. \\
\hline 3.00 & 0.162 & 0.162 & 0.83 & 0.10 & 0.87 & -92.69 & 1.00 & -74 \\
\hline 3.50 & 0.140 & 0.140 & 0.86 & 0.07 & 0.89 & -92.26 & 1.00 & -71 \\
\hline 4.00 & 0.123 & 0.123 & 0.88 & 0.05 & 0.91 & -91.96 & 1.00 & -67. \\
\hline 5.00 & 0.099 & 0.099 & 0.91 & 0.00 & 0.93 & -91.58 & 1.00 & -61. \\
\hline
\end{tabular}


Non Dimensional Ship Motions

ship file: 0591

Shipid: 150,000 ton, Tanker, partial load, 20 tons Gill thruster

Heading (degrees) $=150.0$

Ship speed (knots) $=0.0$

\begin{tabular}{|c|c|c|c|c|c|c|c|c|}
\hline Wave I./ & Omegae & Omega & Hea & & & itch & Su & cge \\
\hline Ship I. & $2 \mathrm{p} i / \mathrm{s}$ & $2 p i / s$ & $2 / h$ & Phase & Theta/k & ch Phase & $x / h$ & Phase \\
\hline 0.40 & 0.741 & 0.741 & 0.04 & 136.52 & 0.02 & -29.34 & 0.02 & 81.22 \\
\hline 0.50 & 0.657 & 0.657 & 0.10 & 90.07 & 0.03 & -79.19 & 0.05 & 88.74 \\
\hline 0.60 & 0.593 & 0.593 & 0.13 & 75.55 & 0.11 & -129.92 & 0.04 & 110.92 \\
\hline 0.80 & 0.495 & 0.495 & 0.20 & 13.34 & 0.37 & -115.10 & 0.10 & -110.60 \\
\hline 1.00 & 0.423 & 0.423 & 0.38 & 1.68 & 0.50 & -104.65 & 0.26 & -98.16 \\
\hline 1,20 & 0.368 & 0.368 & 0.51 & 0.18 & 0.58 & -99.87 & 0.42 & -92.71 \\
\hline 1.40 & 0.324 & 0.324 & 0.60 & -0.05 & 0.63 & -97.33 & 0.58 & -88.94 \\
\hline 1.60 & 0.289 & 0.289 & 0.67 & -0.05 & 0.67 & -95.81 & 0.73 & -85.91 \\
\hline 1.80 & 0.261 & 0.261 & 0.72 & -0.03 & 0.69 & -94.82 & 0.88 & -83.29 \\
\hline 2.00 & 0.237 & 0.237 & 0.75 & -0.01 & 0.71 & -94.13 & 1.00 & -80.95 \\
\hline 2.50 & 0.193 & 0.193 & 0.82 & 0.01 & 0.75 & -93.08 & 1.00 & -75.82 \\
\hline 3.00 & 0.162 & 0.162 & 0.85 & 0.02 & 0.77 & -92.46 & 1.00 & -71.37 \\
\hline 3.50 & 0.140 & 0.140 & 0.88 & 0.02 & 0.78 & -92.07 & 1.00 & -67.37 \\
\hline 4.00 & 0.123 & 0.123 & 0.90 & 0.02 & 0.79 & -91.78 & 1.00 & -63.72 \\
\hline 5.00 & 0.099 & 0.099 & 0.92 & -0.01 & 0.81 & -91.44 & 1.00 & -57.27 \\
\hline
\end{tabular}

$\begin{array}{lrrrr}\text { Wave L./ Omegae } & \text { Omega } & \text { Sway } & \text { Roll } & \text { Yaw } \\ \text { Ship L. } & 2 \mathrm{p} i / \mathrm{s} & 2 \mathrm{p} i / \mathrm{s} & \mathrm{y} / \mathrm{h} & \text { Phase } \mathrm{fi} / \mathrm{kh} \text { Phase psi/kh phase }\end{array}$

$\begin{array}{rrrrrrrrr}0.40 & 0.741 & 0.741 & 0.02 & -50.71 & 0.03 & 1.02 & 0.01 & 1.34 \\ 0.50 & 0.657 & 0.657 & 0.04 & -7.30 & 0.10 & 15.58 & 0.01 & 119.06 \\ 0.60 & 0.593 & 0.593 & 0.03 & 25.52 & 0.11 & 28.17 & 0.04 & 165.96 \\ 0.80 & 0.495 & 0.495 & 0.05 & 87.87 & 0.11 & -148.95 & 0.12 & 174.09 \\ 1.00 & 0.423 & 0.423 & 0.11 & 84.83 & 0.33 & -114.53 & 0.20 & 177.49 \\ 1.20 & 0.368 & 0.368 & 0.20 & 85.69 & 0.44 & -100.92 & 0.27 & 179.69 \\ 1.40 & 0.324 & 0.324 & 0.29 & 87.30 & 0.48 & -94.40 & 0.32 & -179.05 \\ 1.60 & 0.289 & 0.289 & 0.36 & 88.30 & 0.49 & -91.33 & 0.38 & -178.05 \\ 1.80 & 0.261 & 0.261 & 0.43 & 89.00 & 0.49 & -89.46 & 0.43 & -177.29 \\ 2.00 & 0.237 & 0.237 & 0.50 & 89.41 & 0.49 & -88.36 & 0.48 & -176.62 \\ 2.50 & 0.193 & 0.193 & 0.66 & 89.37 & 0.49 & -88.01 & 0.61 & -175.62 \\ 3.00 & 0.162 & 0.162 & 0.82 & 88.65 & 0.48 & -89.15 & 0.73 & -174.74 \\ 3.50 & 0.140 & 0.140 & 0.96 & 88.84 & 0.47 & -88.78 & 0.85 & -173.31 \\ 4.00 & 0.123 & 0.123 & 1.10 & 88.98 & 0.46 & -88.50 & 0.97 & -171.93 \\ 5.00 & 0.099 & 0.099 & 1.34 & 89.18 & 0.44 & -88.12 & 1.19 & -169.27\end{array}$

Viscous contributions for roll motion, Kappa $4=1.50$ 
Non Dimensional Ship Motions

Ship Eile: 0591

Shipid: 150,000 ton, Tanker, partial load, 20 tons Gill thruster

Heading (degrees) $\quad=120.0$

Ship speed (knots) $=0.0$

\begin{tabular}{|c|c|c|c|c|c|c|c|c|}
\hline ave & Omegae & Omega & Hea & & & $t c h$ & $\mathrm{Su}$ & ge \\
\hline iip $\mathrm{L}$. & $2 \mathrm{pi} / \mathrm{s}$ & $2 \mathrm{pi} / \mathrm{s}$ & $2 / h$ & Phase & Theta/ & h Phase & $x / h$ & Phase \\
\hline 0.40 & 0.741 & 0.741 & 0.06 & 3.44 & 0.04 & -153.74 & 0.01 & 65.12 \\
\hline 0.5 & 0.657 & 0.657 & 0.16 & -38.53 & 0.13 & -163.02 & 0.02 & -104.62 \\
\hline 0.60 & 0.593 & 0.593 & 0.37 & -38.38 & 0.26 & -148.14 & 0.06 & -98.78 \\
\hline 0.80 & 0.495 & 0.495 & 0.66 & -16.76 & 0.40 & -117.78 & 0.16 & -91.18 \\
\hline 1.00 & 0.423 & 0.423 & 0.75 & -7.60 & 0.43 & -105.06 & 0.26 & -85.83 \\
\hline 1.20 & 0.368 & 0.368 & 0.80 & -3.96 & 0.44 & -99.60 & 0.35 & -81.49 \\
\hline 1.40 & 0.3 & 0.324 & 0.82 & -2.28 & 0.44 & -96.82 & 0.43 & -77.73 \\
\hline 1.60 & 0.289 & 0.289 & 0.84 & -1.40 & 0.44 & -95.22 & 0.52 & -74.35 \\
\hline 1.80 & 0.261 & 0.261 & 0.86 & -0.90 & 0.45 & -94.21 & 0.61 & -71.26 \\
\hline 2.00 & 0.237 & 0.237 & 0.87 & -0.61 & 0.45 & -93.53 & 0.71 & -68.40 \\
\hline 2.50 & 0.193 & 0.193 & 0.89 & -0.27 & 0.46 & .55 & 0.96 & -62.02 \\
\hline 3.0 & 0.162 & 0.162 & 0.91 & -0.13 & 0.46 & -92.00 & 1.00 & -56.53 \\
\hline 3.50 & 0.140 & 0.140 & 0.92 & -0.07 & 0.47 & -91.65 & 1.00 & -51.77 \\
\hline 4.00 & 0.123 & 0.123 & 0.93 & -0.04 & 0.47 & -91.42 & 1.00 & -47.62 \\
\hline 5.00 & 0.099 & 0.099 & 0.94 & -0.04 & 0.47 & $-91: 14$ & 1.00 & -40.80 \\
\hline
\end{tabular}

\begin{tabular}{|c|c|c|c|c|c|c|c|c|}
\hline Wave & Omegae & Omega & Swa & & & 11 & & Law \\
\hline Ship L. & $2 \mathrm{pi} / \mathrm{s}$ & $2 \mathrm{pi} / \mathrm{s}$ & $y / h$ & Phase & $\mathrm{fi} / \mathrm{kh}$ & Phase & $\mathrm{psi} / \mathrm{kh}$ & Phase \\
\hline 0.40 & 0.741 & 0.741 & 0.04 & 54.53 & 0.03 & 32.66 & 0.05 & 171.49 \\
\hline 0.5 & 0.657 & 0.657 & 0.09 & 117.94 & 0.16 & -176.59 & 0.10 & 174.35 \\
\hline 0.60 & 0.593 & 0.593 & 0.14 & 130.53 & 0.46 & -161.15 & 0.15 & 176.59 \\
\hline 0.80 & 0.495 & 0.495 & 0.18 & 106.84 & 1.03 & -135.35 & 0.23 & 179.68 \\
\hline 1.00 & 0.423 & 0.423 & 0.33 & 88.10 & 1.27 & -112.30 & 0.29 & -177.95 \\
\hline 1.20 & 0.368 & 0.368 & 0.50 & 87.32 & 1.25 & -100.09 & 0.34 & -176.45 \\
\hline 1.40 & 0.324 & 0.324 & 0.64 & 88.42 & 1.17 & -94.05 & 0.38 & -175.28 \\
\hline 1.60 & 0.289 & 0.289 & 0.77 & 89.32 & 1.09 & -90.95 & 0.43 & -174.17 \\
\hline 1.80 & 0.261 & 0.261 & 0.88 & 89.92 & 1.04 & -89.09 & 0.48 & -173.24 \\
\hline 2.00 & 0.237 & 0.237 & 0.99 & 90.24 & 1.00 & -88.04 & 0.52 & -172.37 \\
\hline 2.50 & 0.193 & 0.193 & 1.25 & 90.01 & 0.93 & -87.77 & 0.65 & -170.73 \\
\hline 3.00 & 0.162 & 0.162 & 1.50 & 89.16 & 0.89 & -88.98 & 0.77 & -169.15 \\
\hline 3.50 & 0.140 & 0.140 & 1.74 & 89.28 & 0.85 & -88.64 & 0.89 & -166.99 \\
\hline 4 & 0.123 & 0.123 & 1.97 & 89.38 & 0.82 & -88.37 & 1.02 & -164.87 \\
\hline 5.00 & 0.099 & 0.099 & 2.38 & 89.50 & 0.79 & -88.02 & 1.25 & -160.79 \\
\hline
\end{tabular}

Viscous contributions for roll motion, Kappa4 $=1.50$ 
Non Dimensional Ship Motions

Ship file: 0591

Shipid: 150,000 ton, Tanker, partial load, 20 tons Gill thruster

Heading (degrees) $=90.0$

Ship speed (knots) $=0.0$

\begin{tabular}{lrlrrrrrr} 
Wave L.l & Omegae & \multicolumn{2}{c}{ Omega } & \multicolumn{2}{c}{ Heave } & \multicolumn{2}{c}{ Pitch } & \multicolumn{2}{c}{ Surge } \\
Ship L. & $2 p i / s$ & $2 p i / s$ & $z / h$ & Phase & Theta/kh Phase & \multicolumn{2}{c}{ x/h } & Phase \\
& & & & & & & & \\
0.40 & 0.741 & $0: 741$ & 0.26 & -62.53 & 0.01 & -16.11 & 0.01 & -180.00 \\
0.50 & 0.657 & 0.657 & 0.50 & -64.11 & 0.02 & 2.11 & 0.00 & -180.00 \\
0.60 & 0.593 & 0.593 & 0.78 & -51.34 & 0.03 & 41.18 & 0.00 & 0.00 \\
0.80 & 0.495 & 0.495 & 1.01 & -23.45 & 0.03 & 109.82 & 0.03 & 0.00 \\
1.00 & 0.423 & 0.423 & 1.00 & -10.94 & 0.02 & 139.74 & 0.06 & 0.00 \\
1.20 & 0.368 & 0.368 & 0.97 & -5.79 & 0.02 & 153.36 & 0.10 & 0.00 \\
1.40 & 0.324 & 0.324 & 0.95 & -3.37 & 0.01 & 160.78 & 0.14 & 0.00 \\
1.60 & 0.289 & 0.289 & 0.94 & -2.10 & 0.01 & 165.31 & 0.19 & 0.00 \\
1.80 & 0.261 & 0.261 & 0.93 & -1.38 & 0.01 & 168.34 & 0.25 & 0.00 \\
2.00 & 0.237 & 0.237 & 0.93 & -0.94 & 0.01 & 170.48 & 0.31 & 0.00 \\
2.50 & 0.193 & 0.193 & 0.93 & -0.43 & 0.01 & 173.64 & 0.50 & 0.00 \\
3.00 & 0.162 & 0.162 & 0.94 & -0.22 & 0.01 & 175.51 & 0.73 & 0.00 \\
3.50 & 0.140 & 0.140 & 0.94 & -0.12 & 0.01 & 176.65 & 1.00 & 0.00 \\
4.00 & 0.123 & 0.123 & 0.95 & -0.07 & 0.00 & 177.40 & 0.78 & 0.00 \\
5.00 & 0.099 & 0.099 & 0.95 & -0.06 & 0.00 & 178.45 & 0.51 & 0.00
\end{tabular}

\begin{tabular}{lrlrrrrrr} 
Wave L./ & Omegae & Omega & \multicolumn{2}{c}{ Sway } & \multicolumn{2}{c}{ Roll } & \multicolumn{2}{c}{ Yaw } \\
Ship L. & 2pi/s & 2pi/s & \multicolumn{1}{c}{$y / h$} & Phase & fi/kh & Phase & psi/kh & Phase \\
& & & & & & & & \\
0.40 & 0.741 & 0.741 & 0.41 & 157.02 & 0.69 & -170.48 & 0.01 & -65.05 \\
0.50 & 0.657 & 0.657 & 0.40 & 151.32 & 0.98 & -165.58 & 0.01 & -73.95 \\
0.60 & 0.593 & 0.593 & 0.37 & 147.87 & 1.37 & -157.16 & 0.01 & -85.25 \\
0.80 & 0.495 & 0.495 & 0.31 & 115.88 & 1.90 & -134.12 & 0.02 & -90.71 \\
1.00 & 0.423 & 0.423 & 0.49 & 91.85 & 1.96 & -112.13 & 0.03 & -84.66 \\
1.20 & 0.368 & 0.368 & 0.69 & 89.20 & 1.77 & -99.43 & 0.03 & -82.99 \\
1.40 & 0.324 & 0.324 & 0.84 & 89.85 & 1.56 & -93.65 & 0.04 & -82.65 \\
1.60 & 0.289 & 0.289 & 0.97 & 90.59 & 1.41 & -90.57 & 0.05 & -82.39 \\
1.80 & 0.261 & 0.261 & 1.09 & 91.06 & 1.31 & -88.72 & 0.07 & -82.56 \\
2.00 & 0.237 & 0.237 & 1.21 & 91.28 & 1.24 & -87.69 & 0.08 & -83.70 \\
2.50 & 0.193 & 0.193 & 1.50 & 90.85 & 1.13 & -87.47 & 0.13 & -87.24 \\
3.00 & 0.162 & 0.162 & 1.78 & 89.86 & 1.06 & -88.73 & 0.19 & -90.10 \\
3.50 & 0.140 & 0.140 & 2.05 & 89.89 & 1.01 & -88.42 & 0.25 & -90.07 \\
4.00 & 0.123 & 0.123 & 2.31 & 89.91 & 0.97 & -88.18 & 0.32 & -90.05 \\
$\mathbf{5 . 0 0}$ & 0.099 & 0.099 & 2.78 & 89.94 & 0.92 & -87.87 & 0.49 & -90.03
\end{tabular}

Viscous contributions for roll motion, Kappa4 $=1.50$ 
Non Dimensional Ship Motions

ship file: 0591

Shipid; 150,000 ton, Tanker, partial load, 20 tons Gill thruster

Heading (degrees) $=60.0$

Ship speed (knots) $=0.0$

\begin{tabular}{|c|c|c|c|c|c|c|c|c|}
\hline Wave L./ & Omegae & Omega & Hec & & Pitc & & Su & \\
\hline Ship L. & $2 \mathrm{p} i / \mathrm{s}$ & $2 \mathrm{pi} / \mathrm{s}$ & $2 / h$ & Phase & Theta/kh & Phase & $\mathrm{x} / \mathrm{h}$ & Phase \\
\hline 0.40 & 0.741 & 0.741 & 0.04 & 29.15 & 0.04 & 45.80 & 0.01 & -65.12 \\
\hline 0.50 & 0.657 & 0.657 & 0.10 & -22.92 & 0.13 & 30.39 & 0.02 & 104.62 \\
\hline 0.60 & 0.593 & 0.593 & 0.25 & -41.21 & 0.28 & 43.79 & 0.06 & 98.78 \\
\hline 0.80 & 0.495 & 0.495 & 0.59 & -23.81 & 0.43 & 74.05 & 0.16 & 91.18 \\
\hline 1.00 & 0.423 & 0.423 & 0.73 & -11.43 & 0.44 & 85.30 & 0.26 & 85.83 \\
\hline 1.20 & 0.368 & 0.368 & 0.79 & -6.11 & 0.44 & 89.26 & 0.35 & 81.49 \\
\hline 1.40 & 0.324 & 0.324 & 0.82 & -3.59 & 0.45 & 90.83 & 0.43 & 77.73 \\
\hline 1.60 & 0.289 & 0.289 & 0.84 & -2.26 & 0.45 & 91.50 & 0.52 & 74.35 \\
\hline 1.80 & 0.261 & 0.261 & 0.86 & -1.49 & 0.45 & 91.77 & 0.61 & 71.26 \\
\hline 2.00 & 0.237 & 0.237 & 0.87 &.-1.03 & 0.45 & 91.86 & 0.71 & 68.40 \\
\hline 2.50 & 0.193 & 0.193 & 0.89 & -0.49 & 0.46 & 91.79 & 0.42 & 62.02 \\
\hline 3.00 & 0.162 & 0.162 & 0.91 & -0.25 & 0.46 & 91.62 & 0.34 & 56.53 \\
\hline 3.50 & 0.140 & 0.140 & 0.92 & -0.14 & 0.47 & 91.44 & 0.27 & 51.77 \\
\hline 4.00 & 0.123 & 0.123 & 0.93 & -0.09 & 0.47 & 91.29 & 0.22 & 47.62 \\
\hline 5.00 & 0.099 & 0.099 & 0.94 & -0.07 & 0.47 & 91.04 & 0.16 & 40.80 \\
\hline
\end{tabular}

\begin{tabular}{|c|c|c|c|c|c|c|c|c|}
\hline Wave L./ & Omegae & Omega & Sw & & & 211 & $Y_{2}$ & w \\
\hline Ship L. & $2 \mathrm{pi} / \mathrm{s}$ & $2 \mathrm{pi} / \mathrm{s}$ & $y / h$ & Phase & $\mathrm{fi} / \mathrm{kh}$ & Phase & $\mathrm{psi} / \mathrm{kh}$ & Phase \\
\hline 0.40 & 0.741 & 0.741 & 0.04 & 57.91 & 0.04 & 25.42 & 0.05 & 0.41 \\
\hline 0.50 & 0.657 & 0.657 & 0.09 & 121.12 & 0.13 & -174.54 & 0.10 & -0.41 \\
\hline 0.60 & 0.593 & 0.593 & 0.14 & 134.08 & 0.41 & -159.08 & 0.15 & -0.10 \\
\hline 0.80 & 0.495 & 0.495 & 0.19 & 113.17 & 0.98 & -134.29 & 0.23 & -0.25 \\
\hline 1.00 & 0.423 & 0.423 & 0.33 & 92.95 & 1.22 & -111.66 & 0.29 & -0.58 \\
\hline 1.20 & 0.368 & 0.368 & 0.50 & 90.75 & 1.22 & -99.61 & 0.34 & -1.04 \\
\hline 1.40 & 0.324 & 0.324 & 0.64 & 91.15 & 1.15 & -93.69 & 0.39 & -1.72 \\
\hline 1.60 & 0.289 & 0.289 & 0.76 & 91.70 & 1.08 & -90.55 & 0.44 & -2.48 \\
\hline 1.80 & 0.261 & 0.261 & 0.87 & 92.05 & 1.03 & -88.65 & 0.49 & -3.44 \\
\hline 2.00 & 0.237 & 0.237 & 0.98 & 92.20 & 0.99 & -87.55 & 0.54 & -4.55 \\
\hline 2.50 & 0.193 & 0.193 & 1.25 & 91.65 & 0.93 & -87.26 & 0.65 & -7.77 \\
\hline 3.00 & 0.162 & 0.162 & 1.50 & 90.57 & 0.89 & -88.50 & 0.77 & -10.86 \\
\hline 3.50 & 0.140 & 0.140 & 1.74 & 90.50 & 0.85 & -88.22 & 0.89 & -13.02 \\
\hline 4.00 & 0.123 & 0.123 & 1.97 & 90.46 & 0.82 & -88.00 & 1.02 & -15.13 \\
\hline 5.00 & 0.099 & 0.099 & 2.38 & 90.38 & 0.78 & -87.72 & 1.25 & -19.21 \\
\hline
\end{tabular}

Viscous contributions for roll motion, Kappa4 $=1.50$ 
Non Dimensional Ship Motions

\begin{tabular}{|c|c|c|c|c|c|c|c|c|}
\hline \multicolumn{2}{|c|}{$\begin{array}{l}\text { Shipid: } 150,000 \text { tor } \\
\text { Heading (degrees) } \\
\text { Ship speed (knots) }\end{array}$} & \multicolumn{3}{|c|}{$\begin{array}{l}\text { n, Tanker, partial load, } \\
\quad=30.0\end{array}$} & \multicolumn{2}{|c|}{20 tons Gill } & \multicolumn{2}{|l|}{ hruster } \\
\hline ave L.l & Omegae & Omega & $\mathrm{He}$ & ave & & itch & Sur & rge \\
\hline ip L. & $2 \mathrm{pi} / \mathrm{s}$ & $2 p i / s$ & $z / h$ & Phase & Theta/ & kh Phase & $x / h$ & Phase \\
\hline 0.40 & 0.741 & 0.741 & 0.04 & -179.22 & 0.02 & 175.70 & 0.02 & -81.22 \\
\hline 0.50 & 0.657 & 0.657 & 0.08 & 118.60 & 0.04 & 133.72 & 0.05 & -88.74 \\
\hline 0.60 & 0.593 & 0.593 & 0.11 & 118.23 & 0.10 & 74.52 & 0.04 & -110.92 \\
\hline 0.80 & 0.495 & 0.495 & 0.11 & -20.90 & 0.36 & 80.22 & 0.10 & 110.60 \\
\hline 1.00 & 0.423 & 0.423 & 0.34 & -11.32 & 0.51 & 88.26 & 0.26 & 98.16 \\
\hline 1.20 & 0.368 & 0.368 & 0.50 & -6.16 & 0.58 & 91.19 & 0.42 & 92.71 \\
\hline 1.40 & 0.324 & 0.324 & 0.59 & -3.66 & 0.63 & 92.27 & 0.58 & 88.94 \\
\hline 1.60 & 0.289 & 0.289 & 0.66 & -2.33 & 0.67 & 92.66 & 0.73 & 85.91 \\
\hline 1.80 & 0.261 & 0.261 & 0.71 & -1.56 & 0.69 & 92.75 & 0.88 & 83.29 \\
\hline 2.00 & 0.237 & 0.237 & 0.75 & -1.09 & 0.71 & 92.72 & 1.00 & 80.95 \\
\hline 2.50 & 0.193 & 0.193 & 0.82 & -0.53 & 0.75 & 92.43 & 0.14 & 75.82 \\
\hline 3.00 & 0.162 & 0.162 & 0.85 & -0.28 & 0.77 & 92.14 & 0.11 & 71.37 \\
\hline 3.50 & 0.140 & 0.140 & 0.88 & -0.17 & 0.78 & 91.89 & 0.09 & 67.37 \\
\hline 4.00 & 0.123 & 0.123 & 0.90 & -0.11 & 0.79 & 91.68 & 0.08 & 63.72 \\
\hline 5.00 & 0.099 & 0.099 & 0.92 & -0.08 & 0.81 & 91.35 & 0.06 & 57.27 \\
\hline Wave $\mathrm{L} .1$ & Omegae & Omega & Sw & & & 211 & & Law \\
\hline iip L. & $2 \mathrm{pi} / \mathrm{s}$ & $2 \mathrm{pi} / \mathrm{s}$ & $y / h$ & Phase & $\mathrm{fi} / \mathrm{kh}$ & Phase & psi/kh & Phase \\
\hline 0.40 & 0.741 & 0.741 & 0.02 & -35.74 & 0.02 & 16.78 & 0.01 & -172.61 \\
\hline 0.50 & 0.657 & 0.657 & 0.04 & 0.74 & 0.10 & 22.54 & 0.00 & -32.69 \\
\hline 0.60 & 0.593 & 0.593 & 0.04 & 31.06 & 0.12 & 34.03 & 0.04 & -1.44 \\
\hline 0.80 & 0.495 & 0.495 & 0.05 & 98.38 & 0.08 & -162.14 & 0.12 & 1.32 \\
\hline 1.00 & 0.423 & 0.423 & 0.11 & 93.13 & 0.30 & -116.53 & 0.20 & 2.38 \\
\hline 1.20 & 0.368 & 0.368 & 0.20 & 91.54 & 0.42 & -101.34 & 0.27 & 2.13 \\
\hline 1.40 & 0.324 & 0.324 & 0.28 & 91.95 & 0.47 & -94.48 & 0.33 & 1.67 \\
\hline 1.60 & 0.289 & 0.289 & 0.36 & 92.37 & 0.48 & -91.05 & 0.38 & 1.05 \\
\hline 1.80 & 0.261 & 0.261 & 0.43 & 92.65 & 0.48 & -88.97 & 0.44 & 0.30 \\
\hline 2.00 & 0.237 & 0.237 & 0.50 & 92.78 & 0.48 & -87.71 & 0.49 & -0.55 \\
\hline 2.50 & 0.193 & 0.193 & 0.66 & 92.20 & 0.48 & -87.21 & 0.61 & -2.99 \\
\hline 3.00 & 0.162 & 0.162 & 0.82 & 91.08 & 0.48 & -88.37 & 0.73 & -5.26 \\
\hline 3.50 & 0.140 & 0.140 & 0.96 & 90.95 & 0.46 & -88.09 & 0.85 & -6.69 \\
\hline 4.00 & 0.123 & 0.123 & 1.10 & 90.85 & 0.45 & -87.88 & 0.97 & -8.07 \\
\hline & & 0.099 & & & & & 1.19 & \\
\hline
\end{tabular}

Viscous contributions for roll motion, Kappa4 $=1.50$ 
Non Dimensional Ship Motions

\begin{tabular}{|c|c|c|c|c|c|c|c|c|}
\hline \multirow{2}{*}{$\begin{array}{l}\text { Wave L./ } \\
\text { Ship L. }\end{array}$} & \multirow{2}{*}{$\begin{array}{r}\text { Omegae } \\
2 \mathrm{p} i / \mathrm{s}\end{array}$} & \multirow{2}{*}{$\begin{array}{l}\text { Omega } \\
2 \mathrm{pi} / \mathrm{s}\end{array}$} & \multicolumn{2}{|c|}{ Heave } & \multicolumn{2}{|c|}{ Pitch } & \multicolumn{2}{|c|}{ Surge } \\
\hline & & & $z / h$ & Phase & Theta/k & kh Phase & $x / h$ & Phase \\
\hline 0.40 & 0.741 & 0.741 & 0.04 & -142.30 & 0.01 & -130.58 & 0.01 & -87.25 \\
\hline 0.50 & 0.657 & 0.657 & 0.06 & 150.11 & 0.05 & 175.80 & 0.07 & -88.62 \\
\hline 0.60 & 0.593 & 0.593 & 0.13 & 130.19 & 0.05 & 133.50 & 0.08 & -97.99 \\
\hline 0.80 & 0.495 & 0.495 & 0.02 & 149.61 & 0.27 & 84.74 & 0.05 & 141.86 \\
\hline 1,00 & 0.423 & 0.423 & 0.21 & -11.61 & 0.47 & 89.96 & 0.22 & 104.59 \\
\hline 1.20 & 0.368 & 0.368 & 0.38 & -6.32 & 0.58 & 92.21 & 0.40 & 96.69 \\
\hline 1.40 & 0.324 & 0.324 & 0.50 & -3.76 & 0.66 & 93.01 & 0.59 & 92.24 \\
\hline 1.60 & 0.289 & 0.289 & 0.58 & -2.40 & 0.71 & 93.24 & 0.77 & 88.97 \\
\hline 1.80 & 0.261 & 0.261 & 0.65 & -1.62 & 0.75 & 93.24 & 0.94 & 86.29 \\
\hline 2.00 & 0.237 & 0.237 & 0.70 & -1.14 & 0.79 & 93.13 & 1.00 & 83.95 \\
\hline .50 & 0.193 & 0.193 & 0.78 & -0.55 & 0.84 & 92.75 & 0.53 & 79.00 \\
\hline 3.00 & 0.162 & 0.162 & 0.83 & -0.30 & 0.87 & 92.39 & 0.41 & 74.77 \\
\hline 3.50 & 0.140 & 0.140 & 0.86 & -0.18 & 0.89 & 92.10 & 0.34 & 71.00 \\
\hline .00 & 0.123 & 0.123 & 0.88 & -0.12 & 0.91 & 91.86 & 0.28 & 67.55 \\
\hline 0 & 0.099 & 0.099 & 0.91 & -0.08 & 0.93 & 91.49 & 0.22 & 61.41 \\
\hline
\end{tabular}




\section{APPENDIX III}

\section{RAO's for Buoy}

Heading $180,150,120,90,60,30,0$ degrees at zero speed 
Draft to depth ratio, $h / t=10.00$

Non Dimensional Traffic Ship Motions

Ship file: 1000

Shipid: Suction Mooring Sys

Heading (degrees) $=180.0$

ship speed (knots) $=0.0$

Wave L./ Omegae Omega

Ship I. 2pi/s 2pi/s

Heave

Pitch z/h Phase Theta/kh Phase

Surge
$\mathrm{x} / \mathrm{h}$

0.40

2.389

2.389

0.50

2.136

2.136

$0.00-126.12$

$0.00 \quad 178.14$

$0.00 \quad 89.08$

$0.00 \quad 77.57$

$0.00-85.55$

$0.01-90.23$

$1.689 \quad 1.689$

0.00168 .35

0.80

$0.01 \quad 7.89$

1.511

1. 511

$0.03 \quad 3.05$

$0.06 \quad 0.47$

1.3791 .379

$0.10 \quad-2.05$

1.40

1.60

1.277

1.1941 .194

$0.14-4.72$

$1.126 \quad 1.126$

$\begin{array}{ll}0.18 & -7.32\end{array}$

$1.068 \quad 1.068$

$0.22,-9.41$

$0.33-10.94$

2.00

0.955

$0.872 \quad 0.872$

$0.43-9.17$

$0.51 \quad-7.06$

0.807

0.807

$0.56 \quad-5.42$

$0.04-93.11$

$0.07 \quad-96.62$

$0.11-100.75$

$0.15-104.74$

$0.19-107.45$

$0.25-108.25$

$0.38-104.84$

$0.47-100.42$

$0.54-97.40$

$0.59-95.48$

$0.66 \quad-93.35$

$0.01 \quad 59.53$

$0.03 \quad 114.83$

0.03166 .10

$0.10-99.48$

$0.22-82.97$

$0.33-77.10$

$0.42-74.11$

$0.50-72.23$

$0.57-70.85$

$0.63-69.70$

$0.74-67.21$

$0.83-64.86$

$0.90-62.54$

$0.97-60.24$

5.00

0.676

0.676

$0.65 \quad-3.42$

$\begin{array}{ll}1.00 & -55.77\end{array}$ 
Non Dimensional Traffic Ship Motions

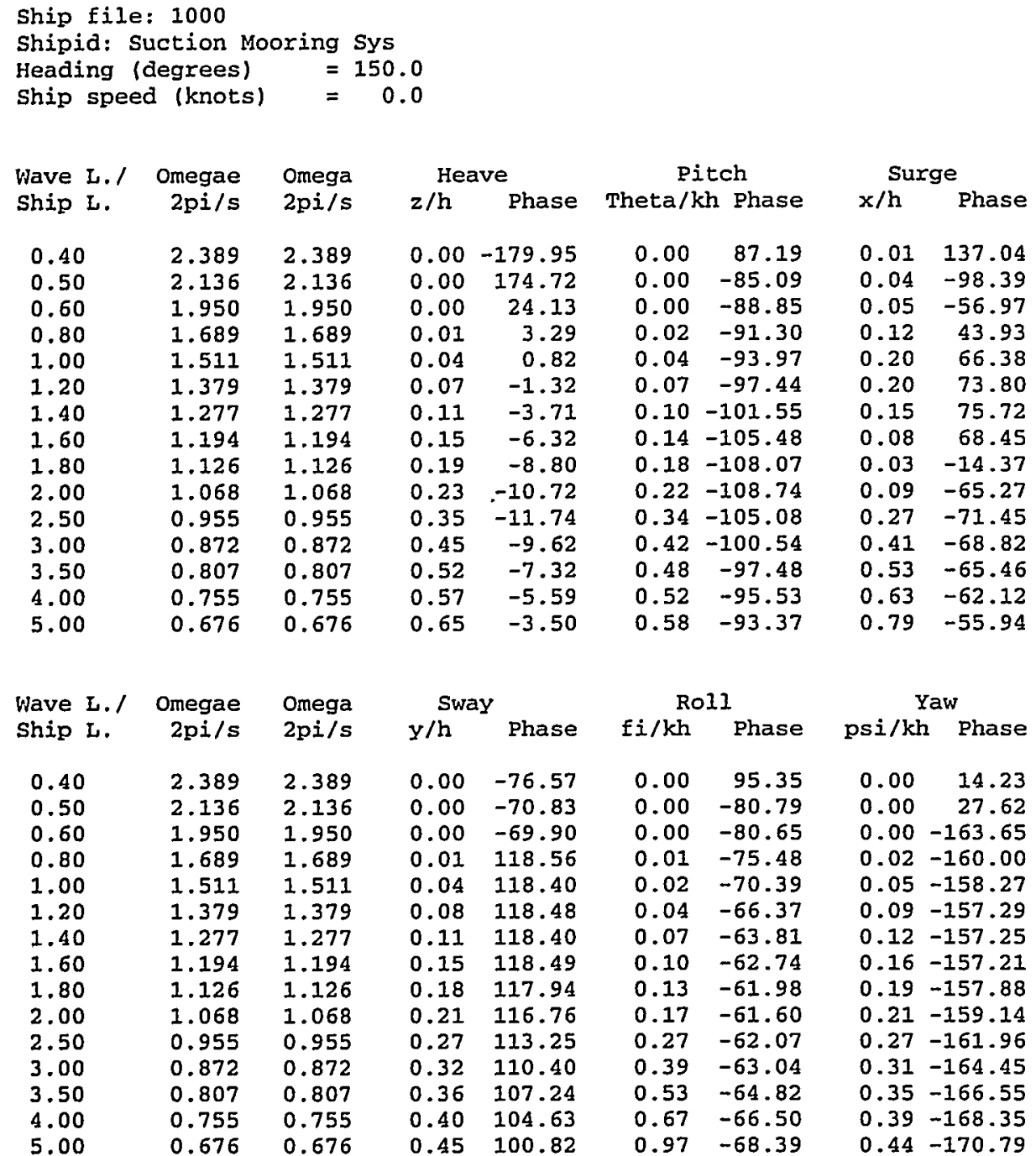

Viscous contributions for roll motion, Kappa4 $=1.50$ 
Non Dimensional Traffic Ship Motions

Ship file: 1000

Shipid: Suction Mooring Sys

Heading (degrees) $=120.0$

Wave L./ Omegae Omega

Ship L. 2pi/s 2pi/s

$\begin{array}{cc}\text { Heave } & \text { Pitch } \\ z / h \quad \text { Phase Theta/kh Phase }\end{array}$

Surge

$2.389 \quad 2.389$

0,50

2.136

2.136

0.00

2.18

$0.00 \quad-89.90$

$\mathrm{x} / \mathrm{h}$ Phase

0.60

$1.950 \quad 1.950$

0.80

$1.689 \quad 1.689$

0.00

$0.00 \quad-90.42$

$0.01 \quad 0.31$

$0.03-0.84$

1.00

1.511

1.511

$1.379 \quad 1.379$

1.40

1.277

$0.01 \quad-91.04$

$0.02 \quad-92.79$

$0.03 \quad-95.38$

$0.05 \quad-98.89$

$0.10-4.27$

$0.14 \quad-6.64$

$0.07-103.02$

$0.09-106.85$

$\begin{array}{lll}1.60 & 1.194 & 1.194\end{array}$

$\begin{array}{lll}1.80 & 1.126 & 1.126\end{array}$

$\begin{array}{lll}2.00 & 1.068 & 1.068\end{array}$

$\begin{array}{lll}2.50 & 0.955 & 0.955\end{array}$

3.00

$0.11-109.24$

$0.14-109.67$

$0.21-105.54$

$0.26 .-13.15$

$0.38-13.24$

$0.47-10.48$

$0.54 \quad-7.83$

$0.25-100.79$

$0.28 \quad-97.62$

4.00

$0.807 \quad 0.807$

5.00

0.755

0.755

$0.60 \quad-5.92$

$0.31 \quad-95.62$

$0.01 \quad-47.24$

$0.00-40.00$

$0.02-65.95$

$0.07 \quad 61.50$

$0.26 \quad 94.32$

$0.42 \quad 106.53$

$0.51 \quad 115.04$

$0.53 \quad 122.12$

$0.52 \quad 128.49$

$0.49 \quad 134.49$

$0.38 \quad 149.35$

$0.27 \quad 167.09$

$0.18-165.68$

$0.15-123.38$

$0.27-65.97$

Wave L./ Omegae Omeg

Omega

Sway

$y / h$ Phase

Roll

Yaw

$\begin{array}{lll}0.40 & 2.389 & 2.389\end{array}$

$\begin{array}{lll}0.50 & 2.136 & 2.136\end{array}$

$0.00 \quad-55.27$

$0.00 \quad 107.60$

Phase

psi/kh Phase

$\begin{array}{lll}0.60 & 1.950 & 1.950\end{array}$

$\begin{array}{lll}0.80 & 1.689 & 1.689\end{array}$

$0.02 \quad 111.04$

$0.07 \quad 114.59$

$0.00 \quad-82.22$

$0.00-166.88$

$0.00-81.00$

$0.00 \quad-78.28$

$0.02 \quad-72.66$

$0.00-164.37$

$0.14 \quad 116.56$

$0.05 \quad-67.91$

$0.01-162.50$

$0.04-159.75$

$\begin{array}{lll}1.00 & 1.511 & 1.511 \\ 1.20 & 1.379 & 1.379\end{array}$

$0.21 \quad 117.24$

$0.09-64.33$

$0.08-158.16$

$1.277 \quad 1.277$

$0.28 \quad 117.50$

$0.14 \quad-62.10$

$0.11-157.24$

$0.34 \quad 117.75$

$0.19-61.25$

$0.15-157.20$

1.60

$1.194 \quad 1.194$

$0.39 \quad 117.35$

$0.25-60.68$

$0.18-157.18$

$0.43 \quad 116.33$

$0.31-60.46$

$0.21-157.87$

2.00

$1.068 \quad 1.068$

$0.52 \quad 112.89$

$0.872 \quad 0.872$

3.00

$0.59 \quad 110.14$

$0.66 \quad 106.99$

$0.49-60.40$

$0.23-159.12$

$\begin{array}{lllll}0.69 & -61.39 & 0.33 & -164.43\end{array}$

$0.807 \quad 0.807$

4.00

0.755

0.755

$0.72 \quad 104.39$

$-63.15$

$0.36-166.54$

$0.39-168.35$

5.00

0.676

0.676

$1.69-67.49$

$0.44-170.79$

Viscous contributions for roll motion, Kappa4 $=1.50$ 
Non Dimensional Traffic Ship Motions

Ship file: 1000

Shipid: Suction Mooring Sys

Heading (degrees) $=90.0$

ship speed (knots) $=0.0$

\begin{tabular}{|c|c|c|c|c|c|c|c|c|}
\hline .1 & Omegae & Omega & He & & Pit & cch & su & ge \\
\hline hip L. & $2 \mathrm{pi} / \mathrm{s}$ & $2 \mathrm{pi} / \mathrm{s}$ & $2 / h$ & Phase & Theta/kh & Phase & $x / h$ & Phase \\
\hline 0.40 & 2.389 & 2.389 & 0.00 & -0.32 & 0.00 & -86.24 & 0.01 & 180.00 \\
\hline 0.5 & 2.136 & 2.136 & 0.01 & -0.59 & 0.00 & -73.55 & 0.01 & 180.00 \\
\hline 0.60 & 1.950 & 1.950 & 0.02 & -0.96 & 0.00 & -77.11 & 0.01 & -180.00 \\
\hline 0.80 & 1.689 & 1.689 & 0.05 & -2.01 & 0.00 & -56.97 & 0.04 & 180.00 \\
\hline 1.00 & 1.511 & 1.511 & 0.08 & -3.51 & 0.00 & -52.52 & 0.13 & 180.00 \\
\hline 1.20 & 1.379 & 1.379 & 0.12 & -5.51 & 0.00 & -23.94 & 0.26 & 180.00 \\
\hline 1.40 & 1.277 & 1.277 & 0.15 & -7.93 & 0.00 & 0.83 & 0.38 & 180.00 \\
\hline 1.60 & 1.194 & 1.194 & 0.19 & -10.50 & 0.00 & -9.31 & 0.47 & 180.00 \\
\hline 1.80 & 1.126 & 1.126 & 0.23 & -12.78 & 0.00 & 56.58 & 0.52 & 180.00 \\
\hline 2.00 & 1.068 & 1.068 & 0.28 &.-14.27 & 0.00 & 44.10 & 0.55 & 180.00 \\
\hline 2.50 & 0.955 & 0.955 & 0.39 & -13.95 & 0.00 & 82.43 & 0.55 & 180.00 \\
\hline 3.00 & 0.872 & 0.872 & 0.49 & -10.88 & 0.00 & 128.14 & 0.48 & 180.00 \\
\hline 3.50 & 0.807 & 0.807 & 0.56 & -8.08 & 0.00 & 173.18 & 0.38 & 180.00 \\
\hline .00 & 0.755 & 0.755 & 0.61 & -6.07 & 0.00 & 111.63 & 0.27 & -180.00 \\
\hline & 0.676 & 0.676 & 0.68 & -3.73 & 0.00 & 154.83 & 0.06 & -180.00 \\
\hline
\end{tabular}

\begin{tabular}{|c|c|c|c|c|c|c|c|c|}
\hline ave & Omegae & Omega & Sw & & & & & raw \\
\hline Ship L & $2 \mathrm{pi} / \mathrm{s}$ & $2 \mathrm{pi} / \mathrm{s}$ & $y / h$ & Phase & $\mathrm{fi} / \mathrm{kh}$ & Phase & $\mathrm{psi} / \mathrm{kh}$ & Phase \\
\hline 0.40 & 2.389 & 2.389 & 0.01 & 106.55 & 0.00 & -85.65 & 0.00 & 176.60 \\
\hline 0.50 & 2.136 & 2.136 & 0.03 & 109.03 & 0.00 & -81.16 & 0.00 & -164.84 \\
\hline 0.60 & 1.950 & 1.950 & 0.05 & 111.08 & 0.01 & -77.42 & 0.00 & -173.49 \\
\hline 0.80 & 1.689 & 1.689 & 0.13 & 114.17 & 0.03 & -71.40 & 0.00 & 168.13 \\
\hline 1.00 & 1.511 & 1.511 & 0.22 & 116.11 & 0.07 & -66.75 & 0.00 & 163.97 \\
\hline 1.20 & 1.379 & 1.379 & 0.30 & 116.84 & 0.11 & -63.36 & 0.00 & -158.11 \\
\hline 1.40 & 1.277 & 1.277 & 0.37 & 117.16 & 0.17 & -61.26 & 0.00 & -155.94 \\
\hline 1.60 & 1.194 & 1.194 & 0.43 & 117.45 & 0.23 & -60.52 & 0.00 & 173.73 \\
\hline 1.80 & 1.126 & 1.126 & 0.49 & 117.10 & 0.30 & -60.03 & 0.00 & -151.23 \\
\hline 2.00 & 1.068 & 1.068 & 0.54 & 116.05 & 0.37 & -59.09 & 0.00 & 170.31 \\
\hline 2.50 & 0.955 & 0.955 & 0.63 & 112.76 & 0.58 & -59.85 & 0.00 & 136.70 \\
\hline 3.00 & 0.872 & 0.872 & 0.71 & 110.06 & 0.81 & -60.91 & 0.00 & -179.95 \\
\hline 3.50 & 0.807 & 0.807 & 0.78 & 106.93 & 1.08 & -62.74 & 0.00 & -154.24 \\
\hline & 55 & 0.755 & 0.84 & 104.34 & 1.36 & -64.40 & 0.00 & -156.02 \\
\hline 5.0 & 0.676 & 0.676 & 0.94 & 100.53 & 1.95 & -66.17 & 0.00 & -81.06 \\
\hline
\end{tabular}

Viscous contributions for roll motion, Kappa4 $=1.50$ 
Non Dimensional Traffic Ship Motions

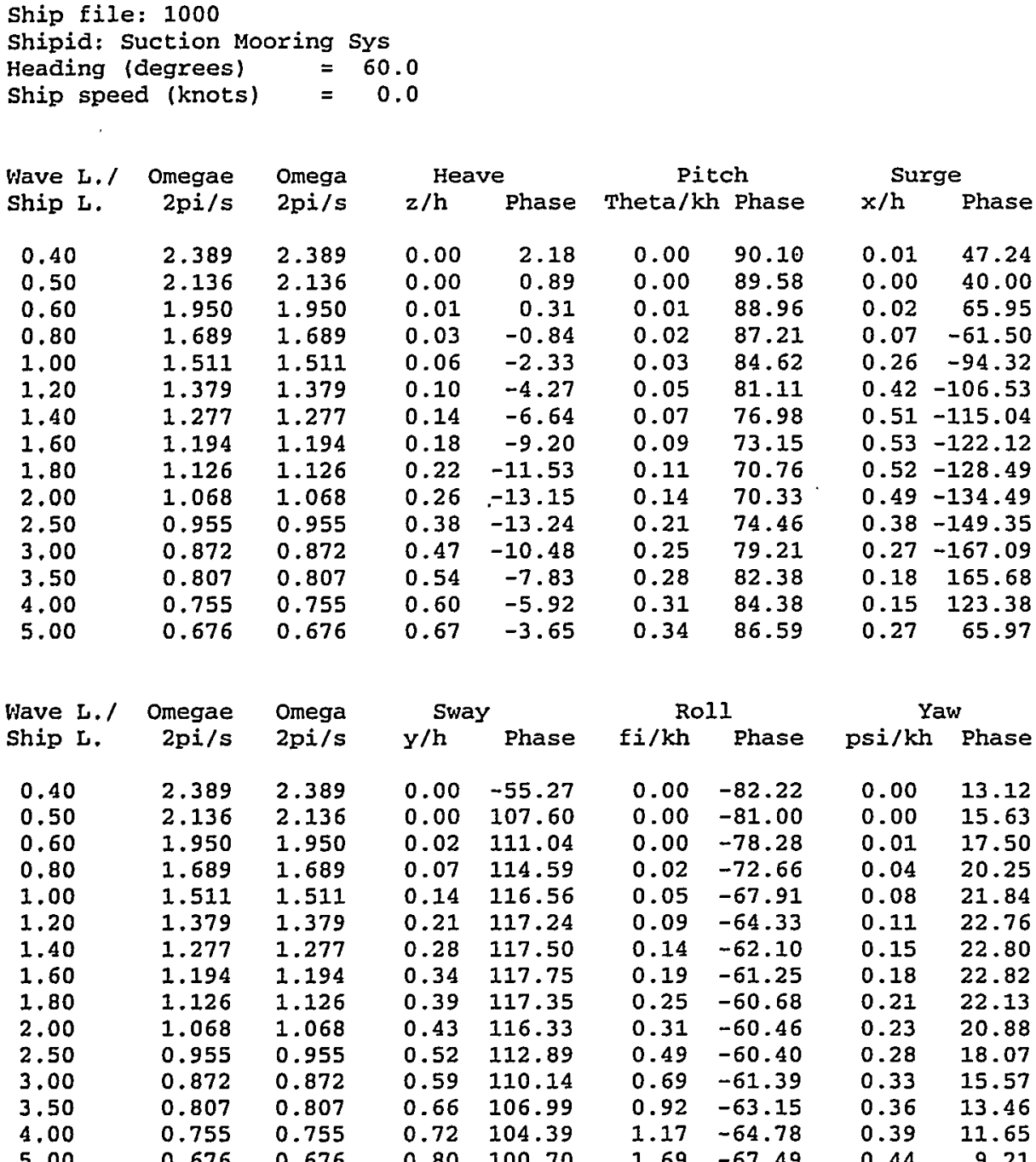

Viscous contributions for roll motion, Kappa4 $=1.50$ 
Ton Dimensional Traffic Ship Motions

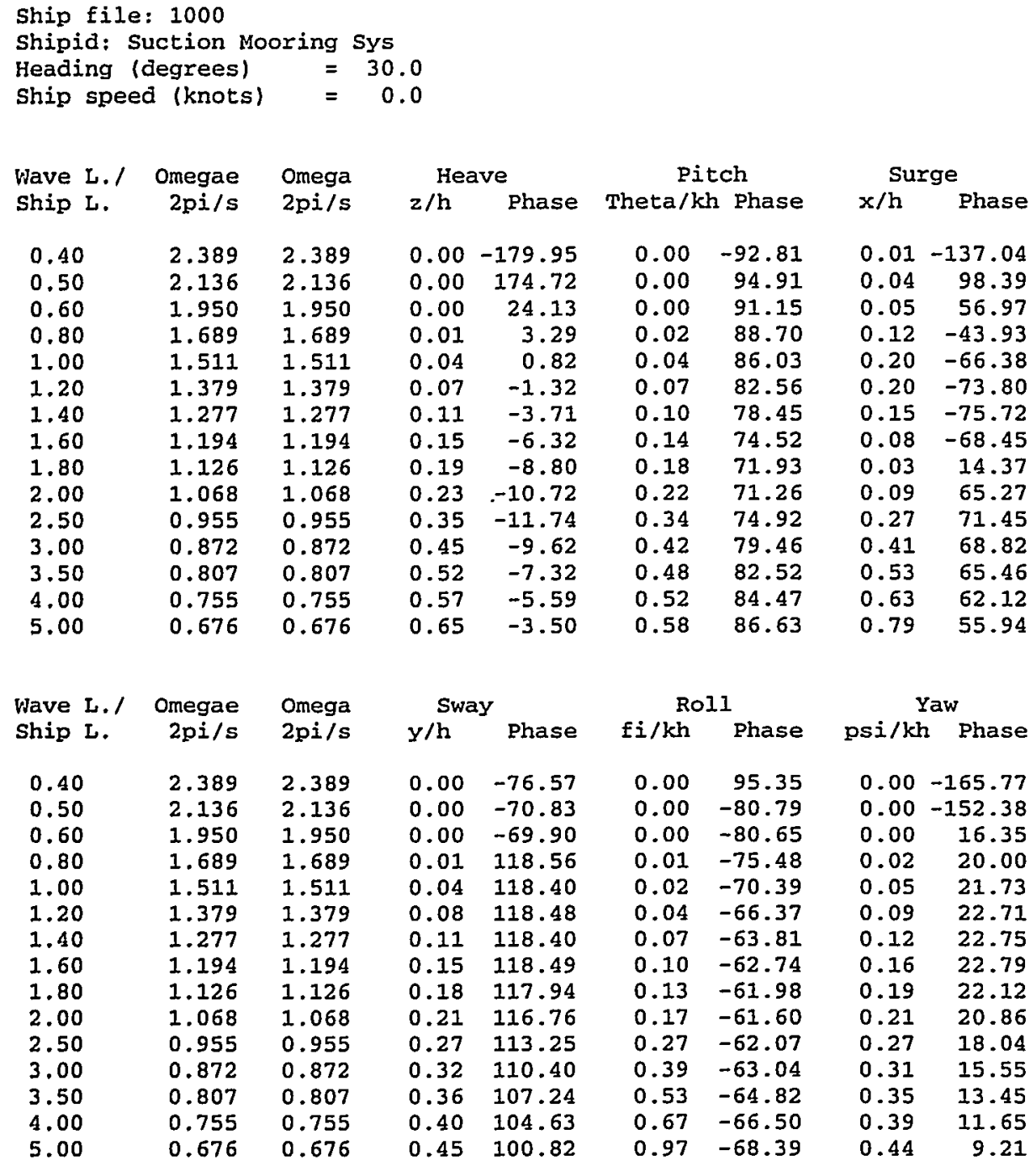

Viscous contributions for roll motion, Rappa4 $=1.50$ 
Non Dimensional Traffic Ship Motions

Ship file: 1000

Shipid: Suction Mooring Sys

Heading (degrees) $=0.0$

Ship speed (knots) $=0.0$

\begin{tabular}{|c|c|c|c|c|c|c|c|c|}
\hline Wave L.I & Omegae & Omega & Hea & ave & $\mathrm{Pi}$ & tch & sur & ge \\
\hline Ship L. & $2 \mathrm{pi} / \mathrm{s}$ & $2 \mathrm{pi} / \mathrm{s}$ & $z / h$ & Phase & Theta/k] & h Phase & $x / h$ & Phase \\
\hline 0.40 & 2.389 & 2.389 & 0.00 & -126.12 & 0.00 & -90.92 & 0.01 & -59.53 \\
\hline 0 . & 2.136 & 2.136 & 0.00 & 178.14 & 0.00 & -102.43 & 0.03 & -114.83 \\
\hline 0.60 & 1.950 & 1.950 & 0.00 & 168.35 & 0.00 & 94.45 & 0.03 & -166.10 \\
\hline 0.80 & 1.689 & 1.689 & 0.01 & 7.89 & 0.01 & 89.77 & 0.10 & 99.48 \\
\hline 1.00 & 1.511 & 1.511 & 0.03 & 3.05 & 0.04 & 86.89 & 0.22 & 82.97 \\
\hline 1.20 & 1.379 & 1.379 & 0.06 & 0.47 & 0.07 & 83.38 & 0.33 & 77.10 \\
\hline 1.40 & 1.277 & 1.277 & 0.10 & -2.05 & 0.11 & 79.25 & 0.42 & 74.11 \\
\hline 1.60 & 1.194 & 1.194 & 0.14 & -4.72 & 0.15 & 75.26 & 0.50 & 72.23 \\
\hline 1.80 & 1.126 & 1.126 & 0.18 & -7.32 & 0.19 & 72.55 & 0.57 & 70.85 \\
\hline 2.00 & 1.068 & 1.068 & 0.22 & -9.41 & 0.25 & 71.75 & 0.63 & 69.70 \\
\hline 2.50 & 0.955 & 0.955 & 0.33 & -10.94 & 0.38 & 75.16 & 0.74 & 67.21 \\
\hline 3.00 & 0.872 & 0.872 & 0.43 & -9.17 & 0.47 & 79.58 & 0.83 & 64.86 \\
\hline 3.50 & 0.807 & 0.807 & 0.51 & -7.06 & 0.54 & 82.60 & 0.90 & 62.54 \\
\hline 4.00 & 0.755 & 0.755 & 0.56 & -5.42 & 0.59 & 84.52 & 0.97 & 60.24 \\
\hline .00 & 0.676 & 0.676 & 0.65 & -3.42 & 0.66 & 86.65 & 1.00 & 55.77 \\
\hline
\end{tabular}




\section{APPENDIXIV}

\section{SIMULATION RESULTS}

Run \# 10 to Run \# 34

Hands-On Simulation

Log Report of Ship Handling

Simulation Track Plot 


\section{HANDS-ON SIMULATION}

\section{SUCTION MOORNG TECHNOLOGY}

Simulation Number: 10

Date: 3 February 1999

Start Time: 08:39

Length of run [secs]: 1808

Ship Condition: draft $=13.72 \mathrm{~m}$

Environment

Wind Speed [knots]:

0 Direction: 0

Sea State, Significant wave height $[\mathrm{m}]: .$.

Current Speed [knots]:

0 Direction: 0

0 Direction: 0

Ship Operator: J.A.

Observer: B.K.J \& J.K.

Final Mating, distance between center of buoy and center of moon pool [m]: 4.1

Evaluation:

Normal deceleration did not happen, because of wrong setting of the thruster at start, otherwise it was a normal approach. The captain made several heading changes to the right to offset some left set (The thruster was I degree off). On the final closure the speed was about 0.2 knots. The center of the buoy was a little to the right. It appeared to come in within the limits but just failed to do so. At this point we were almost stopped and we could easily have pulled the moon pool to the center of the buoy by use of the thruster and stop the engine. 
Ship:

Exercise number:

Scenario: $g$ file

caffic ships:

Initial conditions Exercise identification:
591150,000 ton, Tankex, partial load

750 Suction Mooring System

110 Open Ocean

999 Dummy tug file based on containership

590 Suction Mooring Buoy

590 150k tanker in partial load - Suction Mooring $\operatorname{RuN} \pm 10$

Time Position (m) $\quad$ RPM $\quad$ Speed (Knots) Turn Rudier Gill wind Current HH:MM:SS East North Port Stbd Long Bow Stern Heading Deg/Min Deg $\%$ Deg knots Deg Knots Deg

\begin{tabular}{|c|c|c|c|c|c|c|c|c|c|c|c|c|c|c|c|c|}
\hline $00: 08: 20$ & -1 & -1033 & 26 & 0 & 2.95 & 0.02 & -0.03 & 360 & 0.292 & 3.3 & 98.0 & 181 & 0 & 180 & 0.0 & 180 \\
\hline $00: 08: 30$ & -1 & -1018 & 26 & 0 & 2.91 & 0.02 & -0.04 & 360 & 0.335 & 2.1 & 98.0 & 181 & 0 & 180 & 0.0 & 180 \\
\hline $00: 08 ; 40$ & -1 & -1003 & 26 & 0 & 2.87 & 0.02 & -0.04 & $\cdot 360$ & 0.351 & 1.1 & 98.0 & 181 & 0 & 180 & 0.0 & 180 \\
\hline $00: 08: 50$ & -1 & -989 & 26 & 0 & 2.82 & 0.01 & -0.04 & 360 & 0.340 & 0.4 & 98.0 & 181 & 0 & 180 & 0.0 & 180 \\
\hline $00: 09: 00$ & -1 & -974 & 26 & 0 & 2.78 & 0.01 & -0.04 & 000 & 0.307 & -0.7 & 98.0 & 181 & 0 & 180 & 0.0 & 180 \\
\hline $00: 09: 10$ & -1 & -960 & 26 & 0 & 2.74 & 0.01 & -0.03 & 000 & 0.257 & -1.8 & 98.0 & 181 & 0 & 180 & 0.0 & 180 \\
\hline $00: 09: 20$ & -1 & -946 & 26 & 0 & 2.70 & 0.00 & -0.03 & 000 & 0.192 & -2.3 & 98.0 & 181 & 0 & 180 & 0.0 & 180 \\
\hline $00: 09: 30$ & -2 & -932 & 26 & 0 & 2.66 & 0.00 & -0.02 & 000 & 0.121 & -2.9 & 98.0 & 181 & 0 & 180 & 0.0 & 180 \\
\hline $00: 09: 40$ & -2 & -919 & 26 & 0 & 2.62 & -0.01 & -0.02 & 000 & 0.046 & -2.8 & 98.0 & 181 & 0 & 180 & 0.0 & 180 \\
\hline $00: 09: 50$ & -2 & -905 & 26 & 0 & 2.58 & -0.01 & -0.01 & 000 & -0.022 & -2.8 & 98.0 & 181 & 0 & 180 & 0.0 & 180 \\
\hline $00: 10: 00$ & -2 & -892 & 26 & 0 & 2.54 & -0.02 & 0.00 & 000 & -0.085 & -2.8 & 98.0 & 181 & 0 & 180 & 0.0 & 180 \\
\hline $00: 10: 10$ & -2 & -879 & 26 & 0 & 2.50 & -0.02 & 0.00 & 000 & -0.140 & -2.2 & 98.0 & 181 & 0 & 180 & 0.0 & 180 \\
\hline $00: 10: 20$ & -2 & -866 & 26 & 0 & 2.46 & -0.02 & 0.01 & 000 & -0.181 & -1.6 & 98.0 & 181 & 0 & 180 & 0.0 & 180 \\
\hline $00: 10: 30$ & -2 & -854 & 26 & 0 & 2.42 & -0.02 & 0.01 & 000 & -0.206 & -1.0 & 98.0 & 181 & 0 & 180 & 0.0 & 180 \\
\hline $00: 10: 40$ & -2 & -841 & 26 & 0 & 2.38 & -0.02 & 0.01 & 000 & -0.216 & -0.5 & 98.0 & 181 & 0 & 180 & 0.0 & 180 \\
\hline $00: 10: 50$ & -2 & -829 & 26 & 0 & 2.34 & -0.02 & 0.01 & 000 & -0.211 & 0.1 & 98.0 & 181 & 0 & 280 & 0.0 & 180 \\
\hline $00: 11: 00$ & -2 & -817 & 26 & 0 & 2.31 & -0.02 & 0.01 & 360 & -0.193 & 0.7 & 98.0 & 181 & 0 & 180 & 0.0 & 180 \\
\hline $00 ; 11: 10$ & -2 & -806 & 26 & 0 & 2.28 & -0.02 & 0.01 & 360 & -0.164 & 1.2 & 52.0 & 181 & 0 & 180 & 0.0 & 180 \\
\hline $00: 11: 20$ & -2 & -794 & 26 & 0 & 2.28 & -0.01 & 0.01 & 360 & -0.127 & 1.8 & 52.0 & 181 & 0 & 180 & 0.0 & 180 \\
\hline $00 ; 11: 30$ & -2 & -782 & 26 & 0 & 2.29 & -0.01 & 0.00 & 360 & -0.083 & 1.7 & 25.0 & 181 & 0 & 180 & 0.0 & 180 \\
\hline $00: 11: 40$ & -2 & -770 & 26 & 0 & 2.30 & -0.01 & 0.00 & 360 & -0.039 & 2.3 & 25.0 & 181 & 0 & 180 & 0.0 & 180 \\
\hline $00 ; 11: 50$ & -2 & -758 & 26 & 0 & 2.32 & 0.00 & 0.00 & 360 & 0.011 & 2.2 & 50.0 & 181 & 0 & 180 & 0.0 & 180 \\
\hline $00: 12: 00$ & -2 & -746 & 26 & 0 & 2.32 & 0.00 & -0.01 & 360 & 0.055 & 1.7 & 50.0 & 181 & 0 & 180 & 0.0 & 180 \\
\hline $00: 12: 10$ & -2 & -734 & 26 & 0 & 2.32 & 0.00 & -0.02 & 360 & 0.122 & 9.3 & 50.0 & 181 & 0 & 180 & 0.0 & 180 \\
\hline $00: 12: 20$ & -2 & -722 & 26 & 0 & 2.33 & 0.02 & -0.05 & 360 & 0.489 & 18.9 & 50.0 & 181 & 0 & 180 & 0.0 & 180 \\
\hline $00: 12: 30$ & -2 & -711 & 26 & 0 & 2.33 & 0.04 & -0.09 & 000 & 0.854 & 16.4 & 50.0 & 181 & 0 & 180 & 0.0 & 180 \\
\hline $00: 12: 40$ & -2 & -699 & 26 & 0 & 2.33 & 0.06 & -0.12 & 000 & 1.136 & 13.5 & 50.0 & 181 & 0 & 180 & 0.0 & 180 \\
\hline $00: 12 ; 50$ & -2 & -687 & 26 & 0 & 2.32 & 0.07 & -0.14 & 000 & 1.320 & 9.5 & 75.0 & 181 & 0 & 180 & 0.0 & 180 \\
\hline$n 0: 13: 00$ & -2 & -675 & 26 & 0 & 2.30 & 0.06 & -0.14 & 001 & 1.266 & -7.7 & 75.0 & 181 & 0 & 180 & 0.0 & 180 \\
\hline$: 13: 10$ & -2 & -663 & 26 & 0 & 2.29 & 0.03 & -0.11 & 001 & 0.883 & -15.6 & 75.0 & 181 & 0 & 180 & 0.0 & 180 \\
\hline$. J: 13: 20$ & -2 & -651 & 26 & 0 & 2.25 & 0.00 & -0.07 & 001 & 0.461 & -17.1 & 99.0 & 181 & 0 & 180 & 0.0 & 180 \\
\hline $00: 13: 30$ & -2 & -640 & 26 & 0 & 2.21 & -0.02 & -0.03 & 001 & 0.041 & -18.0 & 99.0 & 181 & 0 & 180 & 0.0 & 180 \\
\hline $00: 13: 40$ & -2 & -628 & 26 & 0 & 2.17 & -0.04 & 0.01 & 001 & -0.348 & -17.3 & 99.0 & 181 & 0 & 180 & 0.0 & 180 \\
\hline $00: 13: 50$ & -2 & -617 & 26 & 0 & 2.13 & -0.06 & 0.05 & 001 & -0.684 & -15.4 & 99.0 & 181 & 0 & 180 & 0.0 & 180 \\
\hline $00: 14: 00$ & -2 & -606 & 26 & 0 & 2.09 & -0.07 & 0.07 & 001 & -0.949 & -13.0 & 99.0 & 181 & 0 & 180 & 0.0 & 180 \\
\hline $00: 14: 10$ & -2 & -596 & 26 & 0 & 2.06 & -0.07 & 0.08 & 001 & -0.986 & 4.6 & 99.0 & 181 & 0 & 180 & 0.0 & 180 \\
\hline $00: 14: 20$ & -2 & -585 & 26 & 0 & 2.03 & -0.05 & 0.06 & 000 & -0.724 & 10.6 & 77.0 & 181 & 0 & 180 & 0.0 & 180 \\
\hline $00: 14: 30$ & -2 & -575 & 26 & 0 & 2.01 & -0.04 & 0.03 & 000 & -0.431 & 12.1 & 77.0 & 181 & 0 & 180 & 0.0 & 180 \\
\hline $00: 14: 40$ & -2 & -565 & 26 & 0 & 2.00 & -0.02 & 0.00 & 000 & -0.133 & 13.0 & 77.0 & 181 & 0 & 180 & 0.0 & 180 \\
\hline $00: 14: 50$ & -2 & -554 & 26 & 0 & 1.98 & 0.00 & -0.02 & 000 & 0.075 & 1.6 & 77.0 & 181 & 0 & 180 & 0.0 & 180 \\
\hline $00: 15: 00$ & -2 & -544 & 26 & 0 & 1.97 & -0.01 & -0.01 & 000 & -0.020 & -5.1 & 77.0 & 181 & 0 & 180 & 0.0 & 180 \\
\hline $00: 15: 10$ & -2 & -534 & 26 & 0 & 1.96 & -0.02 & 0.00 & 000 & -0.130 & -5.0 & 77.0 & 181 & 0 & 180 & 0.0 & 180 \\
\hline $00: 15: 20$ & -2 & -524 & 26 & 0 & 1.94 & -0.02 & 0.01 & 000 & -0.228 & -4.4 & 77.0 & 181 & 0 & 180 & 0.0 & 180 \\
\hline $00 ; 15: 30$ & -2 & -514 & 26 & 0 & 1.93 & -0.02 & 0.02 & 000 & -0.305 & -3.8 & 77.0 & 181 & 0 & 180 & 0.0 & 180 \\
\hline $00: 15: 40$ & -2 & -504 & 26 & 0 & 1.92 & -0.03 & 0.03 & .000 & -0.356 & -2.6 & 77.0 & 181 & 0 & 180 & 0.0 & 180 \\
\hline $00: 15: 50$ & -2 & -494 & 26 & 0 & 1.90 & -0.03 & 0.03 & 000 & -0.382 & -1.5 & 77.0 & 181 & 0 & 180 & 0.0 & 180 \\
\hline $00: 16: 00$ & -1 & -485 & 26 & 0 & 1.89 & -0.03 & 0.03 & 000 & -0.383 & -0.3 & 77.0 & 181 & 0 & 180 & 0.0 & 180 \\
\hline $00: 16: 10$ & -1 & -475 & 26 & 0 & 1.88 & -0.02 & 0.03 & 360 & -0.360 & 0.8 & 77.0 & 181 & 0 & 180 & 0.0 & 180 \\
\hline $00: 16: 20$ & -1 & -465 & 26 & 0 & 1.86 & -0.02 & 0.03 & 360 & -0.311 & 4.7 & 77.0 & 181 & 0 & 180 & 0.0 & 180 \\
\hline $00: 16: 30$ & -1 & -456 & 26 & 0 & 1.85 & 0.00 & 0.00 & 360 & 0.003 & 20.3 & 77.0 & 181 & 0 & 180 & 0.0 & 180 \\
\hline $00 ; 16: 40$ & -2 & -446 & 26 & 0 & 1.84 & 0.02 & -0.04 & 360 & 0.418 & 19.4 & 77.0 & 181 & 0 & 180 & 0.0 & 180 \\
\hline $00: 16: 50$ & -2 & -437 & 26 & 0 & 1.82 & 0.04 & -0.08 & 000 & 0.776 & 17.5 & 77.0 & 181 & 0 & 180 & 0.0 & 180 \\
\hline $00: 17: 00$ & -2 & -427 & 26 & 0 & 1.81 & 0.06 & -0.11 & 000 & 1.060 & 14.5 & 77.0 & 181 & 0 & 180 & 0.0 & 180 \\
\hline $00: 17: 10$ & -2 & -418 & 26 & 0 & 1.80 & 0.06 & -0.12 & 000 & 1.090 & -4.5 & 77.0 & 181 & 0 & 180 & 0.0 & 180 \\
\hline $00: 17: 20$ & -2 & -409 & 26 & 0 & 1.79 & 0.05 & -0.11 & 001 & 1.003 & 7.8 & 77.0 & 181 & 0 & 180 & 0.0 & 180 \\
\hline $00: 17: 30$ & -2 & -400 & 26 & 0 & 1.77 & 0.05 & -0.12 & 001 & 1.076 & 4.9 & 77.0 & 181 & 0 & 180 & 0.0 & 180 \\
\hline $00: 17: 40$ & -2 & -391 & 26 & 0 & 1.76 & 0.05 & -0.12 & 001 & 1.079 & 1.5 & 77.0 & 181 & 0 & 180 & 0.0 & 180 \\
\hline $00: 17: 50$ & -2 & -382 & 26 & 0 & 1.75 & 0.03 & -0.10 & 001 & 0.841 & -17.5 & 77.0 & 181 & 0 & 180 & 0.0 & 180 \\
\hline $00: 18: 00$ & -2 & -373 & 26 & 0 & 1.73 & 0.00 & -0.06 & 001 & 0.372 & -21.0 & 100.0 & 181 & 0 & 180 & 0.0 & 180 \\
\hline $00: 18: 10$ & -2 & -364 & 26 & 0 & 1.69 & -0.03 & -0.01 & 001 & -0.084 & -20.8 & 100.0 & 181 & 0 & 180 & 0.0 & 180 \\
\hline $00: 18: 20$ & -2 & -355 & 26 & 0 & 1.65 & -0.05 & 0.03 & 001 & -0.503 & -19.9 & 100.0 & 181 & 0 & 180 & 0.0 & 180 \\
\hline $00: 18: 30$ & -2 & -347 & 26 & 0 & 1.62 & -0.07 & 0.06 & 001 & -0.827 & -10.5 & 100.0 & 181 & 0 & 180 & 0.0 & 180 \\
\hline $00: 18 ; 40$ & -2 & -339 & 26 & 0 & 1.58 & -0.06 & 0.06 & 001 & -0.816 & 2.9 & 100.0 & 181 & 0 & 180 & 0.0 & 180 \\
\hline $00: 18: 50$ & -2 & -330 & 26 & 0 & 1.55 & -0.06 & 0.06 & 001 & -0.712 & -1.7 & 100.0 & 181 & 0 & 180 & 0.0 & 180 \\
\hline $00 ; 19: 00$ & -1 & -323 & 26 & 0 & 1.52 & -0.06 & 0.07 & 001 & -0.857 & -10.6 & 100.0 & 181 & 0 & 180 & 0.0 & 180 \\
\hline $00: 19: 10$ & -1 & -315 & 26 & 0 & 1.48 & -0.07 & 0.09 & 000 & -0.994 & -7.6 & 100.0 & 181 & 0 & 180 & 0.0 & 180 \\
\hline $00: 19: 20$ & -1 & -307 & 26 & 0 & 1.45 & -0.07 & 0.10 & 000 & -1.062 & -4.2 & 100.0 & 181 & 0 & 180 & 0.0 & 180 \\
\hline $7: 19 ; 30$ & -1 & -300 & 26 & 0 & 1.42 & -0.07 & 0.09 & 000 & -1.014 & 7.4 & 100.0 & 181 & 0 & 180 & 0.0 & 180 \\
\hline$: 19: 40$ & -1 & -293 & 26 & 0 & 1.39 & -0.04 & 0.06 & 360 & -0.653 & 19.1 & 100.0 & 181 & 0 & 180 & 0.0 & 180 \\
\hline vu: 19 ;50 & -1 & -286 & 26 & 0 & 1.35 & -0.02 & 0.02 & 360 & -0.228 & 20.5 & 100.0 & 181 & 0 & 180 & 0.0 & 180 \\
\hline $00: 20: 00$ & -1 & -279 & 26 & 0 & 1.32 & 0.01 & -0.02 & 360 & 0.181 & 20.1 & 100.0 & 181 & 0 & 180 & 0.0 & 180 \\
\hline $00: 20: 10$ & -1 & -272 & 26 & 0 & 1.29 & 0.03 & -0.06 & 360 & 0.548 & 18.6 & .100 .0 & 181 & 0 & 180 & 0.0 & 180 \\
\hline $00: 2$ & & -266 & 26 & 0 & 1.2 & 0.05 & -0.0 & 000 & & . & 100 & 181 & & & 0.0 & 180 \\
\hline
\end{tabular}




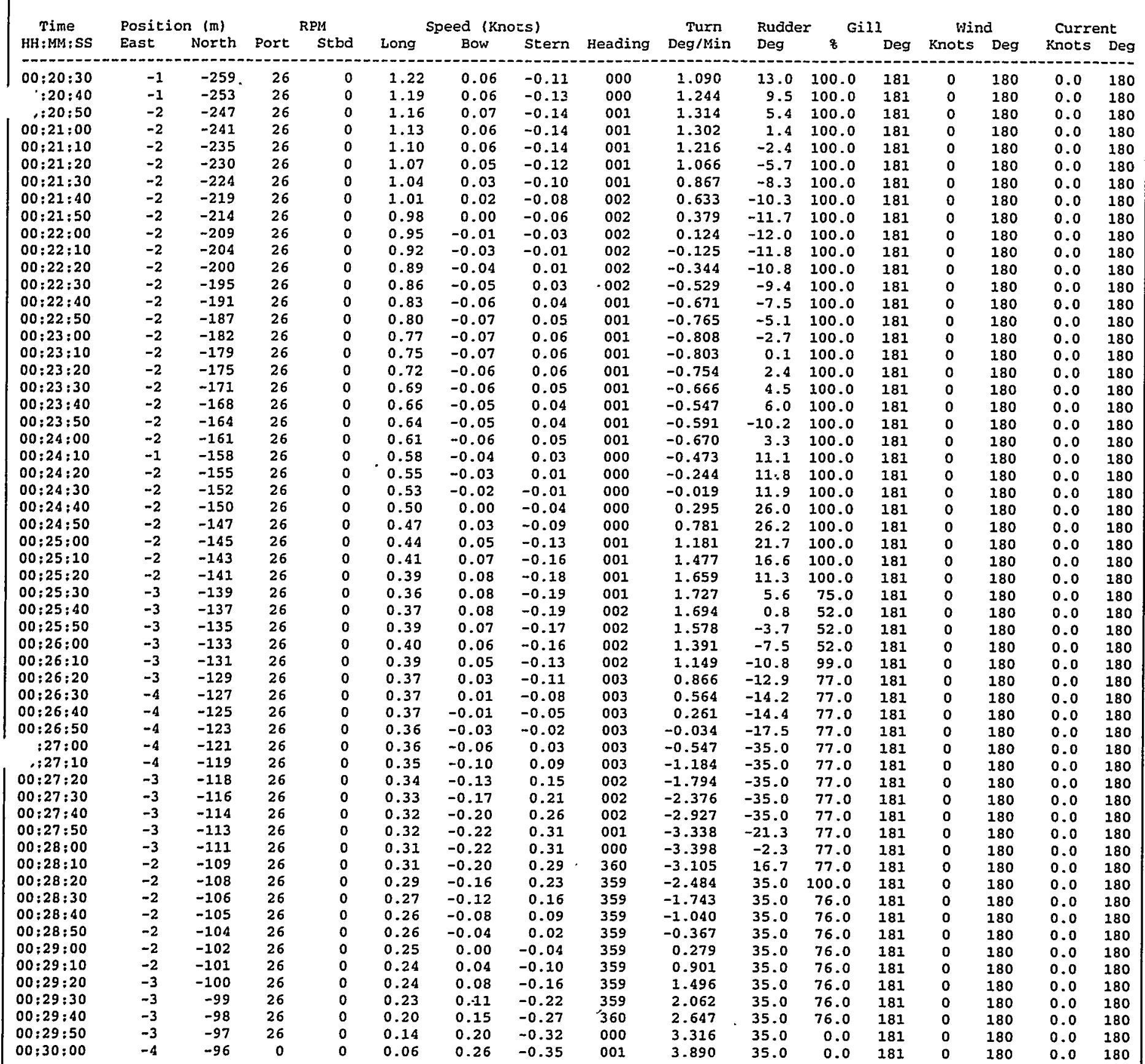


Open Ocean

\section{Simulation Track Plot}

Ship: 150,000 ton, Tanker, partial load

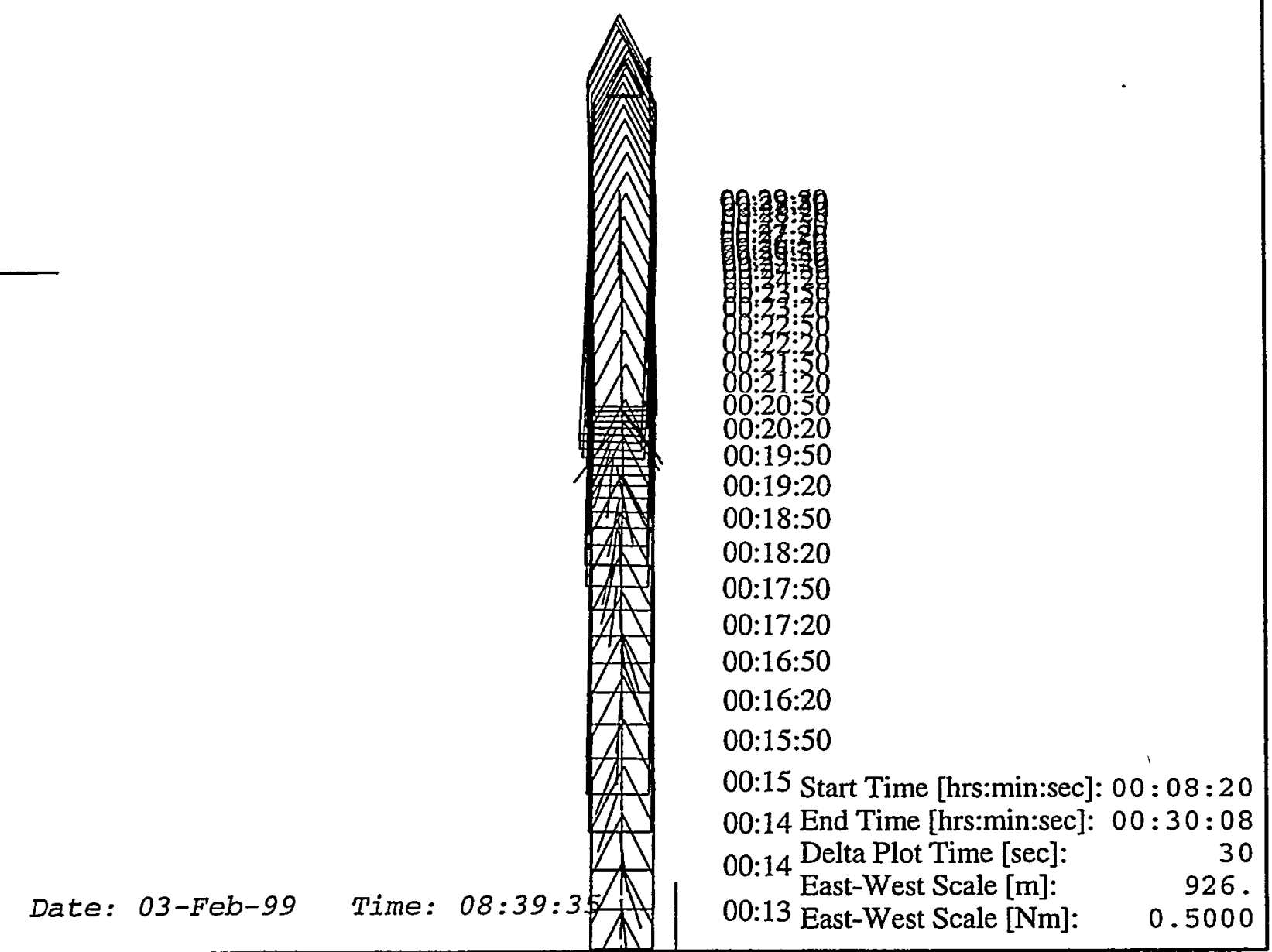




\section{HANDS-ON SIMULATION}

\section{SUCTION MOORNG TECHNOLOGY}

Simulation Number: 11

Date: 3 February 1999

Start Time: 09:24

Length of run [secs]: 2156

Ship Condition: draft $=13.72 \mathrm{~m}$

Environment

Wind Speed [knots]:

0 Direction: 0

Sea State, Significant wave height $[\mathrm{m}]: .$.

0 Direction: 0

Current Speed [knots]:

0 Direction: 0

Ship Operator: J.A.

Observer: B.K.J \& J.K.

Final Mating, distance between center of buoy and center of moon pool [m]: 0.9

Evaluation:

Everything went well. Started deceleration immediately by reducing RPM to 25 and using full reverse thruster. In the latter part of the approach the captain made several small heading adjustments. Deceleration was done in the following way: 3 knots at 1000 $m$ from the buoy to the moon pool, and at $500 \mathrm{~m}$ the speed was 2 knots. Just before attachment the speed was 0.2 knots - perfect mating, about $1 \mathrm{~m}$ off. 
Ship:

Exercise number:

Scenario:

$g$ file:

affic ships: Exercise identification:
591 150,000 ton, Tanker, partial load

750 Suction Mooring System

110 Open Ocean

999 Dummy tug file based on containership

590 Suction Mooring Buoy

590 l50k tankef, in partial load - Suction Mooring

Speed (Knots)

Turn Rudder Gill Wind Current Stern Heading Deg/Min Deg o

\begin{tabular}{|c|c|c|c|c|c|c|c|c|c|c|c|c|c|c|c|c|}
\hline $\begin{array}{l}00: 13: 20 \\
00: 13: 30\end{array}$ & $\begin{array}{l}0 \\
0\end{array}$ & $\begin{array}{l}-789 \\
-776\end{array}$ & $\begin{array}{l}26 \\
26\end{array}$ & $\begin{array}{l}0 \\
0\end{array}$ & $\begin{array}{l}2.39 \\
2.38\end{array}$ & $\begin{array}{l}0.00 \\
0.00\end{array}$ & $\begin{array}{l}0.00 \\
0.00\end{array}$ & $\begin{array}{l}360 \\
360\end{array}$ & $\begin{array}{r}0.002 \\
-0.008\end{array}$ & $\begin{array}{l}0.0 \\
0.5\end{array}$ & $\begin{array}{l}75.0 \\
75.0\end{array}$ & $\begin{array}{l}180 \\
180\end{array}$ & $\begin{array}{l}0 \\
0\end{array}$ & $\begin{array}{l}180 \\
180\end{array}$ & $\begin{array}{l}0.0 \\
0.0\end{array}$ & $\begin{array}{l}180 \\
180\end{array}$ \\
\hline $00: 13: 40$ & 0 & -764 & 26 & 0 & 2.36 & 0.00 & 0.00 & 360 & -0.004 & 0.6 & 75.0 & 180 & 0 & 180 & 0.0 & 180 \\
\hline $00: 13: 50$ & 0 & -752 & 26 & 0 & 2.35 & 0.00 & 0.00 & 360 & 0.000 & 0.6 & 75.0 & 180 & 0 & 180 & 0.0 & 180 \\
\hline $00: 14: 00$ & 0 & -740 & 26 & 0 & 2.33 & 0.00 & 0.00 & 360 & 0.003 & 0.6 & 75.0 & 180 & 0 & 180 & 0.0 & 180 \\
\hline $00: 14: 10$ & 0 & -728 & 26 & 0 & 2.32 & 0.00 & 0.00 & 360 & 0.006 & 0.6 & 75.0 & 180 & 0 & 180 & 0.0 & 180 \\
\hline $00: 14: 20$ & 0 & -716 & 26 & 0 & 2.30 & 0.00 & 0.00 & 360 & 0.000 & 0.0 & 75.0 & 180 & 0 & 180 & 0.0 & 180 \\
\hline $00: 14: 30$ & 1 & -704 & 26 & 0 & 2.29 & 0.00 & 0.00 & 360 & -0.005 & 0.6 & 75.0 & 180 & 0 & 180 & 0.0 & 180 \\
\hline $00: 14 ; 40$ & 1 & -692 & 26 & 0 & 2.27 & 0.00 & 0.00 & 360 & -0.002 & 0.6 & 75.0 & 180 & 0 & 180 & 0.0 & 180 \\
\hline $00: 14: 50$ & 1 & -681 & 26 & 0 & 2.26 & 0.00 & 0.00 & 360 & 0.001 & 0.6 & 75.0 & 180 & 0 & 180 & 0.0 & 180 \\
\hline $00: 15 ; 00$ & 1 & -669 & 26 & 0 & 2.25 & 0.00 & 0.00 & 360 & 0.004 & 0.6 & 75.0 & 180 & 0 & 180 & 0.0 & 180 \\
\hline $00: 15: 10$ & 1 & -658 & 26 & 0 & 2.23 & 0.00 & 0.00 & 360 & 0.002 & 0.0 & 75.0 & 180 & 0 & 180 & 0.0 & 180 \\
\hline $00: 15: 20$ & 1 & -646 & 26 & 0 & 2.22 & 0.00 & 0.00 & 360 & -0.005 & 0.6 & 100.0 & 180 & 0 & 180 & 0.0 & 180 \\
\hline $00: 15 ; 30$ & 1 & -635 & 26 & 0 & 2.18 & 0.00 & 0.00 & 360 & -0.001 & 0.6 & 100.0 & 180 & 0 & 180 & 0.0 & 180 \\
\hline $00: 15: 40$ & 1 & -624 & 26 & 0 & 2.14 & 0.00 & 0.00 & 360 & 0.002 & 0.6 & 100.0 & 180 & 0 & 180 & 0.0 & 180 \\
\hline $00: 15: 50$ & 1 & -613 & 26 & 0 & 2.10 & 0.00 & 0.00 & 360 & 0.006 & 0.6 & 100.0 & 180 & 0 & 180 & 0.0 & 180 \\
\hline $00: 16: 00$ & 1 & -602 & 26 & 0 & 2.06 & 0.00 & 0.00 & 360 & -0.004 & 0.0 & 100.0 & 180 & 0 & 180 & 0.0 & 180 \\
\hline $00: 16: 10$ & 1 & -592 & 26 & 0 & 2.03 & 0.00 & 0.01 & 360 & -0.107 & -0.5 & 100.0 & 180 & 0 & 180 & 0.0 & 180 \\
\hline $00: 16: 20$ & 1 & -581 & 26 & 0 & 1.99 & 0.00 & 0.01 & 360 & -0.096 & 0.6 & 100.0 & 180 & 0 & 180 & 0.0 & 180 \\
\hline $00: 16: 30$ & 1 & -571 & 26 & 0 & 1.95 & 0.00 & 0.01 & 360 & -0.080 & 0.2 & 100.0 & 180 & 0 & 180 & 0.0 & 180 \\
\hline $00: 16: 40$ & 1 & -561 & 26 & 0 & 1.92 & -0.01 & 0.03 & 360 & -0.288 & -16.1 & 100.0 & 180 & 0 & 180 & 0.0 & 180 \\
\hline $00: 16: 50$ & 1 & -551 & 26 & 0 & 1.88 & -0.03 & 0.07 & 360 & -0.616 & -13.9 & 100.0 & 180 & 0 & 180 & 0.0 & 180 \\
\hline $00: 17: 00$ & 1 & -542 & 26 & 0 & 1.84 & -0.03 & 0.07 & 360 & -0.675 & 5.1 & 100.0 & 180 & 0 & 180 & 0.0 & 180 \\
\hline $00: 17: 10$ & 1 & -532 & 26 & 0 & 1.81 & -0.02 & 0.06 & 360 & -0.515 & 7.1 & 100.0 & 180 & 0 & 180 & 0.0 & 180 \\
\hline $00: 17: 20$ & 1 & -523 & 26 & 0 & 1.77 & -0.01 & 0.04 & 360 & -0.336 & 8.1 & 100.0 & 180 & 0 & 180 & 0.0 & 180 \\
\hline $00: 17: 30$ & 1 & -514 & 26 & 0 & 1.74 & 0.00 & 0.03 & 360 & -0.151 & 8.5 & 100.0 & 180 & 0 & 180 & 0.0 & 180 \\
\hline $00: 17: 40$ & 1 & -505 & 26 & 0 & 1.70 & 0.01 & 0.01 & 360 & 0.033 & 9.0 & 100.0 & 180 & 0 & 180 & 0.0 & 180 \\
\hline $00: 17: 50$ & 1 & -497 & 26 & 0 & 1.67 & 0.02 & -0.01 & 360 & 0.199 & 8.3 & 100.0 & 180 & 0 & 180 & 0.0 & 180 \\
\hline $00: 18: 00$ & 1 & -488 & 26 & 0 & 1.63 & 0.03 & -0.02 & 360 & 0.341 & 7.6 & 100.0 & 180 & 0 & 180 & 0.0 & 180 \\
\hline$: 28: 10$ & 1 & -480 & 26 & 0 & 1.60 & 0.04 & -0.03 & 360 & 0.452 & 6.4 & 100.0 & 180 & 0 & 180 & 0.0 & 180 \\
\hline$: 18: 20$ & 1 & -472 & 26 & 0 & 1.56 & 0.03 & -0.03 & 360 & 0.372 & -11.3 & 100.0 & 180 & 0 & 180 & 0.0 & 180 \\
\hline $00: 18: 30$ & 1 & -464 & 26 & 0 & 1.53 & 0.01 & 0.00 & 360 & 0.067 & -13.6 & 100.0 & 180 & 0 & 180 & 0.0 & 180 \\
\hline $00: 18: 40$ & 1 & -456 & 26 & 0 & 1.50 & 0.00 & 0.03 & 360 & -0.222 & -13.3 & 100.0 & 180 & 0 & 180 & 0.0 & 180 \\
\hline $00: 18: 50$ & 1 & -448 & 26 & 0 & 1.46 & -0.01 & 0.04 & 360 & -0.366 & 1.5 & 100.0 & 180 & 0 & 180 & 0.0 & 180 \\
\hline $00: 19: 00$ & 1 & -441 & 26 & 0 & 1.43 & 0.00 & 0.03 & 360 & -0.235 & 6.5 & 100.0 & 180 & 0 & 180 & 0.0 & 180 \\
\hline $00: 19: 10$ & 1 & -434 & 26 & 0 & 1.40 & -0.01 & 0.04 & 360 & -0.298 & -10.4 & 100.0 & 180 & 0 & 180 & 0.0 & 180 \\
\hline $00: 19: 20$ & 1 & -426 & 26 & 0 & 1.37 & -0.02 & 0.06 & 360 & -0.490 & -9.1 & 100.0 & 180 & 0 & 180 & 0.0 & 180 \\
\hline $00: 19: 30$ & 1 & -420 & 26 & 0 & 1.33 & -0.03 & 0.07 & 359 & -0.639 & -7.3 & 100.0 & 180 & 0 & 180 & 0.0 & 180 \\
\hline $00: 19: 40$ & 1 & -413 & 26 & 0 & 1.30 & -0.03 & 0.09 & 359 & -0.739 & -5.0 & 100.0 & 180 & 0 & 180 & 0.0 & 180 \\
\hline $00: 19: 50$ & 1 & -406 & 26 & 0 & 1.27 & -0.03 & 0.09 & 359 & -0.788 & -2.6 & 100.0 & 180 & 0 & 180 & 0.0 & 180 \\
\hline $00: 20: 00$ & 1 & -400 & 26 & 0 & 1.24 & -0.03 & 0.09 & 359 & -0.787 & -0.4 & 100.0 & 180 & 0 & 180 & 0.0 & 180 \\
\hline $00: 20: 10$ & 1 & -393 & 26 & 0 & 1.21 & -0.03 & 0.09 & 359 & -0.740 & 1.8 & 100.0 & 180 & 0 & 180 & 0.0 & 180 \\
\hline $00: 20: 20$ & 1 & -387 & 26 & 0 & 1.18 & -0.02 & 0.08 & 359 & -0.654 & 4.0 & 100.0 & 180 & 0 & 180 & 0.0 & 180 \\
\hline $00: 20: 30$ & 1 & -381 & 26 & 0 & 1.15 & -0.01 & 0.07 & 359 & -0.535 & 5.6 & 100.0 & 180 & 0 & 180 & 0.0 & 180 \\
\hline $00: 20: 40$ & 1 & -375 & 26 & 0 & 1.12 & 0.00 & 0.06 & 359 & -0.395 & 7.2 & 100.0 & 180 & 0 & 180 & 0.0 & 180 \\
\hline $00: 20 ; 50$ & 1 & -370 & 26 & 0 & 1.09 & 0.01 & 0.04 & 359 & -0.188 & 17.6 & 100.0 & 180 & 0 & 180 & 0.0 & 180 \\
\hline $00: 21: 00$ & 1 & -364 & 26 & 0 & 1.05 & 0.04 & -0.01 & 359 & 0.277 & 25.0 & 100.0 & 180 & 0 & 180 & 0.0 & 180 \\
\hline $00: 21: 10$ & 1 & -359 & 26 & 0 & 1.02 & 0.06 & -0.05 & 359 & 0.723 & 23.4 & 100.0 & 180 & 0 & 180 & 0.0 & 180 \\
\hline $00: 21: 20$ & 1 & -354 & 26 & 0 & 0.99 & 0.08 & -0.09 & 359 & 1.095 & 20.1 & 100.0 & 180 & 0 & 180 & 0.0 & 180 \\
\hline $00: 21: 30$ & 1 & -349 & 26 & 0 & 0.96 & 0.10 & -0.12 & 359 & 1.377 & 15.8 & 100.0 & 180 & 0 & 180 & 0.0 & 180 \\
\hline $00: 21: 40$ & 1 & -344 & 26 & 0 & 0.93 & 0.11 & -0.14 & 359 & 1.559 & 11.0 & 100.0 & 180 & 0 & 180 & 0.0 & 180 \\
\hline $00: 21: 50$ & 1 & -339 & 26 & 0 & 0.90 & 0.11 & -0.15 & 360 & 1.638 & 6.3 & 100.0 & 180 & 0 & 180 & 0.0 & 180 \\
\hline $00 ; 22: 00$ & 1 & -334 & 26 & 0 & 0.87 & 0.11 & -0.15 & 360 & 1.618 & 1.2 & 100.0 & 180 & 0 & 180 & 0.0 & 180 \\
\hline $00: 22: 10$ & 0 & -330 & 26 & 0 & 0.84 & 0.10 & -0.14 & 000 & 1.507 & -3.2 & 100.0 & 180 & 0 & 180 & 0.0 & 180 \\
\hline $00: 22: 20$ & 0 & -326 & 26 & 0 & 0.82 & 0.09 & -0.12 & 000 & 1.320 & -7.5 & 100.0 & 180 & 0 & 180 & 0.0 & 180 \\
\hline $00: 22: 30$ & 0 & -322 & 26 & 0 & 0.79 & 0.07 & -0.10 & 001 & 1.073 & -10.6 & 100.0 & 180 & 0 & 180 & 0.0 & 180 \\
\hline $00: 22: 40$ & 0 & -318 & 26 & 0 & 0.78 & 0.05 & -0.07 & 001 & 0.785 & -13.0 & 74.0 & 180 & 0 & 180 & 0.0 & 180 \\
\hline $00: 22: 50$ & 0 & -314 & 26 & 0 & 0.77 & 0.03 & -0.04 & 001 & 0.474 & -14.8 & 74.0 & 180 & 0 & 180 & 0.0 & 180 \\
\hline $00: 23: 00$ & 0 & -310 & 26 & 0 & 0.77 & 0.01 & -0.01 & 001 & 0.160 & -15.4 & 74.0 & 180 & 0 & 180 & 0.0 & 180 \\
\hline $00: 23: 10$ & 0 & -306 & 26 & 0 & 0.77 & -0.01 & 0.02 & 001 & -0.144 & -15.0 & 74.0 & 180 & 0 & 180 & 0.0 & 180 \\
\hline $00: 23: 20$ & 1 & -302 & 26 & 0 & 0.76 & -0.02 & 0.04 & 001 & -0.414 & -13.4 & 74.0 & 180 & 0 & 180 & 0.0 & 180 \\
\hline $00: 23: 30$ & 1 & -298 & 26 & 0 & 0.76 & -0.03 & 0.07 & 001 & -0.651 & -15.8 & 74.0 & 180 & 0 & 180 & 0.0 & 180 \\
\hline $00: 23: 40$ & 1 & -294 & 26 & 0 & 0.76 & -0.06 & 0.11 & 001 & -1.082 & -26.5 & 74.0 & 180 & 0 & 180 & 0.0 & 180 \\
\hline $00: 23: 50$ & 1 & -290 & 26 & 0 & 0.75 & -0.08 & 0.15 & 000 & -1.497 & -21.8 & 74.0 & 180 & 0 & 180 & 0.0 & 180 \\
\hline $00: 24: 00$ & 1 & -286 & 26 & 0 & 0.75 & -0.10 & 0.18 & 000 & -1.795 & -16.3 & 74.0 & 180 & 0 & 180 & 0.0 & 180 \\
\hline $00: 24: 10$ & 1 & -282 & 26 & 0 & 0.74 & -0.11 & 0.20 & 360 & -1.969 & -10.4 & 74.0 & 180 & 0 & 180 & 0.0 & 180 \\
\hline $00: 24: 20$ & 2 & -279 & 26 & 0 & 0.74 & -0.11 & 0.21 & 359 & -2.018 & -4.2 & 74.0 & 180 & 0 & 180 & 0.0 & 180 \\
\hline $0: 24: 30$ & 2 & -275 & 26 & 0 & 0.74 & -0.10 & 0.21 & 359 & -1.951 & 1.4 & 74.0 & 180 & 0 & 180 & 0.0 & 180 \\
\hline $24: 40$ & 2 & -271 & 26 & 0 & 0.73 & -0.09 & 0.19 & 359 & -1.781 & 6.8 & 74.0 & 180 & 0 & 180 & 0.0 & 180 \\
\hline $0: 24: 50$ & 2 & -267 & 26 & 0 & 0.73 & -0.07 & 0.17 & 358 & -1.525 & 11.5 & 74.0 & 180 & 0 & 180 & 0.0 & 180 \\
\hline $00: 25: 00$ & 2 & -263 & 26 & 0 & 0.73 & -0.05 & 0.14 & 358 & -1.206 & 14.9 & 74.0 & 180 & 0 & 180 & 0.0 & 180 \\
\hline $00: 25: 10$ & 2 & -260 & 26 & 0 & 0.73 & -0.03 & 0.10 & 358 & -0.844 & 17.7 & 74.0 & 180 & 0 & 180 & 0.0 & 180 \\
\hline $00: 25: 20$ & 2 & -256 & 26 & 0 & 0.72 & -0.01 & 0.07 & 358 & -0.464 & 18.8 & 74.0 & 180 & 0 & 180 & 0.0 & 180 \\
\hline
\end{tabular}


Speed (Knots)

Turn Rudder Gill

wind

Current

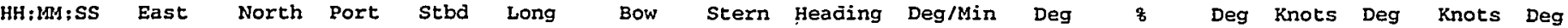

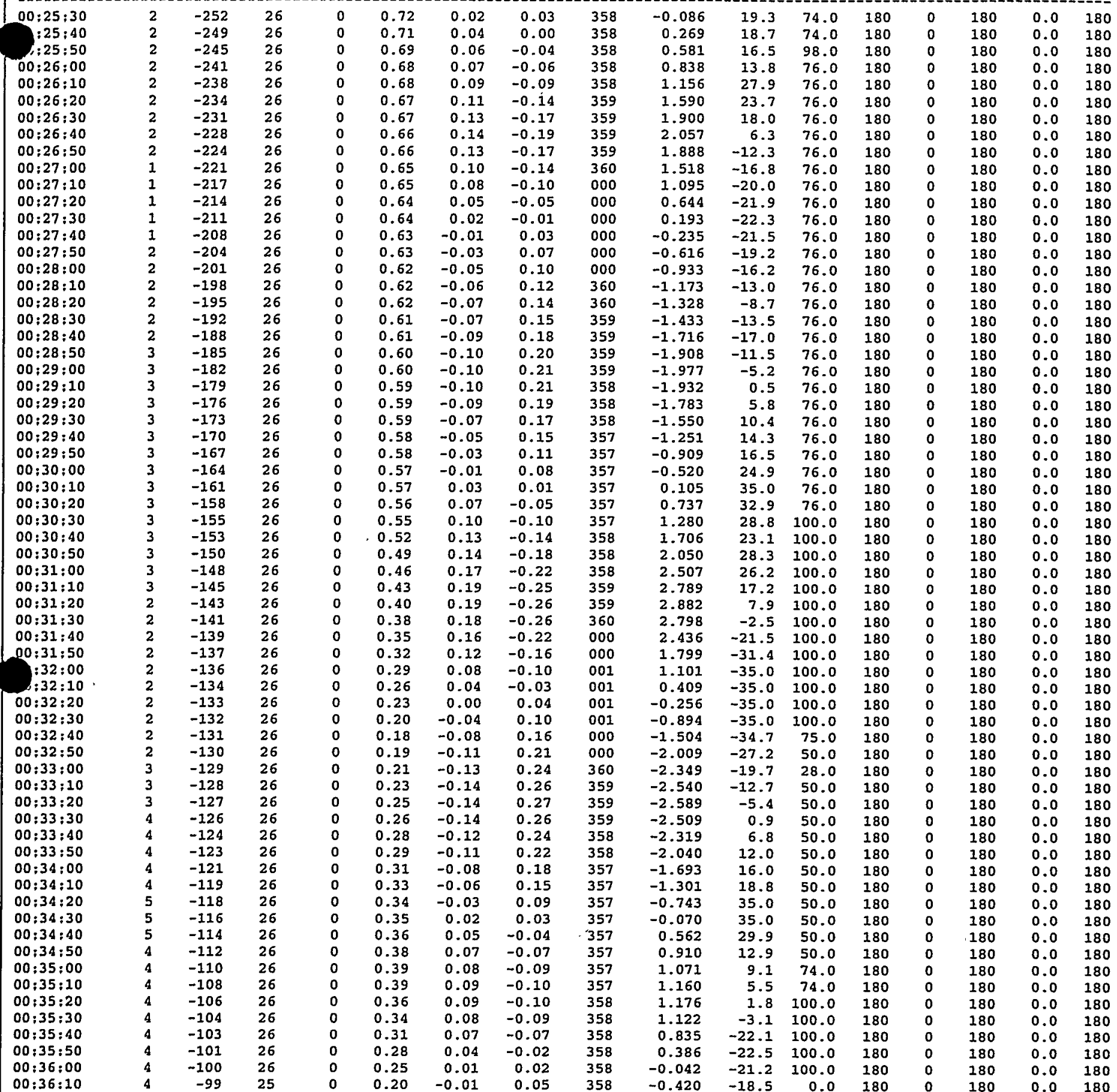


Open Ocean

\section{Simulation Track Plot}

Ship: 150,000 ton, Tanker, partial load
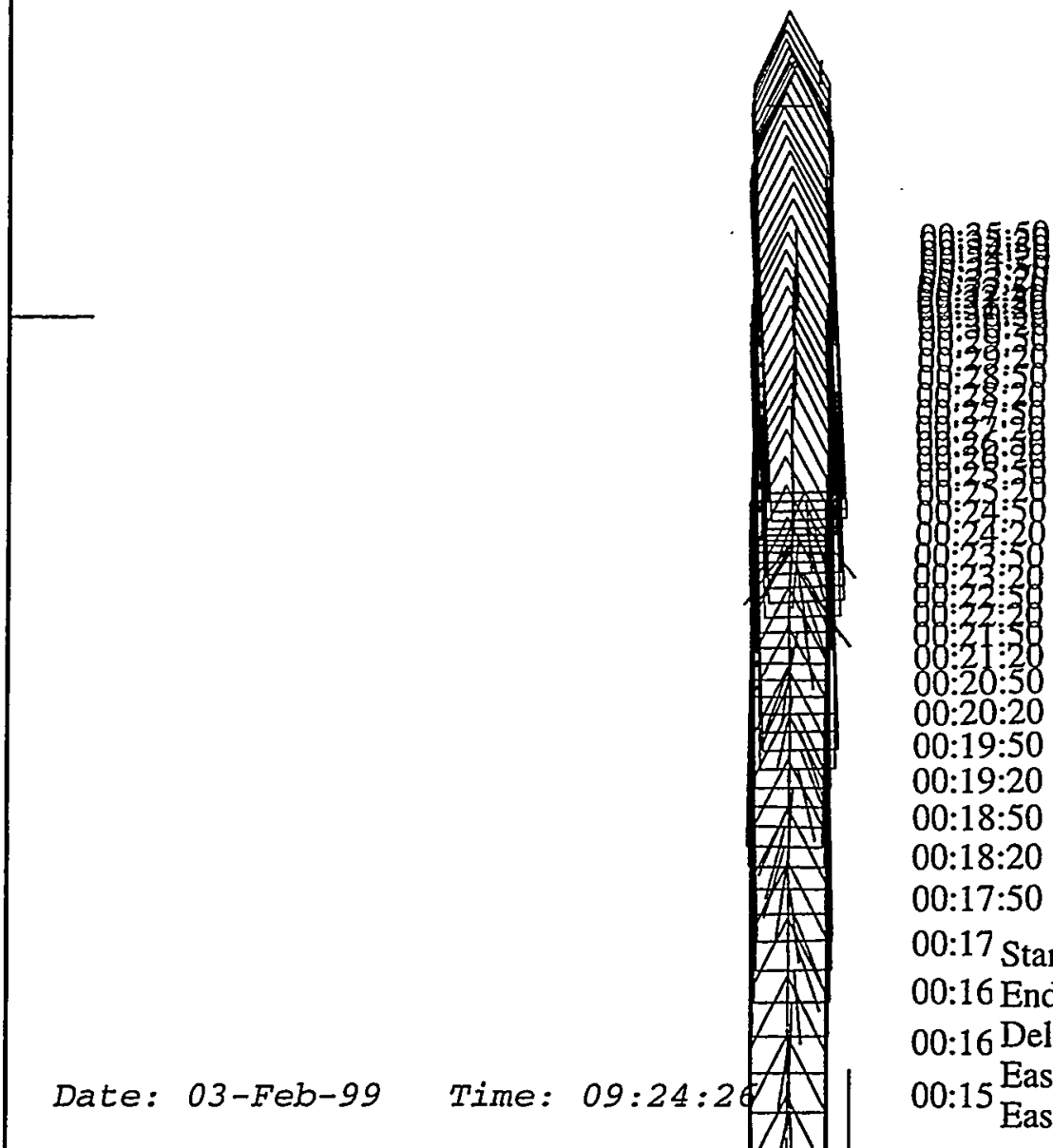

00:17:50

00:17 Start Time [hrs:min:sec]: $00: 13: 20$ 00:16 End Time [hrs:min:sec]: 00:36:15 00:16 Delta Plot Time [sec]: 00:15 East-West Scale [m]: 


\section{HANDS-ON SIMULATION}

\section{SUCTION MOORNG TECHNOLOGY}

Simulation Number: 12

Start Time: 10:06

Date: 3 February 1999

Length of run [secs]: 1650

Ship Condition: draft $=13.72 \mathrm{~m}$

Environment

Wind Speed [knots]:

0 Direction: 0

Sea State, Significant wave height [m]:..

0 Direction: 0

Current Speed [knots]:..................

2 Direction: 180

Ship Operator: J.A.

Observer: B.K.J \& J.K.

Final Mating, distance between center of buoy and center of moon pool [m]: 0.2

Evaluation:

Very successful run.

Very similar to run \# 11. It tracked well. Recommend start of simulation $1700 \mathrm{~m}$ south of the buoy instead of $1500 \mathrm{~m}$. 
Ship:

Exercise number:

Ecenario: g file:

caffic ships:

Initial conditions:

Exercise identification
591150,000 ton, Tanker, partial load

754 Suction Mooring System

112 Open Ocean

999 Dumny tug file based on containership

590 Suction Mooring Buoy

594 150k tanker in partial load - Suction Mooring $\operatorname{RuN} \# 12$

Time Position (m) RPM Speed (Knots) Turn Rucaer Gill Wurrent

\begin{tabular}{|c|c|c|c|c|c|c|c|c|c|c|c|c|c|c|c|c|}
\hline $\begin{array}{l}00: 08: 20 \\
00: 08: 30\end{array}$ & $\begin{array}{l}0 \\
0\end{array}$ & $\begin{array}{l}-596 \\
-587\end{array}$ & $\begin{array}{l}26 \\
26\end{array}$ & $\begin{array}{l}0 \\
0\end{array}$ & $\begin{array}{l}1.83 \\
1.80\end{array}$ & $\begin{array}{l}-0.01 \\
-0.01\end{array}$ & $\begin{array}{l}0.04 \\
0.04\end{array}$ & 000 & & $\begin{array}{r}-0.9 \\
0.9\end{array}$ & 76.0 & 180 & 0 & 000 & 2.0 & 180 \\
\hline $\begin{array}{l}00: 08: 30 \\
00: 08: 40\end{array}$ & 0 & -577 & 26 & 0 & 1.77 & -0.03 & 0.06 & 360 & $\begin{array}{l}-0.321 \\
-0.538\end{array}$ & $\begin{array}{r}0.2 \\
-16.5\end{array}$ & $\begin{array}{l}76.0 \\
76.0\end{array}$ & $\begin{array}{l}180 \\
180\end{array}$ & $\begin{array}{l}0 \\
0\end{array}$ & $\begin{array}{l}000 \\
000\end{array}$ & $\begin{array}{l}2.0 \\
2.0\end{array}$ & $\begin{array}{l}180 \\
180\end{array}$ \\
\hline $00 ; 08: 50$ & 1 & -568 & 26 & 0 & 1.74 & -0.05 & 0.10 & 360 & -0.962 & -14.0 & 76.0 & 180 & 0 & 000 & 2.0 & 180 \\
\hline $00: 09: 00$ & 1 & -560 & 26 & 0 & 1.71 & -0.07 & 0.13 & 360 & -1.267 & -10.4 & 76.0 & 180 & 0 & 000 & 2.0 & 180 \\
\hline $00: 09: 10$ & 1 & -551 & 26 & 0 & 1.68 & -0.08 & 0.15 & 359 & -1.443 & -5.3 & 76.0 & 180 & 0 & 000 & 2.0 & 180 \\
\hline $00: 09: 20$ & 1 & -542 & 26 & 0 & 1.65 & -0.08 & 0.16 & 359 & -1.493 & -1.7 & 76.0 & 180 & 0 & 000 & 2.0 & 180 \\
\hline $00: 09: 30$ & 1 & -534 & 26 & 0 & 1.62 & -0.07 & 0.15 & 359 & -1.425 & 2.7 & 76.0 & 180 & 0 & 000 & 2.0 & 180 \\
\hline $00: 09: 40$ & 1 & -526 & 26 & 0 & 1.59 & -0.06 & 0.14 & 359 & -1.258 & 6.6 & 76.0 & 180 & 0 & 000 & 2.0 & 180 \\
\hline $00: 09: 50$ & 1 & -518 & 26 & 0 & 1.56 & -0.05 & 0.11 & 358 & -1.015 & 9.8 & 76.0 & 180 & 0 & 000 & 2.0 & 180 \\
\hline $00: 10: 00$ & 1 & -510 & 26 & 0 & 1.53 & -0.03 & 0.08 & 358 & -0.721 & 12.4 & 76.0 & 180 & 0 & 000 & 2.0 & 180 \\
\hline $00: 10: 10$ & 1 & -502 & 26 & 0 & 1.50 & -0.02 & 0.05 & 358 & -0.403 & 13.8 & 76.0 & 180 & 0 & 000 & 2.0 & 180 \\
\hline $00: 10: 20$ & 1 & -494 & 26 & 0 & 1.47 & 0.01 & 0.00 & 358 & 0.033 & 26.6 & 76.0 & 180 & 0 & 000 & 2.0 & 180 \\
\hline $00: 10 ; 30$ & 0 & -487 & 26 & 0 & 1.44 & 0.05 & -0.08 & 358 & 0.774 & 30.3 & 76.0 & 180 & 0 & 000 & 2.0 & 180 \\
\hline $00: 10: 40$ & 0 & -479 & 26 & 0 & 1.41 & 0.08 & -0.14 & 358 & 1.402 & 26.4 & 76.0 & 180 & 0 & 000 & 2.0 & 180 \\
\hline $00: 10 ; 50$ & 0 & -472 & 26 & 0 & 1.38 & 0.10 & -0.19 & 359 & 1.868 & 21.0 & 50.0 & 180 & 0 & 000 & 2.0 & 180 \\
\hline $00: 11: 00$ & -1 & -465 & 26 & 0 & 1.37 & 0.11 & -0.23 & 359 & 2.155 & 15.0 & 50.0 & 180 & 0 & 000 & 2.0 & 180 \\
\hline $00: 11: 10$ & -1 & -458 & 26 & 0 & 1.36 & 0.12 & -0.24 & 359 & 2.262 & 8.2 & 50.0 & 180 & 0 & 000 & 2.0 & 180 \\
\hline $00: 11: 20$ & -2 & -451 & 26 & 0 & 1.36 & 0.11 & -0.24 & 360 & 2.199 & 1.5 & 50.0 & 180 & 0 & 000 & 2.0 & 180 \\
\hline $00: 11: 30$ & -2 & -444 & 26 & 0 & 1.35 & 0.10 & -0.23 & 000 & 2.079 & 7.7 & 50.0 & 180 & 0 & 000 & 2.0 & 180 \\
\hline $00: 11: 40$ & -2 & -437 & 26 & 0 & 1.34 & 0.10 & -0.24 & 001 & 2.197 & 6.5 & 50.0 & 180 & 0 & 000 & 2.0 & 180 \\
\hline $00: 11: 50$ & -3 & -430 & 26 & 0 & 1.33 & 0.10 & -0.24 & 001 & 2.157 & -0.1 & 50.0 & 180 & 0 & 000 & 2.0 & 180 \\
\hline $00: 12: 00$ & -3 & -423 & 26 & 0 & 1.33 & 0.09 & -0.22 & 001 & 1.968 & -6.3 & 50.0 & 180 & 0 & 000 & 2.0 & 180 \\
\hline $00: 12: 10$ & -3 & -417 & 26 & 0 & 1.32 & 0.07 & -0.19 & 002 & 1.659 & -11.5 & 50.0 & 180 & 0 & 000 & 2.0 & 180 \\
\hline $00: 12 ; 20$ & -3 & -410 & 26 & 0 & 1.31 & 0.05 & -0.15 & 002 & 1.263 & -15.2 & 50.0 & 180 & 0 & 000 & 2.0 & 180 \\
\hline $00: 12: 30$ & -3 & -403 & 26 & 0 & 1.31 & 0.03 & -0.10 & 002 & 0.817 & -18.2 & 27.0 & 180 & 0 & 000 & 2.0 & 180 \\
\hline $00: 12: 40$ & -3 & -396 & 26 & 0 & 1.31 & 0.00 & -0.05 & 002 & 0.357 & -19.5 & 27.0 & 180 & 0 & 000 & 2.0 & 180 \\
\hline $00: 12: 50$ & -3 & -390 & 26 & 0 & 1.32 & -0.02 & -0.01 & 002 & -0.087 & -19.6 & 27.0 & 180 & 0 & 000 & 2.0 & 180 \\
\hline $0: 13: 00$ & -3 & -383 & 26 & 0 & 1.32 & -0.04 & 0.04 & 002 & -0.486 & -18.2 & 27.0 & 180 & 0 & 000 & 2.0 & 180 \\
\hline$: 13: 10$ & -2 & -376 & 26 & 0 & 1.32 & -0.05 & 0.08 & 002 & -0.814 & -16.2 & 27.0 & 180 & 0 & 000 & 2.0 & 180 \\
\hline S:13:20 & -2 & -369 & 26 & 0 & 1.32 & -0.06 & 0.11 & 002 & -1.058 & -13.1 & 27.0 & 180 & 0 & 000 & 2.0 & 180 \\
\hline $00: 13: 30$ & -2 & -362 & 26 & 0 & 1.33 & -0.07 & 0.13 & 002 & -1.208 & -9.6 & 27.0 & 180 & 0 & 000 & 2.0 & 180 \\
\hline $00: 13: 40$ & -1 & -356 & 26 & 0 & 1.33 & -0.08 & 0.16 & 001 & -1.525 & -23.4 & 27.0 & 180 & 0 & 000 & 2.0 & 180 \\
\hline $00: 13: 50$ & -1 & -349 & 26 & 0 & 1.29 & -0.10 & 0.20 & 001 & -1.918 & -17.9 & 100.0 & 180 & 0 & 000 & 2.0 & 180 \\
\hline $00: 14: 00$ & 0 & -342 & 26 & 0 & 1.24 & -0.11 & 0.23 & 001 & -2.133 & -11.5 & 78.0 & 180 & 0 & 000 & 2.0 & 180 \\
\hline $00: 14: 10$ & 0 & -336 & 26 & 0 & 1.21 & -0.11 & 0.24 & 000 & -2.179 & -4.7 & 78.0 & 180 & 0 & 000 & 2.0 & 180 \\
\hline $00: 14: 20$ & 0 & -330 & 26 & 0 & 1.18 & -0.10 & 0.23 & 000 & -2.068 & 1.5 & 78.0 & 180 & 0 & 000 & 2.0 & 180 \\
\hline $00: 14: 30$ & 1 & -324 & 26 & 0 & 1.16 & -0.08 & 0.21 & 360 & -1.827 & 7.4 & 78.0 & 180 & 0 & 000 & 2.0 & 180 \\
\hline $00: 14: 40$ & 1 & -318 & 26 & 0 & 1.13 & -0.06 & 0.17 & 359 & -1.487 & 12.2 & 78.0 & 180 & 0 & 000 & 2.0 & 180 \\
\hline $00 ; 14: 50$ & 1 & -312 & 26 & 0 & 1.10 & -0.04 & 0.13 & 359 & -1.081 & 15.2 & 78.0 & 180 & 0 & 000 & 2.0 & 180 \\
\hline $00: 15: 00$ & 1 & -307 & 26 & 0 & 1.08 & -0.01 & 0.09 & 359 & -0.644 & 17.6 & 78.0 & 180 & 0 & 000 & 2.0 & 180 \\
\hline $00: 15: 10$ & $i$ & -301 & 26 & 0 & 1.05 & 0.01 & 0.05 & 359 & -0.210 & 18.2 & 78.0 & 180 & 0 & 000 & 2.0 & 180 \\
\hline $00 ; 15: 20$ & 1 & -296 & 26 & 0 & 1.02 & 0.03 & 0.00 & 359 & 0.200 & 17.9 & 78.0 & 180 & 0 & 000 & 2.0 & 180 \\
\hline $00: 15: 30$ & 1 & -291 & 26 & 0 & 1.00 & 0.05 & -0.03 & 359 & 0.552 & 16.3 & 78.0 & 180 & 0 & 000 & 2.0 & 180 \\
\hline $00: 15: 40$ & 1 & -285 & 26 & 0 & 0.98 & 0.07 & -0.07 & .359 & 0.831 & 14.2 & 50.0 & 180 & 0 & 000 & 2.0 & 180 \\
\hline $00: 15: 50$ & 1 & -280 & 26 & 0 & 0.97 & 0.07 & -0.09 & 359 & 1.029 & 11.1 & 74.0 & 180 & 0 & 000 & 2.0 & 180 \\
\hline $00: 16: 00$ & 1 & -275 & 26 & 0 & 0.95 & 0.08 & -0.10 & 360 & 1.140 & 7.6 & 74.0 & 180 & 0 & 000 & 2.0 & 180 \\
\hline $00: 16: 10$ & 1 & -271 & 26 & 0 & 0.93 & 0.08 & -0.11 & 360 & 1.165 & 4.2 & 74.0 & 180 & 0 & 000 & 2.0 & 180 \\
\hline $00: 16: 20$ & 1 & -266 & 26 & 0 & 0.91 & 0.07 & -0.10 & 360 & 1.111 & 0.8 & 74.0 & 180 & 0 & 000 & 2.0 & 180 \\
\hline $00 ; 16 ; 30$ & 1 & -261 & 26 & 0 & 0.89 & 0.06 & -0.09 & 000 & 0.990 & -2.4 & 74.0 & 180 & 0 & 000 & 2.0 & 180 \\
\hline $00: 16: 40$ & 1 & -257 & 26 & 0 & 0.87 & 0.04 & -0.06 & 000 & 0.640 & -20.0 & 74.0 & 180 & 0 & 000 & 2.0 & 180 \\
\hline $00: 16: 50$ & 1 & -252 & 26 & 0 & 0.85 & 0.00 & 0.00 & 000 & 0.030 & -22.6 & 74.0 & 180 & 0 & 000 & 2.0 & 180 \\
\hline $00: 17: 00$ & $I$ & -248 & 26 & 0 & 0.83 & -0.03 & 0.05 & 000 & -0.518 & -21.2 & 74.0 & 180 & 0 & 000 & 2.0 & 180 \\
\hline $00: 17: 10$ & 1 & -244 & 26 & 0 & 0.81 & -0.05 & 0.10 & 000 & -0.974 & -18.3 & 74.0 & 180 & 0 & 000 & 2.0 & 180 \\
\hline $00: 17: 20$ & 1 & -240 & 26 & 0 & 0.79 & -0.07 & 0.13 & 360 & -1.315 & -14.4 & 74.0 & 180 & 0 & 000 & 2.0 & 180 \\
\hline $00: 17: 30$ & 1 & -236 & 26 & 0 & 0.77 & -0.09 & 0.16 & 360 & -1.531 & -9.6 & 74.0 & 180 & 0 & 000 & 2.0 & 180 \\
\hline $00: 17: 40$ & 1 & -232 & 26 & 0 & 0.75 & -0.09 & 0.17 & 359 & -1.621 & -4.8 & 74.0 & 180 & 0 & 000 & 2.0 & 180 \\
\hline $00: 17: 50$ & 1 & -228 & 26 & 0 & 0.73 & -0.09 & 0.16 & 359 & -1.593 & 0.2 & 74.0 & 180 & 0 & 000 & 2.0 & 180 \\
\hline $00: 18: 00$ & 2 & -224 & 26 & 0 & 0.72 & -0.08 & 0.15 & 359 & -1.463 & 4.7 & 74.0 & 180 & 0 & 000 & 2.0 & 180 \\
\hline $00: 18: 10$ & 2 & -220 & 26 & 0 & 0.70 & -0.07 & 0.13 & 359 & -1.251 & 8.3 & 74.0 & 180 & 0 & 000 & 2.0 & 180 \\
\hline $00: 18: 20$ & 2 & -217 & 26 & 0 & 0.68 & -0.05 & 0.10 & 358 & -0.981 & 11.3 & 74.0 & 180 & 0 & 000 & 2.0 & 180 \\
\hline $00: 18: 30$ & 2 & -213 & 26 & 0 & 0.66 & -0.04 & 0.07 & 358 & -0.676 & 13.6 & 66.0 & 180 & 0 & 000 & 2.0 & 180 \\
\hline $00: 18: 40$ & 2 & -210 & 26 & 0 & 0.65 & -0.02 & 0.04 & 358 & -0.362 & 14.2 & 66.0 & 180 & 0 & 000 & 2.0 & 180 \\
\hline $00 ; 18 ; 50$ & 2 & -207 & 26 & 0 & 0.64 & -0.01 & 0.00 & 358 & -0.057 & 14.7 & 66.0 & 180 & 0 & 000 & 2.0 & 180 \\
\hline $00: 19: 00$ & 1 & -203 & 26 & 0 & 0.63 & 0.01 & -0.03 & 358 & 0.221 & 13.7 & 66.0 & 180 & 0 & 000 & 2.0 & 180 \\
\hline $00: 19: 10$ & 1 & -200 & 26 & 0 & 0.62 & 0.02 & -0.05 & 358 & 0.453 & 12.1 & 66.0 & 180 & 0 & 000 & 2.0 & 180 \\
\hline $00: 19: 20$ & 1 & -197 & 26 & 0 & 0.61 & 0.04 & -0.09 & 358 & 0.830 & 27.7 & 66.0 & 180 & 0 & 000 & 2.0 & 180 \\
\hline$: 19: 30$ & 1 & -194 & 26 & 0 & 0.59 & 0.07 & -0.14 & 359 & 1.330 & 23.4 & 66.0 & 180 & 0 & 000 & 2.0 & 180 \\
\hline$: 19: 40$ & 1 & -191 & 26 & 0 & 0.58 & 0.09 & -0.18 & 359 & 1.689 & 18.1 & 66.0 & 180 & 0 & 000 & 2.0 & 180 \\
\hline $0: 19: 50$ & 0 & -188 & 26 & 0 & 0.57 & 0.10 & -0.20 & 359 & 1.898 & 12.4 & 66.0 & 180 & 0 & 000 & 2.0 & 180 \\
\hline $00: 20: 00$ & 0 & -185 & 26 & 0 & 0.56 & 0.10 & -0.21 & 359 & 1.961 & 6.1 & 66.0 & 180 & 0 & 000 & 2.0 & 180 \\
\hline $00 ; 20 ; 10$ & 0 & -182 & 26 & 0 & 0.55 & 0.09 & -0.20 & 360 & 1.891 & 0.4 & 66.0 & 180 & 0 & 000 & 2.0 & 180 \\
\hline & -1 & -179 & 26 & 0 & 0.54 & & -0.19 & 000 & & & & & & & & \\
\hline
\end{tabular}




\begin{tabular}{|c|c|c|c|c|c|c|c|c|c|c|c|c|c|c|c|c|}
\hline \multirow{2}{*}{$\begin{array}{c}\text { Time } \\
H H: M M: S S\end{array}$} & \multicolumn{2}{|c|}{ Position (m) } & \multicolumn{2}{|c|}{ RPM } & \multicolumn{3}{|c|}{ Speed (Knots) } & \multirow[b]{2}{*}{ Heading } & \multirow{2}{*}{$\begin{array}{c}\text { Turn } \\
\text { Deg/Min }\end{array}$} & \multirow{2}{*}{$\begin{array}{l}\text { Rudder } \\
\text { Deg }\end{array}$} & \multicolumn{2}{|c|}{ Gill } & \multicolumn{2}{|c|}{ wind } & \multicolumn{2}{|c|}{ Current } \\
\hline & East & North & Port & stbd & Long & Bow & stern & & & & 8 & Deg & knots & Deg & Knots & Deg \\
\hline $0: 20: 30$ & -1 & -177 & 26 & 0 & 0.52 & 0.07 & -0.16 & 000 & 1.441 & -8.9 & 76.0 & 180 & 0 & 000 & 2.0 & 180 \\
\hline $1: 20: 40$ & -1 & -174 & 26 & 0 & 0.50 & 0.05 & -0.13 & 001 & 1.165 & -2.7 & 76.0 & 180 & 0 & 000 & 2.0 & 180 \\
\hline $30: 20: 50$ & -1 & -172 & 26 & 0 & 0.49 & 0.05 & -0.13 & 001 & 1.170 & 2.2 & 76.0 & 180 & 0 & 000 & 2.0 & 180 \\
\hline $00: 21 ; 00$ & -1 & -169 & 26 & 0 & 0.47 & 0.05 & -0.13 & 001 & 1.131 & -1.1 & 76.0 & 180 & 0 & 000 & 2.0 & 180 \\
\hline $00: 21: 10$ & -2 & -167 & 26 & 0 & 0.46 & 0.05 & -0.11 & 001 & 1.021 & -4.3 & 63.0 & 180 & 0 & 000 & 2.0 & 180 \\
\hline $00: 21: 20$ & -2 & -164 & 26 & 0 & 0.45 & 0.04 & -0.10 & 001 & 0.860 & -6.8 & 63.0 & 180 & 0 & 000 & 2.0 & 180 \\
\hline $00: 21: 30$ & -2 & -162 & 26 & 0 & 0.45 & 0.03 & -0.08 & 001 & 0.664 & -8.5 & 52.0 & 180 & 0 & 000 & 2.0 & 180 \\
\hline $00: 21: 40$ & -2 & -160 & 26 & 0 & 0.45 & 0.02 & -0.05 & 001 & 0.449 & -9.6 & 52.0 & 180 & 0 & 000 & 2.0 & 180 \\
\hline $00: 21: 50$ & -2 & -157 & 26 & 0 & 0.45 & 0.01 & -0.03 & 002 & 0.232 & -10.2 & 52.0 & 180 & 0 & 000 & 2.0 & 180 \\
\hline $00: 22: 00$ & -2 & -155 & 26 & 0 & 0.45 & 0.00 & -0.01 & 002 & 0.025 & -10.2 & 52.0 & 180 & 0 & 000 & 2.0 & 180 \\
\hline $00: 22: 10$ & -2 & -153 & 26 & 0 & 0.45 & -0.01 & 0.02 & 002 & -0.188 & -16.0 & 52.0 & 180 & 0 & 000 & 2.0 & 180 \\
\hline $00 ; 22: 20$ & -2 & -151 & 26 & 0 & 0.45 & -0.04 & 0.07 & 001 & -0.676 & -24.7 & 52.0 & 180 & 0 & 000 & 2.0 & 180 \\
\hline $00: 22: 30$ & -1 & -148 & 26 & 0 & 0.45 & -0.06 & 0.11 & 001 & -1.124 & -20.8 & 52.0 & 180 & 0 & 000 & 2.0 & 180 \\
\hline $00: 22: 40$ & -1 & -146 & 26 & 0 & 0.44 & -0.08 & 0.15 & 001 & -1.449 & -16.4 & 52.0 & 180 & 0 & 000 & 2.0 & 180 \\
\hline $00: 22: 50$ & -1 & -144 & 26 & 0 & 0.44 & -0.09 & 0.17 & 001 & -1.644 & -10.9 & 52.0 & 180 & 0 & 000 & 2.0 & 180 \\
\hline $00: 23: 00$ & -1 & -141 & 26 & 0 & 0.45 & -0.09 & 0.18 & 001 & -1.713 & -5.7 & 52.0 & 180 & 0 & 000 & 2.0 & 180 \\
\hline $00: 23: 10$ & -1 & -139 & 26 & 0 & 0.44 & -0.09 & 0.17 & 000 & -1.667 & -0.6 & 76.0 & 180 & 0 & 000 & 2.0 & 180 \\
\hline $00: 23: 20$ & 0 & -137 & 26 & 0 & 0.43 & -0.08 & 0.16 & 000 & -1.523 & 3.6 & 76.0 & 180 & 0 & 000 & 2.0 & 180 \\
\hline $00: 23: 30$ & 0 & -135 & 26 & 0 & 0.41 & -0.07 & 0.14 & 360 & -1.301 & 7.7 & 76.0 & 180 & 0 & 000 & 2.0 & 180 \\
\hline $00: 23: 40$ & 0 & -133 & 26 & 0 & 0.39 & -0.05 & 0.11 & 360 & -1.025 & 10.4 & 76.0 & 180 & 0 & 000 & 2.0 & 180 \\
\hline $00: 23: 50$ & 0 & -131 & 26 & 0 & 0.38 & -0.03 & 0.08 & 359 & -0.722 & 12.4 & 67.0 & 180 & 0 & 000 & 2.0 & 180 \\
\hline $00: 24: 00$ & 0 & -129 & 26 & 0 & 0.37 & -0.01 & 0.05 & 359 & -0.413 & 13.2 & 67.0 & 180 & 0 & 000 & 2.0 & 180 \\
\hline $00: 24 ; 10$ & 0 & -127 & 26 & 0 & 0.36 & 0.00 & 0.02 & 359 & -0.122 & 12.8 & 67.0 & 180 & 0 & 000 & 2.0 & 180 \\
\hline $00: 24: 20$ & 0 & -125 & 26 & 0 & 0.35 & 0.02 & -0.01 & 359 & 0.138 & 11.9 & 67.0 & 180 & 0 & 000 & 2.0 & 180 \\
\hline $00: 24: 30$ & 0 & -123 & 26 & 0 & 0.34 & 0.03 & -0.03 & 359 & 0.355 & 10.6 & 67.0 & 180 & 0 & 000 & 2.0 & 180 \\
\hline $00: 24: 40$ & 0 & -121 & 26 & 0 & 0.33 & 0.04 & -0.05 & 359 & 0.520 & 8.9 & 67.0 & 180 & 0 & 000 & 2.0 & 180 \\
\hline $00: 24: 50$ & 0 & -120 & 26 & 0 & 0.32 & 0.04 & -0.06 & 360 & 0.630 & 6.7 & 79.0 & 180 & 0 & 000 & 2.0 & 180 \\
\hline $00: 25 ; 00$ & 0 & -118 & 26 & 0 & 0.30 & 0.05 & -0.06 & 360 & 0.684 & 4.1 & 79.0 & 180 & 0 & 000 & 2.0 & 180 \\
\hline $00: 25: 10$ & 0 & -117 & 26 & 0 & 0.29 & 0.04 & -0.06 & 360 & 0.687 & 1.7 & 70.0 & 180 & 0 & 000 & 2.0 & 180 \\
\hline $00: 25: 20$ & 0 & -115 & 26 & 0 & 0.28 & 0.04 & -0.06 & 360 & 0.645 & 0.1 & 63.0 & 180 & 0 & 000 & 2.0 & 180 \\
\hline $00: 25: 30$ & 0 & -114 & 26 & 0 & 0.27 & 0.04 & -0.05 & 360 & 0.571 & -1.3 & 63.0 & 180 & 0 & 000 & 2.0 & 180 \\
\hline $00: 25: 40$ & 0 & -112 & 26 & 0 & 0.27 & 0.03 & -0.04 & 000 & 0.474 & -2.7 & 63.0 & 180 & 0 & 000 & 2.0 & 180 \\
\hline $00: 25: 50$ & 0 & -111 & 26 & 0 & 0.26 & 0.02 & -0.03 & 000 & 0.364 & -3.3 & 63.0 & 180 & 0 & 000 & 2.0 & 180 \\
\hline $00: 26: 00$ & 0 & -110 & 26 & 0 & 0.26 & 0.02 & -0.02 & 000 & 0.247 & -3.9 & 63.0 & 180 & 0 & 000 & 2.0 & 180 \\
\hline $00: 26 ; 10$ & 0 & -108 & 26 & 0 & 0.26 & 0.01 & -0.01 & 000 & 0.135 & -4.0 & 63.0 & 180 & 0 & 000 & 2.0 & 180 \\
\hline $00: 26: 20$ & 0 & -107 & 26 & 0 & 0.25 & 0.00 & 0.00 & 000 & 0.033 & -4.1 & 63.0 & 180 & 0 & 000 & 2.0 & 180 \\
\hline $00: 26: 30$ & 0 & -106 & 26 & 0 & 0.25 & 0.00 & 0.01 & 000 & -0.060 & -3.7 & 63.0 & 180 & 0 & 000 & 2.0 & 180 \\
\hline $00: 26: 40$ & 0 & -104 & 26 & 0 & 0.24 & 0.00 & 0.02 & 000 & -0.127 & -2.8 & 63.0 & 180 & 0 & 000 & 2.0 & 180 \\
\hline$-90: 26: 50$ & 0 & -103 & 26 & 0 & 0.24 & -0.01 & 0.02 & 000 & -0.178 & -1.9 & 63.0 & 180 & 0 & 000 & 2.0 & 180 \\
\hline $0: 27: 00$ & 0 & -102 & 26 & 0 & 0.23 & -0.01 & 0.02 & 000 & -0.210 & -1.2 & 63.0 & 180 & 0 & 000 & 2.0 & 180 \\
\hline $0: 27: 10$ & 0 & -101 & 26 & 0 & 0.23 & -0.01 & 0.03 & 000 & -0.223 & -0.5 & 63.0 & 180 & 0 & 000 & 2.0 & 180 \\
\hline $00: 27: 20$ & 0 & -100 & 26 & 0 & 0.22 & -0.01 & 0.03 & 000 & -0.223 & 0.1 & 63.0 & 180 & 0 & 000 & 2.0 & 180 \\
\hline
\end{tabular}


Open Ocean

\section{Simulation Track Plot}

Ship: 150,000 ton, Tanker, partial load

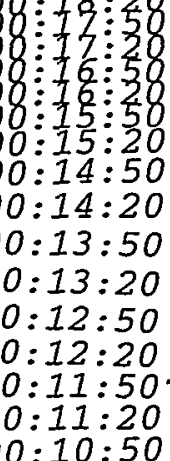

$00: 10: 50$
$00: 10: 20$

$00:$ ( Start Time [hrs:min:sec]: 00:08:20

$00:($ End Time [hrs:min:sec]: $00: 27: 30$

$00:($ Delta Plot Time [sec]:

00:08 East-West Scale [m]:

30

Date: 03-Feb-99

Time: 10:06:4

East-West Scale [Nm]:

926.

0.5000 


\section{HANDS-ON SIMULATION \\ SUCTION MOORNG TECHNOLOGY}

Simulation Number: 13

Date: 3 February 1999

Start Time: 10:46

Length of run [secs]: 1785

Ship Condition: $\mathrm{draft}=13.72 \mathrm{~m}$

Environment

Wind Speed [knots]:

20 Direction: 0

Sea State, Significant wave height $[\mathrm{m}]: .$.

3 Direction: 180

Current Speed [knots]:

0 Direction: 0

Ship Operator: J.A.

Observer: B.K.J \& J.K.

Final Mating, distance between center of buoy and center of moon pool [m]: 1.25

Evaluation:

Successful run.

Everything worked out okay.

A quicker response of the autopilot might help the course keeping.

The deceleration plan worked out well, 0.3 knots at mating. 
Ship: 591150,000 ton, Tanker, partial load

Exercise number: 754 Suction Mooring System

112 Open Ocean

Genario:

999 Dummy tug file based on containership

cafic ships:

590 Suction Mooring Buoy

Initial conditions: $\quad 594$ 150k tanker in partial load - Suction Mooring

Exercise identification: R UN \#13

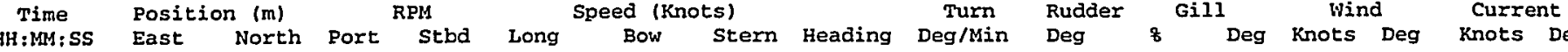

\begin{tabular}{|c|c|c|c|c|c|c|c|c|c|c|c|c|c|c|c|c|}
\hline $\begin{array}{l}00: 08: 20 \\
00: 08: 30\end{array}$ & $\begin{array}{l}0 \\
0\end{array}$ & $\begin{array}{l}-873 \\
-859\end{array}$ & $\begin{array}{l}28 \\
28\end{array}$ & $\begin{array}{l}0 \\
0\end{array}$ & $\begin{array}{l}2.76 \\
2.69\end{array}$ & $\begin{array}{l}0.00 \\
0.00\end{array}$ & $\begin{array}{l}0.01 \\
0.01\end{array}$ & $\begin{array}{l}360 \\
360\end{array}$ & $\begin{array}{l}-0.050 \\
-0.043\end{array}$ & $\begin{array}{l}0.6 \\
0.6\end{array}$ & $\begin{array}{r}100.0 \\
55.0\end{array}$ & $\begin{array}{l}180 \\
180\end{array}$ & $\begin{array}{l}22 \\
23\end{array}$ & $\begin{array}{l}000 \\
000\end{array}$ & $\begin{array}{l}0.0 \\
0.0\end{array}$ & $\begin{array}{l}180 \\
180\end{array}$ \\
\hline $\begin{array}{l}00: 08: 30 \\
00: 08: 40\end{array}$ & $\begin{array}{l}0 \\
0\end{array}$ & $\begin{array}{l}-859 \\
-845\end{array}$ & $\begin{array}{l}28 \\
28\end{array}$ & $\begin{array}{l}0 \\
0\end{array}$ & 2.67 & 0.00 & 0.01 & $\begin{array}{l}360 \\
360\end{array}$ & $\begin{array}{l}-0.043 \\
-0.034\end{array}$ & $\begin{array}{l}0.6 \\
1.1\end{array}$ & $\begin{array}{l}55.0 \\
47.0\end{array}$ & $\begin{array}{l}180 \\
180\end{array}$ & $\begin{array}{l}23 \\
17\end{array}$ & $\begin{array}{l}000 \\
000\end{array}$ & $\begin{array}{l}0.0 \\
0.0\end{array}$ & $\begin{array}{l}180 \\
180\end{array}$ \\
\hline $\begin{array}{l}00: 08: 40 \\
00: 08: 50\end{array}$ & 0 & -832 & 28 & 0 & 2.64 & 0.00 & 0.00 & 360 & -0.014 & 1.1 & 47.0 & 180 & 20 & 000 & 0.0 & $\begin{array}{l}180 \\
180\end{array}$ \\
\hline $00: 09: 00$ & 0 & -818 & 28 & 0 & 2.63 & 0.00 & 0.00 & 360 & 0.003 & 1.1 & 76.0 & 180 & 15 & 000 & 0.0 & 180 \\
\hline $00: 09: 10$ & 0 & -805 & 28 & 0 & 2.61 & 0.00 & 0.00 & 360 & 0.021 & 1.1 & 76.0 & 180 & 14 & 000 & 0.0 & 180 \\
\hline $00: 09: 20$ & 0 & -791 & 28 & 0 & 2.54 & 0.00 & 0.00 & 360 & 0.035 & 0.5 & 76.0 & 180 & 13 & 000 & 0.0 & 180 \\
\hline $00: 09: 30$ & 0 & -778 & 28 & 0 & 2.50 & 0.00 & 0.00 & 360 & 0.036 & 0.5 & 76.0 & 180 & 20 & 000 & 0.0 & 180 \\
\hline $00: 09: 40$ & 0 & -766 & 28 & 0 & 2.45 & 0.01 & 0.00 & 360 & 0.065 & 5.8 & 76.0 & 180 & 18 & 000 & 0.0 & 180 \\
\hline $00: 09: 50$ & 0 & -753 & 28 & 0 & 2.39 & 0.01 & -0.01 & 360 & 0.080 & 0.5 & 76.0 & 180 & 20 & 000 & 0.0 & 180 \\
\hline $00: 10: 00$ & 0 & -741 & 28 & 0 & 2.36 & 0.01 & 0.00 & 360 & 0.069 & 0.0 & 76.0 & 180 & 17 & 000 & 0.0 & 180 \\
\hline $00: 10: 10$ & 0 & -729 & 28 & 0 & 2.33 & 0.00 & 0.00 & 000 & 0.055 & 0.0 & 76.0 & 180 & 18 & 000 & 0.0 & 180 \\
\hline $00: 10: 20$ & 0 & -717 & 28 & 0 & 2.31 & 0.00 & 0.00 & 000 & 0.041 & 0.0 & 76.0 & 180 & 16 & 000 & 0.0 & 180 \\
\hline $00: 10: 30$ & 0 & -705 & 28 & 0 & 2.30 & 0.00 & 0.00 & 000 & 0.027 & -0.6 & 76.0 & 180 & 19 & 000 & 0.0 & 180 \\
\hline $00: 10: 40$ & 0 & -693 & 28 & 0 & 2.26 & 0.00 & 0.00 & 000 & 0.003 & -0.6 & 76.0 & 180 & 17 & 000 & 0.0 & 180 \\
\hline $00: 10: 50$ & 0 & -682 & 28 & 0 & 2.22 & 0.00 & 0.00 & 000 & -0.021 & -0.6 & 50.0 & 180 & 20 & 000 & 0.0 & 180 \\
\hline $00: 11: 00$ & 0 & -670 & 28 & 0 & 2.23 & 0.00 & 0.01 & 000 & -0.034 & 0.0 & 50.0 & 180 & 19 & 000 & 0.0 & 180 \\
\hline $00: 11: 10$ & 0 & -659 & 28 & 0 & 2.22 & 0.00 & 0.01 & 000 & -0.041 & 0.0 & 50.0 & 180 & 21 & 000 & 0.0 & 180 \\
\hline $00: 11: 20$ & 0 & -647 & 28 & 0 & 2.20 & 0.00 & 0.01 & 000 & -0.048 & 0.0 & 50.0 & 180 & 20 & 000 & 0.0 & 180 \\
\hline $00: 11: 30$ & 0 & -636 & 28 & 0 & 2.18 & 0.00 & 0.01 & 360 & -0.054 & 0.0 & 50.0 & 180 & 23 & 000 & 0.0 & 180 \\
\hline $00: 11: 40$ & 0 & -625 & 28 & 0 & 2.16 & 0.00 & 0.01 & 360 & -0.055 & 0.6 & 50.0 & 180 & 22 & 000 & 0.0 & 180 \\
\hline $00: 11: 50$ & 0 & -614 & 28 & 0 & 2.12 & 0.00 & 0.01 & 360 & -0.048 & 0.6 & 50.0 & 180 & 23 & 000 & 0.0 & 180 \\
\hline $00: 12: 00$ & 0 & -603 & 28 & 0 & 2.08 & 0.00 & 0.01 & 360 & -0.041 & 0.6 & 50.0 & 180 & 20 & 000 & 0.0 & 180 \\
\hline $00: 12: 10$ & 0 & -593 & 28 & 0 & 2.05 & 0.00 & 0.01 & 360 & -0.035 & 0.6 & 50.0 & 180 & 19 & 000 & 0.0 & 180 \\
\hline $00: 12: 20$ & 0 & -582 & 28 & 0 & 2.06 & 0.00 & 0.01 & 360 & -0.028 & 0.6 & 50.0 & 180 & 18 & 000 & 0.0 & 180 \\
\hline $00: 12: 30$ & 0 & -571 & 28 & 0 & 2.05 & 0.00 & 0.00 & 360 & -0.010 & 1.1 & 50.0 & 180 & 18 & 000 & 0.0 & 180 \\
\hline $00: 12: 40$ & 0 & -561 & 28 & 0 & 2.04 & 0.00 & 0.00 & 360 & $\cdot 0.007$ & 1.1 & 50.0 & 180 & 17 & 000 & 0.0 & 180 \\
\hline $00: 12: 50$ & 0 & -550 & 28 & 0 & 2.04 & 0.00 & 0.00 & 360 & 0.023 & 1.1 & 50.0 & 180 & 19 & 000 & 0.0 & 180 \\
\hline $0: 13 ; 00$ & 0 & -540 & 28 & 0 & 2.01 & 0.00 & 0.00 & 360 & 0.027 & 0.5 & 75.0 & 180 & 18 & 000 & 0.0 & 180 \\
\hline$: 13: 10$ & 0 & -530 & 28 & 0 & 2.00 & 0.00 & 0.00 & 360 & 0.028 & 0.5 & 75.0 & 180 & 14 & 000 & 0.0 & 180 \\
\hline$d: 13: 20$ & 0 & -519 & 28 & 0 & 1.97 & 0.01 & 0.00 & 360 & 0.065 & 0.5 & 75.0 & 180 & 15 & 000 & 0.0 & 180 \\
\hline $00: 13: 30$ & 0 & -509 & 28 & 0 & 1.95 & 0.01 & 0.00 & 360 & 0.064 & 0.5 & 75.0 & 180 & 17 & 000 & 0.0 & 180 \\
\hline $00: 13: 40$ & 0 & -499 & 28 & 0 & 1.93 & 0.00 & 0.00 & 360 & 0.055 & 0.0 & 75.0 & 180 & 18 & 000 & 0.0 & 180 \\
\hline $00: 13: 50$ & 0 & -489 & 28 & 0 & 1.90 & 0.00 & 0.00 & 000 & 0.042 & 0.0 & 75.0 & 180 & 18 & 000 & 0.0 & 180 \\
\hline $00: 14: 00$ & 0 & -480 & 28 & 0 & 1.84 & 0.00 & 0.00 & 000 & 0.030 & 0.0 & 52.0 & 180 & 21 & 000 & 0.0 & 180 \\
\hline $00: 14: 10$ & 0 & -470 & 28 & 0 & 1.81 & 0.00 & 0.00 & 000 & 0.018 & 0.0 & 52.0 & 180 & 18 & 000 & 0.0 & 180 \\
\hline $00: 14 ; 20$ & 0 & -461 & 28 & 0 & 1.80 & 0.00 & 0.00 & 000 & 0.008 & 0.0 & 52.0 & 180 & 18 & 000 & 0.0 & 180 \\
\hline $00: 14: 30$ & 0 & -452 & 28 & 0 & 1.78 & 0.00 & 0.00 & 000 & -0.002 & 0.0 & 52.0 & 180 & 17 & 000 & 0.0 & 180 \\
\hline $00 ; 14: 40$ & 0 & -443 & 28 & 0 & 1.78 & 0.00 & 0.00 & 000 & -0.011 & 0.0 & 52.0 & 180 & 16 & 000 & 0.0 & 180 \\
\hline $00: 14: 50$ & 0 & -434 & 28 & 0 & 1.77 & 0.00 & 0.00 & 000 & -0.019 & 0.0 & 52.0 & 180 & 16 & 000 & 0.0 & 180 \\
\hline $00: 15: 00$ & 0 & -425 & 28 & 0 & 1.77 & 0.00 & 0.00 & 000 & -0.027 & 0.0 & 52.0 & 180 & 24 & 000 & 0.0 & 180 \\
\hline $00: 15: 10$ & 0 & -416 & 28 & 0 & 1.74 & 0.00 & 0.01 & 000 & -0.034 & 0.0 & 52.0 & 180 & 22 & 000 & 0.0 & 180 \\
\hline $00: 15: 20$ & 0 & -407 & 28 & 0 & 1.73 & 0.00 & 0.01 & 360 & -0.044 & -1.9 & 52.0 & 180 & 20 & 000 & 0.0 & 180 \\
\hline $00: 15: 30$ & 0 & -398 & 28 & 0 & 1.74 & -0.02 & 0.03 & 360 & -0.319 & -17.2 & 52.0 & 180 & 20 & 000 & 0.0 & 180 \\
\hline $00: 15: 40$ & 0 & -389 & 28 & 0 & 1.73 & -0.04 & 0.07 & 360 & -0.701 & -15.3 & 52.0 & 180 & 23 & 000 & 0.0 & 180 \\
\hline $00: 25: 50$ & 1 & -380 & 28 & 0 & 1.74 & -0.06 & 0.10 & 360 & -1.009 & -12.8 & 52.0 & 180 & 17 & 000 & 0.0 & 180 \\
\hline $00: 16 ; 00$ & 1 & -371 & 28 & 0 & 1.71 & -0.07 & 0.13 & 360 & -1.229 & -9.3 & 77.0 & 180 & 19 & 000 & 0.0 & 180 \\
\hline $00: 16: 10$ & 1 & -362 & 28 & 0 & 1.64 & -0.07 & 0.14 & 359 & -1.351 & -5.2 & 100.0 & 180 & 16 & 000 & 0.0 & 180 \\
\hline $00: 16: 20$ & 1 & -354 & 28 & 0 & 1.58 & -0.07 & 0.14 & 359 & -1.380 & -1.2 & 100.0 & 180 & 17 & 000 & 0.0 & 180 \\
\hline $00: 16: 30$ & 1 & -346 & 28 & 0 & 1.52 & -0.07 & 0.14 & 359 & -1.316 & 3.3 & 100.0 & 180 & 17 & 000 & 0.0 & 180 \\
\hline $00: 16 ; 40$ & 1 & -338 & 28 & 0 & 1.45 & -0.05 & 0.12 & 359 & -1.110 & 6.5 & 100.0 & 180 & 21 & 000 & 0.0 & 180 \\
\hline $00: 16: 50$ & 1 & -331 & 28 & 0 & 1.41 & -0.04 & 0.10 & 359 & -0.886 & 9.7 & 100.0 & 180 & 15 & 000 & 0.0 & 180 \\
\hline $00: 17: 00$ & 1 & -324 & 28 & 0 & 1.37 & -0.03 & 0.07 & 358 & -0.657 & 11.8 & 100.0 & 180 & 20 & 000 & 0.0 & 180 \\
\hline $00: 17: 10$ & 1 & -317 & 28 & 0 & 1.30 & -0.01 & 0.04 & 358 & -0.310 & 20.4 & 100.0 & 180 & 24 & 000 & 0.0 & 180 \\
\hline $00: 17: 20$ & 0 & -311 & 28 & 0 & 1.24 & 0.02 & -0.02 & 358 & 0.287 & 30.7 & 100.0 & 180 & 22 & 000 & 0.0 & 180 \\
\hline $00: 17: 30$ & 0 & -304 & 28 & 0 & 1.19 & 0.06 & -0.08 & 358 & 0.897 & 28.4 & 100.0 & 180 & 14 & 000 & 0.0 & 180 \\
\hline $00: 17: 40$ & 0 & -298 & 28 & 0 & 1.18 & 0.08 & -0.14 & 359 & 1.395 & 24.0 & 75.0 & 180 & 18 & 000 & 0.0 & 180 \\
\hline $00 ; 17 ; 50$ & 0 & -292 & 28 & 0 & 1.16 & 0.11 & -0.19 & 359 & 1.901 & 34.5 & 75.0 & 180 & 17 & 000 & 0.0 & 180 \\
\hline $00: 18: 00$ & -1 & -286 & 28 & 0 & 1.15 & 0.14 & -0.25 & 359 & 2.495 & 29.5 & 75.0 & 180 & 18 & 000 & 0.0 & 180 \\
\hline $00: 18: 10$ & -1 & -280 & 28 & 0 & 1.12 & 0.16 & -0.30 & 360 & 2.894 & 21.2 & 75.0 & 180 & 19 & 000 & 0.0 & 180 \\
\hline $00: 18: 20$ & -1 & -275 & 28 & 0 & 1.09 & 0.16 & -0.32 & 000 & 3.076 & 11.9 & 75.0 & 180 & 20 & 000 & 0.0 & 180 \\
\hline $00: 18: 30$ & -2 & -269 & 28 & 0 & 1.05 & 0.16 & -0.32 & 001 & 3.054 & 2.3 & 75.0 & 180 & 17 & 000 & 0.0 & 180 \\
\hline $00: 18: 40$ & -2 & -264 & 28 & 0 & 1.01 & 0.14 & -0.30 & 001 & 2.845 & -6.6 & 75.0 & 180 & 22 & 000 & 0.0 & 180 \\
\hline $00: 18: 50$ & -2 & -259 & 28 & 0 & 0.97 & 0.12 & -0.27 & 002 & 2.461 & -14.2 & 75.0 & 180 & 18 & 000 & 0.0 & 180 \\
\hline $00: 19: 00$ & -3 & -254 & 28 & 0 & 0.97 & 0.09 & -0.22 & 002 & 1.966 & -20.3 & 75.0 & 180 & 13 & 000 & 0.0 & 180 \\
\hline $00: 19: 10$ & -3 & -249 & 28 & 0 & 0.93 & 0.06 & -0.16 & 002 & 1.398 & -24.8 & 75.0 & 180 & 16 & 000 & 0.0 & 180 \\
\hline $00: 19: 20$ & -3 & -244 & 28 & 0 & 0.89 & 0.02 & -0.10 & 002 & 0.750 & -27.5 & 75.0 & 180 & 23 & 000 & 0.0 & 180 \\
\hline $8: 19 ; 30$ & -3 & -240 & 28 & 0 & 0.86 & -0.01 & -0.04 & 003 & 0.165 & -27.8 & 100.0 & 180 & 18 & 000 & 0.0 & 180 \\
\hline$: 19: 40$ & -3 & -235 & 28 & 0 & 0.80 & -0.03 & 0.02 & 003 & -0.364 & -27.1 & 100.0 & 180 & 16 & 000 & 0.0 & 180 \\
\hline $00: 19: 50$ & -2 & -231 & 38 & 0 & 0.77 & -0.08 & 0.10 & 002 & -1.119 & -23.6 & 100.0 & 180 & 17 & 000 & 0.0 & 180 \\
\hline $00: 20 ; 00$ & -2 & -227 & 38 & 0 & 0.77 & -0.12 & 0.17 & 002 & -1.878 & -18.0 & 100.0 & 180 & 18 & 000 & 0.0 & 180 \\
\hline $00: 20: 10$ & -2 & -223 & 38 & 0 & 0.78 & -0.14 & 0.22 & 002 & -2.260 & -11.2 & 100.0 & 180 & 18 & 000 & 0.0 & 180 \\
\hline 00 & - & -219 & 38 & 0 & 0.77 & -0.14 & 0.24 & 001 & -2.381 & -3.8 & 100.0 & 180 & 21 & 000 & 0.0 & 180 \\
\hline
\end{tabular}




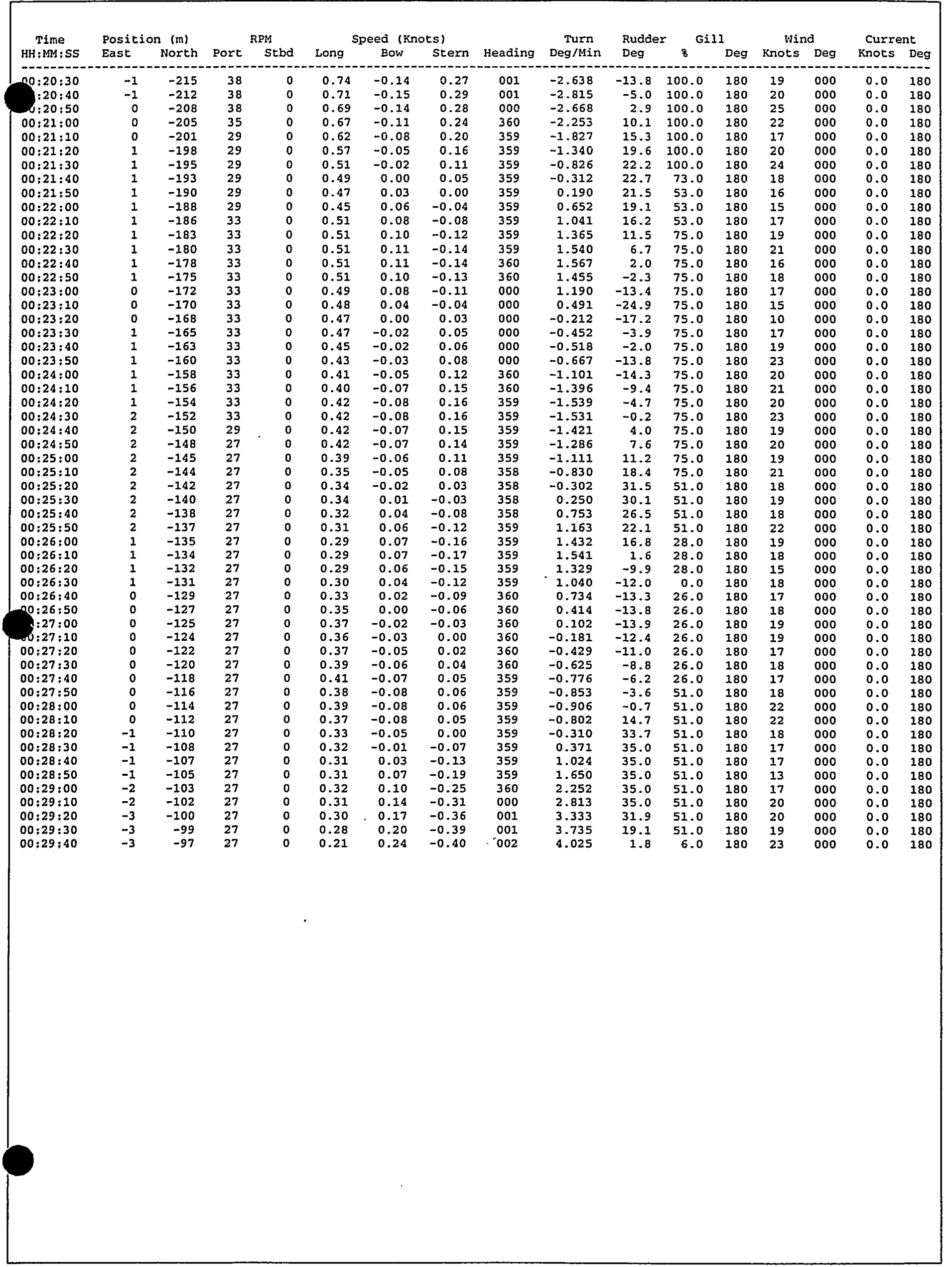


Open Ocean

\section{Simulation Track Plot}

Ship: 150,000 ton, Tanker, partial load

$88 \div 30: 20$

$88 \div \frac{1}{7}: 58$

$00: 78: 38$

$00: 17: 50$

$00: 17: 20$

$00: 16: 50$

$00: 16: 20$

$00: 15: 50$

$00: 15: 20$

$00: 14: 50$

$00: 14: 20$

$00: 13: 50$

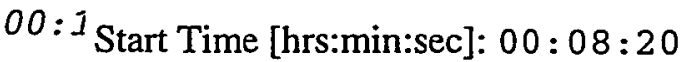
$00: 1$ End Time [hrs:min:sec]: $00: 29: 45$ $00:$ : Delta Plot Time [sec]:

00ast-West Scale [m]: 926.

$00: 1$ East-West Scale [Nm]: $\quad 0.5000$ 


\section{HANDS-ON SIMULATION}

\section{SUCTION MOORNG TECHNOLOGY}

Simulation Number: 14

Date: 3 February 1999

Start Time: 11:27

Length of run [secs]: 3310

Ship Condition: $\mathrm{draft}=13.72 \mathrm{~m}$

Environment

Wind Speed [knots]:

20 Direction: 0

Sea State, Significant wave height $[\mathrm{m}]: .$.

3 Direction: 180

Current Speed [knots]:.

0 Direction: 0

Ship Operator: J.A.

Observer: B.K.J \& J.K.

Final Mating, distance between center of buoy and center of moon pool [m]: 2.9

Evaluation:

Deceleration phase went well.

On the final closure the buoy did not center quite as well as desired (about $5 \mathrm{~m}$ off). The captain released the buoy and maneuvered back. In repositioning the ship it was necessary to maintain heading control within 10 degrees of the up force direction. In order to do this, it was necessary to use propeller, rudder and thruster. The mating was tried three times with the final mating with the buoy $2.9 \mathrm{~m}$ off center. 
Ship:

Exercise number:

591 150,000 ton, Tanker, partial load

Scenario:

grafic ships:

754 Suction Mooring System

112 Open Ocean

999 Dumny tug file based on containership

Initial conditions

590 Suction Mooring Buoy

Exercise identification

$594150 k$ tanker in partial load - Suction Mooring

RUN $\# 14$

Time Position (m) RPM Speed (Knots) Turn Rudder Gill Wind

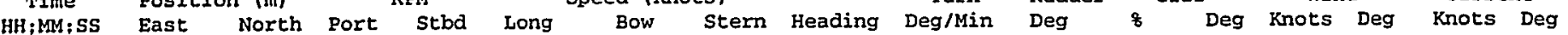

\begin{tabular}{|c|c|c|c|c|c|c|c|c|c|c|c|c|c|c|c|c|}
\hline $00 ; 30 ; 00$ & 1 & -179 & 25 & 0 & 0.57 & 0.01 & 0.01 & 000 & 0.032 & -23.1 & 51.0 & 180 & 20 & 000 & 0.0 & 180 \\
\hline $00: 30: 10$ & 1 & -176 & 25 & 0 & 0.57 & -0.01 & 0.05 & 000 & -0.361 & -22.3 & 51.0 & 180 & 20 & 000 & 0.0 & 180 \\
\hline $00: 30: 20$ & 1 & -173 & 25 & 0 & 0.53 & -0.03 & 0.08 & 000 & -0.706 & -19.8 & 51.0 & 180 & 20 & 000 & 0.0 & 180 \\
\hline $00: 30: 30$ & 1 & -171 & 25 & 0 & 0.49 & -0.05 & 0.11 & 000 & -0.994 & -16.2 & 51.0 & 180 & 16 & 000 & 0.0 & 180 \\
\hline $00: 30: 40$ & 1 & -168 & 25 & 0 & 0.48 & -0.06 & 0.13 & 360 & -1.206 & -12.3 & 51.0 & 180 & 21 & 000 & 0.0 & 180 \\
\hline $00: 30: 50$ & 2 & -166 & 25 & 0 & 0.47 & -0.07 & 0.15 & 360 & -1.344 & -8.0 & 51.0 & 180 & 18 & 000 & 0.0 & 180 \\
\hline $00: 31: 00$ & 2 & -164 & 25 & 0 & 0.45 & -0.07 & 0.15 & 359 & -1.386 & -3.7 & $51.0^{\circ}$ & 180 & 18 & 000 & 0.0 & 180 \\
\hline $00 ; 31 ; 10$ & 2 & -161 & 25 & 0 & 0.45 & -0.07 & 0.15 & 359 & -1.368 & 0.2 & 51.0 & 180 & 22 & 000 & 0.0 & 180 \\
\hline $00 ; 31: 20$ & 2 & -159 & 25 & 0 & 0.45 & -0.06 & 0.14 & 359 & -1.306 & 4.0 & 51.0 & 180 & 25 & 000 & 0.0 & 180 \\
\hline $00: 31: 30$ & 2 & -157 & 25 & 0 & 0.44 & -0.06 & 0.13 & 359 & -1.175 & 7.7 & 51.0 & 180 & 16 & 000 & 0.0 & 180 \\
\hline $00: 31: 40$ & 2 & -154 & 25 & 0 & 0.42 & -0.05 & 0.11 & 359 & -0.997 & 10.7 & 51.0 & 180 & 17 & 000 & 0.0 & 180 \\
\hline $00: 31: 50$ & 2 & -152 & 25 & 0 & 0.43 & -0.04 & 0.09 & 358 & -0.794 & 12.9 & 51.0 & 180 & 15 & 000 & 0.0 & 180 \\
\hline $00: 32 ; 00$ & 3 & -150 & 25 & 0 & 0.43 & -0.02 & 0.06 & 358 & -0.545 & 13.9 & 51.0 & 180 & 15 & 000 & 0.0 & 180 \\
\hline $00: 32: 10$ & 3 & -148 & 25 & 0 & 0.45 & -0.02 & 0.04 & 358 & -0.355 & 15.0 & 51.0 & 180 & 14 & 000 & 0.0 & 180 \\
\hline $00: 32: 20$ & 2 & -145 & 25 & 0 & 0.43 & -0.01 & 0.01 & 358 & -0.137 & 15.0 & 51.0 & 180 & 19 & 000 & 0.0 & 180 \\
\hline $00: 32: 30$ & 2 & -143 & 25 & 0 & 0.41 & 0.00 & -0.01 & 358 & 0.061 & 14.5 & 51.0 & 180 & 15 & 000 & 0.0 & 180 \\
\hline $00: 32: 40$ & 2 & -141 & 25 & 0 & 0.38 & 0.00 & -0.04 & 358 & 0.213 & 14.0 & 51.0 & 180 & 19 & 000 & 0.0 & 180 \\
\hline $00: 32 ; 50$ & 2 & -139 & 25 & 0 & 0.37 & 0.00 & -0.06 & 358 & 0.359 & 12.4 & 51.0 & 180 & 20 & 000 & 0.0 & 180 \\
\hline $00: 33: 00$ & 2 & -137 & 25 & 0 & 0.34 & 0.01 & -0.07 & 358 & 0.546 & 10.5 & 51.0 & 180 & 17 & 000 & 0.0 & 180 \\
\hline $00: 33: 10$ & 2 & -136 & 25 & 0 & 0.33 & 0.02 & -0.08 & 358 & 0.659 & 7.8 & 51.0 & 180 & 13 & 000 & 0.0 & 180 \\
\hline $00: 33: 20$ & 1 & -134 & 25 & 0 & 0.34 & 0.03 & -0.10 & 359 & 0.804 & 19.9 & 51.0 & 180 & 23 & 000 & 0.0 & 180 \\
\hline $00: 33: 30$ & 1 & -132 & 25 & 0 & 0.32 & 0.04 & -0.13 & 359 & 1.099 & 19.6 & 51.0 & 180 & 23 & 000 & 0.0 & 180 \\
\hline $00: 33: 40$ & 1 & -131 & 25 & 0 & 0.30 & 0.05 & -0.16 & 359 & 1.313 & 14.5 & 51.0 & 180 & 20 & 000 & 0.0 & 180 \\
\hline $00: 33: 50$ & 1 & -129 & 25 & 0 & 0.29 & 0.06 & -0.17 & 359 & 1.447 & 10.0 & 51.0 & 180 & 19 & 000 & 0.0 & 180 \\
\hline $00: 34: 00$ & 0 & -128 & 25 & 0 & 0.30 & 0.06 & -0.18 & 359 & 1.477 & 5.5 & 51.0 & 180 & 21 & 000 & 0.0 & 180 \\
\hline $00: 34: 10$ & 0 & -126 & 25 & 0 & 0.30 & 0.06 & -0.17 & 360 & 1.459 & 1.0 & 51.0 & 180 & 19 & 000 & 0.0 & 180 \\
\hline $00: 34: 20$ & 0 & -125 & 25 & 0 & 0.31 & 0.05 & -0.17 & 360 & 1.364 & -2.9 & 51.0 & 180 & 22 & 000 & 0.0 & 180 \\
\hline $00: 34 ; 30$ & -1 & -123 & 25 & 0 & 0.30 & 0.04 & -0.15 & 000 & 1.219 & -5.8 & 51.0 & 180 & 20 & 000 & 0.0 & 180 \\
\hline $00: 34: 40$ & -1 & -122 & 25 & 0 & 0.29 & 0.03 & -0.13 & 000 & 1.032 & -9.2 & 51.0 & 180 & 18 & 000 & 0.0 & 180 \\
\hline$: 34: 50$ & -1 & -120 & 25 & 0 & 0.27 & 0.02 & -0.11 & 000 & 0.823 & -10.8 & $36: 0$ & 180 & 21 & 000 & 0.0 & 180 \\
\hline $10: 35: 00$ & -1 & -119 & 25 & 0 & 0.25 & 0.00 & -0.09 & 001 & 0.587 & -12.4 & 36.0 & 180 & 23 & 000 & 0.0 & 180 \\
\hline $00: 35: 10$ & -2 & -118 & 25 & 0 & 0.24 & -0.01 & -0.06 & 001 & 0.335 & -12.9 & 36.0 & 180 & 17 & 000 & 0.0 & 180 \\
\hline $00: 35: 20$ & -2 & -116 & 25 & 0 & 0.25 & -0.02 & -0.04 & 001 & 0.108 & -12.4 & 36.0 & 180 & 15 & 000 & 0.0 & 180 \\
\hline $00: 35: 30$ & -2 & -115 & 25 & 0 & 0.24 & -0.03 & -0.02 & 001 & -0.095 & -11.4 & 36.0 & 180 & 20 & 000 & 0.0 & 180 \\
\hline $00: 35: 40$ & -2 & -114 & 25 & 0 & 0.23 & -0.04 & 0.00 & 001 & -0.290 & -9.8 & 36.0 & 180 & 19 & 000 & 0.0 & 180 \\
\hline $00 ; 35 ; 50$ & -2 & -113 & 25 & 0 & 0.21 & -0.05 & 0.02 & 001 & -0.426 & -7.8 & 36.0 & 180 & 15 & 000 & 0.0 & 180 \\
\hline $00: 36: 00$ & -2 & -112 & 25 & 0 & 0.23 & -0.06 & 0.03 & 000 & -0.526 & -6.3 & 36.0 & 180 & 13 & 000 & 0.0 & 180 \\
\hline $00: 36: 10$ & -2 & -110 & 25 & 0 & 0.23 & -0.06 & 0.04 & 000 & -0.603 & -4.3 & 26.0 & 180 & 14 & 000 & 0.0 & 180 \\
\hline $00: 36: 20$ & -2 & -109 & 25 & 0 & 0.24 & -0.06 & 0.04 & 000 & -0.640 & -1.9 & 26.0 & 180 & 16 & 000 & 0.0 & 280 \\
\hline $00: 36: 30$ & -2 & -108 & 25 & 0 & 0.28 & -0.05 & 0.03 & 000 & -0.508 & 16.1 & 26.0 & 180 & 21 & 000 & 0.0 & 180 \\
\hline $00: 36: 40$ & -2 & -106 & 25 & 0 & 0.30 & -0.03 & 0.00 & 000 & -0.204 & 16.6 & 0.0 & 180 & 19 & 000 & 0.0 & 180 \\
\hline $00: 36: 50$ & -2 & -105 & 25 & 0 & 0.28 & -0.02 & -0.03 & 000 & 0.077 & 19.0 & 0.0 & 180 & 20 & 000 & 0.0 & 180 \\
\hline $00: 37: 00$ & -3 & -103 & 25 & 0 & 0.29 & 0.01 & -0.07 & 000 & 0.559 & 35.0 & 0.0 & 180 & 22 & 000 & 0.0 & 180 \\
\hline $00: 37: 10$ & -3 & -102 & 25 & 0 & 0.27 & 0.05 & -0.13 & 000 & 1.134 & 35.0 & 0.0 & 180 & 22 & 000 & 0.0 & 180 \\
\hline $00: 37: 20$ & -3 & -101 & 25 & 0 & 0.27 & 0.08 & -0.18 & 001 & 1.687 & 35.0 & 0.0 & 180 & 14 & 000 & 0.0 & 180 \\
\hline $00: 37: 30$ & -3 & -99 & 25 & 0 & 0.27 & 0.12 & -0.23 & .001 & 2.220 & 35.0 & 0.0 & 180 & 15 & 000 & 0.0 & 180 \\
\hline $00: 37: 40$ & -4 & -98 & 25 & 0 & 0.21 & 0.16 & -0.29 & 001 & 2.847 & 35.0 & 0.0 & 180 & 18 & 000 & 0.0 & 180 \\
\hline $00: 37: 50$ & -4 & -97 & 25 & 0 & 0.13 & 0.22 & -0.33 & 002 & 3.483 & 35.0 & 0.0 & 180 & 23 & 000 & 0.0 & 180 \\
\hline $00: 38: 00$ & -4 & -96 & 25 & 0 & 0.13 & 0.25 & -0.37 & 002 & 3.953 & 35.0 & 0.0 & 180 & 20 & 000 & 0.0 & 180 \\
\hline $00: 38: 10$ & -4 & -96 & 25 & 0 & 0.10 & 0.28 & -0.41 & 003 & 4.368 & 35.0 & 50.0 & 180 & 20 & 000 & 0.0 & 180 \\
\hline $00: 38: 20$ & -5 & -95 & 25 & 0 & 0.06 & 0.29 & -0.43 & 004 & 4.612 & 35.0 & 99.0 & 180 & 18 & 000 & 0.0 & 180 \\
\hline $00: 38: 30$ & -5 & -95 & 25 & 0 & 0.04 & 0.32 & -0.45 & 005 & 4.936 & 35.0 & 99.0 & 180 & 22 & 000 & 0.0 & 180 \\
\hline $00: 38 ; 40$ & -5 & -95 & 0 & 0 & -0.01 & 0.34 & -0.44 & 006 & 4.951 & 16.7 & 99.0 & 180 & 19 & 000 & 0.0 & 180 \\
\hline $00: 38: 50$ & -6 & -95 & 0 & 0 & -0.06 & 0.32 & -0.43 & 006 & 4.730 & 13.3 & 99.0 & 212 & 18 & 000 & 0.0 & 180 \\
\hline $00: 39: 00$ & -6 & -95 & 0 & 0 & -0.15 & 0.31 & -0.42 & 007 & 4.609 & 32.3 & 99.0 & 212 & 16 & 000 & 0.0 & 180 \\
\hline $00: 39: 10$ & -6 & -96 & 0 & 0 & -0.18 & 0.32 & -0.41 & 008 & 4.687 & 27.2 & 99.0 & 244 & 21 & 000 & 0.0 & 180 \\
\hline $00: 39: 20$ & -7 & -97 & 0 & 0 & -0.23 & 0.31 & -0.41 & 009 & 4.602 & 19.7 & 99.0 & 266 & 18 & 000 & 0.0 & 180 \\
\hline $00: 39: 30$ & -7 & -99 & 0 & 0 & -0.28 & 0.30 & -0.41 & 009 & 4.511 & 10.4 & 99.0 & 266 & 18 & 000 & 0.0 & 180 \\
\hline $00: 39: 40$ & -8 & -100 & 0 & 0 & -0.29 & 0.25 & -0.41 & 010 & 4.241 & -0.9 & 99.0 & 266 & 16 & 000 & 0.0 & 180 \\
\hline $00: 39: 50$ & -9 & -101 & 33 & 0 & -0.28 & 0.24 & -0.41 & 011 & 4.076 & -12.8 & 99.0 & 266 & 22 & 000 & 0.0 & 180 \\
\hline $00: 40: 00$ & -9 & -103 & 33 & 0 & -0.30 & 0.22 & -0.36 & 011 & 3.687 & -22.5 & 99.0 & 214 & 22 & 000 & 0.0 & 180 \\
\hline $00: 40: 10$ & -10 & -104 & 33 & 0 & -0.34 & 0.19 & -0.29 & 012 & 3.051 & -29.2 & 99.0 & 214 & 22 & 000 & 0.0 & 180 \\
\hline $00: 40: 20$ & -10 & -106 & 33 & 0 & -0.36 & 0.14 & -0.21 & 012 & 2.199 & -33.7 & 99.0 & 272 & 16 & 000 & 0.0 & 180 \\
\hline $00: 40: 30$ & -11 & -108 & 43 & 0 & -0.32 & 0.01 & -0.09 & 013 & 0.637 & -33.4 & 99.0 & 272 & 19 & 000 & 0.0 & 180 \\
\hline $00: 40: 40$ & -12 & -109 & 49 & 0 & -0.23 & -0.11 & 0.07 & 013 & -1.140 & -24.9 & 99.0 & 272 & 19 & 000 & 0.0 & 180 \\
\hline $00: 40: 50$ & -12 & -110 & 43 & 0 & -0.13 & -0.21 & 0.16 & 012 & -2.403 & -6.9 & 99.0 & 272 & 20 & 000 & 0.0 & 180 \\
\hline $00: 41 ; 00$ & -12 & -110 & 30 & 0 & -0.07 & -0.27 & 0.15 & 012 & -2.691 & 12.1 & 99.0 & 272 & 17 & 000 & 0.0 & 180 \\
\hline $0: 41: 10$ & -13 & -111 & 30 & 0 & -0.05 & -0.20 & 0.09 & 012 & -1.842 & 20.9 & 99.0 & 211 & 19 & 000 & 0.0 & 180 \\
\hline$: 41: 20$ & -13 & -111 & 30 & 0 & -0.08 & -0.13 & 0.05 & 011 & -1.187 & 9.6 & 99.0 & 211 & 16 & 000 & 0.0 & 180 \\
\hline $00 ; 41 ; 30$ & -13 & -111 & 30 & 0 & -0.11 & -0.10 & 0.03 & 011 & -0.851 & 5.7 & 99.0 & 211 & 18 & 000 & 0.0 & 180 \\
\hline $00: 41: 40$ & -13 & -112 & 30 & 0 & -0.15 & -0.10 & 0.01 & 011 & -0.676 & 5.3 & 99.0 & 211 & 15 & 000 & 0.0 & 180 \\
\hline $00: 41: 50$ & -14 & -113 & 30 & 0 & -0.13 & -0.06 & -0.01 & 011 & -0.298 & 3.8 & 99.0 & 304 & 14 & 000 & 0.0 & 180 \\
\hline & & & & & & & -0.04 & 011 & 68 & & 99.0 & 304 & 18 & 000 & 0.0 & 180 \\
\hline
\end{tabular}

00.42 .00

$-14--113 \quad 42$ 

HH:MM:SS East North Port Stbd Long

ppeed (Knots)

Turn Rudder Gill

wind

Current

\begin{tabular}{|c|c|c|c|c|c|c|c|c|c|c|c|c|c|c|c|c|}
\hline HH : MM : SS & East & North & Port & stbd & Long & Bow & Stern & Heading & Deg/Min & Deg & 8 & Deg & Knots & Deg & Knots & Deg \\
\hline $20: 42: 10$ & -14 & -113 & 43 & 0 & 0.02 & -0.05 & -0.06 & 011 & 0.031 & 1.6 & 99.0 & 304 & 23 & 000 & 0.0 & 180 \\
\hline$: 42: 20$ & -15 & -113 & 42 & 0 & 0.08 & -0.05 & -0.07 & 011 & 0.136 & 0.1 & 99.0 & 239 & 20 & 000 & 0.0 & 180 \\
\hline $0: 42: 30$ & -15 & -112 & 42 & 0 & 0.11 & -0.05 & -0.08 & 011 & 0.191 & -0.7 & 99.0 & 239 & 17 & 000 & 0.0 & 180 \\
\hline $00: 42 ; 40$ & -15 & -112 & 42 & 0 & 0.13 & -0.07 & -0.08 & 011 & 0.061 & -3.4 & 99.0 & 239 & 19 & 000 & 0.0 & 180 \\
\hline $00: 42: 50$ & -15 & -111 & 43 & 0 & 0.17 & -0.16 & -0.02 & 011 & -0.898 & -22.4 & 99.0 & 239 & 18 & 000 & 0.0 & 180 \\
\hline $00: 43: 00$ & -16 & -110 & 43 & 0 & 0.21 & -0.28 & 0.12 & 011 & -2.538 & -35.0 & 99.0 & 239 & 17 & 000 & 0.0 & 180 \\
\hline $00: 43: 10$ & -16 & -109 & 43 & 0 & 0.24 & -0.41 & 0.27 & 010 & -4.328 & -35.0 & 99.0 & 239 & 20 & 000 & 0.0 & 180 \\
\hline $00: 43: 20$ & -16 & -107 & 43 & 0 & 0.28 & -0.48 & 0.40 & 009 & -5.599 & -35.0 & 99.0 & 239 & 21 & 000 & 0.0 & 180 \\
\hline $00: 43: 30$ & -16 & -106 & 43 & 0 & 0.29 & -0.56 & 0.53 & 008 & -6.914 & -35.0 & 99.0 & 239 & 21 & 000 & 0.0 & 180 \\
\hline $00: 43: 40$ & -16 & -104 & 43 & 0 & 0.31 & -0.63 & 0.61 & 007 & -7.870 & -20.7 & 99.0 & 239 & 25 & 000 & 0.0 & 180 \\
\hline $00: 43 ; 50$ & -15 & -103 & 42 & 0 & 0.35 & -0.57 & 0.62 & 006 & -7.509 & -1.7 & 99.0 & 116 & 22 & 000 & 0.0 & 180 \\
\hline $00: 44: 00$ & -15 & -101 & 42 & 0 & 0.39 & -0.43 & .0 .54 & 004 & -6.180 & 17.3 & 99.0 & 146 & 19 & 000 & 0.0 & 180 \\
\hline $00: 44: 10$ & -15 & -99 & 25 & 0 & 0.34 & -0.34 & 0.45 & 004 & -5.053 & 35.0 & 99.0 & 146 & 20 & 000 & 0.0 & 180 \\
\hline $00: 44: 20$ & -14 & -97 & 25 & 0 & 0.30 & -0.25 & 0.38 & 003 & -3.958 & 35.0 & 99.0 & 167 & 22 & 000 & 0.0 & 180 \\
\hline $00: 44: 30$ & -14 & -96 & 25 & 0 & 0.25 & -0.15 & 0.31 & 002 & -2.945 & 35.0 & 99.0 & 145 & 15 & 000 & 0.0 & 180 \\
\hline $00: 44: 40$ & -13 & -95 & 25 & 0 & 0.18 & -0.08 & 0.26 & 002 & -2.142 & 19.3 & 99.0 & 145 & 16 & 000 & 0.0 & 180 \\
\hline $00: 44: 50$ & -13 & -94 & 25 & 0 & 0.12 & -0.03 & 0.24 & 001 & -1.739 & 0.3 & 99.0 & 145 & 16 & 000 & 0.0 & 180 \\
\hline $00 ; 45 ; 00$ & -12 & -93 & 25 & 0 & 0.09 & -0.01 & 0.26 & 001 & -1.687 & -18.7 & 99.0 & 145 & 15 & 000 & 0.0 & 180 \\
\hline $00: 45: 10$ & -11 & -93 & 25 & 0 & 0.06 & -0.01 & 0.30 & 001 & -1.936 & -35.0 & 99.0 & 145 & 15 & 000 & 0.0 & 180 \\
\hline $00: 45: 20$ & -11 & -93 & 25 & 0 & 0.02 & -0.01 & 0.35 & 001 & -2.254 & -30.8 & 99.0 & 145 & 22 & 000 & 0.0 & 180 \\
\hline $00: 45: 30$ & -10 & -93 & 40 & 0 & 0.01 & -0.01 & 0.41 & 000 & -2.686 & -22.9 & 99.0 & 145 & 18 & 000 & 0.0 & 180 \\
\hline $00: 45: 40$ & -9 & -93 & 42 & 0 & 0.02 & -0.02 & 0.47 & 360 & -3.112 & -13.5 & 99.0 & 145 & 18 & 000 & 0.0 & 180 \\
\hline $00 ; 45: 50$ & -7 & -93 & 41 & 0 & 0.03 & 0.00 & 0.48 & 359 & -3.097 & -5.6 & 99.0 & 145 & 17 & 000 & 0.0 & 180 \\
\hline $00: 46: 00$ & -6 & -92 & 42 & 0 & 0.07 & 0.04 & 0.45 & 359 & -2.619 & 9.9 & 99.0 & 145 & 17 & 000 & 0.0 & 180 \\
\hline $00: 46: 10$ & -5 & -92 & 42 & 0 & 0.08 & 0.10 & 0.40 & 358 & -1.907 & 3.7 & 99.0 & 145 & 13 & 000 & 0.0 & 180 \\
\hline $00: 46: 20$ & -4 & -91 & 29 & 0 & 0.09 & 0.14 & 0.37 & 358 & -1.447 & 6.4 & 99.0 & 145 & 21 & 000 & 0.0 & 180 \\
\hline $00: 46: 30$ & -2 & -91 & 26 & 0 & 0.07 & 0.17 & 0.35 & 358 & -1.143 & 9.1 & 99.0 & 145 & 20 & 000 & 0.0 & 180 \\
\hline $00: 46: 40$ & -1 & -91 & 26 & 0 & 0.01 & 0.19 & 0.32 & 358 & -0.839 & 10.6 & 99.0 & 145 & 20 & 000 & 0.0 & 180 \\
\hline $00: 46: 50$ & 0 & -91 & 0 & 0 & -0.06 & 0.22 & 0.31 & 358 & -0.584 & 11.5 & 99.0 & 145 & 21 & 000 & 0.0 & 180 \\
\hline $00 ; 47 ; 00$ & 2 & -91 & 0 & 0 & -0.14 & 0.23 & 0.32 & 357 & -0.550 & 12.8 & 99.0 & 145 & 23 & 000 & 0.0 & 180 \\
\hline $00: 47: 10$ & 3 & -92 & 0 & 0 & -0.20 & 0.25 & 0.32 & 357 & -0.472 & 13.9 & 99.0 & 178 & 16 & 000 & 0.0 & 180 \\
\hline $00: 47: 20$ & 5 & -93 & 0 & 0 & -0.29 & 0.25 & 0.31 & 357 & -0.388 & 14.6 & 99.0 & 178 & 20 & 000 & 0.0 & 180 \\
\hline $00: 47: 30$ & 6 & -95 & 0 & 0 & -0.36 & 0.24 & 0.30 & 357 & -0.397 & 15.7 & 99.0 & 217 & 22 & 000 & 0.0 & 180 \\
\hline $00: 47: 40$ & 8 & -97 & 0 & 0 & -0.42 & 0.21 & 0.29 & 357 & -0.491 & 16.9 & 99.0 & 217 & 21 & 000 & 0.0 & 180 \\
\hline $00: 47: 50$ & 9 & -99 & 0 & 0 & -0.41 & 0.16 & 0.27 & 357 & -0.673 & 19.1 & 99.0 & 327 & 18 & 000 & 0.0 & 180 \\
\hline $00 ; 48 ; 00$ & 10 & -101 & 0 & 0 & -0.40 & 0.13 & 0.26 & 357 & -0.782 & 21.8 & 99.0 & 327 & 22 & 000 & 0.0 & 180 \\
\hline $00: 48: 10$ & 11 & -103 & 0 & 0 & -0.35 & 0.10 & 0.24 & 357 & -0.894 & 25.2 & 99.0 & 027 & 16 & 000 & 0.0 & 180 \\
\hline $00: 48: 20$ & 12 & -105 & 0 & 0 & -0.39 & 0.14 & 0.25 & 357 & -0.708 & 26.7 & 99.0 & 145 & 17 & 000 & 0.0 & 180 \\
\hline $0: 48: 30$ & 13 & -107 & 0 & 0 & -0.43 & 0.15 & 0.25 & 357 & -0.636 & 28.5 & 99.0 & 065 & 18 & 000 & 0.0 & 180 \\
\hline$: 48: 40$ & 15 & -109 & 0 & 0 & -0.40 & 0.12 & 0.24 & 356 & -0.788 & 30.8 & 99.0 & 304 & 18 & 000 & 0.0 & 180 \\
\hline $00: 48: 50$ & 16 & -111 & 0 & 0 & -0.41 & 0.06 & 0.22 & 356 & -1.063 & 34.4 & 99.0 & 304 & 16 & 000 & 0.0 & 180 \\
\hline $00: 49: 00$ & 16 & -113 & 0 & 0 & -0.42 & 0.00 & 0.21 & 356 & -1.309 & 35.0 & 99.0 & 304 & 22 & 000 & 0.0 & 180 \\
\hline $00: 49: 10$ & 17 & -115 & 29 & 0 & -0.39 & -0.04 & 0.15 & 356 & -1.192 & 30.7 & 99.0 & 304 & 19 & 000 & 0.0 & 180 \\
\hline $00: 49: 20$ & 17 & -117 & 24 & 0 & -0.37 & -0.07 & 0.10 & 356 & -1.048 & 11.7 & 99.0 & 304 & 16 & 000 & 0.0 & 180 \\
\hline $00: 49: 30$ & 17 & -119 & 0 & 0 & -0.36 & -0.09 & 0.08 & 355 & -1.093 & -7.3 & 99.0 & 304 & 19 & 000 & 0.0 & 180 \\
\hline $00: 49: 40$ & 17 & -120 & 30 & 0 & -0.31 & -0.17 & 0.10 & 355 & -1.707 & -26.3 & 99.0 & 304 & 23 & 000 & 0.0 & 180 \\
\hline $00: 49: 50$ & 17 & -122 & 25 & 0 & -0.26 & -0.27 & 0.16 & 355 & -2.782 & -35.0 & 99.0 & 304 & 19 & 000 & 0.0 & 180 \\
\hline $00: 50: 00$ & 17 & -123 & 25 & 0 & -0.24 & -0.35 & 0.22 & 354 & -3.603 & -34.5 & 99.0 & 304 & 17 & 000 & 0.0 & 180 \\
\hline $00: 50: 10$ & 17 & -124 & 0 & 0 & -0.22 & -0.41 & 0.22 & 354 & -4.022 & -15.5 & 99.0 & 358 & 16 & 000 & 0.0 & 180 \\
\hline $00: 50: 20$ & 16 & -125 & 32 & 0 & -0.14 & -0.44 & 0.23 & 353 & -4.215 & 3.5 & 99.0 & 358 & 15 & 000 & 0.0 & 180 \\
\hline $00: 50: 30$ & 16 & -126 & 32 & 0 & -0.04 & -0.42 & 0.20 & 352 & -3.958 & 22.5 & 99.0 & 358 & 18 & 000 & 0.0 & 180 \\
\hline $00: 50: 40$ & 15 & -126 & 32 & 0 & 0.04 & -0.40 & 0.12 & 352 & -3.301 & 26.3 & 99.0 & 358 & 20 & 000 & 0.0 & 180 \\
\hline $00: 50: 50$ & 14 & -126 & 36 & 0 & 0.14 & -0.36 & 0.03 & 351 & -2.479 & 35.0 & 99.0 & 358 & 19 & 000 & 0.0 & 180 \\
\hline $00 ; 51: 00$ & 13 & -125 & 42 & 0 & 0.23 & -0.33 & -0.10 & 351 & -1.414 & 35.0 & 99.0 & 358 & 20 & 000 & 0.0 & 180 \\
\hline $00 ; 51 ; 10$ & 12 & -124 & 41 & 0 & 0.32 & -0.30 & -0.25 & 351 & -0.314 & 35.0 & 99.0 & 358 & 23 & 000 & 0.0 & 180 \\
\hline $00: 51: 20$ & 10 & -122 & 33 & 0 & 0.37 & -0.22 & -0.39 & 351 & 1.092 & 35.0 & 99.0 & 096 & 17 & 000 & 0.0 & 180 \\
\hline $00 ; 51 ; 30$ & 8 & -120 & 25 & 0 & 0.36 & -0.16 & -0.42 & 351 & 1.680 & 35.0 & 99.0 & 096 & 13 & 000 & 0.0 & 180 \\
\hline $00: 51: 40$ & 7 & -119 & 25 & 0 & 0.35 & -0.09 & -0.45 & 351 & 2.287 & 35.0 & 99.0 & 096 & 14 & 000 & 0.0 & 180 \\
\hline $00: 51: 50$ & 5 & -117 & 25 & 0 & 0.36 & -0.04 & -0.48 & 352 & 2.771 & 35.0 & 99.0 & 096 & 19 & 000 & 0.0 & 180 \\
\hline $00: 52: 00$ & 3 & -116 & 27 & 0 & 0.33 & 0.02 & -0.51 & 352 & 3.351 & 35.0 & 99.0 & 145 & 19 & 000 & 0.0 & 180 \\
\hline $00: 52: 10$ & 2 & -114 & 27 & 0 & 0.27 & 0.07 & -0.54 & 353 & 3.881 & 35.0 & 99.0 & 145 & 20 & 000 & 0.0 & 180 \\
\hline $00: 52: 20$ & 1 & -113 & 27 & 0 & 0.25 & 0.09 & -0.57 & 354 & 4.159 & 35.0 & 99.0 & 145 & 18 & 000 & 0.0 & 180 \\
\hline $00: 52: 30$ & -1 & -112 & 27 & 0 & 0.19 & 0.13 & -0.59 & 354 & 4.535 & 35.0 & 99.0 & 145 & 22 & 000 & 0.0 & 180 \\
\hline $00: 52 ; 40$ & -2 & -111 & 27 & 0 & 0.16 & 0.18 & -0.61 & 355 & 4.971 & 35.0 & 99.0 & 145 & 22 & 000 & 0.0 & 180 \\
\hline $00: 52: 50$ & -3 & -110 & 27 & 0 & 0.14 & 0.22 & -0.62 & 356 & 5.327 & 35.0 & 99.0 & 089 & 22 & 000 & 0.0 & 180 \\
\hline $00: 53: 00$ & -4 & -110 & 27 & 0 & 0.17 & 0.26 & -0.62 & 357 & 5.573 & 35.0 & 76.0 & 089 & 17 & 000 & 0.0 & 180 \\
\hline $00: 53: 10$ & -5 & -109 & 27 & 0 & 0.17 & 0.29 & -0.63 & 358 & 5.861 & 35.0 & 76.0 & 089 & 21 & 000 & 0.0 & 180 \\
\hline $00 ; 53 ; 20$ & -6 & -108 & 27 & 0 & 0.16 & 0.32 & -0.64 & 359 & 6.068 & 35.0 & 76.0 & 089 & 21 & 000 & 0.0 & 180 \\
\hline $00: 53: 30$ & -7 & -107 & 27 & 0 & 0.16 & 0.36 & -0.64 & 360 & 6.324 & 35.0 & 76.0 & 089 & 20 & 000 & 0.0 & 180 \\
\hline $00: 53: 40$ & -7 & -106 & 27 & 0 & 0.17 & 0.39 & -0.64 & 001 & 6.580 & 35.0 & 76.0 & 066 & 15 & 000 & 0.0 & 180 \\
\hline $00: 53: 50$ & -8 & -105 & 27 & 0 & 0.18 & 0.43 & -0.65 & 002 & 6.824 & 35.0 & 52.0 & 066 & 18 & 000 & 0.0 & 180 \\
\hline $00: 54: 00$ & -8 & -105 & 27 & 0 & 0.19 & 0.45 & -0.66 & 003 & 7.006 & 34.5 & 52.0 & 066 & 20 & 000 & 0.0 & 180 \\
\hline $00: 54: 10$ & -9 & -103 & 27 & 0 & 0.19 & 0.46 & -0.64 & 004 & 7.012 & 15.5 & 52.0 & 036 & 22 & 000 & 0.0 & 180 \\
\hline $00: 54: 20$ & -9 & -102 & 27 & 0 & 0.19 & 0.45 & -0.60 & 006 & 6.650 & -3.5 & 52.0 & 272 & 15 & 000 & 0.0 & 180 \\
\hline $00: 54: 30$ & -10 & -101 & 27 & 0 & 0.21 & 0.42 & -0.55 & 007 & 6.129 & -7.5 & 52.0 & 272 & 15 & 000 & 0.0 & 180 \\
\hline $00: 54: 40$ & -10 & -100 & 27 & 0 & 0.20 & 0.37 & -0.50 & 008 & 5.504 & -7.0 & 52.0 & 272 & 16 & 000 & 0.0 & 180 \\
\hline $00: 54: 50$ & -10 & -99 & 27 & 0 & 0.22 & 0.32 & -0.45 & 008 & 4.911 & -7.0 & 52.0 & 272 & 19 & 000 & 0.0 & 180 \\
\hline$: 55: 00$ & -10 & -98 & 27 & 0 & 0.27 & 0.31 & -0.42 & 009 & 4.609 & -7.0 & 52.0 & 272 & 15 & 000 & 0.0 & 180 \\
\hline
\end{tabular}


Open Ocean

\section{Simulation Track Plot}

Ship: 150,000 ton, Tanker, partial load

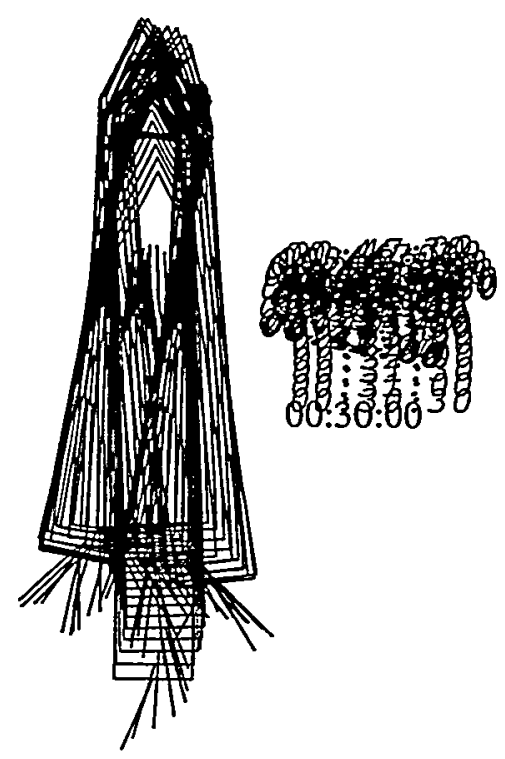

Start Time [hrs:min:sec]: $00: 30: 00$

End Time [hrs:min:sec]: $00: 55: 10$

Delta Plot Time [sec]:

Date: 03-Feb-99 Time: 11:27:14

East-West Scale [m]:

30

East-West Scale [Nm]:

0.5000 


\section{HANDS-ON SIMULATION}

\section{SUCTION MOORNG TECHNOLOGY}

Simulation Number: 15

Date: 3 February 1999

Start Time: 13:31

Length of run [secs]: 3238

Ship Condition: draft $=13.72 \mathrm{~m}$

\section{Environment}

Wind Speed [knots]:

20 Direction: 45

Sea State, Significant wave height $[\mathrm{m}]: .$.

3 Direction: 225

Current Speed [knots]

1 Direction: 180

Ship Operator: J.A.

Observer: B.K.J \& J.K.

Final Mating, distance between center of buoy and center of moon pool [m]: 3.1

Evaluation:

After maneuvering near the approach course the captain turned left to parallel the bow close to the course line (bow was tracking the line). The captain slowed down to experiment with the equilibrium heading for near zero speed - approximately 36 degrees. Then he speeded up a little to approach the buoy and altered the heading to the left for increased speed, and then decelerated again and altered heading to come close to equilibrium. At the final closure to the buoy he altered heading to the left to increase wind and wave forces on the starboard side to offset the current on the port side. The captain performed a sideways closure from port to center. 
Ship:

Exercise number:

591 150,000 ton, Tanker, partial load

754 Suction Mooring System

112 Open Ocean

ig file:

999 Dummy tug file based on containership

rraffic ships:

590 Suction Mooring Buoy

Initial conditions: $\quad 594$ 150k tanker in partial load - Suction Mooring

Exercise identification: $R$ UN 15

RPM

Speed (Knots)

Turn Rudder Gill

Wind Current

\begin{tabular}{|c|c|c|c|c|c|c|c|c|c|c|c|c|c|c|c|c|}
\hline $\begin{array}{l}00: 26: 40 \\
00: 26: 50\end{array}$ & $\begin{array}{l}-31 \\
-29\end{array}$ & $\begin{array}{l}-309 \\
-308\end{array}$ & $\begin{array}{l}51 \\
45\end{array}$ & $\begin{array}{l}0 \\
0\end{array}$ & $\begin{array}{l}0.42 \\
0.42\end{array}$ & $\begin{array}{l}-0.15 \\
-0.05\end{array}$ & $\begin{array}{l}0.41 \\
0.20\end{array}$ & $\begin{array}{l}031 \\
031\end{array}$ & $\begin{array}{l}-3.559 \\
-1.617\end{array}$ & $\begin{array}{l}35.0 \\
35.0\end{array}$ & $\begin{array}{l}100.0 \\
100.0\end{array}$ & $\begin{array}{l}180 \\
180\end{array}$ & $\begin{array}{l}17 \\
18\end{array}$ & $\begin{array}{l}045 \\
045\end{array}$ & $\begin{array}{l}1.0 \\
1.0\end{array}$ & $\begin{array}{l}180 \\
180\end{array}$ \\
\hline $00: 27: 00$ & -28 & -306 & 45 & 0 & 0.43 & 0.01 & 0.02 & 031 & -0.010 & 35.0 & 100.0 & 180 & 19 & 045 & 1.0 & 180 \\
\hline $00: 27: 10$ & -27 & -304 & 39 & 0 & 0.40 & 0.12 & -0.15 & 031 & 1.713 & 35.0 & 100.0 & 180 & 19 & 045 & 1.0 & 180 \\
\hline $00: 27: 20$ & -26 & -302 & 30 & 0 & 0.36 & 0.10 & -0.23 & 031 & 2.091 & 35.0 & 100.0 & 180 & 17 & 045 & 1.0 & 180 \\
\hline $00 ; 27: 30$ & -25 & -301 & 30 & 0 & 0.32 & 0.12 & -0.29 & 032 & 2.568 & 34.7 & 100.0 & 180 & 18 & 045 & 1.0 & 180 \\
\hline $00: 27: 40$ & -25 & -299 & 30 & 0 & 0.28 & 0.11 & -0.33 & 032 & 2.797 & 25.1 & 100.0 & 180 & 17 & 045 & 1.0 & 180 \\
\hline $00: 27: 50$ & -25 & -298 & 30 & 0 & 0.21 & 0.15 & -0.35 & 033 & 3.125 & 11.3 & 100.0 & 180 & 18 & 045 & 1.0 & 180 \\
\hline $00: 28: 00$ & -25 & -296 & 30 & 0 & 0.18 & 0.09 & -0.33 & 033 & 2.633 & 3.6 & 100.0 & 180 & 22 & 045 & 1.0 & 180 \\
\hline $00: 28: 10$ & -25 & -295 & 30 & 0 & 0.12 & 0.03 & -0.30 & 033 & 2.102 & -3.2 & 100.0 & 180 & 22 & 045 & 1.0 & 180 \\
\hline $00: 28: 20$ & -25 & -295 & 30 & 0 & 0.04 & 0.02 & -0.26 & 034 & 1.720 & -10.7 & 100.0 & 180 & 18 & 045 & 1.0 & 180 \\
\hline $00: 28: 30$ & -25 & -294 & 30 & 0 & 0.00 & 0.00 & -0.20 & 034 & 1.222 & -14.1 & 100.0 & 180 & 17 & 045 & 1.0 & 180 \\
\hline $00: 28: 40$ & -26 & -294 & 30 & 0 & -0.04 & -0.01 & -0.14 & 034 & 0.800 & -13.2 & 100.0 & 180 & 17 & 045 & 1.0 & 180 \\
\hline $00: 28: 50$ & -26 & -294 & 30 & 0 & -0.07 & -0.02 & -0.09 & 034 & 0.469 & -10.1 & 100.0 & 180 & 13 & 045 & 1.0 & 180 \\
\hline $00: 29: 00$ & -27 & -295 & 30 & 0 & -0.10 & -0.02 & -0.05 & 034 & 0.189 & -7.3 & 100.0 & 180 & 17 & 045 & 1.0 & 180 \\
\hline $00: 29: 10$ & -27 & -295 & 35 & 0 & -0.14 & -0.06 & -0.02 & 034 & -0.242 & -3.2 & 100.0 & 180 & 20 & 045 & 1.0 & 180 \\
\hline $00 ; 29 ; 20$ & -28 & -295 & 38 & 0 & -0.15 & -0.07 & 0.01 & 034 & -0.454 & -0.5 & 100.0 & 180 & 20 & 045 & 1.0 & 180 \\
\hline $00: 29: 30$ & -28 & -296 & 38 & 0 & -0.16 & -0.12 & 0.02 & 034 & -0.881 & 4.0 & 100.0 & 180 & 19 & 045 & 1.0 & 180 \\
\hline $00: 29: 40$ & -29 & -297 & 38 & 0 & -0.17 & -0.12 & 0.01 & 034 & -0.838 & 6.3 & 100.0 & 180 & 23 & 045 & 1.0 & 180 \\
\hline $00: 29: 50$ & -30 & -297 & 37 & 0 & -0.16 & -0.10 & 0.01 & 034 & -0.661 & 7.3 & 51.0 & 180 & 19 & 045 & 1.0 & 180 \\
\hline $00: 30: 00$ & -30 & -298 & 38 & 0 & -0.13 & -0.12 & -0.01 & 034 & -0.692 & 5.1 & 51.0 & 180 & 20 & 045 & 1.0 & 180 \\
\hline $00: 30: 10$ & -31 & -298 & 38 & 0 & -0.10 & -0.18 & 0.03 & 034 & -1.332 & -13.9 & 51.0 & 180 & 20 & 045 & 1.0 & 180 \\
\hline $00: 30: 20$ & -32 & -298 & 38 & 0 & -0.09 & -0.23 & 0.08 & 033 & -1.970 & -3.8 & 51.0 & 180 & 20 & 045 & 1.0 & 180 \\
\hline $00: 30: 30$ & -32 & -298 & 38 & 0 & -0.10 & -0.24 & 0.09 & 033 & -2.089 & 2.8 & 51.0 & 180 & 16 & 045 & 1.0 & 180 \\
\hline $00: 30: 40$ & -33 & -298 & 37 & 0 & -0.06 & -0.24 & 0.08 & 033 & -2.017 & 1.0 & 51.0 & 180 & 21 & 045 & 1.0 & 180 \\
\hline $00: 30: 50$ & -33 & -298 & 38 & 0 & -0.04 & -0.26 & 0.11 & 032 & -2.381 & -7.5 & 51.0 & 180 & 18 & 045 & 1.0 & 180 \\
\hline $00: 31: 00$ & -34 & -298 & 48 & 0 & -0.01 & -0.20 & 0.10 & 032 & -1.915 & 10.4 & 51.0 & 180 & 18 & 045 & 1.0 & 180 \\
\hline $00: 31 ; 10$ & -34 & -298 & 48 & 0 & 0.07 & -0.18 & 0.08 & 032 & -1.661 & -4.8 & 51.0 & 180 & 22 & 045 & 1.0 & 180 \\
\hline $0: 31: 20$ & -34 & -297 & 55 & 0 & 0.15 & -0.24 & 0.13 & 031 & -2.377 & -5.5 & 51.0 & 180 & 25 & 045 & 1.0 & 180 \\
\hline$p: 31: 30$ & -33 & -296 & 60 & 0 & 0.27 & -0.28 & 0.18 & 031 & -2.886 & -1.9 & 51.0 & 180 & 16 & 045 & 1.0 & 180 \\
\hline $00: 31: 40$ & -33 & -295 & 61 & 0 & 0.39 & -0.28 & 0.18 & 030 & -2.951 & 2.7 & 51.0 & 180 & 17 & 045 & 1.0 & 180 \\
\hline $00: 31: 50$ & -32 & -293 & 61 & 0 & 0.54 & -0.26 & 0.13 & 030 & -2.495 & 7.8 & 51.0 & 180 & 15 & 045 & 1.0 & 180 \\
\hline $00: 32: 00$ & -31 & -290 & 61 & 0 & 0.67 & -0.18 & 0.04 & 030 & -1.402 & 10.9 & 51.0 & 180 & 15 & 045 & 1.0 & 180 \\
\hline $00: 32: 10$ & -29 & -286 & 61 & 0 & 0.83 & -0.18 & -0.06 & 029 & -0.716 & 12.8 & 51.0 & 180 & 14 & 045 & 1.0 & 180 \\
\hline $00: 32: 20$ & -28 & -282 & 61 & 0 & 0.90 & -0.13 & -0.17 & 029 & 0.262 & 12.1 & 98.0 & 180 & 19 & 045 & 1.0 & 180 \\
\hline $00: 32: 30$ & -26 & -277 & 62 & 0 & 0.98 & -0.11 & -0.25 & 029 & 0.904 & 9.6 & 98.0 & 180 & 15 & 045 & 1.0 & 180 \\
\hline $00: 32: 40$ & -24 & -272 & 34 & 0 & 1.00 & -0.14 & -0.28 & 030 & 0.882 & 6.4 & 98.0 & 180 & 19 & 045 & 1.0 & 180 \\
\hline $00: 32: 50$ & -23 & -267 & 29 & 0 & 0.94 & -0.17 & -0.29 & 030 & 0.770 & 4.1 & 98.0 & 180 & 20 & 045 & 1.0 & 180 \\
\hline $00: 33: 00$ & -21 & -263 & 29 & 0 & 0.88 & -0.10 & -0.29 & 030 & 1.185 & 0.7 & 98.0 & 180 & 17 & 045 & 1.0 & 180 \\
\hline $00: 33: 10$ & -20 & -258 & 29 & 0 & 0.82 & -0.09 & -0.28 & 030 & 1.204 & -2.8 & 98.0 & 180 & 13 & 045 & 1.0 & 180 \\
\hline $00: 33: 20$ & -19 & -254 & 25 & 0 & 0.79 & -0.09 & -0.26 & 030 & 1.047 & -6.0 & 98.0 & 180 & 23 & 045 & 1.0 & 180 \\
\hline $00: 33: 30$ & -18 & -250 & 25 & 0 & 0.72 & -0.11 & -0.24 & 030 & 0.811 & -8.6 & 98.0 & 180 & 23 & 045 & 1.0 & 180 \\
\hline $00: 33: 40$ & -17 & -247 & 25 & 0 & 0.65 & -0.12 & -0.21 & 031 & 0.561 & -10.6 & 98.0 & 180 & 20 & 045 & 1.0 & 180 \\
\hline $00: 33: 50$ & -16 & -244 & 25 & 0 & 0.60 & -0.12 & -0.18 & 031 & 0.426 & -15.4 & 98.0 & 180 & 19 & 045 & 1.0 & 180 \\
\hline $00: 34: 00$ & -15 & -241 & 25 & 0 & 0.55 & -0.19 & -0.13 & 031 & -0.407 & -34.4 & 98.0 & 180 & 21 & 045 & 1.0 & 180 \\
\hline $00: 34: 10$ & -14 & -238 & 25 & 0 & 0.50 & -0.19 & -0.06 & 031 & -0.782 & -35.0 & 98.0 & 180 & 19 & 045 & 1.0 & 180 \\
\hline $00: 34: 20$ & -13 & -236 & 25 & 0 & 0.46 & -0.25 & 0.00 & 030 & -1.575 & -35.0 & 98.0 & 180 & 22 & 045 & 1.0 & 180 \\
\hline $00: 34: 30$ & -13 & -233 & 25 & 0 & 0.41 & -0.31 & 0.06 & 030 & -2.373 & -29.7 & 98.0 & 180 & 20 & 045 & 1.0 & 180 \\
\hline $00: 34: 40$ & -12 & -231 & 25 & 0 & 0.35 & -0.38 & 0.10 & 030 & -3.046 & -18.6 & 98.0 & 180 & 18 & 045 & 1.0 & 180 \\
\hline $00: 34: 50$ & -12 & -230 & 25 & 0 & 0.29 & -0.46 & 0.12 & 029 & -3.684 & -16.1 & 98.0 & 180 & 21 & 045 & 1.0 & 180 \\
\hline $00: 35: 00$ & -13 & -228 & 25 & 0 & 0.21 & -0.50 & 0.14 & 028 & -4.087 & -25.1 & 98.0 & 180 & 23 & 045 & 1.0 & 180 \\
\hline $00: 35: 10$ & -13 & -227 & 25 & 0 & 0.16 & -0.46 & 0.15 & 028 & -3.897 & -9.7 & 98.0 & 180 & 17 & 045 & 1.0 & 180 \\
\hline $00: 35: 20$ & -13 & -226 & 25 & 0 & 0.12 & -0.48 & 0.14 & 027 & -3.882 & 3.7 & 98.0 & 180 & 15 & 045 & 1.0 & 180 \\
\hline $00: 35: 30$ & -14 & -225 & 25 & 0 & 0.07 & -0.47 & 0.10 & 026 & -3.638 & 14.6 & 98.0 & 180 & 20 & 045 & 1.0 & 180 \\
\hline $00: 35 ; 40$ & -15 & -224 & 25 & 0 & 0.01 & -0.40 & 0.06 & 026 & -2.923 & 3.9 & 98.0 & 180 & 19 & 045 & 1.0 & 180 \\
\hline $00: 35: 50$ & -15 & -224 & 25 & 0 & -0.05 & -0.43 & 0.06 & 025 & -3.127 & -4.9 & 98.0 & 180 & 15 & 045 & 1.0 & 180 \\
\hline $00: 36: 00$ & -17 & -224 & 25 & 0 & -0.08 & -0.47 & 0.06 & 025 & -3.364 & 3.1 & 98.0 & 180 & 13 & 045 & 1.0 & 180 \\
\hline $00: 36: 10$ & -18 & -224 & 33 & 0 & -0.12 & -0.42 & 0.03 & 024 & -2.892 & 9.8 & 98.0 & 180 & 14 & 045 & 1.0 & 180 \\
\hline $00: 36: 20$ & -19 & -224 & 46 & 0 & -0.12 & -0.38 & -0.02 & 024 & -2.261 & 14.3 & 98.0 & 180 & 16 & 045 & 1.0 & 180 \\
\hline $00: 36: 30$ & -20 & -224 & 57 & 0 & -0.05 & -0.31 & -0.12 & 024 & -1.229 & 14.6 & 98.0 & 180 & 21 & 045 & 1.0 & 180 \\
\hline $00: 36 ; 40$ & -21 & -224 & 60 & 0 & 0.05 & -0.35 & -0.24 & 023 & -0.733 & 14.3 & 98.0 & 180 & 19 & 045 & 1.0 & 180 \\
\hline $00 ; 36 ; 50$ & -23 & -223 & 61 & 0 & 0.12 & -0.33 & -0.33 & 023 & -0.030 & 11.9 & 98.0 & 180 & 20 & 045 & 1.0 & 180 \\
\hline $00: 37: 00$ & -24 & -221 & 61 & 0 & 0.21 & -0.36 & -0.41 & 023 & 0.300 & 11.0 & 98.0 & 180 & 22 & 045 & 1.0 & 180 \\
\hline $00: 37: 10$ & -25 & -219 & 61 & 0 & 0.28 & -0.39 & -0.47 & 023 & 0.501 & 9.5 & 98.0 & 180 & 22 & 045 & 1.0 & 180 \\
\hline $00 ; 37 ; 20$ & -27 & -217 & 61 & 0 & 0.37 & -0.37 & -0.50 & 024 & 0.820 & 6.7 & 98.0 & 180 & 14 & 045 & 1.0 & 180 \\
\hline $00: 37: 30$ & -28 & -214 & 61 & 0 & 0.45 & -0.39 & -0.45 & 024 & 0.347 & -10.4 & 98.0 & 180 & 15 & 045 & 1.0 & 180 \\
\hline $00: 37: 40$ & -29 & -211 & 61 & 0 & 0.52 & -0.53 & -0.21 & 024 & -2.063 & -23.9 & 98.0 & 180 & 18 & 045 & 1.0 & 180 \\
\hline $0 ; 37: 50$ & -29 & -207 & 61 & 0 & 0.60 & -0.62 & -0.02 & 023 & -3.863 & -11.9 & 98.0 & 180 & 23 & 045 & 1.0 & 180 \\
\hline $0: 38: 00$ & -30 & -204 & 28 & 0 & 0.63 & -0.70 & 0.02 & 022 & -4.521 & 1.7 & 98.0 & 180 & 20 & 045 & 1.0 & 180 \\
\hline $00: 38 ; 10$ & -30 & -200 & 26 & 0 & 0.57 & -0.69 & -0.03 & 022 & -4.179 & 16.5 & 98.0 & 180 & 20 & 045 & 1.0 & 180 \\
\hline $00: 38: 20$ & -31 & -197 & 26 & 0 & 0.51 & -0.54 & -0.10 & 021 & -2.746 & 35.0 & 98.0 & 180 & 18 & 045 & 1.0 & 180 \\
\hline $00: 38: 30$ & -31 & -194 & 26 & 0 & 0.48 & -0.50 & -0.19 & 021 & -1.991 & 35.0 & 98.0 & 180 & 22 & 045 & 1.0 & 180 \\
\hline 00: & 2 & -1 & 26 & 0 & 0.44 & -0.46 & -0.27 & 020 & -1.249 & 35 & 98.0 & 180 & 19 & 045 & 1.0 & 8 \\
\hline
\end{tabular}


Time Position (m) RPM

Speed (Knots)

Turn Rudder Gill Wind Current

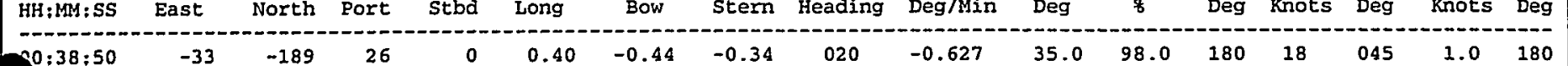

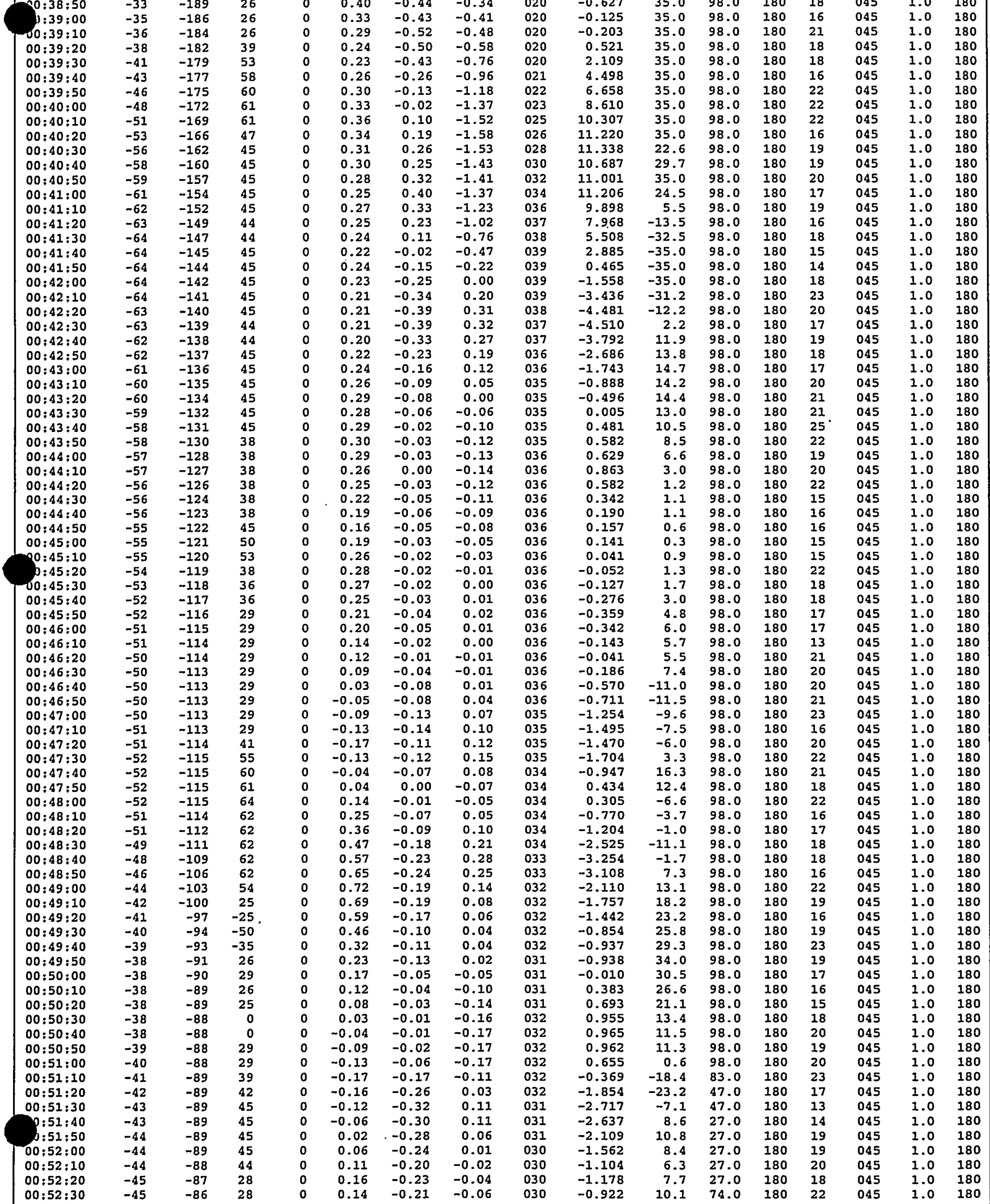




\begin{tabular}{|c|c|c|c|c|c|c|c|c|c|c|c|c|c|c|c|c|}
\hline \multirow{3}{*}{$\begin{array}{c}\text { Time } \\
\text { HH:MM:SS }\end{array}$} & \multicolumn{2}{|c|}{ position (m) } & \multicolumn{2}{|c|}{ RPM } & \multicolumn{3}{|c|}{ Speed (knots) } & \multirow[b]{2}{*}{ Heading } & \multirow{2}{*}{$\begin{array}{c}\text { Turn } \\
\text { Deg/Min }\end{array}$} & \multirow{2}{*}{$\begin{array}{l}\text { Rudder } \\
\text { Deg }\end{array}$} & \multicolumn{2}{|c|}{ Gill } & \multicolumn{2}{|c|}{ wind } & \multicolumn{2}{|c|}{ current } \\
\hline & East & North & Port & Stbd & Long & Bow & stern & & & & 8 & Deg & Knots & Deg & Knots & Deg \\
\hline & & & & & & & & & & & & & & & & \\
\hline $9: 52: 40$ & -45 & -85 & 28 & 0 & 0.11 & -0.16 & -0.09 & 030 & -0.441 & 9.9 & 100.0 & 180 & 22 & 045 & 1.0 & 180 \\
\hline$: 52: 50$ & -45 & -85 & 0 & 0 & 0.08 & -0.13 & -0.11 & 030 & -0.163 & 8.7 & 100.0 & 180 & 22 & 045 & 1.0 & 180 \\
\hline $\begin{array}{l}0: 53: 00 \\
00: 53: 10\end{array}$ & $\begin{array}{l}-46 \\
-47\end{array}$ & $\begin{array}{l}-84 \\
-84\end{array}$ & $\begin{array}{r}0 \\
-28\end{array}$ & $\begin{array}{l}0 \\
0\end{array}$ & $\begin{array}{r}0.02 \\
-0.07\end{array}$ & $\begin{array}{l}-0.19 \\
-0.19\end{array}$ & $\begin{array}{l}-0.12 \\
-0.13\end{array}$ & $\begin{array}{l}030 \\
029\end{array}$ & $\begin{array}{l}-0.481 \\
-0.336\end{array}$ & $\begin{array}{l}12.0 \\
11.8\end{array}$ & $\begin{array}{l}100.0 \\
100.0\end{array}$ & $\begin{array}{l}180 \\
180\end{array}$ & $\begin{array}{l}17 \\
21\end{array}$ & $\begin{array}{l}045 \\
045\end{array}$ & $\begin{array}{l}1.0 \\
1.0\end{array}$ & $\begin{array}{l}180 \\
180\end{array}$ \\
\hline $00: 53: 20$ & -48 & -84 & -51 & 0 & -0.21 & -0.27 & -0.14 & 029 & -0.854 & 14.7 & 100.0 & 180 & 21 & 045 & 1.0 & 180 \\
\hline $00: 53: 30$ & -50 & -85 & -38 & 0 & -0.38 & -0.30 & -0.13 & 029 & -1.066 & 17.0 & 100.0 & 180 & 20 & 045 & 1.0 & 180 \\
\hline $00: 53: 40$ & -52 & -86 & 0 & 0 & -0.47 & -0.32 & -0.13 & 029 & -1.221 & 20.2 & 100.0 & 180 & 15 & 045 & 1.0 & 180 \\
\hline $00: 53: 50$ & -54 & -88 & 0 & 0 & -0.55 & -0.35 & -0.13 & 029 & -1.402 & 24.6 & 100.0 & 180 & 18 & 045 & 1.0 & 180 \\
\hline
\end{tabular}




\section{Open Ocean}

\section{Simulation Track Plot}

Ship: 150,000 ton, Tanker, partial load

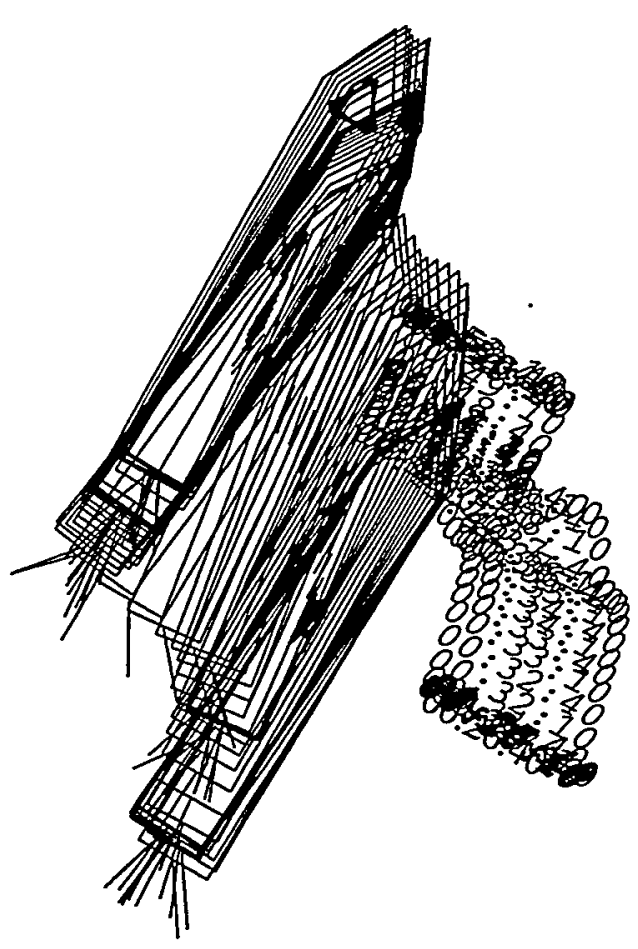

Start Time [hrs:min:sec]: $00: 26: 40$

End Time [hrs:min:sec]: $00: 53: 58$

Delta Plot Time [sec]:

East-West Scale [m]:

30

Date: $03-F e b-99$

Time: $13: 31: 11$

East-West Scale [Nm]: $\quad 0.5000$ 


\section{HANDS-ON SIMULATION}

\section{SUCTION MOORNG TECHNOLOGY}

Simulation Number: 16

Date: 3 February 1999

Start Time: $14: 36$

Length of run [secs]: 2940

Ship Condition: draft $=13.72 \mathrm{~m}$

Environment

Wind Speed [knots] :................... $\quad 20 \quad$ Direction: 45

Sea State, Significant wave height [m]:.. 3 Direction: 225

Current Speed [knots] $: \ldots \ldots \ldots \ldots \ldots \ldots \ldots . \ldots 1$ Direction: 180

Ship Operator: J.A.

Observer: B.K.J \& J.K.

Final Mating, distance between center of buoy and center of moon pool [m]: 0.4

Evaluation:

The final mating was nearly perfect.

It was easier to find the equilibrium because of the experience with the previous exercise, run \# 15. 
Ship:

Exercise number:

Scenario:

If file:

affic ships:

Initial conditions:

Exercise identification:
591150,000 ton, Tanker, partial load

754 Suction Mooring System

112 Open Ocean

999 Dummy tug file based on containership

590 Suction Mooring Buoy

594 150k tanker in partial load - Suction Mooring RUN 416

Time Position (m) RPM Speed (Knots) Turn Rudder Gill Current

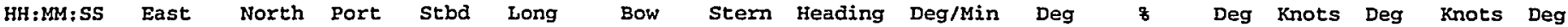

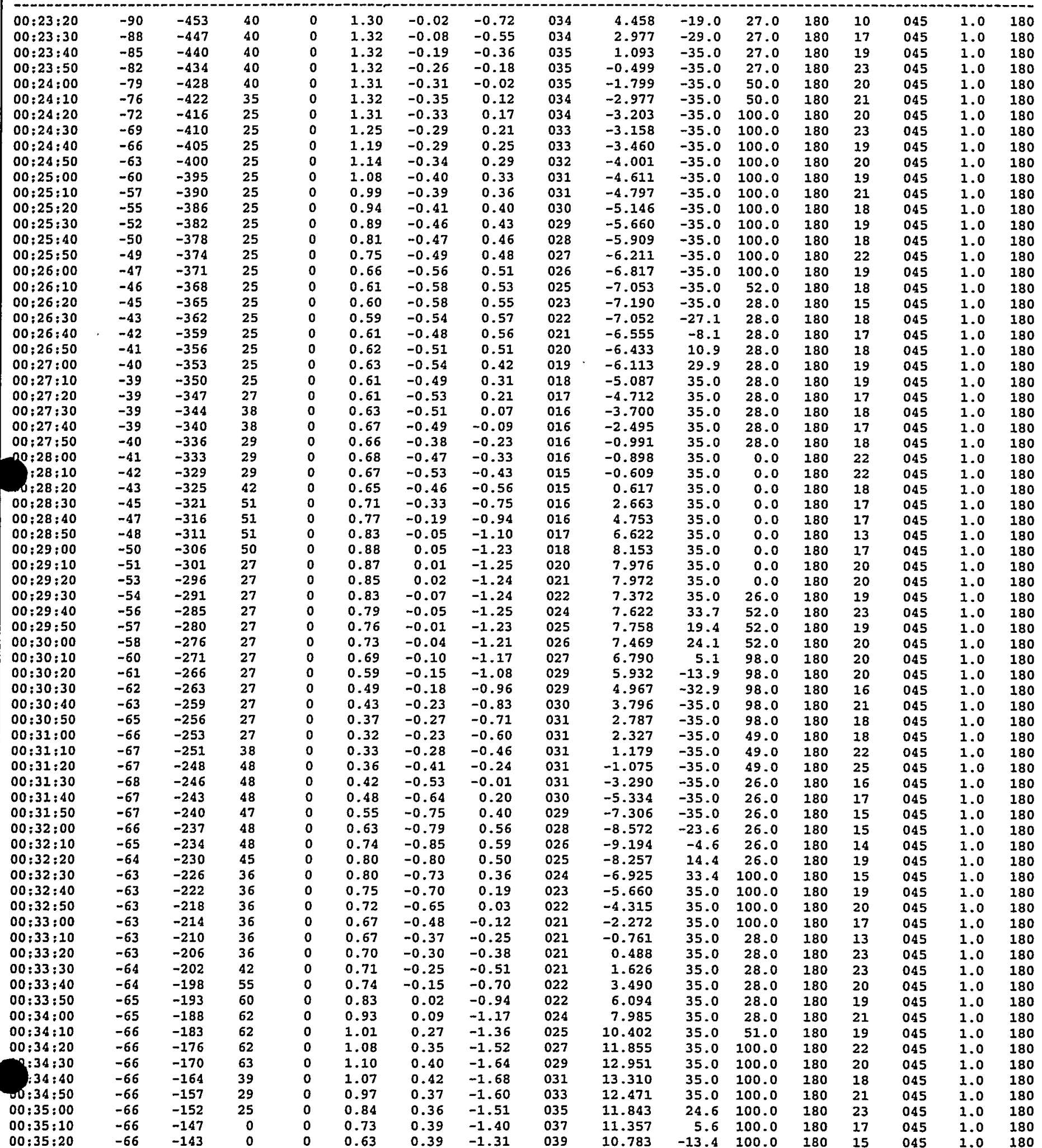


Speed (Knots)

Turn Rudder Gill Winà

Current

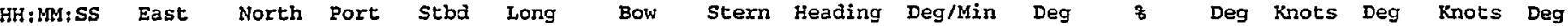

\begin{tabular}{|c|c|c|c|c|c|c|c|c|c|c|c|c|c|c|c|c|}
\hline $\begin{array}{l}0: 35: 30 \\
: 35: 40\end{array}$ & $\begin{array}{l}-65 \\
-65\end{array}$ & $\begin{array}{l}-139 \\
-136\end{array}$ & $\begin{array}{l}31 \\
47\end{array}$ & 0 & $\begin{array}{l}0.54 \\
0.48\end{array}$ & $\begin{array}{l}0.37 \\
0.26\end{array}$ & $\begin{array}{l}-1.16 \\
-0.85\end{array}$ & $\begin{array}{l}041 \\
042\end{array}$ & $\begin{array}{l}9.694 \\
7.019\end{array}$ & $\begin{array}{l}-32.4 \\
-35.0\end{array}$ & $\begin{array}{l}100.0 \\
100.0\end{array}$ & $\begin{array}{l}180 \\
180\end{array}$ & $\begin{array}{l}20 \\
19\end{array}$ & $\begin{array}{l}045 \\
045\end{array}$ & $\begin{array}{l}1.0 \\
1.0\end{array}$ & $\begin{array}{l}180 \\
180 \\
180\end{array}$ \\
\hline $\begin{array}{l}0: 35: 50 \\
00: 36: 00\end{array}$ & $\begin{array}{l}-64 \\
-63\end{array}$ & $\begin{array}{l}-133 \\
-131\end{array}$ & 56 & $\begin{array}{l}0 \\
0\end{array}$ & $\begin{array}{l}0.48 \\
0.53\end{array}$ & $\begin{array}{r}0.08 \\
-0.09\end{array}$ & $\begin{array}{l}-0.48 \\
-0.12\end{array}$ & 043 & $\begin{array}{l}3.524 \\
0.170\end{array}$ & $\begin{array}{l}-35.0 \\
-35.0\end{array}$ & $\begin{array}{l}100.0 \\
100.0\end{array}$ & 180 & $\begin{array}{l}15 \\
13\end{array}$ & $\begin{array}{l}045 \\
045\end{array}$ & 1.0 & $\begin{array}{l}180 \\
180\end{array}$ \\
\hline $00: 36: 10$ & $\begin{array}{l}-63 \\
-61\end{array}$ & $\begin{array}{l}-131 \\
-128\end{array}$ & $\begin{array}{l}46 \\
29\end{array}$ & 0 & $\begin{array}{l}0.53 \\
0.49\end{array}$ & -0.12 & 0.02 & 043 & -0.882 & -35.0 & 100.0 & $\begin{array}{l}180 \\
180\end{array}$ & $\begin{array}{l}13 \\
14\end{array}$ & 045 & 1.0 & $\begin{array}{l}180 \\
180\end{array}$ \\
\hline $00: 36: 20$ & -60 & -127 & 26 & 0 & 0.43 & -0.12 & 0.08 & 043 & -1.265 & -35.0 & 100.0 & 180 & 16 & 045 & 1.0 & 180 \\
\hline $\begin{array}{l}00: 36: 30 \\
00: 36: 40\end{array}$ & $\begin{array}{l}-59 \\
-57\end{array}$ & $\begin{array}{l}-125 \\
-124\end{array}$ & $\begin{array}{l}26 \\
26\end{array}$ & $\begin{array}{l}0 \\
0\end{array}$ & $\begin{array}{l}0.41 \\
0.37\end{array}$ & $\begin{array}{l}-0.11 \\
-0.12\end{array}$ & $\begin{array}{l}0.15 \\
0.21\end{array}$ & $\begin{array}{l}043 \\
043\end{array}$ & $\begin{array}{l}-1.640 \\
-2.095\end{array}$ & $\begin{array}{l}-35.0 \\
-35.0\end{array}$ & $\begin{array}{l}100.0 \\
100.0\end{array}$ & $\begin{array}{l}180 \\
180\end{array}$ & $\begin{array}{l}21 \\
19\end{array}$ & $\begin{array}{l}045 \\
045\end{array}$ & $\begin{array}{l}1.0 \\
1.0\end{array}$ & $\begin{array}{l}180 \\
180\end{array}$ \\
\hline $00: 36: 50$ & -56 & -123 & 26 & 0 & 0.29 & -0.13 & 0.26 & 042 & -2.516 & -35.0 & 100.0 & 180 & 20 & 045 & 1.0 & 180 \\
\hline $00: 37: 00$ & -54 & -122 & 26 & 0 & 0.23 & -0.15 & 0.32 & 042 & -2.982 & -35.0 & 100.0 & 180 & 22 & 045 & 1.0 & 180 \\
\hline $00: 37: 10$ & -53 & -121 & 26 & 0 & 0.16 & -0.17 & 0.38 & 041 & -3.465 & -35.0 & 100.0 & 180 & 22 & 045 & 1.0 & 180 \\
\hline $00: 37: 20$ & -53 & -121 & 26 & 0 & 0.10 & -0.18 & 0.43 & 041 & -3.903 & -35.0 & 100.0 & 180 & 14 & 045 & 1.0 & 180 \\
\hline $00: 37: 30$ & -52 & -121 & 36 & 0 & 0.05 & -0.21 & 0.51 & 040 & -4.533 & -35.0 & 100.0 & 180 & 15 & 045 & 1.0 & 180 \\
\hline $00: 37: 40$ & -51 & -122 & 41 & 0 & 0.03 & -0.28 & 0.65 & 039 & -5.903 & -35.0 & 100.0 & 180 & 18 & 045 & 1.0 & 180 \\
\hline $00: 37: 50$ & -50 & -122 & 41 & 0 & 0.01 & -0.33 & 0.76 & 038 & -6.876 & -19.2 & 100.0 & 180 & 23 & 045 & 1.0 & 180 \\
\hline $00: 38: 00$ & -49 & -123 & 41 & 0 & -0.01 & -0.35 & 0.77 & 037 & -7.137 & -0.2 & 100.0 & 180 & 20 & 045 & 1.0 & 180 \\
\hline $00: 38: 10$ & -48 & -124 & 41 & 0 & -0.03 & -0.31 & 0.70 & 036 & -6.454 & 18.8 & 100.0 & 180 & 20 & 045 & 1.0 & 180 \\
\hline $00: 38: 20$ & -48 & -125 & 41 & 0 & -0.05 & -0.20 & 0.59 & 035 & -4.956 & 15.0 & 100.0 & 180 & 18 & 045 & 1.0 & 180 \\
\hline $00: 38: 30$ & -47 & -125 & 50 & 0 & -0.02 & -0.18 & 0.55 & 034 & -4.639 & -4.0 & 100.0 & 180 & 22 & 045 & 1.0 & 180 \\
\hline $00: 38: 40$ & -46 & -126 & 43 & 0 & 0.02 & -0.18 & 0.53 & 033 & -4.464 & 7.3 & 100.0 & 180 & 19 & 045 & 1.0 & 180 \\
\hline $00: 38: 50$ & -45 & -126 & 35 & 0 & 0.02 & -0.15 & 0.46 & 033 & -3.915 & 19.0 & 100.0 & 180 & 18 & 045 & 1.0 & 180 \\
\hline $00: 39: 00$ & -45 & -127 & 35 & 0 & -0.01 & -0.12 & 0.37 & 032 & -3.122 & 30.2 & 74.0 & 180 & 16 & 045 & 1.0 & 180 \\
\hline $00: 39: 10$ & -44 & -127 & 29 & 0 & -0.02 & -0.15 & 0.28 & 031 & -2.762 & 35.0 & 74.0 & 180 & 21 & 045 & 1.0 & 180 \\
\hline $00: 39: 20$ & -44 & -127 & 29 & 0 & -0.05 & -0.14 & 0.20 & 031 & -2.139 & 35.0 & 100.0 & 180 & 18 & 045 & 1.0 & 180 \\
\hline $00: 39: 30$ & -45 & -127 & 29 & 0 & -0.11 & -0.12 & 0.12 & 031 & -1.520 & 35.0 & 100.0 & 180 & 18 & 045 & 1.0 & 180 \\
\hline $00: 39: 40$ & -45 & -128 & 29 & 0 & -0.15 & -0.07 & 0.04 & 031 & -0.679 & 35.0 & 100.0 & 180 & 16 & 045 & 1.0 & 180 \\
\hline $00: 39: 50$ & -46 & -129 & 29 & 0 & -0.20 & -0.06 & -0.03 & 031 & -0.143 & 35.0 & 100.0 & 180 & 22 & 045 & 1.0 & 180 \\
\hline $00: 40: 00$ & -46 & -130 & 29 & 0 & -0.26 & -0.05 & -0.10 & 031 & 0.324 & 35.0 & 100.0 & 180 & 22 & 045 & 1.0 & 180 \\
\hline $00: 40: 10$ & -47 & -131 & 42 & 0 & -0.27 & -0.01 & -0.19 & 031 & 1.173 & 35.0 & 28.0 & 180 & 22 & 045 & 1.0 & 180 \\
\hline $00 ; 40 ; 20$ & -49 & -131 & 42 & 0 & -0.26 & 0.05 & -0.32 & 031 & 2.356 & 35.0 & 0.0 & 180 & 16 & 045 & 1.0 & 180 \\
\hline $00: 40: 30$ & -50 & -132 & 47 & 0 & -0.21 & 0.16 & -0.43 & 031 & 3.727 & 29.3 & 0.0 & 180 & 19 & 045 & 1.0 & 180 \\
\hline $00: 40: 40$ & -51 & -132 & 48 & 0 & -0.11 & 0.19 & -0.47 & 032 & 4.170 & 10.3 & 0.0 & 180 & 19 & 045 & 1.0 & 180 \\
\hline $00: 40: 50$ & -52 & -132 & 48 & 0 & -0.03 & 0.17 & -0.40 & 033 & 3.606 & -8.7 & 0.0 & 180 & 20 & 045 & 1.0 & 180 \\
\hline $00: 41: 00$ & -52 & -132 & 48 & 0 & 0.05 & 0.11 & -0.23 & 033 & 2.148 & -21.7 & 0.0 & 180 & 17 & 045 & 1.0 & 180 \\
\hline $00: 41: 10$ & -52 & -132 & 39 & 0 & 0.14 & -0.01 & -0.12 & 034 & 0.712 & -2.7 & 30.0 & 180 & 19 & 045 & 1.0 & 180 \\
\hline $00: 41: 20$ & -52 & -131 & 29 & 0 & 0.10 & -0.05 & -0.11 & 034 & 0.396 & 3.7 & 99.0 & 180 & 16 & 045 & 1.0 & 180 \\
\hline $00: 41: 30$ & -52 & -130 & 29 & 0 & 0.05 & -0.06 & -0.11 & 034 & 0.286 & 0.7 & 99.0 & 180 & 18 & 045 & 1.0 & 180 \\
\hline $00: 41: 40$ & -52 & -130 & 29 & 0 & 0.02 & -0.08 & -0.07 & 034 & -0.032 & -18.3 & 23.0 & 180 & 15 & 045 & 1.0 & 180 \\
\hline $40: 41: 50$ & -53 & -129 & 29 & 0 & 0.06 & -0.15 & -0.01 & 034 & -0.868 & -18.3 & 0.0 & 180 & 14 & 045 & 1.0 & 180 \\
\hline$b: 42: 00$ & -53 & -129 & 29 & 0 & 0.06 & -0.16 & 0.03 & 033 & -1.216 & -10.5 & 50.0 & 180 & 18 & 045 & 1.0 & 180 \\
\hline $00: 42: 10$ & -53 & -129 & 29 & 0 & 0.04 & -0.18 & 0.04 & 033 & -1.422 & -0.6 & 50.0 & 180 & 23 & 045 & 1.0 & 180 \\
\hline $00: 42: 20$ & -53 & -128 & 29 & 0 & 0.03 & -0.20 & 0.04 & 033 & -1.508 & 3.8 & 50.0 & 180 & 20 & 045 & 1.0 & 180 \\
\hline $00: 42: 30$ & -53 & -128 & 29 & 0 & 0.02 & -0.21 & 0.03 & 033 & -1.499 & 6.6 & 26.0 & 180 & 17 & 045 & 1.0 & 180 \\
\hline $00: 42: 40$ & -54 & -128 & 29 & 0 & 0.01 & -0.19 & 0.01 & 032 & -1.286 & 8.8 & 26.0 & 180 & 19 & 045 & 1.0 & 180 \\
\hline $00: 42: 50$ & -54 & -127 & 31 & 0 & 0.03 & -0.13 & -0.01 & 032 & -0.823 & 7.8 & 26.0 & 180 & 18 & 045 & 1.0 & 180 \\
\hline $00: 43: 00$ & -54 & -127 & 37 & 0 & 0.07 & -0.11 & -0.02 & 032 & -0.587 & 4.4 & 26.0 & 180 & 17 & 045 & 1.0 & 180 \\
\hline $00: 43: 10$ & -54 & -126 & 38 & 0 & 0.12 & -0.08 & -0.02 & 032 & -0.401 & 2.0 & 26.0 & 180 & 20 & 045 & 1.0 & 180 \\
\hline $00: 43: 20$ & -54 & -125 & 38 & 0 & 0.18 & -0.13 & -0.02 & 032 & -0.696 & 4.2 & 49.0 & 180 & 21 & 045 & 1.0 & 180 \\
\hline $00: 43: 30$ & -54 & -124 & 38 & 0 & 0.19 & -0.14 & -0.03 & 032 & -0.649 & 6.0 & 49.0 & 180 & 21 & 045 & 1.0 & 180 \\
\hline $00: 43: 40$ & -54 & -123 & 38 & 0 & 0.21 & -0.13 & -0.05 & 032 & -0.526 & 6.6 & 49.0 & 180 & 25 & 045 & 1.0 & 180 \\
\hline $00: 43: 50$ & -54 & -122 & 38 & 0 & 0.24 & -0.16 & -0.07 & 032 & -0.571 & 8.1 & 76.0 & 180 & 22 & 045 & 1.0 & 180 \\
\hline $00: 14: 00$ & -53 & -121 & 36 & 0 & 0.24 & -0.17 & -0.10 & 032 & -0.492 & 9.5 & 99.0 & 180 & 19 & 045 & 1.0 & 180 \\
\hline $00: 44: 10$ & -53 & -119 & 33 & 0 & 0.20 & -0.13 & -0.12 & 032 & -0.042 & 8.1 & 99.0 & 180 & 20 & 045 & 1.0 & 180 \\
\hline $00: 44: 20$ & -54 & -118 & 43 & 0 & 0.19 & -0.16 & -0.13 & 032 & -0.158 & 8.6 & 51.0 & 180 & 22 & 045 & 1.0 & 180 \\
\hline $00: 44: 30$ & -54 & -117 & 44 & 0 & 0.23 & -0.18 & -0.16 & 032 & -0.097 & 8.9 & 77.0 & 180 & 15 & 045 & 1.0 & 180 \\
\hline $00: 44: 40$ & -54 & -115 & 44 & 0 & 0.23 & -0.18 & -0.19 & 032 & 0.066 & 8.2 & 77.0 & 180 & 16 & 045 & 1.0 & 180 \\
\hline $00: 44 ; 50$ & -54 & -114 & 44 & 0 & 0.24 & -0.14 & -0.20 & 032 & 0.372 & 6.4 & 77.0 & 180 & 16 & 045 & 1.0 & 180 \\
\hline $00: 45: 00$ & -54 & -112 & 43 & 0 & 0.27 & -0.10 & -0.21 & 032 & 0.684 & 4.2 & 77.0 & 180 & 15 & 045 & 1.0 & 180 \\
\hline $00: 45: 10$ & -54 & -111 & 43 & 0 & 0.31 & -0.09 & -0.20 & 032 & 0.717 & 2.0 & 77.0 & 180 & 15 & 045 & 1.0 & 180 \\
\hline $00: 45: 20$ & -54 & -109 & 38 & 0 & 0.33 & -0.08 & -0.18 & 032 & 0.603 & 0.2 & 77.0 & 180 & 22 & 045 & 1.0 & 180 \\
\hline $00: 45: 30$ & -53 & -107 & 38 & 0 & 0.32 & -0.09 & -0.15 & 032 & 0.370 & -0.8 & 100.0 & 180 & 18 & 045 & 1.0 & 180 \\
\hline $00: 45: 40$ & -53 & -105 & 38 & 0 & 0.31 & -0.13 & -0.13 & 032 & -0.002 & 0.0 & 100.0 & 180 & 18 & 045 & 1.0 & 180 \\
\hline $00: 45: 50$ & -53 & -104 & 38 & 0 & 0.28 & -0.15 & -0.11 & 032 & -0.260 & 1.5 & 100.0 & 180 & 17 & 045 & 1.0 & 180 \\
\hline $00: 46: 00$ & -52 & -102 & 38 & 0 & 0.31 & -0.17 & -0.11 & 032 & -0.397 & 3.1 & 76.0 & 180 & 17 & 045 & 1.0 & 180 \\
\hline $00: 46: 10$ & -52 & -100 & 38 & 0 & 0.30 & -0.16 & -0.11 & 032 & -0.349 & 4.1 & 100.0 & 180 & 13 & 045 & 1.0 & 180 \\
\hline $00: 46: 20$ & -52 & -99 & 29 & 0 & 0.28 & -0.15 & -0.11 & 032 & -0.242 & 4.9 & 100.0 & 180 & 21 & 045 & 1.0 & 180 \\
\hline $00: 46: 30$ & -52 & -97 & 29 & 0 & 0.24 & -0.19 & -0.12 & 032 & -0.452 & 6.3 & 100.0 & 180 & 20 & 045 & 1.0 & 180 \\
\hline $00: 46: 40$ & -52 & -96 & 29 & 0 & 0.18 & -0.24 & -0.13 & 032 & -0.663 & 9.2 & 100.0 & 180 & 20 & 045 & 1.0 & 180 \\
\hline $00: 46: 50$ & -53 & -95 & 29 & 0 & 0.15 & -0.19 & -0.15 & 032 & -0.251 & 9.2 & 0.0 & 180 & 21 & 045 & 1.0 & 180 \\
\hline $00: 47: 00$ & -53 & -93 & 41 & 0 & 0.17 & -0.22 & -0.17 & 032 & -0.310 & 9.9 & 0.0 & 180 & 23 & 045 & 1.0 & 180 \\
\hline $00: 47: 10$ & -53 & -92 & 56 & 0 & 0.26 & -0.19 & -0.21 & 032 & 0.151 & 8.5 & 0.0 & 180 & 16 & 045 & 1.0 & 180 \\
\hline $00: 47: 20$ & -53 & -90 & 34 & 0 & 0.32 & -0.10 & -0.23 & 032 & 0.805 & 5.3 & 0.0 & 180 & 20 & 045 & 1.0 & 180 \\
\hline $00: 47: 30$ & -53 & -88 & 34 & 0 & 0.35 & -0.10 & -0.22 & 032 & 0.783 & 2.6 & 0.0 & 180 & 22 & 045 & 1.0 & 180 \\
\hline $00: 47: 40$ & -53 & -86 & 34 & 0 & 0.38 & -0.09 & -0.21 & 032 & 0.713 & 0.7 & 0.0 & 180 & 21 & 045 & 1.0 & 180 \\
\hline $00: 47: 50$ & -52 & -84 & 34 & 0 & 0.41 & -0.14 & -0.19 & 032 & 0.296 & 0.3 & 0.0 & 180 & 18 & 045 & 1.0 & 180 \\
\hline $00: 48: 00$ & -52 & -82 & 34 & 0 & 0.42 & -0.15 & -0.16 & 032 & 0.066 & -9.4 & 0.0 & 180 & 22 & 045 & 1.0 & 180 \\
\hline $00: 48: 10$ & -51 & -80 & 34 & 0 & 0.46 & -0.20 & -0.08 & 032 & -0.743 & -28.4 & 0.0 & 180 & 16 & 045 & 1.0 & 180 \\
\hline $0: 48: 20$ & -51 & -77 & 34 & 0 & 0.48 & -0.25 & 0.04 & 032 & -1.834 & -35.0 & 0.0 & 180 & 17 & 045 & 1.0 & 180 \\
\hline $0: 48: 30$ & -50 & -75 & 34 & 0 & 0.52 & -0.35 & 0.15 & 031 & -3.144 & -35.0 & 0.0 & 180 & 18 & 045 & 1.0 & 180 \\
\hline $00: 48: 40$ & -49 & -72 & 34 & 0 & 0.55 & -0.42 & 0.25 & 031 & -4.250 & -35.0 & 0.0 & 180 & 18 & 045 & 1.0 & 180 \\
\hline $00: 48: 50$ & -48 & -69 & 34 & 0 & 0.55 & -0.52 & 0.35 & 030 & -5.472 & -35.0 & 0.0 & 180 & 16 & 045 & 1.0 & 180 \\
\hline
\end{tabular}




\section{Simulation Track Plot}

Open Ocean

Ship: 150,000 ton, Tanker, partial load

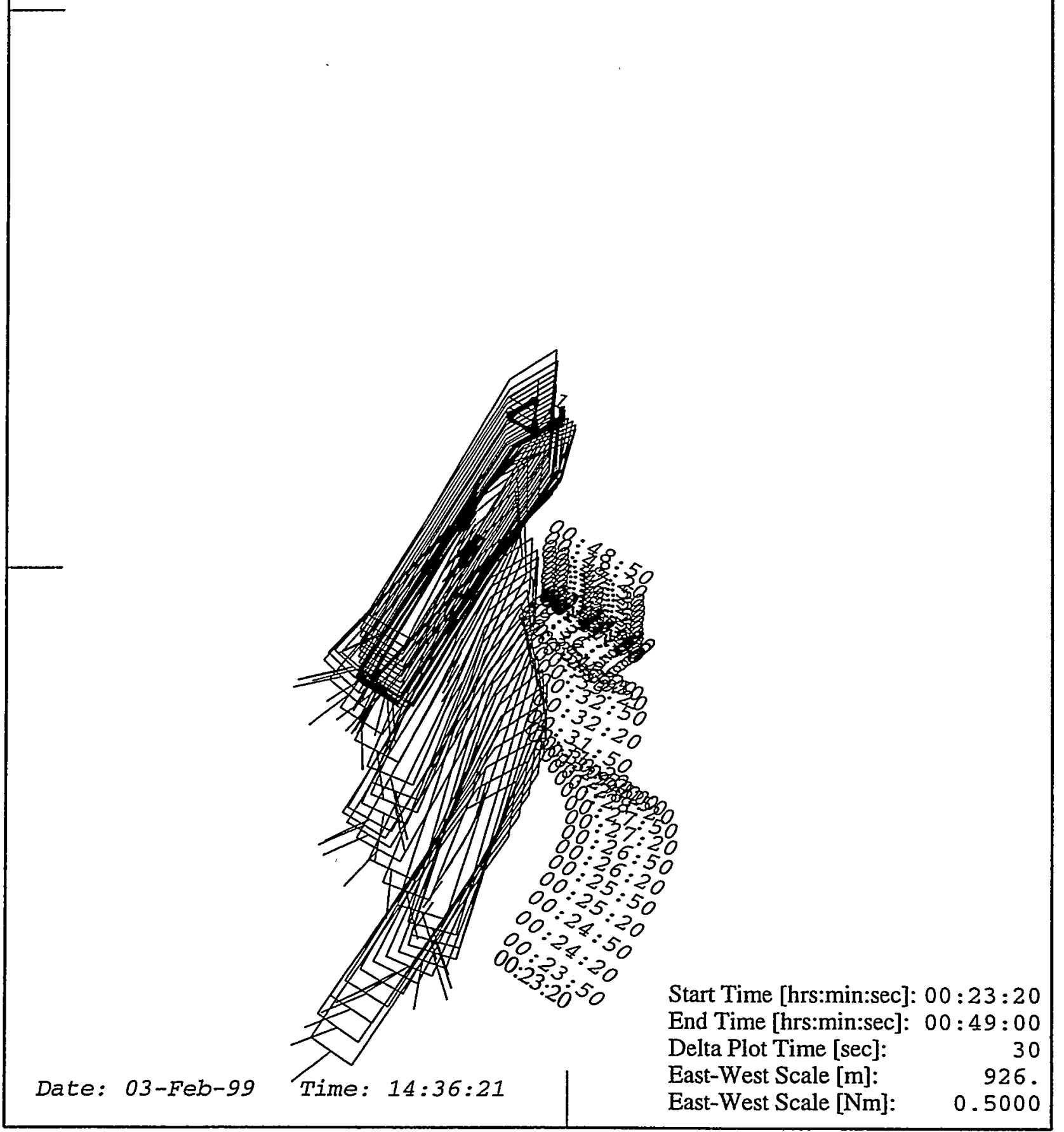




\section{HANDS-ON SIMULATION}

\section{SUCTION MOORNG TECHNOLOGY}

Simulation Number: 17

Date: 3 February 1999

Start Time: $15: 38$

Length of run [secs]: 2872

Ship Condition: draft $=13.72 \mathrm{~m}$

Environment

Wind Speed [knots]

20 Direction: 90

Sea State, Significant wave height [m]:..

3 Direction: 270

Current Speed [knots]:

1 Direction: 180

Ship Operator: J.A.

Observer: B.K.J \& J.K.

Final Mating, distance between center of buoy and center of moon pool [m]: 1.1

Evaluation:

On the final closure at very slow speed (to stop) on 070 degrees heading the ship closed with the buoy at a very slow rate. This seems to indicate that the equilibrium heading was probably between 072 to 075 degrees. The ship behaved very well. The buoy closed from beam on port side. 
Ship:

Exercise number:

Cenario

raffic ships:

Initial conditions:

Exercise identification
591150,000 ton, Tanker, partial load

754 Suction Hooring System

112 Open Ocean

999 Dummy tug file based on containership

590 Suction Mooring Buoy

$594150 k$ tanker in partial load - Suction Mooring RuN \pm 17

Time Position (m) RPM Speed (Knots) Turn Rudder Gill Wind

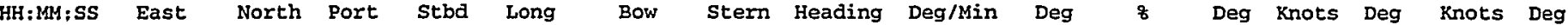

\begin{tabular}{|c|c|c|c|c|c|c|c|c|c|c|c|c|c|c|c|c|}
\hline $\begin{array}{l}00: 23: 20 \\
00: 23: 30 \\
00: 23: 40 \\
00: 23: 50\end{array}$ & $\begin{array}{l}-41 \\
-41 \\
-40 \\
-40\end{array}$ & $\begin{array}{l}-438 \\
-431 \\
-424 \\
-417\end{array}$ & $\begin{array}{l}29 \\
29 \\
29 \\
29\end{array}$ & $\begin{array}{l}0 \\
0 \\
0 \\
0\end{array}$ & $\begin{array}{l}1.23 \\
1.18 \\
1.12 \\
1.06\end{array}$ & $\begin{array}{l}-0.79 \\
-0.75 \\
-0.82 \\
-0.82\end{array}$ & $\begin{array}{l}-0.77 \\
-0.71 \\
-0.70 \\
-0.66\end{array}$ & $\begin{array}{l}036 \\
036 \\
036 \\
036\end{array}$ & $\begin{array}{l}-0.147 \\
-0.254 \\
-0.812 \\
-1.011\end{array}$ & $\begin{array}{l}-35.0 \\
-35.0 \\
-35.0 \\
-35.0\end{array}$ & $\begin{array}{l}100.0 \\
100.0 \\
100.0 \\
100.0\end{array}$ & $\begin{array}{l}180 \\
180 \\
180 \\
180\end{array}$ & $\begin{array}{l}10 \\
17 \\
19 \\
23\end{array}$ & $\begin{array}{l}090 \\
090 \\
090 \\
090\end{array}$ & $\begin{array}{l}1.0 \\
1.0 \\
1.0 \\
1.0\end{array}$ & $\begin{array}{l}180 \\
180 \\
180 \\
180\end{array}$ \\
\hline $00: 24: 00$ & -40 & -411 & 29 & 0 & 1.00 & -0.76 & -0.61 & 035 & -0.953 & -35.0 & 100.0 & 180 & 20 & 090 & 1.0 & 180 \\
\hline $00: 24: 10$ & -40 & -405 & 29 & 0 & 0.95 & -0.73 & -0.57 & 035 & -1.053 & -35.0 & 100.0 & 180 & 21 & 090 & 1.0 & 180 \\
\hline $00: 24: 20$ & -40 & -399 & 29 & 0 & 0.92 & -0.71 & -0.52 & 035 & -1.237 & -35.0 & 100.0 & 180 & 20 & 090 & 1.0 & 180 \\
\hline $00: 24: 30$ & -40 & -393 & 29 & 0 & 0.87 & -0.62 & -0.45 & 035 & -1.051 & -35.0 & 100.0 & 180 & 23 & 090 & 1.0 & 180 \\
\hline $00: 24: 40$ & -39 & -388 & 26 & 0 & 0.82 & -0.65 & -0.43 & 035 & -1.370 & -35.0 & 100.0 & 180 & 19 & 090 & 1.0 & 180 \\
\hline $00: 24: 50$ & -40 & -383 & 26 & 0 & 0.78 & -0.77 & -0.45 & 034 & -2.022 & -35.0 & 100.0 & 180 & 20 & 090 & 1.0 & 180 \\
\hline $00: 25: 00$ & -40 & -378 & 26 & 0 & 0.74 & -0.91 & -0.48 & 034 & -2.718 & -35.0 & 100.0 & 180 & 19 & 090 & 1.0 & 180 \\
\hline $00: 25: 10$ & -41 & -373 & 26 & 0 & 0.68 & -0.89 & -0.46 & 034 & -2.686 & -35.0 & 100.0 & 180 & 21 & 090 & 1.0 & 180 \\
\hline $00: 25: 20$ & -42 & -368 & 26 & 0 & 0.64 & -0.91 & -0.45 & 033 & -2.910 & -35.0 & 100.0 & 180 & 18 & 090 & 1.0 & 180 \\
\hline $00: 25: 30$ & -43 & -363 & 39 & 0 & 0.61 & -1.01 & -0.44 & 033 & -3.657 & -35.0 & 100.0 & 180 & 19 & 090 & 1.0 & 180 \\
\hline $00: 25: 40$ & -45 & -359 & 52 & 0 & 0.62 & -1.09 & -0.27 & 032 & -5.240 & -35.0 & 100.0 & 180 & 18 & 090 & 1.0 & 180 \\
\hline $00: 25: 50$ & -46 & -354 & 58 & 0 & 0.68 & -1.23 & -0.04 & 031 & -7.549 & -26.0 & 100.0 & 180 & 22 & 090 & 1.0 & 180 \\
\hline $00: 26: 00$ & -47 & -349 & 55 & 0 & 0.75 & -1.33 & -0.01 & 029 & -8.367 & -7.0 & 100.0 & 180 & 19 & 090 & 1.0 & 180 \\
\hline $00: 26: 10$ & -48 & -344 & 48 & 0 & 0.80 & -1.22 & -0.12 & 028 & -7.027 & 12.0 & 100.0 & 180 & 18 & 090 & 1.0 & 180 \\
\hline $00: 26: 20$ & -49 & -339 & 29 & 0 & 0.80 & -1.06 & -0.27 & 027 & -5.037 & 31.0 & 100.0 & 180 & 15 & 090 & 1.0 & 180 \\
\hline $00: 26: 30$ & -50 & -334 & 29 & 0 & 0.76 & -0.85 & -0.40 & 026 & -2.903 & 35.0 & 100.0 & 180 & 18 & 090 & 1.0 & 180 \\
\hline $00: 26: 40$ & -51 & -329 & 29 & 0 & 0.73 & -0.65 & -0.50 & 026 & -0.938 & 35.0 & 100.0 & 180 & 17 & 090 & 1.0 & 180 \\
\hline $00: 26: 50$ & -53 & -324 & 29 & 0 & 0.69 & -0.63 & -0.68 & 026 & 0.338 & 35.0 & 100.0 & 180 & 18 & 090 & 1.0 & 180 \\
\hline $00: 27: 00$ & -54 & -320 & 31 & 0 & 0.65 & -0.64 & -0.86 & 026 & 1.406 & 35.0 & 100.0 & 180 & 19 & 090 & 1.0 & 180 \\
\hline $00: 27: 10$ & -56 & -315 & 43 & 0 & 0.63 & -0.52 & -1.00 & 027 & 3.058 & 35.0 & 53.0 & 180 & 19 & 090 & 1.0 & 180 \\
\hline $00: 27: 20$ & -59 & -310 & 47 & 0 & 0.67 & -0.52 & -1.22 & 027 & 4. 481 & 26.4 & 53.0 & 180 & 17 & 090 & 1.0 & 180 \\
\hline $00: 27: 30$ & -61 & -305 & 55 & 0 & 0.73 & -0.50 & -1.31 & 028 & 5.166 & 12.2 & 53.0 & 180 & 18 & 090 & 1.0 & 180 \\
\hline $00: 27: 40$ & -63 & -299 & 58 & 0 & 0.82 & -0.46 & -1.45 & 029 & 6.255 & 31.2 & 53.0 & 180 & 17 & 090 & 1.0 & 180 \\
\hline $00: 27: 50$ & -65 & -293 & 57 & 0 & 0.86 & -0.24 & -1.62 & 030 & 8.761 & 35.0 & 53.0 & 180 & 18 & 090 & 1.0 & 180 \\
\hline $9: 28: 00$ & -67 & -286 & 54 & 0 & 0.91 & -0.25 & -1.82 & 032 & 9.986 & 35.0 & 53.0 & 180 & 22 & 090 & 1.0 & 180 \\
\hline$: 28: 10$ & -69 & -280 & 29 & 0 & 0.86 & -0.30 & -1.87 & 034 & 9.933 & 35.0 & 53.0 & 180 & 22 & 090 & 1.0 & 180 \\
\hline $00: 28: 20$ & -72 & -273 & 29 & 0 & 0.79 & -0.24 & -1.83 & 035 & 10.095 & 35.0 & 53.0 & 180 & 18 & 090 & 1.0 & 180 \\
\hline $00: 28: 30$ & -73 & -267 & 29 & 0 & 0.74 & -0.16 & -1.76 & 037 & 10.159 & 35.0 & 77.0 & 180 & 17 & 090 & 1.0 & 180 \\
\hline $00: 28: 40$ & -75 & -261 & 29 & 0 & 0.67 & -0.08 & -1.67 & 039 & 10.108 & 23.0 & 77.0 & 180 & 17 & 090 & 1.0 & 180 \\
\hline $00: 28: 50$ & -76 & -256 & 29 & 0 & 0.63 & -0.03 & -1.54 & 040 & 9.575 & 4.0 & 48.0 & 180 & 13 & 090 & 1.0 & 180 \\
\hline $00: 29: 00$ & -77 & -251 & 29 & 0 & 0.61 & -0.03 & -1.37 & 042 & 8.529 & -15.0 & 48.0 & 180 & 17 & 090 & 1.0 & 180 \\
\hline $00: 29: 10$ & -77 & -246 & 29 & 0 & 0.59 & -0.17 & -1.21 & 043 & 6.605 & -34.0 & 27.0 & 180 & 20 & 090 & 1.0 & 180 \\
\hline $00: 29: 20$ & -78 & -242 & 29 & 0 & 0.58 & -0.22 & -1.03 & 044 & 5.182 & -35.0 & 27.0 & 180 & 20 & 090 & 1.0 & 180 \\
\hline $00: 29: 30$ & -78 & -237 & 29 & 0 & 0.58 & -0.42 & -0.91 & 045 & 3.109 & -35.0 & 27.0 & 180 & 19 & 090 & 1.0 & 180 \\
\hline $00: 29: 40$ & -78 & -233 & 29 & 0 & 0.58 & -0.47 & -0.79 & 045 & 2.004 & -35.0 & 0.0 & 180 & 23 & 090 & 1.0 & 180 \\
\hline $00: 29: 50$ & -78 & -228 & 29 & 0 & 0.59 & -0.47 & -0.67 & 045 & 1.298 & -35.0 & 0.0 & 180 & 19 & 090 & 1.0 & 180 \\
\hline $00: 30: 00$ & -78 & -224 & 29 & 0 & 0.60 & -0.58 & -0.58 & 045 & 0.001 & -35.0 & 0.0 & 180 & 20 & 090 & 1.0 & 180 \\
\hline $00: 30: 10$ & -78 & -220 & 29 & 0 & 0.61 & -0.73 & -0.51 & 045 & -1.401 & -35.0 & 0.0 & 180 & 20 & 090 & 1.0 & 180 \\
\hline $00: 30: 20$ & -78 & -215 & 29 & 0 & 0.61 & -0.82 & -0.44 & 045 & -2.397 & -35.0 & 0.0 & 180 & 20 & 090 & 1.0 & 180 \\
\hline $00: 30: 30$ & -78 & -211 & 29 & 0 & 0.60 & -0.83 & -0.37 & 045 & -2.919 & -35.0 & 0.0 & 180 & 16 & 090 & 1.0 & 180 \\
\hline $00: 30: 40$ & -78 & -206 & 29 & 0 & 0.62 & -0.86 & -0.31 & 044 & -3.508 & -35.0 & 0.0 & 180 & 21 & 090 & 1.0 & 180 \\
\hline $00: 30: 50$ & -78 & -202 & 37 & 0 & 0.64 & -0.88 & -0.23 & 043 & -4.161 & -35.0 & 0.0 & 180 & 18 & 090 & 1.0 & 180 \\
\hline $00: 31: 00$ & -78 & -198 & 44 & 0 & 0.69 & -0.78 & -0.04 & 043 & -4.691 & -35.0 & 0.0 & 180 & 18 & 090 & 1.0 & 180 \\
\hline $00: 31: 10$ & -77 & -194 & 44 & 0 & 0.76 & -0.81 & 0.09 & 042 & -5.718 & -25.1 & 0.0 & 180 & 22 & 090 & 1.0 & 180 \\
\hline $00: 31: 20$ & -75 & -189 & 44 & 0 & 0.83 & -0.88 & 0.10 & 041 & -6.240 & -7.3 & 0.0 & 180 & 25 & 090 & 1.0 & 180 \\
\hline $00: 31: 30$ & -74 & -185 & 44 & 0 & 0.90 & -0.84 & 0.02 & 040 & -5.454 & 9.4 & 25.0 & 180 & 16 & 090 & 1.0 & 180 \\
\hline $00: 31: 40$ & -73 & -180 & 44 & 0 & 0.95 & -0.74 & -0.12 & 039 & -3.892 & 21.3 & 99.0 & 180 & 17 & 090 & 1.0 & 180 \\
\hline $00: 31: 50$ & -72 & -174 & 44 & 0 & 0.96 & -0.64 & -0.32 & 039 & -2.040 & 28.8 & 99.0 & 180 & 15 & 090 & 1.0 & 180 \\
\hline $00: 32 ; 00$ & -70 & -169 & 44 & 0 & 0.96 & -0.44 & -0.51 & 038 & 0.431 & 35.0 & 99.0 & 180 & 15 & 090 & 1.0 & 180 \\
\hline $00: 32: 10$ & -69 & -163 & 38 & 0 & 0.96 & -0.45 & -0.74 & 039 & 1.819 & 35.0 & 99.0 & 180 & 14 & 090 & 1.0 & 180 \\
\hline $00: 32: 20$ & -69 & -158 & 29 & 0 & 0.90 & -0.42 & -0.86 & 039 & 2.812 & 35.0 & 99.0 & 180 & 19 & 090 & 1.0 & 180 \\
\hline $00: 32: 30$ & -69 & -152 & 29 & 0 & 0.84 & -0.41 & -0.96 & 040 & 3.505 & 35.0 & 99.0 & 180 & 15 & 090 & 1.0 & 180 \\
\hline $00: 32: 40$ & -69 & -146 & 29 & 0 & 0.76 & -0.47 & -1.06 & 040 & 3.741 & 35.0 & 99.0 & 180 & 19 & 090 & 1.0 & 180 \\
\hline $00: 32: 50$ & -70 & -141 & 43 & 0 & 0.71 & -0.46 & -1.16 & 041 & 4.410 & 35.0 & 99.0 & 180 & 20 & 090 & 1.0 & 180 \\
\hline $00: 33: 00$ & -70 & -135 & 56 & 0 & 0.69 & -0.20 & -1.27 & 042 & 6.799 & 35.0 & 99.0 & 180 & 17 & 090 & 1.0 & 180 \\
\hline $00: 33: 10$ & -71 & -130 & 54 & 0 & 0.70 & -0.03 & -1.40 & 043 & 8.655 & 35.0 & 99.0 & 180 & 13 & 090 & 1.0 & 180 \\
\hline $00 ; 33: 20$ & -71 & -125 & 44 & 0 & 0.71 & 0.06 & -1.47 & 045 & 9.737 & 35.0 & 99.0 & 180 & 23 & 090 & 1.0 & 180 \\
\hline $00: 33: 30$ & -71 & -120 & 39 & 0 & 0.65 & 0.09 & -1.46 & 046 & 9.852 & 35.0 & 99.0 & 180 & 23 & 090 & 1.0 & 180 \\
\hline $00: 33: 40$ & -71 & -115 & 39 & 0 & 0.59 & 0.12 & -1.44 & 048 & 9.847 & 35.0 & 99.0 & 180 & 20 & 090 & 1.0 & 180 \\
\hline $00: 33: 50$ & -71 & $-i 11$ & 39 & 0 & 0.54 & 0.19 & -1.39 & 050 & 10.038 & 35.0 & 99.0 & 180 & 19 & 090 & 1.0 & 180 \\
\hline $00: 34: 00$ & -71 & -107 & 30 & 0 & 0.49 & 0.10 & -1.34 & 051 & 9.138 & 35.0 & 99.0 & 180 & 21 & 090 & 1.0 & 180 \\
\hline $00: 34: 10$ & -71 & -103 & 30 & 0 & 0.43 & 0.20 & -1.26 & 053 & 9.307 & 35.0 & 99.0 & 180 & 19 & 090 & 1.0 & 180 \\
\hline $00: 34: 20$ & -71 & -100 & 30 & 0 & 0.38 & 0.16 & -1.20 & 054 & 8.614 & 35.0 & 99.0 & 180 & 22 & 090 & 1.0 & 180 \\
\hline$: 34: 30$ & -71 & -96 & 30 & 0 & 0.32 & 0.10 & -1.14 & 056 & 7.871 & 35.0 & 99.0 & 180 & 20 & 090 & 1.0 & 180 \\
\hline$: 34: 40$ & -71 & -93 & 40 & 0 & 0.27 & 0.04 & -1.11 & 057 & 7.268 & 35.0 & 99.0 & 180 & 18 & 090 & 1.0 & 180 \\
\hline $00: 34: 50$ & -71 & -90 & 38 & 0 & 0.23 & -0.05 & -1.09 & 058 & 6.625 & 35.0 & 99.0 & 180 & 21 & 090 & 1.0 & 180 \\
\hline $00: 35: 00$ & -72 & -87 & 38 & 0 & 0.17 & -0.04 & -1.07 & 059 & 6.488 & 35.0 & 99.0 & 180 & 23 & 090 & 1.0 & 180 \\
\hline $00: 35: 10$ & -73 & -85 & 25 & 0 & 0.12 & 0.06 & -1.02 & 060 & 6.832 & 35.0 & 99.0 & 180 & 17 & 090 & 1.0 & 180 \\
\hline $00: 35: 20$ & -73 & -82 & 25 & 0 & 0.07 & 0.04 & -0.94 & 061 & 6.219 & 35.0 & 99.0 & 180 & 15 & 090 & 1.0 & 180 \\
\hline
\end{tabular}


Time Position (m) RPM Speed (Knots) Turn Rudder Gill wind Current HH:MM:SS East North Port Stbd Long Bow Stern Heading Deg/Min Deg \& Deg Knots Deg Knots Deg

\begin{tabular}{|c|c|c|c|c|c|c|c|c|c|c|c|c|c|c|c|c|}
\hline $0: 35: 30$ & -74 & -80 & 28 & 0 & 0.00 & 0.03 & -0.87 & 062 & 5.732 & 35.0 & 99.0 & 180 & 20 & 090 & 1.0 & 180 \\
\hline$: 35: 40$ & -75 & -79 & 28 & 0 & -0.06 & 0.13 & -0.81 & 063 & 5.922 & 35.0 & 99.0 & 180 & 19 & 090 & 1.0 & 180 \\
\hline $0: 35: 50$ & -77 & -77 & 28 & 0 & -0.13 & 0.10 & -0.74 & 064 & 5.353 & 35.0 & 99.0 & 180 & 15 & 090 & 1.0 & 180 \\
\hline $00: 36: 00$ & -78 & -76 & 28 & 0 & -0.16 & 0.06 & -0.69 & 065 & 4.749 & 35.0 & 99.0 & 180 & 13 & 090 & 1.0 & 180 \\
\hline $00: 36: 10$ & -79 & -75 & 28 & 0 & -0.21 & 0.13 & -0.63 & 066 & 4.825 & 35.0 & 99.0 & 180 & 14 & 090 & 1.0 & 180 \\
\hline $00: 36: 20$ & -81 & -75 & 28 & 0 & -0.26 & 0.16 & -0.58 & 067 & 4.684 & 35.0 & 99.0 & 180 & 16 & 090 & 1.0 & 180 \\
\hline $00: 36: 30$ & -83 & -74 & 28 & 0 & -0.28 & 0.19 & -0.53 & 067 & 4.560 & 35.0 & 99.0 & 180 & 21 & 090 & 1.0 & 180 \\
\hline $00: 36: 40$ & -84 & -74 & 28 & 0 & -0.31 & 0.09 & -0.49 & 068 & 3.678 & 35.0 & 99.0 & 180 & 19 & 090 & 1.0 & 180 \\
\hline $00: 36: 50$ & -86 & -74 & 28 & 0 & -0.37 & 0.06 & -0.44 & 069 & 3.224 & 21.7 & 99.0 & 180 & 20 & 090 & 1.0 & 180 \\
\hline $00: 37 ; 00$ & -89 & -73 & 28 & 0 & -0.41 & 0.02 & -0.43 & 069 & 2.863 & 8.9 & 99.0 & 180 & 22 & 090 & 1.0 & 180 \\
\hline $00: 37: 10$ & -91 & -73 & 28 & 0 & -0.47 & -0.03 & -0.40 & 070 & 2.361 & 3.0 & 99.0 & 180 & 22 & 090 & 1.0 & 180 \\
\hline $00: 37: 20$ & -94 & -73 & 43 & 0 & -0.45 & -0.03 & -0.36 & 070 & 2.113 & -3.3 & 0.0 & 180 & 14 & 090 & 1.0 & 180 \\
\hline $00: 37: 30$ & -96 & -73 & 49 & 0 & -0.37 & -0.02 & -0.30 & 070 & 1.793 & -9.2 & 0.0 & 180 & 15 & 090 & 1.0 & 180 \\
\hline $00 ; 37: 40$ & -98 & -73 & 53 & 0 & -0.28 & -0.09 & -0.15 & 070 & 0.352 & -9.4 & 0.0 & 180 & 18 & 090 & 1.0 & 180 \\
\hline $00: 37: 50$ & -99 & -73 & 54 & 0 & -0.18 & -0.14 & 0.00 & 070 & -0.857 & -1.8 & 0.0 & 180 & 23 & 090 & 1.0 & 180 \\
\hline $00: 38: 00$ & -100 & -73 & 59 & 0 & -0.04 & -0.19 & 0.03 & 070 & -1.382 & 15.0 & 0.0 & 180 & 20 & 090 & 1.0 & 180 \\
\hline $00: 38 ; 10$ & -100 & -72 & 62 & 0 & 0.09 & -0.16 & -0.01 & 070 & -0.910 & 9.4 & 0.0 & 180 & 20 & 090 & 1.0 & 180 \\
\hline $00: 38: 20$ & -99 & -72 & 52 & 0 & 0.22 & -0.06 & 0.01 & 070 & -0.412 & 4.5 & 0.0 & 180 & 18 & 090 & 1.0 & 180 \\
\hline $00: 38: 30$ & -98 & -71 & 34 & 0 & 0.27 & -0.06 & 0.04 & 070 & -0.621 & 6.0 & 50.0 & 180 & 22 & 090 & 1.0 & 180 \\
\hline $00: 38: 40$ & -97 & -70 & 34 & 0 & 0.31 & -0.05 & 0.05 & 070 & -0.651 & 8.2 & 50.0 & 180 & 19 & 090 & 1.0 & 180 \\
\hline $00: 38: 50$ & -95 & -70 & 34 & 0 & 0.34 & -0.05 & 0.06 & 070 & -0.659 & 9.6 & 50.0 & 180 & 18 & 090 & 1.0 & 180 \\
\hline $00: 39: 00$ & -93 & -69 & 34 & 0 & 0.34 & -0.05 & 0.06 & 069 & -0.708 & 12.0 & 50.0 & 180 & 16 & 090 & 1.0 & 180 \\
\hline $00: 39: 10$ & -92 & -69 & 26 & 0 & 0.36 & -0.14 & 0.06 & 069 & -1.238 & 16.4 & 73.0 & 180 & 21 & 090 & 1.0 & 180 \\
\hline $00: 39: 20$ & -90 & -68 & 26 & 0 & 0.33 & -0.15 & 0.05 & 069 & -1.247 & 20.7 & 73.0 & 180 & 18 & 090 & 1.0 & 180 \\
\hline $00: 39: 30$ & -89 & -67 & 26 & 0 & 0.29 & -0.15 & 0.03 & 069 & -1.177 & 23.1 & 73.0 & 180 & 18 & 090 & 1.0 & 180 \\
\hline $00: 39: 40$ & -87 & -66 & 26 & 0 & 0.27 & -0.11 & 0.04 & 069 & -0.920 & 4.1 & 73.0 & 180 & 16 & 090 & 1.0 & 180 \\
\hline $00: 39: 50$ & -86 & -65 & 26 & 0 & 0.24 & -0.14 & 0.07 & 069 & -1.347 & -3.2 & 100.0 & 180 & 22 & 090 & 1.0 & 180 \\
\hline $00: 40: 00$ & -85 & -65 & 26 & 0 & 0.19 & -0.17 & 0.10 & 068 & -1.724 & 5.5 & 100.0 & 180 & 22 & 090 & 1.0 & 180 \\
\hline $00: 40: 10$ & -85 & -64 & 26 & 0 & 0.13 & -0.18 & 0.11 & 068 & -1.818 & 16.0 & 100.0 & 180 & 22 & 090 & 1.0 & 180 \\
\hline $00: 40: 20$ & -84 & -64 & 26 & 0 & 0.05 & -0.17 & 0.09 & 068 & -1.649 & 33.4 & 100.0 & 180 & 16 & 090 & 1.0 & 180 \\
\hline $00: 40 ; 30$ & -84 & -64 & 26 & 0 & 0.00 & -0.08 & 0.06 & 067 & -0.907 & 35.0 & 100.0 & 180 & 19 & 090 & 1.0 & 180 \\
\hline $00: 40: 40$ & -84 & -64 & 26 & 0 & -0.03 & -0.06 & 0.03 & 067 & -0.611 & 35.0 & 70.0 & 180 & 19 & 090 & 1.0 & 180 \\
\hline $00: 40: 50$ & -84 & -64 & 26 & 0 & -0.03 & -0.01 & 0.02 & 067 & -0.166 & 22.7 & 53.0 & 180 & 20 & 090 & 1.0 & 180 \\
\hline $00: 41: 00$ & -85 & -64 & 26 & 0 & -0.03 & 0.05 & 0.03 & 067 & 0.153 & 9.1 & 27.0 & 180 & 17 & 090 & 1.0 & 180 \\
\hline $00: 41: 10$ & -85 & -64 & 26 & 0 & 0.00 & -0.05 & 0.04 & 067 & -0.580 & 26.4 & 27.0 & 180 & 19 & 090 & 1.0 & 180 \\
\hline $00: 41: 20$ & -85 & -64 & 26 & 0 & 0.00 & -0.10 & 0.02 & 067 & -0.772 & 35.0 & 27.0 & 180 & 16 & 090 & 1.0 & 180 \\
\hline $00 ; 41 ; 30$ & -85 & -64 & 26 & 0 & -0.01 & -0.11 & 0.00 & 067 & -0.663 & 31.9 & 51.0 & 180 & 18 & 090 & 1.0 & 180 \\
\hline $00: 41: 40$ & -85 & -64 & 26 & 0 & -0.03 & -0.09 & -0.01 & 067 & -0.464 & 28.5 & 51.0 & 180 & 15 & 090 & 1.0 & 180 \\
\hline $0: 41: 50$ & -85 & -63 & 26 & 0 & -0.01 & -0.15 & -0.01 & 067 & -0.906 & 12.7 & 51.0 & 180 & 14 & 090 & 1.0 & 180 \\
\hline$: 42: 00$ & -85 & -63 & 26 & 0 & -0.02 & -0.15 & 0.02 & 067 & -1.118 & 7.5 & 51.0 & 180 & 18 & 090 & 1.0 & 180 \\
\hline 10;42:10 & -86 & -63 & 26 & 0 & -0.04 & -0.18 & 0.04 & 066 & -1.433 & 13.4 & 26.0 & 180 & 23 & 090 & 1.0 & 180 \\
\hline $00: 42: 20$ & -86 & -62 & 26 & 0 & -0.04 & -0.21 & 0.05 & 066 & -1.695 & 18.3 & 26.0 & 180 & 20 & 090 & 1.0 & 180 \\
\hline $00: 42: 30$ & -86 & -62 & 26 & 0 & -0.05 & -0.24 & 0.05 & 066 & -1.847 & 22.6 & 26.0 & 180 & 17 & 090 & 1.0 & 180 \\
\hline $00: 42: 40$ & -87 & -62 & 26 & 0 & -0.06 & -0.23 & 0.07 & 066 & -1.886 & 3.6 & 26.0 & 180 & 19 & 090 & 1.0 & 180 \\
\hline $00: 42: 50$ & -87 & -62 & 29 & 0 & -0.04 & -0.16 & 0.10 & 065 & -1.633 & 12.2 & 26.0 & 180 & 18 & 090 & 1.0 & 180 \\
\hline $00: 43: 00$ & -87 & -62 & 34 & 0 & -0.01 & -0.11 & 0.07 & 065 & -1.150 & 31.2 & 26.0 & 180 & 17 & 090 & 1.0 & 180 \\
\hline $00 ; 43: 10$ & -87 & -62 & 34 & 0 & 0.02 & -0.02 & 0.01 & 065 & -0.172 & 28.4 & 26.0 & 180 & 20 & 090 & 1.0 & 180 \\
\hline $00: 43: 20$ & -87 & -61 & 28 & 0 & 0.05 & -0.10 & 0.01 & 065 & -0.671 & 9.3 & 26.0 & 180 & 21 & 090 & 1.0 & 180 \\
\hline $00: 43: 30$ & -87 & -61 & 28 & 0 & 0.05 & -0.14 & 0.03 & 065 & -1.101 & 5.1 & 26.0 & 180 & 21 & 090 & 1.0 & 180 \\
\hline $00: 43: 40$ & -87 & -61 & 28 & 0 & 0.06 & -0.16 & 0.05 & 065 & -1.309 & 10.2 & 26.0 & 180 & 25 & 090 & 1.0 & 180 \\
\hline $00: 43: 50$ & -87 & -60 & 28 & 0 & 0.08 & -0.24 & 0.07 & 064 & -1.944 & -3.4 & 26.0 & 180 & 22 & 090 & 1.0 & 180 \\
\hline $00 ; 44: 00$ & -87 & -60 & 28 & o & 0.10 & -0.32 & 0.12 & 064 & -2.793 & -10.1 & 26.0 & 180 & 19 & 090 & 1.0 & 180 \\
\hline $00: 44: 10$ & -86 & -59 & 28 & 0 & 0.10 & -0.28 & 0.15 & 063 & -2.711 & 2.2 & 26.0 & 180 & 20 & 090 & 1.0 & 180 \\
\hline $00 ; 44: 20$ & -86 & -58 & 28 & 0 & 0.11 & -0.36 & 0.16 & 063 & -3.310 & 9.7 & 26.0 & 180 & 22 & 090 & 1.0 & 180 \\
\hline $00: 44: 30$ & -86 & -57 & 25 & 0 & 0.11 & -0.42 & 0.15 & 062 & -3.664 & 19.2 & 26.0 & 180 & 15 & 090 & 2.0 & 180 \\
\hline $00 ; 44: 40$ & -86 & -57 & 25 & 0 & 0.09 & -0.43 & 0.13 & 062 & -3.592 & 30.0 & 50.0 & 180 & 16 & 090 & 1.0 & 180 \\
\hline $00: 44: 50$ & -86 & -56 & 26 & 0 & 0.06 & -0.37 & 0.09 & 061 & -2.954 & 35.0 & 50.0 & 180 & 16 & 090 & 1.0 & 180 \\
\hline $00: 45: 00$ & -86 & -55 & 33 & 0 & 0.07 & -0.26 & 0.03 & 061 & -1.843 & 35.0 & 50.0 & 180 & 15 & 090 & 1.0 & 180 \\
\hline $00: 45: 10$ & -86 & -54 & 26 & 0 & 0.09 & -0.19 & -0.03 & 061 & -0.971 & 35.0 & 50.0 & 180 & 15 & 090 & 1.0 & 180 \\
\hline $00: 45: 20$ & -86 & -54 & 25 & 0 & 0.08 & -0.12 & -0.07 & 060 & -0.369 & 32.9 & 50.0 & 180 & 22 & 090 & 1.0 & 180 \\
\hline $00: 45: 30$ & -86 & -53 & 25 & 0 & 0.07 & -0.10 & -0.09 & 060 & -0.064 & 29.8 & 50.0 & 180 & 18 & 090 & 3.0 & 180 \\
\hline $00: 45: 40$ & -85 & -52 & 25 & 0 & 0.07 & -0.13 & -0.11 & 060 & -0.093 & 30.6 & 50.0 & 180 & 18 & 090 & 1.0 & 180 \\
\hline $00: 45: 50$ & -86 & -52 & 25 & 0 & 0.05 & -0.16 & -0.13 & 060 & -0.163 & 31.0 & 62.0 & 180 & 17 & 090 & 1.0 & 180 \\
\hline $00: 46: 00$ & -86 & -51 & 25 & 0 & 0.05 & -0.20 & -0.16 & 060 & -0.268 & 32.7 & 76.0 & 180 & 17 & 090 & 1.0 & 180 \\
\hline $00: 46: 10$ & -86 & -50 & 25 & 0 & 0.01 & -0.16 & -0.17 & 060 & 0.094 & 30.3 & 76.0 & 180 & 13 & 090 & 1.0 & 180 \\
\hline $00: 46: 20$ & -86 & -49 & 25 & 0 & 0.00 & -0.15 & -0.18 & 060 & 0.230 & 28.7 & 76.0 & 180 & 21 & 090 & 1.0 & 180 \\
\hline $00: 46: 30$ & -87 & -48 & 25 & 0 & -0.02 & -0.23 & -0.20 & 060 & -0.215 & 35.0 & 76.0 & 180 & 20 & 090 & 1.0 & 180 \\
\hline $00: 46: 40$ & -88 & -47 & 25 & 0 & -0.06 & -0.34 & -0.22 & 060 & -0.739 & 35.0 & 76.0 & 180 & 20 & 090 & 1.0 & 180 \\
\hline $00: 46: 50$ & -89 & -46 & 25 & 0 & -0.12 & -0.26 & -0.23 & 060 & -0.164 & 35.0 & 1.0 & 180 & 21 & 090 & 1.0 & 180 \\
\hline $00: 47: 00$ & -90 & -46 & 25 & 0 & -0.12 & -0.33 & -0.24 & 060 & -0.558 & 35.0 & 0.0 & 180 & 23 & 090 & 1.0 & 180 \\
\hline $00: 47: 10$ & -91 & -45 & 25 & 0 & -0.12 & -0.30 & -0.25 & 060 & -0.333 & 35.0 & 0.0 & 180 & 16 & 090 & 1.0 & 180 \\
\hline $00: 47: 20$ & -93 & -44 & 25 & 0 & -0.12 & -0.16 & -0.26 & 060 & 0.596 & 35.0 & 0.0 & 180 & 20 & 090 & 1.0 & 180 \\
\hline $00: 47: 30$ & -94 & -43 & 0 & 0 & -0.12 & -0.15 & -0.26 & 060 & 0.690 & 35.0 & 0.0 & 180 & 22 & 090 & 1.0 & 180 \\
\hline $00: 47: 40$ & -95 & -43 & 0 & 0 & -0.13 & -0.12 & -0.24 & 060 & 0.759 & 35.0 & 0.0 & 180 & 21 & 090 & 1.0 & 180 \\
\hline $00: 47: 50$ & -96 & -42 & 0 & 0 & -0.15 & -0.20 & -0.23 & 060 & 0.183 & 17.4 & 0.0 & 180 & 18 & 090 & 1.0 & 180 \\
\hline
\end{tabular}


Open Ocean

\section{Simulation Track Plot}

Ship: 150,000 ton, Tanker, partial load

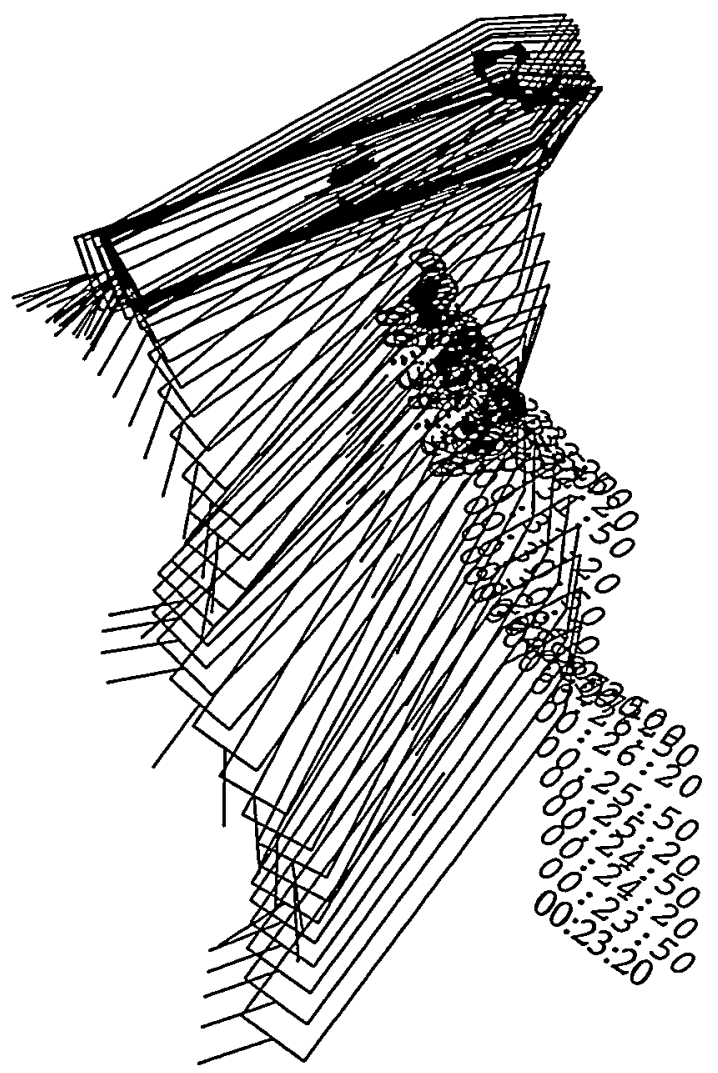

Start Time [hrs:min:sec]: $00: 23: 20$

End Time [hrs:min:sec]: $00: 47: 52$

Delta Plot Time [sec]:

East-West Scale [m]:

30

Date: 03-Feb-99

Time: 15:38:37

East-West Scale [Nm]:

926.

0.5000 


\section{HANDS-ON SIMULATION}

\section{SUCTION MOORNG TECHNOLOGY}

Simulation Number: 18

Date: 3 February 1999

Start Time: $16: 35$

Length of run [secs]: 2812

Ship Condition: draft $=13.72 \mathrm{~m}$

Environment

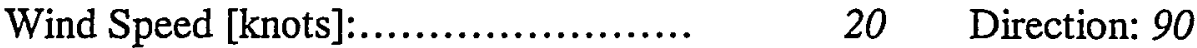

Sea State, Significant wave height $[\mathrm{m}]: . . \quad 3 \quad$ Direction: 270

Current Speed [knots]:.................. 1 Direction: 180

Ship Operator: J.A.

Observer: B.K.J \& J.K.

Final Mating, distance between center of buoy and center of moon pool [m]: 3.8

Evaluation:

A little too much throttle to move ahead and we missed by $3.8 \mathrm{~m}$.

The approach was very controlled. The ship handler had a lapse in discipline specifically in forgetting to maintain a balance of the thruster versus propeller. He momentarily forgot about the axial components of all forces, which were at cute angles. J.A. asked for a rerun, which we never made. 
Ship:

591 150,000 ton, Tanker, partial load

Exercise number:

754 Suction Mooring System

112 Open Ocean

(g)

999 Dummy tug file based on containership

raffic ships:

590 Suction Mooring Buoy

Initial conditions: $\quad 594$ 150k tanker in partial load - Suction Mooring

Exercise identification: $\quad$ Rus $\# 18$

Time Position (m) RPM Speed (Knots) Turn Rudder Gill Wind

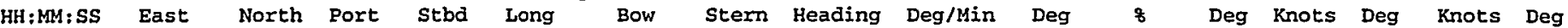

\begin{tabular}{|c|c|c|c|c|c|c|c|c|c|c|c|c|c|c|c|c|}
\hline $00: 23: 20$ & -68 & -276 & 25 & 0 & 0.55 & -0.42 & -1.29 & 038 & 5.521 & 3.4 & 50.0 & 180 & 10 & 090 & 1.0 & 180 \\
\hline $00: 23: 30$ & -70 & -271 & 25 & 0 & 0.53 & -0.38 & -1.24 & 039 & 5.478 & 22.4 & 50.0 & 180 & 17 & 090 & 1.0 & 180 \\
\hline $00: 23: 40$ & -71 & -266 & 25 & 0 & 0.48 & -0.42 & -1.26 & 040 & 5.321 & 35.0 & 76.0 & 180 & 19 & 090 & 1.0 & 280 \\
\hline $00: 23: 50$ & -73 & -262 & 25 & 0 & 0.42 & -0.38 & -1.26 & 041 & 5.555 & 35.0 & 76.0 & 180 & 23 & 090 & 1.0 & 180 \\
\hline $00: 24: 00$ & -75 & -257 & 25 & 0 & 0.37 & -0.30 & -1.24 & 042 & 6.000 & 35.0 & 76.0 & 180 & 20 & 090 & 1.0 & 180 \\
\hline $00: 24: 10$ & -76 & -253 & 25 & 0 & 0.32 & -0.23 & -1.21 & 043 & 6.223 & 35.0 & 76.0 & 180 & 21 & 090 & 1.0 & 180 \\
\hline $00: 24: 20$ & -78 & -250 & 25 & 0 & 0.30 & -0.19 & -1.18 & 044 & 6.322 & 35.0 & 0.0 & 180 & 20 & 090 & 1.0 & 180 \\
\hline $00: 24: 30$ & -79 & -246 & 28 & 0 & 0.29 & -0.06 & -1.14 & 045 & 6.841 & 35.0 & 0.0 & 180 & 23 & 090 & 1.0 & 180 \\
\hline $00: 24: 40$ & -80 & -243 & 41 & 0 & 0.30 & -0.05 & -1.14 & 046 & 6.916 & 35.0 & 0.0 & 180 & 19 & 090 & 1.0 & 180 \\
\hline $00: 24: 50$ & -81 & -240 & 43 & 0 & 0.35 & -0.10 & -1.19 & 048 & 6.912 & 32.7 & 0.0 & 180 & 20 & 090 & 1.0 & 180 \\
\hline $00: 25: 00$ & -82 & -236 & 44 & 0 & 0.39 & -0.21 & -1.18 & 049 & 6.141 & 14.5 & 0.0 & 180 & 19 & 090 & 1.0 & 180 \\
\hline $00: 25: 10$ & -83 & -232 & 44 & 0 & 0.42 & -0.22 & -1.07 & 050 & 5.401 & -1.6 & 24.0 & 180 & 21 & 090 & 1.0 & 180 \\
\hline $00: 25: 20$ & -83 & -228 & 44 & 0 & 0.48 & -0.31 & -0.90 & 050 & 3.757 & -13.8 & 51.0 & 180 & 18 & 090 & 1.0 & 180 \\
\hline $00: 25: 30$ & -83 & -224 & 44 & 0 & 0.52 & -0.46 & -0.73 & 051 & 1.719 & -18.4 & 51.0 & 180 & 19 & 090 & 1.0 & 180 \\
\hline $00: 25: 40$ & -83 & -220 & 44 & 0 & 0.55 & -0.52 & -0.56 & 051 & 0.257 & -19.6 & 51.0 & 180 & 18 & 090 & 1.0 & 180 \\
\hline $00: 25: 50$ & -82 & -216 & 44 & 0 & 0.59 & -0.58 & -0.42 & 051 & -1.013 & -16.5 & 76.0 & 180 & 22 & 090 & 1.0 & 180 \\
\hline $00: 26: 00$ & -81 & -212 & 44 & 0 & 0.60 & -0.70 & -0.34 & 051 & -2.275 & -9.6 & 76.0 & 180 & 19 & 090 & 1.0. & 180 \\
\hline $00: 26: 10$ & -81 & -208 & 44 & 0 & 0.63 & -0.68 & -0.29 & 050 & -2.460 & -2.0 & 76.0 & 180 & 18 & 090 & 1.0 & 180 \\
\hline $00: 26: 20$ & -80 & -204 & 44 & 0 & 0.65 & -0.61 & -0.28 & 050 & -2.099 & 4.8 & 100.0 & 180 & 15 & 090 & 1.0 & 180 \\
\hline $00: 26: 30$ & -79 & -200 & 33 & 0 & 0.62 & -0.48 & -0.29 & 050 & -1.198 & 8.7 & 100.0 & 180 & 18 & 090 & 1.0 & 180 \\
\hline $00: 26: 40$ & -77 & -197 & 33 & 0 & 0.61 & -0.34 & $-0.3 I$ & 050 & -0.230 & 8.9 & 100.0 & 180 & 17 & 090 & 1.0 & 180 \\
\hline $00: 26: 50$ & -76 & -194 & 33 & 0 & 0.58 & -0.36 & -0.35 & 050 & -0.105 & 20.5 & 100.0 & 180 & 18 & 090 & 1.0 & 180 \\
\hline $00: 27: 00$ & -75 & -190 & 33 & 0 & 0.56 & -0.39 & -0.44 & 050 & 0.343 & 35.0 & 100.0 & 180 & 19 & 090 & 1.0 & 180 \\
\hline $00: 27: 10$ & -74 & -187 & 33 & 0 & 0.50 & -0.30 & -0.53 & 050 & 1.455 & 35.0 & 100.0 & 180 & 19 & 090 & 1.0 & 180 \\
\hline $00: 27: 20$ & -74 & -183 & 33 & 0 & 0.47 & -0.34 & -0.61 & 050 & 1.740 & 35.0 & 100.0 & 180 & 17 & 090 & 1.0 & 180 \\
\hline $00: 27: 30$ & -74 & -180 & 33 & 0 & 0.43 & -0.31 & -0.68 & 050 & 2.333 & 35.0 & 100.0 & 180 & 18 & 090 & 1.0 & 180 \\
\hline $00: 27: 40$ & -74 & -177 & 33 & 0 & 0.40 & -0.31 & -0.74 & 051 & 2.708 & 35.0 & 100.0 & 180 & 17 & 090 & 1.0 & 180 \\
\hline $00: 27: 50$ & -74 & -173 & 33 & 0 & 0.34 & -0.19 & -0.78 & 051 & 3.703 & 35.0 & 76.0 & 180 & 18 & 090 & 1.0 & 180 \\
\hline $00: 28: 00$ & -74 & -170 & 33 & 0 & 0.33 & -0.28 & -0.82 & 052 & 3.423 & 35.0 & 52.0 & 180 & 22 & 090 & 1.0 & 180 \\
\hline $0: 28: 10$ & -75 & -167 & 33 & 0 & 0.32 & -0.33 & -0.86 & 052 & 3.374 & 35.0 & 27.0 & 180 & 22 & 090 & 1.0 & 180 \\
\hline $0: 28: 20$ & -75 & -163 & 33 & 0 & 0.29 & -0.26 & -0.88 & 053 & 3.955 & 35.0 & 27.0 & 180 & 18 & 090 & 1.0 & 180 \\
\hline $00: 28: 30$ & -76 & -160 & 33 & 0 & 0.30 & -0.17 & -0.89 & 054 & 4.536 & 35.0 & 27.0 & 180 & 17 & 090 & 1.0 & 180 \\
\hline $00: 28: 40$ & -76 & -157 & 33 & 0 & 0.31 & -0.08 & -0.89 & 055 & 5.137 & 35.0 & 27.0 & 180 & 17 & 090 & 1.0 & 180 \\
\hline $00: 28: 50$ & -76 & -154 & 33 & 0 & 0.32 & 0.02 & -0.88 & 055 & 5.712 & 35.0 & 27.0 & 180 & 13 & 090 & 1.0 & 180 \\
\hline $00: 29: 00$ & -76 & -152 & 33 & 0 & 0.34 & 0.08 & -0.85 & 056 & 5.840 & 16.5 & 27.0 & 180 & 17 & 090 & 1.0 & 180 \\
\hline $00: 29: 10$ & -76 & -149 & 33 & 0 & 0.35 & -0.01 & -0.76 & 057 & 4.790 & -2.5 & 27.0 & 180 & 20 & 090 & 1.0 & 180 \\
\hline $00: 29: 20$ & -75 & -147 & 33 & 0 & 0.36 & -0.04 & -0.63 & 058 & 3.764 & -21.5 & 27.0 & 180 & 20 & 090 & 1.0 & 180 \\
\hline $00: 29: 30$ & -74 & -144 & 33 & 0 & 0.38 & -0.23 & -0.47 & 058 & 1.494 & -35.0 & 27.0 & 180 & 19 & 090 & 1.0 & 180 \\
\hline $00: 29: 40$ & -74 & -142 & 33 & 0 & 0.36 & -0.31 & -0.31 & 059 & 0.002 & -35.0 & 100.0 & 180 & 23 & 090 & 1.0 & 180 \\
\hline $00: 29: 50$ & -73 & -139 & 26 & 0 & 0.31 & -0.32 & -0.21 & 059 & -0.704 & -35.0 & 100.0 & 180 & 19 & 090 & 1.0 & 180 \\
\hline $00: 30: 00$ & -72 & -137 & 26 & 0 & 0.27 & -0.43 & -0.12 & 058 & -1.954 & -35.0 & 100.0 & 180 & 20 & 090 & 1.0 & 180 \\
\hline $00: 30: 10$ & -72 & -136 & 26 & 0 & 0.22 & -0.57 & -0.03 & 058 & -3.388 & -35.0 & 100.0 & 180 & 20 & 090 & 1.0 & 180 \\
\hline $00: 30: 20$ & -72 & -134 & 26 & 0 & 0.16 & -0.66 & 0.04 & 057 & -4.455 & -24.4 & 100.0 & 180 & 20 & 090 & 1.0 & 180 \\
\hline $00: 30: 30$ & -72 & -132 & 26 & 0 & 0.09 & -0.67 & 0.09 & 056 & -4.803 & -7.1 & 100.0 & 180 & 16 & 090 & 1.0 & 180 \\
\hline $00: 30: 40$ & -73 & -131 & 26 & 0 & 0.05 & -0.68 & 0.10 & 056 & -4.962 & 9.3 & 100.0 & 180 & 21 & 090 & 1.0 & 180 \\
\hline $00: 30: 50$ & -74 & -129 & 26 & 0 & 0.01 & -0.66 & 0.09 & 055 & -4.737 & 23.4 & 100.0 & 180 & 18 & 090 & 1.0 & 180 \\
\hline $00: 31: 00$ & -74 & -128 & 26 & 0 & -0.02 & -0.48 & 0.06 & 054 & -3.402 & 35.0 & 35.0 & 180 & 18 & 090 & 1.0 & 180 \\
\hline $00: 31: 10$ & -75 & -127 & 26 & 0 & 0.00 & -0.42 & 0.00 & 054 & -2.665 & 35.0 & 26.0 & 180 & 22 & 090 & 1.0 & 180 \\
\hline $00: 31: 20$ & -76 & -127 & 26 & 0 & 0.01 & -0.45 & -0.06 & 053 & -2.487 & 35.0 & 26.0 & 180 & 25 & 090 & 1.0 & 180 \\
\hline $00: 31: 30$ & -77 & -125 & 26 & 0 & 0.01 & -0.44 & -0.11 & 053 & -2.075 & 35.0 & 26.0 & 180 & 16 & 090 & 1.0 & 180 \\
\hline $00: 31: 40$ & -77 & -124 & 26 & 0 & 0.01 & -0.41 & -0.15 & 052 & -1.615 & 35.0 & 26.0 & 180 & 17 & 090 & 1.0 & 180 \\
\hline $00: 31: 50$ & -78 & -123 & 26 & 0 & 0.03 & -0.41 & -0.20 & 052 & -1.317 & 35.0 & 26.0 & 180 & 15 & 090 & 1.0 & 180 \\
\hline $00: 32: 00$ & -79 & -122 & 26 & 0 & 0.04 & -0.33 & -0.24 & 052 & -0.570 & 35.0 & 26.0 & 180 & 15 & 090 & 1.0 & 180 \\
\hline $00: 32: 10$ & -80 & -120 & 26 & 0 & 0.06 & -0.42 & -0.28 & 052 & -0.841 & 35.0 & 26.0 & 180 & 14 & 090 & 1.0 & 180 \\
\hline $00: 32: 20$ & -81 & -119 & 26 & 0 & 0.05 & -0.42 & -0.33 & 052 & -0.626 & 35.0 & 26.0 & 180 & 19 & 090 & 1.0 & 180 \\
\hline $00: 32: 30$ & -82 & -117 & 26 & 0 & 0.05 & -0.45 & -0.37 & 052 & -0.534 & 35.0 & 26.0 & 180 & 15 & 090 & 1.0 & 180 \\
\hline $00: 32: 40$ & -83 & -115 & 26 & 0 & 0.04 & -0.54 & -0.41 & 052 & -0.816 & 35.0 & 0.0 & 180 & 19 & 090 & 1.0 & 180 \\
\hline $00: 32: 50$ & -84 & -113 & 26 & 0 & 0.05 & -0.57 & -0.44 & 051 & -0.785 & 35.0 & 0.0 & 180 & 20 & 090 & 1.0 & 180 \\
\hline $00: 33: 00$ & -86 & -111 & 32 & 0 & 0.04 & -0.41 & -0.46 & 051 & 0.368 & 35.0 & 0.0 & 180 & 17 & 090 & 1.0 & 180 \\
\hline $00: 33: 10$ & -87 & -109 & 44 & 0 & 0.07 & -0.29 & -0.54 & 052 & 1.583 & 35.0 & 50.0 & 180 & 13 & 090 & 1.0 & 180 \\
\hline $00: 33: 20$ & -88 & -107 & 45 & 0 & 0.13 & -0.20 & -0.66 & 052 & 2.901 & 35.0 & 50.0 & 180 & 23 & 090 & 1.0 & 180 \\
\hline $00: 33: 30$ & -89 & -105 & 51 & 0 & 0.17 & -0.12 & -0.78 & 053 & 4.211 & 35.0 & 50.0 & 180 & 23 & 090 & 1.0 & 180 \\
\hline $00: 33: 40$ & -89 & -102 & 49 & 0 & 0.21 & -0.03 & -0.89 & 053 & 5.480 & 35.0 & 50.0 & 180 & 20 & 090 & 1.0 & 180 \\
\hline $00: 33: 50$ & -90 & -100 & 49 & 0 & 0.25 & 0.10 & -0.97 & 054 & 6.787 & 35.0 & 75.0 & 180 & 19 & 090 & 1.0 & 180 \\
\hline $00: 34: 00$ & -90 & -97 & 50 & 0 & 0.30 & 0.08 & -1.04 & 056 & 7.121 & 35.0 & 53.0 & 180 & 21 & 090 & 1.0 & 180 \\
\hline $00: 34: 10$ & -90 & -94 & 49 & 0 & 0.35 & 0.24 & -1.09 & 057 & 8.438 & 35.0 & 53.0 & 180 & 19 & 090 & 1.0 & 180 \\
\hline $00: 34: 20$ & -89 & -91 & 50 & 0 & 0.40 & 0.26 & -1.12 & 058 & 8.792 & 35.0 & 74.0 & 180 & 22 & 090 & 1.0 & 180 \\
\hline $0: 34: 30$ & -89 & -88 & 49 & 0 & 0.43 & 0.27 & -1.15 & 060 & 8.973 & 35.0 & 74.0 & 180 & 20 & 090 & 1.0 & 180 \\
\hline$: 34: 40$ & -88 & -85 & 50 & 0 & 0.45 & 0.25 & -1.17 & 061 & 8.996 & 35.0 & 74.0 & 180 & 18 & 090 & 1.0 & 180 \\
\hline $00: 34: 50$ & -87 & -82 & 37 & 0 & 0.45 & 0.19 & -1.16 & 063 & 8.554 & 35.0 & 74.0 & 180 & 21 & 090 & 1.0 & 180 \\
\hline $00: 35: 00$ & -86 & -79 & 29 & 0 & 0.37 & 0.17 & -1.09 & 064 & 8.022 & 35.0 & 100.0 & 180 & 23 & 090 & 1.0 & 180 \\
\hline $00: 35: 10$ & -85 & -76 & 29 & 0 & 0.30 & 0.25 & -1.01 & 065 & 7.985 & 35.0 & 100.0 & 180 & 17 & 090 & 1.0 & 180 \\
\hline $00: 35: 20$ & -85 & -74 & 29 & 0 & 0.25 & 0.23 & -0.93 & 067 & 7.414 & 35.0 & 100.0 & 180 & 15 & 090 & 1.0 & 180 \\
\hline
\end{tabular}




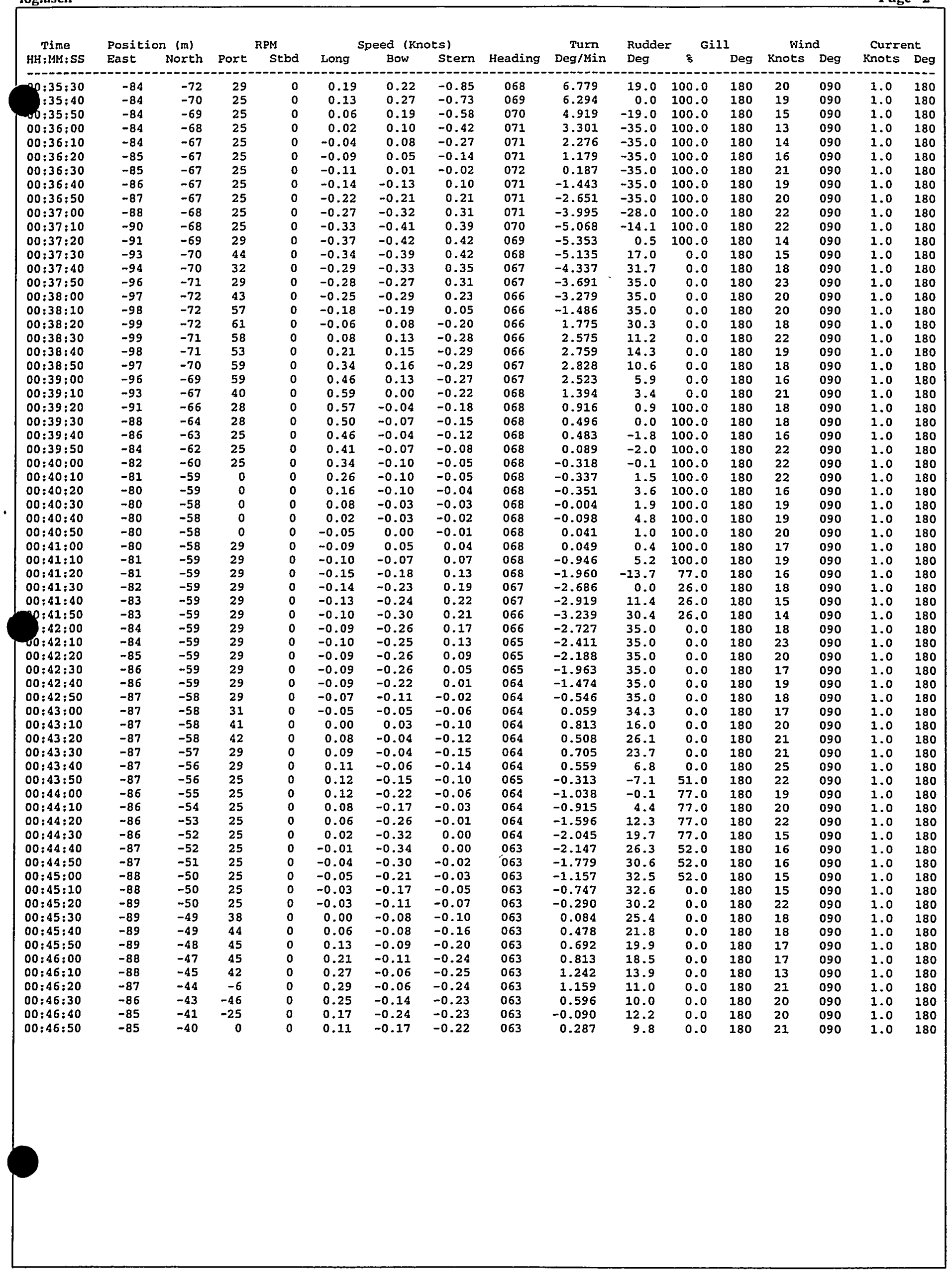


Open Ocean

\section{Simulation Track Plot}

Ship: 150,000 ton, Tanker, partial load

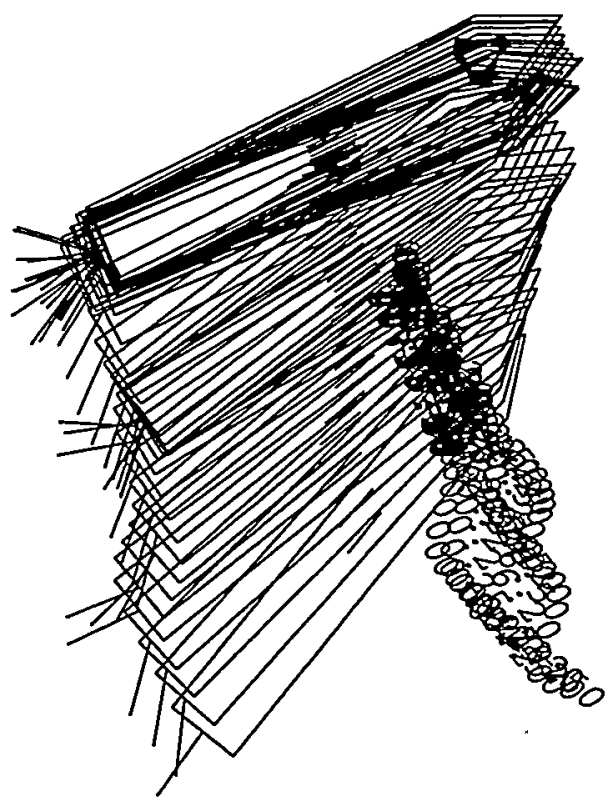

Start Time [hrs:min:sec]: $00: 23: 20$

End Time [hrs:min:sec]: $00: 46: 52$

Delta Plot Time [sec]:

30

East-West Scale [m]:

926.

Date: 03-Feb-99 Time: 16:35:37

East-West Scale [Nm]:

0.5000 


\section{HANDS-ON SIMULATION}

\section{SUCTION MOORNG TECHNOLOGY}

Simulation Number: 19

Date: 4 February 1999

Start Time: 08:00

Length of run [secs]: 2205

Ship Condition: $\mathrm{draft}=13.72 \mathrm{~m}$

Environment

Wind Speed [knots]:....................

25 Direction: 0

Sea State, Significant wave height $[\mathrm{m}]: .$.

5 Direction: 180

Current Speed [knots]:

0 Direction: 0

Ship Operator: J.A.

Observer: B.K.J \& J.K.

Final Mating, distance between center of buoy and center of moon pool [m]: 2.6

Evaluation:

Deceleration and tracking were good up to the point where the buoy came into camera view. When the buoy was in camera view, the ground head speed was about 0.2 knots. At first the buoy was tracking apparently straight aft. Then the buoy began to trend just $a$ little to starboard. The captain started to change the autopilot heading to the right up to 005 degrees but it was not enough. The buoy ended up a little aft and to the starboard, just beyond the limit. It appears that higher rudder rate would help the problem. A high lift rudder would also be very beneficial. 
Ship

Exercise number:

591150,000 ton, Tanker, partial load

Ecenario:

g file:

755 Suction Mooring System

112 Open Ocean

999 Dumny tug file based on containership

affic ships:

590 Suction Mooring Buoy

Initial conditions: 595 150k tanker in partial load - suction Mooring

Exercise identification: RuN \pm 19

Time Position (m) RPM Speed (Knots) Turn Rudder Gill Wind

\begin{tabular}{|c|c|c|c|c|c|c|c|c|c|c|c|c|c|c|c|c|}
\hline Hн;MM: SS & East & North & Port & stbd & Long & Bow & stern & Heading & Deg/Min & Deg & 8 & Deg & Knots & Deg & Knots & Deg \\
\hline & & & & & & & & & & & & & & & & \\
\hline $00: 16: 40$ & 0 & -590 & 28 & 0 & 1.76 & -0.02 & 0.04 & 000 & -0.351 & -6.6 & 0.0 & $\begin{array}{l}180 \\
180\end{array}$ & $\begin{array}{l}32 \\
21\end{array}$ & $\begin{array}{l}000 \\
000\end{array}$ & $\begin{array}{l}0.0 \\
0.0\end{array}$ & $\begin{array}{l}180 \\
180\end{array}$ \\
\hline $00: 16: 50$ & 0 & -581 & 28 & 0 & 1.70 & -0.02 & 0.05 & 000 & -0.462 & $\begin{array}{l}-5.3 \\
-4.1\end{array}$ & 0.0 & $\begin{array}{l}180 \\
180\end{array}$ & 21 & $\begin{array}{l}000 \\
000\end{array}$ & $\begin{array}{l}0.0 \\
0.0\end{array}$ & $\begin{array}{l}180 \\
180\end{array}$ \\
\hline $00: 17: 00$ & 0 & -572 & 28 & 0 & 1.74 & -0.02 & 0.06 & 000 & -0.532 & $\begin{array}{l}-4.1 \\
-2.4\end{array}$ & 0.0 & $\begin{array}{l}180 \\
180\end{array}$ & $\begin{array}{l}29 \\
25\end{array}$ & $\begin{array}{l}000 \\
000\end{array}$ & $\begin{array}{l}0.0 \\
0.0\end{array}$ & $\begin{array}{l}180 \\
180\end{array}$ \\
\hline $00: 17: 10$ & 0 & -563 & 37 & 0 & 1.74 & -0.02 & 0.07 & 000 & & -2.4 & 0.0 & 180 & 25 & 000 & 0.0 & 180 \\
\hline $00: 17: 20$ & 0 & -555 & 41 & 0 & 1.71 & -0.02 & 0.08 & 000 & -0.633 & -0.1 & 0.0 & 180 & 22 & 000 & 0.0 & 180 \\
\hline $00: 17: 30$ & 1 & -546 & 42 & 0 & 1.79 & -0.02 & 0.07 & 360 & -0.579 & 1.5 & 0.0 & 180 & 24 & 000 & 0.0 & 180 \\
\hline $00: 17: 40$ & 1 & -536 & 42 & 0 & 1.83 & -0.01 & 0.06 & 360 & -0.458 & 3.1 & 0.0 & 180 & 16 & 000 & 0.0 & 180 \\
\hline $00: 17: 50$ & 1 & -527 & 33 & 0 & 1.84 & 0.00 & 0.05 & 360 & -0.325 & 4.1 & 0.0 & 180 & 25 & 000 & 0.0 & 180 \\
\hline $00: 18 ; 00$ & 1 & -517 & 33 & 0 & 1.73 & 0.00 & 0.03 & 360 & -0.204 & 4.6 & 0.0 & 180 & 26 & 000 & 0.0 & 180 \\
\hline $00: 18: 10$ & 1 & -509 & 33 & 0 & 1.62 & 0.01 & 0.02 & 360 & -0.047 & 5.1 & 0.0 & 180 & 20 & 000 & 0.0 & 180 \\
\hline $00: 18: 20$ & 1 & -501 & 33 & 0 & 1.59 & 0.01 & 0.00 & 360 & 0.067 & 5.0 & 0.0 & 180 & 23 & 000 & 0.0 & 180 \\
\hline $00: 18: 30$ & 1 & -492 & 33 & 0 & 1.57 & 0.02 & -0.01 & 360 & 0.171 & 1.5 & 0.0 & 180 & 20 & 000 & 0.0 & 180 \\
\hline $00: 18: 40$ & 1 & -484 & 33 & 0 & 1.56 & 0.00 & 0.02 & 360 & -0.107 & -13.0 & 0.0 & 180 & 13 & 000 & 0.0 & 180 \\
\hline $00: 18: 50$ & 1 & -477 & 33 & 0 & 1.52 & 0.00 & 0.03 & 360 & -0.199 & 5.2 & 0.0 & 180 & 25 & 000 & 0.0 & 180 \\
\hline $00: 29: 00$ & 1 & -469 & 39 & 0 & 1.53 & 0.01 & 0.01 & 360 & -0.035 & 5.7 & 0.0 & 180 & 23 & 000 & 0.0 & 180 \\
\hline $00: 19: 10$ & 1 & -461 & 39 & 0 & 1.54 & 0.02 & -0.01 & 360 & 0.176 & 4.9 & 0.0 & 180 & 28 & 000 & 0.0 & 180 \\
\hline $00: 19: 20$ & 1 & -453 & 40 & 0 & 1.59 & 0.00 & 0.01 & 360 & -0.011 & -12.2 & 0.0 & 180 & 27 & 000 & 0.0 & 180 \\
\hline $00: 19: 30$ & 1 & -444 & 40 & 0 & 1.59 & -0.04 & 0.06 & 360 & -0.626 & -11.9 & 0.0 & 180 & 23 & 000 & 0.0 & 180 \\
\hline $00: 19: 40$ & 1 & -436 & 40 & 0 & 1.55 & -0.05 & 0.08 & 360 & -0.804 & 4.8 & 0.0 & 180 & 28 & 000 & 0.0 & 180 \\
\hline $00 ; 19: 50$ & 1 & -428 & 40 & 0 & 1.58 & -0.02 & 0.04 & 359 & -0.387 & 9.9 & 0.0 & 180 & 24 & 000 & 0.0 & 180 \\
\hline $00: 20: 00$ & 1 & -420 & 40 & 0 & 1.66 & 0.00 & -0.01 & 359 & 0.071 & 10.1 & 0.0 & 180 & 23 & 000 & 0.0 & 180 \\
\hline $00: 20: 10$ & 1 & -411 & 40 & 0 & 1.66 & 0.02 & -0.05 & 359 & 0.461 & 9.2 & 0.0 & 180 & 24 & 000 & 0.0 & 180 \\
\hline $00 ; 20 ; 20$ & 1 & -403 & 40 & 0 & 1.73 & 0.04 & -0.08 & 360 & 0.737 & 7.4 & 0.0 & 180 & 26 & 000 & 0.0 & 180 \\
\hline $00: 20 ; 30$ & 0 & -394 & 40 & 0 & 1.78 & 0.04 & -0.10 & 360 & 0.908 & 4.5 & 0.0 & 180 & 20 & 000 & 0.0 & 180 \\
\hline $00: 20: 40$ & 0 & -385 & 40 & 0 & 1.74 & 0.04 & -0.11 & 360 & 0.954 & 1.6 & 0.0 & 180 & 27 & 000 & 0.0 & 180 \\
\hline $00: 20: 50$ & 0 & -376 & 39 & 0 & 1.69 & 0.03 & -0.10 & 000 & 0.879 & -1.2 & 0.0 & 180 & 25 & 000 & 0.0 & 180 \\
\hline $00: 21: 00$ & 0 & -367 & 40 & 0 & 1.65 & 0.02 & -0.09 & 000 & 0.710 & -3.3 & 0.0 & 180 & 19 & 000 & 0.0 & 180 \\
\hline $00: 21: 10$ & 0 & -359 & 40 & 0 & 1.67 & 0.01 & -0.07 & 000 & 0.482 & -4.8 & 0.0 & 180 & 22 & 000 & 0.0 & 180 \\
\hline $90: 21: 20$ & 0 & -350 & 40 & 0 & 1.63 & 0.00 & -0.04 & 000 & 0.214 & -5.8 & 50.0 & 180 & 21 & 000 & 0.0 & 180 \\
\hline$: 21: 30$ & 0 & -342 & 40 & 0 & 1.54 & -0.02 & -0.01 & 000 & -0.093 & -5.6 & 50.0 & 180 & 24 & 000 & 0.0 & 180 \\
\hline$-0: 21: 40$ & 0 & -334 & 39 & 0 & 1.54 & -0.04 & 0.02 & 000 & -0.344 & -4.8 & 75.0 & 180 & 25 & 000 & 0.0 & 180 \\
\hline $00: 21: 50$ & 0 & -326 & 40 & 0 & 1.56 & -0.04 & 0.04 & 000 & -0.509 & -3.6 & 75.0 & 180 & 19 & 000 & 0.0 & 180 \\
\hline $00: 22: 00$ & 0 & -318 & 40 & 0 & 1.54 & -0.05 & 0.05 & 000 & -0.589 & -1.8 & 100.0 & 180 & 23 & 000 & 0.0 & 180 \\
\hline $00: 22: 10$ & 0 & -311 & 38 & 0 & 1.42 & -0.05 & 0.05 & 000 & -0.599 & -0.1 & 100.0 & 180 & 25 & 000 & 0.0 & 180 \\
\hline $00: 22: 20$ & 0 & -303 & 35 & 0 & 1.37 & -0.04 & 0.04 & 360 & -0.540 & 1.6 & 100.0 & 180 & 19 & 000 & 0.0 & 180 \\
\hline $00: 22: 30$ & 0 & -297 & 35 & 0 & 1.28 & -0.03 & 0.04 & 360 & -0.438 & 3.2 & 100.0 & 180 & 33 & 000 & 0.0 & 180 \\
\hline $00 ; 22 ; 40$ & 0 & -290 & 35 & 0 & 1.25 & -0.03 & 0.02 & 360 & -0.306 & 4.2 & 100.0 & 180 & 23 & 000 & 0.0 & 180 \\
\hline $00: 22: 50$ & -1 & -284 & 35 & 0 & 1.25 & -0.02 & 0.01 & 360 & -0.157 & 4.7 & 100.0 & 180 & 19 & 000 & 0.0 & 180 \\
\hline $00: 23: 00$ & -1 & -277 & 35 & 0 & 1.22 & -0.01 & -0.01 & 360 & 0.007 & 5.1 & 100.0 & 180 & 22 & 000 & 0.0 & 180 \\
\hline $00: 23: 10$ & -1 & -271 & 33 & 0 & 1.16 & 0.00 & -0.02 & 360 & 0.121 & 4.4 & 100.0 & 180 & 19 & 000 & 0.0 & 180 \\
\hline $00: 23: 20$ & -1 & -265 & 33 & 0 & 1.16 & 0.00 & -0.03 & 360 & 0.220 & 3.7 & 100.0 & 180 & 25 & 000 & 0.0 & 180 \\
\hline $00: 23: 30$ & -1 & -259 & 33 & 0 & 1.08 & 0.01 & -0.05 & 360 & 0.342 & 11.3 & 100.0 & 180 & 24 & 000 & 0.0 & 180 \\
\hline $00: 23: 40$ & -1 & -254 & 33 & 0 & 0.99 & 0.03 & -0.10 & 360 & 0.830 & 18.7 & 100.0 & 180 & 25 & 000 & 0.0 & 180 \\
\hline $00: 23: 50$ & -1 & -249 & 29 & 0 & 0.87 & 0.05 & -0.13 & 000 & 1.180 & 15.3 & 100.0 & 180 & 27 & 000 & 0.0 & 180 \\
\hline $00: 24: 00$ & -1 & -245 & 29 & 0 & 0.76 & 0.07 & -0.15 & 000 & 1.417 & 10.8 & 52.0 & 180 & 25 & 000 & 0.0 & 180 \\
\hline $00: 24: 10$ & -2 & -241 & 29 & 0 & 0.70 & 0.08 & -0.17 & 001 & 1.545 & 5.8 & 27.0 & 180 & 21 & 000 & 0.0 & 180 \\
\hline $00: 24: 20$ & -2 & -238 & 27 & 0 & 0.63 & 0.08 & -0.17 & 001 & 1.543 & 0.6 & 27.0 & 180 & 28 & 000 & 0.0 & 180 \\
\hline $00: 24: 30$ & -2 & -235 & 25 & 0 & 0.64 & 0.07 & -0.16 & 001 & 1.467 & -4.3 & 27.0 & 180 & 21 & 000 & 0.0 & 180 \\
\hline $00: 24: 40$ & -2 & -231 & 25 & 0 & 0.64 & 0.07 & -0.13 & 001 & 1.229 & -23.3 & 27.0 & 180 & 23 & 000 & 0.0 & 180 \\
\hline $00: 24: 50$ & -2 & -228 & 25 & 0 & 0.54 & 0.04 & -0.08 & 001 & 0.736 & -27.5 & 27.0 & 180 & 27 & 000 & 0.0 & 180 \\
\hline $00: 25: 00$ & -2 & -226 & 25 & 0 & 0.44 & 0.01 & -0.03 & 002 & 0.215 & -28.2 & 27.0 & 180 & 28 & 000 & 0.0 & 180 \\
\hline $00: 25: 10$ & -2 & -223 & 37 & 0 & 0.42 & -0.03 & 0.05 & 002 & -0.475 & -26.3 & 27.0 & 180 & 24 & 000 & 0.0 & 180 \\
\hline $00: 25: 20$ & -2 & -221 & 37 & 0 & 0.48 & -0.05 & 0.13 & 001 & -1.173 & -21.9 & 27.0 & 180 & 21 & 000 & 0.0 & 180 \\
\hline $00 ; 25: 30$ & -2 & -219 & 37 & 0 & 0.43 & -0.09 & 0.19 & 001 & -1.779 & -15.5 & 27.0 & 180 & 19 & 000 & 0.0 & 180 \\
\hline $00: 25: 40$ & -1 & -217 & 37 & 0 & 0.47 & -0.11 & 0.23 & 001 & -2.148 & -9.2 & 27.0 & 180 & 17 & 000 & 0.0 & 180 \\
\hline $00: 25: 50$ & -1 & -214 & 37 & 0 & 0.51 & -0.11 & 0.24 & 000 & -2.242 & -2.7 & 27.0 & 180 & 23 & 000 & 0.0 & 180 \\
\hline $00: 26: 00$ & -1 & -211 & 37 & 0 & 0.58 & -0.10 & 0.23 & 000 & -2.094 & 3.1 & 27.0 & 180 & 19 & 000 & 0.0 & 180 \\
\hline $00: 26: 10$ & 0 & -208 & 37 & 0 & 0.64 & -0.08 & 0.20 & 360 & -1.784 & 7.8 & 27.0 & 180 & 27 & 000 & 0.0 & 180 \\
\hline $00: 26: 20$ & 0 & -205 & 37 & 0 & 0.64 & -0.06 & 0.15 & 359 & -1.329 & 11.9 & 40.0 & 180 & 20 & 000 & 0.0 & 180 \\
\hline $00: 26: 30$ & 0 & -202 & 37 & 0 & 0.59 & -0.03 & 0.10 & 359 & -0.862 & 14.1 & 40.0 & 180 & 19 & 000 & 0.0 & 180 \\
\hline $00 ; 26 ; 40$ & 0 & -199 & 37 & 0 & 0.57 & -0.01 & 0.05 & 359 & -0.357 & 15.4 & 40.0 & 180 & 29 & 000 & 0.0 & 180 \\
\hline $00: 26: 50$ & 0 & -196 & 37 & 0 & 0.52 & 0.01 & -0.01 & 359 & 0.141 & 14.3 & 40.0 & 180 & 25 & 000 & 0.0 & 180 \\
\hline $00: 27: 00$ & 0 & -193 & 37 & 0 & 0.52 & 0.03 & -0.05 & 359 & 0.566 & 12.3 & 40.0 & 180 & 25 & 000 & 0.0 & 180 \\
\hline $00: 27: 10$ & 0 & -190 & 37 & 0 & 0.50 & 0.05 & -0.09 & 359 & 0.864 & 9.8 & 40.0 & 180 & 27 & 000 & 0.0 & 180 \\
\hline $00: 27: 20$ & 0 & -188 & 37 & 0 & 0.47 & 0.06 & -0.11 & 360 & 1.074 & 5.8 & 40.0 & 180 & 23 & 000 & 0.0 & 180 \\
\hline $00: 27: 30$ & 0 & -185 & 37 & 0 & 0.48 & 0.06 & -0.12 & 360 & 1.145 & 2.8 & 40.0 & 180 & 25 & 000 & 0.0 & 180 \\
\hline $00: 27: 40$ & 0 & -183 & 37 & 0 & 0.44 & 0.05 & -0.12 & 360 & 1.083 & -0.7 & 40.0 & 180 & 26 & 000 & 0.0 & 180 \\
\hline $2: 27: 50$ & -1 & -181 & 37 & 0 & 0.40 & 0.04 & -0.10 & 000 & 0.912 & -3.8 & 40.0 & 180 & 23 & 000 & 0.0 & 180 \\
\hline$: 28 ; 00$ & -1 & -179 & 37 & 0 & 0.40 & 0.03 & -0.08 & 000 & 0.681 & -5.4 & 15.0 & 180 & 29 & 000 & 0.0 & 180 \\
\hline$U 0: 28: 10$ & -1 & -177 & 37 & 0 & 0.39 & 0.02 & -0.05 & 000 & 0.416 & -6.4 & 15.0 & 180 & 19 & 000 & 0.0 & 180 \\
\hline $00: 28: 20$ & -1 & -175 & 37 & 0 & 0.34 & 0.00 & -0.02 & 000 & 0.133 & -6.7 & 15.0 & 180 & 24 & 000 & 0.0 & 180 \\
\hline $00: 28: 30$ & -1 & -173 & 40 & 0 & 0.35 & -0.02 & 0.00 & 000 & -0.149 & -5.7 & 15.0 & 180 & 24 & 000 & 0.0 & 180 \\
\hline & -1 & 1 & 40 & 0 & 2 & -0.03 & 0.03 & 000 & -0.355 & -4.3 & 25.0 & 180 & 19 & 000 & 0.0 & 180 \\
\hline
\end{tabular}




\begin{tabular}{|c|c|c|c|c|c|c|c|c|c|c|c|c|c|c|c|c|}
\hline Iog.ascii & \multirow{2}{*}{\multicolumn{2}{|c|}{ Position (m) }} & & & & & & & & & & & & $\mathbf{P a}$ & 2 \\
\hline Time & & & \multicolumn{2}{|r|}{ RPM } & \multicolumn{3}{|c|}{ Speed (Knots) } & \multirow[b]{2}{*}{ Heading } & \multirow{2}{*}{$\begin{array}{c}\text { Turn } \\
\text { Deg/Min }\end{array}$} & \multirow{2}{*}{$\begin{array}{l}\text { Rudder } \\
\text { Deg }\end{array}$} & \multicolumn{2}{|c|}{ Gill } & \multicolumn{2}{|c|}{ wind } & \multicolumn{2}{|c|}{ Current } \\
\hline HH:MM : SS & East & North & Port & Stbd & Long & Bow & Stern & & & & के & Deg & Knots & Deg & Knots & Deg \\
\hline & & & & & & & & & & & & 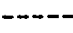 & $-\Delta$ & & & \\
\hline$-0: 28 ; 50$ & -1 & -168. & 38 & 0 & 0.55 & -0.04 & 0.04 & 000 & -0.492 & -2.9 & 39.0 & 180 & 18 & 000 & 0.0 & 180 \\
\hline$: 29: 00$ & -1 & -165 & 36 & 0 & 0.57 & -0.04 & 0.05 & 000 & -0.544 & -1.1 & 39.0 & 180 & 19 & 000 & 0.0 & 180 \\
\hline$-0: 29: 10$ & -1 & -162 & 36 & 0 & 0.55 & -0.03 & 0.04 & 000 & -0.413 & 11.3 & 39.0 & 180 & 24 & 000 & 0.0 & 180 \\
\hline $00: 29: 20$ & -1 & -160 & 36 & 0 & 0.56 & 0.01 & -0.02 & 000 & 0.176 & 17.1 & 39.0 & 180 & 24 & 000 & 0.0 & 180 \\
\hline $00: 29: 30$ & -1 & -157 & 36 & 0 & 0.57 & 0.04 & -0.07 & 000 & 0.726 & 14.8 & 39.0 & 180 & 28 & 000 & 0.0 & 180 \\
\hline $00: 29: 40$ & -1 & -154 & 36 & 0 & 0.52 & 0.06 & -0.11 & 000 & 1.127 & 11.0 & 51.0 & 180 & 24 & 000 & 0.0 & 180 \\
\hline $00: 29: 50$ & -1 & -151 & 36 & 0 & 0.47 & 0.08 & -0.14 & 000 & 1.371 & 6.2 & 51.0 & 180 & 22 & 000 & 0.0 & 180 \\
\hline $00: 30: 00$ & -1 & -149 & 36 & 0 & 0.47 & 0.09 & -0.14 & 001 & 1.449 & 2.0 & 51.0 & 180 & 19 & 000 & 0.0 & 180 \\
\hline $00: 30: 10$ & -1 & -146 & 36 & 0 & 0.45 & 0.09 & -0.13 & 001 & 1.389 & -2.5 & 51.0 & 180 & 23 & 000 & 0.0 & 180 \\
\hline $00: 30: 20$ & -1 & -144 & 36 & 0 & 0.44 & 0.07 & -0.11 & 001 & 1.175 & -6.0 & 51.0 & 180 & 27 & 000 & 0.0 & 180 \\
\hline $00: 30: 30$ & -1 & -142 & 36 & 0 & 0.43 & 0.07 & -0.08 & 001 & 0.954 & -8.7 & 51.0 & 180 & 26 & 000 & 0.0 & 180 \\
\hline $00: 30: 40$ & -1 & -140 & 36 & 0 & 0.35 & 0.06 & -0.04 & 001 & 0.670 & -10.7 & 35.0 & 180 & 22 & 000 & 0.0 & 180 \\
\hline $00: 30: 50$ & -1 & -138 & 36 & 0 & 0.30 & 0.05 & 0.00 & 002 & 0.313 & -11.4 & 25.0 & 180 & 27 & 000 & 0.0 & 180 \\
\hline $00: 31: 00$ & -1 & -137 & 36 & 0 & 0.27 & 0.03 & 0.04 & 002 & -0.056 & -9.9 & 25.0 & 180 & 22 & 000 & 0.0 & 180 \\
\hline $00: 31: 10$ & -1 & -135 & 36 & 0 & 0.26 & 0.00 & 0.09 & 002 & -0.515 & -22.8 & 25.0 & 180 & 21 & 000 & 0.0 & 180 \\
\hline $00: 31: 20$ & 0 & -134 & 36 & 0 & 0.28 & -0.03 & 0.16 & 001 & -1.218 & -19.8 & 0.0 & 180 & 26 & 000 & 0.0 & 180 \\
\hline $00: 31: 30$ & 0 & -132 & 39 & 0 & 0.36 & -0.07 & 0.22 & 001 & -1.808 & -14.5 & 0.0 & 180 & 19 & 000 & 0.0 & 180 \\
\hline $00 ; 31 ; 40$ & 0 & -130 & 39 & 0 & 0.40 & -0.09 & 0.25 & 001 & -2.184 & -8.4 & 0.0 & 180 & 26 & 000 & 0.0 & 180 \\
\hline $00: 31: 50$ & 1 & -128 & 39 & 0 & 0.39 & -0.09 & 0.26 & 000 & -2.217 & -1.3 & 0.0 & 180 & 22 & 000 & 0.0 & 180 \\
\hline $00: 32: 00$ & 1 & -126 & 39 & 0 & 0.49 & -0.08 & 0.25 & 000 & -2.104 & -6.9 & 0.0 & 180 & 28 & 000 & 0.0 & 180 \\
\hline $00: 32: 10$ & 2 & -124 & 36 & 0 & 0.51 & -0.10 & 0.28 & 360 & -2.377 & -7.0 & 47.0 & 180 & 27 & 000 & 0.0 & 180 \\
\hline $00: 32: 20$ & 2 & -121 & 33 & 0 & 0.49 & -0.10 & 0.28 & 359 & -2.414 & 0.1 & 47.0 & 180 & 20 & 000 & 0.0 & 180 \\
\hline $00: 32: 30$ & 3 & -119 & 33 & 0 & 0.40 & -0.10 & 0.25 & 359 & -2.208 & 8.1 & 47.0 & 180 & 23 & 000 & 0.0 & 180 \\
\hline $00: 32: 40$ & 3 & -117 & 33 & 0 & 0.37 & -0.07 & 0.21 & 359 & -1.761 & 13.3 & 47.0 & 180 & 24 & 000 & 0.0 & 180 \\
\hline $00: 32: 50$ & 3 & -115 & 33 & 0 & 0.43 & -0.07 & 0.16 & 358 & -1.459 & 16.4 & 47.0 & 180 & 22 & 000 & 0.0 & 180 \\
\hline $00: 33: 00$ & 3 & -113 & 33 & 0 & 0.40 & -0.05 & 0.10 & 358 & -0.980 & 19.3 & 77.0 & 180 & 22 & 000 & 0.0 & 180 \\
\hline $00: 33: 10$ & 3 & -111 & 33 & 0 & 0.31 & -0.03 & 0.05 & 358 & -0.456 & 20.3 & 77.0 & 180 & 21 & 000 & 0.0 & 180 \\
\hline $00: 33: 20$ & 3 & -109 & 33 & 0 & 0.25 & 0.00 & -0.01 & 358 & 0.062 & 18.9 & 77.0 & 180 & 18 & 000 & 0.0 & 180 \\
\hline $00: 33: 30$ & 3 & -108 & 33 & 0 & 0.18 & 0.00 & -0.06 & 358 & 0.339 & 16.4 & 77.0 & 180 & 21 & 000 & 0.0 & 180 \\
\hline $00: 33: 40$ & 3 & -108 & 33 & 0 & 0.08 & 0.00 & -0.09 & 358 & 0.551 & 9.9 & 77.0 & 180 & 22 & 000 & 0.0 & 180 \\
\hline $00: 33: 50$ & 3 & -107 & 33 & 0 & 0.04 & -0.01 & -0.10 & 358 & 0.613 & 4.4 & 27.0 & 180 & 29 & 000 & 0.0 & 180 \\
\hline $00: 34: 00$ & 2 & -107 & 37 & 0 & 0.01 & -0.01 & -0.11 & 358 & 0.593 & 2.2 & 27.0 & 180 & 22 & 000 & 0.0 & 180 \\
\hline $00: 34: 10$ & 2 & -107 & 37 & 0 & 0.01 & -0.02 & -0.11 & 358 & 0.569 & 1.2 & 27.0 & 180 & 23 & 000 & 0.0 & 180 \\
\hline $00: 34: 20$ & 2 & -107 & 36 & 0 & 0.04 & -0.02 & -0.10 & 358 & 0.544 & 0.8 & 15.0 & 180 & 22 & 000 & 0.0 & 180 \\
\hline $00: 34: 30$ & 1 & -107 & 37 & 0 & 0.11 & -0.01 & -0.10 & 359 & 0.588 & 2.9 & 15.0 & 180 & 24 & 000 & 0.0 & 180 \\
\hline $00: 34: 40$ & 1 & -106 & 37 & 0 & 0.14 & -0.02 & -0.11 & 359 & 0.535 & 2.6 & 15.0 & 180 & 26 & 000 & 0.0 & 180 \\
\hline $00: 34: 50$ & 1 & -105 & 37 & 0 & 0.18 & -0.04 & -0.11 & 359 & 0.410 & 3.9 & 24.0 & 180 & 25 & 000 & 0.0 & 180 \\
\hline $00: 35: 00$ & 0 & -104 & 37 & 0 & 0.19 & -0.03 & -0.15 & 359 & 0.765 & 17.4 & 24.0 & 180 & 32 & 000 & 0.0 & 180 \\
\hline $0: 35: 10$ & 0 & -103 & 37 & 0 & 0.13 & 0.00 & -0.21 & 359 & 1.338 & 27.0 & 24.0 & 180 & 24 & 000 & 0.0 & 180 \\
\hline$: 35: 20$ & -1 & -103 & 37 & 0 & 0.16 & 0.03 & -0.28 & 359 & 1.965 & 20.2 & 24.0 & 180 & 26 & 000 & 0.0 & 180 \\
\hline $0: 35: 30$ & -2 & -102 & 36 & 0 & 0.18 & 0.07 & -0.34 & 360 & 2.605 & 31.6 & 24.0 & 180 & 24 & 000 & 0.0 & 180 \\
\hline $00: 35: 40$ & -2 & -101 & 37 & 0 & 0.24 & 0.13 & -0.44 & 000 & 3.632 & 35.0 & 24.0 & 180 & 21 & 000 & 0.0 & 180 \\
\hline $00: 35: 50$ & -3 & -99 & 37 & 0 & 0.21 & 0.20 & -0.54 & 001 & 4.666 & 35.0 & 24.0 & 180 & 17 & 000 & 0.0 & 180 \\
\hline $00: 36: 00$ & -4 & -99 & 37 & 0 & 0.10 & 0.30 & -0.62 & 002 & 5.828 & 28.5 & 24.0 & 180 & 19 & 000 & 0.0 & 180 \\
\hline $00: 36: 10$ & -5 & -98 & 37 & 0 & 0.07 & 0.45 & -0.64 & 003 & 6.900 & 9.5 & 24.0 & 180 & 23 & 000 & 0.0 & 180 \\
\hline $00: 36: 20$ & -5 & -98 & 37 & 0 & 0.00 & 0.60 & -0.59 & 004 & 7.533 & -9.5 & 24.0 & 180 & 25 & 000 & 0.0 & 180 \\
\hline $00: 36: 30$ & -5 & -98 & 37 & 0 & -0.14 & 0.74 & -0.57 & 005 & 8.302 & -28.5 & 24.0 & 180 & 21 & 000 & 0.0 & 180 \\
\hline $00: 36: 40$ & -4 & -99 & 36 & 0 & -0.24 & 0.74 & -0.48 & 007 & 7.740 & -35.0 & 24.0 & 180 & 22 & 000 & 0.0 & 180 \\
\hline
\end{tabular}




\section{Simulation Track Plot}

Open Ocean

Ship: 150,000 ton, Tanker, partial load

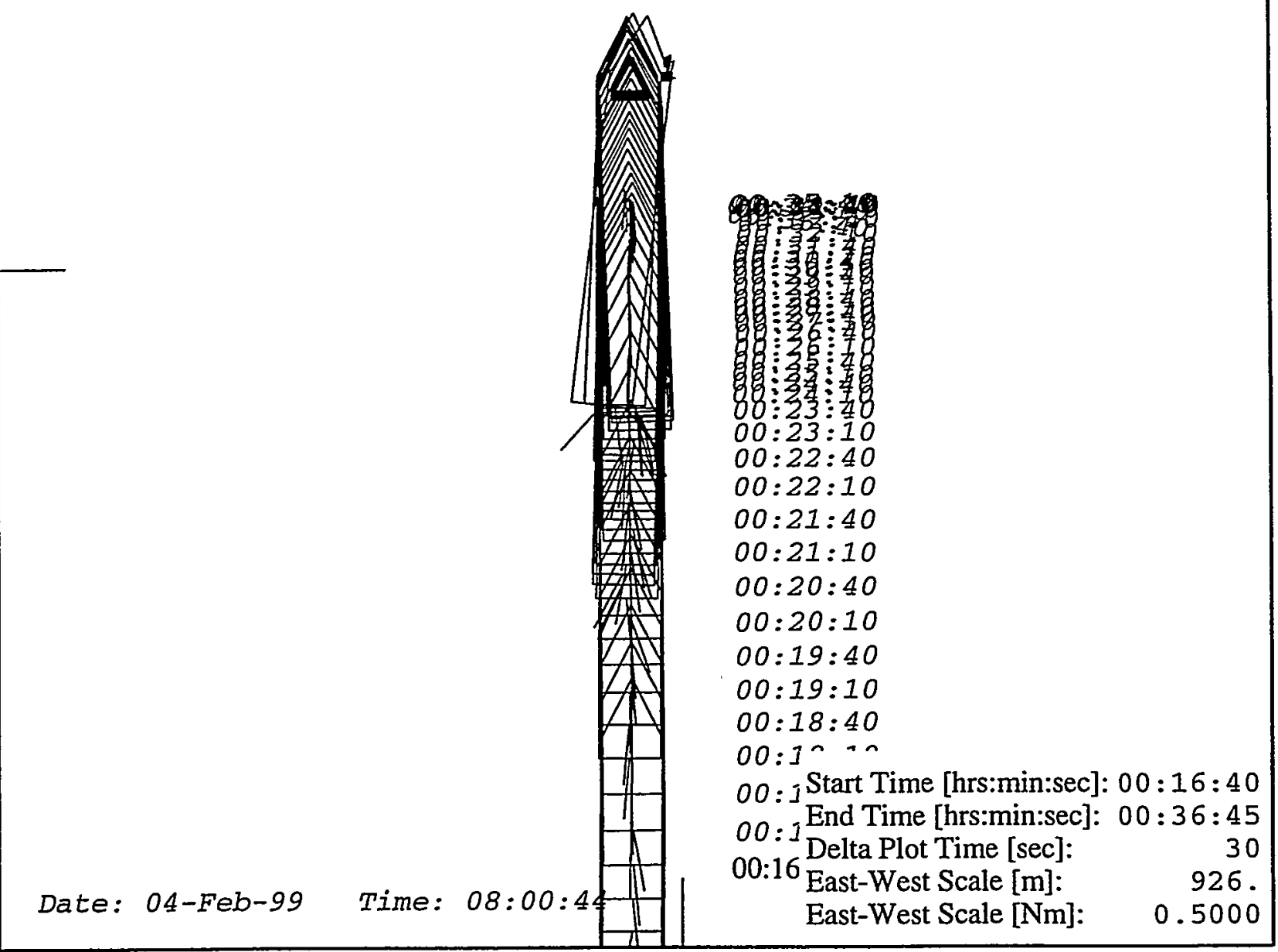




\section{HANDS-ON SIMULATION}

\section{SUCTION MOORNG TECHNOLOGY}

Simulation Number: 20

Date: 4 February 1999

Start Time: 08:50

Length of run [secs]: 2280

Ship Condition: draft $=13.72 \mathrm{~m}$

Environment

Wind Speed [knots]:.

25 Direction: 0

Sea State, Significant wave height $[\mathrm{m}]: .$.

5.1 Direction: 180

Current Speed [knots]:

0 Direction: 0

Ship Operator: J.A.

Observer: B.K.J \& J.K.

Final Mating, distance between center of buoy and center of moon pool [m]: 1.8

Evaluation:

The approach-deceleration and tracking along the course line was no problem. The plan was to bring the ship to a stop over the buoy and hover there by balancing forces, thruster against propeller and achieving lateral motion control by autopilot change of 1 to 2 degrees. The captain believes that this is better than the case of the previous run, where the ship was kept moving ahead over the buoy. The last approach will allow for more mating operations if needed with no difficulty. 
Ship:

591 150,000 ton, Tanker, partial load

Exercise number:

755 Suction Mooring System

112 Open Ocean

g file:

999 Dummy tug file based on containership

raffic ships:

590 Suction Mooring Buoy

Exercise identification: ${ }^{295}$ RuN 20

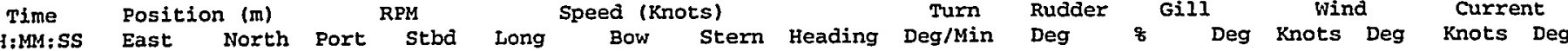

\begin{tabular}{|c|c|c|c|c|c|c|c|c|c|c|c|c|c|c|c|c|}
\hline $\begin{array}{l}00: 16: 40 \\
00: 16: 50\end{array}$ & $\begin{array}{l}-2 \\
-2\end{array}$ & $\begin{array}{l}-399 \\
-388\end{array}$ & $\begin{array}{l}27 \\
27\end{array}$ & $\begin{array}{l}0 \\
0\end{array}$ & $\begin{array}{l}2.15 \\
2.12\end{array}$ & $\begin{array}{r}0.01 \\
-0.01\end{array}$ & $\begin{array}{l}-0.06 \\
-0.01\end{array}$ & $\begin{array}{l}001 \\
001\end{array}$ & $\begin{array}{r}0.441 \\
-0.055\end{array}$ & $\begin{array}{l}-24.4 \\
-24.7\end{array}$ & $\begin{array}{l}25.0 \\
25.0\end{array}$ & $\begin{array}{l}180 \\
180\end{array}$ & $\begin{array}{l}32 \\
21\end{array}$ & $\begin{array}{l}000 \\
000\end{array}$ & $\begin{array}{l}0.0 \\
0.0\end{array}$ & $\begin{array}{l}180 \\
180\end{array}$ \\
\hline $00: 17: 00$ & -2 & -377 & 27 & 0 & 2.03 & -0.03 & 0.05 & 001 & -0.529 & -23.9 & 25.0 & 180 & 29 & 000 & 0.0 & 180 \\
\hline $00: 17: 10$ & -2 & -367 & 27 & 0 & 2.04 & -0.04 & 0.10 & 001 & -0.900 & -21.4 & 25.0 & 180 & 25 & 000 & 0.0 & 180 \\
\hline $00: 17: 20$ & -1 & -356 & 27 & 0 & 2.05 & -0.05 & 0.14 & 001 & -1.238 & -17.9 & 25.0 & 180 & 22 & 000 & 0.0 & 180 \\
\hline $00: 17: 30$ & -1 & -346 & 27 & 0 & 1.96 & -0.06 & 0.18 & 001 & -1.528 & -13.7 & 25.0 & 180 & 24 & 000 & 0.0 & 180 \\
\hline $00: 17: 40$ & 0 & -336 & 27 & 0 & 1.97 & -0.07 & 0.20 & 001 & -1.681 & -8.6 & 25.0 & 180 & 16 & 000 & 0.0 & 180 \\
\hline $00: 17: 50$ & 0 & -325 & 27 & 0 & 1.97 & -0.06 & 0.21 & 000 & -1.720 & -3.5 & 25.0 & 180 & 25 & 000 & 0.0 & 180 \\
\hline $00: 18: 00$ & 1 & -315 & 27 & 0 & 1.95 & -0.05 & 0.21 & 360 & -1.665 & -3.8 & 25.0 & 180 & 26 & 000 & 0.0 & 180 \\
\hline $00: 18: 10$ & 1 & -306 & 27 & 0 & 1.85 & -0.06 & 0.23 & 360 & -1.858 & -11.2 & 25.0 & 180 & 20 & 000 & 0.0 & 180 \\
\hline $00: 18: 20$ & 1 & -296 & 27 & 0 & 1.69 & -0.07 & 0.24 & 359 & -1.955 & -5.4 & 25.0 & 180 & 23 & 000 & 0.0 & 180 \\
\hline $00: 18: 30$ & 2 & -288 & 27 & .0 & 1.62 & -0.07 & 0.24 & 359 & -1.974 & 0.9 & 25.0 & 180 & 20 & 000 & 0.0 & 180 \\
\hline $00: 18 \div 40$ & 2 & -280 & 27 & 0 & 1.57 & -0.07 & 0.22 & 359 & -1.865 & 14.1 & 25.0 & 180 & 13 & 000 & 0.0 & 180 \\
\hline $00: 18: 50$ & 2 & -272 & 27 & 0 & 1.54 & -0.04 & 0.17 & 358 & -1.322 & 29.1 & 25.0 & 180 & 25 & 000 & 0.0 & 180 \\
\hline $00: 19: 00$ & 2 & -264 & 27 & 0 & 1.47 & 0.01 & 0.10 & 358 & -0.548 & 31.4 & 25.0 & 180 & 23 & 000 & 0.0 & 180 \\
\hline $00: 19: 10$ & 2 & -256 & 27 & 0 & 1.44 & 0.05 & 0.03 & 358 & 0.148 & 31.4 & 25.0 & 180 & 28 & 000 & 0.0 & 180 \\
\hline $00: 19: 20$ & 2 & -249 & 27 & 0 & 1.39 & 0.08 & -0.03 & 358 & 0.690 & 29.8 & 25.0 & 180 & 27 & 000 & 0.0 & 180 \\
\hline $00: 19: 30$ & 2 & -242 & 27 & 0 & 1.40 & 0.10 & -0.09 & 358 & 1.202 & 26.6 & 51.0 & 180 & 23 & 000 & 0.0 & 180 \\
\hline $00: 19: 40$ & 2 & -235 & 27 & 0 & 1.33 & 0.10 & -0.13 & 359 & 1.493 & 22.3 & 100.0 & 180 & 28 & 000 & 0.0 & 180 \\
\hline $00: 19: 50$ & 2 & -228 & 27 & 0 & 1.21 & 0.11 & -0.17 & 359 & 1.745 & 16.9 & 100.0 & 180 & 24 & 000 & 0.0 & 180 \\
\hline $00: 20: 00$ & 1 & -222 & 27 & 0 & 1.09 & 0.11 & -0.19 & 359 & 1.887 & 11.0 & 100.0 & 180 & 23 & 000 & 0.0 & 180 \\
\hline $00: 20: 10$ & 1 & -217 & 27 & 0 & 1.09 & 0.11 & -0.20 & 360 & 1.919 & 5.2 & 100.0 & 180 & 24 & 000 & 0.0 & 180 \\
\hline $00: 20: 20$ & 1 & -211 & 27 & 0 & 1.02 & 0.10 & -0.19 & 360 & 1.853 & -0.4 & 100.0 & 180 & 26 & 000 & 0.0 & 180 \\
\hline $00: 20: 30$ & 1 & -206 & 27 & 0 & 0.96 & 0.09 & -0.18 & 000 & 1.690 & -5.9 & 100.0 & 180 & 20 & 000 & 0.0 & 180 \\
\hline $00: 20: 40$ & 0 & -201 & 27 & 0 & 0.96 & 0.07 & -0.16 & 000 & 1.464 & -10.3 & 100.0 & 180 & 27 & 000 & 0.0 & 180 \\
\hline $00: 20: 50$ & 0 & -197 & 27 & 0 & 0.85 & 0.05 & -0.11 & 001 & 1.034 & -27.9 & 100.0 & 180 & 25 & 000 & 0.0 & 180 \\
\hline $00 ; 21: 00$ & 0 & -193 & 27 & 0 & 0.71 & 0.02 & -0.04 & 001 & 0.412 & -33.3 & 100.0 & 180 & 19 & 000 & 0.0 & 180 \\
\hline $00: 21: 10$ & 0 & -189 & 27 & 0 & 0.57 & -0.01 & 0.02 & 001 & -0.208 & -32.2 & 100.0 & 180 & 22 & 000 & 0.0 & 180 \\
\hline $0: 21: 20$ & 0 & -187 & 27 & 0 & 0.46 & -0.04 & 0.08 & 001 & -0.744 & -29.6 & 100.0 & 180 & 21 & 000 & 0.0 & 180 \\
\hline$: 21: 30$ & 0 & -185 & 27 & 0 & 0.38 & -0.05 & 0.13 & 001 & -1.185 & -25.1 & 100.0 & 180 & 24 & 000 & 0.0 & 180 \\
\hline$-0: 21: 40$ & 1 & -183 & 27 & 0 & 0.22 & -0.07 & 0.17 & 000 & -1.538 & -16.5 & 100.0 & 180 & 25 & 000 & 0.0 & 180 \\
\hline $00: 21: 50$ & 1 & -182 & 27 & 0 & 0.10 & -0.08 & 0.18 & 000 & -1.671 & -2.6 & 100.0 & 180 & 19 & 000 & 0.0 & 180 \\
\hline $00 ; 22: 00$ & 1 & -182 & 27 & 0 & 0.06 & -0.08 & 0.17 & 360 & -1.534 & 9.4 & 100.0 & 180 & 23 & 000 & 0.0 & 180 \\
\hline $00: 22: 10$ & 1 & -182 & 27 & 0 & 0.04 & -0.06 & 0.13 & 360 & -1.220 & 18.0 & 100.0 & 180 & 25 & 000 & 0.0 & 180 \\
\hline $00: 22: 20$ & 2 & -182 & 27 & 0 & -0.08 & -0.03 & 0.08 & 359 & -0.712 & 28.5 & 100.0 & 180 & 19 & 000 & 0.0 & 180 \\
\hline $00: 22: 30$ & 2 & -182 & 37 & 0 & -0.18 & 0.00 & 0.03 & 359 & -0.227 & 13.4 & 100.0 & 180 & 33 & 000 & 0.0 & 180 \\
\hline $00 ; 22: 40$ & 2 & -183 & 37 & 0 & -0.20 & 0.02 & -0.01 & 359 & 0.155 & 11.0 & 25.0 & 180 & 23 & 000 & 0.0 & 180 \\
\hline $00: 22: 50$ & 2 & -184 & 37 & 0 & -0.20 & 0.03 & -0.04 & 359 & 0.483 & 8.5 & 0.0 & 180 & 19 & 000 & 0.0 & 180 \\
\hline $00: 23: 00$ & 2 & -285 & 37 & 0 & -0.14 & 0.04 & -0.06 & 359 & 0.669 & 3.9 & 0.0 & 180 & 22 & 000 & 0.0 & 180 \\
\hline $00: 23: 10$ & 2 & -186 & 37 & 0 & -0.06 & 0.03 & -0.05 & 360 & 0.560 & -11.4 & 0.0 & 180 & 19 & 000 & 0.0 & 180 \\
\hline $00 ; 23: 20$ & 2 & -186 & 36 & 0 & -0.06 & 0.00 & 0.00 & 360 & -0.012 & -11.4 & 0.0 & 180 & 25 & 000 & 0.0 & 180 \\
\hline $00: 23: 30$ & 2 & -186 & 37 & 0 & -0.01 & -0.01 & 0.01 & 360 & -0.127 & 7.6 & 27.0 & 180 & 24 & 000 & 0.0 & 180 \\
\hline $00: 23: 40$ & 2 & -186 & 37 & 0 & 0.04 & 0.00 & -0.02 & 360 & 0.119 & 3.6 & 0.0 & 180 & 25 & 000 & 0.0 & 180 \\
\hline $00: 23: 50$ & 2 & -186 & 37 & 0 & 0.02 & 0.00 & -0.02 & 360 & 0.122 & -0.2 & 26.0 & 180 & 27 & 000 & 0.0 & 180 \\
\hline $00: 24: 00$ & 1 & -186 & 36 & 0 & 0.01 & -0.01 & -0.02 & .360 & 0.068 & 1.7 & 26.0 & 180 & 25 & 000 & 0.0 & 180 \\
\hline $00: 24: 10$ & 1 & -186 & 37 & 0 & -0.04 & -0.01 & -0.03 & 360 & 0.102 & 2.8 & 26.0 & 180 & 21 & 000 & 0.0 & 180 \\
\hline $00: 24: 20$ & 1 & -186 & 37 & 0 & -0.08 & -0.02 & -0.01 & 360 & -0.100 & -11.0 & 26.0 & 180 & 28 & 000 & 0.0 & 180 \\
\hline $00: 24: 30$ & $i$ & -187 & 37 & 0 & -0.11 & -0.05 & 0.02 & 360 & -0.441 & -6.4 & 26.0 & 180 & 21 & 000 & 0.0 & 180 \\
\hline $00: 24: 40$ & 1 & -187 & 42 & 0 & -0.13 & -0.06 & 0.05 & 360 & -0.693 & -3.1 & 0.0 & 180 & 23 & 000 & 0.0 & 180 \\
\hline $00 ; 24: 50$ & 1 & -188 & 42 & 0 & -0.05 & -0.06 & 0.04 & 359 & -0.681 & 8.3 & 0.0 & 180 & 27 & 000 & 0.0 & 180 \\
\hline $00: 25: 00$ & 1 & -188 & 41 & 0 & -0.01 & -0.04 & -0.01 & 359 & -0.158 & 7.2 & 0.0 & 180 & 28 & 000 & 0.0 & 180 \\
\hline $00: 25: 10$ & 1 & -188 & 42 & 0 & -0.06 & -0.04 & -0.01 & 359 & -0.189 & -2.1 & 0.0 & 180 & 24 & 000 & 0.0 & 180 \\
\hline $00: 25: 20$ & 1 & -188 & 45 & 0 & -0.08 & -0.05 & 0.00 & 359 & -0.296 & -0.7 & 0.0 & 180 & 21 & 000 & 0.0 & 180 \\
\hline $00: 25: 30$ & 1 & -189 & 45 & 0 & -0.01 & -0.05 & -0.01 & 359 & -0.266 & 6.4 & 0.0 & 180 & 19 & 000 & 0.0 & 180 \\
\hline $00: 25: 40$ & 1 & -189 & 44 & 0 & 0.08 & -0.06 & -0.01 & 359 & -0.283 & 0.6 & 0.0 & 180 & 17 & 000 & 0.0 & 180 \\
\hline $00: 25: 50$ & 0 & -188 & 44 & 0 & 0.06 & -0.05 & -0.02 & 359 & -0.237 & 1.3 & 0.0 & 180 & 23 & 000 & 0.0 & 180 \\
\hline $00: 26: 00$ & 0 & -188 & 44 & 0 & 0.15 & -0.03 & -0.05 & 359 & 0.143 & 14.8 & 0.0 & 180 & 19 & 000 & 0.0 & 180 \\
\hline $00: 26: 10$ & 0 & -187 & 45 & 0 & 0.22 & 0.01 & -0.11 & 359 & 0.754 & 10.3 & 0.0 & 180 & 27 & 000 & 0.0 & 180 \\
\hline $00: 26: 20$ & 0 & -185 & 45 & 0 & 0.32 & 0.02 & -0.15 & 359 & 1.090 & 7.4 & 51.0 & 180 & 20 & 000 & 0.0 & 180 \\
\hline $00 ; 26: 30$ & -1 & -183 & 42 & 0 & 0.39 & 0.02 & -0.17 & 360 & 1.224 & 4.2 & 75.0 & 180 & 19 & 000 & 0.0 & 180 \\
\hline $00 ; 26 ; 40$ & -1 & -181 & 41 & 0 & 0.38 & 0.02 & -0.17 & 360 & 1.216 & 0.8 & 75.0 & 180 & 29 & 000 & 0.0 & 180 \\
\hline $00 ; 26: 50$ & -2 & -180 & 42 & 0 & 0.32 & 0.02 & -0.17 & 360 & 1.182 & 9.0 & 75.0 & 180 & 25 & 000 & 0.0 & 180 \\
\hline $00 ; 27: 00$ & -2 & -178 & 42 & 0 & 0.29 & 0.04 & -0.21 & 000 & 1.587 & 8.5 & 75.0 & 180 & 25 & 000 & 0.0 & 180 \\
\hline $00 ; 27: 10$ & -2 & -177 & 42 & 0 & 0.24 & 0.05 & -0.22 & 000 & 1.706 & 1.8 & 75.0 & 180 & 27 & 000 & 0.0 & 180 \\
\hline $00: 27: 20$ & -3 & -175 & 42 & 0 & 0.25 & 0.05 & -0.20 & 001 & 1.571 & -2.5 & 27.0 & 180 & 23 & 000 & 0.0 & 180 \\
\hline $00: 27: 30$ & -3 & -174 & 42 & 0 & 0.26 & 0.04 & -0.17 & 001 & 1.328 & -5.3 & 27.0 & 180 & 25 & 000 & 0.0 & 180 \\
\hline $00 \div 27: 40$ & -3 & -173 & 42 & 0 & 0.25 & 0.02 & -0.13 & 001 & 0.913 & -7.6 & 27.0 & 180 & 26 & 000 & 0.0 & 180 \\
\hline$: 27: 50$ & -4 & -171 & 42 & 0 & 0.28 & -0.01 & -0.09 & 001 & 0.502 & -7.2 & 27.0 & 180 & 23 & 000 & 0.0 & 180 \\
\hline$: 28: 00$ & -4 & -170 & 41 & 0 & 0.26 & -0.02 & -0.05 & 001 & 0.156 & -6.9 & 27.0 & 180 & 29 & 000 & 0.0 & 180 \\
\hline$\sigma 0: 28: 10$ & -4 & -169 & 42 & 0 & 0.25 & -0.03 & -0.01 & 001 & -0.080 & -5.8 & 27.0 & 180 & 19 & 000 & 0.0 & 180 \\
\hline $00: 28: 20$ & -4 & -167 & 42 & 0 & 0.27 & -0.03 & 0.01 & 001 & -0.249 & -4.5 & 0.0 & 180 & 24 & 000 & 0.0 & 180 \\
\hline $00: 28: 30$ & -4 & -166 & 41 & 0 & 0.28 & -0.03 & 0.03 & 001 & -0.378 & -3.5 & 0.0 & 180 & 24 & 000 & 0.0 & .180 \\
\hline & & & & & & & & & & & & & 19 & 000 & 0.0 & 180 \\
\hline
\end{tabular}

$00,28,40$ 


\begin{tabular}{|c|c|c|c|c|c|c|c|c|c|c|c|c|c|c|c|c|}
\hline \multirow{2}{*}{$\begin{array}{c}\text { Time } \\
H H: M M: S S\end{array}$} & \multicolumn{2}{|c|}{ Position (m) } & \multicolumn{2}{|c|}{ RPM } & \multicolumn{3}{|c|}{ Speed (Krots) } & \multirow[b]{2}{*}{ Heading } & \multirow{2}{*}{$\begin{array}{c}\text { Turn } \\
\text { Deg/Min }\end{array}$} & \multirow{2}{*}{$\begin{array}{l}\text { Ruddex } \\
\text { Deg }\end{array}$} & \multicolumn{2}{|c|}{ Gill } & \multicolumn{2}{|c|}{ Wind } & \multicolumn{2}{|c|}{ Current } \\
\hline & East & North & Port & Stbd & Long & Bow & Stern & & & & 8 & Deg & Knots & Deg & Knots & Deg \\
\hline $0: 28: 50$ & -4 & -163. & 42 & 0 & 0.27 & -0.04 & 0.04 & 001 & -0.533 & 0.1 & 0.0 & 180 & 18 & 000 & 0.0 & 180 \\
\hline$: 29: 00$ & -4 & -161 & 42 & 0 & 0.45 & -0.03 & 0.04 & 001 & -0.450 & 0.6 & 0.0 & 180 & 19 & 000 & 0.0 & 180 \\
\hline $0: 29: 10$ & -4 & -159 & 42 & 0 & 0.51 & -0.02 & 0.03 & 001 & -0.335 & 1.3 & 0.0 & 180 & 24 & 000 & 0.0 & 180 \\
\hline $00: 29: 20$ & -4 & -156 & 39 & 0 & 0.53 & -0.01 & 0.03 & 001 & -0.202 & 2.1 & 75.0 & 180 & 24 & 000 & 0.0 & 180 \\
\hline $00: 29: 30$ & -4 & -154 & 37 & 0 & 0.50 & 0.01 & 0.02 & 001 & -0.086 & 1.9 & 75.0 & 180 & 28 & 000 & 0.0 & 180 \\
\hline $00: 29: 40$ & -3 & -151 & 37 & 0 & 0.48 & 0.02 & 0.01 & 001 & 0.042 & 2.2 & 75.0 & 180 & 24 & 000 & 0.0 & 180 \\
\hline $00: 29: 50$ & -3 & -149 & 37 & 0 & 0.49 & 0.05 & 0.01 & 001 & 0.276 & 1.0 & 51.0 & 180 & 22 & 000 & 0.0 & 180 \\
\hline $00: 30: 00$ & -3 & -146 & 37 & 0 & 0.46 & 0.06 & 0.01 & 001 & 0.328 & -0.3 & 51.0 & 180 & 19 & 000 & 0.0 & 180 \\
\hline $00: 30: 10$ & -3 & -144 & 37 & 0 & 0.41 & 0.06 & 0.01 & 001 & 0.319 & -1.5 & 51.0 & 180 & 23 & 000 & 0.0 & 180 \\
\hline $00: 30: 20$ & -3 & -142 & 37 & 0 & 0.40 & 0.07 & 0.02 & 001 & 0.299 & -2.0 & 34.0 & 180 & 27 & 000 & 0.0 & 180 \\
\hline $00: 30: 30$ & -2 & -140 & 37 & 0 & 0.39 & 0.07 & 0.03 & 001 & 0.251 & -3.0 & 34.0 & 180 & 26 & 000 & 0.0 & 180 \\
\hline $00: 30: 40$ & -2 & -138 & 37 & 0 & 0.38 & 0.07 & 0.04 & 001 & 0.157 & -3.3 & 34.0 & 180 & 22 & 000 & 0.0 & 180 \\
\hline $00: 30: 50$ & -2 & -136 & 37 & 0 & 0.40 & 0.06 & 0.07 & 001 & -0.106 & -18.0 & 34.0 & 180 & 27 & 000 & 0.0 & 180 \\
\hline $00: 31: 00$ & -1 & -134 & 37 & 0 & 0.32 & 0.03 & 0.14 & 001 & -0.691 & -17.3 & 34.0 & 180 & 22 & 000 & 0.0 & 180 \\
\hline $00: 31: 10$ & -1 & -133 & 37 & 0 & 0.27 & 0.01 & 0.19 & 001 & -1.129 & -12.4 & 25.0 & 180 & 21 & $000^{\circ}$ & 0.0 & 180 \\
\hline $00: 31: 20$ & 0 & -131 & 40 & 0 & 0.23 & -0.01 & 0.21 & 001 & -1.392 & -7.0 & 25.0 & 180 & 26 & 000 & 0.0 & 180 \\
\hline $00: 31: 30$ & 0 & -130 & 41 & 0 & 0.24 & -0.01 & 0.22 & 000 & -1.469 & -2.4 & 25.0 & 180 & 19 & 000 & 0.0 & 180 \\
\hline $00: 31: 40$ & 1 & -129 & 40 & 0 & 0.26 & 0.00 & 0.21 & 000 & -1.368 & 1.3 & 25.0 & 180 & 26 & 000 & 0.0 & 180 \\
\hline $00: 31: 50$ & 1 & -127 & 41 & 0 & 0.34 & 0.01 & 0.19 & 000 & -1.167 & 3.3 & 25.0 & 180 & 22 & 000 & 0.0 & 180 \\
\hline $00: 32: 00$ & 2 & -125 & 41 & 0 & 0.41 & 0.03 & 0.17 & 360 & -0.905 & 4.8 & 25.0 & 180 & 28 & 000 & 0.0 & 180 \\
\hline $00: 32: 10$ & 2 & -123 & 41 & 0 & 0.38 & 0.05 & 0.14 & 360 & -0.589 & 6.3 & 75.0 & 180 & 27 & 000 & 0.0 & 180 \\
\hline $00: 32: 20$ & 3 & -121 & 41 & 0 & 0.43 & 0.06 & 0.11 & 360 & -0.366 & -1.7 & 75.0 & 180 & 20 & 000 & 0.0 & 180 \\
\hline $00 ; 32 ; 30$ & 3 & -119 & 38 & 0 & 0.47 & 0.03 & 0.15 & 360 & -0.738 & -8.9 & 75.0 & 180 & 23 & 000 & 0.0 & 180 \\
\hline $00: 32: 40$ & 4 & -116 & 36 & 0 & 0.44 & 0.01 & 0.17 & 359 & -1.002 & -5.2 & 75.0 & 180 & 24 & 000 & 0.0 & 180 \\
\hline $00: 32: 50$ & 4 & -114 & 36 & 0 & 0.34 & -0.01 & 0.17 & 359 & -1.121 & -0.8 & 100.0 & 180 & 22 & 000 & 0.0 & 180 \\
\hline $00: 33: 00$ & 4 & -113 & 36 & 0 & 0.24 & 0.00 & 0.16 & 359 & -0.997 & 3.5 & 100.0 & 180 & 22 & 000 & 0.0 & 180 \\
\hline $00: 33: 10$ & 5 & -112 & 36 & 0 & 0.26 & 0.00 & 0.14 & 359 & -0.876 & 5.0 & 75.0 & 180 & 21 & 000 & 0.0 & 180 \\
\hline $00: 33: 20$ & 5 & -110 & 36 & 0 & 0.28 & 0.00 & 0.11 & 359 & -0.756 & 7.0 & 52.0 & 180 & 18 & 000 & 0.0 & 180 \\
\hline $00: 33: 30$ & 5 & -109 & 36 & 0 & 0.24 & 0.01 & 0.09 & 359 & -0.495 & 8.0 & 52.0 & 180 & 21 & 000 & 0.0 & 180 \\
\hline $00: 33: 40$ & 6 & -108 & 36 & 0 & 0.19 & 0.02 & 0.06 & 359 & -0.245 & 8.3 & 52.0 & 180 & 22 & 000 & 0.0 & 180 \\
\hline $00 \div 33 ; 50$ & 6 & -107 & 36 & 0 & 0.16 & 0.02 & 0.03 & 359 & -0.052 & 7.7 & 52.0 & 180 & 29 & 000 & 0.0 & 180 \\
\hline $00: 34: 00$ & 6 & -106 & 36 & 0 & 0.07 & 0.01 & 0.00 & 359 & 0.035 & 6.8 & 63.0 & 180 & 22 & 000 & 0.0 & 180 \\
\hline $00: 34: 10$ & 6 & -106 & 36 & 0 & 0.03 & 0.01 & 0.00 & 359 & 0.064 & -4.3 & 63.0 & 180 & 23 & 000 & 0.0 & 180 \\
\hline $00: 34: 20$ & 6 & -106 & 36 & 0 & -0.01 & -0.01 & 0.01 & 359 & -0.143 & 4.3 & 26.0 & 180 & 22 & 000 & 0.0 & 180 \\
\hline $00 \div 34: 30$ & 6 & -106 & 36 & 0 & -0.02 & -0.02 & 0.01 & 359 & -0.166 & 0.5 & 26.0 & 180 & 24 & 000 & 0.0 & 180 \\
\hline $00: 34: 40$ & 6 & -106 & 36 & 0 & -0.03 & -0.02 & 0.01 & 359 & -0.214 & -1.3 & 26.0 & 180 & 26 & 000 & 0.0 & 180 \\
\hline $00: 34: 50$ & 6 & -106 & 36 & 0 & 0.03 & -0.01 & 0.00 & 359 & -0.045 & 3.8 & 26.0 & 180 & 25 & 000 & 0.0 & 180 \\
\hline $00: 35: 00$ & 6 & -106 & 36 & 0 & 0.07 & -0.02 & 0.01 & 358 & -0.202 & -5.7 & 26.0 & 180 & 32 & 000 & 0.0 & 280 \\
\hline $9: 35: 10$ & 6 & -105 & 35 & 0 & 0.10 & -0.05 & 0.03 & 358 & -0.500 & -1.9 & 26.0 & 180 & 24 & 000 & 0.0 & 180 \\
\hline$: 35: 20$ & 6 & -105 & 36 & 0 & 0.13 & -0.08 & 0.03 & 358 & -0.682 & -0.3 & 26.0 & 180 & 26 & 000 & 0.0 & 180 \\
\hline $0: 35: 30$ & 5 & -104 & 36 & 0 & 0.10 & -0.08 & 0.03 & 358 & -0.672 & 3.6 & 26.0 & 180 & 24 & 000 & 0.0 & 180 \\
\hline $00: 35: 40$ & 5 & -104 & 36 & 0 & 0.08 & -0.09 & 0.01 & 358 & -0.620 & 6.4 & 26.0 & 180 & 21 & 000 & 0.0 & 180 \\
\hline $00: 35: 50$ & 5 & -103 & 36 & 0 & 0.12 & -0.11 & -0.01 & 358 & -0.624 & 5.7 & 51.0 & 180 & 17 & 000 & 0.0 & 180 \\
\hline $00: 36: 00$ & 5 & -103 & 36 & 0 & 0.13 & -0.11 & -0.03 & 358 & -0.565 & 6.2 & 63.0 & 180 & 19 & 000 & 0.0 & 180 \\
\hline $00: 36: 10$ & 4 & -102 & 36 & 0 & 0.17 & -0.13 & -0.04 & 358 & -0.553 & 6.9 & 75.0 & 180 & 23 & 000 & 0.0 & 180 \\
\hline $00: 36: 20$ & 4 & -101 & 36 & 0 & 0.07 & -0.13 & -0.07 & 358 & -0.391 & 8.4 & 100.0 & 180 & 25 & 000 & 0.0 & 180 \\
\hline $00: 36: 30$ & 3 & -101 & 36 & 0 & 0.03 & -0.12 & -0.09 & 358 & -0.191 & 7.7 & 52.0 & 180 & 21 & 000 & 0.0 & 180 \\
\hline $00: 36: 40$ & 3 & -101 & 36 & 0 & 0.06 & -0.15 & -0.11 & 358 & -0.284 & 8.5 & 52.0 & 180 & 22 & 000 & 0.0 & 180 \\
\hline $00: 36: 50$ & 2 & -101 & 36 & 0 & 0.00 & -0.13 & -0.14 & 358 & 0.014 & 6.7 & 52.0 & 180 & 21 & 000 & 0.0 & 180 \\
\hline $00: 37: 00$ & 1 & -101 & 35 & 0 & -0.12 & -0.07 & -0.17 & 358 & 0.648 & 1.9 & 52.0 & 180 & 18 & 000 & 0.0 & 180 \\
\hline $00: 37: 10$ & 1 & -102 & 36 & 0 & -0.17 & 0.06 & -0.20 & 358 & 1.632 & -7.5 & 52.0 & 180 & 26 & 000 & 0.0 & 180 \\
\hline $00: 37: 20$ & 1 & -103 & 36 & 0 & -0.12 & 0.16 & -0.18 & 358 & 2.175 & -23.7 & 52.0 & 180 & 25 & 000 & 0.0 & 180 \\
\hline $00: 37: 30$ & 1 & -103 & 36 & 0 & -0.05 & 0.24 & -0.11 & 359 & 2.223 & -35.0 & 52.0 & 180 & 28 & 000 & 0.0 & 180 \\
\hline $00: 37: 40$ & 1 & -103 & 36 & 0 & 0.05 & 0.28 & -0.02 & 359 & 1.903 & -32.7 & 52.0 & 180 & 21 & 000 & 0.0 & 180 \\
\hline $00: 37: 50$ & 2 & -103 & 36 & 0 & 0.15 & 0.29 & 0.07 & 359 & 1.386 & -28.6 & 52.0 & 180 & 26 & 000 & 0.0 & 180 \\
\hline
\end{tabular}


Open Ocean

\section{Simulation Track Plot}

Ship: 150,000 ton, Tanker, partial load
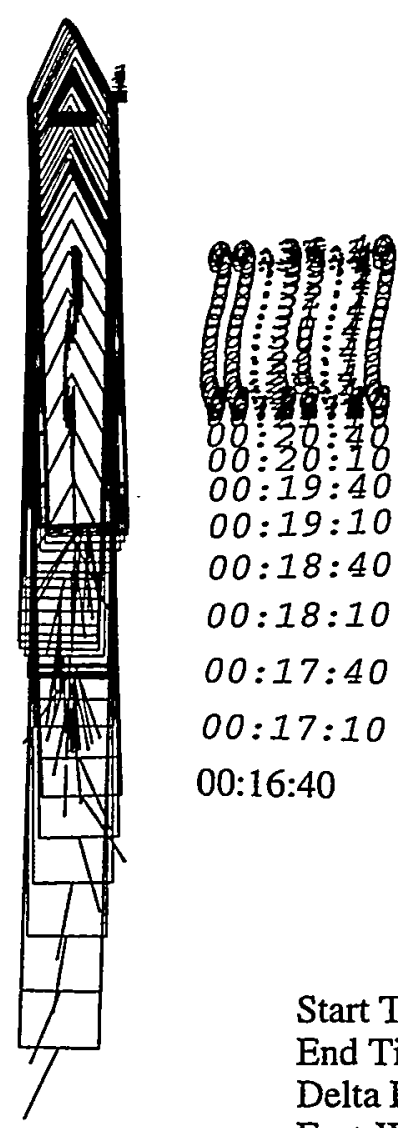

Start Time [hrs:min:sec]: $00: 16: 40$

End Time [hrs:min:sec]: $00: 38: 00$ Delta Plot Time [sec]:

East-West Scale [m]:

30

Date: 04-Feb-99 Time: 08:50:07 


\section{HANDS-ON SIMULATION}

\section{SUCTION MOORNG TECHNOLOGY}

Simulation Number: 21

Start Time: 08:39

Date: 4 February 1999

Length of run [secs]: 3664

Ship Condition: draft $=13.72 \mathrm{~m}$

Environment

Wind Speed [knots]:

25 Direction: 45

Sea State, Significant wave height [m]:

5 Direction: 225

Current Speed [knots]:

1 Direction: 180

Ship Operator: J.A.

Observer: B.K.J \& J.K.

Final Mating, distance between center of buoy and center of moon pool [m]: 2.5

Evaluation:

The approach and deceleration were no problem.

The captain used long time on final mating, part of it was learning time. It was not possible to get a steady longitudinal thrust balance. 
Ship:

Exercise number:

Genario: g Eile:

Initial conditions:

Exercise identification
591 150,000 ton, Tanker, partial load

54 Suction Mooring system

112 Open Ocean

999 Dummy tug file based on containership

590 Suction Mooring Buoy

594 150k tanker in partial load - Suction Mooring RuN \#21

Time Position (m) RPM Speed (Knots) Turn Rudder Gill Wind Current

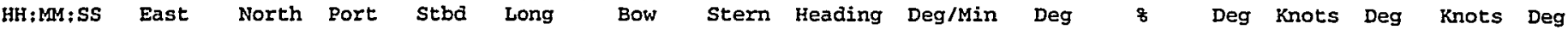

\begin{tabular}{|c|c|c|c|c|c|c|c|c|c|c|c|c|c|c|c|c|}
\hline $\begin{array}{l}00: 33: 20 \\
00: 33: 30\end{array}$ & $\begin{array}{l}-58 \\
-58\end{array}$ & $\begin{array}{r}-100 \\
-99\end{array}$ & $\begin{array}{l}25 \\
25\end{array}$ & $\begin{array}{l}0 \\
0\end{array}$ & $\begin{array}{l}0.23 \\
0.14\end{array}$ & $\begin{array}{r}0.01 \\
-0.01\end{array}$ & $\begin{array}{l}-0.24 \\
-0.24\end{array}$ & $\begin{array}{l}041 \\
041\end{array}$ & $\begin{array}{l}1.573 \\
1.460\end{array}$ & $\begin{array}{l}18.2 \\
11.4\end{array}$ & $\begin{array}{r}100.0 \\
75.0\end{array}$ & $\begin{array}{l}180 \\
180\end{array}$ & $\begin{array}{l}18 \\
21\end{array}$ & $\begin{array}{l}045 \\
045\end{array}$ & $\begin{array}{l}1.0 \\
1.0\end{array}$ & $\begin{array}{l}180 \\
180\end{array}$ \\
\hline $00: 33: 40$ & -58 & -98 & 32 & 0 & 0.03 & -0.01 & -0.22 & 041 & 1.327 & 3.4 & 50.0 & 180 & 22 & 045 & 1.0 & 180 \\
\hline $00: 33: 50$ & -58 & -98 & 33 & 0 & -0.01 & -0.01 & -0.19 & 041 & 1.117 & -0.2 & 50.0 & 180 & 29 & 045 & 1.0 & 180 \\
\hline $00 ; 34 ; 00$ & -59 & -97 & 38 & 0 & -0.05 & -0.01 & -0.15 & 041 & 0.882 & -1.4 & 27.0 & 180 & 22 & 045 & 1.0 & 180 \\
\hline $00: 34: 10$ & -59 & -97 & 39 & 0 & -0.05 & 0.00 & -0.10 & 042 & 0.629 & -2.9 & 27.0 & 180 & 23 & 045 & 1.0 & 180 \\
\hline $00: 34: 20$ & -60 & -97 & 39 & 0 & -0.02 & 0.01 & -0.05 & 042 & 0.411 & -8.3 & 27.0 & 180 & 22 & 045 & 1.0 & 180 \\
\hline $00: 34: 30$ & -60 & -97 & 39 & 0 & 0.05 & 0.01 & 0.02 & 042 & -0.042 & -4.5 & 17.0 & 180 & 24 & 045 & 1.0 & 180 \\
\hline $00: 34: 40$ & -59 & -97 & 39 & 0 & 0.08 & 0.01 & 0.03 & 042 & -0.084 & 6.8 & 17.0 & 180 & 26 & 045 & 1.0 & 180 \\
\hline $00 ; 34: 50$ & -59 & -97 & 39 & 0 & 0.13 & -0.01 & 0.02 & 042 & -0.162 & 7.7 & 17.0 & 180 & 25 & 045 & 1.0 & 180 \\
\hline $00: 35: 00$ & -58 & -96 & 39 & 0 & 0.14 & $=0.01$ & 0.01 & 042 & 0.007 & 6.2 & 17.0 & 180 & 32 & 045 & 1.0 & 180 \\
\hline $00 ; 35: 10$ & -58 & -96 & 39 & 0 & 0.09 & 0.02 & 0.01 & 042 & 0.087 & 5.0 & 27.0 & 180 & 24 & 045 & 1.0 & 180 \\
\hline $00: 35: 20$ & -58 & -96 & 39 & 0 & 0.13 & 0.00 & 0.01 & 042 & -0.035 & 6.5 & 17.0 & 180 & 26 & 045 & 1.0 & 180 \\
\hline $00: 35: 30$ & -57 & -95 & 39 & 0 & 0.15 & 0.01 & 0.01 & 042 & 0.018 & 6.1 & 17.0 & 180 & 24 & 045 & 1.0 & 180 \\
\hline $00: 35: 40$ & -56 & -94 & 38 & 0 & 0.22 & 0.01 & 0.00 & 042 & 0.024 & 2.1 & 27.0 & 180 & 21 & 045 & 1.0 & 180 \\
\hline $00: 35: 50$ & -56 & -93 & 39 & 0 & 0.21 & -0.02 & 0.05 & 042 & -0.472 & -16.9 & 50.0 & 180 & 17 & 045 & 1.0 & 180 \\
\hline $00: 36: 00$ & -55 & -93 & 39 & 0 & 0.14 & -0.04 & 0.15 & 041 & -1.241 & -17.6 & 36.0 & 180 & 19 & 045 & 1.0 & 180 \\
\hline $00: 36: 10$ & -54 & -93 & 39 & 0 & 0.19 & -0.11 & 0.22 & 041 & -2.084 & -9.4 & 36.0 & 180 & 23 & 045 & 1.0 & 180 \\
\hline $00: 36: 20$ & -53 & -92 & 39 & 0 & 0.18 & -0.16 & 0.24 & 041 & -2.526 & 0.6 & 36.0 & 180 & 25 & 045 & 1.0 & 180 \\
\hline $00: 36: 30$ & -53 & -91 & 39 & 0 & 0.10 & -0.17 & 0.21 & 040 & -2.420 & 17.0 & 45.0 & 180 & 21 & 045 & 1.0 & 180 \\
\hline $00: 36: 40$ & -52 & -91 & 39 & 0 & 0.00 & -0.08 & 0.11 & 040 & -1.165 & 35.0 & 45.0 & 180 & 22 & 045 & 1.0 & 180 \\
\hline $00: 36: 50$ & -52 & -91 & 39 & 0 & 0.00 & 0.01 & -0.01 & 040 & 0.124 & 25.5 & 45.0 & 180 & 21 & 045 & 1.0 & 180 \\
\hline $00: 37: 00$ & -52 & -91 & 39 & 0 & -0.01 & 0.05 & -0.05 & 040 & 0.583 & 6.5 & 45.0 & 180 & 18 & 045 & 1.0 & 180 \\
\hline $\begin{array}{l}00: 37: 10 \\
00: 37: 20\end{array}$ & $\begin{array}{l}-52 \\
-52\end{array}$ & $\begin{array}{l}-91 \\
-91\end{array}$ & $\begin{array}{l}39 \\
38\end{array}$ & $\begin{array}{l}0 \\
0\end{array}$ & $\begin{array}{l}0.02 \\
0.04\end{array}$ & $\begin{array}{l}0.05 \\
0.04\end{array}$ & $\begin{array}{r}-0.01 \\
0.05\end{array}$ & $\begin{array}{l}040 \\
040\end{array}$ & $\begin{array}{r}0.336 \\
-0.011\end{array}$ & $\begin{array}{r}-12.5 \\
-3.2\end{array}$ & $\begin{array}{l}52.0 \\
52.0\end{array}$ & $\begin{array}{l}180 \\
180\end{array}$ & $\begin{array}{l}26 \\
25\end{array}$ & $\begin{array}{l}045 \\
045\end{array}$ & $\begin{array}{l}1.0 \\
1.0\end{array}$ & $\begin{array}{l}180 \\
180\end{array}$ \\
\hline $00: 37: 30$ & -52 & -91 & 39 & 0 & 0.06 & 0.04 & 0.07 & 040 & -0.185 & -1.0 & 62.0 & 180 & 28 & 045 & 1.0 & $\begin{array}{l}180 \\
180\end{array}$ \\
\hline $00: 37: 40$ & -51 & -91 & 39 & 0 & 0.04 & 0.01 & 0.08 & 040 & -0.446 & 6.9 & 62.0 & 180 & 21 & 045 & 1.0 & 180 \\
\hline $00: 37: 50$ & -51 & -91 & 39 & 0 & 0.05 & 0.02 & 0.07 & 040 & -0.345 & 5.2 & 62.0 & 180 & 26 & 045 & 1.0 & 180 \\
\hline $0: 38: 00$ & -51 & -91 & 39 & 0 & 0.03 & 0.01 & 0.07 & 040 & -0.348 & 3.9 & 62.0 & 180 & 28 & 045 & 1.0 & 180 \\
\hline$: 38: 10$ & -50 & -91 & 39 & 0 & 0.05 & -0.07 & 0.08 & 040 & -0.944 & 4.7 & 62.0 & 180 & 24 & 045 & 1.0 & 180 \\
\hline $00: 38: 20$ & -50 & -91 & 39 & 0 & 0.06 & -0.08 & 0.08 & 040 & -0.988 & 5.9 & 65.0 & 180 & 26 & 045 & 1.0 & 180 \\
\hline $00: 38: 30$ & -50 & -91 & 39 & 0 & 0.04 & -0.02 & 0.06 & 040 & -0.555 & 5.8 & 65.0 & 180 & 23 & 045 & 1.0 & 180 \\
\hline $00: 38: 40$ & -50 & -91 & 39 & 0 & 0.08 & -0.05 & 0.07 & 039 & -0.746 & 2.3 & 70.0 & 180 & 22 & 045 & 1.0 & 180 \\
\hline $00: 38: 50$ & -50 & -90 & 39 & 0 & 0.06 & -0.09 & 0.06 & 039 & -0.943 & 11.4 & 77.0 & 180 & 21 & 045 & 1.0 & 180 \\
\hline $00: 39: 00$ & -50 & -90 & 38 & 0 & 0.06 & -0.13 & 0.03 & 039 & -0.987 & 11.9 & 77.0 & 180 & 23 & 045 & 1.0 & 180 \\
\hline $00: 39: 10$ & -50 & -90 & 38 & 0 & 0.01 & -0.16 & 0.00 & 039 & -1.011 & 14.2 & 77.0 & 180 & 24 & 045 & 1.0 & 180 \\
\hline $00: 39: 20$ & -50 & -90 & 39 & 0 & -0.13 & -0.14 & -0.02 & 039 & -0.759 & 8.1 & 66.0 & 180 & 24 & 045 & 1.0 & 180 \\
\hline $00: 39: 30$ & -51 & -90 & 39 & 0 & -0.19 & -0.17 & -0.03 & 039 & -0.901 & 9.5 & 66.0 & 180 & 20 & 045 & 1.0 & 180 \\
\hline $00: 39: 40$ & -52 & -90 & 39 & 0 & -0.19 & -0.17 & -0.04 & 039 & -0.807 & 11.8 & 66.0 & 180 & 29 & 045 & 1.0 & 180 \\
\hline $00: 39: 50$ & -53 & -91 & 39 & 0 & -0.18 & -0.17 & -0.06 & 038 & -0.671 & 13.3 & 66.0 & 180 & 23 & 045 & 1.0 & 180 \\
\hline $00: 40: 00$ & -54 & -91 & 39 & 0 & -0.24 & -0.10 & -0.08 & 038 & -0.094 & 11.8 & 0.0 & 180 & 21 & 045 & 1.0 & 180 \\
\hline $00: 40: 10$ & -55 & -92 & 39 & 0 & -0.20 & -0.03 & -0.09 & 038 & 0.363 & 8.2 & 0.0 & 180 & 20 & 045 & 1.0 & 180 \\
\hline $00: 40: 20$ & -56 & -93 & 39 & 0 & -0.15 & -0.03 & -0.09 & 038 & 0.386 & 6.3 & 0.0 & 180 & 20 & 045 & 1.0 & 180 \\
\hline $00: 40: 30$ & -57 & -93 & 39 & 0 & -0.12 & -0.07 & -0.08 & 039 & 0.081 & 7.6 & 0.0 & 180 & 24 & 045 & 1.0 & 180 \\
\hline $00: 40: 40$ & -57 & -93 & 38 & 0 & -0.12 & -0.11 & -0.08 & 039 & -0.177 & 9.8 & 0.0 & 180 & 24 & 045 & 1.0 & 180 \\
\hline $00: 40: 50$ & -58 & -93 & 38 & 0 & -0.11 & -0.07 & -0.09 & 039 & 0.114 & 8.0 & 0.0 & 180 & 29 & 045 & 1.0 & 180 \\
\hline $00: 41: 00$ & -59 & -93 & 39 & 0 & -0.10 & -0.11 & -0.09 & 039 & -0.136 & 9.5 & 0.0 & 180 & 22 & 045 & 1.0 & 180 \\
\hline $00: 41: 10$ & -60 & -93 & 39 & 0 & -0.05 & -0.12 & -0.10 & 038 & -0.093 & 10.0 & 0.0 & 180 & 20 & 045 & 1.0 & 180 \\
\hline $00: 41: 20$ & -60 & -93 & 39 & 0 & -0.01 & -0.07 & -0.10 & 039 & 0.212 & 6.2 & 0.0 & 180 & 18 & 045 & 1.0 & 180 \\
\hline $00: 41: 30$ & -60 & -93 & 42 & 0 & 0.08 & -0.10 & -0.10 & 039 & -0.008 & 8.4 & 0.0 & 180 & 24 & 045 & 1.0 & 180 \\
\hline $00: 41: 40$ & -60 & -92 & 41 & 0 & 0.14 & -0.17 & -0.12 & 038 & -0.313 & 10.9 & 0.0 & 180 & 25 & 045 & 1.0 & 180 \\
\hline $00: 41: 50$ & -61 & -91 & 41 & 0 & 0.09 & -0.16 & -0.15 & 038 & -0.046 & 19.6 & 0.0 & 180 & 24 & 045 & 1.0 & 180 \\
\hline $00 ; 42: 00$ & -61 & -90 & 41 & 0 & 0.09 & -0.14 & -0.23 & 039 & 0.571 & 23.8 & 0.0 & 180 & 27 & 045 & 1.0 & 180 \\
\hline $00: 42: 10$ & -62 & -89 & 44 & 0 & 0.07 & -0.14 & -0.29 & 039 & 0.965 & 19.7 & 0.0 & 180 & 28 & 045 & 1.0 & 180 \\
\hline $00: 42: 20$ & -62 & -88 & 44 & 0 & 0.05 & -0.18 & -0.33 & 039 & 0.984 & 17.5 & 0.0 & 180 & 23 & 045 & 1.0 & 180 \\
\hline $00: 42: 30$ & -63 & -87 & 44 & 0 & 0.07 & -0.16 & -0.36 & 039 & 1.241 & 13.1 & 0.0 & 180 & 27 & 045 & 1.0 & 180 \\
\hline $00: 42: 40$ & -64 & -86 & 44 & 0 & 0.04 & -0.07 & -0.37 & 039 & 1.916 & 19.7 & 0.0 & 180 & 26 & 045 & 1.0 & 180 \\
\hline $00: 42: 50$ & -65 & -85 & 47 & 0 & 0.05 & 0.00 & -0.40 & 040 & 2.495 & 11.0 & 0.0 & 180 & 20 & 045 & 1.0 & 180 \\
\hline $00: 43: 00$ & -65 & -84 & 47 & 0 & 0.10 & 0.01 & -0.37 & 040 & 2.406 & 3.7 & 0.0 & 180 & 25 & 045 & 1.0 & 180 \\
\hline $00: 43: 10$ & -65 & -83 & 46 & 0 & 0.22 & -0.01 & -0.33 & 040 & 2.020 & 2.0 & 0.0 & 180 & 20 & 045 & 1.0 & 180 \\
\hline $00: 43: 20$ & -65 & -82 & 44 & 0 & 0.17 & -0.02 & -0.28 & 041 & 1.633 & -2.8 & 75.0 & 180 & 28 & 045 & 1.0 & 180 \\
\hline $00: 43: 30$ & -65 & -81 & 44 & 0 & 0.14 & -0.04 & -0.20 & 041 & 1.048 & -5.7 & 54.0 & 180 & 20 & 045 & 1.0 & 180 \\
\hline $00: 43: 40$ & -65 & -80 & 44 & 0 & 0.14 & -0.05 & -0.14 & 041 & 0.540 & -5.0 & 54.0 & 180 & 17 & 045 & 1.0 & 180 \\
\hline $00: 43: 50$ & -65 & -79 & 44 & 0 & 0.21 & -0.06 & -0.08 & 041 & 0.147 & -1.1 & 54.0 & 180 & 24 & 045 & 1.0 & 180 \\
\hline $00: 44: 00$ & -64 & -78 & 39 & 0 & 0.24 & -0.07 & -0.10 & 041 & 0.176 & 15.0 & 100.0 & 180 & 19 & 045 & 1.0 & 180 \\
\hline $00: 44: 10$ & -64 & -76 & 34 & 0 & 0.20 & 0.00 & -0.12 & 041 & 0.769 & 10.4 & 100.0 & 180 & 22 & 045 & 1.0 & 180 \\
\hline $00: 44: 20$ & -64 & -76 & 34 & 0 & 0.17 & 0.02 & -0.12 & 041 & 0.901 & 5.8 & 100.0 & 180 & 24 & 045 & 1.0 & 180 \\
\hline$: 44: 30$ & -63 & -75 & 29 & 0 & 0.17 & 0.01 & -0.11 & 041 & 0.755 & 4.0 & 100.0 & 180 & 21 & 045 & 1.0 & 180 \\
\hline$: 44: 40$ & -63 & -74 & 29 & 0 & 0.06 & 0.00 & -0.09 & 042 & 0.560 & -1.0 & 100.0 & 180 & 20 & 045 & 1.0 & 180 \\
\hline $00: 44: 50$ & -63 & -74 & 29 & 0 & 0.01 & -0.03 & -0.06 & 042 & 0.211 & 0.7 & 51.0 & 180 & 25 & 045 & 1.0 & 180 \\
\hline $00: 45: 00$ & -63 & -74 & 29 & 0 & -0.03 & -0.07 & -0.05 & 042 & -0.099 & 7.4 & 74.0 & 180 & 27 & 045 & 1.0 & 180 \\
\hline $00: 45: 10$ & -64 & -74 & 29 & 0 & -0.17 & -0.08 & -0.04 & 042 & -0.202 & 7.5 & 51.0 & 180 & 32 & 045 & 1.0 & 180 \\
\hline $00: 45: 20$ & -65 & -74 & 29 & 0 & -0.24 & -0.07 & -0.03 & 042 & -0.203 & 7.7 & 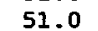 & 180 & 5 & 045 & 1.0 & 180 \\
\hline
\end{tabular}




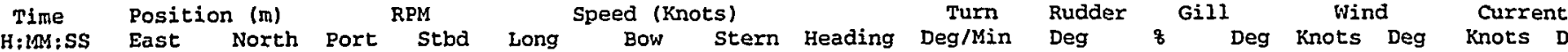

\begin{tabular}{|c|c|c|c|c|c|c|c|c|c|c|c|c|c|c|c|c|}
\hline $\begin{array}{l}0: 45: 30 \\
: 45: 40\end{array}$ & $\begin{array}{l}-66 \\
-67\end{array}$ & $\begin{array}{l}-75 \\
-76\end{array}$ & $\begin{array}{l}29 \\
29\end{array}$ & $\begin{array}{l}0 \\
0\end{array}$ & $\begin{array}{l}-0.28 \\
-0.28\end{array}$ & $\begin{array}{l}-0.03 \\
-0.03\end{array}$ & $\begin{array}{l}-0.03 \\
-0.01\end{array}$ & $\begin{array}{l}042 \\
042\end{array}$ & $\begin{array}{l}-0.038 \\
-0.126\end{array}$ & $\begin{array}{l}7.5 \\
7.8\end{array}$ & $\begin{array}{l}0.0 \\
0.0\end{array}$ & $\begin{array}{l}180 \\
180\end{array}$ & $\begin{array}{l}25 \\
24\end{array}$ & $\begin{array}{l}045 \\
045\end{array}$ & $\begin{array}{l}1.0 \\
1.0\end{array}$ & $\begin{array}{l}180 \\
180\end{array}$ \\
\hline$: 45: 50$ & -68 & -77 & 29 & 0 & -0.29 & -0.04 & -0.01 & 042 & -0.237 & 8.8 & 0.0 & 180 & 22 & 045 & 1.0 & 180 \\
\hline $00 ; 46 ; 00$ & -69 & -79 & 43 & 0 & -0.31 & -0.01 & 0.00 & 042 & -0.051 & 8.7 & 0.0 & 180 & 22 & 045 & 1.0 & 180 \\
\hline $00: 46: 10$ & -70 & -80 & 53 & 0 & -0.29 & 0.03 & -0.01 & 042 & 0.269 & 10.4 & 0.0 & 180 & 26 & 045 & 1.0 & 180 \\
\hline $00: 46: 20$ & -71 & -81 & 48 & 0 & -0.27 & 0.07 & -0.09 & 042 & 1.051 & 19.7 & 0.0 & 180 & 25 & 045 & 1.0 & 180 \\
\hline $00: 46: 30$ & -72 & -82 & 39 & 0 & -0.17 & 0.09 & -0.12 & 042 & 1.326 & 6.3 & 0.0 & 180 & 19 & 045 & 1.0 & 180 \\
\hline $0: 46: 40$ & -72 & -82 & 31 & 0 & -0.19 & 0.08 & -0.08 & 042 & 1.046 & -7.7 & 28.0 & 180 & 28 & 045 & 1.0 & 180 \\
\hline $00: 46: 50$ & -73 & -83 & 39 & 0 & -0.19 & 0.08 & -0.02 & 042 & 0.640 & -7.4 & 28.0 & 180 & 20 & 045 & 1.0 & 180 \\
\hline $00: 47: 00$ & -73 & -84 & 55 & 0 & -0.14 & 0.05 & 0.05 & 042 & -0.017 & -4.5 & 28.0 & 180 & 21 & 045 & 1.0 & 180 \\
\hline $00: 47: 10$ & -73 & -84 & 57 & 0 & 0.03 & 0.05 & 0.11 & 042 & -0.339 & 0.8 & 28.0 & 180 & 19 & 045 & 1.0 & 180 \\
\hline $00: 47: 20$ & -73 & -84 & 48 & 0 & 0.20 & 0.01 & 0.13 & 042 & -0.733 & 1.8 & 28.0 & 180 & 19 & 045 & 1.0 & 180 \\
\hline $00: 47: 30$ & -72 & -83 & 44 & 0 & 0.22 & 0.01 & 0.13 & 042 & -0.745 & 3.8 & 52.0 & 180 & 28 & 045 & 1.0 & 180 \\
\hline $00: 47: 40$ & -71 & -83 & 42 & 0 & 0.17 & 0.01 & 0.12 & 042 & -0.697 & 6.9 & 52.0 & 180 & 25 & 045 & 1.0 & 180 \\
\hline $00: 47: 50$ & -70 & -82 & 41 & 0 & 0.19 & 0.01 & 0.10 & 042 & -0.597 & 7.6 & 52.0 & 180 & 24 & 045 & 1.0 & 180 \\
\hline $00: 48: 00$ & -69 & -82 & 42 & 0 & 0.17 & 0.00 & 0.08 & 042 & -0.481 & 8.9 & 25.0 & 180 & 28 & 045 & 1.0 & 180 \\
\hline $00: 48: 10$ & -68 & -81 & 44 & 0 & 0.14 & 0.02 & 0.05 & 042 & -0.245 & 8.8 & 25.0 & 180 & 19 & 045 & 1.0 & 180 \\
\hline $00: 48: 20$ & -68 & -81 & 44 & 0 & 0.19 & 0.04 & 0.04 & 042 & 0.005 & 7.3 & 17.0 & 180 & 20 & 045 & 1.0 & 180 \\
\hline $00: 48: 30$ & -67 & -80 & 44 & 0 & 0.21 & 0.06 & 0.02 & 042 & 0.230 & 5.5 & 28.0 & 180 & 29 & 045 & 1.0 & 180 \\
\hline $00: 48: 40$ & -66 & -80 & 44 & 0 & 0.21 & 0.07 & 0.02 & 042 & 0.288 & 4.6 & 49.0 & 180 & 20 & 045 & 1.0 & 180 \\
\hline $00: 48: 50$ & -65 & -79 & 44 & 0 & 0.18 & 0.08 & 0.04 & 042 & 0.280 & -6.4 & 49.0 & 180 & 26 & 045 & 1.0 & 180 \\
\hline $00: 49: 00$ & -64 & -79 & 43 & 0 & 0.19 & 0.04 & 0.12 & 042 & -0.475 & -10.3 & 61.0 & 180 & 20 & 045 & 1.0 & 180 \\
\hline $00: 49: 10$ & -63 & -78 & 43 & 0 & 0.19 & -0.01 & 0.17 & 042 & -1.157 & -3.8 & 61.0 & 180 & 24 & 045 & 1.0 & 180 \\
\hline $00: 49: 20$ & -62 & -78 & 43 & 0 & 0.15 & -0.03 & 0.18 & 041 & -1.346 & 3.1 & 61.0 & 180 & 26 & 045 & 1.0 & 180 \\
\hline $00: 49: 30$ & -62 & -78 & 43 & 0 & 0.14 & 0.03 & 0.17 & 041 & -0.905 & 3.5 & 78.0 & 180 & 24 & 045 & 1.0 & 180 \\
\hline $00: 49: 40$ & -61 & -77 & 43 & 0 & 0.12 & 0.07 & 0.17 & 041 & -0.645 & -0.3 & 78.0 & 180 & 26 & 045 & 1.0 & 180 \\
\hline $00: 49: 50$ & -60 & -77 & 43 & 0 & 0.22 & 0.01 & 0.23 & 041 & -1.359 & -9.5 & 90.0 & 180 & 25 & 045 & 1.0 & 180 \\
\hline $00: 50: 00$ & -58 & -77 & 33 & 0 & 0.22 & -0.01 & 0.27 & 041 & -1.763 & -3.2 & 100.0 & 180 & 22 & 045 & 1.0 & 180 \\
\hline $00 ; 50: 10$ & -57 & -76 & 31 & 0 & 0.20 & 0.00 & 0.27 & 040 & -1.747 & 2.7 & 100.0 & 180 & 22 & 045 & 1.0 & 180 \\
\hline $00: 50: 20$ & -56 & -76 & 31 & 0 & 0.12 & -0.03 & 0.26 & 040 & -1.818 & 12.1 & 100.0 & 180 & 29 & 045 & 1.0 & 180 \\
\hline $00: 50: 30$ & -55 & -76 & 36 & 0 & 0.03 & -0.01 & 0.20 & 040 & -1.352 & 20.3 & 100.0 & 180 & 21 & 045 & 1.0 & 180 \\
\hline $00: 50: 40$ & -55 & -76 & 36 & 0 & 0.03 & -0.06 & 0.12 & 040 & -1.137 & 35.0 & 75.0 & 180 & 23 & 045 & 1.0 & 180 \\
\hline $00: 50: 50$ & -55 & -76 & 32 & 0 & -0.02 & -0.04 & 0.03 & 039 & -0.428 & 35.0 & 75.0 & 180 & 25 & 045 & 1.0 & 180 \\
\hline $00: 51: 00$ & -55 & -76 & 32 & 0 & -0.14 & -0.04 & -0.02 & 039 & -0.077 & 16.6 & 75.0 & 180 & 24 & 045 & 1.0 & 180 \\
\hline $00: 51: 10$ & -56 & -77 & 32 & 0 & -0.19 & -0.03 & -0.03 & 039 & 0.026 & 11.1 & 75.0 & 180 & 22 & 045 & 1.0 & 180 \\
\hline $00: 51: 20$ & -57 & -78 & 33 & 0 & -0.19 & -0.02 & -0.04 & 039 & 0.116 & 20.5 & 75.0 & 180 & 20 & 045 & 1.0 & 180 \\
\hline $00: 51: 30$ & -58 & -78 & 33 & 0 & -0.23 & -0.04 & -0.04 & 039 & 0.003 & 10.7 & 26.0 & 180 & 25 & 045 & 1.0 & 180 \\
\hline $00: 51: 40$ & -59 & -79 & 43 & 0 & -0.24 & -0.06 & -0.05 & 039 & -0.087 & 11.7 & 26.0 & 180 & 21 & 045 & 1.0 & 180 \\
\hline $0: 51: 50$ & -60 & -80 & 43 & 0 & -0.26 & -0.05 & -0.06 & 039 & 0.062 & 11.0 & 0.0 & 180 & 20 & 045 & 1.0 & 180 \\
\hline$: 52: 00$ & -61 & -81 & 43 & 0 & -0.25 & -0.06 & -0.07 & 039 & 0.060 & 10.4 & 0.0 & 180 & 24 & 045 & 1.0 & 180 \\
\hline$-0: 52: 10$ & -62 & -82 & 42 & 0 & -0.30 & -0.06 & -0.08 & 039 & 0.126 & 10.2 & 0.0 & 180 & 34 & 045 & 1.0 & 180 \\
\hline $00: 52: 20$ & -63 & -83 & 47 & 0 & -0.29 & -0.03 & -0.09 & 039 & 0.392 & 8.6 & 0.0 & 180 & 19 & 045 & 1.0 & 180 \\
\hline $00: 52: 30$ & -64 & -84 & 50 & 0 & -0.19 & 0.00 & -0.08 & 040 & 0.541 & 5.2 & 0.0 & 180 & 25 & 045 & 1.0 & 180 \\
\hline $00: 52: 40$ & -65 & -84 & 49 & 0 & -0.08 & -0.03 & -0.07 & 040 & 0.250 & 4.3 & 0.0 & 180 & 25 & 045 & 1.0 & 180 \\
\hline $00: 52: 50$ & -65 & -84 & 50 & 0 & -0.01 & 0.00 & -0.04 & 040 & 0.240 & -3.9 & 25.0 & 180 & 23 & 045 & 1.0 & 180 \\
\hline $00 ; 53: 00$ & -65 & -84 & 49 & 0 & 0.03 & 0.00 & 0.04 & 040 & -0.244 & -3.8 & 25.0 & 180 & 28 & 045 & 1.0 & 180 \\
\hline $00: 53: 10$ & -65 & -84 & 50 & 0 & 0.17 & -0.08 & 0.09 & 040 & -1.064 & -2.3 & 25.0 & 180 & 26 & 045 & 1.0 & 180 \\
\hline $00: 53: 20$ & -64 & -83 & 49 & 0 & 0.22 & -0.16 & 0.11 & 039 & -1.736 & 3.6 & 73.0 & 180 & 26 & 045 & 1.0 & 180 \\
\hline $00: 53: 30$ & -63 & -82 & 50 & 0 & 0.23 & -0.19 & 0.08 & 039 & -1.701 & 8.5 & 73.0 & 180 & 27 & 045 & 1.0 & 180 \\
\hline $00: 53: 40$ & -63 & -81 & 49 & 0 & 0.24 & -0.17 & 0.03 & 039 & -1.325 & 10.9 & 85.0 & 180 & 21 & 045 & 1.0 & 180 \\
\hline $00: 53: 50$ & -62 & -79 & 50 & 0 & 0.20 & -0.12 & -0.02 & 039 & -0.637 & 10.9 & 85.0 & 180 & 23 & 045 & 1.0 & 180 \\
\hline $00: 54: 00$ & -62 & -78 & 49 & 0 & 0.27 & -0.16 & -0.07 & 038 & -0.594 & 11.4 & 79.0 & 180 & 23 & 045 & 1.0 & 180 \\
\hline $00: 54: 10$ & -62 & -77 & 48 & 0 & 0.32 & -0.14 & -0.11 & 038 & -0.132 & 11.2 & 95.0 & 180 & 14 & 045 & 1.0 & 180 \\
\hline $00 ; 54: 20$ & -61 & -75 & 45 & 0 & 0.29 & -0.12 & -0.15 & 038 & 0.198 & 9.4 & 95.0 & 180 & 22 & 045 & 2.0 & 180 \\
\hline $00: 54: 30$ & -61 & -74 & 29 & 0 & 0.17 & -0.13 & -0.16 & .038 & 0.145 & 8.8 & 95.0 & 180 & 22 & 045 & 1.0 & 180 \\
\hline $00: 54 ; 40$ & -61 & -73 & 29 & 0 & 0.07 & -0.13 & -0.17 & 038 & 0.258 & 23.8 & 75.0 & 180 & 22 & 045 & 1.0 & 180 \\
\hline $00: 54: 50$ & -62 & -72 & 29 & 0 & -0.02 & -0.13 & -0.20 & 039 & 0.451 & 22.9 & 95.0 & 180 & 21 & 045 & 1.0 & 180 \\
\hline $00: 55: 00$ & -63 & -72 & 29 & 0 & -0.12 & -0.13 & -0.24 & 039 & 0.700 & 35.0 & 95.0 & 180 & 24 & 045 & 1.0 & 180 \\
\hline $00: 55: 10$ & -64 & -72 & 29 & 0 & -0.25 & -0.09 & -0.28 & 039 & 1.211 & 35.0 & 44.0 & 180 & 22 & 045 & 1.0 & 180 \\
\hline $00: 55: 20$ & -66 & -72 & 29 & 0 & -0.26 & -0.01 & -0.32 & 039 & 1.949 & 27.5 & 44.0 & 180 & 26 & 045 & 1.0 & 180 \\
\hline $00: 55: 30$ & -67 & -73 & 29 & 0 & -0.27 & 0.05 & -0.34 & 039 & 2.451 & 35.0 & 21.0 & 180 & 27 & 045 & 1.0 & 180 \\
\hline $00: 55: 40$ & -68 & -74 & 29 & 0 & -0.26 & 0.09 & -0.36 & 040 & 2.877 & 26.9 & 0.0 & 180 & 23 & 045 & 1.0 & 180 \\
\hline $00: 55: 50$ & -70 & -74 & 36 & 0 & -0.25 & 0.14 & -0.36 & 040 & 3.194 & 15.3 & 0.0 & 180 & 26 & 045 & 1.0 & 180 \\
\hline $00: 56: 00$ & -71 & -75 & 36 & 0 & -0.17 & 0.13 & -0.37 & 041 & 3.122 & 29.5 & 0.0 & 180 & 16 & 045 & 1.0 & 180 \\
\hline $00: 56: 10$ & -72 & -75 & 37 & 0 & -0.17 & 0.14 & -0.41 & 041 & 3.479 & 27.1 & 0.0 & 180 & 26 & 045 & 1.0 & 180 \\
\hline $00: 56: 20$ & -73 & -75 & 37 & 0 & -0.26 & 0.18 & -0.41 & 042 & 3.749 & 14.6 & 0.0 & 180 & 29 & 045 & 1.0 & 180 \\
\hline $00: 56: 30$ & -74 & -76 & 40 & 0 & -0.21 & 0.16 & -0.35 & 043 & 3.252 & -4.4 & 0.0 & 180 & 23 & 045 & 1.0 & 180 \\
\hline $00: 56: 40$ & -75 & -76 & 40 & 0 & -0.15 & 0.10 & -0.23 & 043 & 2.062 & -16.8 & 0.0 & 180 & 26 & 045 & 1.0 & 180 \\
\hline $00: 56: 50$ & -76 & -76 & 40 & 0 & -0.12 & 0.04 & -0.10 & 043 & 0.907 & -14.8 & 0.0 & 180 & 24 & 045 & 1.0 & 180 \\
\hline $00: 57: 00$ & -76 & -77 & 41 & 0 & -0.09 & 0.00 & -0.01 & 043 & 0.086 & -9.2 & 0.0 & 180 & 17 & 045 & 1.0 & 180 \\
\hline $00: 57: 10$ & -77 & -77 & 42 & 0 & -0.10 & 0.01 & 0.05 & 043 & -0.300 & -3.9 & 0.0 & 180 & 24 & 045 & 1.0 & 180 \\
\hline $00: 57: 20$ & -77 & -78 & 42 & 0 & -0.07 & -0.04 & 0.13 & 043 & -1.056 & -9.0 & 0.0 & 180 & 21 & 045 & 1.0 & 180 \\
\hline $00: 57: 30$ & -77 & -78 & 44 & 0 & -0.05 & -0.06 & 0.16 & 043 & -1.383 & 2.4 & 0.0 & -180 & 24 & 045 & 1.0 & 180 \\
\hline $00: 57: 40$ & -77 & -78 & 48 & 0 & -0.05 & -0.05 & 0.16 & 043 & -1.311 & 4.8 & 0.0 & 180 & 28 & 045 & 1.0 & 180 \\
\hline $00: 57: 50$ & -77 & -79 & 48 & 0 & -0.09 & -0.03 & 0.15 & 043 & -1.153 & 10.1 & 0.0 & 180 & 20 & 045 & 1.0 & 180 \\
\hline $00: 58 ; 00$ & -77 & -79 & 46 & 0 & -0.09 & 0.03 & 0.09 & 043 & -0.416 & 13.0 & 0.0 & 180 & 32 & 045 & 1.0 & 180 \\
\hline $00: 58: 10$ & -77 & -80 & 46 & 0 & -0.06 & 0.07 & 0.06 & 042 & 0.075 & 8.2 & 0.0 & 180 & 23 & 045 & 1.0 & 180 \\
\hline$: 58: 20$ & -77 & -80 & 45 & 0 & 0.04 & 0.11 & 0.06 & 043 & 0.309 & 4.5 & 0.0 & 180 & 18 & 045 & 1.0 & 180 \\
\hline$: 58: 30$ & -76 & -80 & 46 & 0 & 0.09 & 0.12 & 0.06 & 043 & 0.352 & 4.0 & 0.0 & 180 & 21 & 045 & 1.0 & 180 \\
\hline $00: 58: 40$ & $-75^{\circ}$ & -80 & 46 & 0 & 0.14 & 0.14 & 0.07 & 043 & 0.473 & 3.0 & 0.0 & 180 & 21 & 045 & 2.0 & 180 \\
\hline $00: 58: 50$ & -74 & -80 & 45 & 0 & 0.19 & 0.15 & 0.09 & 043 & 0.388 & -4.5 & 0.0 & 180 & 21 & 045 & 1.0 & 180 \\
\hline $00: 59: 00$ & -73 & -79 & 45 & 0 & 0.26 & 0.09 & 0.17 & 043 & -0.516 & -11.1 & 49.0 & 180 & 26 & 045 & 1.0 & 180 \\
\hline $00: 59: 10$ & -72 & -79 & 46 & 0 & 0.24 & 0.05 & 0.24 & 043 & -1.188 & -5.8 & 49.0 & 180 & 25 & 045 & 1.0 & 180 \\
\hline
\end{tabular}




\begin{tabular}{|c|c|c|c|c|c|c|c|c|c|c|c|c|c|c|c|c|}
\hline \multirow{2}{*}{$\begin{array}{c}\text { pime } \\
H H: M M: s S\end{array}$} & \multicolumn{2}{|c|}{ Position (m) } & \multicolumn{2}{|c|}{$R P M$} & \multicolumn{3}{|c|}{ Speed (Knots) } & \multirow[b]{2}{*}{ Heading } & \multirow{2}{*}{$\begin{array}{c}\text { Turn } \\
\text { Deg/Min }\end{array}$} & \multirow{2}{*}{$\begin{array}{l}\text { Rudider } \\
\text { Deg }\end{array}$} & \multicolumn{2}{|c|}{ Gill } & \multicolumn{2}{|c|}{ Wind } & \multicolumn{2}{|c|}{ Current } \\
\hline & East & North & Port & stbd & Iong & Bow & Stern & & & & 8 & Deg & Knots & Deg & Knots & Deg \\
\hline & & & & & & & & & & & & & & & & \\
\hline $0: 59: 20$ & -70 & -79 & 46 & 0 & 0.29 & 0.03 & 0.27 & 042 & -1.575 & -0.6 & 49.0 & 180 & 20 & 045 & 1.0 & 180 \\
\hline$: 59: 30$ & -68 & -78 & 46 & 0 & 0.37 & 0.02 & 0.28 & 042 & -1.653 & 3.2 & 100.0 & 180 & 22 & 045 & 1.0 & 180 \\
\hline $0: 59: 40$ & -67 & -77 & 46 & 0 & 0.31 & 0.05 & 0.26 & 042 & -1.328 & 7.4 & 100.0 & 180 & 21 & 045 & 1.0 & 180 \\
\hline $00: 59: 50$ & -65 & -76 & 45 & 0 & 0.31 & 0.04 & 0.27 & 042 & -1.458 & -6.3 & 100.0 & 180 & 28 & 045 & 1.0 & 180 \\
\hline $01: 00: 00$ & -63 & -76 & 45 & 0 & 0.34 & 0.02 & 0.30 & 041 & -1.770 & -0.6 & 100.0 & 180 & 26 & 045 & 1.0 & 180 \\
\hline $01: 00: 10$ & -62 & -75 & 46 & 0 & 0.34 & -0.03 & 0.29 & 041 & -2.043 & 6.1 & 100.0 & 180 & 23 & 045 & 1.0 & 180 \\
\hline $01: 00: 20$ & -60 & -74 & 46 & 0 & 0.28 & -0.06 & 0.25 & 041 & -1.948 & 13.4 & 100.0 & 180 & 25 & 045 & 1.0 & 180 \\
\hline $01: 00: 30$ & -59 & -73 & 46 & 0 & 0.14 & 0.01 & 0.17 & 040 & -1.001 & 17.9 & 100.0 & 180 & 29 & 045 & 1.0 & 180 \\
\hline $01: 00: 40$ & -58 & -73 & 46 & 0 & 0.10 & 0.05 & 0.09 & 040 & -0.267 & 15.0 & 0.0 & 180 & 22 & 045 & 1.0 & 180 \\
\hline $01 ; 00: 50$ & -58 & -73 & 31 & 0 & 0.11 & 0.03 & 0.05 & 040 & -0.073 & 13.2 & 0.0 & 180 & 30 & 045 & 1.0 & 180 \\
\hline $01: 01 ; 00$ & -57 & -73 & 0 & 0 & 0.07 & 0.04 & 0.03 & 040 & 0.083 & 11.3 & 0.0 & 180 & 26 & 045 & 1.0 & 180 \\
\hline
\end{tabular}


Open Ocean

\section{Simulation Track Plot}

Ship: 150,000 ton, Tanker, partial load

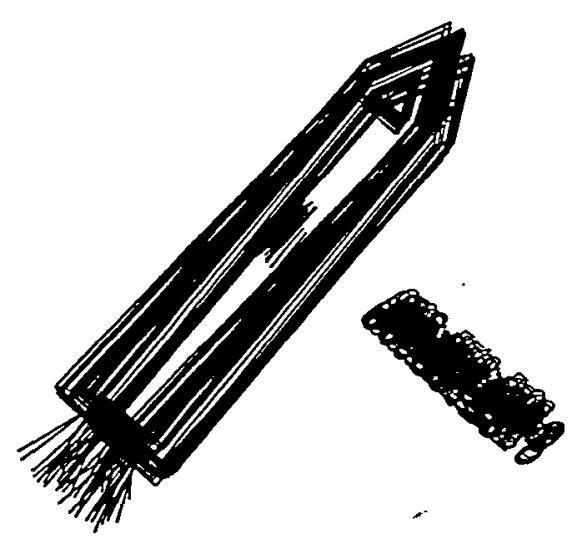

Start Time [hrs:min:sec]: $00: 33: 20$

End Time [hrs:min:sec]: $01: 01: 04$

Delta Plot Time [sec]:

East-West Scale [m]:

Date: $04-F e b-99$

Time: 09:40:51

East-West Scale [Nm]:

926.

0.5000 


\section{HANDS-ON SIMULATION}

\section{SUCTION MOORNG TECHNOLOGY}

Simulation Number: 22

Date: 4 February 1999

Start Time: $10: 50$

Length of run [secs]: 1665

Ship Condition: $\mathrm{draft}=13.72 \mathrm{~m}$

Environment

Wind Speed [knots]:

25 Direction: 45

Sea State, Significant wave height $[\mathrm{m}]: .$.

5 Direction: 225

Current Speed [knots]:

1 Direction: 180

Ship Operator: J.A.

Observer: B.K.J \& J.K.

Final Mating, distance between center of buoy and center of moon pool [m]: 2.5

Evaluation:

Started as the previous run \# 21. The plan was to shorten the approach time first by incepting the course line closer to the buoy and second by waiting until closer to the buoy to get on the equilibrium heading. In doing this, the buoy was slightly overshot when the captain had achieved the intercept heading at close to zero speed. Then to get back, he went slightly to the right of the up wind / up wave direction to get back quicker.

The equilibrium heading at zero speed was about 044 to 045 degrees. 
Ship:

Exercise number: enario: caffic ships:

Initial conditions: Exercise identification:
591150,000 ton, Tanker, partial load

754 Suction Mooring System

112 Open Ocean

999 Dummy tug file based on containership

Suction Mooring Buoy

594 lsok tanker in partial load - Suction Mooring RuN $\# 22$

Time Position ( $m$ ) RPM Speed (Knots) Turn Rudder Gill Current

\begin{tabular}{|c|c|c|c|c|c|c|c|c|c|c|c|c|c|c|c|c|}
\hline $\begin{array}{l}00: 08: 20 \\
00: 08: 30\end{array}$ & $\begin{array}{l}-169 \\
-161\end{array}$ & $\begin{array}{l}-1096 \\
-1076\end{array}$ & $\begin{array}{l}42 \\
42\end{array}$ & $\begin{array}{l}0 \\
0\end{array}$ & $\begin{array}{l}4.09 \\
4.02\end{array}$ & $\begin{array}{l}-1.03 \\
-0.95\end{array}$ & $\begin{array}{l}0.47 \\
0.46\end{array}$ & $\begin{array}{l}027 \\
025\end{array}$ & $\begin{array}{l}-9.549 \\
-8.968\end{array}$ & $\begin{array}{l}-32.6 \\
-13.6\end{array}$ & $\begin{array}{l}0.0 \\
0.0\end{array}$ & $\begin{array}{l}180 \\
180\end{array}$ & $\begin{array}{l}26 \\
19\end{array}$ & $\begin{array}{l}045 \\
045\end{array}$ & $\begin{array}{l}1.0 \\
1.0\end{array}$ & $\begin{array}{l}180 \\
180\end{array}$ \\
\hline $00: 08: 40$ & -154 & -1057 & 42 & 0 & 3.95 & -0.92 & 0.33 & 024 & -7.974 & 5.4 & 0.0 & 180 & 25 & 045 & 1.0 & 180 \\
\hline $00: 08: 50$ & -147 & -1038 & 42 & 0 & 3.96 & -0.63 & 0.10 & 023 & -4.602 & 24.4 & 0.0 & 180 & 24 & 045 & 1.0 & 180 \\
\hline $00: 09: 00$ & -141 & -1018 & 42 & 0 & 3.93 & -0.50 & -0.22 & 022 & -1.830 & 35.0 & 0.0 & 180 & 25 & 045 & 1.0 & 180 \\
\hline $00: 09: 10$ & -135 & -999 & 42 & 0 & 3.87 & -0.43 & -0.53 & 022 & 0.633 & 35.0 & 0.0 & 180 & 29 & 045 & 1.0 & 180 \\
\hline $00: 09: 20$ & -131 & -980 & 42 & 0 & 3.78 & -0.38 & -0.82 & 022 & 2.803 & 35.0 & 0.0 & 180 & 27 & 045 & 1.0 & 180 \\
\hline $00: 09: 30$ & -126 & -961 & 42 & 0 & 3.69 & -0.27 & -1.07 & 023 & 5.075 & 32.2 & 0.0 & 180 & 25 & 045 & 1.0 & 180 \\
\hline $00: 09: 40$ & -122 & -942 & 42 & 0 & 3.57 & -0.29 & -1.23 & 024 & 5.949 & 15.1 & 0.0 & 180 & 30 & 045 & 1.0 & 180 \\
\hline $00: 09: 50$ & -118 & -924 & 42 & 0 & 3.47 & -0.26 & -1.25 & 025 & 6.329 & -3.4 & 0.0 & 180 & 18 & 045 & 1.0 & 180 \\
\hline $00: 10: 00$ & -114 & -906 & 42 & 0 & 3.43 & -0.17 & -1.15 & 026 & 6.223 & -22.4 & 0.0 & 180 & 22 & 045 & 1.0 & 180 \\
\hline $00: 10: 10$ & -109 & -889 & 42 & 0 & 3.43 & -0.32 & -0.95 & 027 & 3.967 & -35.0 & 0.0 & 180 & 26 & 045 & 1.0 & 180 \\
\hline $00: 10: 20$ & -104 & -872 & 42 & 0 & 3.42 & -0.44 & -0.75 & 028 & 1.967 & -35.0 & 0.0 & 180 & 20 & 045 & 1.0 & 180 \\
\hline $00: 10: 30$ & -98 & -855 & 42 & 0 & 3.38 & -0.31 & -0.57 & 028 & 1.644 & -35.0 & 0.0 & 180 & 21 & 045 & 1.0 & 180 \\
\hline $00: 10: 40$ & -91 & -839 & 42 & 0 & 3.37 & -0.36 & -0.39 & 028 & 0.216 & -35.0 & 0.0 & 180 & 18 & 045 & 1.0 & 180 \\
\hline $00: 10: 50$ & -85 & -822 & 42 & 0 & 3.43 & -0.69 & -0.22 & 028 & -2.959 & -35.0 & 0.0 & 180 & 22 & 045 & 1.0 & 180 \\
\hline $00: 11: 00$ & -79 & -806 & 42 & 0 & 3.35 & -0.90 & -0.09 & 027 & -5.119 & -35.0 & 50.0 & 180 & 21 & 045 & 1.0 & 180 \\
\hline $00: 11: 10$ & -74 & -789 & 42 & 0 & 3.33 & -1.00 & 0.01 & 026 & -6.466 & -35.0 & 50.0 & 180 & 26 & 045 & 1.0 & 180 \\
\hline $00: 11: 20$ & -69 & -773 & 42 & 0 & 3.25 & -0.99 & 0.11 & 025 & -6.959 & -35.0 & 50.0 & 180 & 23 & 045 & 1.0 & 180 \\
\hline $00: 11: 30$ & -64 & -757 & 42 & 0 & 3.21 & -1.02 & 0.20 & 024 & -7.752 & -35.0 & 50.0 & 180 & 24 & 045 & 1.0 & 180 \\
\hline $00: 11: 10$ & -59 & -741 & 42 & 0 & 3.25 & -1.26 & 0.28 & 022 & -9.774 & -35.0 & 50.0 & 180 & 19 & 045 & 1.0 & 180 \\
\hline $00: 11: 50$ & -55 & -724 & 42 & 0 & 3.25 & -1.14 & 0.34 & 021 & -9.398 & -35.0 & 50.0 & 180 & 21 & 045 & 1.0 & 180 \\
\hline $00: 12: 00$ & -52 & -708 & 42 & 0 & 3.18 & $-1 \cdot 30$ & 0.40 & 019 & -10.815 & -33.9 & 50.0 & 180 & 30 & 045 & 1.0 & 180 \\
\hline $00: 12: 10$ & -49 & -692 & 42 & 0 & 3.12 & -1.26 & 0.38 & 017 & -10.395 & -14.9 & 50.0 & 180 & 24 & 045 & 1.0 & 180 \\
\hline $00: 12: 20$ & -47 & -676 & 42 & 0 & 3.09 & -1.34 & 0.32 & 015 & -10.584 & -12.6 & 50.0 & 180 & 26 & 045 & 2.0 & 180 \\
\hline $00: 12: 30$ & -45 & -660 & 42 & 0 & 3.06 & -1.32 & 0.17 & 014 & -9.501 & 6.4 & 50.0 & 180 & 28 & 045 & 1.0 & 180 \\
\hline $00: 12: 40$ & -45 & -644 & 42 & 0 & 3.01 & -1.32 & -0.09 & 012 & -7.819 & 25.4 & 50.0 & 180 & 22 & 045 & 1.0 & 180 \\
\hline $00: 12: 50$ & -46 & -628 & 42 & 0 & 2.98 & -0.95 & -0.40 & 011 & -3.460 & 35.0 & 23.0 & 180 & 22 & 045 & 1.0 & 180 \\
\hline $0: 13: 00$ & -46 & -612 & 42 & 0 & 2.98 & -0.56 & -0.68 & 011 & 0.720 & 35.0 & 23.0 & 180 & 24 & 045 & 1.0 & 180 \\
\hline$: 13: 10$ & -46 & -597 & 42 & 0 & 2.97 & -0.41 & -0.93 & 012 & 3.289 & 35.0 & 23.0 & 180 & 18 & 045 & 1.0 & 180 \\
\hline$-00: 13: 20$ & -46 & -581 & 42 & 0 & 2.95 & -0.18 & -1.14 & 012 & 6.085 & 35.0 & 23.0 & 180 & 27 & 045 & 1.0 & 180 \\
\hline $00: 13: 30$ & -46 & -565 & 42 & 0 & 2.94 & -0.20 & -1.32 & 013 & 7.090 & 27.0 & 23.0 & 180 & 20 & 045 & 1.0 & 180 \\
\hline $00: 13: 40$ & -47 & -550 & 42 & 0 & 2.95 & -0.59 & -1.42 & 014 & 5.293 & 8.0 & 23.0 & 180 & 27 & 045 & 1.0 & 180 \\
\hline $00: 13: 50$ & -49 & -534 & 42 & 0 & 2.86 & -0.71 & -1.46 & 015 & 4.800 & 14.2 & 23.0 & 180 & 30 & 045 & 1.0 & 180 \\
\hline $00: 14: 00$ & -50 & -518 & 42 & 0 & 2.76 & -0.63 & -1.58 & 016 & 6.048 & 33.2 & 23.0 & 180 & 17 & 045 & 1.0 & 180 \\
\hline $00: 14: 10$ & -52 & -503 & 42 & 0 & 2.77 & -0.72 & -1.75 & 017 & 6.573 & 35.0 & 23.0 & 180 & 23 & 045 & 1.0 & 180 \\
\hline $00: 14: 20$ & -54 & -487 & 42 & 0 & 2.70 & -0.85 & -1.89 & 018 & 6.625 & 28.1 & 23.0 & 180 & 22 & 045 & 1.0 & 180 \\
\hline $00: 14: 30$ & -56 & -472 & 42 & 0 & 2.67 & -0.95 & -1.93 & 019 & 6.232 & 9.1 & 23.0 & 180 & 22 & 045 & 1.0 & 180 \\
\hline $00: 14: 40$ & -58 & -457 & 42 & 0 & 2.59 & -0.83 & -1.84 & 020 & 6.411 & -9.9 & 23.0 & 180 & 25 & 045 & 1.0 & 180 \\
\hline $00: 14: 50$ & -60 & -442 & 42 & 0 & 2.51 & -0.83 & -1.65 & 021 & 5.162 & -28.6 & 23.0 & 180 & 26 & 045 & 1.0 & 180 \\
\hline $00: 15: 00$ & -61 & -428 & 42 & 0 & 2.46 & -1.35 & -1.41 & 022 & 0.399 & -35.0 & 23.0 & 180 & 20 & 045 & 1.0 & 180 \\
\hline $00: 15: 10$ & -63 & -414 & 42 & 0 & 2.42 & -1.46 & -1.21 & 022 & -1.577 & -32.0 & 23.0 & 180 & 22 & 045 & 1.0 & 180 \\
\hline $00: 15: 20$ & -65 & -400 & 42 & 0 & 2.35 & -1.55 & -1.08 & 021 & -2.999 & -17.7 & 23.0 & 180 & 20 & 045 & 1.0 & 180 \\
\hline $00: 15: 30$ & -67 & -386 & 42 & 0 & 2.27 & -1.40 & -1.07 & 021 & -2.091 & 1.3 & 23.0 & 180 & 24 & 045 & 1.0 & 180 \\
\hline $00: 15: 40$ & -69 & -373 & 42 & 0 & 2.25 & -1.27 & -1.15 & .021 & -0.750 & 20.3 & 23.0 & 180 & 24 & 045 & 1.0 & 180 \\
\hline $00: 15: 50$ & -70 & -360 & 42 & 0 & 2.25 & -0.89 & -1.31 & 021 & 2.721 & 35.0 & 23.0 & 180 & 19 & 045 & 1.0 & 180 \\
\hline $00: 16: 00$ & -71 & -347 & 42 & 0 & 2.25 & -0.72 & -1.48 & 022 & 4.807 & 35.0 & 23.0 & 180 & 22 & 045 & 1.0 & 180 \\
\hline $00: 16: 10$ & -72 & -335 & 42 & 0 & 2.20 & -0.58 & -1.61 & 022 & 6.533 & 35.0 & 23.0 & 180 & 25 & 045 & 1.0 & 180 \\
\hline $00: 16: 20$ & -73 & -322 & 42 & 0 & 2.14 & -0.38 & -1.71 & 024 & 8.443 & 35.0 & 23.0 & 180 & 23 & 045 & 1.0 & 180 \\
\hline $00: 16: 30$ & -73 & -310 & 42 & 0 & 2.10 & -0.41 & -1.78 & 025 & 8.697 & 35.0 & 23.0 & 180 & 25 & 045 & 1.0 & 180 \\
\hline $00: 16: 40$ & -74 & -298 & 42 & 0 & 2.02 & -0.43 & -1.84 & 027 & 8.948 & 35.0 & 23.0 & 180 & 32 & 045 & 1.0 & 180 \\
\hline $00: 16: 50$ & -74 & -286 & 42 & 0 & 1.91 & -0.43 & -1.88 & 028 & 9.171 & 30.8 & 23.0 & 180 & 21 & 045 & 1.0 & 180 \\
\hline $00: 17: 00$ & -75 & -275 & 42 & 0 & 1.92 & -0.47 & -1.88 & 030 & 8.955 & 11.8 & 23.0 & 180 & 29 & 045 & 1.0 & 180 \\
\hline $00: 17: 10$ & -75 & -263 & 42 & 0 & 1.88 & -0.46 & -1.79 & 031 & 8.446 & -7.2 & 23.0 & 180 & 25 & 045 & 1.0 & 180 \\
\hline $00: 17: 20$ & -75 & -252 & 42 & 0 & 1.79 & -0.45 & -1.60 & 032 & 7.265 & -26.2 & 23.0 & 180 & 22 & 045 & 1.0 & 180 \\
\hline $00: 17: 30$ & -74 & -242 & 42 & 0 & 1.80 & -0.54 & -1.33 & 033 & 4.995 & -35.0 & 23.0 & 180 & 24 & 045 & 1.0 & 180 \\
\hline $00: 17: 40$ & -73 & -232 & 42 & 0 & 1.79 & -0.70 & -1.05 & 034 & 2.233 & -35.0 & 23.0 & 180 & 16 & 045 & 1.0 & 180 \\
\hline $00: 17: 50$ & -71 & -221 & 42 & 0 & 1.78 & -0.94 & -0.81 & 034 & -0.818 & -35.0 & 23.0 & 180 & 25 & 045 & 1.0 & 180 \\
\hline $00: 18: 00$ & -70 & -212 & 42 & 0 & 1.64 & -1.05 & -0.61 & 034 & -2.806 & -35.0 & 100.0 & 180 & 26 & 045 & 1.0 & 180 \\
\hline $00: 18: 10$ & -69 & -203 & 42 & 0 & 1.50 & -0.99 & -0.43 & 033 & -3.565 & -35.0 & 100.0 & 180 & 20 & 045 & 1.0 & 180 \\
\hline $00: 18: 20$ & -68 & -194 & 42 & 0 & 1.43 & -1.13 & -0.25 & 033 & -5.618 & -35.0 & 100.0 & 180 & 23 & 045 & 1.0 & 180 \\
\hline $00: 18: 30$ & -67 & -186 & 42 & 0 & 1.38 & -1.20 & -0.09 & 032 & -7.020 & -35.0 & 100.0 & 180 & 20 & 045 & 1.0 & 180 \\
\hline $00: 18: 40$ & -66 & -179 & 42 & 0 & 1.34 & -1.17 & 0.04 & 030 & -7.655 & -26.5 & 100.0 & 180 & 23 & 045 & 1.0 & 180 \\
\hline $00: 18: 50$ & -65 & -172 & 41 & 0 & 1.29 & -0.93 & 0.07 & 029 & -6.344 & -7.4 & 100.0 & 180 & 25 & 045 & 1.0 & 180 \\
\hline $00: 19: 00$ & -63 & -165 & 30 & 0 & 1.27 & -0.88 & 0.02 & 028 & -5.705 & 11.6 & 100.0 & 180 & 23 & 045 & 1.0 & 180 \\
\hline $00: 19: 10$ & -62 & -158 & 29 & 0 & 1.19 & -0.77 & -0.08 & 027 & -4.416 & 30.6 & 100.0 & 180 & 28 & 045 & 1.0 & 180 \\
\hline $00: 19: 20$ & -62 & -152 & 29 & 0 & 1.15 & -0.75 & -0.20 & 027 & -3.506 & 35.0 & 100.0 & 180 & 27 & 045 & 1.0 & 180 \\
\hline$: 19: 30$ & -62 & -146 & 36 & 0 & 1.06 & -0.82 & -0.32 & 026 & -3.196 & 35.0 & 100.0 & 180 & 23 & 045 & 1.0 & 180 \\
\hline$: 19: 40$ & -62 & -140 & 49 & 0 & 0.97 & -0.72 & -0.50 & 026 & -1.382 & 35.0 & 100.0 & 180 & 28 & 045 & 1.0 & 180 \\
\hline $00: 19: 50$ & -63 & -134 & 54 & 0 & 0.97 & -0.63 & -0.76 & 026 & 0.802 & 35.0 & 100.0 & 180 & 24 & 045 & 1.0 & 180 \\
\hline $00: 20: 00$ & -64 & -127 & 54 & 0 & 1.01 & -0.44 & -1.01 & 026 & 3.615 & 35.0 & 100.0 & 180 & 23 & 045 & 1.0 & 180 \\
\hline $00: 20: 10$ & -65 & -121 & 60 & 0 & 1.00 & -0.31 & -1.25 & 027 & 5.928 & 35.0 & 100.0 & 180 & 24 & 045 & 1.0 & 180 \\
\hline $00: 20: 20$ & -67 & -115 & 61 & 0 & 1.04 & -0.30 & -1.47 & 028 & 7.434 & 35.0 & 100.0 & 180 & 26 & 045 & 1.0 & 180 \\
\hline
\end{tabular}




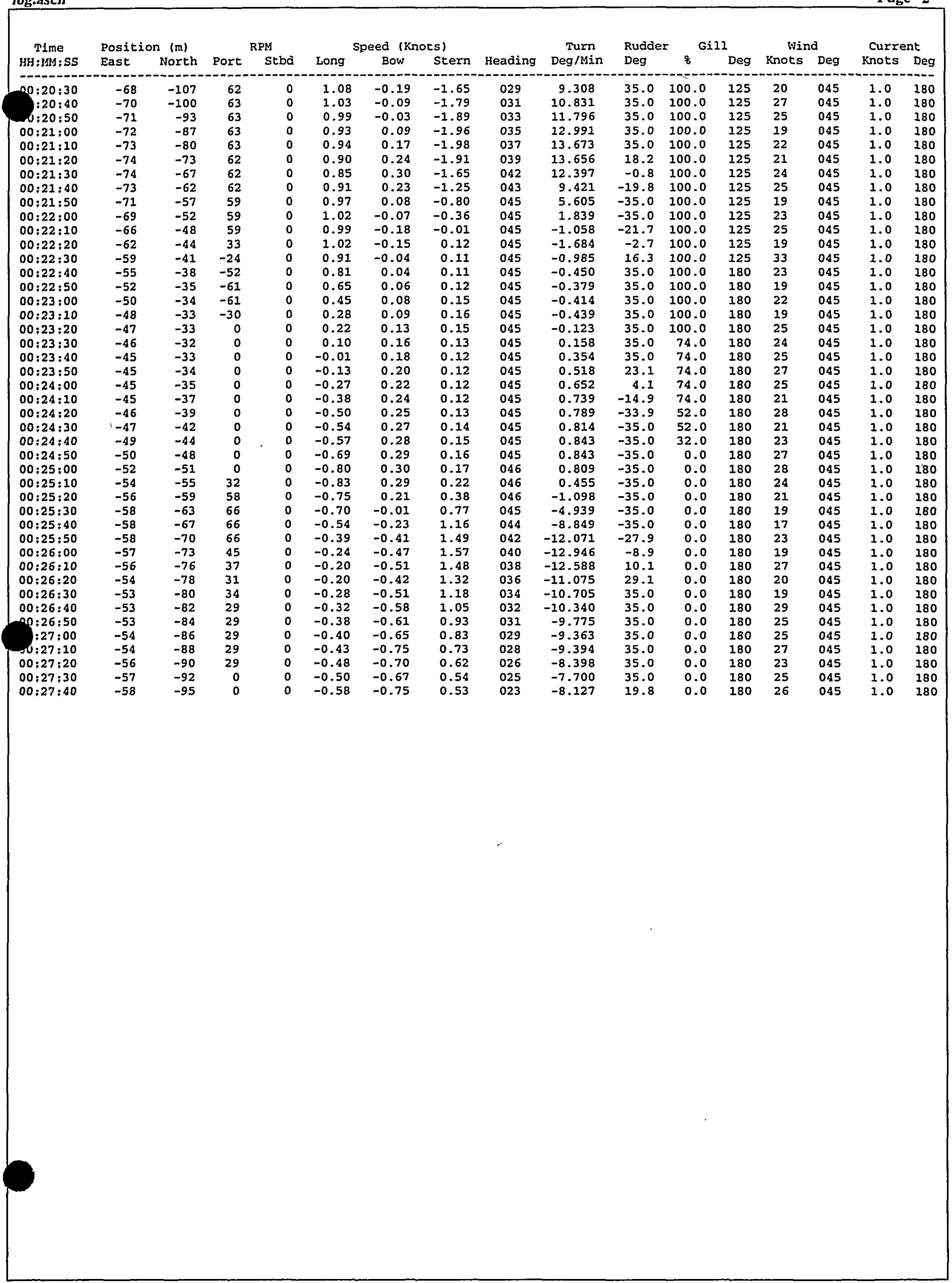


Open Ocean

\section{Simulation Track Plot}

Ship: 150,000 ton, Tanker, partial load

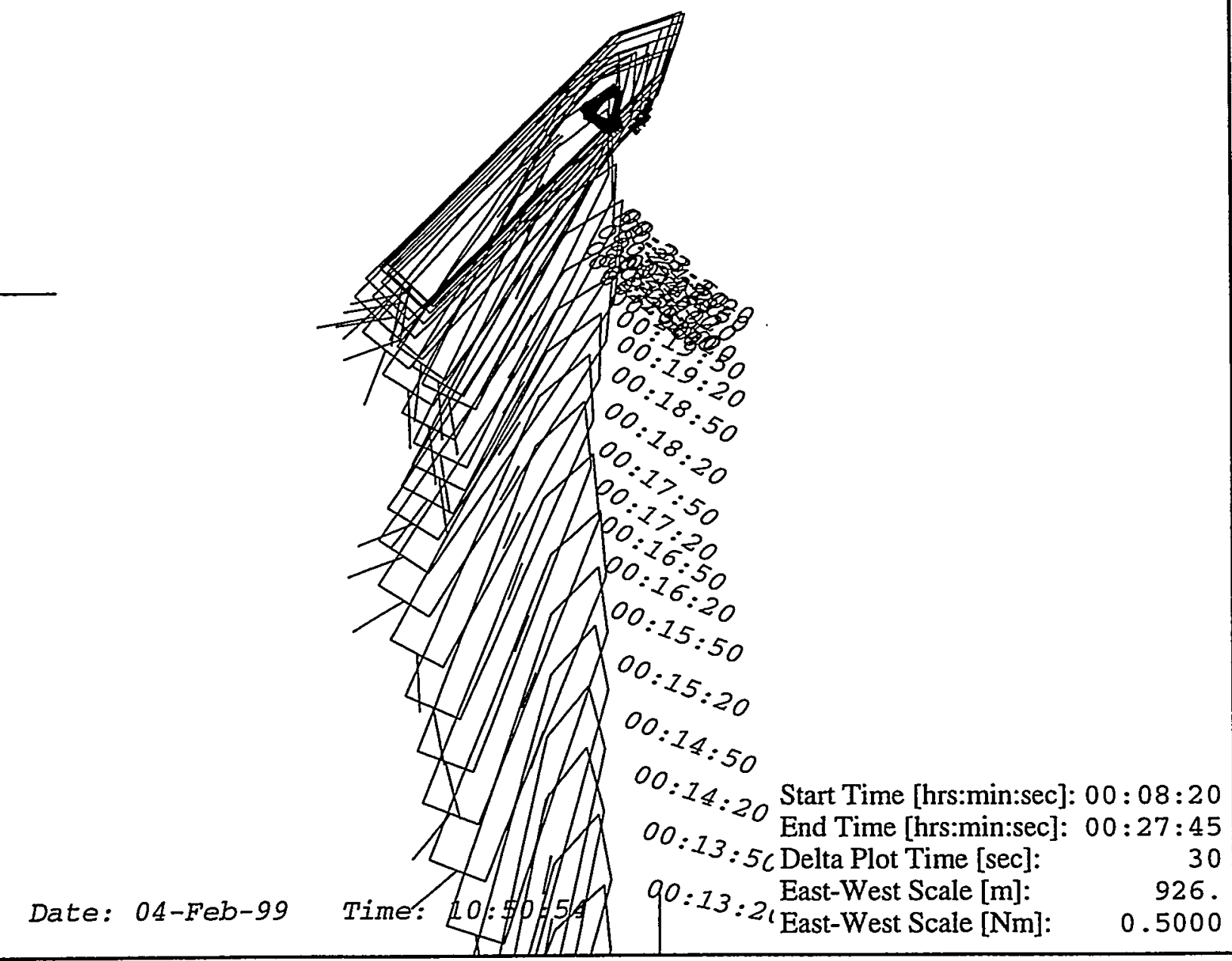




\section{HANDS-ON SIMULATION}

\section{SUCTION MOORNG TECHNOLOGY}

Simulation Number: 23

Date: 4 February 1999

Start Time: 11:32

Length of run [secs]: 2160

Ship Condition: $\mathrm{draft}=13.72 \mathrm{~m}$

Environment

Wind Speed $[$ knots] $] .. \ldots \ldots \ldots \ldots \ldots \ldots \ldots . . \ldots \ldots$

Sea State, Significant wave height $[\mathrm{m}]: . . \quad 5 \quad$ Direction: 270

Current Speed $[\mathrm{knots}]: \ldots \ldots \ldots \ldots \ldots \ldots \ldots . \ldots \ldots$

Ship Operator: J.A.

Observer: B.K.J \& J.K.

Final Mating, distance between center of buoy and center of moon pool [m]: 2.0

Evaluation:

The approach went well. The ship came into the approximate equilibrium heading 080 at zero speed near the buoy. The captain decided not to mate on the first pass through the center of the moon pool. Going to the left and changing the heading to about 085 degrees and then let the current force push the ship sideways. 
Ship:

591150,000 ton, Tanker, partial load

Exercise number:

754 Suction Mooring System

112 Open Ocean

999 Dumny tug file based on containership

g file:

590 Suction Mooring Buoy

Initial conditions: 594 150k tanker in partial load - Suction Mooring

Exercise identification: RUN $\$ 23$

RPM

Speed (Knots)

Turn Rudder

Gill

Wind

Current Bow Stern Heading Deg/Min Deg $z$ Deg Knots Deg knots Deg

\begin{tabular}{|c|c|c|c|c|c|c|c|c|c|c|c|c|c|c|c|c|}
\hline $\begin{array}{l}00: 16: 40 \\
00: 16: 50\end{array}$ & $\begin{array}{l}-85 \\
-85\end{array}$ & $\begin{array}{l}-154 \\
-150\end{array}$ & $\begin{array}{l}36 \\
36\end{array}$ & $\begin{array}{l}0 \\
0\end{array}$ & $\begin{array}{l}0.35 \\
0.29\end{array}$ & $\begin{array}{l}-0.52 \\
-0.65\end{array}$ & $\begin{array}{l}-0.72 \\
-0.60\end{array}$ & $\begin{array}{l}068 \\
068\end{array}$ & $\begin{array}{r}1.273 \\
-0.289\end{array}$ & $\begin{array}{l}-1.0 \\
-0.1\end{array}$ & $\begin{array}{l}83.0 \\
76.0\end{array}$ & $\begin{array}{l}180 \\
180\end{array}$ & $\begin{array}{l}32 \\
21\end{array}$ & $\begin{array}{l}090 \\
090\end{array}$ & $\begin{array}{l}1.0 \\
1.0\end{array}$ & $\begin{array}{l}180 \\
180\end{array}$ \\
\hline $00: 17: 00$ & -85 & -147 & 36 & 0 & 0.33 & -0.83 & -0.51 & 068 & -1.990 & 11.6 & 76.0 & 180 & 29 & 090 & 1.0 & 180 \\
\hline $00: 17 ; 10$ & -85 & -143 & 36 & 0 & 0.32 & -0.82 & -0.50 & 067 & -2.062 & 30.6 & 76.0 & 180 & 25 & 090 & 1.0 & 180 \\
\hline $00: 17: 20$ & -85 & -139 & 36 & 0 & 0.25 & -0.72 & -0.53 & 067 & -1.243 & 35.0 & 76.0 & 180 & 22 & 090 & 1.0 & 180 \\
\hline $00: 17: 30$ & -85 & -136 & 36 & 0 & 0.28 & -0.68 & -0.56 & 067 & -0.810 & 35.0 & 52.0 & 180 & 24 & 090 & 1.0 & 180 \\
\hline $00 ; 17: 40$ & -85 & -132 & 36 & 0 & 0.29 & -0.77 & -0.58 & 067 & -1.173 & 35.0 & 52.0 & 180 & 16 & 090 & 1.0 & 180 \\
\hline $00: 17: 50$ & -85 & -128 & 36 & 0 & 0.29 & -1.06 & -0.60 & 067 & -2.962 & 35.0 & 52.0 & 180 & 25 & 090 & 1.0 & 180 \\
\hline $00: 18: 00$ & -85 & -124 & 36 & 0 & 0.19 & -1.14 & -0.61 & 066 & -3.405 & 35.0 & 75.0 & 180 & 26 & 090 & 1.0 & 180 \\
\hline $00: 18: 10$ & -86 & -119 & 36 & 0 & 0.08 & -0.91 & -0.62 & 066 & -1.880 & 35.0 & 75.0 & 180 & 20 & 090 & 1.0 & 180 \\
\hline $00: 18: 20$ & -88 & -116 & 36 & 0 & 0.06 & -1.07 & -0.62 & 065 & -2.900 & 35.0 & 0.0 & 180 & 23 & 090 & 1.0 & 180 \\
\hline $00: 18: 30$ & -89 & -111 & 36 & 0 & 0.06 & -1.09 & -0.61 & 065 & -3.031 & 35.0 & 26.0 & 180 & 20 & 090 & 1.0 & 180 \\
\hline $00: 18: 40$ & -91 & -107 & 36 & 0 & 0.06 & -0.96 & -0.61 & 064 & -2.202 & 35.0 & 26.0 & 180 & 13 & 090 & 1.0 & 180 \\
\hline $00: 18: 50$ & -92 & -104 & 36 & 0 & 0.04 & -0.58 & -0.62 & 064 & 0.254 & 35.0 & 26.0 & 180 & 25 & 090 & 1.0 & 180 \\
\hline $00: 19: 00$ & -93 & -101 & 44 & 0 & 0.06 & -0.55 & -0.65 & 064 & 0.623 & 35.0 & 26.0 & 180 & 23 & 090 & 1.0 & 180 \\
\hline $00: 19: 10$ & -94 & -98 & 45 & 0 & 0.07 & -0.45 & -0.71 & 064 & 1.645 & 35.0 & 52.0 & 180 & 28 & 090 & 1.0 & 180 \\
\hline $00: 19: 20$ & -95 & -95 & 45 & 0 & 0.11 & -0.46 & -0.76 & 065 & 1.905 & 35.0 & 52.0 & 180 & 27 & 090 & 1.0 & 180 \\
\hline $00: 19: 30$ & -96 & -92 & 44 & 0 & 0.12 & -0.58 & -0.81 & 065 & 1.429 & 35.0 & 52.0 & 180 & 23 & 090 & 1.0 & 180 \\
\hline $00: 19: 40$ & -97 & -89 & 44 & 0 & 0.08 & -0.52 & -0.85 & 065 & 2.099 & 35.0 & 52.0 & 180 & 28 & 090 & 1.0 & 180 \\
\hline $00: 19: 50$ & -98 & -85 & 47 & 0 & 0.11 & -0.54 & -0.88 & 066 & 2.202 & 35.0 & 52.0 & 180 & 24 & 090 & 1.0 & 180 \\
\hline $00: 20: 00$ & -99 & -82 & 47 & 0 & 0.19 & -0.41 & -0.93 & 066 & 3.307 & 35.0 & 52.0 & 180 & 23 & 090 & 1.0 & 180 \\
\hline $00: 20: 10$ & -100 & -78 & 48 & 0 & 0.20 & -0.38 & -0.97 & 067 & 3.734 & 35.0 & 27.0 & 180 & 24 & 090 & 1.0 & 180 \\
\hline $00: 20: 20$ & -100 & -74 & 47 & 0 & 0.27 & -0.48 & -1.00 & 067 & 3.310 & 35.0 & 49.0 & 180 & 26 & 090 & 1.0 & 180 \\
\hline $00: 20: 30$ & -100 & -70 & 48 & 0 & 0.32 & -0.50 & -1.04 & 068 & 3.393 & 35.0 & 49.0 & 180 & 20 & 090 & 1.0 & 180 \\
\hline $00: 20 \div 40$ & -100 & -66 & 48 & 0 & 0.29 & -0.54 & -1.06 & 068 & 3.308 & 35.0 & 49.0 & 180 & 27 & 090 & 1.0 & 180 \\
\hline $00: 20: 50$ & -100 & -61 & 47 & 0 & 0.26 & -0.65 & -1.08 & 069 & 2.716 & 35.0 & 49.0 & 180 & 25 & 090 & 1.0 & 180 \\
\hline $00: 21: 00$ & -101 & -57 & 47 & 0 & 0.23 & -0.66 & -1.07 & 069 & 2.586 & 28.1 & 49.0 & 180 & 19 & 090 & 1.0 & 180 \\
\hline $00: 21: 10$ & -101 & -52 & 47 & 0 & 0.26 & -0.77 & -1.05 & 070 & 1.805 & 35.0 & 49.0 & 180 & 22 & 090 & 1.0 & 180 \\
\hline$-0: 21: 20$ & -102 & -47 & 47 & 0 & 0.23 & -0.78 & -1.06 & 070 & 1.812 & 35.0 & 49.0 & 180 & 21 & 090 & 1.0 & 180 \\
\hline$: 21: 30$ & -102 & -43 & 47 & 0 & 0.18 & -0.44 & -1.05 & 070 & 3.889 & 23.9 & 49.0 & 180 & 24 & 090 & 1.0 & 180 \\
\hline $00: 21: 40$ & -102 & -39 & 47 & 0 & 0.23 & -0.29 & -0.95 & 071 & 4.197 & 12.3 & 25.0 & 180 & 25 & 090 & 1.0 & 180 \\
\hline $00: 21: 50$ & -102 & -36 & 48 & 0 & 0.31 & -0.31 & -0.87 & 072 & 3.549 & 16.8 & 25.0 & 180 & 19 & 090 & 1.0 & 180 \\
\hline $00 ; 22: 00$ & -101 & -32 & 47 & 0 & 0.37 & -0.45 & -0.78 & 072 & 2.126 & 11.1 & 25.0 & 180 & 23 & 090 & 1.0 & 180 \\
\hline $00: 22: 10$ & -100 & -29 & 48 & 0 & 0.34 & -0.29 & -0.75 & 073 & 2.934 & 20.0 & 50.0 & 180 & 25 & 090 & 1.0 & 180 \\
\hline $00: 22: 20$ & -99 & -26 & 48 & 0 & 0.37 & -0.28 & -0.69 & 073 & 2.576 & 12.2 & 74.0 & 180 & 19 & 090 & 1.0 & 180 \\
\hline $00: 22: 30$ & -98 & -23 & 47 & 0 & 0.34 & -0.29 & -0.68 & 073 & 2.475 & 31.2 & 74.0 & 180 & 33 & 090 & 1.0 & 180 \\
\hline $00: 22: 40$ & -97 & -20 & 48 & 0 & 0.37 & -0.23 & -0.75 & 074 & 3.274 & 35.0 & 97.0 & 180 & 23 & 090 & 1.0 & 180 \\
\hline $00: 22: 50$ & -96 & -17 & 48 & 0 & 0.40 & -0.18 & -0.80 & 075 & 3.965 & 35.0 & 97.0 & 180 & 19 & 090 & 1.0 & 180 \\
\hline $00: 23: 00$ & -95 & -14 & 47 & 0 & 0.40 & 0.00 & -0.85 & 075 & 5.420 & 35.0 & 97.0 & 180 & 22 & 090 & 1.0 & 180 \\
\hline $00: 23: 10$ & -93 & -11 & 41 & 0 & 0.37 & -0.03 & -0.87 & 076 & 5.334 & 35.0 & 97.0 & 180 & 19 & 090 & 1.0 & 180 \\
\hline $00: 23: 20$ & -92 & -9 & 33 & 0 & 0.37 & -0.01 & -0.84 & 077 & 5.291 & 35.0 & 97.0 & 180 & 25 & 090 & 1.0 & 180 \\
\hline $00: 23: 30$ & -91 & -6 & 33 & 0 & 0.29 & -0.06 & -0.78 & 078 & 4.566 & 23.2 & 97.0 & 180 & 24 & 090 & 1.0 & 180 \\
\hline $00: 23: 40$ & -90 & -4 & 33 & 0 & 0.20 & -0.23 & -0.69 & 079 & 2.883 & 18.4 & 97.0 & 180 & 25 & 090 & 1.0 & 180 \\
\hline $00: 23: 50$ & -90 & -1 & 33 & 0 & 0.10 & -0.35 & -0.59 & 079 & 1.524 & 15.4 & 97.0 & 180 & 27 & 090 & 1.0 & 180 \\
\hline $00: 24: 00$ & -90 & 1 & 33 & 0 & -0.01 & -0.34 & -0.53 & 079 & 1.206 & 29.4 & 97.0 & 180 & 25 & 090 & 1.0 & 180 \\
\hline $00: 24: 10$ & -91 & 3 & 33 & 0 & -0.09 & -0.33 & -0.48 & 079 & 0.927 & 27.2 & 75.0 & 180 & 21 & 090 & 1.0 & 180 \\
\hline $00 ; 24 ; 20$ & -92 & 5 & 33 & 0 & -0.17 & -0.28 & -0.43 & 080 & 0.916 & 24.2 & 75.0 & 180 & 28 & 090 & 1.0 & 180 \\
\hline $00: 24: 30$ & -93 & 6 & 33 & 0 & -0.17 & -0.26 & -0.37 & 080 & 0.694 & 21.9 & 75.0 & 180 & 21 & 090 & 1.0 & 180 \\
\hline $00: 24: 40$ & -94 & 8 & 33 & 0 & -0.17 & -0.28 & -0.32 & 080 & 0.214 & 31.0 & 52.0 & 180 & 23 & 090 & 1.0 & 180 \\
\hline $00: 24: 50$ & -95 & 9 & 33 & 0 & -0.25 & -0.19 & -0.36 & 080 & 1.069 & 35.0 & 52.0 & 180 & 27 & 090 & 1.0 & 180 \\
\hline $00: 25: 00$ & -97 & 10 & 33 & 0 & -0.34 & -0.07 & -0.42 & 080 & 2.259 & 35.0 & 52.0 & 180 & 28 & 090 & 1.0 & 180 \\
\hline $00: 25: 10$ & -99 & 11 & 33 & 0 & -0.36 & -0.01 & -0.47 & 081 & 2.937 & 35.0 & 52.0 & 180 & 24 & 090 & 1.0 & 180 \\
\hline $00: 25: 20$ & -101 & 12 & 33 & 0 & -0.33 & -0.05 & -0.51 & 081 & 2.943 & 35.0 & 52.0 & 180 & 21 & 090 & 1.0 & 180 \\
\hline $00: 25: 30$ & -103 & 13 & 33 & 0 & -0.40 & 0.05 & -0.55 & 082 & 3.778 & 35.0 & 0.0 & 180 & 19 & 090 & 1.0 & 180 \\
\hline $00: 25: 40$ & -105 & 14 & 43 & 0 & -0.37 & 0.20 & -0.62 & 082 & 5.197 & 34.5 & 0.0 & 180 & 17 & 090 & 1.0 & 180 \\
\hline $00: 25: 50$ & -107 & 15 & 43 & 0 & -0.30 & 0.33 & -0.67 & 083 & 6.297 & 15.5 & 0.0 & 180 & 23 & 090 & 1.0 & 180 \\
\hline $00: 26: 00$ & -108 & 15 & 47 & 0 & -0.19 & 0.34 & -0.62 & 084 & 6.085 & -3.5 & 0.0 & 180 & 19 & 090 & 1.0 & 180 \\
\hline $00: 26: 10$ & -109 & 16 & 38 & 0 & -0.11 & 0.25 & -0.50 & 085 & 4.785 & -22.5 & 0.0 & 180 & 27 & 090 & 1.0 & 180 \\
\hline $00 ; 26 ; 20$ & -110 & 16 & 43 & 0 & -0.09 & 0.20 & -0.32 & 086 & 3.306 & -35.0 & 0.0 & 180 & 20 & 090 & 1.0 & 180 \\
\hline $00: 26: 30$ & -110 & 17 & 43 & 0 & -0.12 & 0.08 & -0.13 & 086 & 1.288 & -35.0 & 0.0 & 180 & 19 & 090 & 1.0 & 180 \\
\hline $00: 26: 40$ & -111 & 17 & 48 & 0 & -0.10 & -0.02 & 0.04 & 086 & -0.322 & -21.5 & 0.0 & 180 & 29 & 090 & 1.0 & 180 \\
\hline $00: 26: 50$ & -111 & 16 & 49 & 0 & -0.08 & -0.05 & 0.13 & 086 & -1.133 & -8.2 & 0.0 & 180 & 25 & 090 & 1.0 & 180 \\
\hline $00: 27: 00$ & -112 & 16 & 49 & 0 & -0.02 & -0.07 & 0.19 & 086 & -1.636 & 7.3 & 0.0 & 180 & 25 & 090 & 1.0 & 180 \\
\hline $00: 27: 10$ & -112 & 16 & 48 & 0 & 0.02 & -0.06 & 0.19 & 086 & -1.557 & 13.6 & 0.0 & 180 & 27 & 090 & 1.0 & 180 \\
\hline $00: 27: 20$ & -111 & 15 & 49 & 0 & 0.05 & -0.02 & 0.20 & 086 & -1.372 & 7.1 & 0.0 & 180 & 23 & 090 & 1.0 & 180 \\
\hline $00: 27: 30$ & -111 & 15 & 49 & 0 & 0.11 & 0.00 & 0.23 & 085 & -1.451 & 5.6 & 26.0 & 180 & 25 & 090 & 1.0 & 180 \\
\hline $00: 27: 40$ & -110 & 14 & 48 & 0 & 0.13 & 0.01 & 0.25 & 085 & -1.502 & 9.1 & 26.0 & 180 & 26 & 090 & 1.0 & 180 \\
\hline$: 27: 50$ & -109 & 14 & 48 & 0 & 0.14 & 0.02 & 0.24 & 085 & -1.449 & 12.8 & 26.0 & 180 & 23 & 090 & 1.0 & 180 \\
\hline$: 28: 00$ & -109 & 13 & 48 & 0 & 0.19 & 0.02 & 0.22 & 085 & -1.265 & 14.4 & 50.0 & 180 & 29 & 090 & 1.0 & 180 \\
\hline $00: 28: 10$ & -107 & 13 & 49 & 0 & 0.21 & 0.06 & 0.19 & 084 & -0.832 & 15.3 & 50.0 & 180 & 19 & 090 & 1.0 & 180 \\
\hline $00: 28: 20$ & -106 & 12 & 48 & 0 & 0.20 & 0.14 & 0.16 & 084 & -0.134 & 13.6 & 75.0 & 180 & 24 & 090 & 1.0 & 180 \\
\hline $00: 28: 30$ & -105 & 11 & 48 & 0 & 0.22 & 0.20 & 0.15 & 084 & 0.377 & 10.8 & 75.0 & 180 & 24 & 090 & 1.0 & 180 \\
\hline & & & & & & & & & & & 75.0 & 180 & 19 & 09 & 1.0 & 180 \\
\hline
\end{tabular}

$00: 28: 40 \quad-103 \quad 11 \quad 49$ 


\begin{tabular}{|c|c|c|c|c|c|c|c|c|c|c|c|c|c|c|c|c|}
\hline \multirow{2}{*}{$\begin{array}{c}\text { Time } \\
H H: M M ; S S\end{array}$} & \multicolumn{2}{|c|}{ Position (m) } & \multicolumn{2}{|c|}{ RPM } & \multicolumn{3}{|c|}{ Speed (Knots) } & \multirow[b]{2}{*}{ Heading } & \multirow{2}{*}{$\begin{array}{c}\text { Turn } \\
\text { Deg/Min }\end{array}$} & \multirow{2}{*}{$\begin{array}{l}\text { Rudder } \\
\text { Deg }\end{array}$} & \multicolumn{2}{|c|}{ Gill } & \multicolumn{2}{|c|}{ Hind } & \multicolumn{2}{|c|}{ Current } \\
\hline & East & North & Port & stbà & Long & Bow & Stern & & & & 8 & Deg & Knots & Deg & Knots & Deg \\
\hline & & & & & & & & & & & & & & & & \\
\hline $00: 28: 50$ & -101 & 10 & 48 & 0 & 0.43 & 0.21 & 0.15 & 084 & 0.434 & 9.0 & 100.0 & 180 & 18 & 090 & 1.0 & 180 \\
\hline$: 29: 00$ & -99 & 9 & 45 & 0 & 0.43 & 0.21 & 0.16 & 085 & 0.351 & 7.6 & 100.0 & 180 & 19 & 090 & 1.0 & 180 \\
\hline $0: 29: 10$ & -97 & 8 & 40 & 0 & 0.38 & 0.22 & 0.17 & 085 & 0.288 & 7.1 & 100.0 & 180 & 24 & 090 & 1.0 & 180 \\
\hline $00: 29: 20$ & -95 & 8 & 34 & 0 & 0.35 & 0.18 & 0.19 & 085 & -0.059 & 7.4 & 100.0 & 180 & 24 & 090 & 1.0 & 180 \\
\hline $00: 29: 30$ & -93 & 7 & 30 & 0 & 0.29 & 0.06 & 0.22 & 085 & -1.038 & 12.7 & 100.0 & 180 & 28 & 090 & 1.0 & 180 \\
\hline $00: 29: 40$ & -92 & 6 & 30 & 0 & 0.19 & 0.07 & 0.22 & 084 & -0.989 & 17.2 & 100.0 & 180 & 24 & 090 & 1.0 & 180 \\
\hline $00: 29: 50$ & -91 & 6 & 30 & 0 & 0.08 & 0.07 & 0.22 & 084 & -0.978 & 22.1 & 100.0 & 180 & 22 & 090 & 1.0 & 180 \\
\hline $00: 30: 00$ & -91 & 5 & 30 & 0 & 0.02 & 0.08 & 0.22 & 084 & -0.888 & 25.2 & 100.0 & 180 & 19 & 090 & 1.0 & 180 \\
\hline $00: 30: 10$ & -91 & 4 & 30 & 0 & -0.06 & 0.08 & 0.21 & 084 & -0.822 & 26.6 & 100.0 & 180 & 23 & 090 & 1.0 & 180 \\
\hline $00: 30: 20$ & -91 & 3 & 30 & 0 & -0.11 & 0.10 & 0.20 & 084 & -0.591 & 26.3 & 54.0 & 180 & 27 & 090 & 1.0 & 180 \\
\hline $00: 30: 30$ & -92 & 3 & 30 & $\mathbf{0}$ & -0.12 & 0.09 & 0.17 & 084 & -0.480 & 27.4 & 52.0 & 180 & 26 & 090 & 1.0 & 180 \\
\hline $00: 30: 40$ & -92 & 2 & 30 & 0 & -0.23 & 0.09 & 0.12 & 084 & -0.166 & 26.1 & 52.0 & 180 & 22 & 090 & 1.0 & 180 \\
\hline $00: 30 ; 50$ & -94 & 1 & 30 & 0 & -0.30 & 0.12 & 0.07 & 084 & 0.295 & 24.3 & 25.0 & 180 & 27 & 090 & 1.0 & 180 \\
\hline $00: 31: 00$ & -95 & 1 & 30 & 0 & -0.35 & 0.18 & 0.04 & 084 & 0.867 & 20.8 & 0.0 & 180 & 22 & 090 & 1.0 & 180 \\
\hline $00: 31: 10$ & -97 & 0 & 40 & 0 & -0.35 & 0.22 & -0.01 & 084 & 1.451 & 15.6 & 0.0 & 180 & 21 & 090 & 1.0 & 180 \\
\hline $00: 31: 20$ & -99 & -1 & 41 & 0 & -0.31 & 0.26 & -0.04 & 084 & 1.930 & 8.2 & 0.0 & 180 & 26 & 090 & 1.0 & 180 \\
\hline $00: 31: 30$ & -100 & -2 & 40 & 0 & -0.21 & 0.29 & -0.04 & 085 & 2.125 & -1.2 & 0.0 & 180 & 19 & 090 & 1.0 & 180 \\
\hline $00: 31: 40$ & -101 & -2 & 41 & 0 & -0.15 & 0.33 & -0.01 & 085 & 2.156 & -9.3 & 0.0 & 180 & 26 & 090 & 1.0 & 180 \\
\hline $00: 31: 50$ & -102 & -3 & 40 & 0 & -0.16 & 0.32 & 0.05 & 085 & 1.737 & -12.0 & 0.0 & 180 & 22 & 090 & 1.0 & 180 \\
\hline $00: 32: 00$ & -102 & -4 & 45 & 0 & -0.04 & 0.23 & 0.14 & 085 & 0.585 & -11.2 & 0.0 & 180 & 28 & 090 & 1.0 & 180 \\
\hline $00: 32: 10$ & -102 & -5 & 45 & 0 & 0.01 & 0.14 & 0.25 & 085 & -0.734 & -3.0 & 0.0 & 180 & 27 & 090 & 1.0 & 180 \\
\hline $00: 32: 20$ & -102 & -6 & 45 & 0 & 0.05 & 0.06 & 0.31 & 085 & -1.578 & 6.1 & 0.0 & 180 & 20 & 090 & 1.0 & 180 \\
\hline $00: 32: 30$ & -101 & -7 & 45 & 0 & 0.02 & 0.06 & 0.32 & 085 & -1.651 & 12.2 & 0.0 & 180 & 23 & 090 & 1.0 & 180 \\
\hline $00: 32: 40$ & -101 & -8 & 44 & 0 & 0.05 & 0.11 & 0.30 & 085 & -1.244 & 12.5 & 0.0 & 180 & 24 & 090 & 1.0 & 180 \\
\hline $00: 32: 50$ & -100 & -9 & 44 & 0 & 0.17 & 0.06 & 0.29 & 084 & -1.469 & 16.8 & 0.0 & 180 & 22 & 090 & 1.0 & 180 \\
\hline $00: 33: 00$ & -99 & -10 & 44 & 0 & 0.20 & 0.08 & 0.24 & 084 & -1.065 & 18.4 & 0.0 & 180 & 22 & 090 & 1.0 & 180 \\
\hline $00: 33: 10$ & -98 & -11 & 29 & 0 & 0.19 & 0.11 & 0.21 & 084 & -0.668 & 19.5 & 0.0 & 180 & 21 & 090 & 1.0 & 180 \\
\hline $00: 33: 20$ & -97 & -11 & 25 & 0 & 0.14 & 0.12 & 0.22 & 084 & -0.594 & 21.2 & 0.0 & 180 & 18 & 090 & 1.0 & 180 \\
\hline $00: 33: 30$ & -97 & -12 & 25 & 0 & 0.08 & 0.07 & 0.22 & 084 & -0.943 & 26.8 & 0.0 & 180 & 21 & 090 & 1.0 & 180 \\
\hline $00: 33: 40$ & -97 & -13 & 25 & 0 & -0.02 & 0.06 & 0.22 & 084 & -0.992 & 32.1 & 0.0 & 180 & 22 & 090 & 1.0 & 180 \\
\hline $00: 33: 50$ & -97 & -14 & 25 & 0 & -0.06 & 0.07 & 0.21 & 084 & -0.878 & 32.8 & 0.0 & 180 & 29 & 090 & 1.0 & 180 \\
\hline $00: 34: 00$ & -97 & -14 & 25 & 0 & -0.12 & 0.09 & 0.20 & 083 & -0.662 & 33.0 & 0.0 & 180 & 22 & 090 & 1.0 & 180 \\
\hline $00: 34: 10$ & -98 & -15 & 25 & 0 & -0.15 & 0.15 & 0.15 & 083 & -0.030 & 30.1 & 0.0 & 180 & 23 & 090 & 1.0 & 180 \\
\hline $00: 34: 20$ & -98 & -16 & 25 & 0 & -0.16 & 0.23 & 0.12 & 083 & 0.700 & 25.3 & 0.0 & 180 & 22 & 090 & 1.0 & 180 \\
\hline $00: 34: 30$ & -99 & -17 & 25 & 0 & -0.12 & 0.30 & 0.09 & 084 & .1 .313 & 19.2 & 0.0 & 180 & 24 & 090 & 1.0 & 180 \\
\hline $00: 34: 40$ & -100 & -18 & 25 & 0 & -0.13 & 0.29 & 0.08 & 084 & 1.352 & 14.3 & 0.0 & 180 & 26 & 090 & 1.0 & 180 \\
\hline $00: 34: 50$ & -100 & -19 & 25 & 0 & -0.12 & 0.23 & 0.08 & 084 & 0.967 & 12.1 & 0.0 & 180 & 25 & 090 & 1.0 & 180 \\
\hline $00: 35: 00$ & -101 & -20 & 25 & 0 & -0.13 & 0.23 & 0.08 & 084 & 1.009 & 9.4 & 0.0 & 180 & 32 & 090 & 1.0 & 180 \\
\hline $00: 35: 10$ & -101 & -21 & 25 & 0 & -0.22 & 0.24 & 0.08 & 084 & 1.005 & 7.3 & 0.0 & 180 & 24 & 090 & 1.0 & 180 \\
\hline $3: 35: 20$ & -102 & -22 & 25 & 0 & -0.21 & 0.18 & 0.09 & 084 & 0.604 & 6.7 & 0.0 & 180 & 26 & 090 & 1.0 & 180 \\
\hline $00: 35: 30$ & -103 & -23 & 25 & 0 & -0.22 & 0.17 & 0.10 & 085 & 0.437 & 5.8 & 0.0 & 180 & 24 & 090 & 1.0 & 180 \\
\hline $00: 35: 40$ & -104 & -23 & 25 & 0 & -0.18 & 0.14 & 0.11 & 085 & 0.230 & 5.5 & 0.0 & 180 & 21 & 090 & 1.0 & 180 \\
\hline $00: 35: 50$ & -105 & -24 & 25 & 0 & -0.22 & 0.13 & 0.12 & 085 & 0.093 & 6.3 & 0.0 & 180 & 17 & 090 & 1.0 & 180 \\
\hline
\end{tabular}




\section{Simulation Track Plot}

Open Ocean

Ship: 150,000 ton, Tanker, partial load

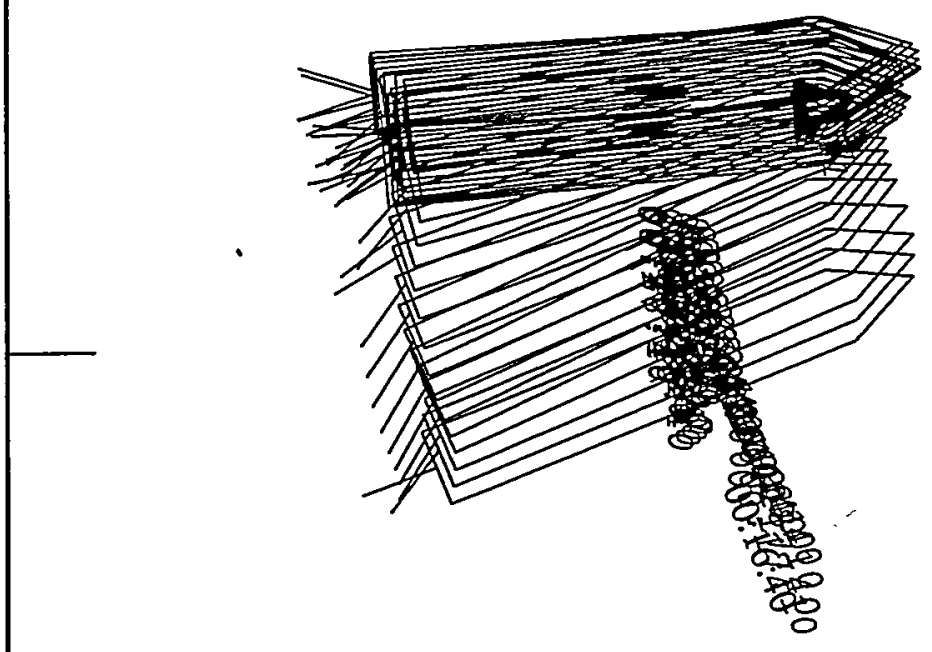

Start Time [hrs:min:sec]: $00: 16: 40$ End Time [hrs:min:sec]: $00: 36: 00$ Delta Plot Time [sec]: 


\section{HANDS-ON SIMULATION}

\section{SUCTION MOORNG TECHNOLOGY}

Simulation Number: 24

Date: 4 February 1999

Start Time: 13:55

Length of run [secs]: 2235

Ship Condition: draft $=13.72 \mathrm{~m}$

Environment

Wind Speed [knots]

25 Direction: 90

Sea State, Significant wave height $[\mathrm{m}]: .$.

5 Direction: 270

Current Speed [knots]:

1 Direction: 180

Ship Operator: J.A.

Observer: B.K.J \& J.K.

Final Mating, distance between center of buoy and center of moon pool [m]: 2.4

Evaluation:

The approach went well.

Fairly close replay of run \# 23.

The captain tried to use the thruster at 120 degrees to get the ship to move faster, and it worked. The captain feels the rudder rate is too slow. 
Ship:

Exercise number:

senario: g file: affic ships: Initial conditions: Exercise identification:
591 150,000 ton, Tanker, partial load

754 Suction Mooring System

112 Op

999 Dummy tug file based on containership

590 Suction Mooring Buoy

594 150k tankex in partial load - Suction Mooring RuN \#24

Time Position (m) $\quad$ RPM $\quad$ Speed (Knots) Turn Rudder Gill Wind

\begin{tabular}{|c|c|c|c|c|c|c|c|c|c|c|c|c|c|c|c|c|}
\hline $00: 16: 40$ & -124 & -152 & 76 & 0 & 1.77 & 0.07 & -2.91 & 049 & 18.932 & 35.0 & 99.0 & 129 & 32 & 090 & 1.0 & 180 \\
\hline $00 ; 16: 50$ & -122 & -141 & 76 & 0 & 1.73 & 0.06 & -2.90 & 052 & 18.738 & 35.0 & 99.0 & 129 & 21 & 090 & 1.0 & 180 \\
\hline $00: 17: 00$ & -119 & -130 & 76 & 0 & 1.78 & -0.04 & -2.88 & 055 & 18.005 & 35.0 & 99.0 & 129 & 29 & 090 & 1.0 & 180 \\
\hline $00: 17: 10$ & -115 & -118 & 76 & 0 & 1.79 & 0.08 & -2.84 & 058 & 18.539 & 35.0 & 99.0 & 129 & 25 & 090 & 1.0 & 180 \\
\hline $00: 17: 20$ & -111 & -108 & 76 & 0 & 1.74 & 0.27 & -2.77 & 061 & 19.299 & 35.0 & 99.0 & 129 & 22 & 090 & 1.0 & 180 \\
\hline $00: 17: 30$ & -105 & -98 & 61 & 0 & 1.77 & 0.35 & -2.65 & 065 & 19.040 & 35.0 & 99.0 & 129 & 24 & 090 & 1.0 & 180 \\
\hline $00: 17: 40$ & -99 & -90 & 54 & 0 & 1.70 & 0.25 & -2.44 & 068 & 17.046 & 35.0 & 99.0 & 129 & 16 & 090 & 1.0 & 180 \\
\hline $00: 17: 50$ & -94 & -81 & 29 & 0 & 1.58 & -0.04 & -2.22 & 070 & 13.775 & 35.0 & 99.0 & 129 & 25 & 090 & 1.0 & 180 \\
\hline $00: 18: 00$ & -88 & -73 & 31 & 0 & 1.36 & -0.10 & -2.02 & 072 & 12.171 & 35.0 & 99.0 & 229 & 26 & 090 & 1.0 & 180 \\
\hline $00: 18: 10$ & -84 & -67 & 8 & 0 & 1.14 & 0.09 & -1.83 & 074 & 12.188 & 35.0 & 99.0 & 129 & 20 & 090 & 1.0 & 180 \\
\hline $00: 18: 20$ & -79 & -61 & -47 & 0 & 0.96 & 0.01 & -1.65 & 076 & 10.526 & 35.0 & 99.0 & 129 & 23 & 090 & 1.0 & 180 \\
\hline $00: 18: 30$ & -76 & -56 & -61 & 0 & 0.74 & 0.02 & -1.46 & 078 & 9.350 & 29.9 & 99.0 & 129 & 20 & 090 & 1.0 & 180 \\
\hline $00: 18: 40$ & -73 & -52 & -61 & 0 & 0.52 & 0.10 & -1.28 & 079 & 8.784 & 10.9 & 99.0 & 129 & 13 & 090 & 1.0 & 180 \\
\hline $00: 18: 50$ & -72 & -49 & -30 & 0 & 0.31 & 0.28 & -1.13 & 081 & 8.968 & -8.1 & 99.0 & 129 & 25 & 090 & 1.0 & 180 \\
\hline $00: 19: 00$ & -71 & -47 & 26 & 0 & 0.22 & 0.29 & -0.94 & 082 & 7.812 & -27.1 & 99.0 & 129 & 23 & 090 & 1.0 & 180 \\
\hline $00: 19: 10$ & -70 & -46 & 37 & 0 & 0.15 & 0.20 & -0.64 & 083 & 5.336 & -35.0 & 99.0 & 176 & 28 & 090 & 1.0 & 180 \\
\hline $00: 19: 20$ & -69 & -45 & 37 & 0 & 0.14 & 0.07 & -0.36 & 084 & 2.762 & -35.0 & 75.0 & 176 & 27 & 090 & 1.0 & 180 \\
\hline $00: 19: 30$ & -69 & -44 & 37 & 0 & 0.11 & -0.07 & -0.12 & 084 & 0.338 & -35.0 & 52.0 & 180 & 23 & 090 & 1.0 & 180 \\
\hline $00: 19: 40$ & -68 & -44 & 37 & 0 & 0.05 & -0.16 & 0.09 & 084 & -1.579 & -35.0 & 52.0 & 180 & 28 & 090 & 1.0 & 180 \\
\hline $00: 19: 50$ & -68 & -44 & 37 & 0 & 0.05 & -0.25 & 0.27 & 084 & -3.351 & -23.6 & 52.0 & 180 & 24 & 090 & 1.0 & 180 \\
\hline $00: 20: 00$ & -68 & -44 & 37 & 0 & 0.11 & -0.31 & 0.44 & 083 & -4.767 & -35.0 & 52.0 & 180 & 23 & 090 & 1.0 & 180 \\
\hline $00: 20: 10$ & -67 & -44 & 37 & 0 & 0.09 & -0.37 & 0.58 & 082 & -6.037 & -16.0 & 74.0 & 180 & 24 & 090 & 1.0 & 180 \\
\hline $00: 20 ; 20$ & -67 & -45 & 37 & 0 & 0.13 & -0.45 & 0.64 & 081 & -6.900 & 3.0 & 74.0 & 180 & 26 & 090 & 1.0 & 180 \\
\hline $00: 20: 30$ & -66 & -45 & 37 & 0 & 0.14 & -0.46 & 0.63 & 080 & -6.898 & 22.0 & 74.0 & 180 & 20 & 090 & 1.0 & 180 \\
\hline $00: 20: 40$ & -65 & -45 & 37 & 0 & 0.06 & -0.46 & 0.56 & 079 & -6.422 & 35.0 & 74.0 & 180 & 27 & 090 & 1.0 & 180 \\
\hline $00: 20: 50$ & -65 & -45 & 37 & 0 & -0.01 & -0.50 & 0.48 & 078 & -6.236 & 35.0 & 74.0 & 180 & 25 & 090 & 1.0 & 180 \\
\hline $00: 21: 00$ & -65 & -45 & 37 & 0 & -0.11 & -0.51 & 0.40 & 077 & -5.799 & 35.0 & 100.0 & 180 & 19 & 090 & 1.0 & 180 \\
\hline $00: 21: 10$ & -66 & -45 & 37 & 0 & -0.11 & -0.60 & 0.34 & 076 & -5.948 & 35.0 & 25.0 & 180 & 22 & 090 & 1.0 & 180 \\
\hline $00: 21 ; 20$ & -67 & -44 & 37 & 0 & -0.15 & -0.64 & 0.28 & 075 & -5.852 & 35.0 & 26.0 & 180 & 21 & 090 & 1.0 & 280 \\
\hline$: 21: 30$ & -68 & -44 & 37 & 0 & -0.22 & -0.40 & 0.21 & 074 & -3.875 & 35.0 & 26.0 & 180 & 24 & 090 & 1.0 & 180 \\
\hline $.0: 21: 40$ & -69 & -44 & 39 & 0 & -0.21 & -0.25 & 0.14 & 074 & -2.498 & 35.0 & 26.0 & 180 & 25 & 090 & 1.0 & 180 \\
\hline $00: 21: 50$ & -70 & -44 & 45 & 0 & -0.14 & -0.24 & 0.04 & 073 & -1.772 & 35.0 & 26.0 & 180 & 19 & 090 & 1.0 & 180 \\
\hline $00: 22: 00$ & -71 & -43 & 49 & 0 & -0.09 & -0.32 & -0.07 & 073 & -1.577 & 35.0 & 26.0 & 180 & 23 & 090 & 1.0 & 180 \\
\hline $00: 22: 10$ & -72 & -42 & 49 & 0 & -0.11 & -0.13 & -0.20 & 073 & 0.411 & 35.0 & 26.0 & 180 & 25 & 090 & 1.0 & 180 \\
\hline $00: 22: 20$ & -73 & -42 & 48 & 0 & -0.07 & -0.08 & -0.31 & 073 & 1.436 & 35.0 & 26.0 & 180 & 19 & 090 & 1.0 & 180 \\
\hline $00: 22: 30$ & -73 & -41 & 48 & 0 & -0.07 & -0.07 & -0.41 & 073 & 2.109 & 35.0 & 78.0 & 180 & 33 & 090 & 1.0 & 180 \\
\hline $00: 22: 40$ & -74 & -40 & 45 & 0 & -0.04 & -0.06 & -0.47 & 074 & 2.555 & 35.0 & 78.0 & 180 & 23 & 090 & 1.0 & 180 \\
\hline $00: 22: 50$ & -74 & -38 & 45 & 0 & 0.01 & -0.06 & -0.52 & 074 & 2.915 & 35.0 & 78.0 & 180 & 19 & 090 & 1.0 & 180 \\
\hline $00: 23: 00$ & -75 & -37 & 45 & 0 & 0.02 & 0.08 & -0.57 & 075 & 4.126 & 35.0 & 78.0 & 180 & 22 & 090 & 1.0 & 180 \\
\hline $00: 23: 10$ & -75 & -36 & 44 & 0 & 0.02 & 0.02 & -0.60 & 075 & 3.930 & 35.0 & 78.0 & 180 & 19 & 090 & 1.0 & 180 \\
\hline $00: 23: 20$ & -75 & -34 & 44 & 0 & 0.07 & 0.05 & -0.63 & 076 & 4.274 & 35.0 & 78.0 & 180 & 25 & 090 & 1.0 & 180 \\
\hline $00: 23: 30$ & -75 & -33 & 44 & 0 & 0.05 & 0.01 & -0.65 & 077 & 4.178 & 35.0 & 78.0 & 180 & 24 & 090 & 1.0 & 180 \\
\hline $00: 23: 40$ & -75 & -31 & 45 & 0 & 0.01 & -0.14 & -0.67 & 077 & 3.361 & 35.0 & 99.0 & 180 & 25 & 090 & 1.0 & 180 \\
\hline $00: 23: 50$ & -76 & -28 & 41 & 0 & -0.07 & -0.23 & -0.67 & 078 & 2.763 & 35.0 & 99.0 & 180 & 27 & 090 & 1.0 & 180 \\
\hline $00: 24: 00$ & -77 & -26 & 38 & 0 & -0.17 & -0.20 & -0.64 & 078 & 2.817 & 35.0 & 99.0 & 180 & 25 & 090 & 1.0 & 180 \\
\hline $00: 24: 10$ & -78 & -25 & 38 & 0 & -0.25 & -0.16 & -0.67 & 079 & 3.260 & 35.0 & 99.0 & 180 & 21 & 090 & 1.0 & 180 \\
\hline $00: 24: 20$ & -80 & -23 & 38 & 0 & -0.35 & -0.03 & -0.74 & 079 & 4.471 & 35.0 & 99.0 & 180 & 28 & 090 & 1.0 & 180 \\
\hline $00: 24: 30$ & -82 & -21 & 38 & 0 & -0.34 & 0.05 & -0.76 & 080 & 5.139 & $18: 9$ & 49.0 & 180 & 21 & 090 & 1.0 & 180 \\
\hline $00: 24: 40$ & -84 & -20 & 38 & 0 & -0.31 & 0.05 & -0.72 & 081 & 4.858 & 3.2 & 49.0 & 180 & 23 & 090 & 1.0 & 180 \\
\hline $00: 24: 50$ & -86 & -18 & 38 & 0 & -0.38 & 0.07 & -0.63 & 082 & 4.467 & -9.6 & 49.0 & 180 & 27 & 090 & 1.0 & 180 \\
\hline $00: 25: 00$ & -89 & -18 & 42 & 0 & -0.43 & 0.09 & -0.51 & 083 & 3.804 & -20.1 & 49.0 & 180 & 28 & 090 & 1.0 & 180 \\
\hline $00: 25: 10$ & -91 & -17 & 45 & 0 & -0.41 & 0.02 & -0.34 & 083 & 2.310 & -26.6 & 24.0 & 180 & 24 & 090 & 1.0 & 180 \\
\hline $00: 25: 20$ & -93 & -16 & 52 & 0 & -0.30 & -0.13 & -0.14 & 083 & 0.040 & -24.5 & 24.0 & 180 & 21 & 090 & 1.0 & 180 \\
\hline $00: 25: 30$ & -94 & -16 & 52 & 0 & -0.28 & -0.18 & 0.02 & 083 & -1.272 & -16.9 & 24.0 & 180 & 19 & 090 & 1.0 & 180 \\
\hline $00: 25: 40$ & -96 & -16 & 52 & 0 & -0.17 & -0.15 & 0.12 & 083 & -1.686 & -5.9 & 24.0 & 180 & 17 & 090 & 1.0 & 180 \\
\hline $00: 25: 50$ & -96 & -16 & 56 & 0 & -0.05 & -0.11 & 0.15 & 083 & -1.656 & 11.7 & 24.0 & 180 & 23 & 090 & 1.0 & 180 \\
\hline $00: 26: 00$ & -96 & -16 & 50 & 0 & 0.10 & -0.07 & 0.09 & 082 & -1.044 & 15.5 & 24.0 & 180 & 19 & 090 & 1.0 & 180 \\
\hline $00: 26: 10$ & -95 & -16 & 48 & 0 & 0.19 & -0.16 & 0.13 & 082 & -1.830 & 7.2 & 74.0 & 280 & 27 & 090 & 1.0 & 180 \\
\hline $00: 26: 20$ & -94 & -16 & 47 & 0 & 0.22 & -0.13 & 0.14 & 082 & -1.731 & 10.9 & 74.0 & 180 & 20 & 090 & 1.0 & 180 \\
\hline $00: 26: 30$ & -93 & -16 & 36 & 0 & 0.14 & -0.22 & 0.14 & 082 & -2.266 & 24.7 & 100.0 & 180 & 19 & 090 & 1.0 & 180 \\
\hline $00: 26: 40$ & -93 & -15 & 36 & 0 & 0.07 & -0.25 & 0.10 & 081 & -2.205 & 35.0 & 100.0 & 180 & 29 & 090 & 1.0 & 180 \\
\hline $00: 26: 50$ & -93 & -15 & 36 & 0 & -0.03 & -0.23 & 0.05 & 081 & -1.764 & 35.0 & 100.0 & 180 & 25 & 090 & 1.0 & 180 \\
\hline $00: 27: 00$ & -93 & -14 & 36 & 0 & -0.09 & -0.22 & 0.01 & 081 & -1.414 & 35.0 & 100.0 & 180 & 25 & 090 & 1.0 & 180 \\
\hline $00: 27: 10$ & -94 & -14 & 36 & 0 & -0.15 & -0.24 & -0.03 & 080 & -1.332 & 34.5 & 100.0 & 180 & 27 & 090 & 1.0 & 180 \\
\hline $00: 27: 20$ & -95 & -13 & 36 & 0 & -0.24 & -0.15 & -0.08 & 080 & -0.463 & 15.5 & 100.0 & 180 & 23 & 090 & 1.0 & 180 \\
\hline $00: 27: 30$ & -96 & -13 & 37 & 0 & -0.27 & -0.11 & -0.08 & 080 & -0.164 & -3.5 & 100.0 & 180 & 25 & 090 & 1.0 & 180 \\
\hline $00: 27: 40$ & -98 & -13 & 40 & 0 & -0.36 & -0.14 & -0.01 & 080 & -0.797 & -22.5 & 100.0 & 180 & 26 & 090 & 1.0 & 180 \\
\hline $0: 27: 50$ & -100 & -13 & 54 & 0 & -0.36 & -0.29 & 0.18 & 080 & -2.995 & -23.4 & 25.0 & 180 & 23 & 090 & 1.0 & 180 \\
\hline$: 28: 00$ & -101 & -13 & 58 & 0 & -0.27 & -0.41 & 0.31 & 079 & -4.583 & -4.7 & 25.0 & 180 & 29 & 090 & 1.0 & 180 \\
\hline $00: 28: 10$ & -103 & -13 & 58 & 0 & -0.18 & -0.40 & 0.30 & 078 & -4.424 & 14.3 & 25.0 & 180 & 19 & 090 & 1.0 & 180 \\
\hline $00: 28: 20$ & -103 & -13 & 57 & 0 & -0.14 & -0.22 & 0.16 & 078 & -2.448 & 33.3 & 25.0 & 180 & 24 & 090 & 1.0 & 180 \\
\hline $00: 28: 30$ & -104 & -13 & 58 & 0 & -0.06 & 0.01 & -0.06 & 078 & 0.406 & 35.0 & 51.0 & 180 & 24 & 090 & 1.0 & 180 \\
\hline $00: 28: 40$ & -104 & -13 & 57 & 0 & 0.13 & 0.11 & -0.25 & 078 & 2.285 & 35.0 & 75.0 & 180 & 19 & 090 & 1.0 & 180 \\
\hline
\end{tabular}


Time Position (m) RPM Speed (Knots) Turn Rudder Gill Wind

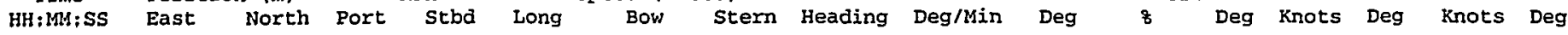

\begin{tabular}{|c|c|c|c|c|c|c|c|c|c|c|c|c|c|c|c|c|}
\hline $0: 28: 50$ & -103 & -12 & 58 & 0 & 0.22 & 0.20 & -0.43 & 078 & 4.008 & 35.0 & 52.0 & 180 & 18 & 090 & 1.0 & 180 \\
\hline$: 29: 00$ & -102 & -11 & 57 & 0 & 0.30 & 0.29 & -0.58 & 079 & 5.506 & 35.0 & 52.0 & 180 & 19 & 090 & 1.0 & 180 \\
\hline$d: 29: 10$ & -100 & -10 & 57 & 0 & 0.33 & 0.40 & -0.70 & 080 & 6.997 & 35.0 & 98.0 & 180 & 24 & 090 & 1.0 & 180 \\
\hline $00: 29: 20$ & -99 & -9 & 38 & 0 & 0.31 & 0.39 & -0.70 & 081 & 6.917 & 35.0 & 98.0 & 180 & 24 & 090 & 1.0 & 180 \\
\hline $00: 29: 30$ & -97 & -8 & 39 & 0 & 0.27 & 0.22 & -0.66 & 082 & 5.587 & 26.9 & 98.0 & 180 & 28 & 090 & 1.0 & 180 \\
\hline $00: 29: 40$ & -96 & -7 & 39 & 0 & 0.19 & 0.21 & -0.58 & 083 & 5.003 & 7.9 & 98.0 & 180 & 24 & 090 & 1.0 & 180 \\
\hline $00: 29: 50$ & -96 & -6 & 40 & 0 & 0.14 & 0.15 & -0.42 & 084 & 3.609 & -11.1 & 74.0 & 180 & 22 & 090 & 1.0 & 180 \\
\hline $00: 30: 00$ & -95 & -5 & 40 & 0 & 0.12 & 0.05 & -0.20 & 085 & 1.619 & -30.1 & 99.0 & 180 & 19 & 090 & 1.0 & 180 \\
\hline $00: 30: 10$ & -95 & -5 & 40 & 0 & 0.06 & -0.07 & 0.03 & 085 & -0.603 & -24.9 & 99.0 & 180 & 23 & 090 & 1.0 & 180 \\
\hline $00: 30: 20$ & -95 & -5 & 40 & 0 & 0.03 & -0.11 & 0.17 & 084 & -1.775 & -5.9 & 99.0 & 180 & 27 & 090 & 1.0 & 180 \\
\hline $00: 30: 30$ & -94 & -5 & 40 & 0 & -0.01 & -0.15 & 0.22 & 084 & -2.377 & 13.1 & 99.0 & 180 & 26 & 090 & 1.0 & 180 \\
\hline $00: 30: 40$ & -95 & -5 & 40 & 0 & -0.12 & -0.17 & 0.23 & 084 & -2.547 & 30.8 & 99.0 & 180 & 22 & 090 & 1.0 & 180 \\
\hline $00: 30: 50$ & -96 & -5 & 40 & 0 & -0.21 & -0.09 & 0.14 & 083 & -1.461 & 21.0 & 99.0 & 180 & 27 & 090 & 1.0 & 180 \\
\hline $00: 31: 00$ & -97 & -6 & 39 & 0 & -0.24 & -0.01 & 0.06 & 083 & -0.460 & 18.1 & 28.0 & 180 & 22 & 090 & 1.0 & 180 \\
\hline $00: 31: 10$ & -98 & -6 & 39 & 0 & -0.23 & 0.05 & 0.01 & 083 & 0.296 & 14.3 & 15.0 & 180 & 21 & 090 & 1.0 & 180 \\
\hline $00: 31: 20$ & -99 & -6 & 40 & 0 & -0.19 & 0.11 & -0.05 & 083 & 1.013 & 23.7 & 15.0 & 280 & 26 & 090 & 1.0 & 180 \\
\hline $00: 31: 30$ & -100 & -6 & 40 & 0 & -0.10 & 0.18 & -0.10 & 083 & 1.806 & 9.6 & 15.0 & 180 & 19 & 090 & 1.0 & 18 \\
\hline $00: 31: 40$ & -100 & -7 & 40 & 0 & -0.06 & 0.21 & -0.03 & 084 & 1.527 & 5.2 & 74.0 & 180 & 26 & 090 & 1.0 & 180 \\
\hline $00: 31: 50$ & -100 & -7 & 40 & 0 & -0.09 & 0.20 & 0.02 & 084 & 1.161 & 8.9 & 49.0 & 180 & 22 & 090 & 1.0 & 180 \\
\hline $00: 32: 00$ & -101 & -8 & 40 & 0 & 0.00 & 0.16 & 0.04 & 084 & 0.746 & 24.6 & 49.0 & 180 & 28 & 090 & 1.0 & 18 \\
\hline $00: 32: 10$ & -100 & -8 & 40 & 0 & 0.00 & 0.13 & 0.02 & 084 & 0.676 & 21.0 & 75.0 & 180 & 27 & 090 & 1.0 & 180 \\
\hline $00: 32: 20$ & -100 & -9 & 39 & 0 & -0.01 & 0.08 & 0.02 & 084 & 0.400 & 20.7 & 52.0 & 180 & 20 & 090 & 1.0 & 180 \\
\hline $00: 32: 30$ & -101 & -9 & 39 & 0 & -0.07 & 0.09 & 0.01 & 084 & 0.514 & 21.6 & 37.0 & 180 & 23 & 090 & 1.0 & 180 \\
\hline $00: 32: 40$ & -101 & -9 & 40 & 0 & -0.06 & 0.15 & 0.01 & 085 & 0.885 & 16.5 & 25.0 & 180 & 24 & 090 & 1.0 & 180 \\
\hline $00: 32: 50$ & -101 & -10 & 40 & 0 & 0.03 & 0.08 & 0.03 & 085 & 0.339 & 17.7 & 19.0 & 180 & 22 & 090 & 1.0 & 18 \\
\hline $00: 33: 00$ & -101 & -10 & 40 & 0 & 0.05 & 0.08 & 0.05 & 085 & 0.204 & 6.3 & 0.0 & 180 & 22 & 090 & 1.0 & 18 \\
\hline $00: 33: 10$ & -101 & -10 & 40 & 0 & 0.03 & 0.05 & 0.13 & 085 & -0.563 & -5.4 & 0.0 & 180 & 21 & 090 & 1.0 & 18 \\
\hline $00: 33: 20$ & -100 & -11 & 40 & 0 & 0.03 & 0.03 & 0.21 & 085 & -1.135 & 1.5 & 0.0 & 180 & 18 & 090 & 1.0 & 180 \\
\hline $00: 33: 30$ & -100 & -11 & 40 & 0 & 0.02 & -0.03 & 0.24 & 084 & -1.734 & 13.4 & 0.0 & 180 & 21 & 090 & 1.0 & 180 \\
\hline $00: 33: 40$ & -100 & -12 & 40 & 0 & -0.03 & -0.03 & 0.23 & 084 & -1.657 & 18.7 & 0.0 & 180 & 22 & 090 & 1.0 & 180 \\
\hline $00: 33: 50$ & -100 & -13 & 39 & 0 & -0.02 & 0.00 & 0.21 & 084 & -1.383 & 19.8 & 0.0 & 180 & 29 & 090 & 1.0 & 180 \\
\hline $00: 34: 00$ & -100 & -13 & 40 & 0 & -0.04 & 0.03 & 0.19 & 084 & -1.052 & 19.2 & 0.0 & 180 & 22 & 090 & 1.0 & 180 \\
\hline $00: 34: 10$ & -100 & -14 & 40 & 0 & -0.03 & 0.08 & 0.18 & 083 & -0.659 & 17.4 & 0.0 & 180 & 23 & 090 & 1.0 & 180 \\
\hline $00: 34: 20$ & -100 & -14 & 40 & 0 & 0.01 & 0.14 & 0.17 & 083 & -0.213 & 14.1 & 0.0 & 180 & 22 & 090 & 1.0 & 18 \\
\hline $00: 34: 30$ & -100 & -15 & 40 & 0 & 0.10 & 0.20 & 0.18 & 083 & 0.128 & 11.7 & 0.0 & 180 & 24 & 090 & 1.0 & 18 \\
\hline $00: 34: 40$ & -99 & -16 & 40 & 0 & 0,14 & 0.18 & 0.19 & 083 & -0.045 & 12.0 & 0.0 & 180 & 26 & 090 & 1.0 & 18 \\
\hline $00: 34: 50$ & -98 & -17 & 40 & 0 & 0.19 & 0.11 & 0.19 & 083 & -0.501 & 15.4 & 0.0 & 180 & 25 & 090 & 1.0 & 18 \\
\hline $00: 35: 00$ & -97 & -18 & 40 & 0 & 0.21 & 0.12 & 0.17 & 083 & -0.345 & 16.2 & 0.0 & 180 & 32 & 090 & 1.0 & 18 \\
\hline $00: 35: 20$ & -96 & -18 & 39 & 0 & 0.17 & 0.13 & 0.16 & 083 & -0.205 & 16.2 & 0.0 & 180 & 24 & 090 & 1.0 & 18 \\
\hline $1: 35: 20$ & -95 & -19 & 40 & 0 & 0.22 & 0.08 & 0.15 & 083 & -0.462 & 18.5 & 0.0 & 180 & 26 & 090 & 1.0 & 18 \\
\hline $0: 35: 30$ & -94 & -19 & 40 & 0 & 0.25 & 0.07 & 0.13 & 083 & -0.350 & 19.0 & 0.0 & 180 & 24 & 090 & 1.0 & 18 \\
\hline $00: 35: 40$ & -92 & -20 & 40 & 0 & 0.33 & 0.06 & 0.11 & 083 & -0.269 & 18.9 & 0.0 & 180 & 21 & 090 & 1.0 & 180 \\
\hline $00: 35: 50$ & -90 & -20 & 40 & 0 & 0.33 & 0.07 & 0.08 & 083 & -0.088 & 19.5 & 0.0 & 180 & 17 & 090 & 1.0 & 18 \\
\hline $00: 36: 00$ & -89 & -20 & 40 & 0 & 0.28 & 0.14 & 0.06 & 083 & 0.498 & 16.6 & 0.0 & 180 & 19 & 090 & 1.0 & 18 \\
\hline $00: 36: 10$ & -87 & -20 & 40 & 0 & 0.34 & 0.08 & 0.06 & 083 & 0.150 & 16.3 & 0.0 & 180 & 23 & 090 & 1.0 & 18 \\
\hline $00: 36: 20$ & -85 & -20 & 40 & 0 & 0.35 & 0.03 & 0.05 & 083 & -0.138 & 17.9 & 0.0 & 180 & 25 & 090 & 1.0 & 18 \\
\hline $00: 36: 30$ & -84 & -20 & 39 & 0 & 0.28 & -0.01 & 0.04 & 083 & -0.298 & 19.3 & 0.0 & 180 & 21 & 090 & 1.0 & 18 \\
\hline $00: 36: 40$ & -83 & -20 & 40 & 0 & 0.20 & 0.06 & 0.03 & 083 & 0.211 & 16.9 & 0.0 & 180 & 22 & 090 & 1.0 & 18 \\
\hline $00: 36: 50$ & -82 & -20 & 40 & 0 & 0.22 & 0.10 & 0.03 & 083 & 0.434 & 14.7 & 0.0 & 180 & 21 & 090 & 1.0 & 18 \\
\hline $00: 37: 00$ & -80 & -21 & 40 & 0 & 0.24 & 0.12 & 0.03 & 083 & 0.536 & 13.2 & 0.0 & 180 & 18 & 090 & 1.0 & 18 \\
\hline $00: 37: 10$ & -79 & -21 & 40 & 0 & 0.28 & 0.16 & 0.05 & 083 & 0.708 & 10.8 & 0.0 & 180 & 26 & 090 & 1.0 & 18 \\
\hline
\end{tabular}




\section{Open Ocean}

\section{Simulation Track Plot}

Ship: 150,000 ton, Tanker, partial load

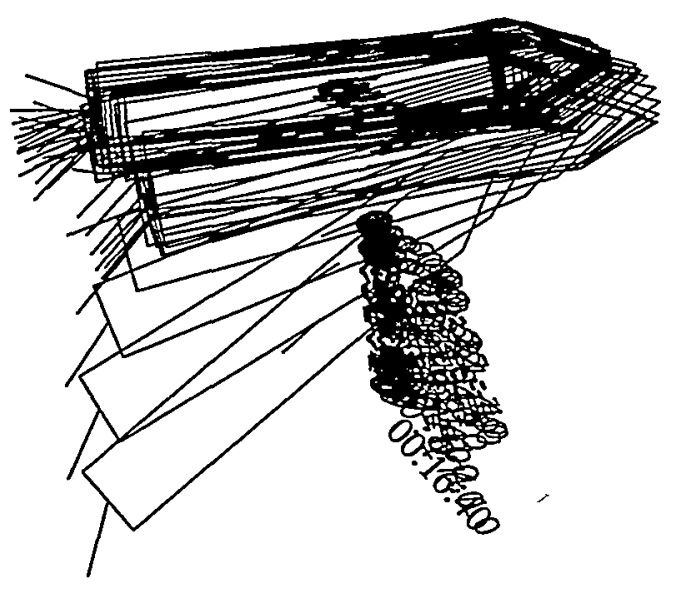

Start Time [hrs:min:sec]: $00: 16: 40$

End Time [hrs:min:sec]: $00: 37: 15$ Delta Plot Time [sec]:

East-West Scale [m]:

30

Date: 04-Feb-99 Time: 13:55:29

East-West Scale [Nm]:

0.5000 


\section{HANDS-ON SIMULATION}

\section{SUCTION MOORNG TECHNOLOGY}

Simulation Number: 25

Date: 4 February 1999

Start Time: 14:49

Length of run [secs]: 3465

Ship Condition: $\operatorname{draft}=13.72 \mathrm{~m}$

Environment

Wind Speed [knots]:

25 Direction: 90

Sea State, Significant wave height $[\mathrm{m}]$ :..

5 Direction: 270

Current Speed [knots]:

2 Direction: 180

Ship Operator: J.A.

Observer: B.K.J \& J.K.

Final Mating, distance between center of buoy and center of moon pool [m]: 1.8

Evaluation:

The approach was similar to the previous run \# 24.

When the captain was $400 \mathrm{~m}$ from the buoy, he backed down, reversed RPM and killed most of the speed. Then he used the thruster at 120 degrees to bring the bow around more quickly. First equilibrium - he tried 070 degrees and it was not an equilibrium heading. With a heading of 055 degrees he was able to close sidewise to the buoy. After that it was maneuvering between 060 and 0.70 degrees heading to play back and forth over the buoy. Higher rudder rate and lift would help. 
Ship:

Exercise number: enario: raffic ships:

Initial conditions: Exercise identification:
591 150,000 ton, Tanker, partial load

754 Suction Mooring System

112 Open Ocean

999 Dumny tug file based on containership

590 Suction Mooring Buoy

594 150k tanker in partial load - Suction Mooring RuN 425

Time Position (m) RPM Speed (Knots) Turn Rudaer Gill Wind Grent

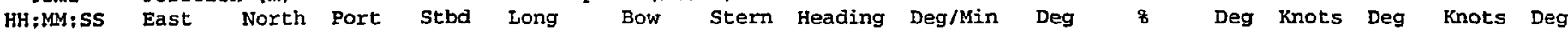

\begin{tabular}{|c|c|c|c|c|c|c|c|c|c|c|c|c|c|c|c|c|}
\hline $00: 33: 20$ & -75 & -102 & 39 & 0 & 0.08 & -0.20 & $\cdot-0.59$ & 053 & 2.462 & 25.6 & 51.0 & 180 & 18 & 090 & 2.0 & 180 \\
\hline $00: 33: 30$ & -76 & -100 & 39 & 0 & 0.04 & -0.41 & -0.58 & 054 & 1.110 & 24.2 & 51.0 & 180 & 21 & 090 & 2.0 & 180 \\
\hline $00: 33: 40$ & -77 & -98 & 39 & 0 & -0.02 & -0.43 & -0.56 & 054 & 0.804 & 22.9 & 51.0 & 180 & 22 & 090 & 2.0 & 180 \\
\hline $00: 33: 50$ & -79 & -96 & 39 & 0 & -0.03 & -0.36 & -0.52 & 054 & 1.027 & 19.2 & 27.0 & 180 & 29 & 090 & 2.0 & 180 \\
\hline $00: 34: 00$ & -80 & -94 & 39 & 0 & -0.05 & -0.27 & -0.47 & 054 & 1.278 & 14.5 & 0.0 & 180 & 22 & 090 & 2.0 & 180 \\
\hline $00: 34: 10$ & -82 & -93 & 39 & 0 & -0.04 & -0.11 & -0.38 & 054 & 1.715 & 6.0 & 0.0 & 180 & 23 & 090 & 2.0 & 180 \\
\hline $00: 34: 20$ & -82 & -93 & 39 & 0 & -0.01 & 0.11 & -0.24 & 055 & 2.175 & -12.0 & 0.0 & 180 & 22 & 090 & 2.0 & 180 \\
\hline $00: 34: 30$ & -82 & -93 & 42 & 0 & -0.06 & 0.24 & -0.04 & 055 & 1.771 & -21.6 & 0.0 & 180 & 24 & 090 & 2.0 & 180 \\
\hline $00: 34: 40$ & -81 & -93 & 42 & 0 & 0.10 & 0.08 & 0.09 & 055 & -0.045 & -2.6 & 0.0 & 180 & 26 & 090 & 2.0 & 180 \\
\hline $00: 34: 50$ & -81 & -92 & 42 & 0 & 0.16 & -0.26 & 0.08 & 055 & -2.135 & 16.3 & 0.0 & 180 & 25 & 090 & 2.0 & 180 \\
\hline $00: 35: 00$ & -80 & -91 & 41 & 0 & 0.18 & -0.22 & 0.03 & 055 & -1.598 & 17.7 & 24.0 & 180 & 32 & 090 & 2.0 & 180 \\
\hline $00: 35: 10$ & -80 & -91 & 42 & 0 & 0.15 & -0.19 & -0.01 & 054 & -1.104 & 19.1 & 51.0 & 180 & 24 & 090 & 2.0 & 180 \\
\hline $00: 35: 20$ & -80 & -89 & 42 & 0 & 0.18 & -0.41 & -0.09 & 054 & -2.047 & 24.7 & 51.0 & 180 & 26 & 090 & 2.0 & 180 \\
\hline $00: 35: 30$ & -80 & -88 & 42 & 0 & 0.20 & -0.42 & -0.17 & 054 & -1.589 & 27.8 & 51.0 & 180 & 24 & 090 & 2.0 & 180 \\
\hline $00: 35: 40$ & -80 & -86 & 41 & 0 & 0.24 & -0.45 & -0.26 & 054 & -1.226 & 29.8 & 100.0 & 180 & 21 & 090 & 2.0 & 180 \\
\hline $00: 35: 50$ & -80 & -83 & 42 & 0 & 0.18 & -0.43 & -0.34 & 053 & -0.544 & 31.4 & 100.0 & 180 & 17 & 090 & 2.0 & 180 \\
\hline $00: 36: 00$ & -81 & -82 & 42 & 0 & 0.09 & -0.13 & -0.37 & 053 & 1.520 & 21.5 & 100.0 & 180 & 19 & 090 & 2.0 & 280 \\
\hline $00: 36: 10$ & -81 & -80 & 42 & 0 & 0.11 & -0.38 & -0.39 & 054 & 0.063 & 24.4 & 51.0 & 180 & 23 & 090 & 2.0 & 180 \\
\hline $00: 36: 20$ & -82 & -78 & 42 & 0 & 0.11 & -0.59 & -0.45 & 054 & -0.912 & 28.5 & 51.0 & 180 & 25 & 090 & 2.0 & 180 \\
\hline $00: 36: 30$ & -83 & -75 & 42 & 0 & 0.04 & -0.71 & -0.52 & 053 & -1.197 & 35.0 & 51.0 & 180 & 21 & 090 & 2.0 & 180 \\
\hline $00: 36 ; 40$ & -85 & -73 & 42 & 0 & -0.03 & -0.38 & -0.55 & 053 & 1.071 & 35.0 & 23.0 & 180 & 22 & 090 & 2.0 & 180 \\
\hline $00: 36: 50$ & -87 & -71 & 41 & 0 & -0.01 & -0.18 & -0.58 & 054 & 2.528 & 35.0 & 0.0 & 180 & 21 & 090 & 2.0 & 180 \\
\hline $00: 37: 00$ & -88 & -70 & 48 & 0 & -0.01 & -0.03 & -0.61 & 054 & 3.652 & 35.0 & 0.0 & 180 & 18 & 090 & 2.0 & 180 \\
\hline $00: 37: 10$ & -88 & -69 & 49 & 0 & 0.05 & 0.21 & -0.65 & 055 & 5.503 & 35.0 & 0.0 & 180 & 26 & 090 & 2,0 & 180 \\
\hline $00: 37: 20$ & -89 & -68 & 50 & 0 & 0.11 & 0.45 & -0.68 & 056 & 7.160 & 29.3 & 0.0 & 180 & 25 & 090 & 2.0 & 180 \\
\hline $00: 37: 30$ & -88 & -67 & 50 & 0 & 0.17 & 0.54 & -0.59 & 057 & 7.177 & 10.3 & 24.0 & 180 & 28 & 090 & 2.0 & 180 \\
\hline $00: 37: 40$ & -87 & -66 & 50 & 0 & 0.20 & 0.29 & -0.41 & 058 & 4.394 & -8.7 & 24.0 & 180 & 21 & 090 & 2.0 & 180 \\
\hline $00: 37: 50$ & -86 & -66 & 49 & 0 & 0.27 & 0.16 & -0.14 & 059 & 1.886 & -20.3 & 24.0 & 180 & 26 & 090 & 2,0 & 180 \\
\hline $20: 38: 00$ & -85 & -65 & 50 & 0 & 0.30 & 0.02 & 0.08 & 059 & -0.399 & -13.4 & 76.0 & 180 & 28 & 090 & 2.0 & 180 \\
\hline $0: 38: 10$ & -84 & -64 & 49 & 0 & 0.34 & -0.43 & 0.26 & 058 & -4.377 & -26.9 & 76.0 & 180 & 24 & 090 & 2.0 & 180 \\
\hline$-00: 38: 20$ & -82 & -63 & 49 & 0 & 0.38 & -0.57 & 0.47 & 057 & -6.601 & -16.4 & 76.0 & 180 & 26 & 090 & 2.0 & 180 \\
\hline $00: 38: 30$ & -81 & -62 & 43 & 0 & 0.35 & -0.32 & 0.52 & 056 & -5.346 & 2.6 & 100.0 & 180 & 23 & 090 & 2.0 & 180 \\
\hline $00: 38: 40$ & -79 & -61 & 38 & 0 & 0.36 & -0.37 & 0.46 & 056 & -5.237 & 21.6 & 100.0 & 180 & 22 & 090 & 2.0 & 180 \\
\hline $00: 38: 50$ & -78 & -60 & 30 & 0 & 0.30 & -0.49 & 0.33 & 055 & -5.221 & 35.0 & 100.0 & 180 & 21 & 090 & 2.0 & 180 \\
\hline $00: 39: 00$ & -77 & -59 & 30 & 0 & 0.25 & -0.70 & 0.21 & 054 & -5.756 & 35.0 & 100.0 & 180 & 23 & 090 & 2.0 & 180 \\
\hline $00: 39: 10$ & -77 & -57 & 30 & 0 & 0.16 & -0.84 & 0.10 & 053 & -5.966 & 35.0 & 100.0 & 180 & 24 & 090 & 2.0 & 180 \\
\hline $00: 39: 20$ & -78 & -55 & 30 & 0 & -0.01 & -0.70 & 0.02 & 052 & -4.570 & 35.0 & 100.0 & 180 & 24 & 090 & 2.0 & 180 \\
\hline $00 ; 39: 30$ & -79 & -54 & 36 & 0 & -0.10 & -0.73 & -0.06 & 051 & -4.276 & 35.0 & 100.0 & 180 & 20 & 090 & 2.0 & 180 \\
\hline $00: 39: 40$ & -81 & -53 & 36 & 0 & -0.14 & -0.66 & -0.14 & 051 & -3.288 & 35.0 & 100.0 & 180 & 29 & 090 & 2.0 & 180 \\
\hline $00: 39: 50$ & -83 & -51 & 36 & 0 & -0.18 & -0.62 & -0.22 & 050 & -2.508 & 35.0 & 100.0 & 180 & 23 & 090 & 2.0 & 180 \\
\hline $00: 40: 00$ & -85 & -51 & 36 & 0 & -0.29 & -0.26 & -0.25 & 050 & -0.054 & 35.0 & 100.0 & 180 & 21 & 090 & 2.0 & 180 \\
\hline $00: 40: 10$ & -87 & -51 & 36 & 0 & -0.32 & 0.08 & -0.26 & 050 & 2.150 & 35.0 & 100.0 & 180 & 20 & 090 & 2.0 & 180 \\
\hline $00: 40: 20$ & -88 & -52 & 36 & 0 & -0.31 & 0.15 & -0.29 & 050 & 2.813 & 35.0 & 0.0 & 180 & 20 & 090 & 2.0 & 180 \\
\hline $00: 40: 30$ & -90 & -53 & 36 & 0 & -0.29 & 0.01 & -0.34 & 051 & 2.215 & 35.0 & 0.0 & 180 & 24 & 090 & 2.0 & 180 \\
\hline $00: 40: 40$ & -92 & -53 & 36 & 0 & -0.31 & $-0,18$ & -0.39 & .051 & 1.343 & 35.0 & 0.0 & 180 & 24 & 090 & 2.0 & 180 \\
\hline $00: 40: 50$ & -94 & -53 & 38 & 0 & -0.31 & 0.02 & -0.40 & 052 & 2.675 & 35.0 & 0.0 & 180 & 29 & 090 & 2.0 & 180 \\
\hline $00: 41: 00$ & -96 & -53 & 44 & 0 & -0.29 & -0.11 & -0.46 & 052 & 2.201 & 35.0 & 0.0 & 180 & 22 & 090 & 2.0 & 180 \\
\hline $00: 41: 10$ & -98 & -52 & 44 & 0 & -0.24 & -0.10 & -0.50 & 052 & 2.542 & 35.0 & 0.0 & 180 & 20 & 090 & 2.0 & 180 \\
\hline $00: 41: 20$ & -99 & -52 & 45 & 0 & -0.20 & 0.15 & -0.52 & 053 & 4.278 & 35.0 & 0.0 & 180 & 18 & 090 & 2.0 & 180 \\
\hline $00: 41: 30$ & -101 & -52 & 46 & 0 & -0.12 & 0.05 & -0.57 & 054 & 3.958 & 35.0 & 0.0 & 180 & 24 & 090 & 2.0 & 180 \\
\hline $00: 41: 40$ & -102 & -50 & 48 & 0 & -0.06 & -0.21 & -0.64 & 054 & 2.778 & 35.0 & 0.0 & 180 & 25 & 090 & 2.0 & 180 \\
\hline $00: 41: 50$ & -104 & -49 & 50 & 0 & -0.08 & -0.15 & -0.70 & 055 & 3.478 & 35.0 & 0.0 & 180 & 24 & 090 & 2.0 & 180 \\
\hline $00: 42: 00$ & -105 & -47 & 53 & 0 & -0.04 & -0.18 & -0.75 & 055 & 3.627 & 35.0 & 0.0 & 180 & 27 & 090 & 2.0 & 180 \\
\hline $00: 42: 10$ & -107 & -45 & 53 & 0 & -0.04 & -0.27 & -0.82 & 056 & 3.495 & 35.0 & 0.0 & 180 & 28 & 090 & 2.0 & 180 \\
\hline $00: 42: 20$ & -109 & -43 & 59 & 0 & -0.02 & -0.45 & -0.89 & 056 & 2.753 & 35.0 & 0.0 & 180 & 23 & 090 & 2.0 & 180 \\
\hline $00: 42: 30$ & -111 & -40 & 58 & 0 & 0.04 & -0.33 & -0.95 & 057 & 3.932 & 35.0 & 0.0 & 180 & 27 & 090 & 2.0 & 180 \\
\hline $00: 42: 40$ & -112 & -37 & 62 & 0 & 0.07 & 0.15 & -1.00 & 058 & 7.253 & 35.0 & 49.0 & 180 & 26 & 090 & 2.0 & 180 \\
\hline $00: 42: 50$ & -112 & -35 & 61 & 0 & 0.11 & 0.45 & -1.01 & 059 & 9.296 & 35.0 & 49.0 & 180 & 20 & 090 & 2.0 & 180 \\
\hline $00: 43: 00$ & -112 & -34 & 61 & 0 & 0.18 & 0.60 & -1.01 & 061 & 10.225 & 32.9 & 49.0 & 180 & 25 & 090 & 2.0 & 180 \\
\hline $00: 43: 10$ & -112 & -33 & 62 & 0 & 0.32 & 0.57 & -0.88 & 063 & 9.227 & 14.9 & 49.0 & 160 & 20 & 090 & 2.0 & 180 \\
\hline $00: 43: 20$ & -110 & -31 & 62 & 0 & 0.36 & 0.52 & -0.63 & 064 & 7.303 & -2.5 & 49.0 & 136 & 28 & 090 & 2.0 & 180 \\
\hline $00: 43: 30$ & -109 & -30 & 60 & 0 & 0.43 & 0.45 & -0.35 & 065 & 5.111 & -2.8 & 49.0 & 136 & 20 & 090 & 2.0 & 180 \\
\hline $00: 43: 40$ & -106 & -30 & 60 & 0 & 0.50 & 0.37 & -0.04 & 066 & 2.654 & -15.6 & 49.0 & 180 & 17 & 090 & 2.0 & 180 \\
\hline $00: 43: 50$ & -103 & -30 & 59 & 0 & 0.64 & 0.27 & 0.24 & 066 & 0.138 & -15.0 & 77.0 & 180 & 24 & 090 & 2.0 & 180 \\
\hline $00: 44: 00$ & -99 & -29 & 30 & 0 & 0.66 & 0.01 & 0.39 & 066 & -2.380 & -6.2 & 99.0 & 180 & 19 & 090 & 2.0 & 180 \\
\hline $00: 44: 10$ & -96 & -29 & 30 & 0 & 0.60 & 0.25 & 0.41 & 065 & -1.031 & -2.7 & 99.0 & 180 & 22 & 090 & 2.0 & 180 \\
\hline $00: 44: 20$ & -92 & -30 & 30 & 0 & 0.57 & 0.30 & 0.45 & 065 & -0.936 & -0.8 & 99.0 & 130 & 24 & 090 & 2.0 & 180 \\
\hline $0: 44: 30$ & -89 & -30 & 25 & 0 & 0.57 & 0.18 & 0.47 & 065 & -1.811 & 4.9 & 99.0 & 130 & 21 & 090 & 2.0 & 180 \\
\hline $0: 44: 40$ & -86 & -30 & 25 & 0 & 0.46 & 0.09 & 0.47 & 065 & -2.413 & 13.1 & 99.0 & 160 & 20 & 090 & 2.0 & 180 \\
\hline $00: 44 ; 50$ & -84 & -31 & 25 & 0 & 0.37 & -0.04 & 0.45 & 064 & -3.150 & 24.8 & 99.0 & 160 & 25 & 090 & 2.0 & 180 \\
\hline $00: 45: 00$ & -82 & -30 & 25 & 0 & 0.29 & -0.29 & 0.41 & 063 & -4.472 & 35.0 & 99.0 & 160 & 27 & 090 & 2.0 & 180 \\
\hline $00: 45: 10$ & -81 & -30 & 25 & 0 & 0.12 & -0.36 & 0.38 & 063 & -4.687 & 35.0 & 99.0 & 160 & 32 & 090 & 2.0 & 180 \\
\hline & 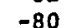 & 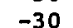 & 25 & 0 & 0.01 & -0.28 & 0.36 & 062 & -4.079 & 35.0 & 99.0 & 160 & 25 & 090 & 20 & 0 \\
\hline
\end{tabular}


Time Position (m) RPM Speed (Knots) Turn Rudder Gill Wind Current HH;MM:SS East North Port Stbd Long Bow Stern Heading Deg/Min Deg \& Deg Knots Deg Knots Deg

\begin{tabular}{|c|c|c|c|c|c|c|c|c|c|c|c|c|c|c|c|c|}
\hline $\begin{array}{l}0: 45: 30 \\
1: 45: 40\end{array}$ & $\begin{array}{l}-80 \\
-80\end{array}$ & $\begin{array}{l}-31 \\
-32\end{array}$ & $\begin{array}{l}25 \\
34\end{array}$ & $\begin{array}{l}0 \\
0\end{array}$ & $\begin{array}{l}-0.09 \\
-0.15\end{array}$ & $\begin{array}{l}-0.02 \\
-0.04\end{array}$ & $\begin{array}{l}0.35 \\
0.32\end{array}$ & $\begin{array}{l}061 \\
061\end{array}$ & $\begin{array}{l}-2.355 \\
-2.334\end{array}$ & $\begin{array}{l}35.0 \\
35.0\end{array}$ & $\begin{array}{l}99.0 \\
99.0\end{array}$ & $\begin{array}{l}160 \\
140\end{array}$ & $\begin{array}{l}25 \\
24\end{array}$ & $\begin{array}{l}090 \\
090\end{array}$ & $\begin{array}{l}2.0 \\
2.0\end{array}$ & $\begin{array}{l}180 \\
180\end{array}$ \\
\hline $0: 45: 50$ & -81 & -33 & 35 & 0 & -0.18 & -0.08 & 0.29 & 061 & -2.342 & 35.0 & 99.0 & 131 & 22 & 090 & 2.0 & 180 \\
\hline $00: 46: 00$ & -81 & -34 & 35 & 0 & -0.24 & 0.13 & 0.26 & 060 & -0.792 & 35.0 & 99.0 & 131 & 22 & 090 & 2.0 & 180 \\
\hline $00: 46: 10$ & -82 & -36 & 35 & 0 & -0.31 & 0.36 & 0.24 & 060 & 0.818 & 35.0 & 99.0 & 131 & 26 & 090 & 2.0 & 180 \\
\hline $00: 46: 20$ & -83 & -38 & 35 & 0 & -0.40 & 0.28 & 0.21 & 060 & 0.438 & 35.0 & 99.0 & 131 & 25 & 090 & 2.0 & 180 \\
\hline $00: 46: 30$ & -84 & -40 & 35 & 0 & -0.39 & 0.14 & 0.20 & 060 & -0.394 & 35.0 & 74.0 & 131 & 19 & 090 & 2.0 & 180 \\
\hline $00: 46 ; 40$ & -85 & -41 & 35 & 0 & -0.42 & 0.24 & 0.19 & 060 & 0.347 & 35.0 & 74.0 & 131 & 28 & 090 & 2.0 & 180 \\
\hline $00: 46: 50$ & -87 & -44 & 35 & 0 & -0.43 & 0.38 & 0.18 & 061 & 1.306 & 35.0 & 74.0 & 131 & 20 & 090 & 2.0 & 180 \\
\hline $00: 47: 00$ & -88 & -46 & 35 & 0 & -0.45 & 0.48 & 0.17 & 061 & 1.972 & 35.0 & 74.0 & 131 & 21 & 090 & 2.0 & 180 \\
\hline $00: 47: 10$ & -89 & -49 & 39 & 0 & -0.40 & 0.67 & 0.18 & 061 & 3.137 & 35.0 & 74.0 & 131 & 19 & 090 & 2.0 & 180 \\
\hline $00 ; 47: 20$ & -90 & -51 & 55 & 0 & -0.30 & 0.37 & 0.09 & 062 & 1.776 & 35.0 & 74.0 & 161 & 19 & 090 & 2.0 & 180 \\
\hline $00: 47: 30$ & -90 & -53 & 56 & 0 & -0.25 & 0.32 & -0.05 & 062 & 2.352 & 35.0 & 74.0 & 236 & 28 & 090 & 2.0 & 180 \\
\hline $00: 47: 40$ & -91 & -54 & 57 & 0 & -0.26 & 0.23 & -0.17 & 062 & 2.531 & 34.0 & 74.0 & 236 & 25 & 090 & 2.0 & 180 \\
\hline $00: 47: 50$ & -92 & -54 & 57 & 0 & -0.17 & 0.10 & -0.24 & 063 & 2.147 & 25.1 & 49.0 & 236 & 24 & 090 & 2.0 & 180 \\
\hline $00: 48 ; 00$ & -93 & -54 & 58 & 0 & -0.12 & -0.04 & -0.24 & 063 & 1.298 & 24.8 & 49.0 & 236 & 28 & 090 & 2.0 & 180 \\
\hline $00: 48: 10$ & -94 & -54 & 55 & 0 & -0.09 & -0.06 & -0.25 & 063 & 1.206 & 22.0 & 49.0 & 226 & 19 & 090 & 2.0 & 180 \\
\hline $00: 48: 20$ & -95 & -53 & 55 & 0 & 0.00 & -0.02 & -0.20 & 064 & 1.130 & 14.6 & 49.0 & 180 & 20 & 090 & 2.0 & 180 \\
\hline $00: 48: 30$ & -95 & -53 & 55 & 0 & 0.06 & 0.04 & -0.09 & 064 & 0.836 & 8.4 & 49.0 & 180 & 29 & 090 & 2.0 & 180 \\
\hline $00 ; 48 ; 40$ & -94 & -52 & 55 & 0 & 0.12 & 0.04 & -0.05 & 064 & 0.559 & 15.8 & 49.0 & 180 & 20 & 090 & 2.0 & 180 \\
\hline $00 ; 48: 50$ & -94 & -52 & 56 & 0 & 0.15 & 0.20 & 0.00 & 064 & 1.245 & 10.2 & 49.0 & 208 & 26 & 090 & 2.0 & 180 \\
\hline $00: 49: 00$ & -93 & -52 & 53 & 0 & 0.22 & 0.20 & 0.06 & 064 & 0.901 & 10.9 & 49.0 & 208 & 20 & 090 & 2.0 & 180 \\
\hline $00: 49: 10$ & -91 & -52 & 42 & 0 & 0.23 & 0.01 & 0.10 & 064 & -0.542 & 16.5 & 49.0 & 208 & 24 & 090 & 2.0 & 180 \\
\hline $00: 19: 20$ & -90 & -52 & 41 & 0 & 0.20 & -0.05 & 0.09 & 064 & -0.857 & 22.2 & 75.0 & 208 & 26 & 090 & 2.0 & 180 \\
\hline $00: 49: 30$ & -89 & -52 & 42 & 0 & 0.15 & 0.29 & 0.10 & 064 & 1.155 & 11.5 & 100.0 & 208 & 24 & 090 & 2.0 & 180 \\
\hline $00: 49: 40$ & -88 & -53 & 42 & 0 & 0.12 & 0.44 & 0.16 & 064 & 1.756 & 5.3 & 100.0 & 239 & 26 & 090 & 2.0 & 180 \\
\hline $00 ; 49: 50$ & -87 & -54 & 41 & 0 & 0.21 & 0.26 & 0.22 & 065 & 0.279 & 7.3 & 100.0 & 239 & 25 & 090 & 2.0 & 180 \\
\hline $00: 50: 00$ & -85 & -54 & 38 & 0 & 0.23 & 0.24 & 0.25 & 065 & -0.054 & 8.7 & 100.0 & 239 & 22 & 090 & 2.0 & 180 \\
\hline $00: 50: 10$ & -83 & -55 & 38 & 0 & 0.24 & 0.27 & 0.28 & 065 & -0.055 & 4.7 & 100.0 & 207 & 22 & 090 & 2.0 & 180 \\
\hline $00: 50: 20$ & -82 & -56 & 38 & 0 & 0.19 & 0.08 & 0.36 & 064 & -1.780 & -14.3 & 100.0 & 207 & 29 & 090 & 2.0 & 180 \\
\hline $00: 50: 30$ & -81 & -57 & 38 & 0 & 0.11 & -0.07 & 0.52 & 064 & -3.708 & -33.3 & 100.0 & 207 & 21 & 090 & 2.0 & 180 \\
\hline $00 ; 50: 40$ & -80 & -57 & 35 & 0 & 0.10 & -0.54 & 0.65 & 063 & -7.538 & -16.3 & 100.0 & 207 & 23 & 090 & 2.0 & 180 \\
\hline $00: 50: 50$ & -79 & -57 & 35 & 0 & 0.06 & -0.68 & 0.71 & 062 & -8.818 & 2.7 & 100.0 & 207 & 25 & 090 & 2.0 & 180 \\
\hline $00: 51 ; 00$ & -79 & -57 & 35 & 0 & -0.05 & -0.74 & 0.69 & 050 & -9.074 & 21.7 & 100.0 & 207 & 24 & 090 & 2.0 & 180 \\
\hline $00 ; 51: 10$ & -80 & -57 & 35 & 0 & -0.11 & -0.63 & 0.63 & 059 & -7.954 & 35.0 & 100.0 & 207 & 22 & 090 & 2.0 & 180 \\
\hline $00: 51: 20$ & -80 & -57 & 35 & 0 & -0.10 & -0.49 & 0.54 & 057 & -6.561 & 35.0 & 53.0 & 179 & 20 & 090 & 2.0 & 180 \\
\hline $00: 51: 30$ & -81 & -58 & 36 & 0 & -0.13 & -0.50 & 0.46 & 056 & -6.085 & 35.0 & 53.0 & 179 & 15 & 090 & 2.0 & 180 \\
\hline $00: 51: 40$ & -81 & -58 & 42 & 0 & -0.14 & -0.53 & 0.33 & 055 & -5.515 & 35.0 & 53.0 & 179 & 21 & 090 & 2.0 & 180 \\
\hline $00: 51: 50$ & -82 & -58 & 36 & 0 & -0.16 & -0.44 & 0.22 & 055 & -4.205 & 35.0 & 25.0 & 179 & 20 & 090 & 2.0 & 180 \\
\hline$: 52 ; 00$ & -84 & -58 & 36 & 0 & -0.18 & -0.48 & 0.14 & 054 & -3.906 & 27.0 & 25.0 & 179 & 24 & 090 & 2.0 & 180 \\
\hline $0: 52: 10$ & -85 & -58 & 44 & 0 & -0.23 & -0.47 & 0.12 & 053 & -3.749 & 8.0 & 25.0 & 179 & 34 & 090 & 2.0 & 180 \\
\hline $00: 52: 20$ & -86 & -58 & 45 & 0 & -0.20 & -0.32 & 0.21 & 053 & -3.377 & -11.0 & 25.0 & 125 & 19 & 090 & 2.0 & 180 \\
\hline $00: 52: 30$ & -87 & -58 & 45 & 0 & -0.13 & -0.23 & 0.33 & 052 & -3.587 & -4.3 & 25.0 & 125 & 25 & 090 & 2.0 & 180 \\
\hline $00: 52: 40$ & -87 & -59 & 45 & 0 & -0.04 & -0.37 & 0.32 & 051 & -4.354 & 14.7 & 25.0 & 125 & 25 & 090 & 2.0 & 180 \\
\hline $00: 52: 50$ & -87 & -59 & 45 & 0 & 0.00 & -0.07 & 0.22 & 051 & -1.847 & 33.7 & 25.0 & 125 & 23 & 090 & 2.0 & 180 \\
\hline $00: 53: 00$ & -87 & -59 & 44 & 0 & 0.02 & 0.31 & 0.08 & 051 & 1.473 & 35.0 & 16.0 & 146 & 28 & 090 & 2.0 & 180 \\
\hline $00: 53: 10$ & -86 & -60 & 44 & 0 & 0.11 & 0.08 & -0.10 & 051 & 1.136 & 35.0 & 16.0 & 146 & 26 & 090 & 2.0 & 180 \\
\hline $00 ; 53: 20$ & -86 & -59 & 45 & 0 & 0.13 & -0.28 & -0.28 & 051 & 0.029 & 35.0 & 16.0 & 146 & 26 & 090 & 2.0 & 180 \\
\hline $00: 53: 30$ & -87 & -57 & 45 & 0 & 0.14 & -0.39 & -0.43 & 051 & 0.262 & 35.0 & 50.0 & 146 & 27 & 090 & 2.0 & 180 \\
\hline $00: 53: 40$ & -87 & -55 & 45 & 0 & 0.14 & -0.35 & -0.54 & 051 & 1.155 & 35.0 & 50.0 & 121 & 21 & 090 & 2.0 & 180 \\
\hline $00: 53: 50$ & -88 & -53 & 43 & 0 & 0.10 & -0.15 & -0.60 & 052 & 2.867 & 35.0 & 50.0 & 121 & 23 & 090 & 2.0 & 180 \\
\hline $00: 54: 00$ & -89 & -51 & 43 & 0 & 0.13 & -0.36 & -0.68 & 052 & 2.044 & 35.0 & 50.0 & 121 & 23 & 090 & 2.0 & 180 \\
\hline $00: 54: 10$ & -90 & -48 & 43 & 0 & 0.18 & -0.27 & -0.73 & 053 & 2.921 & 35.0 & 50.0 & 121 & 14 & 090 & 2.0 & 180 \\
\hline $00: 54: 20$ & -91 & -45 & 38 & 0 & 0.15 & -0.18 & -0.74 & 053 & 3.554 & 35.0 & 50.0 & 121 & 22 & 090 & 2.0 & 180 \\
\hline $00: 54: 30$ & -92 & -43 & 38 & 0 & 0.08 & -0.20 & -0.75 & .054 & 3.462 & 35.0 & 50.0 & 136 & 22 & 090 & 2.0 & 180 \\
\hline $00: 54: 40$ & -93 & -41 & 38 & 0 & 0.05 & -0.16 & -0.74 & 054 & 3.685 & 35.0 & 50.0 & 137 & 22 & 090 & 2.0 & 180 \\
\hline $00: 54: 50$ & -94 & -39 & 38 & 0 & 0.00 & -0.18 & -0.73 & 055 & 3.454 & 35.0 & 50.0 & 137 & 21 & 090 & 2.0 & 180 \\
\hline $00: 55: 00$ & -96 & -37 & 38 & 0 & -0.04 & -0.23 & -0.71 & 055 & 3.028 & 35.0 & 50.0 & 137 & 24 & 090 & 2.0 & 180 \\
\hline $00: 55: 10$ & -97 & -35 & 38 & 0 & -0.11 & -0.11 & -0.67 & 056 & 3.564 & 35.0 & 75.0 & 116 & 22 & 090 & 2.0 & 180 \\
\hline $00: 55: 20$ & -99 & -35 & 38 & 0 & -0.11 & 0.26 & -0.61 & 057 & 5.511 & 35.0 & 99.0 & 088 & 26 & 090 & 2.0 & 180 \\
\hline $00: 55: 30$ & -99 & -34 & 38 & 0 & -0.10 & 0.49 & -0.55 & 058 & 6.554 & 35.0 & 99.0 & 088 & 27 & 090 & 2.0 & 180 \\
\hline $00: 55 ; 40$ & -100 & -35 & 38 & 0 & -0.08 & 0.64 & -0.49 & 059 & 7.162 & 35.0 & 99.0 & 088 & 23 & 090 & 2.0 & 180 \\
\hline $00: 55: 50$ & -100 & -36 & 41 & 0 & -0.05 & 0.84 & -0.42 & 060 & 7.997 & 35.0 & 99.0 & 088 & 26 & 090 & 2.0 & 180 \\
\hline $00: 56: 00$ & -99 & -36 & 40 & 0 & 0.02 & 0.64 & -0.38 & 061 & 6.501 & 35.0 & 77.0 & 088 & 16 & 090 & 2.0 & 180 \\
\hline $00: 56 ; 10$ & -99 & -37 & 41 & 0 & 0.03 & 0.47 & -0.36 & 062 & 5.274 & 35.0 & 77.0 & 089 & 26 & 090 & 2.0 & 180 \\
\hline $00: 56: 20$ & -99 & -37 & 40 & 0 & -0.04 & 0.57 & -0.33 & 063 & 5.747 & 35.0 & 77.0 & 089 & 29 & 090 & 2.0 & 180 \\
\hline $00: 56 ; 30$ & -99 & -38 & 42 & 0 & 0.00 & 0.54 & -0.30 & 064 & 5.374 & 35.0 & 77.0 & 089 & 23 & 090 & 2.0 & 180 \\
\hline $00: 56: 40$ & -98 & -38 & 44 & 0 & 0.06 & 0.44 & -0.29 & 065 & 4.650 & 35.0 & 77.0 & 089 & 26 & 090 & 2.0 & 180 \\
\hline $00: 56: 50$ & -98 & -38 & 44 & 0 & 0.08 & 0.32 & -0.29 & 066 & 3.861 & 35.0 & 26.0 & 089 & 24 & 090 & 2.0 & 180 \\
\hline $00: 57: 00$ & -97 & -38 & 36 & 0 & 0.09 & 0.37 & -0.26 & 066 & 4.010 & 35.0 & 26.0 & 109 & 17 & 090 & 2.0 & 180 \\
\hline $00: 57: 10$ & -97 & -39 & 36 & 0 & 0.05 & 0.60 & -0.22 & 067 & 5.205 & 35.0 & 26.0 & 089 & 24 & 090 & 2.0 & 180 \\
\hline $00: 57: 20$ & -96 & -39 & 36 & 0 & 0.05 & 0.39 & -0.16 & 068 & 3.506 & 23.3 & 26.0 & 059 & 21 & 090 & 2.0 & 180 \\
\hline $00: 57: 30$ & -96 & -39 & 36 & 0 & 0.04 & 0.15 & -0.07 & 068 & 1.382 & 20.1 & 26.0 & 059 & 24 & 090 & 2.0 & 180 \\
\hline $00: 57: 40$ & -96 & -40 & 36 & 0 & 0.01 & 0.14 & -0.01 & 068 & 0.949 & 27.1 & 26.0 & 059 & 28 & 090 & 2.0 & 180 \\
\hline
\end{tabular}




\section{Simulation Track Plot}

Open Ocean

Ship: 150,000 ton, Tanker, partial load

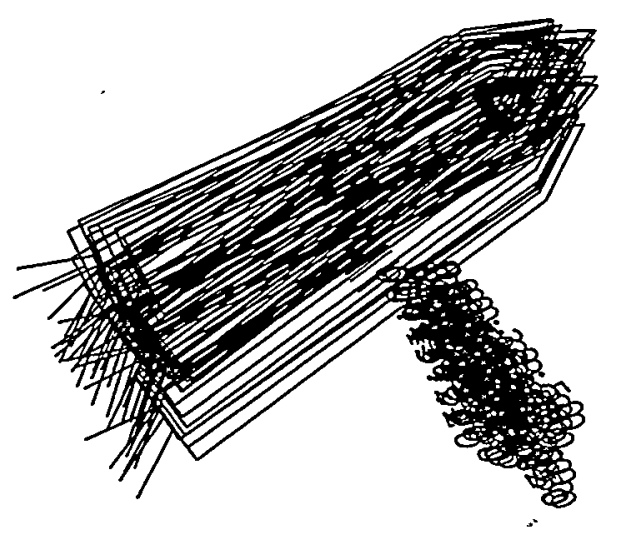

Start Time [hrs:min:sec]: $00: 33: 20$

End Time [hrs:min:sec]: $00: 57: 45$

Delta Plot Time [sec]:

East-West Scale [m]:

30

Date: 04-Feb-99 Time: 14:49:56

East-West Scale [Nm]:

926.

0.5000 


\section{HANDS-ON SIMULATION}

\section{SUCTION MOORNG TECHNOLOGY}

Simulation Number: 26

Date: 4 February 1999

Start Time: 16:29

Length of run [secs]: 3912

Ship Condition: draft $=13.72 \mathrm{~m}$

Environment

Wind Speed [knots]:

25 Direction: 90

Sea State, Significant wave height $[\mathrm{m}]: .$.

5 Direction: 270

Current Speed [knots]:

2 Direction: 180

Ship Operator: J.A.

Observer: B.K.J \& J.K.

Final Mating, distance between center of buoy and center of moon pool [m]: 1.5

Evaluation:

The approach was okay.

The final positioning and mating took a long time maneuvering over the buoy. A reason for this may be found primarily in slow rudder response. There was also problems in keeping an equilibrium speed of zero knots due to the randomness of the wind and waves. 
Ship: Exercise number: file affic ships:

Initial conditions Exercise identification:
591150,000 ton, Tanker, partial load

754 Suction Mooring System

112 Open Ocean

999 Dumny tug file based on containership

590 Suction Mooring Buoy

594 150k tanker in partial load - Suction Mooring RuN $\$ 26$

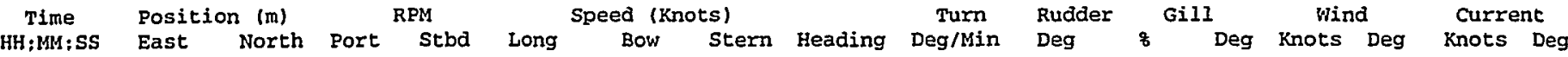

\begin{tabular}{|c|c|c|c|c|c|c|c|c|c|c|c|c|c|c|c|c|}
\hline $00: 41: 40$ & -80 & -44 & 40 & 0 & 0.51 & -0.14 & 0.30 & 063 & -2.768 & 11.4 & 96.0 & 180 & 25 & 090 & 2.0 & 180 \\
\hline $00 ; 41: 50$ & -78 & -43 & 33 & 0 & 0.39 & -0.12 & 0.28 & 062 & -2.529 & 20.5 & 96.0 & 180 & 24 & 090 & 2.0 & 180 \\
\hline $00: 42: 00$ & -76 & -42 & 30 & 0 & 0.31 & -0.18 & 0.24 & 062 & -2.671 & 30.8 & 96.0 & 180 & 27 & 090 & 2.0 & 180 \\
\hline $00: 42: 10$ & -75 & -42 & 25 & 0 & 0.19 & -0.31 & 0.19 & 061 & -3.190 & 35.0 & 96.0 & 180 & 28 & 090 & 2.0 & 180 \\
\hline $00 ; 42: 20$ & -75 & -41 & 25 & 0 & 0.07 & -0.54 & 0.17 & 061 & -4.525 & 35.0 & 96.0 & 180 & 23 & 090 & 2.0 & 180 \\
\hline $00: 42: 30$ & -75 & -40 & 25 & 0 & -0.01 & -0.53 & 0.16 & 060 & -4.352 & 35.0 & 96.0 & 180 & 27 & 090 & 2.0 & 180 \\
\hline $00: 42: 40$ & -76 & -40 & 25 & 0 & -0.14 & -0.18 & 0.16 & 059 & -2.144 & 31.7 & 96.0 & 180 & 26 & 090 & 2.0 & 180 \\
\hline $00: 42: 50$ & -76 & -40 & 25 & 0 & -0.24 & 0.00 & 0.18 & 059 & -1.173 & 20.3 & 96.0 & 180 & 20 & 090 & 2.0 & 180 \\
\hline $00 ; 43 ; 00$ & -77 & -42 & 25 & 0 & -0.30 & 0.04 & 0.20 & 059 & -1.027 & 35.0 & 96.0 & 180 & 25 & 090 & 2.0 & 180 \\
\hline $00: 43: 10$ & -78 & -43 & 25 & 0 & -0.30 & -0.03 & 0.20 & 059 & -1.408 & 35.0 & 51.0 & 180 & 20 & 090 & 2.0 & 180 \\
\hline $00: 43: 20$ & -80 & -44 & 25 & 0 & -0.39 & -0.02 & 0.20 & 059 & -1.378 & 35.0 & 51.0 & 180 & 28 & 090 & 2.0 & 280 \\
\hline $00: 43: 30$ & -81 & -46 & 25 & 0 & -0.46 & -0.01 & 0.21 & 058 & -1.345 & 35.0 & 29.0 & 180 & 20 & 090 & 2.0 & 180 \\
\hline $00: 43: 40$ & -83 & -47 & 25 & 0 & -0.50 & 0.03 & 0.22 & 058 & -1.214 & 35.0 & 29.0 & 180 & 17 & 090 & 2.0 & 180 \\
\hline $00: 43: 50$ & -85 & -49 & 25 & 0 & -0.47 & 0.04 & 0.23 & 058 & -1.203 & 35.0 & 29.0 & 180 & 24 & 090 & 2.0 & 180 \\
\hline $00: 44: 00$ & -87 & -51 & 25 & 0 & -0.48 & -0.23 & 0.23 & 058 & -2.902 & 35.0 & 29.0 & 180 & 19 & 090 & 2.0 & 180 \\
\hline $00: 44: 10$ & -89 & -53 & 27 & 0 & -0.48 & 0.02 & 0.24 & 057 & -1.405 & 35.0 & 0.0 & 180 & 22 & 090 & 2.0 & 180 \\
\hline $00: 44: 20$ & -90 & -55 & 44 & 0 & -0.45 & 0.06 & 0.20 & 057 & -0.853 & 35.0 & 0.0 & 180 & 24 & 090 & 2.0 & 180 \\
\hline $00: 44: 30$ & -92 & -56 & 58 & 0 & -0.34 & -0.06 & 0.05 & 057 & -0.694 & 35.0 & 0.0 & 180 & 21 & 090 & 2.0 & 180 \\
\hline $00 ; 44: 40$ & -93 & -57 & 59 & 0 & -0.27 & -0.10 & -0.15 & 057 & 0.315 & 35.0 & 0.0 & 180 & 20 & 090 & 2.0 & 180 \\
\hline $00: 44: 50$ & -95 & -56 & 55 & 0 & -0.21 & -0.19 & -0.29 & 057 & 0.638 & 35.0 & 0.0 & 180 & 25 & 090 & 2.0 & 180 \\
\hline $00 ; 45 ; 00$ & -96 & -55 & 56 & 0 & -0.14 & -0.43 & -0.43 & 057 & 0.006 & 35.0 & 0.0 & 180 & 27 & 090 & 2.0 & 180 \\
\hline $00: 45: 10$ & -98 & -54 & 62 & 0 & -0.13 & -0.43 & -0.58 & 057 & 0.938 & 35.0 & 0.0 & 180 & 32 & 090 & 2.0 & 180 \\
\hline $00: 45: 20$ & -100 & -52 & 64 & 0 & -0.07 & -0.23 & -0.71 & 057 & 3.068 & 35.0 & 0.0 & 180 & 25 & 090 & 2.0 & 180 \\
\hline $00: 45: 30$ & -101 & -50 & 64 & 0 & 0.01 & 0.17 & -0.82 & 058 & 6.271 & 35.0 & 0.0 & 180 & 25 & 090 & 2.0 & 180 \\
\hline $00 ; 45: 40$ & -102 & -49 & 56 & 0 & 0.12 & 0.23 & -0.86 & 059 & 6.966 & 24.8 & 0.0 & 180 & 24 & 090 & 2.0 & 180 \\
\hline $00: 45: 50$ & -102 & -47 & 48 & 0 & 0.16 & 0.13 & -0.73 & 060 & 5.466 & 11.7 & 0.0 & 180 & 22 & 090 & 2.0 & 180 \\
\hline $00: 46: 00$ & -102 & -46 & 48 & 0 & 0.18 & 0.25 & -0.52 & 061 & 4.884 & -5.9 & 0.0 & 180 & 22 & 090 & 2.0 & 180 \\
\hline $00: 46: 10$ & -101 & -45 & 48 & 0 & 0.19 & 0.32 & -0.23 & 062 & 3.491 & -19.4 & 0.0 & 180 & 26 & 090 & 2.0 & 280 \\
\hline $0: 46: 20$ & -100 & -45 & 49 & 0 & 0.19 & 0.11 & 0.00 & 062 & 0.684 & -8.4 & 0.0 & 180 & 25 & 090 & 2.0 & 180 \\
\hline$: 46: 30$ & -99 & -44 & 49 & 0 & 0.29 & -0.09 & 0.13 & 062 & -1.371 & 3.9 & 0.0 & 180 & 19 & 090 & 2.0 & 180 \\
\hline $0: 46 ; 40$ & -97 & -44 & 43 & 0 & 0.33 & 0.04 & 0.13 & 062 & -0.584 & 21.4 & 0.0 & 180 & 28 & 090 & 2.0 & 180 \\
\hline $00: 46: 50$ & -96 & -44 & 42 & 0 & 0.35 & 0.21 & 0.11 & 062 & 0.623 & 16.7 & 51.0 & 180 & 20 & 090 & 2.0 & 380 \\
\hline $00 ; 47: 00$ & -94 & -44 & 39 & 0 & 0.35 & 0.32 & 0.12 & 062 & 1.287 & 12.2 & 51.0 & 180 & 21 & 090 & 2.0 & 180 \\
\hline $00: 47: 10$ & -91 & -44 & 39 & 0 & 0.42 & 0.50 & 0.16 & 062 & 2.119 & 5.3 & 51.0 & 180 & 19 & 090 & 2.0 & 180 \\
\hline $00: 47: 20$ & -88 & -44 & 39 & 0 & 0.49 & 0.14 & 0.20 & 063 & -0.355 & 7.4 & 99.0 & 180 & 19 & 090 & 2.0 & 180 \\
\hline $00: 47: 30$ & -86 & -44 & 33 & 0 & 0.42 & 0.05 & 0.20 & 062 & -0.954 & 12.1 & 99.0 & 180 & 28 & 090 & 2.0 & 180 \\
\hline $00: 47: 40$ & -84 & -43 & 33 & 0 & 0.30 & -0.08 & 0.20 & 062 & -1.756 & 19.6 & 99.0 & 180 & 25 & 090 & 2.0 & 180 \\
\hline $00: 47: 50$ & -83 & -43 & 33 & 0 & 0.24 & -0.21 & 0.17 & 062 & -2.416 & 31.3 & 99.0 & 180 & 24 & 090 & 2.0 & 180 \\
\hline $00 ; 48 ; 00$ & -82 & -42 & 33 & 0 & 0.15 & -0.33 & 0.12 & 061 & -2.829 & 35.0 & 97.0 & 180 & 28 & 090 & 2.0 & 180 \\
\hline $00: 48: 10$ & -82 & -41 & 33 & 0 & 0.05 & -0.34 & $0.08^{\circ}$ & 061 & -2.677 & 35.0 & 97.0 & 180 & 19 & 090 & 2.0 & 180 \\
\hline $00: 48: 20$ & -82 & -40 & 33 & 0 & 0.00 & -0.28 & 0.06 & 061 & -2.096 & 35.0 & 97.0 & 180 & 20 & 090 & 2.0 & 180 \\
\hline $00: 48: 30$ & -83 & -40 & 33 & 0 & -0.07 & -0.15 & 0.03 & 060 & -1.154 & 35.0 & 97.0 & 180 & 29 & 090 & 2.0 & 180 \\
\hline $00: 48: 40$ & -83 & -40 & 33 & 0 & -0.15 & -0.13 & 0.01 & 060 & -0.950 & 35.0 & 97.0 & 180 & 20 & 090 & 2.0 & 180 \\
\hline $00: 48: 50$ & -84 & -41 & 33 & 0 & -0.24 & 0.05 & 0.01 & 060 & 0.257 & 35.0 & 76.0 & 180 & 26 & 090 & 2.0 & 180 \\
\hline $00 ; 49 ; 00$ & -85 & -41 & 38 & 0 & -0.26 & 0.08 & 0.00 & 060 & 0.466 & 35.0 & 51.0 & 180 & 20 & 090 & 2.0 & 180 \\
\hline $00: 49: 10$ & -86 & -42 & 38 & 0 & -0.28 & -0.11 & -0.03 & 060 & -0.521 & 35.0 & 51.0 & 180 & 24 & 090 & 2.0 & 180 \\
\hline $00: 49: 20$ & -88 & -42 & 39 & 0 & -0.33 & -0.18 & -0.05 & 060 & -0.815 & 35.0 & 51.0 & 180 & 26 & 090 & 2.0 & 180 \\
\hline $00: 49: 30$ & -90 & -43 & 39 & 0 & -0.35 & 0.21 & -0.05 & 060 & 1.697 & 35.0 & 26.0 & 180 & 24 & 090 & 2.0 & 180 \\
\hline $00: 49: 40$ & -91 & -45 & 39 & 0 & -0.35 & 0.45 & -0.06 & 061 & 3.235 & 35.0 & 0.0 & 180 & 26 & 090 & 2.0 & 180 \\
\hline $00 ; 49 ; 50$ & -92 & -46 & 39 & 0 & -0.25 & 0.33 & -0.05 & 061 & 2.410 & 26.1 & 0.0 & 180 & 25 & 090 & 2.0 & 180 \\
\hline $00: 50: 00$ & -92 & -47 & 40 & 0 & -0.20 & 0.34 & -0.02 & 061 & 2.245 & 19.1 & 0.0 & 180 & 22 & 090 & 2.0 & 180 \\
\hline $00: 50: 10$ & -93 & -49 & 50 & 0 & -0.11 & 0.38 & 0.04 & 062 & 2.189 & 11.5 & 0.0 & 180 & 22 & 090 & 2.0 & 180 \\
\hline $00: 50: 20$ & -93 & -50 & 49 & 0 & -0.06 & 0.18 & 0.15 & 062 & 0.204 & -1.1 & 60.0 & 180 & 29 & 090 & 2.0 & 180 \\
\hline $00 ; 50 ; 30$ & -92 & -51 & 50 & 0 & -0.05 & 0.07 & 0.23 & 062 & -1.028 & 8.4 & 22.0 & 180 & 21 & 090 & 2.0 & 180 \\
\hline $00: 50: 40$ & -92 & -51 & 50 & 0 & 0.05 & -0.31 & 0.21 & 062 & -3.304 & 27.4 & 22.0 & 180 & 23 & 090 & 2.0 & 180 \\
\hline $00: 50: 50$ & -92 & -50 & 50 & 0 & 0.09 & -0.33 & 0.08 & 061 & -2.608 & 35.0 & 22.0 & 180 & 25 & 090 & 2.0 & 180 \\
\hline $00 ; 51: 00$ & -92 & -50 & 49 & 0 & 0.06 & -0.29 & -0.04 & 061 & -1.547 & 34.1 & 22.0 & 180 & 24 & 090 & 2.0 & 180 \\
\hline $00 ; 51: 10$ & -92 & -49 & 50 & 0 & 0.09 & -0.12 & -0.15 & 061 & 0.210 & 35.0 & 22.0 & 180 & 22 & 090 & 2.0 & 180 \\
\hline $00 ; 51: 20$ & -92 & -48 & 49 & 0 & 0.16 & 0.06 & -0.25 & 061 & 1.975 & 35.0 & 22.0 & 180 & 20 & 090 & 2.0 & 180 \\
\hline $00: 51: 30$ & -92 & -47 & 50 & 0 & 0.18 & 0.10 & -0.33 & 061 & 2.731 & 35.0 & 22.0 & 180 & 15 & 090 & 2.0 & 180 \\
\hline $00 ; 51 ; 40$ & -91 & -46 & 50 & 0 & 0.20 & 0.10 & -0.40 & 062 & 3.180 & 35.0 & 0.0 & 180 & 21 & 090 & 2.0 & 180 \\
\hline $00: 51: 50$ & -90 & -45 & 50 & 0 & 0.19 & 0.21 & -0.46 & 062 & 4.210 & 35.0 & 50.0 & 180 & 20 & 090 & 2.0 & 180 \\
\hline $00: 52: 00$ & -90 & -44 & 50 & 0 & 0,18 & 0.23 & -0.50 & 063 & 4.632 & 35.0 & 50.0 & 180 & 24 & 090 & 2.0 & 180 \\
\hline $00 ; 52 ; 10$ & -89 & -43 & 49 & 0 & 0.13 & 0.32 & -0.52 & 064 & 5.351 & 35.0 & 50.0 & 180 & 34 & 090 & 2.0 & 180 \\
\hline $00: 52: 20$ & -89 & -42 & 49 & 0 & 0.13 & 0.55 & -0.53 & 065 & 6.833 & 33.5 & 50.0 & 180 & 19 & 090 & 2.0 & 180 \\
\hline $00: 52: 30$ & -88 & -42 & 50 & 0 & 0.20 & 0.73 & -0.50 & 066 & 7.774 & 30.7 & 50.0 & 180 & 25 & 090 & 2.0 & 180 \\
\hline $00: 52: 40$ & -87 & -42 & 49 & 0 & 0.27 & 0.67 & -0.43 & 067 & 6.961 & 21.2 & 50.0 & 180 & 25 & 090 & 2.0 & 180 \\
\hline $9: 52: 50$ & -85 & -43 & 39 & 0 & 0,27 & 0.81 & -0.30 & 068 & 7.043 & 5.7 & 99.0 & 180 & 23 & 090 & 2.0 & 180 \\
\hline :53;00 & -83 & -44 & 39 & 0 & 0.22 & 0.94 & -0.10 & 070 & 6.607 & -12.3 & 99.0 & 180 & 28 & 090 & 2.0 & 180 \\
\hline $60: 53: 10$ & $-8 I$ & -46 & 39 & 0 & 0.26 & 0.65 & 0.16 & 070 & 3.136 & -31.3 & 99.0 & 180 & 26 & 090 & 2.0 & 180 \\
\hline $00: 53 ; 20$ & -79 & -47 & 39 & 0 & 0.21 & 0.26 & 0.42 & 071 & -1.003 & -35.0 & 99.0 & 180 & 26 & 090 & 2.0 & 180 \\
\hline $00: 53: 30$ & -78 & -48 & 39 & 0 & 0.14 & 0.03 & 0.66 & 070 & -3.986 & -35.0 & 99.0 & 180 & 27 & 090 & 2.0 & 180 \\
\hline $00: 53: 40$ & -77 & -50 & 39 & 0 & 0.07 & -0.12 & 0.88 & 069 & -6.292 & -35.0 & 99.0 & 180 & 21 & 090 & 2.0 & 180 \\
\hline
\end{tabular}




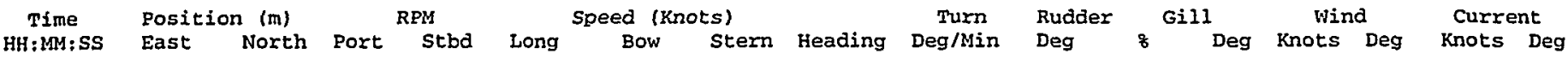

\begin{tabular}{|c|c|c|c|c|c|c|c|c|c|c|c|c|c|c|c|c|}
\hline$\left\{\begin{array}{r}53: 50 \\
: 54: 00 \\
: 54: 10\end{array}\right.$ & $\begin{array}{l}-76 \\
-75\end{array}$ & $\begin{array}{l}-52 \\
-54\end{array}$ & $\begin{array}{l}39 \\
39\end{array}$ & $\begin{array}{l}0 \\
0\end{array}$ & $\begin{array}{l}-0.02 \\
-0.02\end{array}$ & $\begin{array}{l}-0.16 \\
-0.49\end{array}$ & $\begin{array}{l}1.07 \\
1.25\end{array}$ & $\begin{array}{l}068 \\
067\end{array}$ & $\begin{array}{r}-7.790 \\
-11.034\end{array}$ & $\begin{array}{l}-35.0 \\
-35.0\end{array}$ & $\begin{array}{l}99.0 \\
99.0\end{array}$ & $\begin{array}{l}229 \\
229\end{array}$ & $\begin{array}{l}23 \\
23\end{array}$ & 090 & $\begin{array}{l}2.0 \\
2.0\end{array}$ & $\begin{array}{l}180 \\
180\end{array}$ \\
\hline $\begin{array}{l}.0: 54: 10 \\
00: 54: 20\end{array}$ & -75 & -56 & 39 & 0 & -0.01 & $\begin{array}{l}-0.60 \\
-0.69\end{array}$ & $\begin{array}{l}1.41 \\
1.54\end{array}$ & $\begin{array}{l}065 \\
062\end{array}$ & $\begin{array}{l}-12.784 \\
-14.161\end{array}$ & $\begin{array}{l}-35.0 \\
-22.5\end{array}$ & 99.0 & 229 & 14 & 090 & 2.0 & $\begin{array}{l}180 \\
180\end{array}$ \\
\hline $\begin{array}{l}00: 54: 20 \\
00: 54: 30\end{array}$ & $\begin{array}{l}-74 \\
-73\end{array}$ & $\begin{array}{l}-58 \\
-60\end{array}$ & $\begin{array}{l}39 \\
38\end{array}$ & $\begin{array}{l}0 \\
0\end{array}$ & $\begin{array}{l}-0.06 \\
-0.15\end{array}$ & $\begin{array}{l}-0.69 \\
-0.81\end{array}$ & $\begin{array}{l}1.54 \\
1.58\end{array}$ & $\begin{array}{l}062 \\
060\end{array}$ & $\begin{array}{l}-14.161 \\
-15.190\end{array}$ & $\begin{array}{r}-22.5 \\
-3.5\end{array}$ & $\begin{array}{l}99.0 \\
99.0\end{array}$ & $\begin{array}{l}229 \\
229\end{array}$ & $\begin{array}{l}22 \\
22\end{array}$ & $\begin{array}{l}090 \\
090\end{array}$ & $\begin{array}{l}2.0 \\
2.0\end{array}$ & $\begin{array}{l}180 \\
180\end{array}$ \\
\hline $00: 54: 40$ & -73 & -62 & 39 & 0 & -0.19 & -0.84 & 1.53 & 057 & -15.032 & 15.5 & 99.0 & 229 & 22 & 090 & 2.0 & 180 \\
\hline $00: 54: 50$ & -73 & -64 & 39 & 0 & -0.26 & -0.87 & 1.40 & 055 & -14.374 & 34.5 & 99.0 & 177 & 21 & 090 & 2.0 & 180 \\
\hline $00: 55: 00$ & -74 & -65 & 39 & 0 & -0.32 & -0.90 & 1.23 & 053 & -13.519 & 35.0 & 99.0 & 177 & 24 & 090 & 2.0 & 180 \\
\hline $00 ; 55 ; 10$ & -75 & -67 & 39 & 0 & -0.41 & -0.79 & 1.07 & 050 & -11.783 & 35.0 & 99.0 & 177 & 22 & 090 & 2.0 & 180 \\
\hline $00: 55: 20$ & -76 & -69 & 39 & 0 & -0.44 & -0.43 & 0.93 & 049 & -8.637 & 35.0 & 99.0 & 177 & 26 & 090 & 2.0 & 180 \\
\hline $00: 55: 30$ & -76 & -72 & 42 & 0 & -0.43 & -0.22 & 0.77 & 048 & -6.291 & 35.0 & 0.0 & 177 & 27 & 090 & 2.0 & 180 \\
\hline $00: 55: 40$ & -77 & -74 & 43 & 0 & -0.39 & -0.08 & 0.59 & 047 & -4.215 & 35.0 & 0.0 & 177 & 23 & 090 & 2.0 & 180 \\
\hline $00: 55: 50$ & -77 & -77 & 56 & 0 & -0.32 & 0.16 & 0.37 & 046 & -1.328 & 35.0 & 0.0 & 177 & 26 & 090 & 2.0 & 180 \\
\hline $00: 56 ; 00$ & -78 & -78 & 62 & 0 & -0.17 & -0.03 & 0.02 & 046 & -0.295 & 35.0 & 0.0 & 177 & 16 & 090 & 2.0 & 180 \\
\hline $00: 56: 10$ & -79 & -78 & 50 & 0 & -0.10 & -0.21 & -0.26 & 046 & 0.315 & 35.0 & 0.0 & 177 & 26 & 090 & 2.0 & 180 \\
\hline $00: 56: 20$ & -80 & -77 & 57 & 0 & -0.10 & -0.05 & -0.47 & 046 & 2.669 & 35.0 & 0.0 & 177 & 29 & 090 & 2.0 & 180 \\
\hline $00: 56: 30$ & -81 & -76 & 57 & 0 & 0.00 & -0.05 & -0.68 & 047 & 3.949 & 35.0 & 0.0 & 177 & 23 & 090 & 2.0 & 180 \\
\hline $00: 56: 40$ & -83 & -75 & 56 & 0 & 0.09 & -0.18 & -0.87 & 048 & 4.365 & 35.0 & 0.0 & 177 & 26 & 090 & 2.0 & 180 \\
\hline $00 ; 56: 50$ & -84 & -72 & 57 & 0 & 0.15 & -0.31 & -1.03 & 048 & 4.613 & 35.0 & 0.0 & 177 & 24 & 090 & 2.0 & 180 \\
\hline $00: 57: 00$ & -86 & -69 & 57 & 0 & 0.21 & -0.15 & -1.13 & 049 & 6.200 & 35.0 & 0.0 & 177 & 17 & 090 & 2.0 & 180 \\
\hline $00: 57: 10$ & -87 & -66 & 57 & 0 & 0.23 & 0.24 & -1.16 & 050 & 8.917 & 35.0 & 0.0 & 177 & 24 & 090 & 2.0 & 180 \\
\hline $00: 57: 20$ & -87 & -63 & 57 & 0 & 0.27 & 0.01 & -1.22 & 052 & 7.821 & 35.0 & 25.0 & 177 & 21 & 090 & 2.0 & 180 \\
\hline $00: 57: 30$ & -88 & -59 & 57 & 0 & 0.30 & -0.22 & -1.29 & 053 & 6.796 & 35.0 & 25.0 & 147 & 24 & 090 & 2.0 & 180 \\
\hline $00: 57: 40$ & -89 & -55 & 57 & 0 & 0.30 & -0.13 & -1.31 & 054 & 7.467 & 35.0 & 25.0 & 146 & 28 & 090 & 2.0 & 180 \\
\hline $00: 57: 50$ & -90 & -51 & 57 & 0 & 0.25 & 0.00 & -1.30 & 056 & 8.265 & 35.0 & 76.0 & 146 & 20 & 090 & 2.0 & 180 \\
\hline $00: 58: 00$ & -91 & -48 & 57 & 0 & 0.22 & 0.21 & -1.26 & 057 & 9.352 & 35.0 & 76.0 & 146 & 31 & 090 & 2.0 & 180 \\
\hline $00: 58 ; 10$ & -91 & -46 & 57 & 0 & 0.22 & 0.47 & -1.20 & 059 & 10.611 & 35.0 & 76.0 & 146 & 23 & 090 & 2.0 & 180 \\
\hline $00: 58: 20$ & -91 & -44 & 56 & 0 & 0.29 & 0.77 & -1.12 & 061 & 11.956 & 35.0 & 76.0 & 146 & 18 & 090 & 2.0 & 180 \\
\hline $00: 58: 30$ & -90 & -43 & 57 & 0 & 0.32 & 0.83 & -1.04 & 063 & 11.809 & 35.0 & 76.0 & 146 & 21 & 090 & 2.0 & 180 \\
\hline $00 ; 58 ; 40$ & -88 & -42 & 44 & 0 & 0.33 & 1.02 & -0.92 & 065 & 12.260 & 35.0 & 76.0 & 146 & 21 & 090 & 2.0 & 180 \\
\hline $00: 58: 50$ & -87 & -41 & 44 & 0 & 0.32 & 1.04 & -0.76 & 067 & 11.437 & 26.9 & 76.0 & 180 & 21 & 090 & 2.0 & 180 \\
\hline $00: 59: 00$ & -85 & -41 & 44 & 0 & 0.32 & 0.87 & -0.56 & 068 & 9.063 & 7.9 & 100.0 & 180 & 26 & 090 & 2.0 & 180 \\
\hline $00: 59: 10$ & -83 & -42 & 40 & 0 & 0.26 & 0.74 & -0.30 & 070 & 6.556 & -11.1 & 100.0 & 180 & 25 & 090 & 2.0 & 180 \\
\hline $00: 59: 20$ & -82 & -43 & 30 & 0 & 0.21 & 0.53 & -0.07 & 070 & 3.804 & -30.1 & 100.0 & 180 & 20 & 090 & 2.0 & 180 \\
\hline $00: 59: 30$ & -80 & -43 & 30 & 0 & 0.20 & 0.36 & 0.15 & 071 & 1.280 & -35.0 & 100.0 & 228 & 22 & 090 & 2.0 & 180 \\
\hline $00: 59: 40$ & -79 & -45 & 30 & 0 & 0.10 & 0.31 & 0.34 & 071 & -0.174 & -35.0 & 100.0 & 228 & 21 & 090 & 2.0 & 180 \\
\hline $00: 59: 50$ & -78 & -46 & 30 & 0 & 0.06 & 0.21 & 0.51 & 071 & -1.896 & -35.0 & 100.0 & 228 & 28 & 090 & 2.0 & 180 \\
\hline $01: 00: 00$ & -77 & -48 & 30 & 0 & 0.05 & 0.09 & 0.66 & 070 & -3.587 & -35.0 & 100.0 & 228 & 26 & 090 & 2.0 & 180 \\
\hline $21: 00: 10$ & -76 & -49 & 30 & 0 & 0.00 & -0.25 & 0.81 & 070 & -6.714 & -35.0 & 100.0 & 228 & 23 & 090 & 2.0 & 180 \\
\hline$: 00: 20$ & -76 & -51 & 30 & 0 & -0.09 & -0.58 & 0.96 & 068 & -9.746 & -35.0 & 100.0 & 228 & 25 & 090 & 2.0 & 180 \\
\hline $01 ; 00 ; 30$ & -77 & -52 & 30 & 0 & -0.26 & -0.54 & 1.10 & 066 & -10.404 & -35.0 & 100.0 & 228 & 29 & 090 & 2.0 & 180 \\
\hline $01: 00: 40$ & -77 & -54 & 30 & 0 & -0.35 & -0.62 & 1.22 & 065 & -11.672 & -35.0 & 100.0 & 228 & 22 & 090 & 2.0 & 180 \\
\hline $01: 00: 50$ & -78 & -56 & 30 & 0 & -0.42 & -0.89 & 1.34 & 062 & -14.134 & -24.1 & 100.0 & 228 & 30 & 090 & 2.0 & 180 \\
\hline $01 ; 01 ; 00$ & -80 & -58 & 30 & 0 & -0.47 & -0.88 & 1.40 & 060 & -14.458 & -5.1 & 100.0 & 238 & 26 & 090 & 2.0 & 180 \\
\hline $01: 01: 10$ & -81 & -61 & 38 & 0 & -0.51 & -0.63 & 1.40 & 058 & -12.856 & 13.9 & 100.0 & 262 & 19 & 090 & 2.0 & 180 \\
\hline $01 ; 01 ; 20$ & -82 & -64 & 53 & 0 & -0.47 & -0.36 & 1.26 & 056 & -10.311 & 32.9 & 100.0 & 262 & 25 & 090 & 2.0 & 180 \\
\hline $01: 01: 30$ & -83 & -67 & 59 & 0 & -0.41 & -0.31 & 0.96 & 054 & -8.034 & 35.0 & 100.0 & 262 & 17 & 090 & 2.0 & 180 \\
\hline $01: 01 ; 40$ & -83 & -70 & 60 & 0 & -0.30 & -0.11 & 0.64 & 053 & -4.743 & 35.0 & 29.0 & 262 & 23 & 090 & 2.0 & 180 \\
\hline $01: 01: 50$ & -84 & -71 & 61 & 0 & -0.19 & -0.20 & 0.32 & 053 & -3.294 & 35.0 & 0.0 & 180 & 19 & 090 & 2.0 & 180 \\
\hline $01 ; 02 ; 00$ & -85 & -71 & 62 & 0 & -0.11 & -0.09 & 0.03 & 052 & -0.798 & 35.0 & 0.0 & 180 & 18 & 090 & 2.0 & 180 \\
\hline $01: 02: 10$ & -85 & -71 & 64 & 0 & -0.03 & 0.04 & -0.24 & 052 & 1.760 & 35.0 & 0.0 & 180 & 26 & 090 & 2.0 & 180 \\
\hline $01: 02: 20$ & -85 & -71 & 62 & 0 & 0.13 & 0.18 & -0.48 & 053 & 4.204 & 35.0 & 0.0 & 180 & 24 & 090 & 2.0 & 180 \\
\hline $01 ; 02: 30$ & -85 & -70 & 45 & 0 & 0.18 & 0.34 & -0.57 & 054 & 5.761 & 35.0 & 0.0 & 180 & 21 & 090 & 2.0 & 180 \\
\hline $01: 02: 40$ & -85 & -68 & 46 & 0 & 0.21 & 0.23 & -0.63 & 055 & 5.472 & 35.0 & 0.0 & 180 & 26 & 090 & 2.0 & 180 \\
\hline $01: 02: 50$ & -84 & -67 & 46 & 0 & 0.29 & 0.11 & -0.68 & 055 & 5.035 & 35.0 & 0.0 & 180 & 19 & 090 & 2.0 & 180 \\
\hline $01: 03: 00$ & -84 & -65 & 45 & 0 & 0.28 & 0.14 & -0.72 & 056 & 5.435 & 35.0 & 27.0 & 180 & 20 & 090 & 2.0 & 180 \\
\hline $01 ; 03 ; 10$ & -84 & -62 & 46 & 0 & 0.24 & -0.06 & -0.76 & 057 & 4.434 & 35.0 & 52.0 & 180 & 28 & 090 & 2.0 & 180 \\
\hline $01: 03: 20$ & -84 & -60 & 46 & 0 & 0.19 & -0.09 & -0.76 & 058 & 4.301 & 27.4 & 52.0 & 180 & 28 & 090 & 2.0 & 180 \\
\hline $01 ; 03: 30$ & -84 & -58 & 46 & 0 & 0.18 & -0.24 & -0.69 & 059 & 2.811 & 9.5 & 52.0 & 180 & 31 & 090 & 2.0 & 180 \\
\hline $01: 03: 40$ & -85 & -55 & 45 & 0 & 0.20 & -0.48 & -0.51 & 059 & 0.153 & -9.5 & 52.0 & 180 & 23 & 090 & 2.0 & 180 \\
\hline $01 ; 03 ; 50$ & -85 & -52 & 46 & 0 & 0.14 & -0.39 & -0.24 & 059 & -1.009 & -28.5 & 52.0 & 180 & 24 & 090 & 2.0 & 180 \\
\hline $01: 04: 00$ & -85 & -52 & 46 & 0 & 0.14 & -0.10 & 0.09 & 058 & -1.211 & -35.0 & 52.0 & 180 & 22 & 090 & 2.0 & 180 \\
\hline $01: 04: 10$ & -84 & -51 & 46 & 0 & 0.18 & -0.20 & 0.37 & 058 & -3.605 & -35.0 & 52.0 & 180 & 23 & 090 & 2.0 & 180 \\
\hline $01: 04: 20$ & -83 & -51 & 46 & 0 & 0.23 & -0.49 & 0.60 & 057 & -6.902 & -35.0 & 52.0 & 180 & 20 & 090 & 2.0 & 180 \\
\hline $01: 04: 30$ & -82 & -51 & 45 & 0 & 0.21 & -0.59 & 0.80 & 056 & -8.834 & -35.0 & 52.0 & 180 & 25 & 090 & 2.0 & 180 \\
\hline $01: 04: 40$ & -81 & -51 & 46 & 0 & 0.21 & -0.47 & 1.00 & 054 & -9.332 & -35.0 & 52.0 & 180 & 23 & 090 & 2.0 & 180 \\
\hline $01: 04: 50$ & -79 & -51 & 46 & 0 & 0.21 & -0.70 & 1.12 & 053 & -11.524 & -21.7 & 52.0 & 180 & 19 & 090 & 2.0 & 180 \\
\hline $01: 05: 00$ & -77 & -51 & 46 & 0 & 0.25 & -0.68 & 1.12 & 051 & -11.439 & -2.7 & 52.0 & 180 & 28 & 090 & 2.0 & 180 \\
\hline $01: 05: 10$ & -76 & -51 & 45 & 0 & 0.33 & -0.76 & 0.98 & 049 & -11.013 & 16.3 & 52.0 & 180 & 22 & 090 & 2.0 & 180 \\
\hline
\end{tabular}


Open Ocean

\section{Simulation Track Plot}

Ship: 150,000 ton, Tanker, partial load

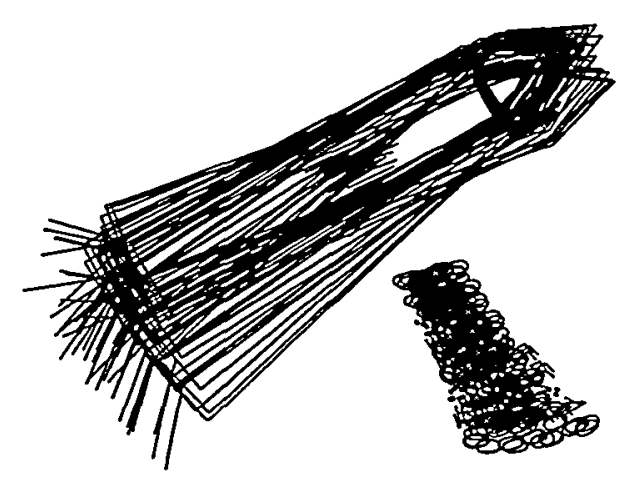

Start Time [hrs:min:sec]: $00: 41: 40$

End Time [hrs:min:sec]: $01: 05: 12$

Delta Plot Time [sec]:

30

East-West Scale [m]:

926.

Date: 04-Feb-99 Time: 16:29:29 


\section{HANDS-ON SIMULATION}

\section{SUCTION MOORNG TECHNOLOGY}

Simulation Number: 27

Date: 5 February 1999

Start Time: 08:06

Length of run [secs]: 5704

Ship Condition: draft $=13.72 \mathrm{~m}$

Environment

Wind Speed [knots]:

40 Direction: 0

Sea State, Significant wave height [m]:..

8 Direction: 180

Current Speed [knots]:

0 Direction: 0

Ship Operator: J.A.

Observer: B.K.J

Final Mating, distance between center of buoy and center of moon pool [m]: 5.1

Evaluation:

Mating $5.1 \mathrm{~m}$ off on the centerline. The challenge is to time when to push the button for release of the buoy during the buoy motion cycle. The captain was two seconds too early in the cycle.

The approach was good until the buoy came into camera view. It was possible to stop the ship entirely through a buoy cycle (approx. 14 secs).

The captain spent considerable time using the thruster only at 180 degrees and varying the heading orders for steering constantly seeking to keep zero ground speed, but it was not possible. Then the captain changed tactics and began experimenting by shifting the thruster vector. This proved to give better control. 
Ship: Exercise number:

acenario: Eile: affic ships:

Initial conditions: Exercise identification
591 150,000 ton, Tanker, partial load

754 Suction Mooring System

999 Dummy tug file based on containership

590 Suction Mooring Buoy

594 150k tanker in partial load - Suction Mooring Rue $\$ 2 \%$

Time position (m) $\quad$ RPM $\quad$ Speed (Knots) $\quad$ Turn Rudder Gill wind current HH:MM; SS East North Port Stbd Long Bow Stern Heading Deg/Min Deg \& Deg Knots Deg Knots Deg

\begin{tabular}{|c|c|c|c|c|c|c|c|c|c|c|c|c|c|c|c|c|}
\hline $00: 58: 20$ & 20 & -96 & 50 & 0 & -0.42 & 0.28 & 0.29 & 357 & -0.036 & 17.6 & 0.0 & 180 & 35 & 000 & 0.0 & 180 \\
\hline $00: 58: 30$ & 22 & -98 & 49 & 0 & -0.46 & 0.32 & 0.19 & 357 & 0.858 & 14.4 & 0.0 & 180 & 39 & 000 & 0.0 & 180 \\
\hline $00: 58: 40$ & 23 & -100 & 49 & 0 & -0.53 & 0.32 & 0.11 & 357 & 1.306 & 10.3 & 0.0 & 180 & 44 & 000 & 0.0 & 180 \\
\hline $00: 58: 50$ & 24 & -103 & 50 & 0 & -0.43 & 0.29 & 0.07 & 358 & 1.418 & 5.4 & 0.0 & 180 & 34 & 000 & 0.0 & 180 \\
\hline $00: 59: 00$ & 25 & -105 & 52 & 0 & -0.35 & 0.26 & 0.05 & 358 & 1.329 & 0.4 & 0.0 & 180 & 34 & 000 & 0.0 & 180 \\
\hline $00: 59: 10$ & 26 & -107 & 51 & 0 & -0.36 & 0.26 & 0.06 & 358 & 1.231 & -3.1 & 0.0 & 180 & 41 & 000 & 0.0 & 180 \\
\hline $00: 59: 20$ & 27 & -108 & 51 & 0 & -0.29 & 0.22 & 0.10 & 358 & 0.791 & -5.1 & 0.0 & 180 & 30 & 000 & 0.0 & 180 \\
\hline $00: 59: 30$ & 28 & -110 & 52 & 0 & -0.26 & 0.16 & 0.19 & 358 & -0.205 & -20.5 & 0.0 & 180 & 40 & 000 & 0.0 & 180 \\
\hline $00: 59: 40$ & 29 & -111 & 56 & 0 & -0.12 & 0.08 & 0.33 & 358 & -1.602 & -11.3 & 0.0 & 180 & 35 & 000 & 0.0 & 180 \\
\hline $00: 59: 50$ & 30 & -111 & 55 & 0 & 0.12 & 0.01 & 0.36 & 358 & -2.228 & -1.2 & 0.0 & 180 & 33 & 000 & 0.0 & 180 \\
\hline $01: 00: 00$ & 31 & -109 & 56 & 0 & 0.27 & -0.05 & 0.33 & 357 & -2.442 & 3.8 & 0.0 & 180 & 46 & 000 & 0.0 & 180 \\
\hline $01: 00: 10$ & 31 & -108 & 56 & 0 & 0.27 & -0.05 & 0.26 & 357 & -1.949 & 9.1 & 0.0 & 180 & 45 & 000 & 0.0 & 180 \\
\hline $01: 00: 20$ & 32 & -107 & 55 & 0 & 0.18 & -0.05 & 0.15 & 357 & -1.280 & 13.7 & 0.0 & 180 & 51 & 000 & 0.0 & 180 \\
\hline $01: 00: 30$ & 32 & -106 & 55 & 0 & 0.23 & -0.07 & 0.04 & 357 & -0.696 & 12.7 & 24.0 & 180 & 35 & 000 & 0.0 & 180 \\
\hline $01: 00: 40$ & 31 & -104 & 55 & 0 & 0.29 & -0.08 & -0.05 & 356 & -0.222 & 11.0 & 24.0 & 180 & 40 & 000 & 0.0 & 180 \\
\hline $01: 00: 50$ & 31 & -103 & 55 & 0 & 0.22 & -0.23 & -0.13 & 356 & 0.002 & 10.7 & 24.0 & 180 & 41 & 000 & 0.0 & 180 \\
\hline $01: 01: 00$ & 30 & -102 & 56 & 0 & 0.19 & -0.14 & -0.19 & 356 & 0.304 & 8.7 & 24.0 & 180 & 43 & 000 & 0.0 & 180 \\
\hline $01: 01 ; 10$ & 29 & -101 & 56 & 0 & 0.27 & -0.16 & -0.24 & 357 & 0.503 & 7.2 & 51.0 & 180 & 29 & 000 & 0.0 & 180 \\
\hline $01: 01: 20$ & 28 & -100 & 55 & 0 & 0.30 & -0.15 & -0.29 & 357 & 0.884 & 15.5 & 51.0 & 180 & 40 & 000 & 0.0 & 180 \\
\hline $01: 01: 30$ & 26 & -98 & 55 & 0 & 0.31 & -0.11 & -0.41 & 357 & 1.904 & 15.2 & 51.0 & 180 & 31 & 000 & 0.0 & 180 \\
\hline $01: 01: 40$ & 25 & -97 & 55 & 0 & 0.29 & -0.10 & -0.46 & 357 & 2.292 & 7.5 & 51.0 & 180 & 39 & 000 & 0.0 & 180 \\
\hline $01: 01: 50$ & 23 & -95 & 55 & 0 & 0.24 & -0.11 & -0.45 & 358 & 2.199 & 0.2 & 51.0 & 180 & 43 & 000 & 0.0 & 180 \\
\hline $01: 02: 00$ & 22 & -94 & 56 & 0 & 0.26 & -0.15 & -0.40 & 358 & 1.562 & -3.2 & 51.0 & 180 & 39 & 000 & 0.0 & 180 \\
\hline $01: 02: 10$ & 20 & -93 & 56 & 0 & 0.26 & -0.23 & -0.34 & 358 & 0.698 & -3.9 & 100.0 & 180 & 40 & 000 & 0.0 & 180 \\
\hline $01: 02: 20$ & 19 & -92 & 55 & 0 & 0.21 & -0.30 & -0.28 & 358 & -0.159 & -2.4 & 100.0 & 180 & 37 & 000 & 0.0 & 180 \\
\hline $01 ; 02 ; 30$ & 17 & -91 & 55 & 0 & 0.12 & -0.34 & -0.25 & 358 & -0.544 & 0.3 & 100.0 & 180 & 42 & 000 & 0.0 & 180 \\
\hline $01: 02: 40$ & 16 & -90 & 55 & 0 & 0.07 & -0.32 & -0.24 & 358 & -0.456 & 1.9 & 100.0 & 180 & 28 & 000 & 0.0 & 180 \\
\hline $01: 02: 50$ & 14 & -90 & 56 & 0 & 0.04 & -0.30 & -0.24 & 358 & -0.343 & 2.6 & 100.0 & 180 & 44 & 000 & 0.0 & 180 \\
\hline ; $03 ; 00$ & 13 & -90 & 55 & 0 & 0.12 & -0.31 & -0.25 & 358 & -0.392 & 3.8 & 100.0 & 180 & 37 & 000 & 0.0 & 180 \\
\hline$: 03: 10$ & 12 & -89 & 55 & 0 & 0.30 & -0.34 & -0.26 & 358 & -0.470 & 5.2 & 100.0 & 180 & 36 & 000 & 0.0 & 180 \\
\hline $1: 03: 20$ & 10 & -87 & 56 & 0 & 0.33 & -0.33 & -0.29 & 358 & -0.246 & 5.7 & 100.0 & 180 & 30 & 000 & 0.0 & 180 \\
\hline $01: 03: 30$ & 8 & -86 & 53 & 0 & 0.41 & -0.31 & -0.31 & 358 & -0.033 & 5.1 & 100.0 & 180 & 38 & 000 & 0.0 & 180 \\
\hline $01: 03: 40$ & 7 & -83 & 45 & 0 & 0.39 & -0.34 & -0.32 & 358 & -0.127 & 5.8 & 100.0 & 180 & 36 & 000 & 0.0 & 180 \\
\hline $01 ; 03 ; 50$ & 5 & -82 & 46 & 0 & 0.24 & -0.36 & -0.33 & 358 & -0.149 & 6.0 & 100.0 & 180 & 38 & 000 & 0.0 & 180 \\
\hline $01: 04: 00$ & 3 & -81 & 46 & 0 & 0.19 & -0.36 & -0.38 & 358 & 0.111 & 21.7 & 100.0 & 180 & 28 & 000 & 0.0 & 180 \\
\hline $01: 04: 10$ & 1 & -80 & 46 & 0 & 0.21 & -0.36 & -0.52 & 358 & 1.019 & 35.0 & 100.0 & 180 & 29 & 000 & 0.0 & 180 \\
\hline $01: 04: 20$ & -2 & -79 & 46 & 0 & 0.16 & -0.30 & -0.66 & 358 & 2.284 & 30.3 & 100.0 & 180 & 33 & 000 & 0.0 & 180 \\
\hline $01: 04: 30$ & -4 & -79 & 45 & 0 & -0.02 & -0.25 & -0.75 & 358 & 3.179 & 20.1 & 100.0 & 180 & 34 & 000 & 0.0 & 180 \\
\hline $01: 04: 40$ & -7 & -79 & 45 & 0 & -0.18 & -0.21 & -0.82 & 359 & 3.889 & 8.5 & 76.0 & 180 & 46 & 000 & 0.0 & 180 \\
\hline $01: 04: 50$ & -10 & -80 & 45 & 0 & -0.19 & -0.19 & -0.83 & 360 & 4.025 & -4.2 & 76.0 & 180 & 29 & 000 & 0.0 & 180 \\
\hline $01: 05: 00$ & -12 & -81 & 46 & 0 & -0.16 & -0.21 & -0.76 & 000 & 3.520 & -14.9 & 76.0 & 180 & 39 & 000 & 0.0 & 180 \\
\hline $01: 05: 10$ & -14 & -82 & 46 & 0 & -0.24 & -0.25 & -0.65 & 001 & 2.589 & -22.4 & 76.0 & 180 & 45 & 000 & 0.0 & 180 \\
\hline $01 ; 05 ; 20$ & -17 & -84 & 46 & 0 & -0.30 & -0.30 & -0.52 & 001 & 1.414 & -25.3 & 76.0 & 180 & 35 & 000 & 0.0 & 180 \\
\hline $01 ; 05: 30$ & -19 & -85 & 46 & 0 & -0.39 & -0.35 & -0.38 & 001 & 0.171 & -25.2 & 52.0 & 180 & 36 & 000 & 0.0 & 180 \\
\hline $01: 05: 40$ & -21 & -88 & 46 & 0 & -0.46 & -0.41 & -0.25 & 001 & -1.041 & -21.5 & 27.0 & 180 & 38 & 000 & 0.0 & 180 \\
\hline $01: 05: 50$ & -22 & -90 & 49 & 0 & -0.50 & -0.45 & -0.14 & 001 & -1.961 & -15.2 & 0.0 & 180 & 28 & 000 & 0.0 & 180 \\
\hline $01: 06: 00$ & -24 & -93 & 53 & 0 & -0.59 & -0.48 & -0.06 & 001 & -2.681 & -7.0 & 0.0 & 180 & 34 & 000 & 0.0 & 180 \\
\hline $01: 06: 10$ & -25 & -96 & 54 & 0 & -0.62 & -0.49 & -0.04 & 000 & -2.887 & 1.7 & 0.0 & 180 & 32 & 000 & 0.0 & 180 \\
\hline $01 ; 06 ; 20$ & -26 & -99 & 54 & 0 & -0.56 & -0.46 & -0.08 & 360 & -2.450 & 9.7 & 0.0 & 180 & 36 & 000 & 0.0 & 180 \\
\hline $01: 06: 30$ & -28 & -102 & 53 & 0 & -0.44 & -0.39 & -0.20 & 359 & -1.177 & 25.4 & 0.0 & 180 & 44 & 000 & 0.0 & 180 \\
\hline $01 ; 06 ; 40$ & -30 & -104 & 59 & 0 & -0.32 & -0.22 & -0.48 & 359 & 1.640 & 35.0 & 0.0 & 180 & 39 & 000 & 0.0 & 180 \\
\hline $01: 06: 50$ & -32 & -105 & 63 & 0 & -0.23 & -0.02 & -0.80 & 360 & 4.934 & 25.5 & 0.0 & 180 & 41 & 000 & 0.0 & 180 \\
\hline $01: 07: 00$ & -34 & -106 & 62 & 0 & -0.16 & 0.09 & -0.95 & 001 & 6.566 & 6.5 & 0.0 & 180 & 40 & 000 & 0.0 & 180 \\
\hline $01: 07: 10$ & -36 & -107 & 62 & 0 & -0.06 & 0.08 & -0.89 & 002 & 6.161 & -12.5 & 0.0 & 180 & 39 & 000 & 0.0 & 180 \\
\hline $01: 07: 20$ & -38 & -106 & 62 & 0 & 0.18 & -0.02 & -0.59 & 003 & 3.604 & -25.0 & 0.0 & 180 & 51 & 000 & 0.0 & 180 \\
\hline $01 ; 07: 30$ & -39 & -105 & 63 & 0 & 0.28 & -0.04 & -0.38 & 003 & 2.192 & -6.0 & 0.0 & 180 & 33 & 000 & 0.0 & 180 \\
\hline $01: 07: 40$ & -40 & -103 & 62 & 0 & 0.31 & 0.02 & -0.34 & 004 & 2.275 & -2.1 & 0.0 & 180 & 33 & 000 & 0.0 & 180 \\
\hline $01: 07: 50$ & -40 & -101 & 62 & 0 & 0.42 & 0.05 & -0.26 & 004 & 1.975 & -6.5 & 51.0 & 180 & 41 & 000 & 0.0 & 180 \\
\hline $01: 08: 00$ & -41 & -99 & 52 & 0 & 0.37 & 0.04 & -0.17 & 004 & 1.364 & -10.5 & 75.0 & 180 & 41 & 000 & 0.0 & 180 \\
\hline $01: 08: 10$ & -41 & -98 & 53 & 0 & 0.29 & 0.01 & -0.07 & 005 & 0.538 & -11.3 & 48.0 & 180 & 40 & 000 & 0.0 & 180 \\
\hline $01: 08: 20$ & $-4 I$ & -96 & 52 & 0 & 0.36 & 0.07 & 0.01 & 005 & 0.371 & -11.3 & 48.0 & 180 & 41 & 000 & 0.0 & 380 \\
\hline $01: 08: 30$ & -40 & -94 & 53 & 0 & 0.48 & 0.13 & 0.10 & 005 & 0.236 & -11.9 & 48.0 & 180 & 37 & 000 & 0.0 & 180 \\
\hline $01 ; 08 ; 40$ & -39 & -91 & 53 & 0 & 0.54 & 0.10 & 0.18 & 005 & -0.505 & -10.2 & 48.0 & 180 & 36 & 000 & 0.0 & 180 \\
\hline $01: 08: 50$ & -38 & -89 & 53 & 0 & 0.47 & 0.15 & 0.23 & 005 & -0.532 & -7.8 & 100.0 & 180 & 42 & 000 & 0.0 & 180 \\
\hline $01 ; 09: 00$ & -37 & -87 & 53 & 0 & 0.32 & 0.19 & 0.27 & 004 & -0.474 & -6.5 & 100.0 & 180 & 36 & 000 & 0.0 & 180 \\
\hline $01: 09: 10$ & -36 & -85 & 53 & 0 & 0.25 & 0.22 & 0.29 & 004 & -0.460 & -4.6 & 100.0 & 180 & 40 & 000 & 0.0 & 180 \\
\hline $01: 09: 20$ & -34 & -84 & 53 & 0 & 0.17 & 0.29 & 0.31 & 004 & -0.132 & -5.2 & 100.0 & 180 & 35 & 000 & 0.0 & 180 \\
\hline$: 09: 30$ & -32 & -84 & 52 & 0 & 0.09 & 0.31 & 0.36 & 004 & -0.299 & -18.2 & 100.0 & 180 & 46 & 000 & 0.0 & 180 \\
\hline$: 09: 40$ & -30 & -84 & 53 & 0 & -0.01 & 0.28 & 0.53 & 004 & -1.576 & -34.4 & 100.0 & 180 & 33 & 000 & 0.0 & 180 \\
\hline $01 ; 09: 50$ & -28 & -84 & 53 & 0 & -0.16 & 0.18 & 0.75 & 004 & -3.648 & -22.3 & 100.0 & 180 & 44 & 000 & 0.0 & 180 \\
\hline $01: 10: 00$ & -26 & -85 & 53 & 0 & -0.18 & 0.09 & 0.86 & 003 & -4.924 & -6.3 & 100.0 & 180 & 41 & 000 & 0.0 & 180 \\
\hline $01: 10: 10$ & -23 & -86 & 53 & 0 & -0.16 & 0.10 & 0.86 & 002 & -4.826 & 3.5 & 100.0 & 130 & 41 & 000 & 0.0 & 180 \\
\hline & & 87 & $5 ?$ & 0 & -0.13 & & & 001 & -5.156 & 126 & & 130 & & & & \\
\hline
\end{tabular}


Time Position (m) RPM Speed (Knots) Turn Rudier Gill Wind

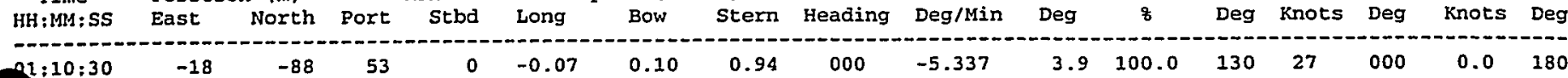

\begin{tabular}{|c|c|c|c|c|c|c|c|c|c|c|c|c|c|c|c|c|}
\hline $\begin{array}{r}1: 10: 30 \\
: 10: 40\end{array}$ & $\begin{array}{l}-18 \\
-16\end{array}$ & $\begin{array}{l}-88 \\
-88\end{array}$ & $\begin{array}{l}53 \\
53\end{array}$ & $\begin{array}{l}0 \\
0\end{array}$ & $\begin{array}{l}-0.07 \\
-0.05\end{array}$ & $\begin{array}{l}0.10 \\
0.17\end{array}$ & $\begin{array}{l}0.94 \\
0.86\end{array}$ & $\begin{array}{l}000 \\
360\end{array}$ & $\begin{array}{l}-5.337 \\
-4.437\end{array}$ & $\begin{array}{r}3.9 \\
16.9\end{array}$ & $\begin{array}{l}100.0 \\
100.0\end{array}$ & $\begin{array}{l}130 \\
130\end{array}$ & $\begin{array}{l}27 \\
37\end{array}$ & $\begin{array}{l}000 \\
000\end{array}$ & $\begin{array}{l}0.0 \\
0.0\end{array}$ & $\begin{array}{l}180 \\
180\end{array}$ \\
\hline $1: 10: 50$ & -13 & -88 & 52 & 0 & 0.05 & 0.29 & 0.69 & 359 & -2.529 & 24.2 & 100.0 & 130 & 31 & 000 & 0.0 & 180 \\
\hline $01: 11: 00$ & -11 & -87 & 52 & 0 & 0.13 & 0.41 & 0.47 & 359 & -0.367 & 23.0 & 100.0 & 130 & 36 & 000 & 0.0 & 180 \\
\hline $01: 11: 10$ & -8 & -87 & 53 & 0 & 0.08 & 0.52 & 0.31 & 359 & 1.384 & 17.1 & 100.0 & 130 & 33 & 000 & 0.0 & 180 \\
\hline $01 ; 11 ; 20$ & -6 & -87 & 53 & 0 & -0.07 & 0.60 & 0.22 & 359 & 2.417 & 7.8 & 100.0 & 130 & 35 & 000 & 0.0 & 180 \\
\hline $01: 11: 30$ & -4 & -87 & 52 & 0 & -0.03 & 0.63 & 0.21 & 360 & 2.649 & -0.6 & 100.0 & 130 & 37 & 000 & 0.0 & 180 \\
\hline $01: 11: 40$ & -2 & -87 & 53 & 0 & 0.09 & 0.63 & 0.24 & 000 & 2.483 & -7.2 & 100.0 & 130 & 52 & 000 & 0.0 & 180 \\
\hline $01: 11: 50$ & 0 & -87 & 53 & 0 & 0.06 & 0.59 & 0.30 & 000 & 1.851 & -12.2 & 100.0 & 180 & 28 & 000 & 0.0 & 180 \\
\hline $01: 12: 00$ & 3 & -86 & 52 & 0 & 0.10 & 0.51 & 0.37 & 001 & 0.922 & -14.3 & 100.0 & 232 & 38 & 000 & 0.0 & 180 \\
\hline $01: 12: 10$ & 5 & -86 & 53 & 0 & 0.12 & 0.42 & 0.43 & 001 & -0.063 & -12.8 & 100.0 & 232 & 33 & 000 & 0.0 & 180 \\
\hline $01: 12 ; 20$ & 7 & -85 & 48 & 0 & 0.04 & 0.34 & 0.47 & 001 & -0.806 & -9.5 & 100.0 & 232 & 32 & 000 & 0.0 & 180 \\
\hline $01: 12: 30$ & 9 & -85 & 48 & 0 & 0.00 & 0.29 & 0.48 & 000 & -1.208 & -5.3 & 100.0 & 213 & 35 & 000 & 0.0 & 180 \\
\hline $01: 12: 40$ & 11 & -85 & 49 & 0 & -0.02 & 0.25 & 0.50 & 000 & -1.566 & 0.4 & 100.0 & 213 & 31 & 000 & 0.0 & 180 \\
\hline $01: 12: 50$ & 13 & -85 & 49 & 0 & -0.07 & 0.21 & 0.48 & 360 & -1.687 & 6.1 & 100.0 & 269 & 37 & 000 & 0.0 & 180 \\
\hline $01: 13: 00$ & 15 & -86 & 49 & 0 & -0.11 & 0.18 & 0.42 & 360 & -1.517 & 10.7 & 100.0 & 269 & 36 & 000 & 0.0 & 180 \\
\hline $01: 13: 10$ & 16 & -87 & 49 & 0 & -0.24 & 0.16 & 0.34 & 359 & -1.117 & 13.4 & 100.0 & 269 & 33 & 000 & 0.0 & 180 \\
\hline $01: 13: 20$ & 17 & -88 & 49 & 0 & -0.36 & 0.15 & 0.24 & 359 & -0.575 & 13.5 & 100.0 & 290 & 39 & 000 & 0.0 & 180 \\
\hline $01: 13: 30$ & 18 & -90 & 48 & 0 & -0.37 & 0.15 & 0.15 & 359 & -0.027 & 13.1 & 100.0 & 296 & 42 & 000 & 0.0 & 180 \\
\hline $01 ; 13 ; 40$ & 19 & -92 & 48 & 0 & -0.34 & 0.14 & 0.06 & 359 & 0.498 & 10.8 & 100.0 & 296 & 35 & 000 & 0.0 & 180 \\
\hline $01: 13: 50$ & 19 & -93 & 49 & 0 & -0.19 & 0.13 & 0.00 & 359 & 0.816 & 5.3 & 100.0 & 296 & 45 & 000 & 0.0 & 180 \\
\hline $01: 14 ; 00$ & 19 & -94 & 49 & 0 & -0.12 & 0.09 & -0.03 & 360 & 0.720 & 1.2 & 100.0 & 270 & 29 & 000 & 0.0 & 180 \\
\hline $01: 14: 10$ & 19 & -95 & 49 & 0 & -0.10 & 0.04 & -0.05 & 360 & 0.520 & -0.2 & 100.0 & 270 & 37 & 000 & 0.0 & 180 \\
\hline $01: 14: 20$ & 19 & -95 & 49 & 0 & -0.08 & -0.01 & -0.06 & 360 & 0.301 & 0.5 & 100.0 & 244 & 49 & 000 & 0.0 & 180 \\
\hline $01: 14: 30$ & 19 & -96 & 48 & 0 & -0.09 & -0.04 & -0.08 & 360 & 0.217 & 1.6 & 100.0 & 218 & $4 I$ & 000 & 0.0 & 180 \\
\hline $01: 14: 40$ & 19 & -96 & 49 & 0 & -0.05 & -0.07 & -0.09 & 360 & 0.154 & 1.4 & 100.0 & 218 & 37 & 000 & 0.0 & 180 \\
\hline $01: 14: 50$ & 18 & -96 & 49 & 0 & -0.11 & -0.09 & -0.11 & 360 & 0.126 & 1.8 & 100.0 & 218 & 38 & 000 & 0.0 & 180 \\
\hline $01: 15: 00$ & 18 & -97 & 48 & 0 & -0.18 & -0.12 & -0.14 & 360 & 0.098 & 1.7 & 100.0 & 272 & 39 & 000 & 0.0 & 180 \\
\hline $01: 15: 10$ & 17 & -98 & 48 & 0 & -0.05 & -0.16 & -0.17 & 360 & 0.026 & 2.1 & 100.0 & 288 & 41 & 000 & 0.0 & 180 \\
\hline $01: 15: 20$ & 16 & -98 & 48 & 0 & 0.10 & -0.21 & -0.19 & 360 & -0.125 & 2.9 & 100.0 & 288 & 36 & 000 & 0.0 & 180 \\
\hline $01: 15: 30$ & 15 & -97 & 49 & 0 & 0.09 & -0.25 & -0.21 & 360 & -0.229 & 3.9 & 100.0 & 278 & 36 & 000 & 0.0 & 180 \\
\hline $01: 15: 40$ & 14 & -97 & 48 & 0 & 0.04 & -0.28 & -0.24 & 360 & -0.290 & 5.2 & 100.0 & 253 & 34 & 000 & 0.0 & 180 \\
\hline $01: 15: 50$ & 12 & -96 & 49 & 0 & 0.16 & -0.28 & -0.25 & 360 & -0.179 & 5.2 & 50.0 & 253 & 40 & 000 & 0.0 & 180 \\
\hline $01 ; 16 ; 00$ & 11 & -95 & 48 & 0 & 0.23 & -0.28 & -0.27 & 360 & -0.068 & 5.3 & 50.0 & 253 & 43 & 000 & 0.0 & 180 \\
\hline $01: 16: 10$ & 9 & -94 & 48 & 0 & 0.26 & -0.29 & -0.29 & 360 & 0.004 & 5.1 & 50.0 & 253 & 44 & 000 & 0.0 & 180 \\
\hline $01: 16: 20$ & 8 & -93 & 49 & 0 & 0.25 & -0.29 & -0.30 & 360 & 0.071 & 4.9 & 50.0 & 205 & 41 & 000 & 0.0 & 180 \\
\hline $01: 16: 30$ & 6 & -92 & 48 & 0 & 0.07 & -0.29 & -0.31 & 360 & 0.117 & 4.1 & 50.0 & 205 & 46 & 000 & 0.0 & 180 \\
\hline $01 ; 16 ; 40$ & 5 & -92 & 49 & 0 & -0.10 & -0.28 & -0.33 & 360 & 0.326 & 2.7 & 50.0 & 242 & 38 & 000 & 0.0 & 180 \\
\hline$-7: 16: 50$ & 3 & -93 & 49 & 0 & -0.13 & -0.27 & -0.35 & 360 & 0.476 & 1.1 & 50.0 & 242 & 33 & 000 & 0.0 & 180 \\
\hline$: 17: 00$ & 2 & -93 & 49 & 0 & -0.15 & -0.28 & -0.36 & 360 & 0.518 & -0.6 & 50.0 & 242 & 37 & 000 & 0.0 & 180 \\
\hline $1: 17: 10$ & 0 & -94 & 49 & 0 & -0.14 & -0.28 & -0.36 & 000 & 0.482 & -1.6 & 50.0 & 242 & 39 & 000 & 0.0 & 180 \\
\hline $01: 17: 20$ & -2 & -95 & 49 & 0 & -0.12 & -0.29 & -0.35 & 000 & 0.398 & -3.1 & 24.0 & 242 & 32 & 000 & 0.0 & 180 \\
\hline $01: 17: 30$ & -3 & -95 & 49 & 0 & -0.16 & -0.29 & -0.33 & 000 & 0.253 & -3.7 & 24.0 & 242 & 26 & 000 & 0.0 & 180 \\
\hline $01: 17: 40$ & -5 & -96 & 48 & 0 & -0.11 & -0.30 & -0.32 & 000 & 0.108 & -3.8 & 24.0 & 140 & 42 & 000 & 0.0 & 180 \\
\hline $01: 17: 50$ & -6 & -97 & 48 & 0 & -0.12 & -0.27 & -0.29 & 000 & 0.113 & -3.8 & 98.0 & 118 & 33 & 000 & 0.0 & 180 \\
\hline $01: 18: 00$ & -8 & -97 & 49 & 0 & -0.10 & -0.24 & -0.25 & 000 & 0.105 & -4.4 & 98.0 & 118 & 47 & 000 & 0.0 & 180 \\
\hline $01: 18: 10$ & -9 & -98 & 49 & 0 & -0.03 & -0.21 & -0.22 & 000 & 0.060 & -4.3 & 99.0 & 118 & 37 & 000 & 0.0 & 180 \\
\hline $01: 18: 20$ & -10 & -98 & 48 & 0 & -0.01 & -0.17 & -0.18 & 000 & 0.068 & -5.0 & 99.0 & 090 & 33 & 000 & 0.0 & 180 \\
\hline $01 ; 18: 30$ & -11 & -98 & 48 & 0 & -0.03 & -0.13 & -0.14 & 000 & 0.020 & -4.7 & 99.0 & 090 & 34 & 000 & 0.0 & 180 \\
\hline $01: 18: 40$ & -11 & -98 & 49 & 0 & -0.10 & -0.10 & -0.09 & 000 & -0.026 & -4.3 & 99.0 & 090 & 42 & 000 & 0.0 & 180 \\
\hline $01: 18: 50$ & -12 & -99 & 48 & 0 & -0.02 & -0.06 & -0.05 & 000 & -0.051 & -3.5 & 99.0 & 090 & 33 & 000 & 0.0 & 180 \\
\hline $01: 19: 00$ & -12 & -98 & 49 & 0 & 0.13 & -0.03 & -0.01 & 000 & -0.078 & -3.2 & 99.0 & 090 & 35 & 000 & 0.0 & 180 \\
\hline $01: 19: 10$ & -12 & -97 & 49 & 0 & 0.14 & 0.00 & 0.02 & 000 & -0.107 & -3.0 & 99.0 & 118 & 31 & 000 & 0.0 & 180 \\
\hline $01 ; 19: 20$ & -12 & -97 & 48 & 0 & 0.16 & 0.04 & 0.05 & 000 & -0.084 & -2.8 & 99.0 & 118 & 36 & 000 & 0.0 & 180 \\
\hline $01: 19: 30$ & -12 & -96 & 48 & 0 & 0.28 & 0.07 & 0.08 & 000 & -0.058 & -3.1 & 99.0 & 118 & 39 & 000 & 0.0 & 180 \\
\hline $01 ; 19: 40$ & -11 & -94 & 48 & 0 & 0.32 & 0.10 & 0.11 & 000 & -0.058 & -3.2 & 76.0 & 118 & 26 & 000 & 0.0 & 180 \\
\hline $01: 19: 50$ & -10 & -93 & 49 & 0 & 0.22 & 0.11 & 0.13 & 000 & -0.105 & -2.3 & 76.0 & 151 & 45 & 000 & 0.0 & 180 \\
\hline $01: 20: 00$ & -10 & -92 & 48 & 0 & 0.12 & 0.13 & 0.14 & 000 & -0.089 & -2.2 & 98.0 & 151 & 28 & 000 & 0.0 & 180 \\
\hline $01: 20: 10$ & -9 & -91 & 48 & 0 & 0.01 & 0.15 & 0.16 & 000 & -0.024 & -2.1 & 52.0 & 090 & 33 & 000 & 0.0 & 180 \\
\hline $01: 20: 20$ & -8 & -91 & 49 & 0 & -0.03 & 0.16 & 0.18 & 000 & -0.132 & -1.4 & 52.0 & 090 & 34 & 000 & 0.0 & 180 \\
\hline $01: 20: 30$ & -7 & -92 & 49 & 0 & 0.02 & 0.16 & 0.19 & 000 & -0.153 & -0.6 & 39.0 & 090 & 36 & 000 & 0.0 & 180 \\
\hline $01: 20: 40$ & -6 & -91 & 49 & 0 & -0.02 & 0.17 & 0.19 & 000 & -0.112 & -0.4 & 39.0 & 090 & 37 & 000 & 0.0 & 180 \\
\hline $01: 20: 50$ & -5 & -92 & 48 & 0 & -0.16 & 0.17 & 0.20 & 000 & -0.200 & -0.2 & 39.0 & 124 & 49 & 000 & 0.0 & 180 \\
\hline $01: 21: 00$ & -4 & -93 & 48 & 0 & -0.21 & 0.17 & 0.21 & 000 & -0.239 & 1.0 & 39.0 & 065 & 33 & 000 & 0.0 & 180 \\
\hline $01: 21: 10$ & -3 & -94 & 48 & 0 & -0.14 & 0.17 & 0.21 & 360 & -0.225 & 1.7 & 39.0 & 065 & 38 & 000 & 0.0 & 180 \\
\hline $01: 21: 20$ & -2 & -94 & 49 & 0 & -0.05 & 0.18 & 0.21 & 360 & -0.177 & 2.2 & 39.0 & 065 & 44 & 000 & 0.0 & 180 \\
\hline $01: 21: 30$ & -1 & -94 & 48 & 0 & 0.04 & 0.19 & 0.20 & 360 & -0.034 & 1.9 & 39.0 & 065 & 40 & 000 & 0.0 & 180 \\
\hline $01: 21: 40$ & 0 & -94 & 48 & 0 & -0.04 & 0.20 & 0.19 & 360 & 0.083 & 0.6 & 39.0 & 153 & 39 & 000 & 0.0 & 180 \\
\hline $01: 21: 50$ & 1 & -95 & 48 & 0 & -0.12 & 0.20 & 0.19 & 360 & 0.072 & 0.7 & 100.0 & 176 & 26 & 000 & 0.0 & 180 \\
\hline $01: 22: 00$ & 2 & -95 & 48 & 0 & -0.14 & 0.20 & 0.20 & 360 & 0.040 & 0.9 & 100.0 & 176 & 37 & 000 & 0.0 & 180 \\
\hline $01: 22: 10$ & 3 & -96 & 49 & 0 & -0.21 & 0.17 & 0.19 & 360 & -0.118 & 1.6 & 100.0 & 242 & 36 & 000 & 0.0 & 180 \\
\hline $01: 22: 20$ & 3 & -97 & 49 & 0 & -0.15 & 0.12 & 0.16 & 360 & -0.270 & 3.2 & 100.0 & 272 & 40 & 000 & 0.0 & 180 \\
\hline $01: 22: 30$ & 4 & -98 & 48 & 0 & -0.05 & 0.08 & 0.13 & 360 & -0.301 & 5.3 & 100.0 & 272 & 42 & 000 & 0.0 & 180 \\
\hline $01: 22: 40$ & 4 & -98 & 48 & 0 & 0.07 & 0.06 & 0.08 & 360 & -0.139 & 5.1 & 100.0 & 242 & 42 & 000 & 0.0 & 180 \\
\hline $01: 22: 50$ & 5 & -97 & 48 & 0 & 0.08 & 0.03 & 0.04 & 360 & -0.040 & 3.9 & 100.0 & 242 & 40 & 000 & 0.0 & 180 \\
\hline $01: 23: 00$ & 5 & -97 & 49 & 0 & 0.06 & 0.00 & 0.00 & 360 & -0.020 & 3.8 & 100.0 & 242 & 47 & 000 & 0.0 & 180 \\
\hline $01: 23: 10$ & 5 & -97 & 49 & 0 & 0.16 & -0.03 & -0.03 & 360 & -0.016 & 3.5 & 100.0 & 211 & 42 & 000 & 0.0 & 180 \\
\hline$: 23: 20$ & 4 & -96 & 48 & 0 & 0.18 & -0.05 & -0.05 & 360 & 0.029 & 3.2 & 100.0 & 211 & 38 & 000 & 0.0 & 180 \\
\hline$: 23: 30$ & 4 & -95 & 49 & 0 & 0.10 & -0.07 & -0.08 & 360 & 0.030 & 3.1 & 100.0 & 211 & 40 & 000 & 0.0 & 180 \\
\hline $01: 23: 40$ & 4 & -94 & 49 & 0 & 0.04 & -0.09 & -0.10 & 360 & 0.007 & 3.3 & 100.0 & 211 & 39 & 000 & 0.0 & 180 \\
\hline $01: 23: 50$ & 3 & -94 & 49 & 0 & -0.03 & -0.12 & -0.12 & 360 & 0.003 & 3.4 & 100.0 & 239 & 31 & 000 & 0.0 & 180 \\
\hline $01: 24: 00$ & 2 & -95 & 49 & 0 & -0.14 & -0.14 & -0.15 & 360 & 0.068 & 3.0 & 51.0 & 239 & 36 & 000 & 0.0 & 180 \\
\hline $01: 24: 10$ & 2 & -96 & 48 & 0 & -0.18 & -0.14 & -0.17 & 360 & 0.196 & 1.9 & 51.0 & 239 & 44 & 000 & 0.0 & 180 \\
\hline
\end{tabular}




\begin{tabular}{|c|c|c|c|c|c|c|c|c|c|c|c|c|c|c|c|c|}
\hline log.ascii & & & & & & & & & & & & & & & Pag & 3 \\
\hline Time & Positi & $n(m)$ & & RPM & & peed ( $\mathrm{K}$ & ts) & & Turn & Rudder & Gi & & win & & Curre: & nt \\
\hline HH:MM:SS & East & North & Port & stbd & Long & Bow & Stern & Heading & Deg/Min & Deg & 8 & Deg & Knots & Deg & Rnots & Deg \\
\hline & & 07 & & & 0 & 03 & & & 0 & 12 & 570 & 230 & & (000 & 00 & 180 \\
\hline$\left\{\begin{array}{r}24: 20 \\
: 24: 30\end{array}\right.$ & $\begin{array}{l}1 \\
0\end{array}$ & $\begin{array}{l}-97 . \\
-98\end{array}$ & $\begin{array}{l}49 \\
48\end{array}$ & $\begin{array}{l}0 \\
0\end{array}$ & $\begin{array}{l}-0.24 \\
-0.30\end{array}$ & $\begin{array}{l}-0.15 \\
-0.15\end{array}$ & $\begin{array}{l}-0.19 \\
-0.20\end{array}$ & $\begin{array}{l}360 \\
360\end{array}$ & $\begin{array}{l}0.268 \\
0.299\end{array}$ & $\begin{array}{l}1.2 \\
0.2\end{array}$ & $\begin{array}{l}51.0 \\
51.0\end{array}$ & $\begin{array}{l}239 \\
249\end{array}$ & $\begin{array}{l}39 \\
34\end{array}$ & $\begin{array}{l}000 \\
000\end{array}$ & $\begin{array}{l}0.0 \\
0.0\end{array}$ & $\begin{array}{l}180 \\
180\end{array}$ \\
\hline$f: 24: 40$ & -1 & -100 & 49 & 0 & -0.25 & -0.16 & -0.20 & 360 & 0.265 & -0.5 & 51.0 & 272 & 37 & 000 & 0.0 & 180 \\
\hline $01: 24: 50$ & -2 & -101 & 49 & 0 & -0.13 & -0.18 & -0.21 & 000 & 0.194 & -1.0 & 51.0 & 272 & 33 & 000 & 0.0 & 180 \\
\hline $01: 25: 00$ & -3 & -101 & 49 & 0 & -0.12 & -0.17 & -0.20 & 000 & 0.180 & -1.5 & 51.0 & 088 & 43 & 000 & 0.0 & 180 \\
\hline $01: 25: 10$ & -4 & -102 & 49 & 0 & -0.23 & -0.16 & -0.18 & 000 & 0.157 & -1.8 & 51.0 & 088 & 37 & 000 & 0.0 & 180 \\
\hline $01: 25: 20$ & -5 & -103 & 48 & 0 & -0.31 & -0.15 & -0.17 & 000 & 0.103 & -2.0 & 51.0 & 059 & 34 & 000 & 0.0 & 180 \\
\hline $01: 25: 30$ & -6 & -105 & 49 & 0 & -0.32 & -0.15 & -0.15 & 000 & 0.045 & -1.8 & 51.0 & 059 & 36 & 000 & 0.0 & 180 \\
\hline $01: 25: 40$ & -6 & -106 & 49 & 0 & -0.16 & -0.12 & -0.13 & 000 & 0.092 & -2.8 & 100.0 & 059 & 36 & 000 & 0.0 & 180 \\
\hline $01 ; 25 ; 50$ & -7 & -107 & 49 & 0 & -0.10 & -0.08 & -0.10 & 000 & 0.114 & -3.3 & 100.0 & 059 & 46 & 000 & 0.0 & 180 \\
\hline $01: 26: 00$ & -7 & -108 & 49 & 0 & -0.16 & -0.05 & -0.07 & 000 & 0.086 & -3.4 & 100.0 & 059 & 35 & 000 & 0.0 & 180 \\
\hline $01 ; 26: 10$ & -7 & -108 & 48 & 0 & -0.06 & -0.03 & -0.03 & 000 & 0.038 & -3.4 & 100.0 & 059 & 32 & 000 & 0.0 & 180 \\
\hline $01: 26: 20$ & -7 & -108 & 49 & 0 & 0.05 & 0.00 & 0.00 & 000 & -0.008 & -2.6 & 100.0 & 059 & 35 & 000 & 0.0 & 180 \\
\hline $01: 26: 30$ & -7 & -108 & 49 & 0 & 0.18 & 0.03 & 0.04 & 000 & -0.013 & -2.7 & 100.0 & 059 & 33 & 000 & 0.0 & 180 \\
\hline $01: 26: 40$ & -7 & -106 & 49 & 0 & 0.35 & 0.07 & 0.07 & 000 & 0.009 & -2.8 & 100.0 & 059 & 22 & 000 & 0.0 & 180 \\
\hline $01: 26: 50$ & -7 & -104 & 48 & 0 & 0.49 & 0.10 & 0.10 & 000 & 0.038 & -2.9 & 100.0 & 059 & 42 & 000 & 0.0 & 180 \\
\hline $01: 27 ; 00$ & -6 & -101 & 48 & 0 & 0.56 & 0.13 & 0.12 & 000 & 0.045 & -2.9 & 100.0 & 059 & 27 & 000 & 0.0 & 180 \\
\hline $01: 27: 10$ & -5 & -99 & 49 & 0 & 0.46 & 0.15 & 0.15 & 000 & 0.016 & -3.4 & 100.0 & 152 & 38 & 000 & 0.0 & 180 \\
\hline $01: 27: 20$ & -4 & -97 & 48 & 0 & 0.36 & 0.17 & 0.17 & 000 & -0.015 & -3.3 & 100.0 & 152 & 38 & 000 & 0.0 & 180 \\
\hline $01: 27: 30$ & -4 & -95 & 48 & 0 & 0.29 & 0.17 & 0.19 & 000 & -0.105 & -2.5 & 100.0 & 184 & 36 & 000 & 0.0 & 180 \\
\hline $01: 27: 40$ & -3 & -94 & 48 & 0 & 0.17 & 0.16 & 0.19 & 000 & -0.183 & -1.9 & 100.0 & 184 & 45 & 000 & 0.0 & 180 \\
\hline $01: 27: 50$ & -2 & -93 & 48 & 0 & 0.11 & 0.16 & 0.19 & 000 & -0.192 & -1.1 & 100.0 & 184 & 41 & 000 & 0.0 & 180 \\
\hline $01: 28 ; 00$ & -1 & -93 & 49 & 0 & 0.08 & 0.16 & 0.18 & 000 & -0.159 & -0.7 & 100.0 & 184 & 39 & 000 & 0.0 & 180 \\
\hline $01: 28: 10$ & 0 & -93 & 48 & 0 & -0.02 & 0.16 & 0.18 & 000 & -0.143 & -0.1 & 100.0 & 184 & 29 & 000 & 0.0 & 180 \\
\hline $01: 28: 20$ & 1 & -93 & 48 & 0 & -0.08 & 0.15 & 0.18 & 000 & -0.224 & 0.9 & 100.0 & 184 & 32 & 000 & 0.0 & 180 \\
\hline $01 ; 28 ; 30$ & 2 & -93 & 48 & 0 & -0.10 & 0.14 & 0.18 & 360 & -0.245 & 1.5 & 100.0 & 184 & 36 & 000 & 0.0 & 180 \\
\hline $01: 28: 40$ & 3 & -94 & 49 & 0 & -0.19 & 0.14 & 0.18 & 360 & -0.225 & 2.2 & 100.0 & 184 & 44 & 000 & 0.0 & 180 \\
\hline $01: 28: 50$ & 3 & -95 & 49 & 0 & -0.27 & 0.17 & 0.18 & 360 & -0.077 & 2.0 & 100.0 & 089 & 25 & 000 & 0.0 & 180 \\
\hline $01 ; 29 ; 00$ & 4 & -97 & 48 & 0 & -0.31 & 0.16 & 0.17 & 360 & -0.082 & 1.9 & 100.0 & 268 & 43 & 000 & 0.0 & 180 \\
\hline $01: 29: 10$ & 5 & -98 & 48 & 0 & -0.26 & 0.12 & 0.15 & 360 & -0.199 & 3.1 & 100.0 & 303 & 37 & 000 & 0.0 & 180 \\
\hline $01: 29: 20$ & 6 & -99 & 48 & 0 & -0.24 & 0.08 & 0.12 & 360 & -0.240 & 3.9 & 100.0 & 303 & 43 & 000 & 0.0 & 180 \\
\hline $01: 29: 30$ & 6 & -101 & 48 & 0 & -0.28 & 0.05 & 0.09 & 360 & -0.219 & 4.3 & 100.0 & 272 & 44 & 000 & 0.0 & 180 \\
\hline $01: 29: 40$ & 6 & -102 & 49 & 0 & -0.33 & 0.03 & 0.05 & 360 & -0.152 & 4.5 & 100.0 & 306 & 40 & 000 & 0.0 & 180 \\
\hline $01: 29: 50$ & 7 & -104 & 48 & 0 & -0.18 & 0.01 & 0.02 & 360 & -0.017 & 4.6 & 100.0 & 336 & 37 & 000 & 0.0 & 180 \\
\hline $01: 30: 00$ & 7 & -104 & 48 & 0 & 0.15 & 0.00 & -0.01 & 360 & 0.062 & 3.4 & 100.0 & 336 & 39 & 000 & 0.0 & 180 \\
\hline $01: 30: 10$ & 6 & -103 & 48 & 0 & 0.25 & -0.03 & -0.04 & 360 & 0.056 & 3.7 & 100.0 & 298 & 43 & 000 & 0.0 & 180 \\
\hline $01: 30: 20$ & 6 & -101 & 49 & 0 & 0.30 & -0.06 & -0.07 & 360 & 0.041 & 3.8 & 100.0 & 298 & 36 & 000 & 0.0 & 180 \\
\hline $01: 30: 30$ & 6 & -100 & 49 & 0 & 0.37 & -0.10 & -0.10 & 360 & 0.018 & 3.9 & 100.0 & 298 & 44 & 000 & 0.0 & 180 \\
\hline $1: 30 ; 10$ & 5 & -98 & 49 & 0 & 0.37 & -0.13 & -0.13 & 360 & -0.001 & 3.9 & 100.0 & 298 & 42 & 000 & 0.0 & 180 \\
\hline$: 30: 50$ & 4 & -96 & 49 & 0 & 0.39 & -0.16 & -0.16 & 360 & -0.008 & 4.0 & 100.0 & 209 & 39 & 000 & 0.0 & 180 \\
\hline o1:31:00 & 4 & -94 & 48 & 0 & 0.39 & -0.17 & -0.17 & 360 & 0.031 & 3.9 & 100.0 & 184 & 32 & 000 & 0.0 & 180 \\
\hline $01 ; 31: 10$ & 3 & -92 & 49 & 0 & 0.32 & -0.17 & -0.18 & 360 & 0.095 & 3.1 & 100.0 & 184 & 42 & 000 & 0.0 & 180 \\
\hline $01: 31: 20$ & 2 & -90 & 49 & 0 & 0.20 & -0.17 & -0.19 & 360 & 0.153 & 2.7 & 100.0 & 184 & 38 & 000 & 0.0 & 180 \\
\hline $01: 31: 30$ & 1 & -90 & 48 & 0 & 0.11 & -0.17 & -0.19 & 360 & 0.133 & 2.1 & 100.0 & 184 & 37 & 000 & 0.0 & 180 \\
\hline $01: 31: 40$ & 0 & -89 & 48 & 0 & 0.09 & -0.17 & -0.19 & 360 & 0.122 & 1.5 & 100.0 & 141 & 31 & 000 & 0.0 & 180 \\
\hline $01: 31: 50$ & -1 & -89 & 49 & 0 & 0.07 & -0.13 & -0.17 & 360 & 0.214 & 0.2 & 100.0 & 119 & 36 & 000 & 0.0 & 180 \\
\hline $01: 32: 00$ & -2 & -89 & 48 & 0 & 0.01 & -0.10 & -0.14 & 360 & 0.227 & -1.1 & 100.0 & 142 & 43 & 000 & 0.0 & 180 \\
\hline $01: 32: 10$ & -2 & -89 & 49 & 0 & 0.02 & -0.09 & -0.12 & 360 & 0.141 & -1.0 & 100.0 & 156 & 42 & 000 & 0.0 & 180 \\
\hline $01: 32: 20$ & -3 & -89 & 49 & 0 & -0.04 & -0.07 & -0.09 & 360 & 0.137 & -1.8 & 100.0 & 153 & 45 & 000 & 0.0 & 180 \\
\hline $01: 32: 30$ & -3 & -89 & 48 & 0 & -0.20 & -0.05 & -0.08 & 000 & 0.159 & -0.9 & 100.0 & 153 & 36 & 000 & 0.0 & 180 \\
\hline $01: 32: 40$ & -3 & -90 & 49 & 0 & -0.31 & -0.03 & -0.06 & 000 & 0.216 & -1.4 & 100.0 & 121 & 32 & 000 & 0.0 & 180 \\
\hline $01: 32: 50$ & -4 & -92 & 48 & 0 & -0.40 & 0.01 & -0.03 & 000 & 0.263 & -2.5 & 100.0 & 121 & 45 & 000 & 0.0 & 180 \\
\hline $01: 33: 00$ & -4 & -95 & 49 & 0 & -0.44 & 0.04 & 0.00 & 000 & 0.257 & -2.9 & 100.0 & 121 & 30 & 000 & 0.0 & 180 \\
\hline $01: 33: 10$ & -3 & -97 & 49 & 0 & -0.44 & 0.07 & 0.04 & 000 & 0.211 & -3.4 & 100.0 & 062 & 29 & 000 & 0.0 & 180 \\
\hline $01: 33: 20$ & -3 & -99 & 49 & 0 & -0.37 & 0.10 & 0.08 & 000 & 0.124 & -3.8 & 100.0 & 062 & 34 & 000 & 0.0 & 180 \\
\hline $01: 33: 30$ & -2 & -101 & 49 & 0 & -0.23 & 0.12 & 0.12 & 000 & 0.017 & -4.0 & 100.0 & 062 & 30 & 000 & 0.0 & 180 \\
\hline $01: 33: 40$ & -2 & -101 & 49 & 0 & -0.07 & 0.15 & 0.16 & 000 & -0.101 & -2.7 & 100.0 & 062 & 33 & 000 & 0.0 & 280 \\
\hline $01: 33: 50$ & -1 & -101 & 49 & 0 & -0.03 & 0.17 & 0.20 & 000 & -0.173 & -2.3 & 100.0 & 119 & 39 & 000 & 0.0 & 180 \\
\hline $01: 34: 00$ & 0 & -102 & 48 & 0 & -0.10 & 0.20 & 0.23 & 000 & -0.208 & -1.8 & 100.0 & 119 & 37 & 000 & 0.0 & 180 \\
\hline $01: 34: 10$ & 1 & -102 & 49 & 0 & 0.01 & 0.23 & 0.26 & 000 & -0.180 & -1.4 & 100.0 & 119 & 42 & 000 & 0.0 & 180 \\
\hline $01: 34: 20$ & 3 & -102 & 49 & 0 & 0.10 & 0.28 & 0.27 & 000 & 0.053 & -1.9 & 100.0 & 119 & 36 & 000 & 0.0 & 180 \\
\hline $01: 34: 30$ & 4 & -101 & 49 & 0 & 0.08 & 0.32 & 0.28 & 000 & 0.215 & -3.1 & 100.0 & 119 & 32 & 000 & 0.0 & 180 \\
\hline $01: 34: 40$ & 6 & -101 & 49 & 0 & 0.05 & 0.35 & 0.30 & 000 & 0.326 & -4.0 & 100.0 & 119 & 40 & 000 & 0.0 & 180 \\
\hline $01: 34: 50$ & 8 & -101 & 49 & 0 & -0.07 & 0.37 & 0.34 & 000 & 0.217 & -4.7 & 100.0 & 119 & 30 & 000 & 0.0 & 180 \\
\hline $01: 35: 00$ & 9 & -102 & 49 & 0 & -0.16 & 0.38 & 0.39 & 000 & -0.051 & -4.4 & 100.0 & 119 & 45 & 000 & 0.0 & 180 \\
\hline
\end{tabular}




\section{Open Ocean}

\section{Simulation Track Plot}

Ship: 150,000 ton, Tanker, partial load

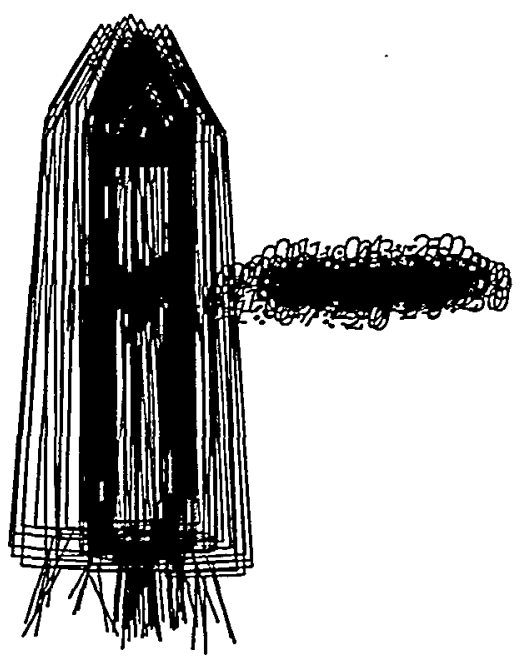

Start Time [hrs:min:sec]: $00: 58: 20$ End Time [hrs:min:sec]: 01:35:04 Delta Plot Time [sec]:

East-West Scale [m]:

East-West Scale [Nm]: $\quad 0.5000$ 


\section{HANDS-ON SIMULATION}

\section{SUCTION MOORNG TECHNOLOGY}

Simulation Number: 28

Date: 5 February 1999

Start Time: 09:53

Length of run [secs]: 2145

Ship Condition: $\mathrm{draft}=13.72 \mathrm{~m}$

Environment

Wind Speed [knots]

40 Direction: 0

Sea State, Significant wave height $[\mathrm{m}]: .$.

8 Direction: 180

Current Speed [knots]:

0 Direction: 0

Ship Operator: J.A.

Observer: B.K.J

Final Mating, distance between center of buoy and center of moon pool [m]: 2.4

Evaluation:

The approach was okay.

The maneuvering over the buoy was done from the start by varying the thruster vector direction and the forces. Throughout the positioning operation the autopilot was kept at 000 degrees and the RPM 44. All maneuvers were controlled by adjusting the thruster vector. 
Ship:

Exercise number:

Fenario:

g file:

atfic ships:

Exercise identification:
591 150,000 ton, Tanker, partial load

754 Suction Mooring System

112 Open Ocean

999 Dummy tug file based on containership

590 Suction Mooring Buoy

594 150k tanker in partial load - Suction Mooring RuN \# 28

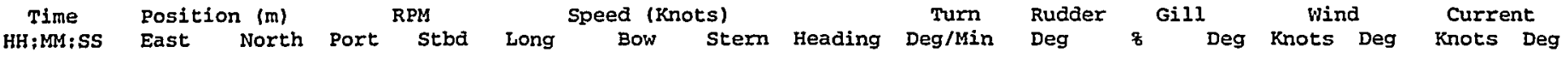

\begin{tabular}{|c|c|c|c|c|c|c|c|c|c|c|c|c|c|c|c|c|}
\hline $\begin{array}{l}00: 16: 40 \\
00: 16: 50\end{array}$ & $\begin{array}{l}10 \\
12\end{array}$ & $\begin{array}{l}-132 \\
-131\end{array}$ & $\begin{array}{l}44 \\
44\end{array}$ & $\begin{array}{l}0 \\
0\end{array}$ & $\begin{array}{l}0.15 \\
0.21\end{array}$ & $\begin{array}{l}0.42 \\
0.39\end{array}$ & $\begin{array}{l}0.37 \\
0.31\end{array}$ & $\begin{array}{l}360 \\
360\end{array}$ & $\begin{array}{l}0.320 \\
0.481\end{array}$ & $\begin{array}{l}4.8 \\
3.1\end{array}$ & $\begin{array}{l}100.0 \\
100.0\end{array}$ & $\begin{array}{l}303 \\
303\end{array}$ & $\begin{array}{l}46 \\
40\end{array}$ & $\begin{array}{l}000 \\
000\end{array}$ & $\begin{array}{l}0.0 \\
0.0\end{array}$ & $\begin{array}{l}180 \\
180\end{array}$ \\
\hline $00 ; 17: 00$ & 14 & -130 & 45 & 0 & 0.26 & 0.35 & 0.27 & 360 & 0.502 & 1.9 & 100.0 & 244 & 43 & 000 & 0.0 & 180 \\
\hline $00: 17: 10$ & 15 & -128 & 45 & 0 & 0.35 & 0.31 & 0.24 & 360 & 0.408 & 0.6 & 100.0 & 244 & 43 & 000 & 0.0 & 180 \\
\hline $00: 17: 20$ & 16 & -126 & 45 & 0 & 0.39 & 0.26 & 0.22 & 360 & 0.259 & -0.1 & 100.0 & 244 & 36 & 000 & 0.0 & 180 \\
\hline $00: 17: 30$ & 18 & -124 & 45 & 0 & 0.27 & 0.19 & 0.24 & 360 & -0.373 & -16.2 & 100.0 & 244 & 47 & 000 & 0.0 & 180 \\
\hline $00: 17: 40$ & 19 & -124 & 45 & 0 & 0.10 & 0.09 & 0.30 & 360 & -1.346 & -22.3 & 100.0 & 244 & 31 & 000 & 0.0 & 180 \\
\hline $00: 17: 50$ & 20 & -123 & 45 & 0 & 0.16 & -0.01 & 0.38 & 359 & -2.464 & -15.0 & 100.0 & 264 & 38 & 000 & 0.0 & 180 \\
\hline $00: 18: 00$ & 20 & -122 & 44 & 0 & 0.26 & -0.10 & 0.39 & 359 & -3.131 & -6.3 & 100.0 & 272 & 27 & 000 & 0.0 & 180 \\
\hline $00: 18: 10$ & 21 & -121 & 44 & 0 & 0.25 & -0.16 & 0.36 & 358 & -3.285 & 5.5 & 100.0 & 242 & 33 & 000 & 0.0 & 180 \\
\hline $00 ; 18: 20$ & 21 & -119 & 44 & 0 & 0.27 & -0.18 & 0.25 & 358 & -2.704 & 23.7 & 100.0 & 242 & 39 & 000 & 0.0 & 180 \\
\hline $00: 18: 30$ & 21 & -118 & 45 & 0 & 0.22 & -0.13 & 0.05 & 358 & -1.155 & 35.0 & 100.0 & 242 & 43 & 000 & 0.0 & 180 \\
\hline $00: 18: 40$ & 21 & -117 & 45 & 0 & 0.10 & -0.05 & -0.14 & 358 & 0.623 & 35.0 & 100.0 & 265 & 27 & 000 & 0.0 & 180 \\
\hline $00: 18: 50$ & 20 & -117 & 45 & 0 & 0.08 & -0.04 & -0.31 & 358 & 1.740 & 28.8 & 100.0 & 265 & 36 & 000 & 0.0 & 180 \\
\hline $00 ; 19 ; 00$ & 19 & -116 & 45 & 0 & 0.08 & -0.09 & -0.44 & 358 & 2.165 & 24.5 & 100.0 & 293 & 39 & 000 & 0.0 & 180 \\
\hline $00: 19: 10$ & 18 & -116 & 45 & 0 & 0.04 & -0.12 & -0.52 & 359 & 2.548 & 17.2 & 100.0 & 293 & 36 & 000 & 0.0 & 180 \\
\hline $00: 19: 20$ & 16 & -116 & 45 & 0 & -0.02 & -0.16 & -0.57 & 359 & 2.641 & 10.1 & 100.0 & 293 & 38 & 000 & 0.0 & 180 \\
\hline $00: 19: 30$ & 14 & -116 & 44 & 0 & -0.17 & -0.16 & -0.61 & 359 & 2.887 & 2.6 & 51.0 & 293 & 31 & 000 & 0.0 & 180 \\
\hline $00: 19: 40$ & 12 & -118 & 44 & 0 & -0.27 & -0.17 & -0.61 & 360 & 2.789 & -5.3 & 75.0 & 316 & 37 & 000 & 0.0 & 180 \\
\hline $00: 19: 50$ & 10 & -119 & 44 & 0 & -0.29 & -0.20 & -0.57 & 000 & 2.303 & -11.3 & 75.0 & 316 & 31 & 000 & 0.0 & 180 \\
\hline $00: 20: 00$ & 8 & -120 & 44 & 0 & -0.24 & -0.26 & -0.50 & 001 & 1.506 & -15.8 & 99.0 & 342 & 44 & 000 & 0.0 & 180 \\
\hline $00: 20: 10$ & 6 & -121 & 45 & 0 & -0.09 & -0.33 & -0.42 & 001 & 0.576 & -17.0 & 99.0 & 303 & 31 & 000 & 0.0 & 180 \\
\hline $00: 20: 20$ & 4 & -122 & 45 & 0 & -0.06 & -0.40 & -0.35 & 001 & -0.293 & -14.3 & 54.0 & 272 & 38 & 000 & 0.0 & 180 \\
\hline $00: 20: 30$ & 2 & -122 & 45 & 0 & -0.06 & -0.43 & -0.29 & 001 & -0.909 & -10.6 & 26.0 & 272 & 34 & 000 & 0.0 & 180 \\
\hline $00: 20: 40$ & 0 & -122 & 45 & 0 & -0.03 & -0.45 & -0.25 & 001 & -1.286 & -5.8 & 72.0 & 000 & 33 & 000 & 0.0 & 180 \\
\hline $00: 20: 50$ & -2 & -122 & 45 & 0 & 0.03 & -0.44 & -0.22 & 000 & -1.408 & -1.2 & 72.0 & 072 & 29 & 000 & 0.0 & 180 \\
\hline $00: 21: 00$ & -3 & -122 & 45 & 0 & 0.11 & -0.41 & -0.20 & 000 & -1.326 & 2.6 & 87.0 & 072 & 35 & 000 & 0.0 & 180 \\
\hline $00: 21: 10$ & -5 & -121 & 44 & 0 & 0.05 & -0.35 & -0.20 & 360 & -0.983 & 5.9 & 99.0 & 072 & 40 & 000 & 0.0 & 180 \\
\hline $0: 21: 20$ & -6 & -121 & 44 & 0 & 0.04 & -0.29 & -0.20 & 360 & -0.572 & 6.7 & 99.0 & 072 & 38 & 000 & 0.0 & 180 \\
\hline $1: 21: 30$ & -7 & $-12 I$ & 44 & 0 & 0.17 & -0.24 & -0.21 & 360 & -0.181 & 5.9 & 99.0 & 072 & 34 & 000 & 0.0 & 180 \\
\hline $00: 21: 40$ & -8 & -119 & 45 & 0 & 0.27 & -0.18 & -0.21 & 360 & 0.161 & 4.5 & 99.0 & 072 & 42 & 000 & 0.0 & 180 \\
\hline $00: 21: 50$ & -9 & -118 & 45 & 0 & 0.17 & -0.14 & -0.20 & 360 & 0.413 & 1.9 & 99.0 & 119 & 49 & 000 & 0.0 & 180 \\
\hline $00: 22: 00$ & -10 & -118 & 45 & 0 & 0.12 & -0.11 & -0.18 & 360 & 0.466 & -0.2 & 53.0 & 119 & 38 & 000 & 0.0 & 180 \\
\hline $00: 22: 10$ & -11 & -117 & 45 & 0 & 0.21 & -0.10 & -0.16 & 360 & 0.391 & -0.2 & 77.0 & 139 & 45 & 000 & 0.0 & 180 \\
\hline $00: 22: 20$ & -11 & -116 & 45 & 0 & 0.25 & -0.10 & -0.15 & 360 & 0.310 & -0.8 & 62.0 & 119 & 31 & 000 & 0.0 & 180 \\
\hline $00: 22: 30$ & -12 & -114 & 45 & 0 & 0.28 & -0.09 & -0.12 & 000 & 0.235 & -1.5 & 62.0 & 087 & 31 & 000 & 0.0 & 180 \\
\hline $00: 22: 40$ & -12 & -113 & 45 & 0 & 0.23 & -0.06 & -0.10 & 000 & 0.248 & -2.3 & 100.0 & 087 & 44 & 000 & 0.0 & 180 \\
\hline $00: 22: 50$ & -13 & -112 & 44 & 0 & 0.03 & -0.02 & -0.05 & 000 & 0.202 & -6.1 & 100.0 & 087 & 35 & 000 & 0.0 & 180 \\
\hline $00: 23: 00$ & -13 & -112 & 44 & 0 & -0.10 & 0.00 & -0.01 & 000 & 0.083 & -3.3 & 97.0 & 061 & 34 & 000 & 0.0 & 180 \\
\hline $00: 23: 10$ & -13 & -113 & 44 & 0 & -0.09 & 0.03 & 0.02 & 000 & 0.078 & -3.2 & 97.0 & 061 & 41 & 000 & 0.0 & 180 \\
\hline $00: 23: 20$ & -13 & -113 & 45 & 0 & -0.11 & 0.06 & 0.05 & 000 & 0.059 & -3.4 & 97.0 & 061 & 39 & 000 & 0.0 & 180 \\
\hline $00: 23: 30$ & -12 & -114 & 45 & 0 & -0.06 & 0.08 & 0.08 & 000 & -0.024 & -2.6 & 97.0 & 031 & 46 & 000 & 0.0 & 180 \\
\hline $00: 23: 40$ & -12 & -114 & 45 & 0 & -0.04 & 0.09 & 0.11 & 000 & -0.090 & -1.3 & 97.0 & 031 & 41 & 000 & 0.0 & 180 \\
\hline $00: 23: 50$ & -11 & -114 & 45 & 0 & -0.04 & 0.11 & 0.13 & 000 & -0.127 & -1.0 & 97.0 & 031 & 43 & 000 & 0.0 & 180 \\
\hline $00: 24: 00$ & -11 & -114 & 46 & 0 & 0.04 & 0.13 & 0.14 & 000 & -0.077 & -1.0 & 97.0 & 031 & 43 & 000 & 0.0 & 180 \\
\hline $00: 24: 10$ & -10 & -114 & 46 & 0 & 0.06 & 0.16 & 0.15 & 000 & 0.040 & -1.9 & 97.0 & 088 & 35 & 000 & 0.0 & 180 \\
\hline $00: 24: 20$ & -9 & -114 & 45 & 0 & 0.12 & 0.17 & 0.16 & 000 & 0.041 & -1.9 & 49.0 & 088 & 35 & 000 & 0.0 & 180 \\
\hline $00: 24: 30$ & -8 & -113 & 45 & 0 & 0.19 & 0.17 & 0.17 & 000 & 0.036 & -2.4 & 49.0 & 088 & 44 & 000 & 0.0 & 180 \\
\hline $00: 24: 40$ & -7 & -112 & 46 & 0 & 0.18 & 0.18 & 0.18 & 000 & 0.022 & -2.4 & 49.0 & 088 & 33 & 000 & 0.0 & 180 \\
\hline $00: 24: 50$ & -6 & -111 & 46 & 0 & 0.13 & 0.18 & 0.18 & 000 & -0.014 & -2.2 & 49.0 & 345 & 45 & 000 & 0.0 & 180 \\
\hline $00: 25: 00$ & -5 & $-i 11$ & 46 & 0 & 0.07 & 0.17 & 0.18 & 000 & -0.089 & -1.1 & 49.0 & 332 & 34 & 000 & 0.0 & 180 \\
\hline $00: 25: 10$ & -4 & -110 & 45 & 0 & 0.21 & 0.16 & 0.18 & 000 & -0.127 & -1.1 & 99.0 & 332 & 30 & 000 & 0.0 & 180 \\
\hline $00: 25: 20$ & -4 & -108 & 45 & 0 & 0.34 & 0.14 & 0.17 & 000 & -0.215 & -0.4 & 51.0 & 269 & 41 & 000 & 0.0 & 180 \\
\hline $00: 25: 30$ & -3 & -107 & 46 & 0 & 0.31 & 0.12 & 0.16 & 000 & -0.246 & 0.3 & 99.0 & 215 & 40 & 000 & 0.0 & 180 \\
\hline $00: 25: 40$ & -2 & -105 & 46 & 0 & 0.32 & 0.10 & 0.14 & 360 & -0.257 & 1.4 & 77.0 & 215 & 34 & 000 & 0.0 & 180 \\
\hline $00: 25: 50$ & -2 & -103 & 46 & 0 & 0.44 & 0.09 & 0.12 & 360 & -0.219 & 1.7 & 77.0 & 215 & 28 & 000 & 0.0 & 180 \\
\hline $00: 26: 00$ & -1 & -101 & 46 & 0 & 0.40 & 0.09 & 0.11 & 360 & -0.127 & 2.1 & 99.0 & 187 & 38 & 000 & 0.0 & 180 \\
\hline $00: 26: 10$ & -1 & -99 & 45 & 0 & 0.25 & 0.09 & 0.09 & 360 & -0.035 & 1.8 & 99.0 & 207 & 36 & 000 & 0.0 & 180 \\
\hline $00: 26: 20$ & 0 & -98 & 46 & 0 & 0.16 & 0.05 & 0.06 & 360 & -0.115 & 3.0 & 99.0 & 260 & 44 & 000 & 0.0 & 180 \\
\hline $00: 26: 30$ & 0 & -98 & 46 & 0 & 0.05 & 0.01 & 0.03 & 360 & -0.121 & 4.8 & 99.0 & 260 & 35 & 000 & 0.0 & 180 \\
\hline $00: 26: 40$ & 0 & -98 & 46 & 0 & 0.00 & -0.02 & -0.02 & 360 & -0.010 & 3.4 & 99.0 & 260 & 41 & 000 & 0.0 & 180 \\
\hline $00: 26: 50$ & 0 & -98 & 46 & 0 & 0.02 & -0.06 & -0.05 & 360 & -0.054 & 3.9 & 99.0 & 260 & 30 & 000 & 0.0 & 180 \\
\hline $00: 27: 00$ & -1 & -98 & 45 & 0 & -0.07 & -0.10 & -0.08 & 360 & -0.075 & 3.6 & 99.0 & 260 & 39 & 000 & 0.0 & 180 \\
\hline $00: 27: 10$ & -1 & -98 & 45 & 0 & -0.23 & -0.13 & -0.12 & 360 & -0.076 & 3.2 & 99.0 & 260 & 30 & 000 & 0.0 & 180 \\
\hline $00: 27: 20$ & -2 & -100 & 46 & 0 & -0.25 & -0.16 & -0.15 & 360 & -0.048 & 3.6 & 99.0 & 260 & 31 & 000 & 0.0 & 180 \\
\hline $00: 27: 30$ & -3 & -101 & 46 & 0 & -0.21 & -0.14 & -0.17 & 360 & 0.199 & 2.5 & 99.0 & 099 & 31 & 000 & 0.0 & 180 \\
\hline $00: 27: 40$ & -3 & -102 & 46 & 0 & -0.10 & -0.10 & -0.16 & 360 & 0.431 & -0.8 & 99.0 & 052 & 33 & 000 & 0.0 & 180 \\
\hline$: 27: 50$ & -4 & -102 & 46 & 0 & -0.01 & -0.07 & -0.14 & 360 & 0.450 & -4.4 & 99.0 & 052 & 35 & 000 & 0.0 & 180 \\
\hline $\int: 28: 00$ & -5 & -102 & 45 & 0 & -0.12 & -0.04 & -0.10 & 000 & 0.385 & -3.9 & 99.0 & 125 & 30 & 000 & 0.0 & 180 \\
\hline $00: 28: 10$ & -5 & -103 & 45 & 0 & -0.18 & -0.01 & -0.07 & 000 & 0.357 & -3.9 & 99.0 & 125 & 50 & 000 & 0.0 & 180 \\
\hline $00: 28: 20$ & -5 & -104 & 46 & 0 & -0.21 & 0.02 & -0.03 & 000 & 0.324 & -4.6 & 99.0 & 086 & 40 & 000 & 0.0 & 180 \\
\hline & -5 & -105 & 46 & 0 & -0.23 & 0.04 & 0.00 & 000 & 0.221 & -4.5 & 99.0 & 036 & 37 & 000 & 0.0 & 180 \\
\hline & & -106 & & 0 & -0.11 & 0.05 & & 000 & 0.063 & & 990 & 036 & & 000 & & \\
\hline
\end{tabular}




\begin{tabular}{|c|c|c|c|c|c|c|c|c|c|c|c|c|c|c|c|c|}
\hline \multirow{2}{*}{$\begin{array}{c}\text { Time } \\
\mathrm{HH} ; M M ; S S\end{array}$} & \multicolumn{2}{|c|}{ Position (m) } & \multicolumn{2}{|c|}{ RPM } & \multicolumn{3}{|c|}{ Speed (Knots) } & \multirow[b]{2}{*}{ Heading } & \multirow{2}{*}{$\begin{array}{c}\text { Turn } \\
\text { Deg/Min }\end{array}$} & \multirow{2}{*}{$\begin{array}{l}\text { Rudder } \\
\text { Deg }\end{array}$} & \multicolumn{2}{|c|}{ Gill } & \multicolumn{2}{|c|}{ Wind } & \multicolumn{2}{|c|}{ Current } \\
\hline & East & North & Port & stbd & Iong & Bow & stern & & & & 8 & Deg & Knots & Deg & Knots & Deg \\
\hline & & & & & & & & & & -3.3 & 99.0 & 036 & 40 & 000 & 0.0 & 180 \\
\hline $\begin{array}{r}n: 28: 50 \\
: 29: 00\end{array}$ & -4 & -106. & & $\begin{array}{l}0 \\
0\end{array}$ & 0.03 & 0.07 & 0.08 & 000 & $\begin{array}{l}-0.041 \\
-0.089\end{array}$ & $\begin{array}{l}-3.3 \\
-3.4\end{array}$ & 99.0 & 036 & 33 & 000 & 0.0 & $\begin{array}{l}180 \\
180\end{array}$ \\
\hline $\begin{array}{r}; 29: 00 \\
\text { vo:29:10 }\end{array}$ & $\begin{array}{l}-4 \\
-3\end{array}$ & $\begin{array}{l}-106 \\
-105\end{array}$ & $\begin{array}{l}45 \\
46\end{array}$ & 0 & 0.20 & 0.11 & 0.12 & 000 & -0.069 & -3.0 & 99.0 & 089 & 44 & 000 & 0.0 & $\begin{array}{l}180 \\
180\end{array}$ \\
\hline $00: 29: 20$ & -3 & -104 & 46 & 0 & 0.23 & 0.15 & 0.15 & 000 & 0.018 & -3.4 & 99.0 & 089 & 28 & 000 & 0.0 & 180 \\
\hline $00: 29 ; 30$ & -2 & -102 & 46 & 0 & 0.36 & 0.19 & 0.18 & 000 & 0.082 & -3.6 & 99.0 & 089 & 34 & 000 & 0.0 & 180 \\
\hline $00: 29: 40$ & -1 & -100 & 46 & 0 & 0.39 & 0.23 & 0.21 & 000 & 0.133 & -4.3 & 99.0 & 089 & 42 & 000 & 0.0 & 180 \\
\hline $00: 29: 50$ & 0 & -98 & 45 & 0 & 0.33 & 0.27 & 0.24 & 000 & 0.178 & -5.0 & 99.0 & 089 & 40 & 000 & 0.0 & 180 \\
\hline $00: 30: 00$ & 2 & -97 & 46 & 0 & 0.32 & 0.28 & 0.26 & 000 & 0.101 & -5.4 & 99.0 & 272 & 44 & 000 & 0.0 & 180 \\
\hline $00: 30: 10$ & 3 & -95 & 46 & 0 & 0.23 & 0.24 & 0.27 & 000 & -0.189 & -3.9 & 99.0 & 216 & 33 & 000 & 0.0 & 180 \\
\hline $00: 30: 20$ & 4 & -95 & 46 & 0 & 0.08 & 0.20 & 0.26 & 000 & -0.364 & -2.2 & 99.0 & 216 & 41 & 000 & 0.0 & 180 \\
\hline $00: 30: 30$ & 6 & -94 & 46 & 0 & -0.01 & 0.18 & 0.25 & 000 & -0.448 & -0.4 & 99.0 & 216 & 43 & 000 & 0.0 & 180 \\
\hline $00: 30: 40$ & 7 & -95 & 45 & 0 & -0.12 & 0.15 & 0.24 & 000 & -0.611 & 2.0 & 99.0 & 216 & 35 & 000 & 0.0 & 180 \\
\hline $00: 30: 50$ & 8 & -96 & 45 & 0 & -0.22 & 0.12 & 0.22 & 360 & -0.659 & 3.9 & 99.0 & 216 & 42 & 000 & 0.0 & 180 \\
\hline $00: 31: 00$ & 8 & -97 & 46 & 0 & -0.21 & 0.10 & 0.20 & 360 & -0.595 & 5.6 & 99.0 & 176 & 47 & 000 & 0.0 & 180 \\
\hline $00: 31: 10$ & 9 & -98 & 46 & 0 & -0.12 & 0.16 & 0.19 & 360 & -0.192 & 5.1 & 99.0 & 065 & 35 & 000 & 0.0 & 180 \\
\hline $00: 31: 20$ & 10 & -98 & 46 & 0 & -0.13 & 0.20 & 0.18 & 360 & 0.136 & 3.3 & 99.0 & 065 & 44 & 000 & 0.0 & 180 \\
\hline $00: 31: 30$ & 11 & -99 & 45 & 0 & -0.24 & 0.18 & 0.16 & 360 & 0.133 & 3.4 & 99.0 & 270 & 39 & 000 & 0.0 & 180 \\
\hline $00: 31: 40$ & 12 & -101 & 45 & 0 & -0.33 & 0.14 & 0.14 & 360 & 0.034 & 3.3 & 99.0 & 270 & 37 & 000 & 0.0 & 180 \\
\hline $00: 31: 50$ & 13 & -102 & 46 & 0 & -0.34 & 0.10 & 0.11 & 360 & -0.046 & 3.5 & 99.0 & 270 & 43 & 000 & 0.0 & 180 \\
\hline $00: 32: 00$ & 13 & -104 & 46 & 0 & -0.21 & 0.06 & 0.08 & 360 & -0.120 & 4.6 & 99.0 & 270 & 34 & 000 & 0.0 & 180 \\
\hline $00: 32: 10$ & 13 & -105 & 46 & 0 & -0.20 & 0.02 & 0.04 & 360 & -0.114 & 4.6 & 99.0 & 300 & 28 & 000 & 0.0 & 180 \\
\hline $00: 32: 20$ & 13 & -106 & 46 & 0 & -0.25 & 0.00 & 0.00 & 360 & -0.055 & 4.8 & 99.0 & 300 & 34 & 000 & 0.0 & 180 \\
\hline $00: 32: 30$ & 13 & -107 & 45 & 0 & -0.14 & -0.03 & -0.03 & 360 & 0.012 & 4.4 & 99.0 & 300 & 39 & 000 & 0.0 & 180 \\
\hline $00: 32: 40$ & 13 & -107 & 45 & 0 & -0.06 & -0.06 & -0.07 & 360 & 0.056 & 3.7 & 99.0 & 300 & 45 & 000 & 0.0 & 180 \\
\hline $00: 32: 50$ & 13 & -107 & 46 & 0 & 0.07 & -0.09 & -0.09 & 360 & 0.016 & 3.8 & 99.0 & 300 & 34 & 000 & 0.0 & 180 \\
\hline $00: 33: 00$ & 12 & -107 & 46 & 0 & 0.21 & -0.13 & -0.12 & 360 & -0.051 & 4.2 & 99.0 & 300 & 40 & 000 & 0.0 & 180 \\
\hline $00: 33: 10$ & 11 & -105 & 46 & 0 & 0.31 & -0.16 & -0.15 & 360 & -0.090 & 4.8 & 99.0 & 240 & 41 & 000 & 0.0 & 180 \\
\hline $00: 33: 20$ & 10 & -104 & 46 & 0 & 0.28 & -0.19 & -0.18 & 360 & -0.091 & 4.8 & 99.0 & 240 & 41 & 000 & 0.0 & 180 \\
\hline $00: 33: 30$ & 9 & -103 & 45 & 0 & 0.17 & -0.23 & -0.21 & 360 & -0.125 & 5.6 & 99.0 & 240 & 36 & 000 & 0.0 & 180 \\
\hline $00: 33: 40$ & 8 & -102 & 46 & 0 & 0.09 & -0.26 & -0.24 & 360 & -0.167 & 6.4 & 99.0 & 240 & 35 & 000 & 0.0 & 180 \\
\hline $00: 33: 50$ & 7 & -102 & 46 & 0 & 0.04 & -0.28 & -0.26 & 360 & -0.112 & 6.1 & 50.0 & 240 & 28 & 000 & 0.0 & 180 \\
\hline $00: 34: 00$ & 5 & -102 & 46 & 0 & -0.02 & -0.28 & -0.29 & 360 & 0.095 & 5.8 & 50.0 & 240 & 30 & 000 & 0.0 & 180 \\
\hline $00: 34: 10$ & 4 & -102 & 46 & 0 & -0.02 & -0.27 & -0.33 & 360 & 0.379 & 3.6 & 50.0 & 240 & 35 & 000 & 0.0 & 180 \\
\hline $00: 34: 20$ & 2 & -102 & 45 & 0 & -0.01 & -0.26 & -0.35 & 360 & 0.551 & 1.8 & 50.0 & 240 & 31 & 000 & 0.0 & 180 \\
\hline $00: 34: 30$ & 1 & -102 & 45 & 0 & -0.09 & -0.26 & -0.36 & 360 & 0.636 & -0.1 & 50.0 & 240 & 36 & 000 & 0.0 & 180 \\
\hline $00: 34: 40$ & -1 & -103 & 46 & 0 & -0.11 & -0.27 & -0.36 & 360 & 0.616 & -2.2 & 50.0 & 240 & 38 & 000 & 0.0 & 180 \\
\hline $00 ; 34 ; 50$ & -3 & -103 & 46 & 0 & -0.09 & -0.22 & -0.37 & 000 & 0.941 & -5.6 & 50.0 & 240 & 40 & 000 & 0.0 & 180 \\
\hline $00: 35: 00$ & -4 & -104 & 46 & 0 & -0.13 & -0.09 & -0.36 & 000 & 1.698 & $-13 \cdot 2$ & 50.0 & 240 & 26 & 000 & 0.0 & 180 \\
\hline $77: 35: 10$ & -5 & -104 & 45 & 0 & -0.14 & 0.07 & -0.31 & 001 & 2.404 & -27.2 & 50.0 & 240 & 46 & 000 & 0.0 & 180 \\
\hline$: 35: 20$ & -5 & -105 & 45 & 0 & -0.10 & 0.22 & -0.19 & 001 & 2.608 & -35.0 & 50.0 & 240 & 35 & 000 & 0.0 & 180 \\
\hline$v 0: 35 ; 30$ & -5 & -105 & 46 & 0 & 0.01 & 0.37 & -0.05 & 002 & 2.667 & -35.0 & 0.0 & 240 & 38 & 000 & 0.0 & 180 \\
\hline $00: 35: 40$ & -3 & -105 & 46 & 0 & 0.08 & 0.44 & 0.10 & 002 & 2.179 & -35.0 & 0.0 & 240 & 32 & 000 & 0.0 & 180 \\
\hline
\end{tabular}




\section{HANDS-ON SIMULATION}

\section{SUCTION MOORNG TECHNOLOGY}

Simulation Number: 29

Date: 5 February 1999

Start Time: 10:41

Length of run [secs]: 2595

Ship Condition: draft $=13.72 \mathrm{~m}$

Environment

Wind Speed [knots]:

40 Direction: 0

Sea State, Significant wave height $[\mathrm{m}]: .$.

8 Direction: 180

Current Speed [knots]:

1 Direction: 135

Ship Operator: J.A.

Observer: B.K.J

Final Mating, distance between center of buoy and center of moon pool [m]: 1.8

Evaluation:

The mating was successful on the third try.

The approach was good up to the buoy, 30 to $40 \mathrm{~m}$ from the buoy.

When the ship was brought to approximately a stop short of the buoy on approximately equilibrium heading, the bow fell off to the port having low RPM, and the rudder power was not sufficient to stop the yaw. After about 25 degrees of yaw the captain put on full power briefly and thruster to 090 degrees at full power. This stopped the yaw and started recovering. From that point there was no particular problem as the bow was brought to starboard to close with the buoy. During the final part of this process the autopilot was kept on about 356 degrees, and later adjusted to 357 degrees, and the propeller RPM was kept at 47 throughout. All adjustments were made with the thruster vector. 
Ship:

591 150,000 ton, Tanker, partial load

Exercise number:

754 Suction Mooring System

112 Open Ocean

999 Dumny tug file based on containership

ig file:

590 Suction Mooring Buoy

rafic ships:

$594150 \mathrm{k}$ tanker in partial load - Suction Mooring

Exercise identification: RUN \#29 HH:MM:SS East North Port Stbd Long Bow Stern Heading Deg/Min Deg \& Deg knots Deg Knots Deg

\begin{tabular}{|c|c|c|c|c|c|c|c|c|c|c|c|c|c|c|c|c|}
\hline $\begin{array}{l}00: 20: 00 \\
00: 20: 10\end{array}$ & $\begin{array}{l}-84 \\
-82\end{array}$ & $\begin{array}{l}-89 \\
-89\end{array}$ & $\begin{array}{l}48 \\
48\end{array}$ & $\begin{array}{l}0 \\
0\end{array}$ & $\begin{array}{r}-0.06 \\
0.03\end{array}$ & $\begin{array}{l}0.27 \\
0.30\end{array}$ & $\begin{array}{l}0.21 \\
0.26\end{array}$ & $\begin{array}{l}358 \\
358\end{array}$ & $\begin{array}{l}0.373 \\
0.256\end{array}$ & -2.4 & $\begin{array}{l}100.0 \\
100.0\end{array}$ & 118 & 44 & 000 & 1.0 & $\begin{array}{l}135 \\
135\end{array}$ \\
\hline $\begin{array}{l}00 ; 20: 10 \\
00 ; 20: 20\end{array}$ & -81 & -89 & 48 & 0 & 0.02 & 0.31 & 0.31 & 358 & 0.010 & -1.7 & 100.0 & $\begin{array}{l}118 \\
118\end{array}$ & 38 & 000 & $\begin{array}{l}1.0 \\
1.0\end{array}$ & $\begin{array}{l}135 \\
135\end{array}$ \\
\hline $00: 20: 30$ & -79 & -89 & 47 & 0 & 0.02 & 0.32 & 0.34 & 358 & -0.123 & -1.3 & 76.0 & 118 & 34 & 000 & 1.0 & 135 \\
\hline $00: 20 ; 40$ & -78 & -89 & 47 & 0 & 0.03 & 0.34 & 0.37 & 358 & -0.174 & -0.6 & 76.0 & 118 & 33 & 000 & 1.0 & 135 \\
\hline $00: 20: 50$ & -76 & -89 & 48 & 0 & 0.06 & 0.34 & 0.40 & 358 & -0.331 & 0.5 & 76.0 & 118 & 29 & 000 & 1.0 & 135 \\
\hline $00 ; 21: 00$ & -74 & -88 & 48 & 0 & 0.11 & 0.34 & 0.41 & 358 & -0.454 & 1.9 & 76.0 & 118 & 35 & 000 & 1.0 & 135 \\
\hline $00: 21: 10$ & -72 & -88 & 47 & 0 & 0.03 & 0.34 & 0.41 & 358 & -0.425 & 3.6 & 76.0 & 118 & 40 & 000 & 1.0 & 135 \\
\hline $00 ; 21 ; 20$ & -70 & -88 & 48 & 0 & 0.00 & 0.35 & 0.41 & 358 & -0.400 & 4.6 & 76.0 & 118 & 38 & 000 & 1.0 & 235 \\
\hline $00: 21: 30$ & -68 & -87 & 47 & 0 & 0.11 & 0.34 & 0.40 & 358 & -0.373 & 5.7 & 76.0 & 118 & 34 & 000 & 1.0 & 135 \\
\hline $00 ; 21: 40$ & -66 & -86 & 48 & 0 & 0.18 & 0.35 & 0.38 & 358 & -0.206 & 6.2 & 76.0 & 118 & 42 & 000 & 1.0 & 135 \\
\hline $00 ; 21 ; 50$ & -64 & -86 & 47 & 0 & 0.09 & 0.38 & 0.37 & 358 & 0.097 & 5.3 & 76.0 & 118 & 49 & 000 & 1.0 & 135 \\
\hline $00: 22: 00$ & -62 & -85 & 48 & 0 & 0.06 & 0.40 & 0.36 & 358 & 0.238 & 4.3 & 76.0 & 118 & 38 & 000 & 1.0 & 135 \\
\hline $00: 22: 10$ & -60 & -85 & 48 & 0 & 0.16 & 0.41 & 0.36 & 358 & 0.319 & 3.1 & 76.0 & 118 & 45 & 000 & 1.0 & 135 \\
\hline $00: 22: 20$ & -58 & -84 & 48 & 0 & 0.19 & 0.41 & 0.37 & 358 & 0.246 & 2.7 & 76.0 & 118 & 31 & 000 & 1.0 & 135 \\
\hline $00: 22: 30$ & -56 & -82 & 47 & 0 & 0.22 & 0.37 & 0.38 & 358 & -0.069 & 3.0 & 76.0 & 158 & 31 & 000 & 1.0 & 135 \\
\hline $00 ; 22: 40$ & -55 & -81 & 48 & 0 & 0.13 & 0.32 & 0.38 & 358 & -0.327 & 4.4 & 99.0 & 161 & 44 & 000 & 1.0 & 135 \\
\hline $00: 22: 50$ & -53 & -81 & 48 & 0 & -0.11 & 0.33 & 0.36 & 358 & -0.222 & 5.2 & 99.0 & 161 & 35 & 000 & 1.0 & 135 \\
\hline $00: 23: 00$ & -51 & -82 & 47 & 0 & -0.29 & 0.37 & 0.36 & 358 & 0.085 & 4.3 & 99.0 & 091 & 34 & 000 & 1.0 & 135 \\
\hline $00: 23: 10$ & -49 & -84 & 47 & 0 & -0.29 & 0.38 & 0.36 & 358 & 0.075 & 4.3 & 99.0 & 060 & 41 & 000 & 1.0 & 135 \\
\hline $00: 23: 20$ & -47 & -85 & 48 & 0 & -0.29 & 0.37 & 0.37 & 358 & 0.018 & 4.5 & 48.0 & 060 & 39 & 000 & 1.0 & 135 \\
\hline $00: 23: 30$ & -45 & -86 & 48 & 0 & -0.27 & 0.37 & 0.36 & 358 & 0.059 & 3.8 & 48.0 & 060 & 46 & 000 & 1.0 & 135 \\
\hline $00: 23: 40$ & -43 & -88 & 47 & 0 & -0.27 & 0.39 & 0.36 & 358 & 0.175 & 3.5 & 100.0 & 060 & 41 & 000 & 1.0 & 135 \\
\hline $00 ; 23: 50$ & -41 & -89 & 48 & 0 & -0.28 & 0.46 & 0.37 & 358 & 0.557 & 1.5 & 100.0 & 034 & 43 & 000 & 1.0 & 135 \\
\hline $00: 24: 00$ & -39 & -90 & 47 & 0 & -0.18 & 0.47 & 0.39 & 358 & 0.484 & -0.1 & 100.0 & 034 & 43 & 000 & 1.0 & 135 \\
\hline $00: 24: 10$ & -37 & -91 & 48 & 0 & -0.19 & 0.46 & 0.42 & 358 & 0.267 & -0.7 & 37.0 & 094 & 35 & 000 & 1.0 & 135 \\
\hline $00: 24: 20$ & -34 & -92 & 47 & 0 & -0.12 & 0.45 & 0.44 & 358 & 0.079 & -0.8 & 27.0 & 058 & 35 & 000 & 1.0 & 135 \\
\hline $00: 24: 30$ & -32 & -92 & 48 & 0 & -0.04 & 0.42 & 0.46 & 358 & -0.255 & 0.1 & 27.0 & 058 & 44 & 000 & 1.0 & 135 \\
\hline $0 ; 24: 40$ & -30 & -92 & 48 & 0 & -0.04 & 0.36 & 0.49 & 358 & -0.830 & -11.8 & 27.0 & 058 & 33 & 000 & 1.0 & 135 \\
\hline$: 24: 50$ & -27 & -92 & 48 & 0 & -0.10 & 0.27 & 0.63 & 358 & -2.269 & -24.8 & 27.0 & 058 & 45 & 000 & 1.0 & 135 \\
\hline $00: 25 ; 00$ & -25 & -93 & 47 & 0 & -0.14 & 0.20 & 0.74 & 357 & -3.465 & -13.7 & 99.0 & 058 & 34 & 000 & 1.0 & 135 \\
\hline $00: 25: 10$ & -23 & -93 & 48 & 0 & 0.00 & 0.19 & 0.79 & 357 & -3.821 & -1.5 & 99.0 & 058 & 30 & 000 & 1.0 & 135 \\
\hline $00: 25: 20$ & -20 & -93 & 48 & 0 & 0.13 & 0.24 & 0.76 & 356 & -3.288 & 7.6 & 99.0 & 058 & 41 & 000 & 1.0 & 135 \\
\hline $00: 25: 30$ & -18 & -92 & 47 & 0 & 0.12 & 0.29 & 0.69 & 355 & -2.501 & 14.8 & 99.0 & 117 & 40 & 000 & 1.0 & 135 \\
\hline $00: 25: 40$ & -15 & -91 & 48 & 0 & 0.16 & 0.30 & 0.58 & 355 & -1.807 & 19.8 & 49.0 & 117 & 34 & 000 & 1.0 & 135 \\
\hline $00: 25: 50$ & -13 & -90 & 48 & 0 & 0.29 & 0.29 & 0.46 & 355 & -1.052 & 22.4 & 49.0 & 117 & 28 & 000 & 1.0 & 135 \\
\hline $00: 26: 00$ & -12 & -88 & 48 & 0 & 0.30 & 0.32 & 0.33 & 355 & -0.061 & 21.6 & 49.0 & 117 & 38 & 000 & 1.0 & 135 \\
\hline $00: 26: 10$ & -10 & -87 & 47 & 0 & 0.20 & 0.30 & 0.22 & 355 & 0.459 & 19.2 & 0.0 & 183 & 36 & 000 & 1.0 & 135 \\
\hline $00: 26: 20$ & -9 & -86 & 48 & 0 & 0.09 & 0.22 & 0.14 & 355 & 0.494 & 16.3 & 100.0 & 183 & 44 & 000 & 1.0 & 135 \\
\hline $00: 26: 30$ & -8 & -85 & 48 & 0 & -0.03 & 0.19 & 0.08 & 355 & 0.666 & 11.8 & 52.0 & 183 & 35 & 000 & 1.0 & 135 \\
\hline $00: 26: 40$ & -8 & -86 & 48 & 0 & -0.10 & 0.12 & 0.05 & 355 & 0.481 & 11.4 & 75.0 & 183 & 41 & 000 & 1.0 & 135 \\
\hline $00: 26: 50$ & -7 & -86 & 47 & 0 & -0.09 & 0.04 & 0.01 & 355 & 0.187 & 12.3 & 52.0 & 183 & 30 & 000 & 1.0 & 135 \\
\hline $00: 27: 00$ & -7 & -87 & 48 & 0 & -0.18 & 0.00 & -0.03 & 355 & 0.180 & 12.9 & 52.0 & 183 & 39 & 000 & 1.0 & 135 \\
\hline $00: 27: 10$ & -7 & -88 & 47 & 0 & -0.34 & 0.08 & -0.06 & 355 & 0.879 & 10.0 & 53.0 & 091 & 30 & 000 & 1.0 & 135 \\
\hline $00: 27: 20$ & -7 & -90 & 48 & 0 & -0.34 & 0.19 & -0.05 & 355 & 1.504 & 4.0 & 100.0 & 062 & 31 & 000 & 1.0 & 135 \\
\hline $00: 27: 30$ & -6 & -92 & 47 & 0 & -0.24 & 0.20 & 0.00 & 356 & 1.274 & -0.6 & 100.0 & 062 & 31 & 000 & 1.0 & 135 \\
\hline $00: 27: 40$ & -6 & -93 & 48 & 0 & -0.13 & 0.18 & 0.07 & 356 & 0.681 & -2.9 & 100.0 & 091 & 33 & 000 & 1.0 & 135 \\
\hline $00: 27: 50$ & -5 & -93 & 48 & 0 & -0.07 & 0.10 & 0.12 & 356 & -0.067 & 1.6 & 52.0 & 091 & 35 & 000 & 1.0 & 135 \\
\hline $00: 28: 00$ & -4 & -94 & 48 & 0 & -0.18 & 0.12 & 0.13 & 356 & -0.097 & 1.7 & 52.0 & 090 & 30 & 000 & 1.0 & 135 \\
\hline $00: 28: 10$ & -4 & -95 & 47 & 0 & -0.21 & 0.14 & 0.15 & 356 & -0.053 & 1.9 & 52.0 & 062 & 50 & 000 & 1.0 & 135 \\
\hline $00: 28: 20$ & -3 & -96 & 48 & 0 & -0.21 & 0.09 & 0.17 & 356 & -0.450 & 4.4 & 52.0 & 062 & 40 & 000 & 1.0 & 135 \\
\hline $00: 28: 30$ & -2 & -97 & 48 & 0 & -0.24 & 0.08 & 0.16 & 356 & -0.525 & 6.2 & 52.0 & 062 & 37 & 000 & 1.0 & 135 \\
\hline $00: 28: 40$ & -1 & -98 & 47 & 0 & -0.15 & 0.09 & 0.16 & 356 & -0.431 & 7.6 & 98.0 & 047 & 35 & 000 & 1.0 & 135 \\
\hline $00: 28: 50$ & -1 & -98 & 48 & 0 & -0.03 & 0.08 & 0.13 & 356 & -0.358 & 10.1 & 51.0 & 090 & 40 & 000 & 1.0 & 135 \\
\hline $00: 29: 00$ & 0 & -98 & 48 & 0 & 0.06 & 0.14 & 0.11 & 356 & 0.155 & 5.5 & 100.0 & 090 & 33 & 000 & 1.0 & 135 \\
\hline $00: 29: 10$ & 1 & -98 & 48 & 0 & 0.06 & 0.19 & 0.12 & 356 & 0.421 & 2.7 & 100.0 & 119 & 44 & 000 & 1.0 & 135 \\
\hline $00: 29: 20$ & 1 & -97 & 47 & 0 & 0.07 & 0.16 & 0.14 & 356 & 0.090 & 4.4 & 100.0 & 119 & 28 & 000 & 1.0 & 135 \\
\hline $00: 29: 30$ & 2 & -97 & 48 & 0 & 0.17 & 0.17 & 0.15 & 356 & 0.097 & 4.0 & 100.0 & 119 & 34 & 000 & 1.0 & 135 \\
\hline $00: 29: 40$ & 3 & -95 & 48 & 0 & 0.17 & 0.17 & 0.17 & 356 & 0.026 & 4.3 & 100.0 & 147 & 42 & 000 & 1.0 & 135 \\
\hline $00: 29: 50$ & 4 & -95 & 48 & 0 & 0.07 & 0.11 & 0.17 & 356 & -0.378 & 7.9 & 100.0 & 147 & 40 & 000 & 1.0 & 135 \\
\hline $00: 30 ; 00$ & 4 & -95 & 47 & 0 & 0.00 & 0.03 & 0.13 & 356 & -0.642 & 16.1 & 100.0 & 147 & 44 & 000 & 1.0 & 135 \\
\hline $00: 30: 10$ & 4 & -95 & 48 & 0 & -0.11 & -0.01 & 0.06 & 356 & -0.446 & 13.3 & 100.0 & 179 & 33 & 000 & 1.0 & 135 \\
\hline $00 ; 30 ; 20$ & 5 & -96 & 47 & 0 & -0.24 & 0.01 & 0.04 & 355 & -0.143 & 9.7 & 100.0 & 063 & 41 & 000 & 1.0 & 135 \\
\hline $00: 30: 30$ & 5 & -97 & 48 & 0 & -0.25 & 0.03 & 0.03 & 355 & 0.002 & 9.6 & 100.0 & 063 & 43 & 000 & 1.0 & 135 \\
\hline $00: 30: 40$ & 5 & -98 & 47 & 0 & -0.29 & 0.07 & 0.03 & 356 & 0.264 & 8.0 & 100.0 & 043 & 35 & 000 & 1.0 & 135 \\
\hline $00: 30: 50$ & 6 & -100 & 48 & 0 & -0.30 & 0.10 & 0.03 & 356 & 0.442 & 6.2 & 100.0 & 035 & 42 & 000 & 1.0 & 135 \\
\hline $00: 31: 00$ & 6 & -101 & 48 & 0 & -0.19 & 0.11 & 0.05 & 356 & 0.439 & 4.2 & 100.0 & 035 & 47 & 000 & 1.0 & 135 \\
\hline $9: 31: 10$ & 6 & -102 & 48 & 0 & -0.06 & 0.10 & 0.07 & 356 & 0.210 & 3.1 & 100.0 & 105 & 35 & 000 & 1.0 & 135 \\
\hline$: 31: 20$ & 7 & -102 & 47 & 0 & -0.15 & 0.07 & 0.07 & 356 & -0.046 & 6.0 & 100.0 & 181 & 44 & 000 & 1.0 & 135 \\
\hline $00: 31: 30$ & 7 & -103 & 48 & 0 & -0.24 & 0.08 & 0.06 & 356 & 0.082 & 5.3 & 100.0 & 357 & 39 & 000 & 1.0 & 135 \\
\hline $00: 31: 40$ & 8 & -104 & 48 & 0 & -0.26 & 0.09 & 0.07 & 356 & 0.113 & 4.6 & 100.0 & 357 & 37 & 000 & 1.0 & 135 \\
\hline $00: 31: 50$ & 8 & -106 & 47 & 0 & -0.21 & 0.07 & 0.07 & 356 & -0.044 & 5.3 & 100.0 & 357 & 43 & 000 & 1.0 & 135 \\
\hline $00: 32: 00$ & 9 & -106 & 48 & 0 & -0.02 & 0.00 & 0.06 & 356 & -0.386 & 14.1 & 100.0 & 357 & 34 & 000 & 1.0 & 135 \\
\hline
\end{tabular}


Time Position (m) RPM Speed (Knots) Turn Rudder Gill Wrent

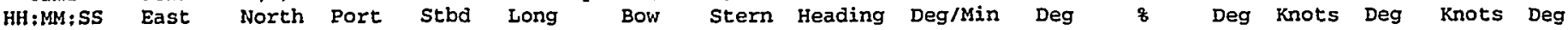

\begin{tabular}{|c|c|c|c|c|c|c|c|c|c|c|c|c|c|c|c|c|}
\hline $90: 32: 10$ & 9 & -106 & 48 & 0 & 0.02 & -0.02 & -0.01 & 356 & -0.052 & 10.3 & 100.0 & 357 & 28 & 000 & 1.0 & 135 \\
\hline$: 32: 20$ & 9 & -106 & 48 & 0 & 0.00 & 0.02 & 0.00 & 356 & 0.070 & -2.6 & 100.0 & 357 & 34 & 000 & 1.0 & 135 \\
\hline 10:32:30 & 9 & -106 & 47 & 0 & 0.14 & 0.05 & 0.01 & 356 & 0.212 & 3.8 & 100.0 & 357 & 39 & 000 & 1.0 & 135 \\
\hline $00: 32: 40$ & 9 & -105 & 48 & 0 & 0.25 & 0.07 & 0.01 & 356 & 0.362 & 3.4 & 100.0 & 027 & 45 & 000 & 1.0 & 135 \\
\hline $00: 32: 50$ & 9 & -103 & 48 & 0 & 0.33 & 0.11 & 0.04 & 356 & 0.466 & 1.7 & 100.0 & 124 & 34 & 000 & 1.0 & 135 \\
\hline $00: 33: 00$ & 9 & -101 & 48 & 0 & 0.43 & -0.01 & 0.05 & 356 & -0.387 & 4.4 & 100.0 & 263 & 40 & 000 & 1.0 & 135 \\
\hline $00: 33: 10$ & 9 & -99 & 47 & 0 & 0.48 & -0.11 & 0.03 & 356 & -0.876 & 7.2 & 100.0 & 214 & 41 & 000 & 1.0 & 135 \\
\hline $00: 33: 20$ & 8 & -97 & 48 & 0 & 0.43 & -0.10 & -0.01 & 356 & -0.559 & 8.9 & 100.0 & 214 & 41 & 000 & 1.0 & 135 \\
\hline $00: 33: 30$ & 8 & -95 & 48 & 0 & 0.29 & -0.18 & -0.06 & 355 & -0.756 & 12.2 & 100.0 & 214 & 36 & 000 & 1.0 & 135 \\
\hline $00: 33: 40$ & 7 & -94 & 48 & 0 & 0.19 & -0.27 & -0.12 & 355 & -0.946 & 15.9 & 100.0 & 214 & 35 & 000 & 1.0 & 135 \\
\hline $00: 33: 50$ & 6 & -93 & 47 & 0 & 0.11 & -0.25 & -0.18 & 355 & -0.409 & 16.1 & 100.0 & 149 & 28 & 000 & 1.0 & 135 \\
\hline $00: 34: 00$ & 5 & -93 & 48 & 0 & 0.01 & -0.21 & -0.22 & 355 & 0.111 & 14.1 & 100.0 & 149 & 30 & 000 & 1.0 & 135 \\
\hline $00: 34: 10$ & 4 & -93 & 48 & 0 & -0.04 & -0.18 & -0.24 & 355 & 0.387 & 11.5 & 100.0 & 149 & 35 & 000 & 1.0 & 135 \\
\hline $00: 34: 20$ & 3 & -93 & 47 & 0 & -0.05 & -0.19 & -0.24 & 355 & 0.345 & 11.1 & 100.0 & 103 & 31 & 000 & 1.0 & 135 \\
\hline $00: 34: 30$ & 2 & -94 & 47 & 0 & -0.15 & -0.14 & -0.23 & 355 & 0.610 & 8.3 & 100.0 & 114 & 36 & 000 & 1.0 & 135 \\
\hline $00: 34: 40$ & 1 & -95 & 48 & 0 & -0.15 & -0.08 & -0.20 & 355 & 0.809 & 4.4 & 51.0 & 083 & 38 & 000 & 1.0 & 135 \\
\hline $00: 34: 50$ & 0 & -96 & 48 & 0 & -0.14 & -0.06 & -0.17 & 356 & 0.668 & 2.3 & 51.0 & 058 & 40 & 000 & 1.0 & 135 \\
\hline $00: 35: 00$ & 0 & -96 & 47 & 0 & -0.19 & -0.07 & -0.13 & 356 & 0.393 & 2.8 & 51.0 & 094 & 26 & 000 & 1.0 & 135 \\
\hline $00: 35 ; 10$ & -1 & -98 & 48 & 0 & -0.25 & -0.10 & -0.10 & 356 & -0.048 & 4.7 & 51.0 & 066 & 46 & 000 & 1.0 & 135 \\
\hline $00: 35: 20$ & -1 & -99 & 47 & 0 & -0.26 & -0.11 & -0.07 & 356 & -0.253 & 6.1 & 99.0 & 066 & 35 & 000 & 1.0 & 135 \\
\hline $00: 35: 30$ & -2 & -100 & 48 & 0 & -0.23 & -0.13 & -0.06 & 356 & -0.430 & 8.2 & 100.0 & 066 & 38 & 000 & 1.0 & 135 \\
\hline $00: 35: 40$ & -2 & -101 & 47 & 0 & -0.24 & -0.08 & -0.05 & 356 & -0.171 & 8.2 & 100.0 & 066 & 32 & 000 & 1.0 & 135 \\
\hline $00: 35: 50$ & -2 & -103 & 48 & 0 & -0.29 & 0.01 & -0.04 & 356 & 0.303 & 6.1 & 100.0 & 066 & 34 & 000 & 1.0 & 135 \\
\hline $00: 36: 00$ & -2 & -104 & 48 & 0 & -0.34 & 0.09 & -0.02 & 356 & 0.658 & 3.3 & 100.0 & 066 & 40 & 000 & 1.0 & 135 \\
\hline $00: 36: 10$ & -1 & -106 & 48 & 0 & -0.16 & 0.13 & 0.03 & 356 & 0.635 & -0.8 & 100.0 & 066 & 42 & 000 & 2.0 & 135 \\
\hline $00: 36: 20$ & -1 & -106 & 47 & 0 & 0.09 & 0.08 & 0.07 & 356 & 0.068 & 1.5 & 52.0 & 066 & 37 & 000 & 1.0 & 135 \\
\hline $00: 36: 30$ & -1 & -105 & 48 & 0 & 0.13 & 0.07 & 0.08 & 356 & -0.091 & 3.5 & 52.0 & 066 & 34 & 000 & 1.0 & 135 \\
\hline $00: 36: 40$ & 0 & -104 & 48 & 0 & 0.17 & 0.06 & 0.09 & 356 & -0.179 & 4.0 & 52.0 & 066 & 34 & 000 & 1.0 & 135 \\
\hline $00: 36: 50$ & 0 & -104 & 47 & 0 & 0.18 & 0.05 & 0.08 & 356 & -0.213 & 5.2 & 52.0 & 066 & 35 & 000 & 1.0 & 135 \\
\hline $00: 37: 00$ & 0 & -103 & 48 & 0 & 0.17 & 0.07 & 0.08 & 356 & -0.054 & 4.3 & 52.0 & 066 & 47 & 000 & 1.0 & 135 \\
\hline $00: 37: 10$ & 1 & -102 & 48 & 0 & 0.22 & 0.01 & 0.08 & 356 & -0.433 & 6.7 & 52.0 & 066 & 25 & 000 & 1.0 & 135 \\
\hline $00: 37: 20$ & 0 & -100 & 48 & 0 & 0.26 & -0.13 & 0.05 & 356 & -1.164 & 11.9 & 52.0 & 066 & 46 & 000 & 1.0 & 135 \\
\hline $00: 37: 30$ & 0 & -99 & 47 & 0 & 0.22 & -0.12 & -0.01 & 355 & -0.720 & 13.9 & 52.0 & 066 & 41 & 000 & 1.0 & 135 \\
\hline $00: 37: 40$ & 0 & -98 & 48 & 0 & 0.14 & -0.06 & -0.06 & 355 & -0.002 & 11.3 & 52.0 & 142 & 40 & 000 & 1.0 & 135 \\
\hline $00: 37: 50$ & -1 & -98 & 47 & 0 & 0.10 & -0.07 & -0.09 & 355 & 0.112 & 10.2 & 73.0 & 089 & 46 & 000 & 1.0 & 135 \\
\hline $00: 38: 00$ & $-I$ & -97 & 48 & 0 & 0.12 & -0.04 & -0.10 & 355 & 0.331 & 7.3 & 73.0 & 089 & 45 & 000 & 1.0 & 135 \\
\hline $00: 38: 10$ & -2 & -97 & 47 & 0 & 0.10 & -0.04 & -0.09 & 355 & 0.321 & 6.3 & 73.0 & 089 & 38 & $000^{\circ}$ & 1.0 & 135 \\
\hline $00: 38: 20$ & -2 & -96 & 48 & 0 & 0.06 & -0.02 & -0.08 & 356 & 0.365 & 3.0 & 73.0 & 118 & 40 & 000 & 1.0 & 135 \\
\hline $0: 38: 30$ & -2 & -96 & 48 & 0 & 0.10 & 0.00 & -0.05 & 356 & 0.288 & 4.0 & 99.0 & 218 & 32 & 000 & 1.0 & 135 \\
\hline $0: 38: 40$ & -2 & -95 & 48 & 0 & 0.03 & -0.02 & -0.03 & 356 & 0.036 & 7.9 & 99.0 & 118 & 34 & 000 & 1.0 & 135 \\
\hline $00: 38: 50$ & -2 & -96 & 48 & 0 & -0.12 & 0.01 & -0.07 & 356 & 0.555 & 19.0 & 99.0 & 118 & 40 & 000 & 1.0 & 135 \\
\hline $00: 39: 00$ & -2 & -97 & 48 & 0 & -0.22 & 0.07 & -0.12 & 356 & 1.198 & 15.1 & 99.0 & 118 & 36 & 000 & 1.0 & 135 \\
\hline $00: 39: 10$ & -2 & -98 & 48 & 0 & -0.32 & 0.15 & -0.13 & 356 & 1.763 & 10.5 & 99.0 & 118 & 41 & 000 & 1.0 & 135 \\
\hline $00: 39: 20$ & -2 & -100 & 47 & 0 & -0.32 & 0.19 & -0.11 & 356 & 1.879 & 4.5 & 99.0 & 065 & 32 & 000 & 1.0 & 135 \\
\hline $00: 39: 30$ & -2 & -102 & 47 & 0 & -0.31 & 0.21 & -0.05 & 357 & 1.666 & 0.0 & 99.0 & 065 & 44 & 000 & 1.0 & 135 \\
\hline $00: 39 ; 40$ & -1 & -103 & 48 & 0 & -0.26 & 0.21 & 0.02 & 357 & 1.205 & -3.4 & 99.0 & 065 & 49 & 000 & 1.0 & 135 \\
\hline $00: 39: 50$ & 0 & -104 & 48 & 0 & -0.10 & 0.20 & 0.10 & 357 & 0.650 & -5.4 & 99.0 & 041 & 38 & 000 & 1.0 & 135 \\
\hline $00: 40: 00$ & 0 & -104 & 47 & 0 & 0.05 & 0.22 & 0.16 & 357 & 0.367 & -5.0 & 99.0 & 041 & 35 & 000 & 1.0 & 135 \\
\hline $00: 40: 10$ & 2 & -103 & 48 & 0 & 0.08 & 0.24 & 0.22 & 357 & 0.093 & -3.9 & 99.0 & 359 & 37 & 000 & 1.0 & 135 \\
\hline $00: 40: 20$ & 3 & -103 & 47 & 0 & 0.11 & 0.20 & 0.26 & 357 & -0.390 & -1.1 & 99.0 & 359 & 34 & 000 & 1.0 & 135 \\
\hline $00: 40: 30$ & 4 & -102 & 48 & 0 & 0.28 & 0.13 & 0.27 & 357 & -0.938 & 2.1 & 99.0 & 182 & 37 & 000 & 1.0 & 135 \\
\hline $00: 40: 40$ & 5 & -100 & 47 & 0 & 0.29 & 0.08 & 0.26 & 357 & -1.169 & 6.3 & 99.0 & 182 & 37 & 000 & 1.0 & 135 \\
\hline $00: 40: 50$ & 5 & -99 & 48 & 0 & 0.22 & 0.04 & 0.23 & 357 & -1.199 & 11.0 & 99.0 & 182 & 34 & 000 & 1.0 & 135 \\
\hline $00: 41: 00$ & 6 & -98 & 48 & 0 & 0.15 & 0.01 & 0.16 & 356 & -0.976 & 15.8 & 99.0 & 182 & 32 & 000 & 1.0 & 135 \\
\hline $00: 41: 10$ & 6 & -97 & 47 & 0 & -0.04 & 0.05 & 0.08 & 356 & -0.158 & 14.2 & 99.0 & 182 & 39 & 000 & 1.0 & 135 \\
\hline $00: 41: 20$ & 7 & -98 & 48 & 0 & -0.12 & 0.11 & 0.04 & 356 & 0.388 & 7.5 & 99.0 & 182 & 33 & 000 & 1.0 & 135 \\
\hline $00: 41: 30$ & 7 & -98 & 48 & 0 & -0.04 & 0.05 & 0.04 & 356 & 0.066 & 8.1 & 99.0 & 182 & 34 & 000 & 1.0 & 135 \\
\hline $00: 41: 40$ & 7 & -98 & 48 & 0 & -0.01 & 0.00 & 0.00 & 356 & 0.002 & 11.0 & 99.0 & 182 & 36 & 000 & 1.0 & 135 \\
\hline $00: 41: 50$ & 7 & -99 & 47 & 0 & -0.10 & 0.01 & -0.02 & 356 & 0.176 & 7.5 & 99.0 & 182 & 37 & 000 & 1.0 & 135 \\
\hline $00 ; 42 ; 00$ & 7 & -100 & 47 & 0 & -0.19 & -0.03 & -0.04 & 356 & 0.042 & 8.7 & 99.0 & 272 & 28 & 000 & 1.0 & 135 \\
\hline $00: 42: 10$ & 7 & -101 & 48 & 0 & -0.22 & -0.06 & -0.06 & 356 & 0.002 & 9.0 & 99.0 & 272 & 38 & 000 & 1.0 & 135 \\
\hline $00: 42: 20$ & 6 & -102 & 48 & 0 & -0.24 & -0.12 & -0.09 & 356 & -0.245 & 10.6 & 99.0 & 272 & 39 & 000 & 1.0 & 135 \\
\hline $00: 42: 30$ & 6 & -103 & 47 & 0 & -0.36 & -0.21 & -0.12 & 356 & -0.627 & 12.6 & 99.0 & 272 & 44 & 000 & 1.0 & 135 \\
\hline $00: 42: 40$ & 5 & -106 & 48 & 0 & -0.43 & -0.24 & -0.15 & 356 & -0.571 & 13.8 & 99.0 & 272 & 36 & 000 & 1.0 & 135 \\
\hline $00: 42: 50$ & 4 & -108 & 47 & 0 & -0.44 & -0.26 & -0.19 & 356 & -0.441 & 15.1 & 99.0 & 272 & 30 & 000 & 1.0 & 135 \\
\hline $00: 43: 00$ & 3 & -110 & 48 & 0 & -0.49 & -0.27 & -0.23 & 356 & -0.250 & 15.7 & 99.0 & 272 & 40 & 000 & 1.0 & 135 \\
\hline $00: 43: 10$ & 2 & -113 & 47 & 0 & -0.48 & -0.27 & -0.27 & 356 & 0.006 & 15.8 & 99.0 & 272 & 29 & 000 & 1.0 & 135 \\
\hline
\end{tabular}




\section{Open Ocean}

\section{Simulation Track Plot}

Ship: 150,000 ton, Tanker, partial load

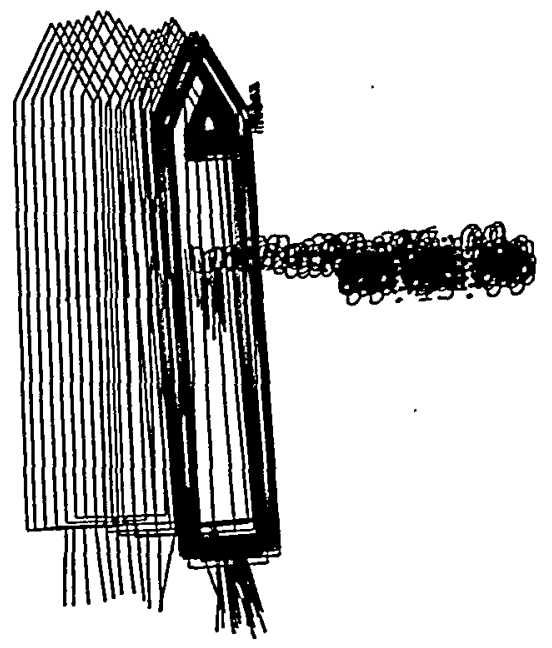

Start Time [hrs:min:sec]: 00:20:00

End Time [hrs:min:sec]: $00: 43: 15$

Delta Plot Time [sec]:

Date: 05-Feb-99 Time: 10:41:18

East-West Scale [m]:

30

East-West Scale [Nm]:

926.

0.5000 


\section{HANDS-ON SIMULATION}

\section{SUCTION MOORNG TECHNOLOGY}

Simulation Number: 30

Date: 5 February 1999

Start Time: 11:37

Length of run [secs]: 1530

Ship Condition: $\operatorname{draft}=13.72 \mathrm{~m}$

Environment

Wind Speed [knots]

40 Direction: 0

Sea State, Significant wave height [m]:..

8 Direction: 180

Current Speed [knots]:

1 Direction: 135

Ship Operator: J.A.

Observer: B.K.J

Final Mating, distance between center of buoy and center of moon pool [m]: 2.0

Evaluation:

Mating in the first try.

The captain had a fast approach by holding speed larger and backing down at $150 \mathrm{~m}$ from the buoy. First he positioned the moon pool a little on the current side and with an equilibrium heading on the autopilot at 357 degrees and RPM 45, then he made all other maneuvers with the thruster vector.

The simulation ran 15 min too long. 
Ship:

Exercise number:

Scenario

ig Eile

raffic ships: Exercise identification:
591 150,000 ton. Tanker, partial load

754 Suction Mooring System

112 Open Ocean

999 Dumny tug file based on containership

590 Suction Mooring Buoy

594 150k tanker in partial load - Suction Mooring Run 430

Time position (m) RPM Speed (Knots) Turn Rudder Gill Wind

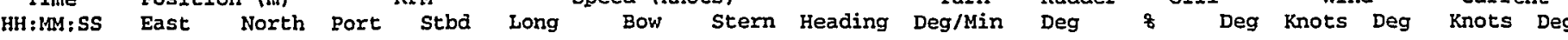

\begin{tabular}{|c|c|c|c|c|c|c|c|c|c|c|c|c|c|c|c|c|}
\hline $00: 03: 20$ & 12 & -581 & 52 & 0 & 4.64 & 0.30 & 0.13 & 356 & 1.115 & -27.3 & 0.0 & 180 & 40 & 000 & 1.0 & 135 \\
\hline $00: 03: 30$ & 12 & -557 & 52 & 0 & 4.55 & 0.18 & 0.35 & 355 & -1.084 & -26.7 & 100.0 & 180 & 32 & 000 & 1.0 & 135 \\
\hline $00: 03: 40$ & 11 & -534 & 52 & 0 & 4.42 & 0.16 & 0.53 & 355 & -2.325 & -21.3 & 100.0 & 180 & 41 & 000 & 1.0 & 135 \\
\hline $00: 03: 50$ & 11 & -511 & 52 & 0 & 4.29 & 0.13 & 0.64 & 355 & -3.204 & -12.6 & 100.0 & 180 & 31 & 000 & 1.0 & 135 \\
\hline $00 ; 04 ; 00$ & 11 & -489 & 52 & 0 & 4.22 & 0.12 & 0.65 & 354 & -3.371 & -2.4 & 100.0 & 180 & 36 & 000 & 1.0 & 135 \\
\hline $00: 04: 10$ & 11 & -468 & 52 & 0 & 4.19 & 0.16 & 0.57 & 354 & -2.614 & 6.9 & 100.0 & 180 & 45 & 000 & 1.0 & 135 \\
\hline $00: 04: 20$ & 10 & -446 & 52 & 0 & 4.09 & 0.30 & 0.44 & 353 & -0.879 & 11.5 & 100.0 & 180 & 35 & 000 & 1.0 & 135 \\
\hline $00: 04: 30$ & 10 & -425 & 52 & 0 & 4.05 & 0.38 & 0.30 & 353 & 0.491 & 11.7 & 100.0 & 180 & 30 & 000 & 1.0 & 135 \\
\hline $00: 04: 40$ & 9 & -404 & 52 & 0 & 4.02 & 0.48 & 0.19 & 354 & 1.864 & 7.9 & 100.0 & 180 & 27 & 000 & 1.0 & 135 \\
\hline $00: 04: 50$ & 8 & -383 & 48 & 0 & 4.06 & 0.45 & 0.15 & 354 & 1.922 & 1.7 & 100.0 & 180 & 36 & 000 & 1.0 & 135 \\
\hline $00: 05: 00$ & 8 & -363 & 48 & 0 & 4.03 & 0.35 & 0.14 & 354 & 1.326 & -2.6 & 100.0 & 180 & 34 & 000 & 1.0 & 135 \\
\hline $00: 05: 10$ & 7 & -342 & 48 & 0 & 3.89 & 0.32 & 0.16 & 354 & 1.017 & -6.5 & 100.0 & 180 & 32 & 000 & 1.0 & 135 \\
\hline $00: 05: 20$ & 6 & -322 & 30 & 0 & 3.75 & 0.25 & 0.17 & 354 & 0.482 & -8.6 & 100.0 & 180 & 34 & 000 & 1.0 & 135 \\
\hline $00: 05: 30$ & 5 & -303 & 29 & 0 & 3.63 & 0.22 & 0.17 & 355 & 0.299 & -9.7 & 100.0 & 180 & 37 & 000 & 1.0 & 135 \\
\hline $00: 05: 40$ & 4 & -285 & 29 & 0 & 3.42 & 0.18 & 0.17 & 355 & 0.078 & -10.2 & 100.0 & 180 & 40 & 000 & 1.0 & 135 \\
\hline $00: 05: 50$ & 4 & -268 & 29 & 0 & 3.29 & 0.17 & 0.19 & 355 & -0.140 & -25.8 & 100.0 & 180 & 43 & 000 & 1.0 & 135 \\
\hline $00: 06: 00$ & 3 & -251 & 29 & 0 & 3.15 & 0.14 & 0.26 & 354 & -0.799 & -35.0 & 100.0 & 180 & 40 & 000 & 1.0 & 235 \\
\hline $00: 06: 10$ & 3 & -236 & 29 & 0 & 3.00 & 0.10 & 0.34 & 354 & -1.539 & -35.0 & 100.0 & 180 & 36 & 000 & 1.0 & 135 \\
\hline $00: 06: 20$ & 2 & -220 & 29 & 0 & 2.83 & 0.08 & 0.41 & 354 & -2.100 & -35.0 & 100.0 & 180 & 34 & 000 & 1.0 & 135 \\
\hline $00: 06: 30$ & 2 & -206 & 28 & 0 & 2.68 & 0.07 & 0.48 & 354 & -2.605 & -35.0 & 100.0 & 180 & 37 & 000 & 1.0 & 135 \\
\hline $00: 06: 40$ & 2 & -193 & 0 & 0 & 2.55 & 0.03 & 0.51 & 353 & -3.019 & -35.0 & 100.0 & 180 & 29 & 000 & 1.0 & 135 \\
\hline $00: 06: 50$ & 2 & -180 & 0 & 0 & 2.38 & -0.03 & 0.49 & 353 & -3.270 & -35.0 & 100.0 & 180 & 35 & 000 & 1.0 & 135 \\
\hline $00: 07: 00$ & 1 & -168 & -21 & 0 & 2.19 & 0.05 & 0.45 & 352 & -2.597 & -35.0 & 100.0 & 180 & 42 & 000 & 1.0 & 135 \\
\hline $00: 07: 10$ & 1 & -157 & -51 & 0 & 1.99 & 0.16 & 0.40 & 352 & -1.545 & -30.6 & 100.0 & 180 & 37 & 000 & 1.0 & 135 \\
\hline $00: 07: 20$ & 1 & -147 & -61 & 0 & 1.82 & 0.18 & 0.33 & 352 & -0.940 & -27.0 & 100.0 & 180 & 32 & 000 & 1.0 & 135 \\
\hline $00: 07: 30$ & 1 & -138 & -62 & 0 & 1.66 & 0.16 & 0.25 & 351 & -0.608 & -24.9 & 100.0 & 180 & 49 & 000 & 1.0 & 135 \\
\hline $00: 07: 40$ & 1 & -130 & -62 & 0 & 1.43 & 0.21 & 0.17 & 351 & 0.239 & -24.9 & 100.0 & 180 & 40 & 000 & 1.0 & 135 \\
\hline $00: 07: 50$ & 1 & -123 & -62 & 0 & 1.22 & 0.18 & 0.10 & 351 & 0.495 & -26.3 & 100.0 & 180 & 46 & 000 & 1.0 & 135 \\
\hline $00: 08: 00$ & 0 & -118 & -62 & 0 & 0.92 & 0.16 & 0.03 & 352 & 0.866 & -29.1 & 100.0 & 180 & 38 & 000 & 1.0 & 135 \\
\hline$: 08: 10$ & 0 & -114 & -62 & 0 & 0.71 & 0.09 & -0.04 & 352 & 0.808 & -32.2 & 100.0 & 180 & 33 & 000 & 1.0 & 135 \\
\hline $0 ; 08 ; 20$ & -1 & -111 & -62 & 0 & 0.50 & -0.02 & -0.11 & 352 & 0.576 & -33.9 & 100.0 & 180 & 43 & 000 & 1.0 & 135 \\
\hline $00: 08: 30$ & -1 & -109 & -49 & 0 & 0.22 & -0.01 & -0.19 & 352 & 1.128 & -35.0 & 100.0 & 180 & 40 & 000 & 1.0 & 135 \\
\hline $00: 08: 40$ & -2 & -108 & -17 & 0 & 0.01 & -0.05 & -0.21 & 352 & 0.984 & -35.0 & 100.0 & 180 & 45 & 000 & 1.0 & 135 \\
\hline $00: 08: 50$ & -2 & -109 & 46 & 0 & 0.00 & -0.10 & -0.10 & 352 & -0.030 & -35.0 & 0.0 & 180 & 36 & 000 & 1.0 & 135 \\
\hline $00: 09: 00$ & -3 & -108 & 74 & 0 & 0.11 & -0.35 & 0.24 & 352 & -3.775 & -18.8 & 0.0 & 180 & 36 & 000 & 1.0 & 135 \\
\hline $00: 09: 10$ & -3 & -107 & 67 & 0 & 0.27 & -0.48 & 0.37 & 351 & -5.451 & 0.2 & 0.0 & 180 & 40 & 000 & 1.0 & 335 \\
\hline $00: 09: 20$ & -4 & -106 & 44 & 0 & 0.26 & -0.48 & 0.29 & 350 & -4.886 & 19.2 & 100.0 & 180 & 44 & 000 & 1.0 & 135 \\
\hline $00: 09: 30$ & -5 & -105 & 46 & 0 & 0.21 & -0.42 & 0.12 & 350 & -3.314 & 35.0 & 100.0 & 180 & 26 & 000 & 1.0 & 135 \\
\hline $00: 09: 40$ & -6 & -104 & 47 & 0 & 0.11 & -0.33 & -0.08 & 349 & -1.596 & 35.0 & 100.0 & 120 & 46 & 000 & 1.0 & 135 \\
\hline $00: 09: 50$ & -7 & -104 & 47 & 0 & 0.08 & -0.17 & -0.25 & 349 & 0.535 & 35.0 & 100.0 & 091 & 32 & 000 & 1.0 & 135 \\
\hline $00: 10: 00$ & -8 & -104 & 48 & 0 & 0.12 & 0.05 & -0.41 & 349 & 2.913 & 35.0 & 100.0 & 091 & 36 & 000 & 1.0 & 135 \\
\hline $00: 10: 10$ & -9 & -103 & 47 & 0 & 0.20 & 0.13 & -0.55 & 350 & 4.319 & 35.0 & 100.0 & 091 & 42 & 000 & 1.0 & 135 \\
\hline $00: 10: 20$ & -10 & -102 & 47 & 0 & 0.23 & 0.26 & -0.67 & 351 & 5.910 & 35.0 & 100.0 & 091 & 40 & 000 & 1.0 & 135 \\
\hline $00: 10: 30$ & -12 & -101 & 47 & 0 & 0.22 & 0.45 & -0.77 & 352 & 7.801 & 35.0 & 100.0 & 118 & 44 & 000 & 1.0 & 135 \\
\hline $00: 10: 40$ & -12 & -100 & 48 & 0 & 0.25 & 0.50 & -0.80 & 353 & 8.264 & 18.4 & 100.0 & 145 & 40 & 000 & 1.0 & 135 \\
\hline $00: 10 ; 50$ & -13 & -99 & 47 & 0 & 0.28 & 0.39 & -0.71 & -355 & 6.988 & 0.5 & 100.0 & 145 & 36 & 000 & 1.0 & 135 \\
\hline $00: 11: 00$ & -14 & -97 & 48 & 0 & 0.24 & 0.28 & -0.53 & 356 & 5.182 & -15.8 & 100.0 & 145 & 30 & 000 & 1.0 & 135 \\
\hline $00: 11 ; 10$ & -15 & -96 & 48 & 0 & 0.22 & 0.16 & -0.33 & 356 & 3.127 & -21.3 & 100.0 & 153 & 38 & 000 & 1.0 & 135 \\
\hline $00: 11: 20$ & -15 & -95 & 47 & 0 & 0.14 & 0.08 & -0.13 & 357 & 1.325 & -23.6 & 100.0 & 153 & 32 & 000 & 1.0 & 135 \\
\hline $00: 11: 30$ & -15 & -95 & 48 & 0 & 0.14 & 0.01 & 0.03 & 357 & -0.145 & -13.1 & 100.0 & 121 & 35 & 000 & 1.0 & 135 \\
\hline $00: 11: 40$ & -15 & -94 & 48 & 0 & 0.20 & -0.03 & 0.12 & 357 & -0.954 & -6.9 & 100.0 & 154 & 20 & 000 & 1.0 & 135 \\
\hline $00: 11: 50$ & -15 & -93 & 47 & 0 & 0.20 & -0.01 & 0.18 & 356 & -1.156 & -2.9 & 100.0 & 136 & 39 & 000 & 1.0 & 135 \\
\hline $00: 12: 00$ & -14 & -92 & 46 & 0 & 0.13 & 0.00 & 0.20 & 356 & -1.260 & 4.2 & 100.0 & 136 & 39 & 000 & 1.0 & 135 \\
\hline $00: 12: 10$ & -14 & -91 & 47 & 0 & 0.08 & 0.04 & 0.19 & 356 & -0.925 & 6.2 & 100.0 & 136 & 39 & 000 & 1.0 & 135 \\
\hline $00: 12: 20$ & -13 & -91 & 47 & 0 & 0.03 & 0.06 & 0.17 & 356 & -0.702 & 7.4 & 100.0 & 136 & 38 & 000 & 1.0 & 135 \\
\hline $00: 12: 30$ & -13 & -91 & 47 & 0 & 0.00 & 0.08 & 0.16 & 356 & -0.475 & 6.9 & 100.0 & 150 & 37 & 000 & 1.0 & 135 \\
\hline $00: 12: 40$ & -12 & -91 & 46 & 0 & -0.06 & 0.11 & 0.15 & 356 & -0.270 & 5.6 & 100.0 & 118 & 35 & 000 & 1.0 & 135 \\
\hline $00: 12: 50$ & -11 & $-9 i$ & 46 & 0 & -0.06 & 0.19 & 0.15 & 356 & 0.239 & 11.8 & 100.0 & 118 & 35 & 000 & 1.0 & 135 \\
\hline $00: 13: 00$ & -10 & -91 & 47 & 0 & -0.05 & 0.28 & 0.09 & 356 & 1.166 & 12.6 & 100.0 & 151 & 39 & 000 & 1.0 & 135 \\
\hline $00: 13: 10$ & -9 & -92 & 47 & 0 & -0.03 & 0.32 & 0.08 & 356 & 1.524 & 6.3 & 100.0 & 151 & 30 & 000 & 1.0 & 135 \\
\hline $00: 13: 20$ & -8 & -92 & 46 & 0 & -0.02 & 0.35 & 0.09 & 356 & 1.662 & 1.9 & 100.0 & 151 & 34 & 000 & 1.0 & 135 \\
\hline $00: 13: 30$ & -7 & -92 & 46 & 0 & 0.03 & 0.33 & 0.13 & 357 & 1.288 & -0.4 & 100.0 & 151 & 38 & 000 & 1.0 & 135 \\
\hline $00: 13: 40$ & -6 & -91 & 47 & 0 & 0.05 & 0.29 & 0.18 & 357 & 0.727 & -0.9 & 100.0 & 151 & 33 & 000 & 1.0 & 135 \\
\hline $00: 13: 50$ & -5 & -91 & 47 & 0 & -0.04 & 0.27 & 0.21 & 357 & 0.409 & -0.9 & 100.0 & 179 & 35 & 000 & 1.0 & 135 \\
\hline $00: 14: 00$ & -3 & -91 & 47 & 0 & -0.07 & 0.25 & 0.23 & 357 & 0.106 & 0.0 & 100.0 & 179 & 45 & 000 & 1.0 & 135 \\
\hline $00: 14: 10$ & -2 & -92 & 46 & 0 & -0.03 & 0.21 & 0.25 & 357 & -0.222 & 1.7 & 100.0 & 179 & 36 & 000 & 1.0 & 135 \\
\hline $00: 14: 20$ & -1 & -92 & 45 & 0 & -0.05 & 0.18 & 0.25 & 357 & -0.445 & 4.0 & 100.0 & 179 & 34 & 000 & 1.0 & 135 \\
\hline $9: 14: 30$ & 0 & -92 & 45 & 0 & -0.05 & 0.17 & 0.24 & 357 & -0.434 & 5.3 & 100.0 & 179 & 35 & 000 & 2.0 & 135 \\
\hline$: 14: 40$ & 1 & -92 & 46 & 0 & -0.11 & 0.20 & 0.23 & 357 & -0.181 & 4.9 & 100.0 & 179 & 39 & 000 & 1.0 & 135 \\
\hline $00: 14: 50$ & 2 & -93 & 46 & 0 & -0.14 & 0.18 & 0.22 & 357 & -0.215 & 5.5 & 77.0 & 179 & 46 & 000 & 1.0 & 135 \\
\hline $00: 15: 00$ & 3 & -93 & 46 & 0 & -0.15 & 0.13 & 0.20 & 357 & -0.464 & 8.1 & 77.0 & 179 & 39 & 000 & 1.0 & 135 \\
\hline $00: 15: 10$ & 4 & -94 & 46 & 0 & -0.19 & 0.13 & 0.19 & 357 & -0.406 & 0.4 & 77.0 & 179 & 39 & 000 & 1.0 & 135 \\
\hline $00: 15: 20$ & 5 & -95 & 46 & 0 & -0.26 & 0.07 & 0.24 & 356 & -1.072 & -4.9 & 52.0 & 179 & 43 & 000 & 1.0 & 135 \\
\hline
\end{tabular}




\begin{tabular}{|c|c|c|c|c|c|c|c|c|c|c|c|c|c|c|c|c|}
\hline \multirow{2}{*}{$\begin{array}{c}\text { Time } \\
\text { HH:MM;:SS }\end{array}$} & \multicolumn{2}{|c|}{ Rosition (m) } & \multicolumn{2}{|r|}{ RPM } & \multicolumn{3}{|c|}{ Speed (Knots) } & \multirow[b]{2}{*}{ Heading } & \multirow{2}{*}{$\begin{array}{l}\text { Turn } \\
\text { Deg/Min }\end{array}$} & \multirow{2}{*}{$\begin{array}{l}\text { Rudider } \\
\text { Deg }\end{array}$} & \multicolumn{2}{|c|}{ Gill } & \multicolumn{2}{|c|}{ Wind } & \multicolumn{2}{|c|}{ Current } \\
\hline & East & North & Port & stbd & Long & Bow & Stern & & & & 8 & Deg & Knots & Deg & Knots & Deg \\
\hline$-20: 15: 30$ & 6 & -97 & 45 & 0 & -0.29 & 0.06 & 0.28 & 356 & -1.398 & -0.3 & 0.0 & 129 & 36 & 000 & 1.0 & 135 \\
\hline$: 15: 40$ & 7 & -98 & 45 & 0 & -0.28 & 0.05 & 0.28 & 356 & -1.479 & 4.7 & 48.0 & 000 & 51 & 000 & 1.0 & 135 \\
\hline 50 & 8 & -99 & 46 & 0 & -0.19 & 0.09 & 0.26 & 356 & -1.079 & 7.8 & 48.0 & 000 & 34 & 000 & 1.0 & 135 \\
\hline $00: 16: 00$ & 9 & -100 & 46 & 0 & -0.12 & 0.10 & 0.22 & 356 & -0.772 & 10.2 & 48.0 & 000 & 30 & 000 & 1.0 & 135 \\
\hline $00: 16: 10$ & 10 & -101 & 46 & 0 & -0.06 & 0.13 & 0.18 & 356 & -0.289 & 9.4 & 48.0 & 000 & 39 & 000 & 1.0 & 135 \\
\hline $00: 16: 20$ & 10 & -101 & 46 & 0 & -0.01 & 0.16 & 0.15 & 356 & 0.049 & 7.5 & 48.0 & 000 & 37 & 000 & 1.0 & 135 \\
\hline $00: 16: 30$ & 11 & -100 & 46 & 0 & 0.07 & 0.14 & 0.13 & 356 & 0.044 & 7.5 & 48.0 & 000 & 34 & 000 & 1.0 & 135 \\
\hline $00: 16: 40$ & 12 & -100 & 45 & 0 & 0.08 & 0.13 & 0.11 & 356 & 0.104 & 6.5 & 48.0 & 000 & 46 & 000 & 1.0 & 135 \\
\hline $00: 16: 50$ & 12 & -99 & 46 & 0 & 0.12 & 0.10 & 0.10 & 356 & 0.047 & 6.9 & 48.0 & 000 & 40 & 000 & 1.0 & 135 \\
\hline $00: 17: 00$ & 13 & -99 & 46 & 0 & 0.23 & 0.08 & 0.08 & 356 & 0.018 & 7.2 & 48.0 & 000 & 43 & 000 & 1.0 & 135 \\
\hline $00: 17: 10$ & 13 & -97 & 46 & 0 & 0.25 & 0.09 & 0.06 & 356 & 0.139 & 6.1 & 48.0 & 000 & 43 & 000 & 1.0 & 135 \\
\hline $00: 17: 20$ & 13 & -96 & 46 & 0 & 0.28 & 0.08 & 0.06 & 356 & 0.172 & 5.5 & 48.0 & 000 & 36 & 000 & 1.0 & 135 \\
\hline $00: 17: 30$ & 14 & -94 & 46 & 0 & 0.37 & 0.08 & 0.05 & 356 & 0.173 & 5.1 & 48.0 & 000 & 47 & 000 & 1.0 & 135 \\
\hline $00: 17: 40$ & 14 & -92 & 45 & 0 & 0.43 & 0.03 & 0.05 & 356 & -0.104 & 5.9 & 48.0 & 000 & 31 & 000 & 1.0 & 135 \\
\hline $00: 17: 50$ & 14 & -90 & 45 & 0 & 0.45 & -0.03 & 0.04 & 356 & -0.425 & 7.8 & 48.0 & 000 & 38 & 000 & 1.0 & 135 \\
\hline $00: 18: 00$ & 13 & -88 & 46 & 0 & 0.38 & -0.02 & 0.02 & 356 & -0.205 & 8.5 & 48.0 & 000 & 27 & 000 & 1.0 & 135 \\
\hline $00: 18: 10$ & 13 & -86 & 46 & 0 & 0.37 & 0.02 & 0.00 & 356 & 0.104 & 7.5 & 48.0 & 000 & 33 & 000 & 1.0 & 135 \\
\hline
\end{tabular}


Open Ocean

\section{Simulation Track Plot}

Ship: 150,000 ton, Tanker, partial load

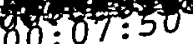

$00: 07: 20$

$00: 06: 50$

$00: 06: 20$

$00: 05: 50$

$00: 05: 20$

$00: 04: 50$

$00: 04: 20$

$00: 0.3: 50$

Start Time [hrs:min:sec]: $00: 03: 20$

End Time [hrs:min:sec]: $00: 18: 20$ 00:0 Delta Plot Time [sec]:

East-West Scale [m]: 


\section{HANDS-ON SIMULATION}

\section{SUCTION MOORNG TECHNOLOGY}

Simulation Number: 31

Date: 5 February 1999

Start Time: 13:18

Length of run [secs]: 4566

Ship Condition: draft $=13.72 \mathrm{~m}$

Environment

Wind Speed [knots]:

40 Direction: 0

Sea State, Significant wave height $[\mathrm{m}]: .$.

8 Direction: 180

Current Speed [knots]:

$1 \quad$ Direction: 90

Ship Operator: J.A.

Observer: B.K.J

Final Mating, distance between center of buoy and center of moon pool [m]: 1.9

Evaluation:

The approach and initial positioning near the buoy was no problem. The same applies to get into the camera view. It took many cycles of ship motion to get small waves when the buoy was near the center of the moon pool. 
Ship:

Exercise number:

Gcenario: 19 file:

affic ships:

Initial conditions: Exercise identification:
591 150,000 ton, Tanker, partial load

754 Suction Mooring Systen

112 Open Ocean

999 Dummy tug file based on containership

Suction Hooring Buoy

594 150k tanker in partial load - Suction Mooring RuN 431

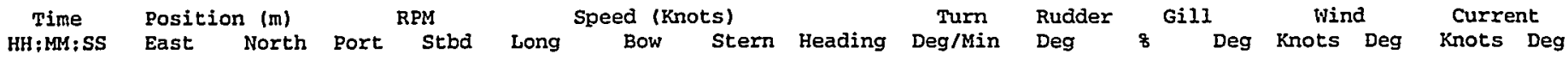

\begin{tabular}{|c|c|c|c|c|c|c|c|c|c|c|c|c|c|c|c|c|}
\hline $00: 41: 40$ & 10 & -102 & 45 & 0 & 0.34 & -0.14 & -0.14 & 354 & -0.046 & 24.9 & 53.0 & 000 & 36 & 000 & 1.0 & 090 \\
\hline $00: 41: 50$ & 9 & -100 & 45 & 0 & 0.29 & -0.07 & -0.17 & 354 & 0.660 & 21.8 & 99.0 & 091 & 37 & 000 & 1.0 & 090 \\
\hline $00: 42: 00$ & 8 & -99 & 45 & 0 & 0.21 & -0.02 & -0.17 & 354 & 0.952 & 16.6 & 99.0 & 091 & 28 & 000 & 1.0 & 090 \\
\hline $00: 42: 10$ & 8 & -98 & 44 & 0 & 0.14 & 0.04 & -0.13 & 354 & 1.059 & 10.3 & 99.0 & 089 & 38 & 000 & 1.0 & 090 \\
\hline $00: 42: 20$ & 7 & -98 & 44 & 0 & 0.11 & 0.02 & -0.07 & 354 & 0.559 & 9.8 & 99.0 & 089 & 39 & 000 & 1.0 & 090 \\
\hline $00: 42: 30$ & 7 & -97 & 44 & 0 & -0.02 & -0.04 & -0.03 & 354 & -0.094 & 17.8 & 99.0 & 089 & 44 & 000 & 1.0 & 090 \\
\hline $00: 42: 40$ & 7 & -98 & 44 & 0 & -0.11 & 0.01 & -0.04 & 354 & 0.289 & 12.8 & 99.0 & 063 & 36 & 000 & 1.0 & 090 \\
\hline $00: 42: 50$ & 7 & -98 & 45 & 0 & -0.10 & 0.04 & 0.00 & 354 & 0.265 & 11.5 & 99.0 & 063 & 30 & 000 & 1.0 & 090 \\
\hline $00: 43: 00$ & 7 & -99 & 45 & 0 & -0.14 & 0.14 & -0.07 & 354 & 1.353 & 19.3 & 99.0 & 063 & 40 & 000 & 1.0 & 090 \\
\hline $00: 43: 10$ & 8 & -100 & 45 & 0 & -0.13 & 0.25 & -0.12 & 355 & 2.305 & 6.5 & 99.0 & 088 & 29 & 000 & 1.0 & 090 \\
\hline $00: 43: 20$ & 8 & -100 & 45 & 0 & -0.14 & 0.34 & -0.11 & 355 & 2.832 & -1.5 & 99.0 & 058 & 40 & 000 & 1.0 & 090 \\
\hline $00: 43: 30$ & 9 & -101 & 45 & 0 & -0.04 & 0.40 & -0.03 & 356 & 2.691 & -8.4 & 99.0 & 058 & 30 & 000 & 1.0 & 090 \\
\hline $00: 43: 40$ & 10 & -100 & 45 & 0 & 0.11 & 0.42 & 0.11 & 356 & 1.931 & -7.1 & 99.0 & 058 & 29 & 000 & 1.0 & 090 \\
\hline $00: 43: 50$ & 11 & -100 & 44 & 0 & 0.16 & 0.33 & 0.21 & 356 & 0.774 & -5.9 & 99.0 & 273 & 37 & 000 & 1.0 & 090 \\
\hline $00 ; 44: 00$ & 13 & -99 & 44 & 0 & 0.17 & 0.18 & 0.28 & 356 & -0.638 & -0.8 & 99.0 & 227 & 49 & 000 & 1.0 & 090 \\
\hline $00: 44: 10$ & 14 & -98 & 44 & 0 & 0.18 & 0.07 & 0.30 & 356 & -1.510 & 6.8 & 99.0 & 227 & 34 & 000 & 1.0 & 090 \\
\hline $00 ; 44: 20$ & 15 & -97 & 45 & 0 & 0.13 & 0.05 & 0.29 & 356 & -1.560 & 13.4 & 99.0 & 227 & 49 & 000 & 1.0 & 090 \\
\hline $00: 44: 30$ & 15 & -96 & 45 & 0 & -0.02 & 0.04 & 0.25 & 355 & -1.348 & 20.0 & 99.0 & 227 & 40 & 000 & 1.0 & 090 \\
\hline $00: 44: 40$ & 16 & -97 & 45 & 0 & -0.06 & -0.03 & 0.19 & 355 & -1.360 & 24.1 & 99.0 & 227 & 39 & 000 & 1.0 & 090 \\
\hline $00 ; 44: 50$ & 16 & -97 & 45 & 0 & -0.04 & -0.08 & 0.13 & 355 & -1.319 & 30.4 & 99.0 & 227 & 41 & 000 & 1.0 & 090 \\
\hline $00 ; 45 ; 00$ & 16 & -97 & 45 & 0 & -0.06 & -0.09 & 0.07 & 355 & -1.036 & 24.2 & 99.0 & 227 & 33 & 000 & 1.0 & 090 \\
\hline $00 ; 45: 10$ & 16 & -97 & 45 & 0 & 0.01 & -0.13 & 0.01 & 355 & -0.841 & .22 .5 & 99.0 & 227 & 44 & 000 & 1.0 & 090 \\
\hline $00: 45: 20$ & 16 & -97 & 44 & 0 & 0.01 & -0.18 & -0.02 & 355 & -1.011 & 20.4 & 99.0 & 292 & 32 & 000 & 1.0 & 090 \\
\hline $00: 45: 30$ & 15 & -97 & 44 & 0 & -0.11 & -0.15 & -0.05 & 354 & -0.624 & 16.6 & 99.0 & 292 & 37 & 000 & 1.0 & 090 \\
\hline $00: 45: 40$ & 15 & -98 & 44 & 0 & -0.10 & -0.12 & -0.12 & 354 & 0.030 & 11.6 & 99.0 & 292 & 37 & 000 & 1.0 & 090 \\
\hline $00: 45: 50$ & 14 & -99 & 45 & 0 & -0.01 & -0.28 & -0.11 & 354 & -1.132 & 20.3 & 99.0 & 292 & 42 & 000 & 1.0 & 090 \\
\hline $00: 46: 00$ & 13 & -99 & 45 & 0 & -0.03 & -0.35 & -0.12 & 354 & -1.473 & 24.8 & 52.0 & 292 & 41 & 000 & 1.0 & 090 \\
\hline $00: 46: 10$ & 12 & -99 & 45 & 0 & -0.16 & -0.38 & -0.17 & 354 & -1.356 & 27.0 & 52.0 & 292 & 49 & 000 & 1.0 & 090 \\
\hline $00: 46: 20$ & 10 & -101 & 45 & 0 & -0.36 & -0.31 & -0.28 & 354 & -0.147 & 25.2 & 52.0 & 091 & 28 & 000 & 1.0 & 090 \\
\hline $0: 46: 30$ & 9 & -103 & 45 & 0 & -0.43 & -0.08 & -0.36 & 354 & 1.733 & 18.0 & 99.0 & 091 & 40 & 000 & 1.0 & 090 \\
\hline $00: 46: 40$ & 8 & -105 & 45 & 0 & -0.43 & -0.02 & -0.38 & 354 & 2.340 & 9.9 & 52.0 & 091 & 44 & 000 & 1.0 & 090 \\
\hline $00: 46: 50$ & 8 & -108 & 45 & 0 & -0.43 & 0.04 & -0.37 & 355 & 2.614 & 1.7 & 99.0 & 041 & 40 & 000 & 1.0 & 090 \\
\hline $00: 47: 00$ & 7 & $-110^{\circ}$ & 44 & 0 & -0.33 & 0.09 & -0.32 & 355 & 2.579 & -8.0 & 99.0 & 000 & 39 & 000 & 1.0 & 090 \\
\hline $00 ; 47: 10$ & 7 & -111 & 44 & 0 & -0.24 & 0.09 & -0.23 & 355 & 2.026 & -16.1 & 99.0 & 036 & 36 & 000 & 1.0 & 090 \\
\hline $00: 47: 20$ & 7 & -112 & 44 & 0 & -0.14 & 0.11 & -0.10 & 356 & 1.357 & -23.5 & 99.0 & 036 & 40 & 000 & 1.0 & 090 \\
\hline $00: 47: 30$ & 7 & -113 & 45 & 0 & -0.07 & 0.07 & 0.05 & 356 & 0.109 & -17.5 & 99.0 & 000 & 37 & 000 & 1.0 & 090 \\
\hline $00: 47: 40$ & 7 & -113 & 45 & 0 & -0.06 & -0.03 & 0.18 & 356 & -1.376 & 1.5 & 99.0 & 000 & 41 & 000 & 1.0 & 090 \\
\hline $00: 47: 50$ & 8 & -113 & 45 & 0 & 0.03 & -0.05 & 0.20 & 355 & -1.600 & 17.3 & 99.0 & 000 & 33 & 000 & 1.0 & 090 \\
\hline $00: 48: 00$ & 8 & -113 & 45 & 0 & 0.09 & -0.02 & 0.20 & 355 & -1.412 & 10.3 & 99.0 & 000 & 32 & 000 & 1.0 & 090 \\
\hline $00 ; 48 ; 10$ & 8 & -112 & 45 & 0 & 0.15 & -0.06 & 0.21 & 355 & -1.696 & 13.6 & 99.0 & 000 & 32 & 000 & 1.0 & 090 \\
\hline $00: 48: 20$ & 9 & -111 & 45 & 0 & 0.26 & -0.15 & 0.19 & 355 & -2.194 & 17.5 & 74.0 & 000 & 35 & 000 & 1.0 & 090 \\
\hline $00: 48: 30$ & 8 & -109 & 45 & 0 & 0.31 & -0.16 & 0.15 & 354 & -1.966 & 21.7 & 49.0 & 000 & 32 & 000 & 1.0 & 090 \\
\hline $00: 48: 40$ & 8 & -108 & 44 & 0 & 0.27 & -0.03 & 0.09 & 354 & -0.801 & 21.5 & 27.0 & 000 & 39 & 000 & 1.0 & 090 \\
\hline $00: 48: 50$ & 8 & -107 & 44 & 0 & 0.30 & 0.02 & 0.04 & 354 & -0.156 & 20.3 & 52.0 & 000 & 41 & 000 & 1.0 & 090 \\
\hline $00: 49: 00$ & 8 & -105 & 45 & 0 & 0.39 & -0.01 & 0.01 & 354 & -0.101 & 20.0 & 27.0 & 000 & 39 & 000 & 1.0 & 090 \\
\hline $00 ; 49: 10$ & 8 & -103 & 45 & 0 & 0.36 & -0.05 & -0.03 & 354 & -0.136 & 20.7 & 27.0 & 033 & 33 & 000 & 1.0 & 090 \\
\hline $00: 49: 20$ & 7 & -101 & 45 & 0 & 0.37 & -0.10 & -0.05 & 354 & -0.310 & 21.8 & 99.0 & 086 & 39 & 000 & 1.0 & 090 \\
\hline $00: 49: 30$ & 7 & -99 & 45 & 0 & 0.37 & -0.12 & -0.08 & 354 & -0.277 & 22.8 & 99.0 & 121 & 42 & 000 & 1.0 & 090 \\
\hline $00: 49: 40$ & 6 & -98 & 45 & 0 & 0.18 & -0.10 & -0.10 & 354 & -0.023 & 22.7 & 99.0 & 121 & 30 & 000 & 1.0 & 090 \\
\hline $00: 49: 50$ & 6 & -97 & 45 & 0 & -0.06 & -0.03 & -0.09 & 354 & 0.424 & 15.2 & 99.0 & 090 & 28 & 000 & 1.0 & 090 \\
\hline $00: 50: 00$ & 5 & -98 & 45 & 0 & -0.11 & -0.01 & -0.10 & 354 & 0.599 & 14.7 & 99.0 & 062 & 32 & 000 & 1.0 & 090 \\
\hline $00: 50: 10$ & 5 & -99 & 44 & 0 & -0.08 & 0.00 & -0.08 & 354 & 0.515 & 11.5 & 99.0 & 062 & 26 & 000 & 1.0 & 090 \\
\hline $00: 50: 20$ & 5 & -99 & 44 & 0 & 0.01 & 0.01 & 0.01 & 354 & 0.031 & -0.9 & 99.0 & 062 & 45 & 000 & 1.0 & 090 \\
\hline $00: 50: 30$ & 5 & -99 & 44 & 0 & 0.00 & 0.01 & 0.06 & 354 & -0.321 & 18.1 & 99.0 & 062 & 36 & 000 & 1.0 & 090 \\
\hline $00: 50: 40$ & 6 & -99 & 45 & 0 & -0.11 & 0.17 & 0.05 & 354 & 0.755 & 12.3 & 99.0 & 091 & 33 & 000 & 1.0 & 090 \\
\hline $00: 50: 50$ & 6 & -99 & 45 & 0 & -0.07 & 0.29 & 0.06 & 354 & 1.475 & 4.3 & 99.0 & 091 & 40 & 000 & 1.0 & 090 \\
\hline $00: 51: 00$ & 7 & -99 & 45 & 0 & -0.02 & 0.31 & 0.13 & 354 & 1.103 & 3.6 & 99.0 & 059 & 41 & 000 & 1.0 & 090 \\
\hline $00: 51: 10$ & 9 & -99 & 45 & 0 & 0.06 & 0.30 & 0.19 & 355 & 0.690 & 4.0 & 99.0 & 059 & 41 & 000 & 1.0 & 090 \\
\hline $00: 51: 20$ & 10 & -99 & 45 & 0 & 0.10 & 0.23 & 0.25 & 355 & -0.096 & 6.5 & 99.0 & 059 & 38 & 000 & 1.0 & 090 \\
\hline $00: 51: 30$ & 11 & -98 & 45 & 0 & 0.08 & 0.20 & 0.27 & 355 & -0.494 & 9.7 & 99.0 & 059 & 36 & 000 & 1.0 & 090 \\
\hline $00: 51: 40$ & 12 & -97 & 44 & 0 & 0.10 & 0.21 & 0.27 & 355 & -0.374 & 10.1 & 99.0 & 263 & 39 & 000 & 1.0 & 090 \\
\hline $00: 51: 50$ & 13 & -97 & 44 & 0 & 0.07 & 0.14 & 0.25 & 354 & -0.698 & 13.4 & 99.0 & 263 & 38 & 000 & 1.0 & 090 \\
\hline $00: 52: 00$ & 14 & -96 & 44 & 0 & 0.10 & 0.03 & 0.21 & 354 & -1.126 & 21.4 & 99.0 & 238 & 32 & 000 & 1.0 & 090 \\
\hline $00: 52: 10$ & 15 & -96 & 45 & 0 & 0.10 & 0.04 & 0.14 & 354 & -0.650 & 21.3 & 99.0 & 220 & 51 & 000 & 1.0 & 090 \\
\hline $00: 52: 20$ & 15 & -95 & 45 & 0 & 0.08 & 0.08 & 0.10 & 354 & -0.099 & 17.9 & 99.0 & 220 & 32 & 000 & 1.0 & 090 \\
\hline $00: 52: 30$ & 15 & -95 & 45 & 0 & 0.15 & 0.03 & 0.07 & 354 & -0.222 & 18.5 & 99.0 & 220 & 43 & 000 & 1.0 & 090 \\
\hline $00: 52: 40$ & 15 & -94 & 45 & 0 & 0.22 & -0.08 & 0.04 & 354 & -0.736 & 22.3 & 99.0 & 205 & 36 & 000 & 1.0 & 090 \\
\hline $0: 52: 50$ & 15 & -93 & 45 & 0 & 0.12 & -0.13 & -0.02 & 354 & -0.693 & 26.5 & 99.0 & 205 & 33 & 000 & 1.0 & 090 \\
\hline$b: 53: 00$ & 14 & -93 & 45 & 0 & -0.04 & -0.13 & -0.08 & 354 & -0.324 & 25.6 & 99.0 & 221 & 41 & 000 & 1.0 & 090 \\
\hline $00: 53: 10$ & 14 & -93 & 44 & 0 & -0.10 & -0.18 & -0.11 & 354 & -0.387 & 25.7 & 99.0 & 210 & 46 & 000 & 1.0 & 090 \\
\hline $00: 53: 20$ & 13 & -94 & 44 & 0 & -0.22 & -0.18 & -0.22 & 354 & 0.268 & 22.6 & 99.0 & 210 & 36 & 000 & 1.0 & 090 \\
\hline $00: 53: 30$ & 12 & -96 & 44 & 0 & -0.36 & -0.26 & -0.31 & 354 & 0.351 & 21.6 & 99.0 & 261 & 37 & 000 & 1.0 & 090 \\
\hline $00: 53: 40$ & 11 & -98 & 44 & 0 & -0.36 & -0.32 & -0.39 & 354 & 0.497 & 20.2 & 99.0 & 323 & 46 & 000 & 1.0 & 090 \\
\hline
\end{tabular}


Time position (m) RPM Speed (Knots) Turn Rudder Gill Wind Current HH;KM:SS East North Port Stbd Long Bow Stern Heading Deg/Min Deg 8 Deg Knots Deg Knots Deg

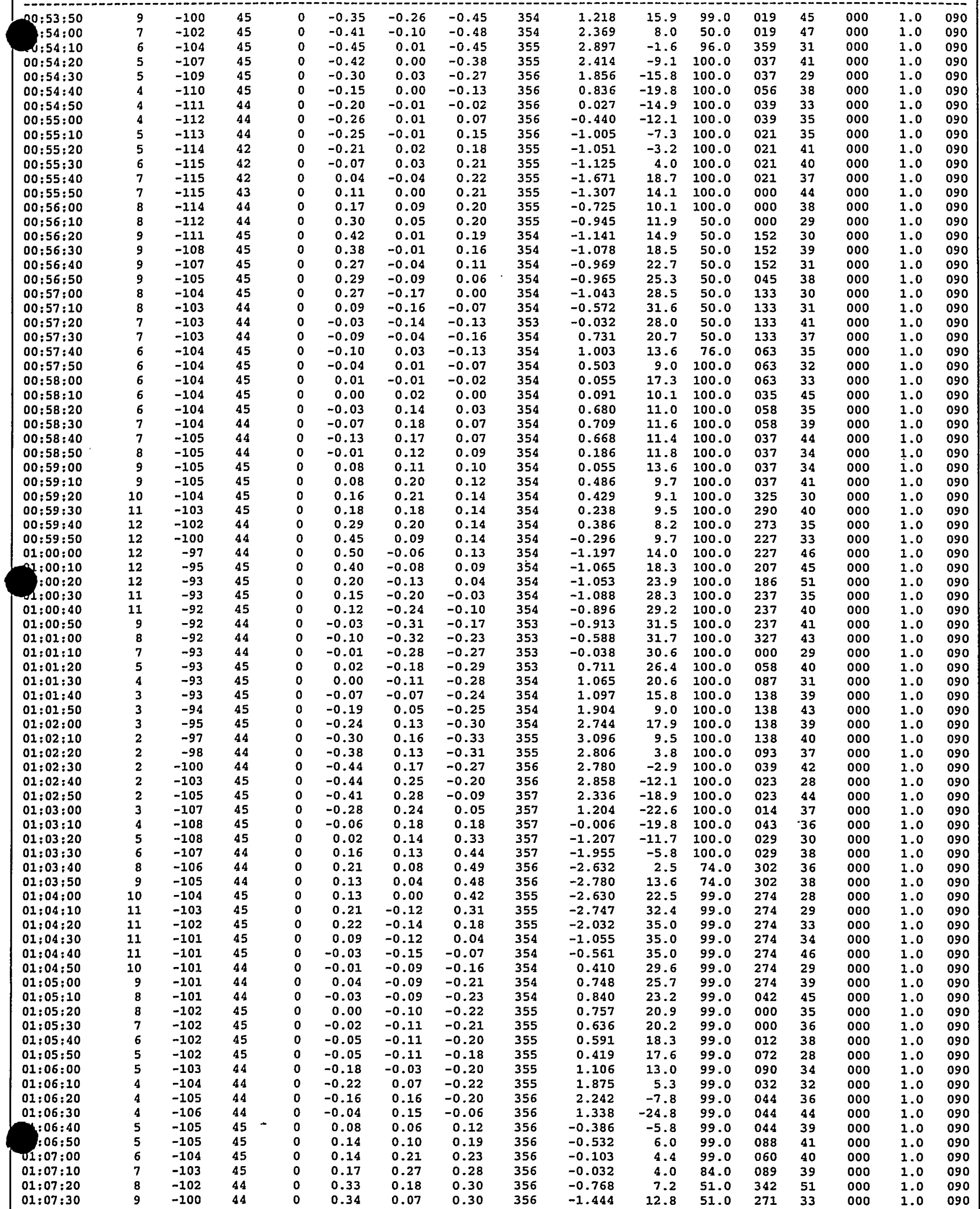




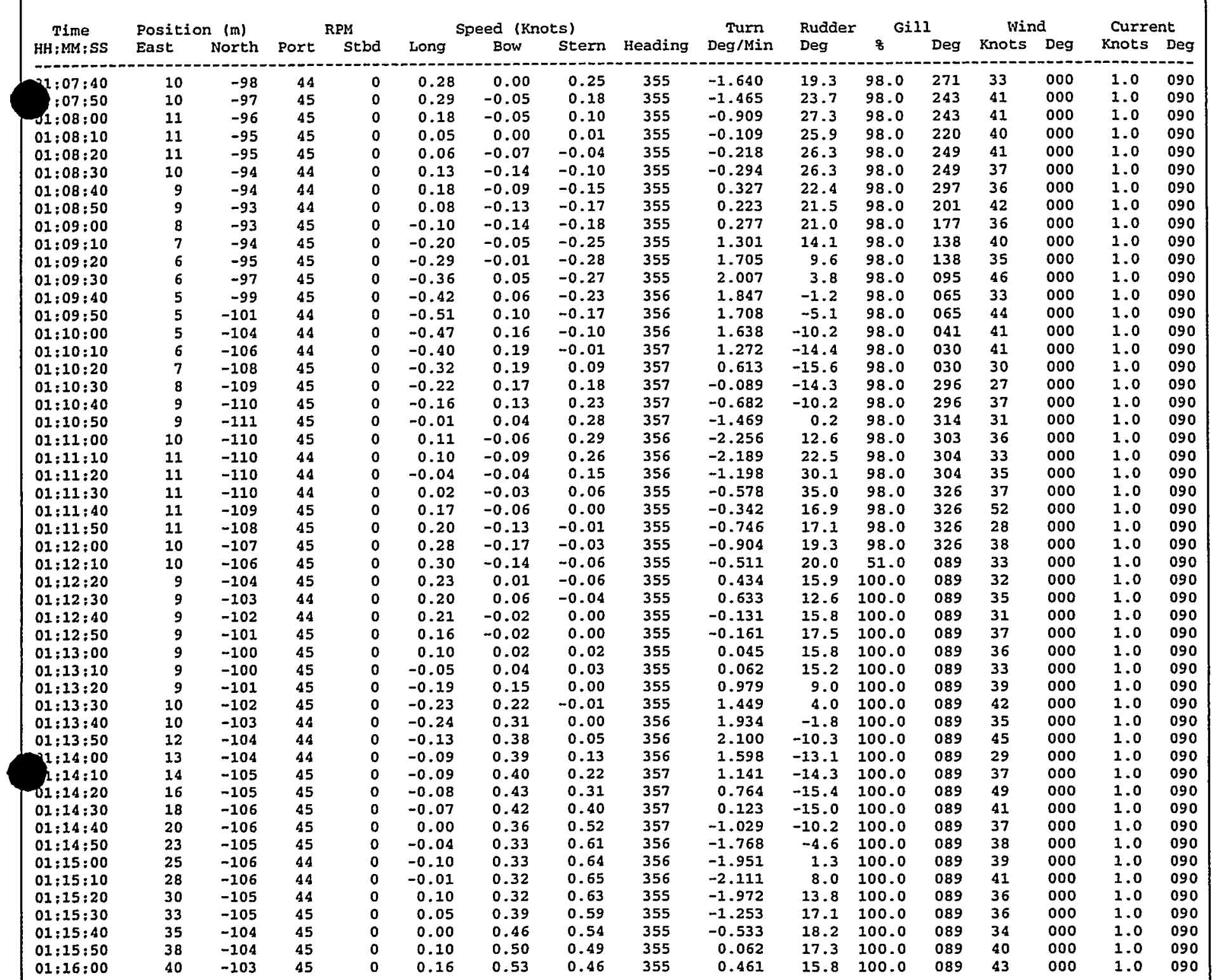




\section{Simulation Track Plot}

Open Ocean

Ship: 150,000 ton, Tanker, partial load

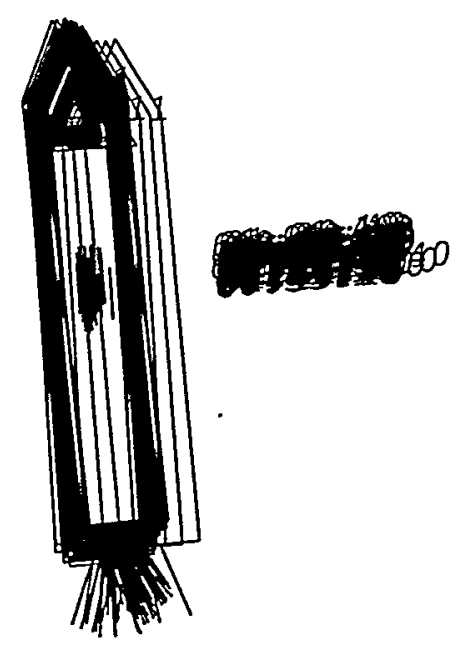

Start Time [hrs:min:sec]: $00: 41: 40$

End Time [hrs:min:sec]: $01: 16: 06$

Delta Plot Time [sec]:

East-West Scale [m]:

30

Date: 05-Feb-99 Time: 13:18:37

East-West Scale [Nm]:

926 .

0.5000 


\section{HANDS-ON SIMULATION \\ SUCTION MOORNG TECHNOLOGY}

Simulation Number: 32

Date: 5 February 1999

Start Time: 14:37

Length of run [secs]: 2760

Ship Condition: $\mathrm{draft}=13.72 \mathrm{~m}$

Environment

Wind Speed [knots]

40 Direction: 0

Sea State, Significant wave height $[\mathrm{m}]: .$.

8 Direction: 180

Current Speed [knots]:

1 Direction: 90

Ship Operator: J.A.

Observer: B.K.J

Final Mating, distance between center of buoy and center of moon pool [m]: 1.5

Evaluation:

This run was very similar to run \# 31.

The captain had to wait for a long time for small waves to occur while the buoy was near the center of the moon pool. 
Ship:

Exercise number:

Scenario: ig file:

Initial condicions:

Exercise identification
591 150,000 ton, Tanker, partial load

754 Suction Mooring System

112 Open Ocean

999 Dummy tug file based on containership

Suction Mooring Buoy

594 150k tanker in partial load - suction Mooring

Time Position (m) RPM Speed (Knots) Gurn Rudder Gill tind

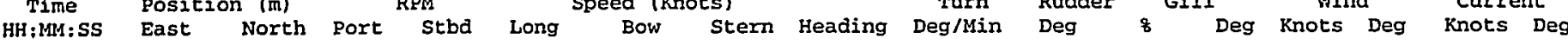

\begin{tabular}{|c|c|c|c|c|c|c|c|c|c|c|c|c|c|c|c|c|}
\hline $\begin{array}{l}00: 25: 00 \\
00: 25: 10\end{array}$ & $\begin{array}{l}13 \\
14\end{array}$ & $\begin{array}{l}-115 \\
-116\end{array}$ & $\begin{array}{l}43 \\
43\end{array}$ & $\begin{array}{l}0 \\
0\end{array}$ & $\begin{array}{l}-0.21 \\
-0.05\end{array}$ & $\begin{array}{l}0.04 \\
0.06\end{array}$ & $\begin{array}{l}0.29 \\
0.24\end{array}$ & $\begin{array}{l}356 \\
356\end{array}$ & $\begin{array}{l}-1.559 \\
-1.130\end{array}$ & $\begin{array}{r}9.2 \\
14.6\end{array}$ & $\begin{array}{l}99.0 \\
99.0\end{array}$ & $\begin{array}{l}000 \\
340\end{array}$ & $\begin{array}{l}34 \\
30\end{array}$ & $\begin{array}{l}000 \\
000\end{array}$ & $\begin{array}{l}1.0 \\
1.0\end{array}$ & $\begin{array}{l}090 \\
090\end{array}$ \\
\hline $00: 25: 20$ & 15 & -115 & 43 & 0 & 0.09 & 0.08 & 0.21 & 355 & -0.879 & 15.1 & 99.0 & 331 & 41 & 000 & 1.0 & 090 \\
\hline $00: 25: 30$ & 16 & -115 & 43 & 0 & 0.12 & 0.06 & 0.19 & 355 & -0.820 & 17.5 & 99.0 & 306 & 40 & 000 & 1.0 & 090 \\
\hline $00: 25: 40$ & 16 & -114 & 43 & 0 & 0.20 & 0.00 & 0.14 & 355 & -0.944 & 20.0 & 99.0 & 306 & 34 & 000 & 1.0 & 090 \\
\hline $00: 25: 50$ & 16 & -113 & 43 & 0 & 0.34 & -0.06 & 0.10 & 355 & -1.015 & 21.0 & 99.0 & 273 & 28 & 000 & 1.0 & 090 \\
\hline $00: 26: 00$ & 16 & -111 & 43 & 0 & 0.33 & -0.08 & 0.04 & 355 & -0.735 & 22.9 & 99.0 & 243 & 38 & 000 & 1.0 & 090 \\
\hline $00: 26: 10$ & 16 & -109 & 44 & 0 & 0.19 & -0.12 & -0.02 & 355 & -0.654 & 25.7 & 99.0 & 243 & 36 & 000 & 1.0 & 090 \\
\hline $00: 26: 20$ & 15 & -109 & 44 & 0 & 0.07 & -0.20 & -0.08 & 355 & -0.729 & 29.7 & 99.0 & 243 & 44 & 000 & 1.0 & 090 \\
\hline $00: 26: 30$ & 14 & -109 & 44 & 0 & -0.08 & -0.22 & -0.14 & 355 & -0.469 & 28.8 & 99.0 & 243 & 35 & 000 & 1.0 & 090 \\
\hline $00: 26: 40$ & 13 & -110 & 44 & 0 & -0.10 & -0.21 & -0.25 & 355 & 0.280 & 25.2 & 99.0 & 348 & 41 & 000 & 1.0 & 090 \\
\hline $00: 26: 50$ & 12 & -110 & 44 & 0 & -0.04 & -0.26 & -0.26 & 355 & 0.016 & 25.8 & 99.0 & 348 & 30 & 000 & 1.0 & 090 \\
\hline $00: 27: 00$ & 11 & -110 & 46 & 0 & -0.07 & -0.27 & -0.27 & 355 & -0.011 & 26.0 & 99.0 & 348 & 39 & 000 & 1.0 & 090 \\
\hline $00: 27: 10$ & 9 & -111 & 46 & 0 & -0.18 & -0.10 & -0.37 & 355 & 1.681 & 17.6 & 99.0 & 308 & 30 & 000 & 1.0 & 090 \\
\hline $00 ; 27: 20$ & 8 & -112 & 46 & 0 & -0.14 & 0.05 & -0.42 & 355 & 2.927 & 2.4 & 99.0 & 334 & 31 & 000 & 1.0 & 090 \\
\hline $00: 27: 30$ & 8 & -113 & 46 & 0 & -0.04 & 0.05 & -0.30 & 356 & 2.241 & -12.1 & 99.0 & 049 & 31 & 000 & 1.0 & 090 \\
\hline $00 ; 27: 40$ & 7 & -113 & 47 & 0 & 0.09 & -0.02 & -0.10 & 356 & 0.515 & -4.6 & 99.0 & 000 & 33 & 000 & 1.0 & 090 \\
\hline $00: 27: 50$ & 7 & -112 & 47 & 0 & 0.20 & -0.09 & -0.02 & 356 & -0.452 & 6.5 & 99.0 & 037 & 35 & 000 & 1.0 & 090 \\
\hline $00: 28: 00$ & 7 & -111 & 46 & 0 & 0.12 & -0.02 & 0.03 & 356 & -0.315 & 8.8 & 99.0 & 058 & 30 & 000 & 1.0 & 090 \\
\hline $00: 28: 10$ & 7 & -110 & 46 & 0 & 0.12 & 0.06 & 0.08 & 356 & -0.089 & 6.9 & 99.0 & 058 & 50 & 000 & 1.0 & 090 \\
\hline $00: 28: 20$ & 7 & -110 & 47 & 0 & 0.11 & 0.05 & 0.12 & 356 & -0.436 & 10.4 & 52.0 & 085 & 40 & 000 & 1.0 & 090 \\
\hline $00: 28: 30$ & 8 & -109 & 47 & 0 & 0.08 & 0.06 & 0.12 & 356 & -0.428 & 12.8 & 100.0 & 033 & 37 & 000 & 1.0 & 090 \\
\hline $00: 28: 40$ & 8 & -109 & 46 & 0 & 0.19 & 0.08 & 0.14 & 355 & -0.347 & 10.9 & 100.0 & 033 & 35 & 000 & 1.0 & 090 \\
\hline $00: 28: 50$ & 8 & -107 & 47 & 0 & 0.34 & 0.08 & 0.15 & 355 & -0.412 & 12.0 & 100.0 & 000 & 40 & 000 & 1.0 & 090 \\
\hline $00: 29: 00$ & 9 & -105 & 46 & 0 & 0.49 & 0.12 & 0.13 & 355 & -0.062 & 11.4 & 100.0 & 267 & 33 & 000 & 1.0 & 090 \\
\hline $00: 29: 10$ & 9 & -102 & 47 & 0 & 0.48 & 0.12 & 0.11 & 355 & 0.079 & 11.0 & 100.0 & 231 & 44 & 000 & 1.0 & 090 \\
\hline $00: 29: 20$ & 10 & -100 & 46 & 0 & 0.46 & 0.06 & 0.10 & 355 & -0.257 & 11.9 & 100.0 & 205 & 28 & 000 & 1.0 & 090 \\
\hline $00: 29: 30$ & 10 & -97 & 47 & 0 & 0.53 & 0.05 & 0.08 & 355 & -0.211 & 12.9 & 100.0 & 205 & 34 & 000 & 1.0 & 090 \\
\hline $00: 29: 40$ & 10 & -95 & 45 & 0 & 0.51 & 0.04 & 0.06 & 355 & -0.149 & 12.7 & 100.0 & 202 & 42 & 000 & 1.0 & 090 \\
\hline $0: 29: 50$ & 10 & -92 & 42 & 0 & 0.42 & -0.06 & 0.04 & 355 & -0.650 & 15.3 & 100.0 & 269 & 40 & 000 & 1.0 & 090 \\
\hline $50: 30: 00$ & 9 & -90 & 43 & 0 & 0.37 & -0.20 & 0.02 & 355 & -1.342 & 20.4 & 100.0 & 269 & 44 & 000 & 1.0 & 090 \\
\hline $00: 30: 10$ & 9 & -89 & 43 & 0 & 0.27 & -0.26 & -0.03 & 355 & -1.456 & 26.4 & 100.0 & 200 & 33 & 000 & 1.0 & 090 \\
\hline $00: 30: 20$ & 8 & -88 & 43 & 0 & 0.09 & -0.21 & -0.09 & 355 & -0.784 & 29.5 & 100.0 & 200 & 41 & 000 & 1.0 & 090 \\
\hline $00: 30: 30$ & 7 & -88 & 43 & 0 & -0.03 & -0.20 & -0.14 & 355 & -0.372 & 29.1 & 100.0 & 154 & 43 & 000 & 1.0 & 090 \\
\hline $00: 30: 40$ & 6 & -88 & 43 & 0 & -0.16 & -0.10 & -0.19 & 355 & 0.587 & 23.5 & 100.0 & 154 & 35 & 000 & 1.0 & 090 \\
\hline $00: 30: 50$ & 6 & -90 & 42 & 0 & -0.29 & 0.01 & -0.26 & 355 & 1.735 & 16.2 & 100.0 & 144 & 42 & 000 & 1.0 & 090 \\
\hline $00: 31: 00$ & 5 & -91 & 42 & 0 & -0.25 & 0.11 & -0.28 & 355 & 2.440 & 5.6 & 100.0 & 093 & 47 & 000 & 1.0 & 090 \\
\hline $00: 31: 10$ & 5 & -92 & 43 & 0 & -0.18 & 0.15 & -0.23 & 356 & 2.387 & -6.3 & 100.0 & 093 & 35 & 000 & 1.0 & 090 \\
\hline $00: 31: 20$ & 5 & -93 & 43 & 0 & -0.24 & 0.16 & -0.16 & 356 & 1.991 & -7.9 & 100.0 & 093 & 44 & 000 & 1.0 & 090 \\
\hline $00: 31: 30$ & 5 & -95 & 43 & 0 & -0.38 & 0.22 & -0.08 & 356 & 1.955 & -9.4 & 100.0 & 093 & 39 & 000 & 1.0 & 090 \\
\hline $00: 31: 40$ & 6 & -97 & 43 & 0 & -0.45 & 0.26 & 0.00 & 357 & 1.618 & -13.1 & 100.0 & 036 & 37 & 000 & 1.0 & 090 \\
\hline $00: 31: 50$ & 7 & -99 & 42 & 0 & -0.43 & 0.19 & 0.08 & 357 & 0.723 & -14.9 & 100.0 & 306 & 43 & 000 & 1.0 & 090 \\
\hline $00: 32: 00$ & 7 & -101 & 42 & 0 & -0.28 & 0.07 & 0.15 & 357 & -0.505 & -11.3 & 100.0 & 306 & 34 & 000 & 1.0 & 090 \\
\hline $00: 32: 10$ & B & -102 & 43 & 0 & -0.27 & -0.01 & 0.19 & 357 & -1.305 & -4.7 & 100.0 & 333 & 28 & 000 & 1.0 & 090 \\
\hline $00: 32: 20$ & 9 & -104 & 44 & 0 & -0.30 & 0.01 & 0.21 & 356 & -1.271 & -1.5 & 100.0 & 333 & 34 & 000 & 1.0 & 090 \\
\hline $00: 32: 30$ & 9 & -105 & 47 & 0 & -0.17 & 0.04 & 0.20 & 356 & -1.025 & 3.0 & 100.0 & 333 & 39 & 000 & 1.0 & 090 \\
\hline $00: 32: 40$ & 10 & -106 & 46 & 0 & -0.06 & 0.10 & 0.13 & 356 & -0.231 & 3.5 & 100.0 & 333 & 45 & 000 & 1.0 & 090 \\
\hline $00: 32: 50$ & 11 & -105 & 46 & 0 & 0.09 & 0.07 & 0.18 & 356 & -0.665 & 6.1 & 100.0 & 333 & 34 & 000 & 1.0 & 090 \\
\hline $00: 33: 00$ & 11 & -104 & 47 & 0 & 0.26 & -0.03 & 0.20 & 356 & -1.417 & 10.0 & 100.0 & 300 & 40 & 000 & 1.0 & 090 \\
\hline $00: 33: 10$ & 11 & -103 & 47 & 0 & 0.35 & -0.11 & 0.17 & 355 & -1.789 & 15.0 & 100.0 & 234 & 41 & 000 & 1.0 & 090 \\
\hline $00: 33: 20$ & 11 & -101 & 46 & 0 & 0.31 & -0.08 & 0.13 & 355 & -1.272 & 18.4 & 100.0 & 220 & 41 & 000 & 1.0 & 090 \\
\hline $00: 33: 30$ & 11 & -100 & 46 & 0 & 0.17 & -0.14 & 0.07 & 355 & -1.309 & 26.0 & 100.0 & 220 & 36 & 000 & 1.0 & 090 \\
\hline $00: 33: 40$ & 11 & -99 & 47 & 0 & 0.08 & -0.21 & -0.02 & 355 & -1.208 & 31.6 & 100.0 & 220 & 35 & 000 & 1.0 & 090 \\
\hline $00: 33: 50$ & 10 & -99 & 47 & 0 & 0.03 & -0.22 & -0.11 & 355 & -0.674 & 29.1 & 100.0 & 302 & 28 & 000 & 1.0 & 090 \\
\hline $00 ; 34 ; 00$ & 9 & -99 & 46 & 0 & -0.01 & -0.20 & -0.17 & 355 & -0.221 & 26.8 & 100.0 & 230 & 30 & 000 & 1.0 & 090 \\
\hline $00: 34: 10$ & 8 & -99 & 46 & 0 & -0.05 & -0.20 & -0.21 & 355 & 0.070 & 25.5 & 100.0 & 147 & 35 & 000 & 1.0 & 090 \\
\hline $00: 34: 20$ & 7 & -100 & 47 & 0 & -0.05 & -0.18 & -0.21 & 355 & 0.239 & 23.9 & 100.0 & 060 & 31 & 000 & 1.0 & 090 \\
\hline $00: 34: 30$ & 6 & -100 & 47 & 0 & -0.10 & -0.10 & -0.23 & 355 & 0.842 & 19.3 & 63.0 & 083 & 36 & 000 & 1.0 & 090 \\
\hline $00: 34: 40$ & 5 & -101 & 46 & 0 & -0.10 & 0.04 & -0.27 & 355 & 2.004 & 4.2 & 99.0 & 083 & 38 & 000 & 1.0 & 090 \\
\hline $00: 34: 50$ & 5 & -101 & 46 & 0 & -0.10 & 0.09 & -0.19 & 355 & 1.771 & -4.8 & 99.0 & 118 & 40 & 000 & 1.0 & 090 \\
\hline $00: 35: 00$ & 5 & -102 & 47 & 0 & -0.17 & 0.14 & -0.14 & 356 & 1.753 & -4.2 & 99.0 & 059 & 26 & 000 & 1.0 & 090 \\
\hline $00: 35: 10$ & 5 & -103 & 47 & 0 & -0.19 & 0.14 & -0.09 & 356 & 1.427 & -5.3 & 99.0 & 059 & 46 & 000 & 1.0 & 090 \\
\hline $00: 35: 20$ & 5 & -104 & 46 & 0 & -0.19 & 0.14 & -0.03 & 356 & 1.101 & -6.5 & 99.0 & 059 & 35 & 000 & 1.0 & 090 \\
\hline $00: 35: 30$ & 6 & -105 & 46 & 0 & -0.13 & 0.11 & 0.03 & 356 & 0.547 & -7.3 & 99.0 & 022 & 38 & 000 & 1.0 & 090 \\
\hline $00: 35: 40$ & 6 & -105 & 47 & 0 & -0.11 & 0.12 & 0.07 & 356 & 0.275 & -6.2 & 99.0 & 000 & 32 & 000 & 1.0 & 090 \\
\hline $00: 35: 50$ & 7 & -106 & 47 & 0 & -0.13 & 0.15 & 0.11 & 356 & 0.257 & -6.2 & 99.0 & 000 & 34 & 000 & 1.0 & 090 \\
\hline $00: 36: 00$ & 8 & -106 & 46 & 0 & -0.15 & 0.17 & 0.14 & 356 & 0.208 & -6.8 & 99.0 & 337 & 40 & 000 & 1.0 & 090 \\
\hline $0: 36: 10$ & 9 & -107 & 46 & 0 & 0.06 & 0.15 & 0.20 & 356 & -0.324 & -3.7 & 99.0 & 337 & 42 & 000 & 1.0 & 090 \\
\hline $0: 36: 20$ & 9 & -106 & 47 & 0 & 0.34 & 0.07 & 0.26 & 356 & -1.232 & 0.4 & 99.0 & 297 & 37 & 000 & 1.0 & 090 \\
\hline $00: 36: 30$ & 10 & -104 & 47 & 0 & 0.38 & 0.01 & 0.28 & 356 & -1.713 & 6.1 & 99.0 & 269 & 34 & 000 & 1.0 & 090 \\
\hline $00: 36: 40$ & 10 & -102 & 46 & 0 & 0.41 & -0.03 & 0.26 & 356 & -1.850 & 11.8 & 99.0 & 269 & 34 & 000 & 1.0 & 090 \\
\hline $00: 36: 50$ & 11 & -99 & 46 & 0 & 0.39 & -0.04 & 0.22 & 355 & -1.650 & 16.7 & 99.0 & 224 & 35 & 000 & 1.0 & $\begin{array}{l}090 \\
090\end{array}$ \\
\hline & 11 & -98 & 47 & 0 & 0.32 & -0.01 & 0.15 & 355 & -1.043 & 20.1 & 99.0 & 224 & 47 & 000 & & \\
\hline
\end{tabular}




\begin{tabular}{|c|c|c|c|c|c|c|c|c|c|c|c|c|c|c|c|c|}
\hline Time & Positi & a $(m)$ & & $P M$ & & eed $(\mathrm{Kr}$ & Es) & & Turn & Rudde & Gi & & Wis & & Curre & \\
\hline $\mathrm{HH}$ :MM ; SS & East & North & Port & stbd & Long & Bow & Stern & Heading & Deg/Min & Deg & 8 & Deg & Knots & Deg & Knots & Deg \\
\hline & & & & & & & & 355 & -1025 & 229 & 990 & 215 & & & 10 & קח \\
\hline $\begin{array}{l}10 \\
20\end{array}$ & 11 & -96 & 45 & $\begin{array}{l}0 \\
0\end{array}$ & $\begin{array}{l}0.32 \\
0.29\end{array}$ & $\begin{array}{l}-0.07 \\
-0.23\end{array}$ & 0.09 & $\begin{array}{l}355 \\
355\end{array}$ & $\begin{array}{l}-1.025 \\
-1.655\end{array}$ & $\begin{array}{l}22.9 \\
29.4\end{array}$ & 99.0 & $\begin{array}{l}215 \\
215\end{array}$ & $\begin{array}{l}25 \\
46\end{array}$ & 000 & $\begin{array}{l}1.0 \\
1.0\end{array}$ & 090 \\
\hline $0: 37: 30$ & 10 & -93 & 45 & 0 & 0.18 & -0.22 & -0.07 & 354 & -0.977 & 32.7 & 99.0 & 215 & 41 & 000 & 1.0 & 090 \\
\hline $00: 37: 40$ & 9 & -93 & 45 & 0 & 0.04 & -0.15 & -0.15 & 354 & 0.015 & 28.7 & 99.0 & 215 & 40 & 000 & 1.0 & 090 \\
\hline $00: 37: 50$ & 8 & -93 & 45 & 0 & -0.05 & -0.17 & -0.19 & 354 & 0.173 & 27.1 & 99.0 & 215 & 46 & 000 & 1.0 & 090 \\
\hline $00: 38: 00$ & 7 & -93 & 45 & 0 & -0.09 & -0.14 & -0.22 & 354 & 0.522 & 24.5 & 99.0 & 122 & 45 & 000 & 1.0 & 090 \\
\hline $00: 38 ; 10$ & 7 & -94 & 45 & 0 & -0.15 & -0.05 & -0.29 & 355 & 1.511 & 16.8 & 49.0 & 122 & 38 & 000 & 1.0 & 090 \\
\hline $00: 38: 20$ & 6 & -95 & 45 & 0 & -0.18 & 0.03 & -0.33 & 355 & 2.263 & 7.5 & 49.0 & 122 & 40 & 000 & 1.0 & 090 \\
\hline $00: 38: 30$ & 5 & -96 & 44 & 0 & -0.14 & 0.06 & -0.31 & 355 & 2.324 & -3.1 & 49.0 & 122 & 32 & 000 & 1.0 & 090 \\
\hline $00: 38: 40$ & 5 & -97 & 44 & 0 & -0.20 & 0.02 & -0.26 & 356 & 1.759 & -3.5 & 49.0 & 122 & 34 & 000 & 1.0 & 090 \\
\hline $00: 38: 50$ & 4 & -98 & 44 & 0 & -0.33 & 0.00 & -0.21 & 356 & 1.348 & -4.1 & 49.0 & 122 & 40 & 000 & 1.0 & 090 \\
\hline $00: 39: 00$ & 4 & -100 & 45 & 0 & -0.42 & 0.00 & -0.16 & 356 & 0.999 & . -5.6 & 49.0 & 122 & 36 & 000 & 1.0 & 090 \\
\hline $00: 39: 10$ & 4 & -102 & 45 & 0 & -0.48 & 0.04 & -0.09 & 356 & 0.821 & -7.6 & 100.0 & 053 & 41 & 000 & 1.0 & 090 \\
\hline $00: 39: 20$ & 4 & -105 & 45 & 0 & -0.46 & 0.07 & -0.01 & 356 & 0.540 & -8.7 & 100.0 & 053 & 32 & 000 & 1.0 & 090 \\
\hline $00: 39: 30$ & 4 & -107 & 50 & 0 & -0.43 & 0.08 & 0.06 & 356 & 0.151 & -8.9 & 100.0 & 022 & 44 & 000 & 1.0 & 090 \\
\hline $00: 39: 40$ & 5 & -109 & 49 & 0 & -0.34 & 0.08 & 0.13 & 356 & -0.328 & -7.4 & 100.0 & 022 & 49 & 000 & 1.0 & 090 \\
\hline $00: 39: 50$ & 6 & -110 & 49 & 0 & -0.15 & 0.09 & 0.18 & 356 & -0.558 & -3.5 & 100.0 & 022 & 38 & 000 & 1.0 & 090 \\
\hline $00: 40 ; 00$ & 6 & -111 & 50 & 0 & 0.02 & 0.12 & 0.23 & 356 & -0.707 & -0.5 & 100.0 & 000 & 35 & 000 & 1.0 & 090 \\
\hline $00: 40: 10$ & 7 & -110 & 49 & 0 & 0.07 & 0.13 & 0.29 & 356 & -1.015 & 2.5 & 100.0 & 298 & 37 & 000 & 1.0 & 090 \\
\hline $00: 40: 20$ & 9 & -110 & 50 & 0 & 0.08 & 0.07 & 0.30 & 356 & -1.433 & 9.3 & 100.0 & 299 & 34 & 000 & 1.0 & 090 \\
\hline $00: 40: 30$ & 9 & -109 & 48 & 0 & 0.28 & -0.01 & 0.28 & 356 & -1.830 & 12.6 & 100.0 & 299 & 37 & 000 & 1.0 & 090 \\
\hline $00: 40: 40$ & 10 & -107 & 45 & 0 & 0.36 & -0.08 & 0.23 & 355 & -2.015 & 18.2 & 100.0 & 269 & 37 & 000 & 1.0 & 090 \\
\hline $00: 40: 50$ & 10 & -105 & 45 & 0 & 0.34 & -0.15 & 0.16 & 355 & -1.996 & 24.8 & 100.0 & 269 & 34 & 000 & 1.0 & 090 \\
\hline $00: 41: 00$ & 9 & -104 & 46 & 0 & 0.29 & -0.21 & 0.06 & 355 & -1.760 & 30.6 & 100.0 & 237 & 32 & 000 & 1.0 & 090 \\
\hline $00: 41: 10$ & 9 & -103 & 46 & 0 & 0.11 & -0.17 & -0.05 & 354 & -0.736 & 33.3 & 100.0 & 237 & 39 & 000 & 1.0 & 090 \\
\hline $00: 41: 20$ & 8 & -102 & 46 & 0 & 0.04 & -0.07 & -0.13 & 354 & 0.382 & 24.2 & 100.0 & 237 & 33 & 000 & 1.0 & 090 \\
\hline $00: 41: 30$ & 8 & -102 & 46 & 0 & 0.13 & -0.15 & -0.17 & 355 & 0.143 & 26.1 & 100.0 & 237 & 34 & 000 & 1.0 & 090 \\
\hline $00: 41: 40$ & 6 & -101 & 45 & 0 & 0.17 & -0.23 & -0.22 & 355 & -0.085 & 27.4 & 100.0 & 215 & 36 & 000 & 1.0 & 090 \\
\hline $00: 41: 50$ & 5 & -101 & 45 & 0 & 0.10 & -0.14 & -0.25 & 355 & 0.674 & 22.9 & 100.0 & 090 & 37 & 000 & 1.0 & 090 \\
\hline $00: 42: 00$ & 4 & -101 & 46 & 0 & 0.01 & -0.04 & -0.28 & 355 & 1.491 & 30.2 & 100.0 & 090 & 28 & 000 & 1.0 & 090 \\
\hline $00: 42: 10$ & 4 & -101 & 46 & 0 & -0.04 & 0.05 & -0.26 & 355 & 1.998 & 15.4 & 100.0 & 080 & 38 & 000 & 1.0 & 090 \\
\hline $00: 42: 20$ & 3 & -101 & 46 & 0 & -0.03 & 0.04 & -0.18 & 355 & 1.395 & 9.5 & 100.0 & 056 & 39 & 000 & 1.0 & 090 \\
\hline $00: 42: 30$ & 3 & -101 & 46 & 0 & -0.13 & 0.03 & -0.18 & 356 & 1.309 & 16.7 & 100.0 & 056 & 44 & 000 & 1.0 & 090 \\
\hline $00: 42: 40$ & 3 & -102 & 46 & 0 & -0.18 & 0.12 & -0.23 & 356 & 2.185 & 8.7 & 100.0 & 056 & 36 & 000 & 1.0 & 090 \\
\hline $00: 42: 50$ & 2 & -103 & 45 & 0 & -0.17 & 0.18 & -0.21 & 356 & 2.450 & -2.7 & 100.0 & 056 & 30 & 000 & 1.0 & 090 \\
\hline $00: 43: 00$ & 3 & -104 & 45 & 0 & -0.18 & 0.21 & -0.15 & 357 & 2.294 & -7.9 & 100.0 & 033 & 40 & 000 & 1.0 & 090 \\
\hline $00: 43: 10$ & 3 & -105 & 46 & 0 & -0.13 & 0.23 & -0.07 & 357 & 1.912 & -13.5 & 100.0 & 033 & 29 & 000 & 1.0 & 090 \\
\hline $00: 43: 20$ & 3 & -105 & 46 & 0 & -0.11 & 0.24 & 0.02 & 357 & 1.445 & -13.9 & 100.0 & 340 & 40 & 000 & 1.0 & 090 \\
\hline $90: 43: 30$ & 4 & -106 & 46 & 0 & 0.00 & 0.21 & 0.11 & 357 & 0.598 & -12.0 & 100.0 & 275 & 30 & 000 & 1.0 & 090 \\
\hline $1: 43: 40$ & 5 & -105 & 46 & 0 & 0.13 & 0.14 & 0.22 & 357 & -0.476 & -5.4 & 100.0 & 305 & 29 & 000 & 1.0 & 090 \\
\hline $00: 43: 50$ & 6 & -104 & 46 & 0 & 0.21 & 0.09 & 0.28 & 357 & -1.202 & -0.2 & 100.0 & 274 & 37 & 000 & 1.0 & 090 \\
\hline $00: 44: 00$ & 7 & -103 & 45 & 0 & 0.29 & 0.01 & 0.31 & 357 & -1.930 & 6.3 & 100.0 & 324 & 49 & 000 & 1.0 & 090 \\
\hline $00: 44: 10$ & 7 & -101 & 45 & 0 & 0.37 & -0.05 & 0.30 & 357 & -2.215 & 12.4 & 100.0 & 289 & 34 & 000 & 1.0 & 090 \\
\hline $00: 44: 20$ & B & -99 & 46 & 0 & 0.37 & -0.04 & 0.24 & 356 & -1.832 & 17.9 & 100.0 & 289 & 49 & 000 & 1.0 & 090 \\
\hline $00: 44: 30$ & 8 & -98 & 46 & 0 & 0.27 & -0.03 & 0.17 & 356 & -1.297 & 22.3 & 100.0 & 289 & 40 & 000 & 1.0 & 090 \\
\hline $00 ; 44 ; 40$ & 8 & -96 & 46 & 0 & 0.29 & -0.07 & 0.09 & 356 & -1.064 & 24.6 & 100.0 & 289 & 39 & 000 & 2.0 & 090 \\
\hline $00: 44: 50$ & 8 & -94 & 46 & 0 & 0.37 & -0.09 & 0.01 & 356 & -0.686 & 25.3 & 100.0 & 289 & 41 & 000 & 1.0 & 090 \\
\hline $00: 45: 00$ & 8 & -93 & 45 & 0 & 0.41 & -0.09 & -0.06 & 356 & -0.133 & 24.6 & 100.0 & 289 & 33 & 000 & 1.0 & 090 \\
\hline $00: 45: 10$ & 7 & -90 & 45 & 0 & 0.53 & -0.10 & -0.13 & 356 & 0.235 & 23.7 & 100.0 & 289 & 44 & 000 & 1.0 & 090 \\
\hline $00: 45: 20$ & 6 & -87 & 46 & 0 & 0.54 & -0.10 & -0.19 & 356 & 0.581 & 21.9 & 100.0 & 289 & 32 & 000 & 1.0 & 090 \\
\hline $00: 45: 30$ & 5 & -85 & 46 & 0 & 0.41 & -0.05 & -0.23 & 356 & 1.116 & 17.4 & 100.0 & 289 & 37 & 000 & 1.0 & 090 \\
\hline $00: 45: 40$ & 4 & -83 & 46 & 0 & 0.41 & -0.05 & -0.23 & 356 & 1.163 & 13.6 & 100.0 & 289 & 37 & 000 & 1.0 & 090 \\
\hline $00 ; 45: 50$ & 3 & -81 & 46 & 0 & 0.50 & -0.15 & -0.22 & 356 & 0.450 & 13.0 & 100.0 & 289 & 42 & 000 & 1.0 & 090 \\
\hline
\end{tabular}


Open Ocean

\section{Simulation Track Plot}

Ship: 150,000 ton, Tanker, partial load

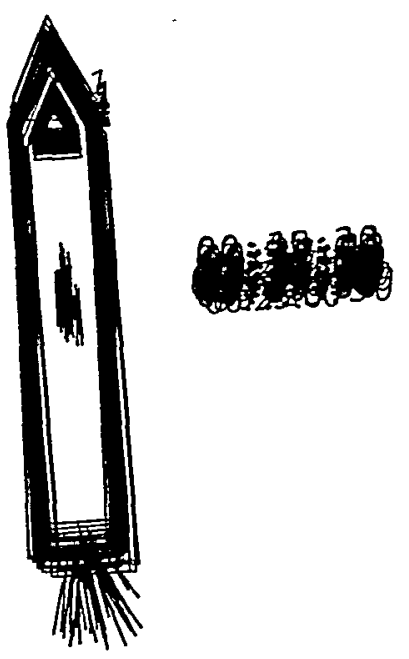

Date: 05-Feb-99

Time: 14:37:00

Start Time [hrs:min:sec]: 00:25:00 End Time [hrs:min:sec]: $00: 46: 00$ Delta Plot Time [sec]:

East-West Scale [m]: 30 East-West Scale [Nm]: 926. 0.5000 


\section{HANDS-ON SIMULATION}

\section{SUCTION MOORNG TECHNOLOGY}

Simulation Number: 33

Date: 5 February 1999

Start Time: 15:29

Length of run [secs]: 3065

Ship Condition: $\mathrm{draft}=13.72 \mathrm{~m}$

Environment

Wind Speed [knots]

40 Direction: 0

Sea State, Significant wave height $[\mathrm{m}]$...

8 Direction: 180

Current Speed [knots]:

2 Direction: 90

Ship Operator: J.A.

Observer: B.K.J

Final Mating, distance between center of buoy and center of moon pool [m]: 1.9

Evaluation:

Mating was made in the second try.

Wind and waves caught the bow and blew it to the port close to 20 degrees. The captain used full ahead and reversed the thruster and brought the ship back with no particular problem. Most of the time was used waiting for small waves to occur when the buoy was near the center of the moon pool. 
Ship:

591150,000 ton, Tanker, partial load

Exercise number:

754 Suction Hooring System

Scenar

112 Open Ocean

g file:

Dummy tug file based on containership

Initial conditions:

590 Suction Mooring Buoy

Exercise identification: RuN $\$ 33$

RPM

Speed (Knots)

Turn Rudder Gill Wind

Current

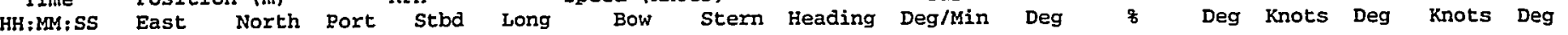

\begin{tabular}{|c|c|c|c|c|c|c|c|c|c|c|c|c|c|c|c|c|}
\hline $00: 16: 40$ & -20 & -127 & 74 & 0 & 0.12 & 1.06 & $\begin{array}{l}-1.11 \\
-1.06\end{array}$ & $\begin{array}{l}340 \\
343\end{array}$ & $\begin{array}{l}13.786 \\
14.857\end{array}$ & $\begin{array}{l}35.0 \\
35.0\end{array}$ & $\begin{array}{l}98.0 \\
98.0\end{array}$ & $\begin{array}{l}157 \\
157\end{array}$ & $\begin{array}{l}46 \\
40\end{array}$ & $\begin{array}{l}000 \\
000\end{array}$ & $\begin{array}{l}2.0 \\
2.0\end{array}$ & $\begin{array}{l}090 \\
090\end{array}$ \\
\hline $00: 16: 50$ & $\begin{array}{l}-20 \\
-70\end{array}$ & $\begin{array}{l}-126 \\
-125\end{array}$ & $\begin{array}{l}74 \\
74\end{array}$ & 0. & $\begin{array}{l}0.21 \\
0.32\end{array}$ & $\begin{array}{l}1.28 \\
1.46\end{array}$ & $\begin{array}{l}-1.06 \\
-0.98\end{array}$ & $\begin{array}{l}343 \\
345\end{array}$ & $\begin{array}{l}14.857 \\
15.535\end{array}$ & $\begin{array}{l}35.0 \\
35.0\end{array}$ & $\begin{array}{l}98.0 \\
98.0\end{array}$ & $\begin{array}{l}157 \\
157\end{array}$ & 43 & 000 & 2.0 & $\begin{array}{l}090 \\
090\end{array}$ \\
\hline $\begin{array}{l}00: 17 ; 00 \\
00: 17 ; 10\end{array}$ & $\begin{array}{l}-19 \\
-18\end{array}$ & -122 & 64 & 0 & 0.48 & 1.32 & -0.82 & 348 & 13.560 & 20.5 & 98.0 & 157 & 43 & 000 & 2.0 & 090 \\
\hline $00: 17: 20$ & -18 & -120 & 50 & 0 & 0.53 & 1.03 & -0.50 & 350 & 9.749 & 1.5 & 98.0 & 157 & 36 & 000 & 2.0 & 090 \\
\hline $00: 17: 30$ & -17 & -117 & 49 & 0 & 0.40 & 0.87 & -0.15 & 351 & 6.518 & -17.5 & 98.0 & 157 & 47 & 000 & 2.0 & 090 \\
\hline $00: 17: 40$ & -15 & -115 & 50 & 0 & 0.23 & 0.75 & 0.26 & 352 & 3.136 & -35.0 & 98.0 & 074 & 31 & 000 & 2.0 & 090 \\
\hline $00: 17: 50$ & -12 & -113 & 45 & 0 & 0.35 & 0.56 & 0.63 & 352 & -0.424 & -34.3 & 98.0 & 038 & 38 & 000 & 2.0 & 090 \\
\hline $00: 18: 00$ & -9 & -111 & 45 & 0 & 0.48 & 0.39 & 0.90 & 352 & -3.224 & -24.6 & 98.0 & 038 & 27 & 000 & 2.0 & 090 \\
\hline $00: 18: 10$ & -6 & -108 & 45 & 0 & 0.48 & 0.25 & 1.07 & 351 & -5.226 & -9.1 & 98.0 & 187 & 33 & 000 & 2.0 & 090 \\
\hline $00: 18: 20$ & -3 & -105 & 44 & 0 & 0.46 & 0.16 & 1.13 & 350 & -6.133 & 9.7 & 98.0 & 180 & 39 & 000 & 2.0 & 090 \\
\hline $00: 18: 30$ & 0 & -102 & 44 & 0 & 0.38 & 0.25 & 1.07 & 349 & -5.184 & 24.1 & 98.0 & 180 & 43 & 000 & 2.0 & 090 \\
\hline $00: 18: 40$ & 3 & -100 & 45 & 0 & 0.24 & 0.43 & 1.04 & 348 & -3.848 & 5.1 & 98.0 & 180 & 27 & 000 & 2.0 & 090 \\
\hline $00: 18: 50$ & 6 & -98 & 45 & 0 & 0.17 & 0.33 & 1.06 & 348 & -4.668 & 15.3 & 98.0 & 180 & 36 & 000 & 2.0 & 090 \\
\hline $00: 19: 00$ & 9 & -96 & 45 & 0 & 0.09 & -0.02 & 1.01 & 347 & -6.531 & 34.2 & 98.0 & 180 & 39 & 000 & 2.0 & 090 \\
\hline $00: 19: 10$ & 11 & -96 & 45 & 0 & -0.02 & -0.02 & 0.92 & 346 & -5.934 & 35.0 & 98.0 & 127 & 36 & 000 & 2.0 & 090 \\
\hline $00: 19: 20$ & 14 & -95 & 45 & 0 & -0.15 & -0.01 & 0.83 & 345 & -5.364 & 35.0 & 98.0 & 127 & 38 & 000 & 2.0 & 090 \\
\hline $00: 19: 30$ & 16 & -96 & 44 & 0 & -0.35 & 0.02 & 0.77 & 344 & -4.751 & 35.0 & 98.0 & 127 & 31 & 000 & 2.0 & 090 \\
\hline $00: 19: 40$ & 19 & -98 & 44 & 0 & -0.50 & 0.18 & 0.71 & 343 & -3.365 & 35.0 & 98.0 & 077 & 37 & 000 & 2.0 & 090 \\
\hline $00: 19: 50$ & 22 & -99 & 44 & 0 & -0.51 & 0.21 & 0.65 & 343 & -2.833 & 35.0 & 98.0 & 031 & 31 & 000 & 2.0 & 090 \\
\hline $00: 20: 00$ & 25 & -101 & 47 & 0 & -0.46 & 0.46 & 0.58 & 342 & -0.727 & 35.0 & 98.0 & 031 & 44 & 000 & 2.0 & 090 \\
\hline $00: 20: 10$ & 28 & -102 & 46 & 0 & -0.31 & 0.65 & 0.49 & 342 & 0.966 & 35.0 & 98.0 & 031 & 31 & 000 & 2.0 & 090 \\
\hline $00: 20: 20$ & 31 & -103 & 46 & 0 & -0.28 & 0.59 & 0.41 & 342 & 1.113 & 35.0 & 98.0 & 269 & 38 & 000 & 2.0 & 090 \\
\hline $00: 20: 30$ & 34 & -103 & 47 & 0 & -0.28 & 0.64 & 0.33 & 343 & 1.990 & 35.0 & 98.0 & 269 & 34 & 000 & 2.0 & 090 \\
\hline $00: 20: 40$ & 37 & -104 & 48 & 0 & -0.23 & 0.78 & 0.26 & 343 & 3.303 & 35.0 & 98.0 & 359 & 33 & 000 & 2.0 & 090 \\
\hline $00: 20: 50$ & 40 & -104 & 48 & 0 & -0.14 & 0.74 & 0.21 & 344 & 3.386 & 34.5 & 98.0 & 359 & 29 & 000 & 2.0 & 090 \\
\hline $00: 21: 00$ & 42 & -104 & 49 & 0 & -0.01 & 0.60 & 0.23 & 344 & 2.332 & 15.5 & 98.0 & 359 & 35 & 000 & 2.0 & 090 \\
\hline $00: 21: 10$ & 44 & -103 & 49 & 0 & -0.01 & 0.47 & 0.33 & 344 & 0.897 & 6.2 & 98.0 & 359 & 40 & 000 & 2.0 & 090 \\
\hline $00: 21: 20$ & 46 & -103 & 48 & 0 & 0.02 & 0.32 & 0.38 & 345 & -0.379 & 24.1 & 98.0 & 268 & 38 & 000 & 2.0 & 090 \\
\hline $0: 21: 30$ & 47 & -102 & 49 & 0 & 0.13 & 0.16 & 0.30 & 344 & -0.934 & 35.0 & 98.0 & 268 & 34 & 000 & 2.0 & 090 \\
\hline $0: 21: 40$ & 48 & -101 & 49 & 0 & 0.20 & 0.11 & 0.21 & 344 & -0.593 & 35.0 & 98.0 & 268 & 42 & 000 & 2.0 & 090 \\
\hline $00 ; 21 ; 50$ & 48 & -100 & 49 & 0 & 0.12 & 0.23 & 0.11 & 344 & 0.720 & 35.0 & 98.0 & 268 & 49 & 000 & 2.0 & 090 \\
\hline $00: 22: 00$ & 49 & -99 & 48 & 0 & 0.09 & 0.26 & 0.04 & 344 & 1.396 & 35.0 & 98.0 & 268 & 38 & 000 & 2.0 & 090 \\
\hline $00: 22: 10$ & 50 & -98 & 48 & 0 & 0.18 & 0.30 & -0.02 & 345 & 2.001 & 35.0 & 98.0 & 268 & 45 & 000 & 2.0 & 090 \\
\hline $00 ; 22: 20$ & 50 & -97 & 48 & 0 & 0.23 & 0.21 & -0.03 & 345 & 1.549 & 27.2 & 98.0 & 268 & 31 & 000 & 2.0 & 090 \\
\hline $00: 22: 30$ & 50 & -96 & 48 & 0 & 0.25 & -0.07 & -0.05 & 345 & -0.076 & 35.0 & 98.0 & 268 & 31 & 000 & 2.0 & 090 \\
\hline $00: 22: 40$ & 49 & -95 & 49 & 0 & 0.17 & -0.29 & -0.08 & 345 & -1.297 & 35.0 & 98.0 & 232 & 44 & 000 & 2.0 & 090 \\
\hline $00: 22: 50$ & 48 & -95 & 48 & 0 & -0.05 & -0.21 & -0.10 & 345 & -0.699 & 35.0 & 98.0 & 262 & 35 & 000 & 2.0 & 090 \\
\hline $00: 23 ; 00$ & 47 & -96 & 48 & 0 & -0.15 & 0.05 & -0.11 & 345 & 1.035 & 35.0 & 98.0 & 324 & 34 & 000 & 2.0 & 090 \\
\hline $00: 23: 10$ & 47 & -96 & 48 & 0 & -0.13 & -0.05 & -0.10 & 345 & 0.348 & 35.0 & 98.0 & 324 & $4 I$ & 000 & 2.0 & 090 \\
\hline $00 ; 23: 20$ & 47 & -97 & 49 & 0 & -0.13 & -0.16 & -0.10 & 345 & -0.403 & 35.0 & 98.0 & 340 & 39 & 000 & 2.0 & 090 \\
\hline $00: 23: 30$ & 47 & -98 & 48 & 0 & -0.07 & -0.05 & -0.10 & 345 & 0.317 & 35.0 & 98.0 & 291 & 46 & 000 & 2.0 & 090 \\
\hline $00: 23: 40$ & 46 & -98 & 48 & 0 & -0.08 & 0.12 & -0.12 & 345 & 1.489 & 30.7 & 98.0 & 291 & 41 & 000 & 2.0 & 090 \\
\hline $00: 23: 50$ & 47 & -99 & 49 & 0 & -0.10 & 0.45 & -0.09 & 346 & 3.440 & 24.5 & 98.0 & 301 & 43 & 000 & 2.0 & 090 \\
\hline $00: 24: 00$ & 48 & -99 & 49 & 0 & -0.02 & 0.49 & -0.02 & 346 & 3.233 & 17.5 & 98.0 & 318 & 43 & 000 & 2.0 & 090 \\
\hline $00: 24: 10$ & 49 & -98 & 49 & 0 & 0.02 & 0.43 & 0.08 & 347 & 2.227 & 15.3 & 98.0 & 318 & 35 & 000 & 2.0 & 090 \\
\hline $00: 24: 20$ & 51 & -98 & 49 & 0 & 0.12 & 0.48 & 0.16 & 347 & 2.043 & 12.0 & 98.0 & 318 & 35 & 000 & 2.0 & 090 \\
\hline $00: 24: 30$ & 52 & -96 & 48 & 0 & 0.24 & 0.35 & 0.26 & 347 & 0.571 & 3.7 & 98.0 & 318 & 44 & 000 & 2.0 & 090 \\
\hline $00: 24: 40$ & 53 & -95 & 49 & 0 & 0.25 & 0.13 & 0.40 & 347 & -1.684 & 3.7 & 98.0 & 270 & 33 & 000 & 2.0 & 090 \\
\hline $00: 24: 50$ & 54 & -93 & 49 & 0 & 0.21 & 0.06 & 0.53 & 347 & -3.013 & -2.6 & 98.0 & 270 & 45 & 000 & 2.0 & 090 \\
\hline $00: 25: 00$ & 56 & -92 & 49 & 0 & 0.15 & -0.01 & 0.61 & 346 & -3.948 & 11.5 & 98.0 & 270 & 34 & 000 & 2.0 & 090 \\
\hline $00: 25: 10$ & 57 & -91 & 49 & 0 & 0.27 & -0.03 & 0.60 & 345 & -3.998 & 22.5 & 98.0 & 270 & 30 & 000 & 2.0 & 090 \\
\hline $00: 25: 20$ & 58 & -89 & 48 & 0 & 0.36 & 0.10 & 0.52 & 345 & -2.641 & 26.9 & 98.0 & 243 & 41 & 000 & 2.0 & 090 \\
\hline $00: 25: 30$ & 59 & -86 & 49 & 0 & 0.33 & 0.14 & 0.41 & 345 & -1.730 & 31.7 & 98.0 & 243 & 40 & 000 & 2.0 & 090 \\
\hline $00: 25: 40$ & 60 & -85 & 48 & 0 & 0.35 & 0.06 & 0.30 & 344 & -1.536 & 35.0 & 98.0 & 243 & 34 & 000 & 2.0 & 090 \\
\hline $00: 25: 50$ & 60 & -82 & 49 & 0 & 0.46 & -0.03 & 0.21 & 344 & -1.539 & 26.0 & 98.0 & 243 & 28 & 000 & 2.0 & 090 \\
\hline $00: 26: 00$ & 59 & -80 & 49 & 0 & 0.43 & -0.02 & 0.17 & 344 & -1.236 & 25.8 & 98.0 & 221 & 38 & 000 & 2.0 & 090 \\
\hline $00: 26: 10$ & 59 & -78 & 45 & 0 & 0.29 & -0.11 & 0.13 & 344 & -1.543 & 32.5 & 98.0 & 221 & 36 & 000 & 2.0 & 090 \\
\hline $00: 26: 20$ & 59 & -77 & 46 & 0 & 0.16 & -0.29 & 0.08 & 343 & -2.383 & 35.0 & 98.0 & 241 & 44 & 000 & 2.0 & 090 \\
\hline $00: 26: 30$ & 58 & -77 & 46 & 0 & 0.02 & -0.31 & 0.04 & 343 & -2.267 & 35.0 & 98.0 & 241 & 35 & 000 & 2.0 & 090 \\
\hline $00: 26: 40$ & 57 & -77 & 46 & 0 & -0.06 & -0.43 & 0.02 & 342 & -2.878 & 35.0 & 98.0 & 241 & 41 & 000 & 2.0 & 090 \\
\hline $00: 26: 50$ & 56 & -78 & 46 & 0 & -0.08 & -0.61 & 0.02 & 342 & -4.006 & 35.0 & 98.0 & 241 & 30 & 000 & 2.0 & 090 \\
\hline $00: 27: 00$ & 55 & -79 & 52 & 0 & -0.19 & -0.65 & 0.01 & 341 & -4.193 & 35.0 & 98.0 & 208 & 39 & 000 & 2.0 & 090 \\
\hline $00: 27: 10$ & 54 & -81 & 52 & 0 & -0.37 & -0.25 & -0.04 & 341 & -1.362 & 35.0 & 98.0 & 208 & 30 & 000 & 2.0 & 090 \\
\hline $00: 27 ; 20$ & 54 & -83 & 52 & 0 & -0.42 & 0.22 & -0.08 & 341 & 1.897 & 35.0 & 98.0 & 208 & 31 & 000 & 2.0 & 090 \\
\hline $00: 27: 30$ & 56 & -85 & 51 & 0 & -0.40 & 0.31 & -0.10 & 341 & 2.618 & 35.0 & 98.0 & 208 & 31 & 000 & 2.0 & 090 \\
\hline $00: 27: 40$ & 57 & -86 & 51 & 0 & -0.36 & 0.25 & -0.12 & 342 & 2.387 & 35.0 & 98.0 & 208 & 33 & 000 & 2.0 & 090 \\
\hline $0: 27: 50$ & 57 & -88 & 51 & 0 & -0.35 & 0.07 & -0.13 & 342 & 1.267 & 35.0 & 98.0. & 208 & 35 & 000 & 2.0 & 090 \\
\hline$b: 28: 00$ & 58 & -90 & 51 & 0 & -0.48 & 0.22 & -0.15 & 342 & 2.306 & 35.0 & 98.0 & 258 & 30 & 000 & 2.0 & 090 \\
\hline $00: 28: 10$ & 59 & -92 & 52 & 0 & -0.49 & 0.41 & -0.16 & 343 & 3.615 & 35.0 & 98.0 & 295 & 50 & 000 & 2.0 & 090 \\
\hline $00: 28: 20$ & 60 & -95 & 51 & 0 & -0.46 & 0.25 & -0.13 & 343 & 2.449 & 28.0 & 98.0 & 295 & 40 & 000 & 2.0 & 090 \\
\hline $00: 28: 30$ & 61 & -97 & 51 & 0 & -0.46 & 0.19 & -0.08 & 343 & 1.707 & 23.8 & 98.0 & 295 & 37 & 000 & 2.0 & 090 \\
\hline & 62 & -99 & 52 & 0 & -0.35 & 0.14 & 0.00 & 344 & 0.914 & 21.1 & 98.0 & 295 & 35 & 000 & 2.0 & 090 \\
\hline
\end{tabular}




\begin{tabular}{|c|c|c|c|c|c|c|c|c|c|c|c|c|c|}
\hline & Positi & , IIII & & RPM & & Speed (Kn & ts) & & Turn & Rudder & Gi & & winc \\
\hline HH:MM:SS & East & North & Port & stbd & Long & Bow & Stern & Heading & Deg/Min & Deg & 8 & Deg & Knots \\
\hline $30: 28: 50$ & 63 & -100 . & 51 & 0 & -0.20 & 0.02 & 0.06 & 344 & -0.218 & 24.3 & 98.0 & 299 & 40 \\
\hline$: 29: 00$ & 63 & -101 & 52 & 0 & -0.06 & 0.15 & 0.07 & 344 & 0.459 & 19.0 & 98.0 & 299 & 33 \\
\hline$d: 29: 10$ & 64 & -100 & 51 & 0 & -0.01 & 0.22 & 0.13 & 344 & 0.610 & 16.5 & 98.0 & 299 & 44 \\
\hline $00: 29: 20$ & 65 & -100 & 52 & 0 & 0.06 & 0.04 & 0.14 & 344 & -0.658 & 28.4 & 98.0 & 299 & 28 \\
\hline $00: 29: 30$ & 65 & -99 & 51 & 0 & 0.20 & 0.05 & 0.11 & 344 & -0.388 & 25.3 & 98.0 & 274 & 34 \\
\hline $00: 29: 40$ & 65 & -98 & 52 & 0 & 0.25 & 0.04 & 0.08 & 344 & -0.297 & 24.9 & 98.0 & 274 & 42 \\
\hline $00: 29: 50$ & 65 & -97 & 51 & 0 & 0.21 & -0.18 & 0.04 & 344 & -1.369 & 33.1 & 98.0 & 274 & 40 \\
\hline $00 ; 30: 00$ & 64 & -96 & 51 & 0 & 0.19 & -0.47 & -0.03 & 343 & -2.844 & 35.0 & 98.0 & 274 & 44 \\
\hline $00: 30: 10$ & 62 & -96 & 52 & 0 & 0.14 & -0.60 & -0.08 & 343 & -3.318 & 35.0 & 98.0 & 274 & 33 \\
\hline $00 \div 30 \div 20$ & 60 & -96 & 52 & 0 & 0.06 & -0.47 & -0.13 & 342 & -2.123 & 35.0 & 98.0 & 300 & 41 \\
\hline $00: 30: 30$ & 59 & -96 & 52 & 0 & 0.05 & -0.42 & -0.18 & 342 & -1.571 & 35.0 & 98.0 & 300 & 43 \\
\hline $00: 30 \div 40$ & 57 & -96 & 51 & 0 & 0.02 & -0.28 & -0.22 & 342 & -0.388 & 35.0 & 98.0 & 300 & 35 \\
\hline $00: 30: 50$ & 56 & -97 & 51 & 0 & -0.01 & -0.11 & -0.26 & 342 & 0.964 & 35.0 & 98.0 & 300 & 42 \\
\hline $00: 31: 00$ & 55 & -97 & 52 & 0 & 0.08 & 0.01 & -0.30 & 342 & 1.954 & 35.0 & 98.0 & 300 & 47 \\
\hline $00 ; 31 ; 10$ & 54 & -96 & 51 & 0 & 0.19 & 0.03 & -0.33 & 342 & 2.327 & 35.0 & 98.0 & 300 & 35 \\
\hline $00: 31: 20$ & 53 & -96 & 51 & 0 & 0.18 & 0.03 & -0.35 & 343 & 2.400 & 28.4 & 98.0 & 300 & 44 \\
\hline $00: 31: 30$ & 53 & -95 & 51 & 0 & 0.09 & 0.20 & -0.27 & 343 & 2.926 & 9.9 & 98.0 & 300 & 39 \\
\hline $00: 31: 40$ & 53 & -95 & 51 & 0 & 0.06 & 0.22 & -0.06 & 344 & 1.807 & -0.5 & 98.0 & 300 & 37 \\
\hline $00: 31: 50$ & 53 & -94 & 52 & 0 & 0.10 & 0.17 & 0.06 & 344 & 0.715 & 15.0 & 98.0 & 300 & 43 \\
\hline $00 ; 32: 00$ & 53 & -93 & 52 & 0 & 0.27 & -0.04 & 0.09 & 344 & -0.836 & 24.4 & 98.0 & 300 & 34 \\
\hline $00: 32: 10$ & 53 & -92 & 51 & 0 & 0.29 & -0.14 & 0.05 & 344 & -1.244 & 29.9 & 98.0 & 300 & 28 \\
\hline $00: 32 ; 20$ & 52 & -91 & 51 & 0 & 0.21 & 0.14 & 0.02 & 344 & 0.762 & 21.6 & 98.0 & 243 & 34 \\
\hline $00: 32: 30$ & 53 & -89 & 52 & 0 & 0.29 & 0.32 & 0.04 & 344 & 1.768 & 15.2 & 98.0 & 243 & 39 \\
\hline $00: 32: 40$ & 53 & -87 & 52 & 0 & 0.33 & 0.40 & 0.11 & 344 & 1.849 & 2.2 & 98.0 & 243 & 45 \\
\hline $00: 32: 50$ & 54 & -85 & 51 & 0 & 0.42 & 0.36 & 0.28 & 344 & 0.473 & -7.4 & 98.0 & 243 & 34 \\
\hline $00 ; 33 ; 00$ & 55 & -82 & 51 & 0 & 0.52 & 0.00 & 0.42 & 344 & -2.728 & 2.2 & 98.0 & 223 & 40 \\
\hline $00: 33 ; 10$ & 55 & -79 & 51 & 0 & 0.58 & -0.21 & 0.45 & 344 & -4.185 & 16.3 & 98.0 & 223 & 41 \\
\hline $00: 33: 20$ & 55 & -76 & 52 & 0 & 0.55 & -0.05 & 0.37 & 343 & -2.703 & 24.1 & 98.0 & 223 & 41 \\
\hline $00: 33: 30$ & 54 & -74 & 52 & 0 & 0.42 & -0.24 & 0.27 & 343 & -3.220 & 35.0 & 98.0 & 210 & 36 \\
\hline $00: 33: 40$ & 53 & -72 & 52 & 0 & 0.34 & -0.48 & 0.16 & 342 & -4.070 & 35.0 & 98.0 & 210 & 35 \\
\hline $00: 33: 50$ & 52 & -71 & 52 & 0 & 0.27 & -0.45 & 0.06 & 341 & -3.200 & 35.0 & 98.0 & 210 & 28 \\
\hline $00: 34 ; 00$ & 51 & -70 & 52 & 0 & 0.17 & -0.38 & -0.03 & 341 & -2.217 & 35.0 & 98.0 & 210 & 30 \\
\hline $00: 34: 10$ & 50 & -70 & 52 & 0 & 0.13 & -0.33 & -0.10 & 341 & -1.435 & 35.0 & 98.0 & 210 & 35 \\
\hline $00: 34: 20$ & 48 & -70 & 52 & 0 & 0.11 & -0.41 & -0.16 & 340 & -1.617 & 35.0 & 98.0 & 210 & 31 \\
\hline $00: 34: 30$ & 47 & -70 & 51 & 0 & -0.01 & -0.29 & -0.21 & 340 & -0.491 & 35.0 & 98.0 & 230 & 36 \\
\hline $00: 34: 40$ & 46 & -71 & 52 & 0 & -0.04 & -0.07 & -0.26 & 340 & 1.196 & 35.0 & 98.0 & 209 & 38 \\
\hline $00: 34: 50$ & 45 & -71 & 50 & 0 & -0.08 & 0.09 & -0.29 & 340 & 2.432 & 35.0 & 98.0 & 209 & 40 \\
\hline $00: 35: 00$ & 45 & -72 & 49 & 0 & -0.19 & 0.17 & -0.30 & 341 & 2.959 & 35.0 & 98.0 & 209 & 26 \\
\hline $0: 35: 10$ & 45 & -73 & 50 & 0 & -0.30 & 0.08 & -0.28 & 341 & 2.283 & 35.0 & 98.0 & 240 & 46 \\
\hline$: 35: 20$ & 45 & -75 & 50 & 0 & -0.34 & 0.00 & -0.27 & 342 & 1.727 & 35.0 & 98.0 & 271 & 35 \\
\hline $0: 35: 30$ & 45 & -77 & 50 & 0 & -0.32 & -0.12 & -0.26 & 342 & 0.837 & 35.0 & 98.0 & 297 & 38 \\
\hline $00: 35: 40$ & 44 & -79 & 49 & 0 & -0.33 & -0.07 & -0.24 & 342 & 1.041 & 32.1 & 98.0 & 297 & 32 \\
\hline $00: 35: 50$ & 44 & -80 & 49 & 0 & -0.37 & 0.13 & -0.19 & 342 & 2.043 & 24.4 & 98.0 & 297 & 34 \\
\hline $00: 36: 00$ & 45 & -82 & 50 & 0 & -0.40 & 0.32 & -0.10 & 343 & 2.647 & 16.2 & 98.0 & 312 & 40 \\
\hline $00: 36: 10$ & 46 & -84 & 49 & 0 & -0.19 & 0.36 & 0.04 & 343 & 2.030 & 7.7 & 98.0 & 324 & 42 \\
\hline $00: 36: 20$ & 47 & -84 & 49 & 0 & 0.09 & 0.19 & 0.16 & 343 & 0.175 & 11.7 & 98.0 & 324 & 37 \\
\hline $00: 36: 30$ & 48 & -83 & 50 & 0 & 0.17 & 0.14 & 0.21 & 343 & -0.483 & 15.8 & 98.0 & 324 & 34 \\
\hline $00: 36: 40$ & 49 & -81 & 50 & 0 & 0.21 & 0.10 & 0.23 & 343 & -0.850 & 19.4 & 98.0 & 223 & 34 \\
\hline $00: 36: 50$ & 49 & -80 & 50 & 0 & 0.19 & 0.08 & 0.22 & 343 & -0.933 & 23.2 & 98.0 & 215 & 35 \\
\hline $00: 37: 00$ & 50 & -79 & 50 & 0 & 0.12 & 0.16 & 0.21 & 343 & -0.269 & 21.7 & 98.0 & 215 & 47 \\
\hline $00: 37: 10$ & 50 & -78 & 50 & 0 & 0.14 & -0.07 & 0.19 & 343 & -1.647 & 32.1 & 98.0 & 241 & 25 \\
\hline $00: 37: 20$ & 50 & -78 & 50 & 0 & 0.14 & -0.62 & 0.13 & 342 & -4.737 & 35.0 & 98.0 & 241 & 46 \\
\hline $00: 37: 30$ & 48 & -78 & 49 & 0 & 0.08 & -0.61 & 0.07 & 341 & -4.312 & 35.0 & 98.0 & 241 & 41 \\
\hline $00: 37: 40$ & 47 & -78 & 50 & 0 & -0.02 & -0.39 & 0.00 & 341 & -2.480 & 35.0 & 98.0 & 241 & 40 \\
\hline $00: 37: 50$ & 46 & -79 & 49 & 0 & -0.10 & -0.44 & -0.05 & 340 & -2.467 & 35.0 & 98.0 & 211 & 46 \\
\hline $00: 38: 00$ & 45 & -80 & 50 & 0 & -0.13 & -0.36 & -0.09 & 340 & -1.729 & 35.0 & 98.0 & 211 & 45 \\
\hline $00: 38: 10$ & 44 & -81 & 50 & 0 & -0.19 & -0.36 & -0.12 & 340 & -1.503 & 35.0 & 98.0 & 211 & 38 \\
\hline $00: 38: 20$ & 43 & -82 & 49 & 0 & -0.26 & -0.23 & -0.14 & 340 & -0.564 & 35.0 & 98.0 & 211 & 40 \\
\hline $00: 38: 30$ & 43 & -84 & 50 & 0 & -0.24 & -0.18 & -0.16 & 340 & -0.149 & 35.0 & 98.0 & 268 & 32 \\
\hline $00: 38: 40$ & 43 & -85 & 50 & 0 & -0.29 & -0.36 & -0.17 & 340 & -1.219 & 35.0 & 98.0 & 268 & 34 \\
\hline $00: 38: 50$ & 42 & -87 & 50 & 0 & -0.40 & -0.46 & -0.17 & 339 & -1.838 & 35.0 & 98.0 & 268 & 40 \\
\hline $00: 39: 00$ & 41 & -90 & 49 & 0 & -0.42 & -0.44 & -0.17 & 339 & -1.733 & 35.0 & 98.0 & 329 & 36 \\
\hline $00: 39: 10$ & 41 & -93 & 49 & 0 & -0.44 & -0.24 & -0.17 & 339 & -0.457 & 35.0 & 98.0 & 329 & 41 \\
\hline $00: 39: 20$ & 41 & -95 & 50 & 0 & -0.40 & -0.12 & -0.17 & 339 & 0.345 & 35.0 & 98.0 & 322 & 32 \\
\hline $00: 39: 30$ & 41 & -97 & 49 & 0 & -0.37 & -0.01 & -0.19 & 339 & 1.125 & 35.0 & 98.0 & 345 & 44 \\
\hline $00: 39: 40$ & 41 & -99 & 49 & 0 & -0.29 & 0.05 & -0.21 & 339 & 1.618 & 35.0 & 98.0 & 000 & 49 \\
\hline $00: 39: 50$ & 41 & -100 & 52 & 0 & -0.13 & 0.15 & -0.23 & 339 & 2.399 & 35.0 & 98.0 & 000 & 38 \\
\hline $00: 40: 00$ & 41 & -100 & 52 & 0 & 0.03 & 0.43 & -0.25 & 340 & 4.305 & 22.2 & 98.0 & 000 & 35 \\
\hline $00: 40: 10$ & 42 & -100 & 53 & 0 & 0.06 & 0.64 & -0.24 & 341 & 5.621 & 26.0 & 98.0 & 272 & 37 \\
\hline $00: 40: 20$ & 43 & -99 & 52 & 0 & 0.08 & 0.56 & -0.17 & 342 & 4.659 & 15.1 & 98.0 & 298 & 34 \\
\hline $00: 40: 30$ & 43 & -98 & 52 & 0 & 0.28 & 0.27 & -0.10 & 342 & 2.362 & 21.0 & 98.0 & 298 & 37 \\
\hline $00: 40: 40$ & 43 & -96 & 53 & 0 & 0.38 & 0.02 & -0.05 & 343 & 0.458 & 7.8 & 98.0 & 298 & 37 \\
\hline $00: 40: 50$ & 42 & -94 & 52 & 0 & 0.38 & -0.25 & 0.04 & 342 & -1.796 & 15.6 & 98.0 & 208 & 34 \\
\hline $00: 41: 00$ & 41 & -93 & 53 & 0 & 0.33 & -0.45 & 0.04 & 342 & -3.150 & 27.4 & 98.0 & 208 & 32 \\
\hline $00: 41: 10$ & 39 & -92 & 52 & 0 & 0.17 & -0.31 & -0.04 & 342 & -1.724 & 35.0 & 98.0 & 208 & 39 \\
\hline $00: 41: 20$ & 38 & -91 & 52 & 0 & 0.11 & 0.02 & -0.08 & 341 & 0.650 & 21.1 & 98.0 & 208 & 33 \\
\hline $00: 41: 30$ & 38 & -91 & 53 & 0 & 0.22 & -0.20 & -0.08 & 342 & -0.734 & 30.1 & 98.0 & 215 & 34 \\
\hline $9: 41: 40$ & 36 & -90 & 51 & 0 & 0.26 & -0.43 & -0.14 & 341 & -1.862 & 35.0 & 98.0 & 207 & 36 \\
\hline$: 41: 50$ & 35 & -89 & 51 & 0 & 0.18 & -0.23 & -0.21 & 341 & -0.160 & 35.0 & 98.0 & 207 & 37 \\
\hline $00: 42: 00$ & 33 & -89 & 50 & 0 & 0.06 & -0.16 & -0.23 & 341 & 0.487 & 31.5 & 98.0 & 182 & 28 \\
\hline $00: 42: 10$ & 33 & -89 & 50 & 0 & -0.03 & -0.04 & -0.20 & 341 & 1.014 & 22.4 & 98.0 & 182 & 38 \\
\hline $00: 42: 20$ & 32 & -90 & 49 & 0 & -0.10 & -0.14 & -0.14 & 341 & 0.020 & 29.3 & 98.0 & 178 & 39 \\
\hline $00: 42: 30$ & 32 & -91 & 50 & 0 & -0.27 & -0.35 & -0.13 & 341 & -1.399 & 35.0 & 98.0 & 178 & 44 \\
\hline
\end{tabular}




\begin{tabular}{|c|c|c|c|c|c|c|c|c|c|c|c|c|c|c|c|c|}
\hline \multirow{3}{*}{$\begin{array}{c}\text { Time } \\
\mathrm{HH}: \mathrm{MM} ; \mathrm{SS}\end{array}$} & \multicolumn{2}{|c|}{ Position (m) } & \multicolumn{2}{|c|}{ RPM } & \multicolumn{3}{|c|}{ Speed (Knots) } & \multirow[b]{2}{*}{ Heading } & \multirow{2}{*}{$\begin{array}{c}\text { Turn } \\
\text { Deg/Min }\end{array}$} & \multirow{2}{*}{$\begin{array}{l}\text { Rudder } \\
\text { Deg }\end{array}$} & \multicolumn{2}{|c|}{ Gill } & \multicolumn{2}{|c|}{ Wind } & \multicolumn{2}{|c|}{ Current } \\
\hline & East & North & Port & Stbd & Long & Bow & stern & & & & 8 & Deg & Knots & Deg & Knots & Deg \\
\hline & & & & & & & & & & & & & & & & \\
\hline $90: 42: 40$ & 31 & -93 & 49 & 0 & -0.37 & -0.26 & -0.12 & 341 & -0.908 & 35.0 & 98.0 & 000 & 36 & 000 & 2.0 & 090 \\
\hline$: 42: 50$ & 31 & -95 & 50 & 0 & -0.32 & -0.18 & -0.12 & 341 & -0.400 & 35.0 & 98.0 & 000 & 30 & 000 & 2.0 & 090 \\
\hline $.0: 43: 00$ & 31 & -97 & 50 & 0 & -0.32 & -0.07 & -0.12 & 341 & 0.302 & 35.0 & 98.0 & 000 & 40 & 000 & 2.0 & 090 \\
\hline $00: 43: 10$ & 31 & -98 & 49 & 0 & -0.27 & 0.08 & -0.12 & 341 & 1.270 & 30.5 & 98.0 & 000 & 29 & 000 & 2.0 & 090 \\
\hline $00: 43: 20$ & 31 & -99 & 50 & 0 & -0.23 & 0.27 & -0.08 & 341 & 2.224 & 20.1 & 98.0 & 000 & 40 & 000 & 2.0 & 090 \\
\hline $00: 43: 30$ & 33 & -100 & 50 & 0 & -0.13 & 0.51 & 0.03 & 342 & 3.030 & 10.8 & 98.0 & 002 & 30 & 000 & 2.0 & 090 \\
\hline $00: 43: 40$ & 34 & -100 & 49 & 0 & 0.00 & 0.62 & 0.15 & 342 & 2.995 & 4.2 & 98.0 & 211 & 29 & 000 & 2.0 & 090 \\
\hline $00: 43: 50$ & 36 & -99 & 49 & 0 & 0.06 & 0.53 & 0.29 & 343 & 1.558 & 1.6 & 98.0 & 273 & 37 & 000 & 2.0 & 090 \\
\hline $00: 44: 00$ & 38 & -98 & 50 & 0 & 0.14 & 0.14 & 0.38 & 343 & -1.520 & 11.4 & 98.0 & 295 & 49 & 000 & 2.0 & 090 \\
\hline $00: 44: 10$ & 39 & -97 & 49 & 0 & 0.23 & -0.09 & 0.36 & 342 & -2.865 & 25.8 & 98.0 & 285 & 34 & 000 & 2.0 & 090 \\
\hline $00: 44: 20$ & 39 & -95 & 50 & 0 & 0.21 & -0.01 & 0.28 & 342 & -1.818 & 28.9 & 98.0 & 246 & 49 & 000 & 2.0 & 090 \\
\hline $00: 44: 30$ & 39 & -94 & 49 & 0 & 0.09 & 0.04 & 0.19 & 342 & -0.979 & 32.0 & 98.0 & 246 & 40 & 000 & 2.0 & 090 \\
\hline $00: 44: 40$ & 39 & -94 & 50 & 0 & 0.07 & -0.16 & 0.11 & 341 & -1.707 & 35.0 & 98.0 & 246 & 39 & 000 & 2.0 & 090 \\
\hline $00: 44: 50$ & 39 & -94 & 50 & 0 & 0.11 & -0.28 & 0.03 & 341 & -1.982 & 35.0 & 98.0 & 246 & 41 & 000 & 2.0 & 090 \\
\hline $00 ; 45 ; 00$ & 38 & -93 & 50 & 0 & 0.12 & -0.27 & -0.04 & 341 & -1.462 & 35.0 & 98.0 & 246 & 33 & 000 & 2.0 & 090 \\
\hline $00: 45: 10$ & 37 & -93 & 49 & 0 & 0.20 & -0.33 & -0.10 & 341 & -1.426 & 35.0 & 98.0 & 246 & 44 & 000 & 2.0 & 090 \\
\hline $00: 45: 20$ & 35 & -92 & 50 & 0 & 0.18 & -0.35 & -0.16 & 340 & -1.211 & 35.0 & 98.0 & 208 & 32 & 000 & 2.0 & 090 \\
\hline $00: 45: 30$ & 34 & -92 & 49 & 0 & 0.00 & -0.09 & -0.21 & 340 & 0.743 & 35.0 & 98.0 & 208 & 37 & 000 & 2.0 & 090 \\
\hline $00: 45: 40$ & 34 & -93 & 50 & 0 & -0.05 & 0.03 & -0.20 & 341 & 1.479 & 25.3 & 98.0 & 258 & 37 & 000 & 2.0 & 090 \\
\hline $00: 45: 50$ & 33 & -93 & 49 & 0 & 0.02 & -0.35 & -0.21 & 341 & -0.902 & 35.0 & 98.0 & 272 & 42 & 000 & 2.0 & 090 \\
\hline $00 ; 46: 00$ & 31 & -93 & 50 & 0 & 0.01 & -0.54 & -0.23 & 340 & -1.957 & 35.0 & 98.0 & 272 & 41 & 000 & 2.0 & 090 \\
\hline $00: 46: 10$ & 29 & -94 & 50 & 0 & -0.11 & -0.71 & -0.25 & 340 & -2.905 & 35.0 & 98.0 & 272 & 49 & 000 & 2.0 & 090 \\
\hline $00: 46: 20$ & 27 & -96 & 49 & 0 & -0.28 & -0.66 & -0.24 & 339 & -2.652 & 35.0 & 98.0 & 352 & 28 & 000 & 2.0 & 090 \\
\hline $00: 46: 30$ & 26 & -98 & 49 & 0 & -0.33 & -0.19 & -0.22 & 339 & 0.159 & 35.0 & 98.0 & 091 & 40 & 000 & 2.0 & 090 \\
\hline $00: 46: 40$ & 26 & -100 & 50 & 0 & -0.32 & -0.09 & -0.21 & 339 & 0.773 & 35.0 & 98.0 & 091 & 44 & 000 & 2.0 & 090 \\
\hline $00: 46: 50$ & 26 & -102 & 49 & 0 & -0.34 & 0.06 & -0.20 & 340 & 1.677 & 35.0 & 98.0 & 091 & 40 & 000 & 2.0 & 090 \\
\hline $00: 47: 00$ & 26 & -103 & 50 & 0 & -0.26 & 0.30 & -0.20 & 340 & 3.186 & 35.0 & 98.0 & 043 & 39 & 000 & 2.0 & 090 \\
\hline $00: 47: 10$ & 27 & -104 & 50 & 0 & -0.18 & 0.49 & -0.20 & 341 & 4.367 & 35.0 & 98.0 & 038 & 36 & 000 & 2.0 & 090 \\
\hline $00: 47: 20$ & 28 & -205 & 50 & 0 & -0.06 & 0.70 & -0.21 & 341 & 5.790 & 35.0 & 98.0 & 000 & 40 & 000 & 2.0 & 090 \\
\hline $00: 47: 30$ & 30 & -104 & 50 & 0 & 0.02 & 0.75 & -0.18 & 342 & 5.904 & 22.1 & 98.0 & 000 & 37 & 000 & 2.0 & 090 \\
\hline $00: 47: 40$ & 31 & -104 & 50 & 0 & 0.05 & 0.53 & -0.05 & 343 & 3.699 & 5.1 & 98.0 & 000 & 41 & 000 & 2.0 & 090 \\
\hline $00: 47: 50$ & 32 & -103 & 49 & 0 & 0.15 & 0.35 & 0.13 & 344 & 1.377 & 0.9 & 98.0 & 269 & 33 & 000 & 2.0 & 090 \\
\hline $00: 48: 00$ & 33 & -102 & 50 & 0 & 0.17 & 0.33 & 0.26 & 344 & 0.418 & 2.4 & 98.0 & 269 & 32 & 000 & 2.0 & 090 \\
\hline $00: 48: 10$ & 34 & -100 & 49 & 0 & 0.20 & 0.11 & 0.36 & 344 & -1.555 & 10.8 & 98.0 & 269 & 32 & 000 & 2.0 & 090 \\
\hline $00: 48: 20$ & 35 & -99 & 49 & 0 & 0.24 & -0.21 & 0.36 & 343 & -3.603 & 29.1 & 98.0 & 220 & 35 & 000 & 2.0 & 090 \\
\hline $00: 48: 30$ & 34 & -98 & 50 & 0 & 0.24 & -0.23 & 0.26 & 343 & -3.115 & 35.0 & 98.0 & 220 & 32 & 000 & 2.0 & 090 \\
\hline $00: 48: 40$ & 34 & -97 & 50 & 0 & 0.16 & 0.10 & 0.15 & 342 & -0.339 & 31.6 & 98.0 & 225 & 39 & 000 & 2.0 & 090 \\
\hline $00: 48: 50$ & 35 & -96 & 50 & 0 & 0.17 & 0.19 & 0.10 & 342 & 0.550 & 25.7 & 98.0 & 269 & 41 & 000 & 2.0 & 090 \\
\hline 49:00: 0 : & 35 & -94 & 50 & 0 & 0.25 & 0.06 & 0.07 & 342 & -0.068 & 27.8 & 98.0 & 223 & 39 & 000 & 2.0 & 090 \\
\hline$: 49: 10$ & 35 & -93 & 49 & 0 & 0.19 & -0.07 & 0.03 & 342 & -0.628 & 32.4 & 98.0 & 208 & 33 & 000 & 2.0 & 090 \\
\hline$-0: 49: 20$ & 34 & -93 & 50 & 0 & 0.16 & -0.25 & -0.03 & 342 & -1.419 & 35.0 & 98.0 & 208 & 39 & 000 & 2.0 & 090 \\
\hline $00: 49: 30$ & 33 & -92 & 49 & 0 & 0.14 & -0.41 & -0.08 & 342 & -2.101 & 35.0 & $98: 0$ & 208 & 42 & 000 & 2.0 & 090 \\
\hline $00: 49: 40$ & 32 & -92 & 50 & 0 & -0.06 & -0.45 & -0.11 & 342 & -2.146 & 35.0 & 98.0 & 208 & 30 & 000 & 2.0 & 090 \\
\hline $00: 49: 50$ & 31 & -94 & 50 & 0 & -0.30 & -0.28 & -0.12 & 341 & -1.006 & 35.0 & 98.0 & 208 & 28 & 000 & 2.0 & 090 \\
\hline $00: 50: 00$ & 30 & -96 & 49 & 0 & -0.41 & -0.34 & -0.11 & 341 & -1.445 & 35.0 & 98.0 & 208 & 32 & 000 & 2.0 & 090 \\
\hline $00: 50: 10$ & 30 & -98 & 50 & 0 & -0.43 & -0.37 & -0.09 & 341 & -1.719 & 35.0 & 98.0 & 208 & 26 & 000 & 2.0 & 090 \\
\hline $00: 50: 20$ & 30 & -101 & 50 & 0 & -0.41 & -0.27 & -0.09 & 341 & -1.089 & 35.0 & 98.0 & 208 & 45 & 000 & 2.0 & 090 \\
\hline $00: 50: 30$ & 29 & -103 & 49 & 0 & -0.49 & -0.27 & -0.09 & 340 & -1.147 & 35.0 & 98.0 & 208 & 36 & 000 & 2.0 & 090 \\
\hline $00: 50: 40$ & 30 & -106 & 49 & 0 & -0.64 & 0.10 & -0.10 & 340 & 1.272 & 35.0 & 98.0 & 208 & 33 & 000 & 2.0 & 090 \\
\hline $00: 50: 50$ & 31 & -109 & 50 & 0 & -0.63 & 0.39 & -0.10 & 341 & 3.111 & 35.0 & 98.0 & 208 & 40 & 000 & 2.0 & 090 \\
\hline $00: 51 ; 00$ & 33 & -112 & 49 & 0 & -0.62 & 0.43 & -0.09 & 341 & 3.297 & 35.0 & 98.0 & 208 & 41 & 000 & 2.0 & 090 \\
\hline
\end{tabular}


Open Ocean

\section{Simulation Track Plot}

Ship: 150,000 ton, Tanker, partial load

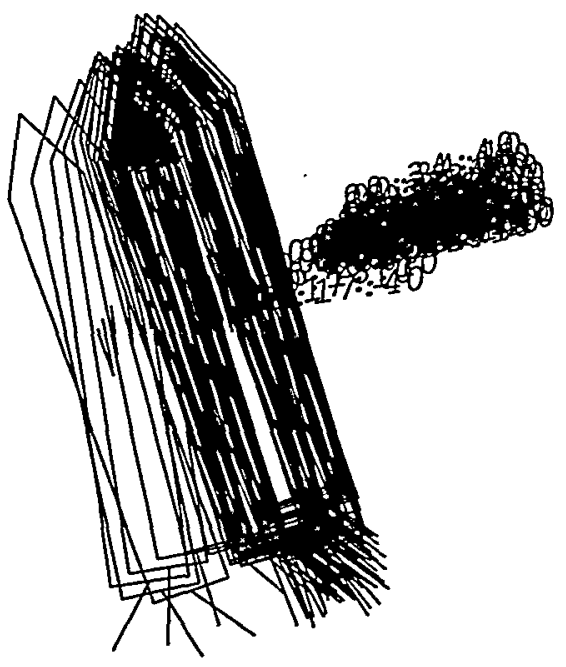

Start Time [hrs:min:sec]: $00: 16: 40$

End Time [hrs:min:sec]: $00: 51: 05$

Delta Plot Time [sec]:

Date: 05-Feb-99 Time: 15:29:38

East-West Scale [m]:

East-West Scale [Nm]: 


\section{HANDS-ON SIMULATION}

\section{SUCTION MOORNG TECHNOLOGY}

Simulation Number: 34

Date: 5 February 1999

Start Time: $16: 28$

Length of run [secs]: 3540

Ship Condition: draft $=13.72 \mathrm{~m}$

Environment

Wind Speed [knots]

Direction: 0

Sea State, Significant wave height [m]...

8 Direction: 180

Current Speed [knots]:

2 Direction: 90

Ship Operator: J.A.

Observer: B.K.J

Final Mating, distance between center of buoy and center of moon pool [m]: 1.7

Evaluation:

Mating was made in the first try.

The captain did not decelerate fast enough. Almost at the buoy the captain backed to kill way and in so doing the current took the ship. He ended up using full ahead and hard left. The ship sailed away, and the captain brought it back and then it took long time to wait for small waves while the buoy was near to the center of the moon pool. During this exercise the captain was able to move the ship sideways with no forward speed. The RPM was set to 47, the rudder 35 degrees starboard, and the thruster about 225 degrees with 100 percent power. 
Ship:

Exercise number:

591 150,000 ton, Tanker, partial Ioad

Cenario:

754 Suction Mooring System

112 Open Ocean

999 Dumny tug file based on containership

file: 590 Suction Mooring Buoy

Initial conditions: $\quad 594$ 150k tanker in partial load - Suction Mooring

Exercise identification: Run 34

Time Position (m) RPM Speed (Knots) Turn Rudder Gill Wind Wint

\begin{tabular}{|c|c|c|c|c|c|c|c|c|c|c|c|c|c|c|c|c|}
\hline $\mathrm{HH} ; \mathrm{MM}: \mathrm{SS}$ & East & North & Port & stbd & Long & Bow & stern & Heading & Deg/Min & Deg & 8 & Deg & Knots & Deg & Knots & Deg \\
\hline $00: 33: 20$ & 42 & -89 & 45 & 0 & 0.32 & -0.11 & 0.06 & 341 & -1.087 & 31.0 & 100.0 & 214 & 41 & 000 & 2.0 & 090 \\
\hline $00: 33: 30$ & 41 & -88 & 46 & 0 & 0.17 & -0.35 & 0.01 & 340 & -2.297 & 35.0 & 100.0 & 214 & 36 & 000 & 2.0 & 090 \\
\hline $00: 33: 40$ & 40 & -88 & 46 & 0 & 0.07 & -0.68 & -0.02 & 340 & -4.217 & 35.0 & 100.0 & 214 & 35 & 000 & 2.0 & 090 \\
\hline $00: 33: 50$ & 38 & -88 & 46 & 0 & -0.01 & -0.69 & -0.04 & 339 & -4.140 & 35.0 & 100.0 & 188 & 28 & 000 & 2.0 & 090 \\
\hline $00 ; 34 ; 00$ & 36 & -89 & 46 & 0 & -0.12 & -0.61 & -0.04 & 338 & -3.647 & 35.0 & 100.0 & 137 & 30 & 000 & 2.0 & 090 \\
\hline $00: 34: 10$ & 35 & -90 & 45 & 0 & -0.14 & -0.54 & -0.04 & 338 & -3.224 & 35.0 & 100.0 & 096 & 35 & 000 & 2.0 & 090 \\
\hline $00: 34: 20$ & 34 & -91 & 45 & 0 & -0.13 & -0.63 & -0.03 & 337 & -3.775 & 35.0 & 100.0 & 096 & 31 & 000 & 2.0 & 090 \\
\hline $00: 34: 30$ & 33 & -93 & 46 & 0 & -0.23 & -0.48 & -0.04 & 337 & -2.804 & 35.0 & 100.0 & 121 & 36 & 000 & 2.0 & 090 \\
\hline $00: 34: 40$ & 32 & -95 & 46 & 0 & -0.28 & -0.22 & -0.05 & 336 & -1.098 & 35.0 & 100.0 & 121 & 38 & 000 & 2.0 & 090 \\
\hline $00: 34: 50$ & 33 & -96 & 46 & 0 & -0.31 & -0.03 & -0.07 & 336 & 0.222 & 35.0 & 100.0 & 121 & 40 & 000 & 2.0 & 090 \\
\hline $00: 35: 00$ & 33 & -98 & 47 & 0 & -0.40 & 0.07 & -0.08 & 336 & 0.954 & 35.0 & 100.0 & 121 & 26 & 000 & 2.0 & 090 \\
\hline $00: 35: 10$ & 34 & -100 & 51 & 0 & -0.49 & -0.03 & -0.10 & 336 & 0.447 & 35.0 & 100.0 & 121 & 46 & 000 & 2.0 & 090 \\
\hline $00: 35: 20$ & 35 & -103 & 51 & 0 & -0.50 & -0.08 & -0.12 & 337 & 0.240 & 35.0 & 100.0 & 031 & 35 & 000 & 2.0 & 090 \\
\hline $00: 35: 30$ & 35 & -105 & 51 & 0 & -0.43 & -0.21 & -0.14 & 337 & -0.455 & 35.0 & 100.0 & 031 & 38 & 000 & 2.0 & 090 \\
\hline $00: 35: 40$ & 35 & -107 & 50 & 0 & -0.42 & -0.11 & -0.15 & 336 & 0.259 & 35.0 & 100.0 & 063 & 32 & 000 & 2.0 & 090 \\
\hline $00: 35: 50$ & 36 & -110 & 50 & 0 & -0.46 & 0.22 & -0.16 & 337 & 2.443 & 35.0 & 100.0 & 063 & 34 & 000 & 2.0 & 090 \\
\hline $00: 36: 00$ & 37 & -112 & 51 & 0 & -0.50 & 0.56 & -0.18 & 337 & 4.682 & 35.0 & 100.0 & 063 & 40 & 000 & 2.0 & 090 \\
\hline $00: 36: 10$ & 39 & -113 & 51 & 0 & -0.30 & 0.76 & -0.21 & 338 & 6.116 & 35.0 & 100.0 & 063 & 42 & 000 & 2.0 & 090 \\
\hline $00: 36: 20$ & 41 & -114 & 52 & 0 & -0.06 & 0.65 & -0.20 & 339 & 5.401 & 35.0 & 100.0 & 063 & 37 & 000 & 2.0 & 090 \\
\hline $00: 36: 30$ & 42 & -113 & 48 & 0 & -0.02 & 0.64 & -0.18 & 340 & 5.199 & 27.6 & 100.0 & 063 & 34 & 000 & 2.0 & 090 \\
\hline $00 \div 36: 40$ & 43 & -113 & 48 & 0 & 0.01 & 0.58 & -0.10 & 341 & 4.311 & 18.2 & 100.0 & 306 & 34 & 000 & 2.0 & 090 \\
\hline $00: 36: 50$ & 44 & -112 & 47 & 0 & 0.05 & 0.43 & -0.01 & 341 & 2.832 & 13.0 & 100.0 & 298 & 35 & 000 & 2.0 & 090 \\
\hline $00 ; 37 ; 00$ & 45 & -112 & 48 & 0 & 0.06 & 0.41 & 0.07 & 342 & 2.131 & 10.4 & 100.0 & 298 & 47 & 000 & 2.0 & 090 \\
\hline $00: 37: 10$ & 46 & -111 & 49 & 0 & 0.14 & 0.07 & 0.15 & 342 & -0.500 & 19.1 & 100.0 & 298 & 25 & 000 & 2.0 & 090 \\
\hline $00: 37: 20$ & 45 & -110 & 49 & 0 & 0.18 & -0.55 & 0.13 & 342 & -4.320 & 35.0 & 100.0 & 315 & 46 & 000 & 2.0 & 090 \\
\hline $00: 37: 30$ & 44 & -110 & 49 & 0 & 0.19 & -0.53 & 0.06 & 341 & -3.781 & 35.0 & 100.0 & 000 & 41 & 000 & 2.0 & 090 \\
\hline $00: 37: 40$ & 43 & -109 & 49 & 0 & 0.17 & -0.27 & -0.01 & 340 & -1.695 & 35.0 & 100.0 & 000 & 40 & 000 & 2.0 & 090 \\
\hline $00: 37: 50$ & 42 & -109 & 49 & 0 & 0.18 & -0.30 & -0.07 & 340 & -1.451 & 35.0 & 100.0 & 000 & 46 & 000 & 2.0 & 090 \\
\hline $0: 38: 00$ & 40 & -108 & 49 & 0 & 0.25 & -0.19 & -0.13 & 340 & -0.386 & 35.0 & 100.0 & 000 & 45 & 000 & 2.0 & 090 \\
\hline$: 38: 10$ & 39 & -107 & 49 & 0 & 0.25 & -0.13 & -0.18 & 340 & 0.346 & 35.0 & 100.0 & 060 & 38 & 000 & 2.0 & 090 \\
\hline $0: 38: 20$ & 38 & -106 & 48 & 0 & 0.27 & 0.06 & -0.22 & 340 & 1.773 & 35.0 & 100.0 & 033 & 40 & 000 & 2.0 & 090 \\
\hline $00: 38: 30$ & 37 & -104 & 49 & 0 & 0.36 & 0.16 & -0.26 & 341 & 2.667 & 35.0 & 100.0 & 355 & 32 & 000 & 2.0 & 090 \\
\hline $00: 38: 10$ & 36 & -103 & 49 & 0 & 0.33 & 0.00 & -0.29 & 341 & 1.857 & 31.0 & 100.0 & 329 & 34 & 000 & 2.0 & 090 \\
\hline $00: 38: 50$ & 35 & -102 & 48 & 0 & 0.24 & -0.03 & -0.26 & 341 & 1.496 & 25.7 & 100.0 & 342 & 40 & 000 & 2.0 & 090 \\
\hline $00: 39: 00$ & 34 & -101 & 48 & 0 & 0.18 & -0.02 & -0.22 & 341 & 1.287 & 21.4 & 100.0 & 093 & 36 & 000 & 2.0 & 090 \\
\hline $00 ; 39: 10$ & 33 & -100 & 49 & 0 & 0.15 & 0.14 & -0.12 & 342 & 1.649 & 10.4 & 100.0 & 000 & 41 & 000 & 2.0 & 090 \\
\hline $00: 39: 20$ & 33 & -99 & 49 & 0 & 0.20 & 0.20 & -0.01 & 342 & 1.321 & 12.0 & 100.0 & 000 & 32 & 000 & 2.0 & 090 \\
\hline $00 \div 39 ; 30$ & 34 & -98 & 49 & 0 & 0.25 & 0.25 & 0.07 & 342 & 1.105 & 11.2 & 100.0 & 322 & 44 & 000 & 2.0 & 090 \\
\hline $00: 39: 40$ & 34 & -97 & 48 & 0 & 0.29 & 0.20 & 0.13 & 342 & 0.450 & 11.2 & 100.0 & 274 & 49 & 000 & 2.0 & 090 \\
\hline $00: 39: 50$ & 34 & -95 & 49 & 0 & 0.41 & 0.18 & 0.17 & 342 & 0.123 & 12.1 & 100.0 & 274 & 38 & 000 & 2.0 & 090 \\
\hline $00: 10: 00$ & 35 & -92 & 44 & 0 & 0.49 & 0.36 & 0.19 & 342 & 1.038 & 8.7 & 100.0 & 238 & 35 & 000 & 2.0 & 090 \\
\hline $00: 40: 10$ & 36 & -89 & 45 & 0 & 0.43 & 0.49 & 0.25 & 343 & 1.529 & 3.6 & 100.0 & 213 & 37 & 000 & 2.0 & 090 \\
\hline $00: 40: 20$ & 37 & -87 & 45 & 0 & 0.35 & 0.40 & 0.34 & 343 & 0.396 & 2.3 & 100.0 & 213 & 34 & 000 & 2.0 & 090 \\
\hline $00: 40: 30$ & 38 & -84 & 45 & 0 & 0.44 & 0.09 & 0.45 & 343 & -2.331 & -7.2 & 100.0 & 213 & 37 & 000 & 2.0 & 090 \\
\hline $00: 40: 40$ & 38 & -82 & 45 & 0 & 0.45 & -0.18 & 0.53 & 342 & -4.543 & 9.0 & 100.0 & 213 & 37 & 000 & 2.0 & 090 \\
\hline $00 ; 40 ; 50$ & 38 & -80 & 44 & 0 & 0.37 & -0.42 & 0.51 & 341 & -5.938 & 28.0 & 100.0 & 213 & 34 & 000 & 2.0 & 090 \\
\hline $00: 41: 00$ & 37 & -78 & 44 & 0 & 0.28 & -0.63 & 0.43 & 340 & -6.741 & 35.0 & 100.0 & 192 & 32 & 000 & 2.0 & 090 \\
\hline $00 ; 41 ; 10$ & 37 & -77 & 43 & 0 & 0.08 & -0.51 & 0.35 & 339 & -5.473 & 35.0 & 100.0 & 225 & 39 & 000 & 2.0 & 090 \\
\hline $00: 41: 20$ & 36 & -77 & 43 & 0 & 0.00 & -0.19 & 0.26 & 338 & -2.866 & 35.0 & 100.0 & 225 & 33 & 000 & 2.0 & 090 \\
\hline $00 ; 41: 30$ & 36 & -77 & 43 & 0 & 0.05 & -0.45 & 0.19 & 338 & -4.062 & 35.0 & 100.0 & 225 & 34 & 000 & 2.0 & 090 \\
\hline $00: 41: 40$ & 35 & -77 & 43 & 0 & 0.06 & -0.77 & 0.15 & 337 & -5.835 & 35.0 & 100.0 & 179 & 36 & 000 & 2.0 & 090 \\
\hline $00 ; 41: 50$ & 34 & -78 & 43 & 0 & -0.05 & -0.59 & 0.10 & 336 & -4.337 & 35.0 & 100.0 & 179 & 37 & 000 & 2.0 & 090 \\
\hline $00: 42 ; 00$ & 33 & -79 & 43 & 0 & -0.18 & -0.54 & 0.07 & 335 & -3.817 & 35.0 & 100.0 & 145 & 28 & 000 & 2.0 & 090 \\
\hline $00: 42: 10$ & 32 & -80 & 43 & 0 & -0.28 & -0.39 & 0.05 & 335 & -2.779 & 35.0 & 100.0 & 145 & 38 & 000 & 2.0 & 090 \\
\hline $00: 42: 20$ & 32 & -82 & 43 & 0 & -0.34 & -0.48 & 0.03 & 334 & -3.205 & 35.0 & 100.0 & 111 & 39 & 000 & 2.0 & 090 \\
\hline $00: 42: 30$ & 32 & -85 & 44 & 0 & -0.49 & -0.79 & 0.02 & 334 & -5.159 & 35.0 & 100.0 & 111 & 44 & 000 & 2.0 & 090 \\
\hline $00: 42: 40$ & 31 & -88 & 55 & 0 & -0.58 & -0.71 & -0.02 & 333 & -4.336 & 35.0 & 100.0 & 180 & 36 & 000 & 2.0 & 090 \\
\hline $00: 42: 50$ & 31 & -92 & 55 & 0 & -0.61 & -0.63 & -0.08 & 332 & -3.441 & 35.0 & 100.0 & 180 & 30 & 000 & 2.0 & 090 \\
\hline $00: 43: 00$ & 31 & -96 & 55 & 0 & -0.68 & -0.52 & -0.14 & 332 & -2.384 & 35.0 & 100.0 & 180 & 40 & 000 & 2.0 & 090 \\
\hline $00: 43: 10$ & 31 & -100 & 57 & 0 & -0.71 & -0.34 & -0.20 & 332 & -0.903 & 35.0 & 100.0 & 180 & 29 & 000 & 2.0 & 090 \\
\hline $00: 43: 20$ & 32 & -103 & 73 & 0 & -0.69 & 0.01 & -0.37 & 332 & 2.361 & 35.0 & 100.0 & 180 & 40 & 000 & 2.0 & 090 \\
\hline $00: 43: 30$ & 33 & -107 & 73 & 0 & -0.57 & 0.45 & -0.54 & 332 & 6.260 & 35.0 & 100.0 & 180 & 30 & 000 & 2.0 & 090 \\
\hline $00: 43: 40$ & 34 & -109 & 73 & 0 & -0.39 & 0.84 & -0.67 & 334 & 9.528 & 35.0 & 100.0 & 180 & 29 & 000 & 2.0 & 090 \\
\hline $00: 43: 50$ & 35 & -110 & 66 & 0 & -0.30 & 0.92 & -0.73 & 335 & 10.462 & 35.0 & 100.0 & 180 & 37 & 000 & 2.0 & 090 \\
\hline $00: 44: 00$ & 36 & -111 & 62 & 0 & -0.25 & 0.55 & -0.71 & 337 & 8.054 & 35.0 & 100.0 & 180 & 49 & 000 & 2.0 & 090 \\
\hline $00: 44: 10$ & 36 & -113 & 62 & 0 & -0.21 & 0.32 & -0.72 & 338 & 6.632 & 35.0 & 100.0 & 180 & 34 & 000 & 2.0 & 090 \\
\hline $00: 44: 20$ & 35 & -114 & 61 & 0 & -0.21 & 0.41 & -0.67 & 339 & 6.823 & 22.1 & 100.0 & 180 & 49 & 000 & 2.0 & 090 \\
\hline $8: 44: 30$ & 35 & -115 & 61 & 0 & -0.30 & 0.35 & -0.46 & 340 & 5.158 & 10.6 & 100.0 & 180 & 40 & 000 & 2.0 & 090 \\
\hline$: 44: 40$ & 36 & -117 & 72 & 0 & -0.25 & 0.03 & -0.26 & 341 & 1.846 & 12.1 & 100.0 & 180 & 39 & 000 & 2.0 & 090 \\
\hline $00: 44: 50$ & 35 & -118 & 73 & 0 & -0.10 & -0.15 & -0.17 & 341 & 0.125 & 17.1 & 100.0 & 180 & 41 & 000 & 2.0 & 090 \\
\hline $00: 45: 00$ & 35 & -118 & 74 & 0 & 0.02 & -0.14 & -0.14 & 341 & -0.020 & 18.1 & 100.0 & 180 & 33 & 000 & 2.0 & 090 \\
\hline $00: 45: 10$ & 34 & -118 & 74 & 0 & 0.22 & -0.20 & -0.15 & 341 & -0.279 & 19.2 & 100.0 & 180 & 44 & 000 & 2.0 & 090 \\
\hline $00: 45: 20$ & 32 & -117 & 68 & 0 & 0.29 & -0.21 & -0.18 & 341 & -0.170 & 19.8 & 100.0 & 180 & 32 & 000 & 2.0 & 090 \\
\hline
\end{tabular}


$\begin{array}{llccccc}\text { Time } & \text { Position (m) } & \text { RPM } & \text { Speed (Knots) } & \text { Turn Rudder Gill Wind } & \text { Current }\end{array}$

\begin{tabular}{|c|c|c|c|c|c|c|c|c|c|c|c|c|c|c|c|c|}
\hline $\begin{aligned} 9: & : 45: 30 \\
& : 45: 40\end{aligned}$ & $\begin{array}{l}31 \\
31\end{array}$ & $\begin{array}{l}-116 \\
-115\end{array}$ & $\begin{array}{l}69 \\
69\end{array}$ & $\begin{array}{l}0 \\
0\end{array}$ & $\begin{array}{l}0.21 \\
0.26\end{array}$ & $\begin{array}{l}0.02 \\
0.08\end{array}$ & $\begin{array}{l}-0.16 \\
-0.05\end{array}$ & $\begin{array}{l}341 \\
341\end{array}$ & $\begin{array}{l}1.170 \\
0.808\end{array}$ & $\begin{array}{l}12.1 \\
10.2\end{array}$ & $\begin{array}{l}100.0 \\
100.0\end{array}$ & $\begin{array}{l}180 \\
180\end{array}$ & $\begin{array}{l}37 \\
37\end{array}$ & $\begin{array}{l}000 \\
000\end{array}$ & $\begin{array}{l}2.0 \\
2.0\end{array}$ & $\begin{array}{l}090 \\
090\end{array}$ \\
\hline $\int: 45: 50$ & 30 & -114 & 68 & 0 & 0.39 & -0.27 & -0.02 & 341 & -1.623 & 19.7 & 100.0 & 180 & 42 & 000 & 2.0 & 090 \\
\hline $00: 46: 00$ & 28 & -112 & 62 & 0 & 0.39 & -0.40 & -0.08 & 341 & -2.035 & 26.6 & 100.0 & 180 & 41 & 000 & 2.0 & 090 \\
\hline $00: 46: 10$ & 26 & -111 & 61 & 0 & 0.27 & -0.48 & -0.18 & 340 & -1.907 & 32.3 & 100.0 & 180 & 49 & 000 & 2.0 & 090 \\
\hline $00 ; 46 ; 20$ & 24 & -111 & 61 & 0 & 0.07 & -0.34 & -0.29 & 340 & -0.312 & 32.9 & 100.0 & 180 & 28 & 000 & 2.0 & 090 \\
\hline $00: 46: 30$ & 23 & -111 & 62 & 0 & 0.01 & 0.08 & -0.31 & 340 & 2.455 & 24.4 & 100.0 & 180 & 40 & 000 & 2.0 & 090 \\
\hline $00: 46: 40$ & 23 & -111 & 62 & 0 & 0.05 & 0.08 & -0.21 & 341 & 1.849 & 11.4 & 100.0 & 180 & 44 & 000 & 2.0 & 090 \\
\hline $00: 46: 50$ & 22 & -111 & 68 & 0 & 0.07 & 0.11 & -0.10 & 341 & 1.292 & 10.1 & 100.0 & 180 & 40 & 000 & 2.0 & 090 \\
\hline $00: 47: 00$ & 23 & -110 & 68 & 0 & 0.17 & 0.28 & -0.06 & 341 & 2.152 & 15.7 & 100.0 & 180 & 39 & 000 & 2.0 & 090 \\
\hline $00: 47: 10$ & 23 & -109 & 71 & 0 & 0.27 & 0.41 & -0.02 & 342 & 2.727 & 11.5 & 100.0 & 180 & 36 & 000 & 2.0 & 090 \\
\hline $00: 47: 20$ & 24 & -107 & 71 & 0 & 0.41 & 0.54 & 0.06 & 342 & 3.055 & 5.8 & 100.0 & 180 & 40 & 000 & 2.0 & 090 \\
\hline $00: 47: 30$ & 25 & -104 & 71 & 0 & 0.51 & 0.52 & 0.21 & 343 & 1.958 & 0.7 & 100.0 & 180 & 37 & 000 & 2.0 & 090 \\
\hline $00: 47: 40$ & 25 & -101 & 63 & 0 & 0.52 & 0.28 & 0.35 & 343 & -0.417 & 3.0 & 100.0 & 180 & 41 & 000 & 2.0 & 090 \\
\hline $00: 47: 50$ & 26 & -98 & 58 & 0 & 0.57 & 0.18 & 0.41 & 343 & -1.425 & 8.3 & 100.0 & 180 & 33 & 000 & 2.0 & 090 \\
\hline $00: 48: 00$ & 27 & -94 & 56 & 0 & 0.57 & 0.28 & 0.42 & 342 & -0.854 & 10.5 & 100.0 & 180 & 32 & 000 & 2.0 & 090 \\
\hline $00: 48: 10$ & 28 & -91 & 35 & 0 & 0.49 & 0.14 & 0.44 & 342 & -1.899 & 16.2 & 100.0 & 180 & 32 & 000 & 2.0 & 090 \\
\hline $00: 48 ; 20$ & 28 & -89 & 35 & 0 & 0.45 & -0.19 & 0.44 & 342 & -3.970 & 31.2 & 100.0 & 180 & 35 & 000 & 2.0 & 090 \\
\hline $00: 48: 30$ & 28 & -86 & 34 & 0 & 0.38 & -0.25 & 0.40 & 341 & -4.088 & 35.0 & 100.0 & 180 & 32 & 000 & 2.0 & 090 \\
\hline $00: 48: 40$ & 28 & -85 & 34 & 0 & 0.24 & 0.06 & 0.35 & 341 & -1.816 & 35.0 & 100.0 & 180 & 39 & 000 & 2.0 & 090 \\
\hline $00: 48: 50$ & 29 & -83 & 34 & 0 & 0.15 & 0.16 & 0.31 & 340 & -0.912 & 35.0 & 100.0 & 180 & 41 & 000 & 2.0 & 090 \\
\hline $00: 49: 00$ & 29 & -82 & 34 & 0 & 0.13 & 0.01 & 0.28 & 340 & -1.720 & 35.0 & 100.0 & 180 & 39 & 000 & 2.0 & 090 \\
\hline $00: 49: 10$ & 30 & -82 & 30 & 0 & 0.01 & -0.18 & 0.28 & 340 & -2.884 & 35.0 & 100.0 & 180 & 33 & 000 & 2.0 & 090 \\
\hline $00: 49: 20$ & 30 & -82 & 31 & 0 & -0.09 & -0.47 & 0.30 & 339 & -4.882 & 35.0 & 100.0 & 180 & 39 & 000 & 2.0 & 090 \\
\hline $00: 49: 30$ & 29 & -83 & 46 & 0 & -0.15 & -0.71 & 0.29 & 338 & -6.364 & 35.0 & 100.0 & 180 & 42 & 000 & 2.0 & 090 \\
\hline $00: 49: 40$ & 28 & -84 & 58 & 0 & -0.32 & -0.76 & 0.20 & 337 & -6.089 & 35.0 & 100.0 & 180 & 30 & 000 & 2.0 & 090 \\
\hline $00: 49: 50$ & 28 & -87 & 63 & 0 & -0.50 & -0.53 & 0.07 & 336 & -3.763 & 35.0 & 100.0 & 180 & 28 & 000 & 2.0 & 090 \\
\hline $00: 50: 00$ & 28 & -90 & 64 & 0 & -0.55 & -0.55 & -0.06 & 336 & -3.067 & 35.0 & 100.0 & 180 & 32 & 000 & 2.0 & 090 \\
\hline $00: 50: 10$ & 27 & -93 & 61 & 0 & -0.52 & -0.53 & -0.17 & 335 & -2.314 & 35.0 & 100.0 & 180 & 26 & 000 & 2.0 & 090 \\
\hline $00: 50: 20$ & 27 & -96 & 65 & 0 & -0.45 & -0.36 & -0.28 & 335 & -0.524 & 35.0 & 100.0 & 180 & 45 & 000 & 2.0 & 090 \\
\hline $00: 50: 30$ & 26 & -99 & 65 & 0 & -0.47 & -0.31 & -0.40 & 335 & 0.588 & 35.0 & 100.0 & 180 & 36 & 000 & 2.0 & 090 \\
\hline $00: 50: 40$ & 26 & -102 & 68 & 0 & -0.55 & 0.22 & -0.50 & 335 & 4.591 & 35.0 & 100.0 & 180 & 33 & 000 & 2.0 & 090 \\
\hline $00: 50: 50$ & 27 & -105 & 68 & 0 & -0.47 & 0.64 & -0.58 & 337 & 7.751 & 35.0 & 100.0 & 180 & 40 & 000 & 2.0 & 090 \\
\hline $00: 51: 00$ & 28 & -107 & 72 & 0 & -0.39 & 0.75 & -0.63 & 338 & 8.786 & 35.0 & 100.0 & 180 & 41 & 000 & 2.0 & 090 \\
\hline $00: 51: 10$ & 29 & -108 & 73 & 0 & -0.28 & 0.84 & -0.71 & 339 & 9.811 & 35.0 & 100.0 & 180 & 41 & 000 & 2.0 & 090 \\
\hline $00: 51: 20$ & 30 & -109 & 73 & 0 & -0.20 & 0.61 & -0.66 & 341 & 8.077 & 19.0 & 100.0 & 180 & 38 & 000 & 2.0 & 090 \\
\hline $00: 51: 30$ & 30 & -110 & 73 & 0 & -0.14 & 0.36 & -0.39 & 342 & 4.764 & 0.0 & 100.0 & 180 & 36 & 000 & 2.0 & 090 \\
\hline $00: 51: 40$ & 30 & -110 & 69 & 0 & -0.03 & 0.33 & -0.03 & 343 & 2.287 & 1.0 & 100.0 & 180 & 39 & 000 & 2.0 & 090 \\
\hline$-0: 51: 50$ & 31 & -110 & 38 & 0 & -0.05 & 0.26 & 0.11 & 343 & 0.917 & 13.1 & 100.0 & 180 & 38 & 000 & 2.0 & 090 \\
\hline$: 52: 00$ & 32 & -110 & 49 & 0 & -0.09 & 0.04 & 0.19 & 343 & -0.932 & 27.3 & 100.0 & 180 & 32 & 000 & 2.0 & 090 \\
\hline $.00: 52: 10$ & 33 & -111 & 58 & 0 & -0.04 & 0.14 & 0.15 & 343 & -0.029 & 21.9 & 100.0 & 330 & 51 & 000 & 2.0 & 090 \\
\hline $00: 52: 20$ & 34 & -110 & 52 & 0 & 0.09 & 0.32 & 0.16 & 343 & 0.966 & 15.2 & 100.0 & 000 & 32 & 000 & 2.0 & 090 \\
\hline $00: 52: 30$ & 35 & -109 & 48 & 0 & 0.27 & 0.24 & 0.21 & 343 & 0.172 & 16.7 & 100.0 & 000 & 43 & 000 & 2.0 & 090 \\
\hline $00: 52: 40$ & 35 & -107 & 48 & 0 & 0.45 & -0.05 & 0.22 & 343 & -1.681 & 24.0 & 100.0 & 331 & 36 & 000 & 2.0 & 090 \\
\hline $00: 52: 50$ & 34 & -105 & 48 & 0 & 0.46 & -0.14 & 0.16 & 343 & -1.909 & 30.8 & 100.0 & 331 & 33 & 000 & 2.0 & 090 \\
\hline $00: 53: 00$ & 34 & -102 & 49 & 0 & 0.41 & -0.07 & 0.08 & 342 & -0.931 & 32.6 & 100.0 & 327 & 41 & 000 & 2.0 & 090 \\
\hline $00: 53: 10$ & 33 & -100 & 49 & 0 & 0.43 & -0.12 & 0.00 & 342 & -0.721 & 34.6 & 100.0 & 306 & $46^{\prime}$ & 000 & 2.0 & 090 \\
\hline $00 ; 53 ; 20$ & 32 & -99 & 48 & 0 & 0.38 & -0.20 & -0.09 & 342 & -0.675 & 35.0 & 100.0 & 335 & 36 & 000 & 2.0 & 090 \\
\hline $00: 53: 30$ & 30 & -97 & 49 & 0 & 0.31 & -0.38 & -0.13 & 342 & -1.591 & 35.0 & 74.0 & 063 & 37 & 000 & 2.0 & 090 \\
\hline $00: 53: 40$ & 28 & -96 & 49 & 0 & 0.28 & -0.46 & -0.16 & 342 & -1.912 & 35.0 & 74.0 & 084 & 46 & 000 & 2.0 & 090 \\
\hline $00: 53: 50$ & 26 & -95 & 49 & 0 & 0.25 & -0.31 & -0.19 & 341 & -0.740 & 35.0 & 99.0 & 084 & 45 & 000 & 2.0 & 090 \\
\hline $00: 54: 00$ & 25 & -95 & 53 & 0 & 0.15 & 0.14 & -0.24 & 341 & 2.419 & 32.6 & 99.0 & 122 & 47 & 000 & 2.0 & 090 \\
\hline $00: 54: 10$ & 25 & -94 & 56 & 0 & 0.10 & 0.48 & -0.20 & 342 & 4.318 & 13.6 & 99.0 & 122 & 31 & 000 & 2.0 & 090 \\
\hline $00: 54: 20$ & 26 & -93 & 56 & 0 & 0.10 & 0.46 & -0.03 & 343 & 3.108 & 7.8 & 99.0 & 122 & 41 & 000 & 2.0 & 090 \\
\hline $00: 54: 30$ & 27 & -92 & 56 & 0 & 0.20 & 0.44 & 0.09 & 343 & 2.215 & 7.1 & 99.0 & 237 & 29 & 000 & 2.0 & 090 \\
\hline $00: 54: 40$ & 28 & -91 & 56 & 0 & 0.34 & 0.28 & 0.19 & 343 & 0.583 & 8.9 & 99.0 & 217 & 38 & 000 & 2.0 & 090 \\
\hline $00: 54: 50$ & 29 & -89 & 49 & 0 & 0.23 & 0.21 & 0.24 & 343 & -0.194 & 11.4 & 99.0 & 217 & 33 & 000 & 2.0 & 090 \\
\hline $00: 55: 00$ & 30 & -88 & 46 & 0 & 0.09 & 0.26 & 0.30 & 343 & -0.276 & 12.3 & 99.0 & 217 & 35 & 000 & 2.0 & 090 \\
\hline $00: 55: 10$ & 31 & -87 & 47 & 0 & 0.02 & 0.16 & 0.34 & 343 & -1.140 & 18.9 & 99.0 & 233 & 35 & 000 & 2.0 & 090 \\
\hline $00: 55: 20$ & 32 & -87 & 47 & 0 & -0.02 & 0.24 & 0.34 & 343 & -0.653 & 19.0 & 99.0 & 210 & 41 & 000 & 2.0 & 090 \\
\hline $00: 55: 30$ & 34 & -86 & 46 & 0 & 0.04 & 0.21 & 0.35 & 343 & -0.898 & 21.6 & 99.0 & 235 & 40 & 000 & 2.0 & 090 \\
\hline $00: 55: 40$ & 35 & -86 & 46 & 0 & 0.06 & -0.07 & 0.32 & 343 & -2.458 & 25.2 & 99.0 & 235 & 37 & 000 & 2.0 & 090 \\
\hline $00: 55: 50$ & 35 & -85 & 47 & 0 & 0.08 & 0.01 & 0.26 & 342 & -1.548 & 26.6 & 99.0 & 273 & 44 & 000 & 2.0 & 090 \\
\hline $00: 56: 00$ & 36 & -84 & 47 & 0 & 0.06 & 0.28 & 0.26 & 342 & 0.097 & 13.7 & 99.0 & 236 & 38 & 000 & 2.0 & 090 \\
\hline $00: 56: 10$ & 37 & -84 & 46 & 0 & 0.14 & 0.13 & 0.29 & 342 & -1.044 & 19.3 & 99.0 & 268 & 29 & 000 & 2.0 & 090 \\
\hline $00: 56: 20$ & 38 & -82 & 46 & 0 & 0.26 & -0.06 & 0.27 & 342 & -2.059 & 28.4 & 99.0 & 268 & 30 & 000 & 2.0 & 090 \\
\hline $00: 56: 30$ & 37 & -81 & 47 & 0 & 0.22 & -0.16 & 0.19 & 342 & -2.224 & 35.0 & 99.0 & 208 & 39 & 000 & 2.0 & 090 \\
\hline $00: 56: 40$ & 37 & -80 & 47 & 0 & 0.08 & -0.27 & 0.12 & 341 & -2.475 & 35.0 & 99.0 & 208 & 31 & 000 & 2.0 & 090 \\
\hline $00: 56: 50$ & 36 & -80 & 46 & 0 & 0.06 & -0.46 & 0.08 & 341 & -3.454 & 35.0 & 99.0 & 208 & 38 & 000 & 2.0 & 090 \\
\hline $00: 57 ; 00$ & 35 & -80 & 46 & 0 & -0.01 & -0.74 & 0.05 & 340 & -5.037 & 35.0 & 99.0 & 208 & 30 & 000 & 2.0 & 090 \\
\hline $00: 57: 10$ & 33 & -82 & 47 & 0 & -0.20 & -0.79 & 0.05 & 339 & -5.311 & 35.0 & 99.0 & 134 & 31 & 000 & 2.0 & 090 \\
\hline $00: 57 ; 20$ & 32 & -84 & 47 & 0 & -0.32 & -0.72 & 0.06 & 338 & -4.951 & 35.0 & 99.0 & 112 & 41 & 000 & 2.0 & 090 \\
\hline $00: 57: 30$ & 32 & -86 & 53 & 0 & -0.38 & -0.39 & 0.04 & 338 & -2.736 & 35.0 & 99.0 & 112 & 37 & 000 & 2.0 & 090 \\
\hline $00: 57: 40$ & 32 & -88 & 57 & 0 & -0.37 & -0.08 & -0.05 & 337 & -0.200 & 35.0 & 99.0 & 112 & 35 & 000 & 2.0 & 090 \\
\hline $00: 57: 50$ & 32 & -90 & 61 & 0 & -0.31 & -0.06 & -0.13 & 337 & 0.426 & 35.0 & 99.0 & 112 & 32 & 000 & 2.0 & 090 \\
\hline $00: 58: 00$ & 32 & -91 & 64 & 0 & -0.24 & -0.07 & -0.24 & 337 & 1.103 & 35.0 & 99.0 & 112 & 33 & 000 & 2.0 & 090 \\
\hline $00: 58: 10$ & 32 & -92 & 63 & 0 & -0.23 & 0.11 & -0.35 & 338 & 2.924 & 35.0 & 99.0 & 112 & 45 & 000 & 2.0 & 090 \\
\hline$: 58: 20$ & 32 & -94 & 63 & 0 & -0.25 & 0.58 & -0.44 & 339 & 6.464 & 35.0 & 99.0 & 112 & 35 & 000 & 2.0 & 090 \\
\hline$: 58: 30$ & 33 & -95 & 64 & 0 & -0.27 & 0.75 & -0.47 & 340 & 7.690 & 28.4 & 99.0 & 112 & 39 & 000 & 2.0 & 090 \\
\hline $00: 58: 40$ & 34 & -96 & 64 & 0 & -0.28 & 0.60 & -0.34 & 341 & 5.966 & 14.3 & 99.0 & 112 & 44 & 000 & 2.0 & 090 \\
\hline $00: 58: 50$ & 35 & -97 & 64 & 0 & -0.14 & 0.35 & -0.12 & 342 & 2.981 & 3.9 & 99.0 & 112 & 34 & 000 & 2.0 & 090 \\
\hline
\end{tabular}




\section{Open Ocean}

\section{Simulation Track Plot}

Ship: 150,000 ton, Tanker, partial load

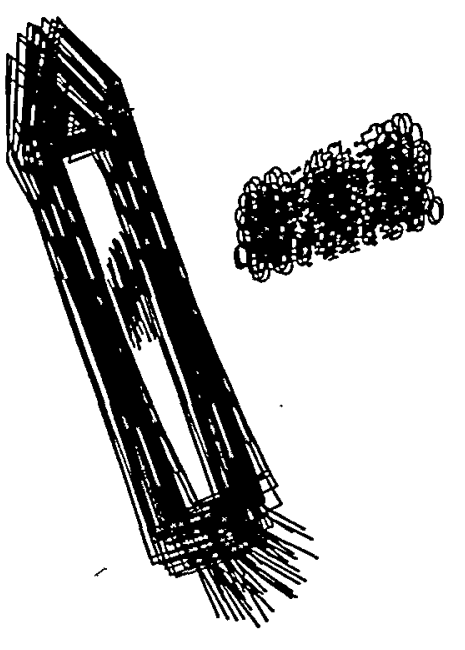

Start Time [hrs:min:sec]: $00: 33: 20$

End Time [hrs:min:sec]: $00: 59: 00$

Delta Plot Time [sec]:

East-West Scale [m]:

30

Date: 05-Feb-99 Time: 16:28:24

East-West Scale [Nm]: 


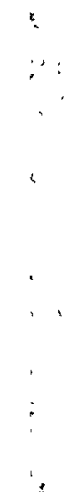


Appendix B REPORT ON APPLICATION TO THE
LIVERPOOL BAY PROJECT 
1.0 Introduction $1-1$

2.0 Summary and Conclusions $2-1$

3.0 Mooring Buoy and system of Operations 3-1

$\begin{array}{lll}3.1 & \text { Buoy Configuration } & 3-1 \\ 3.2 & \text { System of Operation } & 3-3 \\ 3.3 & \text { Model Vessel Configuration } & 3-4\end{array}$

4.0 Impacts between the Buoy and the Vessel 4-1

5.0 Vessel remaining moored by Differential Pressure and Friction 5-1

\section{Figures}

3-1 Specified Mooring Buoy 3-2

3-2 Model Buoy Used 3-2

4-1 Fendering Buoy with Cone

4-2 Fendering Flat Top Buoy

5-1 Buoy Mooring System 5-2

5-2 Mooring Force-Deflection Curve 5-2

\section{Tables}

2-1 Test Wave Conditions 2-3

2-2 Mooring Test Results 2-3

2-3 Results of Mooring/Unmooring Tests $\quad \cdot 2-4$

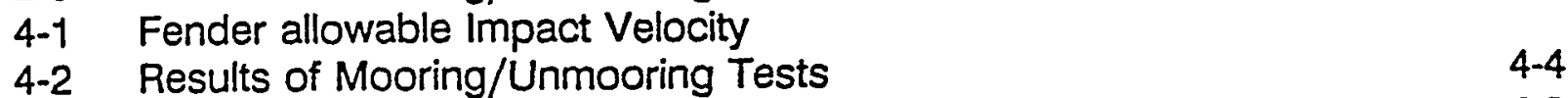

5-1 Mooring Test Results, Maximum Horizontal Buoy Force 5-3

5-2 Maximum Force in the Heaviest Loaded Leg 5-4

\section{Enclosures}

Enclosure A - Photographic Record of Buoy Vessel Impacts and Analyses

Enclosure B - Brochure for The Suction Mooring Technology

Enclosure C - Brochure for Elliot Turbo Machinery Bow Thruster

Enclosure D - Report from the Danish Maritime Institute 


\subsection{Introduction}

This report is the result of Service Order SOW/0321 entered into between Hamilton Oil Co. Ltd. and Jens Korsgaard P.E. on April 14, 1994.

The service order relates to the mooring system for the Oil Loading Unit (OLU) for the Liverpool Bay Development Project.

The objectives of the work were:

o To model test and assess the feasibility of a novel synthetic fiber rope mooring for a submersible mooring buoy

o To model test and determine the operational limits for the mooring technology developed by Mr. Jens Korsgaard called the Suction Mooring Technology (SMT). SMT consists of mooring a vessel to a submersible buoy by means of a combination of hydrostatic pressure differentials and friction.

The model tests were carried out at the Danish Maritime Institute (DMI) May 8 through May 101994 under a contract between Jens Korsgaard and DMI. 


\subsection{Summary and Conclusions}

In a series of communications between Hamilton Oil Co. Ltd. and Jens Korsgaard P.E. in the period September 93 to March 94, conceptual designs for disconnectable moorings for either a shuttle tanker or a Floating Storage Unit (FSU) to be moored in Liverpool Bay for the Liverpool Bay Development Project had been presented. The designs had two novel aspects:

1. The mooring system is a standard submersible Catenary Anchor Leg Mooring (CALM) with a radially deployed anchor line mooring system. However, while the standard system employs only wire rope and/or chain in the anchor line, the anchor line in the new system was comprised of synthetic polyester rope and chain.

2. The mooring is based on the suction mooring technology in which the vessel at least for part of the time is held by a buoy which is forced onto the hull of the tanker by differential hydrostatic pressure, obtained by having a pump take suction in the isolated volume between the top of the buoy and the hull of the vessel.

The first of the above innovations makes it possible to employ submersible CALM buoys in shallow water, it also causes the elastic response of the buoy to become virtually linear in contrast to the strongly non-linear response which is typical for chain or steel wire/chain mooring systems. A secondary benefit of employing synthetic rope is that such rope is nearly weightless in sea water, therefore the synthetic rope exhibits very little catenary action. This factor makes it possible to deploy this type of mooring in wide range of water depths and yet:

o The elastic characteristics of the mooring changes vary little with water depth. This factor reduces the need for specific water depth related designs and makes it possible to standardize the mooring design and make moorings made up entirely of a few standardized components, capable of being used in wide range of water depths.

o The mooring may be reused in another project with approximately the same design forces, but with a much different water depth by changing its configuration very little or not at all.

The patented second innovation makes it possible to moor and unmoor in extreme waves at an unprecedented speed. The actual mooring process lasts less than a minute from there being no physical contact or connection between the vessel and the mooring system to the vessel being completely securely moored. Because of this extreme speed of mooring the vessel need not keep station at the point of mooring, it is sufficient that the mooring area on the vessel drifts slowly through the target area. This factor also 
contributes to the low cost and reliability of the system.

In the course of the discussions with Hamilton Oil Co. several version were presented including some which have normal structural connections between the buoy and the vessel, however, the systems which were model tested were those in which the vessel is moored solely by the friction between the vessel and the buoy. The buoy is held securely to the bottom of the vessel by differential hydrostatic pressure.

The modeled buoy had 16 mooring legs, represented in the model by 6 legs such that the 6-leg assembly was equivalent in stiffness to the prototype 16 leg system. Although the prototype system will include means to lower the pressure between the buoy and the vessel hull to below atmospheric pressure, the model did not have this capability. In the model the lowest possible pressure at the top of the buoy was atmospheric pressure at a level slightly above the bottom plating elevation of the vessel.

The tests were deliberately planned so that the model set-up would have inferior capabilities to the prototype system with the objective of establishing the capability limits. In spite of this no failure or difficulty occurred in any of the tests.

The tests were conducted in the towing tank at the Danish Maritime Institute in Lyngby, Denmark. The prototype water depth in all tests was 29.8 meters. The scale was 1:50. A stock tanker of 125,000 DWT size was used. The tanker was modified to incorporate the suction mooring technology mooring system. The tanker was used in two loading conditions, fully loaded with a displacement of 149,000 tonnes and in ballasted condition with a displacement of 60,000 tonnes.

The towing tank was too deep for the planned tests and therefore the carriage was fitted with a false bottom to create shallow water. Current was generated in the model by towing the model set-up through the tank at appropriate speeds. Wind was simulated by applying a constant force. All environmental actions were collinear in the tests.

The test report from the Danish Maritime Institute is included in Enclosure $D$ and it describes the test set-up and test results in detail.

The metacentric height of the buoy while being raised without a retrieval line was lower than intended due to a misunderstanding by the model tests laboratory, therefore the rolling and pitching of the buoy while being raised by compressed air is larger than the prototype buoy. While the model buoy performed satisfactorily during all tests without a retrieval line, the corresponding prototype buoy can be expected to perform significantly better. The model buoy being retrieved with a retrieval line always had the proper metacentric height. 
The wave conditions used during the tests are given below in table 2-1.

$\begin{array}{ccc}\text { Wave ID } & \mathrm{H}_{\mathrm{s}}(\mathrm{m}) & \mathrm{T}_{z}(\mathrm{sec}) \\ \text { A } & 6.6 & 10.1 \\ \text { B } & 7.7 & 13.1 \\ \text { C } & 5.6 & 9.3 \\ \text { D } & 5.0 & 8.9 \\ \text { E } & 4.7 & 8.0 \\ \text { F } & 4.1 & 7.7\end{array}$

Table 2-1 Test Wave Conditions

A list of all tests with the object of determining the mooring force and main results are given below in table $2-2$.

\begin{tabular}{|c|c|c|c|c|c|c|}
\hline $\begin{array}{l}\text { Run } \\
\text { No. }\end{array}$ & $\begin{array}{l}\text { Wave } \\
\text { ID }\end{array}$ & $\begin{array}{l}\text { Vessel } \\
\text { Displacement } \\
\% \text { of full }\end{array}$ & $\begin{array}{l}\text { Current } \\
\text { Speed } \\
+ \text { head } \\
\text { - follow } \\
(\mathrm{m} / \mathrm{sec})\end{array}$ & $\begin{array}{l}\text { Wind } \\
\text { Force } \\
\text { tonnes }\end{array}$ & Mooring & $\begin{array}{l}\text { Maximum } \\
\text { Horizontal } \\
\text { Mooring } \\
\text { Force } \\
\text { tonnes }\end{array}$ \\
\hline 1100 & A & 100 & 0 & 51 & Intact & 365 \\
\hline 1110 & A & 100 & -1.07 & 51 & Intact & 432 \\
\hline 1120 & A & 100 & +1.07 & 51 & Intact & 389 \\
\hline 1200 & B & 100 & 0 & 51 & Intact & 523 \\
\hline 1300 & C & 100 & 0 & 51 & Intact & 306 \\
\hline 1400 & D & 100 & 0 & 51 & Intact & 365 \\
\hline 1410 & D & 100 & -1.07 & 51 & Intact & 295 \\
\hline 1420 & $D$ & 100 & +1.07 & 51 & Intact & 352 \\
\hline 1500 & $E$ & 100 & 0 & 51 & Intact & 277 \\
\hline 1600 & $F$ & 100 & 0 & 51 & Intact & 172 \\
\hline 2100 & A & 100 & 0 & 51 & - one leg & $210 * *$ \\
\hline 2110 & A & 100 & -1.07 & 51 & - one leg & $178 * \star$ \\
\hline 2120 & A & 100 & +1.07 & 51 & - one leg & $252 * \star$ \\
\hline 5110 & A & 40 & -1.07 & 54 & Intact & 366 \\
\hline 5120 & A & 40 & +1.07 & 54 & Intact & 377 \\
\hline 6100 & A & 40 & 0 & 54 & - one leg & 202 ** \\
\hline 6110 & A & 40 & -1.07 & 54 & - one leg & $191 \star \star$ \\
\hline 6111 & A & 40 & -1.07 & 0 & - one leg & $203 * \star$ \\
\hline 6120 & A & 40 & +1.07 & 54 & - one leg & $209 * \star$ \\
\hline
\end{tabular}

Table 2-2 Mooring Test Results 
A list of all tests with the object of determining the feasibility of the mooring process is given below in table 2-3.

\begin{tabular}{|c|c|c|c|c|c|c|}
\hline $\begin{array}{l}\text { Run } \\
\text { No. }\end{array}$ & $\begin{array}{l}\text { Wave } \\
\text { Height } \\
\text { m }\end{array}$ & $\begin{array}{l}\text { Vessel } \\
\text { Displacement } \\
\% \text { of full }\end{array}$ & $\begin{array}{l}\text { Retrieval } \\
\text { Line }\end{array}$ & Cone & $\begin{array}{l}\text { Number } \\
\text { of } \\
\text { Matings }\end{array}$ & $\begin{array}{l}\text { Largest } \\
\text { Impact } \\
\% \text { of allow. }\end{array}$ \\
\hline 7100 & 6.6 & 40 & no & no & 3 & 20 \\
\hline 7110 & 6.6 & 40 & no & no & 4 & low \\
\hline 7200 & 7.7 & 40 & no & no & 4 & 58 \\
\hline 7400 & 5.0 & 40 & no & no & 5 & 10 \\
\hline 7420 & 5.0 & 40 & no & no & 5 & 65 \\
\hline 7500 & 4.7 & 40 & no & no & 4 & low \\
\hline 7520 & 4.7 & 40 & no & no & 3 & 38 \\
\hline 7600 & 4.1 & 40 & no & no & 4 & 5 \\
\hline 8100 & 6.6 & 40 & yes & yes & 5 & 258 \\
\hline 8101 & 6.6 & 40 & yes & no & 8 & 24 \\
\hline 8400 & 5.0 & 40 & yes & yes & 6 & low \\
\hline 8401 & 5.0 & 40 & yes & no & 7 & 11 \\
\hline 8500 & 4.7 & 40 & yes & yes & 6 & 195 \\
\hline 8501 & 4.7 & 40 & yes & no & 7 & 6 \\
\hline 8520 & 4.7 & 40 & yes & yes & 4 & 75 \\
\hline 8600 & 4.1 & 40 & yes & yes & 7 & 123 \\
\hline 3100 & 6.6 & 100 & no & yes & 1 & 43 \\
\hline 3600 & 4.1 & 100 & no & yes & 2 & 46 \\
\hline 3601 & 4.1 & 100 & no & no & 2 & low \\
\hline 4600 & 4.1 & 100 & yes & yes & 2 & low \\
\hline
\end{tabular}

\section{Table 2-3 Results of Mooring/Unmooring Tests}

The main conclusions are the following:

o A tanker moored solely by differential hydrostatic pressure and the resulting friction between the buoy and the vessel remains safely moored in all tested environmental conditions (waves up to $H_{s}=7.7$ meters).

o An actual full scale system could easily incorporate double the friction compared to that obtained in the model thereby ensuring an adequate safety factor in actual use.

o The best and safest mooring configuration is one in which the buoy has a flat top and is not fitted with a retrieval line. This configuration performed very well in all mooring tests. 
o The next best configuration is a flat top buoy with a retrieval line. This configuration also performed well in all mooring tests and exhibited the lowest impact energies.

- A buoy with an upward pointing male cone, joining a corresponding female cone in the hull, suffered damaging horizontal impacts between the male and female cones in all wave heights tested. This configuration is not recommended.It is probable that a buoy could be constructed of this type which could safely moor in wave heights up to 5 meters.

o The linear characteristics of the tested mooring system causes very substantial reductions in the mooring forces experienced compared to an equivalent non-linear wire rope chain system. Therefore the tested system will require reduced anchor and chain dimensions compared to the traditional wire rope/chain system. 


\subsection{Mooring Buoy and System of Operations.}

The system tested is a mooring system of the suction mooring technology type. The Suction Mooring Technology is described in more detail in enclosure B. The buoy is designed to resist a mooring force of 1200 tonnes and is fitted with 16 anchor legs.

The tested system is near the limit of what can practically be achieved using a submersible buoy. The prototype water depth in the tests was 29.8 meters, leaving only a couple of meters clearance between the buoy and the sea bed when a fully loaded tanker was moored at extreme wave conditions and experienced extreme downward excursions.

\subsection{Buoy Configuration.}

The tested buoy is shown on figure 3-2. The buoy is comprised of three parts:

A. The part attached to the vessel. This part is cushioned on the top by standard dock fenders acting as, fenders, compression elements, and friction pads. In addition this part is fitted with concentrically deployed pliable fluid seals. Part $A$ is fitted with permanent buoyancy and deballasting tanks are normally not fitted in this part.

B. The part anchored to the sea bed. This part includes all anchor line connections, riser connections, and fluid and signal connections between the vessel and the sea bed. Fluid swivels are not included in the buoy but are located on the vessel. This part is a cylindrical drum which is used routinely as a deballasting chamber. Part B usually also includes compressed air storage tanks.

C. The bearing tetween parts $A$ and $B$. This bearing enables the ship to weather vane.

Because of the shallow water depth the buoy would normally descend all the way to the sea bed when not moored to a vessel. This is in contrast to buoys in deeper water which are normally designed to descend to a predetermined water depth rather than all the way to the bottom.

The model test facility had been requested to make the model buoy in accordance with fig 3-1. In reality they made the buoy as indicated on fig 3-2. This deviation resulted in the model buoy having a lower metacentric height when partly ballasted. This had only a practical effect in the cases in which the buoy was deballasted by compressed air. In the case of using retrieval lines to retrieve the buoy there was no internal free water surface, therefore these cases had approximately the intended metacentric height. 


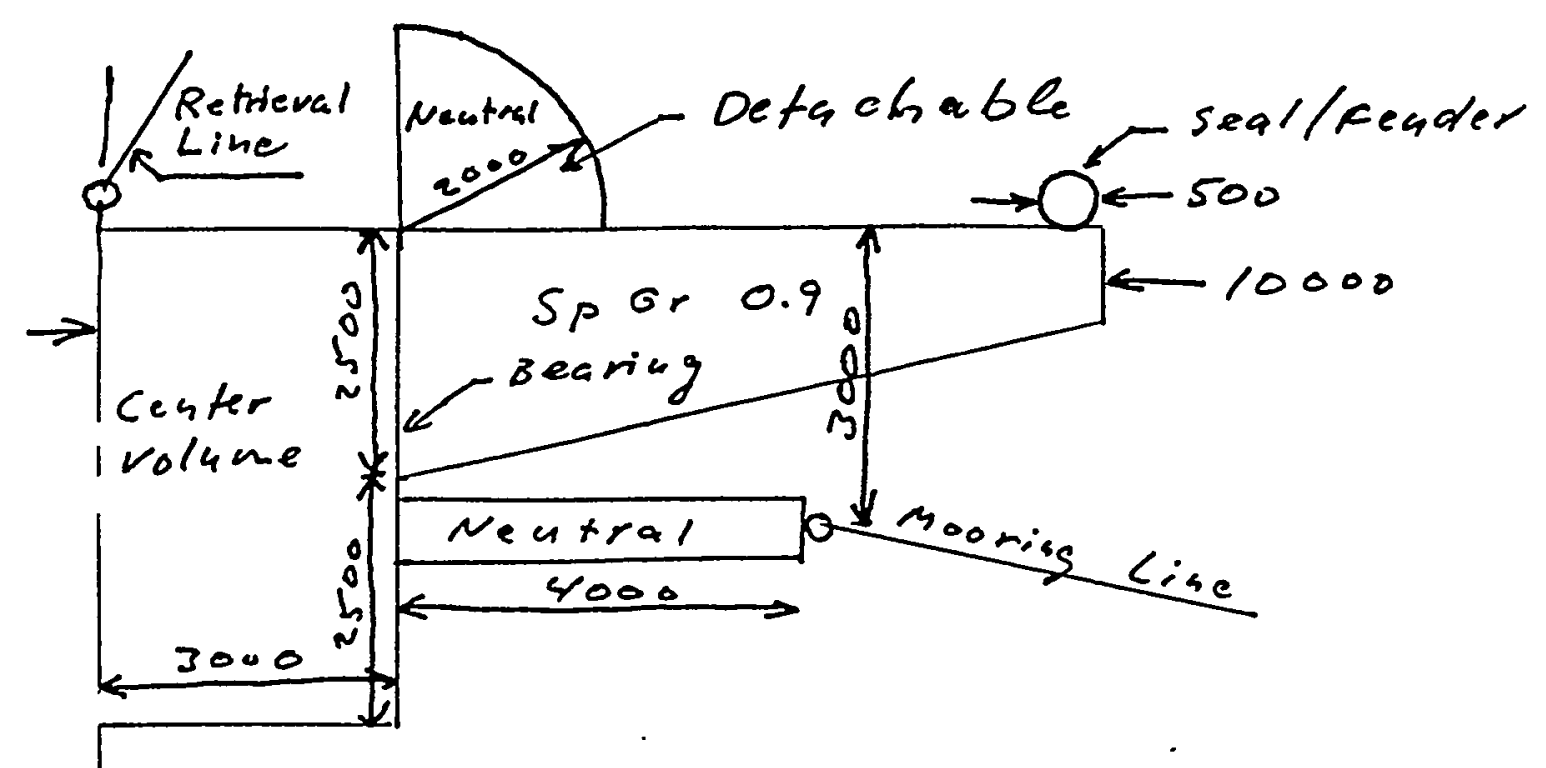

Center volume: S. Gr. 1.5 custer filled S. Gr. 0.8 air filled All meas. in mm

Fig. 3-1 Specified Model Buoy

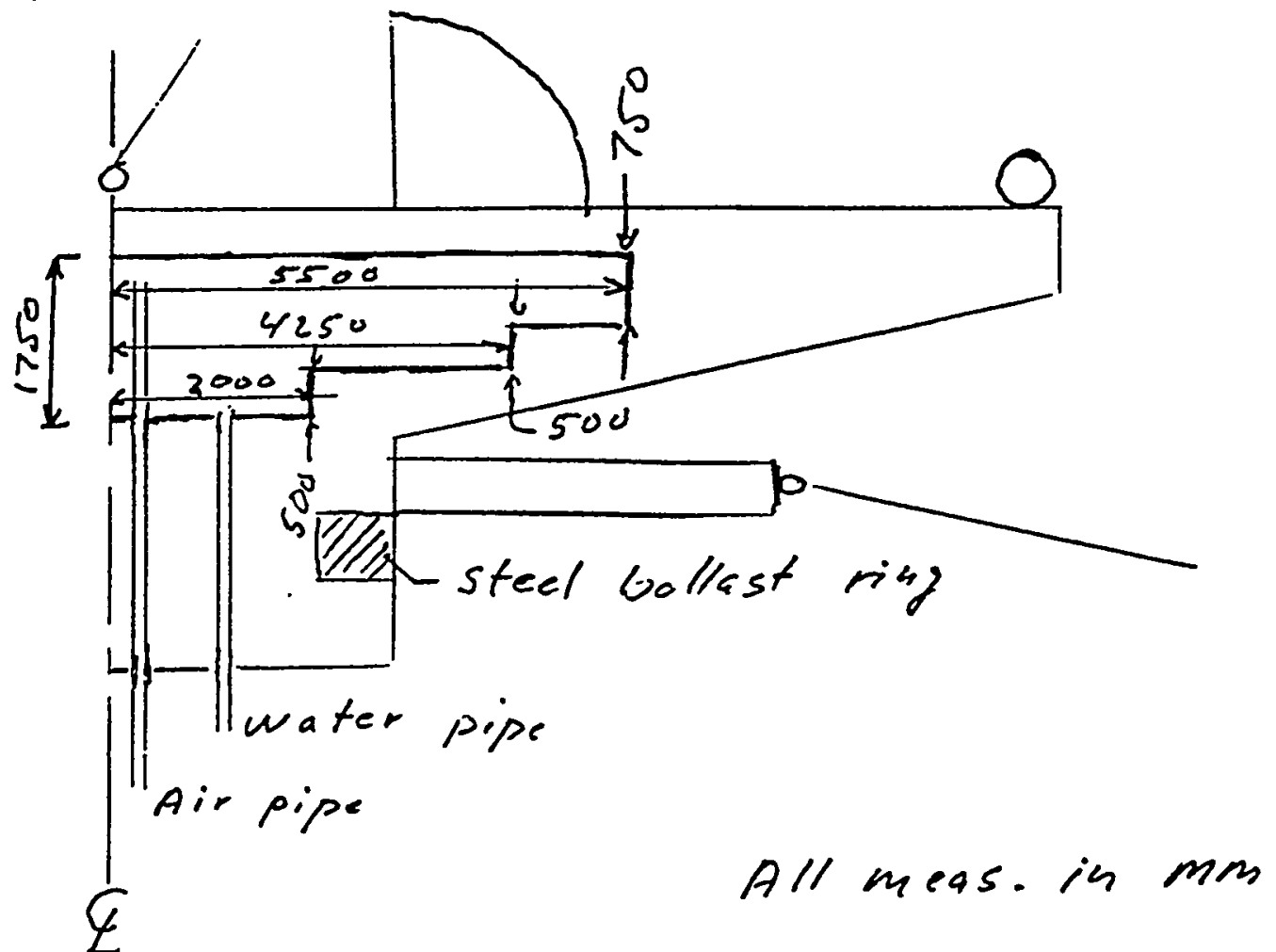

Fig. 3-2 Model Buoy Used

3-2 
Two buoy configurations were tested as indicated on figure 3-1. A configuration with the top conical guide, fitting a corresponding female guide in the vessel was tested, and the alternative configuration with a flat top on the buoy.

\subsection{System of Operation}

Three ways of mooring the vessel were tested:

A. Buoy configuration with a male cone retrieved by means of a buoyant retrieval line.

B. Flat top buoy retrieved by means of a buoyant retrieval line.

C. Flat top buoy retrieved by deballasting with compressed air.

Three different deballasting systems were used during the tests mentioned under $\mathrm{C}$ above:

1. Blowing compressed air into the deballasting chamber to force the ballast water out. Ballast water was let back in by removing the compressed air supply.

2. Same as one, however, compressed air of equal pressure was applied to both the inlet and outlet to stop the water flow. Ballast water was let back in by removing the compressed air supply.

3. A reversible P-D pump was connected to the ballast chamber water connection. The air supply connection was connected to the atmosphere.

System 1 was used in all cases when mooring a fully loaded vessel. System 2 was used for the initial tests with a ballasted vessel, and system 3 was used for the last tests with a ballasted vessel. System 1 could not be properly controlled, whereas systems 2 and 3 could be controlled properly, however, it took too long to reballast the buoy during unmooring using system 2 , therefore a switch was made to system 3.

In all cases using systems 2 and 3 the system was tuned to produce a prototype ascent rate of $0.1 \mathrm{~m} / \mathrm{sec}$ of the buoy in the water column.

In all mooring attempts in the model a dynamic positioning system was approximately emulated by controlling the bow of the vessel by three soft elastic ropes attempting by this means to hold the mooring area of the vessel in proper relations to the buoy. 
The actual procedure was equivalent to the planned prototype procedure in which the vessel while still some distance away commands the buoy acoustically to rise to a level about 2 to 3 meters below the keel of the vessel. When the buoy had risen to this level further deballasting is stopped until the movement of the vessel in relation to the buoy is judged to be opportune and the command is issued to resume the ascent. Approximately 30 seconds later (prototype time) the buoy makes contact with the vessel and blocks the bow thruster intake. About 10 seconds later (prototype time) the buoy is securely held onto the vessel by the differential hydrostatic pressure caused by the bow thruster suction.

\subsection{Model Vessel Configuration}

The model vessel had the following characteristics:

$\begin{array}{ll}L_{\rho p}: & 258 \text { meters } \\ \text { B: } & 40.6 \text { meters } \\ \text { Draft: } & 16.5 \text { meters (fully loaded) } \\ \text { Displacement: } & 148,950 \text { tonnes (fully loaded) }\end{array}$

Additional data on the model vessel is available in enclosure D.

The vessel was equipped with two pumps emulating a bow thruster of the Omnithrusters type or the Elliot Turbo Machinery type. These thrusters take water in at the keel and expel it remotely from the intake area. The prototype pumps would have pumping capacities between 6 and $11 \mathrm{~m}^{3} / \mathrm{sec}$ whereas the model pumps had a combined capacity corresponding to a prototype pumping rate of $4.5 \mathrm{~m}^{3} / \mathrm{sec}$. Thus the model vessel capabilities were significantly below those of the prototype vessel.

A brochure by Elliot Turbo Machinery is enclosed as Enclosure C. 


\subsection{Impacts between the Buoy and the Vessel}

The impacts which were experienced between the buoy and the vessel during the tests depended strongly upon the configuration of the buoy and the method of retrieval. As indicated in the previous chapter three combinations of buoy configuration and retrieval system were tested:

1. Buoy with a conical mating structure and a constant tension winch and a retrieval line.

2. Flat top buoy and a constant tension winch and a retrieval line.

3. Flat top buoy and no retrieval line using compressed air deballasting of the buoy as the means of raising it to the ship's bottom.

System 1 permits horizontal impacts between the conical mating structure on the buoy and the corresponding female cone in the vessel when the male cone enters the female cone in the vessel. All systems permit vertical impacts between the buoy and the bottom of the vessel. Systems 2 and 3 do not permit horizontal impacts between the buoy and the vessel but only vertical impacts.

While the model did not include fendering with the proper stiffness characteristics the model dimensions were based on the provision of specific dock fender units as indicated in figs. 4-1 and 4-2. In the model it was assumed that the fender was already compressed. The model fender was very stiff compared to the prototype fender and therefore only compressed very little during the tests.

The actual performance of the prototype fenders are given in table 4-1.

The performance figures are based on the recommendations by the fender manufacturers and conform to the practice for dock fenders.

For purposes of this report the maximum energy experienced in a test is arbitrarily set to the manufacturer's recommended allowable energy. From this criteria the actual energy experienced in each impact has been caculated as a percentage of the allowable energy.

The actual impact energies experienced during the tests were determined through examination and analysis of the video tapes produced during the tests. An underwater camera had a nearly stationary position relative to the buoy and far removed from the buoy. By examining the video frames from this camera one by one (every 0.02 secs) both the relative angular and translational velocities between the vessel and the buoy can be closely estimated. By analyzing individual frames on the video the impact energies can be estimated with an accuracy of about $+-20 \%$ from the velocities immediately prior to the impact. 


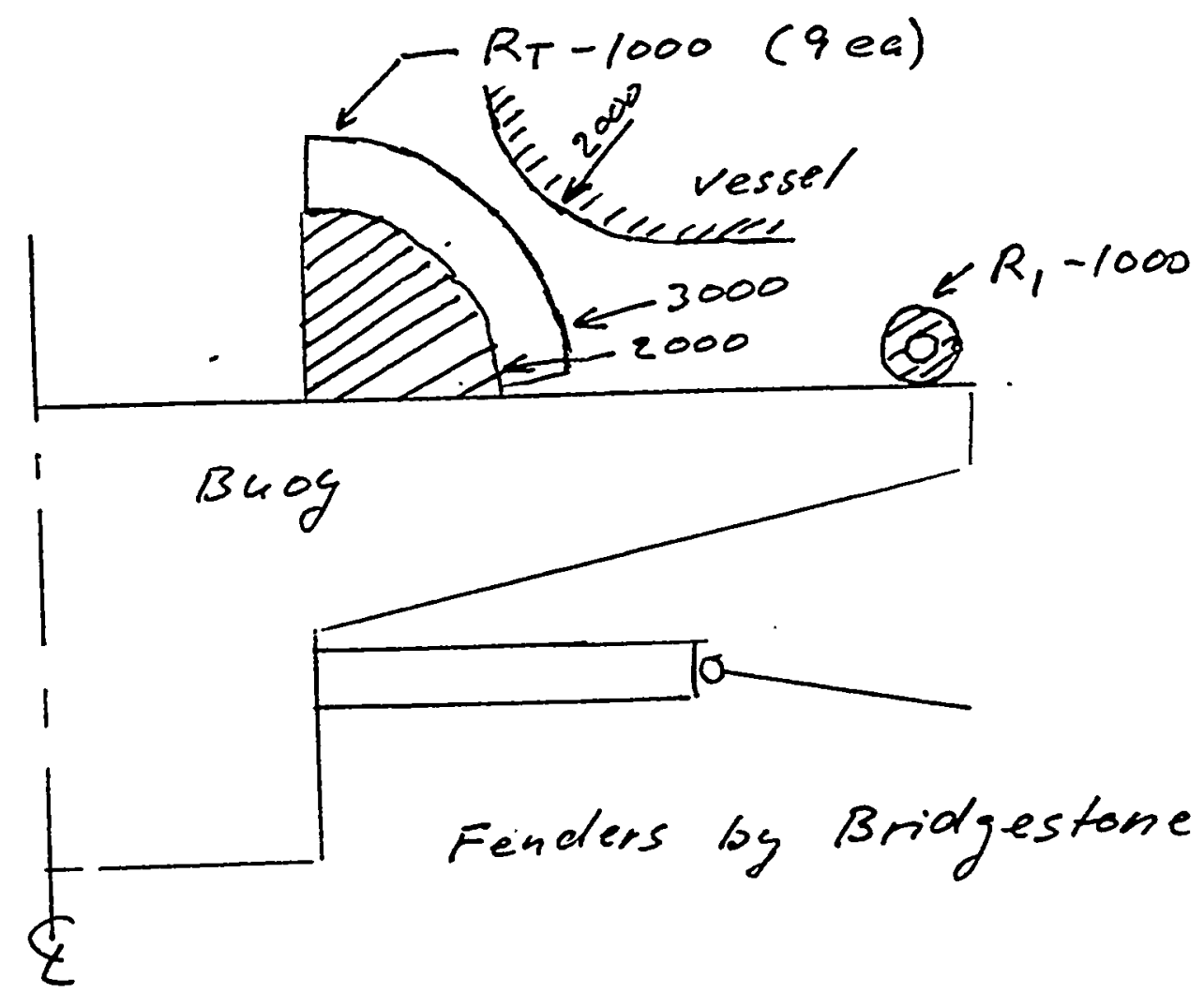

Fig. 4-1 Fenders Buoy with Cone

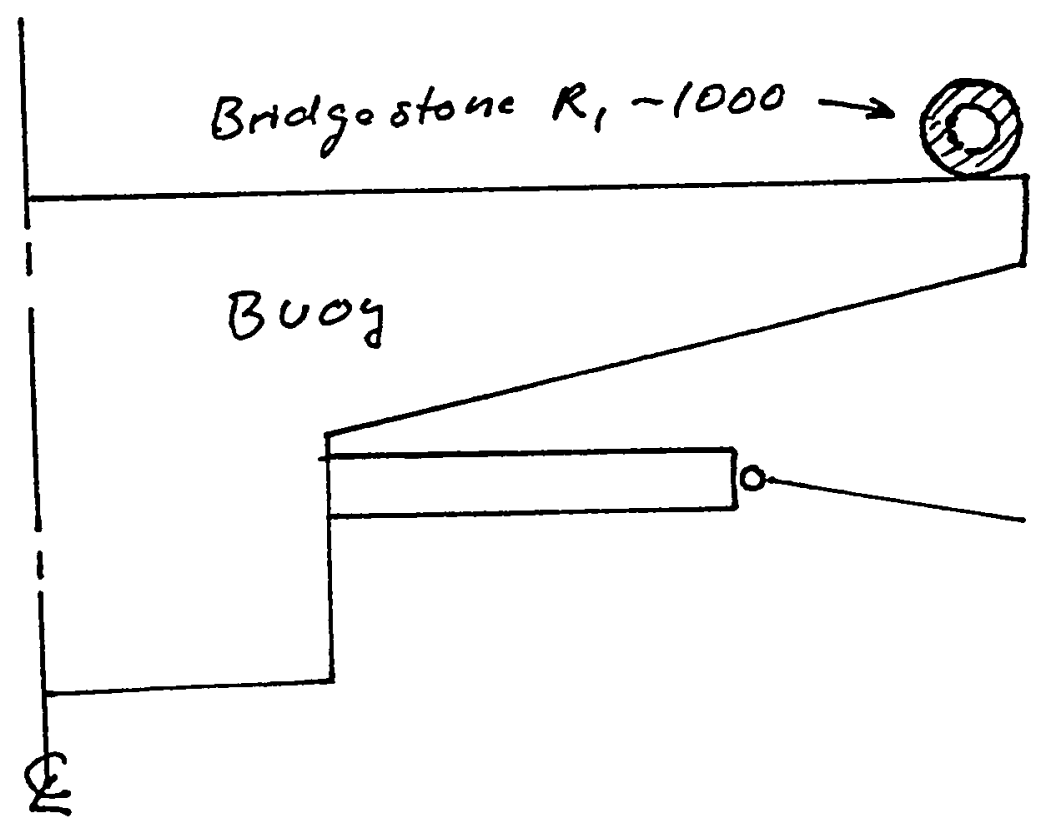

Fig. 4-2 Fenders Flat Top Buoy

4-2 


$\begin{array}{llll}\text { Type of Buoy } & \text { Tilt } & \begin{array}{l}\text { Maximum } \\ \text { Horizontal } \\ \text { Impact } \\ \text { Velocity } \\ \text { (m/sec) }\end{array} & \begin{array}{l}\text { Maximum } \\ \text { Vertical } \\ \text { Impact } \\ \text { Velocity } \\ \text { (m/sec) }\end{array} \\ \text { With cone } & \text { N.A. } & 0.81 & \text { see below } \\ \text { Flat Top } & 0.05 & \text { N.A. } & 2.4 \\ \text { Flat Top } & 0.10 & \text { N.A. } & 1.95 \\ \text { Flat Top } & 0.15 & \text { N.A. } & 1.55 \\ \text { Flat Top } & 0.20 & \text { N.A. } & 1.30 \\ \text { Flat Top } & 0.25 & \text { N.A. } & 1.15\end{array}$

\section{Table 4-1 Allowable Fender Impact Velocities}

All the photos taken from the video tapes are shown in enclosure A. The geometric computations are shown in handwriting on the pictures and the resulting distances and/or angles are shown below each photo. The distances are derived from the known dimensions of the buoy and by using these as scales for the distance measurements.

When a buoy with a male cone as per system 1 impacts the female cone in the vessel both central and eccentric impacts are possible. In consequence all impacts have been conservatively estimated to be central impacts. Vertical central impacts between the buoy and the vessel bottom are possible.

Flat top buoys as per system 2 and system 3 cannot impact the vessel horizontally, only vertical impacts are possible. Central vertical impacts are possible, but cannot cause damage because these impacts are cushioned by the water between the buoy and the vessel hull. The water pressures which would build up would cause large accelerations of the buoy thereby preventing complete compression of the fenders.

Only eccentric impacts with flat top buoys as per systems 2 and 3 can cause damage to the buoy or the vessel.

The analyses of the eccentric impacts were made on the basis that the buoy movement consisted of pure rotation. On the average this assumption is correct, however, for any one specific impact the assumption is conservative if the c.g. of the buoy moves away from the vessel during the impact, and non-conservative if the c.g. of the buoy moves toward the vessel during the impact. The resolution of the photos in general did not permit an evaluation of this factor, it was therefore ignored. 
In the analysis of the impacts the reduction in the energy to be absorbed by the fender due to some of the rotational movement being converted to translational movement was ignored. This is a very conservative assumption which in almost all cases leads to an over estimation of forces and energies to be absorbed by the fenders.

In the analysis of the runs a few instances were found in which there is a discrepancy between the second digit in the run number. The confusion only exists between the digits 5 and 6 and therefore the discrepancy may result in the wave heights $4.1 \mathrm{~m}$ and $4.7 \mathrm{~m}$ being confused in some data. For the purpose of this report this discrepancy has not been resolved, rather the information given in enclosure $D$ (the DMI report) has been assumed to be correct. The discrepancy has no impact on the conclusions to be drawn from the tests.

The results of the tests are given below in table 4-2.

$\begin{array}{ccccccc}\begin{array}{c}\text { Run } \\ \text { No. }\end{array} \begin{array}{c}\text { Wave } \\ \text { Height }\end{array} & \begin{array}{c}\text { Vessel } \\ \text { Displacement Line } \\ \text { m of full }\end{array} & \begin{array}{c}\text { Retrieval } \\ \text { Lene }\end{array} & \begin{array}{c}\text { Cone } \\ \text { Number } \\ \text { of } \\ \text { Matings }\end{array} & \begin{array}{c}\text { Largest } \\ \text { Impact } \\ \% \text { of allow. }\end{array} \\ 7100 & 6.6 & 40 & \text { no } & \text { no } & 3 & 20 \\ 7110 & 6.6 & 40 & \text { no } & \text { no } & 4 & \text { low } \\ 7200 & 7.7 & 40 & \text { no } & \text { no } & 4 & 58 \\ 7400 & 5.0 & 40 & \text { no } & \text { no } & 5 & 10 \\ 7420 & 5.0 & 40 & \text { no } & \text { no } & 5 & 65 \\ 7500 & 4.7 & 40 & \text { no } & \text { no } & 4 & \text { low } \\ 7520 & 4.7 & 40 & \text { no } & \text { no } & 3 & 38 \\ 7600 & 4.1 & 40 & \text { no } & \text { no } & 4 & 5 \\ 8100 & 6.6 & 40 & \text { yes } & \text { yes } & 5 & 258 \\ 8101 & 6.6 & 40 & \text { yes } & \text { no } & 8 & 24 \\ 8400 & 5.0 & 40 & \text { yes } & \text { yes } & 6 & \text { low } \\ 8401 & 5.0 & 40 & \text { yes } & \text { no } & 7 & 11 \\ 8500 & 4.7 & 40 & \text { yes } & \text { yes } & 6 & 195 \\ 8501 & 4.7 & 40 & \text { yes } & \text { no } & 7 & 6 \\ 8520 & 4.7 & 40 & \text { yes } & \text { yes } & 4 & 75 \\ 8600 & 4.1 & 40 & \text { yes } & \text { yes } & 7 & 123 \\ 3100 & 6.6 & 100 & \text { no } & \text { yes } & 1 & 43 \\ 3600 & 4.1 & 100 & \text { no } & \text { yes } & 2 & 46 \\ 3601 & 4.1 & 100 & \text { no } & \text { no } & 2 & \text { low } \\ 4600 & 4.1 & 100 & \text { yes } & \text { yes } & 2 & \text { low }\end{array}$

Table 4-2 Results of Mooring Tests 


\subsection{Vessel remaining Moored by Differential Pressure and Friction.}

A series of tests was carried out in which the vessel was moored and remained moored by the action of differential hydrostatic pressure and friction only. The differential hydrostatic pressure was created by having two pumps with a combined capacity of 4.5 $\mathrm{m}^{3} / \mathrm{sec}$ take suction in the mooring well. Atmospheric air had free access to the well, therefore the minimum possible absolute pressure inside the well was atmospheric pressure at a level immediately above the keel of the vessel.

The external pressure in the $40 \%$ displacement situation was equal to atmospheric pressure plus on the average 4.5 meters head. This modest pressure proved sufficient in all cases to hold the buoy securely attached to the vessel. This was a surprising result because in some cases the bow of the vessel slammed and in at least one case atmospheric air reached the buoy. In this case it would appear that the internal pressure would be the same or larger than the external pressure and therefore the buoy should release immediately. It is believed that the reason it did not happen is that the continuity equation requires that the water filling the expanded volume between the buoy and the vessel be accelerated to move into the void, and the time available did not permit this. For the same reason the friction between the vessel and the buoy hardly diminished either. since the model laws governing the model tests (Froude's Law) also governs this phenomenon this result would also be obtained in the prototype.

In all the tests the buoy was held by pressure and friction only. In no tests did the buoy ever slip. Considering the very low differential pressure used in all $40 \%$ displacement tests it can be concluded that with a pump aboard the vessel capable of lowering the pressure between the buoy and the vessel to say $50 \mathrm{kPa}$ that an adequate safety factor against slipping is assured and this type of mooring is safe in all wave heights tested.

The Mooring system modeled is comprised of 16 mooring legs made up of a combination of polyester rope and chain as indicated in Figure 5-1. The mooring rope is almost neutrally buoyant and therefore does not provide any significant catenary action. The elasticity of the system is in consequence derived from a combination of the non-linear elastic extension of the synthetic rope and the non-linear catenary action of the chain legs. The combination of all these elastic elements result in a nearly linear stress-strain curve as indicated on figure 5-2. 


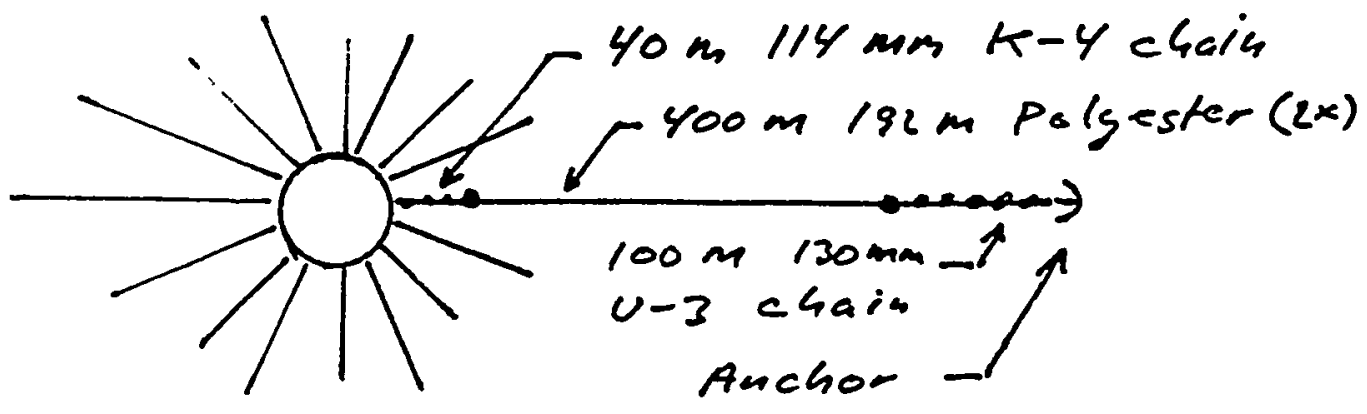

Fig. 5-1 Buoy Mooring System

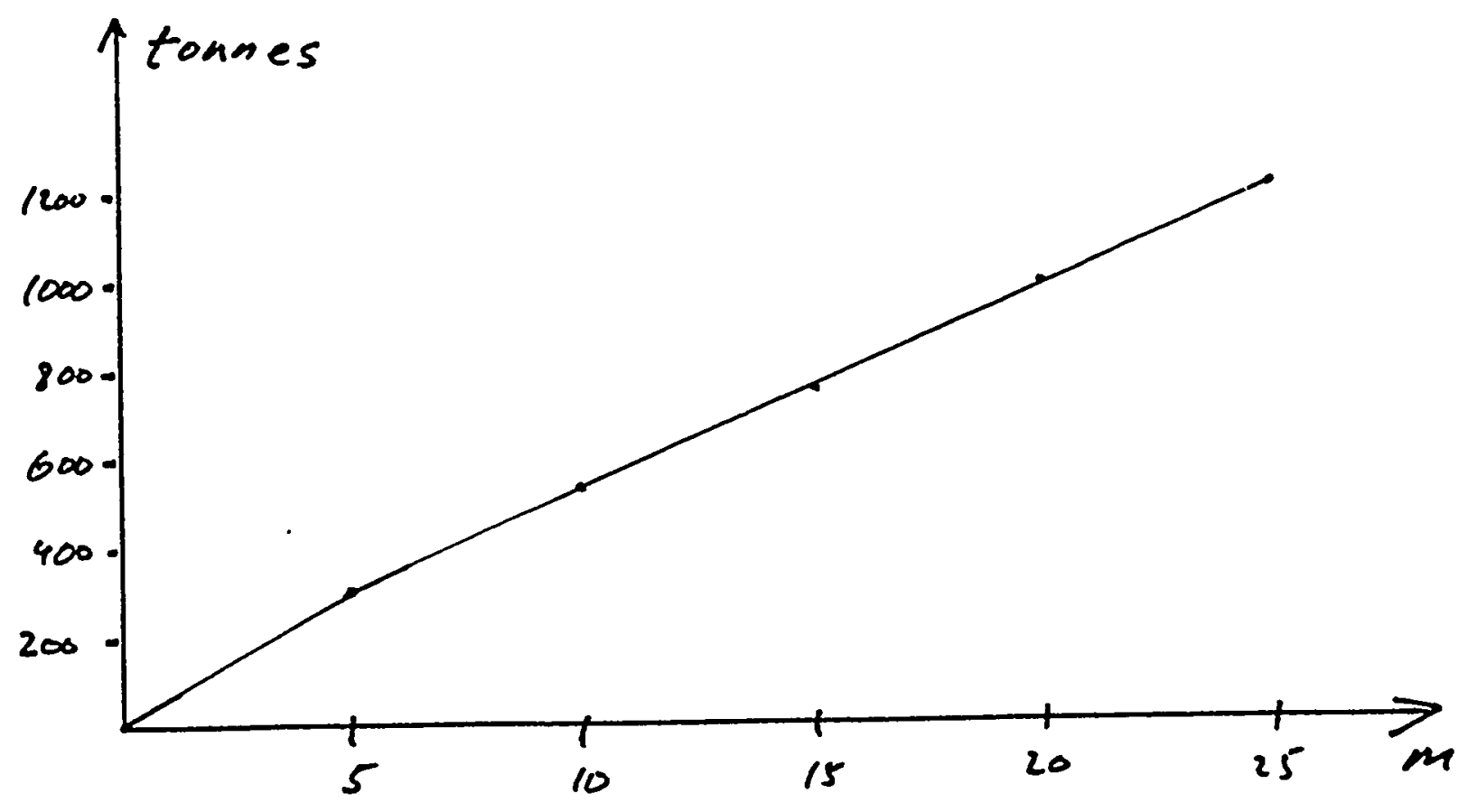

Fig. 5-2 Mooring Force-Deflection Curve

5-2 
The tests are described in more detail in Enclosure $D$, the report from the Danish Maritime Institute. The details are not repeated here but the results are summarized below in table 5-1.

\begin{tabular}{|c|c|c|c|c|c|c|c|}
\hline $\begin{array}{l}\text { Run } \\
\text { No. }\end{array}$ & $\begin{array}{l}\text { Wave } \\
\text { ID }\end{array}$ & \multicolumn{2}{|c|}{$\begin{array}{l}\text { Vessel } \\
\text { Displacement } \\
\% \text { of full }\end{array}$} & $\begin{array}{l}\text { Current } \\
\text { Speed } \\
+ \text { head } \\
\text { - follow } \\
\text { (m/sec) }\end{array}$ & $\begin{array}{l}\text { Wind } \\
\text { Force } \\
\text { tonnes }\end{array}$ & Mooring & $\begin{array}{l}\text { Maximum } \\
\text { Horizontal } \\
\text { Mooring } \\
\text { Force } \\
\text { tonnes }\end{array}$ \\
\hline 1100 & A & 6.6 & 100 & 0 & 51 & Intact & 365 \\
\hline 1110 & A & & 100 & -1.07 & 51 & Intact & 432 \\
\hline 1120 & A & & 100 & +1.07 & 51 & Intact & 389 \\
\hline 1200 & B & 7.7 & 100 & 0 & 51 & Intact & 523 \\
\hline 1300 & C & 5.6 & 100 & 0 & 51 & Intact & 306 \\
\hline 1400 & D & 5.0 & 100 & 0 & 51 & Intact & 365 \\
\hline 1410 & $D$ & & 100 & -1.07 & 51 & Intact & 295 \\
\hline 1420 & $D$ & & 100 & +1.07 & 51 & Intact & 352 \\
\hline 1500 & $E$ & 4.7 & 100 & 0 & 51 & Intact & 277 \\
\hline 1600 & $F$ & 4.1 & 100 & 0 & 51 & Intact & 172 \\
\hline 2100 & A & & 100 & 0 & 51 & - one leg & $\star \star$ \\
\hline 2110 & A & & 100 & -1.07 & 51 & - one leg & ** \\
\hline 2120 & A & & 100 & +1.07 & 51 & - one leg & $\star \star *$ \\
\hline 5110 & A & & 40 & -1.07 & 54 & Intact & 366 \\
\hline 5120 & A & & 40 & +1.07 & 54 & Intact & 377 \\
\hline 6100 & A & & 40 & 0 & 54 & - one leg & $\star \star$ \\
\hline 6110 & A & & 40 & -1.07 & 54 & - one leg & $\star \star *$ \\
\hline 6111 & A & & 40 & -1.07 & 0 & - one leg & *夫 \\
\hline 6120 & A & & 40 & +1.07 & 54 & - one leg & 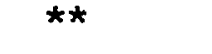 \\
\hline
\end{tabular}

$\star \star$ Not measured

Table 5-1 Mooring Test Results, Maximum Horizontal Buoy Force

In table 5-2 below is given the largest force in the heaviest loaded leg of the 6-leg system tested. 


\begin{tabular}{|c|c|c|c|c|c|c|}
\hline $\begin{array}{l}\text { Run } \\
\text { No. }\end{array}$ & $\begin{array}{l}\text { Wave } \\
\text { ID }\end{array}$ & $\begin{array}{l}\text { Vessel } \\
\text { Displacement } \\
\% \text { of full }\end{array}$ & $\begin{array}{l}\text { Current } \\
\text { Speed } \\
+ \text { head } \\
\text { - follow } \\
(\mathrm{m} / \mathrm{sec})\end{array}$ & $\begin{array}{l}\text { Wind } \\
\text { Force } \\
\text { tonnes }\end{array}$ & Mooring & $\begin{array}{l}\text { Maximum } \\
\text { Horizontal } \\
\text { Force in } \\
\text { One Leg } \\
\text { tonnes }\end{array}$ \\
\hline 1100 & A & 100 & 0 & 51 & Intact & 237 \\
\hline 1110 & $A$ & 100 & -1.07 & 51 & Intact & 264 \\
\hline 1120 & A & 100 & +1.07 & 51 & Intact & 245 \\
\hline 1200 & B & 100 & 0 & 51 & Intact & 308 \\
\hline 1300 & C & 100 & 0 & 51 & Intact & 206 \\
\hline 1400 & D & 100 & 0 & 51 & Intact & 234 \\
\hline 1410 & $D$ & 100 & -1.07 & 51 & Intact & 202 \\
\hline 1420 & $\mathrm{D}$ & 100 & +1.07 & 51 & Intact & 222 \\
\hline 1500 & $E$ & 100 & 0 & 51 & Intact & 192 \\
\hline 1600 & $\bar{F}$ & 100 & 0 & 51 & Intact & 160 \\
\hline 2100 & A & 100 & 0 & 51 & - one leg & 210 \\
\hline 2110 & A & 100 & -1.07 & 51 & - one leg & 178 \\
\hline 2120 & A & 100 & +1.07 & 51 & - one leg & 252 \\
\hline 5110 & A & 40 & -1.07 & 54 & Intact & 229 \\
\hline 5120 & A & 40 & +1.07 & 54 & Intact & 233 \\
\hline 6100 & A & 40 & 0 & 54 & - one leg & 202 \\
\hline 6110 & $A$ & 40 & -1.07 & 54 & - one leg & 191 \\
\hline 6111 & A & 40 & -1.07 & 0 & - one leg & 203 \\
\hline 6120 & A & 40 & +1.07 & 54 & - one leg & 209 \\
\hline
\end{tabular}

Table 5-2 Maximum Force in the Heaviest Loaded Leg 


\section{Appendix C}

\section{MAIN REPORT FROM PHYSICAL MODEL TESTS AT THE DANISH MARITIME INSTITUTE}


1. INTRODUCTION $\ldots \ldots \ldots \ldots \ldots \ldots \ldots \ldots \ldots \ldots \ldots \ldots \ldots$

2. SUMMARY AND CONCLUSION $\ldots \ldots \ldots \ldots \ldots \ldots \ldots \ldots$

3. DESCRIPTION OF EXPERIMENTAL FACILITIES

AND MODELS $\ldots \ldots \ldots \ldots \ldots \ldots \ldots \ldots \ldots \ldots \ldots \ldots \ldots \ldots \ldots$

3.1 Vessel Particulars and Loading Conditions ......... 3

$3.2 \quad$ Physical Modelling $\ldots \ldots \ldots \ldots \ldots \ldots \ldots \ldots . .6$

3.3 Experimental Models $\ldots \ldots \ldots \ldots \ldots \ldots \ldots \ldots .4$

3.3.1 Vessel $\ldots \ldots \ldots \ldots \ldots \ldots \ldots \ldots \ldots \ldots, 4$

3.3.2 Buoy $\ldots \ldots \ldots \ldots \ldots \ldots \ldots \ldots \ldots \ldots, 4$

3.3.3 Mooring system $\ldots \ldots \ldots \ldots \ldots \ldots \ldots . \quad 7$

3.4 Tank Facility $\ldots \ldots \ldots \ldots \ldots \ldots \ldots \ldots \ldots \ldots$

3.5 Instrumentation $\ldots \ldots \ldots \ldots \ldots \ldots \ldots \ldots \ldots, 9$

3.6 Data Logging $\ldots \ldots \ldots \ldots \ldots \ldots \ldots \ldots \ldots$

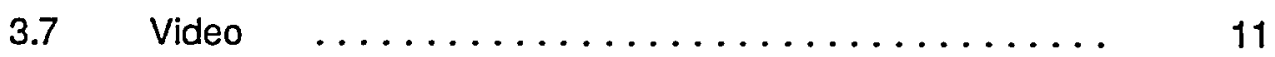

4. TEST SET-UP AND PROCEDURES $\ldots \ldots \ldots \ldots \ldots \ldots \ldots$.

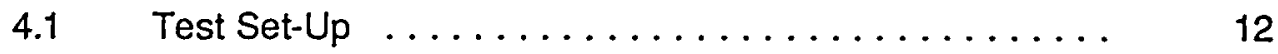

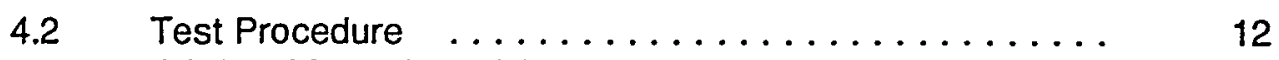

4.2.1 Moored condition tests $\ldots \ldots \ldots \ldots \ldots \ldots \ldots \quad 12$

4.2.2 Mating / decoupling tests ............ 13

5. TEST PROGRAMME $\ldots \ldots \ldots \ldots \ldots \ldots \ldots \ldots \ldots \ldots \ldots \ldots$

$5.1 \quad$ Environmental Conditions $\ldots \ldots \ldots \ldots \ldots \ldots \ldots$

5.1 .1 Waves $\ldots \ldots \ldots \ldots \ldots \ldots \ldots \ldots \ldots \ldots \ldots \ldots \ldots$

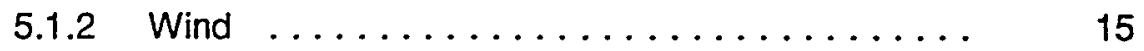

5.1 .3 Current $\ldots \ldots \ldots \ldots \ldots \ldots \ldots \ldots \ldots \ldots .16$

5.1.4 Water depth $\ldots \ldots \ldots \ldots \ldots \ldots \ldots \ldots . \quad 16$

$5.2 \quad$ Test Numbers $\ldots \ldots \ldots \ldots \ldots \ldots \ldots \ldots \ldots \ldots \ldots$ 


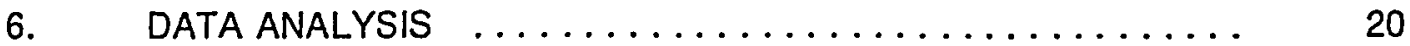

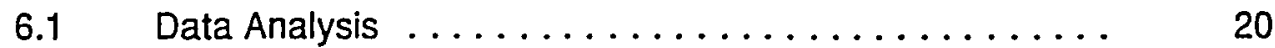

$6.2 \quad$ Presentation of Results $\ldots \ldots \ldots \ldots \ldots \ldots \ldots \ldots \ldots$

7. EVALUATION OF MEASUREMENTS AND RESULTS $\ldots \ldots \ldots .22$

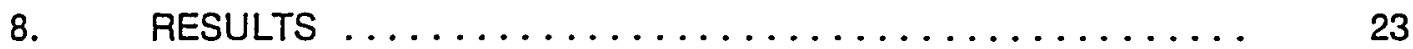

8.1 Moored Vessel Tests $\ldots \ldots \ldots \ldots \ldots \ldots \ldots \ldots \ldots$

8.2 Mating / Decoupling Tests .............. 25

8.3 Retrieval Line Load Versus Buoy Level $\ldots \ldots \ldots \ldots \ldots . . \ldots 24$

\section{APPENDICES:}

Appendix A : Video Tapes.

Appendix B : Results.

Appendix C : Waves.

THESE APPENDICES ARE NOT INCLUDED

IN THIS DOCUMENT

Appendix D : Model Details. 


\section{INTRODUCTION}

This report presents the main results obtained from a study of a disconnectable mooring system developed primarily for shuttle tankers. The study has been carried out for Mr. J Korsgaard, U.S.A. by Danish Maritime Institute.

The concept utilizes a moored buoy with a buoyancy chamber enabling the level of the buoy below the surface to be controlled. The buoy is fixed to the vessel by the surrounding water pressure, i.e. once the buoy has made contact with the vessel the water which is in the moonpool and above the buoy is removed, thereby establishing a pressure difference. The water from the moonpool is removed by the bow thruster which has its intake close to the moonpool orifice. Water leaking past the buoy/vessel sealing is removed continuously by a drainage pump. Decoupling is performed by pumping water into the moonpool thereby removing the pressure difference which otherwise holds the buoy to the vessel.

The objective of the study was primarily to investigate the feasibility of the concept from a technical point of view, i.e. if it worked or not when exposed to harsh as well as moderate environmental conditions and to clarify any limiting effects.

Physical model tests with the concept have been carried out with a typical vessel loaded to two different draughts corresponding to fully and partially loaded. Both tests with the vessel moored to the buoy and the mating / decoupling phase have been tested.

The tests where carried out in May 1994 and were supervised by Mr. J. Korsgaard. 


\section{SUMMARY AND CONCLUSIONS}

A series of physical model tests have been carried out with a disconnectable mooring system developed primarily for shuttle tankers. The mooring system consists of a moored buoy which when not in use is kept submerged at a certain level below the sea-surface. Once the shuttle tanker has arrived and is positioned above the buoy, ballast water is removed from the buoy which then rises up under the vessel. At the same time the fore thruster is used to pump water from the moonpool thereby causing the buoy to be sucked to the vessel due to the pressure difference. A large seal at the outer edge of the buoy prevents damage to the vessel and buoy and ensures that a minimum amount of water is able to flood into the moonpool. Water, which enters the moonpool, is continuously removed by drainage pumps. The reverse situation where the vessel is to decouple from the buoy involves pumping water into the moonpool thereby eliminating the holding pressure thus causing the buoy to release.

A number of tests have been carried out with a typical vessel loaded to two different conditions corresponding to fully and partially loaded. The tests encompassed both the condition where the vessel is moored to the buoy and held on station solely by the mooring system as well as the mating / decoupling situations. In both types of tests the vessel was exposed to a range of different environmental conditions covering both moderate and very harsh situations.

The results from the tests are presented in the form of statistical values for each run, time series of e.g. mooring line forces and video tapes of all the tests.

None of the tests with the vessel attached to the buoy showed any indication of the buoy slipping regardless of the environmental conditions which the vessel was exposed to.

Numerous mating and decoupling tests were carried out using either a retrieval line or controlled buoyancy to bring the buoy up under the vessel. The tests showed that this operation could be performed successfully with both methods under both moderate and severe environmental conditions. Tests were carried out with and without the torus fender included. These tests showed that the mating operation is easier to perform without the torus fender as the fender tends to displace the buoy when the fender collides with the moonpool orifice. 


\section{DESCRIPTION OF EXPERIMENTAL FACILITIES AND MODELS}

This Chapter describes the vessel, buoy and various techniques used in this study. Furthermore, the documentation for the anchor system is included in this Chapter, while all other results are presented in Chapter 8 and Appendix B.

\subsection{Vessel Particulars and Loading Conditions}

The main dimensions of the vessel are listed in Table 3.1 together with the two loading conditions used.

\begin{tabular}{||lc|c|c|}
\hline \multicolumn{1}{|c|}{ Dimension } & & $100 \%$ Loaded & $40 \%$ Loaded \\
\hline \hline Displacement & $\left(\mathrm{m}^{3}\right)$ & 148950 & 60200 \\
Length (Lpp) & $(\mathrm{m})$ & 258.3 & 258.3 \\
Breadth & $(\mathrm{m})$ & 40.6 & 40.6 \\
Depth & $(\mathrm{m})$ & 21.9 & 21.9 \\
Draught, fore & $(\mathrm{m})$ & 16.5 & 5.95 \\
Draught, aft & $(\mathrm{m})$ & 16.5 & 7.95 \\
Radii of gyration, $r_{x}$ (roll) & $(\mathrm{m})$ & 16.3 & 15.6 \\
Radii of gyration, $r_{y}$ (pitch) & $(\mathrm{m})$ & 62.4 & 63.6 \\
Radii of gyration, $r_{z}$ (yaw) & $(\mathrm{m})$ & 62.4 & 64.5 \\
Vertical distance to cog $\mathrm{V}_{\mathrm{cg}}$ & $(\mathrm{m})$ & 11.5 & 12.1 \\
Longitudinal distance to cog $\mathrm{L}_{\mathrm{cg}}$ & $(\mathrm{m})$ & 8.6 & 6.7 \\
\hline
\end{tabular}

Table 3.1. Vessel Particulars and Loading Conditions.

\subsection{Physical Modelling}

The model design is based on Froude scaling, which ensures that the gravitational and inertia forces are modelled correctly if the Froude number is the same in the model as in full-scale. Froude's number is defined as:

$$
F=\frac{V}{\sqrt{g \cdot L}}
$$

where $g$ is the acceleration of gravity, $L$ a characteristic length and $V$ a characteristic velocity. 
Froude modelling leads to the following scales from full size to model:

$\begin{array}{lll}\text { Length } & : & 1: 50 \\ \text { Time } & : & 1: 50^{1 / 2} \\ \text { Mass } & : & 1:\left(50^{3} \cdot 1.025\right) \\ \text { Force } & : & 1:\left(50^{3} \cdot 1.025\right)\end{array}$

The factor of 1.025 is used to compensate for the different water densities due to the fact that the water in the tank is non-saline.

Froude's model law assumes that the fluid is ideal, i.e. viscous forces are not modelled correctly. Hence its validity is restricted to the potential flow regime. Therefore skin friction and drag forces - depending of the geometry - are not modelled entirely correctly.

\subsection{Experimental Models}

This section gives a brief description of how the various models were built.

\subsubsection{Vessel}

A modified stock tanker was used for these tests. The modifications consisted of installing a moonpool (10 $\mathrm{m}$ diameter) $40 \mathrm{~m}$ from the fore perpendicular.

The moonpool consisted of two tubes inserted into each other. The outer tube was fixed to the vessel by a ring with a curved inner side which was mounted flush with the bottom of the hull. The inner tube which could be moved up and down had a clear ceiling enabling the motion of the buoy relative to the vessel to be monitored. The purpose of the ceiling was primarily to reduce the volume of water which had to be removed. A small air-vent tube was led through the ceiling thus ensuring that the minimum pressure in the chamber above the buoy corresponded to the surrounding atmosphere. This tube was further used for the retrieval line. 
Two holes were made side by side and just in front of the moonpool where the curved ring turns into the flat bottom of the vessel. Tubes were glued into the holes and led to pumps which could remove the water in the moonpool and discharge it at the stern. The pumps simulated the intake of the thruster though providing somewhat less capacity than the proposed thruster is capable of. The capacity of the pumps was measured to approximately $4.5 \mathrm{~m}^{3} / \mathrm{s}$. The design originally called for $6 \mathrm{~m}^{3} / \mathrm{s}$ which later was revised to $11 \mathrm{~m}^{3} / \mathrm{s}$.

The principle is illustrated in Figure 3.1 below.

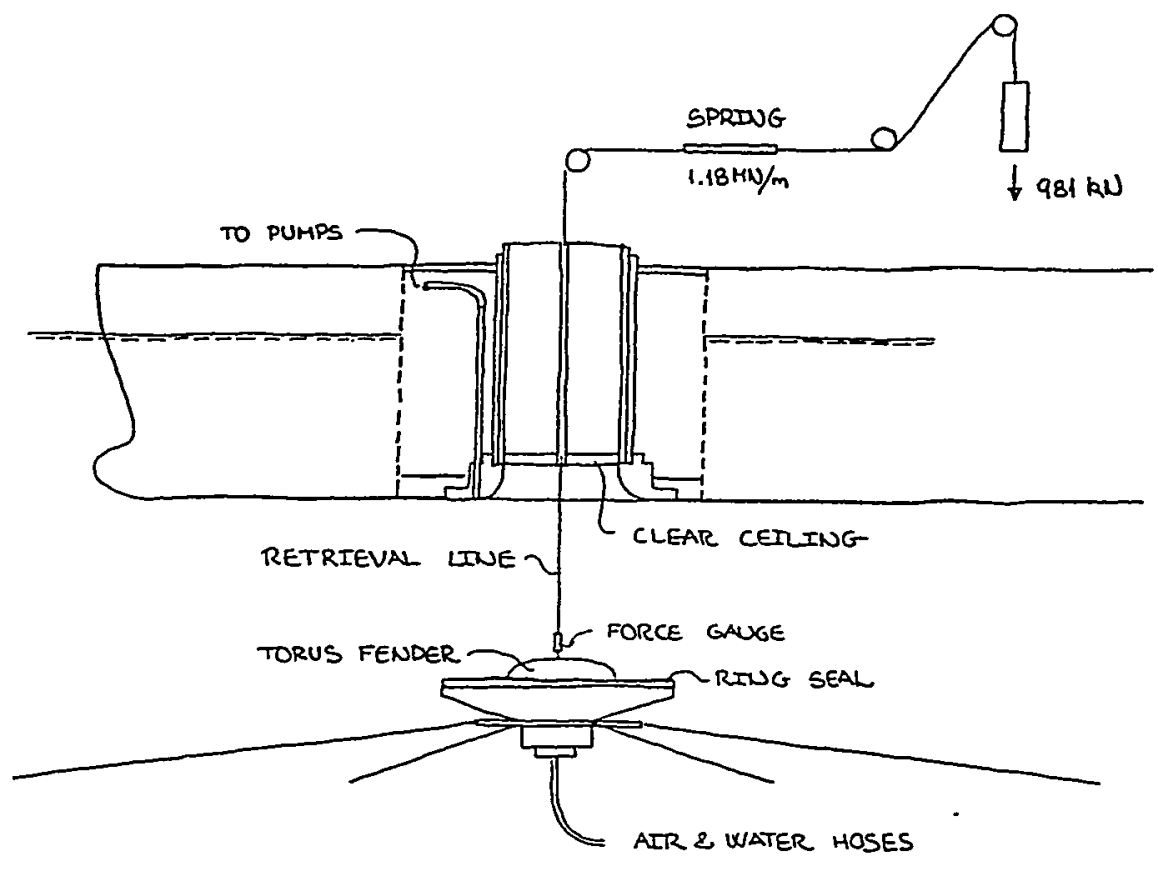

Figure 3.1. Moonpool.

The clear ceiling was for the majority of the tests placed $3 \mathrm{~m}$ above the bottom of the hull. However, for the tests where a force gauge was placed in the retrieval line (Run Nos. 81008600 ) the height of the ceiling above the bottom of the hull was increased to $5.2 \mathrm{~m}$. 


\subsubsection{Buoy}

The buoy was designed based on information provided by Mr. J. Korsgaard in fax to DMI dated 1994-04-04. Figure 3.2 shows the main dimensions of the buoy. In order to protect the protruding air / water attachments an additional and neutrally buoyant protection ring was mounted under the buoy. The detachable torus fender mounted on top of the buoy was made of foam-rubber, while the sealing at the outer edge of the buoy was made of a latex hose in order to provide sufficiently soft characteristics.

Various systems were devised to control the amount of water in the internal buoyancy chamber. In the final version a water pump was used to either suck out or pump in the water. This was done by attaching a flexible and submerged hose to the water outlet pipes leading into the chamber while the similar air pipe was attached to the water pump. This set-up provided an efficient control of the buoy level.

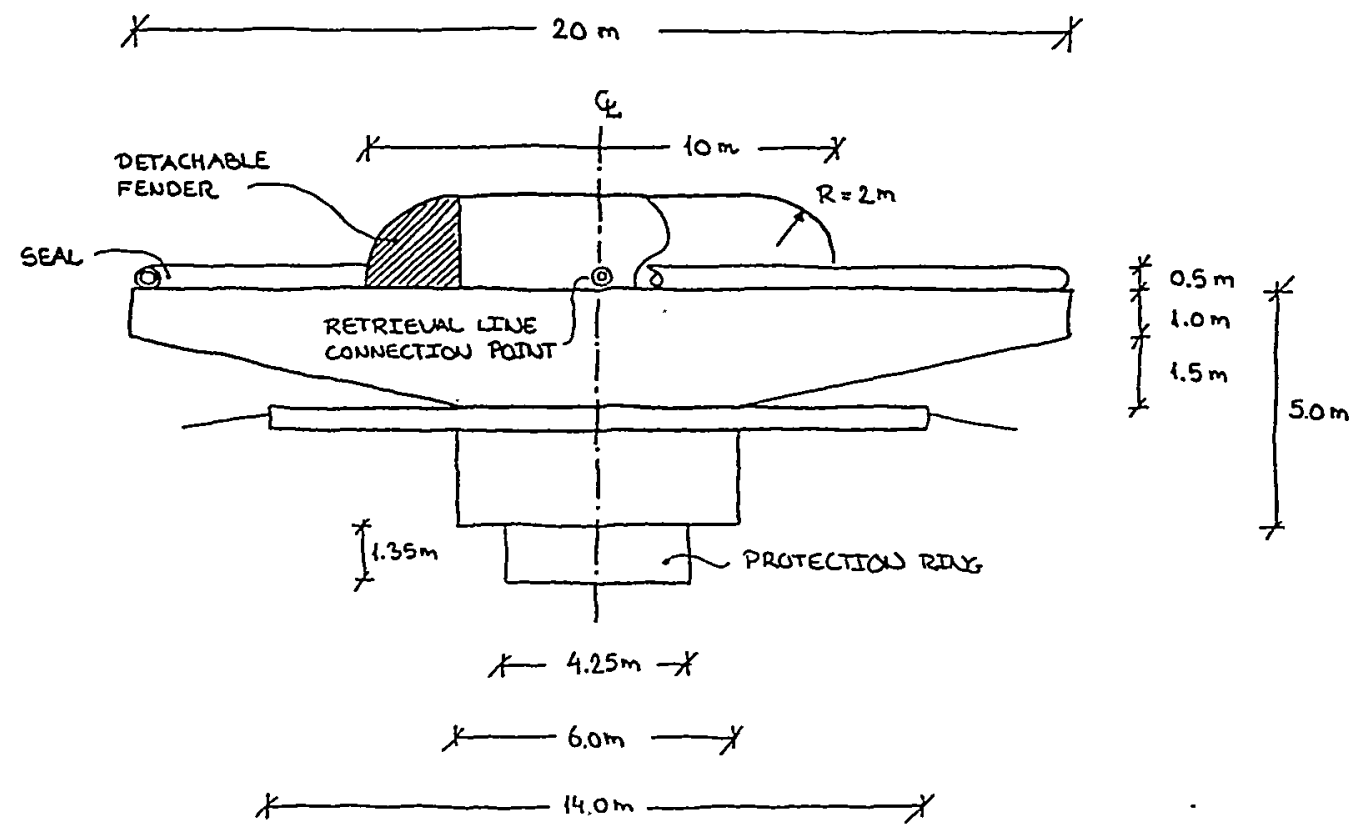

Figure 3.2. Buoy with Detachable Fender.

See also Appendix $D$ for further details. 


\subsubsection{Mooring system}

The system as designed utilizes 16 lines. These were for practical reasons represented by 6 lines, i.e. each line corresponds to approximately 2.7 lines. In order to obtain the desired stiffness of the mooring system springs are inserted into the lines. The characteristics of the springs are obtained by measuring the elongation versus load and adjusting the spring length until the desired stiffness has been obtained.

Figure 3.3 shows the target and obtained stiffness for the system (100\% loading condition).

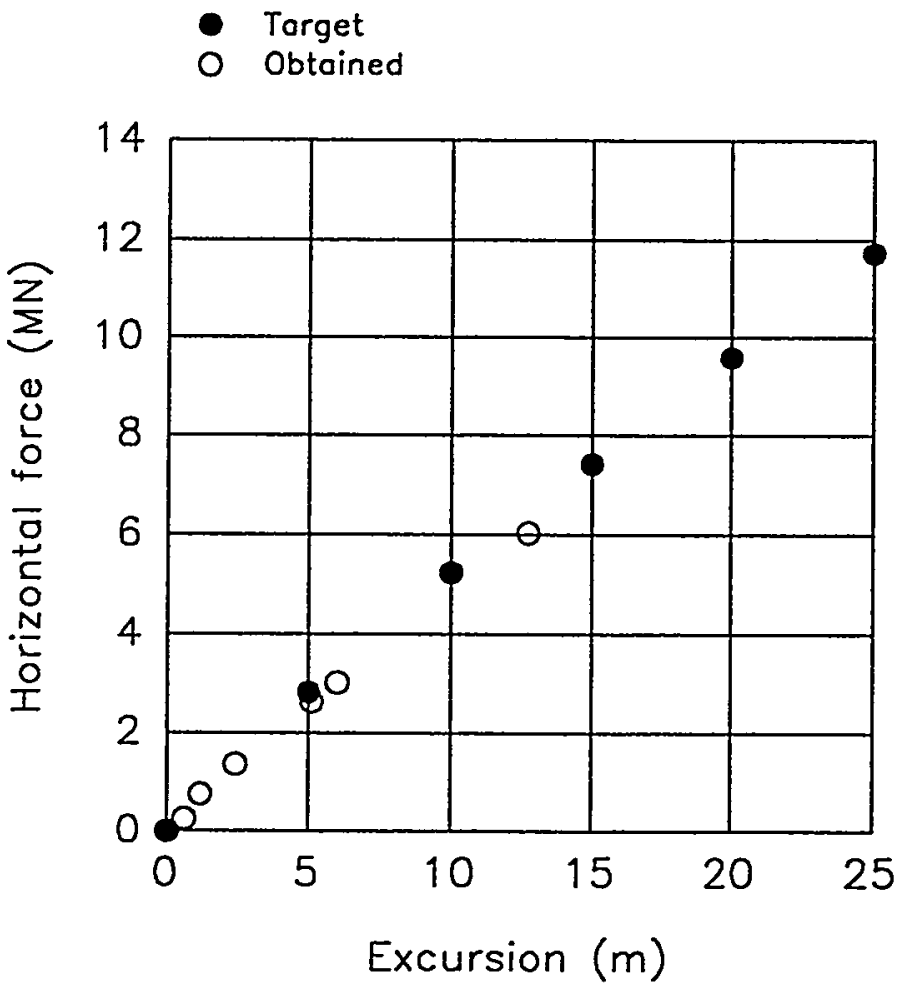

Figure 3.3. Desired and Obtained Mooring System Characteristics.

The 6 lines are oriented in a symmetrical layout with $60^{\circ}$ between the lines as depicted in Figure 3.4 . 


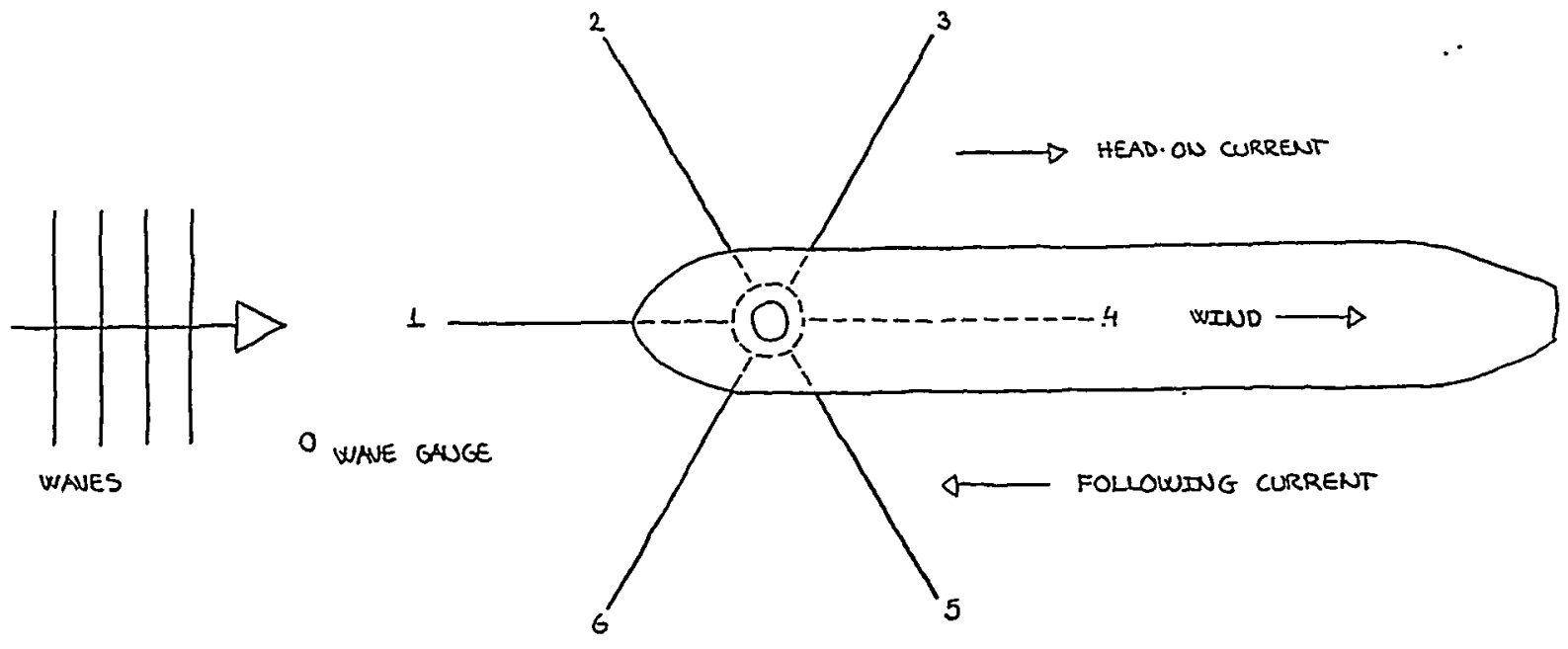

Figure 3.4. Mooring System.

A pretension corresponding to $1.04 \mathrm{MN}$ was specified for each line. The pretension in each line was obtained by leading the lines through blocks mounted just over the sea bed to blocks above the water and attaching appropriate clump weights (corresponding to $1.04 \mathrm{MN}$ ) to the end of the lines. This system was used to position the buoy correctly with the correct pretension.

Once positioned, the lines were locked at the above-water block, thereby transferring buoy and vessel motion to the stiffness model, i.e. the springs mounted in the lines.

\subsection{Tank Facility}

The tests were conducted in DMl's large towing and wave generation tank, which is $240 \mathrm{~m}$ long, $12 \mathrm{~m}$ wide and has a normal water depth of $5.3 \mathrm{~m}$. 
The tank is equipped with wave generation facilities at one end and a wave absorbing beach at the opposite end. The wave generator consists of a double hinged paddle which is controlled remotely, enabling either regular or irregular waves to be generated in the tank.

The wave time series were synthesized using a method developed by M.S.Longuett-Higgens (c.f. "On the Joint Distribution of Periods and Amplitudes of Sea Waves", J.Geophysical

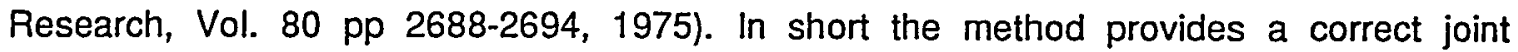
distribution of wave heights and periods while at the same time fulfilling the condition that the resulting time series must not contain any regular events which could otherwise occur from an inverse Fourier expansion with regular frequency intervals. In the present case 500 components were used in the generation of each of the wave time-series.

A current is simulated by towing the whole test set-up through the tank. The test set-up is mounted under a carriage running on rails above the tank. Due to the limited water depth required for these tests ( $29.8 \mathrm{~m}$ in full-scale) a $12 \times 12 \mathrm{~m}$ artificial bed was mounted on the carriage. The size of the bed corresponds to $600 \times 600 \mathrm{~m}$ in full-scale.

\section{$3.5 \quad$ Instrumentation}

\section{Line Forces:}

Haul-in line as well as mooring-line forces are measured by inserting a force transducer in each line, i.e. the force is transferred from the vessel/buoy through the gauge to the line. The transducers are calibrated prior to mounting by hanging them vertically and attaching a number of calibrated weights, thus obtaining the relation between signal output and force imposed. During the tests the gauges are kept under water, thus ensuring that no temperature drift, etc., occurs. The integrity of the transducers is checked regularly under calm conditions.

\section{Wave Gauges:}

Waves or surface elevation is measured with conductance-type gauges, which are calibrated by immersing the gauges in steps, thus obtaining the relation between water level and conductance. 
While calibrating the waves three gauges were placed across the centre of the artificial bed, where the position of the centre gauge corresponds to the position of the moonpool under calm conditions.

The centre wave gauge was moved to a position up front of the vessel and to the port side so as not to interfere with the mooring system and station-keeping system.

\subsection{Data Logging}

Time series are sampled at $30 \mathrm{~Hz}$ using a PC equipped with A/D facilities. Prior to logging the signals are amplified within a range of $\pm 10 \mathrm{~V}$ and filtered by analog low-pass filters to prevent folding of undesired frequency components in the analog/digital data logger.

Data logging consists of three sequences: "zero-check", "span/gain-check" and actual measurement of the instantaneous signal.

The "zero-check", which represents a transducer offset, is logged for 60 seconds before a test and under calm conditions. For all channels the mean value obtained during the "zero-check" is subtracted from the time series during the data processing.

The "span/gain-check serves as calibration check of amplifiers, filters, transmissions, interface and the data-acquisition computer before a test. The "span/gain-check" is logged for 60 seconds after the "zero-check" has been logged. The obtained values are used in the data processing for calibration or conversion purposes.

Both these checks are performed when the model has been changed or some time has elapsed, thus ensuring that the signals are converted correctly. The checks are, however, not performed when running a number of tests in a rapid succession where the model is not interfered with. In this case the values from the "zero-check" and "span/gain-check" are inherited from one run to the next until these checks are performed again. 


\section{$\underline{3.7 \quad \text { Video }}$}

Video recordings are made of all tests using the PAL-VHS system. Four cameras are used:

- Moonpool Camera: Mounted inside the moonpool and giving a view of the buoy from above.

- Underwater Camera: Giving a side view of the buoy and vessel (port side of the vessel).

- Top Camera:

Mounted above the vessel. A longitudinal and transversal scale is mounted close to the moonpool giving an approximate indication of the motions. The distance between the marks (from right edge to right edge) is $5 \mathrm{~cm}$ corresponding to $2.5 \mathrm{~m}$ in full-scale.

- Front/Side Camera: $\quad$ Mounted above water and giving an upfront view of the vessel from the port side.

All video recordings are made in model scale, i.e. $50^{1 / 2}$ times faster than in full-scale. Once data logging is started, a digital clock is activated. This shows the time in model scale and is in synchronization with the time series of the various parameters presented. The latter are, however, shown with a full-scale time-axis, i.e. $50^{1 / 2}$ slower than in model scale. 


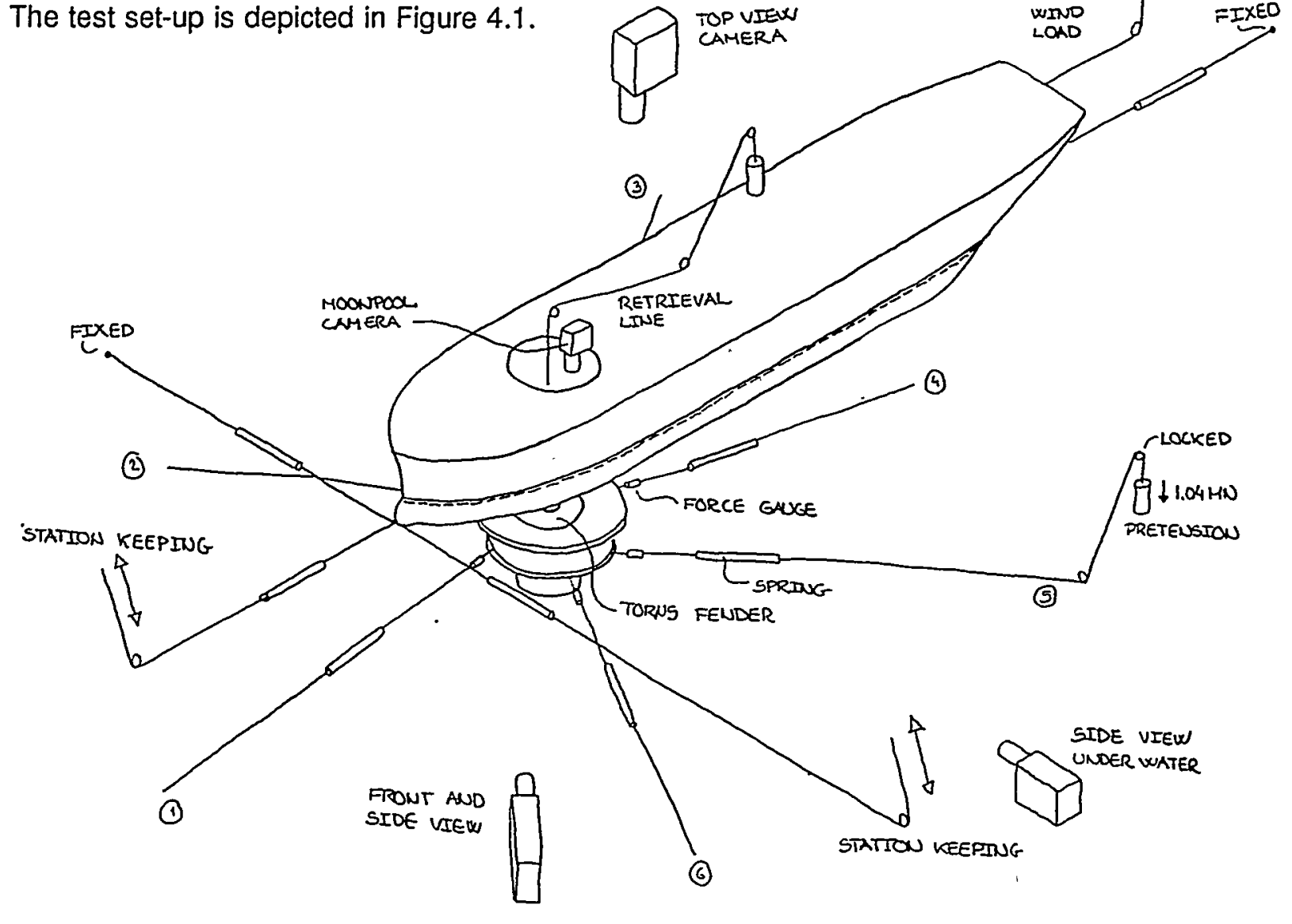

\subsection{Test Procedures}

\subsubsection{Moored condition tests}

During the moored condition tests the vessel was held by the buoy solely by water pressure, i.e. no measures were taken to ensure that the buoyancy chamber was filled with air. Water leaking past the outer ring seal was removed by the moonpool pumps which ran continuously. The majority of these tests were carried out with the mooring system intact. However, some tests were deliberately carried out with one line missing. In these tests Line No. 1 was removed leaving the buoy moored by Line Nos. 2, 3, 4, 5 \& 6 . \\ 4. TEST SET-UP AND PROCEDURES}

\section{$4.1 \quad$ Test Set-Up}

The test set-up is depicted in Figure 4.1.

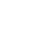

Figure 4.1. Test Set-Up.

Figure 4.1. Test Set-Up.

$$
\text { removed leaving the buoy moored by Line Nos. } 2,3,4,5 \& 6 .
$$




\subsubsection{Mating/decoupling tests}

The position of the vessel was controlled manually by hauling in or letting out lines attached to the bow of the vessel while a non-controllable line was attached to the bow. In order to give a reasonably realistic vessel response soft latex springs were mounted in all lines attached to the vessel, thus enabling the vessel to respond to the environmental loads. With no tension on the control lines the vessel was offset downwards and to the starboard side of the buoy. Hence, before the mating procedure was initiated the vessel was hauled into the approximate position using the moonpool video camera and underwater side-view video camera as aids.

Two different methods for mating were tested:

- While submerged in the holding or stand-by position water was removed from the buoyancy chamber and replaced by air causing the buoy to rise upwards towards the vessel.

While submerged in the holding or stand-by position a near constant tension force was applied to the retrieval line causing the buoy to be pulled up to vessel.

In both cases the moonpool water pumps were activated just prior to mating ensuring that a pressure difference was established quickly. Once mated the continuous removal of water leaking past the seal and into the moonpool was removed thus ensuring that the buoy was kept in place.

Similar, the decoupling procedure was performed as follows:

Water was led into the buoyancy chamber and through the air-vent tube in the moonpool ceiling thereby increasing the pressure until the buoy was released.

- The tension in the retrieval line was reduced while water was being pumped into the moonpool until the buoy was released. 
In both cases the moonpool water pumps were turned off prior to commencing the decoupling procedure. The control lines were only held loosely during the decoupling sequences thus letting the vessel fall back when released.

Mating/decoupling tests were repeated as often as possible within the available one hour (fullscale) test time. 


\section{TEST PROGRAMME}

This chapter describes the environmental conditions and the number system used to identify the individual test.

\subsection{Environmental Conditions}

\subsubsection{Waves}

The waves generated in the tank for this particular series of tests are based on the JONSWAP spectrum. Obtained values based on the no current situation are presented in Table 5.1 below:

\begin{tabular}{|c|lll||}
\hline Wave ID & $\begin{array}{l}\mathrm{H}_{\mathrm{s}} \\
(\mathrm{m})\end{array}$ & $\begin{array}{l}\mathrm{T}_{2} \\
(\mathbf{s})\end{array}$ & $\begin{array}{l}\mathrm{T}_{\mathrm{p}} \\
(\mathbf{s})\end{array}$ \\
\hline \hline $\mathrm{A}$ & 6.6 & 10.1 & 12.1 \\
$\mathrm{~B}$ & 7.7 & 13.1 & 13.6 \\
$\mathrm{C}$ & 5.6 & 9.3 & $8.7 /(12.0) \star$ \\
$\mathrm{D}$ & 5.0 & 8.9 & 9.1 \\
$\mathrm{E}$ & 4.7 & 8.0 & 8.8 \\
$\mathrm{~F}$ & 4.1 & 7.7 & 8.4 \\
\hline
\end{tabular}

* A second peak exists at $12.0 \mathrm{~s}$.

Table 5.1. Obtained Wave Parameters.

The abrupt transition from deep water to shallow water makes it difficult to generate prespecified wave conditions. The above values where obtained during a number of trial and error tests.

The above conditions are documented in Appendix C.

\subsubsection{Wind}

A simple pulley and weight system was used to provide a wind load corresponding to $51 \mathrm{t}$ for the $100 \%$ load condition and $54 \mathrm{t}$ for the $40 \%$ load condition and acting at the stern at deck level. 


\subsubsection{Current}

A head-on and following current corresponding to $1.07 \mathrm{~m} / \mathrm{s}$ was generated by moving the entire test set-up through the tank while generating waves.

\subsubsection{Water depth}

A water depth of $29.8 \mathrm{~m}$ was maintained over an area of $600 \times 600 \mathrm{~m}$ during the tests.

\subsection{Test Numbers}

A four digit number is used to identify the content/configuration of the individual runs. The significance of the individual digit - from left to right - is as follows:

\section{First Digit (Configuration):}

1 : 100\% loaded - intact mooring system

$2: 100 \%$ loaded - one leg out

3 : 100\% loaded - mating/decoupling with air assistance

4 : $100 \%$ loaded - mating/decoupling with retrieval line

$1: 40 \%$ loaded - intact mooring system

2 : $40 \%$ loaded - one leg out

3 : $40 \%$ loaded - mating/decoupling with air assistance

4 : $40 \%$ loaded - mating/decoupling with retrieval line

9 : Other

\section{Second Digit (Waves):}

$$
\begin{aligned}
& 1: A-H_{s}=6.6 \mathrm{~m} \mathrm{~T}_{z}=10.1 \mathrm{~s} T_{p}=12.1 \mathrm{~s} \\
& 2: B-H_{s}=7.7 \mathrm{~m} \mathrm{~T}_{2}=13.1 \mathrm{~s} T_{p}=13.6 \mathrm{~s} \\
& 3: C-H_{s}=5.6 \mathrm{~m} \mathrm{~T}_{2}=9.3 \mathrm{~s} \mathrm{~T}_{\mathrm{p}}=8.7 / 12.0 \mathrm{~s} \\
& 4: D-H_{s}=5.0 \mathrm{~m} T_{2}=8.9 \mathrm{~s} T_{p}=9.1 \mathrm{~s} \\
& 5: E-H_{s}=4.7 \mathrm{~m} \mathrm{~T}_{z}=8.0 \mathrm{~s} \mathrm{~T}_{\mathrm{p}}=8.8 \mathrm{~s} \\
& 6: F-H_{s}=4.1 \mathrm{~m} \mathrm{~T}_{2}=7.7 \mathrm{~s} \mathrm{~T}_{\mathrm{p}}=8.4 \mathrm{~s}
\end{aligned}
$$


Third Digit (Current):

0 : None

1 : Following $(1.07 \mathrm{~m} / \mathrm{s})$

2 : Head-on $(1.07 \mathrm{~m} / \mathrm{s})$

\section{Fourth Digit (Run number):}

0-9 : Individual run-number. See tables for further information. 


\begin{tabular}{|c|c|c|c|l||}
\hline \hline Run No. & \multicolumn{2}{|c|}{ Waves, current and wind } & Comments \\
\hline \hline & & & & Mooring system: Intact: \\
1120 & A & Head-on & $51 \mathrm{t}$ & \\
1100 & A & None & $51 \mathrm{t}$ & \\
110 & A & Following & $51 \mathrm{t}$ & \\
1200 & B & None & $51 \mathrm{t}$ & \\
1300 & C & None & $51 \mathrm{t}$ & \\
1420 & D & Head-on & $51 \mathrm{t}$ & \\
1400 & D & None & $51 \mathrm{t}$ & \\
1410 & D & Following & $51 \mathrm{t}$ & \\
1500 & E & None & $51 \mathrm{t}$ & \\
1600 & F & None & $51 \mathrm{t}$ & \\
& & & & \\
2120 & A & Head-on & $51 \mathrm{t}$ & Mooring system: One leg out (No.1): \\
2100 & A & None & $51 \mathrm{t}$ & \\
2110 & A & Following & $51 \mathrm{t}$ & \\
\hline \hline
\end{tabular}

Table 5.2. Load condition: $100 \%$ - Vessel Attached to Buoy - Torus Fender Included

\begin{tabular}{|c|c|c|c|c|}
\hline Run No. & \multicolumn{3}{|c|}{ Waves, current and wind } & Comments \\
\hline 3100 & A & None & None & $\begin{array}{l}\text { Mating/decoupling w/o retrieval line: } \\
\text { Torus fender excluded. }\end{array}$ \\
\hline $\begin{array}{l}3600 \\
3601\end{array}$ & $\begin{array}{l}F \\
F\end{array}$ & $\begin{array}{l}\text { None } \\
\text { None }\end{array}$ & $\begin{array}{l}\text { None } \\
\text { None }\end{array}$ & $\begin{array}{l}\text { Mating/decoupling with retrieval line: } \\
\text { Torus fender included }\end{array}$ \\
\hline 4600 & $F$ & None & None & \\
\hline
\end{tabular}

Table 5.3. Load Condition: 100\% - Mating / Decoupling. 


\begin{tabular}{||c|c|c|c|c|}
\hline Run No. & \multicolumn{2}{|c|}{ Waves, current and wind } & Comments \\
\hline \hline & & & Mooring system: Intact: \\
5120 & A & Head-on & $54 \mathrm{t}$ & \\
5110 & $\mathrm{~A}$ & Following & $54 \mathrm{t}$ & Mooring system: One leg out (No.1): \\
6120 & $\mathrm{~A}$ & Head-on & $54 \mathrm{t}$ & \\
6100 & $\mathrm{~A}$ & None & $54 \mathrm{t}$ & \\
6110 & $\mathrm{~A}$ & Following & $54 \mathrm{t}$ & \\
6111 & $\mathrm{~A}$ & Following & None & \\
\hline
\end{tabular}

Table 5.4. . Load Condition: 40\% - Vessel Attached to Buoy - Torus Fender Excluded.

\begin{tabular}{||c|c|c|c|c||}
\hline Run No. & \multicolumn{2}{|c|}{ Waves, current and wind } & Comments \\
\hline \hline 7100 & A & None & None & Mating/decoupling w/o retrieval line: \\
7110 & A & Following & None & \\
7200 & B & None & None & \\
7400 & D & None & None & \\
7420 & D & Head-on & None & \\
7500 & E & None & None & \\
7520 & E & Head-on & None & \\
7600 & F & None & None & \\
\hline
\end{tabular}

Table 5.5. Load Condition: 40\% - Mating / Decoupling. Torus Fender Excluded.

\begin{tabular}{|c|c|c|c|c|}
\hline Run No. & \multicolumn{3}{|c|}{ Waves, current and wind } & Comments \\
\hline 8100 & $A$ & None & None & $\begin{array}{l}\text { Mating/decoupling with retrieval line: } \\
\text { Torus fender included }\end{array}$ \\
\hline 8101 & A & None & None & Torus fender excluded \\
\hline 8400 & $\mathrm{D}$ & None & None & Torus fender included \\
\hline 8401 & $D$ & None & None & Torus fender excluded \\
\hline 8500 & $E$ & None & None & Torus fender included \\
\hline 8501 & $E$ & None & None & Torus fender excluded \\
\hline 8520 & $E$ & Head-on & None & Torus fender included \\
\hline 8600 & $F$ & None & None & Torus fender included \\
\hline
\end{tabular}

Table 5.6. Load Condition: 40\% - Mating / Decoupling with Retrieval Line. 


\section{DATA ANALYSIS}

This Chapter gives a brief description of the how the obtained data is analysed and presented. All values presented have been converted to full-scale if not specifically mentioned otherwise.

\subsection{Data Analysis}

Based on the logged time series of the various signals, i.e. surface elevation and line forces, the mean, minimum, maximum and rms values are calculated for the various parameters. The minimum and maximum values are relative to zero and represent the extreme values encountered within the duration of a test. The mean and rms values for the various parameters are calculated as:

$$
\begin{aligned}
& \bar{x}=\frac{1}{N} \sum_{i=1}^{N} x_{i} \\
& x_{r m s}=\sqrt{\frac{1}{N} \sum_{i=1}^{N}\left(x_{i}-\bar{x}\right)^{2}}
\end{aligned}
$$

where:

$N$ : Total number of data points.

$x_{1}$ : Value of data point number $i$.

The various wave cases are further documented in form of wave energy spectra and Weibull plots showing the distribution of the wave heights within each condition.

\subsection{Presentation of Results}

Results from the tests are presented in the form of time series and tables containing statistical values as outlined in the previous paragraph. All mooring-line forces presented include the pretension of $1.04 \mathrm{MN}$. 
It should be noted that the mooring-line forces presented from the tests where mating I decoupling was performed are influenced by the station-keeping system and therefore do not necessarily represent the actual loads which may be encountered if the vessel is held solely on station by the buoy. The time series from these tests have been included as they very clearly show when the mating/decoupling has occurred.

All tests are further documented by video recordings.

Furthermore, the horizontal force on the buoy is presented as the vectorial sum of the line forces. It should be stressed that this force is only approximate as the instantaneous position of the buoy is not measured, i.e. it is assumed that the angles between the lines remain constant $\left(60^{\circ}\right)$. 


\section{EVALUATION OF MEASUREMENTS AND RESULTS}

It proved difficult to obtain the originally specified wave conditions in the tank due to the abrupt transition from deep to shallow water and back to deep water again. A considerable more elaborate set-up would be required in order to improve the ability of achieving the target values. However, for evaluating the feasibility of the concept the conditions produced in the tank provide ample and valuable information.

In general the equipment used for the tests worked as intended. With the $100 \%$ loading condition tests frequent shipping of green water was observed. During these tests the integrity of the vessel was checked regularly and any bilge water was removed.

The buoy had when held by the pretensioned mooring lines not originally sufficient buoyancy to float it to the surface. The available buoyancy was, however, sufficient to perform all the $100 \%$ loading conditions tests. In order to perform the $40 \%$ loading condition tests the ballast in the buoy was reduced, without changing the outer geometry of the buoy.

The capacity of the moonpool pumps used for the tests was somewhat below what had been anticipated. An increased capacity would have meant a faster discharge rate thereby establishing the necessary pressure difference at an earlier stage. 


\section{RESULTS}

The results are presented in Appendix B which for each run describes the configuration, environmental conditions and obtained results.

\subsection{Moored Vessel Tests}

In general the vessel responded as expected and no unusual observations were made though frequent shipping of 'green water' occurred during the tests with the vessel loaded to the $100 \%$ condition.

In the test cases with a following current the wind load maintained the orientation of the vessel and no abnormal behaviour was observed. It should be noted that no information was readily available as regards the pressure centre of the wind. In these tests the load centre was placed at deck level at the stern.

None of the tests showed any indication that the buoy was unable to hold the vessel as no relative motion between the vessel and buoy was observed regardless of the load condition and environmental conditions.

Similar results were obtained for the tests with one leg out. The missing leg caused the vessel to fall back, thereby occasionally lowering the line force of Line No 4 below the pretension value. Again no relative motion between the vessel and buoy was observed.

Figures 8.1 and 8.2 show the maximum encountered mooring line forces for the $100 \%$ and $40 \%$ load condition as function of Run Number.

Approximately $35 \mathrm{l} / \mathrm{s}$ leaked passed the vessel/buoy seal when then vessel was moored to the buoy. 


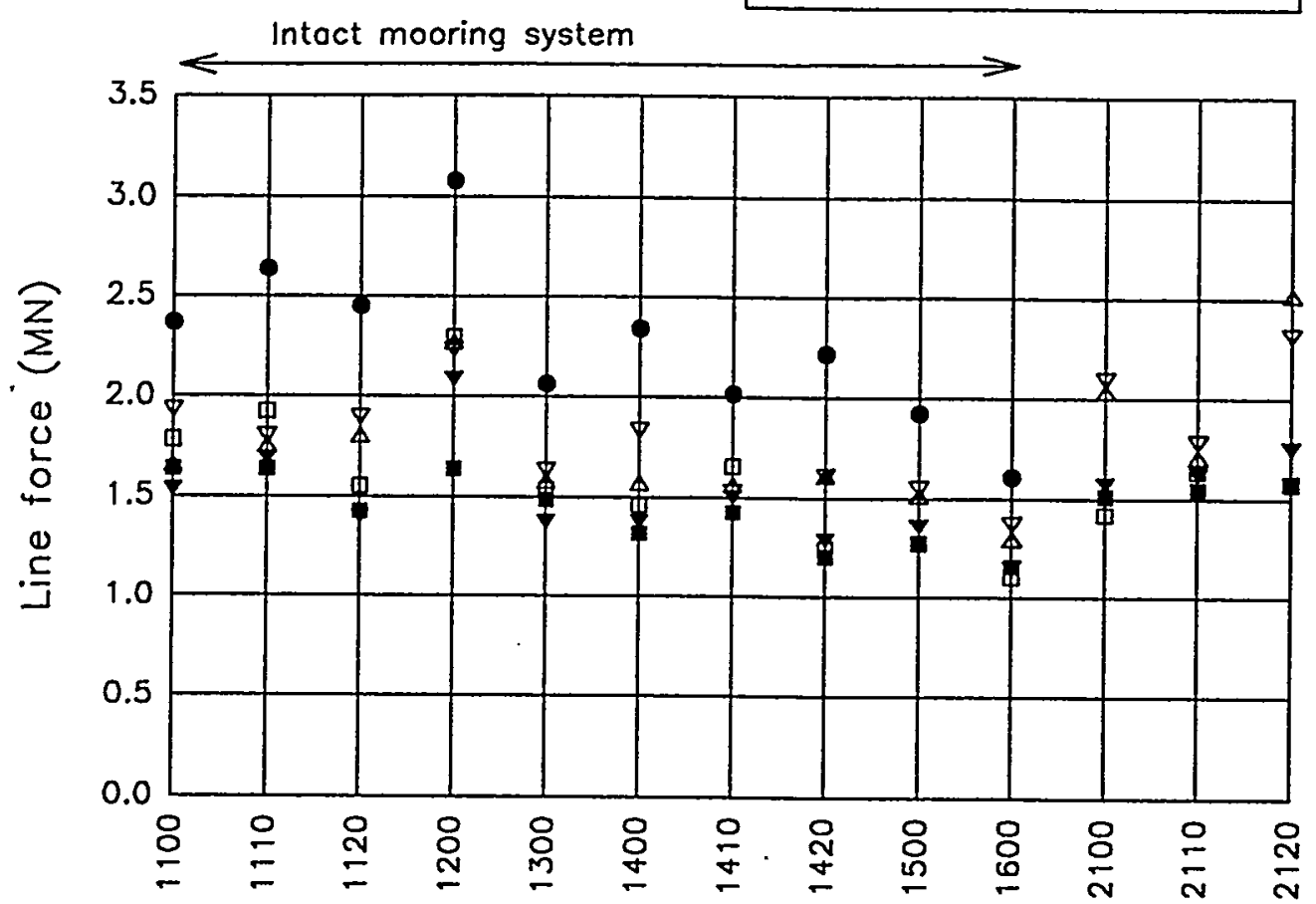

Run number

Figure 8.1. Maximum Mooring Line Forces - Load Condition: $100 \%$.

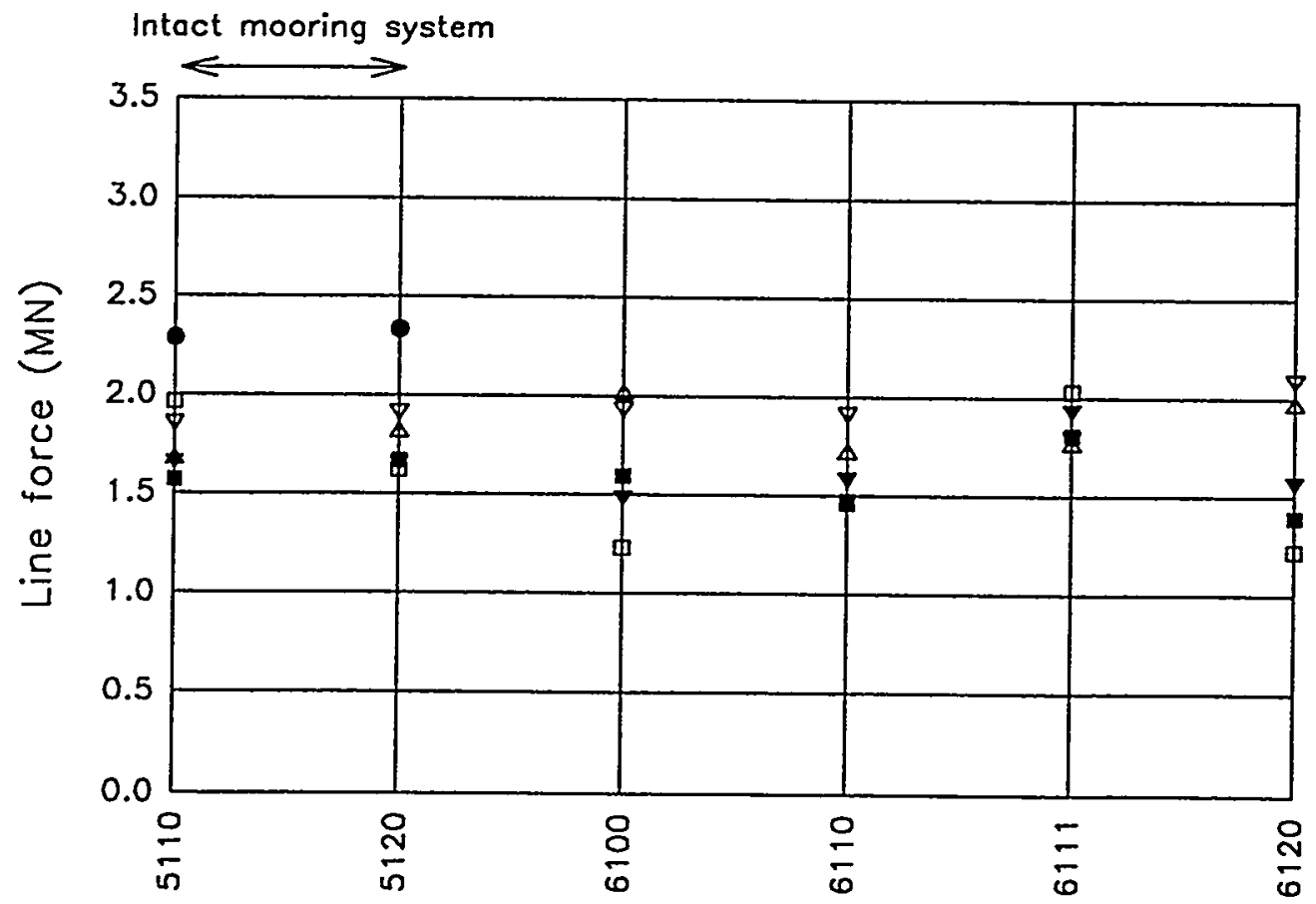

Run number

Figure 8.2. Maximum Mooring Line Forces - Load Condition: $40 \%$. 


\subsection{Mating / Decoupling Tests}

Numerous mating/decoupling tests were made under both moderate and severe conditions. The tendency for the buoy to pitch/roll is reduced significantly as the buoy approaches the vessel and in general the mating was well controlled. The torus fender did at times make it more difficult to perform the mating as it tended to collide with the moonpool orifice thus displacing the buoy before sufficient suction was available. Removing the torus fender speeded up the mating process and in general made it easier to perform the task.

It should be noted that the mooring line forces presented from the tests where mating / decoupling was performed are influenced by the station-keeping system, and therefore do not necessarily represent the actual loads which may be encountered if the vessel is held solely on station by the buoy. A number of these time-series have, however, been included as they very clearly show when the mating / decoupling has occurred.

\subsection{Retrieval Line Load Versus Buoy Level}

Two indicative tests were carried out where the load in the retrieval line was measured as function of the depth of the buoy below the hull. The results from these tests are presented in Figure 8.3.

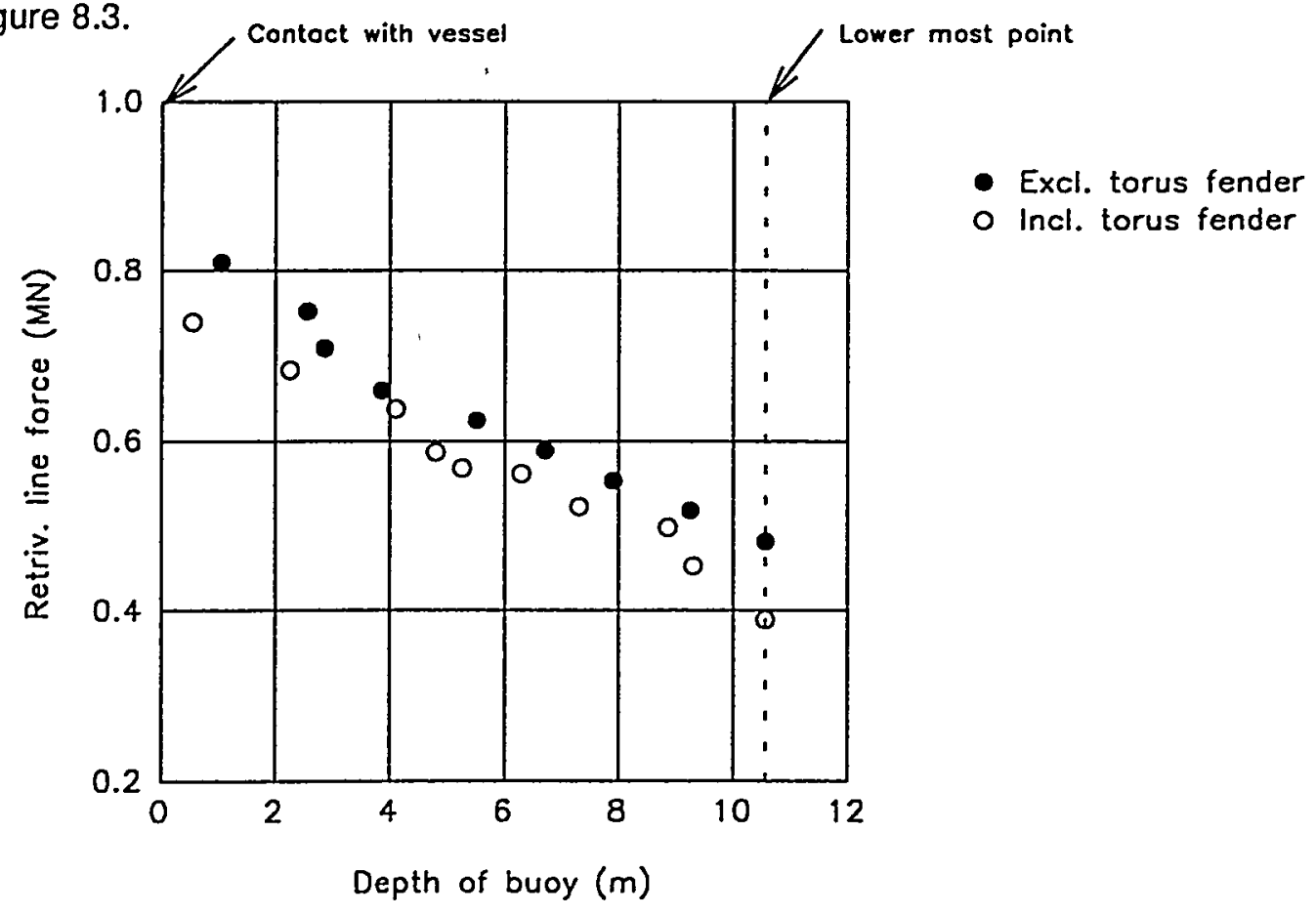

Figure 8.3. Retrieval Line Load Versus Depth of Buoy Below Hull. 


\section{The Hydrostatic Mooring System}

Final Technical Report Appendices D, F, G, H, \& I

Volume 2 of 2

Jens Korsgaard

Jens Korsgaard

Date Published August 8, 2000

Prepared for the United States

Department of Energy

Under Cooperative Agreement

No. DE-FG36-98G010321 


\section{THE HYDROSTATIC MOORING SYSTEM}

\section{TABLE OF CONTENTS}

Page No.

TRANSMITTAL LETTER

EXECUTIVE SUMMARY

LIST OF FIGURES

LIST OF TABLES

vii

1. INTRODUCTION

$1-1$

2. OVERVIEW OF THE HYDROSTATIC MOORING SYSTEM

2.1 CONCEPT

$2-1$

2.2 APPLICATION EXAMPLE

$2-4$

2.2.1 Met-Ocean Conditions Ashkelon

$2-4$

2.2.2 Mooring Design Conditions

$2-5$

2.2.3 Cargo Transfer

$2-7$

2.3 PETROLEUM CARGO TRANSFER

$2-8$

3. MANEUVERING TESTS

4. PRELIMINARY DESIGN 4-1

4.1 SEALING SYSTEM BETWEEN THE HULL AND THE BUOY 4-1

4.2 BUOYANCY CONTROL SYSTEM

4.3 MAIN BEARING

4.4 STRUCTURAL DESIGN

4.5 FAILURE TREE ANALYSIS 


\section{TABLE OF CONTENTS (Cont'd)}

APPENDICES:

A - MANEUVERING TEST REPORT BY NICHOLS

B - REPORT ON APPLICATION TO THE LIVERPOOL BAY PROJECT

C - MAIN REPORT FROM PHYSICAL MODEL TESTS AT THE DANISH MARITIME INSTITUTE

D - SUBMITTAL TO THE AMERICAN BUREAU OF SHIPPING

F - CORRESPONDENCE WITH THE AMERICAN BUREAU OF SHIPPING

G - EVALUATION OF THE MOORING BY THE NAVIGATOR, CAPTAIN JAMES ATKINSON

H - OFFSHORE TECHNOLOGY CONFERENCE PAPER NUMBER 7711

I- HYDROSTATIC MOORING BROCHURE 


\section{Appendix D}

\section{SUBMITTAL TO THE AMERICAN BUREAU OF SHIPPING}




\section{Table of Contents}

Table of Contents

The Hydrostatic Mooring - A Brief Overview

1. Concept

2. Application Example

2.1 Met-Ocean Condition Ashkelon

2.2 Mooring Design Conditions

2.3 Cargo Transfer

3. Petroleum Transfer System

1. Mooring System

1.1 General Arrangement

1.2 Compression elements

1.3 Sealing Units

$1-3$

1.4 Structural bearing

2. Structural Design

2.1 Float units (Sealing Units)

6 pages

2.2 Steel Structure

41 pages

2.3 Bearing

12 pages

3. Mechanical Electrical design

3.1 Compressed air system

3.2 Back-up calculations Compressed air system

6 pages

41 pages

4. Risk Analysis

4.1 Operational Risk Analysis

4-1

4.2 Buoy equipment Failure

4-6

4.3 Stay Moored and Unmooring

4-9

4.4 Fluid Connection System

5. Applicable Standards 
The Hydrostatic Mooring - A Brief Overview.

\section{Concept}

The Hydrostatic Mooring is a single point mooring of the type that attaches to the keel of the vessel. Existing similar concepts comprise the Submerged Turret Loading (STL) provided by Advanced Product Loading and the Turret-Collet type buoy moorings provided by Single Buoy Moorings and SOFEC. These existing systems rely on structural connectors of the hydraulic clamp type to effect the structural connection between the buoy and the vessel. The STL has the structural swivel built into the buoy whereas the turret-collet type buoy mooring has the structural swivel built into the vessel.

The Hydrostatic Mooring has the structural swivel built into the buoy, but in contrast to the other submerged systems relies on a combination of compressive and friction forces between the hull of the vessel and the buoy to transmit the mooring forces from the vessel to the buoy. The Hydrostatic Mooring is shaped like a giant suction cup and reducing the hydrostatic pressure in the volume between the buoy and the hull creates a large compressive force between the buoy and the vessel hull. The mooring is attached to the ship's hull as shown on Figure 1 below.

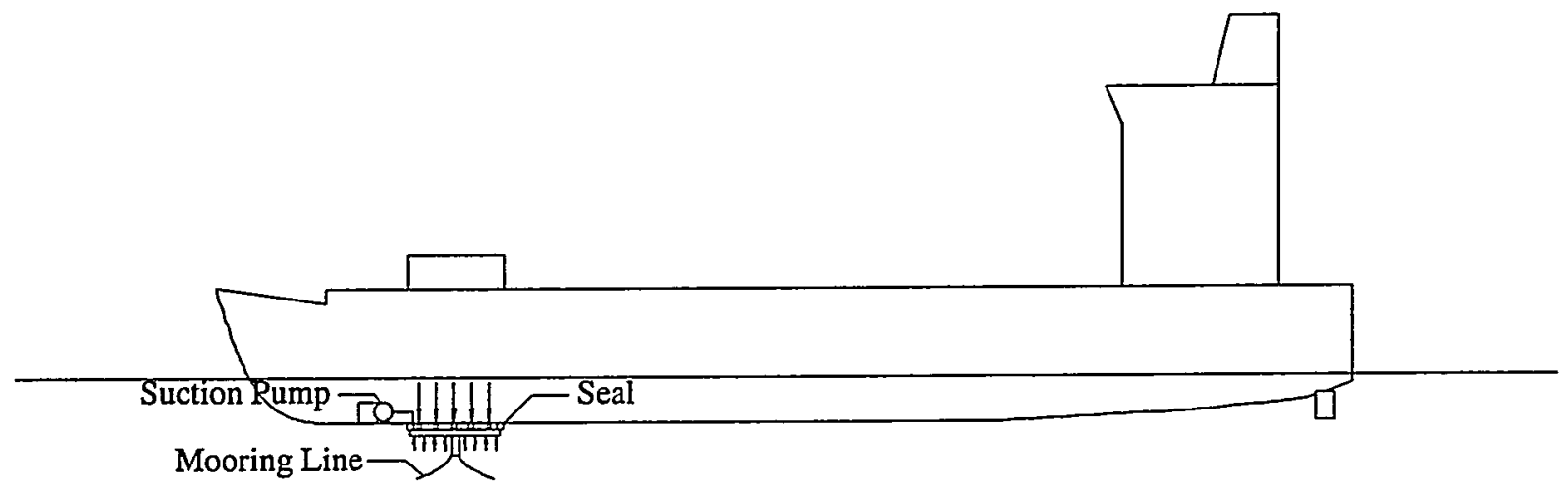

Figure 1

The volume between the ship's hull and the mooring is isolated from the sea by the circular seal shown on figure 1 . The suction pump takes suction within this volume and lowers the absolute pressure to for example $50 \mathrm{kPa}$ within the volume. The exterior pressure is at least atmospheric ( $100 \mathrm{kPa})$ thus the disk is pressed upward as indicated by the upward arrows. This upward force is resisted by the compression elements within the volume. The downward pointing arrows illustrate the compressive forces. These compressive forces resist any vertical mooring forces. There is friction between the hull and the compression elements. This friction provides the means to resist horizontal forces between the mooring buoy and the hull. The mooring lines shown in figure 1 provide the means to transmit the mooring forces to the seabed. 
The potential of this mooring system is illustrated by the following numerical example:

Ships draft in the mooring area: 5 meters

Sealing ring diameter

20 meters

Mooring pressure:

$50 \mathrm{kPa}$ (absolute)

External pressure:

$150 \mathrm{kPa}$ (absolute)

Friction coefficient:

$20 \%$

Mooring area calculated: $\quad \pi / 4 * 20^{\wedge} 2=314 \mathrm{~m} 2$

Compressive force calculated: $\quad 314 *(150-50)=31400 \mathrm{kN}$

Max. horizontal force calculated: $\quad 20 \% * 31400=6280 \mathrm{kN}$

The maximum horizontal force in this example is comparable to a normal design force for a FPSO in an extreme ocean environment. Several avenues are available to increase the force capability of the above sample mooring. The sealing ring diameter can in most cases be doubled, the friction coefficient can be increased by proper materials selection and finally ballasting operations can increase the pressure differential. In this manner this mooring can resist even extreme ice forces. Consequently, the hydrostatic mooring has the capability of mooring all large ships in the Arctic or ocean environment.

A more detailed view of the Mooring concept is shown in Figure 2 below:

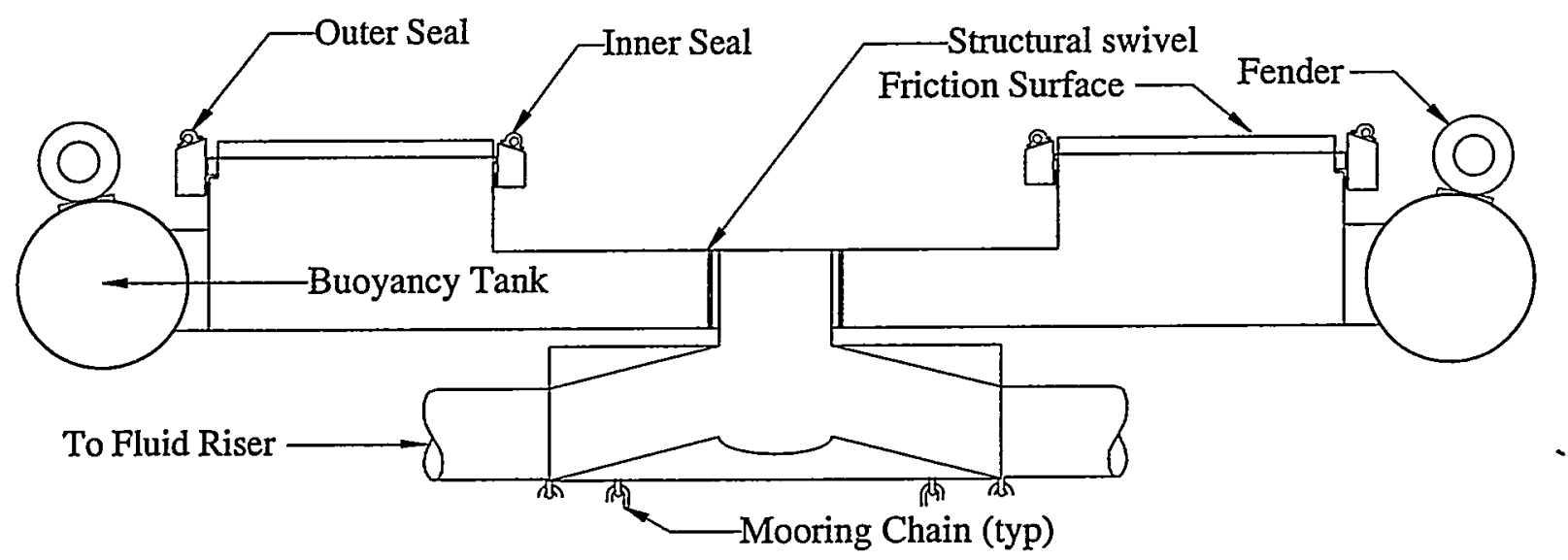

Figure 2

This more detailed view shows two annular seals between which the hydrostatic pressure is lowered. This reduces the compressive force somewhat, but then permits the maintenance of for example atmospheric pressure in the center area of the buoy. The fluid connection is made within the structural swivel area. The fluid swivel is on the vessel. This is similar to the STL arrangement. Also note the fenders that prevent damaging impacts between the buoy and the vessel. 
The Hydrostatic Mooring is under continuous development. One standardized system is sought fitting virtually all situations from the deepest water to depths that are barely enough for the vessel.

\section{Application Example.}

The application example is for a transport system in which water is transported from Manavgat in Turkey to Ashkelon in Israel. The vessels used are either VLCC or ULCC.

The following describes only the Ashkelon mooring. The mooring in Turkey is similar. A buoy that has an outer sealing ring diameter of $21 \mathrm{~m}$ is chosen for either class of vessel. The vessel must have flat bottom plating in the mooring area with a width on the order of $28 \mathrm{~m}$ or more for this to work effectively and safely. Tests show that the mooring operation can easily be performed with a precision of 2 meters in conditions with wave heights of $\mathrm{Hs}=5 \mathrm{~m}$ or below regardless of cross currents and wind. Mooring is consistently possible on the first attempt in all wave heights up to $\mathrm{Hs}=8$ meters regardless of wind and cross current conditions. The accuracy deteriorates to a typical error of 3 meters at 8 meter wave heights. Mooring operations are probably feasible in even higher wave heights, but this may be of theoretical interest only, because operations on the vessel will be curtailed in any event at these extreme conditions.

\subsection{Met-Ocean Conditions Ashkelon}

The environmental condition in Ashkelon and in Manavgat is described by the following:

- $\mathrm{Hs}=2.0$ meters

- Hs=5.0 meters

- $\mathrm{Hs}=6.0$ meters

- $\mathrm{Hs}=8.0$ meters
Exceeded 50 days per year

Exceeded 3.8 days per year

Exceeded 0.6 days per year

Return Period 50 years

The current at Ashkelon is reported to be usually north going with a speed of 0.5 knots. For design purposes at this time a design current of 1.5 knots is assumed.

The wind speed is generally quite low at Ashkelon and the maximum-recorded wind speed is 35 knots.

\subsection{Mooring Design Conditions.}

A system designed for a maximum wave height of $\mathrm{Hs}=5.0 \mathrm{~m}$ will have $99 \%$ availability and weather delays is not a concern. The required precision for mooring can be set to be $2.0 \mathrm{~m}$ in accordance with the results from the maneuvering tests performed. In the actual design a 3.0 meter tolerance will be provided.

For purposes of this document the mooring is designed for a maximum horizontal mooring force of $6000 \mathrm{kN}$. 
The buoy is secured to the ship by differential hydrostatic pressure. The present buoy is designed for water transport. It has two sealing rings, one at $10.5 \mathrm{~m}$ radius and another at 4 meter radius. The pressure in the area between the two sealing rings is typically maintained at $50 \mathrm{kPa}$ (absolute). This is done through pumps aboard the moored vessel that take suction within the space between the two sealing rings. The vessels to be moored by this buoy must have a minimum width of flat bottom plate of 28 meters $(60,000 \mathrm{DWT})$. There is no upper limit on ship size other than the limit on force caused by the combination of ship size and environmental conditions and potential water depth limitations.

The submergence of the hull may vary between 4 meters and 25 meters depending on the ship size and the loading condition. This corresponds to absolute hydrostatic pressures at the keel between $140 \mathrm{kPa}$ and $290 \mathrm{kPa}$. The pressure within the sealing area is $50 \mathrm{kPa}$.

Assuming a 4 meter submergence the differential hydrostatic pressure is $90 \mathrm{kPa}$. The area is $\pi^{*}\left(10.5^{*} 10.5-4^{*} 4\right)=295 \mathrm{~m} 2$. The resulting compressive force between the buoy and the vessel is then $27000 \mathrm{kN}$. The net buoyancy of the combined buoy/mooring system must be added. This is normally negative but close to zero and is ignored. Consequently, a friction coefficient of 0.3 between the keel and the buoy permits a horizontal force of $8100 \mathrm{kN}$ at a draft of 4 meters. At larger drafts the possible horizontal force increases to as much as $18000 \mathrm{kN}$. In consequence the vessel can control the friction capacity of the buoy and may in principle avoid dangerous combinations of force capability versus possible mooring force by increasing its draft.

The friction coefficient between the ship's bottom and the buoy is believed to be in the $50 \%$ to $80 \%$ range. Standard ablating anti-fouling paint coats the ship's bottom. The surfaces on the buoy that touch the ship are primarily composed of rubber fender elements. The rubber in these fender elements is specially formulated to have a large friction coefficient relative to wet anti-fouling paint.

The friction coefficient that is assumed for computational purposes is ordinarily in the $10 \%$ to $30 \%$ range, thereby providing an adequate safety factor against mooring failure. It should be noted that it is possible to determine the actual friction coefficient during unmooring by recording the combination of mooring force and pressure at the time of initiation of the separation process, i.e., when the buoy starts moving on the bottom of the vessel. Therefore, it is possible to monitor the efficacy of the system by measuring the buoy loading condition at initiation of motion upon separation at unmooring. This measurement may be done at each unmooring thereby providing for each ship that uses the mooring a continuous record of the friction coefficient between the buoy and the vessel.

The vessels arriving in Ashkelon are loaded and will discharge their cargo. For purposes of this document it is assumed that the maximum draft of the arriving vessel is 25 meters. The maximum wave condition for approach and mooring is set to Hs $=5$ meters in this case. A safety margin between the vessel and the buoy of $7.0 \mathrm{~m}$ is set. The buoy is 8 meters high requiring a minimum water depth in the mooring area of $25+7+8=40 \mathrm{~m}$. 
The water depth in the mooring area is only about $37 \mathrm{~m}$. . Therefore, in order to provide space for the buoy a circular area with a diameter of $100 \mathrm{~m}$ will be dredged to a depth of $44 \mathrm{~m}$.. The additional $3 \mathrm{~m}$ over depth allows for sedimentation for several years until maintenance dredging is required.

\subsection{Cargo Transfer.}

The cargo to be transferred in the case at hand is fresh water and therefore unique in the sense that it is nonpolluting and normally can be discharged freely to the sea. This makes it possible to use simpler designs in the cargo transfer system with fewer safeguards against leakage and spills compared to petroleum transfer systems. Later in this section a normal petroleum or gas transfer system will be described.

The proposed water transfer system is shown in Figure 3.

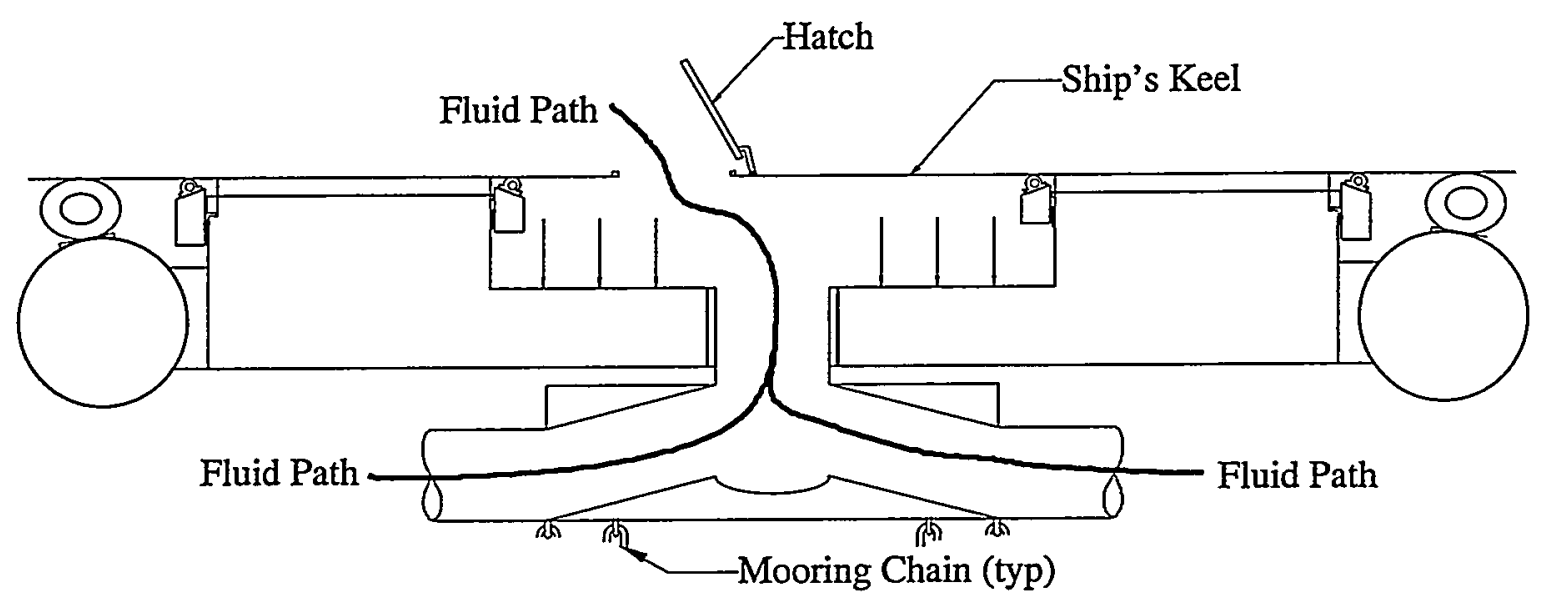

Figure 3

The water is conveyed through the buoy as shown by the "Fluid Path" signature on Figure 3. Consequently, the cargo transfer connection is automatically established as soon as the buoy is securely attached to the ship. One disadvantage of the arrangement shown in Figure 3 is the downward pressure indicated by the arrows in the center of the buoy. This pressure counteracts the suction that presses the buoy onto the ship's keel. However, it acts on a much smaller area than the suction. As an example consider that the suction acts between two sealing circles with diameters $21 \mathrm{~m}$ and $8 \mathrm{~m}$ respectively. Assume that the differential hydrostatic pressure is $-90 \mathrm{kPa}$, then the compressive force is $27000 \mathrm{kN}$ as previously calculated. Assume further that the differential hydrostatic pressure in the center of the buoy is $+200 \mathrm{kPa}$, then the repulsive force in the center of the buoy is $\pi * 4 * 4 * 200=10000 \mathrm{kN}$ resulting in a minimum net attractive force between the buoy and the vessel of $17000 \mathrm{kN}$. The latter only occurs at the end of the discharge process or in the beginning of the loading process when the vessel is ballasted. At any other time the compressive force and thereby the mooring capacity is larger. 
The vessel monitors the mooring force at all times. This is done by monitoring the mooring buoy position through the GPS positioning system. The known mooring response curve then provides the force. Therefore, the vessel can continuously monitor dangerous combinations of center well pressure and mooring capacity. In the event that such dangerous combination is approached then the vessel may take action by reducing the center well pressure. This would in turn reduce the cargo transfer rate.

\section{Petroleum Cargo Transfer.}

A more common use of the buoy is for the transfer of oil and/or gas between the vessel and the riser. In this case the pressure in the center well of the buoy would normally be maintained at close to atmospheric pressure. In this case the center of the buoy would provide additional compressive force and thereby assist in the mooring.

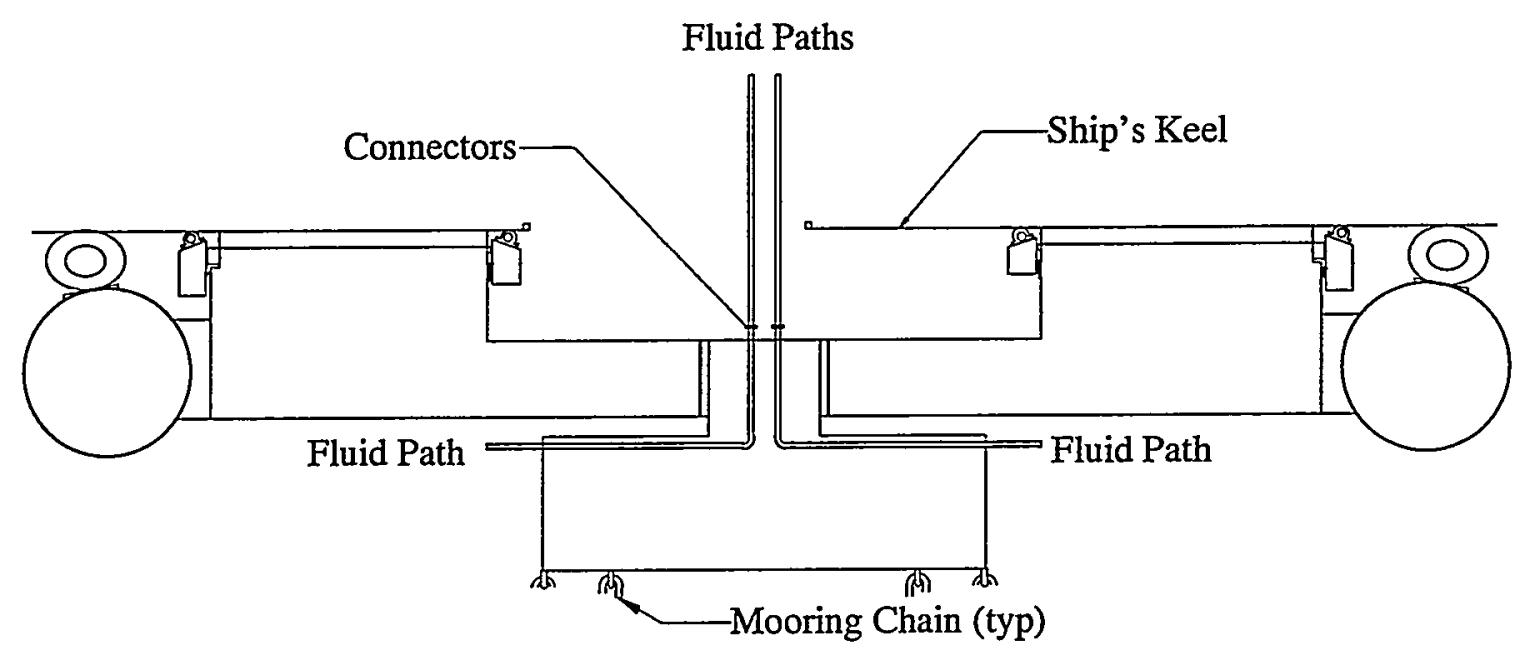

Figure 4

The mooring shown in figure 4 is similar to the mooring shown in figure 3 except that the fluids are transferred in separate pipes (Fluid Paths) as shown on Figure 4. In this case a fluid connection is established after mooring by connecting the pipes in the vessel to the pipes in the buoy at the connectors shown on figure 4 . Note that the buoy is not centered in the opening in the ship's bottom. Positioning the ship over the buoy for the mooring during the mooring operation cannot be done with complete accuracy. This is particularly so because of the 13 seconds delay between the decision to make the mooring and the actual mating between the buoy and the vessel. Test performed at Nichol's Advanced Marine in Arlington showed that the mating can be consistently performed with an accuracy of about 3 meters in conditions with $\mathrm{Hs}=8 \mathrm{~m}$. In lower wave heights the operation can be performed with better accuracy. It is expected that experienced operators of the system will consistently be able to perform the mooring with a deviation between the center of the buoy and the center of the hull opening of less than 2 meters. 
Assume that the fluid pipe assembly has a diameter of $1.5 \mathrm{~m}$ and that the diameter of the inner sealing circle is $10 \mathrm{~m}$. An opening in the bottom of the vessel of $5.8 \mathrm{~m}$ then assures that it is possible to make the fluid connection whenever the deviation of the buoy is less than $2.1 \mathrm{~m}$ from the centered position. Check calculations: Opening $2 * 2.1 \mathrm{~m}+1.5 \mathrm{~m}=5.7$ $\mathrm{m}<5.8 \mathrm{~m}$, Sealing circle $2 * 2.1 \mathrm{~m}+5.8 \mathrm{~m}=10 \mathrm{~m}$.

A $13.5 \mathrm{~m}$ diameter inner sealing circle with $7.5 \mathrm{~m}$ opening in the bottom of the ship would in this case ensures a $3 \mathrm{~m}$ tolerance on the positioning of the buoy on the bottom of the ship.

The fluid paths rotate relative to the ship when the ship changes heading. Therefore, a fluid swivel is required. This is located on the vessel. This fluid swivel may be an ordinary swivel stack permitting unlimited rotation. Alternatively, the fluid swivel may be of the limited number of rotations kind. There are several such types. The limit on the number of revolutions is 1.5 in the case of a drag chain and may be 12 or more in the case of an SMT swivel. Power to turn the swivel must be provided. A powered servomechanism may be used or torque may be transferred from the buoy to the swivel. The system proposed here use torque transfer. A long member of triangular cross section will serve the dual purpose of supporting the fluid paths and to serve as a torque bar. The buoy is placed on the bottom of the ship at random within the mooring area. Therefore, the swivel can be moved such that it is always placed vertically above the center of the buoy.

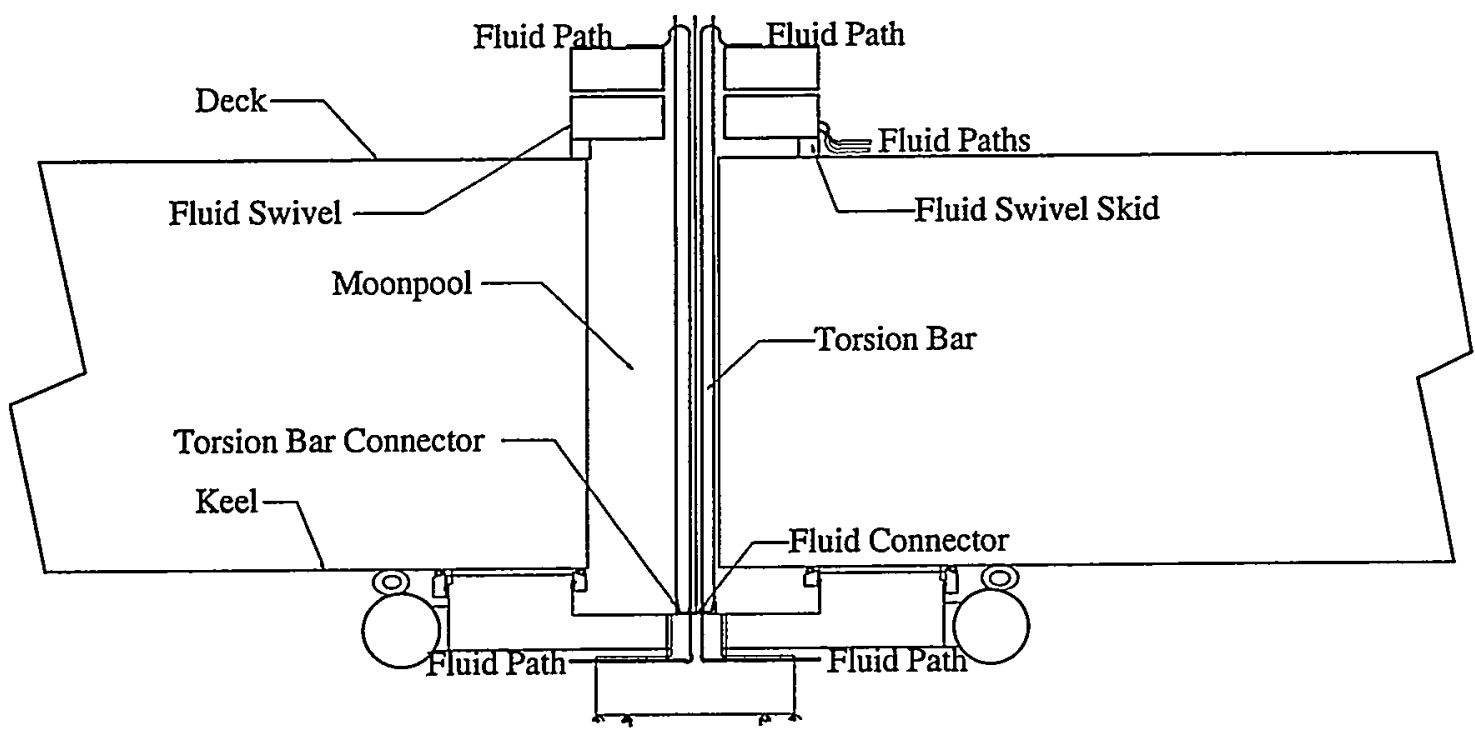

Figure 5

Figure 5 shows the general arrangement of the fluid transfer system. A fluid swivel on the deck of the ship is connected through a moonpool to the center of the buoy by means of a triangular torsion bar. The torsion bar is mated at the buoy to a triangular female receptacle. The fluid pipes are held to the torsion bar by sleeves. Thus alignment with the 
fluid connectors on the buoy is automatically achieved. The torsion bar can be raised and lowered relative to the fluid swivel. The fluid pipes can be raised or lowered relative to the torsion bar. During the mooring operation the torsion bar and the pipes are all in their upper position. Following the mooring the fluid swivel skid is centered above the buoy. Then the torsion bar is turned to align with the buoy orientation. The torsion bar is then guided into and seated in the torsion bar receptacle on the buoy. The fluid pipes are now lowered individually to each connector and connected. Each pipe is pressurized with nitrogen and the seal integrity is tested. Following the seal integrity test the nitrogen pressure is increased further. The sealing area is in fluid communication with the valve actuator in the corresponding valve in the buoy. The nitrogen pressure actuates the valve to open. The valve is equipped with spring return for automatic closure upon loss of sealing pressure.

At disconnect the operation is reversed. Instead of the testing of seal integrity the pipes are flushed and cleaned such that no product escapes upon disconnect.

The fluid pipes are shown to located on the exterior surface of the torsion bar in Figure 5. However, they would ordinarily be located inside the torsion bar. This location inside the torsion bar creates several advantages:

- The fluid pipes are shielded from impact damage

- The atmosphere inside the torsion bar may be maintained inert at low cost and with high reliability.

- Any leaking fluid is contained within the torsion bar. This provides a double barrier against escape.

- The torsion bar may have a larger cross section for the same overall assembly diameter. This enhances the structural capacity of the torsion bar.

The fluid pipes are equipped with several safety devices that ensure against all but minimal spills during emergency and/or accidental disconnect. Controlled emergency disconnect is initiated if:

- The buoy moves on the bottom of the ship. This is detected two ways, optically by an automatic monitoring system and/or mechanically by a continuous comparison between tilt sensors within the torsion bar and on deck.

- The mooring maintenance pressure rises to above a preset level. This pressure is recorded continuously at all 3 pressure maintenance pumps.

- The vessel has an excessive excursion, either due to chain failure or due to excessive weather conditions. This is detected by the differential GPS system, which determines buoy deflection. Back-up systems may consist of acoustic systems detecting movement relative to beacons on the seabed or electronic triangulation systems such as for example Motorola Miniranger.

- Emergency conditions aboard the vessel such as fire or collision.

Controlled emergency disconnect comprises the following steps:: 
- Reducing nitrogen seal pressure. This causes closure of the valves on both sides of the connector, because these valves are maintained open by the seal pressure.

- If time permits purging of the fluid paths by nitrogen

- Blow down of Seal nitrogen and release of couplers

The vessel may now disconnect the mooring. In the event of accidental violent moves of the mooring buoy the nitrogen seal pressure maintenance system will automatically release all pressure and automatically cause closure of both the automatic valves on both sides of the coupler and the release of the coupler. This is automatic upon loss of sealing pressure. The content of approximately $20 \mathrm{~m}$ of pipe will be released within the torsion bar. Please note that all or nearly all this liquid will be trapped within the mooring moonpool and may be cleaned up by the ship's crew before the next mooring.

Uncontrolled disconnect is forced by the release of the seal pressure, either through rupture of the nitrogen piping or through the activation of emergency blowdown systems. The seal pressure release causes automatic valve closure $(7-10 \mathrm{sec})$ and automatic release of couplers. The pipes within the torsion bar may optionally be equipped with break-away couplers that are actuated by the torsion bar being let down below a preset level. If so equipped, the actuation causes a short time release of the fluids $(7-10 \mathrm{sec})$, closure of valves on both sides of the coupler and release of the couplers. All couplers will likely be lost in this event, the torsion bar will likely be damaged, and there may be some superficial hull damage in the moonpool area. The buoy will in this case drop to its storage position well clear of the vessel. 


\section{Mooring System}

The present mooring system is a single point mooring attached to the keel of the moored vessel. The mooring buoy is held firmly to the bottom of the vessel by differential hydrostatic pressure. A structural swivel is built into the mooring buoy that permits the moored ship to weathervane in relation to the mooring chains.

Pressure maintenance pumps aboard the vessel maintain the differential pressure. These pumps take suction within the volume isolated by the top of the buoy and the hull of the vessel.

\subsection{General Arrangement}

The function and basic general arrangement is explained in the previous section. Single point moorings are typically moored to the seabed through a series of radial deployed catenary mooring lines terminating in drag anchors or stake piles in the seabed. This standard arrangement is illustrated on Figure 1-2 (Hydrostatic Mooring General Arrangement -2).

A special mooring system has been developed that takes advantage of the unique properties inherent in the present mooring system as shown on figure 1-1 (Hydrostatic Mooring General Arrangement -1). This system is comprised of a series of heavy weights that are lifted by the excursions of the mooring buoy. When the buoy deflects horizontally the connecting chain is bent over a fairlead in the top of the stake pile. In the proposed system that is specifically designed for the water transport system at Askelon Israel six stake piles are provided. This system maintains a higher fraction of its initial capabilities than the traditional system shown in Figure 1-2 when one mooring line fails.

Because of the small amount of chain that is used in mooring concept 1 it is not a significant economic penalty to oversize the mooring chains to compensate for the wear that will occur at the fairlead. It is the intent to frequently inspect the system and to replace chain and fairleads when significant wear has occurred.

This is the preferred system partly because of significant cost reductions and partly due to its much superior force deflection characteristics as illustrated in figure 1-4.

The graph in Figure 1-3 depicts the chain force as a function of the horizontal excursion of the vessel. Because the chain force is supplied by a hanging weight it is nearly constant. It is, however, modified above the fairlead by the friction in the fairlead. The present calculation is based on a friction coefficient of $10 \%$ as being the steel on steel friction coefficient. Figure 1-4 shows the resulting component of the chain force for a single chain. There are six chains, therefore the actual mooring force is six times the force shown on Figure 1-4. 
The principal buoyancy is provided in an exterior near toroidal shaped ring beam. This ring beam serves three main purposes:

1. It protects the mooring surface of the buoy against vessel impact

2. It serves as the main buoyancy and trim volume in the buoy

3. It provides low-pressure air storage tanks for the rapid ascent system used in the mooring process.

This is shown in more detail in figure 1-5. The ring beam is in the shape of a 12-sided polygon. Each side of the polygon is protected against ship impact by a 2 meter long standard cylindrical ship fender $1400 \mathrm{~mm}$ by $700 \mathrm{~mm}$ as shown in more detail on Figure 1-6. Later in this section an analysis is presented showing that this level of fendering is sufficient to protect both the ship and the buoy against damaging impact during the mooring and the unmooring processes.

The metacentric height of the buoy in concept 1 is obviously strongly positive. The vertical loads of the mooring weights are applied at the bottom of the buoy and therefore bring the apparent center of gravity down in the lower third of the buoy. The center of buoyancy is about at the mid point of the buoy. A detailed analysis has not been made however it is believed that concept 1 has a positive metacenter height in all conditions.

Concept 2 was used in the model tests in Denmark. Ballasting in the bottom of the buoy is always possible, however, for the model tested buoy it was necessary to keep the diameter of the rapid ascent volume at 6 meters in order to keep the metacentric height positive during the ascent.

\subsection{Compression Elements}

Figure 1-5 also shows the main compression elements between the buoy surface and the vessel hull. These are comprised of a series of Trellex Morse Fender bar units deployed in a radial pattern as shown. Because of the density of fender bars they may for computational purposes be treated as a continuos surface. The force capacity of the fender bars is 54 tonnes or $530 \mathrm{kN}$ per meter. There are a total of 360 meters of fender bars on the buoy surface. The total theoretical capacity of these units are to resist a a total compressive force of $191,000 \mathrm{kN}$ ( 19,500 tonnes). The maximum possible compression is obtained when absolute vacuum exists above the buoy while submerged to its deepest submergence. The area within the outer seal ring is $346 \mathrm{~m} 2$.

In the case that the pressure above the buoy is 0 absolute the maximum possible differential load is 35 tonnes $/ \mathrm{m} 2$ resulting in a total load of 12,100 tonnes. The mooring load is at most applied 8 meters below the fender surface. Assume 600 tonnes horizontal load, then the resulting moment is 4800 tonnes meters. The fendering circle ring has an OD of $18.5 \mathrm{~m}$ and an ID of $10.5 \mathrm{~m}$. The section modulus of this ring is $\| / 32 *\left(18.5^{\wedge} 3\right.$ $\left.10.5^{\wedge} 3\right)=508 \mathrm{~m} 3$. The resulting pressure differential is $4800 / 508=9.45$ tonnes $/ \mathrm{m} 2$. The 
allowable is the difference between the stress resulting from 12,100 tonnes and 19,500 tonnes or 21.4 tonnes per $\mathrm{m} 2$.

This margin is required to cope with the ship's bottom being out plane. The total allowable stress is $19,500 / 346=56.4$ tonnes $/ \mathrm{m} 2$. The allowable margin is $21.4-9.45=$ 11.9 tonnes $/ \mathrm{m} 2$ or $21 \%$ of the fully allowed deflection. Since the allowable deflection is $150 \mathrm{~mm}$ the system can cope with $2 * 0.21 * 150=63 \mathrm{~mm}$ out plane deviation within the mooring area. Note, however, that from a practical point of view significantly higher out of plane deviation would at most cause some minor damage to the fender bars, and then only in the event that full vacuum, maximum submergence, and full horizontal mooring force all occur simultaneously.

Figures 1-9 through 1-12 shows catalogue cuts of the various fendering elements were selected for this application.

\subsection{Sealing Units}

The sealing units contact the keel plating in the ship and impede the flow of water from the sea to the volume in which the low pressure is maintained. There are two annular seals an inner and an outer as for example illustrated in Figure 2 in the Brief overview.

Because the pressure is maintained low above the buoy through continuous removal of water by pumps aboard the ship the seals need not be perfect. Rather, their function is to impede the water flow sufficiently that the pumps may reliably maintain the pressure low above the buoy.

Figure 1-6 shows an outer sealing element. This sealing elements must be capable of the following:

- To maintain contact with the hull for all pressure differentials ranging from a low 50 $\mathrm{kPa}$ to a high of $350 \mathrm{kPa}$.

- To maintain sealing pressure in the presence of unevenness of the hull.

- To be able to deflect without damage when a maximum impact between the buoy and the vessel deflects the exterior fenders fully.

- To be able to slide on the bottom plating of the vessel without damage.

- To resist safely all possible levels of differential pressure

- To provide the highest practical level of sealing.

A range of materials were investigated and the result was that the steel unit shown on figures 1-6, 1-7, and 1-8 was the only unit that was able to fulfill all criteria. Figure 1-7 shows in more detail how the unit pivots. Figure 1-8 shows the structural make-up of an exterior sealing unit. The structural design of the unit is presented later in this document.

The sealing units are seated in "boxes" at the edge of the buoy. Each "box" holds one sealing unit. The sealing unit fits loosely in its "box" and is prevented from sliding out by 
a tab on the underside. The curved surface shown on the top of the unit is bolted on after the unit is placed in the "box". The units are spaced some $25 \mathrm{~mm}$ apart along the perimeter. A special Trellex Morse extruded fender is placed in a continuous ring above the units and is bolted onto each unit and is held securely by bolted on angle irons. On the exterior and below the sealing element there is a continuous rubber sheet made from conveyor belting. This rubber sheet seals the gaps between the sealing elements. It also seals the gap between the hull of the buoy and the sealing element.

The conveyor belting would typically be capable of resisting differential pressures in excess of $1000 \mathrm{kPa}$ in this application. Thus there is no issue with the structural strength of the rubber sheet. Both the rubber sheet and the extruded fender will be stretched when the sealing ring is depressed. However the stretch is less than $5 \%$, which is well within the capabilities of these elements.

The sealing elements are cantilevered. The purpose of the cantilever is to make it possible for the sealing element to deflect vertically and also to have the hydrostatic pressure differential increase the sealing pressure as the pressure differential increases.

Figure 1-8 shows an exterior sealing element. The interior sealing element is similar, however, the taper is in the opposite direction. In consequence the interior seal "box" design is with parallel sides and the unit also has parallel sides for the part that is placed within the "box"

\subsection{Structural Bearing}

The structural bearing is continually submerged. However, because of the use of compressed air to regulate the buoyancy during the rapid ascent to effect the mooring it is possible to have the bearing in air if it is placed in a volume in the buoy that is only open downward.

Because the bearing will be at least occasionally immersed in seawater a standard large diameter roller bearing is not practical. The strongly varying hydrostatic pressure will almost certainly drive salt water into such a bearing with rapid failure being the result.

A separate problem is the marine growth that will accumulate in continuously immersed areas. This may also be a problem for friction bearings such as Lubron bearings.

Therefore a bearing based on the technology used to move heavy objects on endless track roller units has been adopted (Hillman Rollers). These roller units are highly resistant to dirt. By placing the bearing most of the time within an air pocket with only occasional wetting, marine growth problems are avoided. The roller units will be manufactured from relatively noble materials such as stainless steel or Monel. The roller units will roll on a 3 sided circular track with one vertical and two horizontal surfaces. 
The roller units are mounted that they may be individually removed and mounted while the buoy is surfaced for maintenance. The units are backed by Belleville springs that have a maximum deflection of $19 \mathrm{~mm}$. This feature reduces the accuracy required on the bearing rolling surfaces, permitting a relatively primitive manufacturing process.

\subsection{Rapid Ascent Buoyancy}

Rapid ascent of the mooring buoy is achieved by introducing air into a downward facing "can" with a volume of $120 \mathrm{~m} 3$. By filling this volume with air the buoyancy increases by approximately $1200 \mathrm{kN}$.

The rapid ascent buoyancy is only needed for 13 seconds. Therefore there is no need that the rapid ascent buoyancy be airtight. Indeed it is undesirable that the rapid ascent buoyancy be airtight because of the danger that gases developed by biological processes accumulate in this volume and thereby cause that the buoy surfaces unexpectedly. In consequence the rapid ascent buoyancy is equipped with bleed holes that causes the air to bleed out in approximately 15 minutes.

The rapid ascent buoyancy is also equipped with ports that can be opened that bleed the air out in 20 seconds. These are actuated automatically in the event that the stay-closed signal is lost or in the event that an active "open" signal is received. This system is actuated in case the vessel fails to capture the buoy. In this case the buoy will descend again within 20 seconds which will normally be before the vessel moves sufficiently that the buoy would surface and thereby potentially impact the vessel on the side.

The normal process is that the ascent signal serves, as the stay-closed signal is well. When the mooring is secure this signal is shut off and the rapid descent system is automatically actuated and the rapid ascent air is released. Thus following the mooring the buoy is immediately ready to be released. Even in the event that this fails the buoy is ready for release 15 minutes later. 


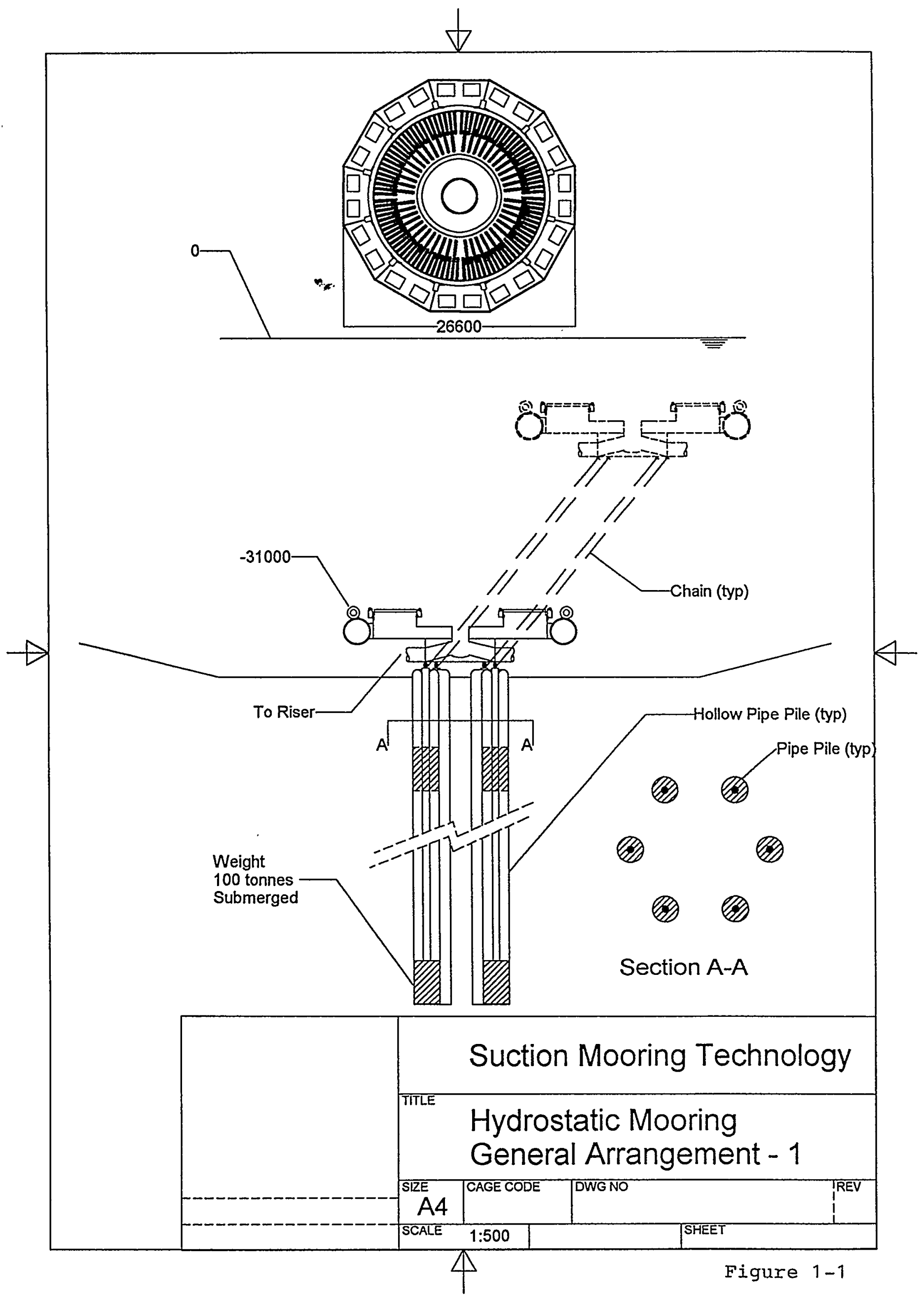




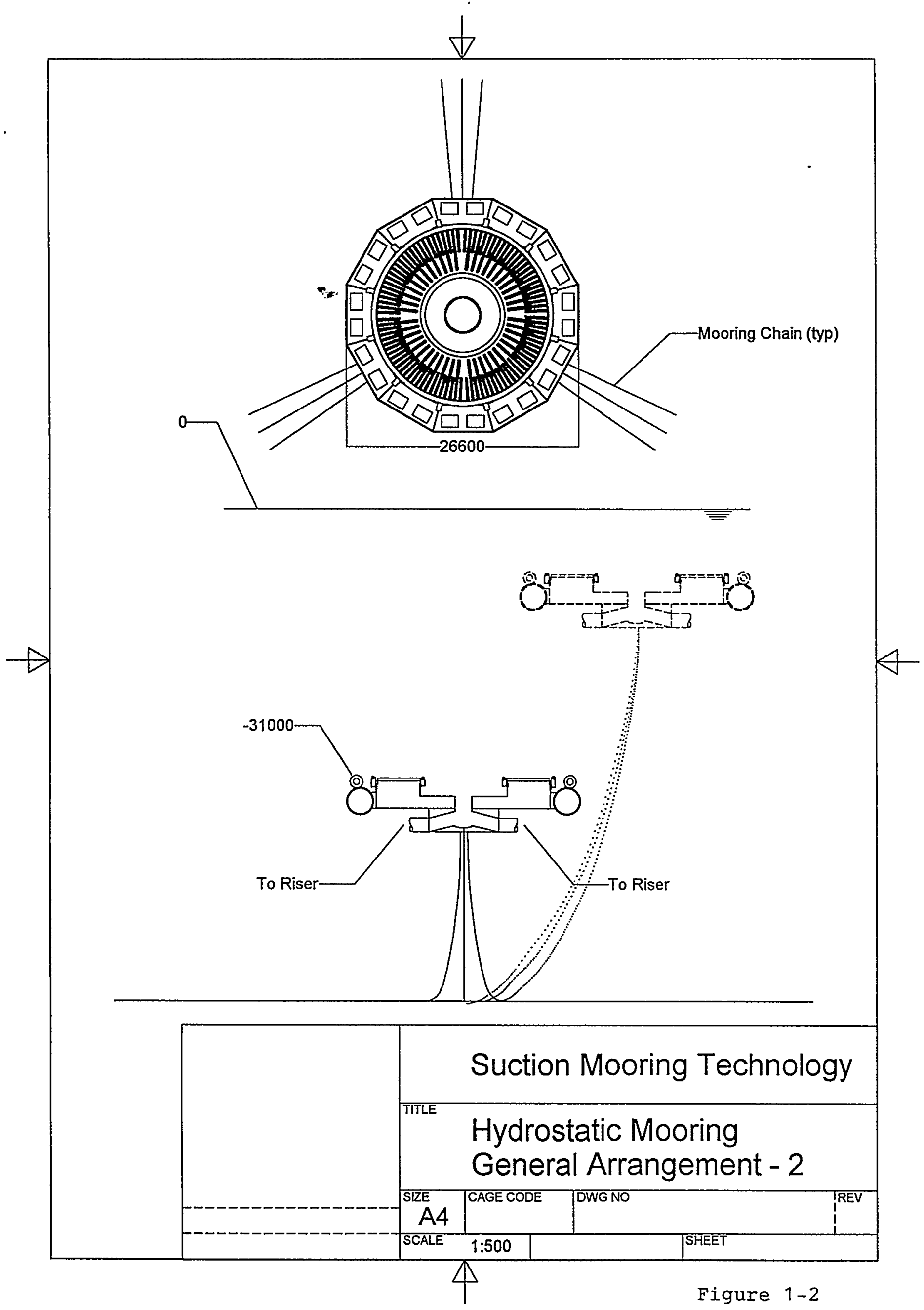




\section{- SAWM Line force Askelon}

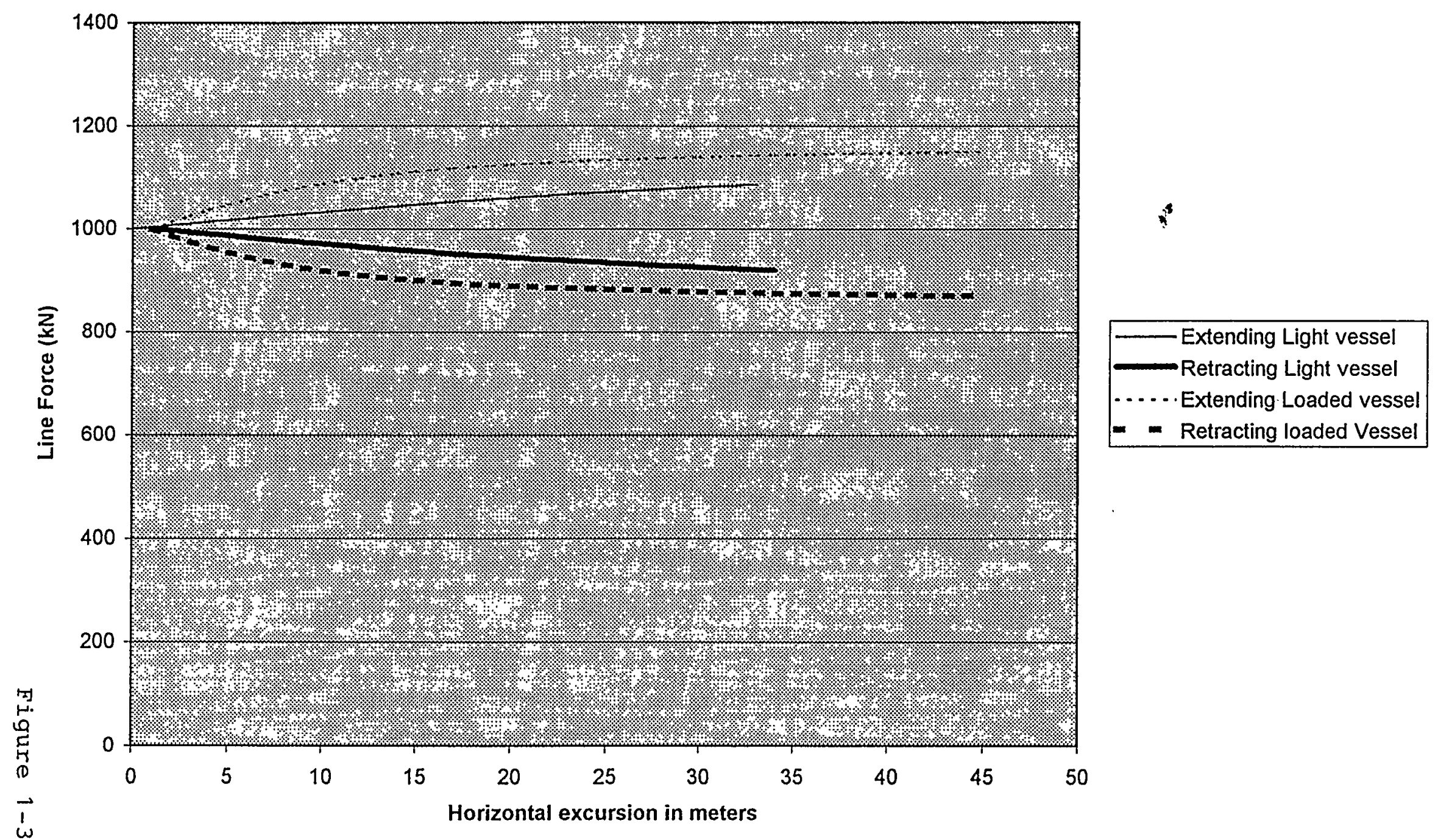




\section{Horizontal Mooring Force Askelon (1/6 of force)}
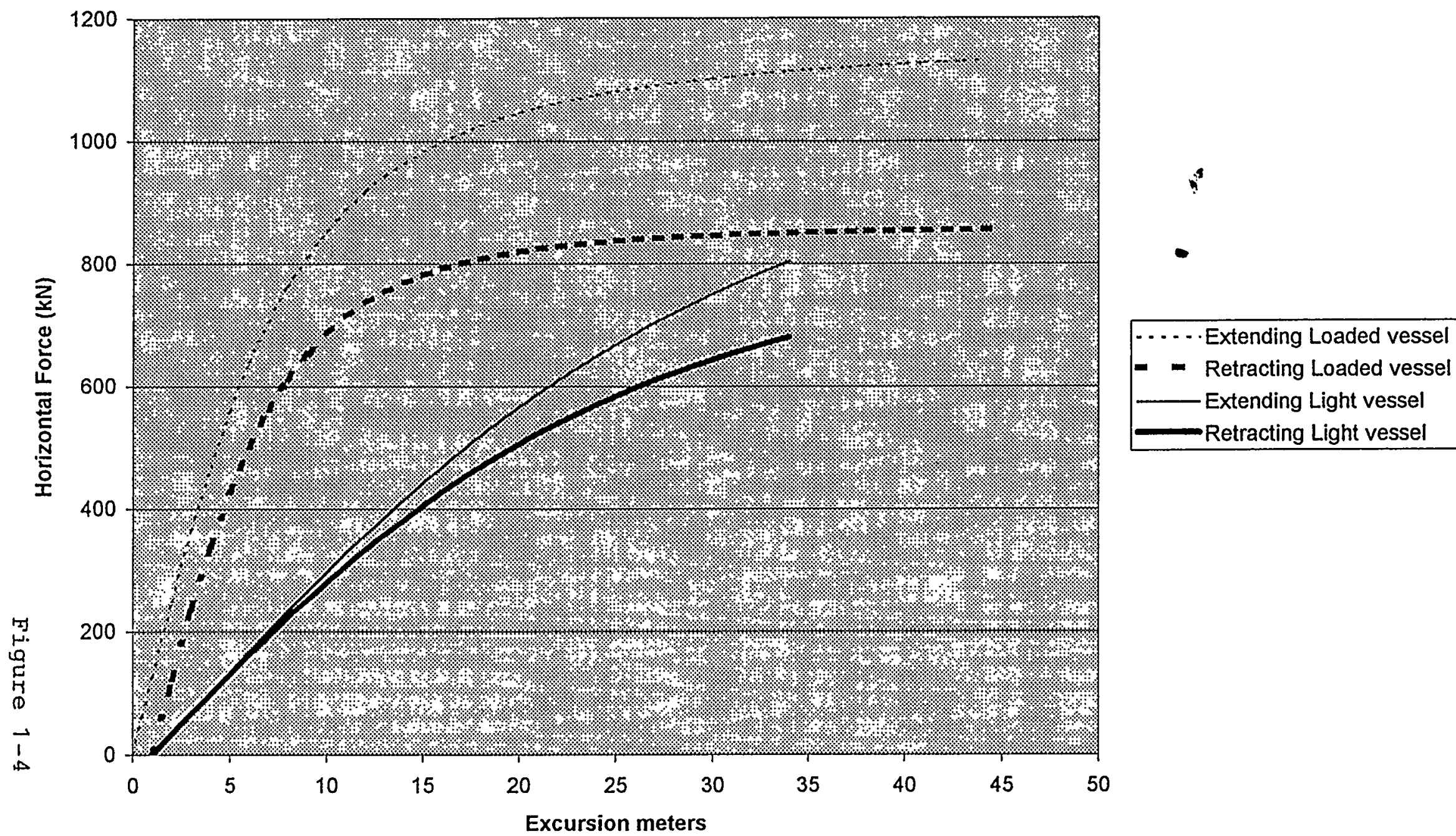


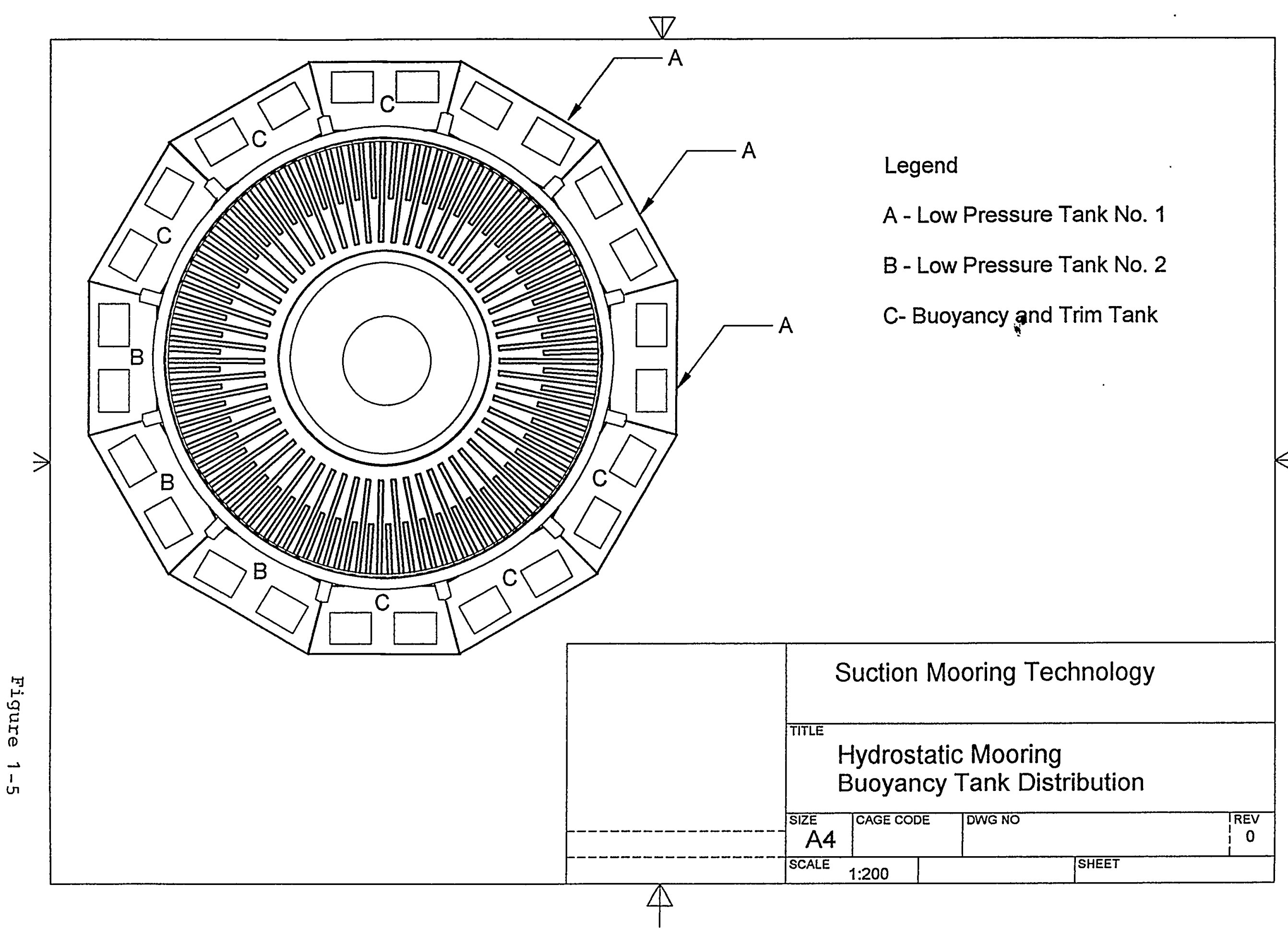




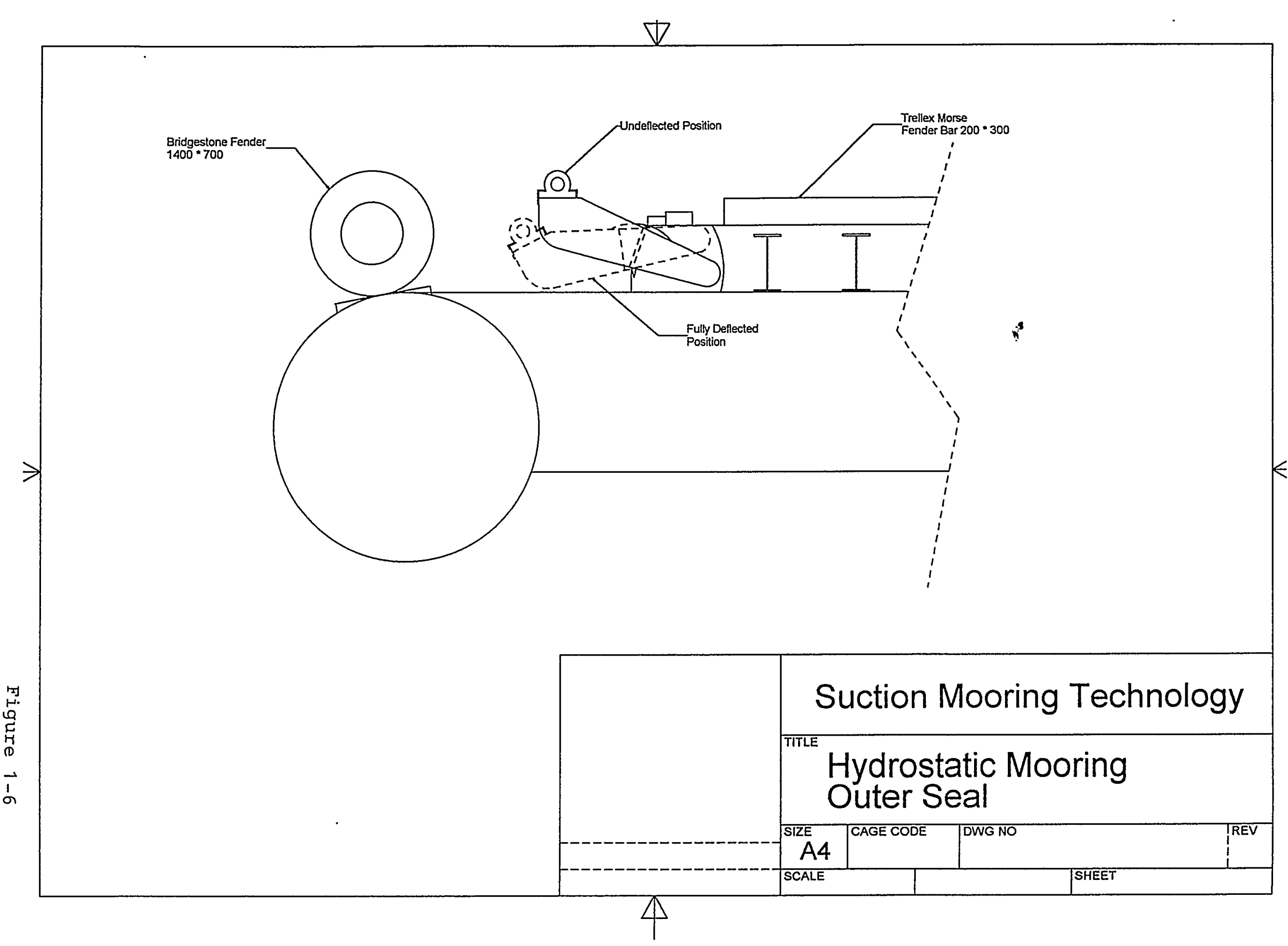




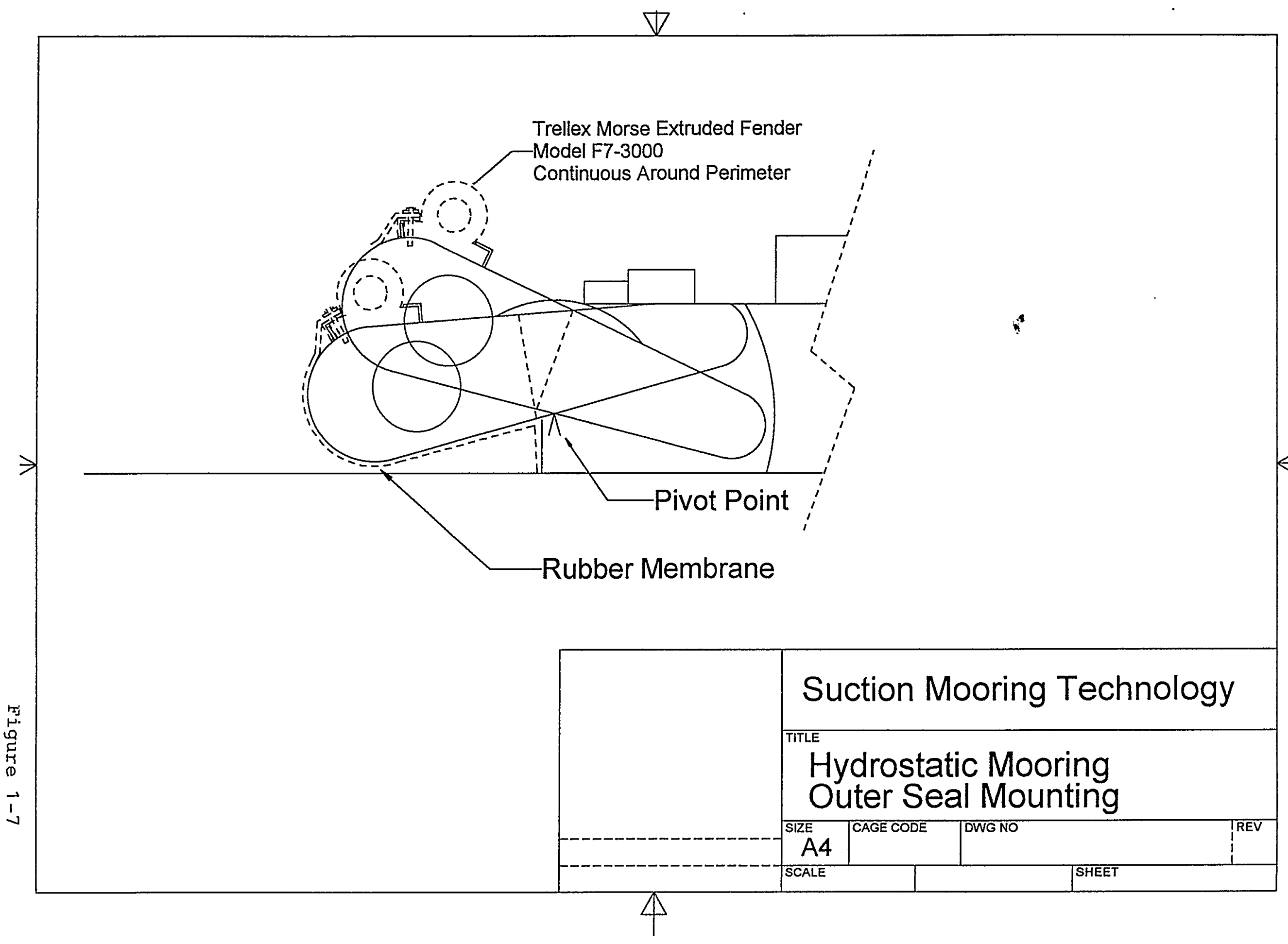




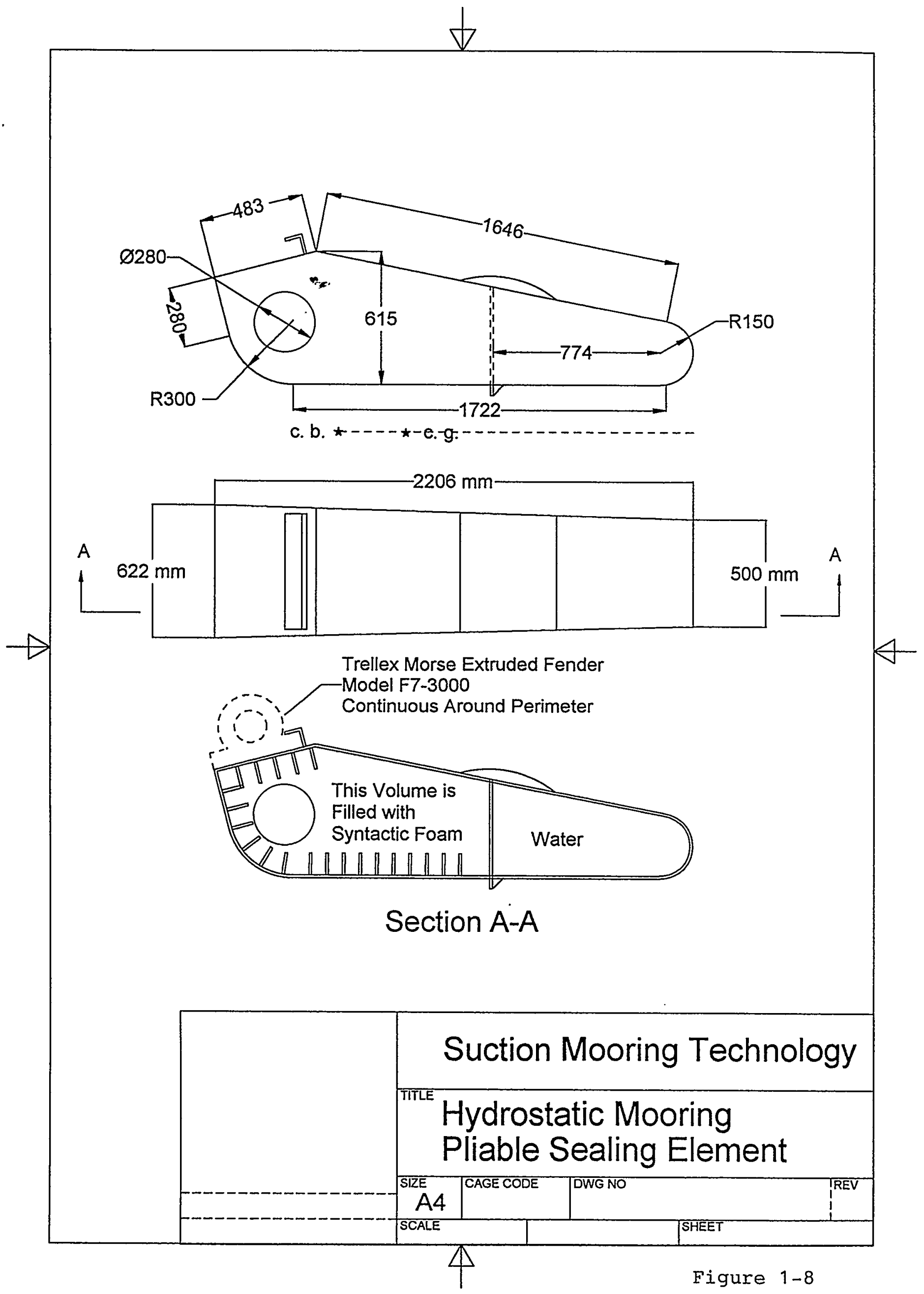


(Length $=1 \mathrm{~m}$ )

\begin{tabular}{|c|c|c|c|c|c|c|c|c|}
\hline \multirow{2}{*}{$\begin{array}{l}\text { Deflection } \\
\text { peöformence } \\
\text { Size }(m \mathrm{~m})\end{array}$} & \multicolumn{2}{|c|}{$40 \%$} & \multicolumn{2}{|c|}{$45 \%$} & \multicolumn{2}{|c|}{$50 \%$} & \multicolumn{2}{|c|}{$\cdot(70 x)$} \\
\hline & $\mathbf{R}$ & $\boldsymbol{E}$ & $\mathbf{R}$ & E & A & $\mathbf{E}$ & $\mathbf{R}$ & E \\
\hline $900_{\phi} \times 450_{\phi}$ & 18.6 & 3.40 & 22.3 & 4.30 & 27.3 & 5.40 & (546) & $(40.50)$ \\
\hline $1000_{\phi} \times 500 \phi$ & 20.6 & 4.20 & 24.8 & 5.30 & 30.3 & 6.70 & $(606)$ & $(50.25)$ \\
\hline $1100 \phi \times 550 \phi$ & 22.7 & 5.10 & 27.3 & 6.50 & 33.3 & 8.10 & (666) & (60.75) \\
\hline $1200 \phi \times 600 \phi$ & 24.8 & 6.10 & 29.8 & 7.70 & 36.3 & 9.70 & (726) & $(72.75)$ \\
\hline
\end{tabular}

- Figures at $70 \%$ deflection are for reference.

\section{REACTION FORCE}

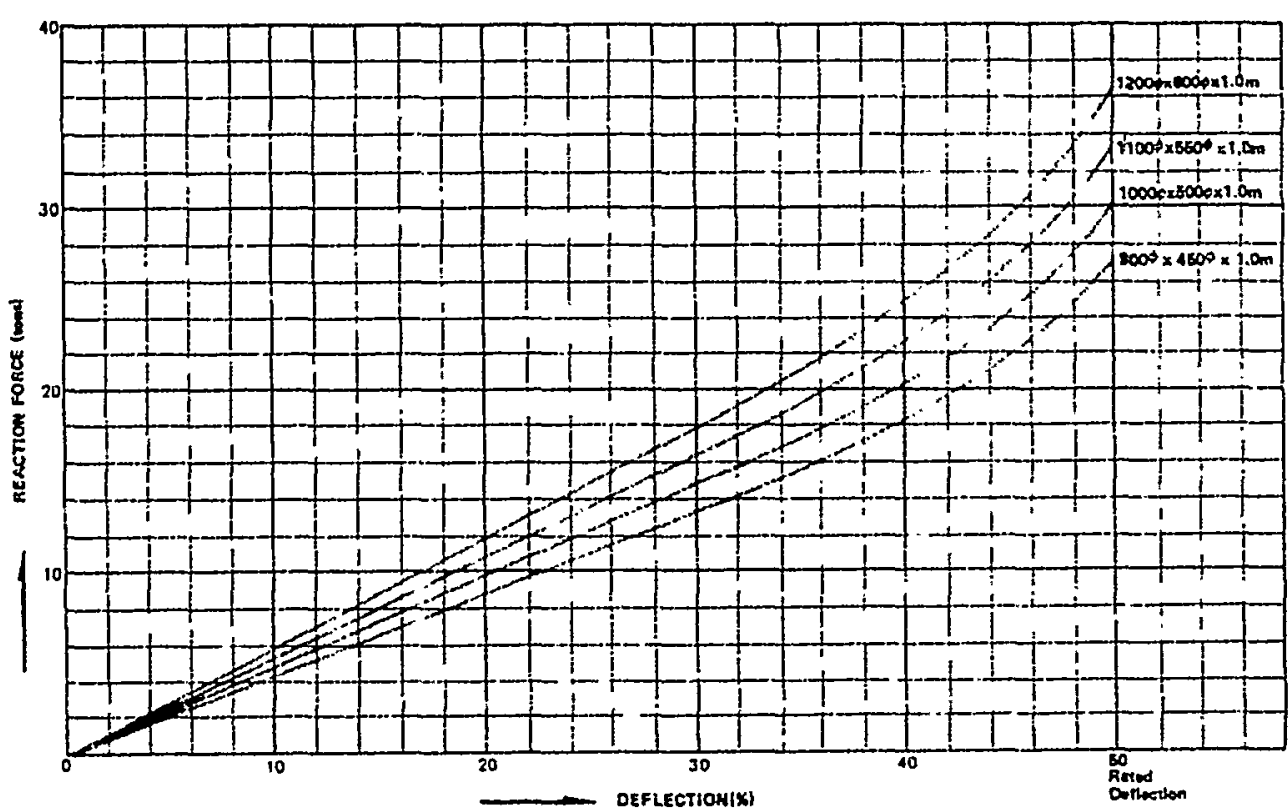

ENERGY ABSORPTION

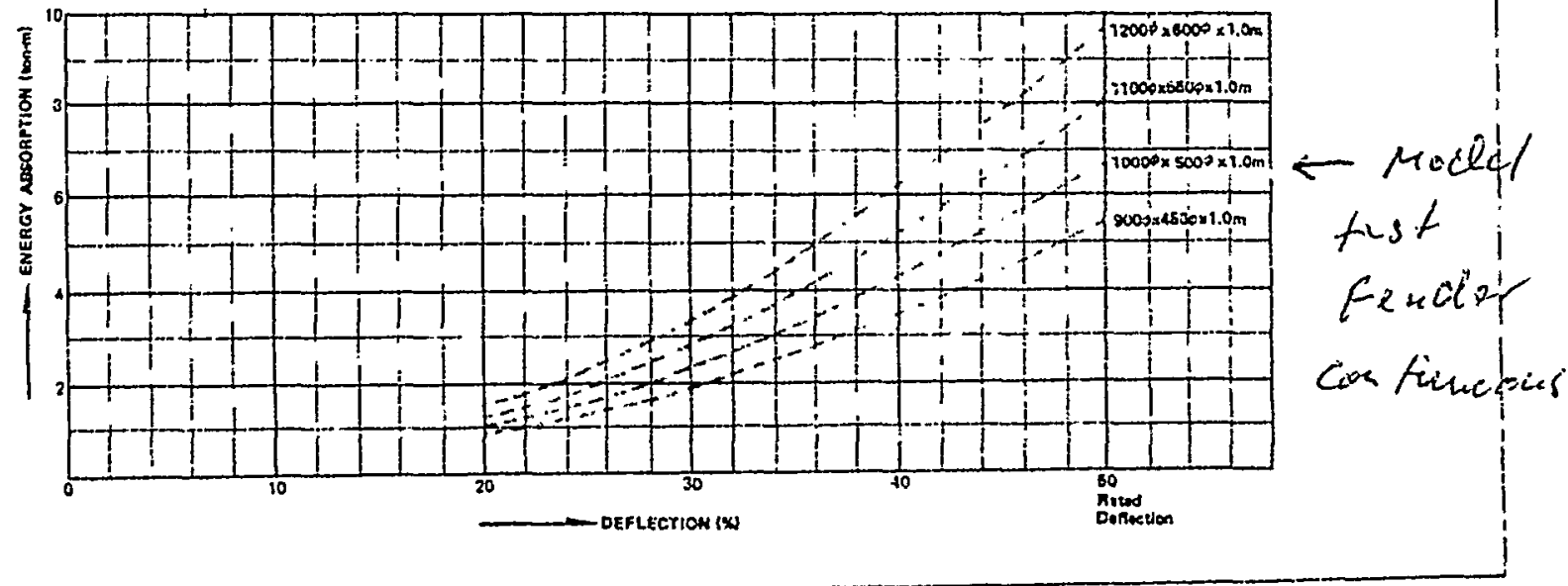




\begin{tabular}{|c|c|c|c|c|c|c|c|c|}
\hline \multirow{3}{*}{$\begin{array}{l}\text { Doffaction } \\
\text { Porformmenco } \\
\text { Size }(\mathrm{mm})\end{array}$} & \multirow{2}{*}{\multicolumn{2}{|c|}{$40 \%$}} & & & & & & ength $=1$ \\
\hline & & & \multicolumn{2}{|c|}{$45 \%$} & \multicolumn{2}{|c|}{$50 \%$} & \multicolumn{2}{|c|}{$\cdot(70 \%)$} \\
\hline & $\mathbf{R}$ & E & $\mathbf{R}$ & $E$ & $\mathbf{R}$ & $E$ & R & E \\
\hline $1300 \phi \times 650 \phi$ & 26.8 & $\begin{array}{r}7.10 \\
-\quad 10\end{array}$ & 32.2 & 9.00 & 39.4 & 11.40 & (788) & $(85.50)$ \\
\hline $1400 \phi \times 700_{\phi}$ & 28.9 & 8.30 & 34.7 & 10.50 & 42.4 & 13.20 & (848) & $(99.00)$ \\
\hline $1500_{\phi} \times 750 \phi$ & 31.0 & 9.50 & 37.2 & 12.10 & 45.4 & 15.10 & (908) & $(113.25)$ \\
\hline $1600 \phi \times 800 \phi$ & 33.0 & 10.75 & 39.7 & 13.57 & 48.4 & 17.18 & $(968)$ & $(128.85)$ \\
\hline
\end{tabular}

\section{REACTION FORCE}

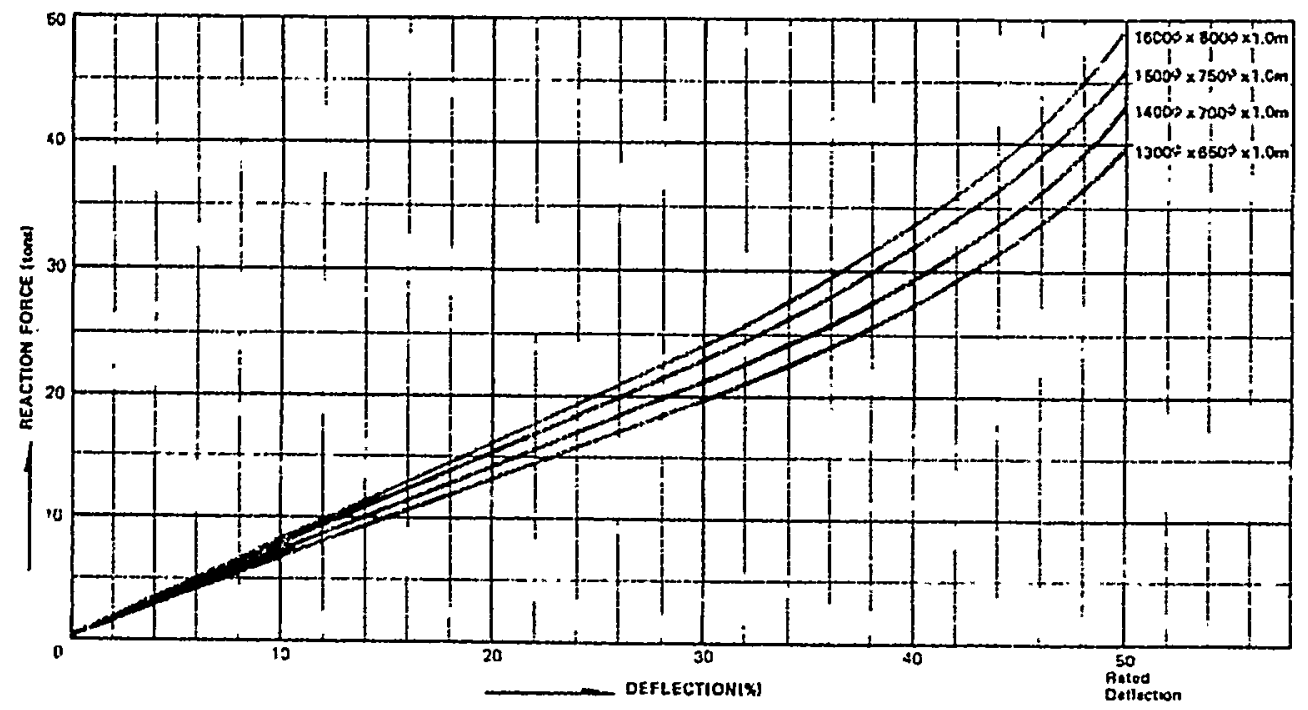

\section{ENERGY ABSORPTION}

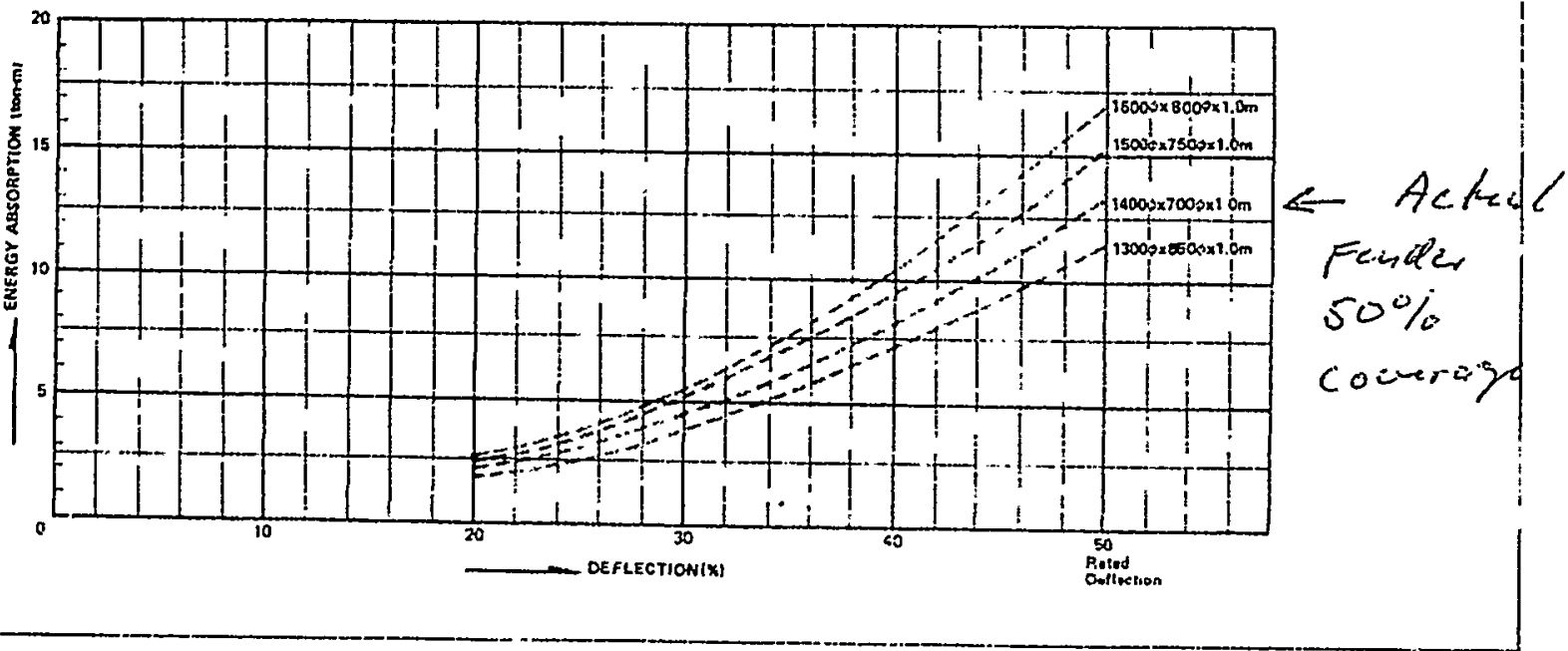


LENGTHS UP TO $20 \mathrm{FT}$. OTHER SIZES AVAILABLE ON REQUEST.

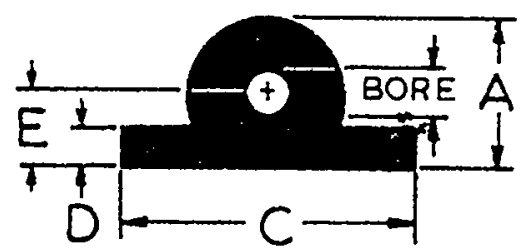

\begin{tabular}{|c|c|c|c|c|c|c|c|}
\hline PART No & $A$ & BORE & & 0 & $E$ & ${ }^{n} t / F t$ & $R / E$ \\
\hline UNITS & \multicolumn{5}{|c|}{ INCHES } & IDS & Kipk $F, K_{1} p$ \\
\hline$F 7-0000$ & 3 & 1 & 6 & .75 & 1.5 & 4.3 & 25 \\
\hline$F 7-8000$ & 4 & 2 & 7.5 & 1.0 & 2.25 & 10 & 195 \\
\hline$F 7.1000$ & 6 & 3 & 9 & 1.5 & 3 & 15.5 & 135 \\
\hline$F 8-1000$ & 8 & 4 & 12 & 20 & 4 & 30 & 11 \\
\hline F8-4000 & 10 & 5 & 15 & 25 & 5 & 44 & 9 \\
\hline F7-3000 & 12 & 6 & 18 & 3.0 & 6 & 585 & 6.3 \\
\hline
\end{tabular}

APPROXIMATE LOAD/ENERGY, DEFLECTION CURVES FOR MORSE STANDARD DEFLECTION (INCHES) CYLINDRICAL WING $\operatorname{selichey~}$

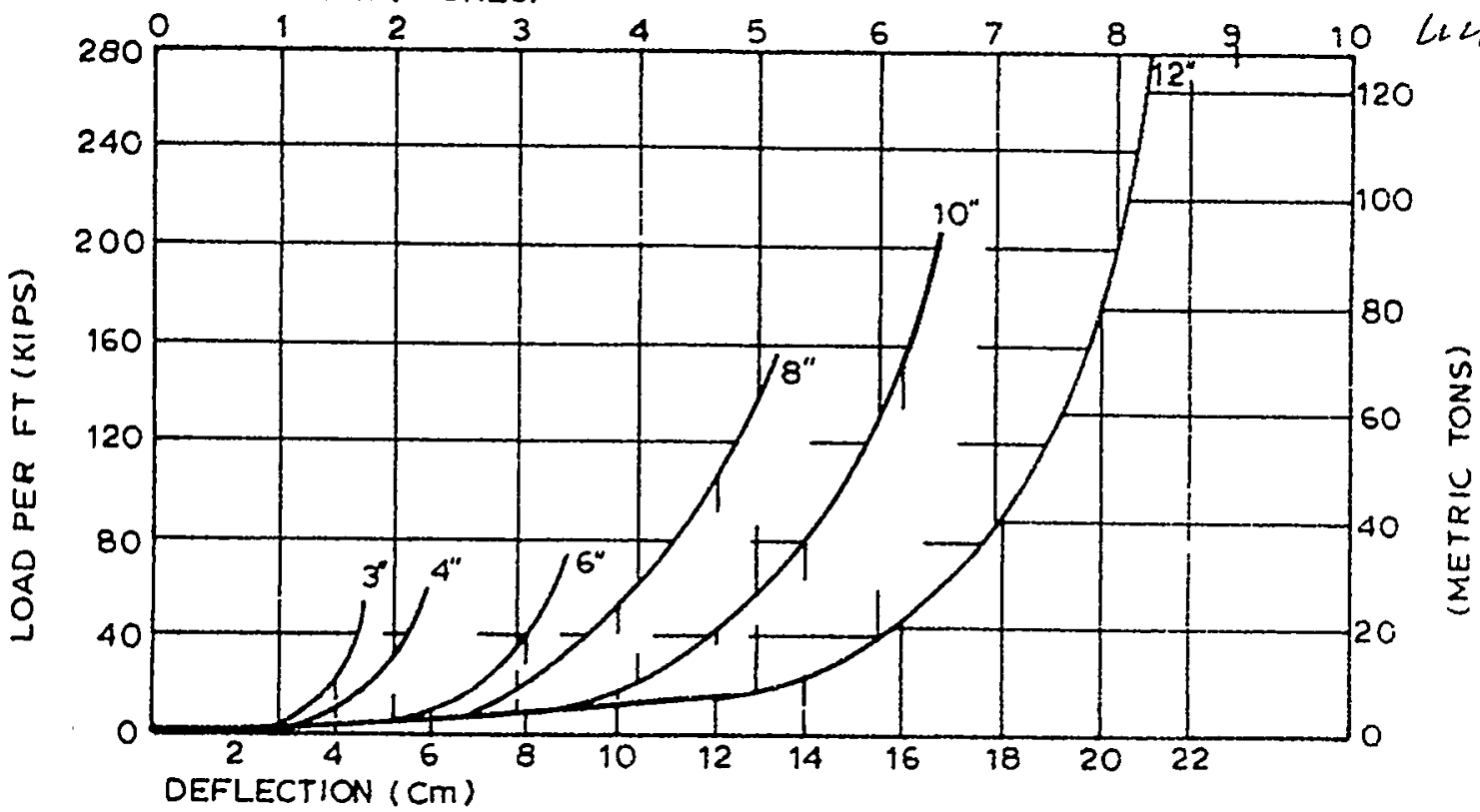
DEFLECTION (INCHES)

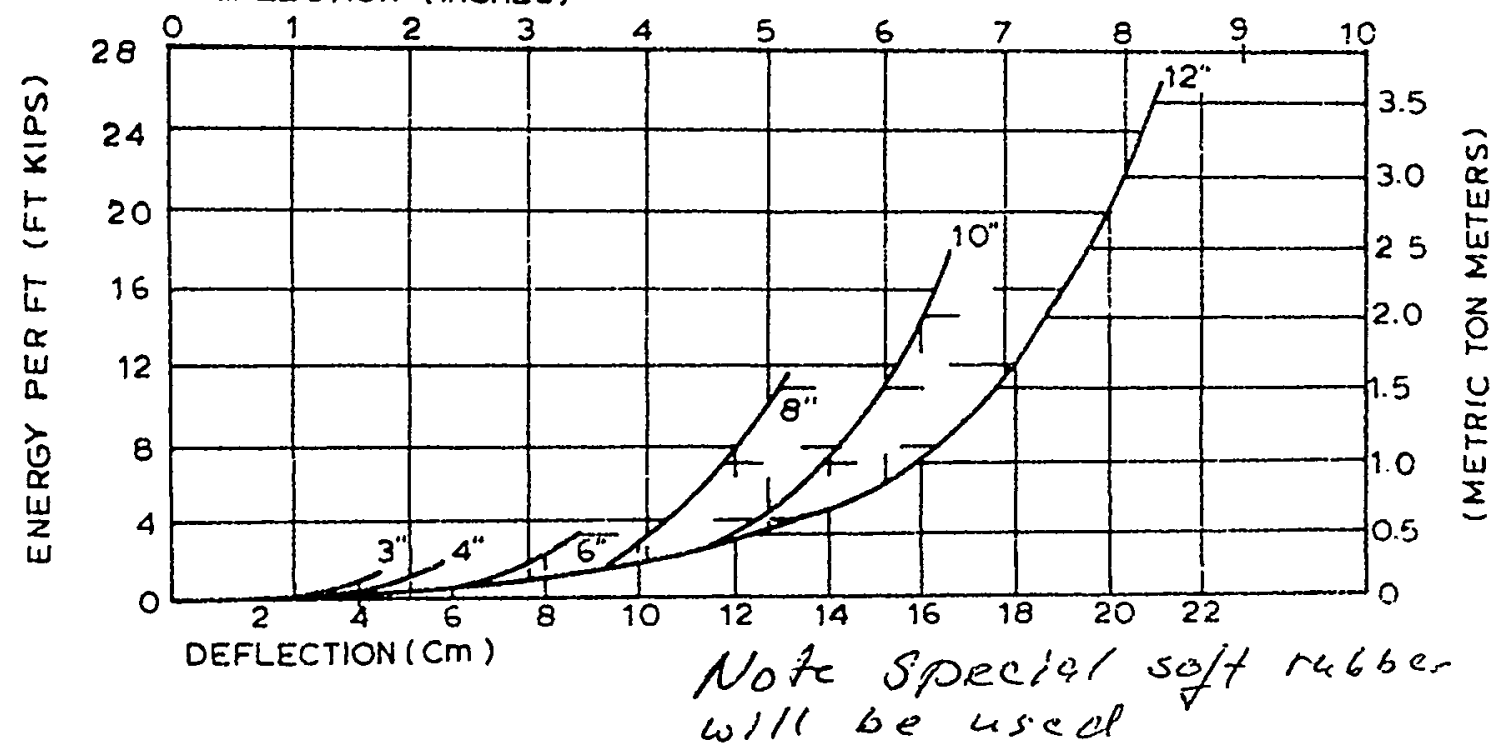

$$
\begin{aligned}
& \text { ifs of o static moorting seal } \\
& 3 \text { Kackies } 50 \% \text { of the above } \\
& \text { Eis } 1-i
\end{aligned}
$$


FERDER BAR SELECTION

\begin{tabular}{|c|c|c|c|c|c|c|}
\hline \multicolumn{4}{|c|}{ AnD roptams } & \multicolumn{3}{|c|}{ 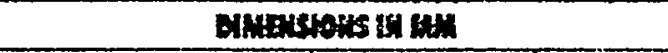 } \\
\hline \multicolumn{2}{|c|}{ Emey Meconerd } & \multicolumn{2}{|c|}{ mucmon pexa } & \multirow{2}{*}{$\underset{W \times M \times L}{\operatorname{man}}$} & \multirow[t]{2}{*}{ ancuon } & \multirow[t]{2}{*}{ 2n } \\
\hline Then in & $n+x+a s$ & To:unus & wis & & & \\
\hline 2.7 & $\begin{array}{l}12.3 \\
18.4\end{array}$ & $\begin{array}{l}65 \\
98 \\
\end{array}$ & $\begin{array}{r}143 \\
216\end{array}$ & $\begin{array}{l}150 \times 150 \times 1000 \\
150 \times 150 \times 1500 \\
\end{array}$ & M24 & $\frac{2}{3}$ \\
\hline $\begin{array}{l}1.7 \\
2.5 \\
\end{array}$ & $\begin{array}{l}12.3 \\
18.4 \\
\end{array}$ & $\begin{array}{l}45 \\
68 \\
\end{array}$ & $\begin{array}{r}99 \\
150 \\
\end{array}$ & $\begin{array}{l}150 \times 200 \times 1000 \\
150 \times 200 \times 1500\end{array}$ & M24 & $\frac{2}{3}$ \\
\hline $\begin{array}{l}2.7 \\
4.1\end{array}$ & $\begin{array}{l}19.5 \\
29.7\end{array}$ & $\begin{array}{r}84 \\
126 \\
\end{array}$ & $\begin{array}{l}185 \\
278\end{array}$ & $\begin{array}{l}200 \times 210 \times 1000 \\
200 \times 200 \times 1500\end{array}$ & M 30 & $\frac{2}{3}$ \\
\hline $\begin{array}{l}2.7 \\
4.1 \\
\end{array}$ & $\begin{array}{l}19.5 \\
29.7 \\
\end{array}$ & $\begin{array}{r}6 \hat{r}^{*} \\
101\end{array}$ & $\begin{array}{l}148 \\
.223 \\
\end{array}$ & $\begin{array}{l}200 \times 250 \times 1000 \\
200 \times 250 \times 1500 \\
\end{array}$ & 1130 & $\frac{2}{3}$ \\
\hline $\begin{array}{l}2.7 \\
4.1\end{array}$ & $\begin{array}{l}19.5 \\
29.7\end{array}$ & $\begin{array}{l}54 \\
81\end{array}$ & $\begin{array}{l}119 \\
179\end{array}$ & $\begin{array}{l}200 \times 300 \times 1000 \\
200 \times 300 \times 1500\end{array}$ & $M 30$ & $\frac{2}{3}$ \\
\hline
\end{tabular}

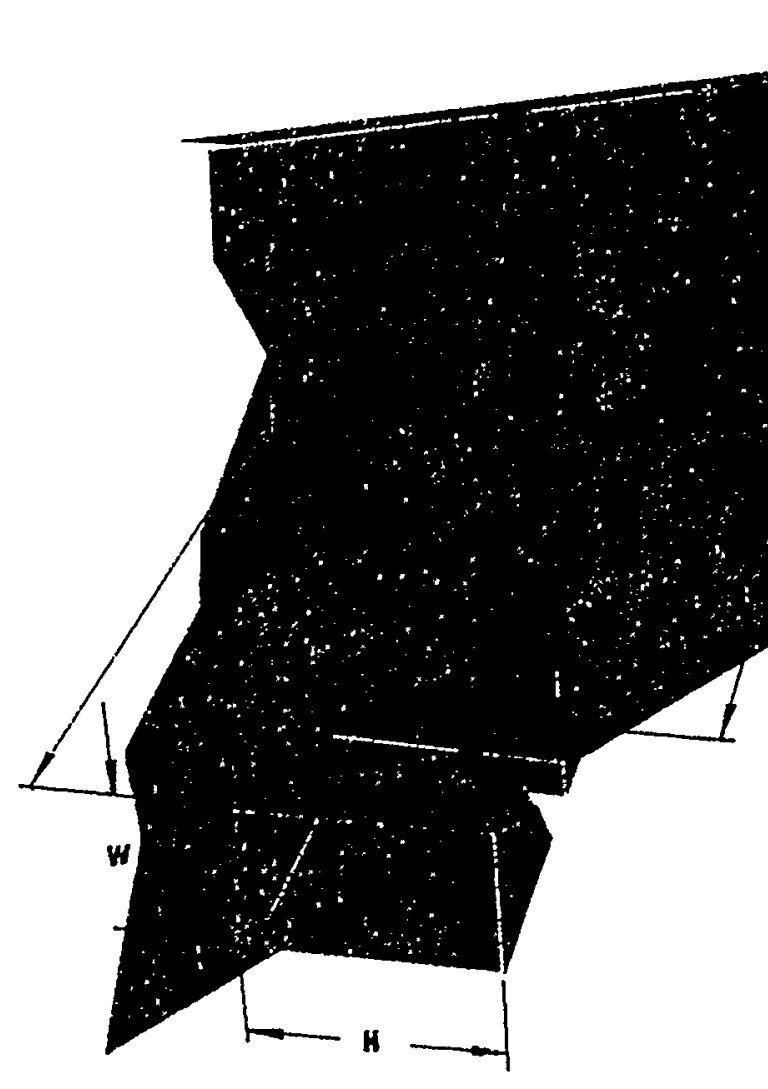

selecital

Uait
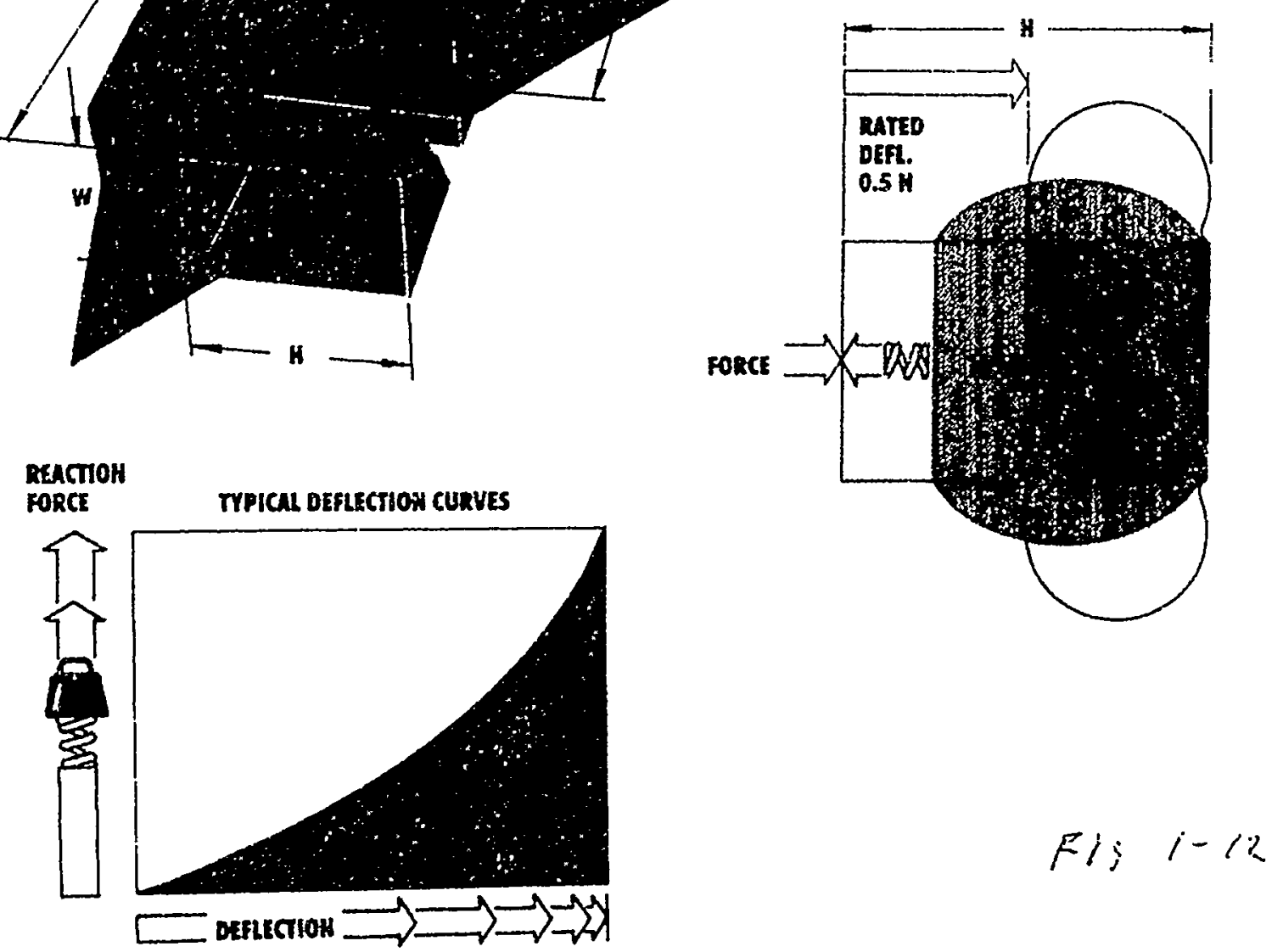
Hydrostatic mooring with one main weight

origin of coordinate system is at seabed veritcally under main weight

Spread sheet only applicable to single Stakepile anchor

\begin{tabular}{|c|c|}
\hline le & \\
\hline $\begin{array}{l}\text { leg length } \\
\text { Top of Stakepile elevation }\end{array}$ & $\mathrm{l}$ \\
\hline Elevation Top of chain & \% 10 meters \\
\hline Weight elevation & -55 meters \\
\hline water chain lght & 30 meters \\
\hline Main weight submerged & $000 \mathrm{kN}$ \\
\hline riction coeff & \\
\hline
\end{tabular}

Point

number

meters

0
1
2
3
4
5
6
7
8
9
10
11
12
13
14
15
16
17
18

\begin{tabular}{|c|c|c|c|c|c|c|c|}
\hline $\begin{array}{l}\text { Elevation } \\
\text { of weight }\end{array}$ & $\begin{array}{l}\text { Length of } \\
\text { chain in } \\
\text { water }\end{array}$ & $\begin{array}{l}\text { Legal } \\
\text { points. } \\
\text { Top of } \\
\text { stakepile } \\
\text { above } \\
\text { weight }\end{array}$ & $\begin{array}{l}\text { Angle to } \\
\text { attach } \\
\text { point from } \\
\text { horizontal }\end{array}$ & $\begin{array}{l}\text { chain } \\
\text { force } \\
\text { extending }\end{array}$ & $\begin{array}{l}\text { Chain } \\
\text { Force } \\
\text { retracting }\end{array}$ & $\begin{array}{l}\text { Horizontal } \\
\text { force } \\
\text { Extending }\end{array}$ & $\begin{array}{l}\text { Horizontal } \\
\text { Force } \\
\text { retracting }\end{array}$ \\
\hline e & c & $e>z$ & alfa & $k N$ & $\mathrm{kN}$ & $\mathrm{kN}$ & $\mathrm{kN}$ \\
\hline-55.00 & 30.00 & yes & & 1000 & 1000 & 0 & 0 \\
\hline-54.98 & 30.02 & yes & 1.537475 & 1003.338 & 996.6734 & 33.42602 & 33.20401 \\
\hline-54.93 & 30.07 & yes & 1.504228 & 1006.679 & 993.3653 & 66.96329 & 66.07768 \\
\hline-54.85 & 30.15 & yes & 1.471128 & 1010.017 & 990.0826 & 100.5004 & 98.5169 \\
\hline-54.73 & 30.27 & yes & 1.438245 & 1013.343 & 986.8323 & 133.9272 & 130.4234 \\
\hline-54.59 & 30.41 & yes & 1.405648 & 1016.652 & 983.6208 & 167.1366 & 161.7063 \\
\hline-54.41 & 30.59 & yes & 1.373401 & 1019.936 & 980.454 & 200.0258 & 192.2828 \\
\hline-54.19 & 30.81 & yes & 1.341564 & 1023.188 & 977.3375 & 232.4986 & 222.08 \\
\hline-53.95 & 31.05 & yes & 1.310194 & 1026.403 & 974.2764 & 264.4657 & 251.0346 \\
\hline-53.68 & 31.32 & yes & 1.27934 & 1029.575 & 971.275 & 295.8461 & 279.0938 \\
\hline-53.38 & 31.62 & yes & 1.249046 & 1032.698 & 968.3371 & 326.5679 & 306.2151 \\
\hline-53.05 & 31.95 & yes & 1.219352 & 1035.769 & 965.4659 & 356.5684 & 332.3661 \\
\hline-52.69 & 32.31 & yes & 1.19029 & 1038.784 & 962.6642 & 385.7946 & 357.5245 \\
\hline-52.30 & 32.70 & yes & 1.161888 & 1041.738 & 959.934 & 414.2029 & 381.6769 \\
\hline-51.89 & 33.11 & yes & 1.134169 & 1044.63 & 957.2768 & 441.7588 & 404.8184 \\
\hline-51.46 & 33.54 & yes & 1.107149 & 1047.456 & 954.6937 & 468.4367 & 426.952 \\
\hline-51.00 & 34.00 & yes & 1.080839 & 1050.216 & 952.1852 & 494.2192 & 448.0871 \\
\hline-50.52 & 34.48 & yes & 1.055247 & 1052.907 & 949.7515 & 519.0964 & 468.2394 \\
\hline-50.01 & 34.99 & yes & 1.030377 & 1055.529 & 947.3924 & 543.0651 & 487.4293 \\
\hline
\end{tabular}




19
20
21
22
23
24
25
26
27
28
29
30
31
32
33
34
35
36
37
38
39
40
41
42
43
44
45
46
47
48
49
50
51
52
53
54
55
56
57
58
59

$\begin{array}{ll}-49.49 & 35.51 \text { yes } \\ -48.94 & 36.06 \text { yes } \\ -48.38 & 36.62 \text { yes } \\ -47.80 & 37.20 \text { yes } \\ -47.20 & 37.80 \text { yes } \\ -46.58 & 38.42 \text { yes } \\ -45.95 & 39.05 \text { yes } \\ -45.30 & 39.70 \text { yes } \\ -44.64 & 40.36 \text { yes } \\ -43.96 & 41.04 \text { yes } \\ -43.27 & 41.73 \text { yes } \\ -42.57 & 42.43 \text { yes } \\ -41.86 & 43.14 \text { yes } \\ -41.14 & 43.86 \text { yes } \\ -40.40 & 44.60 \text { yes } \\ -39.66 & 45.34 \\ -38.90 & 46.10 \\ -38.14 & 46.86 \\ -37.37 & 47.63 \\ -36.59 & 48.41 \\ -35.80 & 49.20 \\ -35.00 & 50.00 \\ -34.20 & 50.80 \\ -33.39 & 51.61 \\ -32.57 & 52.43 \\ -31.75 & 53.25 \\ -30.92 & 54.08 \\ -30.08 & 54.92 \\ -29.24 & 55.76 \\ -28.40 & 56.60 \\ -27.55 & 57.45 \\ -26.69 & 58.31 \\ -25.83 & 59.17 \\ -24.97 & 60.03 \\ -24.10 & 60.90 \\ -23.23 & 61.77 \\ -22.35 & 62.65 \\ -21.47 & 63.53 \\ -20.59 & 64.41 \\ -19.70 & 65.30 \\ -18.81 & 66.19\end{array}$

$\begin{array}{lllll}1.006227 & 1058.081 & 945.1072 & 566.1285 & 505.6816\end{array}$ $\begin{array}{llllll}0.982794 & 1060.563 & 942.8951 & 588.2947 & 523.0241\end{array}$ $\begin{array}{llllll}0.96007 & 1062.976 & 940.7549 & 609.5768 & 539.4875\end{array}$ $\begin{array}{llllll}0.938047 & 1065.32 & 938.6854 & 629.9913 & 555.1044\end{array}$ $\begin{array}{lllll}0.916714 & 1067.595 & 936.685 & 649.5584 & 569.9087\end{array}$ $\begin{array}{llllll}0.896055 & 1069.803 & 934.7519 & 668.3004 & 583.9349\end{array}$ $\begin{array}{llllll}0.876058 & 1071.944 & 932.8845 & 686.2418 & 597.2181\end{array}$ $\begin{array}{llllll}0.856706 & 1074.02 & 931.0809 & 703.4088 & 609.7933\end{array}$ $\begin{array}{llllll}0.837981 & 1076.033 & 929.3392 & 719.8284 & 621.6951\end{array}$ $0.819867 \quad 1077.984 \quad 927.6573 \quad 735.5284 \quad 632.9575$ $\begin{array}{llllll}0.802346 & 1079.875 & 926.0333 & 750.5368 & 643.6136\end{array}$ $\begin{array}{llllll}0.785398 & 1081.706 & 924.4653 & 764.8819 & 653.6956\end{array}$ $\begin{array}{llllll}0.769006 & 1083.481 & 922.9511 & 778.5918 & 663.2346\end{array}$ $\begin{array}{llllll}0.753151 & 1085.2 & 921.4889 & 791.6939 & 672.2605\end{array}$ $\begin{array}{llllll}0.737815 & 1086.866 & 920.0768 & 804.2155 & 680.8017\end{array}$ 0.722979

0.708626

0.694738

0.681298

0.668289

0.655696

0.643501

0.631691

0.620249

0.609163

0.598419

0.588003

0.577902

0.568105

0.558599

0.549374

0.54042

0.531724

0.523278

0.515073

0.507099

0.499347

0.491809

0.484478

0.477345

0.470404 
Hydrostatic mooring with one main weight

origin of coordinate system is at seabed veritcally under main weight

Spread sheet only applicable to single Stakepile anchor

leg length

Top of Stakepile elevation

Elevation Top of chain

Weight elevation

water chain lght

Main weight submerged

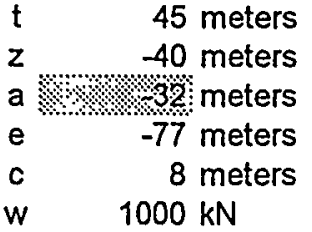

friction coefficient

$f$

Point

number

meters

0

7
0.1

\section{Legal}

points.

Top of

$\begin{array}{llll}\text { Length of stakepile attach chain } & \text { Chain Horizontal Horizontal } \\ \text { Elevation chain in above point from force } & \text { Force force Force }\end{array}$

of weight water weight horizontal extending retracting Extending retracting

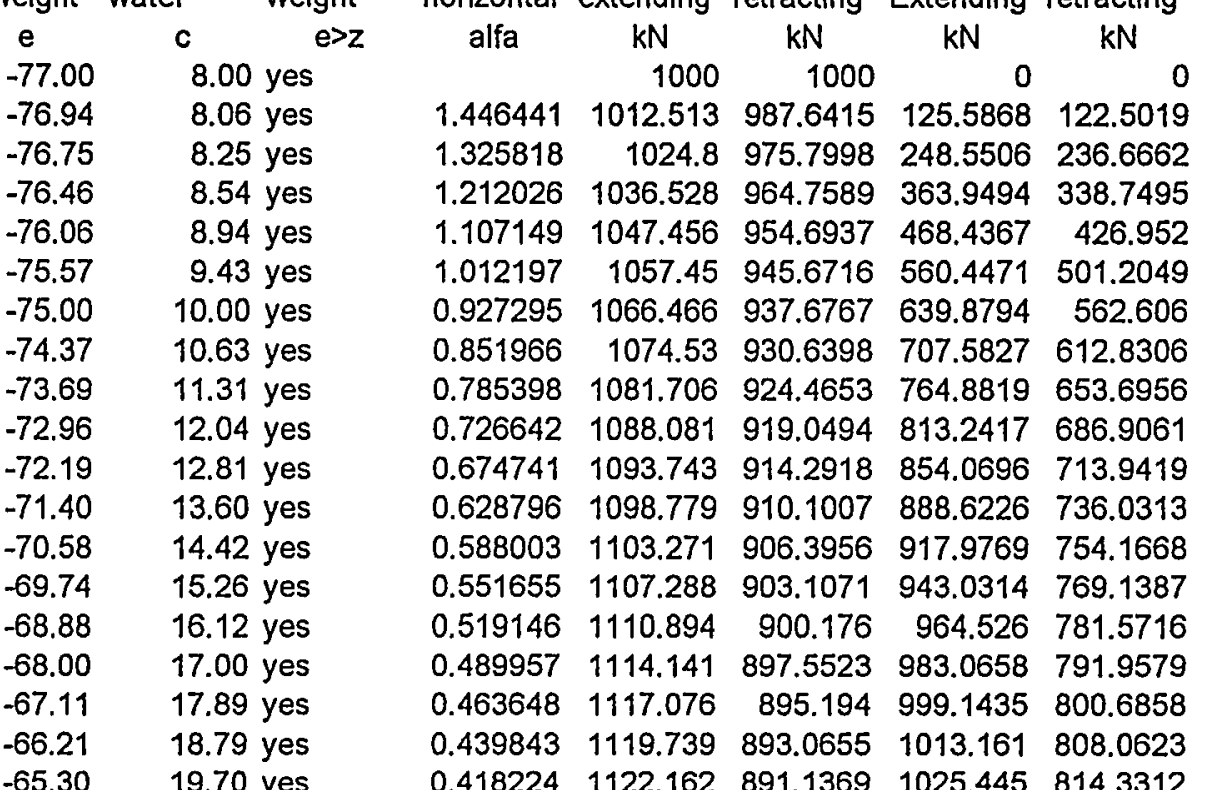


$\begin{array}{lllll}0.398522 & 1124.375 & 889.3829 & 1036.264 & 819.6868\end{array}$ $\begin{array}{llllll}0.380506 & 1126.403 & 887.7821 & 1045.839 & 824.2849\end{array}$ $\begin{array}{llllll}0.363979 & 1128.266 & 886.316 & 1054.351 & 828.2514\end{array}$ $\begin{array}{llllll}0.348771 & 1129.983 & 884.9691 & 1061.951 & 831.6882\end{array}$ $\begin{array}{llllll}0.334737 & 1131.57 & 883.728 & 1068.764 & 834.6783\end{array}$ $\begin{array}{lllll}0.321751 & 1133.04 & 882.5811 & 1074.896 & 837.29\end{array}$ $\begin{array}{llllll}0.309703 & 1134.406 & 881.5185 & 1080.436 & 839.5795\end{array}$ $\begin{array}{llllll}0.298499 & 1135.678 & 880.5314 & 1085.457 & 841.5934\end{array}$ $\begin{array}{llllll}0.288055 & 1136.865 & 879.6123 & 1090.024 & 843.3706\end{array}$ $\begin{array}{llllll}0.2783 & 1137.974 & 878.7545 & 1094.189 & 844.9435\end{array}$ $\begin{array}{llllll}0.269167 & 1139.014 & 877.9524 & 1098.001 & 846.3396\end{array}$ $\begin{array}{llllll}0.260602 & 1139.99 & 877.2008 & 1101.498 & 847.582\end{array}$ $\begin{array}{llllll}0.252554 & 1140.908 & 876.4951 & 1104.715 & 848.6903\end{array}$ $\begin{array}{llllll}0.244979 & 1141.772 & 875.8313 & 1107.682 & 849.6812\end{array}$ $\begin{array}{llllll}0.237836 & 1142.588 & 875.206 & 1110.424 & 850.569\end{array}$ $\begin{array}{lllll}0.231091 & 1143.359 & 874.6158 & 1112.965 & 851.366\end{array}$ $\begin{array}{llllll}0.224711 & 1144.089 & 874.058 & 1115.325 & 852.0829\end{array}$ $\begin{array}{llllll}0.218669 & 1144.78 & 873.5301 & 1117.52 & 852.7287\end{array}$ $\begin{array}{llllll}0.212938 & 1145.437 & 873.0296 & 1119.566 & 853.3115\end{array}$ $\begin{array}{lllllll}0.207496 & 1146.06 & 872.5546 & 1121.477 & 853.8381\end{array}$ $\begin{array}{llllll}0.202322 & 1146.653 & 872.1032 & 1123.265 & 854.3147\end{array}$

$\begin{array}{llllll}0.197396 & 1147.218 & 871.6737 & 1124.94 & 854.7464\end{array}$

$\begin{array}{llllll}0.192701 & 1147.757 & 871.2646 & 1126.513 & 855.138\end{array}$

$\begin{array}{llllll}0.188222 & 1148.271 & 870.8744 & 1127.991 & 855.4935\end{array}$

$\begin{array}{llllll}0.183943 & 1148.763 & 870.5019 & 1129.383 & 855.8166\end{array}$ $\begin{array}{lllll}0.179853 & 1149.232 & 870.146 & 1130.695 & 856.1105\end{array}$

\subsection{4}

0.172191

0.168597

0.165149

0.161837

0.158655

0.155595

0.152649

0.149812

0.147078

0.144442

0.141897

0.13944

0.137066

0.134771 
Content of chapter 2.

Section 1: This is the structural calculation of the sealing units.

Section 2: This is the structural calculation of the buoy and the bearing system performed by Han Padron Associates.

Subsequent to this design a number of changes was made that have only negligible impact on the structural system. These were:

- The watertight plate was moved from the underside of the buoy to the top of the buoy. The reason for this was to improve the structural force transfer between the fenders and the structure. The timber in-fill now serves the prevention of trapping air below the buoy rather than the function of preventing the accumulation of sediments on top of the buoy, which was the previous function..

- The center 8-meter diameter plate was changed to be able to resist the maximum physically possible hydrostatic pressure differential of $350 \mathrm{kPa}$.

- The bearing was changed from a Lubron bearing to a Hillman roller bearing. A revised bearing design is presented in section 3 .

Section 3: This is the final concept for the mooring chain attachments and the bearing. 


\section{Content of chapter 2 .}

Section 1: This is the structural calculation of the sealing units.

Section 2: This is the structural calculation of the buoy and the bearing system performed by Han Padron Associates.

Subsequent to this design a number of changes was made that have only negligible impact on the structural system. These were:

- The watertight plate was moved from the underside of the buoy to the top of the buoy. The reason for this was to improve the structural force transfer between the fenders and the structure. The timber in-fill now serves the prevention of trapping air below the buoy rather than the function of preventing the accumulation of sediments on top of the buoy, which was the previous function.

- The center 8-meter diameter plate was changed to be able to resist the maximum physically possible hydrostatic pressure differential of $350 \mathrm{kPa}$.

- The bearing was changed from a Lubron bearing to a Hillman roller bearing. A revised bearing design is presented in section 3 .

Section 3: 'This is the final concept for the mooring chain attachments and the bearing. 
Results:

Horizontal projection:

Moment, vetical axis:

Moment, horizontal axis:

Results;

\section{Pressure Design:}

Curved panel:

Bottom sheet:

Stiffener $75 \times 12 \mathrm{~mm}$ :

External sheet:

Support plate for fender:

Stiffener $140 \times 20 \mathrm{~mm}$ : $\left.\sin \left(13.508^{\circ}\right)\right)+\mathrm{H} \times\left(1.060 \times \cos \left(2.54^{\circ}\right)-1.867 \times \sin \left(2.54^{\circ}\right)\right)$

$\mathrm{V}_{1}=227 \mathrm{kN}$

$\mathrm{V}_{2}=19 \mathrm{kN}$

$\mathrm{H}=232 \mathrm{kN}$

$1 / 2 \times 227:=Q_{1}-Q_{2}-Q_{3}$

$1 / 2 \times 227 \times 0.929=0.9375 \times Q_{3}$

$1 / 2 \times 227 \times 1.018=0.4995 \times Q_{1}+0.0415 \times Q_{3}$

$\mathrm{Q}_{1}=222 \mathrm{kN}$

$\mathrm{Q}_{2}=-4 \mathrm{kN}$

$\mathrm{Q}_{3}=112 \mathrm{kN}$
Membrane stress: $f_{n}=p \times R / t=0.35 \times 300 / 12=8.8 \mathrm{MPa}$

$\mathrm{R} / \mathrm{t}=300 / 12=25$, no stability problem.

Area between stiffeners: $231 \times 598 \mathrm{~mm}$. Carrying capacity only considered in one direction. $M=\mathrm{p} \mathrm{xl}^{2} / 12=0.35 \times 231^{2} / 12=1556 \mathrm{~N}$

$\mathrm{f}_{\mathrm{b}}=6 \times 1556 / 12^{2}=65 \mathrm{Mpa}$

Area: $219 \times 12+87 \times 12=2628+1044=3672 \mathrm{~nm}^{2}$

Center of gravity: $\mathrm{a}=(2628 \times 6+1044 \times 43.5) / 3672=16.7 \mathrm{~nm}$

$\mathrm{I}=2628 \times 10.7^{2}+1044 \times 26.8^{2}+2628 \times 12^{2} / 12+1044 \times 87^{2} / 12=1.741 \mathrm{E} 6 \mathrm{~mm}^{4}$.

$\mathrm{S}_{\mathrm{b}}=1.741 \mathrm{E} 6 / 70.3=24.77 \mathrm{E} 3 \mathrm{~mm}^{3}$.

$\mathrm{S}_{\mathrm{l}}=1.741 \mathrm{E} 6 / 16.7=104.3 \mathrm{E} 3 \mathrm{~mm}^{3}$.

Moment in stiffener: $\mathrm{M}=0.35 \times 231 \times 598^{2} / 8=3.614 \mathrm{E} 6 \mathrm{Nmm}$

$\mathrm{f}_{\mathrm{bb}}=3.614 \mathrm{E} 6 / 24.77 \mathrm{E} 3=146 \mathrm{Mpa}<149$.

$\mathrm{f}_{\mathrm{bt}}=3.614 \mathrm{E} 6 / 104.3 \mathrm{E} 3=35 \mathrm{Mpa}$.

Area between stiffeners: $280 \times 628 \mathrm{~mm}$. Carrying capacity only considered in one direction. $M=p \times 1^{2} / 12=0.35 \times 280^{2} / 12=2287 \mathrm{~N}$

$f_{\mathrm{b}}=6 \times 2287 / 12^{2}=95 \mathrm{Mpa}$

Moment in stiffener: $\mathrm{M}=0.35 \times 1 / 2 \times 280 \times 628^{2} / \mathrm{S}=2.416 \mathrm{E} 6 \mathrm{Nmm}<3.614 \mathrm{E} 6$

Hydraulic pressure + reaction on fender (load case 1 )

$\mathrm{p}=0.35+424000 /(400 \times 628)=2.038 \mathrm{MPa}$

Area between stiffeners: $161 \times 628 \mathrm{~mm}$. Carrying capacity only considered in one direction. $\mathrm{M}=\mathrm{p} \mathrm{x} \mathrm{I}^{2} / 8=2.038 \times 161^{2} / 10=5283 \mathrm{~N}$

$\mathrm{f}_{\mathrm{b}}=6 \times 5283 / 20^{2}=79 \mathrm{Mpa}<149$.

Area: $141 \times 20+160 \times 20=2820+3200=6020 \mathrm{~mm}^{2}$

Center of gravity: $\mathrm{a}=(2820 \times 10+3200 \times 80) / 6020=47.2 \mathrm{~mm}$

$\mathrm{I}=2820 \times 37.2^{2}+3200 \times 32.8^{2}+2820 \times 20^{2} / 12+3200 \times 160^{2} / 12=14.27 \mathrm{E} 6 \mathrm{~mm}^{4}$.

$\mathrm{S}_{\mathrm{b}}=14.27 \mathrm{E} 6 / 112.8=126.5 \mathrm{E} 3 \mathrm{~mm}^{3}$.

$\mathrm{S}_{\mathrm{l}}=14.27 \mathrm{E} 6 / 47.2=302.3 \mathrm{E} 3 \mathrm{~mm}^{3}$.

Moment in stiffener: $\mathrm{M}=2.038 \times 161 \times 1.1 \times 628^{2} / 8=17.79 \mathrm{E} 6 \mathrm{Nmm}$

$\mathrm{f}_{\mathrm{bb}}=17.79 \mathrm{E} 6 / 126.5 \mathrm{E} 3=141 \mathrm{Mpa}<149$.

$\mathrm{f}_{\mathrm{bt}}=17.79 \mathrm{E} 6 / 302.3 \mathrm{E} 3=59 \mathrm{Mpa}$. 


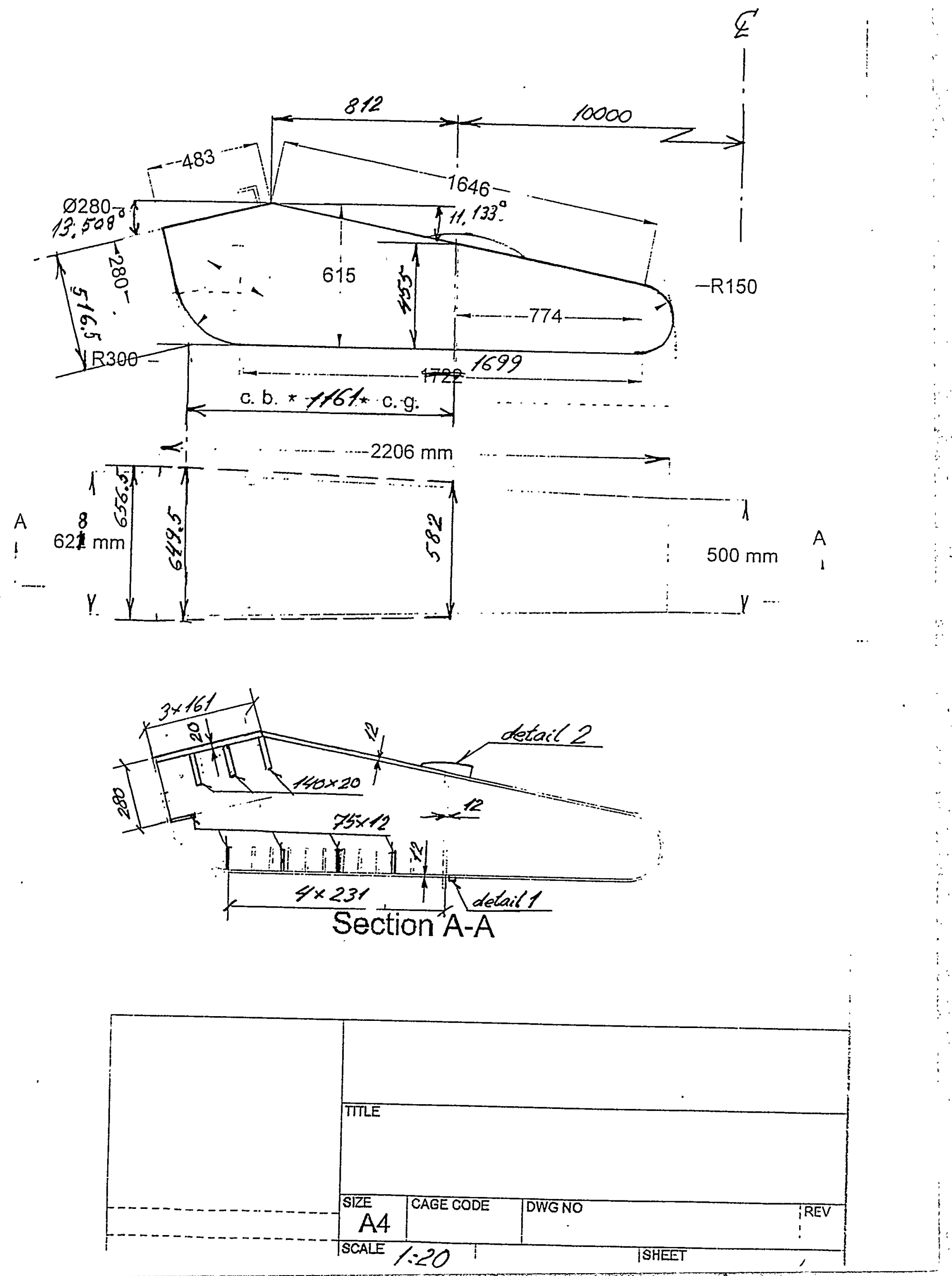




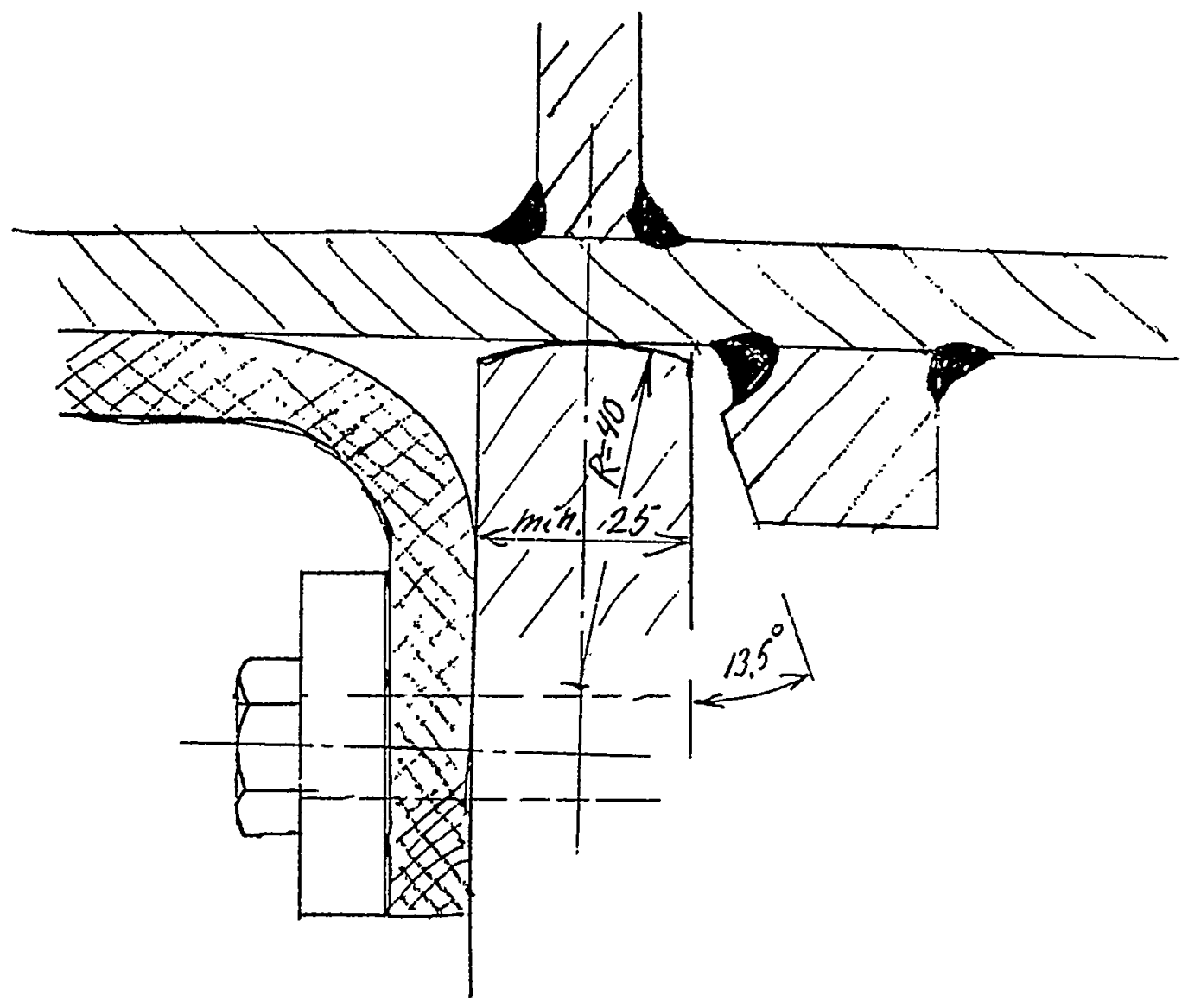

Detait 1,101 


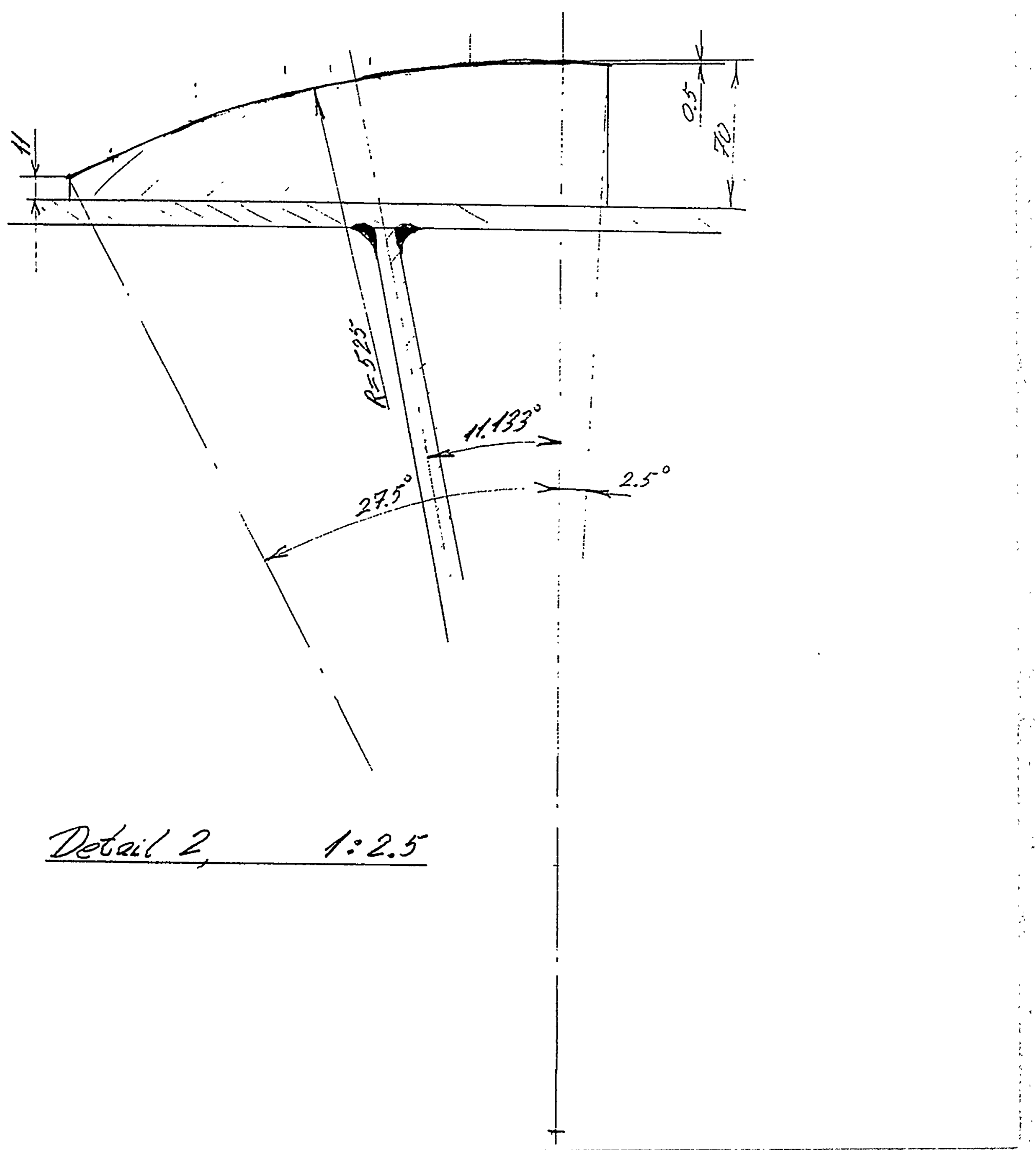




\section{\%o position \\ lond case}

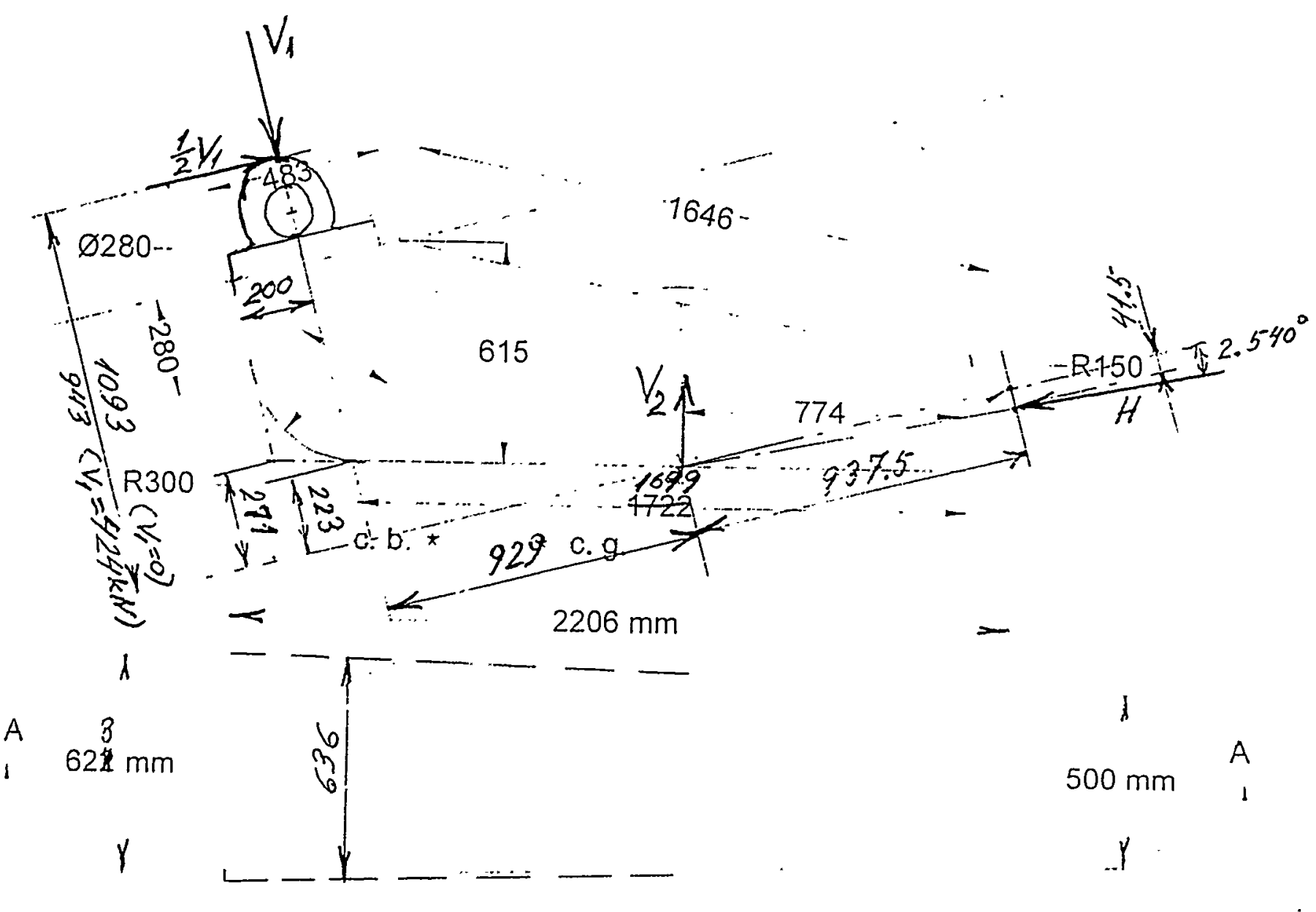

\section{Section A-A}

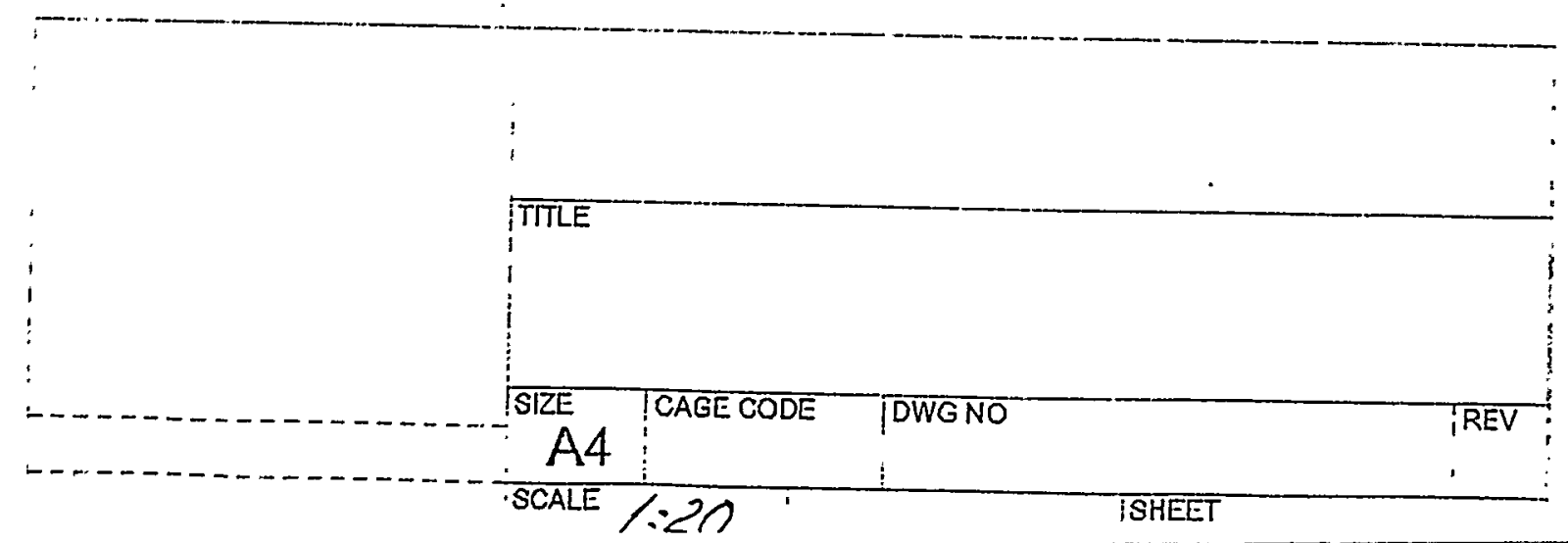




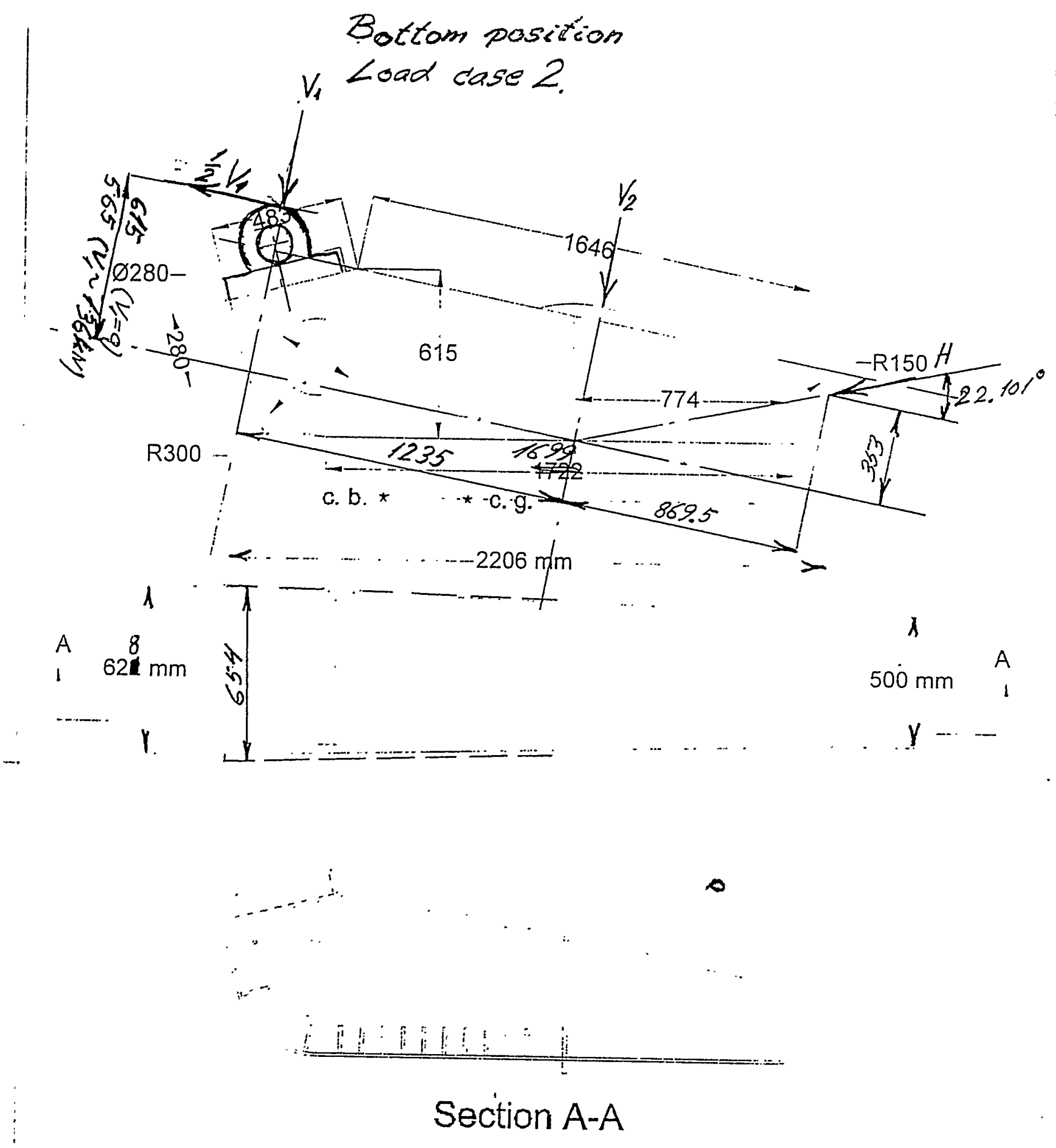


hoad case 3
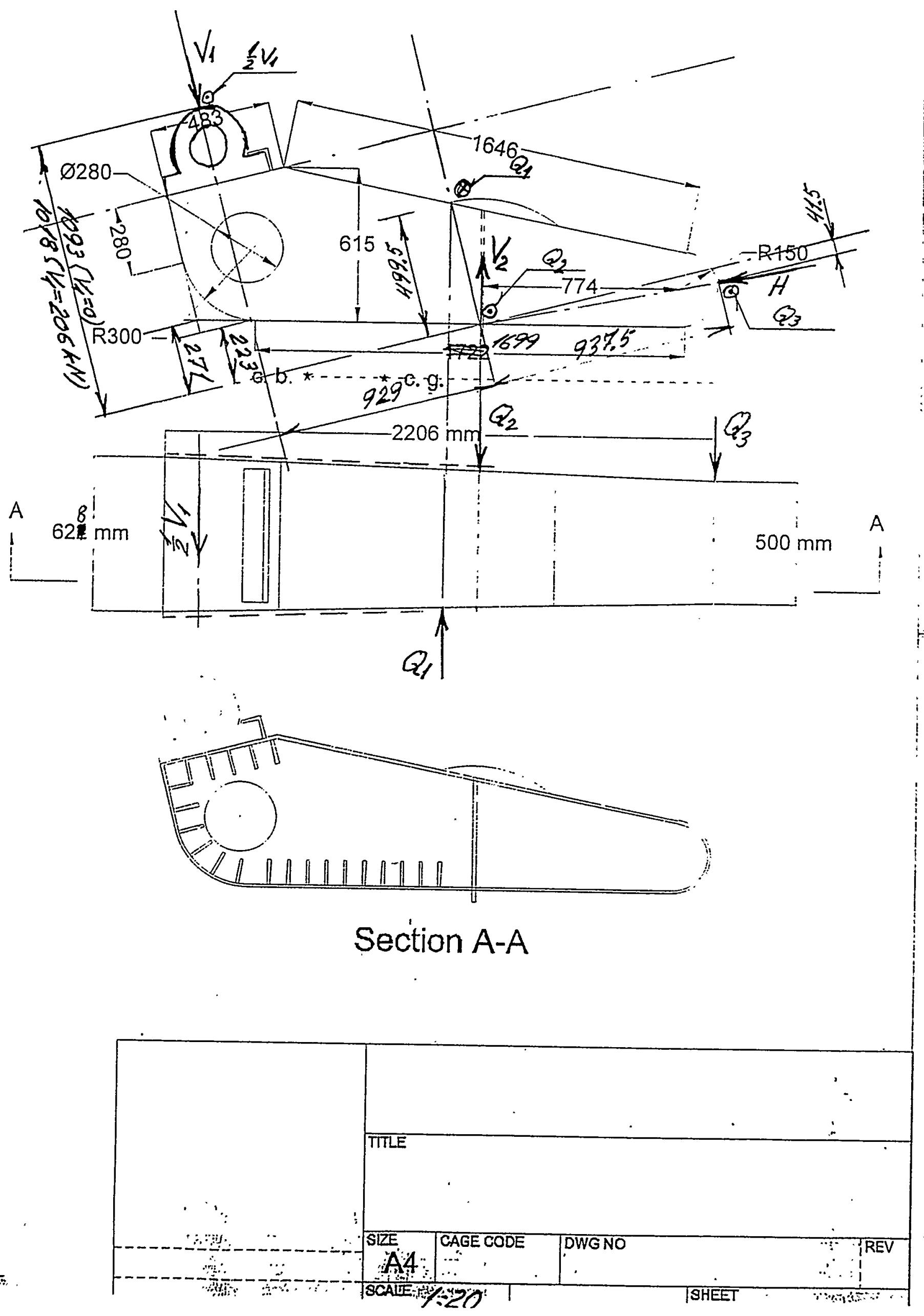
H(P)A $\frac{\text { HAN - PATRON ASSOCIATES }}{\text { CONSULTING ENGINEERS }}$

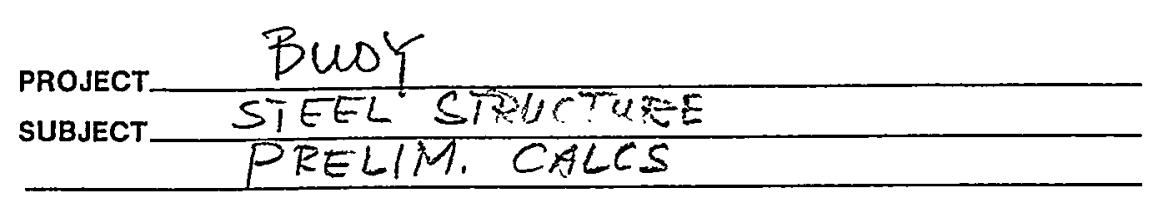

SHEET NO 1 OF 6 JOB NO. $869,0 \%$ JOB NO. $869,0 Z$
MADE BY LL DATE $0 \% / 07 / 00$
CHKD. BY DATE

DESIGN LOADS

1. SMIP RESISTING LOAOS

$$
\begin{aligned}
& \text { HORIZONTAL COMPONENT - 600.0T (METRIC) } \\
& \text { VERTCAL COMPONENT T - } 400.07 \text { (METRIC) }
\end{aligned}
$$

2. HYDRAULL PRESSURE ON $5 M \pm$ PLATE UNIT

Assumptions

1. ALC COADS FRON SHIP ARE TRANSFERING BY FRICTION ATS FUNCTION OF HYDRAULIC PRESSURE TO THE PLATE UNIT.

FROM THE PLATE UNIT LOADS ARE TRANSFERING TO THE XINCHOD UNIT BY SLIDING RING BELONGING TO PLATE UNIT TO SUPPORTS BELONGING TO ANCHOR UNIT. ANCHOR UNIT IS KEEPING IN PLAT BY. CHAINS.

2. ASSUMPTION FOR COMPUTER MOAEL OF THE ANCHOR UNIT

2:\% HORIZONTAL AND VERTICAL LOAAS FROM PLATE UNIT IS TOKEN RY TWO SUPPORTS, EVERY ONE TA KS $1 / 2$ OF TOTAL HORIZONTAL OR VERTICAL LOAD.

2.2. ALL MEMBETS WAS THKEN AS HOT ROLLED ELEMENTS WITH FY $=36 \mathrm{kSI}$ ( $5000 \mathrm{KG} / \mathrm{cm}^{2}$ ). ALL MEMBER ON DESIGN STAGE SHALL BE TAKEN AS WELDED ELEMENTS WITH CHARACTERISTICS (A, I, S) CLOSE TO TAKEN FOR RUNS "BOY" AND' $3 D$ MODEL SHALL BE BUILT.

2.3. MEMER 17 IS TAKEN CONDITIONALS TO INDICATE THAT TE SYSTEM (UNER SHIP) CPA NOT MOVE LIP. LINER APPLIED LOADS. 
(HA) AAN-PADRON ASSOCIATES
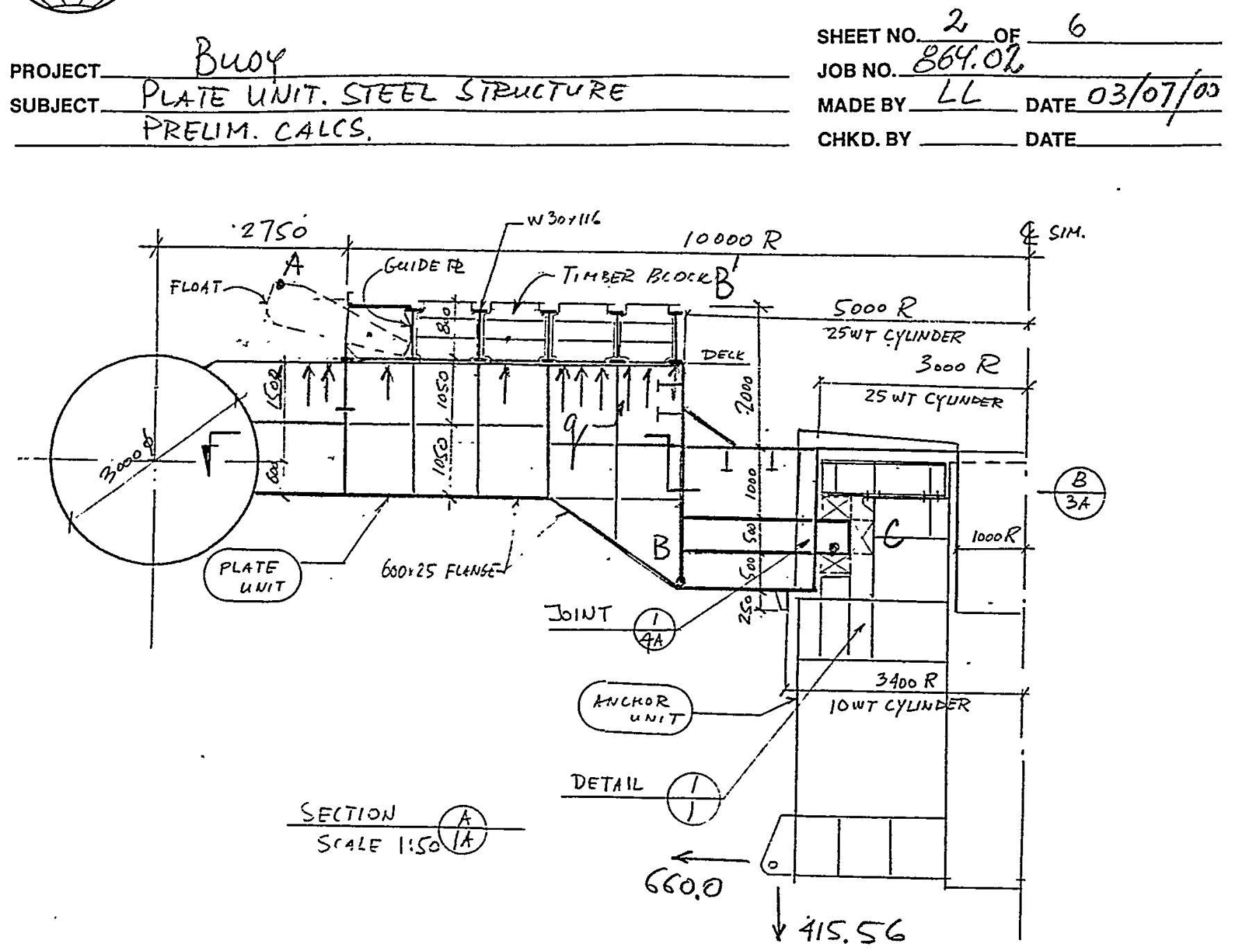

MAIN GIRDER

SUPPOPE REACTIONS"660.0 kPS \& $415.56 \mathrm{mps}$ SEE RUN "BUOYI" (*

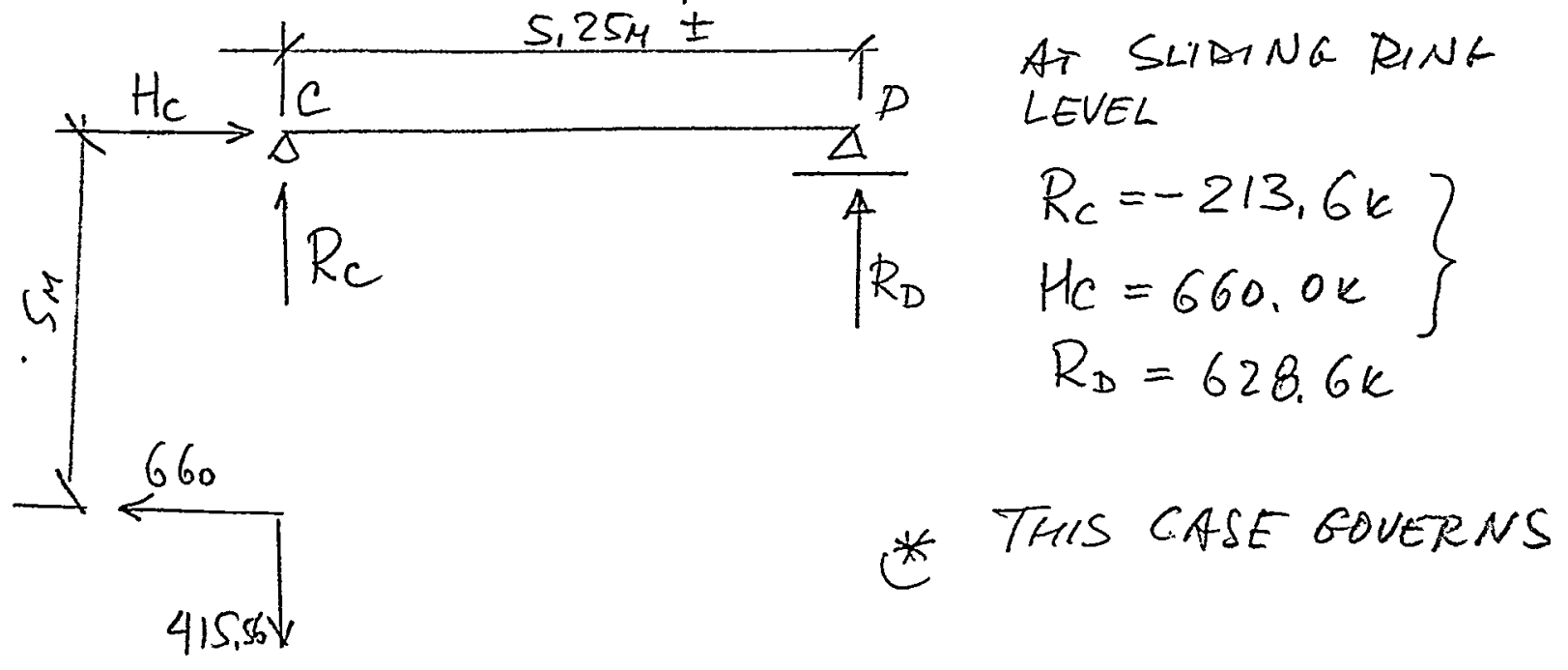


H(P)A HAN-PADRON ASSOCIATES

Buoy

PROJECT PLATE UNIT. STEEL STRUCTURE.

SUBJECT PRELIM, CALCS.

$$
\text { SHEET NO. } \frac{3}{869} \text { OF } 6
$$$$
\text { MADE BY LL DATE } 03 / 07 / 00
$$

CHKD. BY DATE

MOMENT AT SECTION $B B^{\prime}$

$$
M_{L}=660 \times 0,35 \times 3.28+213 \times 2.25 \times 3.28=2330 \mathrm{kFT}
$$

FOR LEFT GIRDER AND FOR RIGHT GIRDER

$M_{R}=628,6 \times 2,25 \times 3,28=4640 \mathrm{kFT}$ - GOVERNS

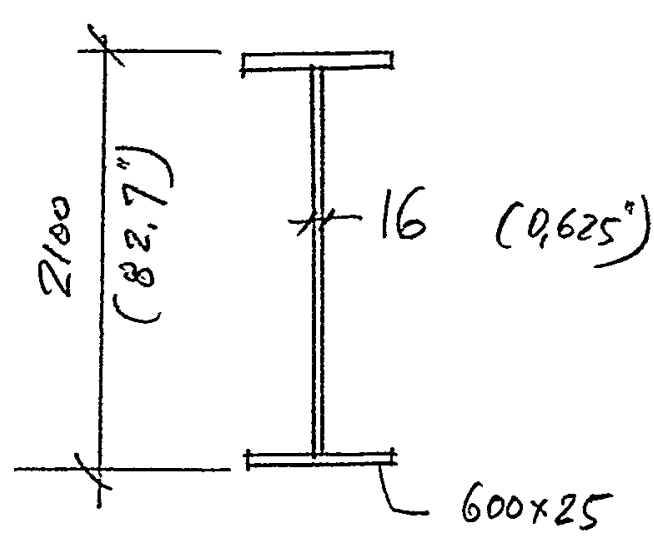

$$
\begin{aligned}
& I=\frac{0.625 \times 80,7^{3}}{12}+23,6 \times 41^{2} \times 2 \\
& I=106,716 \mathrm{~N}^{4} \\
& S=\frac{106716}{41,35}=2580.81 \mathrm{~N}^{3}
\end{aligned}
$$

$\left(23,6^{\prime \prime} \times 1^{\prime \prime}\right)$

BENIN STER

$$
f_{b}=\frac{4640 \times 12}{2580.8}=21.6 \mathrm{ks} 1<24.0
$$

$0, k$.

FROM HYDRAULiC PRESSURE.

$$
\begin{aligned}
& A V E R, q=35 \mathrm{~T} / \mathrm{M}^{2} \times \frac{5+2.5}{2}=131.25 \mathrm{~T} / \mathrm{M} \quad \text { OR } \\
& q=88.0 \mathrm{k} / \mathrm{FT} \\
& M=\frac{88 \times(6 \times 3.28)^{2}}{8}=4260.0 \mathrm{kFT}<4640 \mathrm{KFT} \\
& 0 . K \text {. } \\
& \text { SHEAR - } V=628.6 k-A=0.625 \times 80.7+2 \times 23.6=97.6 \mathrm{~m}^{2} \\
& f_{u}=\frac{628.6}{97.6}=6.4 \mathrm{ks} 1<14.4 \mathrm{kccow} \text {. }
\end{aligned}
$$


HAN-PADRON ASSOCIATES

CONSULTING ENGINEERS

$$
\begin{aligned}
& \text { PROJECT } \frac{\text { BUOY }}{\text { PLATE UNIT. STEEL STRUCTURE. }} \\
& \text { SUBSET }
\end{aligned}
$$

SHEET NO $\frac{4}{B C Y}$ OF 6 JOB NO. 864,02 MADE BY LL DATE $03 / 07 / 00$ CHKD. BY DATE

PECKING

ASSUME, THAT TIMBER BLOCKS WITHSTAND HYDRAULIC PRESSURE IN SPAN BETWEEN BEAMS, SO THICKNESS OF STEEL PLATE CAN BE TAKEN $W / O$ CALCULATIONS OF 14 TO $16 \mathrm{~mm}$.

PECK BEAM.

LONGEST REAM HAS LENGTH $\frac{\pi \times 19}{12}-0,3=4,67 \mathrm{M}$ SPACING - 1,OM DISTR. LORD PER BEAM IS 35,0T/M OR 23.5K/FT

$$
\begin{aligned}
& M=\frac{23.5 \times(4.67 \times 3.28)^{2}}{8}=689.2 \text { EFT } \\
& S_{\text {REQ }}=\frac{689.2 \times 12}{24}=344.6 \mathrm{~N}^{3}
\end{aligned}
$$

USE $W 30 \times 124-S=3551 N^{3}$ FOR CONGESTS TAN WB 116 FOR AU THE OTTERS

SLIDING RING

$\left.+x^{30^{\circ}}{ }^{4}\right) \mathrm{V}$

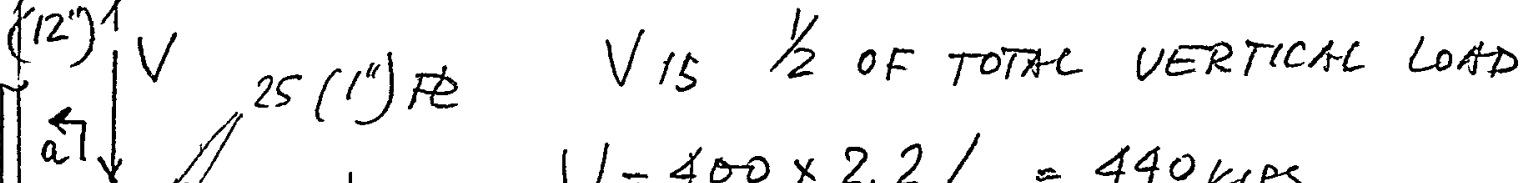

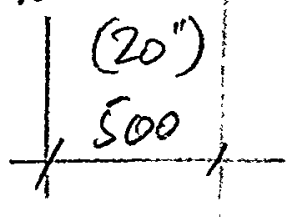

VERTICAL LOAD IS DISTR. TO TWO STIFF. RES, PER ONE te $V_{1}=440 / 2=220.0 \mathrm{kiPS}$ 
H(PAA HAN-PADRON ASSOCIATES

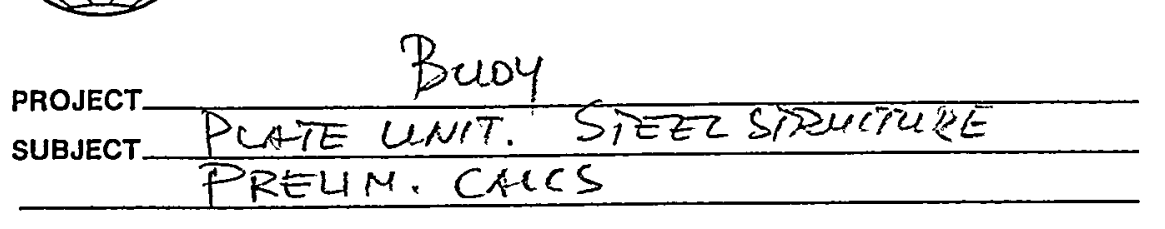

$$
\begin{aligned}
& \text { SHEET NO. } \frac{5}{86 \%} \text { OF } \frac{6}{6}, 0 \\
& \text { JOB NO. DATE } 03 / 07 / 09 \\
& \text { MADE BY } \angle L \text { DATE } \\
& \text { CHKD.BY }
\end{aligned}
$$

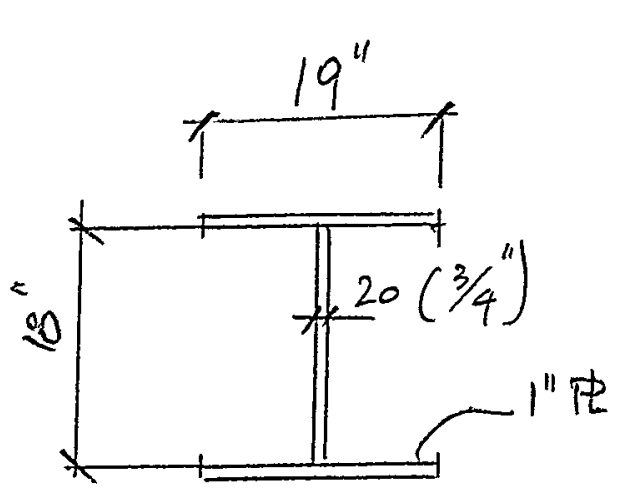

SECTION $a-a$

$$
\begin{aligned}
& I=\frac{0,75 \times 18^{3}}{12}+19 \times 9,5^{2} \times 2=3790 \mathrm{IN}^{4} \\
& S=379 \%=379 \mathrm{~N}^{3} \\
& M=220 \mathrm{kFT} \\
& f_{b}=\frac{220 \times 12}{379}=7.0 \mathrm{ks} \\
& 0 . \mathrm{K} .
\end{aligned}
$$

Vertical weLD FOR STIFF. IT

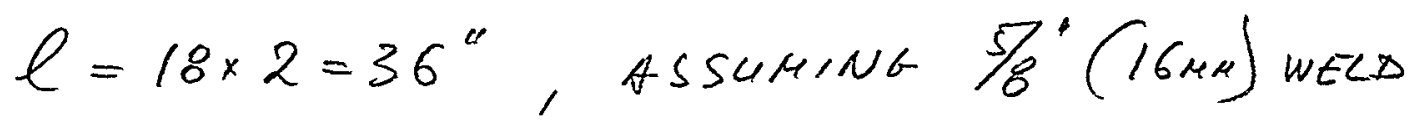

CAPACITY $0,927 \times 10=9.27 \mathrm{k} / 11 \mathrm{~s}$

weLd capaCITy $P_{w}=9,27 \times 36=334$ mas $>220$

$0, k$. 
HP) HAN-PADRON ASSOCIATES

$\frac{\text { BuOY }}{\text { SubJeCt PLATE UNIT. STEEL STRUTIURE. }}$

SHEET NO $\frac{6}{86}$ OF 6 JOB NO. $86 \% .0 \%$ JOB NO. $864.02, \angle L \quad$ DATE $03 / 07 /$ DO

TIMBER blOCKS UNDER HYDRRUCIC PRESSURE

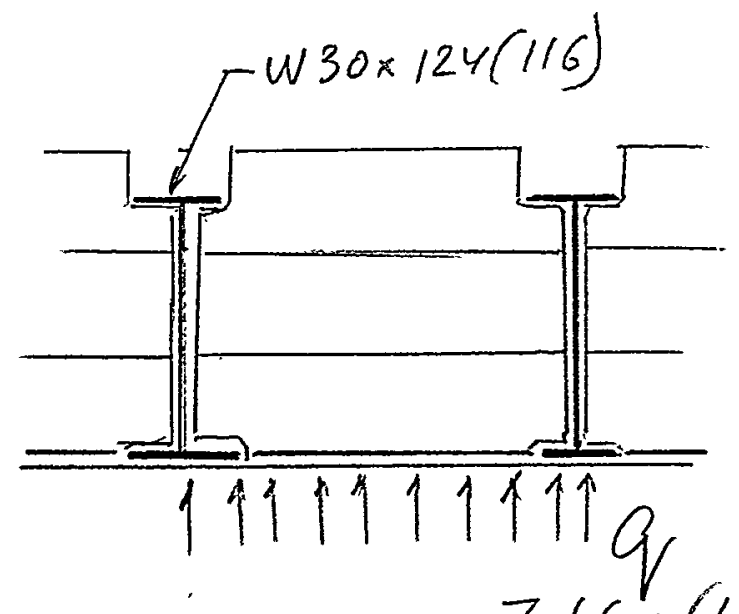

$q=35 T / H^{2}$ OR

$7.16 \mathrm{ksF}$

ASSUME 3 TMBER BLocks SHALL WITHSTAND UPLIFT PRESSCCRE

SAY $12^{\prime \prime} \times 12^{\prime \prime}$ TAKERS

TOTAL

$$
\begin{aligned}
& M=\frac{7.16 \times(1 \times 3,28)^{2}}{8}=9.62 \mathrm{kFT} / \mathrm{FT} \\
& V=7.16 \times \frac{3.28}{2}=11.7 \mathrm{kPS}-\text { SHEAR }
\end{aligned}
$$

MOMENT PER ONE BLOCK - 3.21KFT

$$
\begin{aligned}
& S \cong 0,9 \times \frac{12^{3}}{6} \cong 259.21 \mathrm{~N}^{3} \\
& f_{6}=\frac{3.21 \times 12}{259.2}=0.15 \mathrm{ks} \quad \text { o. K. } \\
& S_{H E A R} f_{=}=\frac{3}{2} \times \frac{11700}{28 \times 12}=52_{p S 1}<100 \text { ps. } \quad 0 . \mathrm{K} .
\end{aligned}
$$


$S T A A D-111$

Revision 22.3

Proprietary Program of

Research Engineers, Inc.

Date $=$ MAR 6,2000

Time $=14: 1: 5$

USER ID: HAN-PADRON ASSOCIATES

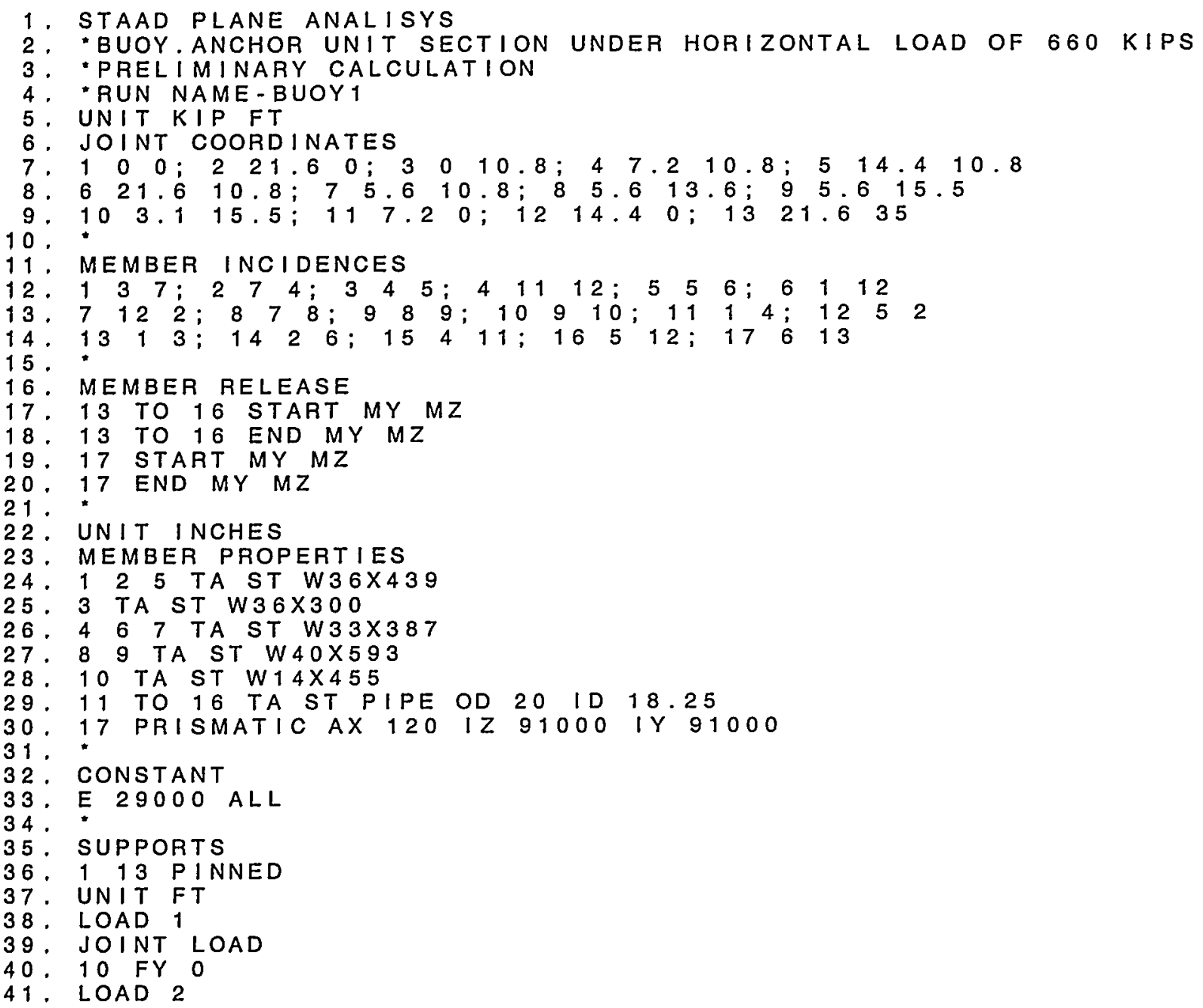




\section{ANAL I SYS}

- BUOY. ANCHOR UNIT SECTION UNDER HORIZONTAL L

42. 8 FX 660

43 .

44. LOAD COMBINATION 3

$45,11.021 .0$

46. PERFORM ANALYSIS

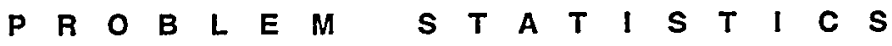

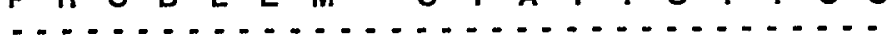

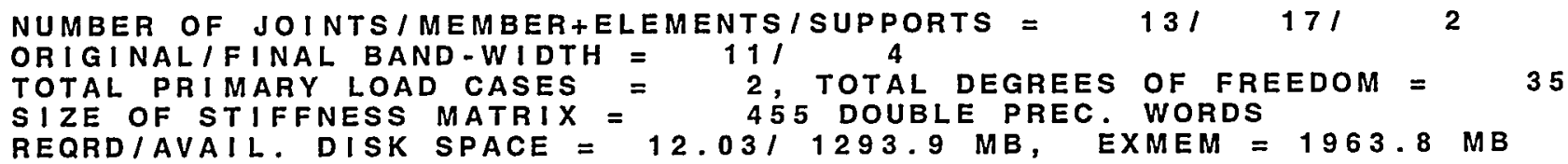

++ Processing Element Stiffness Matrix.

++ Processing Global stiffness Matrix.

++ Processing Triangular Factorization.

+ Calculating Joint Displacements.

++ Calculating Member Forces.
$13: 12: 39$

$13: 12: 39$

$13: 12: 39$

$13: 12: 39$

$13: 12: 40$

47. PARAMETER

48. RATIO 1.0 ALL

49. TRACK 1.0 ALL

50. CHECK CODE ALL 
- BUOY. ANCHOR UNIT SECTION UNDER HORIZONTAL L

\section{STAAD-III CODE CHECKING - (AISC)}

ALL UNITS ARE - KIP FEET (UNLESS OTHERWISE NOTED)

\begin{tabular}{|c|c|c|c|c|c|c|}
\hline MEMBER & TABLE & $\begin{array}{l}\text { RESULT I } \\
\text { FX }\end{array}$ & $\begin{array}{r}\text { CRIT ICAL } \\
M Y\end{array}$ & CONDI & $\begin{array}{c}\text { RAT } 10 I \\
M Z\end{array}$ & $\begin{array}{l}\text { LOAD ING I } \\
\text { LOCATION }\end{array}$ \\
\hline
\end{tabular}

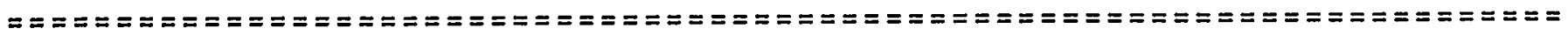
1 ST W $36 \times 439$
PASS
AISC- $\mathrm{H} 1 \cdot 3$
067
2 .00 .00
213.67
5.60

$M E M=$

1, UNIT KIP-INCH,

$L=67.2$

$67.2 \quad A X=128.00$

$S Z=1620.5 \quad S Y=234.6$

I $K L / R-Y=17.0 \quad C B=1.00 \quad Y L D=36.00 \quad A L L O W A B L E$ STRESSES:

I FTZ $=23.76$ FCY $=27.00 \quad F T Y=27.00 \quad F A=20.77 \quad F T=21.60$

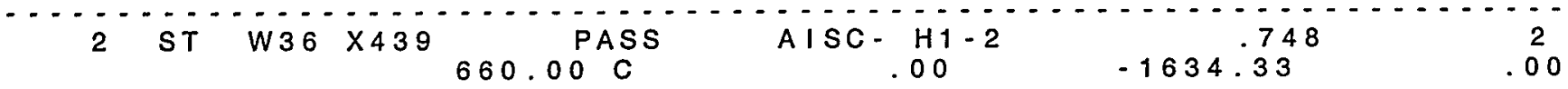
I MEM $=2, U N|T K / P-| N C H, L=19.2 \quad A X=128.00 \quad S Z=1620.5 \quad S Y=234.6$
$K L / R-Y=4.9 \quad C B=1.00 \quad Y L D=36.00$ ALLOWABLE STRESSES: FCZ= 23.76
I FTZ $=23.76$ FCY $=27.00 \quad F T Y=27.00 \quad F A=21.39 \quad F T=21.60 \quad F V=14.40$
3 ST W36
S 300 $377.08 \mathrm{C}$
$00 \quad-1737.01$
.00

MEM $=3$, UNITKIP-INCH, $L=86.4 \quad A X=88.30 \quad S Z=1105.1 \quad S Y=156.1$

I $K L / R-Y=22.5 \quad C B=1.00 \quad Y L D=36.00 \quad A L L O W A B L E$ STRESSES: FCZ= 23.76

I FTZ=23.76 FCY $=27.00$ FTY $=27.00 \quad F A=20.44 \quad F T=21.60 \quad F V=14.40$
4 ST W33 $\times 387$
PASS
AISC- $\mathrm{H} 1-3$
.242
2 .00

.00

647.57

7.20

I $M E M=$

86. $4 \quad A X=113.00$

$S Z=13519 \quad S Y=2000$

$K L / R-Y=22^{\prime} .8 \quad C B=1.00$ ' $Y L D=36.00$ ALLOWABLE STRESSES: FCZ= 23.76

$F T Z=23.76 \quad F C Y=27.00 \quad F T Y=27.00 \quad F A=20.42 \quad F T=21.60 \quad F V=14.40$

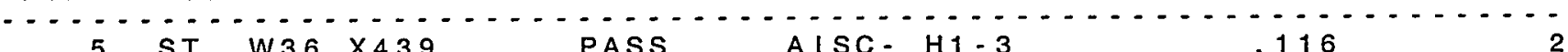

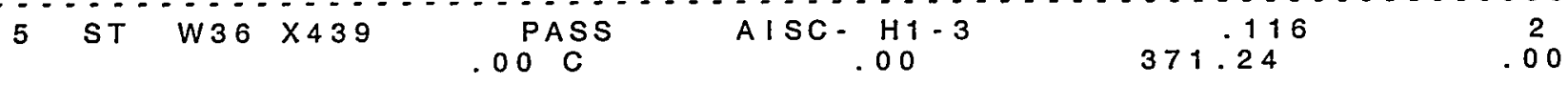

MEM $=$ 5, UNITKIP-INCH, L= 86.4 AX=128.00 SZ=1620.5 SY= 234.6

$K L / A-Y=21.9 \quad C B=1.00$ YLD=36.00 ALLOWABLE STRESSES: FCZ= 23.76

I FTZ $=23.76$ FCY $=27.00 \quad F T Y=27.00 \quad F A=20.48 \quad F T=21.60 \quad F V=14.40$

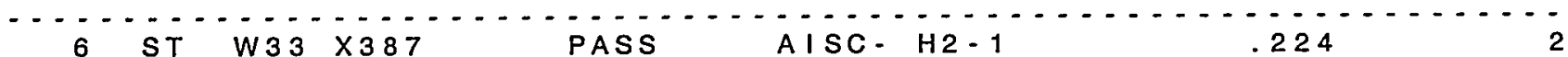

$377.08 T$

.00

$-186 \cdot 11$

.00

MEM $\quad 6, \quad U N I T K I P-I N C H, \quad L=172.8 \quad A X=113.00 \quad S Z=1351.9 \quad S Y=200.0$

$K L / R-Y=45.6 \quad C B=1.00 \quad Y L D=36.00 \quad A L L O W A B L E$ STRESSES: FCZ= 23.76

$F T Z=23.76$ FCY $=27.00$ FTY $=27.00$ FA= 18.72 FT $=21.60 \quad F V=14.40$ 
"BUOY. ANCHOR UNIT SECTION UNDER HORIZONTAL L

ALL UNITS ARE - KIP FEET (UNLESS OTHERWISE NOTED)

MEMBER

TABLE

RESULT I

CRITICAL C CONDI

RATIOI

$M Z$

LOAD ING /

FX MY MZ LOCATION

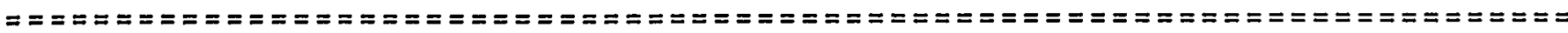

7 ST $\quad$ W $33 \times 387$

PASS AISC- $\mathrm{H2}-1$

.336

2

$377.08 \mathrm{~T}$

.00

484.69

00

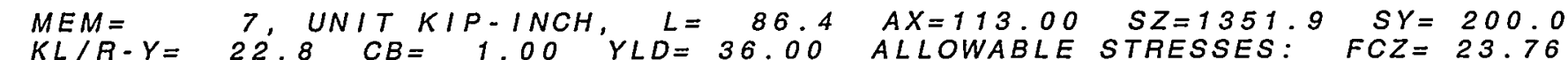

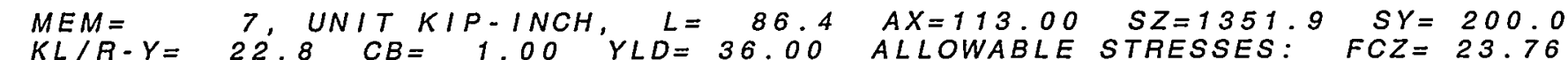

AX

-...

22.8 $C B=1.00 \quad Y L D=36.00 \quad A L L O W A B L E$ STRESSES: FCZ= 23.7
$A=20.42$
$F T=21.60$
$F V=14.40$

$F T Z=23$.

FTY $=27.0$

8 ST 40 X 593

PASS

$.00 \mathrm{~T}$

SHEAR

$\begin{array}{rrrrr}.00 \mathrm{~T} & .00 & 1848.00 & .00\end{array}$

I $M E M=$

8, UNITKIP-INCH, L= $\quad$, 3.6 00

$K L / R-Y=$

$F T Z=23.76$ FCY $=27.0$

$.00 Y L D=36$

$A X=174.00$

$S Z=2344.7$

$S Y=302.0$

$F T Y=27.00 \quad F A=21.21 \quad F T=21.60 \quad F V=14.40$

9 ST W40 $\times 593$

PASS

A I SC - H1 - 3

000

1

$.00 \mathrm{C}$

00

.00

.00

I $M E M=$

9, UNITKIP-INCH, $L=22.8 \quad A X=174.00$

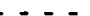

$K L / R-Y=6.0 \quad C B=1.00$ Y $Y L D=36.00 \quad$ ALLOWABLE STRESSES.7 SY= 302.0

I FTZ $=23.76 \quad F C Y=27.00 \quad F T Y=27.00 \quad F A=21.34 \quad F T=21.60 \quad F V=14.40$

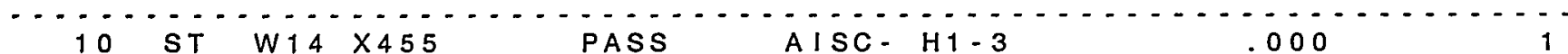

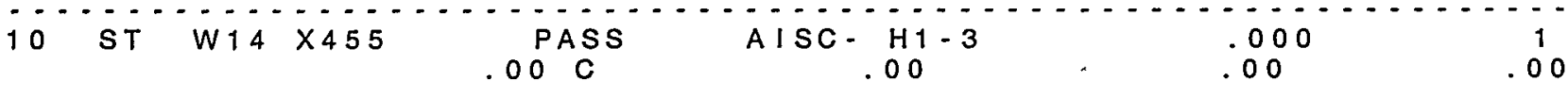

$L=30.0 \quad A X=134.00 \quad S Z=756.0 \quad S Y=304.1$

$K L / A-Y=6{ }^{\prime} .9 C B=1.00$ ' $Y L D=36.00$ ALLOWABLE STRESSES: FCZ= $23.76 \quad$

I FTZ $=23.76 \quad F C Y=27.00 \quad F T Y=27.00 \quad F A=21.30 \quad F T=21.60 \quad F V=14.40$

11 ST PIP E PASS AISC

186.11

00

469.61

00

MEM $=$ 11, UNITKIP-INCH, $L=155.8$ AX $=52.57$ SZ 240.9 SY 240.9

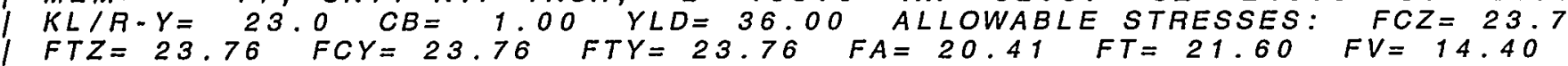

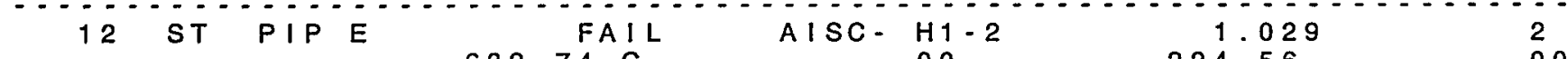

$633.74 \mathrm{C}$

.00

224.56

.00

$M E M=$

12 UNIT KIP-INCH

AX $\ldots . \cdots$

.....

$Y=240.9$

$K L / R \cdot Y=23.0 \quad C B=1.00$ ' $Y L D=36.00$ ALLOWABLE STRESSES: FCZ= 23.76

$F T Z=23.76 \quad F C Y=23.76 \quad F T Y=23.76 \quad F A=20.41 \quad F T=21.60 \quad F V=14.40$

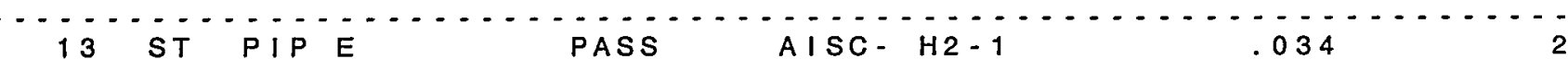

$38.16 \mathrm{~T}$

AISC- H2-1

.00

.00 
- BUOY. ANCHOR UNIT SECTION UNDER HORIZONTAL L

\section{ALL UNITS ARE - KIP FEET (UNLESS OTHERWISE NOTED)}

$\begin{array}{cccccc}\text { MEMBER TABLE } & \text { RESULTI } & \text { CRITICAL CONDI } & \text { RATIOI MY MAINGI } \\ & & \text { FX } & \text { MOCATION }\end{array}$

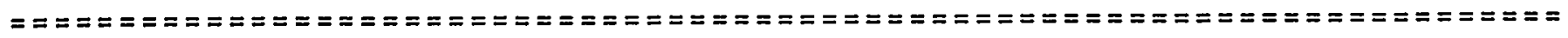

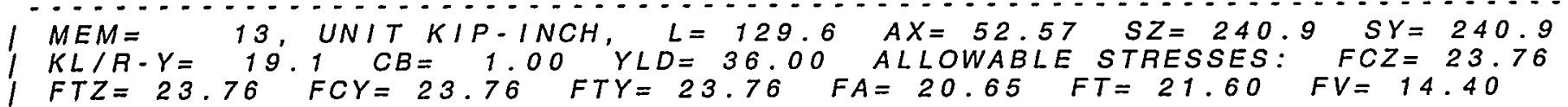

14
$\mathrm{ST}$ PIP E

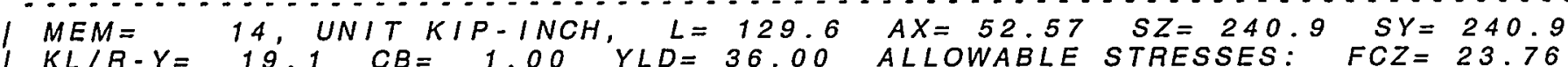

I FTZ $=23.76$ FCY $=23.76$ FTY $=23.76$ FA= $20.65 \quad F T=21.60 \quad F V=14.40$

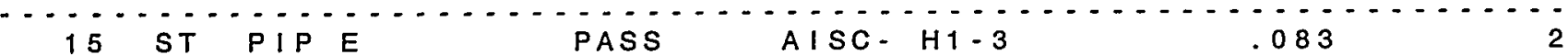

$89.94 \mathrm{C} \quad .00 \quad .00$

.00

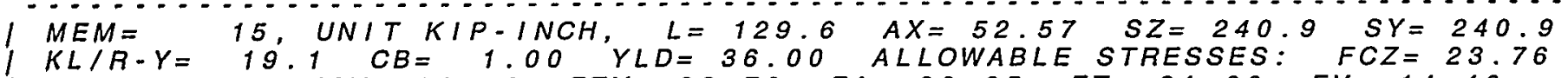

$F T Z=23.76$ FCY $=23.76$ FTY 23.76 FA= 20.65 FT= $21.60 \quad F V=14.40$

16 ST PIP E PASS AISC- H2 $134.71 \mathrm{~T}$

. 00

.00

.00

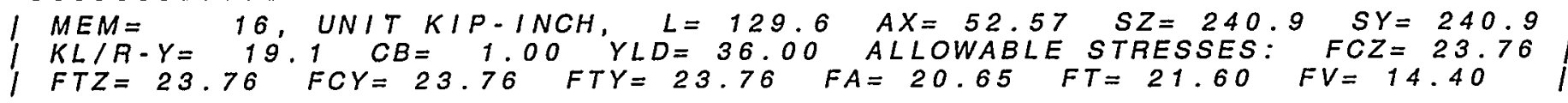

51. PRINT ANALYSIS RESULTS 
- BUOY. ANCHOR UNIT SECTION UNDER HORIZONTAL L

JOINT DISPLACEMENT (INCH RADIANS) STRUCTURE TYPE = PLANE

$\cdots \cdots \cdots \cdot \ldots \cdot \cdots \cdot \cdots$

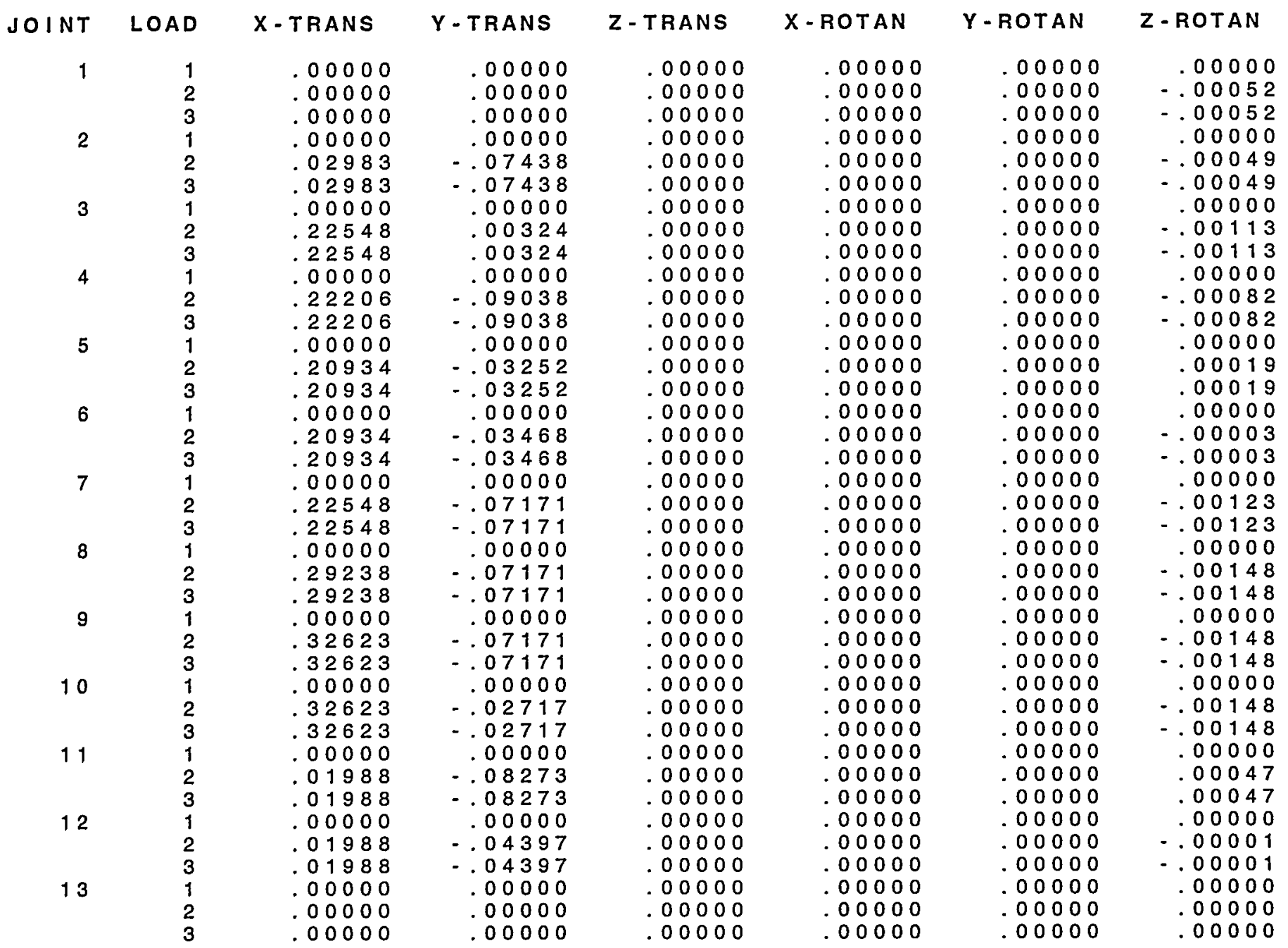


ANALISYS

- BUOY. ANCHOR UNIT SECTION UNDER HORIZONTAL L

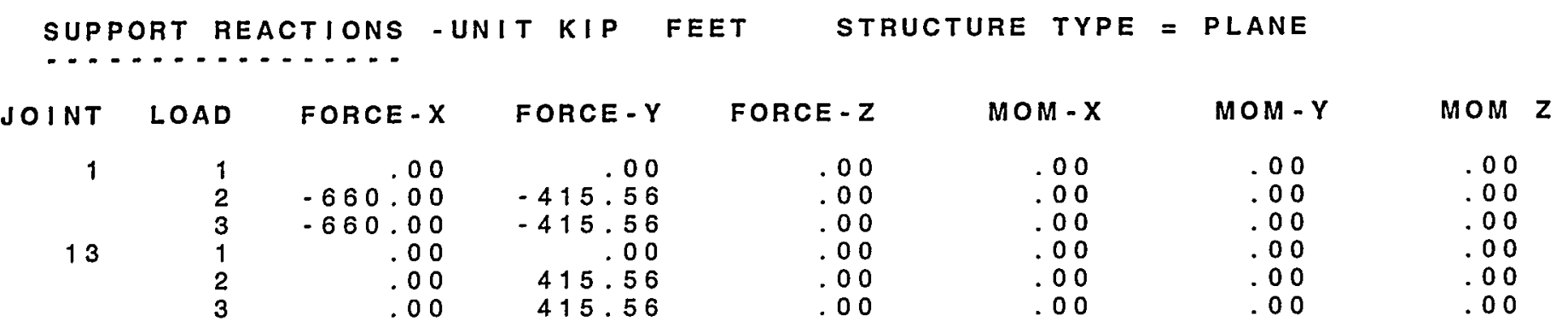


- BUOY. ANCHOR UNIT SECTION UNDER HORIZONTAL L

\section{MEMBER END FORCES STRUCTURE TYPE = PLANE}

ALL UNITS ARE - KIP FEET

MEMBER LOAD JT AXIAL

$\begin{array}{lll}1 & 1 & 3 \\ & 2 & 7 \\ & 3 \\ & 3 & 3 \\ & 3\end{array}$

$$
\begin{array}{lll}
.0 & 0 \\
.0 & 0 \\
.0 & 0 \\
0 & 0 \\
.0 & 0 \\
.0 & 0
\end{array}
$$

2

$\begin{array}{ll}1 & 7 \\ & 4 \\ 2 & 7 \\ & 4 \\ & 7 \\ & 4\end{array}$

31
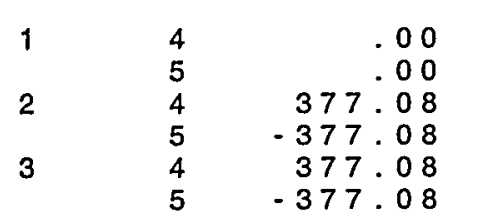

4

$\begin{array}{ll}4 \quad 1 & 11 \\ & 12 \\ & 11 \\ & 12 \\ 3 & 11 \\ & 12\end{array}$

51

1
2

3

6

6

$\begin{array}{lr}1 & 1 \\ & 12 \\ 3 & 1 \\ & 12 \\ & 12\end{array}$

71

12
$-\quad 2$
.00

660.00

.660 .00

660.00

$-660.00$

$$
\begin{array}{r}
.00 \\
.00 \\
-38.16 \\
38.16 \\
-38.16 \\
38.16
\end{array}
$$

.00

.00

.00

.00

.00

.00

$-38.16$

38.16

$-38.16$

38.16

$$
\begin{array}{r}
.00 \\
.00 \\
-324.00 \\
324.00 \\
-324.00 \\
324.00 \\
\\
.00 \\
.00 \\
-89.94 \\
89.94 \\
-89.94 \\
89.94
\end{array}
$$

.00$$
.00
$$$$
.00
$$$$
.00
$$$$
.00
$$

$$
.00
$$

.00

.00

.00

.00

.00

.00
.00
51.56
-51.56
51.56
-51.56

$$
\begin{array}{r}
.00 \\
.00 \\
-377.08 \\
377.08 \\
-377.08 \\
377.08
\end{array}
$$

.00

$$
\begin{array}{r}
.00 \\
.00 \\
-1.61 \\
1.61 \\
-1.61 \\
1.61 \\
.00 \\
.00
\end{array}
$$$$
.00
$$

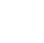


* BUOY. ANCHOR UNIT SECTION UNDER HORIZONTAL L

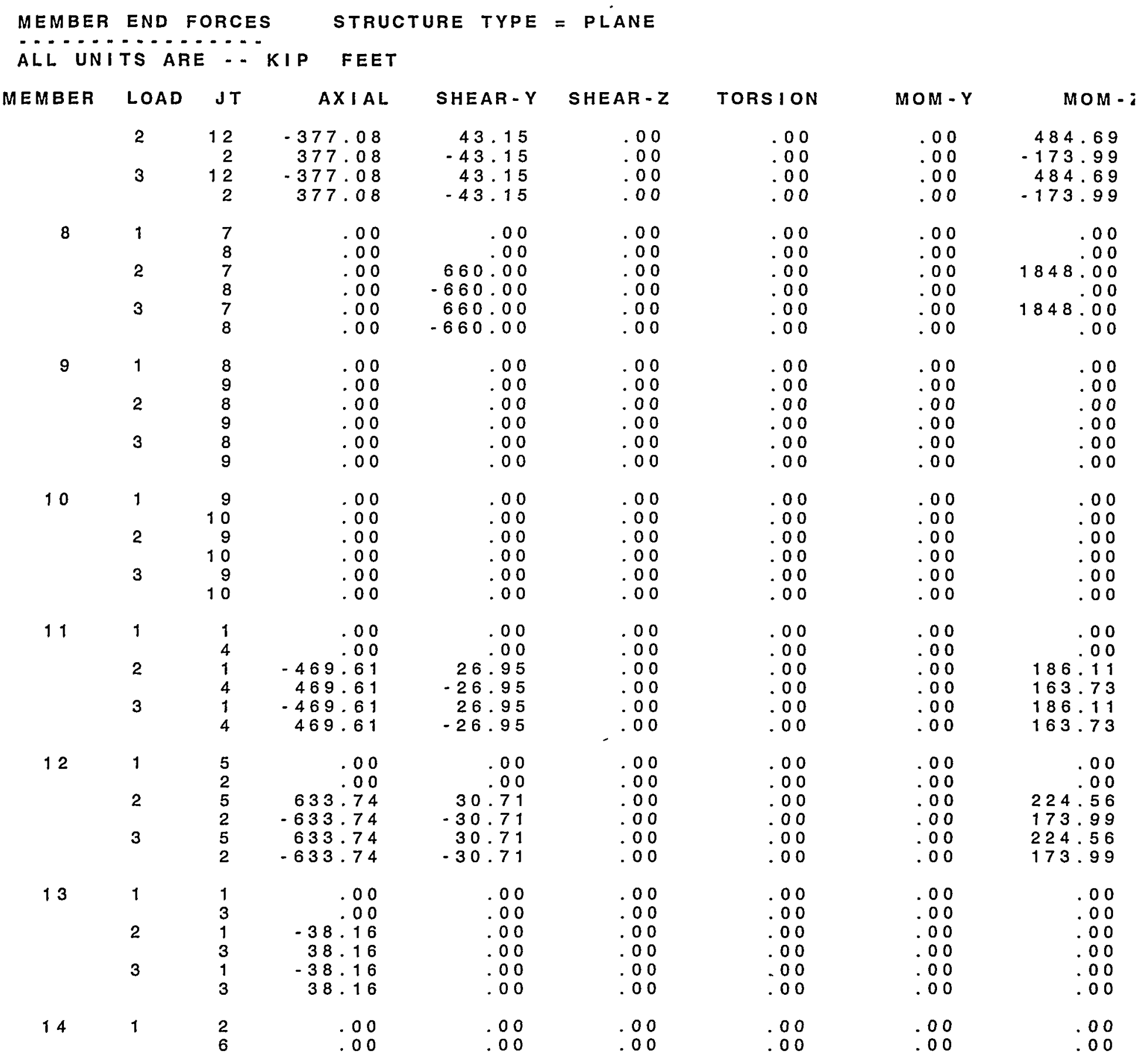


- BUOY. ANCHOR UNIT SECTION UNDER HORIZONTAL L

\begin{tabular}{|c|c|c|c|c|c|c|c|c|}
\hline \multirow[t]{2}{*}{ MEMBER } & LOAD & J T & $A X \mid A L$ & SHEAR - Y & SHEAR - Z & TORSION & $M O M-Y$ & MOM - \\
\hline & $\begin{array}{l}2 \\
3\end{array}$ & $\begin{array}{l}2 \\
6 \\
2 \\
6\end{array}$ & $\begin{array}{r}-467 \cdot 12 \\
467 \cdot 12 \\
-467.12 \\
467.12\end{array}$ & $\begin{array}{ll}.0 & 0 \\
.0 & 0 \\
.0 & 0 \\
.0 & 0\end{array}$ & $\begin{array}{ll}.0 & 0 \\
.0 & 0 \\
.0 & 0 \\
.0 & 0\end{array}$ & $\begin{array}{ll}.0 & 0 \\
.0 & 0 \\
.0 & 0 \\
.0 & 0\end{array}$ & $\begin{array}{ll}.0 & 0 \\
.0 & 0 \\
.0 & 0 \\
.0 & 0\end{array}$ & $\begin{array}{l}.00 \\
.00 \\
.00 \\
.00 \\
.00\end{array}$ \\
\hline 15 & $\begin{array}{l}1 \\
2 \\
3\end{array}$ & $\begin{array}{r}4 \\
11 \\
4 \\
11 \\
4 \\
11\end{array}$ & $\begin{array}{r}.00 \\
.00 \\
89.94 \\
-89.94 \\
89.94 \\
-89.94\end{array}$ & $\begin{array}{lll}.0 & 0 & 0 \\
.0 & 0 \\
.0 & 0 \\
.0 & 0 \\
.0 & 0 \\
.0 & 0\end{array}$ & $\begin{array}{ll}.0 & 0 \\
.0 & 0 \\
.0 & 0 \\
.0 & 0 \\
.0 & 0 \\
.0 & 0\end{array}$ & $\begin{array}{lll}.0 & 0 \\
.0 & 0 \\
.0 & 0 \\
.0 & 0 \\
.0 & 0 \\
.0 & 0\end{array}$ & $\begin{array}{lll}.0 & 0 \\
.0 & 0 & 0 \\
.0 & 0 \\
.0 & 0 \\
.0 & 0 \\
.0 & 0\end{array}$ & $\begin{array}{ll}.0 & 0 \\
.0 & 0 \\
.0 & 0 \\
.0 & 0 \\
.0 & 0 \\
.0 & 0\end{array}$ \\
\hline 16 & $\begin{array}{l}1 \\
2 \\
3\end{array}$ & $\begin{array}{r}5 \\
12 \\
5 \\
12 \\
5 \\
12\end{array}$ & $\begin{array}{r}.00 \\
.000 \\
-134.71 \\
134.71 \\
-134.71 \\
134.71\end{array}$ & $\begin{array}{lll}.0 & 0 \\
.0 & 0 \\
.0 & 0 \\
.0 & 0 \\
.0 & 0 \\
.0 & 0\end{array}$ & $\begin{array}{lll}. & 0 & 0 \\
.0 & 0 \\
.0 & 0 \\
.0 & 0 \\
.0 & 0 \\
.0 & 0\end{array}$ & $\begin{array}{lll}.0 & 0 & 0 \\
.0 & 0 \\
.0 & 0 \\
.0 & 0 \\
.0 & 0 \\
.0 & 0\end{array}$ & $\begin{array}{lll}. & 0 & 0 \\
.0 & 0 & 0 \\
.0 & 0 & 0 \\
.0 & 0 \\
.0 & 0 \\
.0 & 0\end{array}$ & $\begin{array}{ll}.0 & 0 \\
.0 & 0 \\
.0 & 0 \\
.0 & 0 \\
.0 & 0 \\
.0 & 0\end{array}$ \\
\hline 17 & $\begin{array}{l}1 \\
2 \\
3\end{array}$ & $\begin{array}{r}6 \\
13 \\
6 \\
13 \\
6 \\
13\end{array}$ & $\begin{array}{r}.00 \\
.00 \\
-415.56 \\
415.56 \\
-415.56 \\
415.56\end{array}$ & 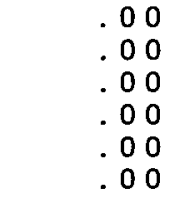 & $\begin{array}{lll}.0 & 0 \\
.0 & 0 \\
.0 & 0 \\
0 & 0 \\
.0 & 0 \\
0 & 0 \\
0 & 0\end{array}$ & $\begin{array}{lll}. & 0 & 0 \\
.0 & 0 \\
.0 & 0 \\
.0 & 0 \\
.0 & 0 \\
.0 & 0\end{array}$ & $\begin{array}{ll}.0 & 0 \\
.0 & 0 \\
.0 & 0 \\
.0 & 0 \\
.0 & 0 \\
0 & 0 \\
0 & 0\end{array}$ & $\begin{array}{ll}.0 & 0 \\
.0 & 0 \\
.0 & 0 \\
.0 & 0 \\
.0 & 0 \\
0 & 0 \\
0 & 0\end{array}$ \\
\hline
\end{tabular}

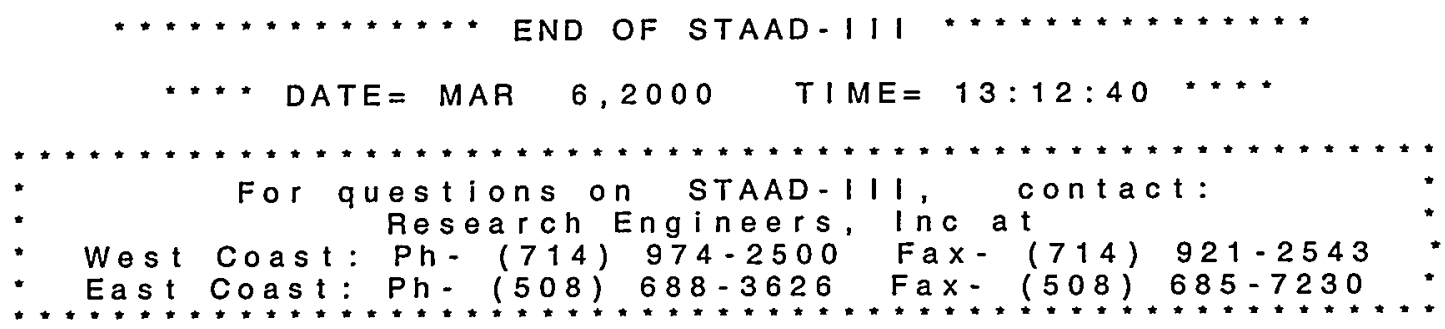




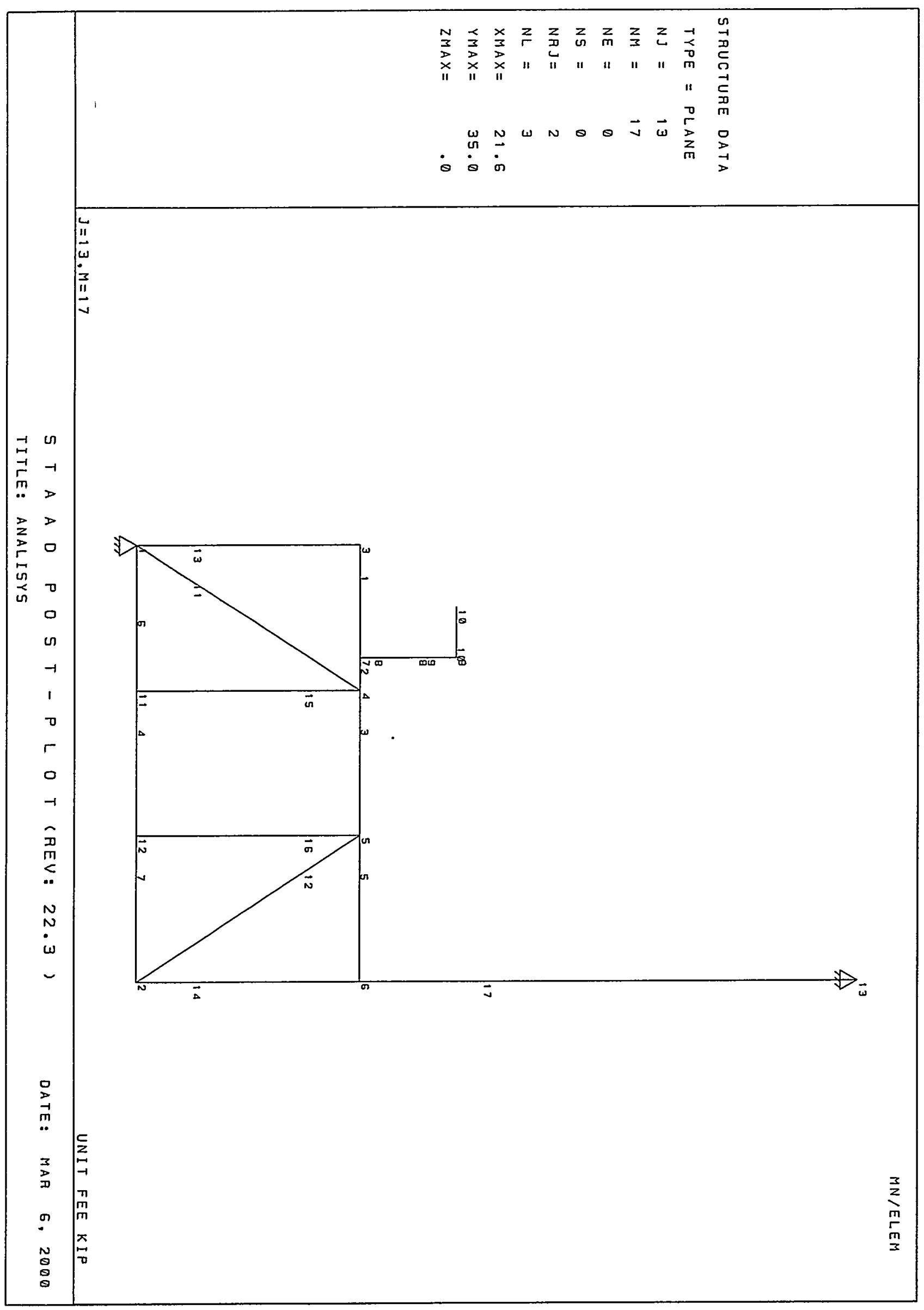


$S T A A D-111$

Revisjon 22.3

Proprietary Program of

Research Engineers, Inc.

Date $=$ MAR 6,2000

Time $=\quad 13: 58: 51$

USER ID: HAN-PADRON ASSOCIATES

1. STAAD PLANE ANALISYS

2. BUOY. ANCHOR UNIT SECTION UNDER VERTICAL LOAD OF $440 \mathrm{KIPS}$

3. PREL I MINARY CALCULATION

4. "RUN NAME-BUOY2

5. UNIT KIP FT

6. JOINT COORDINATES

7. $1000 ; 221.60 ; 30010.8 ; 447.210 .8 ; 5514.410 .8$

8. $621.610 .8 ; 75.610 .8 ; 8$ 5.6 $13.6 ; 955.615 .5$

9. $103.115 .5 ; 117.20 ; 1214.40 ; 1321.635$

10 .

11. MEMBER INCIDENCES

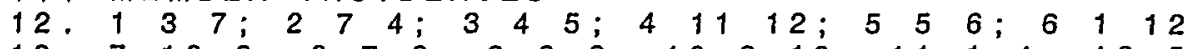

13: 712 2; 878 8; $989 ; \quad 109910 ; 11$ 1 $4 ; 1252$

$14.1313 ; 1426 ; 15411 ; 165 ; 12 ; 176613$

15 .

16. MEMBER RELEASE

17. 13 TO 16 START MY MZ

18. 13 TO 16 END $M Y M Z$

19. 17 START MY MZ

20. 17 END $M Y M Z$

$21 . *$

22: UNIT INCHES

23. MEMBER PROPERTIES

24. 125 TA ST W36 2439

25. 3 TA ST W36 3300

26. 4 . 67 TA ST W3 $3 \times 387$

$27: 89$ TA ST W40X593

28,10 TA ST W14X455

29. 11 TO 16 TA ST PIPE OD 20 ID 18.25

30. 17 PRISMATIC AX 120 IZ 91000 IY 91000

31 .

32. CONSTANT

33. E $29000 \mathrm{ALL}$

34 .

35 , SUPPORTS

$36: 113$ I I NNED

37. UNIT FT

38 . LOAD 1

39. JOINT LOAD

$40: 10 \mathrm{FY}=440$

41. LOAD 2 
- BUOY. ANCHOR UNIT SECTION UNDER HORIZONTAL L

42. $8 F \times \quad 0$

43.

44. LOAD COMBINATION 3

45. 11.021 .0

46. PERFORM ANALYSIS

$$
\text { P R O B }
$$

NUMBER OF JOINTS/MEMBER+ELEMENTS/SUPPORTS = $13 / 17 / 2$ ORIGINAL /FINAL BAND-WIDTH = TOTAL PRIMARY LOAD CASES = 111 2 , TOTAL DEGREES SIZE OF STIFFNESS MATRIX $=$
REQRD/AVAIL. DISK SPACE $=12.03 / 1292.7 \mathrm{MB}$,

OF FREEDOM = 35 EXMEM $=1963.6 \mathrm{MB}$

++ Processing Element Stiffness Matrix.

++ Processing Global stiffness Matrix.

++ Processing Triangular Factorization.

+ Calculating Joint Displacements.

+ Calculating Member Forces.

$13: 31: 7$

$13: 31: 7$

$13: 31: 7$

$13: 31: 7$

$13: 31: 7$

47. PARAMETER

48. RATIO 1.0 ALL

49. TRACK 1:0 ALL

50. CHECK CODE ALL 
-BUOY. ANCHOR UNIT SECTION UNDER HORIZONTAL L

\section{STAAD-III CODE CHECKING - (AISC)}

ALL UNITS ARE - KIP FEET (UNLESS OTHERWISE NOTED)

\begin{tabular}{|c|c|c|c|c|}
\hline MEMBER & TABLE & $\begin{array}{l}\text { RESULT I } \\
\text { FX }\end{array}$ & $\begin{array}{r}\text { CRIT I CAL } \\
M Y\end{array}$ & COND I \\
\hline
\end{tabular}

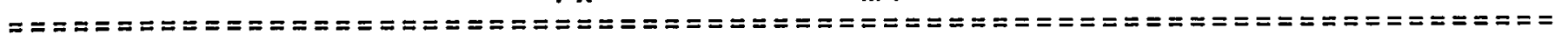
1 ST W $36 \times 439$
PASS
A ISC- H1-3
.423
00
$-1358.11$
5. 1 0

$\left\{\begin{array}{l}M E M=1, U N I T K I P-I N C H, \quad L=67.2 \quad A X=128.00 \\ K L / R-Y=17.0 \quad C B=1.00 \quad Y L D=36.00 \text { ALLOWABLE STRESSES: FCZ= } 23.76\end{array}\right.$

00

$-1358 \cdot 11$

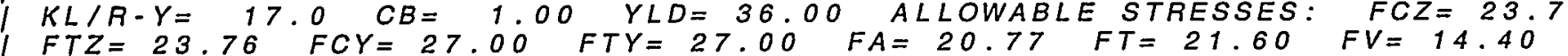

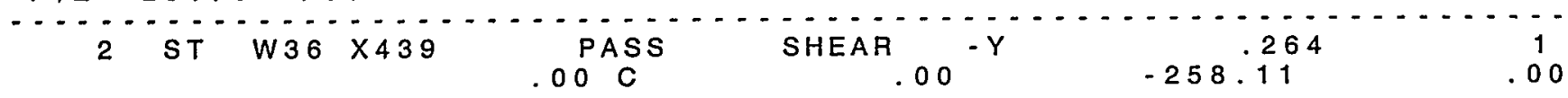

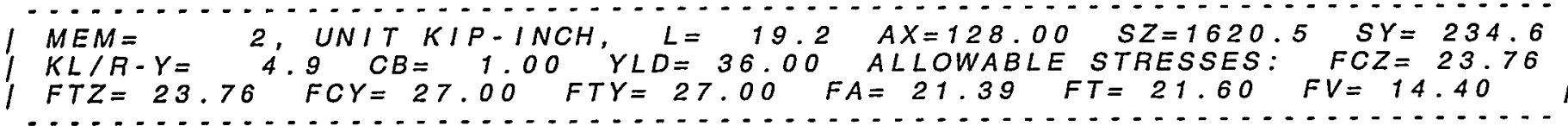
3 ST W36 $\times 300$
PASS
AISC- HI- 3
. 136
210.99
7.20
$71.50 \mathrm{C}$
00

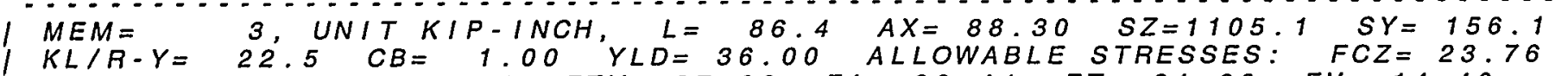

$S Z=1105.1$

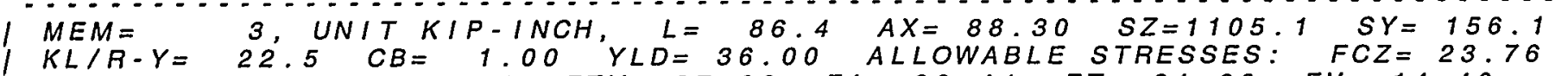

I FTZ=23.76 FCY $=27.00$ FTY $=27.00 \quad F A=20.44$ FT $=21.60 \quad F V=14.40$
ST W33 $\times 387$
PASS
AISC-H1-3
.092
1
.00
245.72
7.20

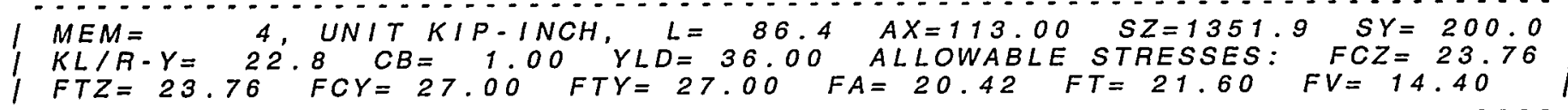

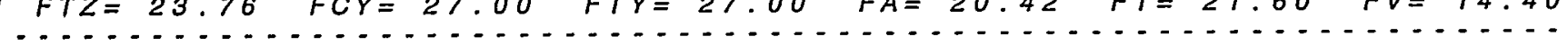

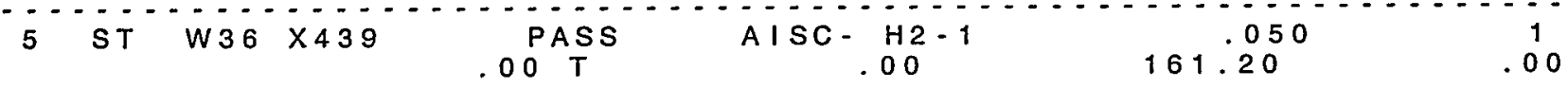

$\left\{\begin{array}{l}M E M= \\ K L\end{array}\right.$

$\left\{\begin{array}{l}K L / R-Y=21.9 \quad C B=21.00 \text { 'YLD=36.00 ALLOWABLE STRESSES: FCZ= } 23.7 \\ F T Z=23.76 \text { FCY } 27.00 \text { FTY } 27.00 \text { FA= } 20.48 \text { FT } 21.60 \quad F V=14.40\end{array}\right.$

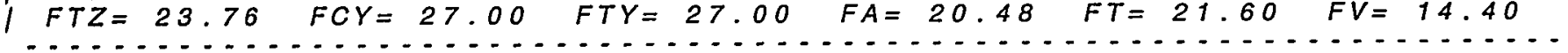
6 ST W33 6387

PASS

A ISC- $\mathrm{H} 2-1$

.081

$71.50 \mathrm{~T}$

.00

$-139.13$

14.40

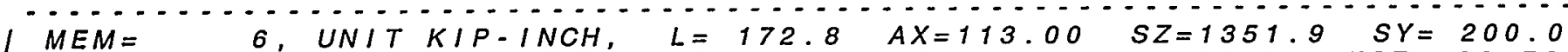

I $K L / R-Y=45.6$ '.6 $C B=1.00$ ' $Y L D=36.00$ ALLOWABLE STRESSES: FCZ= 23.76

$F T Z=23.76$ FCY $=27.00$ FTY $=27.00$ FA= 18.72 FT $=21.60$ FV 14.40 
- BUOY. ANCHOR UNIT SECTION UNDER HORIZONTAL L

ALL UNITS ARE - KIP FEET (UNLESS OTHERWISE NOTED)

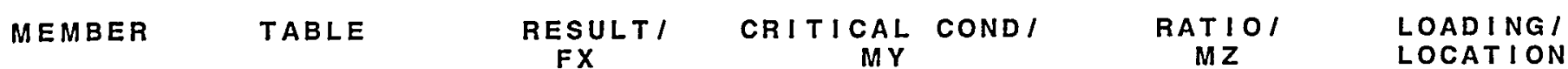

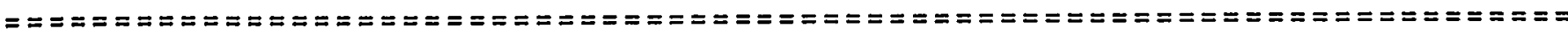

$\begin{array}{llll}7 & \mathrm{ST} & \mathrm{W} 33 \times 387\end{array}$
$71.50 \mathrm{~T}$
A ISC-H2-1 .00
.069

106.59
1

\section{$M E M=$}

7, UNIT KIP-INCH,

$L=86.4$

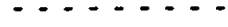

1 $K L / R-Y=22.8 \quad C B=1.00 \quad Y L D=36$
$F T Z=23.76 \quad F C Y=27.00 \quad F T Y=$

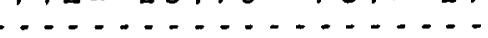
8 ST W40 $\times 593$

$440.00 \mathrm{C}$

00

$A X=113.00$

$S Z=1351.9$

$S Y=200.0$ $A L L O W A B L E$ STRESSES: $F C Z=23$.
$F A=20.42 \quad F T=21.60 \quad F V=14.40$

AISC- $\mathrm{H} 1-3$ 356 $-1100.00$ 1 .00 MEM $=\quad 8$, UNITKIP-INCH, L= 33.6 AX=174.00 SZ=2344.7 SY= 302.0 $K L / R \cdot Y=8.8 \quad C B=1.00 \quad Y L D=36.00 \quad A L L O W A B L E$ STRESSES: FCZ= 23.76 I FTZ $=23.76$ FCY $=27.00$ FTY $=27.00 \quad F A=21.21 \quad F T=21.60 \quad F V=14.40$ $9 \mathrm{ST}$ W40 X593 $\quad$ PASS AISC Hi 3 $440.00 \mathrm{C}$ 00 $-1100.00$ .00

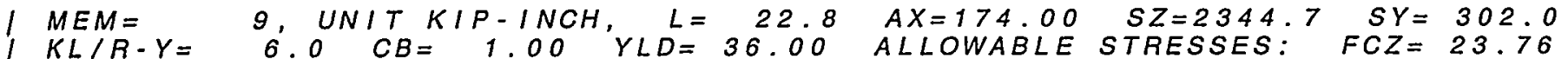
$1=25$ $--$ 1100.00 -... I FTZ $=23.76 \quad F C Y=27.00 \quad F T Y=27.00$ $A=21.34 \quad F T=21.60 \quad F V=14.40$ 10 ST W14 $\times 455$ PASS $00 \mathrm{~T}$ SHEAR $-Y$ .00 .797 1100.00 1 .00 I MEM $=10$, UNITKIP-INCH, $L=30.0$ AX=134.00 SZ=756.0 SY= 304.1

I $K L / R-Y=6.9 \quad C B=1.00 \quad Y L D=36.00$ ALLOWABLE STRESSES: I FTZ $=23.76$ FCY $=27.00$ FTY $=27.00 \quad F A=21.30 \quad F T=21.60$

11 ST PIP E

PASS

AISC- $\mathrm{H} 1-3$

.338

$-96.16$

1

$146.77 \mathrm{C}$

00

$S Z=240.9$

12.98

I $M E M=$ 11, UNIT KIP-INCH

$L=155.8$

$A X=52.57$

$S Z=240$.

$K L / R-Y=23.0 \quad C B=1.00 \quad Y L D=36.00$ ALLOWABLE STRESSES:

$F T Z=23.76$

$F C Y=23.7$

$Y L D=36$

$F A=20.41$

$F T=21.60$

\begin{tabular}{l}
$S Y=240,9$ \\
\hline
\end{tabular}

12 ST PIP E PASS AISC H H

$118,85 \mathrm{C}$ 00

49.79

00

I $M \bar{M}=$ 12 UNIT KIP.INCH

$L=155.8 \quad A X=52.57$

l

$F T Z=23$

12, UNIT

$I P-I N C H$

$L=155.8$

$A X=52.57 \quad S Z=240.9$

$S Y=240.9$

.... $23.76 \quad F C Y=23.76 \quad F T Y=23.7$

13 ST PIP E

PASS

AISC- $\mathrm{H}_{1}-1$

.00

$242.52 \mathrm{C}$

223

$F C Z=23$.

$T=21.60 \quad F V=14.40$ 
- BUOY. ANCHOR UNIT SECTION UNDER HORIZONTAL L

ALL UNITS ARE - KIP FEET (UNLESS OTHERWISE NOTED)

MEMBER

TABLE

\section{RESULT /}

$F X$
CRITICAL CONDI

$M Y$
RATIOI

$M Z$
LOADING I LOCATION

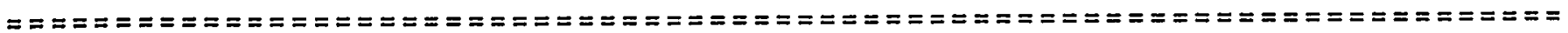

I MEM = 13, UNITKIP-INCH

$L=129.6$

$A X=52.57$

SY

$M E M=$

19.1 ' $C B=1.00$ Y Y $=36.00$ ALLOWABLE STRESSES

$K L / R-Y=$
$F T Z=23.76 \quad F C Y=23.76 \quad F T Y=23.7$

- - - - - - - - -

PASS

$85.54 \mathrm{~T}$

A I SC-H2-1

. 00

$S Y=240.9$
$C Z=23.76$

14 ST PIP E

14, UNITKIP-INCH, L=129.6

$A X=52.57 \quad S Z=240.9$

$M E M=$

19,1 , 190

$Y L D=36.00$

$K L / R-Y=$
$F T Z=23.7$

$19 \cdot 1 \quad C B=$

1.00

ALLOWABLE STRESSES:

- - - -

15 ST P P E

PASS

A I SC - $\mathrm{H} 1-3$

.00

031

.00 $F V=14.40$

$34.13 \mathrm{C}$

I $M E M=$ 15, UN/TKIP-INCH $L=129.6$ $A X=52.57$

$Z=240.9$

$Y Z=$

$\left\{\begin{array}{l}K L / R-Y=19.1 \quad C B=1.00 \\ F T Z=23.76 \quad F C Y=23.76\end{array}\right.$

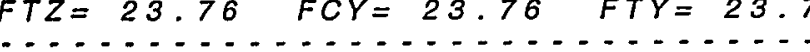

16 ST PIP E PASS

$38.16 \mathrm{~T}$

A ISC- H2 - 1 .00

$F V=14.40$ - -

I MEM $=16$, UNIT KIP-INCH, $L=129.6$ 1 00

$Y L D=36.00$ $A X=52.57 \quad S Z=240.9$ $A X=52.57$ SZ
$A L L O W A B L E$ STRESSES:

$K L / R-Y=$ $F T Z=2 \vec{\theta} .76$

76 FCY $=2 B=1.0$ $F T Y=23.76$ $F A=20.65$

$F T=21.60$ $S Y=240.9$
$F C Z=23.76$
$F V=14.40$

\section{PRINT ANALYSIS RESULTS}


- BUOY. ANCHOR UNIT SECTION UNDER HORIZONTAL L

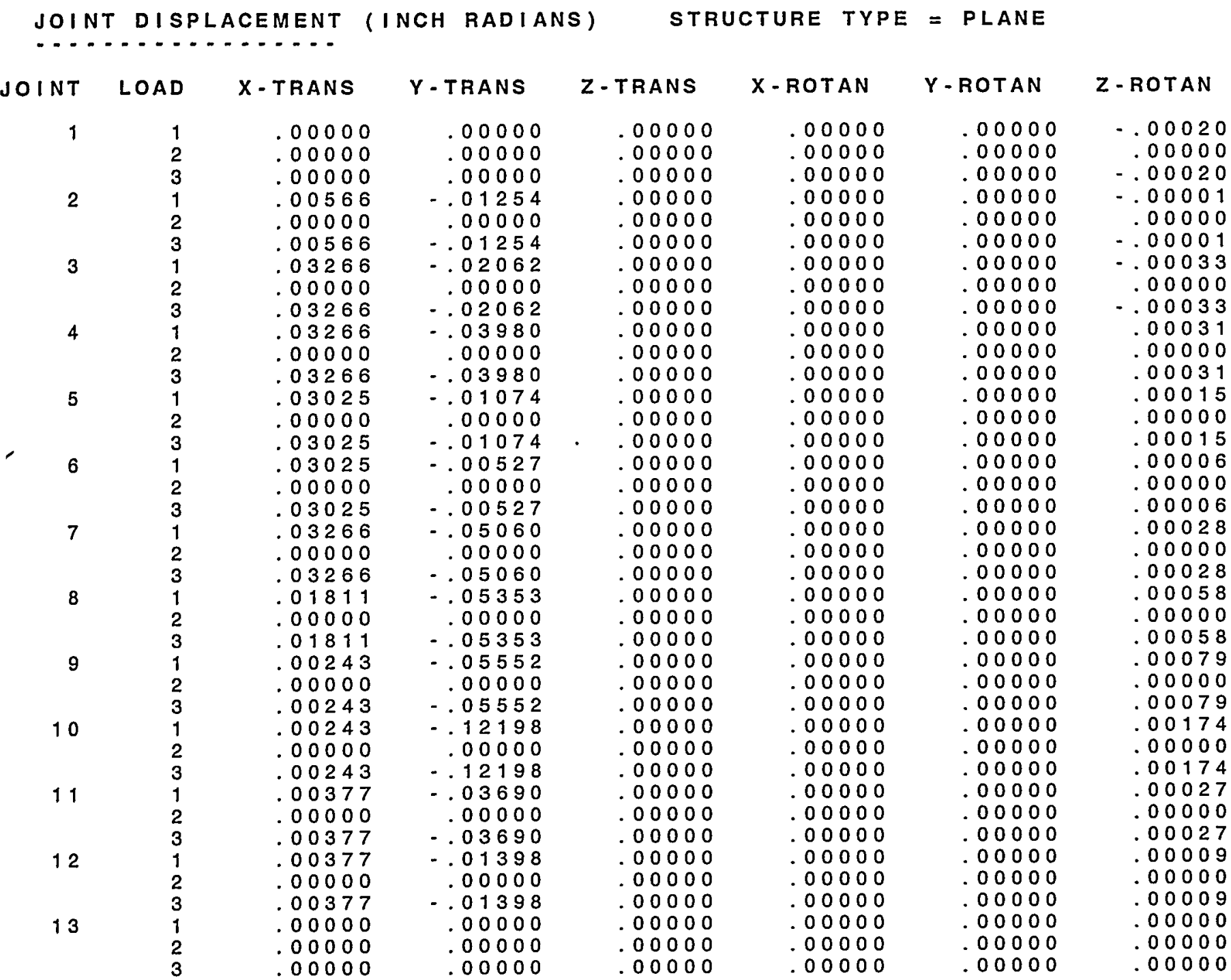


ANALISYS

* BUOY. ANCHOR UNIT SECTION UNDER HORIZONTAL L

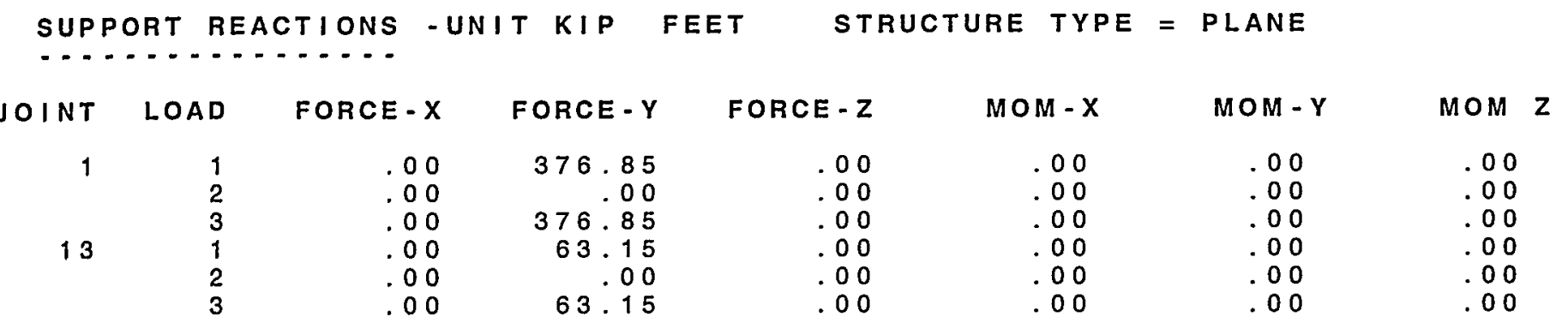


"BUOY. ANCHOR UNIT SECTION UNDER HORIZONTAL L

MEMBER END FORCES STRUCTURE TYPE = PLANE

ALL UNITS ARE - KIP FEET

MEMBER LOAD JT

$A X I A L$

SHEAR - Y

SHEAR - Z

TORSION

MOM - Y

MOM - ;

$\begin{array}{ll}1 & 1 \\ 2 & 3\end{array}$

3
7
3
7
3
7

.00
.00
.00
.00
.000
.00

242.52
-242.52
.00
.00
242.52

.00

$-242.52$

.00

.00

.00

00
.00

.00

.00

.00

$-197.48$

.00

.00

.00

.00

.00

$\begin{array}{rl}0 & 0 \\ .0 & 0\end{array}$

.00

2

.00

197.48

.00

.00

.00

.00

.00

.00

$-197.48$

197.48

.00

.00

.00

.00

.00

.00

$-34.62$

.00

.00

3

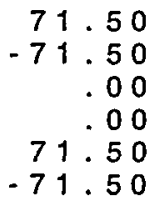

34.62
.00

.00

$-34.62$

34.62

.00

.00

.00

.00

.00

.00

.00

$-34.13$

.00

.00

.00

.00

.00

.00

.00

.00

.00

.00

.00

.00

.00

.00

$-34.13$

34.13

.00

.00

22.39

-22.39
00

.00

.00

.00

.00

.00

.00

22.39

$-22.39$

.00

.00

.00

.00

.00

.00

.00

1358.11

.00

.00

1358.11

$-258.11$

$-57.85$

.00

$-258.11$

$-57.85$

$-38.31$

$-210.99$

.00

.00

$-38.31$

$-210.99$

.00

.00

.00

.00

.00

.00

$-245.72$

.00

.00

.00

.00

.00

.00

5.60
-5.60

.00

.00

161.20
.00

$\begin{array}{rrrr}6 & 1 & 1 & -71.50 \\ & 12 & 71.50 \\ & 2 & 1 & .00 \\ & 12 & .00 \\ & 3 & 1 & -71.50 \\ & & 12 & 71.50\end{array}$

.5 .60
.00

.00

.00

.00

.00

.00

.00

5. 60

.00

.00

$-5.60$

00

.00

.00
.00

71

$\begin{array}{rr}12 & -71.50 \\ 2 & 71.50\end{array}$

9.63

.00

.00

.00

$-58.42$

139.13

.00

$-58.4$

139.13

.00

.00

106.59

$-37.24$ 
- BUOY. ANCHOR UNIT SECTION UNDER HORIZONTAL L

\begin{tabular}{|c|c|c|c|c|c|c|c|c|}
\hline \multirow[t]{2}{*}{ MEMBER } & LOAD & $\mathbf{J} \mathbf{T}$ & $A X \perp A L$ & SHEAR - Y & SHEAR - Z & TORSION & $M O M-Y$ & MOM - i \\
\hline & $\begin{array}{l}2 \\
3\end{array}$ & $\begin{array}{r}12 \\
2 \\
12 \\
2\end{array}$ & $\begin{array}{r}.00 \\
.00 \\
-71.50 \\
71.50\end{array}$ & $\begin{array}{r}.00 \\
.00 \\
9.63 \\
-9.63\end{array}$ & $\begin{array}{ll}.0 & 0 \\
.0 & 0 \\
.0 & 0 \\
.0 & 0\end{array}$ & $\begin{array}{ll}.0 & 0 \\
.0 & 0 \\
0 & 0 \\
.0 & 0\end{array}$ & 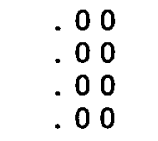 & $\begin{array}{r}.00 \\
.00 \\
106.59 \\
-37.24\end{array}$ \\
\hline 8 & $\begin{array}{l}1 \\
2 \\
3\end{array}$ & $\begin{array}{l}7 \\
8 \\
7 \\
8 \\
7 \\
8\end{array}$ & $\begin{array}{r}440.00 \\
-440.00 \\
.00 \\
.00 \\
440.000 \\
-440.00\end{array}$ & $\begin{array}{ll}.0 & 0 \\
.0 & 0 \\
.0 & 0 \\
.0 & 0 \\
.0 & 0 \\
.0 & 0\end{array}$ & $\begin{array}{ll}.0 & 0 \\
.0 & 0 \\
.0 & 0 \\
0 & 0 \\
.0 & 0 \\
.0 & 0\end{array}$ & $\begin{array}{lll}. & 0 & 0 \\
.0 & 0 \\
.0 & 0 \\
.0 & 0 \\
.0 & 0 \\
0 & 0 \\
0 & 0\end{array}$ & $\begin{array}{lll}. & 0 & 0 \\
.0 & 0 & 0 \\
.0 & 0 \\
.0 & 0 \\
.0 & 0 \\
.0 & 0\end{array}$ & 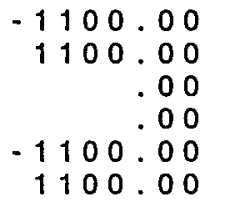 \\
\hline 9 & $\begin{array}{l}1 \\
2 \\
3\end{array}$ & $\begin{array}{l}8 \\
9 \\
8 \\
9 \\
8 \\
9\end{array}$ & $\begin{array}{r}440.00 \\
-440.00 \\
.00 \\
.00 \\
440.00 \\
-440.00\end{array}$ & $\begin{array}{ll}.0 & 0 \\
.0 & 0 \\
.0 & 0 \\
.0 & 0 \\
.0 & 0 \\
.0 & 0\end{array}$ & $\begin{array}{ll}.0 & 0 \\
.0 & 0 \\
.0 & 0 \\
0 & 0 \\
.0 & 0 \\
.0 & 0\end{array}$ & $\begin{array}{lll}. & 0 & 0 \\
.0 & 0 & 0 \\
.0 & 0 \\
.0 & 0 \\
.0 & 0 \\
.0 & 0\end{array}$ & $\begin{array}{lll}. & 0 & 0 \\
. & 0 & 0 \\
.0 & 0 \\
.0 & 0 \\
0 & 0 \\
0 & 0 \\
.0 & 0\end{array}$ & 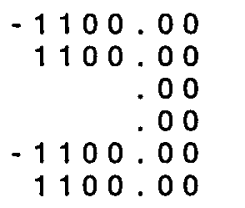 \\
\hline 10 & $\begin{array}{l}1 \\
2 \\
3\end{array}$ & $\begin{array}{r}9 \\
10 \\
9 \\
10 \\
9 \\
10\end{array}$ & $\begin{array}{ll}.0 & 0 \\
.0 & 0 \\
.0 & 0 \\
.0 & 0 \\
.0 & 0 \\
.0 & 0\end{array}$ & $\begin{array}{r}440.00 \\
-440.00 \\
.00 \\
.000 \\
440.000 \\
-440.00\end{array}$ & 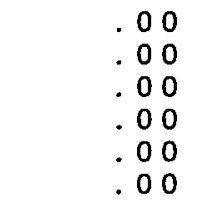 & $\begin{array}{lll}.0 & 0 \\
.0 & 0 \\
.0 & 0 \\
.0 & 0 \\
.0 & 0 \\
.0 & 0\end{array}$ & $\begin{array}{lll}. & 0 & 0 \\
.0 & 0 & 0 \\
.0 & 0 \\
.0 & 0 \\
.0 & 0 & 0 \\
. & 0 \\
.0 & 0\end{array}$ & $\begin{array}{rrr}1100.0 & 0 \\
.00 & 0 \\
.00 & 0 \\
.0 & 0 \\
1100.0 & 0 \\
.0 & 0\end{array}$ \\
\hline 11 & $\begin{array}{l}1 \\
2 \\
3\end{array}$ & $\begin{array}{l}1 \\
4 \\
1 \\
4 \\
1 \\
4\end{array}$ & $\begin{array}{r}146.77 \\
-146.77 \\
.00 \\
.00 \\
146.77 \\
-146.77\end{array}$ & $\begin{array}{r}11.91 \\
-11.91 \\
.00 \\
.00 \\
11.91 \\
-11.91\end{array}$ & $\begin{array}{ll}.0 & 0 \\
.0 & 0 \\
.0 & 0 \\
0 & 0 \\
.0 & 0 \\
.0 & 0\end{array}$ & $\begin{array}{ll}.0 & 0 \\
.0 & 0 \\
.0 & 0 \\
.0 & 0 \\
.0 & 0 \\
.0 & 0\end{array}$ & 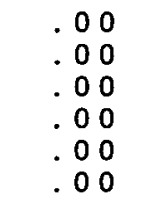 & $\begin{array}{r}58.42 \\
96.16 \\
.00 \\
.00 \\
58.42 \\
96.16\end{array}$ \\
\hline 12 & $\begin{array}{l}1 \\
2 \\
3\end{array}$ & $\begin{array}{l}5 \\
2 \\
5 \\
2 \\
5 \\
2\end{array}$ & $\begin{array}{r}118.85 \\
-118.85 \\
.00 \\
.00 \\
118.85 \\
-118.85\end{array}$ & $\begin{array}{r}6.71 \\
-6.71 \\
.00 \\
.00 \\
6.71 \\
-6.71\end{array}$ & $\begin{array}{lll}. & 0 & 0 \\
.0 & 0 & 0 \\
.0 & 0 \\
0 & 0 & 0 \\
.0 & 0 \\
0 & 0 & 0\end{array}$ & $\begin{array}{ll}.0 & 0 \\
.0 & 0 \\
.0 & 0 \\
0 & 0 \\
.0 & 0 \\
.0 & 0\end{array}$ & $\begin{array}{lll}.0 & 0 \\
.0 & 0 \\
.0 & 0 \\
.0 & 0 \\
.0 & 0 \\
.0 & 0\end{array}$ & $\begin{array}{r}49.79 \\
37.24 \\
.00 \\
.00 \\
49.79 \\
37.24\end{array}$ \\
\hline 13 & $\begin{array}{l}1 \\
2 \\
3\end{array}$ & $\begin{array}{l}1 \\
3 \\
1 \\
3 \\
1 \\
3\end{array}$ & $\begin{array}{r}242.52 \\
-242.52 \\
.00 \\
.00 \\
242.52 \\
-242.52\end{array}$ & $\begin{array}{lll}. & 0 & 0 \\
.0 & 0 \\
.0 & 0 \\
0 & 0 & 0 \\
.0 & 0 \\
0 & 0 & 0\end{array}$ & $\begin{array}{lll}.0 & 0 \\
.0 & 0 \\
.0 & 0 \\
.0 & 0 \\
.0 & 0 \\
0 & 0 & 0\end{array}$ & $\begin{array}{ll}.0 & 0 \\
.0 & 0 \\
.0 & 0 \\
0 & 0 \\
.0 & 0 \\
0 & 0\end{array}$ & $\begin{array}{lll}. & 0 & 0 \\
.0 & 0 & 0 \\
.0 & 0 \\
.0 & 0 \\
.0 & 0 \\
.0 & 0 & 0\end{array}$ & $\begin{array}{ll}.0 & 0 \\
.0 & 0 \\
.0 & 0 \\
.0 & 0 \\
.0 & 0 \\
.0 & 0\end{array}$ \\
\hline 14 & 1 & $\begin{array}{l}2 \\
6\end{array}$ & $\begin{array}{r}-85.54 \\
85.54\end{array}$ & $\begin{array}{l}.00 \\
.00\end{array}$ & $\begin{array}{l}.00 \\
.00\end{array}$ & $\begin{array}{l}.00 \\
.00\end{array}$ & $\begin{array}{l}.00 \\
.00\end{array}$ & $\begin{array}{l}.00 \\
.00\end{array}$ \\
\hline
\end{tabular}


"BUOY. ANCHOR UNIT SECTION UNDER HORIZONTAL L

\begin{tabular}{|c|c|c|c|c|c|c|c|c|}
\hline \multirow[t]{2}{*}{ UEMBER } & LOAD & JT & $A X I A L$ & SHEAR - Y & SHEAR - $Z$ & TORSION & $M O M-Y$ & $M O M-i$ \\
\hline & $\begin{array}{l}2 \\
3\end{array}$ & $\begin{array}{l}2 \\
6 \\
2 \\
6\end{array}$ & $\begin{array}{r}.00 \\
.00 \\
-85.54 \\
85.54\end{array}$ & $\begin{array}{ll}.0 & 0 \\
.0 & 0 \\
.0 & 0 \\
.0 & 0\end{array}$ & $\begin{array}{ll}.0 & 0 \\
.0 & 0 \\
.0 & 0 \\
.0 & 0\end{array}$ & 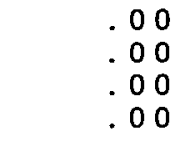 & $\begin{array}{l}.00 \\
.00 \\
.00 \\
.00\end{array}$ & $\begin{array}{l}.00 \\
.00 \\
.00 \\
.00\end{array}$ \\
\hline 15 & $\begin{array}{l}1 \\
2 \\
3\end{array}$ & $\begin{array}{r}4 \\
11 \\
4 \\
11 \\
4 \\
11\end{array}$ & $\begin{array}{r}34.13 \\
-34.13 \\
.00 \\
.00 \\
34.13 \\
-34.13\end{array}$ & $\begin{array}{ll}.0 & 0 \\
.0 & 0 \\
.0 & 0 \\
0 & 0 \\
.0 & 0 \\
.0 & 0\end{array}$ & $\begin{array}{ll}.0 & 0 \\
.0 & 0 \\
.0 & 0 \\
.0 & 0 \\
.0 & 0 \\
.0 & 0\end{array}$ & $\begin{array}{ll}.0 & 0 \\
.0 & 0 \\
.0 & 0 \\
.0 & 0 \\
.0 & 0 \\
.0 & 0\end{array}$ & $\begin{array}{ll}.0 & 0 \\
.0 & 0 \\
.0 & 0 \\
.00 & 0 \\
.0 & 0 \\
.0 & 0\end{array}$ & $\begin{array}{ll}.0 & 0 \\
.0 & 0 \\
.0 & 0 \\
.0 & 0 \\
.0 & 0 \\
.0 & 0\end{array}$ \\
\hline 16 & $\begin{array}{l}1 \\
2 \\
3\end{array}$ & $\begin{array}{r}5 \\
12 \\
5 \\
12 \\
5 \\
12\end{array}$ & $\begin{array}{r}-38.16 \\
38.16 \\
.00 \\
.00 \\
-38.16 \\
38.16\end{array}$ & $\begin{array}{ll}.0 & 0 \\
.0 & 0 \\
.0 & 0 \\
.0 & 0 \\
.0 & 0 \\
.0 & 0\end{array}$ & $\begin{array}{ll}.0 & 0 \\
.0 & 0 \\
.0 & 0 \\
.0 & 0 \\
.0 & 0 \\
.0 & 0\end{array}$ & $\begin{array}{lll}. & 0 & 0 \\
.0 & 0 \\
.0 & 0 \\
.0 & 0 \\
.0 & 0 \\
.0 & 0 & 0\end{array}$ & $\begin{array}{ll}.0 & 0 \\
.0 & 0 \\
.0 & 0 \\
.0 & 0 \\
.0 & 0 \\
.0 & 0\end{array}$ & $\begin{array}{ll}.0 & 0 \\
.0 & 0 \\
.0 & 0 \\
.0 & 0 \\
.0 & 0 \\
.0 & 0\end{array}$ \\
\hline 17 & $\begin{array}{l}1 \\
2 \\
3\end{array}$ & $\begin{array}{r}6 \\
13 \\
6 \\
13 \\
6 \\
13\end{array}$ & $\begin{array}{r}-63.15 \\
63.15 \\
.00 \\
.00 \\
-63.15 \\
63.15\end{array}$ & $\begin{array}{r}.00 \\
.00 \\
.00 \\
.00 \\
.00 \\
.00\end{array}$ & 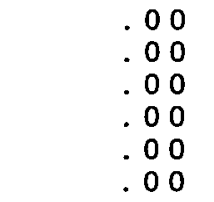 & $\begin{array}{ll}.0 & 0 \\
.0 & 0 \\
.0 & 0 \\
.0 & 0 \\
.0 & 0 \\
.0 & 0\end{array}$ & $\begin{array}{lll}.0 & 0 \\
.0 & 0 \\
.0 & 0 \\
.0 & 0 \\
.0 & 0 \\
.0 & 0\end{array}$ & $\begin{array}{lll}.0 & 0 \\
.0 & 0 \\
.0 & 0 \\
.0 & 0 \\
.0 & 0 \\
.0 & 0\end{array}$ \\
\hline
\end{tabular}

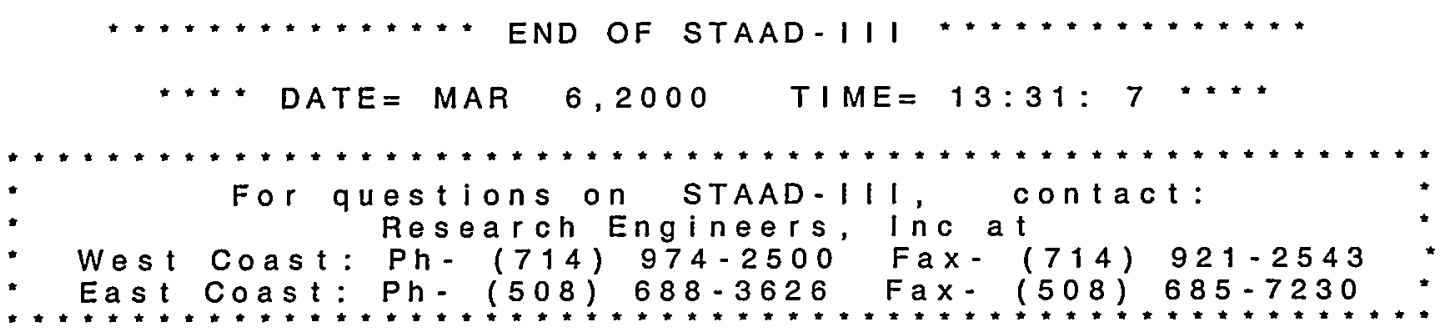




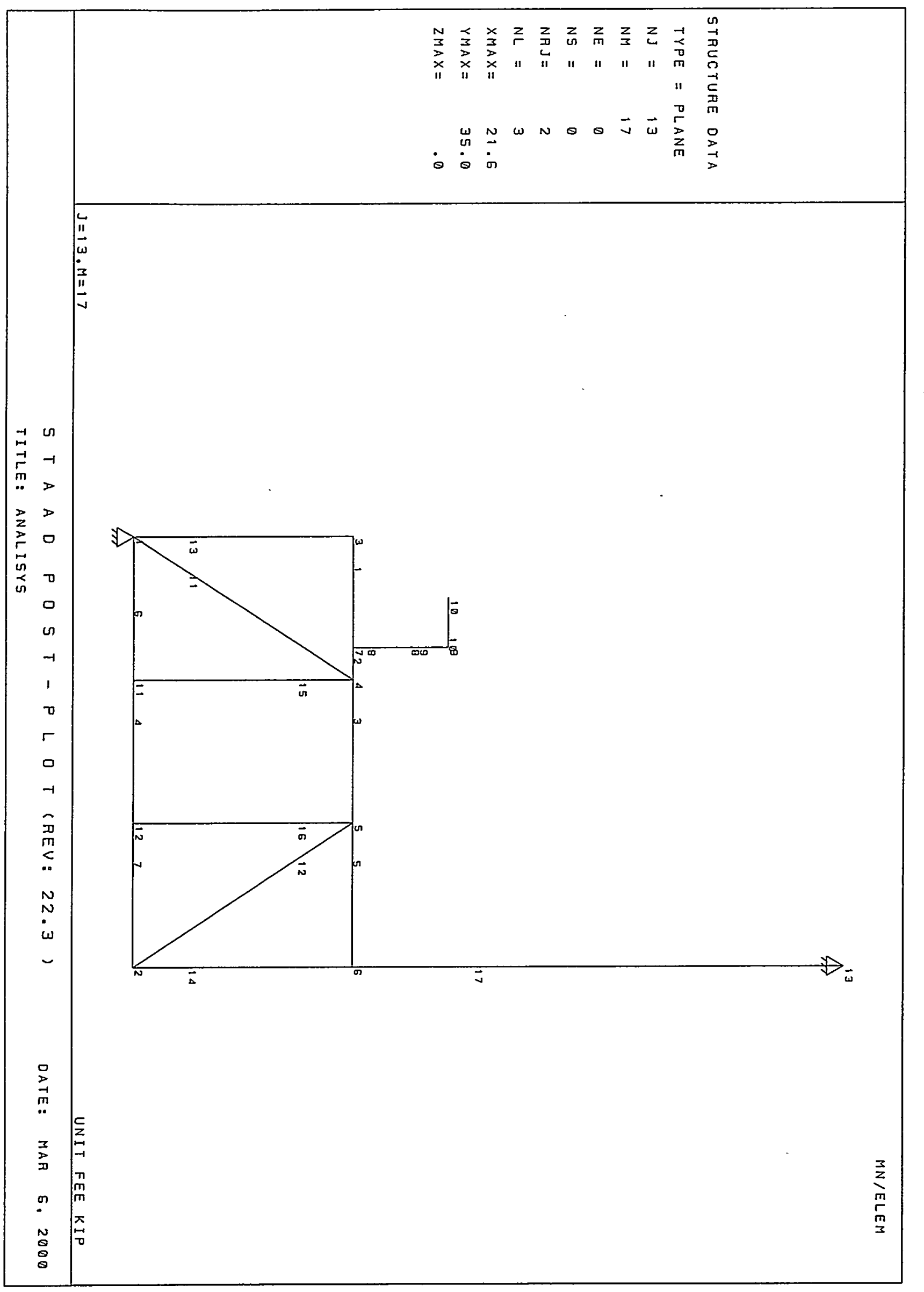




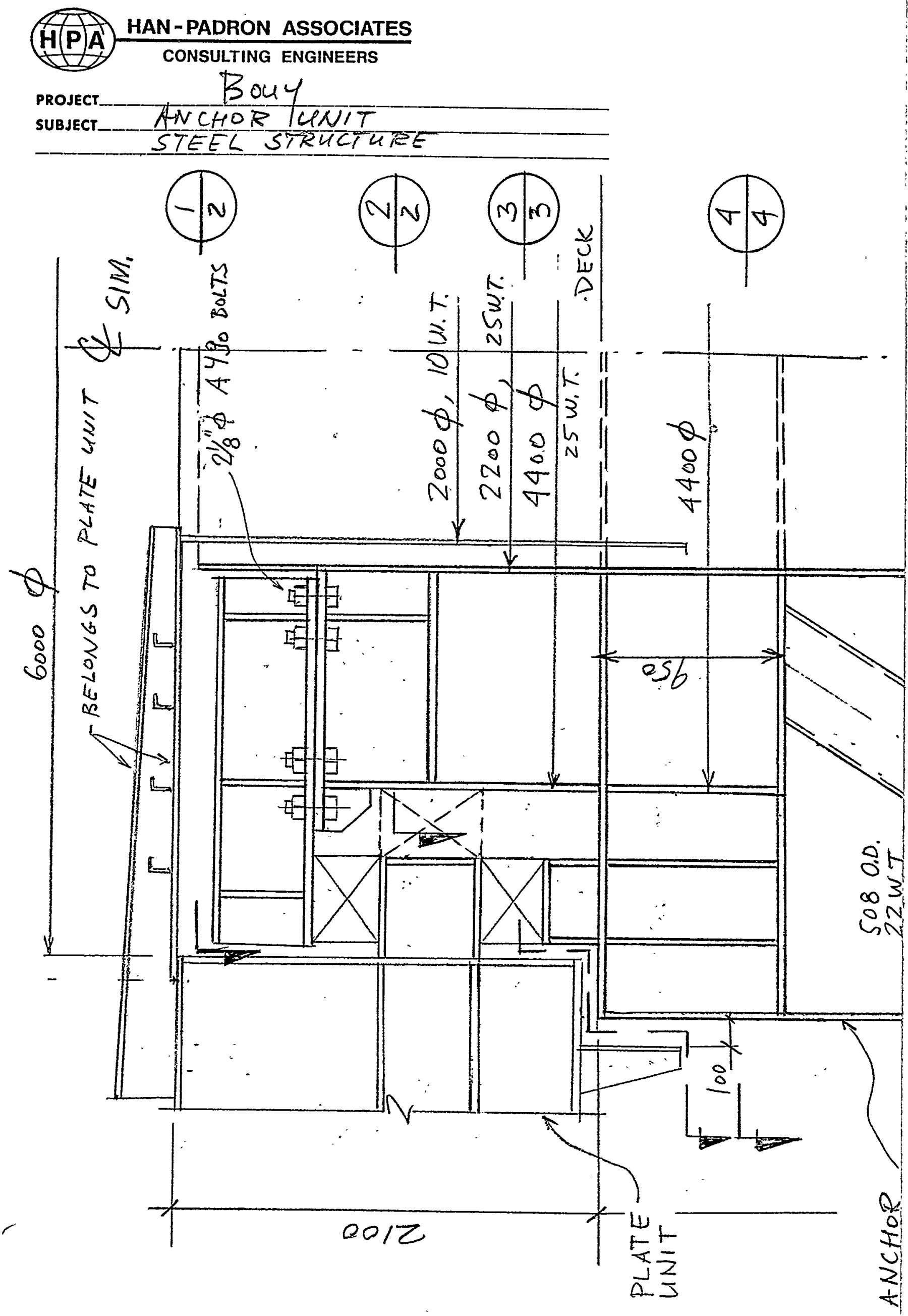




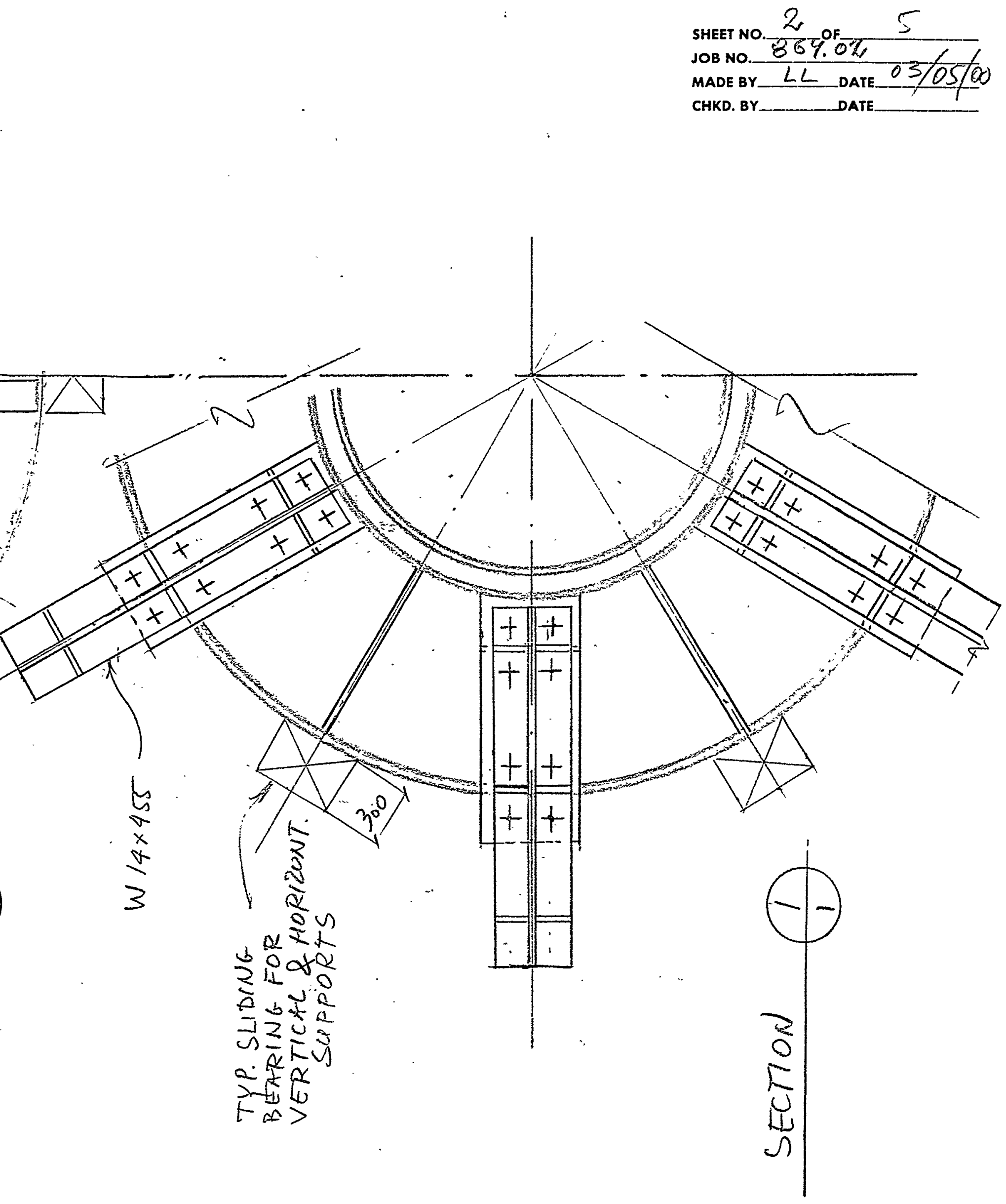


H(PAA) HAN-PADRON ASSOCIATES
CONSULTING ENGINEERS
BUOY
PROJECT_CANJECT_ANYUR UNTT
STEEL STRUCTURE

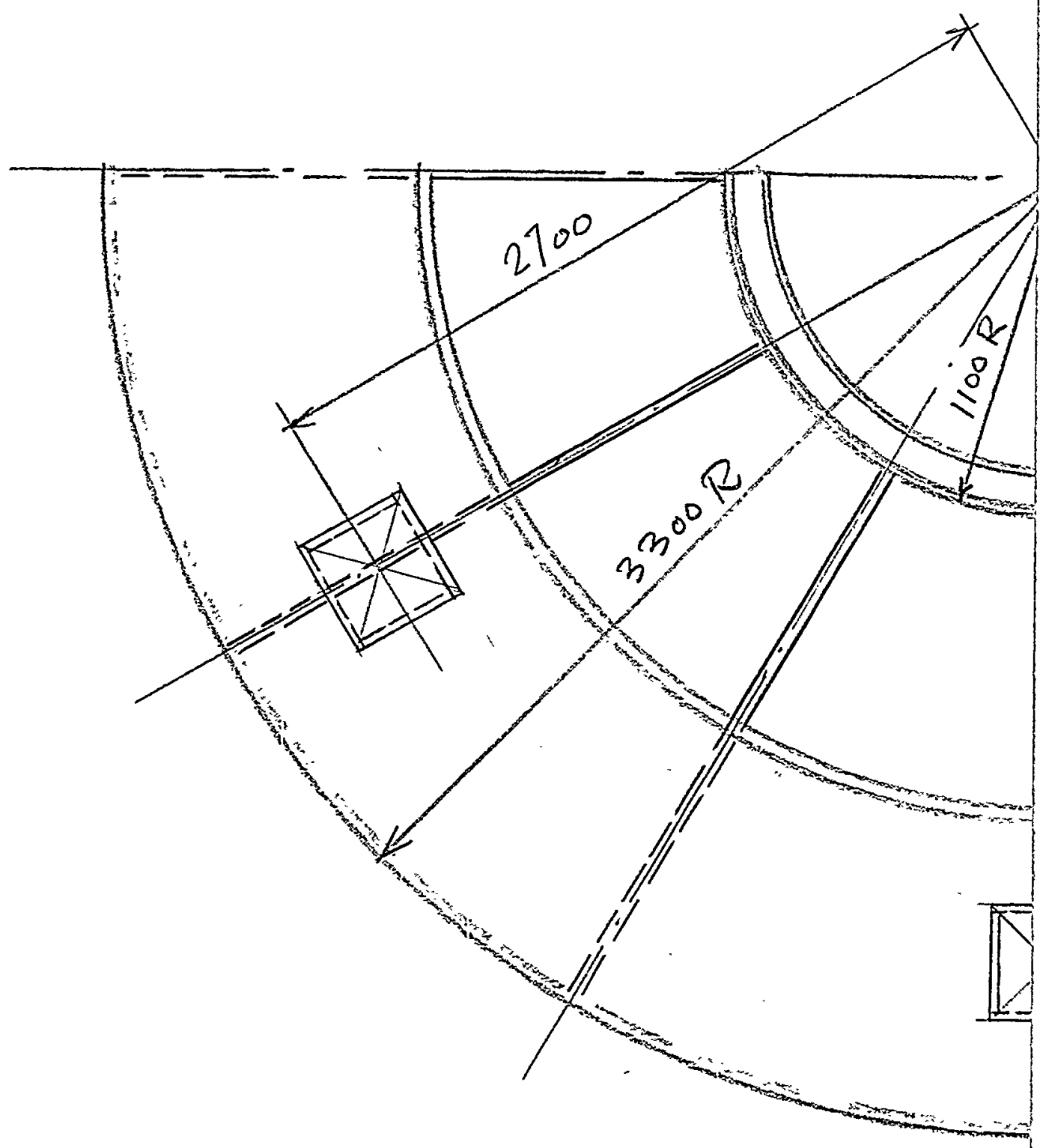



H(PAA HAN-PADRON ASSOCIATES

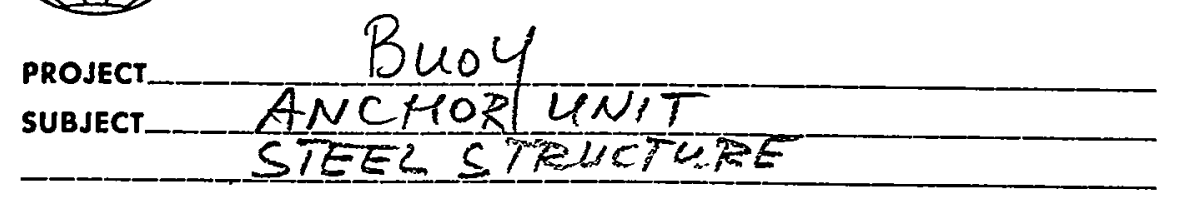

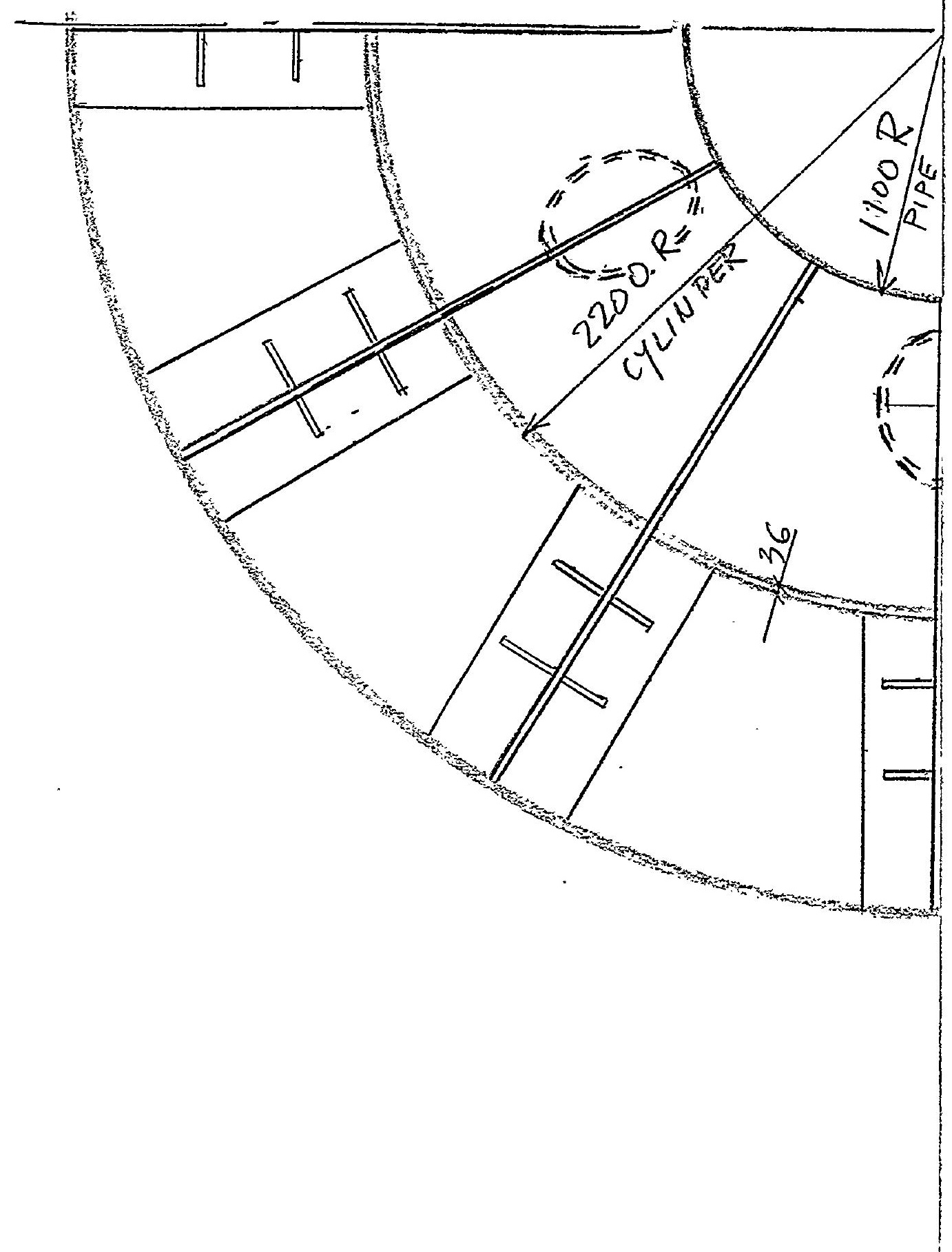



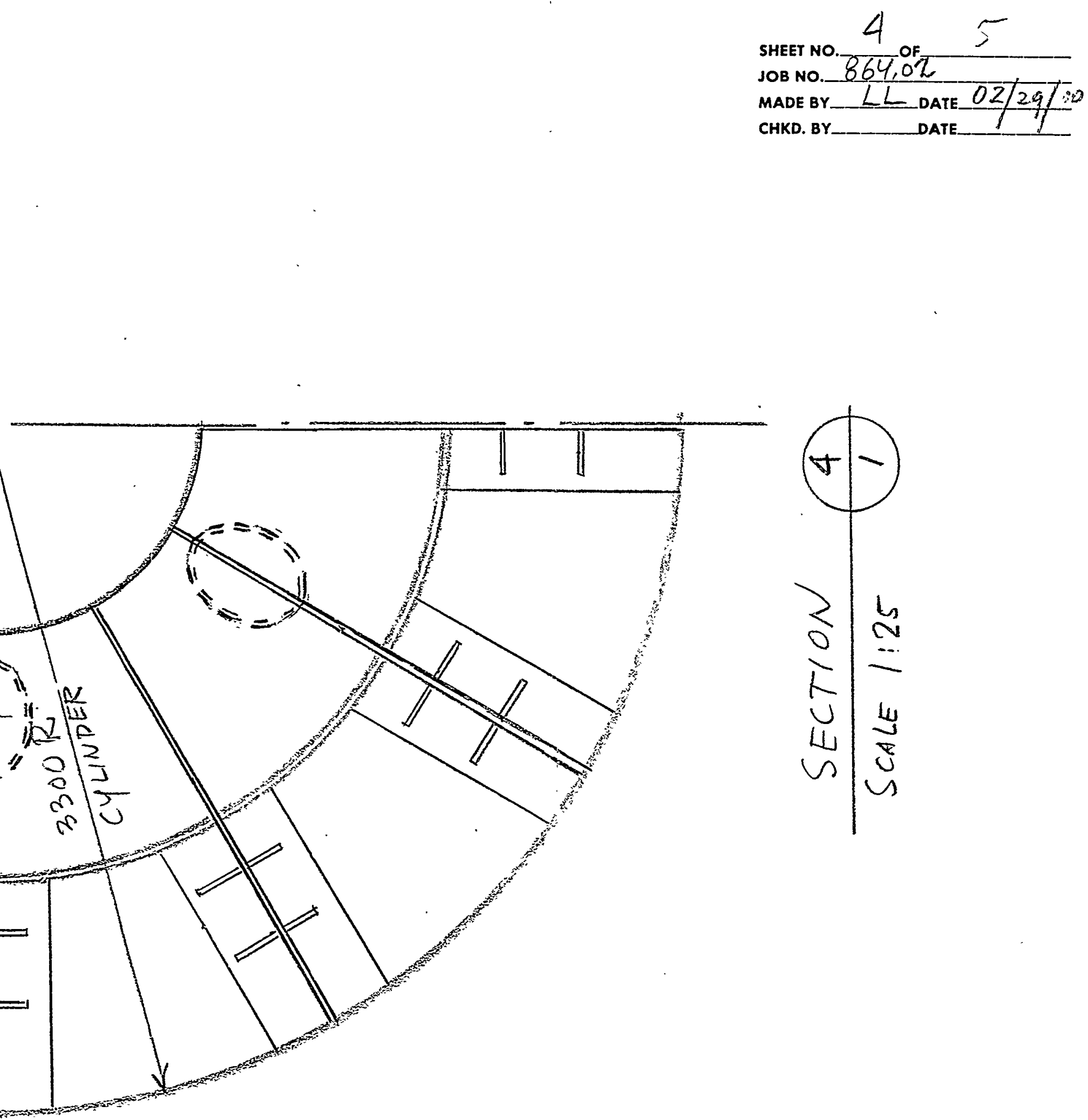
H(PA) HAN-PADRON ASSOCIATES

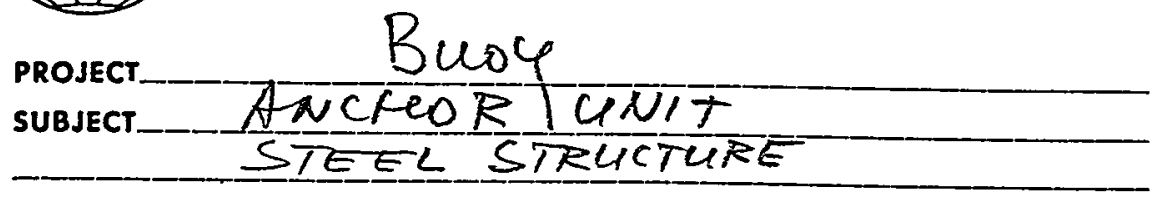

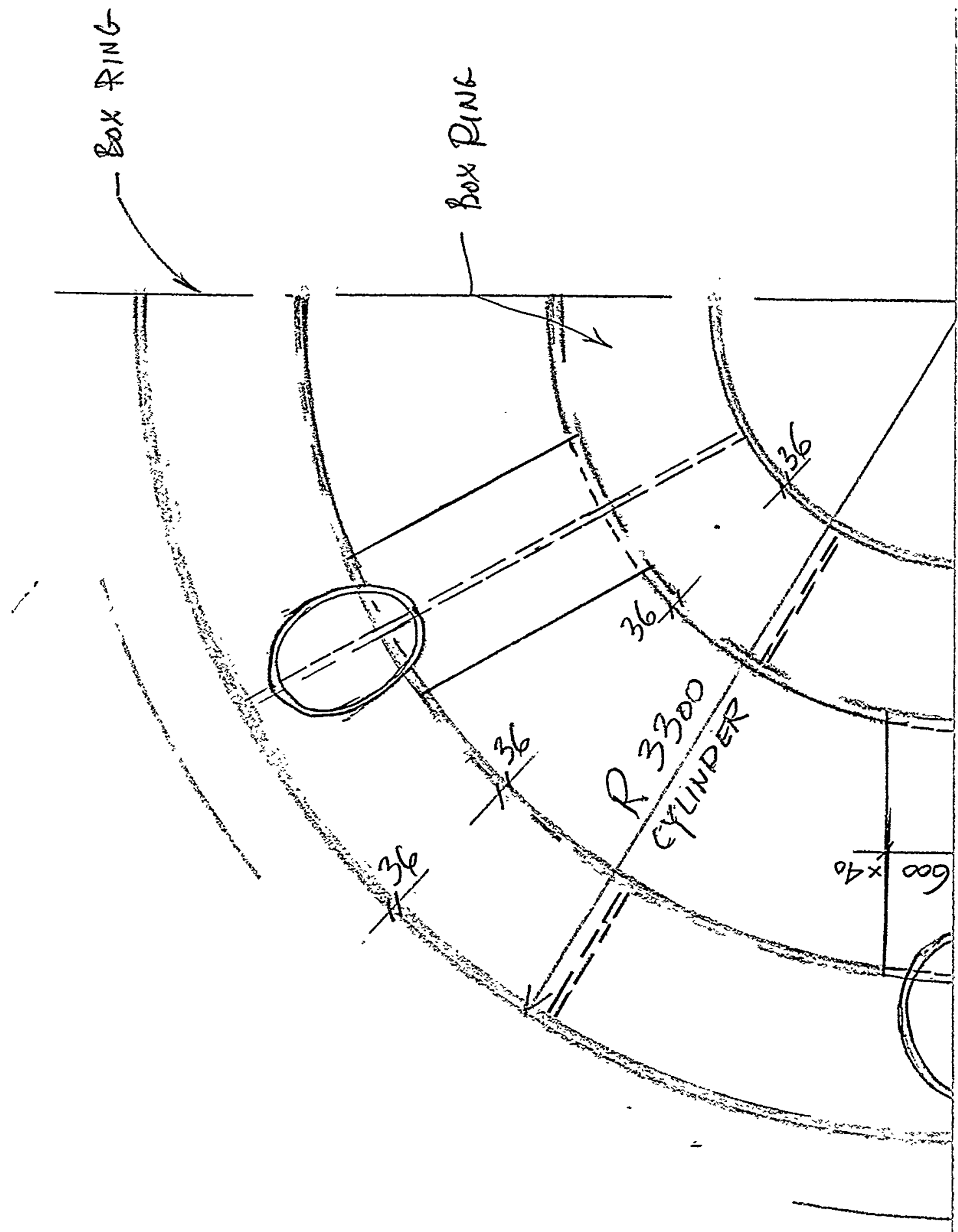



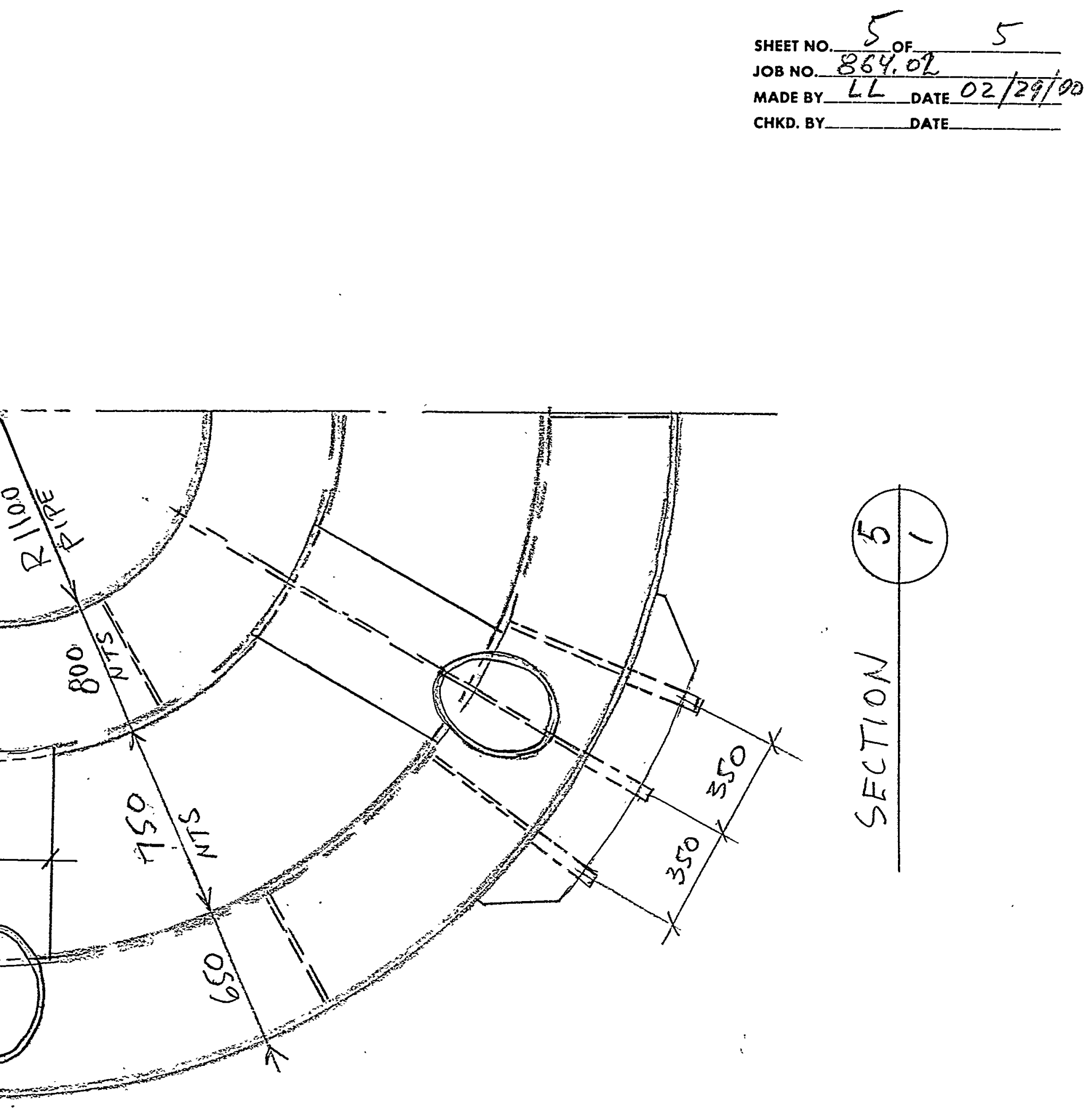
H(PA) HAN-PADRON ASSOCIATES

\begin{tabular}{l} 
BUDY \\
PROJECT_-PLAECT_COTE UNIT \\
\hline STEEL STRUCTURE
\end{tabular}

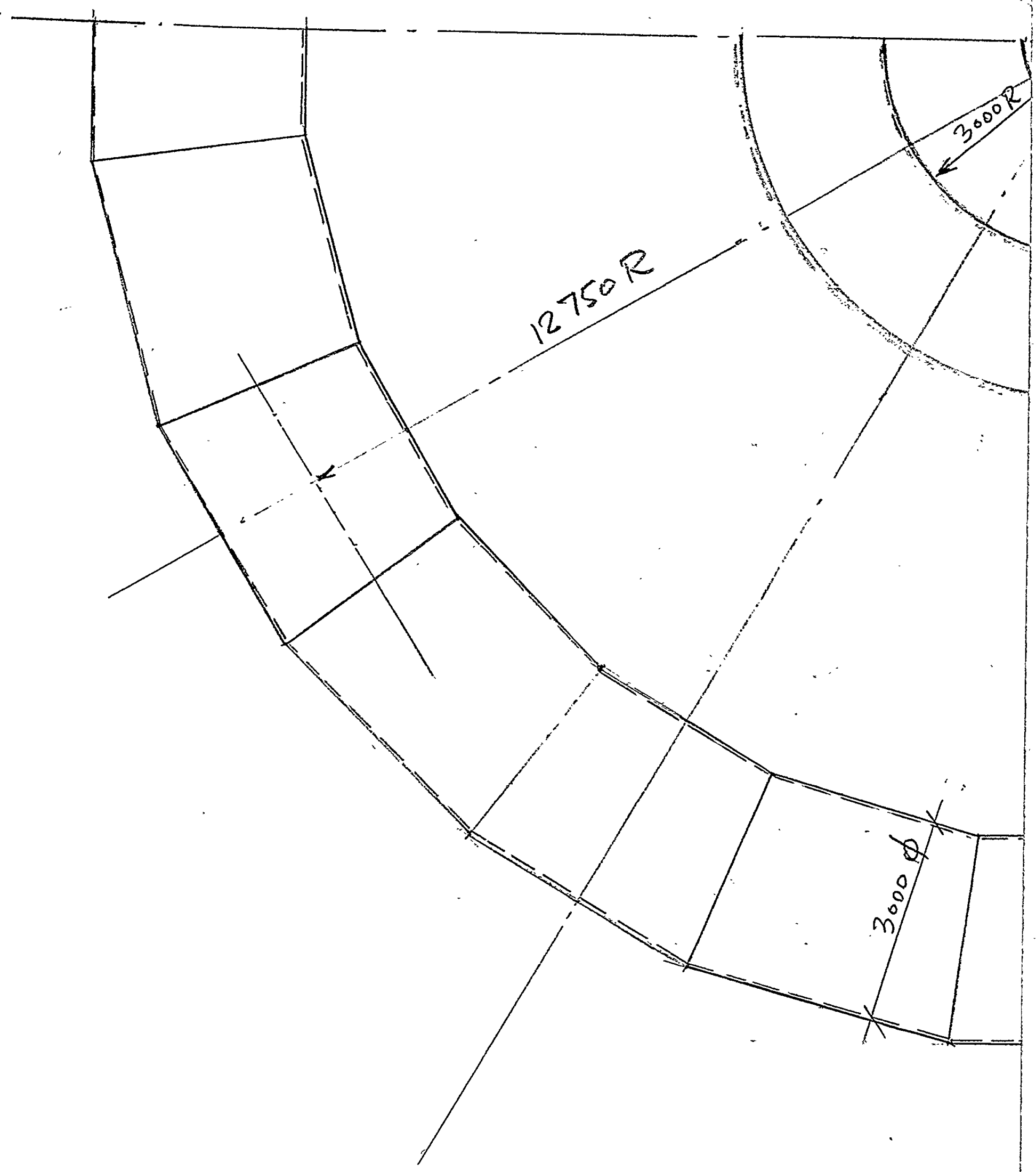




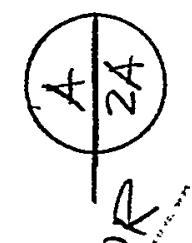

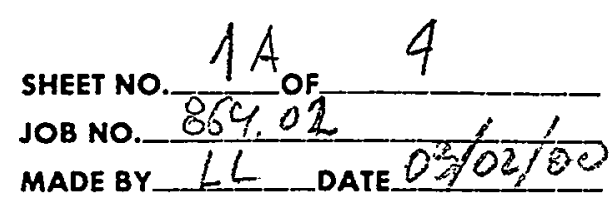

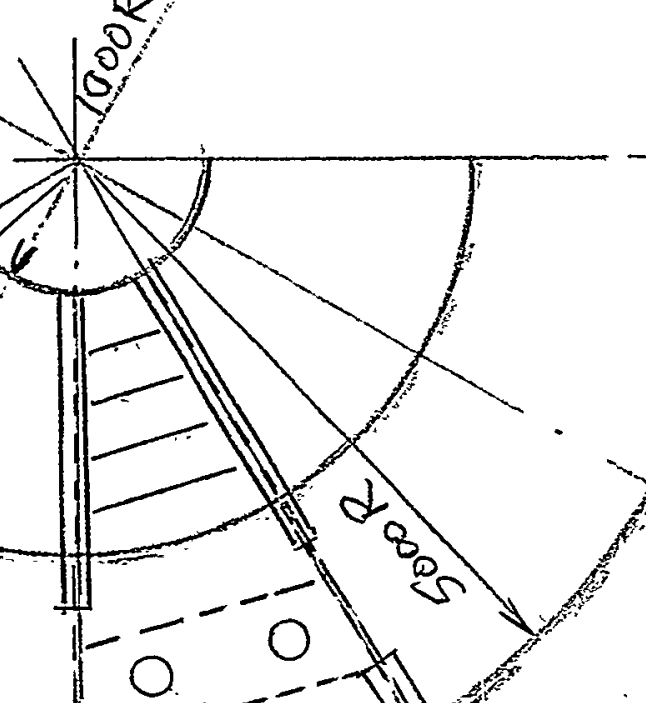

CHKD. BY

DATE

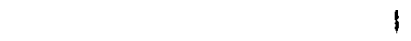


H(PA) $\frac{\text { HAN-PADRON ASSOCIATES }}{\text { CONSULTING ENGINEERS }}$

RuOJECr-TLY
SUBJECT-PLATE UNIT
STEEL STPACTUPE
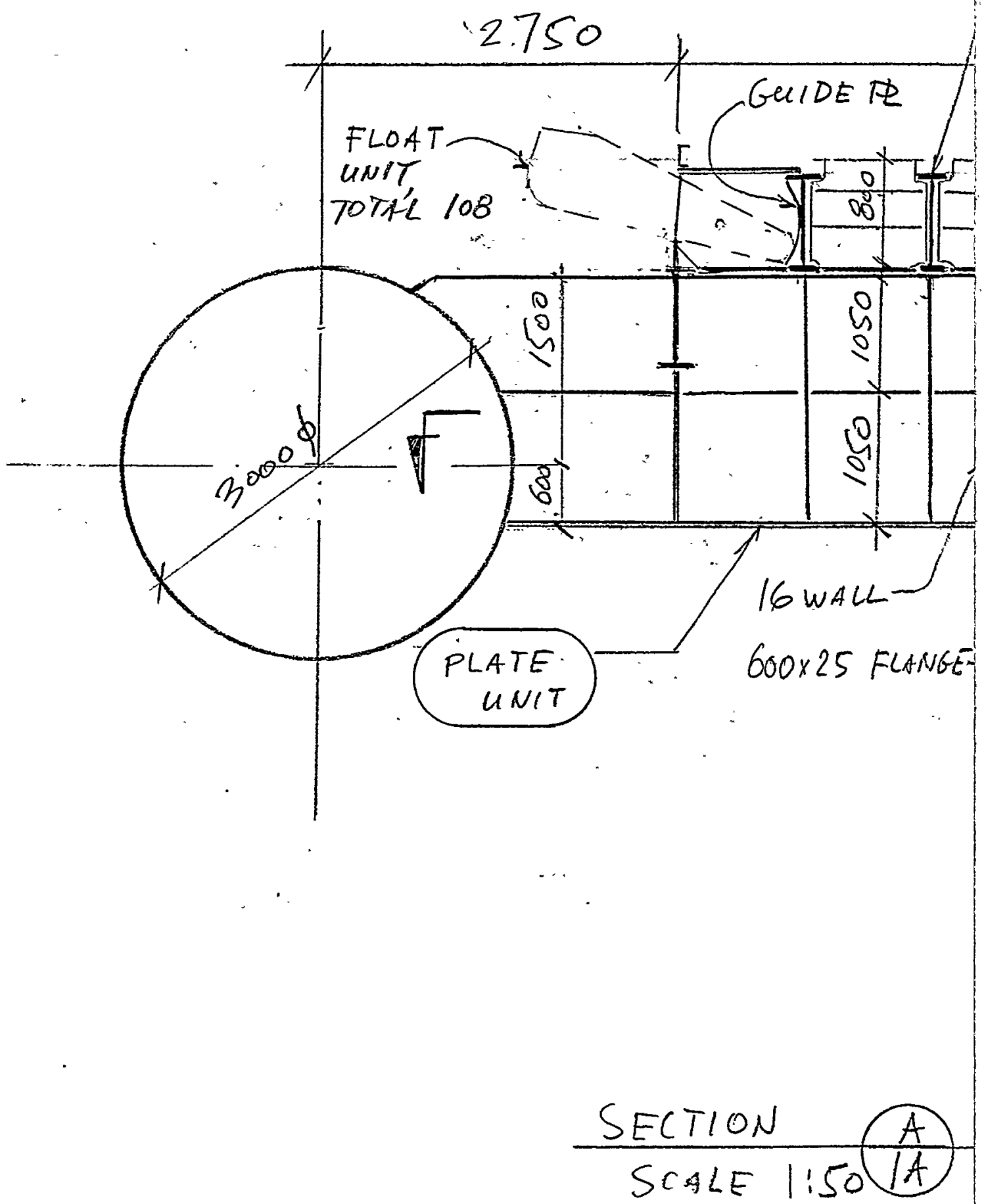


$$
\begin{aligned}
& \text { SHEet No. 2 } 2 \text { A } 4 \text { OF } 4 \\
& \text { JOB NO. 864,02 } \\
& \begin{array}{l}
\text { MADE BY_LL DATE- } 03 / 02 / 00 \\
\text { CHKD.BY___ DATE- }
\end{array}
\end{aligned}
$$

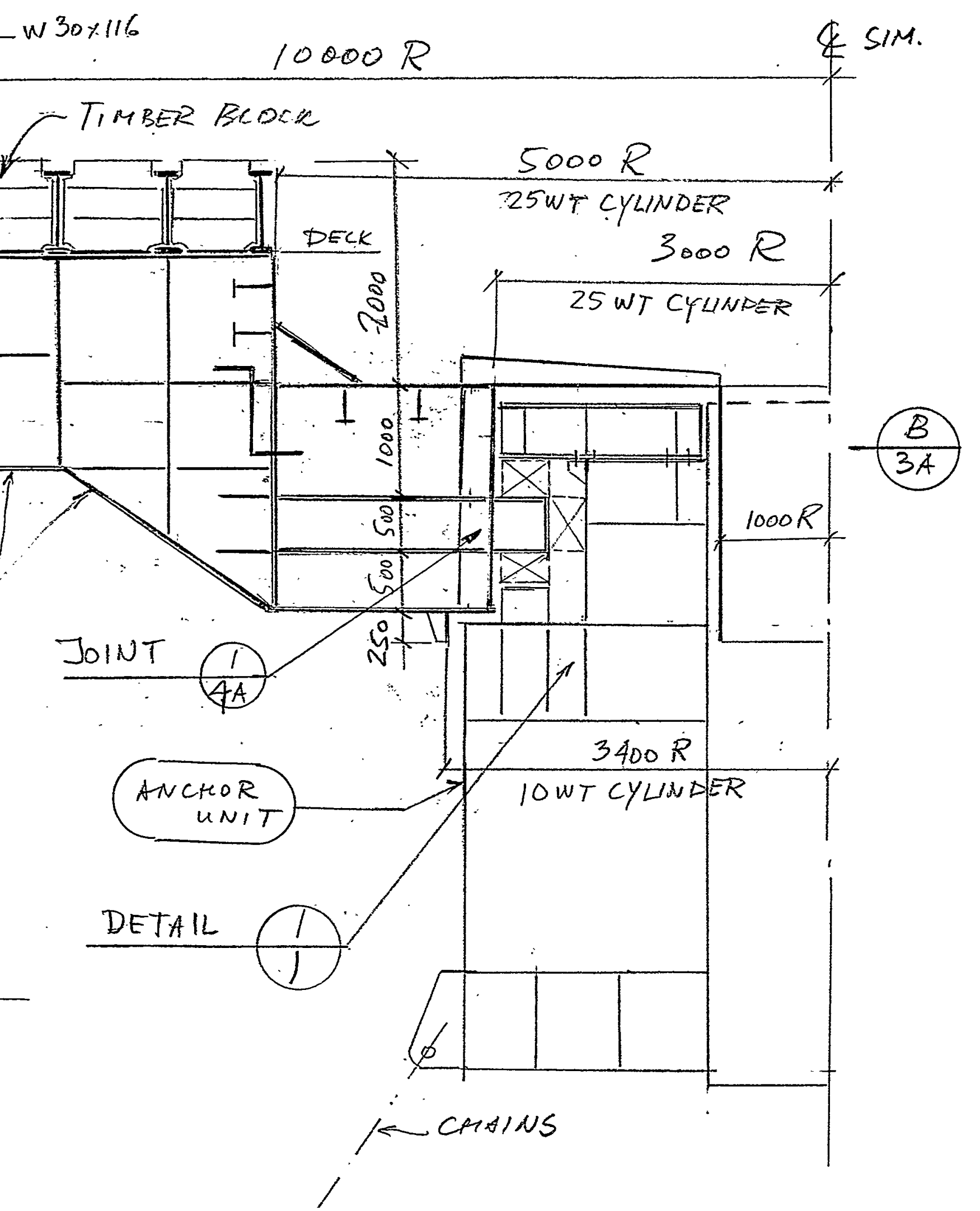


H(PA) HAN-PADRON ASSOCIATES

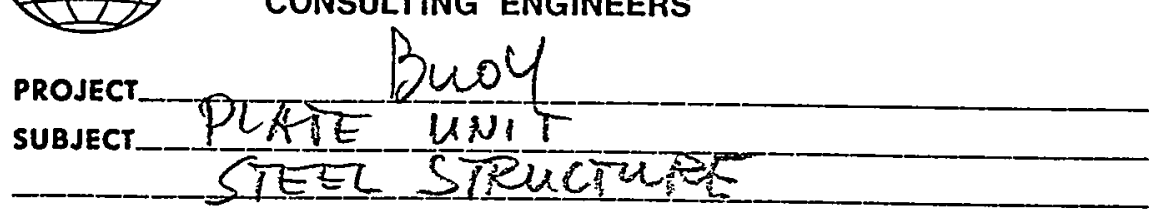

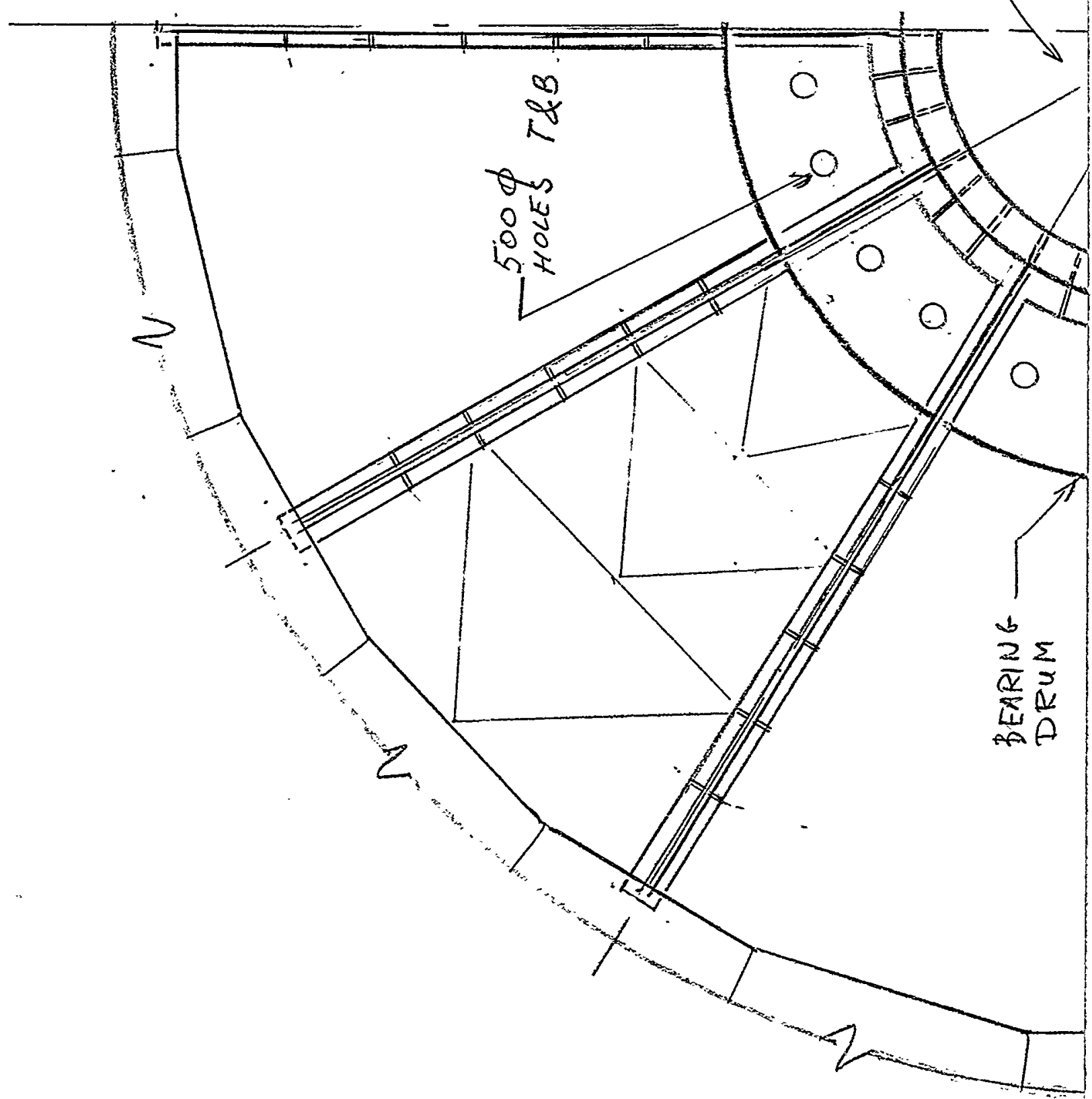


$(\mathbb{A})$ 

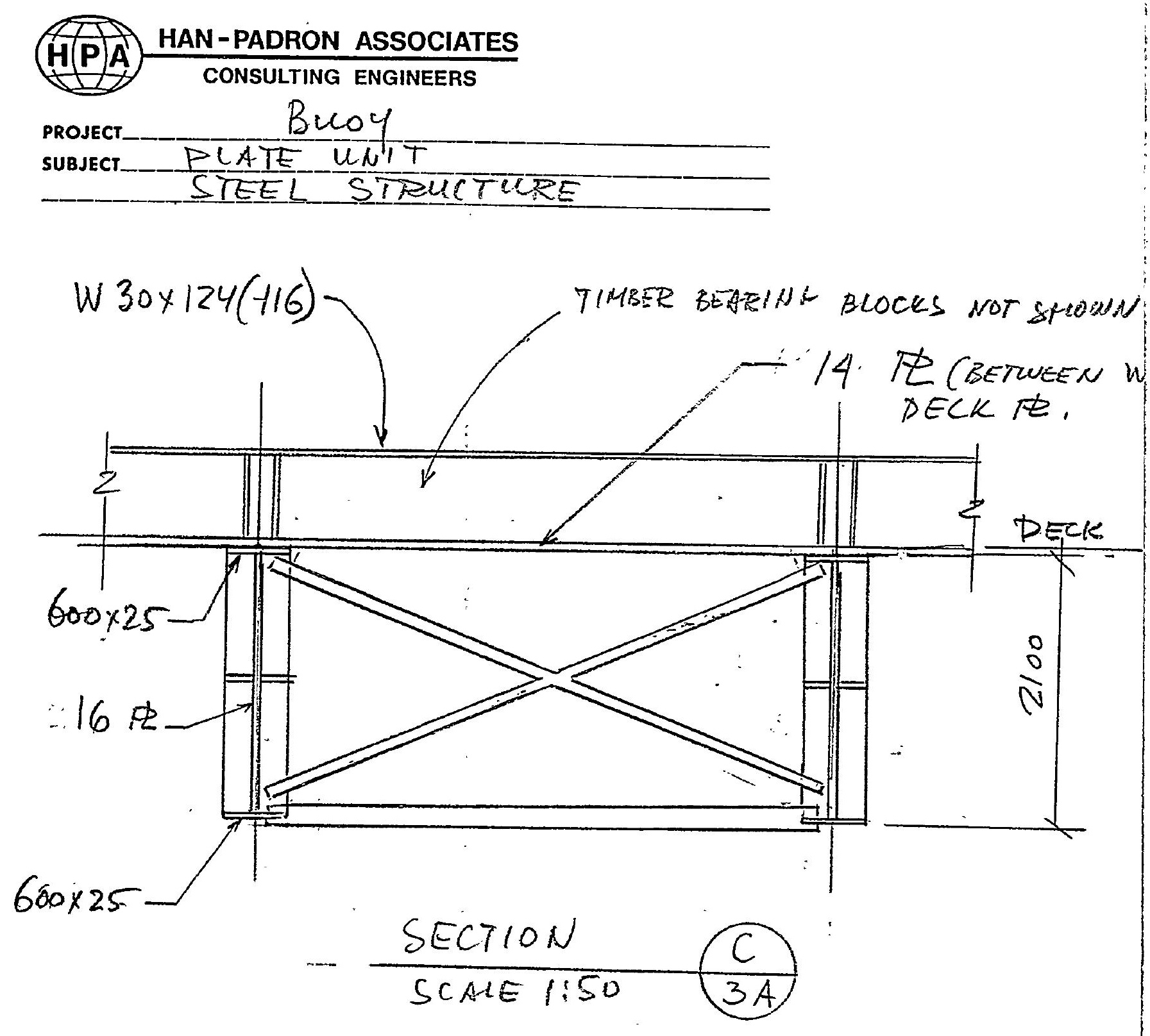


$$
\begin{aligned}
& \begin{array}{l}
\text { SHEET NO. } 4 \pi \text { OF- } 4 \\
\text { JOB NO. } 864 \text {. OF- }
\end{array} \\
& \begin{array}{l}
\text { JOB NO. } 869.0 \\
\text { MADE BY LL DATE } 03 / 03 / 30
\end{array}
\end{aligned}
$$

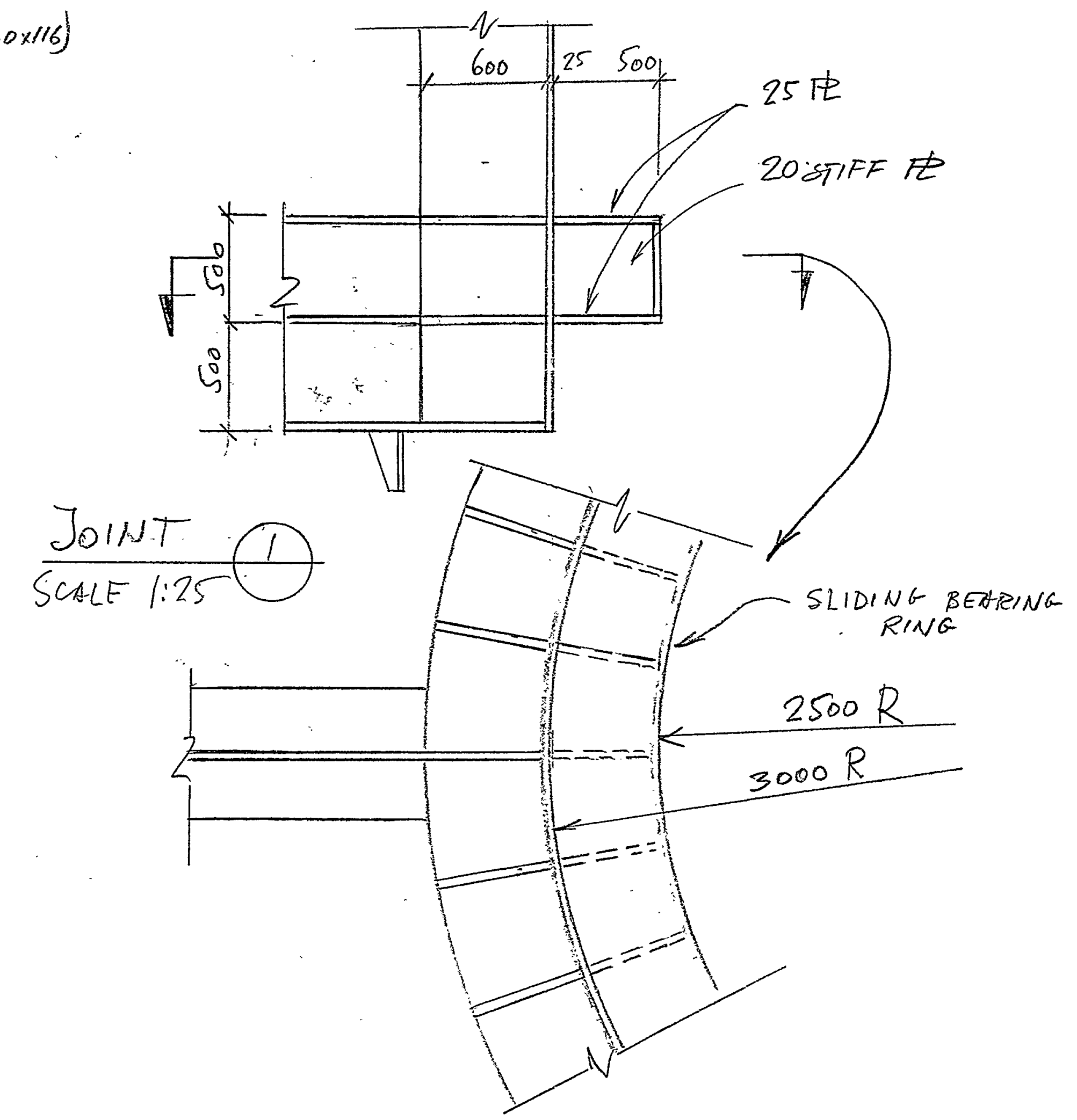


1. July 2000

\section{Preliminary calculations of bearings and anchor unit}

\section{Alternative Design}

Design Loads:

$\begin{array}{ll}\text { Horizontal load from chains: } & 6000\end{array}$

$\mathrm{kN}$

Differential pressure on water pipe:

$200 \mathrm{kPa}$

Material and design stress:

All steel parts are made from hot rolled sheets with $\mathrm{f}_{\mathrm{y}}=36 \mathrm{ksi}\left(\mathrm{f}_{\mathrm{y}}=248 \mathrm{MPa}\right)$

Design stress in bending: $248 / 1.67=$

$\mathrm{MPa}$

Design stress in shear: $248 / 2.50=$

$99 \mathrm{MPa}$

Bolt quality 8.8 (tensile strength $=800 \mathrm{MPa}$, yield stress $=640 \mathrm{MPa}$ )

M54, effective cross section in tension: $1890 \mathrm{~mm}^{2}$

Prestress: $1890 \times 800 \times 0.7 \times 10^{-3}=$

1058

$\mathrm{kN}$

Design strenght: $1890 \times 800 \times 10^{-3} / 2=$

$756 \mathrm{kN}$

Bearings:

Bearings between plate unit and anchor unit are Mammoth rollers from Hilman Equipment Co., New Jersey.

Bearing for horizontal load is a modified type 7X with a capacity of 500 US tons $=4448$ $\mathrm{kN}$

Bearing for vertical load is a modified type $6 \mathrm{X}$ with a capacity of $300 \mathrm{US}$ tons $=2669 \mathrm{kN}$ Bearings between chains and anchor unit are LUBRON AQ flange bushings. Lubron alloy 862 is used with a recommended bearing load of $6000 \mathrm{psi}=41.4 \mathrm{MPa}$. Stainless steel is intended as material in mating surface.

\section{Assumptions:}

1. 6 parallel chains are connected to weights. The submerged weight of each is $1000 \mathrm{kN}$.

The weights are moving in vertical tubes sunk into the sea bed.

2. Horizontal force is assumed evenly distributed between the chains.

3. Vertical loads are only considered in the design of the lubron bearings.

4. Approximate geometry is used in the calculations. 
Extent of design:

Only the lower part of the anchor unit is considered. The upper part is not different from the "standard" design.

Calculation of bearing loads on Lubon bushings:

Horizontal load:

$=$

Bearing stress on bushing:

$0.550^{2}$ ) $=$

Vertical load:

Bearing stress on flange:

41.4 $\mathrm{MPa}$

Design of bottom ring beam:

Horizontal forces:

$\mathrm{kN}$

forces as a sinus

force in the cylindrical wall. Diagonal

considered.

From Roark's Formulas for Stress \& Strain, 6th. edition, table 17, case 1, case 4 and case

20:

Moment around vertical axis:

$3 / \pi)=$

Excentricity of chain force: $\mathrm{e}=550 / 2+200=475 \mathrm{~mm}$

Moment around horizontal axis: $\quad M=1000 \times 0.475 / 2=138 \mathrm{kNm}$

Torsional moments are carried by the diagonal beams.

Cross sectional properties:

$\mathrm{mm}^{4}$

Stress:

$149 \mathrm{MPa}$

Shear stress:
$R=1000 \mathrm{kN}$

Moment at center of bearing : $M=1000 \times 0.475$

$475 \mathrm{kNm}$

$f=1.000 /(0.55 \times 0.275)+0.475 \times 6 /(0.275 \times$

$40.9 \mathrm{MPa}<41.4 \mathrm{MPa}$

$\mathrm{N}=1000 \mathrm{kN}$

$\mathrm{f}=1.000 \times 4 /\left(\pi\left(0.375^{2}-0.325^{2}\right)\right)=36.4 \mathrm{MPa}<$

The ring is loaded with six parallel forces of 1000 each. The ring must redistribute the distributed shear beams are not 
Design of diagonal beam in bottom ring:

Moment:

Shear force:

Cross sectional properties:

$10^{6} \mathrm{~mm}^{4}$

Normal stress:

Shear stress:
$M=1.000 \times 0.475=475 \mathrm{kNm}$ at edge

$\mathrm{Q}=475 / 3.5=136 \mathrm{kN}$

$\mathrm{A}=2 \times 300 \times 20+510 \times 15=19650 \mathrm{~mm}^{2}$

$I=1 / 2 \times 300 \times 20 \times 530^{2}+15 \times 510^{3} / 12=1009 \times$

$\mathrm{S}=\mathrm{I} / 275=3.667 \times 10^{6} \mathrm{~mm}^{3}$

$\mathrm{f}=475 / 3.667=130 \mathrm{MPa}<149 \mathrm{MPa}$

$\tau=136000 /(550 \times 15)=16.5 \mathrm{MPa}$

Design of exterior cylindrical shell:

Shear stress:

$$
f=W /(\pi \times R \times t)=6000 \times 10^{3} /(\pi \times 3500 \times 20)
$$

$=27 \mathrm{MPa}$

Moment:

$$
M=6000 \times 2.650=15900 \mathrm{kNm}
$$

Bending stress (neglecting vertical stiffeners): $f=M /\left(\pi \times R^{2} \times t\right)=15900 \times 10^{6} /(\pi \times$ $\left.3500^{2} \times 20\right)=$

$21 \mathrm{MPa}$

Critical elastic shear stress according to ECCS: "Buckling of Steel Shells"

$\mathrm{x}$

\section{$332 \mathrm{MPa} \gg>99 \mathrm{MPa}>27$}

$$
\mathrm{f}=0.75 \times \mathrm{Ex}(\mathrm{R} /)^{0.5} \times(\mathrm{t} / \mathrm{R})^{1.25}=0.75 \times 2.1 \times 10^{5}
$$
$(3500 / 1950)^{0.5} \times(20 / 3500)^{1.25}=$

Critical elastic compression stress according to API RP 2A:

$20 / 7000=$

$$
f_{x c}=2 \times 0.3 \times E \times t / D=0.6 \times 2.1 \times 10^{5} \times
$$

$360 \mathrm{MPa}$

Design compression stress: $\mathrm{f}_{\mathrm{xc}}=149\left(1.64-0.23 \times(7000 / 20)^{0.25}\right)=96>21 \mathrm{Mpa}$ 
1. July 2009

\section{Preliminary calculations of bearings and anchor unit}

\section{Design Loads:}

Horizontal load from chains:

6000

$\mathrm{kN}$

Differential pressure on water pipe:

$200 \mathrm{kPa}$

Material and design stress:

All steel parts are made from hot rolled sheets with $\mathrm{f}_{\mathrm{y}}=36 \mathrm{ksi}\left(\mathrm{f}_{\mathrm{y}}=248 \mathrm{MPa}\right)$

Design stress in bending: $248 / 1.67=$

$\mathrm{MPa}$

Design stress in shear: $248 / 2.50=$

Bolt quality 8.8 (tensile strength $=800 \mathrm{MPa}$, yield stress $=640 \mathrm{MPa}$ )

$99 \mathrm{MPa}$

M54, effective cross section in tension: $1890 \mathrm{~mm}^{2}$

Prestress: $1890 \times 800 \times 0.7 \times 10^{-3}=$

$\mathrm{kN}$

Design strenght: $1890 \times 800 \times 10^{-3} / 2=$

\section{Bearings:}

Bearings between plate unit and anchor unit are Mammoth rollers from Hilman Equipment Co., New Jersey.

Bearing for horizontal load is a modified type $7 \mathrm{X}$ with a capacity of 500 US tons $=4448$ $\mathrm{kN}$

Bearing for vertical load is a modified type $6 \mathrm{X}$ with a capacity of 300 US tons $=2669 \mathrm{kN}$

\section{Assumptions:}

1. 6 chains are spread out radial from the buoy, each of them anchored to a concrete block on the sea bed.

2.The horizontal force is assumed transmitted by only one chain.

3. Vertical loads from chains are neglected. On the safe side, as vertical chain load reduces moment at level of bearings.

4. Approximate geometry is used in the calculations.

Calculation of bearing loads:

Horizontal load:

$\mathrm{kN}$

Vertical load:
$\mathrm{R}=6000 /\left(1+2 \times \cos \left(60^{\circ}\right)^{2}\right)=4000 \mathrm{kN}<4448$

Moment at level of bearings : $M=6000 \times 3.81=$ $22860 \mathrm{kNm}$

Diameter of centerline of bearings : $6.69 \mathrm{~m}$

$\mathrm{R}=\mathrm{M} /(1.5 \times 6.69)=2278 \mathrm{kN}<2669 \mathrm{kN}$ 
Design of bottom ring beam:

Horizontal forces:

$6000 \mathrm{kN}$.

distributed

diagonal beam

considered.
The ring is loaded with one horizontal force of The ring must redistribute the force as a sinus shear force in the cylindrical wall. Only the in the direction of the force is

From Roark's Formulas for Stress \& Strain, 6th. edition, table 17, case 1 and case 20:

$1516 \mathrm{kNm}$

$\mathrm{M}=\mathrm{W} \times \mathrm{R} /(4 \times \pi)=6000 \times 3.175 / 12.566=$

Cross sectional properties:

$\mathrm{N}=0.75 \times \mathrm{W} / \pi=0.75 \times 6000 / \pi=1432 \mathrm{kN}$

$\mathrm{A}=900 \times 650-840 \times 600=81000 \mathrm{~mm}^{2}$

$\mathrm{mm}^{4}$

$I=900 \times 650^{3} / 12-840 \times 600^{3} / 12=5477 \times 10^{6}$

Stress:

$\mathrm{S}=\mathrm{I} / 325=16.85 \times 10^{6} \mathrm{~mm}^{3}$

$\mathrm{MPa}$

$f=1432 / 81+1516 / 16.85=108 \mathrm{MPa}<149$

Design of diagonal beam in bottom ring:

connection is

Moment from the excentricity of the chain carried by the diagonal beams

Normal force:

$\mathrm{N}=1 / 2 \times 6000=3000 \mathrm{kN}$

Moment:

Excentricity of horizontal chain force: 900/2 -

$200=$

edge of ring

$250 \mathrm{~mm}$

$\mathrm{M}=6000 \times 0.250=1500 \mathrm{kNm}$ at edge

$\mathrm{M}=1500 \times 6350 / 7.7000=1361 \mathrm{kNm}$ at.inner

beam

$\pi / 4=$

Differential pressure on water pipe: $200 \times 2.2^{2} \mathrm{x}$

$760 \mathrm{kN}$

1361 at center

$M=760 \times 7.0 /(3 \times 4)+1 / 2 \times 1500=1193<$

Shear force:

$\mathrm{Q}=1500 / 7.0+760 / 6=341 \mathrm{kN}$

Cross sectional properties:

$\mathrm{A}=2 \times 500 \times 30+840 \times 25=51000 \mathrm{~mm}^{2}$

$10^{6} \mathrm{~mm}^{4}$

$I=1 / 2 \times 500 \times 30 \times 870^{2}+25 \times 840^{3} / 12=6912 \times$

Normal stress:

$\mathrm{S}=\mathrm{I} / 450=15.36 \times 10^{6} \mathrm{~mm}^{3}$

$\mathrm{MPa}$

$f=3000 / 51.00+1361 / 15.36=147 \mathrm{MPa}<149$

Shear stress:

$\mathrm{f}=341000 /(900 \times 25)=15 \mathrm{MPa}$ 


\section{Design of exterior cylindrical shell:}

Shear stress:

$$
f=W /(\pi \times R \times t)=6000 \times 10^{3} /(\pi \times 3500 \times 25)
$$

$=22 \mathrm{MPa}$

Moment:

$$
\mathrm{M}=6000 \times 2.650=15900 \mathrm{kNm}
$$

Bending stress (neglecting vertical stiffeners): $f=M /\left(\pi \times R^{2} \times t\right)=15900 \times 10^{6} /(\pi \times$ $\left.3500^{2} \times 25\right)=$ $17 \mathrm{MPa}$

Critical elastic shear stress according to ECCS: "Buckling of Steel Shells"

$\mathrm{x}$

$$
\mathrm{f}=0.75 \times \mathrm{Ex}(\mathrm{R} /)^{0.5} \times(\mathrm{t} / \mathrm{R})^{1.25}=0.75 \times 2.1 \times 10^{5}
$$

$438 \mathrm{MPa} \gg 99 \mathrm{MPa}$

$$
(3500 / 1950)^{0.5} \times(25 / 3500)^{1.25}=
$$

Critical elastic compression stress according to API RP 2A:

$$
f_{x c}=2 \times 0.3 \times E \times t / D=0.6 \times 2.1 \times 10^{5} \times
$$

$25 / 7000=$

$$
450 \mathrm{MPa}
$$

Design compression stress: $\mathrm{f}_{\mathrm{xc}}=149\left(1.64-0.23 \times(7000 / 25)^{0.25}\right)=104>17 \mathrm{Mpa}$

Design of upper flat plate:

The part of the plate between the radial stiffeners is approximated with a cirkular plate with clamped edges, $R=650 \mathrm{~mm}$.

Moment per unit length:

$$
\begin{aligned}
& \mathrm{m}=\mathrm{p} \mathrm{R} \mathrm{R}^{2} / 8=200 \times 0.65^{2} / 8=10.56 \mathrm{kN} \\
& \mathrm{f}=6 \times 10560 / 30^{2}=70 \mathrm{MPa}<149 \mathrm{MPa}
\end{aligned}
$$

Design of beam over bearings:

Vertical load from bearing:

Tension in bolts:

Vertical reaction at inner end of beam:

Tension in one bolt:

Shear in beam:

Moment in beam:

Shear stress:

Cross sectional properties:

$20800+$

Depth of Center of gravity:

$580) / 48800=$

$+20800 x$

Bending stress, top:

Bending stress, bottom:

Considering holes for bolts:

\section{$2278 \mathrm{kN}$}

$\mathrm{N}_{\mathrm{t}}=2278 \times 2000 / 1515=3007 \mathrm{kN}$

$\mathrm{N}_{\mathrm{c}}=3007-2278=729 \mathrm{kN}$

$\mathrm{N}=3007 / 4=752 \mathrm{kN}<756 \mathrm{MPa}$

$\mathrm{Q}=2278 \mathrm{kN}$

$\mathrm{M}=2278 \times 0.380=866 \mathrm{kNm}$

$\mathrm{f}=2278 \times 10^{3} /(40 \times 600)=95 \mathrm{MPa}<99 \mathrm{MPa}$

$\mathrm{A}=400 \times 40+520 \times 40+300 \times 40=16000+$ $12000=48800 \mathrm{~mm}^{2}$

$\eta=(16000 \times 20+20800 \times 300+12000 \times$

$277.0 \mathrm{~mm}$

$\mathrm{I}=16000 \times 257^{2}+20800 \times 23^{2}+12000 \times 303^{2}$

$.520^{2} / 12=2638 \times 10^{6} \mathrm{~mm}^{4}$

$\mathrm{S}=\mathrm{I} / 323=8167 \times 10^{3} \mathrm{~mm}^{3}$ (top)

$\mathrm{S}=\mathrm{I} / 277=9524 \times 10^{3} \mathrm{~mm}^{3}$ (bottom)

$\mathrm{f}=866 / 8.167=106 \mathrm{MPa}<149 \mathrm{MPa}$

$\mathrm{f}=866 / 9.524=91 \mathrm{MPa}$

$\mathrm{f}=91 \times 400 / 280=130 \mathrm{MPa}<149 \mathrm{MPa}$ 
Anchor Verit, 1:25-

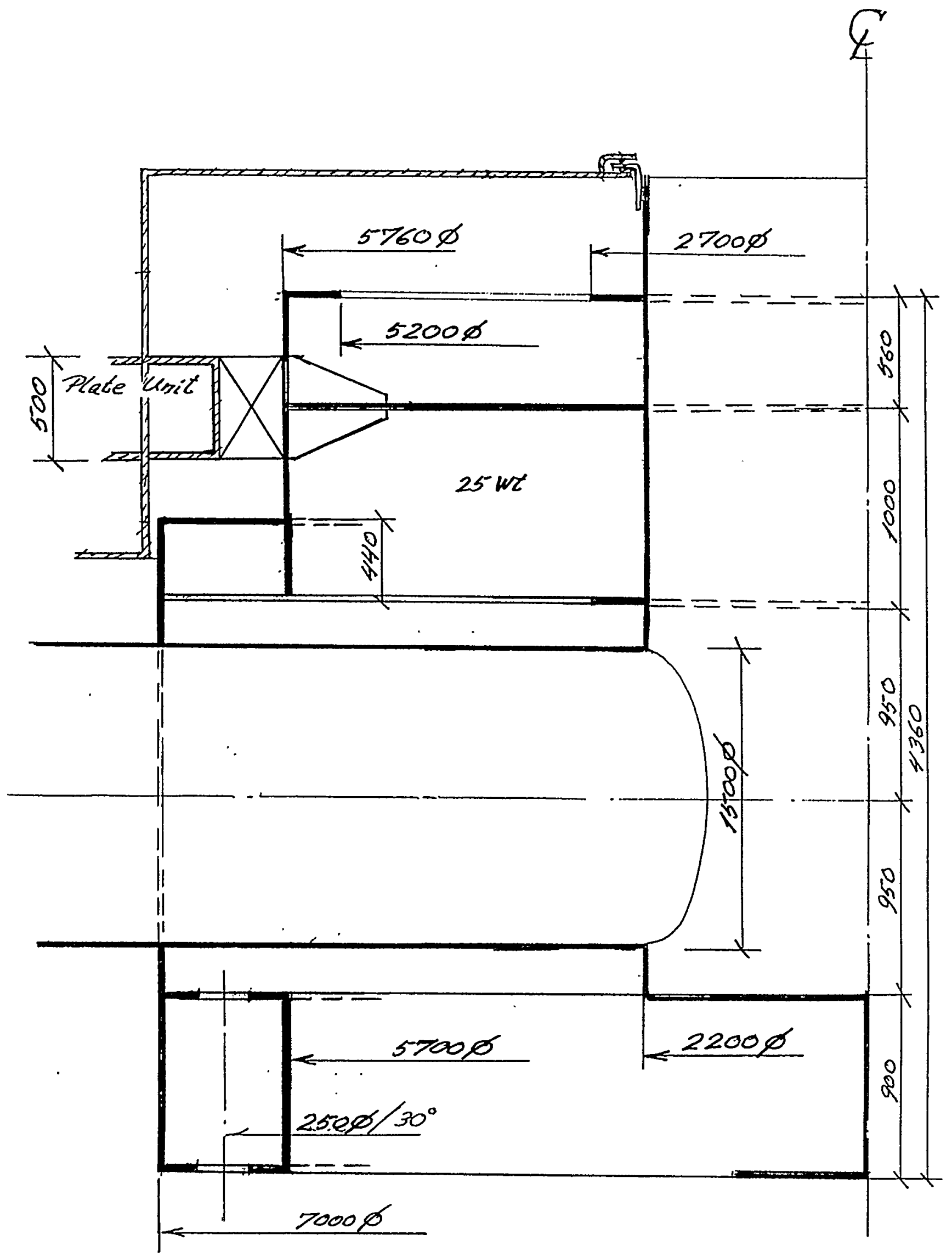


Anctor Unit, 1=25-

26.06 .2000

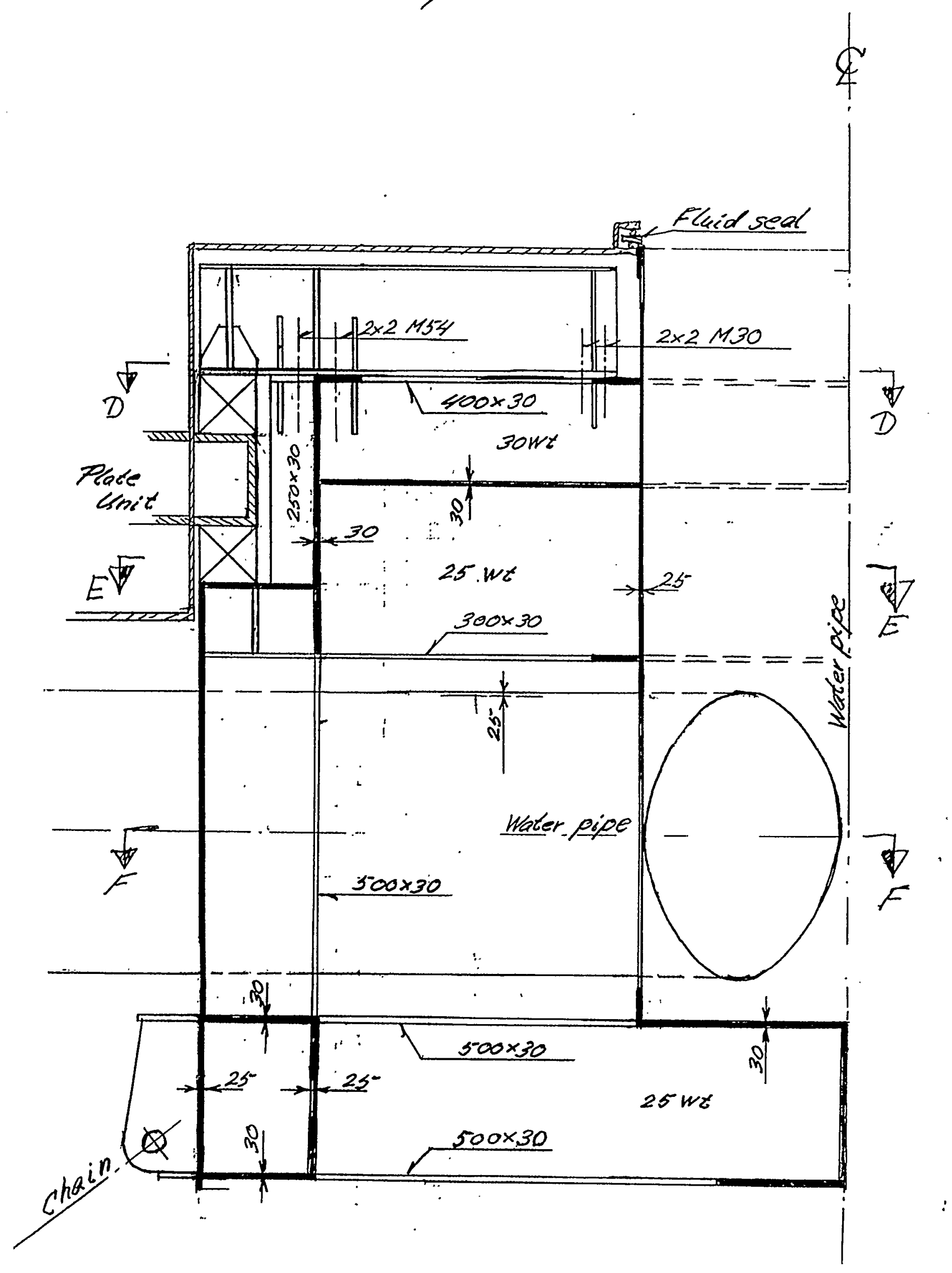




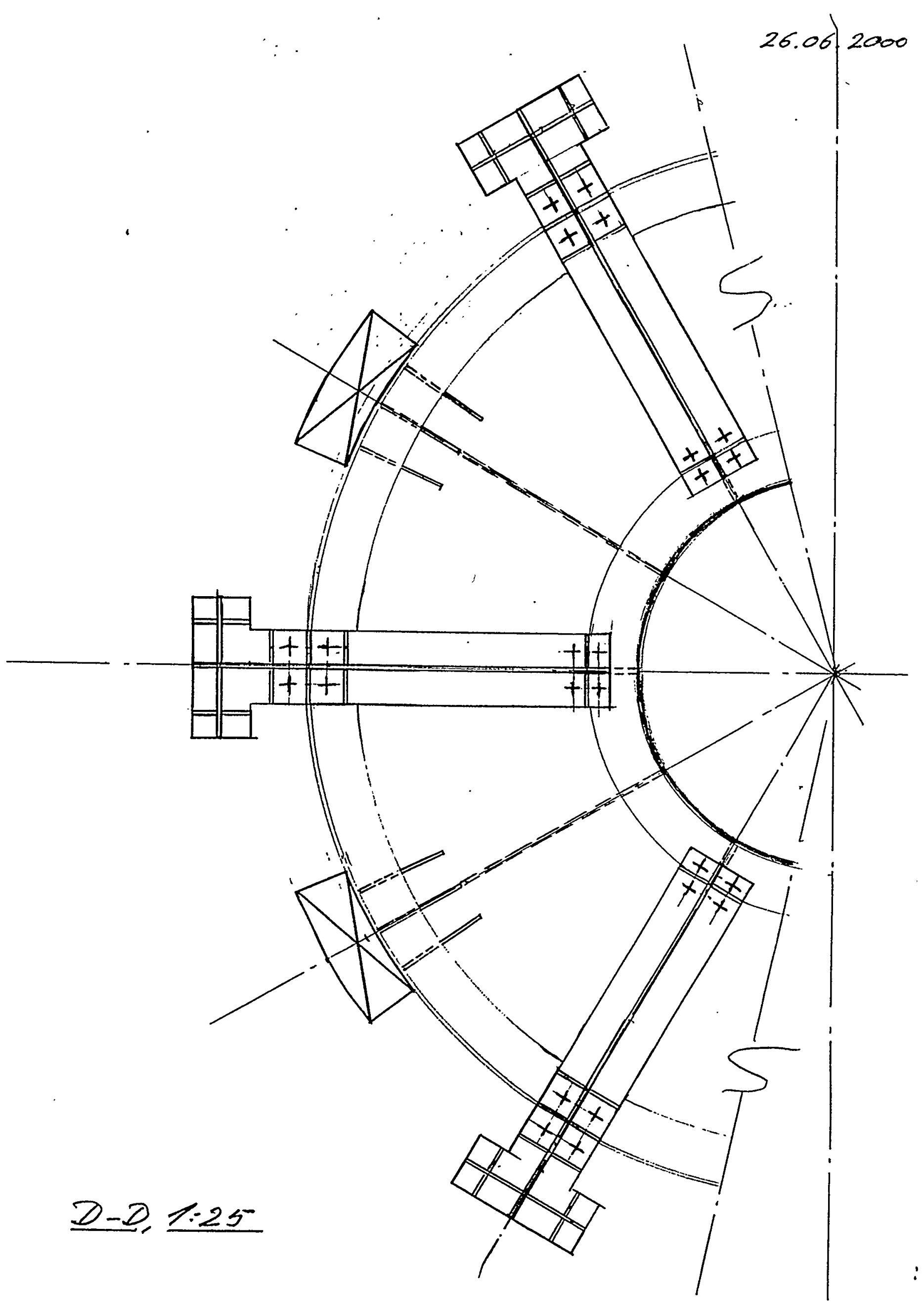




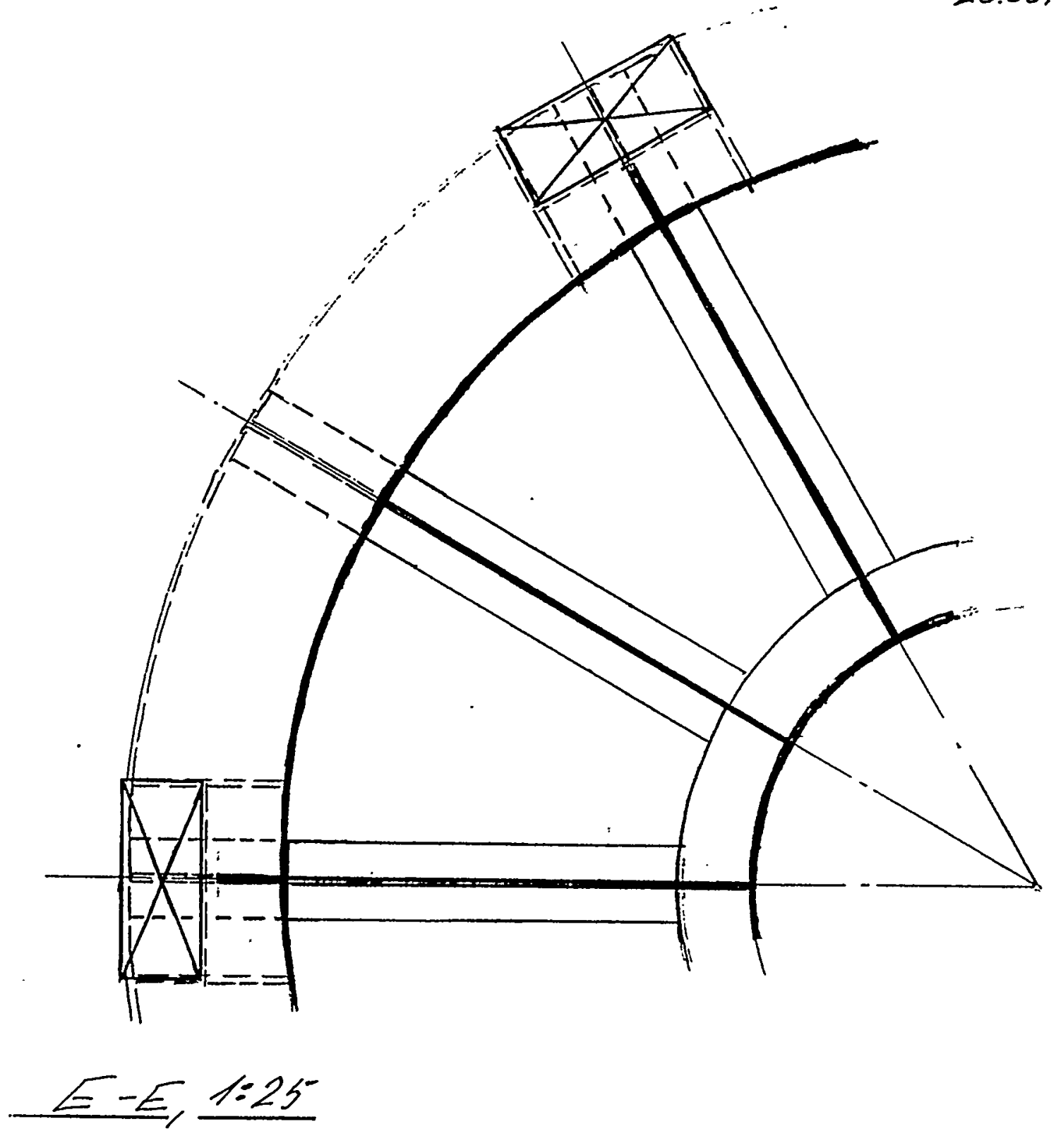




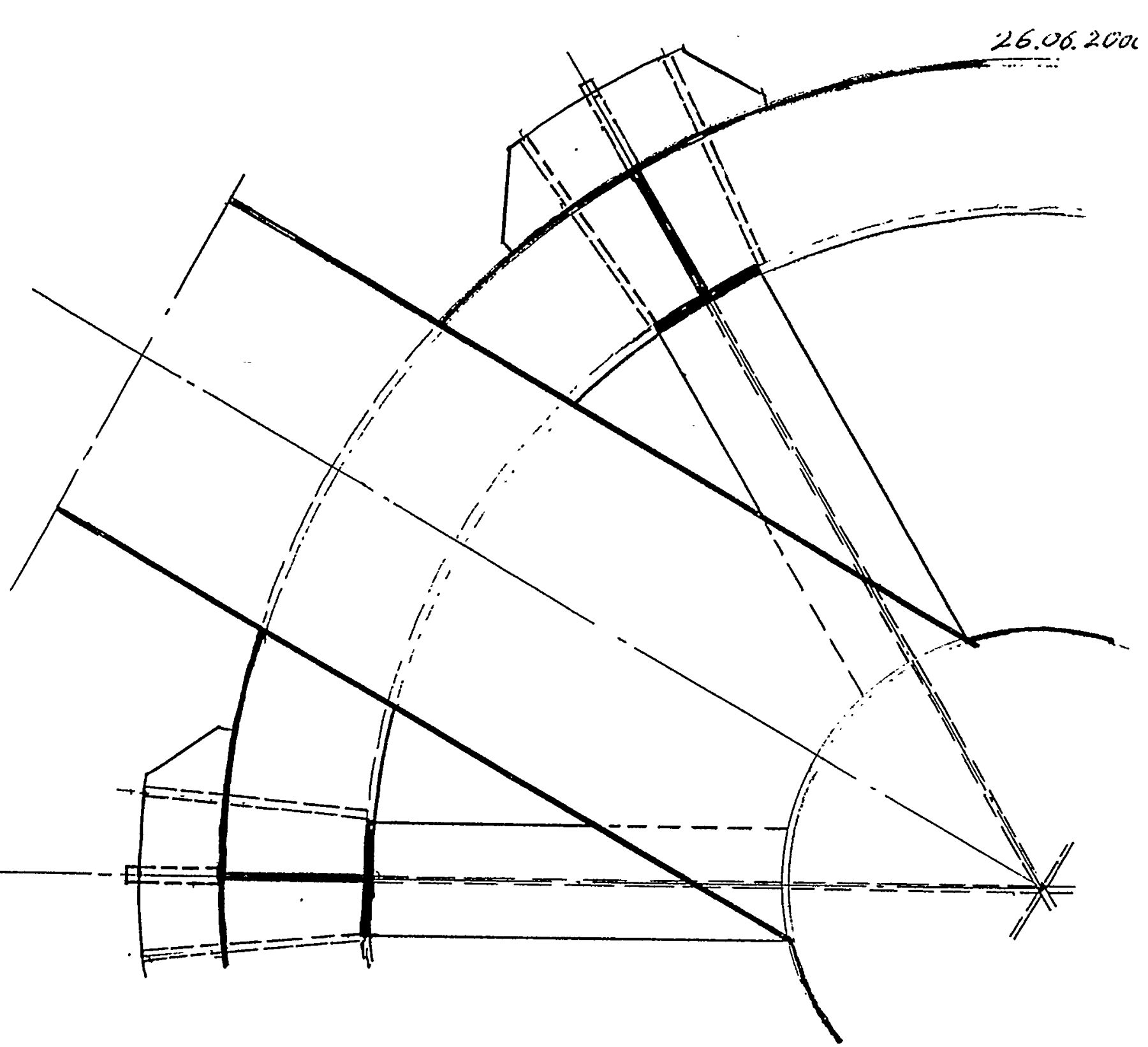

$E-F, 1=25$ 


\section{Beam overbearing}
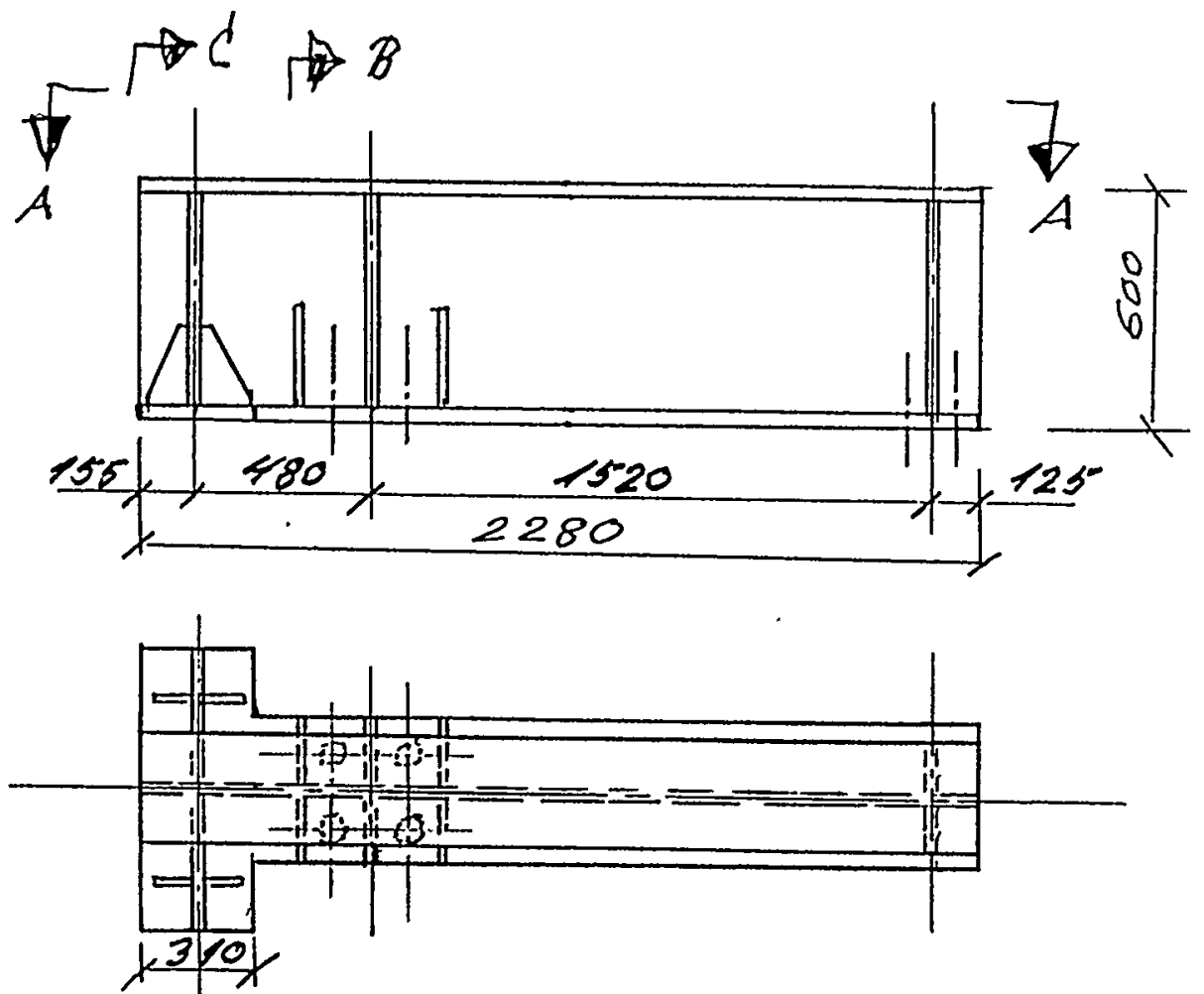

$A-A, 1=25$
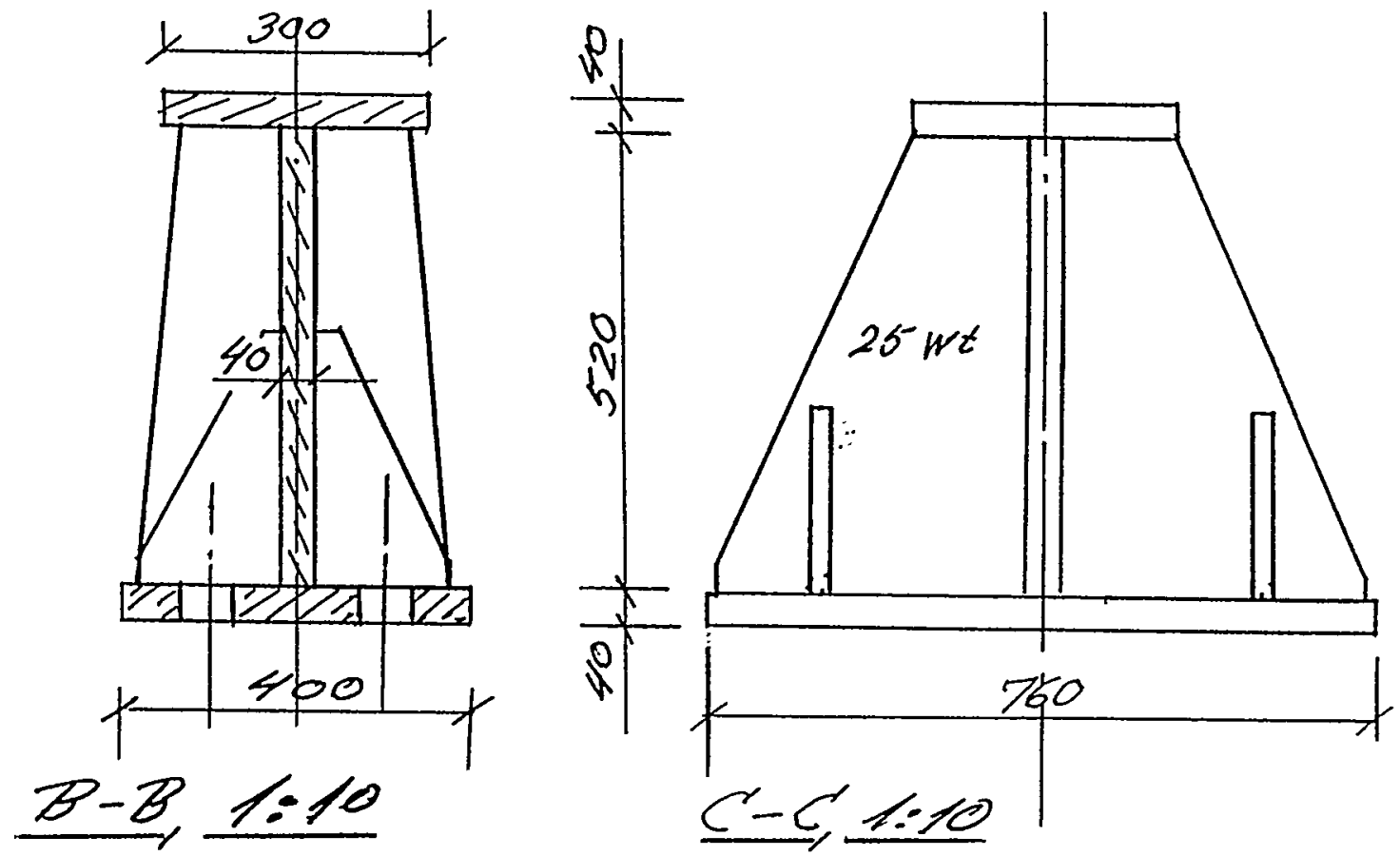


\section{Content of Chapter 3}

The content of this chapter is the design by Han-Padron Associates of the rapid ascent system. The high-pressure tanks are not detailed. They consist of standard 10-inch pipe that are coiled at convenient locations on the underside of the buoy.

The capacity to perform multiple mooring attempts is ensured by making the 10-inch tanks long enough. A standard pressure of $3000 \mathrm{psi}$ is used for these tanks. 50 meters of pipe contain sufficient air for one mooring attempt. Thus $2 * 200$ meters of piping is adequate to give the buoy the capability to perform 8 mooring attempts without having the high-pressure air replenished.

The electrical system has not been detailed. However it is based on 24 volt DC maintained by battery packs that are recharged each time a ship is present. The charging power is provided through an electromagnetic coupler surrounding the coupling for the high-pressure air supply from the ship.

The electrical system is duplicated (A system and B system) These systems have no common parts and are connected to their respective buoyancy control system. Each system has an acoustic receiver that "wakes the system up" when the ship approaches and sends the signal. An acoustic confirmation signal is repeatedly emitted in response. Following wake-up vertical lights are lit to assist in centering the buoy.

Following wake-up PLCs initiate a series of events as follows:

- Pressure in the low pressure tank is raised if required to $700 \mathrm{kPa}$ above ambient external pressure

- The leak proof valve is opened

- The pilot for the rapid opening valve is energized

The command to ascend rapidly is received as a modulated infrared signal from the ship. This causes actuation of the rapid opening valve. If this signal is lost for more than 30 seconds then the rapid descent system is automatically activated. This will for example be the case each time the mooring is successfully moored and the vessel shuts off the signal.

Rapid descent is also activated by an acoustic signal received by the wake-up unit.

The unit automatically goes into sleeping mode one hour after "wake up' or when battery charging starts. 
January 14,2000

Job No. 864-01

Mr. Jens Korsgaard

Suction Mooring Technology

318 North Post Road

Princeton, NJ 08550

Dear Jens,

Han-Padron Associates LLP is pleased to submit the attached report in conclusion of the design/analysis work of the SMT Pneumatic Deployment (Ascend)/Rapid Descend System. The report consists of a summary of the process data, simplified P\&ID, detailed calculations, valve catalog cuts and model numbers, and a budgetary estimate by Daniel Valve Company.

We appreciate the opportunity to carry out this work and we look forward to performing any subsequent work on the subject matter. Please call me should you have any question or comment.

Very truly yours,

HAN-PADRON ASSOCIATES, LLP

Yehoshua Gilad, P.E.

Mechanical Department Manager

Project Mánager

YG/yg

p:1800s 1864 Imechanicaltreportslfinalreport.doc

c: M. Faeth 
Suction Mooring Technology

Page 2

$\operatorname{Jan} 14,2000$

SMT Pneumatic Deployment (Ascend)/Rapid Descend System

Process Data and Description

1. General Description

The suction mooring pneumatic system consists of three compressed air tanks interconnected with pipes and valves to provide the mooring deployment motion (ascend), the emergency disengagement motion (rapid descend), and all associated compressed air services (refill, pressure maintenance, control functions, etc.). The three tanks include a high-pressure tank (HPT), a low-pressure tank (LPT), and a bottomless buoyancy tank (BBT).

The LPT supplies the necessary compressed air to purge the water from the BBT during deployment (ascend) and to purge the air from the BBT during rapid descent. Both operations are performed separately and by separate pipelines and control valves. The HPT serves as a reservoir used to replenish the LPT. All operations are controlled remotely from the moored vessel.

Compressed air is supplied to the mooring system by the vessel through a quickconnect coupling. It is assumed that periodic maintenance. will be performed on the mooring system. Thus, the air lines feeding the valve pilots, where instrument air is needed, are equipped with a filter/regulator/lubricator and a desiccant cartridge.

\section{Deployment System}

The LPT is designed to provide compressed air to the BBT for four separate deployment attempts. It has a design operating pressure range of 80.5 psia to 175 psia. Compressed air is transferred to the BBT through a 6 inch pipeline equipped with an 8 inch control valve. The volumes of the LPT and BBT are $140 \mathrm{cu}-\mathrm{m}$ and $150 \mathrm{cu}-\mathrm{m}$, respectively. The control valve is fully open/close within 2 seconds, and remains open continuously for 8 seconds (per deployment attempt). The incoming compressed air drives the water out of the BBT causing the mooring system to ascend to the vessel. The maximum pressure in the BBT is 66 psia which is the static pressure at the design depth of $35 \mathrm{~m}$. With each deployment attempt, the mass flowrate and remaining mass in the LPT change as do the pressure and temperature inside the LPT. In all cases, the flow through the interconnecting pipe is subsonic.

The conditions for each deployment attempt are shown in the following table: 


\begin{tabular}{|l|l|l|}
\hline \multicolumn{1}{|c|}{ Stage } & \multicolumn{1}{|c|}{ State Variable } & Mass Transfer \\
\hline Preliminary & & \\
\hline & Air Mass $=4,489 \mathrm{lb}_{\mathrm{m}}$ & \\
\hline & Pressure $=175 \mathrm{psia}$ & \\
\hline & Temperature $=60^{\circ} \mathrm{F}$ & \\
\hline After Deployment 1 & & \\
\hline & Air Mass $=4,035 \mathrm{lb}_{\mathrm{m}}$ & Air Mass $=454 \mathrm{lb}_{\mathrm{m}}$ \\
\hline & Pressure $=150.5 \mathrm{psia}$ & \\
\hline & Temperature $=38^{\circ} \mathrm{F}$ & \\
\hline After Deployment 2 & & \\
\hline & Air Mass $=3,639 \mathrm{lb}$ & Air Mass $=396 \mathrm{lb}$ \\
\hline & Pressure $=130.2 \mathrm{psia}$ & \\
\hline & Temperature $=18^{\circ} \mathrm{F}$ & \\
\hline & & \\
\hline & Air Mass $=3,295 \mathrm{lb}_{\mathrm{m}}$ & Air Mass $=344 \mathrm{lb}_{\mathrm{m}}$ \\
\hline & Pressure $=113.3 \mathrm{psia}$ & \\
\hline After Deployment 3 & Temperature $=-1^{\circ} \mathrm{F}$ & \\
\hline & & \\
\hline & Air Mass $=2,999 \mathrm{lb}_{\mathrm{m}}$ & Air Mass $=296 \mathrm{lb}_{\mathrm{m}}$ \\
\hline & Pressure $=99.4 \mathrm{psia}$ & \\
\hline & Temperature $=-17^{\circ} \mathrm{F}$ & \\
\hline
\end{tabular}

The final conditions after each deployment attempt were assumed to be the initial conditions for the following attempt.

\section{Rapid Descent System}

In case of an emergency, the disengagement of the mooring system involves a rapid submergence of the system back to the sea floor. In order to attain rapid submergence, the LPT purges the air from the BBT by a siphon effect. This is done through a $2 \frac{1}{2}$ inch pipeline and a 3 inch control valve. The pipeline and valve are sized to maintain a flowrate in the proximity of 70.64 SCFS. The pressure in the BBT is assumed to be atmospheric. In order to size components based on the worst case, the conditions in the LPT prior to the BBT rapid descent are assumed to correspond to the conditions immediately following the fourth deployment attempt, the lowest reserve of compressed air at the lowest pressure. During a rapid descent activation, the valve remains open for a period of 20 seconds.

After the rapid descent, the conditions in the LPT are as follows: 


$$
\begin{aligned}
& \text { Air Mass } \approx 2,889 \mathrm{lb}_{\mathrm{m}} \\
& \text { Pressure }=94.3 \mathrm{psia} \\
& \text { Temperature }=-24^{0} \mathrm{~F} \\
& \text { Mass Discharged }=110 \mathrm{lb} \mathrm{m} \\
& \text { Volumetric Flowrate }=72.12 \text { SCFS (Sonic) }
\end{aligned}
$$

The minimum design pressure of the LPT is 80.5 psia. The lowest possible operating pressure of 94.3 psia is greater than the minimum design pressure.

\section{Replenishment System}

The LPT is replenished by the HPT. After the fourth deployment attempt, the HPT replenishes the LPT to its maximum operating.pressure. The HPT is designed for two replenishment cycles before itself needing to be recharged. Recharging takes place at the surface and is performed by the vessel. The maximum and mimimum operating pressures of the HPT are 2,987 psia and 2,175 psia, respectively (set by the Scope of Work). The HPT is sized so that the pressure after the second replenishment is slightly above the minumum operating pressure.

The conditions for each recharging of the LPT are as shown in the following table:

\begin{tabular}{|l|l|c|}
\hline \multicolumn{1}{|c|}{ Stage } & \multicolumn{1}{|c|}{ State Variable } & Volumetric Flowrate \\
\hline Preliminary & & \\
\hline & Pressure $=2,987 \mathrm{psia}$ & \\
\hline & Temperature $=60^{\circ} \mathrm{F}$ & \\
\hline After Replenishment 1 & - & \\
\hline & Pressure $=2,571 \mathrm{psia}$ & Flowrate $=1,299$ SCFS \\
\hline & Temperature $=26^{\circ} \mathrm{F}$ & (Sonic Flow) \\
\hline After Replenishment 2 & & \\
\hline & Pressure $=2,182 \mathrm{psia}$ & Flowrate $=1,156$ SCFS \\
\hline & Temperature $=-8^{\circ} \mathrm{F}$ & (Sonic Flow) \\
\hline
\end{tabular}

The valve which opens/closes instantaneously, was calculated to remain open for 15 seconds for the first replenishment and 18 seconds for the second.

The LPT is designed with a pressure relief valve and was sized for the worst case possibility of the replenishment control valve being stuck open. In which case the relief valve needs to vent the mass flowrate from the HPT to the LPT under choked flow. The valve size was calculated to be 8 inch $\times 10$ inch, with a $10 \%$ overpressure.

For all systems, the control valves are electronically controlled from the vessel and pneumatically actuated. Instrument air is supplied by the HPT which is filtered and 
regulated to the operating pressure for each control valve. The quantities of instrument air employed throughout a full deployment/replenishment/descent cycle are assumed to be negligible.

The air flowrates, the resulting air masses, pressures, and temperatures inside the HPT and LPT were calculated assuming adiabatic expansion. The actual pressures and temperatures of the HPT and LPT in between and after deployment attempts and replenishments are higher due to the warming of the HPT and LPT by the ambient sea water. Any heat transfer from the seawater to the HPT and LPT were neglected. 


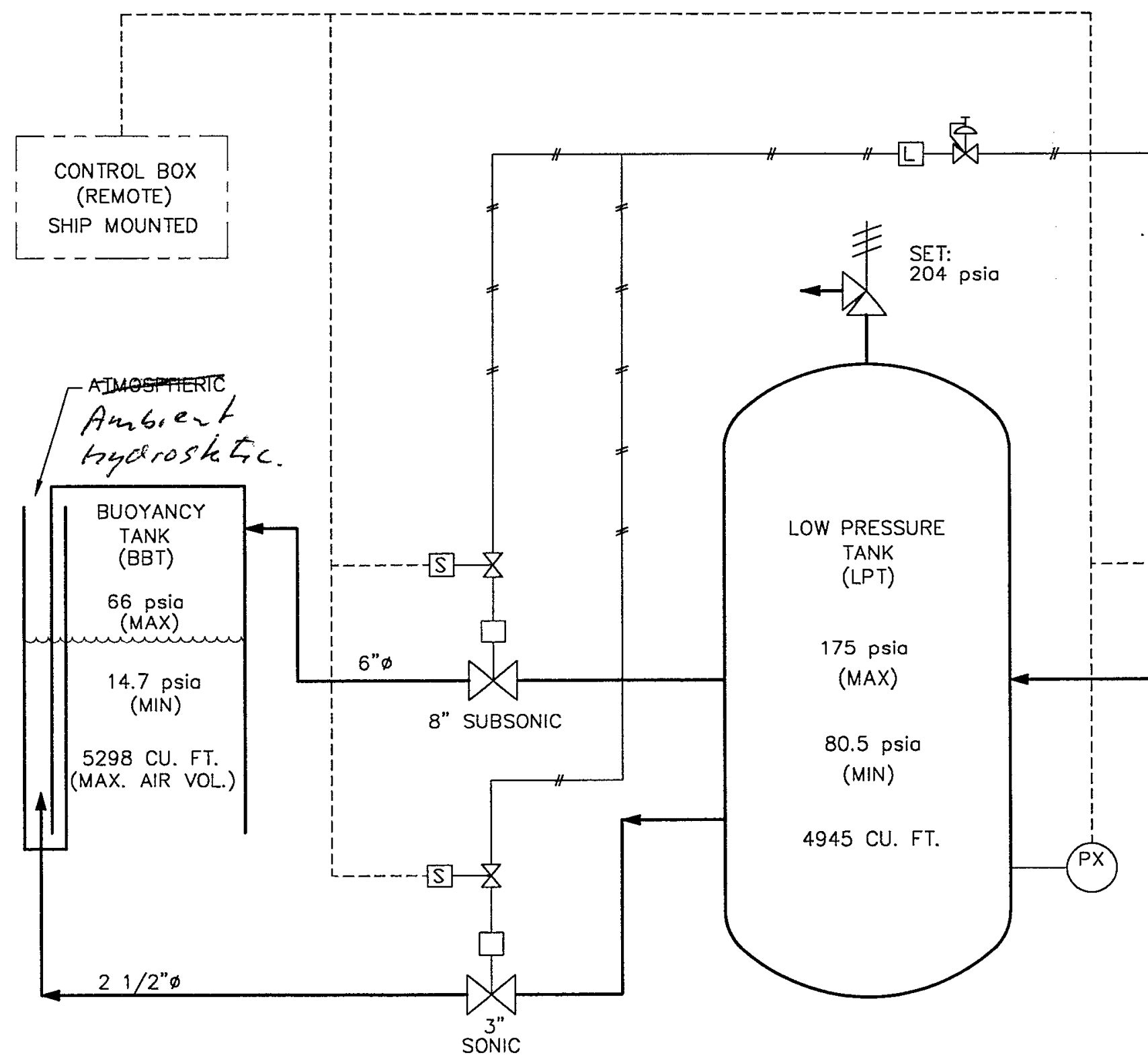




\section{Mooring System (LPT Replenishment)}

\section{Objective:}

The purpose is to size the High Pressure Tank (HPT), connecting piping, and control valve between the HPT and the Low Pressure Tank (LPT) in order to replenish the depleted LPT, after four deployment attempts, raising the mass and pressure inside the LPT to its maximum operating pressure. Determine the time required to replenish the LPT.

Given:

1) Air mass required to replenish LPT (from Filename: "Deployment-1")

2) LPT temperature, pressure, and air mass prior to replenishment (from Filename: "Deployment-1")

3) HPT initial temperature and operating pressure range

4) Required time to execute one replenishment

5) Pipe length

Find:

1) Pipe and contol valve diameters and flow conditions; subsonic or sonic velocity

2) Air flowrate for each replenishment

3) Resulting temperature, pressure, and air mass inside HPT after each replenishment (compare to minimum operating pressure for HPT)

4) Minimum required control valve $C_{V}$ (compare to actual manufacurer's $C_{V}$ )

\section{Methodology:}

The resulting pressure, temperature, and air mass inside the LPT after the fouth deployment attempt are known from the previous calculation (Filename: "Deployment-1"). The mass of each replenishment is equal to the sum of the masses discharged from the LPT during the four deployment attempts. The air flowrate between the HPT and LPT was determined based on:

1) The difference in pressure between the HPT and LPT

2) The pressure drop for an assumed pipe size, length, and number and type of fittings

3) An initial guess for the volume of the HPT

4) An assumed nominal pipe and valve diameter of 2 inches

The intial conditions of the LPT and HPT prior to the first replenishment, the final condition required for the LPT, and the required mass transfer are given. The flowrate and time required for replenishment were calculated and compared to the specified time for replenishment.

In order to accurately obtain the air flowrate and valve open duration for each replenishment, a correction factor, $Y$, was determined graphically based on the parameters for each replenishment, and input individually for each attempt. The air flowrate, the resulting air mass, pressure, and temperature inside the HPT were calculated, assuming adiabatic expansion. The resulting conditions inside the HPT after the first replenishment were used as the initial conditions inside the HPT for the second replenishment.

Following the above calculations, the minimum required $\mathrm{C} v$ for the control valve, installed in the line connecting the two tanks, was calculated based on data obtained from Daniel Valve Co. This minimum required $\mathrm{C} v$ was compared to the actual $\mathrm{C} v$ for the valve selected, from the Daniel product line, to determine the adequacy of the valve. 


\section{Assumptions:}

1) Air is an ideal gas

2) Process is assumed to be isentropic, reversible adiabatic, closed system

3) Due to a rapid open/close time of the control valve, 2 seconds, neglect flow while control valve is opening or closing

4) Range of water temperature given as 10 to 20 degree $C$ : assume 15 degrees $C$ for calculations

6) Neglect temperature and pressure increase in HPT between replenishments due to HPT warming by the sea water.

\section{References:}

1) Crane, Flow of Fluids, Technical Paper No. 410, 1982

2) Mechanical Engineering Reference Manual for the PE exam, 10th edition, 1998

3) Perry's Chemical Engineers' Handbook, 6th edition, 1984

4) Daniel Valve Co., fax dated 11/24/99, from Jim Wass

\section{Conclusions:}

(Refer to Summary on page 12 of this calculation)

The piping connecting the HPT to the LPT is a 2 inch diameter schedule 40 pipe. The control valve is also 2 inch. In both replenishments, the flow is sonic.

Each replenishment delivers the same mass of air to the LPT. The time neccessary to replenish the LPT the first time is 15 seconds and the second is 18 seconds. The second replenishment lasts longer due to the depletion of air in the HPT by the first replenishment. The specified replenishment time is 20 minutes, or 1200 seconds. In order to achieve the specified time, the pipe size would have to be approximately $3 / 8^{\prime \prime}$ in diameter or the resistance in the 2 inch line would need to be significantly increased. Possible options may be to insert an orifice with a pinhole near the LPT or to substantially increase the length of the 2 inch piping by spiralling it around the BBT.

The pressure and temperature drops inside the HPT as a result of the two replenishments, assuming adiabatic expansion, are $805 \mathrm{psi}$ and $68^{\circ} \mathrm{F}$, respectively. Assuming adiabatic expansion is conservative. The actual pressure and temperature are higher due to the warming of the HPT by the ambient sea water during the time intervals between replenishments.

The HPT was sized so that at the completion of the two replenishments, the resulting pressure of the HPT would approach its minimum operating pressure at which point it would need to be recharged. The HPT volume was calculated to be $1200 \mathrm{cu}-\mathrm{ft}$.

The minimum required valve Cv was calculated to be 97 which is well within the actual Cv of 120 . 


\section{Letter Symbols for Variables:}

\section{Piping Parameters}

ID Inside diameter of pipe (in)

$f \quad$ Pipe friction factor (Crane, page A-26)

$L_{\text {pipe }} \quad$ Length of pipe (ft)

$\mathrm{N}_{\text {elbow }} \quad$ Number of $90^{\circ}$ elbows

$\mathrm{C}_{v} \quad$ Valve coefficient of friction (Daniel Valve Co.)

$\mathrm{K}_{\text {entrance }}$ Resistance coefficient for pipe entrance losses (Crane, page A-29)

$\mathrm{K}_{\text {exit }} \quad$ Resistance coefficient for pipe exit losses (Crane, page A-29)

$\mathrm{K}_{\mathrm{plpe}} \quad$ Resistance coefficient for pipe (Crane, page 2-8)

$K_{\text {valve }} \quad$ Resistance coefficient control valve (Daniel Valve Co.)

$\mathrm{K}_{\text {elbow }} \quad$ Resistance coefficient for elbow (Crane, page A-29)

$K_{\text {total }} \quad$ Sum of above coefficients

$N_{\text {II }} \quad$ Number of friction factors

\section{Process Parameters}

$V_{\text {HPT }} \quad H P T$ volume (cu-ft) (GUESS)

$\mathrm{V}_{\text {LPT }} \quad$ LPT volume (cu-ft)

$\Delta t_{\text {actual_1 }}$

Duration of control valve in open position for first replenishment - actual (sec)

$\Delta t_{\text {actual_2 }}$

Duration of control valve in open position for second replenishment - actual (sec)

$\Delta \mathrm{t}_{\text {specified }}$

Duration of control valve in open position - specified (sec)

$T_{w}$

$P_{\text {HPT_min }}$

Average water temperature and initial temperature of air in HPT ( ${ }^{\circ}$, R)

$R_{\text {air }}$ Minimum design pressure in HPT (psia)

k Individual gas constant for air ( $\mathrm{ft}^{\star} \mathrm{lbf} / \mathrm{b}^{\star} \mathrm{R}$ ) (Crane, page $\mathrm{A}-8$ )

S Specific heats' ratio for air, taken at max.HPT operating pressure and temperature (200 atm and 600 F) (Crane, page A-8, Perry's, page 3-144)

$P_{\text {HPT_1a }} \quad$ Density of air in HPT prior to first replenishment of LPT (Ib/cu. ft)

$\rho_{\text {HPT_2a }} \quad$ Density of air in HPT prior to secondt replenishment of LPT (lb/cu. $\mathrm{ft}$ )

$\rho_{\text {STP }} \quad$ Density of air at STP (standard temperature and pressure) (lb/cu. ft) (Crane, page A-10)

$\mathrm{K}_{\mathrm{d}} \quad$ Ratio of densities $\left(\rho_{\text {STP } /} \rho_{\text {HPT_1a }}\right)$

$P_{\text {LPT_4b_a }} \quad$ Pressure in LPT after fourth deployment attempt (psia) (from Filename: "Deployment-1")

$P_{\text {LPT_Ab_g }} \quad$ Pressure in LPT after fourth deployment attempt (psig)

$T_{\text {LPT_4b }}$ Temperature in LPT after fourth deployment attempt (psia) (from Filename: "Deployment-1")

$\rho_{\mathrm{w}} \quad$ Density of sea water $(\mathrm{gr} / \mathrm{cu}-\mathrm{cm})$

$\mathrm{P}_{\mathrm{a}} \quad$ Atmospheric pressure (14.7 psi)

\section{Pressure}

$P_{\text {HPT_1a_9 }}$

PHPT_1a_a

PLPT_2a_g

$P_{\text {HPT_tb_a }}$ and $P_{\text {LPT_2a_a }}$

$P_{\text {HPT_2b_a }}$
HPT pressure prior to first replenishment (psig)

HPT pressure prior to first replenishment (psia)

HPT pressure prior to second replenishment (psig)

HPT pressure prior to second replenishment (psia)

HPT pressure after second replenishment (psia) 


\section{Temperature}

$T_{\text {HPT_1a }}$

HPT temperature prior to first replenishment $\left({ }^{0} \mathrm{R}\right)$

$T_{\text {HPT_tb }}$ and $T_{\text {HPT_2a }}$

$T_{\text {HPT_2b }}$

HPT temperature prior to second replenishment $\left({ }^{\circ} \mathrm{R}\right)$

HPT temperature after second replenishment $\left({ }^{\circ} \mathrm{R}\right)$

Mass

$m_{\text {LPT_1a }}$

HPT mass prior to first replenishment (lb)

$m_{\text {LPT_1b }}$ and $P_{\text {LPT_2a }}$ HPT mass prior to second replenishment (lb)

$m_{\text {LPT_2b }}$

HPT mass after second replenishment (lb)

$m_{\text {replenishment_1 }}$

Mass of first replenishment (lb)

$m_{\text {replenishment_2 }}$

Mass of second replenishment (lb)

\section{Discharge Flowrates}

$q_{1} \quad$ Replenishment 1 flowrate (Standard cu-ft per sec - SCFS)

$\mathrm{q}_{2}$ Replenishment 2 flowrate (Standard cu-ft per sec - SCFS)

\section{Air Density and Specific Volume Parameters}

$\rho_{1 \mathrm{a}} \quad$ Density of air in HPT prior to first replenishment (lb/cu. $\left.\mathrm{ft}\right)$

$\rho_{1 b}$ and $\rho_{2 a} \quad$ Density of air in HPT prior to second replenishment (lb/cu. ft)

$\rho_{2 b} \quad$ Density of air in HPT after second replenishment (lb/cu. ft)

$v_{1 a} \quad$ Specific volume of air in HPT prior to first replenishment (cu. $\mathrm{ft} / \mathrm{b}$ )

$v_{1 b}$ and $v_{2 a} \quad$ Specific volume of air in HPT prior to second replenishment (cu. ft/lb)

$v_{2 b} \quad$ Specific volume of air in HPT after second replenishmen (cu. $\mathrm{ft} / \mathrm{lb}$ )

\section{Graphical Flowrate Correction Factor}

$Y_{1} \quad$ Correction factor for replenishment 1

$\mathrm{Y}_{2} \quad$ Correction factor for replenishment 2 


\section{Cv Calculation Parameters}

$X_{1} \quad$ Critical pressure drop ratio - given - (per Daniel Valve Co.)

$q_{1} \quad$ Maximun discharge flowrate (standard cu-ft per second - SCFS) (corresponding to replenishment 1 flowrate)

Q Maximun discharge flowrate (standard cu-ft per hour - SCFH)

$\Delta \mathrm{P}_{\min } \quad$ Minimum $\triangle \mathrm{P}$ across control valve between HPT and LPT

$X \quad$ Actual pressure drop ratio (per Daniel Valve Co.)

$Y \quad$ Expansion factor (per Daniel Valve Co.)

$\mathrm{C}_{\mathrm{Vrequired} \text { min }} \quad$ Minimum required $\mathrm{G}_{\mathrm{V}}$ for control valve

$\mathrm{C}_{\text {vactual }}$

Design $\mathrm{C}_{v}$ for control valve (per Daniel Valve Co.)

$\mathrm{gr}=1 \cdot \frac{\mathrm{kg}}{1000}$ 


\section{INPUTS}

$\mathrm{ID}:=2.067$ in

$f:=0.019$

$\mathrm{L}_{\text {pipe }}:=50 \cdot \mathrm{ft}$

$N_{\text {elbow }}:=2$

$N_{f f}:=14$

$C_{v}:=120$

$\mathrm{K}_{\text {entrance }}:=0.5$

$\mathrm{K}_{\text {exit }}:=1.0$

\section{$\mathrm{V}_{\mathrm{HPT}}:=1200 \cdot \mathrm{ft}^{3} \quad$ Guess}

$\mathrm{V}_{\mathrm{LPT}}:=4945 \cdot \mathrm{ft}^{3}$

$\Delta t_{\text {specified }}:=1200 \cdot \mathrm{s}$

$\mathrm{T}_{W}:=60 \cdot \mathrm{R}$

$P_{\text {HPT_1a_a }}:=2987 \cdot p s i$

$\mathrm{P}_{\text {HPT_min }}:=2175 \cdot \mathrm{psi}$

$R_{\text {air }}:=53.3 \cdot \frac{\mathrm{ft} \cdot \mathrm{lbf}}{\mathrm{lb} \cdot \mathrm{R}}$

$\mathrm{k}:=1.8$

$\mathrm{m}_{\text {replenishment_1 }}:=1490 \cdot \mathrm{lb}$

$P_{\text {LPT_4b_a }}:=99.4 \cdot p s i$

$T_{L P T \_4 b}:=443 \cdot R$

$\rho_{\mathrm{STP}}:=0.0763 \cdot \frac{\mathrm{lb}}{\mathrm{ft}^{3}}$

$\rho_{\mathrm{w}}:=1.03 \cdot \frac{\mathrm{gr}}{\mathrm{cm}^{3}}$

$P_{a}:=14.7 \cdot p s i$ 


\section{ONE-TIME CALCULATIONS}

\section{Initial Temperature:}

$\mathrm{T}_{\text {HPT_1a }}:=\mathrm{T}_{\mathrm{W}}+460 \cdot \mathrm{R} \quad \mathrm{T}_{\text {HPT_1a }}=520 \circ \mathrm{R}$

Initial pressure in HPT prior to first replenishment of LPT:

$\mathrm{P}_{\text {HPT_1a_a }}=2987 \circ \mathrm{psi}$

HPT pressure (psia)

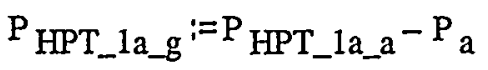

$P_{\text {HPT_la_g }}=2972 \circ \mathrm{psi}$

HPT pressure (psig)

Calculating air density in HPT prior to first replenishment of LPT:

$\rho_{\text {HPT_1a }}:=\frac{P_{\text {HPT_1a_a }}}{R_{\text {air }} \cdot T_{\text {HPT_la }}} \quad \rho_{H P T \_1 a}=15.5190 \frac{\mathrm{lb}}{\mathrm{ft}^{3}} \quad$ (ME reference manual, page 24-14)

\section{Conversion between Standard Cubic Feet (SCF) and Actual Cubic Feet (ACF):}

$$
\begin{aligned}
& \rho_{\mathrm{STP}}:=0.0763 \cdot \frac{\mathrm{lb}}{\mathrm{ft}^{3}} \\
& \mathrm{~K}_{\mathrm{d}}:=\frac{\rho_{\mathrm{STP}}}{\rho_{\mathrm{HPT}} 1 \mathrm{a}} \quad \mathrm{K}_{\mathrm{d}}=0.0049
\end{aligned}
$$

Calculating total Resistance Coefficient (Ktotal) for pipe, fittings, valve, etc.:

$$
\begin{array}{lll}
\mathrm{K}_{\text {elbow }}:=\mathrm{N}_{\text {elbow }} \cdot \mathrm{N}_{\text {ff }} \cdot \mathrm{f} & \mathrm{K}_{\text {elbow }}=0.532 & \mathrm{~K}_{\text {entrance }}:=0.5 \\
\mathrm{~K}_{\text {pipe }}:=\mathrm{f} \cdot\left(\frac{\mathrm{L}_{\text {pipe }}}{\mathrm{ID}}\right) & \mathrm{K}_{\text {pipe }}=5.515 & \mathrm{~K}_{\text {exit }}:=1.0
\end{array}
$$

$\mathrm{K}_{\text {valve }}:=1443 \cdot\left[\frac{\frac{\pi}{4} \cdot\left(\frac{\mathrm{ID}}{\mathrm{in}}\right)^{2}}{\mathrm{C}_{\mathrm{v}}}\right]^{2} \quad \mathrm{~K}_{\text {valve }}=1.128 \quad \begin{aligned} & \text { (Daniel Valve Co., fax 11/24/99) } \\ & \text { (Equation is valid for SONIC and SUBSONIC flow) }\end{aligned}$

$\mathrm{K}_{\text {total }}:=\mathrm{K}_{\text {entrance }}+\mathrm{K}_{\text {exit }}+\mathrm{K}_{\text {elbow }}+\mathrm{K}_{\text {pipe }}+\mathrm{K}_{\text {valve }} \quad \mathrm{K}_{\text {total }}=8.676$ 


\section{Calculations for Replenishment 1}

$P_{\text {HPT_1a_a }}=2987 \circ$ psi

$\mathrm{T}_{\text {HPT_1a }}=520 \circ \mathrm{R}$

$\mathrm{m}_{\text {replenishment_1 }}=1490 \mathrm{olb}$

\section{Calculating Correction Factor Y1 using DeltaP1:}

$P_{L P T \_4 b \_g}:=P_{L P T} 4 b \_a-P_{a}$

$\mathrm{P}_{\text {LPT_4b_g }}=84.7$ opsi

$\Delta \mathrm{P}_{1}:=\mathrm{P}_{\mathrm{HPT}} \mathrm{Ha}_{\mathrm{g}} \mathrm{g}-\mathrm{P}_{\text {LPT_4b_g }}$

$\Delta \mathrm{P}_{1}=2888$ opsi

$\mathrm{R}_{\text {calculated_1 }}:=\frac{\Delta \mathrm{P}_{1}}{\mathrm{P}_{\text {HPT_1a_a }}}$

$\mathrm{R}_{\text {calculated_1 }}=0.97$

\section{REFER TO CRANE page A-22}

Based on: $\quad \mathrm{K}_{\text {total }}=8.7$

If $R_{\text {calculated_1 }}>R_{\text {graphical_max }}$ then flow is SONIC; use $R_{\text {graphical_max }}$ to determine $Y_{1}$ \& new $\Delta P_{1}$

If $R_{\text {calculated_1 }}<R_{\text {graphical_max }}$, then flow is SUBSONIC; use $R_{\text {calculated_1 }}$ to determine $Y_{1}$

$\mathrm{R}_{\text {calculated_1 }}=0.97$

$\mathrm{R}_{\text {graphical_max }}:=0.775 \quad \underline{\text { SONIC }}$

\section{INPUT}

$Y_{1}:=0.6875$

$\mathrm{R}_{\text {graphical_max }}:=0.775$

New $\Delta P_{1}:=R_{\text {graphical_max }} \cdot P_{H P T}{ }_{-1 a}$ a

New $\Delta P_{1}=2315 \circ p s i$

\section{Calculating First Replenishment Flowrate in (SCFS):}

(Crane, page 3-4 and 4-14)

$\mathrm{q}_{1}:=11.3 \cdot \mathrm{Y}_{1} \cdot\left(\frac{\mathrm{ID}}{\mathrm{in}}\right)^{2} \cdot\left[\frac{\left(\frac{\mathrm{New} \Delta \mathrm{P}_{1}}{\mathrm{psi}}\right) \cdot\left(\frac{\mathrm{P}_{\mathrm{HPT} \_1 \mathrm{a} \_\mathrm{a}}}{\mathrm{psi}}\right)}{\mathrm{K}_{\text {total }} \cdot\left(\frac{\mathrm{T}_{\mathrm{HPT} \_1 \mathrm{a}}}{\mathrm{R}}\right)}\right]^{0.5} \cdot \frac{\mathrm{ft}^{3}}{\mathrm{~s}}$

$q_{1}=1299 \cdot \frac{\mathrm{ft}^{3}}{\mathrm{~s}}$

(SCFS) 


$$
\begin{aligned}
& \Delta \mathrm{t}_{\text {actual_1 }}:=\frac{\mathrm{m}_{\text {replenishment_1 }}}{\mathrm{q}_{1} \cdot \mathrm{K}_{\mathrm{d}} \cdot \rho_{\mathrm{HPT}} \text { _la }} \\
& \Delta \mathrm{t}_{\text {actual_l }}=15 \mathrm{~s} \quad \text { Actual At ID }=2.067 \text { in } \\
& \Delta \mathrm{t}_{\text {specified }}=1200 \mathrm{~s} \quad \text { Specified }
\end{aligned}
$$

\section{Calculating mass of air in HPT prior to first replenishment of LPT (based on quess of HPT volume):}

$$
\begin{aligned}
& \mathrm{m}_{\text {HPT_la }}:=\rho_{\text {HPT_1a }} \cdot \mathrm{V}_{\text {HPT }} \\
& \mathrm{m}_{\text {HPT_1a }}=18623 \mathrm{olb}
\end{aligned}
$$

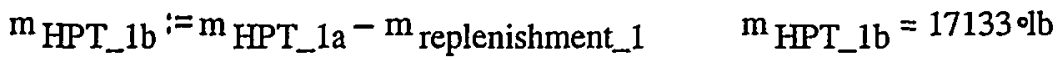

\section{Calculating specific volume of air in HPT before and after first replenishment of LPT:}

$$
\begin{aligned}
& \rho_{1 \mathrm{a}}:=\rho_{\text {HPT_1a }} \\
& \rho_{1 \mathrm{a}}=15.519 \mathrm{o} \frac{\mathrm{lb}}{\mathrm{ft}^{3}} \\
& \mathrm{v}_{1 \mathrm{a}}:=\frac{1}{\rho_{1 \mathrm{a}}} \\
& \mathrm{v}_{1 \mathrm{a}}=0.064 \frac{\mathrm{\textrm {ft } ^ { 3 }}}{\mathrm{lb}} \\
& \rho_{1 b}:=\frac{m_{H P T} 1 b}{V_{H P T}} \\
& \rho_{1 b}=14.277 \frac{\mathrm{ob}}{\mathrm{ft}^{3}} \\
& v_{1 b}:=\frac{1}{\rho_{1 b}} \\
& v_{1 b}=0.07 \frac{\mathrm{ot}^{3}}{1 b}
\end{aligned}
$$

\section{Calculating temperature and pressure of air in HPT after first replenishment of LPT:}

$\mathrm{T}_{\text {HPT_1b }}:=\mathrm{T}_{\text {HPT_1a }} \cdot\left(\frac{\mathrm{v}_{1 \mathrm{a}}}{\mathrm{v}_{1 \mathrm{~b}}}\right)^{\mathrm{k}-1} \quad \mathrm{~T}_{\mathrm{HPT}_{-} 1 \mathrm{~b}}=486 \mathrm{R} \quad \quad \quad$ (ME reference manual, page 25-8)

$\mathrm{P}_{\text {HPT_1b_a }}:=\mathrm{P}_{\text {HPT_1a_a }} \cdot\left(\frac{\mathrm{v}_{1 \mathrm{a}}}{\mathrm{v}_{1 \mathrm{~b}}}\right)^{\mathrm{k}} \quad \mathrm{P}_{\text {HPT_1b_a }}=2571$ opsi $\quad$ (ME reference manual, page 25-8)

$$
\mathrm{P}_{\text {HPT_min }}=2175 \text { psi }
$$




\section{Calculations for Replenishment 2}
$\mathrm{P}_{\text {HPT_2a_a }}:=\mathrm{P}_{H P T \_1 b \_a}$
$P_{\text {HPT_2a_a }}=2571$ opsi
$\mathrm{T}_{\text {HPT_2a }}:=\mathrm{T}_{\text {HPT_1b }}$
$\mathrm{T}_{\text {HPT_2a }}=486 \circ$
$\mathrm{m}_{\text {HPT_2a }}:=\mathrm{m}_{\text {HPT_lb }}$
$\mathrm{m}_{\text {HPT_2a }}=17133 \mathrm{olb}$
$\mathrm{m}_{\text {replenishment_2 }}:=\mathrm{m}_{\text {replenishment_1 }}$
$\mathrm{m}_{\text {replenishment_2 }}=1490 \mathrm{olb}$
$\rho_{\text {HPT_2a }}:=\rho_{1 b}$
$\rho_{\text {HPT_2a }}=14.28 \frac{\mathrm{ob}}{\mathrm{ft}^{3}}$

\section{Calculating Correction Factor Y2 using DeltaP2:}
$\mathrm{P}_{H P T \_2 a \_g}:=\mathrm{P}_{H P T \_2 a \_a}-\mathrm{P}_{\mathrm{a}}$
$\mathrm{P}_{\text {HPT_2a_g }}=2556 \circ \mathrm{psi}$

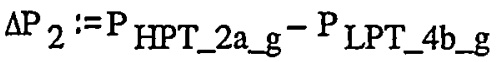
$\Delta \mathrm{P}_{2}=2471$ opsi
$\mathrm{R}_{\text {calculated_2 }}:=\frac{\Delta \mathrm{P}_{2}}{\mathrm{P}_{\text {HPT_2a_a }}}$
$\mathrm{R}_{\text {calculated_2 }}=0.96$

\section{REFER TO CRANE page A-22}

Based on: $\quad \mathrm{K}_{\text {total }}=8.7$

If $R_{\text {calculated_2 }}>R_{\text {graphical_max }}$, then flow is SONIC; use $R_{\text {graphical_max }}$ to determine $Y_{2}$ \& new $\Delta P_{2}$

If $R_{\text {calculated_2 }}<R_{\text {graphical_max }}$ then flow is SUBSONIC; use $R_{\text {calculated_2 }}$ to determine $Y_{2}$

$R_{\text {calculated_2 }}=0.96$

$\mathrm{R}_{\text {graphical_max }}:=0.775 \quad \underline{\text { SONIC }}$

\section{INPUT}

$Y_{2}:=0.6875$

$\mathrm{R}_{\text {graphical_max }}:=0.775$

New $\Delta P_{2}:=R_{\text {graphical_max }} \cdot P_{H P T}$ _2a_a $\quad$ New $\Delta P_{2}=1992{ }^{\circ p s i}$

Calculating Second Replenishment Flowrate in (SCFS): $\quad$ (Crane, page 3-4 and 4-14)

$$
\mathrm{q}_{2}:=11.3 \cdot \mathrm{Y}_{2} \cdot\left(\frac{\mathrm{D}}{\mathrm{in}}\right)^{2} \cdot\left[\frac{\left(\frac{\mathrm{New} \Delta \mathrm{P}_{2}}{\mathrm{psi}}\right) \cdot\left(\frac{\mathrm{P}_{\mathrm{HPT} \_2 \mathrm{a} \_\mathrm{a}}}{\mathrm{psi}}\right)}{\mathrm{K}_{\text {total }} \cdot\left(\frac{\mathrm{T}_{\mathrm{HPT} \_2 \mathrm{a}}}{\mathrm{R}}\right)}\right]^{0.5} \cdot \frac{\mathrm{ft}^{3}}{\mathrm{~s}} \quad \mathrm{q}_{2}=1156 \cdot \frac{\mathrm{ft}^{3}}{\mathrm{~s}} \quad \text { (SCFS) based on } \mathrm{D}=2.067 \text { oin }
$$


$\Delta \mathrm{t}_{\text {actual_2 }}:=\frac{\mathrm{m}_{\text {replenishment_2 }}}{\mathrm{q}_{2} \cdot \mathrm{K}_{\mathrm{d}} \cdot \rho_{\mathrm{HPT} \_2 \mathrm{a}}}$

$\Delta t_{\text {actual_2 }}=18 \mathrm{~s} \quad$ Actual $\quad$ At $\mathrm{ID}=2.067$ in

$\Delta \mathrm{t}_{\text {specified }}=1200 \mathrm{~s} \quad$ Specified

Calculating mass of air in HPT prior to second replenishment of LPT (based on quess of HPT volume):

$m_{H P T} 2 \mathrm{a}=17133 \mathrm{olb}$

$m_{H P T \_2 b}:=m_{H P T \_2 a}-m_{\text {replenishment_2 }} \quad m_{H P T \_2 b}=15643$ olb

Calculating specific volume of air in HPT before and after second replenishment of LPT:
$\rho_{2 a}:=\rho_{\text {HPT_ }} 2 \mathrm{a}$
$\rho_{2 \mathrm{a}}=14.277 \frac{\mathrm{lb}}{\mathrm{ft}^{3}}$
$\mathrm{v}_{2 \mathrm{a}}:=\frac{1}{\rho_{2 \mathrm{a}}}$
$v_{2 a}=0.07 \frac{\mathrm{ft}^{3}}{\mathrm{lb}}$
$\rho_{2 b}:=\frac{m_{H P T} 2 b}{V_{\text {HPT }}}$
$\rho_{2 b}=13.036 \circ \frac{\mathrm{lb}}{\mathrm{ft}^{3}}$
$v_{2 b}:=\frac{1}{\rho_{2 b}}$
$v_{2 b}=0.077 \circ \frac{\mathrm{ft}^{3}}{\mathrm{lb}}$

\section{Calculating temperature and pressure of air in HPT after second replenishment of LPT:}

$$
\begin{aligned}
& T_{\text {HPT_2b }}:=T_{\text {HPT_2a }} \cdot\left(\frac{v_{2 a}}{v_{2 b}}\right)^{k-1} \\
& \mathrm{~T}_{\text {HPT_2b }}=452 \cdot \mathrm{R} \\
& \text { (ME reference manual, page 25-8) } \\
& P_{\text {HPT_2b_a }}:=P_{\text {HPT_2a_a }} \cdot\left(\frac{v_{2 a}}{v_{2 b}}\right)^{k} \\
& P_{H P T \_2 b \_a}=2182 \text { opsi } \\
& \text { (ME reference manual, page 25-8) } \\
& P_{\text {HPT_min }}:=2175 \cdot \mathrm{psi}
\end{aligned}
$$




\section{Summary}

\section{HPT Resultant Temperature}

$\mathrm{T}_{\text {HPT_1a }}=520 \circ \mathrm{R}$

$\mathrm{T}_{\text {HPT_2a }}=486^{\circ} \mathrm{R}$

$\mathrm{T}_{\text {HPT_2b }}=452 \circ$

\section{Replenishment Mass}

$\mathrm{m}_{\text {replenishment_1 }}=1490 \mathrm{Olb}$

$\mathrm{m}_{\text {replenishment_2 }}=1490 \mathrm{\circ lb}$

\section{HPT Resultant Pressure}

$\mathrm{P}_{\text {HPT_la_a }}=2987$ opsi

$\mathrm{P}_{\text {HPT_2a_a }}=2571$ psi

$\mathrm{P}_{\text {HPT_2b_a }}=2182$ opsi

$\mathrm{P}_{\text {HPT_min }}:=2175 \cdot \mathrm{psi}$

\section{Replenishment Flowrate}

$q_{1}=12990 \frac{\mathrm{ft}^{3}}{\mathrm{~s}}$

$q_{2}=1156 \cdot \frac{\mathrm{ft}^{3}}{\mathrm{~s}}$ 


\section{Cv Requirement (Replenishment)}

Reference: (Refer to Daniel Valve Co. fax of 11/24/99)

Inputs: $\quad \mathrm{ID}=2.067$ in

$$
\begin{aligned}
& \mathrm{T}_{\text {HPT_1a }}=520 \circ \mathrm{R} \\
& \mathrm{X}_{\mathrm{t}}:=0.38 \\
& \mathrm{q}_{1}=1299 \frac{\mathrm{ft}^{3}}{\mathrm{~s}} \\
& \mathrm{P}_{\mathrm{HPT} \_2 \mathrm{a} \_\mathrm{a}}=25710 \mathrm{psi} \\
& \mathrm{P}_{\text {LPT_4b_a }}=99.4 \circ \mathrm{psi} \\
& \mathrm{K}_{\text {total }}=8.7 \\
& \mathrm{~K}_{\text {valve }}=1.1
\end{aligned}
$$

\section{Calculation:}

$$
\begin{aligned}
& \Delta \mathrm{P}_{\min }:=\frac{\left(\mathrm{P}_{\mathrm{HPT} \_2 \mathrm{a}_{-} \mathrm{a}}-\mathrm{P}_{\mathrm{LPT}_{-} 4 \mathrm{~b}_{-} \mathrm{a}}\right) \cdot \mathrm{K}_{\text {valve }}}{\mathrm{K}_{\text {total }}} \quad \Delta \mathrm{P}_{\min }=321.4 \circ \mathrm{psi} \\
& \mathrm{X}:=\frac{\Delta \mathrm{P}_{\min }}{\mathrm{P}_{\text {HPT_2a_a }}} \quad \mathrm{X}=0.13 \\
& \mathrm{Y}:=1-\frac{\mathrm{X}}{3 \cdot \mathrm{X}_{\mathrm{t}}} \quad \mathrm{Y}=0.89
\end{aligned}
$$

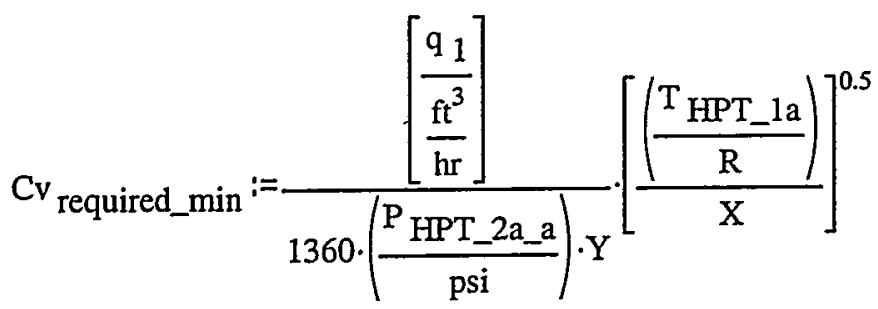




\section{Mooring System (Rapid Descent)}

\section{Obiective:}

To size the connecting piping and control valve between the Low Pressure Tank (LPT) and the Bottomless Buoyancy Tank (BBT) for the rapid descent system.
Given:
1) LPT temperature, pressure, and air mass prior to rapid descent (assuming LPT conditions after fourth deployment attempt) (from Filename: "Deployment-1")
2) Pressure inside BBT prior to rapid descent (assuming surface conditions of one atmosphere)
3) Specified flow rate for design to achieve.
4) Control valve stroke time of 20 seconds
5) Pipe length

\section{Find:}

1) Pipe and contol valve diameters and flow conditions; subsonic or sonic velocity

2) Air flowrate and mass discharged for rapid descent (compare to specified flowrate)

3) Resulting temperature, pressure, and mass inside LPT after rapid descent (compare to minimum operating pressure for LPT)

4) Minimum required control valve $C_{V}$ (compare to actual manufacurer's $C_{V}$ )

\section{Methodology:}

The resulting pressure, temperature, and air mass inside the LPT after the fouth deployment attempt are known from the previous calculation (Filename: "Deployment-1"). The air flowrate between the LPT and BBT was determined based on:

1) The difference in pressure between the LPT and BBT

2) The pressure drop for an assumed pipe size, length, and number and type of fittings

3) An assumed nominal pipe diameter of $21 / 2$ inches and valve diameter of 3 inch

The intial conditions of the LPT and BBT prior to rapid descent are known. The flowrate and time required for rapid descent were calculated and compared to the specified flowrate and time. The final conditions of the LPT were calculated. The resulting final LPT pressure was compared to the minimum working pressure for the LPT.

In order to accurately obtain the air flowrate, a correction factor, $\mathrm{Y}$, was determined graphically based on the parameters for the rapid descent. The air flowrate, the resulting air mass, pressure, and temperature inside the LPT were calculated, assuming adiabatic expansion.

Following the above calculations, the minimum required $\mathrm{Cv}$ for the control valve, installed in the line connecting the two tanks, was calculated based on data obtained from Daniel Valve Co. This minimum required Cv was compared to the actual $\mathrm{C} v$ for the valve chosen, from the Daniel product line, to determine the adequacy of the valve chosen. 


\section{Assumptions:}

1) Air is an ideal gas

2) Process is assumed to be isentropic, reversible adiabatic, closed system

3) Due to a rapid open/close time of the control valve, 2 seconds, neglect flow while control valve is opening or closing

4) Range of water temperature given as 10 to 20 degree $C$ : assume 15 degrees $C$ for calculations

5) Neglect pressure difference between top and bottom of buoyancy tank.

6) Neglect temperature and pressure increase in LPT after rapid descent due to LPT warming by the sea water.

\section{References:}

1) Crane, Flow of Fluids, Technical Paper No. 410, 1982

2) Mechanical Engineering Reference Manual for the PE exam, 10th edition, 1998

3) Perry's Chemical Engineers' Handbook, 6th edition, 1984

4) Daniel Valve Co., fax dated 11/24/99, from Jim Wass

\section{Conclusions:}

(Refer to Summary on page 9 of this calculation)

The piping connecting the LPT to the BBT is a $21 / 2$ inch diameter schedule 40 pipe. The control valve is a 3 inch valve. The increase in valve size was neccessary to accommodate the minimum required valve $\mathrm{Cv}$. The flow is sonic.

The rapid descent discharge flowrate is 72.12 SCFS and deviates approximately $2.1 \%$ from the specified flowrate of 70.64 SCFS. The air mass discharged from the LPT was calculated to be $110 \mathrm{lb}$.

The pressure and temperature drops inside the LPT as a result of the rapid deployment, assuming adiabatic expansion, are $5.1 \mathrm{psi}$ and $70 \mathrm{~F}$, respectively. Assuming adiabatic expansion is conservative. The actual pressure and temperature are higher due to the warming of the LPT by the ambient sea water. The lowest calculated pressure of 94.3 psia inside the LPT exceeds the minimum operating pressure of 80.5 psia.

The minimum required valve $\mathrm{Cv}$ was calculated to be 161 which is well within the actual $\mathrm{Cv}$ of 330 . 


\section{Letter Symbols for Variables:}

\section{Piping Parameters}

$I D_{\text {pipe }} \quad$ Inside diameter of pipe (in)

$I_{\text {valve }} \quad$ Inside diameter of control valve (in)

$f \quad$ Pipe friction factor (Crane, page A-26)

$L_{\text {pipe }} \quad$ Length of pipe (ft)

$\mathrm{N}_{\text {elbow }} \quad$ Number of $90^{\circ}$ elbows

$\mathrm{C}_{v} \quad$ Valve coefficient of friction (Daniel Valve Co.)

$\mathrm{K}_{\text {entrance }} \quad$ Resistance coefficient for pipe entrance losses (Crane, page A-29)

$\mathrm{K}_{\text {exit }}$

$\mathrm{K}_{\text {pipe }}$

$K_{\text {valve }}$

Resistance coefficient for pipe exit losses (Crane, page A-29)

Resistance coefficient for pipe (Crane, page 2-8)

$K_{\text {elbow }}$

$K_{\text {total }}$

Resistance coefficient control valve (Daniel Valve Co.)

$N_{11}$

Resistance coefficient for elbow (Crane, page A-29)

Sum of above coefficients

Number of friction factors

\section{Process Parameters}

$V_{\text {LPT }} \quad$ LPT volume (cu-ft)

$\Delta t_{\text {speciiied }} \quad$ Duration of control valve in open position - specified (sec)

$R_{\text {air }} \quad$ Individual specific gas constant for air ( $\left.f^{\star} \mid b f / / b^{*} R\right)$ (Crane, page A-8)

$k \quad$ Specific heats' ratio for air, taken at 1 atm and 60\% (Crane, page A-8, Perry's, page 3-144)

$\rho_{\text {LPT_1 }} \quad$ Density of air in LPT after fourth deployment attempt (lb/cu. ft) (From Calc: Deployment-1)

$\rho_{\text {STP }} \quad$ Density of air at STP (standard temperature and pressure) (Ib/cu. ft) (Crane, page A-10)

$K_{d} \quad$ Ratio of densities $\left(\rho_{\text {STP/PPT }} \rho_{\text {LP }}\right)$

$P_{\text {LPT_1_a }} \quad$ Pressure in LPT after fourth deployment attempt (psia) (from Calc: Deployment-1)

$\mathrm{T}_{\text {LPT_1 }} \quad$ Temperature in LPT after fourth deployment attempt (psia) (from Calc: Deployment-1)

$\mathrm{P}_{\mathrm{a}} \quad$ Atmospheric pressure (14.7 psi)

\section{Pressure}

$P_{\text {BBT_g }} \quad$ Pressure inside BBT prior to rapid descent (psig)

$P_{B B T_{\_} a} \quad$ Pressure inside BBT prior to rapid descent (psia)

$P_{\text {LPT_1_g }} \quad$ LPT pressure prior to rapid descent (psig)

$P_{\text {LPT_1_a }} \quad$ LPT pressure prior to rapid descent (psia)

$P_{\text {LPT___a }} \quad$ LPT pressure after rapid descent (psia)

\section{Temperature}

$T_{L_{\text {PT_1 }}} \quad$ LPT temperature prior to rapid descent $\left({ }^{\circ} \mathrm{R}\right)$

$T_{\text {LPT_2 }} \quad$ LPT temperature after rapid descent $\left({ }^{\circ} \mathrm{R}\right)$ 
Mass

$m_{\text {LPT_1 }} \quad$ LPT mass prior to rapid descent (lb)

$m_{\text {LPT_2 }} \quad$ LPT mass after rapid descent $(l b)$

$m_{\text {cischarge }} \quad$ Mass of discharged air from LPT during rapid descent (Ib)

$V_{\text {discharge_stP }}$ Volume of discharged air from LPT during rapid descent (Standard cu-ft - SCF)

\section{Discharge Flowrates}

$\mathrm{q}_{\text {actual }} \quad$ Actual rapid descent flowrate (Standard cu-ft per sec - SCFS)

$q_{\text {specilied }} \quad$ Specified rapid descent flowrate (Standard cu-ft per sec - SCFS)

Air Density and Specific Volume Parameters

$\rho_{1} \quad$ Density of air in LPT prior to rapid descent (lb/cu. $\mathrm{ft}$ )

$\rho_{2} \quad$ Density of air in LPT after rapid descent (lb/cu. ft)

$v_{1} \quad$ Specific volume of air in LPT prior to rapid descent (cu. $\mathrm{ft} / \mathrm{lb}$ )

$v_{2} \quad$ Specific volume of air in LPT after rapid descentt (cu. $\mathrm{tt} / \mathrm{lb}$ )

\section{Graphical Flowrate Correction Factor}

Y Correction factor for rapid descent

\section{$\underline{\text { Cv Calculation Parameters }}$}

$X_{1} \quad$ Critical pressure drop ratio - given - (per Daniel Valve Co.)

$T_{\text {LPT_max }} \quad$ Maximum temperature inside LPT $\left({ }^{\circ} R\right)$, ambient water temperature of $60{ }^{\circ} \mathrm{F}$

$\mathrm{q}_{\text {actual }} \quad$ Rapid descent discharge flowrate (standard cu-ft per second - SCFS)

$Q \quad$ Rapid descent discharge flowrate (standard cu-ft per hour - SCFH)

$\Delta \mathrm{P}_{\min } \quad$ Minimum $\triangle \mathrm{P}$ across control valve between LPT and BBT

$X \quad$ Actual pressure drop ratio (per Daniel Valve Co.)

$Y \quad$ Expansion factor (per Daniel Valve Co.)

$\mathrm{C}_{\text {Vrequired_min }} \quad$ Minimum required $\mathrm{C}_{\mathrm{V}}$ for control valve

$\mathrm{C}_{\text {Vactual }} \quad$ Design $\mathrm{C}_{\mathrm{V}}$ for control valve (per Daniel Valve Co.) 


\section{INPUTS}

$\mathrm{ID}_{\text {pipe }}:=2.469$ in

ID valve $:=3.068$ in

$f:=0.018$

$L_{\text {pipe }}:=50 \cdot \mathrm{ft}$

$N_{\text {elbow }}:=2$

$\mathrm{N}_{\mathrm{ff}}:=14$

$C_{V}:=330$

$\mathrm{K}_{\text {entrance }}:=0.5$

$\mathrm{K}_{\text {exit }}:=1.0$

$\mathrm{V}_{\text {LPT }}:=4945 \cdot \mathrm{ft}^{3}$

$\Delta t_{\text {specified }}:=20 \cdot \mathrm{s}$

$\mathrm{T}_{\text {LPT_max }}:=520 \cdot \mathrm{R}$

$\mathrm{P}_{\mathrm{BBT}_{\_} \mathrm{a}}:=14.7 \cdot \mathrm{psi}$

$q_{\text {specified }}:=70.64 \cdot \frac{\mathrm{ft}^{3}}{\mathrm{~s}}$

$R_{\text {air }}:=53.3 \cdot \frac{\mathrm{ft} \cdot \mathrm{lbf}}{\mathrm{lb} \cdot \mathrm{R}}$

$k:=1.4$

PLPT_1_a $_{\text {LP }}: 99.4 \cdot \mathrm{psi}$

$\mathrm{T}_{\mathrm{LPT}_{-} 1}:=443 \cdot \mathrm{R}$

$\rho_{\text {LPT_L }_{\text {I }}}:=0.607 \cdot \frac{\mathrm{lb}}{\mathrm{ft}^{3}}$

$\rho_{\mathrm{STP}}:=0.0763 \cdot \frac{\mathrm{lb}}{\mathrm{ft}^{3}}$

$\mathrm{P}_{\mathrm{a}}:=14.7 \cdot \mathrm{psi}$ 


\section{ONE-TIME CALCULATIONS}

\section{Initial Temperature:}

$\mathrm{T}_{\mathrm{LPT} \_1}=443 \circ \mathrm{R}$

Initial pressure in LPT after fourth deployment attempt and before rapid descent:

$\mathrm{P}_{\text {LPT_1_a }}=99.40 \mathrm{psi}$

LPT pressure (psia)

$P_{\text {LPT_1_g }}:=P_{\text {LPT_1_a }}-P_{a}$

$P_{\text {LPT_1_g }}=84.7$ opsi

LPT pressure (psig)

Conversion between Standard Cubic Feet (SCF) and Actual Cubic Feet (ACF):

$\rho_{\mathrm{STP}}:=0.0763 \cdot \frac{\mathrm{lb}}{\mathrm{ft}^{3}}$

$\mathrm{K}_{\mathrm{d}}:=\frac{\rho_{\mathrm{STP}}}{\rho_{\text {LPT_1 }}} \quad \mathrm{K}_{\mathrm{d}}=0.1257$

\section{Calculating total Resistance Coefficient (Ktotal) for pipe, fittings, valve, etc.:}

$$
\begin{aligned}
& \mathrm{K}_{\text {entrance }}:=0.5 \\
& \mathrm{~K}_{\text {exit }}:=1.0 \\
& \mathrm{~K}_{\text {elbow }}:=\mathrm{N}_{\text {elbow }} \cdot \mathrm{N}_{\mathrm{ff}} \cdot \mathrm{f} \quad \mathrm{K}_{\text {elbow }}=0.504 \\
& K_{\text {pipe }}:=f \cdot\left(\frac{L_{\text {pipe }}}{\mathrm{I}_{\text {pipe }}}\right) \quad K_{\text {pipe }}=4.374 \\
& \mathrm{~K}_{\text {valve }}:=1443 \cdot\left[\frac{\frac{\pi}{4} \cdot\left(\frac{\mathrm{I} \text { valve }}{\text { in }^{5}}\right)^{2}}{C_{\mathrm{v}}}\right]^{2} \quad \mathrm{~K}_{\text {valve }}=0.724
\end{aligned}
$$$$
\text { (Daniel Valve Co., fax 11/24/99) }
$$$$
\text { (Equation is valid for SONIC and SUBSONIC flow) }
$$

$$
\mathrm{K}_{\text {total }}:=\mathrm{K}_{\text {entrance }}+\mathrm{K}_{\text {exit }}+\mathrm{K}_{\text {elbow }}+\mathrm{K}_{\text {pipe }}+\mathrm{K}_{\text {valve }} \quad \mathrm{K}_{\text {total }}=7.102
$$




\section{Calculations for Rapid Descent}

$$
\begin{aligned}
& P_{\text {LPT_1_a }}=99.40 \mathrm{psi} \\
& \mathrm{T}_{\text {LPT_1 }_{1}}=443 \circ \mathrm{R}
\end{aligned}
$$

\section{Calculating Correction Factor Y1 using DeltaP1:}

$\mathrm{P}_{\mathrm{BBT} \_\mathrm{g}}:=\mathrm{P}_{\mathrm{BBT}_{\_} \mathrm{a}}-\mathrm{P}_{\mathrm{a}} \quad \mathrm{P}_{\mathrm{BBT} \_\mathrm{g}}=0$ opsi

$\Delta \mathrm{P}:=\mathrm{P}_{\mathrm{LPT} \_1 \_\mathrm{g}}-\mathrm{P}_{\mathrm{BBT} \_\mathrm{g}} \quad \Delta \mathrm{P}=84.7$ opsi

$\mathrm{R}_{\text {calculated }}:=\frac{\Delta \mathrm{P}}{\mathrm{P}_{\text {LPT_1_a }}} \quad \mathrm{R}_{\text {calculated }}=0.85$

\section{BEFER TO CRANE page A-22}

Based on: $\quad \mathrm{K}_{\text {total }}=7.1$

If $R_{\text {calculated }}>R_{\text {graphical_max }}$, then flow is SONIC; use $R_{\text {graphical_max }}$ to determine $Y$ \& new $\Delta P$

If $R_{\text {calculated }}<R_{\text {graphical_max }}$, then flow is SUBSONIC; use $R_{\text {calculated }}$ to determine $Y$

$\mathrm{R}_{\text {calculated }}=0.85$

$\mathrm{R}_{\text {graphical_max }}:=0.755 \quad \underline{\text { SONIC }}$

INPUT

$Y:=0.680$

$\mathrm{R}_{\text {graphical_max }}:=0.755$

$\Delta P_{\text {new }}:=R_{\text {graphical_max }} \cdot P_{\text {LPT_1_a }} \quad \Delta P_{\text {new }}=75 \circ$ psi

\section{Calculating First Replenishment Flowrate in (SCFS):}

$\mathrm{q}_{\text {actual }}:=11.3 \cdot \mathrm{Y} \cdot\left(\frac{\mathrm{D} \text { pipe }}{\text { in }}\right)^{2} \cdot\left[\frac{\left(\frac{\Delta \mathrm{P}_{\text {new }}}{\mathrm{psi}}\right) \cdot\left(\frac{\mathrm{P}_{\mathrm{LPT} \_1 \_a}}{\mathrm{psi}}\right)}{\mathrm{K}_{\text {total }} \cdot\left(\frac{\mathrm{T}_{\mathrm{LPT} \_1}}{\mathrm{R}}\right)}\right]^{0.5} \frac{\mathrm{ft}^{3}}{\mathrm{~s}}$

(Crane, page 3-4 and 4-14)

based on

$\mathrm{ID}_{\text {pipe }}=2.469$ in 
$q_{\text {actual }}=72.12 \frac{\mathrm{ft}^{3}}{\mathrm{~s}} \quad$ Actual (SCFS)

$\mathrm{q}_{\text {specified }}:=70.64 \cdot \frac{\mathrm{ft}^{3}}{\mathrm{~s}}$ Specified

\section{Calculating mass of discharged air for rapid descent:}

$\mathrm{V}_{\text {discharge_STP }}:=\mathrm{q}_{\text {actual }} \cdot \Delta \mathrm{t}$ specified

$V_{\text {discharge_STP }}=1442 \mathrm{oft}^{3}$

$\rho_{\mathrm{STP}}:=0.0763 \cdot \frac{\mathrm{lb}}{\mathrm{ft}^{3}}$

$\mathrm{m}_{\text {discharge }}:=\rho_{\mathrm{STP}} \cdot \mathrm{V}_{\text {discharge_STP }} \quad \mathrm{m}_{\text {discharge }}=110 \mathrm{ll}$

\section{Calculating mass of air in LPT after rapid descent after fourth deployment attempt}

$$
\begin{aligned}
& \mathrm{m}_{\mathrm{LPT} \_1}:=\rho{ }_{\mathrm{LPT} \_1} \cdot \mathrm{v}_{\mathrm{LPT}} \quad \mathrm{m}_{\mathrm{LPT} \_1}=3002 \mathrm{olb} \\
& \mathrm{m}_{\mathrm{LPT} \_2}:=\mathrm{m}_{\mathrm{LPT} \_1}-\mathrm{m}_{\text {discharge }} \quad \mathrm{m}_{\mathrm{LPT} \_2}=2892 \mathrm{olb}
\end{aligned}
$$

Calculating specific volume of air in LPT before and after rapid descent attempt:
$\rho_{1}:=\rho_{\text {LPT_1 }}$
$\rho_{1}=0.607 \frac{\mathrm{lb}}{\mathrm{ft}^{3}}$
$v_{1}:=\frac{1}{\rho_{1}}$
$v_{1}=1.647 \frac{\mathrm{ft}^{3}}{\mathrm{lb}}$
$\rho_{2}:=\frac{m_{\text {LPT_2 }}}{v_{\text {LPT }}}$
$\rho_{2}=0.585^{\circ} \frac{\mathrm{lb}}{\mathrm{ft}^{3}}$
$v_{2}:=\frac{1}{\rho_{2}}$
$v_{2}=1.71 \frac{0 \mathrm{ft}^{3}}{\mathrm{lb}}$

Calculating temperature and pressure of air in LPT after rapid descent (after 4th deployment attempt:
$\mathrm{T}_{\text {LPT_2 }_{2}}:=\mathrm{T}_{\text {LPT_1 }_{1}} \cdot\left(\frac{\mathrm{v}_{1}}{\mathrm{v}_{2}}\right)^{\mathrm{k}-1}$
$\mathrm{T}_{\text {LPT_2 }}=436 \cdot \mathrm{R}$
(ME reference manual, page 25-8)
$\mathrm{P}_{\text {LPT_2_a }}:=\mathrm{P}_{\text {LPT_1_a }} \cdot\left(\frac{\mathrm{v}_{1}}{\mathrm{v}_{2}}\right)^{\mathrm{k}}$
$\mathrm{P}_{\text {LPT_2_a }}=94.30 \mathrm{psi}$
(ME reference manual, page 25-8) 


\section{Summary}

\section{LPT Resultant Temperature}

$\mathrm{T}_{\text {LPT_1 }}=443 \circ \mathrm{R}$

$\mathrm{T}_{\mathrm{LPT}_{2} 2}=436{ }^{\circ} \mathrm{R}$

\section{Replenishment Mass}

$\mathrm{m}_{\text {discharge }}=110 \mathrm{lb}$

\section{LPT Resultant Pressure}

$P_{\text {LPT_1_a }}=99.4$ psi

$\mathrm{P}_{\text {LPT_2_a }}=94.3 \mathrm{psi}$

\section{Replenishment Flowrate}

$\mathrm{q}_{\text {actual }}=72.120 \frac{\mathrm{ft}^{3}}{\mathrm{~s}} \quad$ Actual

$\mathrm{q}_{\text {specified }}:=70.64 \cdot \frac{\mathrm{ft}^{3}}{\mathrm{~s}} \quad$ Specified 


\section{Cv Requirement (Rapid Descent)}

Reference: (Refer to Daniel Valve Co. fax of 11/24/99)

Inputs: $\quad \mathrm{D}_{\text {valve }}=3.068$ in

$\mathrm{T}_{\text {LPT_max }}=520 \circ \mathrm{R}$

$X_{t}:=0.38$

$q_{\text {actual }}=72.12 \cdot \frac{\mathrm{ft}^{3}}{\mathrm{~s}}$

$P_{\text {LPT_1_a }}=99.4$ psi

$\mathrm{P}_{\mathrm{BBT}_{-} \mathrm{a}}=14.7 \mathrm{psi}$

$\mathrm{K}_{\text {total }}=7.102$

$\mathrm{K}_{\text {valve }}=0.724$

\section{Calculation:}

$\Delta \mathrm{P}_{\min }:=\frac{\left(\mathrm{P}_{\text {LPT_1_a }}-\mathrm{P}_{\text {BBT_a }}\right) \cdot \mathrm{K}_{\text {valve }}}{\mathrm{K}_{\text {total }}} \quad \Delta \mathrm{P}_{\min }=8.636 \circ \mathrm{psi}$

$\mathrm{X}:=\frac{\Delta \mathrm{P}_{\min }}{\mathrm{P}_{\text {LPT_1_a }}} \quad \mathrm{X}=0.09$

$\mathrm{Y}:=1-\frac{\mathrm{X}}{3 \cdot \mathrm{X}_{\mathrm{t}}} \quad \mathrm{Y}=0.924$

$C v_{\text {required_min }}:=\frac{\left[\frac{\mathrm{q}_{\text {actual }}}{\mathrm{ft}^{3}} \frac{[\mathrm{hr}}{\mathrm{hr}}\right]}{1360 \cdot\left(\frac{\mathrm{P}_{\mathrm{LPT} \_1 \_\mathrm{a}}}{\mathrm{psi}}\right) \cdot \mathrm{Y}} \cdot\left[\frac{\left(\frac{\mathrm{T}_{\mathrm{LPT} \_ \text {max }}}{\mathrm{R}}\right)}{\mathrm{X}}\right]^{0.5} \quad \mathrm{Cv}_{\text {required_min }}=161$ 


\section{Mooring System (LPT Relief Valve Sizing)}

\section{Objective:}

To size the pressure relief valve located on the Low Pressure Tank (LPT) and to select a model(s).

\section{Given:}

1) LPT maximum design pressure

2) Relief valve overpressure percentage

3) Water depth and temperature

4) Maximum air mass flowrate into LPT (per Filename: LPT Replenishment)

5) Maximum air volume flowrate into LPT (per Filename: LPT Replenishment)

Find:

1) Relief valve inlet pressure and back pressure

2) Minimum required relief valve inlet diameter based on mass flowrate and volume flowrate

3) Model number(s) of relief valves satisfying the minimum requirements

\section{Methodology:}

The size of the LPT relief valve was determined using the sizing calculation from Crosby Valves, Catalog No. 318 . It was assumed that the greatest overpressurization of the LPT would occur if the control valve between the LPT and the High Pressure Tank (HPT) was accidentally stuck open and the HPT was fully charged. The LPT's and HPT's maximum operating pressures are $175 \mathrm{psia}$ and $2987 \mathrm{psia}$, respectively. This would result in a tremendous air flowrate from the HPT into the LPT, considerably increasing LPT pressure. Refering to calculation with filename: LPT Replenishment, the maximum air flowrate into the LPT is known. Also known are the LPT interior and exterior ambient conditions. From these conditions, the relief valve inlet and back pressures were calculated. Substituting into Crosby's sizing calculation yielded the minimum valve inlet area from which the minimum diameter was determined. The sizing calculation was performed by two methods as a check. One method was based on the mass flowrate and the other was based on the volume flowrate.

\section{Assumptions:}

1) Air is an ideal gas

2) Range of water temperature given as 10 to 20 degree $C$ : assume 15 degrees $C$ for calculations

3) Temperature of air entering the relief valve is the same temperature as the ambient water temperature

\section{References:}

1) Crosby Valve \& Gage Company, "Crosby JPV Series Pilot Operated Pressure Relief Valves Styles JPV and JPVM," Catalog No. 318, December 1986.

2) Mechanical Engineering Reference Manual for the PE exam, 10th edition, 1998 (Section 24-19)

3) Suction Mooring Technology, Engineering Calculation, (Filename: LPT Replenishment) 


\section{Conclusions:}

The relief valve inlet pressure was calculated to be the sum of the set pressure plus the overpressure plus atmospheric pressure and equaled 222.6 psia. Based on the static head of the water, the back pressure was calculated to be 66.0 psia. The minimum valve inlet area was calculated using two approaches, the second to check the first. One required the air mass flowrate and yielded an effective area of $19.627 \mathrm{sq}$. in and the other required the volume flowrate yielding an effective area of $19.595 \mathrm{sq}$. in. The minimum diameter was determined using the greater of the two areas. The minimum required diameter is 4.999 inches.

In addition to the water temperature, this calculation was also run for temperatures $-200 \mathrm{~F}$ and $1000 \mathrm{~F}$ to determine the effect of temperature in the sizing process. The results yielded a minimum diameter of 4.794 inches and 5.092 inches, respectively. With a difference of approximately $6 \%$, temperature is not very significant in sizing the relief valve.

The JPV 8T10 Style 15 or the JPVM 8T10 Style 15, both having ANSI B16.5 Class 150 inlet and outlet flanges, are adequate.

\section{Attachments:}

1) Crosby Valve \& Gage Company, "Crosby JPV Series Pilot Operated Pressure Relief Valves Styles JPV and JPVM," Catalog No. 318, December 1986. (pages 18, 20, 22, 32) 


\section{Letter Symbols for Variables:}

Input:

CxK

$M$

Pdesign_max

Patm_a Overpressure $_{\%}$

$\mathrm{H}_{\text {stalic }}$

$T$

W

SCFM

Z

$\rho_{\text {water }}$

\section{Calculation:}

Pback_pressure_a

$P_{\text {set_g }}$

Pover_g

Pinlet_a

Back_Pressure $\%$

$\mathrm{K}_{\mathrm{b}}$

A mass_rate

AsCFM

$I D_{\text {min }}$
Variable factor multiplied by the coefficient of discharge (page 32)

Molecular weight of air

LPT maximum design pressure (psig)

Atmosheric pressure (14.7 psia)

Relief valve overpressure percentage (\%)

Depth of water $(m)$

Absolute temperature of air at relief valve inlet $\left({ }^{0} R\right)$

Required relieving capacity in (lbm/hr) (per Filename: LPT Replenishment)

Required relieving capacity in (standard cu. $\mathrm{ft} / \mathrm{min}$ ) (per Filename: LPT Replenishment)

Compressibility factor (see: Mechanical Engineering Reference Manual)

Density of sea water ( $\mathrm{lbm} / \mathrm{cu} . \mathrm{ft})$

\section{Back pressure (psia)}

Relief valve set pressure (psig) $\left(P_{\text {design_max }}+\right.$ Hydrostatic Head)

Relief valve overpressure (psig)

Relieving pressure (set pressure + overpressure + atmospheric pressure) (psia)

Back pressure percentage (\%)

Capacity correction factor due to back pressure for use when superimposed (constant) back pressure exceeds critical pressure (page 32 )

Minimum required effective valve discharge area (sq. in) - using W

Minimum required effective valve discharge area (sq. in) - using SCFM

Minimum diameter of relief valve discharge (in) 


\section{INPUTS:}

$$
\begin{aligned}
& \text { CxK: }=345 \\
& M:=28.97 \\
& P_{\text {design_max }}:=137.75 \cdot p s i \\
& \mathrm{P}_{\text {atm_a }}:=14.7 \cdot \mathrm{psi} \\
& \text { Overpressure } \%:=10 . \% \\
& \mathrm{H}_{\text {static }}:=35 \cdot \mathrm{m} \\
& \mathrm{T}:=520 \cdot \mathrm{R} \\
& \mathrm{W}:=357600 \cdot \frac{\mathrm{lb}}{\mathrm{hr}} \\
& \mathrm{SCFM}:=77940 \cdot \frac{\mathrm{ft}^{3}}{\min } \\
& \mathrm{Z}:=0.99 \\
& \rho_{\text {water }}:=1.03 \cdot \frac{\mathrm{gr}}{\mathrm{cm}^{3}} \\
& \mathrm{gr} \equiv 1 \cdot \frac{\mathrm{kg}}{1000}
\end{aligned}
$$




\section{Determining minimum area of relief valve inlet:}

\section{Back Pressure:}

$P_{\text {back_pressure_a }}:=\rho_{\text {water }} \cdot \mathrm{g} \cdot \mathrm{H}_{\text {static }}+\mathrm{P}_{\text {atm_a }}$

$\mathrm{P}_{\text {back_pressure_a }}=65.975{ }^{\circ} \mathrm{psi}$

\section{Set Pressure:}

$$
\mathrm{P}_{\text {set_g }}:=\mathrm{P}_{\text {design_max }}+\rho_{\text {water }} \cdot \mathrm{g} \cdot \mathrm{H}_{\text {static }} \quad \mathrm{P}_{\text {set_g }}=189.03 \circ \mathrm{psi}
$$

Overpressure:

$$
\mathrm{P}_{\text {over_g }}:=\mathrm{P}_{\text {set_g }} \cdot \text { Overpressure } \% \quad \mathrm{P}_{\text {over_g }}=18.9 \text { opsi }
$$

Inlet Pressure:

$$
P_{\text {inlet_a }}:=P_{\text {set_g }}+P_{\text {over_g }}+P_{\text {atm_a }} \quad P_{\text {inlet_a }}=222.60 p s i
$$

Capacity Correction Factor, (Kb):

$$
\text { Back_Pressure }_{\%}:=\left(\frac{P_{\text {back_pressure_a }}}{P_{\text {inlet_a }}}\right) \quad \text { Back_Pressure } \%=29.6 \%
$$

$$
\text { At Back_Pressure } \%_{0}=29.6 \% \quad \mathrm{~K}_{\mathrm{b}}:=1.00 \quad \text { (page 32) }
$$

Calculating minimum valve inlet area based on MASS flowrate in $\mathrm{lb} / \mathrm{hr}$.

$$
A_{\text {mass_rate }}:=\frac{\left[\frac{\mathrm{W}}{\left(\frac{\mathrm{lb}}{\mathrm{hr}}\right)}\right] \cdot\left[\left(\frac{\mathrm{T}}{\mathrm{R}}\right) \cdot \mathrm{Z}\right]^{0.5}}{\left(\frac{\mathrm{P}_{\text {inlet_a }}}{\mathrm{psi}}\right) \cdot(\mathrm{CxK}) \cdot \mathrm{K}_{\mathrm{b}} \cdot(\mathrm{M})^{0.5}} \cdot \mathrm{in}^{2} \quad \mathrm{~A}_{\text {mass_rate }}=19.627 \text { in }^{2}
$$




\section{Calculating minimum valve inlet area based on VOLUME flowrate in SCFM:}

$$
\mathrm{A}_{\text {SCFM }}:=\frac{\left[\frac{\mathrm{SCFM}}{\left(\frac{\mathrm{ft}^{3}}{\mathrm{~min}}\right)}\right) \cdot\left[\left(\frac{\mathrm{T}}{\mathrm{R}}\right) \cdot \mathrm{Z}\right]^{0.5}}{1.175 \cdot\left(\frac{\mathrm{P}_{\text {inlet_a }}}{\mathrm{psi}}\right) \cdot(\mathrm{CXK}) \cdot \mathrm{K}_{\mathrm{b}}} \cdot \mathrm{in}^{2}
$$

$$
\mathrm{A}_{\mathrm{SCFM}}=19.595 \text { in }^{2}
$$

Calculating minimum valve inlet diameter:

$$
\mathrm{I}_{\min }:=\left(\frac{\mathrm{A}_{\text {mass_rate }}}{\pi}\right)^{0.5} \cdot 2 \quad \mathrm{ID}_{\min }=4.999 \text { oin }
$$

\section{Selecting a relief valve: $\quad$ (Refer to pages 18 and 22 of the Crosby Valve Catalog)}

Refering to page 22 of the attachments, for an effective area of 19.627 sq. in, an air flowrate of 77,940 SCFM, and a set pressure of 189 psig, only the orifice with the letter designation of " $T$ ", having an effective area of $26 \mathrm{sq}$. in., is applicable. One size lower, orifice " $\mathrm{R}$ " has an effective area of $16 \mathrm{sq}$. in which is inadequate. Refering to page 18, the only valve size to accomodate the "T" orifice is $8 \times 10$. With a pressure/temperature limit of $285 \mathrm{psig}$ at $1000 \mathrm{~F}$, and the actual pressure of $189 \mathrm{psig}$, the $150 \mathrm{lb}$ flange class is adequate for both the inlet and outlet flanges.

The JPVM 8T10 Style 15, having ANSI B16.5 Class 150 inlet and outlet flanges, is adequate. Style JPVM is a non-flowing modulating pilot operated pressure relief valve. The JPV style valve is a snap-acting pilot operated pressure relief valve which is inadequate for this application. 


\section{Flanges and Flange Facings}

Dimensions of flanges conform to current ANSI Standards. On valve inlets, however, thicknesses are greater than the minimum required by ANSI Standards. Dimension $x$ shown below should be used to determine bolt length. Table dimensions are in inches.

Center-to-Face dimensions are subject to tolerances of plus/minus $1 / 16^{\prime \prime}$ for valves through $4^{\prime \prime}$ inlet size and plus/minus $1 / 8^{\prime \prime}$ for valves with an inlet size larger than $4 "$.

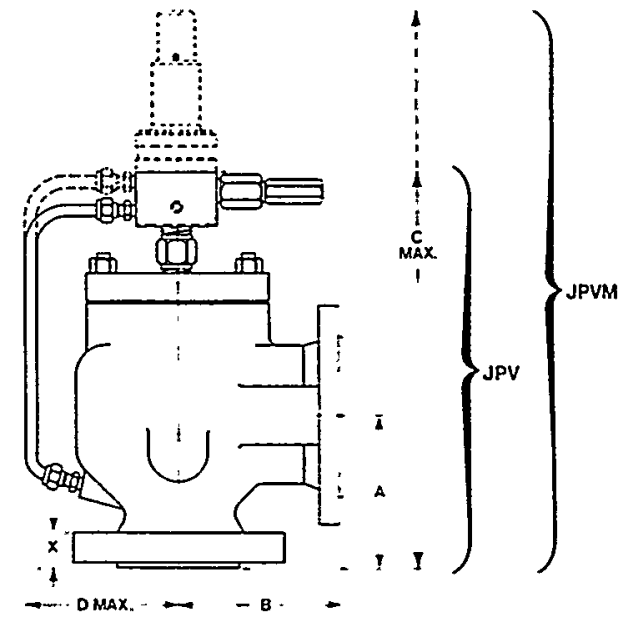

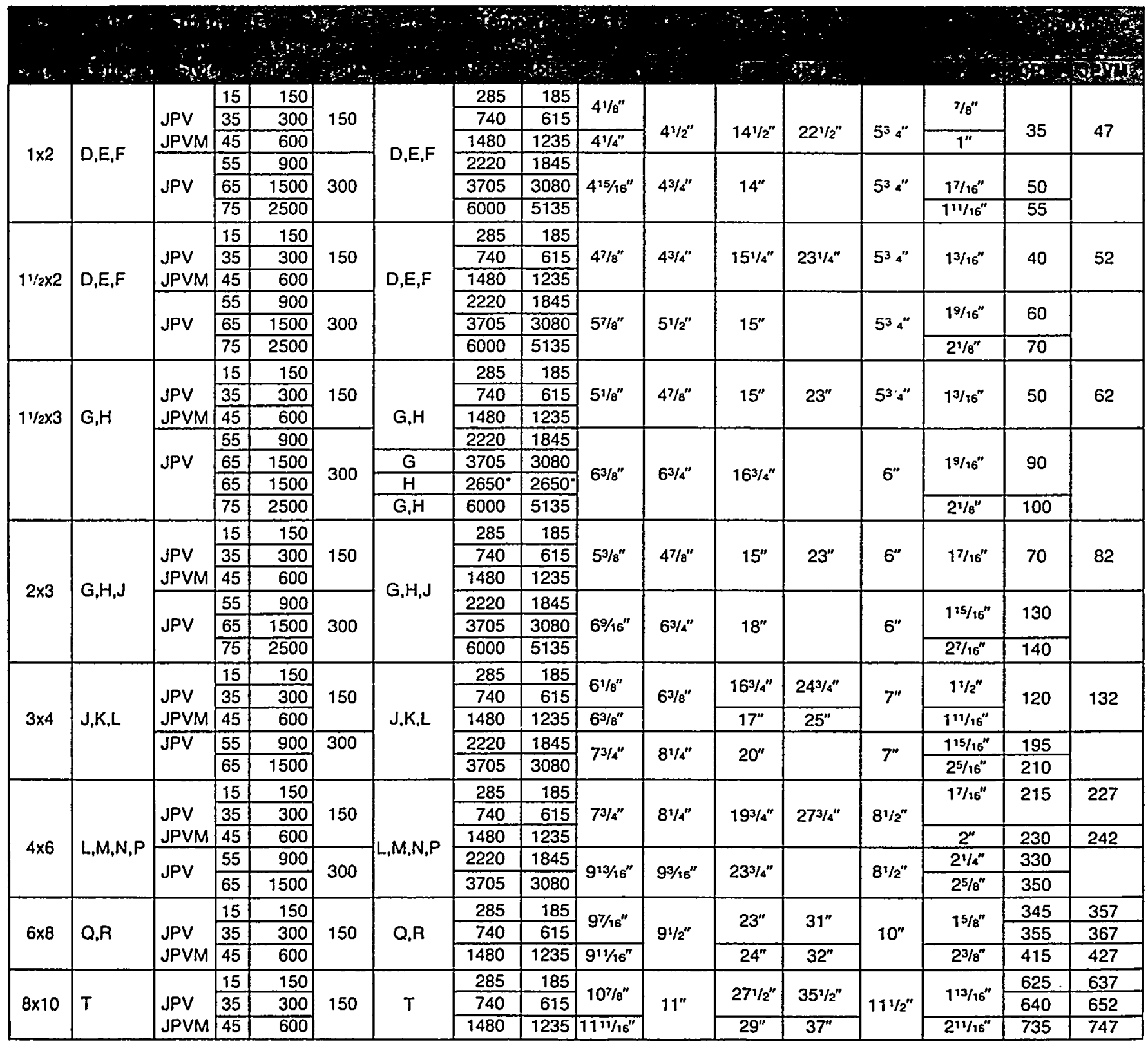

tWhen specifying valve size, the orifice letter must be included. For example a $1^{\prime \prime} \times 2^{\prime \prime}$ valve is expressed as 1D2, $1 E 2$, or 1 F2.

"The pressure limitation on this size is less than the inlet flange rating. See pressure/temperature limit charts on Page 19. Valve temperature limited by elastomer.

" Dimensions and weights do not include accessories and options.

NOTE: Back pressures are restricted to the pressure/temperature limits of the ANSI rating of the outlet flange. 


\section{Gas, Air and Vapor Sizing (English Units) - Styles JPV and JPVM}

\section{Gas and Vapor Sizing - LBS/HR - 10\% Overpressure}

The following is used for sizing valves for gases and vapor when the required flow is expressed as a mass flow rate in pounds per hour. Correction factors are included to account for the effects of backpressure, compressibility and subcritical flow conditions.

$$
A=\frac{W \sqrt{T Z}}{P_{1}(C \times K) K_{b} \sqrt{M}}
$$

Where:

$$
\begin{gathered}
A=\text { Minimum required effective discharge area, } \\
\text { square inches. }
\end{gathered}
$$

$\mathrm{C} \times \mathrm{K}=$ Variable factor, depending upon ratio of specific heats, multiplied by the coefficient of discharge. (See Table on page 32.) Use $\mathrm{C} \times \mathrm{K}=306$ if value is unknown.

$\mathrm{K}_{\mathrm{b}}=$ Capacity correction factor due to back pressure for use when superimposed (constant) back pressure exceeds critical pressure. (See Table on page 32.)

$M=$ Molecular weight of the gas or vapor obtained from standard tables or Table on page 32.

$P_{1}=$ Relieving pressure in pounds per square inch absolute. This is the set pressure + overpressure + atmospheric pressure.

$T=$ Absolute temperature of the fluid at the valve inlet $\left({ }^{\circ} \mathrm{F}+460\right)$.

$W=$ Required relieving capacity in pounds per hour.

$Z$ = Compressibility factor. Use $Z=1.0$ if value is unkown. (See Crosby Engineering Handbook for further information.)

\section{Gas and Vapor Sizing - SCFM - 10\% Overpressure}

The following formula is used for sizing valves for gases and vapor when the required flow is expressed as a volumetric flow rate in SCFM. Correction factors are included to account for the effects of backpressure, compressibility and subcritical flow.

$$
A=\frac{S C F M \sqrt{T G Z}}{1.175(C \times K) P_{1} K_{b}}
$$

Where:

$$
\begin{aligned}
& A=\text { Minimum required effective discharge area, } \\
& \text { square inches. }
\end{aligned}
$$

$\mathrm{C} \times \mathrm{K}=$ Variable factor, depending upon ratio of specific heats, multiplied by the coefficient of discharge. (See Table on page 32.) Use $\mathrm{C} \times \mathrm{K}=306$ if value is unknown.

$\mathrm{G}=$ Specific gravity of the gas and vapor at flowing conditions.

$\mathrm{K}_{\mathrm{b}}=$ Capacity correction factor due to back pressure for use when superimposed (constant) back pressure exceeds critical pressure. (See Table on page 32.)

$P_{1}=$ Relieving pressure in pounds per square inch absolute. This is the set pressure + overpressure + atmospheric pressure.

$\mathrm{T}=$ Absolute temperature of the fluid at the inlet $\left({ }^{\circ} \mathrm{F}\right.$ $+460)$.

SCFM = Required relieving capacity in standard cubic feet per minute.

$Z=$ Compressibility factor. Use $Z=1.0$ if value is unknown. (See Crosby Engineering Handbook for further information.) 


\section{Air Capacities (English Units) - Styles JPV and JPVM}

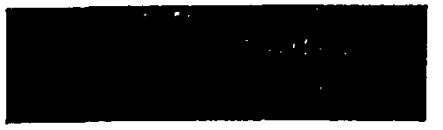

\section{Standard cubic feet per minute at $60^{\circ} \mathrm{F}$ \\ Overpressure $10 \%$ and Above}

Meets Requirements of ASME Boiler and Pressure Vessel Code, Section VIII. Capacities Certified by National Board of Boiler and Pressure Vessel Inspectors.

\begin{tabular}{|c|c|c|c|c|c|c|c|c|c|c|c|c|c|c|}
\hline $\begin{array}{l}\text { si incr. } \\
\text { si incr. }\end{array}$ & $\begin{array}{c}2 \\
11\end{array}$ & $\begin{array}{c}4 \\
19\end{array}$ & $\begin{array}{c}6 \\
30\end{array}$ & $\begin{array}{l}10 \\
49\end{array}$ & $\begin{array}{c}15.5 \\
77\end{array}$ & $\begin{array}{c}25 \\
126\end{array}$ & $\begin{array}{c}36 \\
180\end{array}$ & $\begin{array}{c}56 \\
280\end{array}$ & $\begin{array}{c}70 \\
352\end{array}$ & $\begin{array}{c}85 \\
425\end{array}$ & $\begin{array}{l}125 \\
624\end{array}$ & $\begin{array}{c}216 \\
1082\end{array}$ & $\begin{array}{c}313 \\
1566\end{array}$ & $\begin{array}{c}509 \\
2545\end{array}$ \\
\hline 30 & 44 & 167 & 262 & 429 & 670 & 1098 & 1568 & 2434 & 3072 & 3703 & 5462 & 9429 & 13652 & 22185 \\
\hline 50 & 137 & 244 & 382 & 626 & 977 & 1601 & 2287 & 3550 & 4479 & 5400 & 7914 & 13750 & 19910 & 32354 \\
\hline 75 & 190 & 339 & 531 & 871 & 1358 & 2226 & 3180 & 4935 & 6229 & 7509 & 11039 & 19118 & 27682 & 44985 \\
\hline 80 & 201 & 358 & 560 & 918 & 1433 & 2350 & 3356 & 5209 & 6572 & 7923 & 11648 & 20174 & 29211 & 47468 \\
\hline 100 & 244 & 434 & 681 & 1115 & 1740 & 2853 & 4074 & 6324 & 7980 & 9621 & 14143 & 24495 & 35468 & 57636 \\
\hline 120 & 287 & 511 & 801 & 1312 & 2047 & 3356 & $47 \overline{493}$ & 7440 & 9388 & 11318 & 16638 & 28817 & 41726 & 67805 \\
\hline 140 & 330 & 588 & 921 & 1508 & 2354 & 3860 & 5512 & 8556 & 10796 & 13015 & 19133 & 33138 & 47983 & 77973 \\
\hline 160 & 373 & 664 & 1041 & 1705 & 2661 & 4363 & 6231 & 9672 & 12204 & 14713 & 21629 & 37460 & 54241 & 88141 \\
\hline 180 & 416 & 741 & 1161 & 1902 & 2968 & 4866 & 6950 & 10788 & 13612 & 16410 & 24124 & 41782 & 60498 & 98310 \\
\hline 200 & 459 & 818 & 1281 & 2099 & 3275 & 5370 & 7669 & 11903 & 15020 & 18107 & 26619 & 46103 & 66756 & 108478 \\
\hline 220 & 502 & 894 & 1401 & 2295 & 3582 & 5873 & 8387 & 13019 & 16428 & 19805 & 29114 & 50425 & 73013 & 118646 \\
\hline 240 & 15 & 971 & 1521 & 2492 & 3889 & 6376 & 9106 & 14135 & 17836 & 21502 & 31609 & 54746 & 79271 & 128815 \\
\hline 260 & 588 & 1048 & 1641 & 2689 & 4196 & 6880 & 9825 & 15251 & 19244 & 23199 & 34104 & 59068 & 85528 & 38983 \\
\hline 280 & 631 & 1124 & 1761 & 2886 & 4503 & 7383 & 10544 & 16367 & 20652 & 24897 & 36599 & 63389 & 91786 & 149151 \\
\hline 300 & 674 & 1201 & 1881 & 3082 & 4810 & 7886 & 11263 & 17482 & 22060 & 26594 & 39095 & 67711 & 98043 & 59320 \\
\hline 320 & 717 & 8 & 2001 & 3279 & 5117 & 8390 & 982 & 8598 & 23468 & 28291 & 41590 & 72032 & 04300 & 69488 \\
\hline 340 & & 54 & 2121 & 3476 & 5424 & 8893 & 12700 & 19714 & 24876 & 29989 & 44085 & 76354 & 0558 & 179657 \\
\hline 360 & 803 & 1431 & 2241 & 3672 & 5731 & 9396 & 13419 & 20830 & 26283 & 31686 & 46580 & 80676 & 16815 & 189825 \\
\hline 380 & 846 & 1508 & 2361 & 3869 & 6038 & 9900 & 14138 & 21945 & 27691 & 33384 & 49075 & 84997 & 123073 & 199993 \\
\hline 400 & 889 & 1584 & 2482 & 4066 & 6345 & 10403 & 14857 & 23061 & 29099 & 35081 & 51570 & 89319 & 129330 & 210162 \\
\hline 420 & 932 & 1661 & 2602 & 4263 & 6652 & 10906 & 5576 & 177 & 30507 & 36778 & 54066 & 93640 & 588 & 20330 \\
\hline 440 & 975 & 38 & 2722 & 4459 & 6959 & 11410 & 16294 & 293 & 31915 & 38476 & 56561 & 97962 & 1845 & 230498 \\
\hline 460 & 018 & 1814 & 2842 & 4656 & 7266 & 11913 & 17013 & 26409 & 33323 & 40173 & 59056 & 102283 & 8103 & 240667 \\
\hline 480 & 61 & 1891 & 2962 & 4853 & 7573 & 12416 & 17732 & 27524 & 34731 & 41870 & 61551 & 106605 & 1360 & 250835 \\
\hline & 04 & 1968 & 3082 & 5049 & 7880 & 920 & 18451 & 28640 & 36139 & 3568 & 64046 & 110927 & 618 & 61004 \\
\hline 520 & 47 & 3044 & 3202 & 5246 & 8187 & 13423 & 19170 & 29756 & 37547 & 45265 & 66541 & 115248 & 66875 & 271172 \\
\hline & & 21 & 3322 & 5443 & 8494 & 13926 & 19889 & 30872 & 38955 & 46962 & 69037 & 119570 & 132 & 1340 \\
\hline 560 & 33 & 2198 & 3442 & 5640 & 8801 & 14430 & 20607 & 31987 & 40363 & 48660 & 71532 & 123891 & 390 & 291509 \\
\hline 580 & 76 & 2274 & 3562 & 5836 & 9108 & 14933 & 21326 & 33103 & 41771 & 50357 & 74027 & 128213 & 647 & 1677 \\
\hline & & 51 & 3682 & 6033 & 415 & 15 & 22045 & 219 & 43179 & 52054 & 76522 & 34 & 905 & \\
\hline 620 & & 2427 & 3802 & 6230 & 22 & 40 & 22764 & 335 & 4587 & 3752 & 17 & 356 & & 014 \\
\hline & & & 3922 & 6426 & 29 & 16443 & 23483 & 6451 & 45994 & 5449 & & 177 & 420 & 332182 \\
\hline 660 & & 81 & 4042 & 6623 & 336 & 16946 & 24202 & 37566 & 47402 & 57146 & 84008 & & 677 & 342351 \\
\hline 680 & & 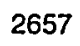 & 4162 & 6820 & 10643 & 17450 & 24920 & 38682 & 48810 & 58844 & & & & 519 \\
\hline & & 34 & 4282 & 7017 & 350 & 17953 & 25639 & 39798 & 50218 & 60541 & 98 & & 92 & 362687 \\
\hline 720 & & & 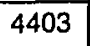 & 7213 & 11257 & , & 26358 & 914 & 1626 & 2238 & 93 & 464 & 29450 & 372856 \\
\hline & & & & 7410 & 564 & 18960 & 27077 & 42030 & 53034 & 3936 & 93988 & 85 & 707 & 383024 \\
\hline & & 2964 & 4643 & 7607 & 11871 & 19463 & 27796 & 43145 & 54442 & 65633 & 96483 & 07 & 365 & 192 \\
\hline 780 & & 304 & 4763 & 7803 & & 19966 & 28515 & 44261 & 55850 & 67330 & & & & 361 \\
\hline 800 & 50 & 17 & 4883 & 8000 & 12485 & 20470 & 29233 & 45377 & 57258 & 69028 & & 750 & & 529 \\
\hline & 33 & & 003 & 8197 & 12792 & 20973 & 29952 & 46493 & 58666 & 70725 & & 371 & 737 & 423697 \\
\hline 840 & & & 123 & 8394 & & 21476 & 30671 & 47608 & 60074 & 72422 & & & & 433866 \\
\hline 860 & 79 & & 243 & 8590 & 106 & 21980 & 31390 & 48724 & 61482 & 20 & & 15 & 252 & 444034 \\
\hline 880 & & 3 & 5363 & 87,87 & & 22483 & 32109 & 49840 & 62890 & & & & & 03 \\
\hline 900 & 65 & 3501 & 5483 & 8984 & 14020 & 22986 & 32827 & 50956 & 64298 & 77514 & & & 767 & 464371 \\
\hline 920 & & & 603 & 9181 & & 23490 & 33546 & 52072 & 65705 & 9212 & 16445 & 79 & 24 & 539 \\
\hline 940 & & & 23 & & & 993 & 265 & 3187 & 67113 & 80909 & & & & 708 \\
\hline 960 & & 3 & 43 & 9574 & & 496 & 984 & 4303 & 68521 & 82606 & 335 & 322 & 539 & 876 \\
\hline on & & & 63 & 9771 & & 2 & 03 & 55419 & 69929 & 84304 & 123930 & 9644 & 797 & 505044 \\
\hline & & & 6083 & 9967 & & 503 & 36422 & 56535 & 71337 & 86001 & 126425 & 218965 & 317054 & 515213 \\
\hline & & 67 & 6684 & 951 & 7090 & 8020 & 40016 & 62114 & 78377 & 94488 & 138901 & 240573 & 348341 & 566055 \\
\hline & & & & & & & & 67693 & 85416 & 102974 & 151377 & 262181 & 379629 & 616896 \\
\hline & & 5034 & 7884 & 12918 & 20161 & 33053 & 47204 & 73271 & 92456 & 111461 & 163853 & 283789 & 410916 & 667738 \\
\hline 1400 & & 5417 & 8485 & 13902 & 21696 & 35570 & 50798 & 78850 & 99496 & 119948 & 176329 & & 442203 & 718580 \\
\hline 1480 & 3212 & 5724 & 8965 & 14689 & 22924 & 37583 & 53673 & 83313 & 105127 & 126737 & 186309 & 322683 & 467233 & 75925 \\
\hline
\end{tabular}

NOTE; See Pages 18 and 19 for Pressure Limits. 


\section{Sizing Correction Factors and Multipliers \\ Flow Factor for Vapors and Gases - $K_{b}$ for Back Pressures Exceeding Critical}

Back pressure generally has no effect on the set pressure or flow capacity of Crosby's Pilot Operated Pressure Relief Valves except when flow is expected to be subcritical (absolute back pressure exceeds $55 \%$ of the absolute relieving pressure). In this case the flow correction factor $\mathrm{K}_{\mathrm{b}}$ must be applied. If the ratio of absolute back pressure to absolute relieving pressure is less than $55 \%$, no special correction factor is required: $K_{b}=1.00$.

Note: Consult the factory for back pressure greater than $70 \%$ of set pressure.

$$
\begin{aligned}
& \mathrm{Pb}_{\mathrm{b}} / \mathrm{P}_{1}=\text { BACK PRESSURE PERCENTAGE }=\frac{\text { BACK PRESSURE, (PSIA or kPa or bar Absolute) }}{\text { ACCUMULATED INLET PRESSURE (PSIA or kPa or bar Absolute) }} \times 100
\end{aligned}
$$

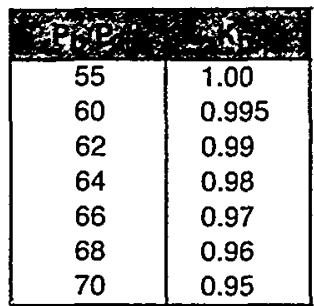

Example for determination of Flow Factor $\mathrm{K}_{\mathrm{b}}$ :

Set Pressure $=150$ psig

Back Pressure $=100$ psig

$\mathrm{P}_{\mathrm{b}} / \mathrm{P}_{1}=$ Back Pressure Percentage $=\frac{100+14.7}{150(1.1)+14.7} \times 100$

$$
=\frac{114.7}{179.7} \times 100=64 \%
$$

Where $\mathrm{Pb}_{\mathrm{b}} / \mathrm{P}_{1}=64 \%, \mathrm{~K}_{\mathrm{b}}=0.98$

\section{Typical Properties of Gases}

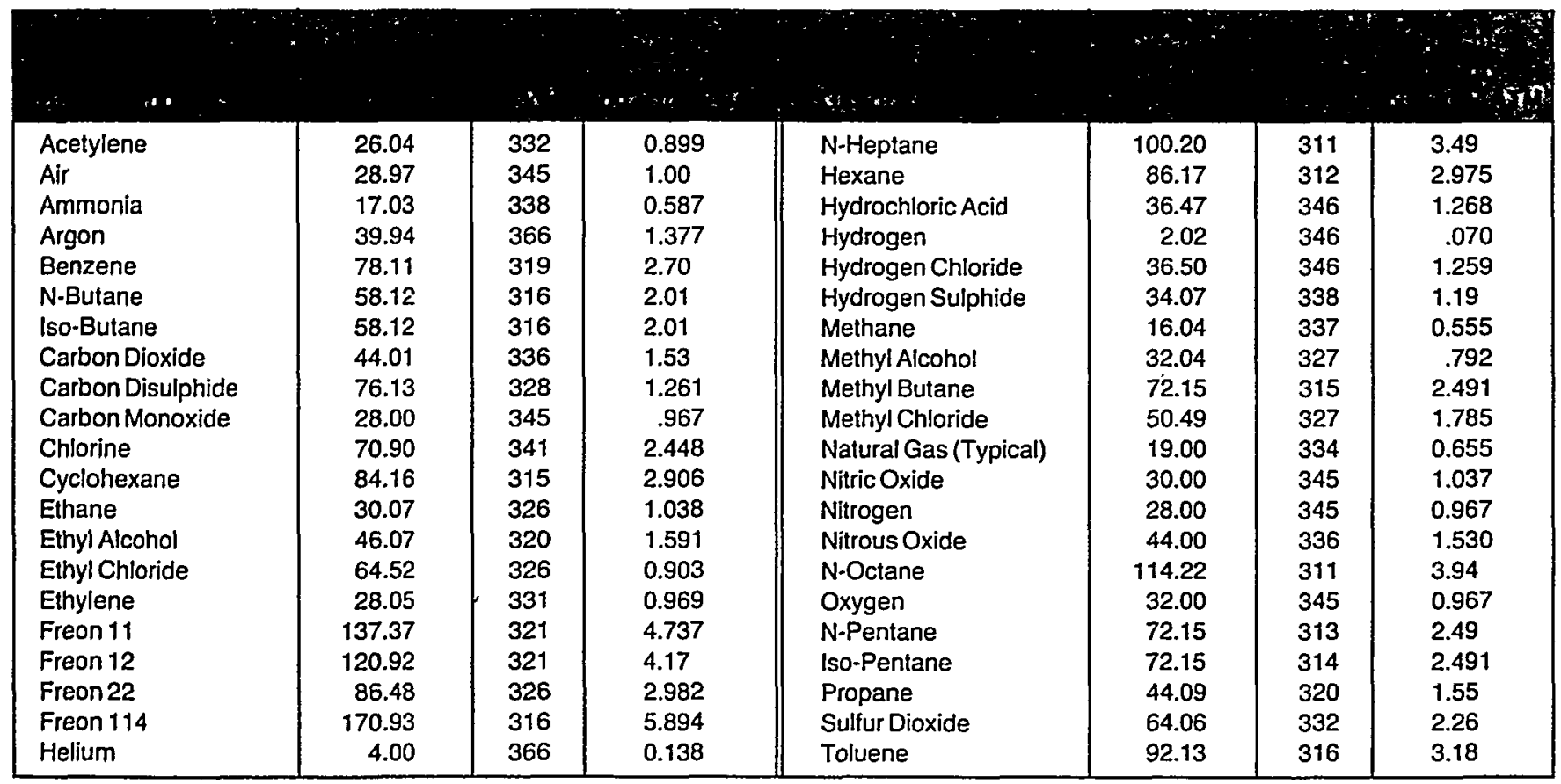




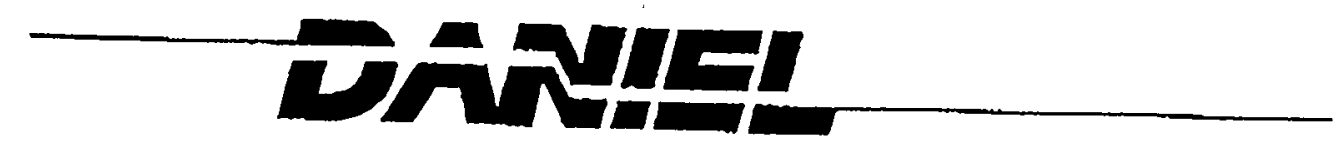

Daniel Valve Company PO Box 40421

Houston, TX 77240

Telephone: (281) 469-0550

Facsimile: (281) 894-1332

http://www.danielind.com

\section{FAX TRANSMITTAL}

\begin{tabular}{llll} 
Company: & HAN-PADRON ASSOCIATES & Date: & January 10, 2000 \\
\hline To: & MR. ROBERT BURY & From: & Ron Kennedy \\
\hline Fax Number: & 212-629-4406 & \multicolumn{2}{c}{ Danflo Sales Mgr. } \\
\hline Phone Number: & 212-736-5466 & Total pages, including cover: \\
\hline Subject: & DVC QUOTE 00-7768-08-104/YOUR JOB 864-01 \\
\hline \multicolumn{3}{l}{ DVC FAX MESSATE 00-0110-02 } & \\
\hline Robert: & DU & \\
\hline
\end{tabular}

Per your request, we offer the following budget quote for the Danflo valves listed on your suction
mooring technology compressed air system:

Valve 1 N/C solenoid valve, General Purpose Solenoid Valve

Unit Budget Price: $\$ 16,550.00$ (USD)

Valve 2

2" ANSI 150\#RF 2-411-ALN-50 Solenoid valve operated Danflo valve with one (1) each NO and one
N/C solenoid valve, General Purpose Solenoid Velve Unit Budget Price: \$10,865.00 (USD)

\section{Valve 3}

3" ANSI 150\#RF 3-411-ALN-50 Solenoid valve operated Danflo valve with one (1) each NO and one N/C solenoid valve, General Purpose Solenoid Valve

Unit Budget Price: \$ 12,310.00 (USD)

Delivery: $12-14$ weeks 


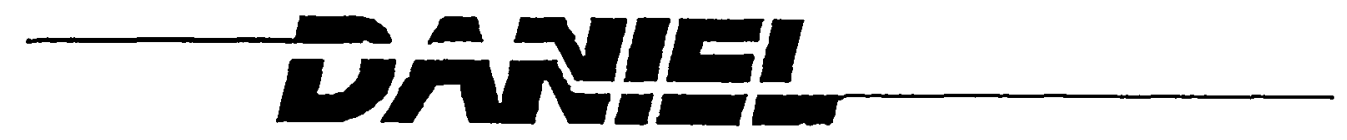

Daniel Valve Company PO Box 40421 Houston, $T \times 77240$ Telephone: (281) 489-0550 Facsimile: (281) 894-1332 http:/hwww.danielind.com

We re quoting our standard Danflo carbon steel valves with soft seats. The leakage rate is classified as Class $\mathrm{N}$, but for all intensive purposes, it is leak tight.

I would like to point out Mr. Bury that I believe that the application that you have illustrated should use ball valves with either hydraulic or spring return actuators in lieu of what we are offering. You should be able to get the same two (2) second or less full closing/opening cycle. This type of valve is used for ESD valves on pipelines often.

With regards to the drafting symbol for the valve, what you have shown on your drawing is okay and acceptable.

Call me if you need any additional information.

Sincerely yours,

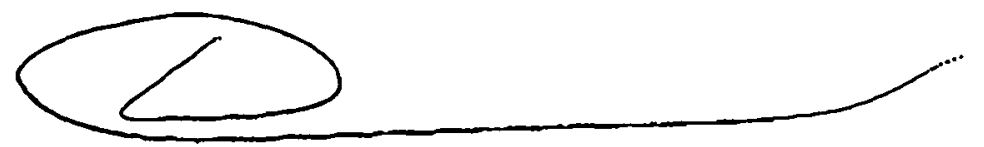

Ron Kennedy.

Danflo Sales Manager

Cc: C. Brown

J. Wass 


\section{The}

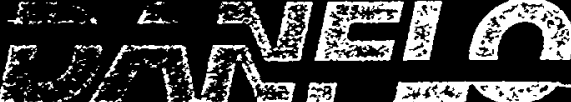 \\ family of \\ CONTROL VALVES}

8

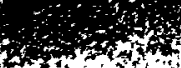

$\therefore$
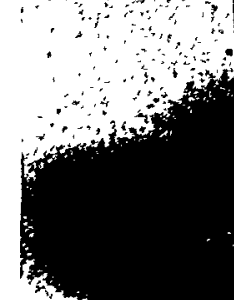

in
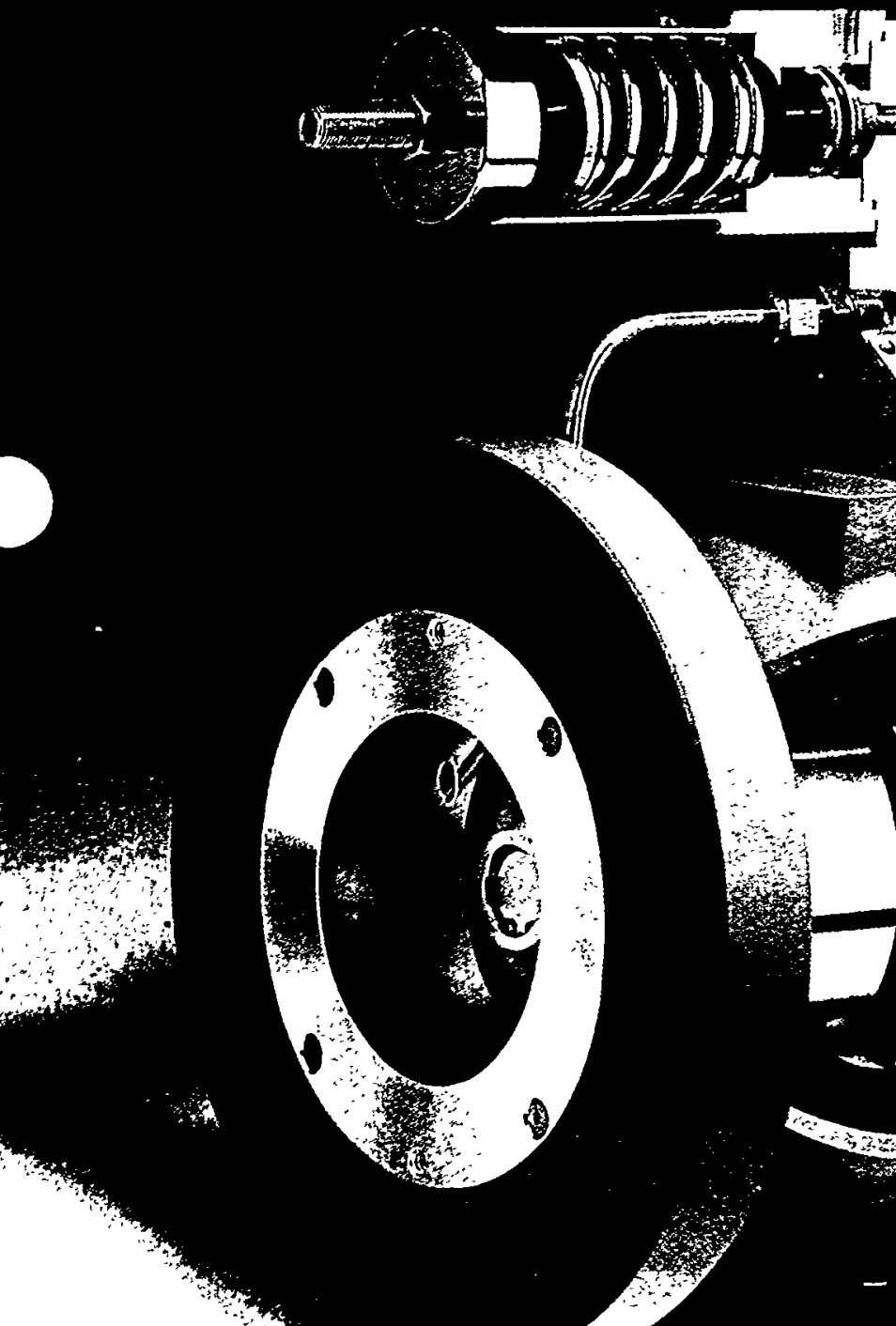

tyon 


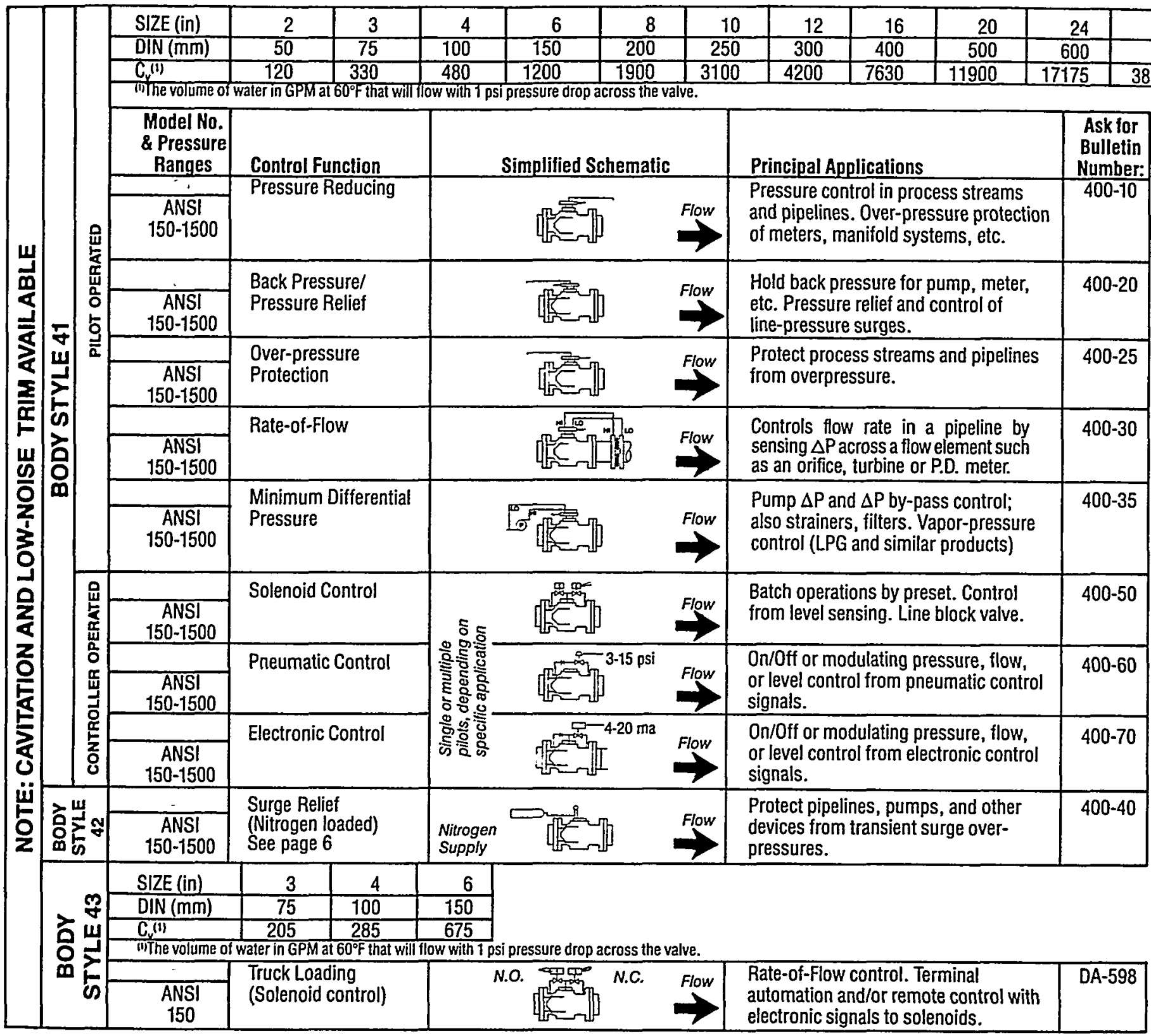

+...

$\left[\begin{array}{lll} & & \end{array} \quad \begin{array}{l}\text { Similar to } 400 \text { series but uses a "pressure- } \\ \text { balanced" plug with power piston. }\end{array}\right.$

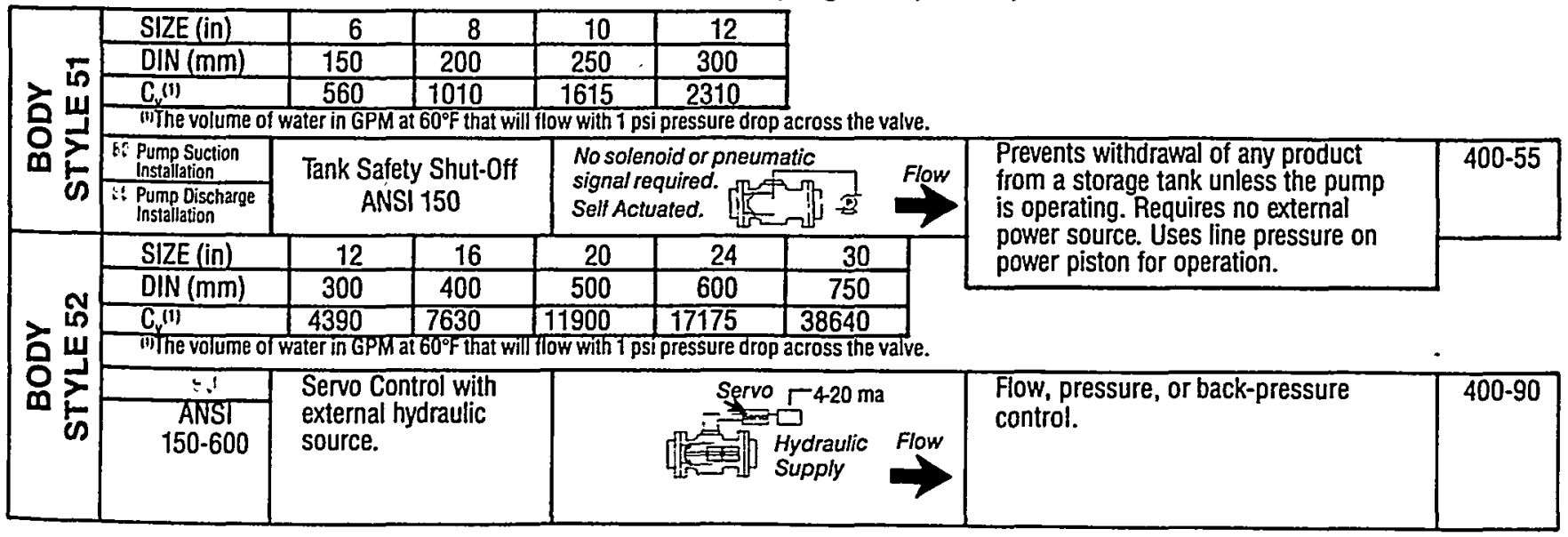




\section{SERIES}

A 400 Series DANFLO valve, in its simplest form, consists of a dynamically balanced-plug assembly that slides back and forth easily and rapidly to open, close, and throttle.

- Pressure in the plug cavity closes the valve.

- With no pressure in the cavity, the valve opens.

Pressures are applied through three ports: upstream, downstream, and to the plug cavity "behind the plug."

Downstream port provides for venting plugcavity pressures rapidly to speed up valve response. The port leads to the point of lowest internal pressure in the valve.

For convenience, the ports (and related pressures) are identified:

$P_{u}=$ Upstream

$P_{d}=$ Downstream

$P_{c}=$ Cavity

\section{GGRL VALVE GCTIOLI}

$P_{c}$ is connected to $P_{u}$. This equalizes pressures on upstream and downstream faces of the plug. The internal-spring force closes the valve.

By connecting $P_{c}$ to $P_{d}$, the plug cavity is vented. Upstream line pressure overcomes the spring force, and the plug is forced open.

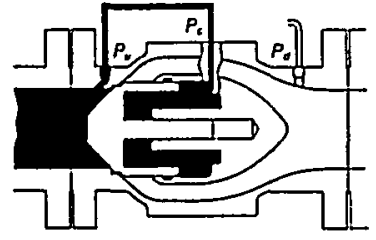

Parts P.and P.connectod Upstroam prossura is oqualized across the vativ SPAng pressure lorces plug to soat
VALVE CLOSED

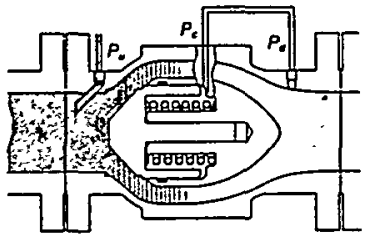

Pons Pand P fonnocted A differentiel prossure axists across the plug Upstream prossure greater than mochanical spring pressure moves

\section{PILOT OPERATION}

\section{SERIES - BODY STYLE 41}

The pilot connects $P_{c}$ to either $P_{u}$ or $P_{d}$ to close or open the valve. The connection is made in the pilot by an internal poppet that shuttles back and forth depending on whether force from the pressure provided by a "sense line" is larger or smaller than the pilot spring force. (Differential-pressure pilots are also available.)

An easy way to remember how the pilot works is that the valve, after the initial start-up sequence, will normally be closed if the spring "points" upstream (back-pressure or relief operation), normally open if "pointed" downstream (pressure-reducing service).

Use of the pilot is therefore:

- "Point" the spring so the valve is normally open or closed, as desired.

- Connect the "sense line" upstream or downstream to "sense" the pressure you want to actuate the pilot.
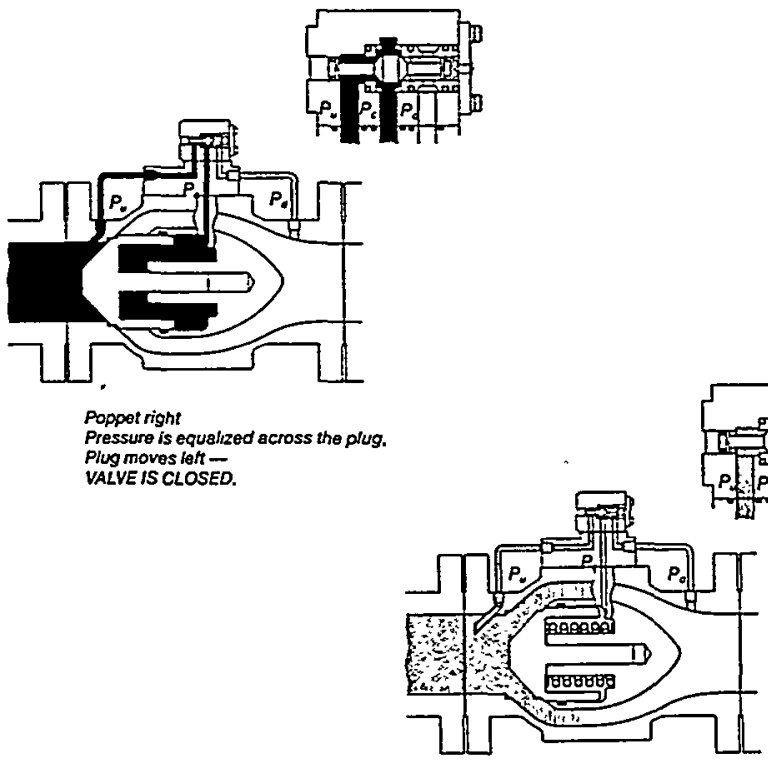

Poppet toh Pressura differential is created Piug moves right

\section{SERIES}

500 Series valves also use axial flow for high flow capacity and are very similar to the 400 Series valves. However, a pressurebalanced plug design has ports to equalize effects of line pressure on each side of the plug - and the plug is connected to a power piston which forces it open or closed.

Principal applications for 500 Series are Tank Safety Shut-Off and Electrohydraulic Servo Control.

\section{TANK SAFETY SHUT-OFF} 500 SERIES - BODY STYLE 51

- Automatic operation.

- When pump is running, valve is open.

- If pump stops or pressure equalizes across pump, pressure in sense line is no longer sufficient to hold valve open. Valve closes to prevent lost product and spills.

\section{MODEL 80, LOCATE IN PUM MODEL 85, LOCATE IN PUM}

Operation:

- Line pressure applied to both faces of power piston.

- Spring forces plug CLOSED.

- Pump starts; discharge pressure on power piston forces plug OPEN.

- Pump stops; pressure again equal across power piston.

- Spring forces plug CLOSED. 


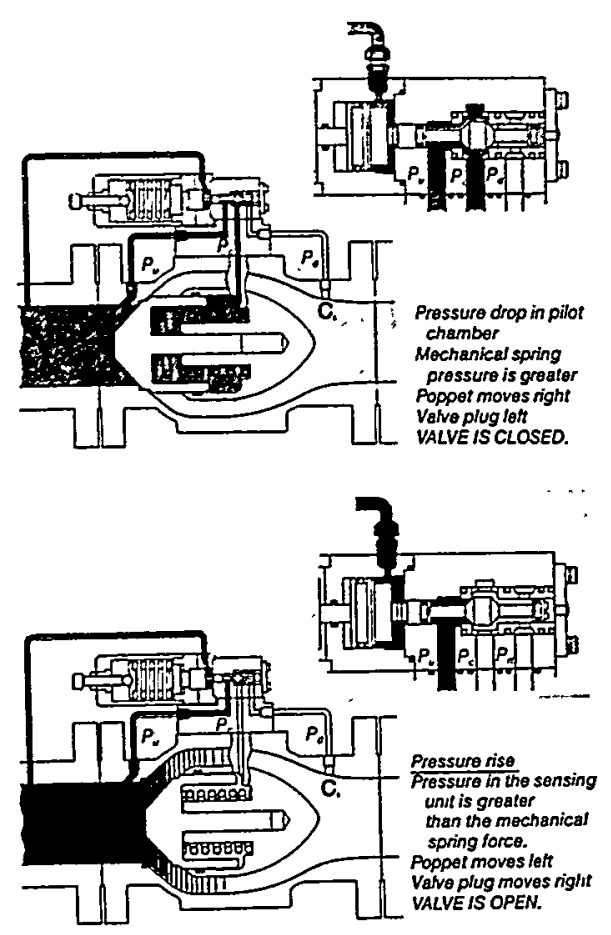

THROTTLML WUTROLLER OPERATIOA

\section{SERIES -BODY STYLE 41}

By connecting $P_{c}$ to $P_{u}$ through a small orifice (to "smooth out" control action) and also connecting $P_{c}$ to $P_{d}$ via a single-seat globe valve with a pneumatic (3-15\# or $6-30 \#)$ or electronic (4-20 Ma) control signal, the valve will throttle in response to the pneumatic or electronic signal from the controller.
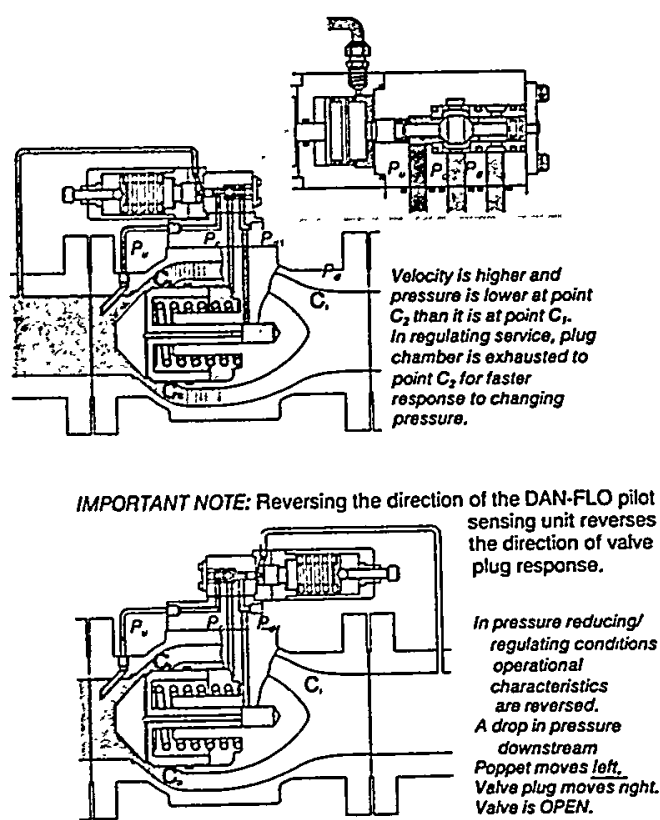

\section{PNEUMATIC CONTROL}

MODEL 60

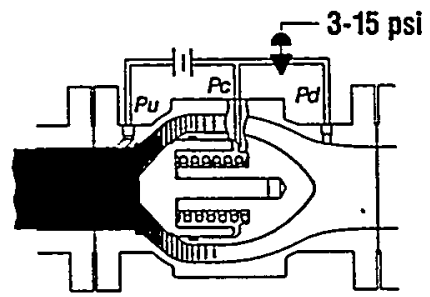

\section{ELECTRONIC CONTROL}

MODEL 70

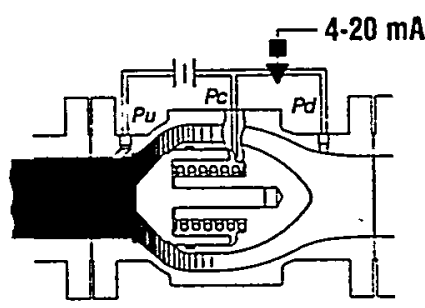

\section{E IN PUMP SUCTION}

\section{E IN PUMP DISCHARGE}
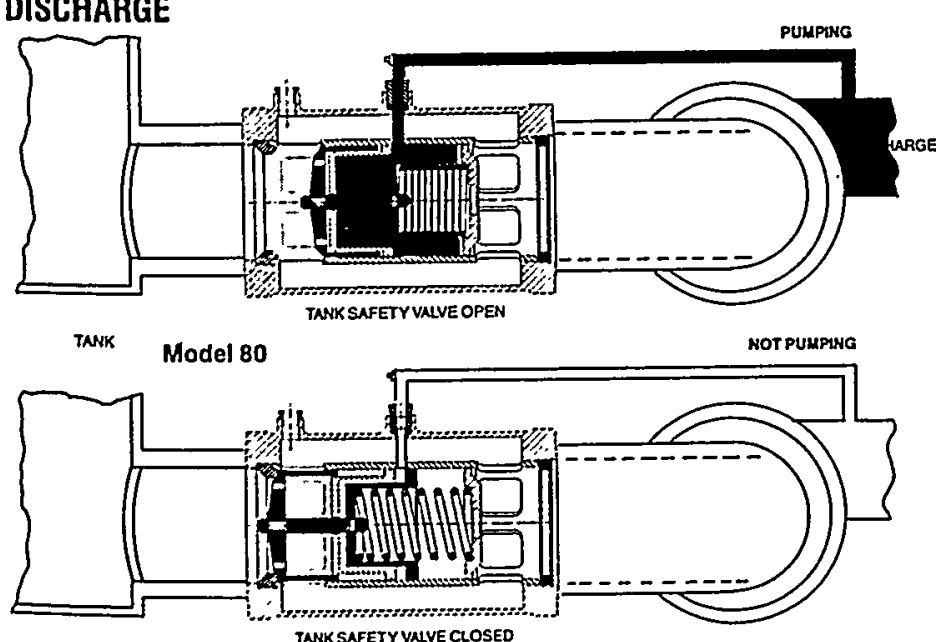

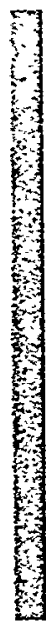

Page 4

\section{ELECTRDHYORAULL SERVO CONTA: 500 SERIES-BODY STYLE 52-MODEL 90
SIZES 12" THRU 30"}

The DANFLO Model 90 valve is designed for use with an external hydraulic supply with suitable piping to apply hydraulic force on each side of the valve's power piston. Control over the two hydraulic lines comes from a servo control board in response to standard low-milliamp or low-voltage signals.

The major use of this efficient valve is for flow, back-pressure, or pressure control. An indicator to show plug position is standard on Model 90 valves.

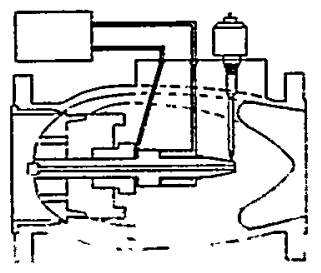




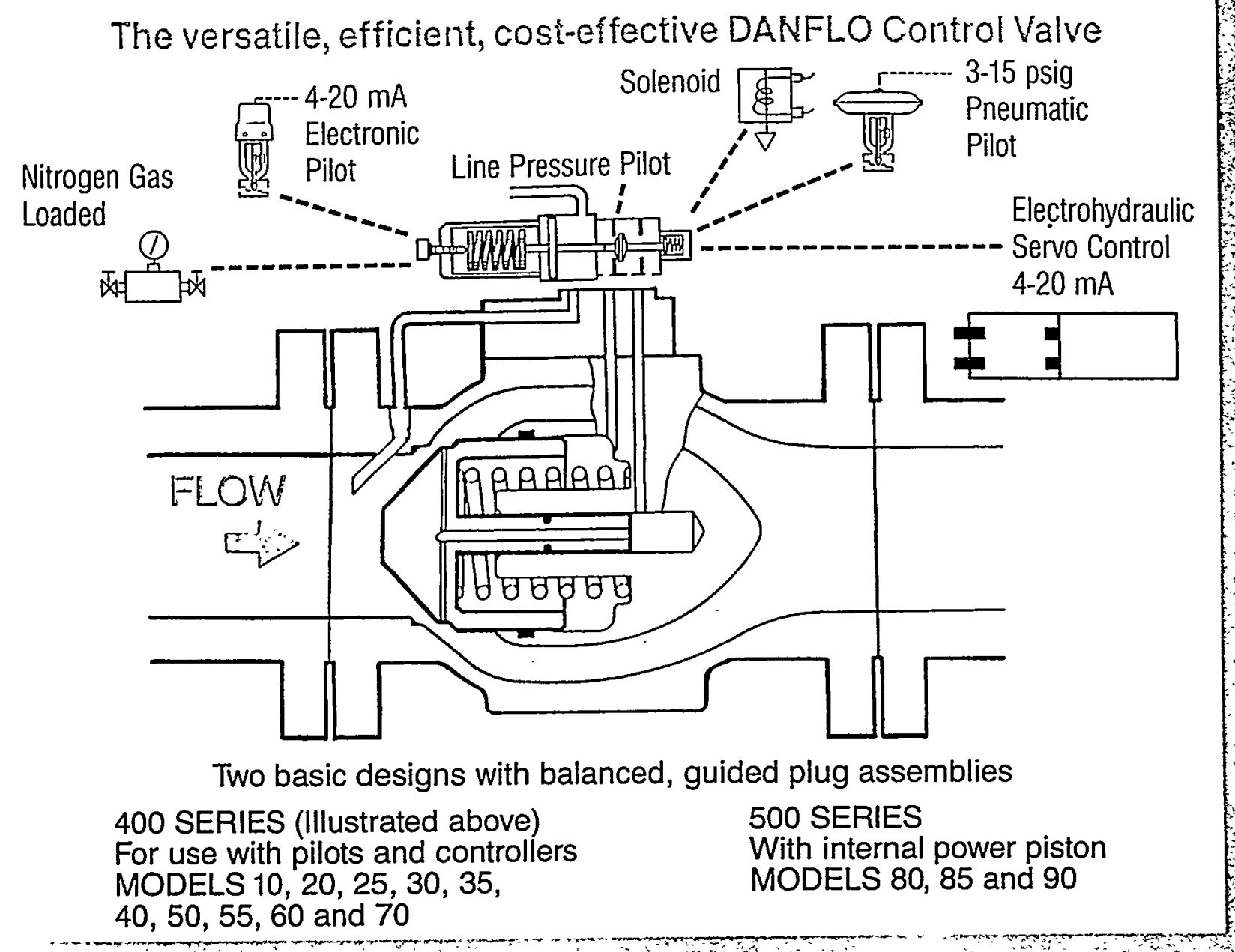

\section{TILEERRINELO DESIGK}

The 400 and 500 series DANFLO valves (with body styles $41,42,43,51$, and 52 ) all share two important characteristics:

- Flow through the valve when open is via a large, venturi-type flow path that provides a high flow coefficient $\left(\mathrm{C}_{v}\right)$ and low pressure drop across the valve.

- Flow is controlled with a guided-plug assembly that moves in response to upstream or downstream line pressure according to the valve application.

\section{TYPICAL DARELO VALLVE}

(Model 20 in Back Pressure/Pressure Relief Service)

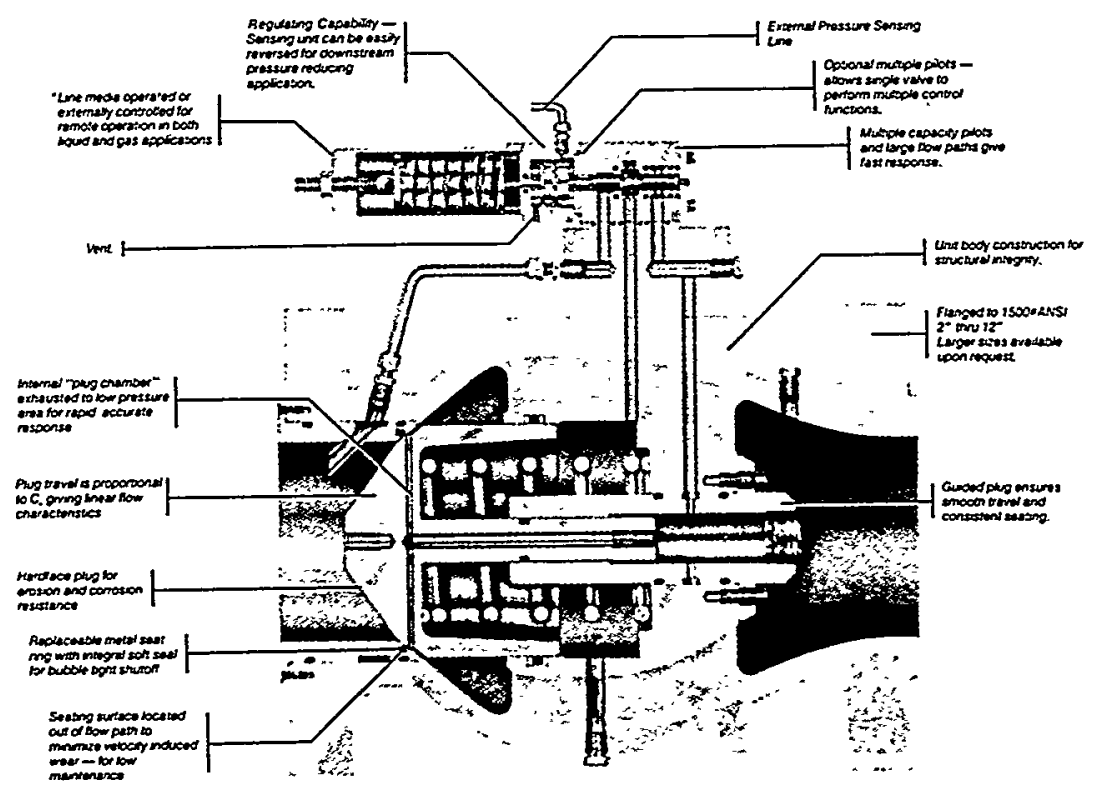

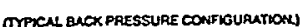

Note: Details of valve body and piping configuration depend on the specific application. 
PRESET TRUCK-LOADING/BATGI-OPERATION 400 SERIES - BODY STYLE 43-MODEL 55

An interesting form of flow control can be achieved with DANFLO valves by using a pair of solenoid valves as shown in the accompanying sketch. They operate in synchronism, alternately tending to open/close the valve but pulsed at such a rapid rate that the plug dynamically "floats" in the correct position to maintain a desired flow rate.

Daniel makes use of this unique digital control in its truckloading systems by coupling DANFLO valves with Daniel LR turbine meters (made specifically for high repeatability measurement in light-hydrocarbon service).

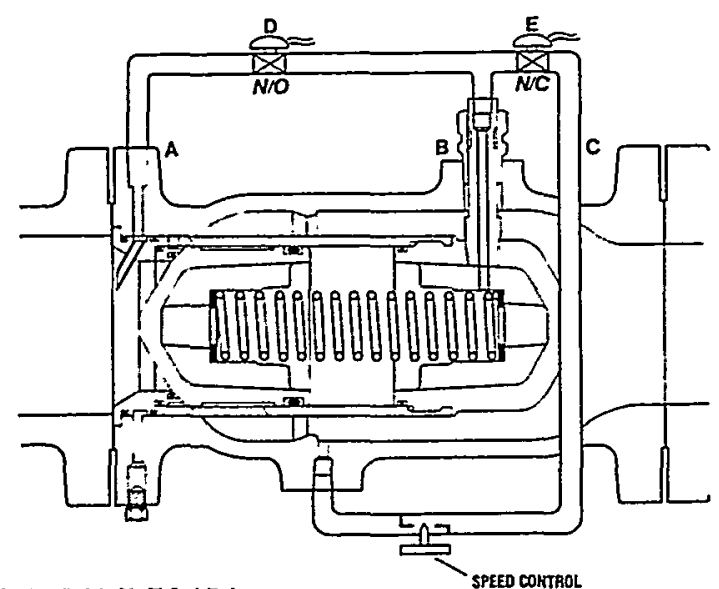

SURGERELLIEE

(Nitrogen pressure loaded)

400 SERIES - BODY STYLE 42-MODEL 40

One of the major uses of DANFLO valves in recent years has been for surge relief in liquid pipelines. Only the $P_{c}$ connection is used, and through it a "set" pressure (from a nitrogen charging system) is applied to the plug cavity.

When a transient surge occurs, line pressure exceeds the "set" pressure and forces the valve plug open instantaneously. The pressure surge volume is dumped to a collection tank or bypassed to downstream. When line pressure drops below the "set" pressure, the valve quickly but smoothly closes. The valve "tracks" all subsequent transient surges until linepressure equilibrium returns.

Operation is fast, automatic, and requires no attention from operating personnel. There is nothing to reset or replace after surge-relief action. The DANFLO Surge valve is always ready for immediate action to prevent damage to equipment and/or harm to personnel.
ORDERING GUIOE

Because of the many variations available in DANFLO valves, it is important that the factory know as much as possible about the intended installation, including:

- Line size and pipe schedule

- Pressure rating (ANSI)

- Maximum line pressures (inlet and outlet)

- Max/min differential pressures

- Max/min line-media temperatures

- Max/min line flow rates

- Composition of line media

- Specific Gravity of media

- Vapor pressure of media

- Batch or continuous operation

- Operational mode and type control device to be used (pilot, controller, servo, etc.)

- Low-noise or cavitation trim needed

- Special considerations.

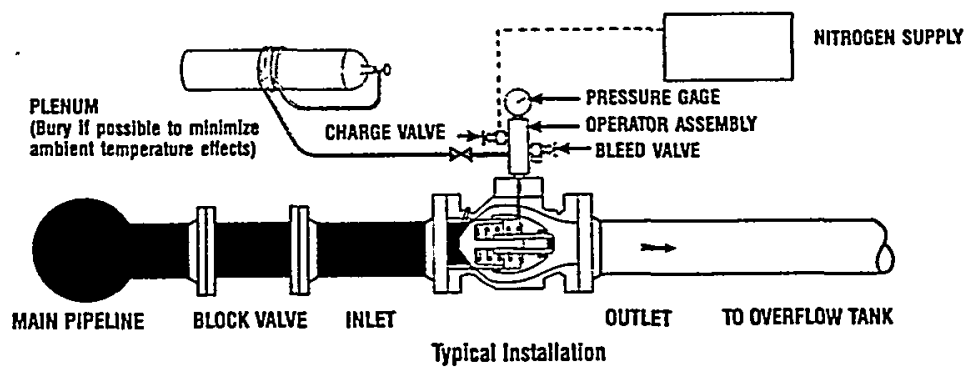

Cavity behind plug filled with nitrogen gas. Valve closed until pressure surge in line exceeds "set" pressure. Valve immediately opens to abate surge, closes when line pressure drops below "set" pressure.

Page 6

DANIEL VALVE COMPANY 19191 Hempstead Highway P.O. Box 40421

Houston TX 77240

Phone: (713) 469-0550

Outside Texas: 1-800-231-3690

Telex: $762-400$

Fax: (713) 894-1332
DANIEL VALVE COMPANY

Library House

The Green

Datchet, Slough

Berkshire, England

SL3 9AU

Phone: (011-44) 753-548173

Fax: $(011-44) 753-582765$
DANIEL INDUSTRIES CANADA

\#114-4215-72 Avenue, S.E. Calgary, Alberta, Canada T2C $2 \mathrm{G} 5$

Phone: (403) 279-1879

Telex: 03-827701

Fax: (403) 236-1337 


\section{Risk Analysis}

In this chapter a failure tree analysis is presented examining the risks inherent in the use of the mooring as described in the previous sections. The risks are evaluated by examining a primary failure and then subsequent failure of each of the systems used following the primary failure and a listing of the consequence of each successive failure.

\subsection{Operational Mooring Risks}

This section examines the risks that are inherent in the mooring operation:

In a successful mooring attempt the following systems are used in sequence aboard the ship:

- The navigation system (differential GPS and back-up (e.g. Miniranger))

- The bow thruster on the vessel

- The autopilot

- The main propulsion machinery

- The underwater video equipment

- The acoustic communication system

- The optical communication system

- The releasable hatch in the bottom of the ship

- The pressure maintenance pumps

The following equipment is used aboard the mooring buoy:

- The buoy wake-up system (acoustic)

- The low pressure tank charging system (A and B system)

- The optical communication system

- The Rapid Ascent system (A or B system)

In the event of failure to capture the buoy the following system will be used:

- Emergency descent system.

The failure tree for the failure of shipborne equipment is shown in figure 4-1. The most serious consequence of the worst scenario sequence of events is the damaging of the hull of the vessel and of the buoy due to lateral impacts as the buoy surfaces next to the ship. In extreme cases the damage to the hull of the ship may consist of holing through of the side plating in the vicinity of the mooring equipment. It then depends on what is behind the plating whether this represents pollution incident or not. The damage so caused is not likely to cause serious problems concerning stability or seaworthiness to the vessel. 
Damage to the buoy would cause it to lose buoyancy and descend to the seabed. If severely damaged salvage equipment may be required to effect repairs because the buoy's ability to surface may no longer exist. 


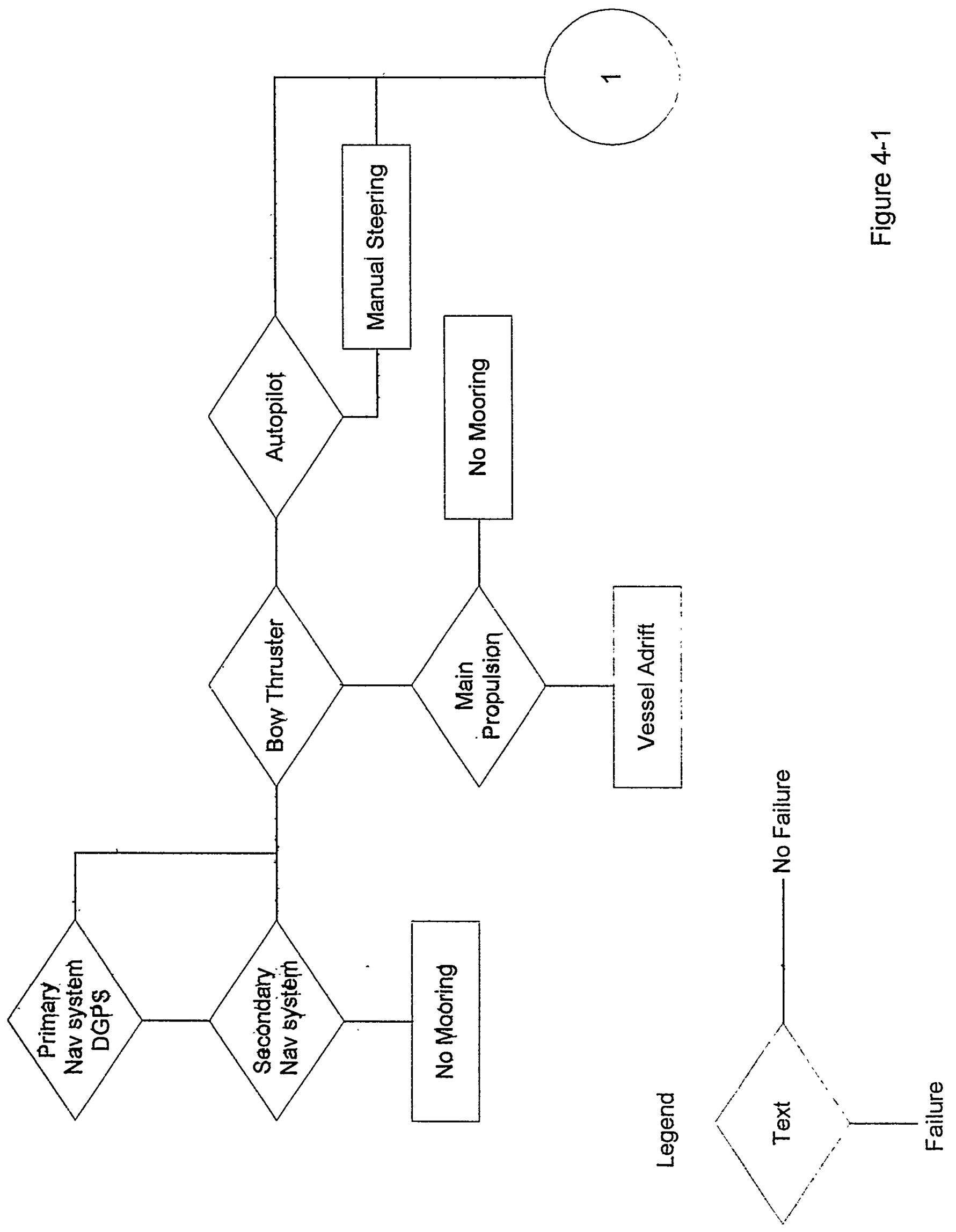

4-3 


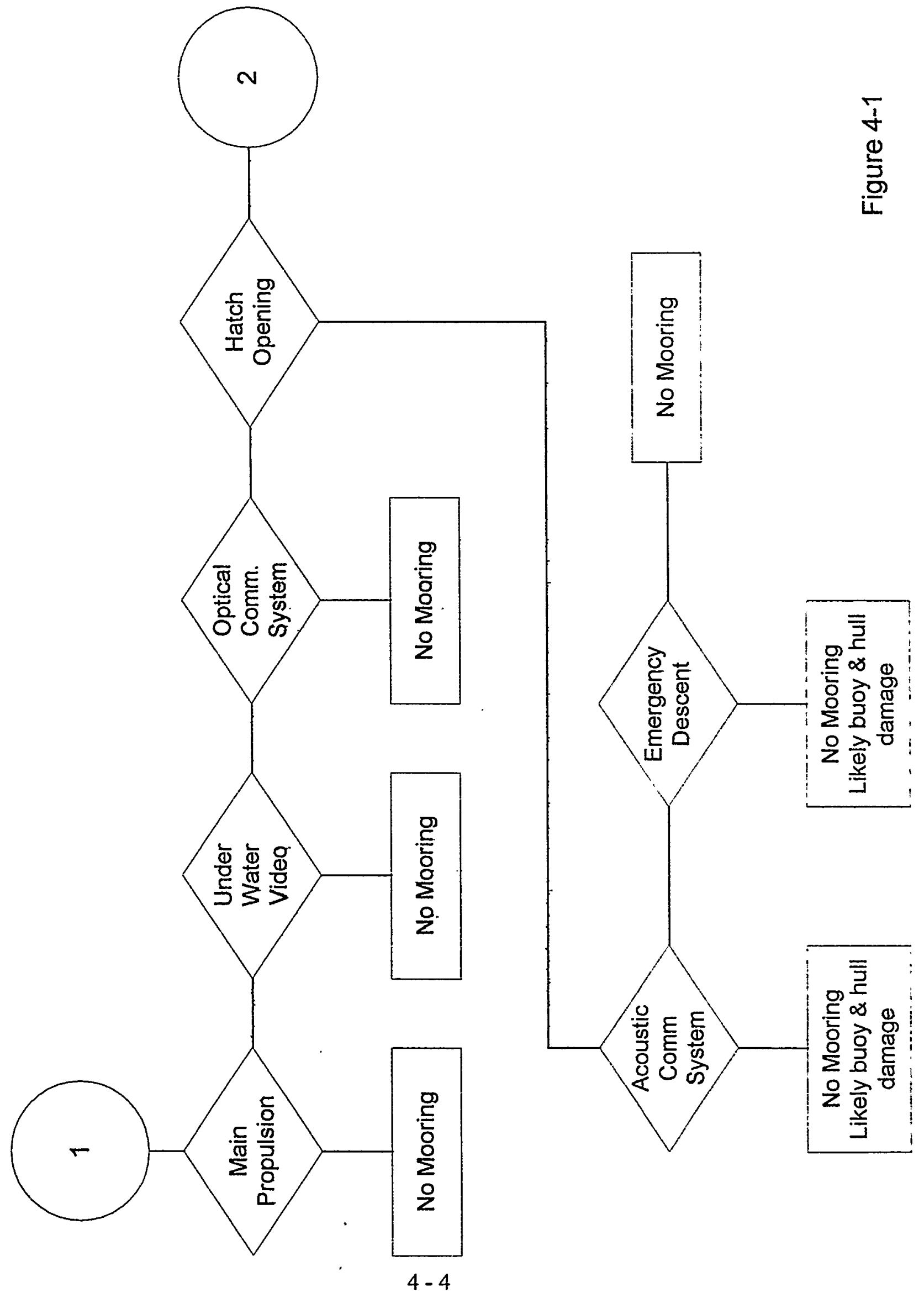




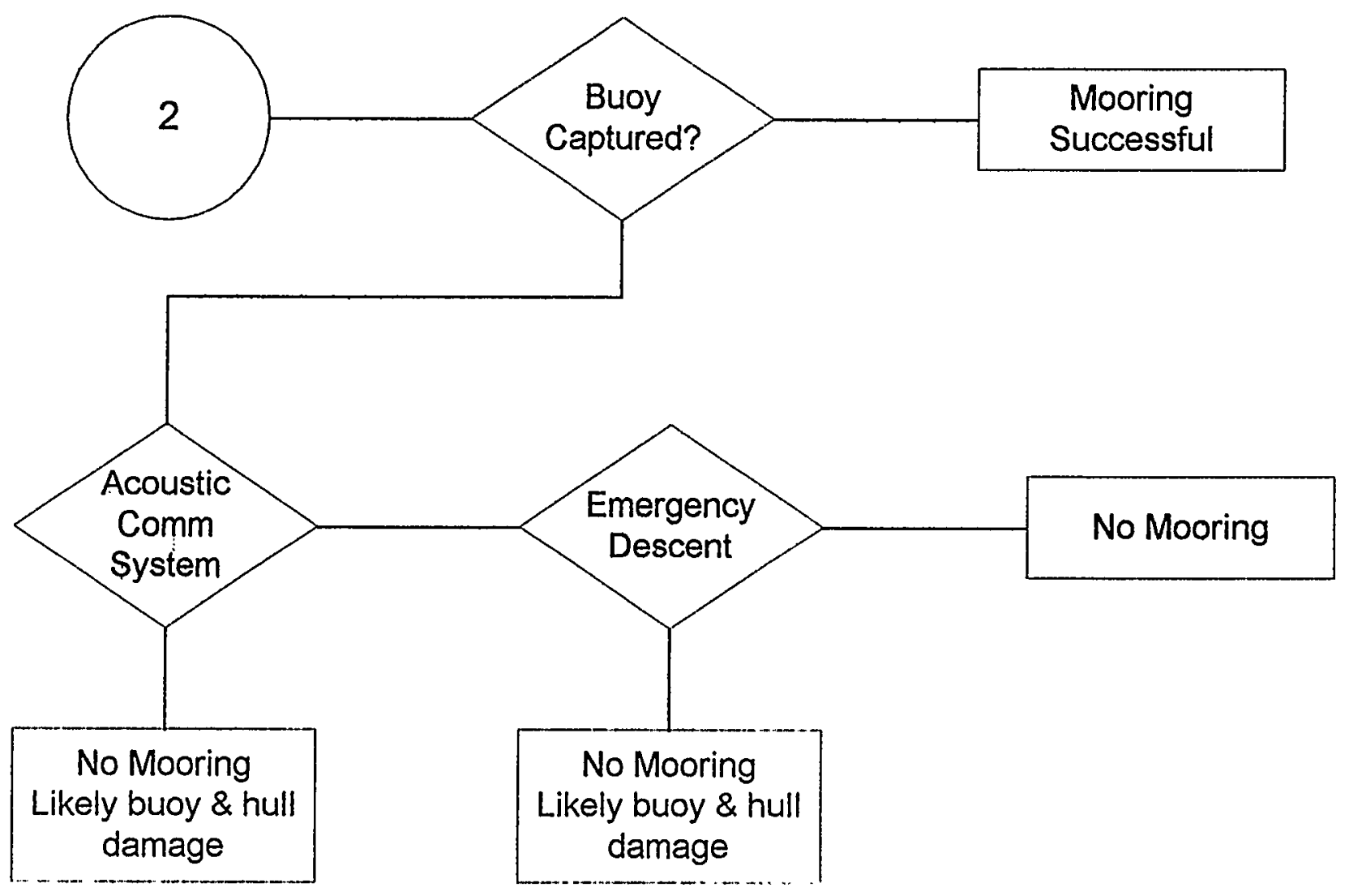

Figure 4-1 


\subsection{Buoy Equipment Failure}

The failure tree of equipment aboard the buoy is shown on figure 4-2. The most serious consequence of a series of failures is the over pressurization of a low-pressure tank used for the rapid ascent system. This may lead to an explosion of the subject tank or rupture of it. This will lead to the buoy sinking to the seabed and making mooring attempts impossible. 


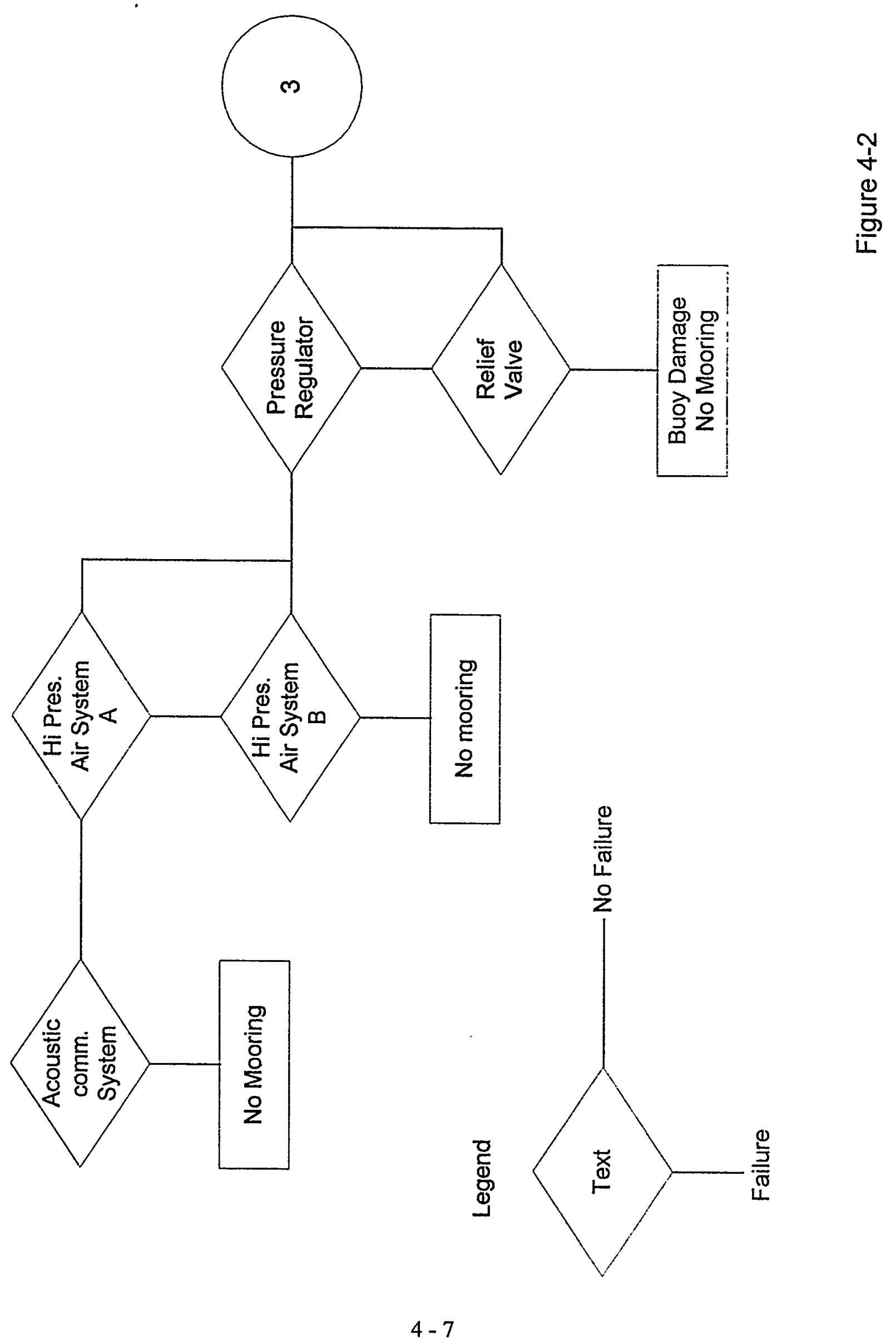




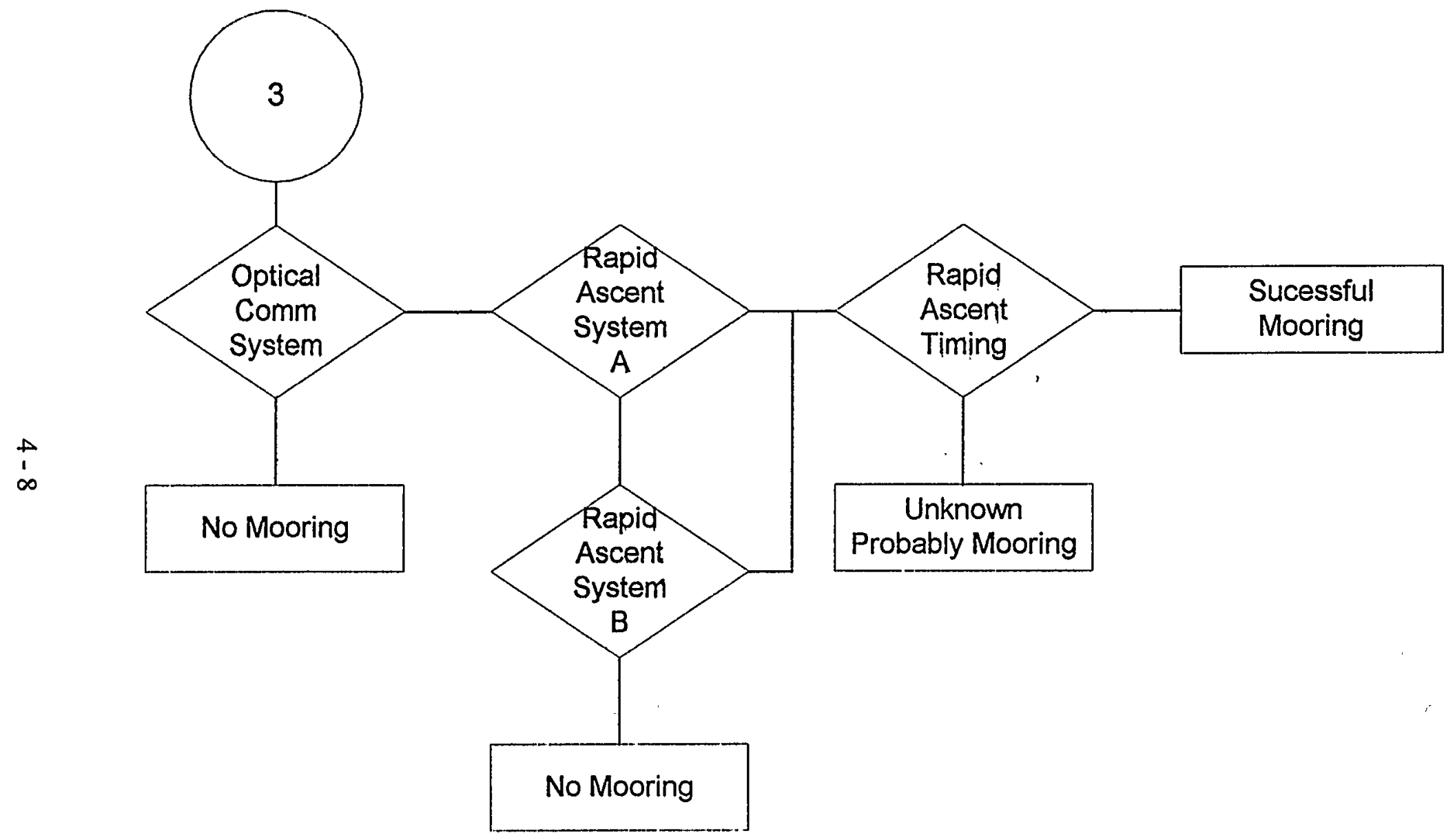

Figure 4-2 


\subsection{Stay Moored and Unmooring.}

While the buoy is moored to the vessel all systems are inactive with the exception of the high pressure tank re-charge and the charging of the batteries in the electrical system.

The emergency descent system is used following each successful mooring to bring the buoy to a eondition that it will descend following release from the vessel. Because the rapid ascent buoyancy volume is not airtight it will fill with water in any event even if the emergency descent system fails to actuate. Therefore no later than 15 minutes after a successful mooring the buoy is always poised to descend when released.

Unmooring is provoked by the simple action of reversing the pressure maintenance pumps. These will pump in approximately $0.3 \mathrm{~m} 3 / \mathrm{sec} .300$ seconds later $90 \mathrm{~m} 3$ has been added to the volume above the buoy. At this point the exterior seal will be broken and the buoy will fall away.

A failure tree analysis of the stay-moored condition is shown in figure 4-3.

A special note is required concerning the unmooring process following the breakage of one of the mooring chains. In the case of a 6 or 9.point spread mooring the buoy may or may not be strongly buoyant. In the case, however, of general arrangement number one where the mooring is secured by lifting weights the buoy will be strongly buoyant following a mooring chain failure. The safe unmooring procedure in this case is to release the buoy with the vessel hovering above the buoy's neutral position. Following release of the buoy the vessel is accelerated astern such that momentum is gained before the buoy surfaces next the vessel. 


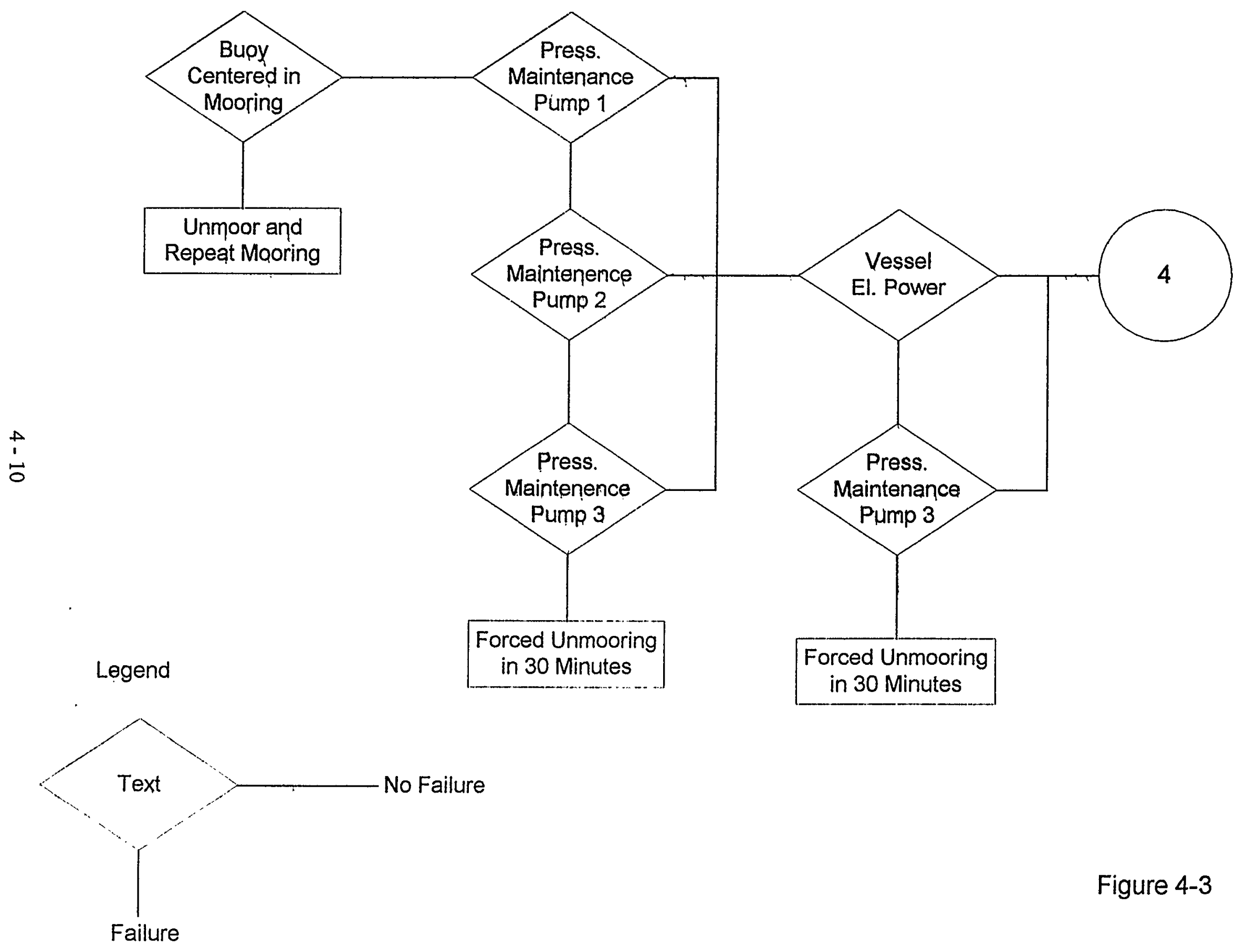




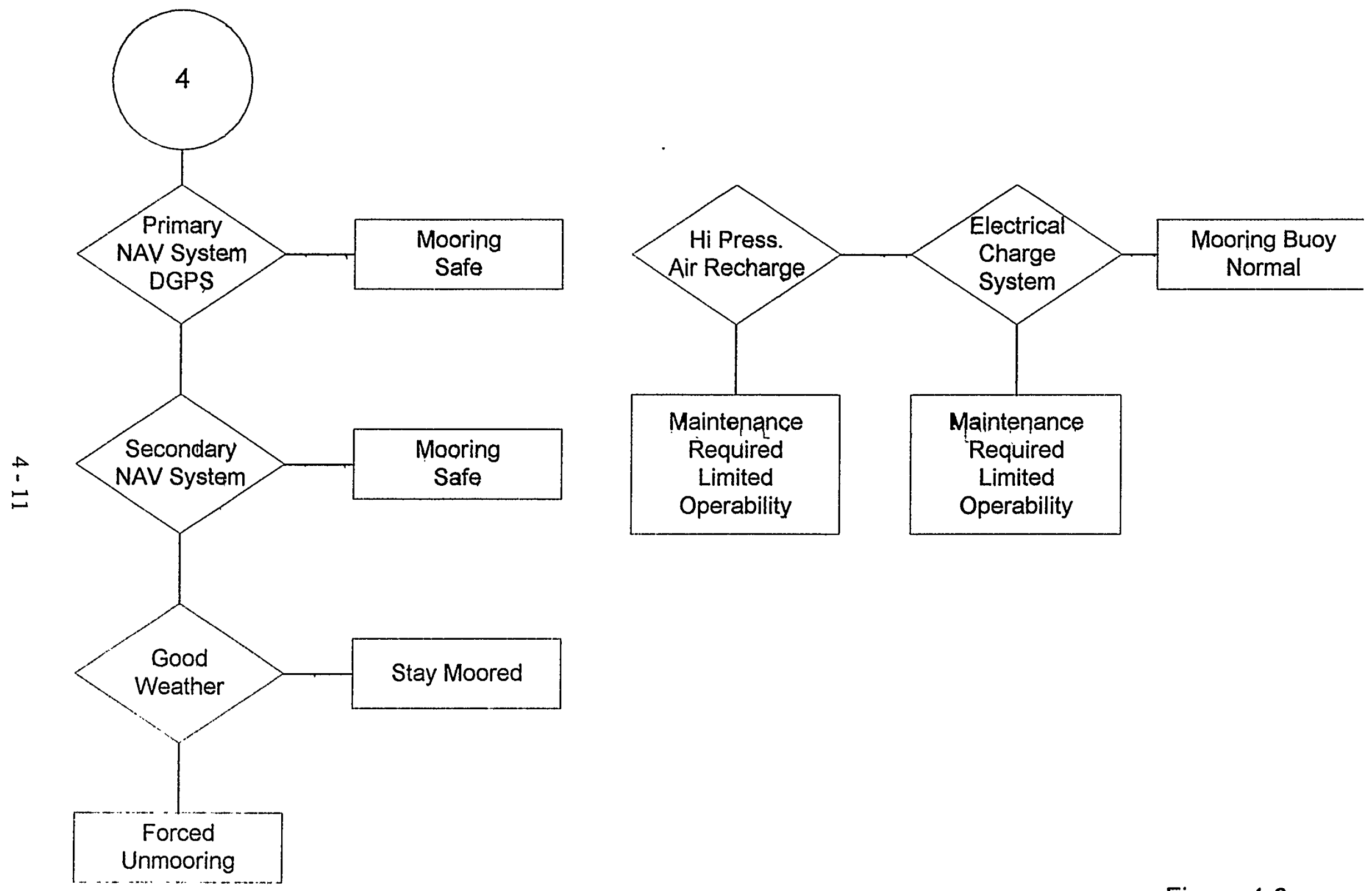

Figure 4-3 


$$
\begin{aligned}
& \frac{9}{1} \\
& 4 \\
& 0 \\
& \frac{0}{3} \\
& \frac{0}{4}
\end{aligned}
$$

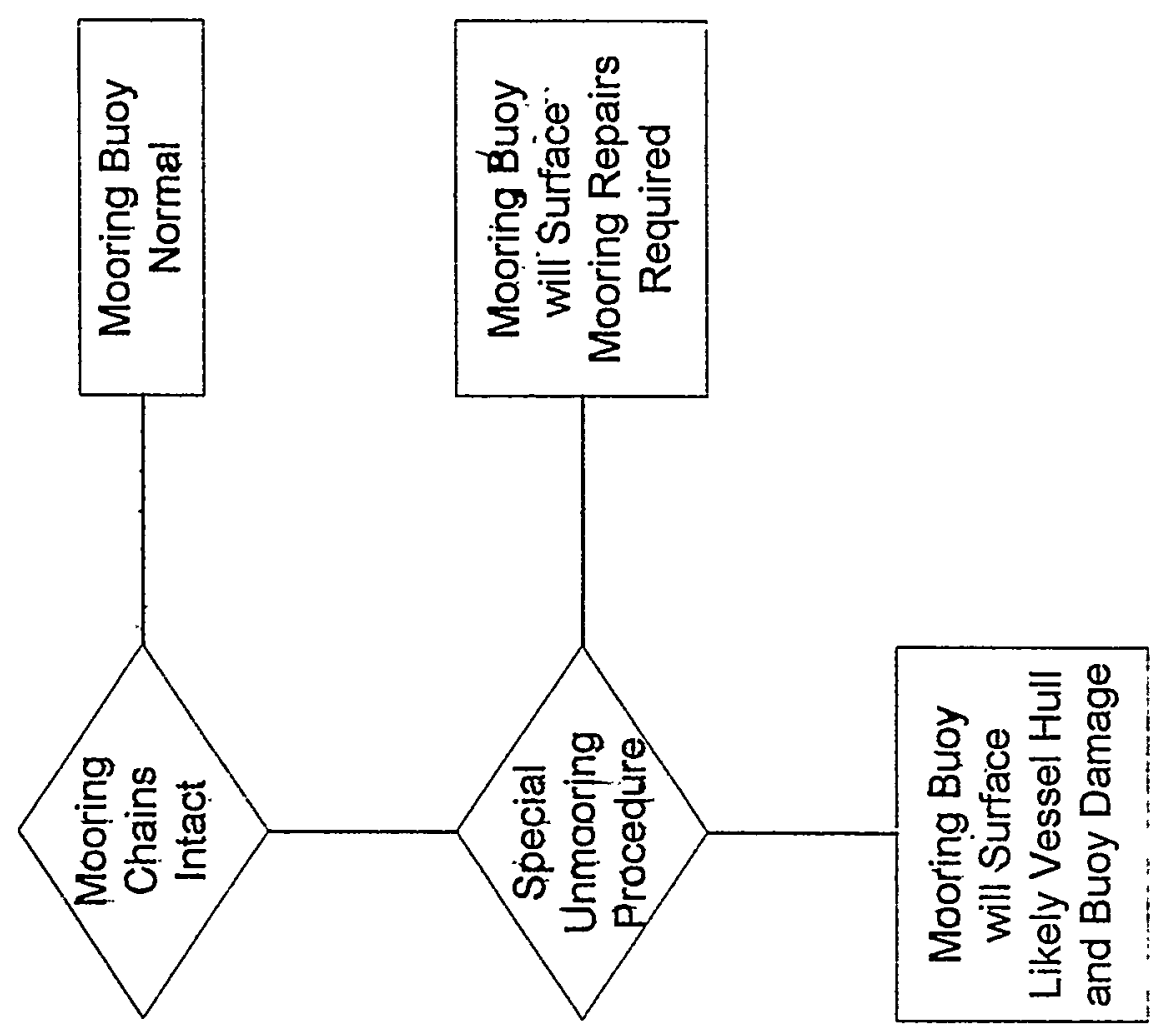




\subsection{Fluid Connection System.}

The fluid connection system is comprised of a number of fluid conductors each individually connected by a quick connector with a double seal charged with nitrogen between the two seals. The nitrogen pressure is maintained above the fluid pressure in the conductor, thereby positively preventing leaks in normal operations.

Each individual conductor is shown in concept below in Figure 4-4. The all valves shown are auto-return valves that automatically shut when the stay open signal is lost.

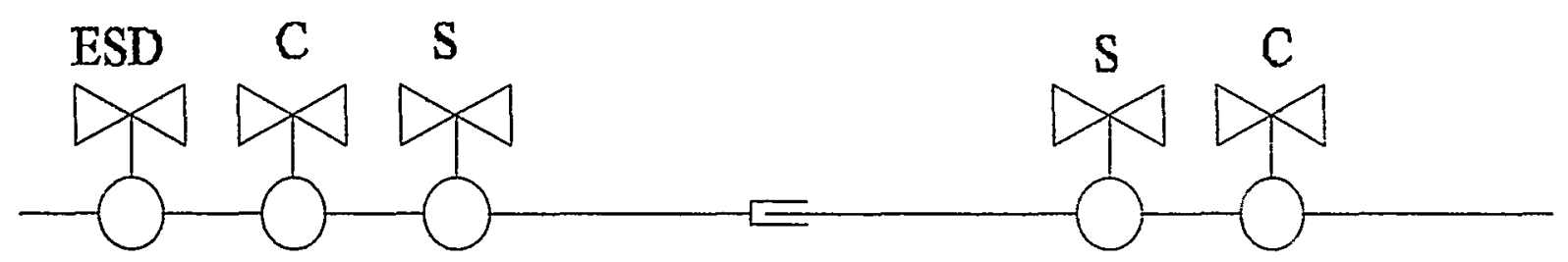

Figure 4-4. Fluid connector system.

The valves labeled ESD and C are electrically controlled valves. These are opened when the fluid connection is desired. The seal pressure in the connector operates the valves $\mathrm{S}$. Thus the normal operating sequence is that the connector is made up and the seals are pressurized. If the seals leak at a rate below some established value the valves $\mathrm{S}$ open. Following the opening of the valves $S$ the valves.C.and ESD-are opened by electrical command. The electrical connection is established through a separate electrical connector.

At disconnect the-fluid conductor is flushed by a separate system that is not illustrated. Thus at normal disconnect no hydrocarbons are released. 


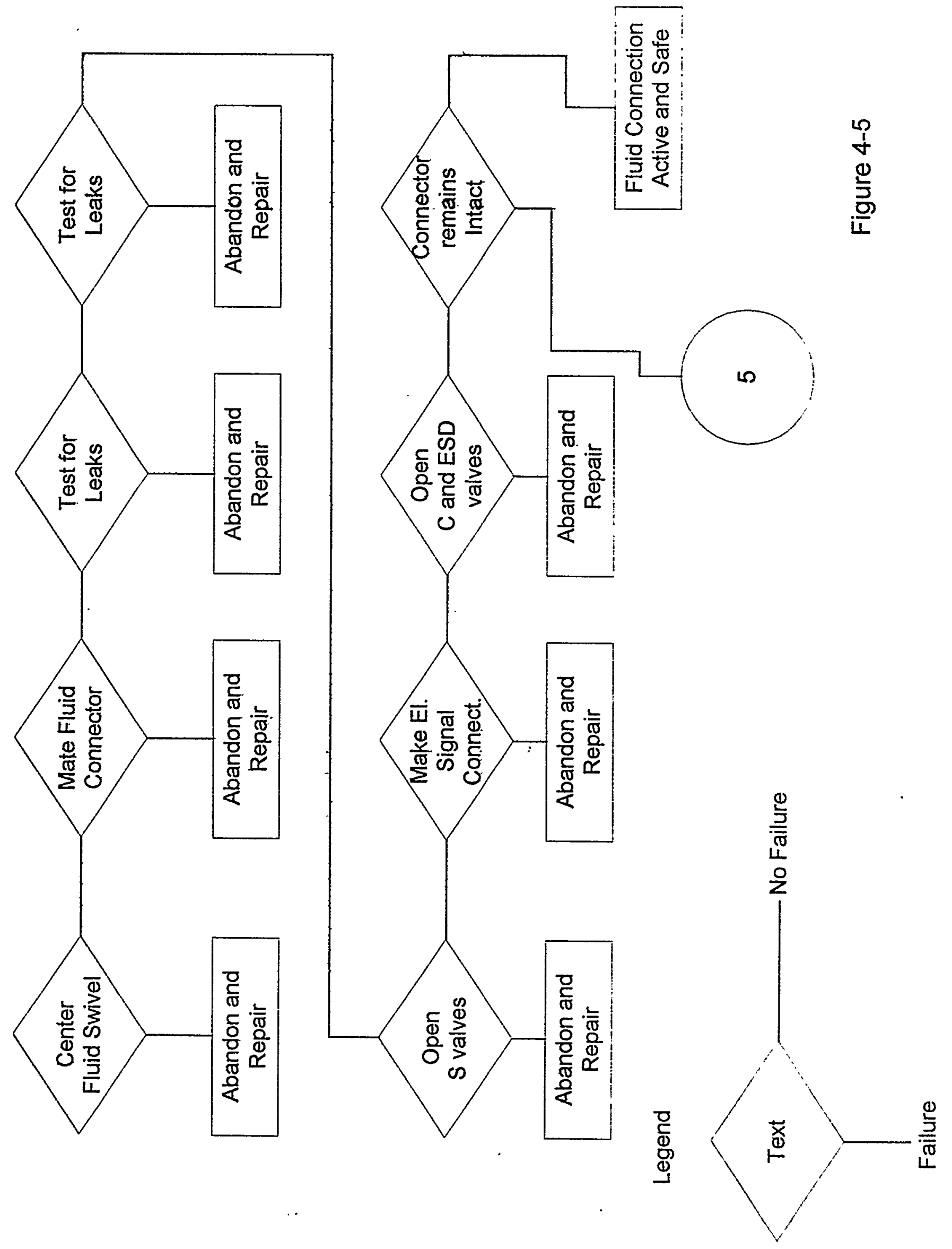




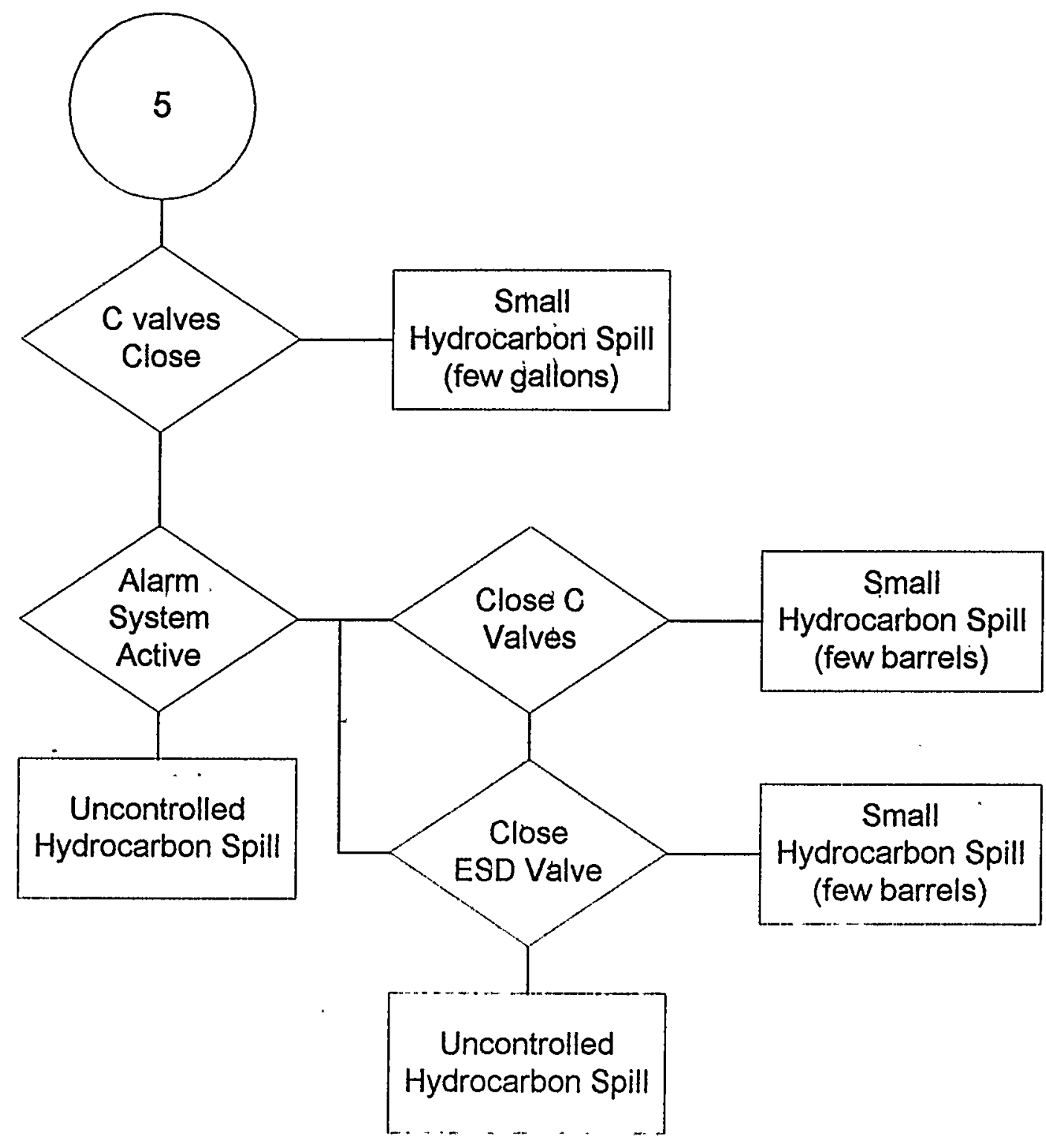




\section{Appendix F}

\section{CORRESPONDENCE WITH THE AMERICAN BUREAU OF SHIPPING}




\section{From: MLee@eagle.org<MLee@eagle.org> \\ To: edbo@ix.netcom.com <edbo@ix.netcom.com> \\ Cc: rdeschamps@eagle.org <rdeschamps@eagle.org>; hpatel@eagle.org <hpatel@eagle.org>; wma@eagle.org<wma@eagle.org> \\ Date: Thursday, August 03, 2000 3:17 PM \\ Subject: Re: Hydrostatic Mooring: Approval in Principle}

We acknowledged your reply, and are awaiting your additional calculations. As to your inquiries regarding corrosion allowance and bearing safety factor, we offer:

\section{Corrosion Allowance}

Section 3/3.3.3 of the ABS SPM Rules provides some specific requirements on corrosion control. Alternatively, we'll accept a corrosion allowance in the weight considerations and member calculations. However, the minimum member size after corrosion consideration should be the size used in the structural calculations while the original member size (before corrosion) should be used in the buoyancy calculations.,

\section{Bearing S.F.}

Section 4/1.7.3 a) of the ABS SPM Rules specifies a safety factor of not less than 2 without yielding. We'll be happy to consider your design modification and/or further studies to increase the system reliability.

Regards,

Ming.Yao Lee

Offshore Engineering Department

ABS Americas (281) 877.6735

edbo@ix.netcom.com

31.07.00 11:13 PM

\author{
To: <MLee@eagle.org> \\ cc: \\ Subject: Re: Hydrostatic Mooring: Approval in Principle
}

Sorry for being a little tardy in responding.

1. The existing model tests already in your possession had a force deflection curve very close to both the mooring systems, which we will demonstrate shortly.

2. Stability calculations will be done and submitted on the actual buoy. We have stabiliity calcs on two prior versions and do not expect any problems in this area.

3. Would it be ok to simply include a corrosion thickness allowance in all weight and buoyancy calculations? 
4. Pile interaction calcs will be furnished.

5. We use a proprieetary riser that may designed to not contact the seabed. We will furnish documentation. It will be clear from this documentation that any amount and size of riser may be accomodated.

Concerning risk assessment. The only element that is non redundant is the main bearing. There is possibly enough space too include structural elements that take over in the event of a bearing element failure. We will check this. Another possibility is to increase the factor of safety on the structural elements in the bearing (25\% ?? 50\%??). Can I have guidance here?

You could take the position that the fluid seal is non redundant. Failure of this is however gradual, such that there would be warnings and enough time to disconnect in the usual fashion.

Best regards

Jens Korsgaard

\section{-Original Message-_-}

From: MLee@eagle.org <MLee@eagle.org>

To: edbo@ix.netcom.com <edbo@ix.netcom.com>

Cc:wma@eagle.org <wma@eagle.org>; rdeschamps@eagle.org <rdeschamps@eagle.org>; Isamuelsson@eagle.org <lsamuelsson@eagle.org>; hpatel@eagle.org <hpatel@eagle.org>

Date: Thursday, July 27, 2000 5:50 PM

Subject: Hydrostatic Mooring: Approval in Principle

Dear Jens,

Per your request, we have the following comments based on a cursory review completed so far:

\section{Mooring and Buoy Design:}

1. Mooring design loads of anchor legs and mooring elements between the buoy and the vessel should be established through proper physical model testing and/or analyses taken into account design safety factors.

2. Stability calculations should be carried out to demonstrate that the system is installable, repairable, and that the buoy is inspectable on the surface.

3. Corrosion protection and/or provisions should be included in the scantling calculations.

4. Calculations verifying the piles and its interactions with mooring legs, both structurally and geotechnically, need to be included.

5. The mechanical wear from the risers in contact with the sea floor, the mooring chains or the buoy body should be investigated. The risers should also be verified for the riser minimum bending radius requirements.

\section{Piping/Mechanical:}

(Yet to be furnished by our machinery group)

Risk Assessment:

Where parts of the system are identified as non-redundant and where redundancy is not possible, we 
suggest that quantitative rather than qualitative analyses be performed to ensure that the risks are minimized.

Please consider the above comments as preliminary at this time. We'll provide a more detailed list of comments and/or suggest areas for further engineering once we complete our review work.

Regards,

Ming-Yao Lee

Offshore Engineering Department

ABS Americas (281) 877.6735 
From: edbo@ix.netcom.com <edbo@ix.netcom.com>

To: MLee@eagle.org <MLee@eagle.org>

Date: Tuesday, August 01, 2000 12:13 AM

Subject: Re: Hydrostatic Mooring: Approval in Principle

Sorry for being a little tardy in responding.

1. The existing model tests already in your possession had a force deflection curve very close to both the mooring systems, which we will demonstrate shortly.

2. Stability calculations will be done and submitted on the actual buoy. We have stabiliity calcs on two prior versions and do not expect any problems in this area.

3. Would it be ok to simply include a corrosion thickness allowance in all weight and buoyancy calculations?

4. Pile interaction calcs will be furnished.

5. We use a proprieetary riser that may designed to not contact the seabed. We will furnish documentation. It will be clear from this documentation that any amount and size of riser may be accomodated.

Concerning risk assessment. The only element that is non redundant is the main bearing. There is possibly enough space too include structural elements that take over in the event of a bearing element failure. We will check this. Another possibility is to increase the factor of safety on the structural elements in the bearing (25\% ?? 50\%??). Can I have guidance here?

You could take the position that the fluid seal is non redundant. Failure of this is however gradual, such that there would be warnings and enough time to disconnect in the usual fashion.

Best regards

Jens Korsgaard

\section{-..... Original Message}

From: MLee@eagle.org <MLee@eagle.org>

To: edbo@ix.netcom.com <edbo@ix.netcom.com>

Cc:wma@eagle.org <wma@eagle.org>; rdeschamps@eagle.org <rdeschamps@eagle.org>;

Isamuelsson@eagle.org <Isamuelsson@eagle.org>; hpatel@eagle.org <hpatel@eagle.org>

Date: Thursday, July 27, 2000 5:50 PM

Subject: Hydrostatic Mooring: Approval in Principle

Dear Jens,

Per your request, we have the following comments based on a cursory review completed so far:

\section{Mooring and Buoy Design:}

1. Mooring design loads of anchor legs and mooring elements between the buoy and the vessel should be established through proper physical model testing and/or analyses taken into account design safety factors. 
2. Stability calculations should be carried out to demonstrate that the system is installable, repairable, and that the buoy is inspectable on the surface.

3. Corrosion protection and/or provisions should be included in the scantling calculations.

4. Calculations verifying the piles and its interactions with mooring legs, both structurally and geotechnically, need to be included.

5. The mechanical wear from the risers in contact with the sea floor, the mooring chains or the buoy body should be investigated. The risers should also be verified for the riser minimum bending radius requirements.

Piping/Mechanical:

(Yet to be furnished by our machinery group)

Risk Assessment:

Where parts of the system are identified as non-redundant and where redundancy is not possible, we suggest that quantitative rather than qualitative analyses be performed to ensure that the risks are minimized.

Please consider the above comments as preliminary at this time. We'll provide a more detailed list of comments and/or suggest areas for further engineering once we complete our review work.

Regards,

Ming-Yao Lee

Offshore Engineering Department

ABS Americas (281) 877.6735 


\section{From: MLee@eagle.org <MLee@eagle.org>}

To: edbo@ix.netcom.com <edbo@ix.netcom.com>

Cc: wma@eagle.org<wma@eagle.org>; rdeschamps@eagle.org <rdeschamps@eagle.org>; Isamuelsson@eagle.org <lsamuelsson@eagle.org>; hpatel@eagle.org <hpatel@eagle.org>

Date: Thursday, July 27, 2000 5:50 PM

Subject: Hydrostatic Mooring: Approval in Principle

Dear Jens,

Per your request, we have the following comments based on a cursory review completed so far:

\section{Mooring and Buoy Design:} 1. Mooring design loads of anchor legs and mooring elements between the buoy and the vessel
should be established through proper physical model testing and/or analyses taken into account
design safety factors.

2. Stability calculations should be carried out to demonstrate that the system is installable, repairable, and that the buoy is inspectable on the surface.

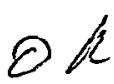

3. Corrosion protection and/or provisions should be included in the scantling calculations.

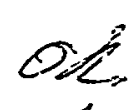

4. Calculations verifying the piles and its interactions with mooring legs, both structurally and

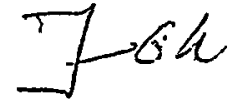
geotechnically, need to be included.

5. The mechanical wear from the risers in contact with the sea floor, the mooring chains or the buoy body should be investigated. The risers should also be verified for the riser minimum bending radius requirements.

\section{Piping/Mechanical:}

(Yet to be furnished by our machinery group)

\section{Risk Assessment:}

Where parts of the system are identified as non-redundant and where redundancy is not possible, we suggest that quantitative rather than qualitative analyses be performed to ensure that the risks are minimized.

Please consider the above comments as preliminary at this time. We'll provide a more detailed list of comments and/or suggest areas for further engineering once we complete our review work.

Regards,

Ming.Yao Lee

Offshore Engineering Department

ABS Americas (281) 877.6735 
Jens Korsgaard

318 North Post Road

Princeton Junction NJ 08550

Phone: 609-936-8543

Fax: 609-936-9373

$07 / 17 / 00$

Mr. Ming-Yao Lee

ABS Americas

16856 Northchase Drive

Houston TX 77060-6796

File Ref: 1014/DBL/RQ

Dear Ming-Yao:

This refers to our submittal for approval in principle for the Hydrostatic Mooring

Here are the details of Section 3 Electrical/Mechanical design to be inserted following the summary report by Han Padron Associates.

Section 5 is still pending.

Best regards

Jens Korsgaard 
Jens Korsgaard

318 North Post Road

Princeton Junction NJ 08550

Phone: 609-936-8543

Fax: 609-936-9373

07/07/00

Mr. Ming-Yao Lee

ABS Americas

16856 Northchase Drive

Houston TX 77060-6796

File Ref: 1014/DBL/RQ

Dear Ming-Yao:

Here is our submittal for approval in principle for the hydrostatic mooring. I have completed this on a trip.

The submittal lacks section 5, Applicable Standards. This will be sent to you on or about July 16 after my return to USA.

Section 3 Electrical/Mechanical design contains only the summary report by Han Padron Associates. The detailed calculations will be submitted with Section 5 .

Please make a fast review in order to identify potential items that are missing. We will furnish these as fast as possible following identification. We are of course available with respect to any clarifications required. If needed we are also available to meet with you and your staff in Houston

Best regards

Jens Korsgaard 
A ONISION OF THE AMERICAN BUREAU OF SHIPPING

16855 NORTHCHASE DR, HOUSTON. TEXAS 77060.60288

TEt: 281.877 .8000

FAX: $281-877-8796$

\section{TELEFAX MESSAGE}

Page 1 of 1

To:

SUCTION MOORING TECHNOLOGY

Date: 25 August 1999

Attn:

Jens Korsgaard

Fax No: 6099389373

Fax No:

From: David Lorenz

Branch code: $\quad 86203$

File Ref: $\quad$ 1014/DBL/RO

PID

\section{Subject: Concept Evaluation}

Mr. Korsgaard.

We hereby accept your conditions for the Approval in Principle of the Hydrostatic Mooring, as detailed in your letter of 28 July 1999.

An invoice will be sent to you for the first payment next week.

You may begin by submitting drawings and calculations as described in our proposal at your earliest convenience.

Please forward all submittals through me.

Thank you for your business.

Best regards,

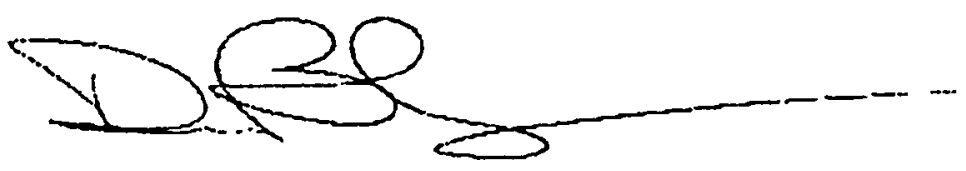

David B Lorenz

Principal Engineer

Project Manager

Offshore Engineering Department 


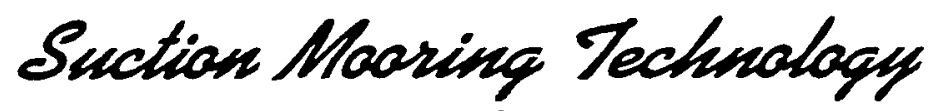

$$
\begin{gathered}
\text { Offshore Mooring Systems } \\
318 \text { North Post Road } \\
\text { Princeton Jct. NJ } 08550 \\
\text { Phone: }(609) 936-9568 \\
\text { Fax: (609) } 936-9373
\end{gathered}
$$

Mr. David A. Crouch, EE-24

July 13, 1999

US Dept. Of Energy

Mailstop 5E-052

Washington DC 20585

By fax to 202-586-3180 (12 pages total)

Reference: Invention 703

Dear Mr. Crouch:

Attached is the proposal from American Bureau of Shipping.

I have clarified the proposal over the phone with ABS, see handwritten notes in the margin of the proposal.

The words "Concept Evaluation" are the same as "Approval in Principle" thus the proposal covers what I desire.

The cost is higher than the basis for my budget. One reason for this is the "Risk Assessment Evaluation". This item was not present in prior proposals from ABS. ABS claims that this item is required and that it was mistake not to include it in prior proposals.

I intend to accept the proposal as modified. I believe that I need your blessing before formal acceptance.

I have a problem with the timeframe. Before we actually have an executed contract and start work it is the end of the month. The ABS budget has increased and the work required from me is increased by virtue of the "Risk Assessment Evaluation". I may need an extension of time, say 3 months, with respect to overall completion.

Because of the increased ABS budget and the increased requirements I may want to move monies from the HPA budget to the ABS budget. I presume that I can do that because this is within the same work item.

Please review the attached for acceptability.

Sincerely yours
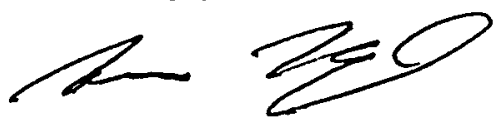

Jens Korsgaard 


\section{Mooring System}

The present mooring system is a single point mooring attached to the keel of the moored vessel. The mooring buoy is held firmly to the bottom of the vessel by differential hydrostatic pressure. A structural swivel is built into the mooring buoy that permits the moored ship to weathervane in relation to the mooring chains.

Pressure maintenance pumps aboard the vessel maintain the differential pressure. These pumps take suction within the volume isolated by the top of the buoy and the hull of the vessel.

\subsection{General Arrangement}

The function and basic general arrangement is explained in the previous section. Single point moorings are typically moored to the seabed through a series of radially deployed catenary mooring lines terminating in drag anchors or stake piles in the sea bed. This standard arrangement is illustrated on Figure 1-2 (Hydrostatic Mooring General Arrangement -2).

A special mooring system has been developed that take advantage of the unique properties inherent in the present mooring system as shown on figure 1-1 (Hydrostatic Mooring General Arrangement -1). This system is comprised of a series of heaty weights that are lifted by the excursions of the mooring buoy. When the buoy deflects horizontally the connecting chain is bent over a fairlead in the top of the stake pile. In the proposed system that is specifically designed for the water transport system at Askelon Israel six stake piles are provided. This system maintains a higher fraction of its initial capabilities than the traditional system shown in Figure 1-2 when one mooring line fails.

Because of the small amount of chainfit is not a significant economic penalty to oversize the mooring chains to compensate for the wear that will occur at the fairlead. It is the intent to frequently inspect the system and to replace chain and fairleads when significant wear has occurred.

This is the preferred system partly because of significant cost reductions and partly due to its much superior force deflection characteristics as illustrated in figures 1-4 and 1-5.

The graph in Figure 1-3 depicts the chain force as a function of the horizontal excursion of the vessel. Because the chain force is supplied by a hanging weight it is nearly constant. It is, however, modified above the fairlead by the friction in the fairlead. The present calculation is based on a friction coefficient of $10 \%$ as being the steel on steel friction coefficient. Figure 1-4 shows the resulting component of the chain force for a single chain. There are six chains, therefore the actual mooring force is six times the force shown on Figure 1-4. 
The principal buoyancy is provided in an exterior near toroidal shaped ring beam. This ring beam serves three main purposes:

1. It protects the mooring surface of the buoy against vessel impact

2. It serves as the main buoyancy and trim volume in the buoy

3. It provides low-pressure air storage tanks for the rapid ascent system used in the mooring process.

This is shown in more detail in figure 1-5. The ring beam is in the shape of a 12-sided polygon. Each side of the polygon is protected against ship impact by a 2 meter long standard cylindrical ship fender $1400 \mathrm{~mm}$ by $700 \mathrm{~mm}$ as shown in more detail on Figure 1-6. Later in this section an analysis is presented showing that this level of fendering is sufficient to protect both the ship and the buoy against damaging impact during the mooring and the unmooring processes.

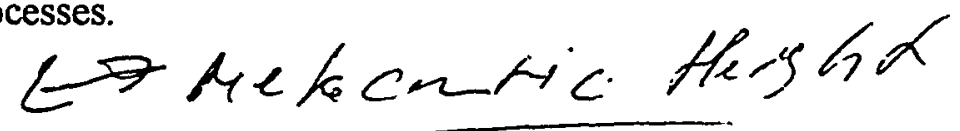

\subsection{Compression Elements}

Figure 1-5 also shows the main compression elements between the buoy surface and the vessel hull. These are comprised of a series of Trellex Morse Fender bar units deployed in a radial pattern as shown. Because of the density of fender bars they may for computational purposes be treated as a continuos surface. The force capacity of the fender bars is 54 tonnes or $530 \mathrm{kN}$ per meter. There are a total of 360 meters of fender bars on the buoy surface. The total theoretical capacity of these units are to resist a a total compressive force of $191,000 \mathrm{kN}$ ( 19,500 tonnes). The maximum possible compression is obtained when absolute vacuum exists above the buoy while submerged to its deepest submergence. The area within the outer seal ring is $346 \mathrm{~m} 2$.

In the case that the pressure above the buoy is 0 absolute the differential load is 35 tonnes/ $\mathrm{m} 2$ resulting in a total load of 12,100 tonnes. The mooring load is at most applied 8 meters below the fender surface. Assume 600 tonnesj then the resulting moment is 4800 . tonnes meters. The fendering circle ring has an OD of $18.5 \mathrm{~m}$ and an $1 D$ of $10.5 \mathrm{~m}$. The section modulus of this ring is $\mathrm{pi} / 32^{*}\left(18.5^{\wedge} 3-10.5^{\wedge} 3\right)=508 \mathrm{~m} 3$. The resulting pressure differential is $4800 / 508=9.45$ tonnes $/ \mathrm{m} 2$. The allowalle is the difference between the stress resulting from 12,100 tonnes and 19,500 tonnes br 21.4 tonnes per $\mathrm{m} 2$. uoriz.ithl unewiy

This margin is required to cope with the ship's bottom being out plane. The total allowable stress is $19,500 / 346=56.4$ tonnes $/ \mathrm{m} 2$. The allowable margin is $21.4-9.45=$ 11.9 tonnes $/ \mathrm{m} 2$ or $21 \%$ of the fully allowed deflection. Since the allowable deflection is $150 \mathrm{~mm}$ the system can cope with $2 * 0.21 * 150=63 \mathrm{~mm}$ out plane deviation within the mooring area. Note, however, that from a practical point of view significantly higher out of plane deviation would at most cause some minor damage to the fender bars, and this only in the event that full vacuum, maximum submergence and full horizontal mooring force all occur simultaneously. 


\subsection{Sealing Units}

The sealing units contact the keel plating in the ship and impede the flow of water from the sea to the volume in which the low pressure is maintained. There are two annular seals an inner and an outer as for example illustrated in Figure 2 in the Brief ofverview. Becayse the pressure is maintained low above the buoy through continuous removal of water the seals need not be perfect. Rather, their function is to impede the water flow sufficiently that the pumps may reliably maintain thefpressure above the buoy.

$$
\text { Lous }
$$

Figure 1-6 shows an outer sealing element. This sealing elements must be capable of the following: - To maintain contact with the hull for all pressure differentia $\sqrt{\text { ranging from a low } 50}$
$\mathrm{kPa}$ to a high of $350 \mathrm{kPa}$.

- To maintain sealing pressure in the presence of unevenness of the hull.

- To be able to deflect without damage when a maximum impact between the buoy and the vessel deflects the exterior fenders fully.

- To be able to slide on the bottom plating of the vessel without damage.

- To resist safely all possible levels of differential pressure

- To provide the highest practical level of sealing.

A range of materials were investigated and the result was that the steel unit shown on figures 1-6, 1-7, and 1-8 was the only unit that was able to fulfill all crireria. Figure 1-7 shows in more detail how the unit pivots. Figure 1-8 shows the structural make-up of an

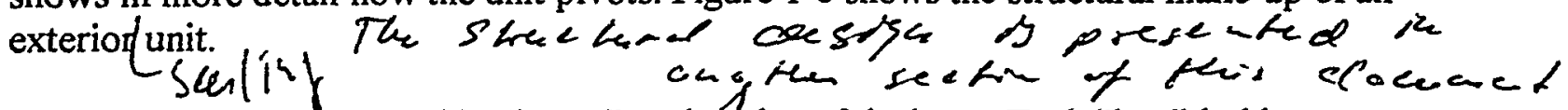

The sealing units are seated in "boxes" at the dige of the buoy. Each "box" holds one sealing unit. The sealing unit fits loosely/n its "box" and is prevented from sliding out by $\mathrm{a}$ tab on the underside. The curved sufface shown on the top of the unit is bolted on after the unit is placed in the "box". Thelunits afe spaced some $25 \mathrm{~mm}$ apart along the perimeter. A special Trellex Mofse extruded fender is placed in a continuous ring above the units and is bolted onto thounit and is held securely by bolted on angle irons. On the exterior and below the sealing element there is a continuous rubber sheet made from conveyor belting. This rubber sheet seals the gaps between the sealing elements. The conveyor belting would typically be capable of resisting differential pressures in excess of $1000 \mathrm{kPa}$ in this application. Thus there is no issue with the structural strength of the rubber sheet. Both the rubber sheet and the extruded fender will be stretched when the sealing ring is depressed. However the stretch is less than $5 \%$ well within the capabilities of these elements. Which is

The sealing elements are cantilevered. The purpose of the cantilever is to make it possible for the sealing element to deflect vertically and also to have the hydrostatic pressure differential increase the sealing pressure as the pressure differential increasses. 


\section{qu}

Figure 1-8 shows the exterior sealing element. The interior sealing element is similar, however, the taper is in the opposite direction. In consequence the "box" design is with parallel sides and the unit also has parallel sides for the part that is placed within the "box" 
TO: SUCTION MOORING TECHNOLOGY

Attn: Jens Korsgaard

Date:

9 July 1999

From: David Lorenz

Fax No: 2126294406

File Ref: 1014/DBL/RQ

Fax No: 6099369373

Branch code:

86203

PID

\section{$\#$}

Subject: Panama Approval

Mr. Korsgàard,

Please find attached our proposal for concept evaluation of the hydrostatic mooring. Also please find attached our terms and conditions.

If you have any questions, please call. I look forward to hearing from you.

Best regards,

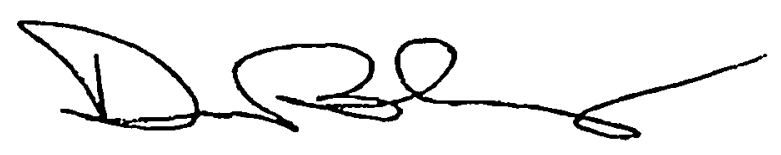

David B Lorenz

Principal Engineer

Project Manager

Offshore Engineering Department

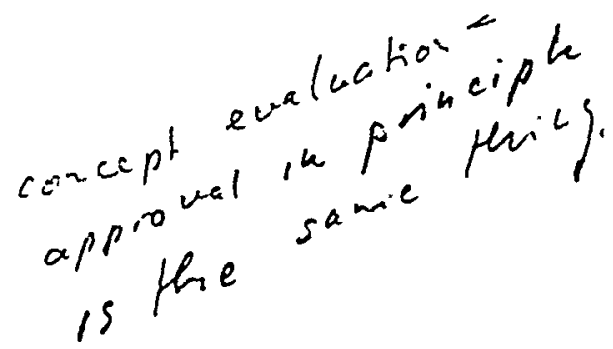

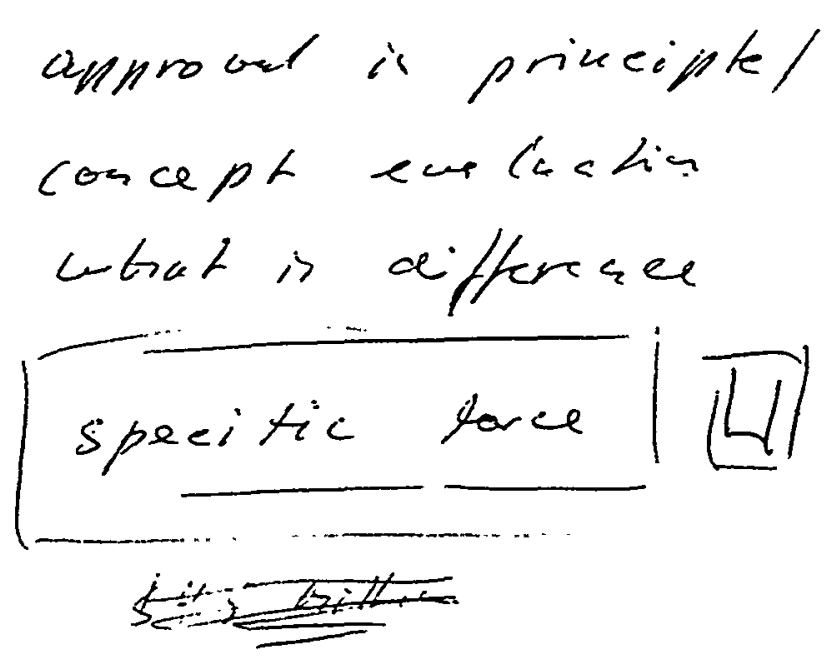




\section{PROPOSAL FOR \\ CONCEPT EVALUATION OF HYDROSTATIC MOORING FOR SUCTION MOORING TECHNOLOGY}

\section{GENERAL}

The American Bureau of Shipping (ABS) proposes to conduct a Concept Evaluation for Suction Mooring Technology for their Hydrostatic Mooring System. This evaluation will consist of a Mooring review, buoy structural review, standards review, risk analysis review, and applicable mechanical systems review. A final report will be issued at the completion of the study.

\section{DISCUSSION}

The Hydrostatic Mooring system proposed by Suction Mooring Technology is basically an underwater buoy that is raised to a position undemeath a ship or barge and is attached and maintained by hydrostatic pressure to the bottom of the hull.

This system can be used to connect a tanker to an underwater pipeline or PLEM in order to transfer water, oil or gas to either the tanker or from the tanker to the shore.

Therefore, in order to evaluate this concept, the following must be reviewed:

1. Mooring System

2. Buoy mechanical and structural components

3. Shipboard mechanical and piping components

4. Control systems

5. Risk review due to potential pollution hazards.

In addition, a list of proposed design standards, such as ASME, ANSI, or others must be listed and evaluated.

\section{SCOPE OF WORK}

\section{Mooring Evaluation}

The mooring will be evaluated by reviewing the mooring design calculations and two sets of model tests. This review will consist of

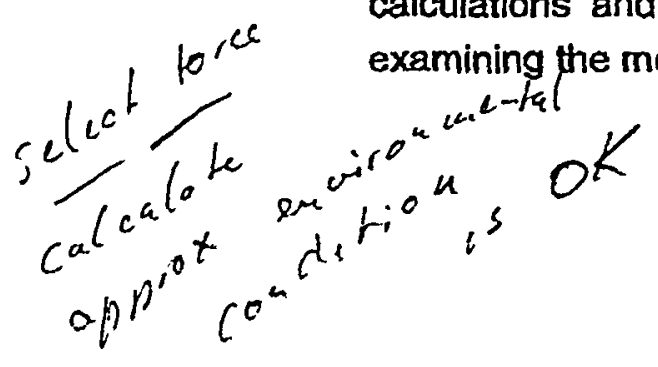


themselves for completeness and accuracy, a review of the design loads, and a review of the mooring system for one specific sea-state and vessel.

A report will be written for this work and incorporated into the final report.

\section{Buoy Structural Evaluation}

The buoy structural elements will be examined for adequacy against the proposed loads imposed both by the mooring system and by the vessel. The loads will be taken from the mooring analysis and hydrostatic suction loads in both symmetric and non-symmetric loading cases.

This review will be for one specified sea state and one specific vessel (the same that is proposed for the mooring study).

A report will be written for this work and incorporated into the final report.

\section{Mechanical and Controf Evaluation}

The piping, mechanical and control systems will be reviewed to insure that the capacities and machinery are suitable for their intended use. They will also be reviewed to ensure that they have the capacity to perform adequately in the chosen environmental conditions.

The control system will be reviewed with respect to its logic and components.

A report will be written for this work and incorporated into the final report.

\section{Risk Assessment Evaluation}

A risk assessment provided by Suction Mooring Technology will be reviewed to determine the risks associated with the operation of the system. This analysis will be examined to ensure that the risks to personnel, the vessel and buoy and the environment are minimized.

A report will be written for this work and incorporated into the final report.
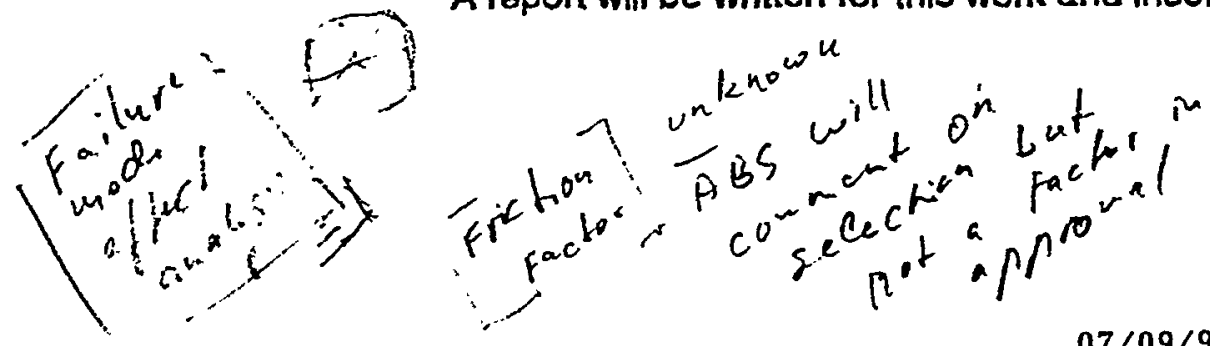


\section{Standards Evaluation}

The various standards for the components and the design criteria will be examined to ensure that they are suitable for marine use and are suitable for this application.

A report will be written for this work and incorporated into the final report.

\section{Project Management.}

Since the review of this concept cuts across many disciplines and departments within $A B S$, the project will be assigned a project manager to coordinate these efforts and to make sure that the project is delivered on schedule.

The project manager will also act as liaison with the customer.

\section{SUBMITTAL REQUIREMENTS}

The following drawings, drawings and calculations must be submitted to ABS for the work to be completed.

\section{Mooring Evaluation \\ Mooring Calculations \\ Model Test Reports \\ Design Assumptions \\ Environmental conditions \\ Derivation of loads}

Buoy Structural Evaluation

Structural Drawings and Plans

Structural calculations, including derivation of loads

Mechanical and Control Evaluation

Piping Diagrams

List of Equipment

Control Logic description or diagram 


\section{PROPOSAL FOR CONCEPT EVALUATION}

07/09/99

Risk Assessment Evaluation

Failure Mode and Effect Analysis or other suitable risk assessment technique.

Standards Evaluation

A list of standards (ANSI, ASME, AISC, etc.) to be used in the design and the selection of equipment.

\section{SCHEDULE}

The Evaluation will be completed 6 weeks after the delivery of the final documents by Suction Mooring Technology.

\section{cósts}

The following hours will be necessary to complete this project:

TASK

Mooring Evaluation

Buoy Structural Evaluation

Mechanical and Control Evaluation

Risk Assessment Evaluation

Standards Evaluation

Project Management

Final Report

TOTAL
Hours

60

40

40

24

Included above

10

16

190

ABS Fees for these hours will be at a rate of $\$ 105.00$ per hour, making the total for the project $\$ 19,950.00$.

\section{TERMS}

Payment for these services is to be made in two (2) Installments. The first payment of $50 \%$ of the fee $(\$ 9,975.00)$ is due upon acceptance of this proposal and prior to the commencement of work. The remainder of the fee must be paid prior to the issuance of the final report. 


\section{PAOPOSAL FOA CONCEPT EVALUATION}

07/09/99

The work described and priced in this proposal includes one submittal only. In the event that re-submittals are required, this work will be additional and billed at the rate of $\$ 105.00$ per hour.

The standard ABS Terms and conditions are attached. 


\section{TERMS AND CONDITTONS}

\section{ABS SERVICES}

a) The work shall be performed solely under the terms and conditions of this Agreement, and any printed terms or conditions found in other documents used to implement this Agreement shall be void and shall not affect or extend any rights or obligations under this Agreement nor create any ncw rights or obligations.

b) In performance of this Agreement, ABS shall be and remain, at all times, an independent contractor und neither ABS nor any of its officers, employees, servants, agents or subcontractors shall be or act as the employee, servant or agent of any other party hereto in its performance of any of the terms and conditions of this Agreement.

c) All work performed on behalf of flag administrations shall be governed by the terms and conditions of this Agreement unless the flag administration specifies otherwise.

d) Client shall verify to ABS that the materials and components intended for classification or certification meet the requirements of the Rules by producing certificates for such materials and components.

e) If any part of the vessel's construction is subcontracted Client shall provide ABS with the names of the subcontractor(s), and address of the subcontractor's site(s), and shall provide the subcontractors with correct and adequate information regarding ABS requirements and any statutory requirements. ABS shall be cntitled to rely on certificates provided by such subcontractors as it would rely on Client's certificates. Clicnt stands surety for the acts and debts of its subcontractors. The cost of services at a subcontractor are in addition to those stated in section 3a), and will be billed directly to the subcontractor.

\section{PAYMENT CONDITIONS}

a) Fees are to be paid in two installments as described in the proposal, unless alternate arrangements have been agreed upon by both the Client and ABS.

b) Unless otherwise directed by American Bureau of Shipping, all fees are to be remitted upon receipt of invoice in U. S. Dollars to Amcrican Bureau of Shipping, P.O. Box 201614, Howston, Texas 77216-1614 U.S.A. by check and shall refer to the ABS issued invoice number. Wire transfers may be made through Texas Commerce Bank, Houston, Texas 77252-8025 U.S.A., account numher 0010-088-8180.

c) Unless otherwise provided by Agreement or prohibited or restricted by law, interest will be charged at a rate of $11 / 2 \%$ per month on any amounts not paid within 30 days from invoice date.

d) The Client shall be liable to ABS for the payment of all charges including but not limited to cnurt costs, expenses and reusonable attorney's fees incurred in collecting sums due ABS, and ABS may take judgment for the entire amount due. Payment to anyone other than ABS or its authorized agent shall not be deemed payment and shall be made at payor's sole risk. 


\section{DEFAULT}

In the event of a default in the payment of any fees assessed in accordance with this Agreement, ABS shall have the right to terminate this Agreement and cease work, as well as all plans, drawings, specifications, information and reports in possession of ABS. shall be subject to a lien for the payment of all fees and expenses due and owing by virtue of this Agreement and the terminition or default hereof.

\section{ASSIGNMENT \& SALE}

Any attempt to subcontract, assign, delegate, sublet or transfer this Agreement wilhout prior written notice to ABS shall at ABS's option render this Agreement void. ABS may deem the classification of any vessel canceled upon the vessel's sale or transfer without prior written notice to ABS.

If Client shall breach any provision hereof or shall become insolvent, enter voluntary or involuntary bankruptcy or reccivership proceedings, or make an assignment for the benefit of creditors, or should the vessel be arrested or sold at auction or at a Marshall's sale ABS shall have the right (without limiting any other rights or remedies which it may have hereunder or by operation of law) to terminale this Agreement, whereupon ABS shall be relieved of any and all further obligation hereunder and Client shall be liable to ABS for all resulting damnges. ABS's right to require strict performance of any obligation hereunder shall not be affected by any previous waiver, forbearance or course of dealing.

\section{CONFIDENTIALITY}

Subject to sections 3 and 4 hereof, all plans, drawings, specifications and infomution given to and reports prepared by ABS in connection with performance under this Agrecment shall be treated as confidential by ABS and shall not be used for any other purposes than those for which furnished without prior written consent, except as required by judicial order, governmental order or regulation, by subpoena or by direction of a governmental agency with subpocna power.

By signing this Agreement Client authorizes ABS to disclose to future owncrs, if any, the design assumptions on which classification was based and, in accordance with the IACS Transfer of Class Agreement, to provide future classification societies, if any. with any information, plans or calculations reasonably requested in connection with their classification duties.

\section{5. $\quad$ ACCESS}

ABS, its officers, employees, servants, agents or subcontractors shall have access to all drawings, plans, records, other items neccssary to complete the requested services

Client shall provide ABS with the following documentation before issuance of a full-term classificution certificate:

a) Plans and calculutions required by the Proposal 


\section{NON-WAIVER AND SEVERABILITY}

No waiver by either party of any breach of any of the terms of this Agreernent shall be constned as a waiver of any subsequent breach, whether of the same or of any other term hereof. If a provision of this Agreement is held invalid all valid provisions that are severable from the invalid provision remain in effect.

\section{FORCE MAJEURE}

Upon prompt notification of the other party by fax, telex or letter communication, neither party hereto shall be liable to the other for default or delay in performing its obligations hereunder if such default or delay is caused by fire, strike, riot, war, act of God, delay of carricrs, governmental order or regulations and/or any other similar or different occurrence beyond the reasonable control of the party so defaulting or delaying, except that cancellation for such callses may not be made without reimbursement to ABS for expenditures actually incurred for labor and matcrials upon the authority of this Agreement prior to receipt of such notice.

\section{TERM}

This Agreement shall remain in force until terminated pursuant to the terms of this Agreement or by either Client or ABS upon thirty days' prior written notice.

\section{RESPONSIBILITY AND LIABILTTY}

It is understood and agreed that any report, statement, notation of plan review or certificate (hereafter referred to collectively as "certificate") issued as part of the services rendered under this Agreement is a representation solely to the signatory to this Agreement and only that at the time of survey the vessel; structure, item of material, equipment or machinery or any other item covered by a certificate has met one or more of the Rules or standards of American Bureau of Shipping and is issued solely for the use of ABS, its committees, clients or other authorized entities. ABS is not an insurer or guarantor of the integrity or safety of a vessel ur of any of its equipment or machinery. The validity, applicability and interpretation of a certificate issued under the terms of or in contemplation of this Agreement is governed by the Rules and standirds of American Burean of Shipping who shall remain the sole judge thereof. Nothing contained herein or in such a certificate or in any report issued in contemplation of such a certificate shall be deemed to relieve any designer, builder, owner, manufacturer, seller, supplier, repairer, operator, insurer or other entity of any duty to inspect or any other duty or warranty express or implied. Except for such entity, nothing in this Agreement or in any cerrificate or report issucd under this Agreement shall be dcemed to create any interesh right, claim or benefit in any insurct or other thind party. It is understood and agreed that nothing expressed hercin is intended or shall be construed to give any person, firm or corporation, other than the signatories hereto and any identified "Owncr", any right, remedy or claim hereunder or under any provisions herein contained; all provisions hereof are for the sole and exclusive benefit of the parties hereto.

11. LIMITATION

ABS MAKES NO REPRESENTATIONS BEYOND THOSE CONTAINED IN SECTIONS 1 AND 11 HEREOF REGARDING ITS REPORTS, STATEMENTS, PLAN REVIEW, SURVEYS, CERTIFICATES OR OTHER SERVICES. 


\section{HOLD HARMLESS}

The party requesting scrvices hereunder, or his assignee or successur in interest, agrecs to release $\mathbf{A B S}$ and to indemnify and hold harmless ABS from and ugainst any and all claims, demands, lawsuits or actions for damages, including legal fees, to persons and/or property, tangible, intangible or otherwise which may be brought against ABS incidental to, arising out of or in connection with this Agreement, the work to be donc, services to be performed or material to be furnished hereunder, exccpt for those claims caused solely and completcly by the negligence of $\mathbf{A B S}$, its agents, employees, officers, directors or subcontractors. The parties agree that for the purposes of the Convention on Limitation of Liability for Maritime Claims, 1976, $A B S$ is a person for whose acts the shipowner is responsible.

Any other individual, corporation, partnership or other entity who is a party hereto or who in any way participates in, is engaged in connection with or is a beneficiary of, any portion of the services described herein shall also release ABS and shall indemnify and hold ABS harmless from and against all claims, demands, lawsuits or actions for damages, including legal fees, to persons and/or property, tangible, intangible or otherwise, which may be brought against ABS by any person or entity as a result of the services performed pursugnt to this Agreement, except for those claims caused solely and completely by the negligence of ABS, its agents, employees, omcers, directors or subcontractors. Said individual or party shall assert no claims, directly or indircetly, against ABS.

\section{ARBPTRATION}

Any and all differences and disputes of whatsoever nature arising out of this Agreement shall be put to arbitration in the City of New York pursuant to the laws relating to arbitration there in force, before a bourd of three persons, consisting of one arbitrator to be appointed by ABS, one by Client, and one by the two so chosen. The decision of any two of the three on any point or points shall be final. Until such time as the arbitrators finally close the hearings either party shall have the right by written notice served on the arbitrators and on an officcr of the other party to specify further disputes or differences under this Agreement for hearing and determination. The arbitration is to be conducted in accordance with the rules of the Society of Maritime Arbitrators, Inc. The arbicrators may grant any relief other than punitive damages which they, or a majority of them, deem just and equitable and within the scope of the Agreement of the parties, including, but not limited to, specific performance. Awards made in pursuance to this clause may include costs including a reasonable allowance for attorney's fees and judgment may be entered upon any award made hcreunder in any court having jurisdiction. ABS and Client hereby mutually waive any and all claims to punitive damuges in any forum.

Client shall be required to notify ABS within thirty (30) days of the commencement of my arbitration between it and third parties which may concem ABS's work in connection with this Agreement and shall afford ABS an opportunity, at ABS's solc option, to participate in the arbitration.

14. TIME BAR TO LEGAL ACTION

Any statutes of limilation notwithstanding, Client expressly agrees that its right to bring or to asscrt aguinst ABS any and all claims, demands or proceedings whether in arbitration or otherwise shall be waived unlcss (a) notice is received by ABS within ninety $(90)$ days after Client had notice of or should reasonably have been expected to have had notice of the basis for such claims; and (b) arbitration or legal proceedings, if any, based on such claims or deminds of whatever nature are commenced within one (1) year of the date of such notice to ABS. 


\section{LIMITATION OF LIABILTTY}

The combined liability of American Bureau of Shipping, its officers, employees, agents or subcontractors for any loss, claim, or damage arising trom negligent performance or non-performance of any services under this Agrecment, or from breach of any implied or express warranty of workmanlike performance in connection with the services, or from any other reason, to Client or any other person, corporation, partnership, business entity, sovereign, country or nation, will be limited to the greater of a) $\$ 100,000$ or b) an amount equal to ten times the sum actually paid for the services alleged to be deficient.

The limitation of liability may be increased up to an amount twenty-five timcs that sum paid for services upon receipt of Client's written request at or before the time of performance of services and upon payment by Client of an additional fee of $\$ 10.00$ for every $\$ 1,000.00$ increase in the limitation.

THE UNDERSIGNED PARTIES ACKNOWLEDGE THAT ALL OF THE TERMS AND CONDITIONS CONTANED IN PAGES I THROUGH 5 HEREOF AND THE PROPOSAL HAVE BEEN REVIEWED. THIS AGREEMENT REPRESEN'IS 'IHE HNTLRE INTEGRATED AGREEMENT BETWEEN THE PARTIES; THERE ARE NO OTHER REPRESENTTATIONS OR ORAL AGREEMENTS. ALL SERVICES RENDERED IN CONNECTION WITH THIS CLASSIFTCATION AGREEMENT ARE GOVERNED BY THE TERMS AND CONDITIONS CONTAINED IIEREIN.

Name of Client

(Signed)

(Title)

(Address)

AMERICAN BURFAU OF SHIPPING

Sígrarure 
Appendix G

\section{EVALUATION OF THE MOORING BY THE NAVIGATOR, CAPTAIN JAMES ATKINSON}




\author{
Captain James A. Atkinson \\ USCG (Retired) \\ Marine Consultant . \\ 4 Pelham Greene West \\ Waynesboro, VA 22980 \\ (540) $946-8237$
}

12. February 1999

Mr. Jens Korsgaard

President

Suction Mooring Technology

318 North Post Road

Princeton Junction, NJ 08550

Dear Mr. Korsgaard:

The week $I$ just spent running simulated mooring approaches to your suction mooring system in the ship maneuvering simulator at Nichols Advanced Marine was challenging, stimulating, and a very satisfying learning experience. I am impressed with your concept and with how you have attacked its vaxious aspects, and I could not help becoming excited by the potential that it offers for liquid or gas transfer in exposed marine locations, even under harsh conditions.

The past week of simulations convinced me that a tanker can be positioned very closely over the submerged buoy under any combination of winds up to about 50 knots, waves up to 8 meters of significant height, and cursents up to two knots. Indeed, $X$ found little difference between 40 and 50 knots of wind, and see no reason why currents higher than two knots cannot be coped with without much difficulty.

The concept that I used most of last week is one I developed specifically for your moorings; I call it the balance-of-forces concept. It depends upon having an omnidirectional thruster of sufficient thrust at the bow, which your selection of the Elliott White Gill thruster fulfills, and the fact that your mooring does not restrain the ship to any particular orientation, during the mating process, or once moored.

The balance-of-forces concept includes achieving a balance of forces between currents, on one hand, and wind and waves, on the other, when they are not from the same direction; between propeller thrust and bow thruster, between propelier thrust and a combination of bow thruster and the axial components of environmental forces; and between propeller-and-rudder and bow thruster vectors. Once an equilibrium heading is established, where the lateral components of current on one side are about equal to the lateral components of winds and waves on the other, with the ship at or near zero groundspeed, but with bow thruster at or near $180^{\circ}$, balanced against propeller thrust, permitting sufficient propeller thrust for effective steering, very small imbalances in lateral forces are used to move the ship to port or starboard, achieved by 
very small heading changes; while very small imbalances in axial force; achieved mostly by small changes in thruster force, are used to move the ship very slowly ahead or astern. When all the environmental forces are from the same direction, propeller thrust is balanced against the envirommental forces, plus added thruster force, applied to permit greater propeller thrust for improved steering, and to permit very slow forc-and-aft movement, mostly by varying the thruster force. When there are little or no environmental forces, thruster vectors are worked against propeller and rudder for all movements until stopped, with the moon pool very close to the buoy; thence one can either continue that scheme until mated, or simply stop and use the bow thruster to pull the moon pool over the buoy, with no regard for orientation.

This is the concept. To apply it requires a little practice in several specific tactics, and that is how I spent most of last week. To apply the tactics, I found it useful, first, to work out in my mind the precepts of shiphandling fundamentals that seemed applicable, in one way or another, to this maneuvering concept. Because it is virtually impossible to perform calculations while shiphandling-a high-stress occupation by any measure-I also found it helpful to have at hand a few simple reference tables, to help me in positioning the moon pool and in determining the precise moment to press the buoy-release button.

Since my involvement began, I have been developing a sort of manual for shiphandlers, including the concept, the relevant precepts, and the reference materials that seem to be useful. Initially, this document is for my own reference and review; it will be expanded to include diagrams that can be used to indoctrinate others into the use of this maneuvering concept, and the mating process. I will add a few approach plans, to assist in the deceleration process for straightforward approaches, or in approaches where there may be geographical or navigational restrictions of one sort or another. This document could constitute the basis for site-specific or ship-specific maneuvering manuals, should the need arise for such manuals.

In the course of last week, I managed to experiment with most of the tactics that I had in mind for the balance-of-forces concept, but not all. On the final day, Friday, I did begin to shorten up the approaches, by starting a bit closer in and decelerating more rapidly. I also took one opportunity to try moving the ship more quickly sideways, by using thruster vectors angled aft and to port, opposed to ahead propeller thrust and a hard-right rudder. It worked very well, but I need more practice with it, not only for moving the ship sideways while holding a heading, but for stopping that movement by reversing those angles of force.

Early on, we found that a 15-tonne thruster is not strong enough for the candidate 150,000-dwt ship, as ballasted, but that a 20 -tonne thruster is sufficient. I also found that the minimal nudder rate of the candidate tanker, $2.4^{\circ}$ per second (the least allowed by the classification society), and the standard rudder, do not provide the steering response or rudder authority that is needed, and their lack costs considerable time in the final positioning. To double the rudder rate is not usually a very costly provision. For more rudder force, a high-lift rudder is needed. A flap-rudder, such as the Becker system, or a Schilling rudder would seem to be a good choice. 
available for all but the first two runs on Friday, and I believe they belped me significantly. That day, I missed the mark on the first run; the remaining seven fell inside the 2.5 meter radius, but not all on the first try. I believe a couple took three releases.

The experience of the four days of meaningful simulation convinced me that it is extremely difficult to make a proper mating when the larger waves of the wave spectrum are at play. It is much better to wait for the smaller waves, which require that the moon pool be in the proper place when they occur. Sometimes that takes a few minutes. And this is what is wrong with simply steering to pass over the buoy while the ship is moving ahead; you just have to be lucky and miss the larger waves as you move across, if fairly large waves are in effect. Otherwise, the approach is missed. But that tactic can be used in smooth seas.

On Friday, I also experimented with the use of thruster vectors in various directions while moving the moon pool over the buoy for the mating. I found that, with the autopilot set for a heading that would keep the rudder hard-over in one direction, and with a propeller thrust that was very nearly in balance with the external forces, I could use thruster vectors both for fore-andaft and lateral movement, keeping the axial speed at or near zero by using thruster angles a little ahead or aft of abeam. But I also found that as the wave heights and their energies vary over the wave spectrum, the ship accelerates both ahead and astern, sometimes too much to control by the thruster alone, so it is sometimes necessary to use propeller changes. In large waves, I found considerable power changes useful, even necessary, if time is to be saved.

In waves of any appreciable size, because of the accelerations, both negative and positive, I found that equilibrium is not possible, so one has to settle for near-equilibrium balances, which also work. But that is reality.

Another thing I experimented with on the last day or two was to initiate the approach upwind/up-wave, rather than up-current. What led me to do this was, first, the thought that this would require less turning during the final phase of the approach, and, second, that this approach would hold the wind and waves at a smaller angle to the bow, which would reduce the tendency for the wind and waves to take over the steering, providing better steering as slower speeds in high winds and large waves. It had become clear that in currents of no more than two knots, the ship, at or near an equilibrium heading in high wind and waves, would be pointing considerably closer to the wind and waves than to the current.

I found out very quickly that I could not steer adequately and at the same time take care of conning the ship and all that that entails. Not having a human helmsman, I had to rely upon the autopilot, and that worked out reasonably well. It might have worked a little better had I been able to apply response tuning to the autopilot, as one normally can do with most autopilots. 
The simulator operators were understandably reluctant to tweak the autopilot, as they had no knobs on the console for that purpose.

I learned a good deal in a week. But I do not feel I have exhausted the learning experience. Nevertheless, we did quite enough to convince me that the candidate ship, with a 20tonne omnidirectional bow thruster, can be mated with the suction mooxing under all the conditions we simulated. With improved steering, it could be done more efficiently. Smaller ships might be more problematical, as they tend to be more livcly in movement in a seaway. Mass may be a problem for shiphandling, and it is, but it also has some advantages too.

Each site and choice of shuttle tanker needs to be simulated, to determine the design features related to navigation, maneuvering and mating with the buoy. Once the shuttle tanker has been simulated and its manevering characteristics determined, the first masters should be indoctrinated into the maneuvering concept and mating process, utilizing a simulator. This should involve discussions, aided by diagrams, and simulations. To save on simulator time, a day or two of discussion should precede the simulator exercises. Then the simulator exercises should be coached, with debriefings after each run. If I am to do the training, I would work up discussion guidelines and talking points. It might be a good idea to allow an extra day for the first such scssion, to provide some extra time as the instruction scheme is refined for future use. Eventually, I now believe, about five days will suffice, the first day being committed to discussion of precepts and tactics, and possibly an introduction to the simulator, followed by four days of simulation exercises, with a debriefing after each run.

In summary, the approaches and mating are clearly feasible. The concept uses fundamental shiphandling pxinciples, but in a different way than one usually uses them in port, or than is dealt with in the texts. But it all is learnable, and the Nichols simulator is an excellent device for the requisite training.

Again, I enjoyed the challenge of this project. For your suction mooring system concept, I believe its time has come.

Sincerely,

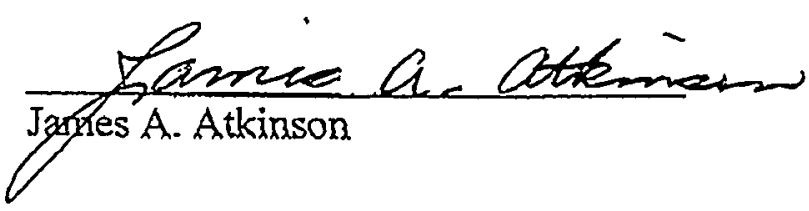




\section{Appendix $\mathrm{H}$}

\section{OFFSHORE TECHNOLOGY CONFERENCE PAPER NUMBER 7711}




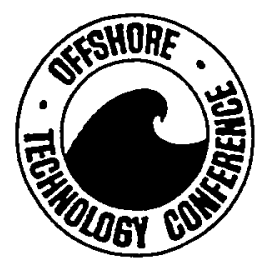

\section{OTC 7711}

\section{THE SUCTION MOORING, A NEW TYPE OF OFFSHORE SINGLE POINT MOORING} Jens Korsgaard and Louis Hutter, Han-Padron Associates

Copyright 1995, Olishore Technology Conference

This paper was presented at the 27th Annual OTC in Houston, Texas, U.S.A., 1-4 May 1995.

This paper was selecled lor presentation by the OTC Program Committee following review of intormation contained in an abstract submilted by the author(s). Contents of the paper. as presented, have not been reviewed by the Olfshore Technology Conference and are subject to correction by the author(s). The material. as presented, does not necessarily reflect any position of the Olishore Technology Conference or its officers. Permission to copy is restricted to an abstract of not more than 300 words. illustrations may not be copied. The abstract should contain consplcuous acknowledgment of where and by whom the paper is presented.

\section{ABSTRACT}

Existing systems for mooring offshore oil production vessels of the ship type only permit the mooring or tying up of the vessel in quite low wave conditions. Virtually all existing systems do not permit the vessel to moor in wave conditions exceeding $H_{s}=4.5 \mathrm{~m}$. Most systems require wave heights smaller yet to moor. This is a problem in cases where frequent mooring operations are required such as for shuttle tankers and for disconnectable oil production or storage vessels moored in areas with a high incidence of tropical storms. The wait for the waves to subside following the storm causes additional production losses due to the oil field being shut in.

A newly developed mooring system, the suction mooring, promises to overcome this problem by permitting the mooring of the vessels in wave heights to at least $H_{s}=8 \mathrm{~m}$. The principle of the mooring consists of using the hydrostatic pressure available at the keel of the vessel to press a mooring buoy onto the bottom and holding the vessel by the resulting friction. The action of the mooring buoy is similar to that of a suction cup.
The pressure above the buoy is lowered and maintained low by means of pumps aboard the vessel.

This paper reports on model tests carried out to prove the feasibility of conducting the mooring operations in high waves. Three different configurations of the mooring buoy were tested. For two of the configurations it was found that the mooring process could be carried out safely in all wave heights tested i.e. up to $H_{s}=7.7 \mathrm{~m}$.

The impact upon offshore operations of this capability was investigated by performing Monte Carlo simulations on a hypothetical oil field in the center North Sea and in the northern North Sea. An actual center North Sea wave record was used in the simulations.

The suction mooring system was compared to two other high performance mooring systems. The results show that weather related oil field shut-ins are reduced an order of magnitude by application of the suction mooring compared to the other systems simulated.

References, nomenclature, and figures at end of paper. 


\section{MOORING PRINCIPLE}

The suction mooring principle involves mooring a submersible buoy to the bottom of a vessel utilizing differential hydrostatic pressure, in a manner similar to a suction cup.

A mooring buoy is brought into contact with the flat portion of the bottom of the ship either by means of a retrieval line such as commonly used for the Buoyant Turret Mooring (BTM) and Submerged Turret Loading (STL) type mooring or by means of reduced buoyancy utilizing a system aboard the buoy to control the buoyancy.

The reduction in hydrostatic pressure above the buoy is provided by pumps aboard the ship. A bowthruster pump of the Omni-thruster or the Elliott Turbo Machinery type having its intake at the keel in the mooring area is used to achieve the initial pressure reductions. This type of pump has a capacity from 4 to $11 \mathrm{~m}^{3} / \mathrm{sec}$. When the buoy comes into contact with the keel of the vessel, it blocks the thruster pump intake. This lowers the pressure above the buoy and is causing the buoy to be pressed against the bottom of the vessel.

As illustrated on Figure 1, the top surface of the buoy is equipped with fenders and seals which prevent damaging impacts between the buoy and the vessel. After contact has been achieved, the seals limit the leakage of water to the top of the buoy to acceptably low values. The leakage rate following the mooring is two orders of magnitude less than the flow rate required to make the initial contact between the buoy and the vessel. This allows the bow thruster pump to be stopped after the mooring has been achieved and the leakage is removed using only pressure maintenance pumps.

A typical buoy has a circular upper surface with a diameter of approximately $20 \mathrm{~m}$. Differential pressures of 100 to $200 \mathrm{kPa}$ are normally achievable, resulting in a vertical attractive force between the buoy and the vessel of 30,000 to $60,000 \mathrm{kN}$.
The horizontal mooring forces between the buoy and the vessel are then resisted by the friction between the buoy and the bottom of the ship. Horizontal forces in excess of $10,000 \mathrm{kN}$ may be resisted by this system.

\section{APPLICATIONS OF THE MOORING SYSTEM}

The mooring system is applicable to ship type vessels engaged in offshore petroleum production such as floating production, storage, and offloading vessels (FPSO), floating storage and offloading vessels (FSO), and shuttle tankers engaged in transporting the crude oil ashore.

It is also expected that the system will be applicable to the ordinary maritime transportation of crude oil and refined products employing single point moorings.

\section{MODEL TESTS}

The new mooring system is presently under development and has in the development period being considered for several offshore fields. Specific designs were developed for an FSO for the conditions offshore Liverpool in Liverpool Bay in the UK. As part of this design process, model tests were carried out at the Danish Maritime Institute in Lyngby, Denmark to assess the feasibility of mooring the FSO to a buoy based on the suction mooring principle.

The FSO was a 125,000 dwt vessel which had not been selected. The model tests were carried out for a generic 125,000 dwt tanker with the properties listed in Table 1.

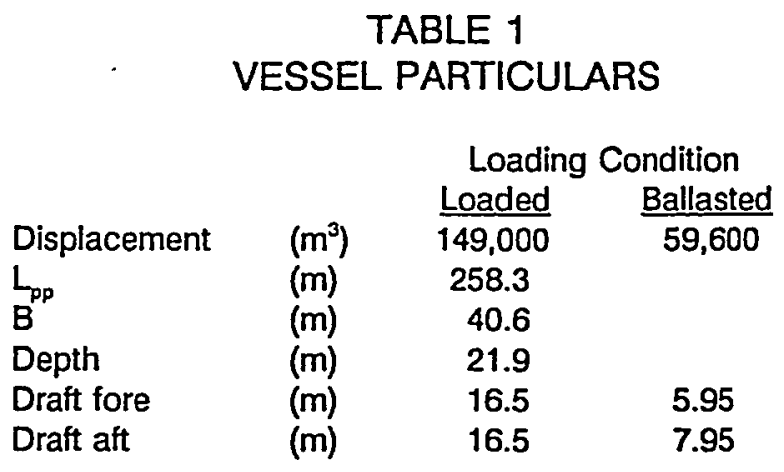

TABLE 1

\section{VESSEL PARTICULARS}


The tests were carried out for two loading conditions. Namely, a fully loaded vessel and a ballasted vessel with a displacement of 0.4 times the fully loaded displacement.

The tests had two objectives:

- To test the feasibility of mooring operations in extreme wave conditions using the suction mooring principle.

- To test a mooring in which the mooring legs were partially made up from synthetic rope instead of wire rope or chain.

The latter was necessary because of the shallow depth of $30 \mathrm{~m}$ which did not permit the application of chain and wire rope catenaries. If the mooring system had been comprised entirely of wire rope and chain, sufficient resiliency was not obtainable without the application of buoys and sinkers in each mooring leg to enhance the elasticity of the mooring leg. The application of buoys and sinkers would cause navigational problems in the area due to the danger of the submerged buoys coming into contact with the ship's hull.

Only the tests performed for the first objective, determining the feasibility of the mooring operations, are reported in this paper.

\section{TEST SET UP}

The tests were conducted in the towing tank at the Danish Maritime Institute as shown in Figure 2.

The tests involved the testing of vessels subjected to combined wave, current, and wind. Due to limitations in the test set up, only co-linear actions of these forces were considered. Wind and waves always acted in the same direction whereas currents acted either opposite to or in the same direction as the wind. The site has very high tidal currents and therefore the vessel would frequently be subjected to the winds and waves going in one direction and the currents going in the opposite direction.
In the test set up, this was emulated by having the carriage travel through the towing tank at the appropriate speed either toward or away from the wave maker.

The tests were conducted in a scale of 1:50. The towing tank has a water depth of $5.3 \mathrm{~m}$. This would correspond to a prototype water depth of $265 \mathrm{~m}$. Therefore, in order to conduct the tests at the shallow water depth at the test site of $30 \mathrm{~m}$, a false floor was erected underneath the towing carriage as shown on Figure 2.

The leading and trailing edges of the false floor represent abrupt depth change in the model. This caused problems in modeling the waves because the waves hitting the leading edge of the false floor was partially reflected from the edge. The same also took place at the trailing edge. It was, therefore, necessary to experiment with the setting of wave generator in order to obtain the appropriate spectrum in the test area.

The wave conditions used are shown below in Table 2.

TABLE 2

TEST WAVE CONDITIONS

$\begin{array}{cccc}\text { ID } & \begin{array}{c}\mathrm{H}_{3} \\ (\mathrm{~m})\end{array} & \begin{array}{c}\mathrm{Tz} \\ (\mathrm{sec})\end{array} & \begin{array}{c}\mathrm{Tp} \\ (\mathrm{sec})\end{array} \\ \mathrm{A} & 6.6 & 10.1 & 12.1 \\ \mathrm{~B} & 7.7 & 13.1 & 13.6 \\ \mathrm{C} & 5.6 & 9.3 & 8.7^{\star} \\ \mathrm{D} & 5.0 & 8.9 & 9.1 \\ \mathrm{E} & 4.7 & 8.0 & 8.8 \\ \mathrm{~F} & 4.1 & 7.7 & 8.6\end{array}$

*Secondary peak at $12.0 \mathrm{sec}$.

The wave maker was initially programmed to make a Jonswap spectrum with a $\gamma$ factor of 3.3. However, due to the distortions taking place at the leading and trailing edges of the false floor, the wave maker was ultimately programmed to generate waves which caused the best obtainable wave spectrum at the vessel. 
The model tests were documented through four video cameras as indicated on Figure 3 . In addition, the mooring ropes and the retrieval line were instrumented with strain gages. The wave action was recorded by four wave gages, one at the wave maker, one placed ahead of the test area on the false bottom, and two wave gages placed laterally to the test area on the false bottom. The measurements from all these gages were recorded on a computer with a frequency of 30 hertz and they were synchronized with the video recordings.

Three different buoy configuration were tested. Buoy configuration 1 is shown on Figure 4. This buoy has an upward protruding $1 / 4$ toroidal "cone" to mate with a corresponding female toroidal recess in the vessel. The buoy's toroidal surfaces were protected by ordinary cylindrical dock fenders with a diameter of $1 \mathrm{~m}$ mounted vertically. The exterior fender seal element on the buoy serves as a seal after mooring and protects against impact during the mooring process. This was also a circular cylindrical fender with a diameter of $1 \mathrm{~m}$. The elasticity of the fendering elements as not represented in the model. Rather the exterior fender was represented by a rubber hose having a diameter equal to that of a compressed fender/seal element. The upward protruding "cone" was not equipped with any fender elements in the model. This buoy configuration was equipped with a retrieval line. The buoy was mated with the vessel by pulling on the retrieval line from the deck of the vessel with a constant force of $981 \mathrm{kN}$.

The dock fenders used were Bridgestone cylindrical fenders $1,000 \mathrm{~mm}$ diam using rubber type R1. The resulting maximum allowable horizontal impact velocity between the cone and the recess was $0.81 \mathrm{~m} / \mathrm{sec}$. The maximum allowable vertical impact velocity between the buoy and the vessel depends on the tilt of the buoy at impact. The higher the tilt the smaller a portion of the fender would be engaged and consequently the allowable impact velocity reduced. The allowable values are stated in Table 3.
TABLE 3

\section{ALLOWABLE IMPACT VELOCITIES}

\begin{tabular}{lc} 
Tilt of & $\begin{array}{c}\text { Allowable Vertical } \\
\text { Impact Velocity } \\
\mathrm{m} / \mathrm{sec}\end{array}$ \\
\hline Buoy & 2.4 \\
0.05 & 1.95 \\
0.10 & 1.55 \\
0.15 & 1.30 \\
0.20 & 1.18 \\
0.25 &
\end{tabular}

The second configuration of the buoy was a flat top buoy equipped with an exterior fender/seal element at a radius of $10 \mathrm{~m}$ from the axis of symmetry similar to buoy configuration 1 . This buoy is illustrated on Figure 5 . This buoy was also brought into contact with the vessel by means of a retrieval line pulled in by a constant tension winch on the deck of the vessel. The retrieval force in this case was also $981 \mathrm{kN}$.

The third configuration of the buoy, shown on Figure 6, was equal to the second configuration with the exception that the buoy was not fitted with any retrieval line. Rather the buoy contained an internal buoyancy tank which during the mooring process was filled with air thereby increasing the buoyancy of the buoy causing it to rise in the water to contact the bottom of the vessel. This retrieval system requires that the vessel be navigated in such a manner that the buoy is properly positioned relative to the vessel during the mating. The positioning system was emulated in the model by two elastic ropes which were controlled by a technician during the test. In this manner, the navigation of the vessel was emulated, however, no attempts were made to make the emulation conform to any particular bow thruster/propulsion system performance.

The configuration 3 buoy is equipped with internal buoyancy chambers. The chambers were made with a larger than specified diameter during the manufacture of the model buoy. Therefore, the buoy had a lower than intended metacentric height. It was intended that the metacentric height for the buoys with configurations 2 and 3 would be approximately equal. The actual 
metacentric height of the configuration 3 buoy was very close to zero. Therefore, during the tests, the buoy exhibited somewhat unstable behavior. Nevertheless, as can be seen from the results mooring and unmooring of the configuration 3 buoy never resulted in impacts between the buoy and the vessel exceeding the capacity of the exterior fender.

The means of creating the suction on the vessels was in the model two positive displacement pumps pumping from a moon pool at a rate of $4.5 \mathrm{~m}^{3} / \mathrm{sec}$. A cross section of the moon pool located $40 \mathrm{~m}$ aft of the forward perpendicular and the emulation of the constant tension retrieval winch is shown on Figure 7. Note that the pump intake is located nearly flush with the bottom of the vessel, therefore, when the pumps start sucking air, the space between the buoy and the vessel is still water filled. Atmospheric air has free access to the mooring area. Therefore, in the model, the lowest possible differential hydrostatic pressure on the buoy is equal to the head from the draft of the vessel. In the prototype the mooring recess area will be closed to the access of atmospheric air. Therefore, in the prototype higher differential pressures can be obtained than in the model.

\section{MODEL TEST RESULTS}

The model tests were made for two purposes.

- Assess the forces in the elastomeric mooring arrangement.

- Assess the feasibility of the mooring and unmooring process in high waves.

Only the second part is reported in this paper. A total of 83 mooring and unmoorings were performed with the ballasted vessel in the model. These were distributed among the three different buoy configurations and the various wave heights tested in the model as indicated below in Table 4.

\section{TABLE 4 \\ IMPACTS DURING \\ MOORING AND UNMOORING}

\begin{tabular}{llcc}
$\begin{array}{l}\text { Buoy } \\
\text { Type }\end{array}$ & $\begin{array}{l}\mathrm{H}_{\mathbf{s}} \\
\mathrm{m}\end{array}$ & $\begin{array}{l}\text { No. of } \\
\text { Tests }\end{array}$ & $\begin{array}{c}\text { Max. Impact } \\
\text { \% of Allowable }\end{array}$ \\
\hline 1 & 6.6 & 5 & 258 \\
1 & 5.0 & 6 & $<20$ \\
1 & 4.7 & 6 & 195 \\
1 & 4.7 & 4 & 75 \\
1 & 4.1 & 7 & 123 \\
2 & 6.6 & 8 & 24 \\
2 & 5.0 & 7 & 11 \\
2 & 4.7 & 7 & 6 \\
3 & 7.7 & 4 & 58 \\
3 & 6.6 & 4 & $<10$ \\
3 & 6.6 & 4 & 20 \\
3 & 5.0 & 5 & 10 \\
3 & 5.0 & 5 & 65 \\
3 & 4.7 & 4 & $<10$ \\
3 & 4.7 & 3 & 38 \\
3 & 4.1 & 4 & 5
\end{tabular}

The impacts between the buoy and the vessel were analyzed by studying the underwater video recordings frame by frame and determining the tilt and position of the buoy in each frame. The vessel model was equipped with a characteristic mark on the side of the vessel enabling the continuous determination of the relative position between the vessel and the buoy.

By analyzing the frames on the video recording, it is possible to determine the relative velocity between the buoy and the vessel with a tolerance of approximately 20 percent. This tolerance translates into a tolerance of 40 percent on the recorded energies. The most important results from these tests is that the buoy with the upward protruding toroidal mating cone suffered damaging impacts for most wave heights tested, i.e., $H_{s}=4.1 \mathrm{~m}$ to $H_{s}=6.6 \mathrm{~m}$. The model buoy with a flat top surface retrieved by a retrieval line in no instance had impacts above 25 percent of the allowable impacts for the selected fender. The configuration 3 buoy was raised with compressed air. This buoy had despite the low metacentric height in all cases smaller than allowable impact between the buoy and the vessel. In the extreme case of $\mathrm{H}_{\mathrm{s}}=7.7 \mathrm{~m}$, an impact of up to 58 percent of the allowable impact 
was experienced. Also at a lower wave height of $H_{s}=5.0 \mathrm{~m}$ an impact of $65 \%$ of the allowable was recorded.

Regardless of the method of retrieval the flattop buoy can safely mate with the vessel for all wave heights tested, i.e., from $\mathrm{H}_{3}=4.1 \mathrm{~m}$ to $\mathrm{H}_{3}=7.7$ $\mathrm{m}$. $\mathrm{H}_{\mathrm{s}}=7.7 \mathrm{~m}$ was the highest obtainable wave height in the test set up. It is likely that mooring and unmooring can be performed in substantially higher wave heights than those tested.

The navigation of the vessel to the buoy and the maintenance of the position of the vessel relative to the buoy during the mooring process was not tested in this series of tests. These tests only show that the physical process of bringing the buoy into contact with the vessel and of releasing the buoy upon departure of the vessel can be safely done in the wave heights tested. Whether or not the vessel can maneuver into the proper position in high wave heights depends on the configuration of the vessel propulsion and thruster systems. These systems were not tested in this series of tests.

The conclusion from the tests is that mooring and unmooring of the flat top type suction mooring buoy may safely take place in all wave heights up to at least $H_{s}=8 \mathrm{~m}$.

\section{OPERATIONS ANALYSES}

The fact that the suction mooring buoys can moor and unmoor in very high wave heights has implications for the type of operations which may be conducted offshore when planning for FPSO, FSO, and shuttle tanker operations. To examine the implications of this capability a series of Monte Carlo simulations were performed for two hypothetical oil production systems located either in the middle North Sea or in the northern North Sea in more severe environmental conditions. The two hypothetical systems are illustrated on Figures 8 and 9 . The first system consists of two buoys connected to an oil field with no storage aboard the production vessel or platform. Two shuttle tankers bring the oil to a port 500 nautical miles distant. This system is compared with an alternative system with a small storage and one mooring buoy. The operation of the first system is that whenever a shuttle ship arrives it ties up to the idle buoy. At an appropriate moment when the shuttle ship that was receiving the oil is full, the production is switched to the second vessel and the loaded vessel leaves for port.

The suction mooring (SM) system was compared to two other shuttle mooring systems. System 1 (S1), capable of mooring at $\mathrm{H}_{\mathrm{s}}=4.5 \mathrm{~m}$ and of remaining on the mooring up to $\mathrm{H}_{\mathrm{s}}=8 \mathrm{~m}$. These values have been derived from Reference 1 . Reference 1 claims a higher disconnect limit than $8 \mathrm{~m}$. However, the highest wave height at which the vessel can remain moored is also a function of the cost of the mooring system. Therefore, for purposes of this paper a maximum disconnect wave height of $8 \mathrm{~m}$ has been selected for all mooring systems that are capable of maintaining the ship moored at that wave height or beyond.

Another shuttle mooring system System 2 (S2) has connect limits of $\mathrm{H}_{3}=4.5 \mathrm{~m}$ and disconnect limits of $\mathrm{H}_{\mathrm{s}}=6 \mathrm{~m}$. These values were derived from Reference 2.

The corresponding values for the suction mooring buoy are a connect limit of $\mathrm{H}_{\mathrm{s}}=8 \mathrm{~m}$ and disconnect limit of $H_{s}=8 \mathrm{~m}$. A common feature of all three systems is that petroleum transfer can take place at all wave heights in which the ship can remain moored.

A record of the winds and waves from the central North Sea was obtained for the purpose of making a detailed operations analysis. The record spans a continuous three year period. The record consists of hourly measurements from two different types of wave gages, a waverider type wave gage and a radar wave gage. The measurements from each of the two wave gages were in general agreement. However, at any specific time they might disagree by as much as 25 percent. For purposes of the Monte Carlo simulations, the two records were unified by taking the average value of the two wave 
recordings in each hour. At certain times the record from only one of the two wave gages was available. In these instances, the available record was used for the value of the wave height. Small gaps exist in the wave record where both gages did not record any data. For purposes of continuity in the simulation, these gaps were filled in by a straight line interpolation between the last recorded wave height prior to the gap and the first recorded wave height following the gap.

Although the weather record also contains information about the velocity and direction of the wind, this data was not taken into account in order to simplify the simulations. It is, however, recognized that for certain combinations of winds and waves, the wave height limits may be lower than those utilized in the simulation.

The simulations simulated a hypothetical shuttle tanker round trip of 500 nautical miles each way to an oil receiving port some place in the North Sea area. The speed with which the shuttle tanker can transit to and from the receiving port also depends on the weather. During high waves, the speed of the vessel must be either be involuntarily or voluntarily reduced. For the purposes of this paper, it is has been assumed that the oil tanker will have speed reduction characteristics similar to the bulk carrier Mineral Sering. The Mineral Sering was a $65,000 \mathrm{dwt}$ bulk carrier for which extensive speed reduction studies were made in the late 1960s. The data reported in reference 3 is the basis for the speed reduction function utilized in the simulations. The speed reduction function is given in Figure 10.

For purposes of the simulation, it was assumed that the vessel was subject to the speed reduction whenever it was within 300 nautical miles of the oil field. The characteristics of the round trip for the vessel is given below in Table 5 .

\begin{tabular}{|c|c|c|}
\hline \multicolumn{3}{|c|}{$\begin{array}{l}\text { TABLE } 5 \\
\text { VESSEL ROUND TRIP }\end{array}$} \\
\hline & Time & Speed \\
\hline Mooring & $1.5 \mathrm{H}$ & \\
\hline $\begin{array}{l}\text { Loading } \\
\text { Unmooring }\end{array}$ & $\begin{array}{l}\text { variable } \\
075 \mathrm{H}\end{array}$ & \\
\hline $\begin{array}{l}\text { Sail First } 300 \mathrm{NM} \\
\text { Next } 200 \mathrm{NM}\end{array}$ & & $\begin{array}{l}\text { reduced speed } \\
15 \text { knots }\end{array}$ \\
\hline Enter port & $12 \mathrm{H}$ & \\
\hline Discharge & variable & \\
\hline Leave port & $4 \mathrm{H}$ & \\
\hline First $200 \mathrm{NM}$ & & 15 knots \\
\hline Last $300 \mathrm{NM}$ & & reduced speed \\
\hline
\end{tabular}

It was assumed that the suction mooring system and Systems 1 and 2 had similar times for hook up and disconnect. While it is believed that the suction mooring system is somewhat faster than the other systems, the same times were assumed for the purpose of having uniform conditions among each of the tested systems in the Monte Carlo simulations.

System 1, System 2, and the suction mooring vessels were treated equally. They were all assumed to have the same rate of transfer between production equipment and the vessel while moored in the oil field and having the same characteristics when delivering the cargo in port.

The actual central North Sea weather record was used in the simulations. No random events were simulated in these simulations. It was assumed that the hypothetical oil field was in production at the beginning of the weather record and had a constant production of 100,000 bpd throughout the three years that the weather record lasted. The results of the simulation were the measurements of the lost production for each of the scenarios simulated. An initial set of simulations was made with the case of having two buoys with no storage at the production facilities, as shown on Figure 8. For this scenario, vessels with a capacity from 350,000 barrels to 500,000 barrels were considered in 25,000 barrel increments. 
A second production scenario, shown on Figure 9 , was also simulated. This scenario has only one mooring buoy and a small in-line storage between the production equipment and the single point mooring buoy. The storage assumed for these simulations was 15,000 barrels. The range of vessel sizes was the same as for the two-buoy case.

The storage basically permits continuation of the production during the time it takes for the vessel to unmoor and the following vessel to moor and get ready receive the cargo.

Following the initial simulations, a series of simulations was made for the one buoy case using a constant size shuttle vessel of 450,000 barrels but, varying the amount of storage available at the production facilities between 0 and 120,000 barrels in 30,000 barrels increments.

In addition to the actual mid North Sea weather record, a transformed record was made by multiplying all the wave heights by 1.2 and adding $.25 \mathrm{~m}$. In this manner, the wave record was transformed in such a way that it acquired the characteristics of a northern North Sea wave record as reported in Reference 4 . It is not certain that the transformed record has the proper persistence characteristics. However, for the purposes of the simulations, comparing the performance of the three types of mooring systems, the northern North Sea weather is adequately represented by the artificial wave records so generated.
The wave conditions actually used are stated below in Table 6.

TABLE 6

MID NORTH SEA WAVE STATISTICS

\begin{tabular}{cr}
$\mathrm{H}_{s}(\mathrm{~m})$ & Frequency \\
\hline $0-1$ & $24.06 \%$ \\
$1-2$ & $36.13 \%$ \\
$2-3$ & $20.58 \%$ \\
$3-4$ & $10.97 \%$ \\
$4-5$ & $4.91 \%$ \\
$5-6$ & $2.16 \%$ \\
$6-7$ & $0.73 \%$ \\
$7-8$ & $0.27 \%$ \\
$8-9$ & $0.08 \%$ \\
$9-10$ & $0.06 \%$ \\
$10-11$ & $0.03 \%$
\end{tabular}

The northern North Sea waves used are shown in Table 7. Table 7 also shows the wave statistics reported in Reference 4 for the northern North Sea.

TABLE 7

NORTHERN NORTH SEA WAVE STATISTICS

$\begin{array}{ccc}\mathrm{H}_{s}(\mathrm{~m}) & \begin{array}{c}\text { Frequency } \\ \text { Used }\end{array} & \begin{array}{c}\text { Frequency } \\ \text { Ref } 4\end{array} \\ 1-1 & 8.29 \% & 8.64 \% \\ 2-3 & 33.91 \% & 32.16 \% \\ 3-4 & 25.55 \% & 25.92 \% \\ 4-5 & 14.93 \% & 15.44 \% \\ 5-6 & 8.71 \% & 9.12 \% \\ 6-7 & 4.51 \% & 4.83 \% \\ 7-8 & 2.35 \% & 2.33 \% \\ 8-9 & 1.04 \% & 1.03 \% \\ 9-10 & 0.35 \% & 0.42 \% \\ 10-11 & 0.22 \% & 0.16 \% \\ 11-12 & 0.05 \% & 0.06 \% \\ 12-13 & 0.05 \% & 0.02 \% \\ 13-14 & 0.02 \% & 0.01 \% \\ & 0.02 \% & 0\end{array}$

\section{SIMULATION MODEL}

The simulation model was written in the GPSSH language. A simplified logic diagram of the simulation model is shown on Figure 11. 
In contrast to the usual Monte Carlo simulation models, this model had no random variations in it at all. It was based on assuming that the process simulated took place during the period at which the actual weather record utilized was recorded. In consequence, the results are simply the results which would have been obtained had the specific production scenario taken place during the period of record. The results, therefore, do not represent a statistical average but rather the actual outcome of an assumed production scenario taking place over the three years covered by the weather record. The result which was recorded in the model was the lost production due to weather.

Other causes for lost production such as downtime due to maintenance or malfunctioning of the mooring, production difficulties, and downtime or malfunction of shipboard equipment, were not simulated. The results reported in this paper are caused solely by the different mooring limits that each of three mooring systems modeled have.

\section{OPERATIONS ANALYSES RESULTS}

The operations analyses were in all cases made on a hypothetical field producing a constant $100,000 \mathrm{bpd}$ oil throughout a three year period.

Figures 12 and 13 show the results from simulation of the case of two mooring buoys, no storage and two shuttle tankers. The only storage, in the system is the shuttle tanker moored to a buoy. The size of the shuttle tankers were varied in 25,000 barrel increments between 350,000 barrels and 500,000 barrels. The results of the center North Sea simulation are shown on Figure 12 and the results from the northern North Sea simulation are shown on Figure 13. In both cases, the lost production when utilizing the suction mooring systems is 20 to $25 \%$ of the nearest competing system.

For the case with only one buoy and two shuttle tankers, but with a storage of 15,000 barrels at the oil production facilities, the performance was somewhat better than for the two buoy case. Figure 14 shows the center North Sea results and Figure 15 shows the northern North Sea results. In this case, the suction mooring has production losses 10 to $20 \%$ of the nearest competing system.

The effect of varying the size of the storage was examined for the case of having one buoy and two 450,000 barrel shuttle tankers. The results from center North Sea for as little as 30,000 barrels storage at the oil production facilities, shown on Figure 16, show that the lost production for the suction mooring case is negligible whereas the $\mathbf{S 1}$ and $\mathbf{S} 2$ systems show losses in the order of 300,000 to 800,000 barrels per year. For the northern North Sea case, shown on Figure 17, a storage at the oil field facilities of 30,000 barrels resulted in the suction mooring system causing losses of approximately 150,000 barrels per year whereas systems 1 and 2 experienced well in excess of 1 million barrels per year losses.

These simulations indicate that in general the lost production due to weather outages could be reduced by approximately one order of magnitude by application of the suction mooring compared to the competing mooring systems simulated.

\section{NOMENCLATURE}

$$
\begin{aligned}
& \text { bpd }=\text { Barrels per day } \\
& \text { bph }=\text { Barrels per hour } \\
& L_{p p}=\text { Length between perpendiculars } \\
& B
\end{aligned}
$$

\section{ACKNOWLEDGEMENT}

We wish to thank the Hamilton Oil Co. Ltd. for their generous financial support of the model tests. 


\section{REFERENCES}

1. STL: A Key to Offshore Development, Submerged Turret Loading. STATOIL (undated)

2. UKOLS: Ugland Kongsberg Offshore Loading System. Brief Description, Ugland Engineering A/S (August 1994).

3. G. Aertssen: "Service Performance and Seakeeping Trials on a Large Ore Carrier," Transactions Royal Institute of Naval Architects (1969) Vol. 111, No. 2.

4. O.M. Faltinsen: "Sea Loads on Ships and Offshore Structures," Cambridge University Press (1990).

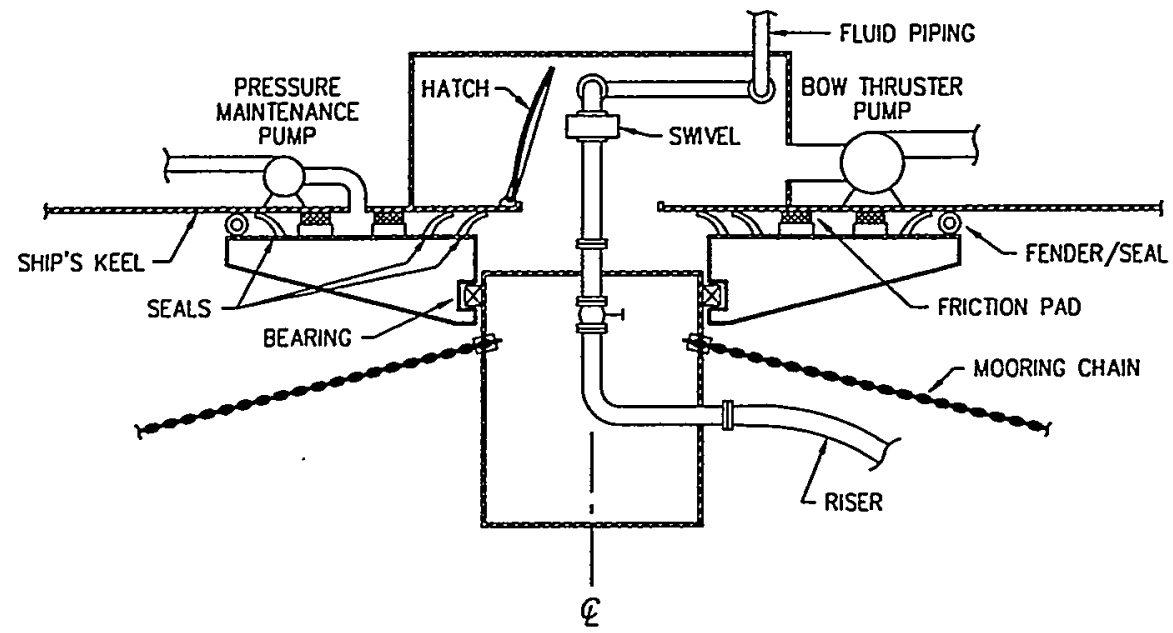

Fig. 1 Section of Mooring System

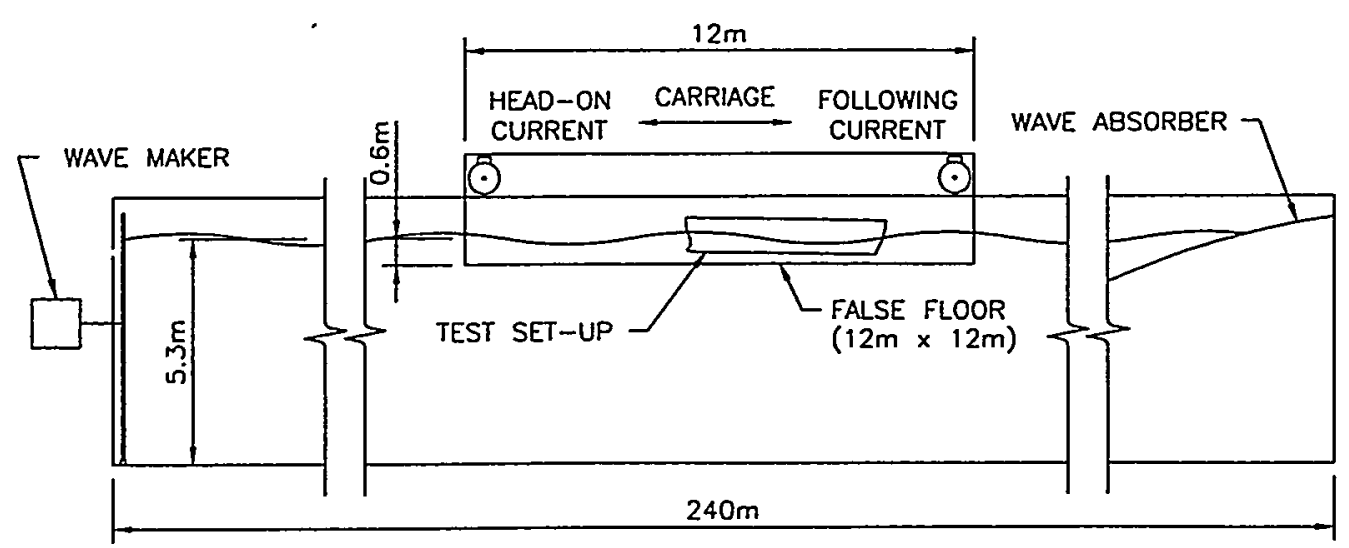

Fig. 2 Test Bosin 


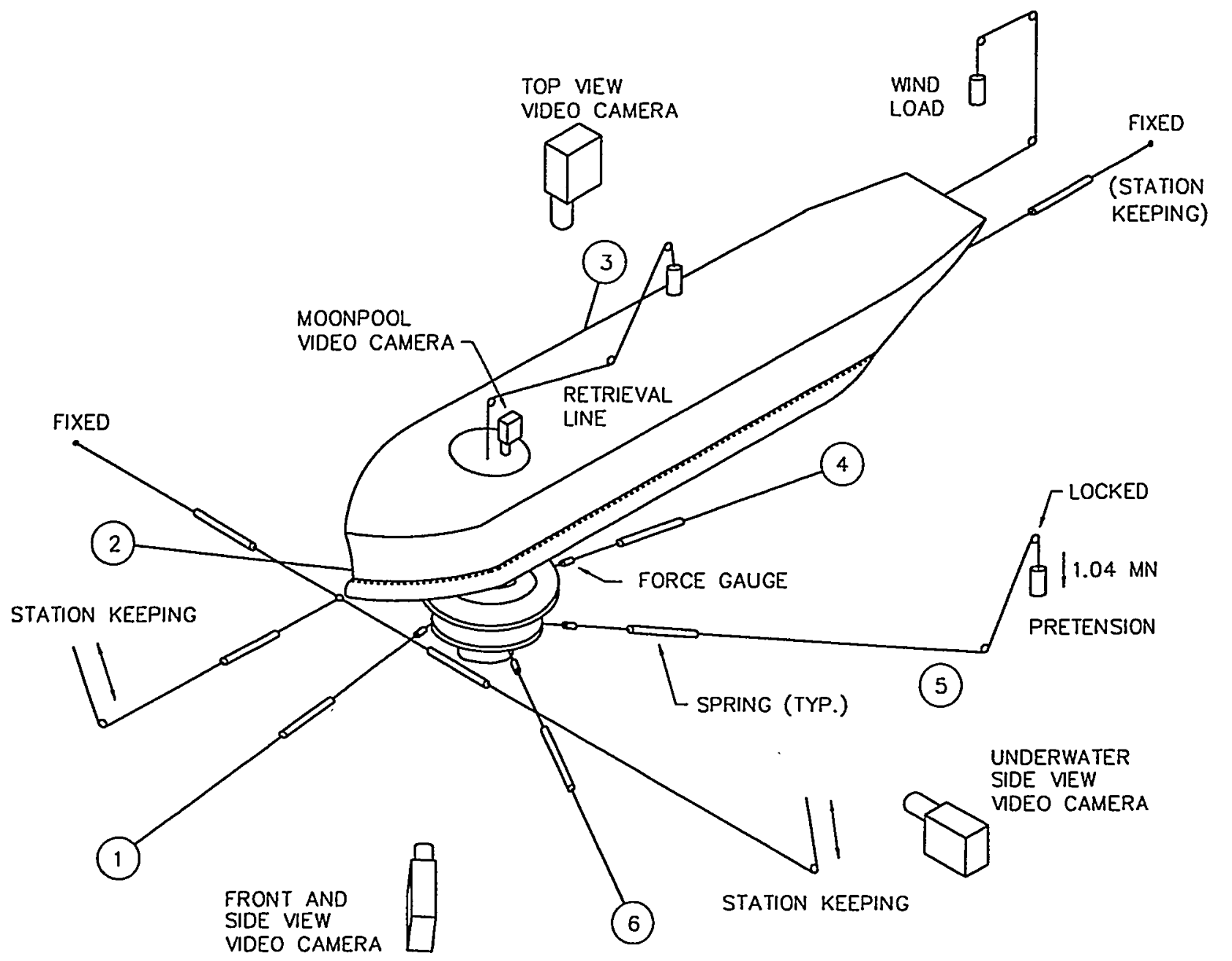

FIG. 3 Test Set-Up

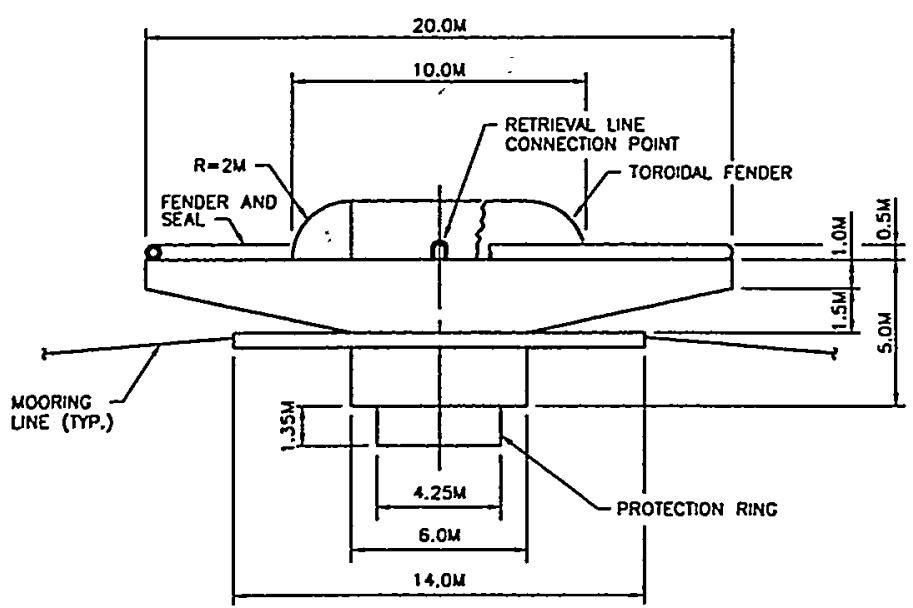

FIG. 4 Mooring Buoy Configuration 1 

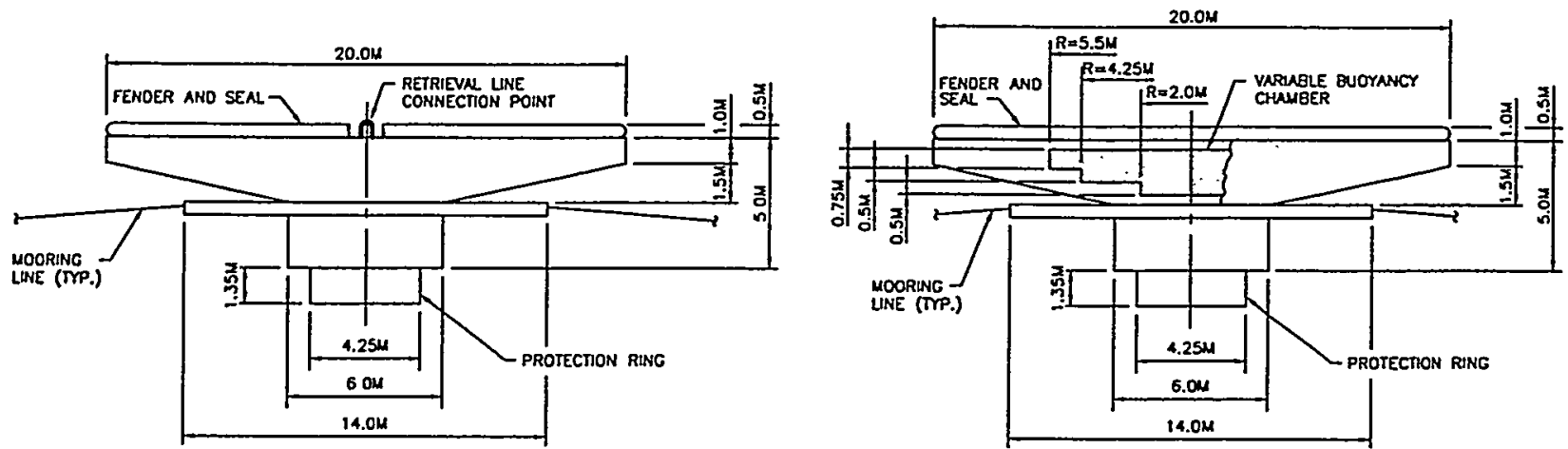

Fig. 5 Mooring Buoy Configuration 2

Fig. 6 Mooring Buoy Configuration 3

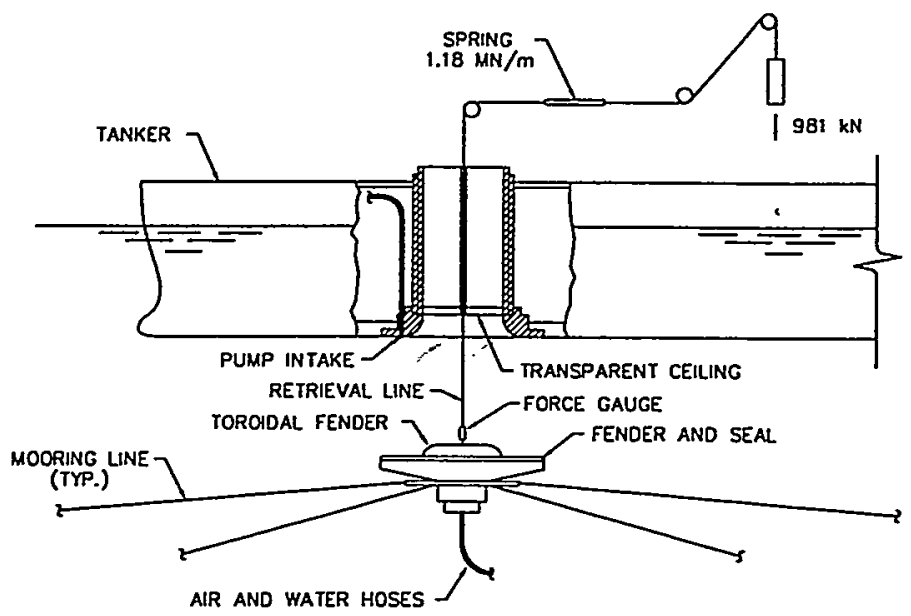

Fig. 7 Moonpood 
EXPORT BUOY 1

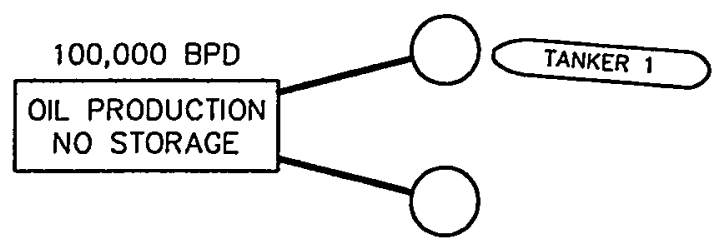

EXPORT BUOY 2<smiles>C[CH-]C</smiles>

FIG. 8 Oil Production Scenorio 1

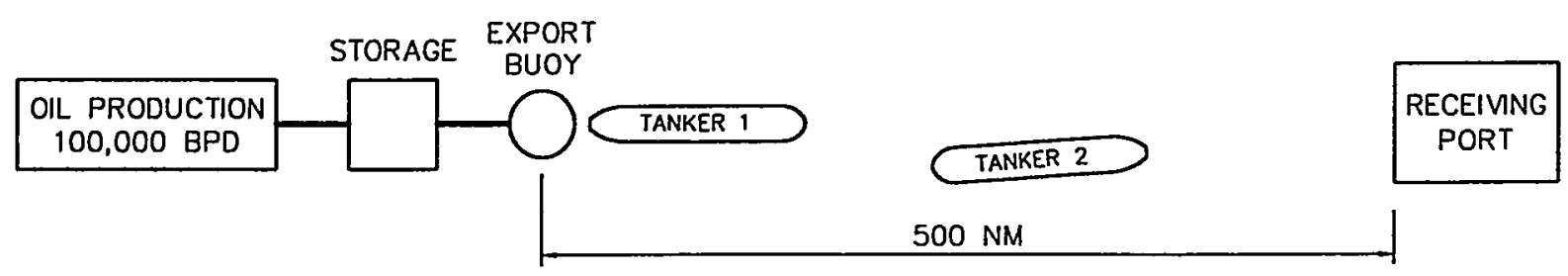

FIG. 9 Oil Production Scenario 2
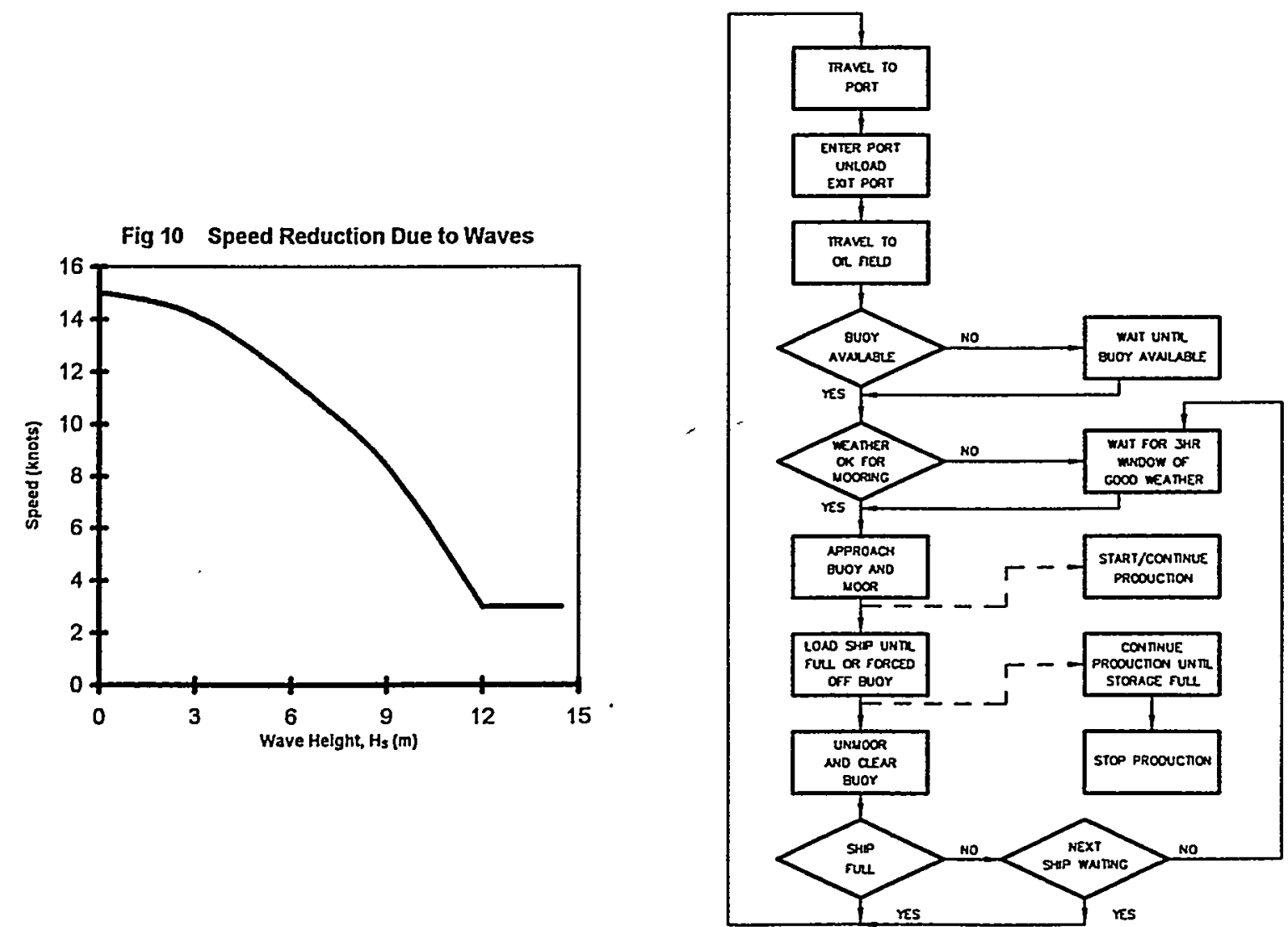

FIG. 11 Simplified Simulation Model Diagram 


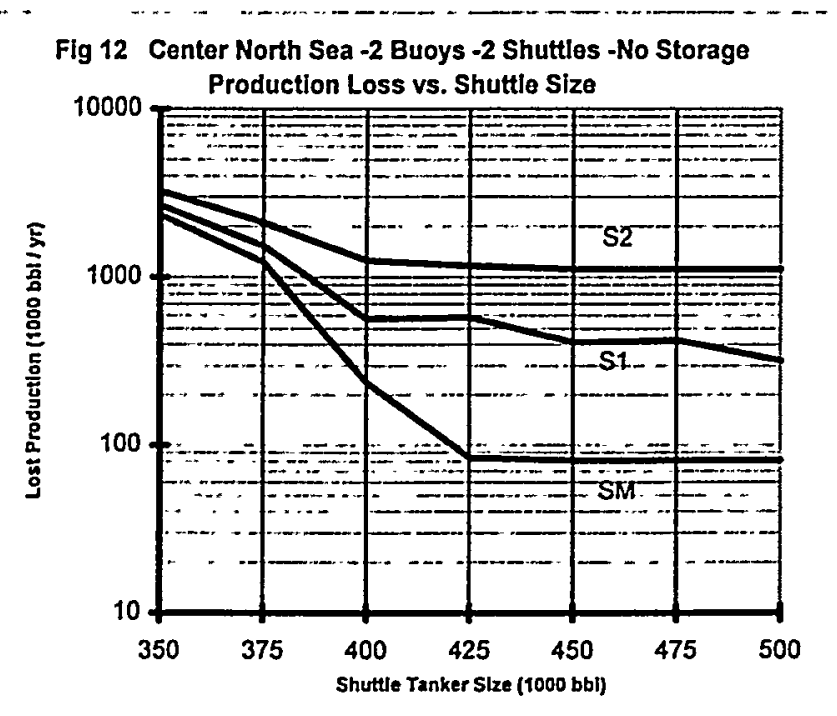

Fig 13 Northern North Sea -2 Buoys -2 Shuttles -No Storage
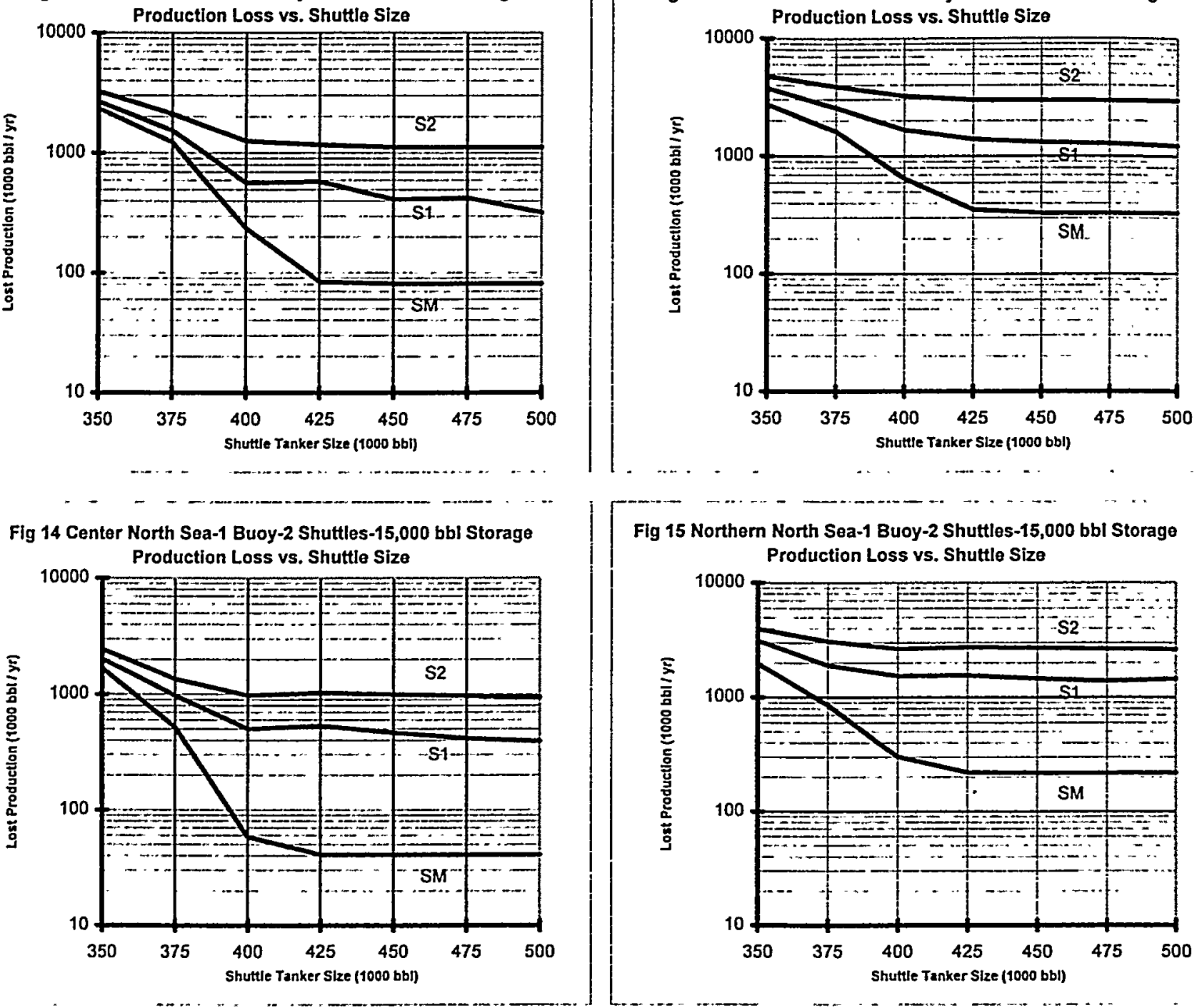

Fig 15 Northern North Sea-1 Buoy-2 Shuttles-15,000 bbl Storage

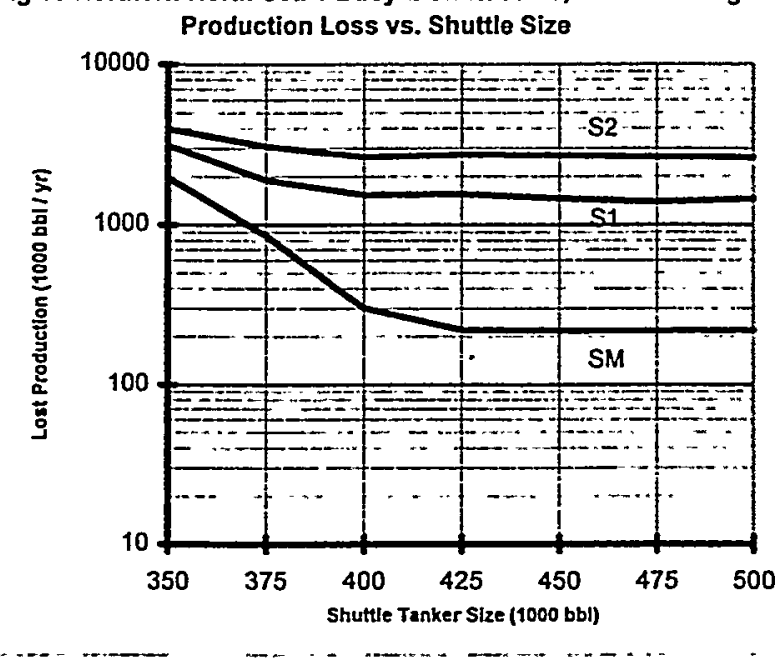

Fig 16 Center North Sea-1 Buoy -2 Shuttles $(450,000$ bbl each) Production Loss vs. Storage

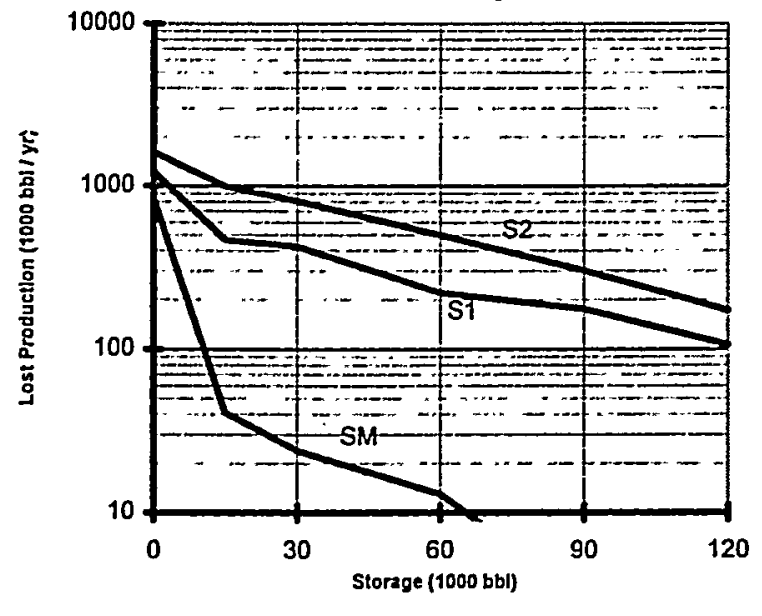

Fig 17 Northern North Sea -1 Buoy -2 Shuttles $(450,000$ bbl each)

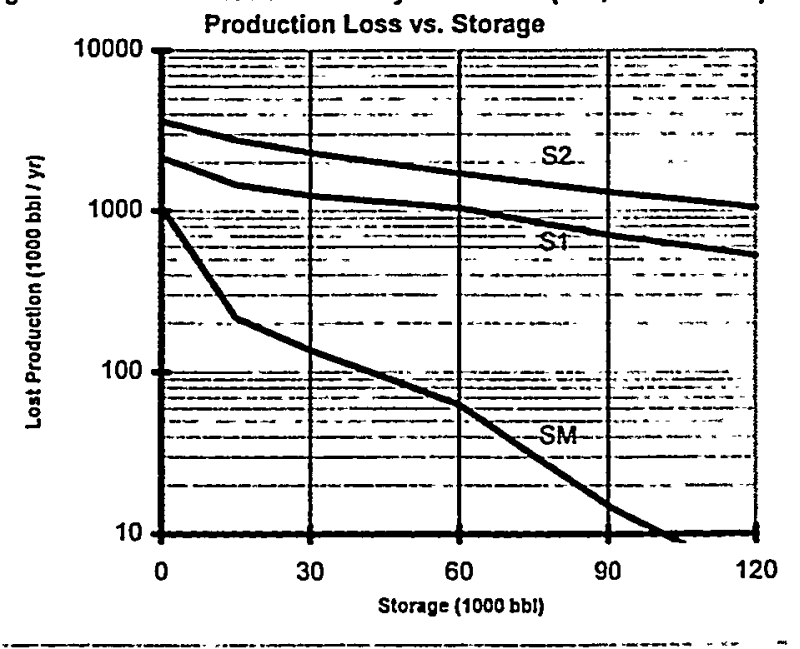


Appendix I HYDROSTATIC MOORING BROCHURE 


\section{THE HYDROSTATIC}

\section{MOORING SYSTEM}

\section{A new era in reoghng.}

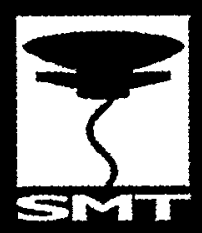

Suction Mooring Technology

Offshore Mooring Systems
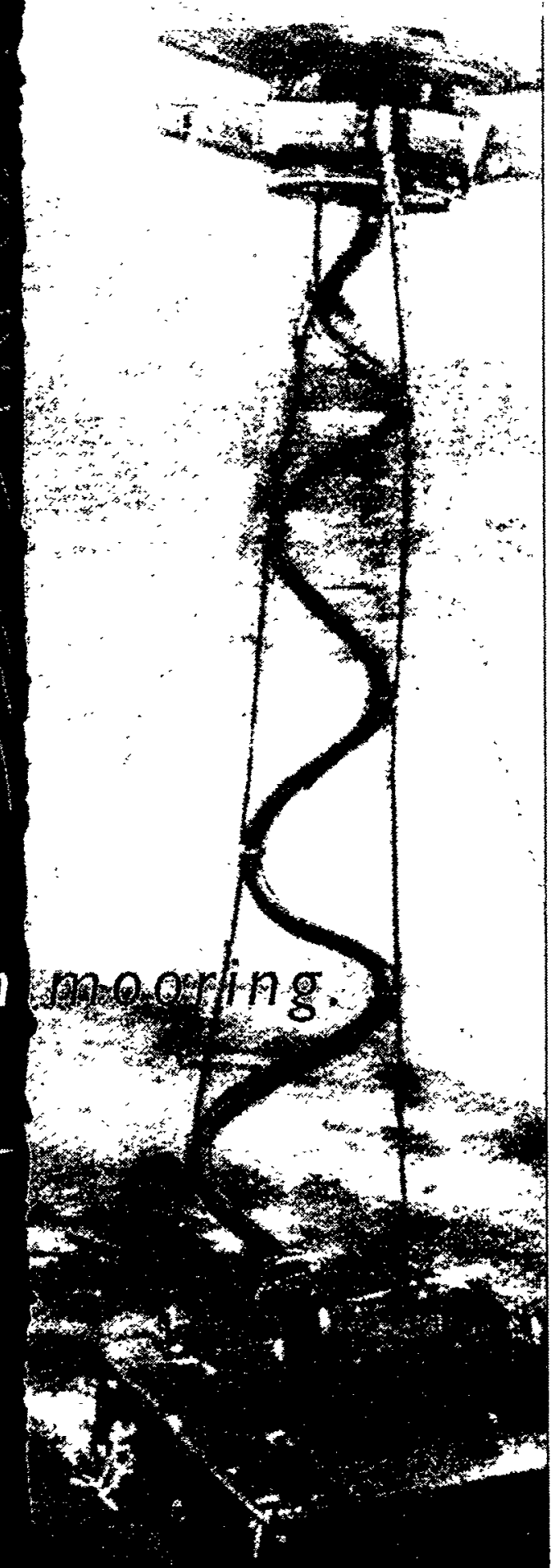


\section{The Hydrostatic Mooring System A New Era in Mooring}

Maybe once in a lifetime you are

presented with an opportunity that

undeniably and positively impacts

every aspect of your mooring

operations. The Hydrostatic

Mooring System is your passport

to success. Relying on outmoded,

inadeduate, limited, dangerous and

expensive mooring systems is

ancient bistory. And bere's why...

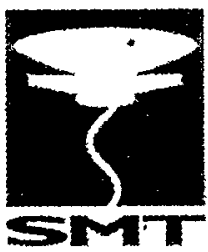

Suction Mooring Technalogy Offshore Mooring Systems

\section{What other \\ Mooring Systems \\ Offer You}

Costly downtime due to weather conditions.

Retrieval lines and hawsers between the tanker and the mooring device that snag and break.

- Weather-related downtime costing nillions of dollars per year.

Mechanical connectors that fail and delay successful mooring and tanker departure.

Personnel and vessel hazards such as line handling, tugboats, hawsers, winches and other equipment elements that are always required to operate current mooring systems.

- The need for special vessel maneuvers during rourine and emergency departures.

- The potential for explosions.

Unproductive, lengthy and dangerous mooring and release time. Expensive maintenance vessels. The potential for pollution.

Expensive vessel retrofit and fit-out.

\section{What Will the Hydrostatic Mooring System Offer You}

\section{No Matter the Weather}

When the tanker can operate it can moor to the Hydrostatic Mooring System. And operations will not be limited, curtailed or abandoned when sea states reach $\mathrm{H}$ s above $\mathrm{B}$ meters. Only the Hydrostatic Mooring System is capable of safely and effectively handling tanker mooring in these extremes.

There is no weather-related downtime typical among competing systems. This unique ability may. allow you to recoup the mooring costs within months.

\section{Safezy First}

All mooring operations are remotely controlled from the bridge of the tanker.

The Hydrostatic Mooring System can be safely released under maximum weather stresses.

Inlike other mooring devices, the Hydrostatic Mooring System presents no risk to passing vessels. Even the deepest draft vessels pass safely over the Hydrostatic Mooring System's resting position. 
The fluid transfer equipment in a

Hydrostatic Mooring System is continually immersed in water mitigating any chance of explosion.

The Hydrostatic Mooring System is virtually risk free and eliminates hazardous elements.

\section{Lightning Fast Speed}

The speed of the Hydrostatic Mooring System is extraordinary. The entire mooring operation lasts only nine seconds - a key sequirement for mooring in extreme wave heights!

Unmooring lasts only three minutes!

Obviously, tanker productivity particularly' on short shuttle runs with less than 24 hour sailing time - is unparalleled, due to the Hydrostatic Mooring System's extreme efficiency of operation.

\section{Classification Society Rule Compliance}

The Hydrostatic Mooring System complies with all current Classification Society rules including;

- The American Bureau of Shipping "Rules for Single Point Moorings," 1996

- The American Bureau of Shipping "Rules for Underwater Vehicles, Systems, Hyperbaric Facilities, 1990

\section{Unprecedented \\ Performance and \\ Performance \\ Monitoring}

The Hydrostatic Mooring System resides below the surface, below the keel of the deepest draft vessels. Its strategic placement reduces survival condition loads. Yet, the Hydrostatic Mooring System easily surfaces upon command. (See Figure 1)

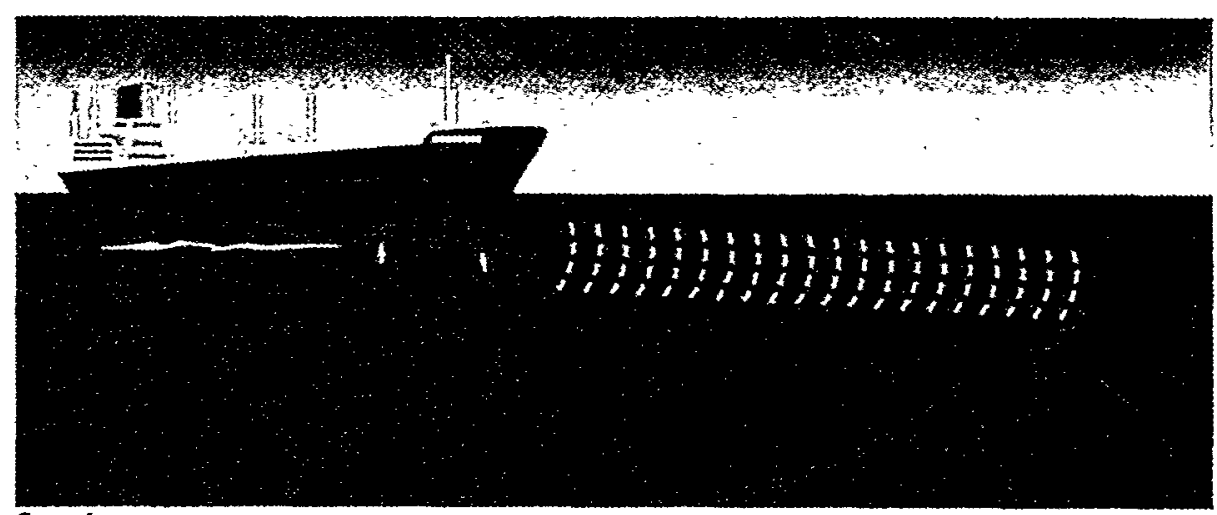

Step I

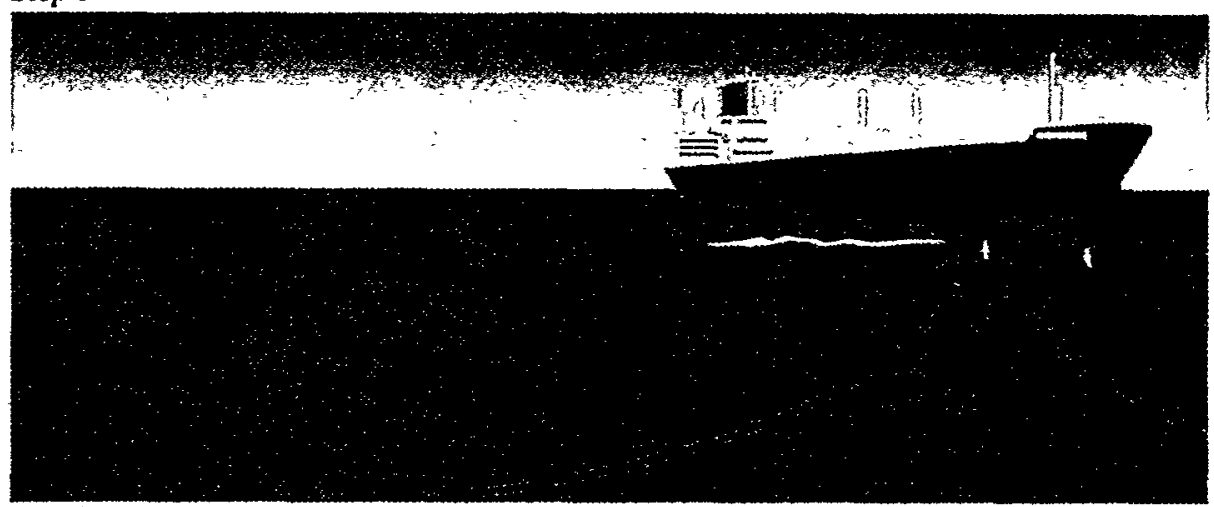

Step 2

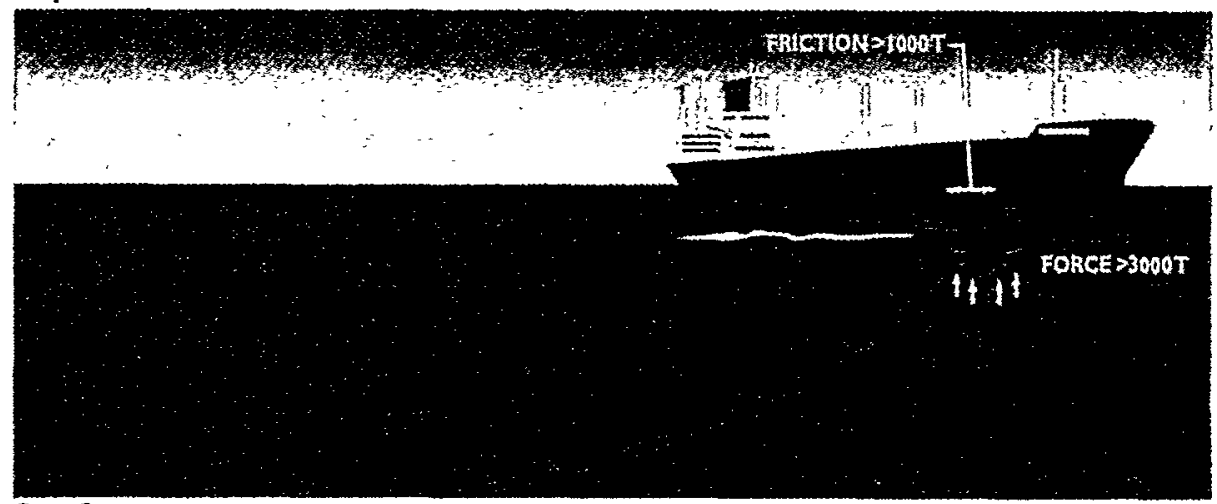

All maintenance is done on the surface with light duty vessels.

Any stom a tanker can weather, the Hydrostatic Mooring System can weather too. Unlike other mooring systems, the Hydrostatic Mooring System continues to operate in the most severe conditions.

The cost to modify a tanker to accept the Hydrostatic Mooring System is 5 to 10 times less than the modification costs associated with other high performance mooring systems. 


\section{Unprecedented \\ Performance and \\ Performance Monitoring \\ Cont'd}

a The Hydrostatic Mooring System reduces time on station because it offers the lowest possible hydraulic power consumption in the piping system.

whe Hydrostatic Mooring System has a greater capacity to carry risers than other submerged mooring systems, increasing the range of water depths and riser diamerers.

Monitoring by Differential Ceo. Positional Satellite System (GPS) - . automatically warns you of design limit violations as well as immediately revealing any anchoring component failures, allowing you to take action before damage accurs.

\section{Environmental Responsibility}

The Hydrostatic Mooring System isolates fluid couplings from the ocean.

- The Hydrostatic Mooring System maintains a low pressure in the mooringtanker interface, allowing ocean water to flow toward the mooring preventing leaking fluids from escaping.

- The fluid swivel is located on the tanker further reducing the potential for fluid to escape.

\section{Standard Construction}

- The Hydrostatic Mooring System is comprised entirely of proven standard components, readily available from a number of different-oil industry suppliers.

- The installation of the Hydrostatic Mooring System is the same as an ordinary single point mooring. The required construction spread depends somewhat on local conditions, but typically involves crane vessels, anchor handling vessels and diving support vessels.

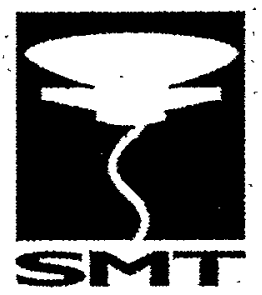

\section{Suction Mooring Technology Offshore Mooring Systems}

318 North Post Road 